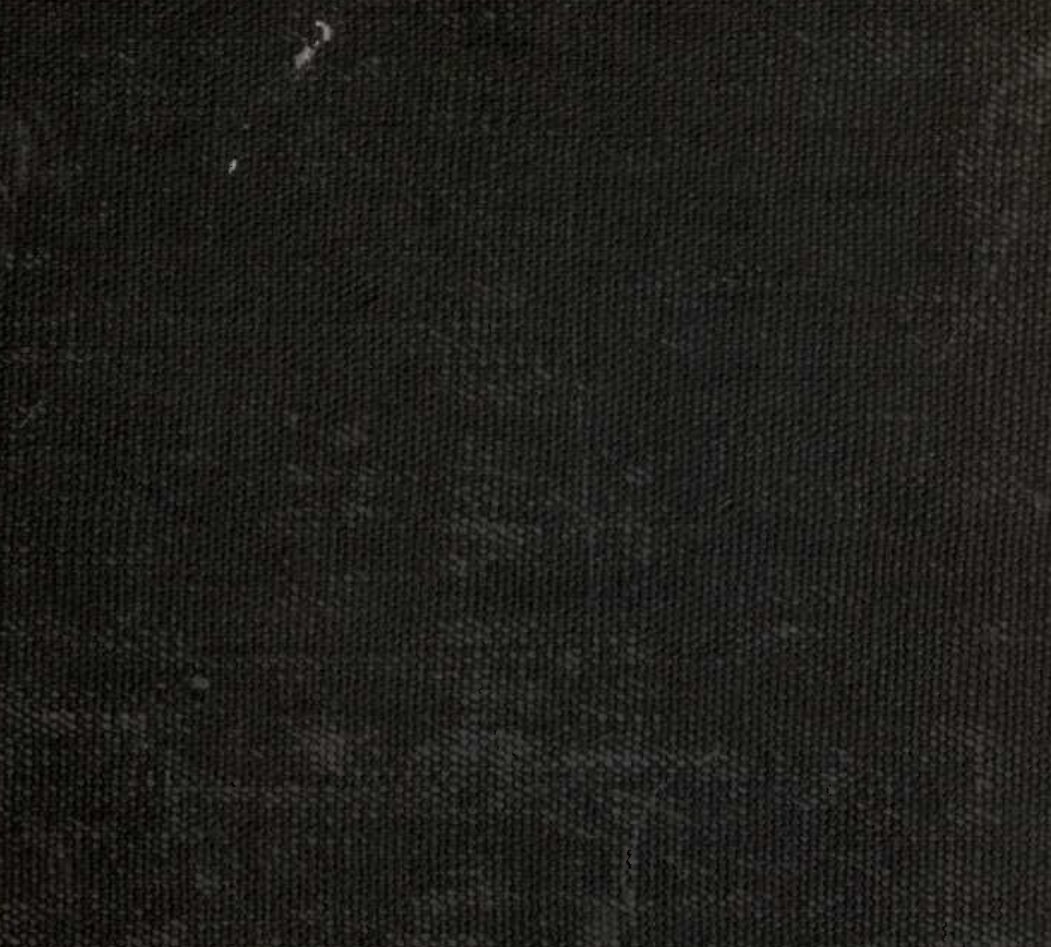

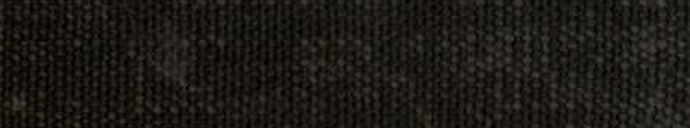
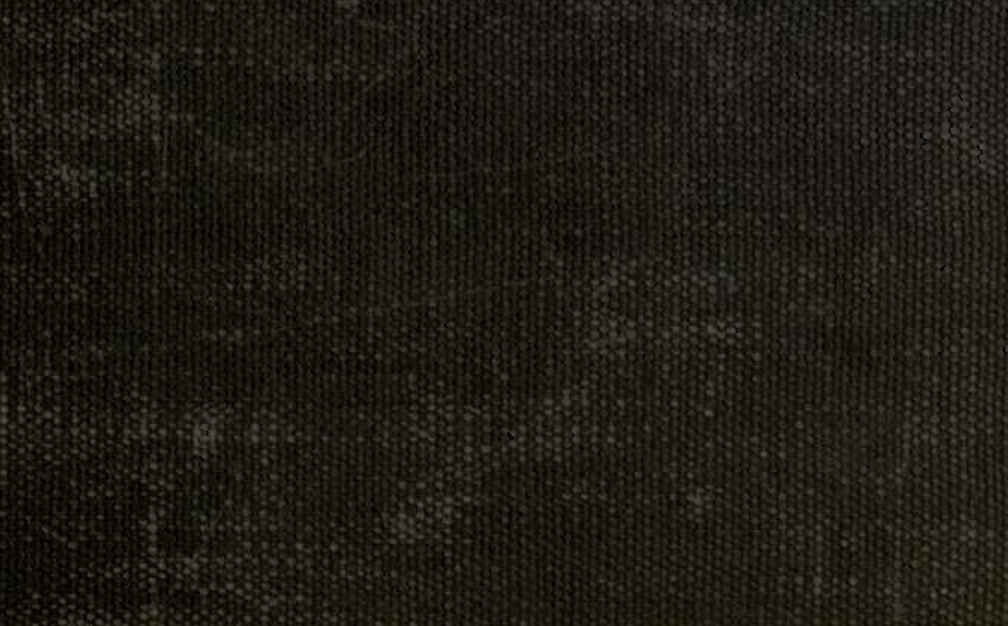

39 


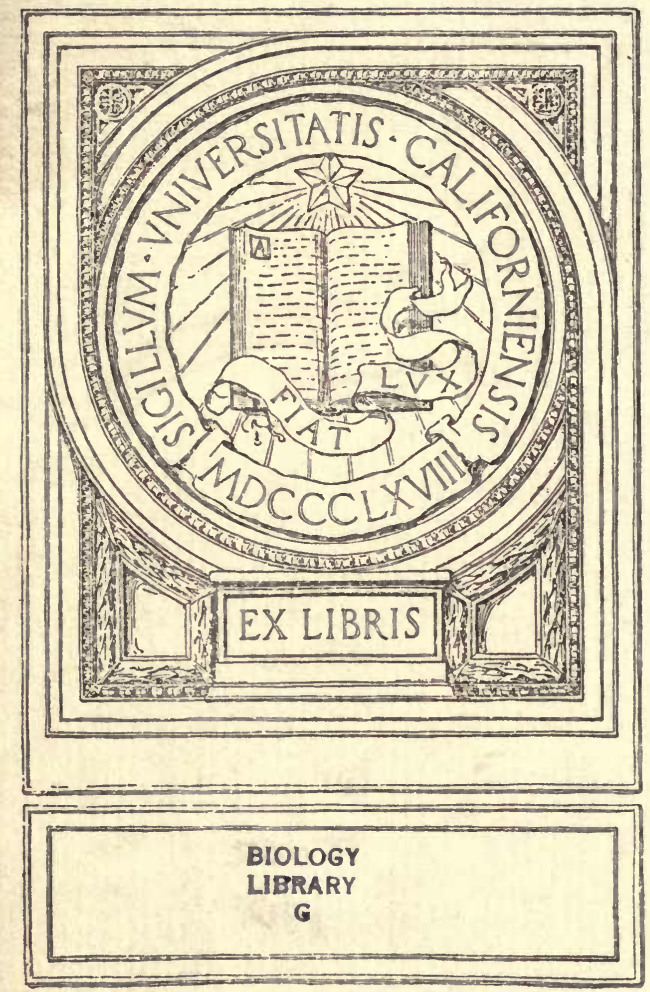

8
18
20
27 






\section{A PRACTICAL TEXT-BOOK}

\section{OF \\ INFECTION, IMMUNITY AND SPECIFIC THERAPY}

WITH SPECIAL REFERENCE TO IMMUNOLOGIC TECHNIC

BY

JOHN A. KOLMER, M.D., DR.P.H.

Instructor of Experimental Pathology, University of Pennsylvania;

Professor of Pathology and Bacteriology, Philadelphia Polyclinic, and

Pathologist to the Department of Dermatologic Research; Pathologist to the Philadelphia Hospital for Contagious Diseases

WITH AN INTRODUCTION BY

ALLEN J. SMITH, M.D., Sc.D., LL.D.

Professor of Pathology, University of Pennsylvania

WITH I43 ORIGINAL ILLUSTRATIONS, 43 IN COLORS

By ERWIN F. FABER

Instructor of Medical Drawing, University of Pennsylvania

PHILADELPHIA AND LONDON

W. B. SAUNDERS COMPANY 1915 


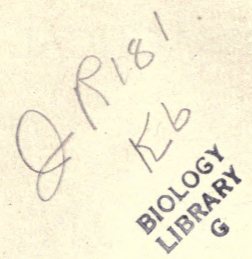

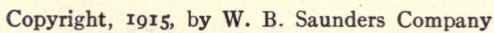

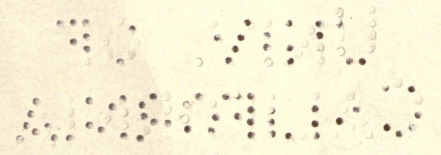

PRINTED IN AMERICA

PRESS OF

W. B. SAUNDERS COMPANY

PHILADELPHIA 
RICHARD M. PEARCE, M.D.

PROFESSOR OF RESEARCH MEDICINE IN THE UNIVERSITY OF PENNSYLVANIA

IN RECOGNITION OF HIS SERVICES TO THE SCIENCE OF MEDICINE

BY

THE AUthor 



\section{INTRODUCTION}

THE last quarter of a century has witnessed an almost marvelous development of knowledge in the domain of medicine and the allied sciences, only a part, of course, of the extensive progress made in the field of general science. A striking portion of this advance has tended to broaden our knowledge of the principles and of the essential details of the processes of infection and immunity, until these branches have today come to form almost a special science in themselves - an imperium in imperio. Aside from the personal factor, the writer's immediate interest in the present volume, as originally projected, arose from the fact that he was desirous of having appear a series of exercises illustrative of the principles of immunology - a class-book intended to set forth in permanent form the very excellent course of instruction that Dr. Kolmer has been giving during the past few years to selected groups of interested students and occasional post-graduate workers in the Medical School of the University of Pennsylvania. That it should have surpassed the original simple plan and grown into a volume of the present proportions is scarcely to be wondered at, if the temptation to elaborate the individual exercises by explanations and cognate considerations was in the slightest to be yielded to. This is due to the fact that in its growth the subject has acquired so much of undoubted importance in the form of isolated observed facts, and itself presents so many analogies and has led to so extensive a terminology, that the author who would attempt to link the observed facts into anything like logical sequence or to add in the least to the bare cook-book-like series of illustrative exercises any explanatory paragraphs, cannot avoid the fullness that Dr. Kolmer has found inevitable in presenting the subject.

The branch of immunology, including primarily infection, and its ramifications into diagnosis and the actual treatment of disease, has brought to the parent subject of preventive medicine the greatest offering of the decades of its growth. Itself contributing to world expansion, it has nowhere found a greater stimulusthan in the field of exotic pathology; and this last, in turn, has enriched internal medicine, even in its most common aspects. The first step in immunology may properly be ascribed to Jenner, with his bovine vaccine for smallpox, a step followed 
only after a long lapse of years by Pasteur. On the heels of the latter there appeared at once, and has since then followed, an army of men whose names crowd the history of the subject, and which many of these are bound permanently to adorn. The old vague theories of infection have taken form, and to observed facts has been added productive theory. The great danger attending this luxurious development is that, temporarily at least, the simpler, and perhaps the more obvious, facts are likely to be neglected; and, also, that symbolization by theories elaborated to harmonize with discovered facts will be accepted too fully as explanatory when in reality it does not explain, and that, as a result, investigation will finally be hampered instead of aided. In the almost universal drift of experimental studies to internal stereochemical factors, are we not in danger of placing too little stress upon actual and possible physical factors? Is there no danger that, by failing to lay stress upon the obvious importance of the turbinate mechanism in the nose as a natural anatomic factor, our rhinologists may at least feel justified in sacrificing this mechanism too readily for what may be but trivial local reasons? Can we insist that every phenomenon described with facility in terms of the side-chain theory is really a manifestation of chemism, when perhaps, with added investigation along lines of physical absorption and the physical properties of colloids, an equally satisfying conception may be had, and possibly new facts be developed? Are we not blundering in rushing madly after matters of specificity as determined by antigen, when perhaps in reality we are confronted by potential and kinetic modifications due to peculiarities of diet or environmental circumstances? The verity of phagocytosis is open to proof by observation, and its variation $\approx$ are likewise to be demonstrated. Is the explanation of opsonins so convincing that merely the word itself is enough to satisfy the investigator?

Infection and immunity constitute a definite chapter in pathologic science. The processes 'ack the dignity of a separate science only in that they present variations, and the fact that these are glossed over by brilliant theories and conceptions cannot prevent the deliberate recognition of serious incompleteness. Yet this criticism can be applied to the growth of every branch of scientific knowledge. It in no wise militates against the right and the need for setting forth the subject in the light that, for the time, is afforded it. The importance of the criticism lies only in its acknowledgment, lest the subject as at present understood be accepted as fixed. With this danger obviated, and with all theories accepted for the time only as working theories, and their adoption not 
urged to curtail investigations based on other views, their prosecution can be heartily applauded.

This is the view that the writer believes that Dr. Kolmer has had in mind in his presentation of the subject as here set down. It is certainly true of the chapters that the present writer has had opportunity of examining. In such a sense, therefore, the work is urged on the appreciation of the student, whether a laboratory worker or a mere seeker of knowledge.

I have often been asked to what extent I believe it profitable to present the subject to the undergraduate student. I do not hesitate to answer that so far as the roster of the medical curriculum will permit, the laboratory demonstrations and exercises should form a part of the required course; and that, with all due caution to emphasize the fact that our present theory is not known to be final, and is offered merely tentatively, the verbal picture of the subject should be outlined before these beginners. To form some conception is necessary; and it is better, provided the mind be kept receptive, to follow a certain theory, even if it is unproved, than to do nothing at all or to work in confusion. Our American medical curriculum for undergraduates is so crowded with absolute essentials that the present subject is habitually neglected, save for a rapid lecture outline; this is an injustice to the student and to American medicine. I have tried to minimize this by providing, through Dr. Kolmer's aid, a reasonable laboratory course in the essentials of the branch to volunteer classes at first, at hours that did not interfere with the regular curriculum -at present during periods open to election. Nevertheless, the subject, influencing as it does every branch of medical practice, must take its place with other commendable additions to the required schedule. That this can be done only by lengthening the course of study, either in the annual session or by adding a year to our present four-year course, is obvious, and to that end we are rapidly approaching.

Allen J. Smith. 



\section{PREFACE}

For the past twenty years the science of immunity has been one of the most progressive and most active branches in the department of medicine. An enormous literature has accumulated; many new terms have been coined, and numerous theories have been adduced; indeed, the subject has acquired an aspect of complexity that is confusing to those not specially interested or engaged in this work.

The purpose of this book is a threefold one, namely:

1. To give to practitioners and students of medicine a connected and concise account of our present knowledge regarding the manner in which the body may become infected, and the method, in turn, by which the organism serves to protect itself against infection, or strives to overcome the infection if it should occur, and also to present a practical application of this knowledge to the diagnosis, prevention, and treatment of disease.

2. To give to physicians engaged in laboratory work and special workers in this field a book to serve as a guide to the various immunologic methods.

3. To outline a laboratory course in experimental infection and immunity for students of medicine and those especially interested in these branches.

1. The subject of infection is intimately connected with that of immunity, and this is especially emphasized in those diseases for which a specific therapy exists, for a knowledge of the nature of the infection is of paramount importance in controlling the dosage and indicating the method of administration of a specific therapeutic agent. By describing principles and technic with considerable detail, a special effort has been made to render Part IV of this book of particular value to practitioners of medicine.

The day is past when the physician and surgeon can relegate the things of immunity. entirely to the laboratory. Diagnostic methods and reactions and the field of specific therapy,--vaccine, serum, and chemo-,are subjects of such practical importance that it is obvious that the physician and the student of medicine can no longer be merely mildly interested onlookers. The physician who injects salvarsan, a serum, or a vaccine, or who uses a diagnostic reaction, must be prepared to explain to his patient the nature of the therapy he employs and the sig- 
nificance of the reaction. This he can do only by equipping himself with the knowledge of the fundamental factors of immunity, or he will be forced into the position of a passive transmitter of ideas entirely beyond his own knowledge.

2. An effort has been made to include data of both practical and theoretic importance, and in some instances tests are described that are more of theoretic than of practical import, especially in research work.

It is obviously impossible, in a single volume, to include the very large number of tests and modifications that have been advocated from time to time, and, as a matter of course, most attention has been given those methods that have been shown to be of practical value or that give promise of becoming so. So far as possible original methods are given, these being, in the larger proportion, more or less important modifications devised as the result of my own experience in hospital and teaching laboratories.

The technic of the various tests and reactions is described in great detail, thus tending the better to secure accuracy, simplicity, and definiteness, and to serve as an opening wedge to those about to enter this special field.

3. The value of the experimental method in the teaching of certain branches of medicine is now well recognized. In no department, however, is this method of greater value than in the study of infection and immunity. A working knowledge of these subjects is so valuable in the practice of medicine and surgery that the student should be well versed in at least their primary principles and practical applications in the prophylaxis, diagnosis, and treatment of diseas

The laboratory course given in Part V is based upon the courses given by me in the Laboratory of Experimental Pathology at the University of Pennsylvania, and in the laboratories of the Philadelphia Polyclinic and College for Graduates in Medicine. In including them in this volume I am carrying out my original plan, for in many of the experiments the exact technic of a given test is described, making a separate book devoted to this part of the subject unnecessary. Future experience may, however, show the necessity of having this portion of the book form a separate laboratory manual. I shall appreciate the opinions of educators who may have occasion to consult the course herein outlined.

Since the larger portion of our knowledge of infection and immunity has been gained from studies upon the lower animals, it is not strange that these were early and directly benefited by a practical application 
of this knowledge to the prophylaxis, diagnosis, and treatment of many of the diseases to which these animals are subject. I have, therefore, included in this volume an account of those immunologic diagnostic reactions and applications of specific therapy that have a direct bearing upon veterinary medicine.

No attempt has been made to cover all literature references on the subject. An effort has been made to state well-established facts concisely, and, in the case of the more recent subjects, to give the principal references to the literature. I have drawn largely from German; French, and English sources, and have endeavored, wherever possible, to give proper and due credit to each author. In order to keep the work up to the times, I would ask the authors of reprints on immunologic subjects to send me copies.

The illustrations, all of which have been made by Mr. Erwin F. Faber, will, it is hoped, serve the purpose for which they are intended, namely, to elucidate the text and to teach, rather than merely to embellish.

It is with deep and sincere appreciation that I acknowledge the encouragement and aid given me by Professor Allen J. Smith, who has written the introduction and reviewed several chapters. My thanks are also due to Professor Richard M. Pearce for reviewing the chapters on Infection; to my assistant, Dr. Anna M. Raiziss, for a number of translations from foreign literature, and to the publishers, whose kind and unvarying courtesy has greatly simplified the work.

J. A. K.

McManes Laboratory of Experimental Pathology,

University of Pennsylvania, January, 1915. 


\section{CONTENTS}

PART I

GENERAL IMMUNOLOGIC TECHNIC

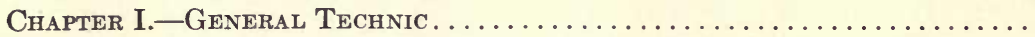

Care of centrifuge, 17-Making a simple capillary pipet, 18-Making looped pipets, 20-Graduated pipets, 21-Making Wright blood-capsules, 23Making vaccine ampules, 24-Preparation of test-tubes for immunologic work, 25-Selection of a satisfactory syringe, 26-Solutions, 27.

Chapter II.-Methods of Obtaining Human and Animal Blood..........

Obtaining corpuscles, 28-Washing erythrocytes, 28-Obtaining serum, 30 -Obtaining corpuscles and serum, 30-Obtaining blood plasma, 30-Obtaining small amounts of human blood, 32-Obtaining larger amounts of human blood (phlebotomy, wet-cupping, placental blood), 33-Obtaining cerebrospinal fluid (technic of spinal puncture), 37-Obtaining small amounts of animal blood (rabbit, guinea-pig, sheep), 41-Obtaining large amounts of animal blood (rabbit, guinea-pig, rat, sheep, hog, monkey, dog, and horse), 42.

Chapter III.-Technic of Antmal Inoculation. . . . . . . . . . . . . . .

General rules, 53-Method of subcutaneous inoculation (fluid and solid inocula), 54-Method of intramuscular inoculation, 56-Methods of intravenous inoculation (rabbit, guinea-pig, mice and rats, horse, sheep, goat, dog), 56Method of intracardial inoculation, 62-Methods of intraperitoneal inoculation (rabbit, guinea-pig), 64 .

Chapter IV.-Methods for Fffecting Active Immunization of Animals. .

Antigens and active immunization, 65-General technic, 66-Production of antitoxins, 68-Production of agglutinins (intravenous and intraperitoneal inoculation), 68-Production of immune opsonins, 69-Production of bacteriolysins, 69-Production of precipitins, 70-Production of hemolysins (intravenous and intraperitoneal inoculations), 71-Production of cytotoxins, 73.

Chapter V.-Preservation of Serums-Methods..................

Methods for the preservation of normal serums, 75-Methods for the preservation of immune serum in fluid form with antiseptics, 76-In fluid form by bacteria-free filtrations, 76-In fluid form by freezing, 78-Preservation in powder form, 79-Preservation in dried paper form, 79 .

\section{PART II}

\section{PRINCIPLES OF INFECTION}

Chapter VI.-Infection. . . . . . . . . . . . . . . . . . . . . .

Definition, 81-Relation of infection to immunity, 83-Source of infection, 83-Contagious and infectious diseases, 84-Exogenous and endogenous infection, 85-Avenues of infection, 86-Normal defenses against bacterial invasion, 90-Mechanism of bacterial invasion, 91-Mechanism of infection, 94The avenue of infection and tissue susceptibility, 97-The numeric relationship of bacteria to infection, 99-General susceptibility in relation to infection, 99The defensive mechanism of the microörganism in relation to infection, 102Mixed infection, 105-Summary, 106. 
Chapter VII.-Infection (continued). Production of Disease.........

Toxins, 108-Extracellular bacterial toxins, 109-General properties of soluble toxins, 109 -Structure of soluble toxins, 110-Nature of soluble toxins, 110-Selective action of soluble toxins, 111. Special Properties of the Principal Soluble Toxins, 112-Diphtheria toxin, 112-The guinea-pig test for virulence of diphtheria bacilli, 113-Tetanus toxin, 115-Botulism toxin, 116-Dysentery toxin, 116-Staphylotoxin, 117-Streptotoxin, 117. Toxins of the Higher Plants and Animals, 118-Phytotoxins, 118-Pollen toxin, 119-Zoötoxins, 119 - Snake venom, 119. Endotoxins, 120-Methods of studying endotoxins, 121Nature of endotoxins, 121-Aggressins, 122-Bail's classification of bacteria, 124-Nature of aggressins, 124-Anti-aggressins, 126. Bacterial Proteins, 126Bacterial split protein, 126-Nature of bacterial proteins, 127-Action of bacterial proteins, 127-Theory of Vaughan, 128. Ptomains, 129. Mechanical Action of Bacteria, 131-Infection with Animal Parasites, 132-The Course of Infection, 134-Stages of infection, 134-Grades of infection, 136-Systemic reaction to infection, 137 .

\section{PART III}

PRINCIPLES OF IMMUNITY AND SPECIAL IMMUNOLOGIC TECHNIC

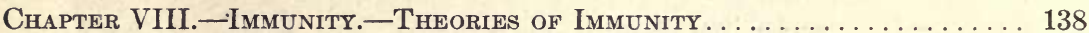

Definition, 140 -Historic, 140-Exhaustion theory of immunity, 141Retention theory of immunity, 144-Theory of phagocytosis, 144-Side-chain theory of immunity, 146-Compatibility of the phagocytic and side-chain theories, 155-Antigens, 159-Antibodies, 161.

Chapter IX.-Various Types of Immunity. .

Natural Immunity, 165-Explanation of natural immunity, 166-Nonspecific immunity, 166-Local immunity, 167-Phagocytosis and natural immunity, 168-Natural antitoxic immunity, 169-Natural bacteriolytic immunity, 169-Natural anti-aggressin immunity, 169-Athreptic immunity, 170. Acquired Immunity, 170-Active acquired immunity, 170-Passive acquired immunity, 172.

Chapter X.-Phagocttosis . . . . . . . . . . . . . . . . . . .

Historic, 175-The original theory of phagocytosis, 176-Kinds of phagocytosis, 177-The relation of the cell types to infection, 178-Chemotaxis, $179-$ Positive chemotaxis, 179-Negative chemotaxis, 182-Results of phagocytosis, 182-The relation of body fluids to phagocytosis, 184-Revised theory of phagocytosis, 186.

Chapter XI.-Opsonins. . . . . . . . . . . . . . . . . . . . .

Historic, 187-Definition, 188-Properties and nature of opsonins, 188-Susceptibility to opsonification, 189-Effect of opsonins on bacteria, 189-Rôle of opsonins in immunity, 190.

Chapter XII.-Opsonic Index. . . . . . . . . . . . . . . . . . . .

Principles involved, 191-Definition, 191-Purpose of the opsonic index, 192-Limitations of the method, 192-Precautions in technic, 193-Technic of the opsonic index (Wright), 193-Technic of quantitative estimation of bacteriotropins in immune serum (Neufeld), 200-Practical value of the opsonic index, 203 - Value of the index in diagnosis, 204-In prognosis, 204-As a guide to bacterial vaccine therapy, 205 .

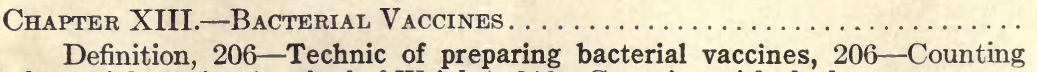
a bacterial vaccine (method of Wright), 210 -Counting with the hemocytometer chamber, 211-Method of Kolle, 212-Method of Hopkins, 212-Preparation of "sensitized" bacterial vaccine, 216-The administration of a bacterial vaccine,. 217-Making the inoculation, 217-Effects of inoculation, 218-Frequency and dosage of inoculation, 218-Ordinary adult doses of the common vaccines, 218. 
Chapter XIV.-Antitoxins ............................. 220

Definition, 220-Historic, 220-Formation of antitoxins, 221-Structure of antitoxins, 223-Properties of antitoxins, 223-Natural antitoxins, 224-Specificity of antitoxins, 224-Nature of the toxin-antitoxin reaction, 224. Production of Diphtheria Antitoxin, 227-Production of diphtheria toxin, 277-Testing the toxin, 228-Immunizing the animals, 228-Collecting the serum, 230-Standardizing the serum, 231. Production of Tetanus Antitoxin, 234-Tetanus toxin, 234-Immunizing the animals, 234-Collecting the serum, 234-Standardizing the serum, 234. Botulinus Antitoxin, 236. Antidysentery Serum, 236The culture, 236-Immunizing the animals, 237-Collecting and testing the serum, 238. Antistaphylococcus Serum, 238-Preparation, 239-Technic of the anti-lysin test, 239. Production of Antivenin, 241. Production of Pollen Antitoxin, 242. The Measure of Antitoxins, 242-A unit, 242-Unit of diphtheria antitoxin, 242-Unit of tetanus antitoxin, 242.

Chapter XV.-Ferments and Anti-Ferments.

Bacterial ferments, 244 - Similarity between toxins and ferments, 244 -Antiferments, 246-Antibodies and antiferments, 247-Antiferments in disease, 247Ferments in pregnancy and disease, 248. Ferment Reactions, 250-Antitrypsin test, 250-Abderhalden's serodiagnosis of pregnancy, 252-Practical value of Abderhalden's test, 263-Sero-enzymes in cancer, 264-In mental diseases, 265 -In syphilis, 265-In tuberculosis and acute infection, 265.

Chapter XVI.-Agglutinins. .

Definition, 266-Historic, 266-Normal and immune agglutinins, 268Formation of agglutinins, 268-Origin of agglutinins, 269-Properties and nature of agglutinins, 270-Mechanism of agglutination, 270-Specificity of agglutinins, 271-Absorption methods for differentiating between a mixed and single infection, 272-Hemagglutinins, 272-Non-agglutinable races of bacteria, 274 -Variation in agglutinating strength of a serum, 274-Rôle of agglutinins in immunity, 274. Practical Applications, 275-In the diagnosis of typhoid fever, 275-Paratyphoid fever, 275-Dysentery, 276-Cholera, 277-Cerebrospinal meningitis, 277-Plague, 277-Malta fever, 277-Glanders, 277-In the differentiation of bacteria, 277-In the diagnosis of single and mixed infection, 278. The Agglutination Reaction, 278-Microscopic method with serum, 282-Microscopic method with dried blood, 283-Macroscopic method, 284-The saturation test of Castellani, 288. Tests before Blood Transfusion for Isohemagglutinins and Isohemolysins, 290.

Chapter XVII.-Precipitins.

Definition, 292-Historic, 292-Nomenclature, 294-Structure and proportion of precipitins, 294-Formation of precipitins, 294-Mechanism of precipitation, 296-Specificity of precipitins, 296-Rôle of precipitins in immunity, 297. Practical Applications, 298-Bacterial precipitins, 298- Fornet ring test, 299 Porges-Meier reaction, 299-Herman-Perutz reaction, 299-Noguchi globulin reaction, 300-Differentiation of proteins, 301. Technic of the Precipitin Reactions, 303-Differentiation of human and animal bloods, 303-Detection of meat adulteration, 310-Bacterial precipitins, 313-Precipitin test in cancer, 314.

Chapter XVIII.-Cytolysins. Amboceptors and Complements..........

Definition, 317-Kinds of cytolysins, 318-Nomenclature, 318. Amboceptors, 319-Historic, 319-Structure of amboceptors, 320-General properties of amboceptors, 321-Mechanism of the action of amboceptors, 321-Formation of amboceptors, 322-Quantitative estimation of amboceptors, 325-Titration of hemolytic amboceptor, 325-Titration of bacteriolytic amboceptor, 325 . Complements, 326-Historic, 326-Definition, 326-Structure and general properties of complement, 326-Anticomplements, 327-Origin of complements, 328 -Multiplicity of complements, 328-Endocomplements, 330-Complementsplitting, 331-Complement fixation, 332-Complement deviation, 333-Quantitative titration of complement, 334 .

Chapter XIX.-Bacteriolysins.

Historic, 336-Definition, 337-Origin of bacteriolysins, 338-Leukins and leukocytic extracts, 338-Method of preparing leukocytic extracts, 339-Mechanism of bacteriolysis, 340 - General properties of bacteriolysins, 341 -Normal bac- 
teriolysins, 341-Specificity of bacteriolysins, 341. Practical Applications, 341Technic of the Pfeiffer test, 342-Bacteriolytic test in vivo for the identification of bacteria, 343-Bacteriolytic test in vivo in the diagnosis of disease, 348-Bacteriolytic test in vitro (method of Stern and Körte), 349-Bacteriolytic test in vitro (method of Wright), 335.

Chapter XX.-Hemolysins

Historic, 361-Definition, 362-Nomenclature, 363-Nature of hemolysins, 363-Analogy between bacteriolysis and hemolysis, 367-Specificity of hemolysins, 368-Normal hemolysins, 368-Production of immune hemolysins, 371General properties of hemolysins, 371-Source of hemolysins, 372. Practical Applications, 373-Quantitative reactions between hemolytic amboceptor and complement, 374-Method of titration of hemolysin, 375-Method for removing hemolysin from a serum, 378-Method of determining natural hemolysins in serum, 378-The serum diagnosis of paroxysmal hemoglobinuria, 379-Method of determining the resistance of red blood-corpuscles, 380 .

Chapter XXI.-Venom Hemolysis.

Historic-nature of venom hemolysis, 383-Venom hemolysis in syphilis, 385-Practical value of the venom test in syphilis, 388-The psycho-reaction of Much in syphilis, 388-Venom hemolysis in tuberculosis, 390-Venom hemolysis in cancer, 390 .

Chapter XXII.-Principles of the Phenomenon of Complement Fixation

Historic, 391-The original complement-fixation method of Bordet, 394Mechanism of complement fixation, 395-Non-specific complement fixation, 396-Quantitative factors in complement-fixation tests, 397-Practical applications, 399 .

Chapter XXIII.-The Technic of Complement-fixation Reactions..... .

The Wassermann Syphilis Reaction, 401-Historic, 401-Principles and theories of the syphilis reaction, 404-General technic, 404-Preparation of the fluid to be tested, 408-Preparation and titration of complement, 411-Preparation and titration of hemolytic amboceptor, 415-Preparation of blood-corpuscles, 416-Preparation and standardization of antigens, 417-Technic of the First Method, 435-Technic of the Second Method, 441-Technic of the Third Method, 443-Technic of the Fourth Method, 446. Modifications of the Wassermann Reaction, 449-Technic of the Noguchi modification, 449-Technic of the Hecht-Weinburg modification, 457-Other modifications, 458. The Wassermann Reaction in the Various Stages of Syphilis, 459-The specificity of the Wassermann reaction, 465-The effect of treatment upon the Wassermann reaction, 466-The Practical Value of the Wassermann reaction, 469.

Chapter XXIV.-Complement-fixation Reactions (continued).........

Specific complement fixation in bacterial diseases, 473-Preparation of bacterial antigens, 473-Standardizing bacterial antigens, 475-Principles of complement fixation with bacterial antigens, 476. Complement Fixation in Gonococcus Infections, 477. Complement Fixation in Glanders, 484 . Complement Fixation in Contagious Abortion, 486. Complement Fixation in Dourine, 487. Complement Fixation in Typhoid Fever, 489. Complement Fixation in Tuberculosis, 490. Complement Fixation in the Standardization of Immune Serums, 491. Complement Fixation in Echinococcus Disease, 492. Complement Fixation in the Differentiation of Proteins (blood-stains, meats, bacteria), 494. Complement Fixation in Cancer, 499.

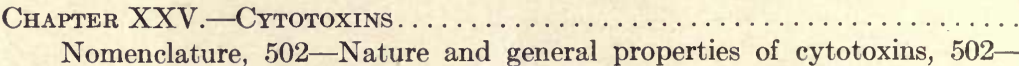
Preparation of cytotoxins, 503-Methods of studying cytotoxins, 503-Specificity of cytotoxins, 504-Autocytotoxins, 505-Isocytotoxins, 506-Anticytotoxic serums, 506-Kinds of cytotoxins, 506-Spermatotoxin, 506-Epitheliotoxin, 506-Leukotoxin, 506-Nephrotoxin, 506-Hepatotoxin, 507-Gastrotoxin, 507-Syncytotoxin, 507-Neurotoxin, 508-Thyrotoxin, 508-Rôle of cytotoxins in immunity, 508-Practical applications, 508-Cytotoxic cancer reaction, 509 . 
Chapter XXVI.-The Relation of Colloids and Lipoids to Immunity. . . 511

Kinds of colloids, 511-Nature and properties of colloids, 511-Analogy between the reactions of immunity and those of colloidal chemistry, 515-Antitoxins, 516-Agglutinins and precipitins, 517-Hemolysins, 518-Complement fixation as a colloid reaction, 519-The relation of lipoids to immunity, 520The epiphanin reaction, 522-The miostagmin reaction, 526.

Chapter XXVII.-ANaphylaxis. .

Historic, 532-Definition, 535-Phenomena of anaphylaxis, 536-Mechanism of anaphylaxis, 541-Anaphylactogens or allergens, 543-Anaphylatoxin, 548-Anaphylactin (allergin), 552-Theories of anaphylaxis, 556-Passive anaphylaxis, 559-Anti-anaphvlaxis, 561-Specificity of anaphylaxis, 563.

\section{PART IV}

APPLIED IMMUNITY IN THE PROPHYLAXIS, DIAGNOSIS, AND TREATMENT OF DISEASE-SPECIFIC THERAPY

Chapter XXVIII.-Anaphylaxis in Its Relation to Infection and ImmUNity. ANAPhylactic or Allergic Reactions................ 565

Relation of anaphylaxis to infectious diseases, 566-Relation of anaphylaxis to non-infectious diseases, 570-Serum disease, 571-Idiosyncrasies, 577Relation of anaphylaxis to immunity, 580-Anaphylactic or allergic reactions, 582-Subcutaneous tuberculin reaction, 592-Intracutaneous tuberculin reaction, 595-Cutaneous tuberculin reaction, 596-Conjunctival tuberculin reaction, 598-Percutaneous tuberculin reaction, 599-Tuberculin reactions among the lower animals, 600 -The luetin reaction, 601 - The mallein reaction, 606-Allergic reactions in typhoid fever, 607-Allergic reactions in other diseases, 609 -Allergic reactions as a measure of immunity, 610 .

Chapter XXIX.-Active Immunization. Vaccines in the Prophylaxis and Treatment of Disease. Vaccine Therapy..............

Historic, 611-Nomenclature, 613-Method of preparing vaccines, 614Mechanism of active immunization, 616-Living versus dead vaccines, 620 Sensitized vaccines, 620 -Autogenous versus stock bacterial vaccines, 620 - The negative phase, 621-Contraindications to active immunization, 622. Prophylactic Immunization or Vaccination, 623-In small-pox, 623-In rabies, 636-In typhoid fever, 643-In plague, 647-In cholera, 650-In dysentery, 652-In cerebrospinal meningitis, 652 - In scarlet fever, 652-In anthrax, 653-In black-leg, 655. Therapeutic Immunization. Bacterial Vaccine Therapy, 655Principles, 655-Diseases of the skin, 656-Diseases of the genito-urinary system, 657 - Diseases of the respiratory system, 659-Acute general infections, 660 Tuberculin therapy, 661 .

Chapter XXX.-Passive Immunization. Serums in the Prophylaxis and

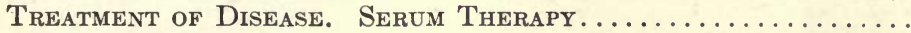

Definition, 681-Purposes of passive immunization, $682-$ Kinds of passive immunity, 683-Indications for passive immunization, 684-Contraindications to passive immunization, 686-Technic of subcutaneous inoculation, 688Technic of intramuscular inoculation, 690-Technic of intravenous inoculation, 690-Technic of subdural inoculation, 694. Serum Treatment and Prophylaxis of Diphtheria, 702. Serum Treatment and Prophylaxis of Tetanus, 719. Serum Treatment and Prophylaxis of Dysentery, 730. Serum Treatment and Prophylaxis of Hog Cholera, 732. Serum Treatment of Snake Bites, 733. Serum Treatment and Prophylaxis of Hay-fever, 734. Serum Treatment and Prophylaxis of Meningococcus Meningitis, 736. Serum Treatment of Influenzal Meningitis, 749. Serum Treatment of Pneumococcus Meningitis, 751. Serum Treatment of Other Localized Pneumococcus Infections, 754. Serum Treatment of Lobar Pneumonia, 754. Serum Treatment of Streptococcus Infections, 760. Serum Treatment of Gonococcus Infections, 765. Serum Treatment of Staphylococcus Infections, 766. Serum Treatment of Anthrax, 767. Serum -Treatment of Typhoid Fever, 768. Serum Treatment of Plague, 769. Serum Treatment of Cholera, 770. Serum Treatment of Tuberculosis, 771. 
Chapter XXXI.-Serum Therapy (continued)

Normal Serum Therapy, 772-Serum treatment of hemorrhage, 772-Serum treatment of the toxicoses of pregnancy, 773-Serum treatment of skin diseases, 774. Autoserum Therapy, 775-Autoserum treatment of skin diseases, 775Autoserum treatment of acute infectious diseases, 775-Autoserum treatment of syphilis (salvarsanized serum), 776-Autoserum treatment of tuberculosis of serous membranes, 781-Autoserum treatment of non-tuberculous effusions, 782.

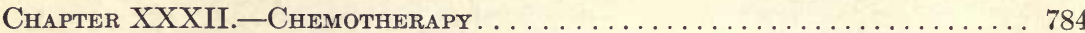

Principles of Chemotherapy, 785-Organotropism and parasitotropism, 785 -Chemoreceptors, 787-Drug "fastness," 789-Therapia magna sterilisans, 791. Salvarsan and Neosalvarsan in the Treatment of Syphilis, 792-Historic, 792Properties of salvarsan, 794-Properties of neosalvarsan, 795-Methods for preparing salvarsan for administration, 795-Methods for preparing neosalvarsan for administration, 795-Administration of salvarsan and neosalvarsan by intravenous injection, 797-By intramuscular injection, 805-By intraspinous injection, 806-Contraindications and precautions in salvarsan therapy, 808Value of salvarsan and neosalvarsan in the treatment of syphilis, 809. Salvarsan in the Treatment of Non-syphilitic Diseases, 810. Chemotherapy in Bacterial Diseases, 811. Chemotherapy in Malignant Diseases, 812.

\section{PART V}

\section{EXPERIMENTAL INFECTION AND IMMUNITY}

Introductory, 814-Methods, 814-The Student, 814-Records, 815-Animal Experiments and Autopsies, 815.

Exercise 1.-Active Immunization of Animals, 815.

Experiment 1, Production of Agglutinins, Bacteriolysins and Opsonins, 815-

Experiment 2, Production of Precipitins, 816-Experiment 3, Production of Hemolysins, 816-Experiment 4, Production of Cytotoxin, 816.

Exercise 2.--Infection, 816.

Experiment 5, Experimental Pneumonia, 816.

Exercise 3.-Toxins, 817.

Experiment 6, Diphtheria Toxin, 817-Experiment 7, Method of Testing the Virulence and Toxicity of Diphtheria Bacilli, 818-Experiment 8, Tetanus Toxin (in vivo), 819 -Experiment 9, Tetanus Toxin (in vitro), 819.

Exercise 4.-Toxins (continued), 820.

Experiment 10, Botulism Toxin, 820-Experiment 11, Dysentery Toxin, 820Experiment 12, Staphylotoxin (in vivo), 821-Experiment 13, Staphylotoxin (in vitro), 821.

Exercise 5.-Toxins (continued) Plant and Animal Toxins, 822.

Experiment 14, Streptotoxin, 822-Experiment 15, Phytotoxins (in vivo and in vitro), 822-Experiment 16, Zoötoxin, Cobra venom (in vivo and in vitro), 823.

Exercise 6.-Endotoxins and Aggressins, 823.

Experiment 17, Endotoxins, 823-Experiment 18, Natural Aggressins, 824.

Exercise 7.-Bacterial Protein. Ptomains. Mechanical Action of Bacteria, 824. Experiment 19, Bacterial Protein, 824-Experiment 20, Ptomains, 825-Experiment 21, Mechanical Action of Bacteria, 826.

Exercise 8.-Kinds of Immunity. Natural Immunity, 826.

Experiment 22, Phagocytosis in Natural Immunity, 826-Experiment 23, Natural Antibacterial Immunity, 826-Experiment 24, Relative Factors in Natural Immunity, 827-Experiment 25, Influence of Temperature Upon Natural Immunity, 827.

Exercise 9.-Acquired Immunity, 827.

Experiment 26, Acquired Active (Antibacterial) Immunity, 827-Experiment 27, Acquired Passive (Antitoxic) Immunity, 828-Experiment 28, Acquired Passive (Antitoxic) Immunity, 828-Experiment 29, Acquired Passive (Anti-. bacterial) Immunity, 828 . 
Exercise 10.-Phagocytosis, 829.

Experiment 30, Phagocytosis (macrophages), 829-Experiment 31, Phagocytosis (microphages), 829-Experiment 32, Phagocytosis, 830.

Exercise 11.-Phagocytosis. Chemotaxis, 830.

Experiment 33, Positive Chemotaxis, 830-Experiment 34, Negative Chemotaxis, 830.

Exercise 12.-Opsonins, 831.

Experiment 35, Normal Opsonins, 831-Experiment 36, Immune Opsonin (Bacteriotropin), 832-Experiment 37, Hemopsonin, 832.

ExerCise 13.-Opsonins (continued), 832.

Experiment 38, Mechanism of Action of Opsonins, 832-Experiment 39, Specificity of Opsonins, 833.

Exercise 14.-Opsonic Index, 834.

Experiment 40, Determining the Opsonic Index, 834-Experiment 41, Quantitative Estimation of Bacteriotropins, 834.

Exercise 15.-Bacterial Vaccines, 834.

Experiment 42, Preparation of Typhoid Vaccine, 834-Experiment 43, Preparation of Staphylococcus Vaccine, 835.

Exercise 16.-Antitoxins, 835.

Experiment 44, Standardizing Diphtheria Antitoxin, 835-Experiment 45, Standardizing Tetanus Antitoxin, 836.

Exercise 17.-Antitoxins (continued), 836.

Experiment 46, Specificity of Antitoxins, 836-Experiment 47, Nature of the Toxin-Antitoxin Reaction. Action of Anti-tetanolysin, 836-Experiment 48, Antistaphylolysin, 837.

Exercise 18.-Ferments and Antiferments, 838.

Experiment 49, Tryptic Ferment of Leukocytes, 838-Experiment 50, Testing the Antitryptic Power of Blood-serum, 838.

Exercise 19.-Ferments (continued), 839.

Experiment 51, Abderhalden Sero-enzyme Reaction in Pregnancy, 839.

ExerCise 20.-Agglutinins, 840.

Experiment 52, Gruber-Widal Reaction in Typhoid Fever ("Wet" Method), 840 -Experiment 53, Gruber-Widal Reaction in Typhoid Fever ("Dry" Method), 840.

Exercise 21.-Agglutinins (continued), 841.

Experiment 54, Macroscopic Agglutination Reaction, 841-Experiment 55, Macroscopic Agglutination Reaction (Kolle), 842-Experiment 56, Macroscopic Agglutination Reaction (Killed Cultures), 842.

Exercise 22.-Agglutinins (continued), 842.

Experiment 57, Group Agglutination, 842-Experiment 58, Pro-agglutination (Agglutinoids), 843-Experiment 59, The Absorption or Saturation Agglutination Reaction, 843.

Exercise 23.-Agglutinins (continued), 843.

Experiment 60, Hemagglutinins, 843-Experiment 61, Blood Transfusion Tests, 844.

Exercise 24.-Precipitins, 844.

Experiment 62, Titration of a Precipitin Serum, 844-Experiment 63, Titration of a Precipitin Serum, 844-Experiment 64, Specificity of Precipitins, 844.

Exercise 25.-Precipitins, 845.

Experiment 65, Forensic Blood Test, 845.

Exercise 26.-Precipitins, 846.

Experiment 66, Lactoserums, 846-Experiment 67, Bacterial Precipitins, 846Experiment 68, Noguchi Globulin Reaction, 846.

Exercise 27.-Amboceptors and Complements. Hemolysins, 847.

Experiment 69, Non-specific Hemolysins, 847-Experiment 70, Serum Hemolysis in vitro, 848-Experiment 71, Serum Hemolysis in vivo, 848.

Exercise 28.-Amboceptor and Complements. Hemolysins, 848.

Experiment 72, Titration of a Hemolytic Amboceptor, 848-Experiment 73, Quantitative Factors in Serum Hemolysis, 849. 
Exercise 29.-Amboceptors and Complements. Hemolysins, 850 .

Experiment 74, Rôle of Amboceptor and Complement in Hemolysis, 850-Experiment 75, Specificity of Amboceptors, 850-Experiment 76, General Properties of Amboceptors, 851.

Exercise 30.-Amboceptors and Complements. Hemolysins, 851.

Experiment 77, Mechanism of Amboceptor Action, 851-Experiment 78, A Further Study of the Mechanism of Amboceptors, 851.

Exercise 31.-Amboceptors and Complements. Hemolysins, 852.

Experiment 79, Natural Hemolysins. Removal of Natural Hemolysins, 852.

Exercise 32.-Amboceptors and Complements, 853.

Experiment 80.-Hemolytic Complement, 853-Experiment 81, Inactivation and Reactivation of Complement, 853-Experiment 82, General Properties of Complement, 854 .

Exercise 33.-Amboceptors and Complements, 854.

Experiment 83, Titration of Hemolytic Complement, 854-Experiment 84, Phenomenon of Complement Fixation, 855.

Exercise 34.-Antigens for the Wassermann Reaction, 856.

Experiment 85, Preparation of Antigens for the Wassermann Reaction, 856.

Exercise 35.-Antigens, 856.

Experiment 86, Method of Titration of Antigens, 856.

Exercise 36.-Wassermann Reaction, 857.

Experiment 87, Anticomplementary Action of Serums, 857.

Exercise 37.-Wassermann Reaction, 857.

Experiment 88, Wassermann Reaction (First Method), 857.

ExerCISE 38.-Wassermann Reaction, 858.

Experiment 89, Wassermann Reaction (Second Method), 858.

Exercise 39.-Wassermann Reaction, 858.

Experiment 90, Wassermann Reaction (Third Method), 858.

Exercise 40.-Wassermann Reaction, 858.

Experiment 91, Wassermann Reaction (Fourth Method), 858.

ExerCise 41.-Noguchi Modification of the Wassermann Reaction, 859.

Experiment 92, Titration of Antihuman Hemolysin, 859-Experiment 93, Technic of the Noguchi Modification, 859.

Exercise 42.-Wassermann and Noguchi Reactions, 859.

Experiment 94, Comparison of Methods, 859.

Exercise 43.-Gonococcus Complement-fixation Reaction, 860.

Experiment 95, Titration of Gonococcus Antigen, 860.

Exercise 44.-Gonococcus Complement-fixation Reaction, 860.

Experiment 96, Technic of the Gonococcus Reaction, 860.

Exercise 45.-Gonococcus Complement-fixation Reaction, 860.

Experiment 97, Technic of the Gonococcus Reaction, 860.

Exercise 46.-Complement Fixation in the Differentiation of Proteins, 861.

Experiment 98, Titration of Immune Serums, 861.

Exercise 47.-Complement Fixation in the Differentiation of Proteins, 861.

Experiment 99, Technic of the Forensic Blood Test, 861.

Exercise 48.-Venom Hemolysis, 861.

Experiment 100, Venom Hemolysis in Syphilis, 861.

Exercise 49.-Bacteriolysis, 862.

Experiment 101, Pfeiffer Bacteriolytic Test, 862.

EXERCise 50.-Bacteriolysis, 862.

Experiment 102, Microscopic Method of Measuring the Bacteriolytic Power of Blood, 862.

Exercise 51.-Bacteriolysis, 863.

Experiment 103, Method of Measuring the Bacteriolytic Activity of the Blood in vitro (Method of Stern and Körte), 863.

Exercise 52.-Cytotoxins, 863.

Experiment 104, Action of Nephrotoxin, 863. 
Exercise 53.-Miostagmin Reaction, 864.

Experiment 105, Technic of the Miostagmin Reaction, 864.

Exercise 54.-Anaphylaxis, 864.

Experiment 106, Anaphylaxis in the Guinea-pig. Specificity of Anaphylaxis, 864.

Exercise 55.-Anaphylaxis, 865.

Experiment 107, Nature of the Anaphylatoxin, 865.

Exercise 56.-Anaphylaxis, 865.

Experiment 108, Anaphylaxis in the Dog, 865.

Exercise 57.-Anaphylaxis, 866.

Experiment 109, Passive Anaphylaxis, 866.

EXERCise 58.-Anaphylaxis, 866.

Experiment 110, Anti-anaphylaxis, 866.

Exercise 59.-Anaphylaxis, 867.

Experiment 111, Local Anaphylactic Reactions, 867.

Exercise 60.-Chemotherapy, 867.

Experiment 112, Salvarsan, 867.

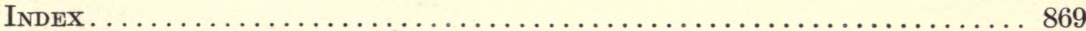





\section{LIST OF ILLUSTRATIONS}

FIG.

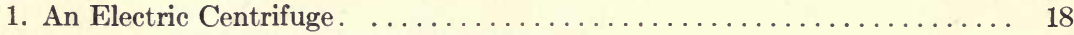

2. Method of Making a Simple Capillary Pipet................. 20

3. Method of Making a Looped Pipet. . . . . . . . . . . . . . . . . . . . 21

4. Graduated Pipets. ............................. 22

5. Method of Making a Wright Blood Capsule................. 23

6. Method of Making a Vaccine Ampule of Glass Tubing. . . . . . . . . . . . 24

7. Method of Making a Large Vaccine Ampule of a Test-tube . . . . . . . 25

8. A Satisfactory Syringe. . . . . . . . . . . . . . . . . . 26

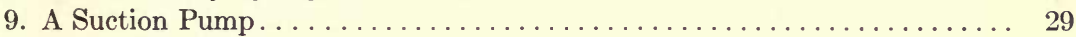

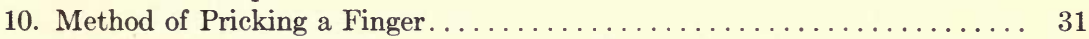

11. Method of Obtaining a Small Amount of Human Blood . . . . . . . . . 32

12. Collecting Blood in a Wright Capsule. .................... 33

13. Removing Serum from a Wright Capsule..................... 34

14. Method of Sealing a Wright Capsule....................... 34

15. Methods for Securing Blood by Puncture of Vein .............. 35

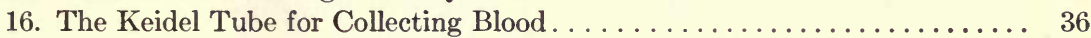

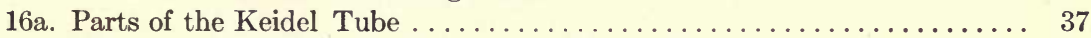

17. A Wet-cup for Securing Blood from Children (Blackfan) . . . . . . . 38

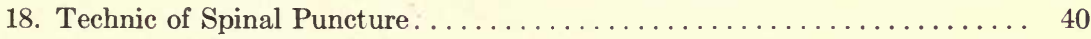

19. Method of Bleeding a Rabbit from the Ear . . . . . . . . . . . . . . 43

20. A Dissection of the Neck of a Rabbit to Show Relation of the Carotid Artery 44

21. Method of Bleeding a Rabbit from the Carotid Artery . . . . . . . . . . 45

22. Method of Bleeding a Rabbit from the Carotid Artery (Pasteur Institute

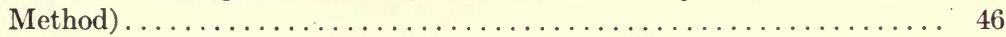

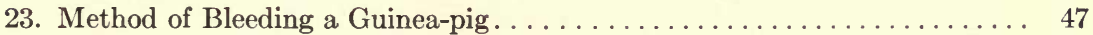

24. A Dissection of the Neck of a Sheep to show the Relations of the External Jugular Vein. . . . . . . . . . . . . . . . . . . . . . . . . . . 48

25. Method of Bleeding a Sheep from the External Jugular Vein. . . . . . . . 49

26. Method of Bleeding a Horse from the Jugular Vein . . . . . . . . . . 51

27. Method of Subcutaneous Inoculation of a Guinea-pig. . . . . . . . . 55

28. Method of Intravenous Inoculation of a Rabbit . . . . . . . . . . . 57

29. A Dissection of the Neck of a Guinea-pig to show the Relations of the Ex-

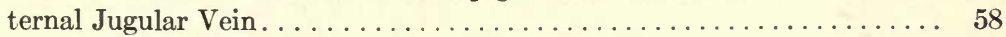

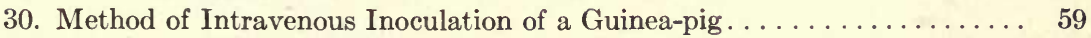

31. Method of Intravenous Inoculation of a Rat ................ 60

32. Method of Intravenous Inoculation of a Horse . . . . . . . . . . . 61

33. Method of Intraperitoneal Inoculation of a Rabbit . . . . . . . . . . 63

34. A Small Berkefeld Filter .............................. 77

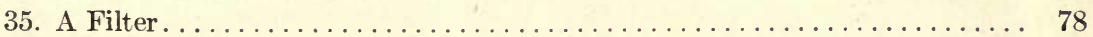

36. Abdominal Wall of Guinea-pig showing Diphtheric Edema........Facing 112

37. Normal Adrenal Gland of a Guinea-pig. . . . . . . . . . . . . Facing 114 
FIG.

38. Adrenal Gland of a Guinea-pig after Fatal Diphtheric Intoxication . . . Facing 114

39. Ehrlich's Side-chain Theory. Formation of Antitoxins . . . . . . . . 150

40. Theoretic Structure of a Molecule of Toxin and Toxoid ............ 151

41. Ehrlich's Side-chain Theory. Formation of Agglutinins and Precipitins.... 153

42. Ehrlich's Side-chain Theory. Formation of Cytolysins (Bacteriolysins, Hemolysins, etc.) . . . . . . . . . . . . . . . . . . 155

43. General Scheme of Antigens and Their Antibodies.............. 163

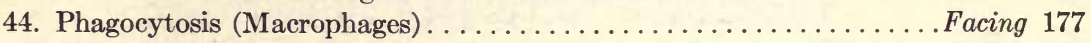

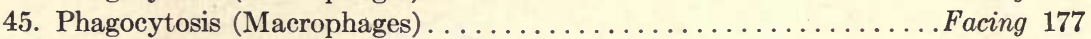

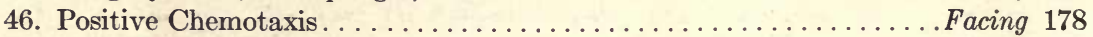

47. Negative Chemotaxis. . . . . . . . . . . . . . . . . . . Facing 182

48. Capillary Pipet for Opsonic Index Determinations . . . . . . . . . . . . 196

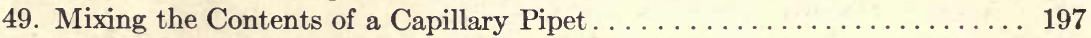

50. Method of Sealing a Capillary Pipet. . . . . . . . . . . . . . . . . . 198

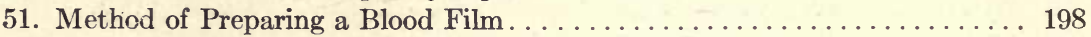

52. Blood Films for Phagocytic Counts. . . . . . . . . . . . . . . 199

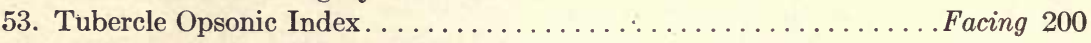

54. An Unsatisfactory Film for Phagocytic Count............... Facing 200

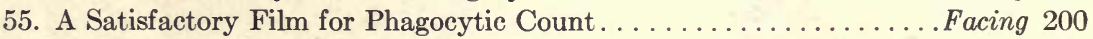

56. An Opsonic Index Chart. . . . . . . . . . . . . . . . . . . . . . . 204

57. Preparation of a Bacterial Vaccine. . . . . . . . . . . . . . . 208

58. A Shaking Apparatus. . . . . . . . . . . . . . . . . . . 209

59. Capillary Pipet for Counting Bacterial Vaccines ............... 210

60. A Satisfactory Preparation for Counting a Bacterial Vaccine........ Facing 211

61. An Unsatisfactory Preparation for Counting a Bacterial Vaccine . . . . Facing 211

62. Instrument for the Standardization of a Platinum Loop. . . . . . . . . . 212

63. Hopkins' Tube for Standardizing a Bacterial Vaccine. . . . . . . . . . . 213

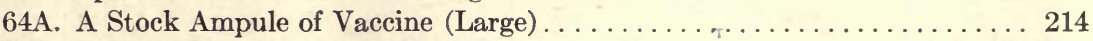

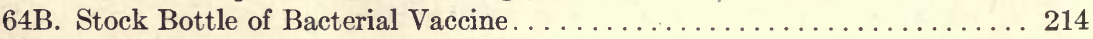

65. A Small Vaccine Ampule. . . . . . . . . . . . . . . . . . . . . . 215

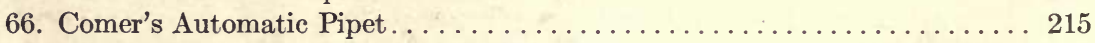

67. Theoretic Formation of an Antitoxin . . . . . . . . . . . . . . 222

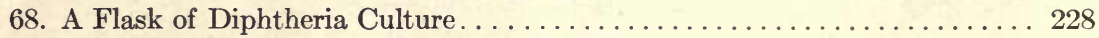

69. A Large Toxin Filter. . . . . . . . . . . . . . . . . . . . . . . . 229

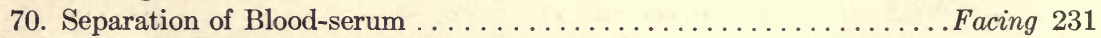

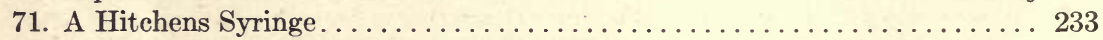

72. A Battery of Hitchens' Syringes........................ 233

73. A Dialysing Cylinder for the Abderhalden Ferment Test. . . . . . . . . . 254

74. The Ninhydrin Reaction (Abderhalden Ferment Test)..........Facing 255

75. Theoretic Formation of Agglutinins . . . . . . . . . . . . . 267

76. Theoretic Structure of Agglutinin and Agglutinoid ............ 268

77. Diagrammatic Illustration of the Action of Agglutinin and Agglutinoids... 269

78. A Satisfactory Culture for the Microscopic Agglutination Reaction . . . . . 280

79. An Unsatisfactory Culture for the Microscopic Agglutination Reaction . . . . 280

80. A Positive Agglutination (Widal) Reaction in Typhoid Fever . . . . . . . 283

81. Microscopic Agglutination Test with Dried Blood.............Facing 284

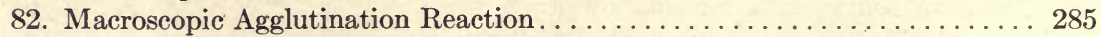

83. Macroscopic Agglutination Test. Pro-agglutination. . . . . . . . . . 286

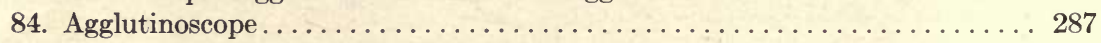

85. The Noguchi Butyric Acid Test for Globulins ............... 301

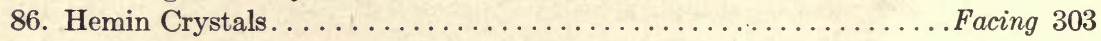


87. Precipitin Test. Preparation of Extract of Blood-stains . . . . . . F Facing 304

88. Uhlenhuth's Filter. . . . . . . . . . . . . . . . . . . . . . 305

89. A Rack for Precipitin and Agglutination Reactions. . . . . . . . . . . . 306

90. Titration of a Precipitin (Serum) . . . . . . . . . . . . . . . . 307

91. A Precipitin Reaction. Biologic Blood Test . . . . . . . . . . . . . . . 309

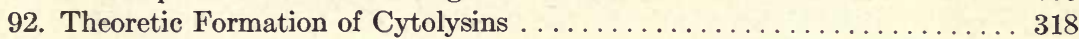

93. Theoretic Structure of a Polyceptor . . . . . . . . . . . . . . 321

94. Scheme showing Mechanism of Complement Fixation . . . . . . . . . . 333

95. Theoretic Structure of a Bacteriolytic Amboceptor . . . . . . . . . . . . 340

96. Method of Removing Exudate from the Peritoneal Cavity of a Guinea-pig. . 346

97. Culture of Cholera Undergoing Bacteriolysis. A Positive Pfeiffer Reaction 347

98. Stained Preparation of Cholera Undergoing Bacteriolysis . . . . . . . Facing 347

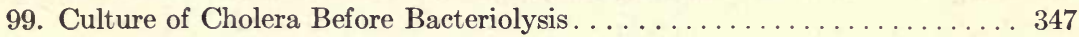

100. Stained Preparation of Cholera Before Bacteriolysis . . . . . . . . . Facing 347

101. Bactericidal Test (Looped Pipet Method of Wright) . . . . . . . F Facing 357

102. Theoretic Structure of a Hemolytic Amboceptor . . . . . . . . . . . . . . . 364

103. Titration of Hemolytic Amboceptor.................. Facing 377

104. Venom Hemolysis. . . . . . . . . . . . . . . . . . . . Facing 387

105. A Vial to Contain Blood for the Wassermann Reaction. . . . . . . . . . . 409

106. Outfit for Collecting Blood for the Wassermann Reaction . . . . . . . . . . 410

107. Titration of Hemolytic Complement. . . . . . . . . . . . . Facing 413

108. Titration of Antigen for Anticomplementary Unit. . . . . . . . . Facing 430

109. Titration of Antigen for Antigenic Unit. . . . . . . . . . . . . Facing 430

110. Wassermann Reaction (First Method) . . . . . . . . . . . . . Facing 438

111. Reading the Wassermann Reaction....................Facing 440

112. Wassermann Reaction (Second Method)...................Facing 444

113. Wassermann Reaction (Third Method) . . . . . . . . . . . . . Facing 445

114. Wassermann Reaction (Fourth Method) ..................Facing 449

115. Titration of Anti-huma. Hemolytic Amboceptor . . . . . . . . . . . Facing 454

116. Noguchi Modification of the Wassermann Reaction............Facing 454

117. Anticomplementary Titration of a Gonococcus Antigen. . . . . . . Facing 479

118. Gonococcus Complement-fixation Reaction. . . . . . . . . . . . Facing 481

119. Urticarial Rash of Serum Sickness. . . . . . . . . . . . . . . . Facing 574

120. Multiform Rash of Serum Sickness. . . . . . . . . . . . . . . . Facing 574

121. Local Serum Anaphylactic Reactions......................Facing 576

122. Method of Performing the Cutaneous Tuberculin Test (von Pirquet) ... 597

123. A Positive Cutaneous Tuberculin Reaction (von Pirquet).........Facing 598

124. A Positive Conjunctival Tuberculin Reaction (Wolff-Eisner-Calmette)

125. Facing 598

125. A Positive Percutaneous Tuberculin Reaction (Moro) . . . . . . . . Facing 599

126. A Positive Luetin Reaction. . . . . . . . . . . . . . . . . . . . Facing 604

127. Production of Cow-pox Vaccine. . . . . . . . . . . . . . . . . . . . 628

128. Method of Vaccination Against Smallpox $\ldots \ldots \ldots \ldots \ldots \ldots \ldots \ldots \ldots \ldots \ldots \ldots$

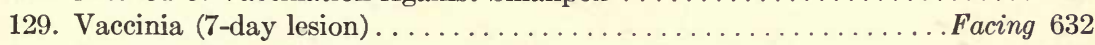

130. Vaccinia (9-day lesion) ... . . . . . . . . . . . . . Facing 632

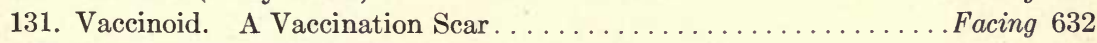

132. Preparation of Rabies Vaccine. . . . . . . . . . . . . . . . . . . 641

133. Preparation of Tuberculin . . . . . . . . . . . . . . . . . . . 665

134. Method of Subcutaneous Injection . . . . . . . . . . . . . . . . . 689

135. Method of Intravenous Injection by Means of a Syringe . . . . . . . . 692

136. Method of Intravenous Injection by Gravity . . . . . . . . . . . 693 
FIG.

137. Outfit for Intraspinal Injection of Antimeningitis Serum by Gravity

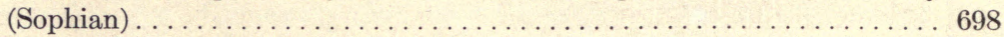

138. Method of Intraspinal Injection by Gravity . . . . . . . . . . . . . . 699

139. Method of Intraspinal Injection by Means of a Syringe . . . . . . . . . . 701

140. Blood of Rat Infected with Sp. recurrentis (dark ground illumination) . . . . 791

141. Same After Administration of Salvarsan (dark ground illumination) . . . . . 792

142. Method of Intravenous Injection of Salvarsan . . . . . . . . . . . . . . 800

143. Method of Intravenous Injection of Neosalvarsan . . . . . . . . . . . . 801 


\section{INFECTION, IMMUNITY, AND SPECIFIC THERAPY}

\section{PART I \\ CHAPTER I \\ GENERAL TECHNIC}

IN this chapter simple methods are described for preparing capillary pipets and similar apparatus usually made in the laboratory, and a few general directions are given concerning the preparation of glassware and other material employed in the various methods described in succeeding chapters and in experimental work.

It may be well here to utter a word of caution to the inexperienced against observing undue haste in performing the manipulations of immunologic technic. Careful and painstaking work is essential in order to secure reliable and successful results, and should never be sacrificed for speed, the latter being attained only by experience.

\section{CENTRIFUGE}

1. A good centrifuge is one of the chief requisites of a laboratory equipment. While any good instrument will answer, preference should be given to the larger types, fitted for holding both 15 c.c. and 50 c.c. centrifuge tubes, propelled by electricity, and mounted on a concrete block in the laboratory (Fig. 1).

2. The machine must be well oiled.

3 . The counter tubes should be of the same weight-it is our custom to weigh the tubes on a small balance, and adjust the counter tubes until both are of equal weight.

4. The centrifuge tubes should rest loosely upon a rubber disc or wad of cotton in the bottom of the metal tube or cup; otherwise centrifuge tubes are quite likely to be broken, especially if the machine is run at high speed.

5. The machine should be started and stopped slowly, and unnecessary speed and long running time should be avoided.

6. Never centrifugalize with cotton plugs in the centrifuge tubes. 
If the latter must be sealed, as when working aseptically, rubber stoppers should be used. However, if cotton plugs are large and fit tightly, they may be prevented from becoming displaced by passing through them two cross-pins in such manner that the ends will rest upon the edge of the tube. The plugs are thus prevented from being thrown to the bottom of the tube.

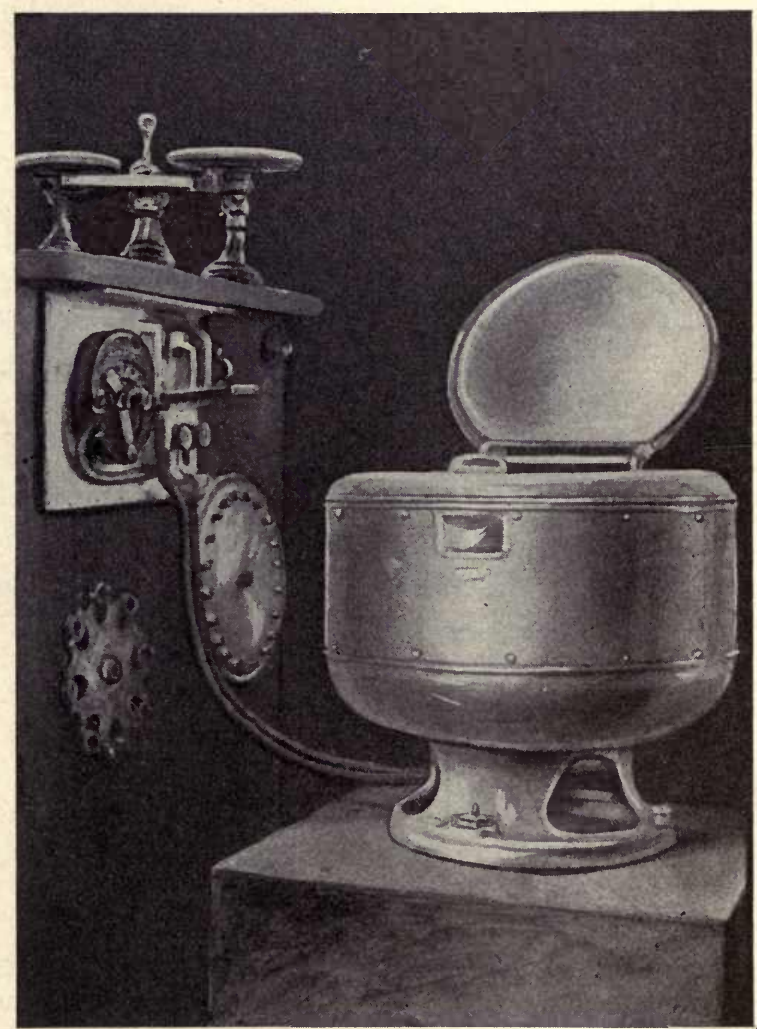

Fig. 1.-Electric Centrifuge.

Mounted on a concrete block. The scales are for the purpose of weighing and counterbalancing the tubes.

7. If the centrifuge is out of order, however slightly, it should not be used, but repaired at once, or else it may be ruined.

\section{PIPETS}

1. Simple Capillary Pipets.-These are made of soft glass tubing in the following way: 
Tubing having a caliber of $6 \mathrm{~mm}$., with thin walls, that does not become opaque, brittle, or "run" on heating, and that does not contain lead, may be used. The question of alkalinity is also of importance in connection with the tubing. Many of the cheaper grades undergo disintegrative changes, which are accompanied by the setting free of alkali, especially when the glass is heated. Glass of this kind should be discarded, as it may introduce an element of error into our experiments and observations.

2. A convenient length of tubing - about 10 to 12 inches - is chosen; this will make two pipets. If a sufficient length of tubing for both sides is not available, one end may be heated and drawn out with forceps, or a handle may be added by fusing to this short end an odd piece of glass.

It is convenient to have on hand a supply of tubes cut to correct lengths, plugged at each end with a ball of cotton, and sterilized in a hot-air sterilizer. They are then ready to be drawn out as needed, thus furnishing sterile pipets with cotton plugs that tend to prevent contamination.

3. The flame must be so regulated as to play upon only so much of the tube as will suffice to furnish the glass required for drawing out the tubing. If a Bunsen flame is used, the tip of the inner greenish flame should be applied. The margins of the flame are the hottest, and for this reason the tube must be shifted from side to side and be constantly rotated.

4. In order to secure uniform heating and satisfactory pipets the tube must be kept constantly rotated from the moment it enters until it leaves the flame. The two ends of the tube are to rest upon the middle finger of each hand while the thumb and forefinger hold the tube in position at either side and impart the rotatory movement. It is also necessary that the tube be displaced laterally from time to time, so as to bring each portion of the middle segment of the tube in turn into the edge of the flame (Fig. 2). If the latter precaution is omitted, we shall obtain a pipet with a central bulb or thicker segment and with thinner segments on each side corresponding to the portions of the tube which lie in the edges or hottest portion of the flame.

5. The tube is heated in this manner until the glass is quite plastic. No attempt is made to draw out the tube until it has been entirely withdrawn from the flame, as otherwise a portion becomes unduly thin and plastic and divides, leaving a small, bent, and very poor pipet in each hand. 
The rapidity and force with which the tube is drawn out determine the caliber of the capillary stem. By drawing rapidly a tapering capillary tube is obtained; by drawing slowly a larger capillary tube of more uniform caliber is obtained. Of course, the worker cannot take too much time, as the glass hardens quickly. With a little practice this part of the technic is soon mastered. Thorough and uniform heating and careful, steady pulling when the tube is sufficiently plastic are of primary importance.

When, owing to an error in judgment in heating the tube, it is with-. drawn before it is sufficiently plastic and begins to harden, the situation cannot be remedied by drawing out the tube quickly with a jerk. Similarly, when a tube has been partially drawn and hardens it cannot, as a rule, be reheated and drawn out to make a satisfactory pipet.
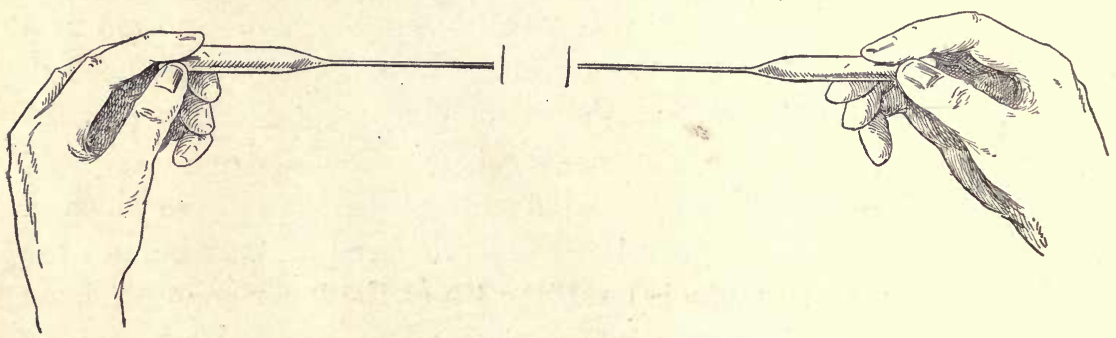

Fig. 2.-Method of Making a Simple Capillary Pipet.

Shows manner of holding tubing in a flame and drawing into capillary tubes. A large portion has been removed from the center.

6. After drawing out the pipets the hands should be held steady for a few seconds, i.e., until the glass has hardened; otherwise the tubes will bend and be less satisfactory.

7. Capillary pipets are manipulated with rubber teats, which should be of the best soft vulcanized rubber, and should fit snugly upon the pipet, rendering it air-tight.

2. Looped Pipets.-Looped pipets find their main application in the measurement of the bactericidal power of the blood, after the method of Sir A. Wright. ${ }^{1}$

The essential features of these pipets are: (a) The capillary stem, which serves for measuring and mixing the bacterial emulsion and serum; (b) the portion that serves first as a chamber for the sterile nutrient broth and later as a cultivation chamber for determining whether the

1 "Technique of the Teat and Capillary Glass Tube," 1912. Constable and Company, London. 
microbes that have been mixed with the serum have or have not been killed by it; $(c)$ the glass loop, which acts as a trap, preventing extraneous contamination; and $(d)$ the handle, upon which the rubber teat can be fitted. With a little practice these pipets are readily made.

1. Select glass tubing about 6 inches in length.

2. Heat one portion about two inches from the end, and when sufficiently plastic, draw it out for about two or three inches or until it is long enough to give a spiral loop of the desired dimensions (Fig. 3). While the glass is still plastic hold the left hand steady, and with the right hand lower the tubing and make a spiral loop in such manner that the loop is closely applied, but does not touch the sides of the upper and lower segments of the tube. Actual contact with the sides must be
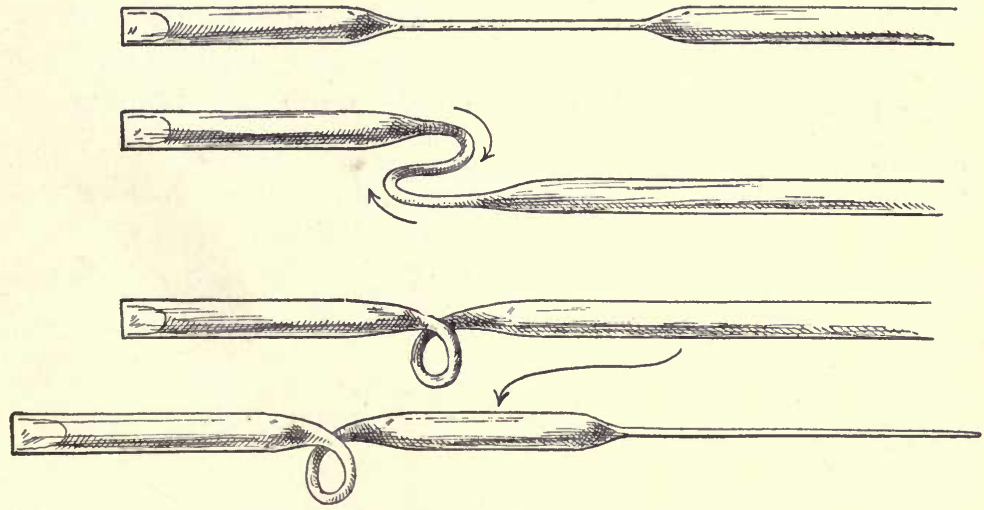

Fig. 3.-Method of Making a Looped Pipet.

avoided, for this would produce strain and predispose the tube to fracture.

3. The longer portion of tubing is now heated and drawn out to a capillary pipet and broken through at the desired point.

4. Instead of this method, the capillary portion may be drawn before the loop is made.

3. Graduated Pipets.-1. In this work 1 c.c. pipets graduated into $\frac{1}{10}$ c.c. $; 5$ and 10 c.c. pipets graduated into $\frac{1}{10}$ c.c. will render satisfactory service. The pipets should be calibrated to the tip, and should preferably be long, with a narrow lumen, rather than short, with a wide lumen, as the latter renders the markings too close to one another. For pipeting small amounts, as in certain complement fixation tests, a 0.2 c.c. pipet graduated to $\frac{1}{100}$ c.c. will be found quite serviceable, permitting 


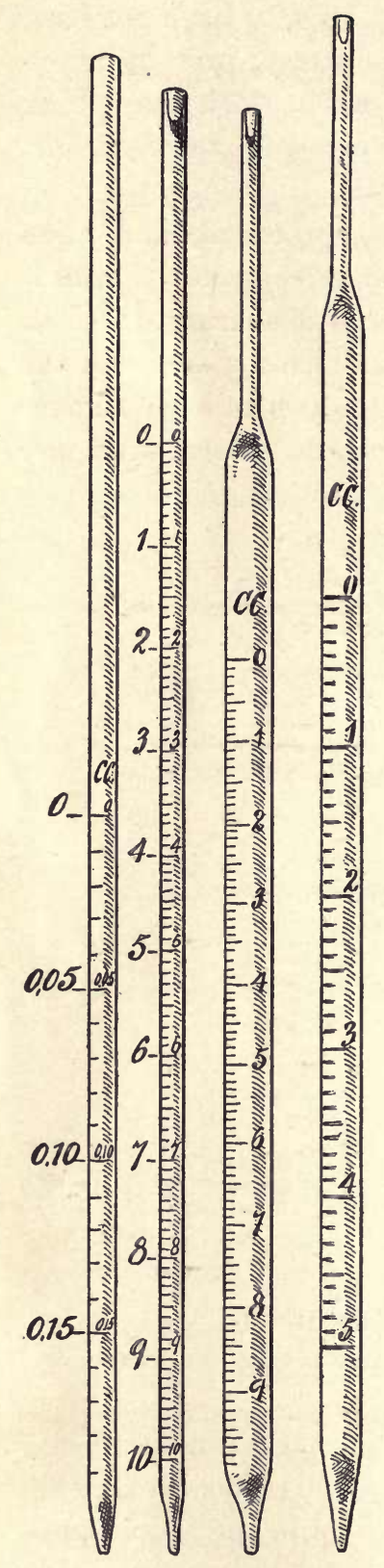

Fig. 4.- Graduated Pipets.

These pipets are generally used in immunologic work. Note the small cotton plugs in the mouth ends. The 1 c.c. pipet (second from the left) is graduated near to, but does not actually include, the tip; this feature is highly desirable, as it permits measuring small amounts of fluid and the pipet is not necessarily ruined, even though a portion of the tip were chipped off. accurate measurement of small amounts of fluid. The entire length of these pipets is equal to the ordinary 1 c.c. pipet which renders the subdivisions far apart and quite easy to read (Fig.4). These pipets are made by competent dealers upon special request.

2. These pipets should be perfectly clean and clear, sterilized, and have sharp, easily read markings. Pipets with broken tips are difficult to handle, and if calibrated to the tip, are inaccurate.

3. The'worker should practise methods of making accurate measurements. The slightest slip may mean an inaccurate measurement and produce untoward results. The mouth end and the pipeting finger should be dry, otherwise, on measuring small amounts, the delivery will be jerky and usually unsatisfactory.

4. After pipets have held infectious material they should be placed at once in a jar containing 1 per cent. formalin solution. After pipeting blood, milk, or serum, the pipets should be rinsed or placed in a jar containing water or a weak lysol solution, as the formalin solution tends to harden these substances and renders cleaning quite difficult. They should be washed thoroughly, the mouth end being plugged neatly and firmly with a bit of cotton, and then placed in a metal box or wrapped in newspaper and sterilized in the hot-air oven. Unless all the serum, blood, milk, etc., are well washed out, the pipets may become occluded and discolored. If this occurs, they should be soaked for twenty-four hours in strong nitric (50 per cent.) or sulphuric acid, and washed thoroughly and sterilized. 
5. The jar for holding soiled pipets should contain a layer of cotton, otherwise, the tip may be broken off when the pipets are dropped in.

\section{BLOOD CAPSULES}

Blood capsules were devised by Sir A. Wright for collecting small amounts of blood for examination. The essential features of a capsule are: (a) The upper straight limb, which can be drawn out to serve as a needle for puncturing; (b) the recurved limb, which makes it possible to fill the capsule by gravity, without risk of the inflow being arrested by the blood running down and blocking the straight limb, which provides an outlet for the air.

These capsules are easily made and prove quite serviceable, es-
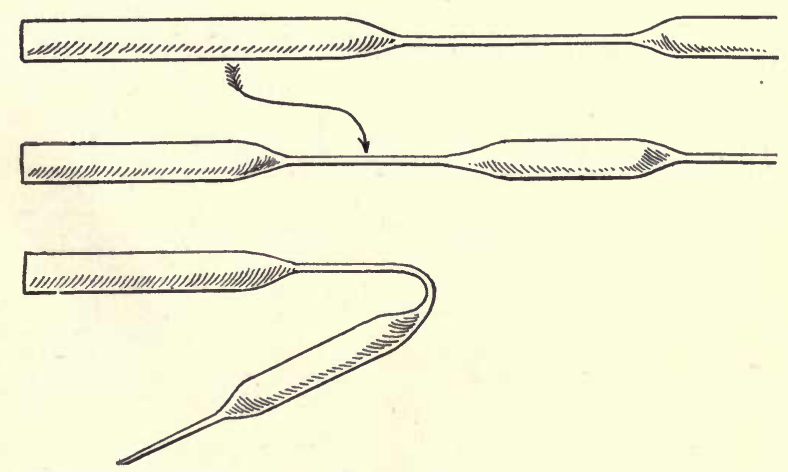

Fig. 5.-Method of Making a Wright Blood Capsule.

pecially for collecting small amounts of blood for making agglutination tests, opsonic measurements, etc.

1. Take a piece of soft glass tubing about 10 or $12 \mathrm{~cm}$. in length, and having an internal diameter of at least $5 \mathrm{~mm}$.

2. Draw one end out into a capillary stem, and break this at an appropriate point (Fig. 5).

3. Then reinsert the tube into the flame, and, leaving a portion at least $3 \mathrm{~cm}$. in length to serve as the barrel of the capsule, draw out the tube into a capillary stem about $8 \mathrm{~cm}$. in length, and bend it so as to form a stout recurved limb lying in the horizontal plane; now, before the glass has lost its plasticity, draw the capsule gently upward so that its long axis will be at an angle of about $30^{\circ}$ horizontally. Finally, separate the capsule from the main tube by filing it across the capillary portion at the distance indicated in the accompanying illustration (Fig. 5). 
4. The straight limb may now be drawn to a sharp point and used as a needle.

\section{VACCINE BULBS}

These may be made of glass tubing, to hold 1 c.c. or more, although when a large number are required, it is cheaper to purchase ready-made ampules, such as are shown on p. 215.

1. Take a piece of glass tubing at least $10 \mathrm{~cm}$. in length, and having an internal diameter of 6 or $7 \mathrm{~mm}$.

2. Seal one end by heating it in a hot Bunsen or blow-pipe flame.

3. After cooling, drop in one or two small glass beads, which serve later to aid in mixing the vaccine before it is injected (Fig. 6).

4. Heat the opposite end and draw out into a wide and stout capillary end.

5. This end may be broken through; the whole ampule is now ster-
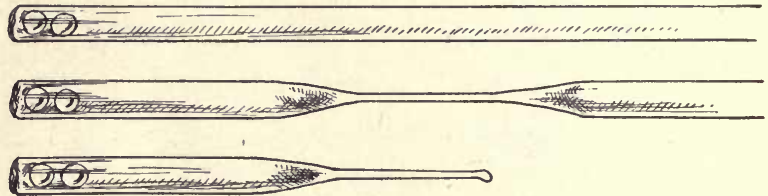

Fig. 6.-Method of Making a Vaccine Ampule of Ordinary Glass Tubing.

ịlized by heat, and after it is cooled, the required amount of vaccine is inserted.

6. The open end is then sealed in the flame by warming the air in the bulb above the surface of the liquid and finally sealing the tip, otherwise the air may expand after heating and cause an explosion at the tip. Care must be exercised not to heat the glass down to the level of the fluid, as this tends to produce steam and to crack the bulb.

Test-tubes may be drawn out and converted into ampules for holding vaccines, serums, or other fluids.

1. Thin-walled and sterilized test-tubes of appropriate size are chosen.

2. The tube is heated at a point near the open end in the Bunsen flame, in the same manner as the glass tubing, i.e., by keeping the tube constantly rotating with a lateral movement to insure uniform heating.

3. When the glass has become plastic, it is drawn out into a stout stem. 
4. After cooling, it is filed through at an appropriate place, being careful to leave a somewhat long stem (Fig. 7).

5. The open end may now serve as a funnel for filling the bulb.

6. The bulb is now sealed by warming the air above the level of the fluid and then sealing the tip. With a long stem, in order to secure a portion of the contents, the sealed end may be broken off from time to time; it is readily resealed.

7. Instead of this procedure the fluid may be placed in the testtube at once, the upper end being heated in the usual manner and drawn out; the stem is broken through, and the tip sealed. If the tube is small or the contents are such as will almost fill a tube, this method may not be successful, owing to the production of steam on heating the
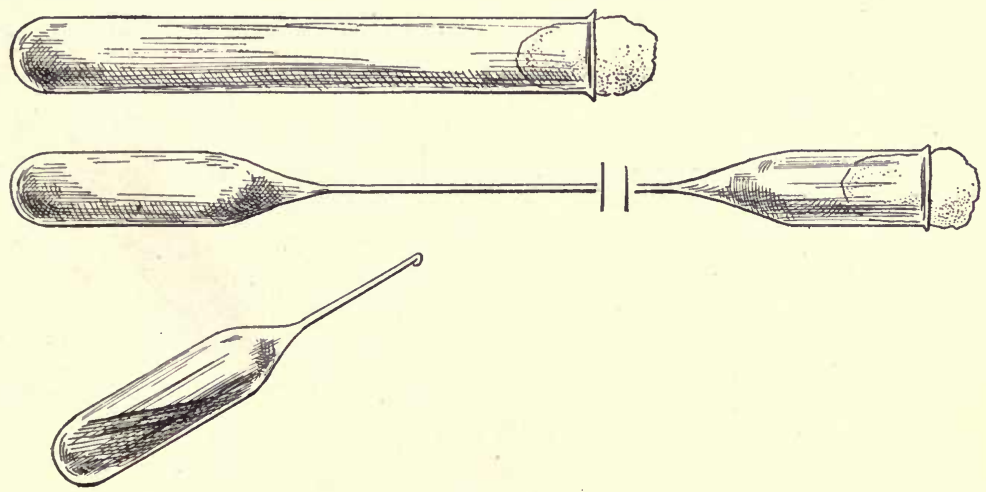

Fig. 7.-Method of Making a Large Vaccine Ampdle of a Test-tube.

fluid, which either cracks the bulb or causes the tip to explode at the time of sealing.

\section{TEST-TUBES}

1. In this work test-tubes of various sizes are used mainly for making the agglutination, precipitin, complement fixation, and other tests. They should be made of good glass, with round bottoms, and be well annealed.

2. Test-tubes should be thoroughly clean, clear, and steritized, preferably by dry heat. It is not necessary to plug them with cotton unless they are to be used for bacteriologic work, for when a large number of tubes are used, this is a waste of time and of material. If the tubes are to be used within from twenty-four to forty-eight hours, merely placing the tube mouth end downward in the wire basket is sufficient. A 
piece of fresh cotton should be placed in the oven at the time of sterilization, and when this has turned a light-brown color, sterilization is completed.

3. After a tube has been used for holding living and infectious or-

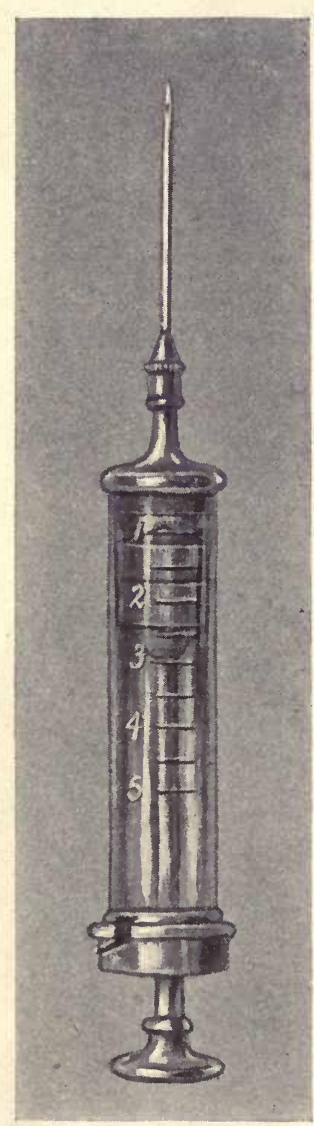

Fig. 8.-A SAtisfaCTORY SYRINGE (Record).

This syringe has a glass barrel and metal plunger. It is easily sterilized, durable, and works smoothly and accurately. ganisms, as in making bacteriologic, agglutination, and opsonic tests, etc., the tube and its plug should be boiled in water or a 1 per cent. solution of caustic soda before it is again handled. In making hemolytic experiments, the material is not usually infectious, and it is sufficient to wash the tubes without previous boiling. In every case all traces of acids or alkalis must be removed by copious washing in plain water, as the slightest trace of either may interfere with the accuracy of some of the tests. Cheaper grades of glass may contain relatively large amounts of alkali, which would introduce a disturbing element in the reactions.

\section{SYRINGES}

A good syringe is indispensable in performing bacteriologic and immunologic work. Various sizes should be at hand, but usually a graduated 5 c.c. syringe answers most purposes.

1. Many kinds of syringes are on the market. Those with rubber or leather plungers and packings are unsatisfactory, as they cannot be sterilized by boiling and soon leak. Nothing is more exasperating than a leaking syringe, as with the leakage unknown quantities of inoculum are lost, not to mention the possible dangers of contaminating the fingers, the animal, and the laboratory.

Syringes with metal or glass plungers are to be preferred, as are also those upon which the needle may be fitted without screwing (Fig. 8).

The old Koch syringe is fitted with a rubber bulb for filling and expelling the fluid. This arrangement is well adapted for making subcutaneous injections, but is somewhat dangerous for purposes of making intravenous injection, on account of the danger of injecting air. 
3. Syringes may be sterilized by filling them with 1 per cent. formalin solution for a few minutes, followed by several washings with sterile water or salt solution. This method is good for syringes having leather or rubber packings and plungers. It is not safe for blood cultures, as spore-forming bacteria may escape the sterilizing process.

4. With all glass or metal syringes, it is best to boil the syringe, especially if a careful aseptic technic is to be employed. The plunger should be removed from the barrel, or else, whether it be of glass or metal, it will expand more rapidly than the accommodation of the barrel will permit. All parts should be placed in a pan or wrapped in gauze, warm water added, and boiling allowed to take place for a minute or so. After cooling the parts are adjusted.

Wright's method for sterilizing a hypodermic syringe for the administration of vaccines is given on p. 217.

5. If infectious material has been used, the syringe, after using, should be washed out and sterilized. The needles should be dried and wired, and a small amount of vaselin rubbed over to prevent rusting. The plunger may likewise be occasionally rubbed with a small quantity of vaselin. Needles may be kept in oil or in absolute alcohol; usually thorough drying and wiring preserves them in good condition.

\section{SOLUTIONS}

1. Salt Solution. -0.85 per cent. sodium chlorid in distilled water is best adapted for immunologic work. This solution is prepared readily by dissolving 8.5 grams of salt in a liter of water, filtering, and sterilizing in an Arnold sterilizer for at least one hour.

2. Sodium citrate in 1 or 2 per cent. solution, made with normal salt solution and not with plain or distilled water, is used to prevent the formation of fibrin in drawn blood and exudates. 


\section{CHAPTER II}

\section{METHODS OF OBTAINING HUMAN AND ANIMAL BLOOD}

As a general rule, when blood is withdrawn to obtain serum a careful aseptic technic should be employed. Similarly, when erythrocytes are to be obtained for purposes of immunization it is necessary to avoid contamination by proper cleansing of the parts and the use of sterile needles, containers, and solutions. In obtaining erythrocytes for making hemolytic tests it is not necessary that the blood be absolutely sterile, the ordinary precautions against gross contamination being sufficient.

Blood may be withdrawn to obtain the corpuscles or serum or both.

\section{OBTAINING CORPUSCLES}

Red blood-corpuscles are usually obtained and washed free of serum for the purpose of making hemolytic reactions and experiments.

Leukocytes are usually obtained for the purpose of estimating the opsonic index. The special technic for obtaining leukocytes for this test is given on p. 195 .

Larger quantities of leukocytes are obtained by injecting sterile irritants, such as sterile aleuronat suspension, into the pleural or abdominal cavities of suitable animals. (See p. 201.)

(a) Blood may be withdrawn and placed at once in two or three volumes of 1 per cent. sodium citrate in 0.85 per cent. salt solution. Clotting is prevented, and the corpuscles are secured by centrifugalization or by sedimentation in the refrigerator.

(b) Blood may be drawn into a beaker or flask and defibrinated at once by whipping with a rod or shaking with glass beads. When the fibrin has been removed, the corpuscles and serum are secured by centrifugalization.

\section{WASHING ERYTHROCYTES}

For purposes of immunization or in making hemolytic tests red blood-corpuscles are washed free of serum before being used.

1. Place the citrated blood, which has previously been diluted with sufficient salt solution, in centrifuge tubes. Defibrinated blood may 
be placed in centrifuge tubes and five to ten volumes of sterile normal salt solution added and thoroughly mixed. Tubes are then carefully balanced and centrifuged at moderate speed for five minutes.

2. Remove the supernatant fluid down to the corpuscles with a sterile pipet. Add an equal volume of salt solution; mix the corpuscles

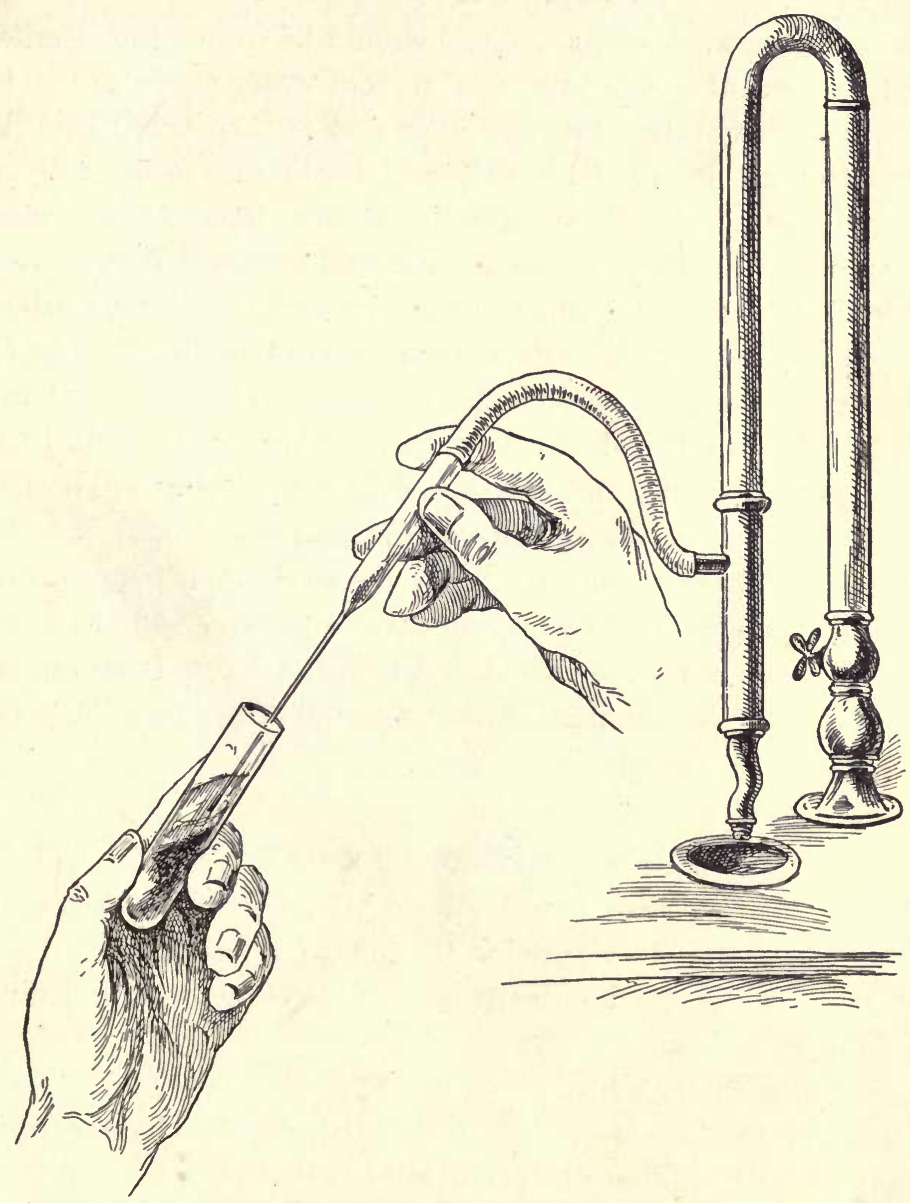

Fig. 9.-A Suction Pump.

By attaching a capillary pipet to the rubber tubing fluid may be removed without disturbing the sediment.

and centrifuge. This process should be repeated once more in order to insure thorough washing of the corpuscles to remove all traces of serum. For removing supernatant fluids which are to be discarded, the suction pump shown in Fig. 9 will be found very useful. It is well to fit the 
rubber tubing with a capillary pipet, which permits the supernatant fluid flush with the sediment to be removed.

3. After the last washing the supernatant salt solution should be carefully removed, when the corpuscles are ready for use.

\section{OBTAINING BLOOD-SERUM}

If serum is desired at once, blood should be drawn into sterile centrifuge tubes and the tube immersed in cold water for from five to ten minutes; this facilitates clotting. The clot is then broken up with a sterile platinum wire or glass rod, and the serum secured by rapid centrifugalization. Or blood may be drawn into sterile cylinders, Petri dishes, or centrifuge tubes, and allowed to stand at room temperature for a few hours, after which they should be placed in a refrigerator until the serum separates. Blood never should be drawn into Erlenmeyer flasks because of the difficulty of drawing off serum without disturbing the clot. When drawn into Petri dishes, care should be taken that the layer of blood is not too thin, otherwise drying will occur with poor separation of the serum. As a rule, the best results are secured by placing blood in centrifuge tubes, for if separation is poor or does not occur at all, the clot may be broken up and serum secured by centrifugalization. So far as possible, avoid drawing blood from an animal immediately after feeding, as under these circumstances the serum is likely to be milky or opalescent.

\section{OBTAINING CORPUSCLES AND SERUM}

For certain purposes it may be desirable to obtain both serum and corpuscles; these may be secured in the following way:

1. Place blood in a large centrifuge tube or cylinder, and defibrinate with rods or glass beads.

2. Centrifuge thoroughly.

3. Remove the serum, which is slightly discolored on account of defibrination, with capillary tube and rubber teat.

4. Filter the corpuscles into a centrifuge tube through a wisp of cotton in a funnel to remove small particles of fibrin.

5. Add normal salt solution, and proceed with the washing process.

\section{OBTAINING BLOOD PLASMA}

In obtaining blood plasma it is necessary to avoid coagulation of blood by securing and handling the blood with the least amount of 
trauma to leukocytes (paraffined tubes), and centrifuging rapidly and at once in cold centrifuge tubes.

1. After warming a centrifuge tube immerse in hot molten paraffin. Remove, drain, and allow the paraffin to harden. This produces a thin coat of paraffin within the tube; if a thicker one is desired, immerse again until a coat of the desired thickness is obtained; chill the tube thoroughly in cracked ice, but avoid getting water inside the tube.

2. Blood should be secured quickly by venipuncture, using a dry sterile needle, and passing the blood into a paraffined tube.

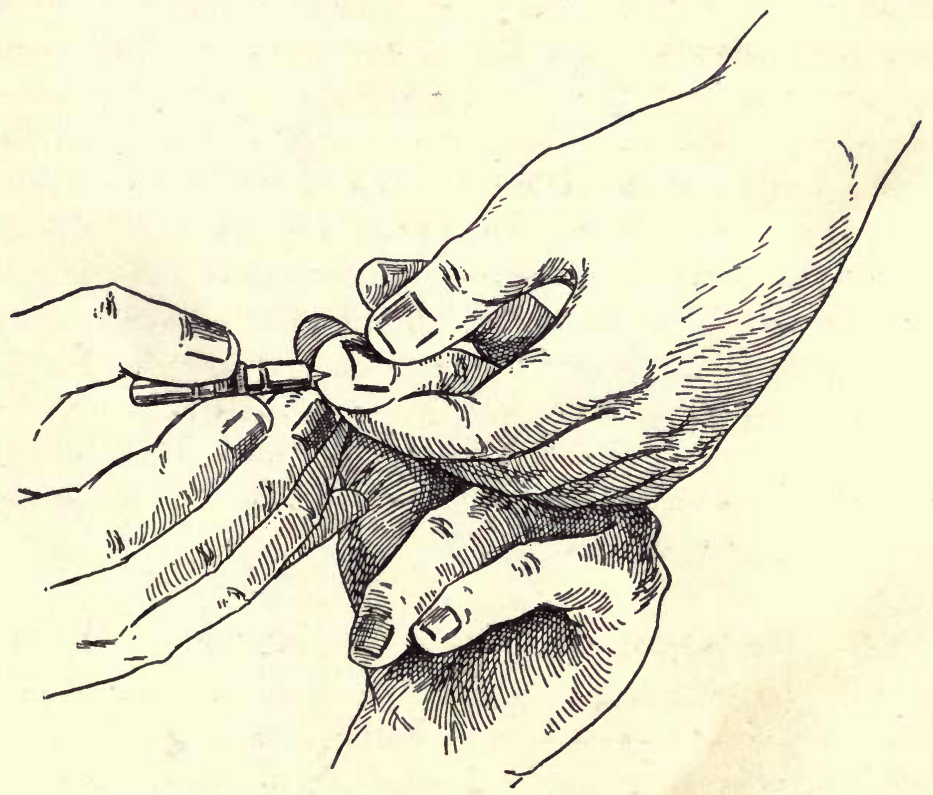

Fig. 10.-Method of Pricking a Finger.

The patient's finger is grasped firmly and lanced with a Daland lancet across the folds of skin. When lanced parallel with the skin-folds, the wound is likely to close before sufficient blood is secured.

3. Centrifuge at once and at high speed. If this is not possible, pack the tube in a large centrifuge cup, and surround with finely cracked ice. This permits of more prolonged centrifugalization, and at lower speed, and yields a hemoglobin-free plasma.

4. The clear yellow plasma is removed at once with pipet and nipple. 


\section{OBTAINING SMALL AMOUNTS OF HUMAN BLOOD}

For obtaining small amounts of blood-up to 2 or 3 c.c.-for the Widal reaction, complement fixation, and other tests, the following method is satisfactory:

1. Wash the last joint of the middle finger with alcohol. If the hand is cold, it should be warmed by immersing it in hot water. Before puncturing compress the finger and squeeze in such a manner as to drive the blood toward the end of the finger.

2. Prick deeply with a broad blood lancet, Hagedorn needle, or scalpel (Fig. 10).

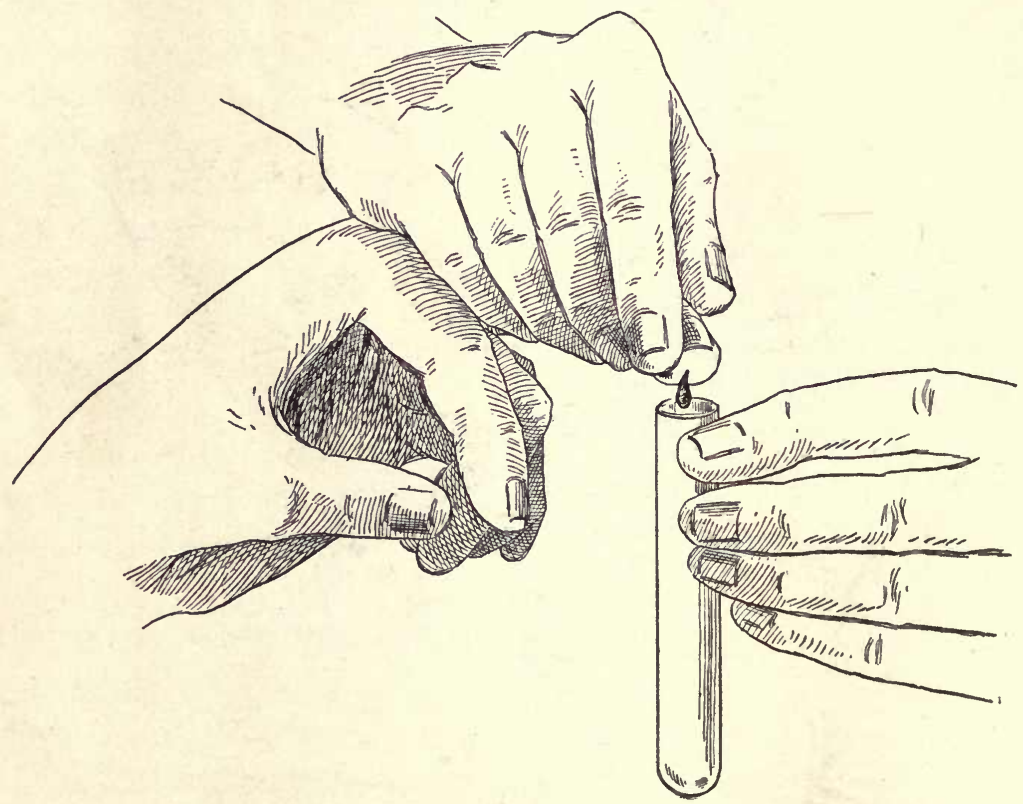

Fig. 11.-Method of Securing a Small Amount of Human Blood.

By pricking the finger deeply across the lines of the skin with a broad lancet, two or more cubic centimeters of blood are easily collected in a small test-tube. Do not use a large tube, as blood may be lost on the sides of the tube.

3. Collect the blood in a small test-tube,-about $8 \mathrm{~cm}$. by $1 \mathrm{~cm}$., - such as is used in performing the Noguchi reaction for the serum diagnosis of syphilis (Fig. 11).

4. By squeezing the finger, sufficient blood can usually be obtained from one puncture practically to fill a tube of the size mentioned. One to two cubic centimeters of serum are easily obtained in this manner, and this is sufficient for conducting the ordinary serum reactions. When 
the treatment of syphilis is being guided by the Wassermann reaction, frequent tests are necessary, and a patient may object to submitting to repeated venipuncture. The method for securing blood just described is so simple and efficient that objections to it are never made.

5. Blood may also be drawn in a Wright capsule, made by drawing out ordinary thin glass tubing in the Bunsen burner. (See p. 23.) After sufficient blood has been collected (Fig. 12), the straight empty end is sealed with a flame and then cooled (Fig. 14). The blood is then shaken into this sealed end, and the bent end, in turn, sealed with the flame. Care should be taken not to heat the blood. When the serum has separated, the tube is opened by filing at a point above the clot and breaking, protecting the hands with a towel. The serum is carefully removed with a capillary pipet and nipple (Fig. 13).

6. To obtain blood from infants and small children the large toe may be punctured, but as a rule better results are obtained by wetcupping or by puncturing a vein.

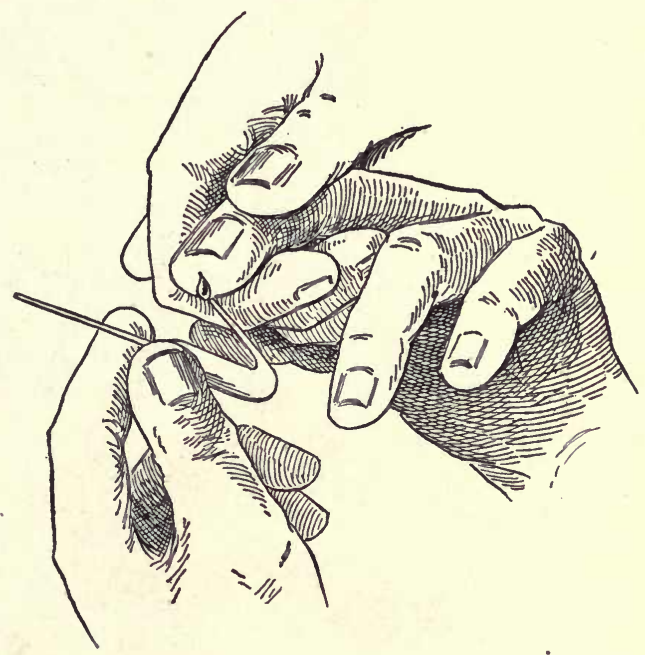

Fig. 12.-Collecting Blood in a Wright Capsule.

\section{OBTAINING LARGE AMOUNTS OF HUMAN BLOOD}

Larger quantities of human blood may be required for making complement fixation reactions, the Abderhalden ferment test, etc.

(a) Phlebotomy.-1. In adults, a prominent vein at the elbow, such as the median basilic, is usually chosen. In children less than a year old this vein is not suitable, better results being obtained when the external jugular or a temporal vein is used.

2. Place a rubber tourniquet or a few firm turns of a wide muslin bandage above the elbow.

3. Apply tincture of iodin to the skin over the vein. The vein may be rendered more prominent by directing the patient to open and close the hand several times. 
4. Steady the skin over the vein, and insert the needle in the direction of the blood-current (Fig. 15). It is more awkward, and of no practical advantage, to puncture in a downward direction toward the hand. The needle should be sharp, and of a size midway between the ordinary hypodermic and a large antitoxin needle, as the former is too small and the latter is unnecessarily large. The blood is then allowed to drop into a sterile tube. It is not necessary to attach

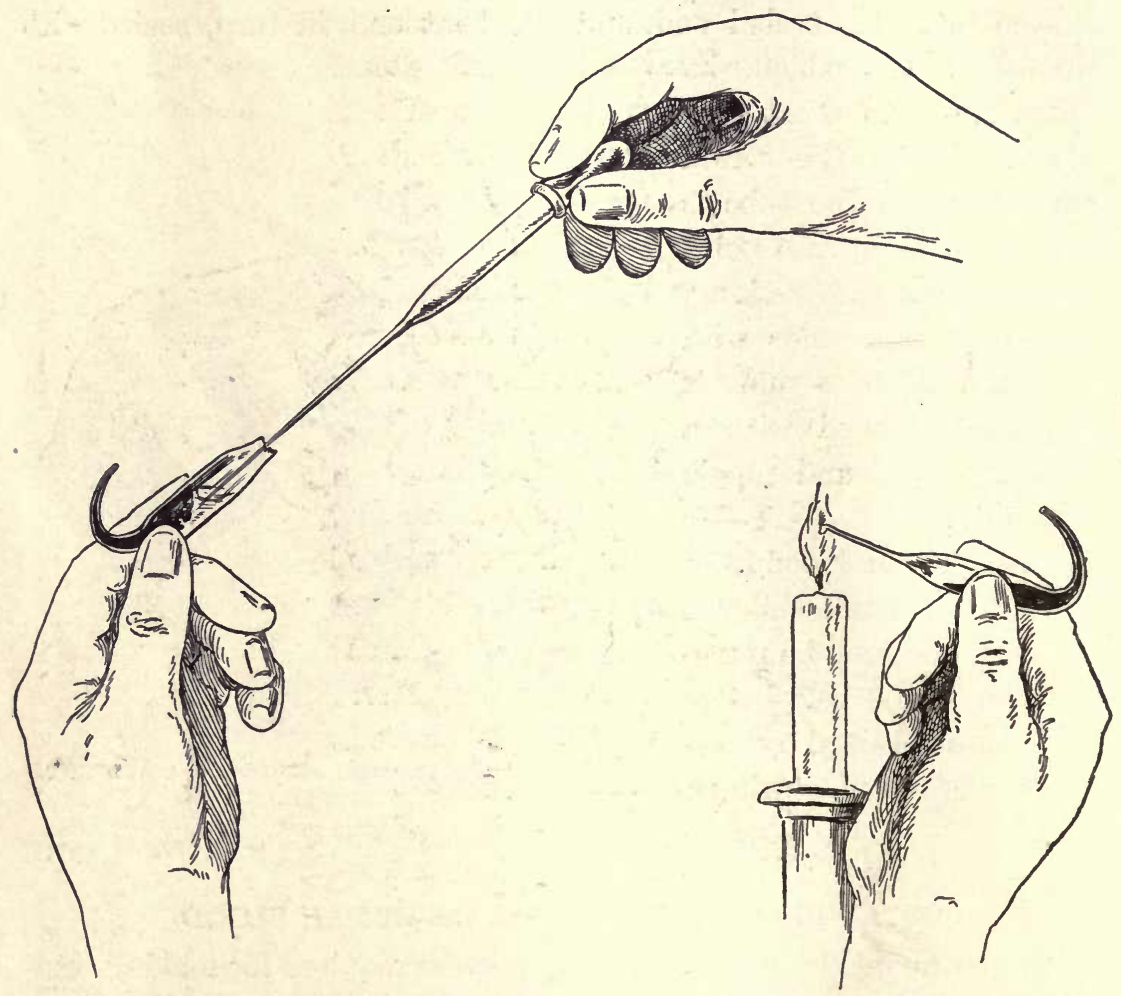

Fig. 13.-Removing Serum from a Wright Capsule.

Fig. 14.-Method of Sealing a Wright Capsule.

a syringe, although 5 to 10 c.c. of blood are obtained more quickly by this means on account of the possible gentle suction. Needle and syringe should be sterilized by boiling. When larger quantities of human serum are required as in auto-serum therapy, a platinumiridium needle should be used, as coagulation in the needle is less likely to occur; besides, these needles are readily sterilized by heating in the flame. 
5. Loosen the tourniquet, withdraw the needle quickly, and seal the wound with a touch of flexible collodion.

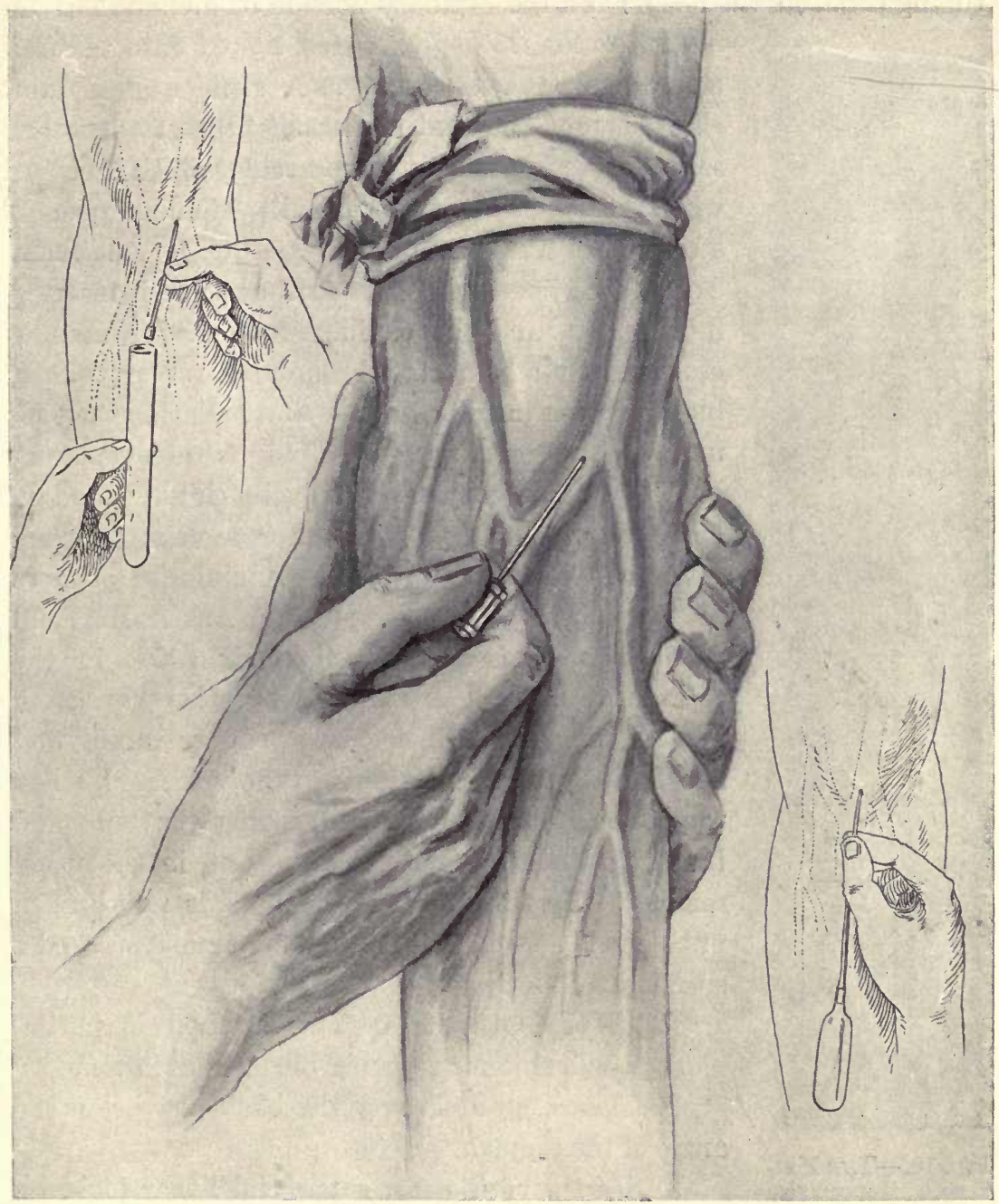

Fig. 15.-Methods for Securing Blood by Puncture of a Vein.

The middle figure shows distention of the veins of the arm about the elbow. The needle is entered by a quick upward thrust. Practically any prominent and firm vein may be used. The upper right-hand figure shows collection of blood in a testtube. Usually 10 c.c. or more are easily collected before clotting occurs. To secure large amounts, use a larger needle with a smooth bore (preferably a platinum-iridium needle). The lower right-hand figure shows collection of blood in a Keidel tube.

6. Instead of a syringe, the 5 c.c. vacuum bulb devised by Keidel has proved quite satisfactory (Fig. 16). This apparatus consists of a 
5 c.c. ampule with arm drawn out to a capillary tip and sealed after a vacuum has been created by heating (Fig. $16 a, B)$. A short piece of rubber tubing connects the needle and the capillary portion of the ampule. A

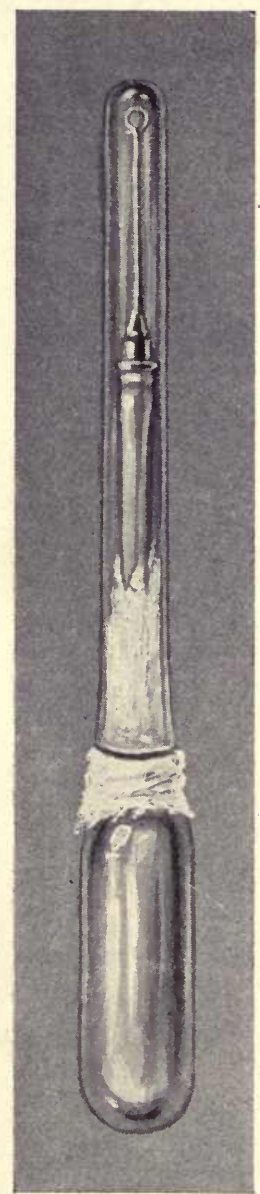

Fig. 16.-THe KeIDEL TUBE FOR C O L L E C T I N G Blood. (Manufactured by the Steele Glass Co., of Philadelphia.) needle of No. 25 gage is fitted tightly into the free end of the rubber tubing. A slender glass tube closed at one end and flaring slightly at the other serves as a protection for the needle, which it covers when the apparatus is sterilized. The apparatus is sterilized in a hot-air oven at $150^{\circ} \mathrm{C}$. for one hour. To obtain a specimen of blood the needle is inserted into a vein, and the capillary end of the ampule crushed with a hemostat through the rubber tubing, blood flowing into the ampule and replacing the vacuum. The protecting glass tubing is then replaced.

Not infrequently, especially in children and in obese adults, one fails to enter a vein. Several attempts to do so may result in ruining one or more of the tubes. My colleague, Dr. Alfred Reginald Allen, has devised a useful modification in the technic of using this handy tube; this consisting in detaching the bulb from the rubber tubing and needle, inserting the latter into the vein, and, when the blood appears, quickly attaching the bulb and breaking the neck with a hemostat, in the usual manner. By this method the bulb is not broken until one is sure he has entered a vein and secured a specimen of blood.

(b) Wet Cupping.-1. This method is particularly applicable for securing blood from infants.

2. Cleanse an area over the back just below the angle of the scapula.

3. Scarify with a few superficial linear incisions or with a special scarifier.

4. Apply a cup and exhaust the air with special syringe. The vacuum produces marked congestion of the skin with a ready flow of blood.

5. Carefully release the cup and pour blood into a tube.

6. The apparatus devised by Blackfan, and shown in the accompanying illustration (Fig. 17), is quite satisfactory and collects blood in a sterile tube. 
(c) Placental Blood.-For purposes of immunization corpuscles may be obtained by collecting placental blood.

1. After tying and cutting the cord, the placental end is placed carefully in a 150 c.c. flask or bottle containing from 25 to 50 c.c. of sterile 2 per cent. sodium citrate in physiologic salt solution. To avoid contamination, the cord may be lightly sponged with 1 per cent. formalin solution and severed with sterile scissors.

2. By exerting pressure on the uterus blood may be squeezed out of the placenta. The flask is then sealed with a sterile cotton plug and gently shaken.

3. The corpuscles are obtained by centrifugalization or sedimentation.

\section{METHOD OF SECURING CEREBRO-} SPINAL FLUID (RACHICENTESIS)

The chief purpose in making spinal puncture is to obtain and examine cerebrospinal fluid as an aid to the diagnosis of cerebrospinal diseases. It is mainly of value in neurologic and psychiatric practice, for the purpose of securing fluid for making the Wassermann reaction, for a study of cytologic changes, alterations in protein content, and the like. Not infrequently the procedure is required as an aid to establishing a diagnosis of meningeal diseases in children, particularly tuberculous meningitis, epi-

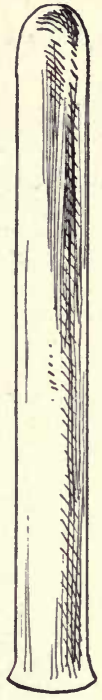

$B$. demic cerebrospinal meningitis, meningeal irritation, "serous meningitis," etc.

Contraindications.-Ordinarily, when skilfully performed, spinal puncture is a harmless procedure. Unless the necessity for obtaining fluid is very urgent, the operation should not be done on persons in poor 
physical condition. Kaplan has cautioned against making lumbar puncture in the presence of tumors of the posterior fossa, particularly of the cerebellum. When it is highly desirable to study the fluid of such cases, 2 c.c. may be withdrawn, and immediately replaced with sterile normal salt solution, or if no immediate effects are observed, the patient may be kept in bed for the next twenty-four hours.

Preparation of Patient.-In bed-fast patients the puncture may be

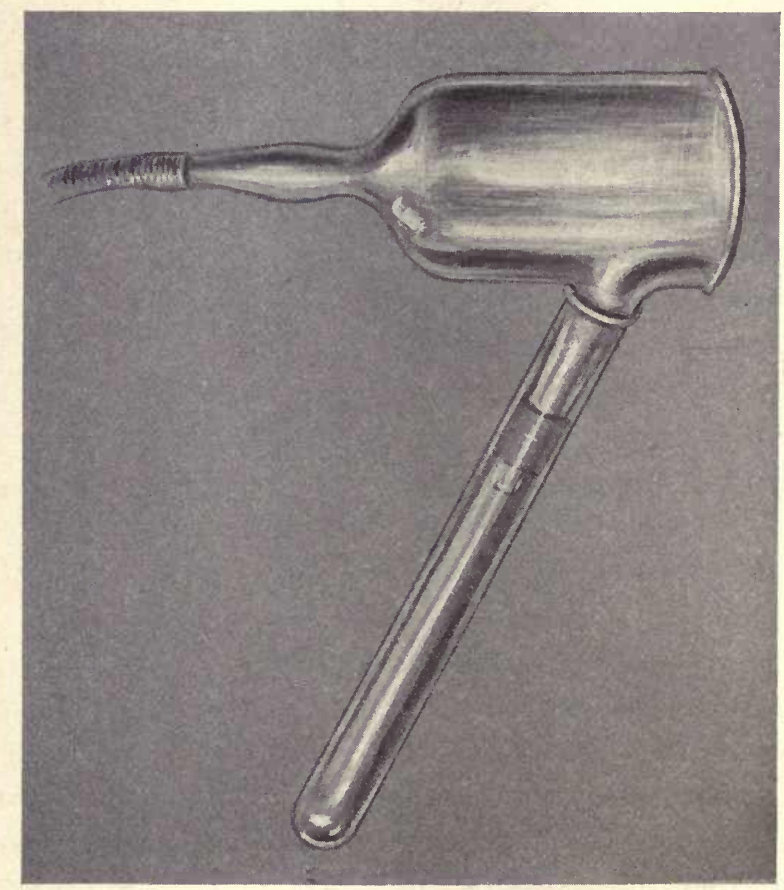

Fig. 17.-A Wet Cup for Securing Blood from Children. (Devised by Blackfan.)

The cup is held in this position over a scarified area; air is exhausted by means of a pump attached to the rubber tubing; blood collects in the small test-tube.

made at any time; with ambulatory patients, however, the most suitable time is late in the afternoon, so as to permit the patient to rest overnight.

The ordinary preparations consist in scrubbing the skin of the lumbar region with green soap and hot water, using gauze sponges, followed by washing with alcohol and ether. The area is then covered with sterile gauze, and, just before the puncture is made, an application of 10 per cent. tincture of iodin is made; or the preliminary cleansing may be 
omitted, two or three coats of the iodin being sufficient. After the fluid has been secured, the iodin may be removed with alcohol and gauze. The operator's hands should be cleansed carefully and washed in alcohol and bichlorid solution or weak formalin, or he may put on sterilized rubber gloves before handling the needle and performing the operation itself.

Anesthesia.-In the majority of instances an anesthetic is not necessary. In tabes dorsalis and general paralysis (two conditions most frequently requiring spinal puncture) the operation is peculiarly painless. Sick children are apparently not greatly disturbed, but in adults it may be necessary to infiltrate the skin about the site of puncture with 1 per cent. eucain (sterile) or cocain solution. Ethyl chlorid is much less satisfactory, except for the mental effect it has upon the patient. Children may receive a few drops of ether. With nervous patients it is good practice to obviate nervous shock by adopting a few simple precautions against causing unnecessary pain.

Technic of Lumbar Puncture.-The patient may either sit in a chair and bend forward, or lie on the left side on the edge of a bed or table. In the case of sick persons, particularly children, the latter position is necessary; it is also advisable with nervous patients, as they are likely to bend backward suddenly or jump up when the needle is inserted, and I have known the needle to be broken off at such a time. (See p. 700.) The back should be arched backward, the patient bending forward, and the knees being drawn up over the abdomen.

The needle should be made of flexible, not rigid, material; for adults, a needle $10 \mathrm{~cm}$. long, having a bore of 1 to $1.5 \mathrm{~mm}$. and furnished with a stilet, will be found satisfactory. For children, a shorter needle may be used, but the bore should be about the same as that used for adults. The needle should be sterilized carefully by boiling in water for several minutes.

The operator now selects a "soft spot" for puncture. By running the finger along the spines of the vertebræ, this will be found to be between the third and fourth lumbar spinous processes, about on a level with the posterior superior spines of the ilia. The needle is grasped firmly and inserted with a sudden thrust, exactly in the median line, and straight forward. The thrust should be sufficient to push the needle through the skin and muscles into the spinous ligaments; it may then be inserted more slowly, a sudden "give way" indicating that the canal has been entered (Fig. 18). This route is better than the lateral route, as there is less danger of striking vertebral processes or 
other obstructions. The stilet is now withdrawn. Usually the first fluid to appear is stained with blood and should be collected in a separate tube. From 5 to 10 c.c. of fluid are then collected in a second sterile tube, the needle is quickly withdrawn, and the puncture wound sealed with collodion and cotton or with adhesive plaster.

It sometimes happens that, on withdrawing the stilet, no fluid issues forth. In this case the patient is instructed to take a deep

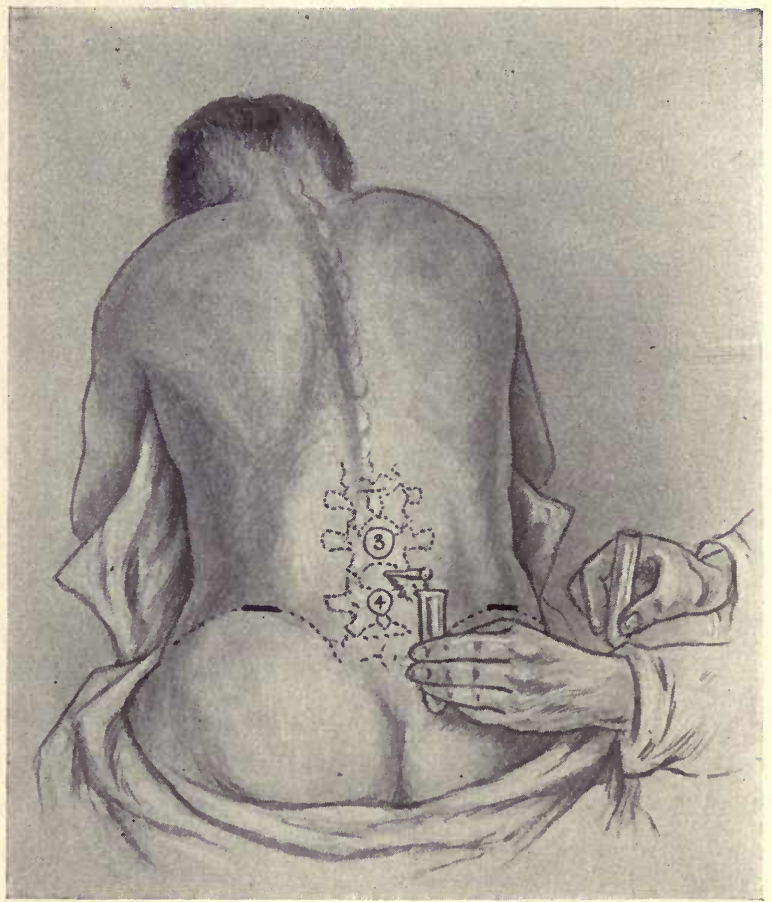

Fig. 18.-Technic of Spinal Puncture.

The patient is sitting on the edge of a chair and is bent forward; the crests of the ilia are indicated by black lines, and are on a level with the spinous process of the fourth lumbar vertebra; the "soft spot" is found just above. The needle is shown in Figs. 137 and 138. The first tube receives the first few drops of fluid, which are usually blood tinged.

breath, and if fluid does not appear now, the stilet may be inserted gently to dislodge any material that may be occluding the needle, or the needle may be withdrawn a trifle if it has been inserted too far, or may be advanced a little if it has not entered the canal. If, however, the tap proves a dry one, or if only a few drops of blood are obtained, it is not advisable to make another puncture, as the second attempt is likely to prove as unsuccessful as the first. 
After-treatment of the Patient.-Occasionally the needle may strike a nerve filament, which occurrence is followed by more or less pain along the course of its distribution; puncture of the bone is likely to be followed by pain for several hours. The majority of patients are so little affected by lumbar puncture that no precautions as regards the aftertreatment are necessary. As previously stated, it is advisable for the patient to rest overnight. Sudden release of pressure or the nervous shock may give rise to severe headache of one or of several days' duration; persons of hysteric temperament may, in addition, suffer from diarrhea and vomiting. Rest in bed, the application of ice-bags, and the administration of sedatives are usually sufficient to relieve these after effects.

Disposal of the Fluid.-As a general rule, the fluid should be sent at once to a laboratory, as total cell counts and bacteriologic cultures are best made with fresh fluid. For the Wassermann reaction it is not advisable or necessary to add a preservative, as the fluid will keep for several days in a good refrigerator; if, however, the fluid is to be kept for longer periods of time, 0.1 c.c. of a 1 per cent. solution of phenol may be added to each cubic centimeter of fluid.

\section{OBTAINING SMALL AMOUNTS OF ANIMAL BLOOD}

Rabbit.-1. Flip an ear vigorously with the hand, and rub with xylol and alcohol. The xylol produces marked congestion and afterward should be carefully removed with alcohol and water, as it produces a low-grade inflammatory reaction.

2. Puncture a marginal vein with a large needle. The blood will flow quickly in drops and practically any amount up to 10 c.c. or even more may be collected in a centrifuge or test-tube (Fig. 19). For making preliminary tests of serum during immunization 2 c.c. of blood is usually sufficient. Bleeding may be checked by making firm pressure over the puncture.

Guinea-pig.-1. Blood may readily be removed directly from the heart by anesthetizing the animal with ether, and inserting a sterile needle into the heart at the point of maximum pulsation. A syringe for aspiration may be attached, but better results are secured by adjusting a suction apparatus. By means of a short piece of rubber tubing the needle may be connected to a test-tube so arranged that a partial vacuum is created by attaching to a water suction pump. As soon as the heart has been entered, blood is seen to flow into the tube 
and the constant suction prevents clot formation in the needle. In this manner 5 c.c. of blood may readily be obtained.

2. Blood may also be secured by aspirating the external jugular vein. The vein is exposed by making a small incision, as in giving intravenous injections.

3. Sufficient blood to make many complement fixation tests may be secured from a large pig by rubbing the ear vigorously with xylol and making a small incision in the margin. Bleeding is facilitated by attaching a small test-tube with a side arm to a suction pump. When the

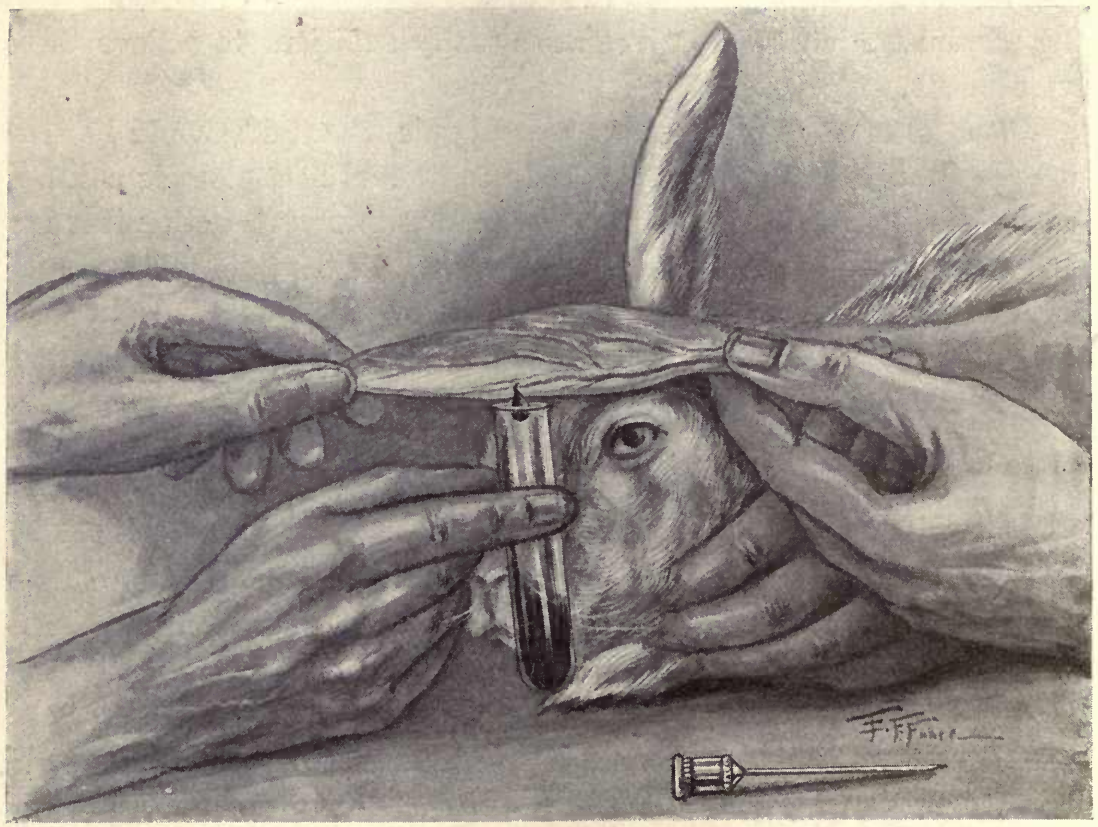

Fig. 19.-Method of Bleeding a Rabbit from the Ear.

proper tube is held firmly over the ear, 5 c.c. of blood may be obtained by this method.

Sheep.-1. Small amounts of blood may be obtained by puncturing one of the ear veins.

\section{OBTAINING LARGE AMOUNTS OF ANIMAL BLOOD}

Rabbit.-After immunization of a rabbit has been completed, the animal is usually bled to death, the object being to secure the maximum quantity of serum in a sterile condition. Various methods may be used. The animal should be anesthetized by ether or high rectal 
injection of a gram of chloral hydrate in 10 c.c. of water, deep sleep being induced by the latter in from five to ten minutes, and lasting for several hours, during which time operative procedures produce no pain.

First Method (Nuttall). - The animal is fastened to an operating board, or, preferably, held by an assistant, and the hair over the neck and thorax is moistened with a 1 per cent. lysol solution.' By means

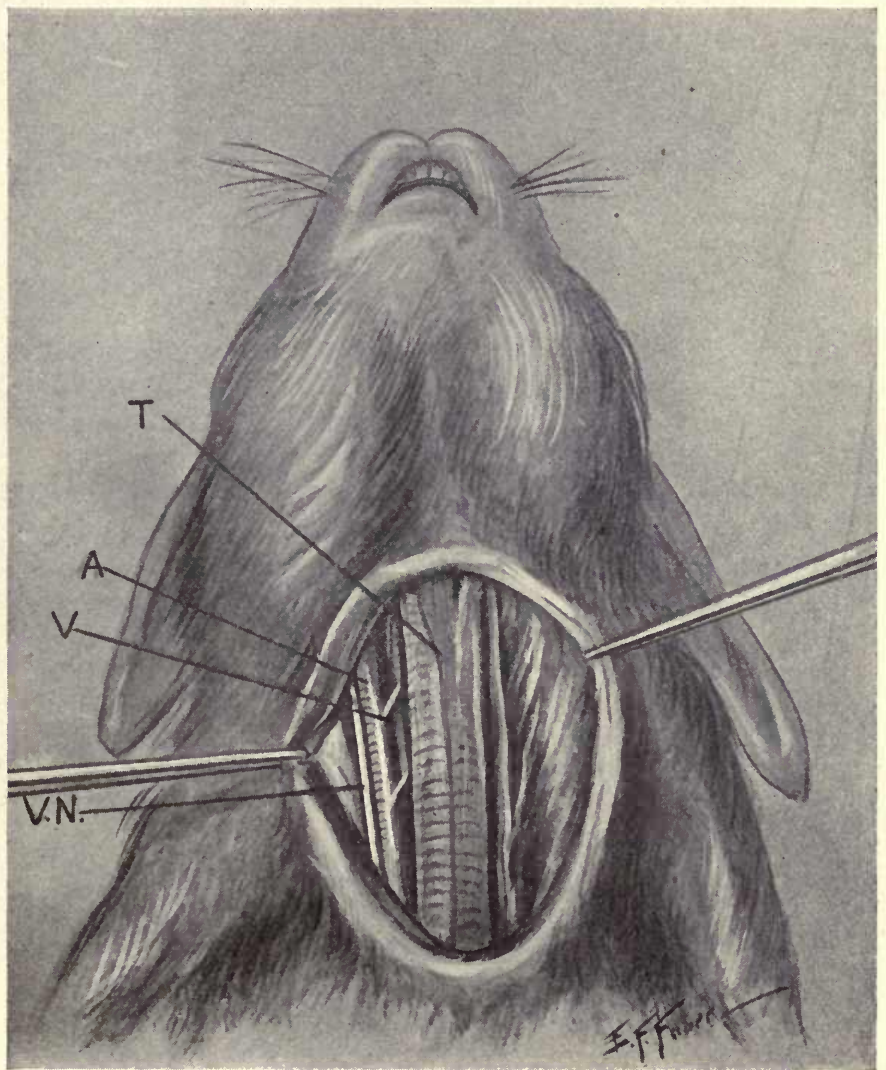

Fig. 20.-A Dissection of the Neck of a Rabbit to Show the Relations of the Carotid Artery.

$\mathrm{T}$, trachea; A, carotid artery; V, internal jugular vein; V.N., vagus nerve.

of a sterile knife the skin is cut longitudinally and the neck muscles exposed for a considerable distance. The animal is then held upright by the assistant over a sterile dish or a large sterile funnel, emptying into a cylinder or 50 c.c. centrifuge tube. The operator stretches the neck by carrying the head backward, and severs the large vessels on one or both sides of the neck with a sharp sterile scalpel or razor, avoiding 
opening the trachea and esophagus. After bleeding, the dish is covered or the tube plugged and set aside for the serum to separate. This method is quite simple, may be employed by the inexperienced, and usually yields a large amount of sterile serum.

Second Method.-The animal is fastened to the operating board and the neck is stretched by placing a roller beneath it. The hair over the neck is clipped close, and the skin moistened with alcohol and 1 per

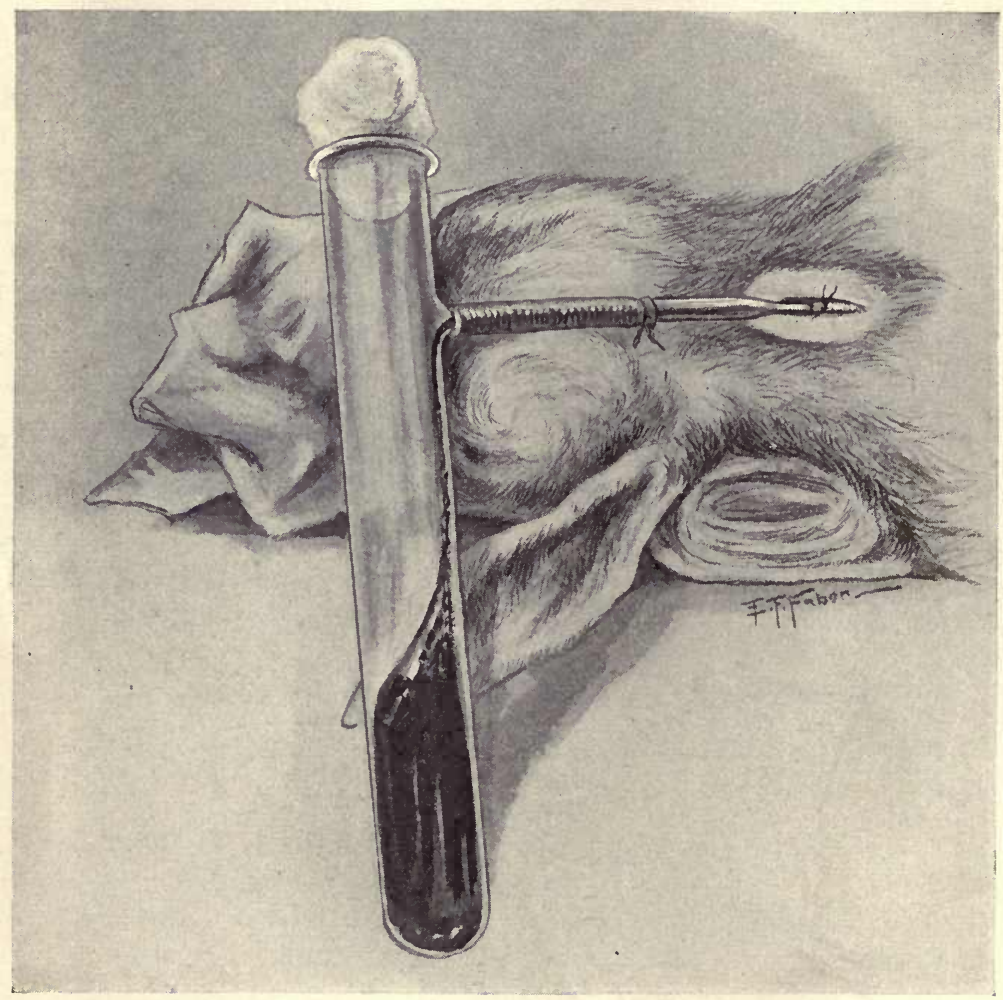

Fig. 21.-Method of Bleeding a Rabbit from the Carotid Artery, SECOND METHOD.

cent. lysol solution. The carotid artery of one side is exposed by making a straight incision through the skin over the trachea and skinning well to one side, exposing the sternohyoid muscles and external jugular vein. The carotid artery, internal jugular vein, and pneumogastric nerve are to be found at the outer border of the sternohyoid muscles (Fig. 20). By means of blunt dissection the artery is exposed and carefully isolated. Two small spring clamps or hemostats are then applied close together 
at the distal end, and the artery divided between them. The proximal end is then held with forceps within the mouth of a sterile cylinder or large centrifuge tube. The wall of the artery is incised with fine scissors proximal to the forceps, and the blood is allowed to flow into the vessel. The yield of blood may be increased somewhat by exerting pressure on the animal's abdomen and thorax.

To avoid the risk of contamination in the foregoing method, the apparatus shown in Fig. 21 may be used. The whole apparatus is

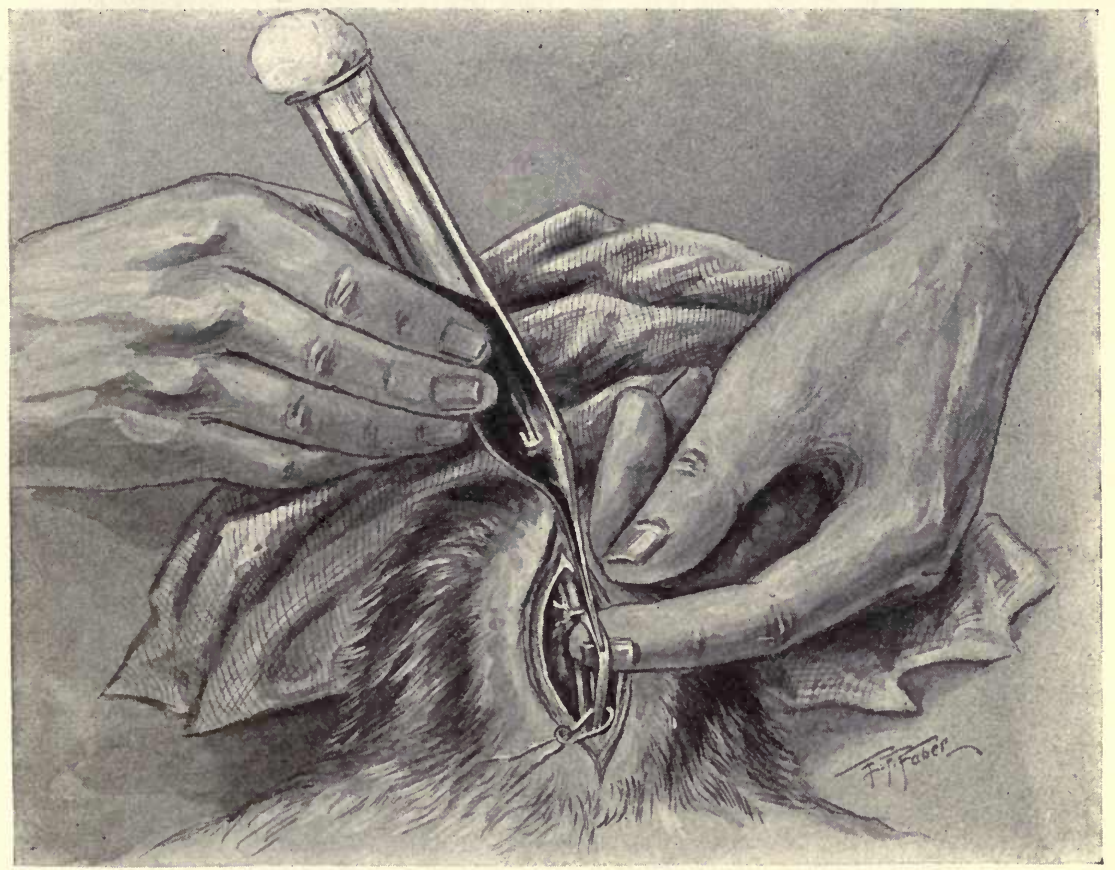

Fig. 22.-Method of Bleeding a Rabbit from the Carotid Artery, ThIRD METHOD.

sterilized in the autoclav before using. After the artery has been exposed and isolated, a temporary clamp is applied to the proximal end. A small incision is made in the wall of the artery, and the cannula inserted and fastened with a ligature. The clamp is then removed, and blood collected in a large tube.

Third Method.-The following method, employed at the Pasteur Institute at Paris, has been found very useful. The animal-a rabbit or a guinea-pig -is anesthetized, and secured to an operating-table. The carotid artery is carefully and aseptically exposed, and separated 
from the tissues for a distance of at least one inch; a ligature is now tied securely about the artery, at the distal end of exposure; a second

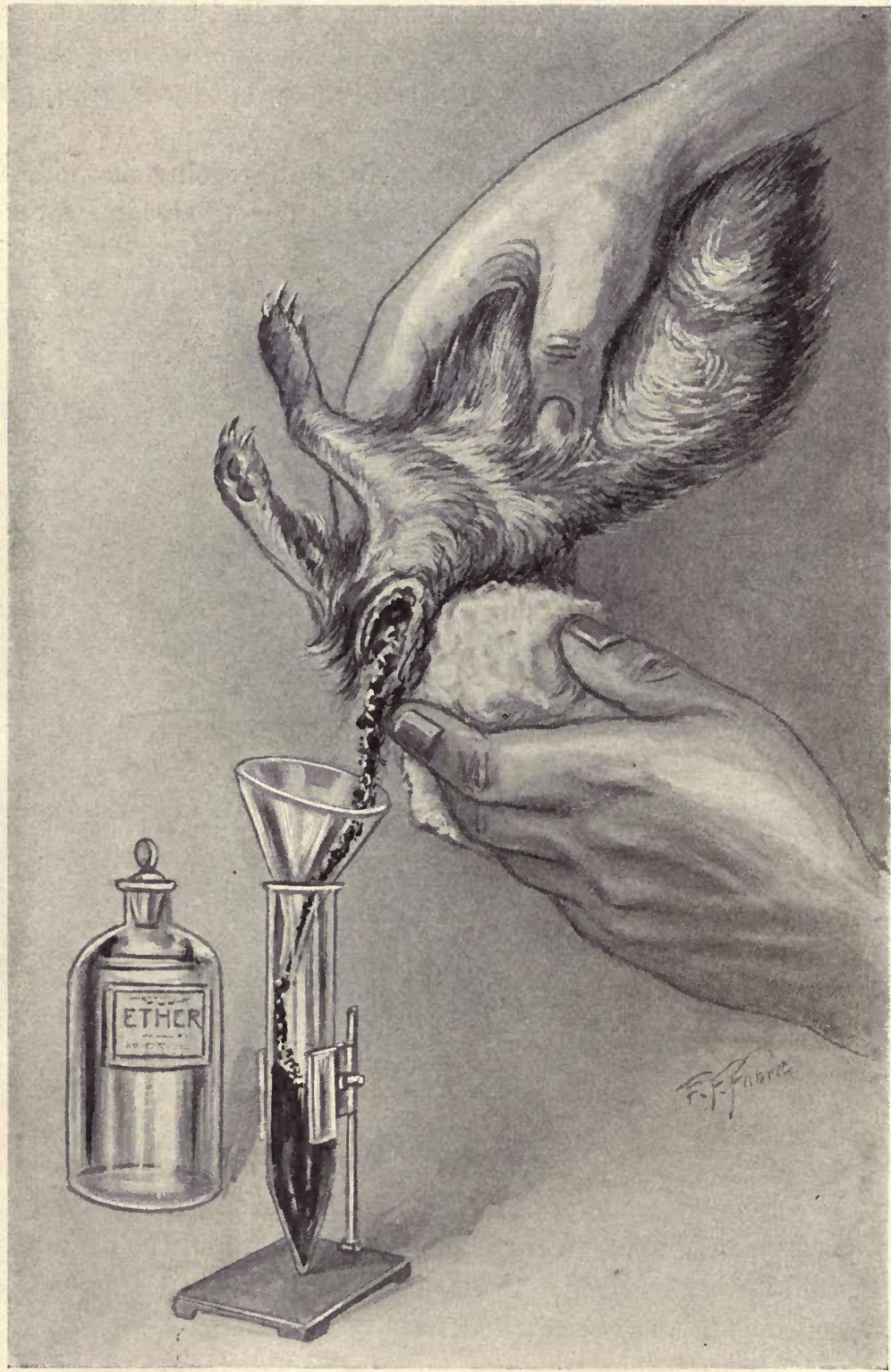

Fig. 23.-Method of Bleeding a Guinea-pig.

ligature is placed in position, and looped loosely, ready to tie about the proximal end (Fig. 22). 
The bottom of a large sterile test-tube is heated and drawn out to a fine point, as shown in the illustration (Fig. 22), and the tip is broken off. The operator now places his moistened forefinger under the artery, elevating it up and rendering it taut; the tip of the tube is then passed through the wall into the interior of the vessel toward the heart. The moment the vessel is entered the blood-pressure drives the blood into the tube, so that 20 c.c. are soon secured. An assistant now ties the ligature below the site of puncture; the tube is withdrawn, and the tip sealed in a flame. The ends of the ligatures are cut short and the wound is stitched. Healing usually occurs at once, and if subsequent study of the blood is required, the other carotid and the femorals can be used similarly for securing it.

Fourth Method.-The animal is fastened to the operating board, and the hair over the neck and thorax moistened with alcohol or lysol solution. The right thorax is then incised and held open by an assistant. The right lung is seized with sterile forceps and quickly severed at the base with sterile scalpel or scissors. The heart is then punctured, and the blood is quickly removed from the thoracic cavity with a sterile 25 c.c. pipet with a large opening. Unless the lung is removed, it tends to float and block the end of the pipet. Everything must be in readiness, as otherwise blood will be lost, flooding the thoracic cavity.

Guinea-pig.-Pig serum is usually secured to furnish complement in hemolytic tests, and should be used within twenty-four or forty-eight hours after bleeding. Precautions to insure sterility are not, therefore, usually necessary.

1. The animal is anesthetized with ether and the large vessels of the neck on one side are exposed by a longitudinal incision. These are severed, and the blood is collected in a Petri dish or in a centrifuge tube by means of a funnel (Fig. 23).

2. By means of a sharp-pointed scissors the vessels on one or both sides of the neck may be incised transversely at one cut, inserting the blade deeply and close to, but avoiding, the trachea and esophagus.

Rats.-1. Small quantities of blood may be obtained by snipping off the tip of the tail of the animal and milking blood into an appropriate sterilized tube containing glass beads, or 2 per cent. sodium citrate solution. In this manner one or more cubic centimeters of blood are easily obtained, and at once defibrinated and injected into the peritoneal cavities of other animals, as in inoculating trypanosomes, etc.

2. Large quantities of blood are obtained by severing the large vessels of the neck, under anesthesia. 
Sheep.-Blood may easily be obtained from a freshly killed animal. The first flow of blood is discarded, and a portion of the remainder is collected in a large, sterile, thick-walled flask containing glass beads

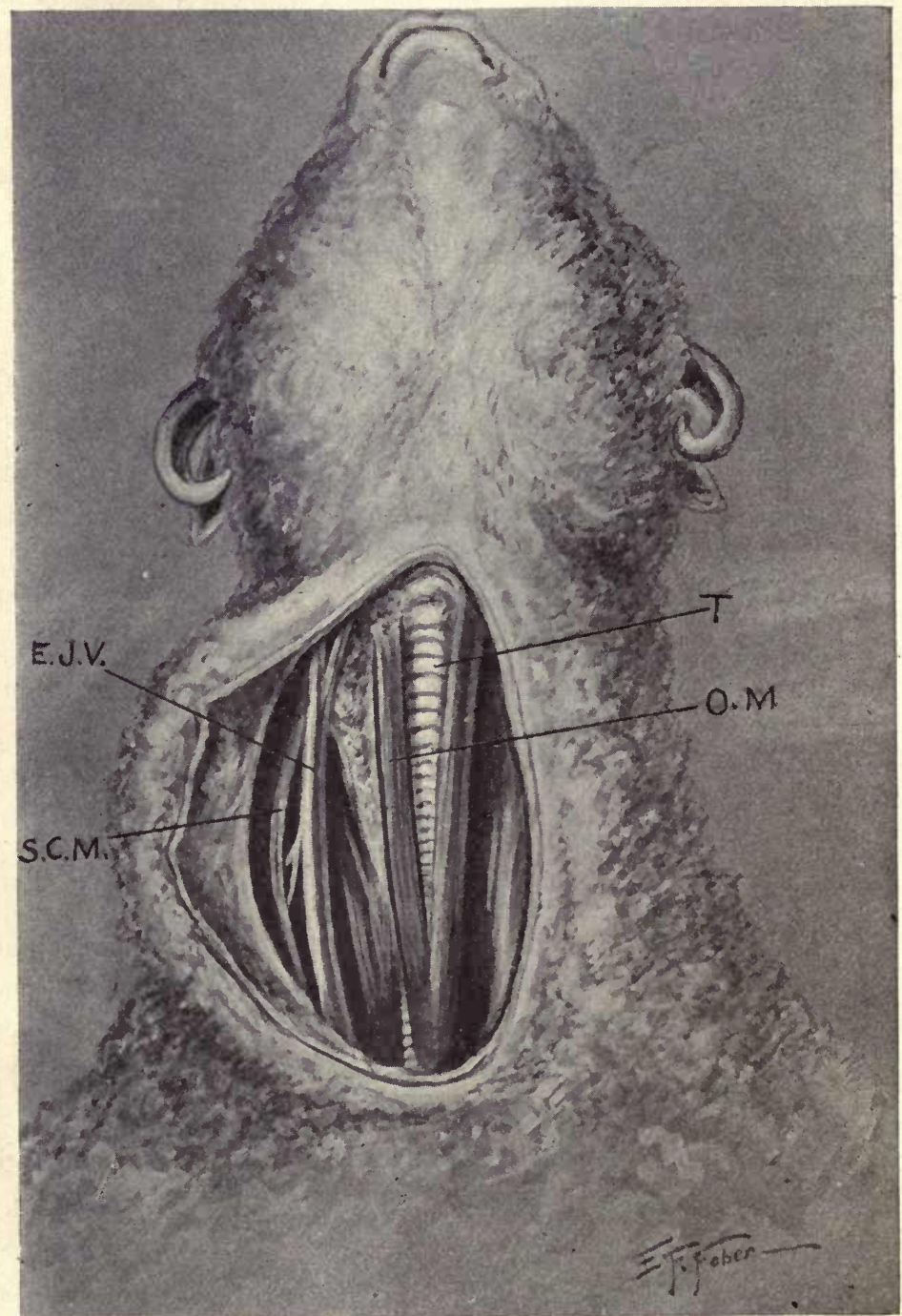

Fig. 24.-A Dissection of the Neck of a Sheep to Show the Relations of the External Jugular Vein.

T, trachea; O.M., omo-hyoid muscle; E.J.V., external jugular vein; S.C.M., sterno-cleido-mastoid muscle. This dissection was made soon after natural death and shows the position and size of the vein with the head held backward as it is when blood is removed according to the technic described in the text. When distended, the vein is even larger than shown; it is quite superficial and is usually palpable when pressure is made over the vein at the base of the neck. 
By shaking vigorously the blood is defibrinated, if one desires to obtain corpuscles, or the blood may be collected in a cylinder and defibrinated by whipping with glass rods.

It is usual, however, in large laboratories, to keep a sheep and remove the blood as it may be required. Small amounts may be obtained from the ear vein, larger quantities being secured from an external jugular vein in the following manner:

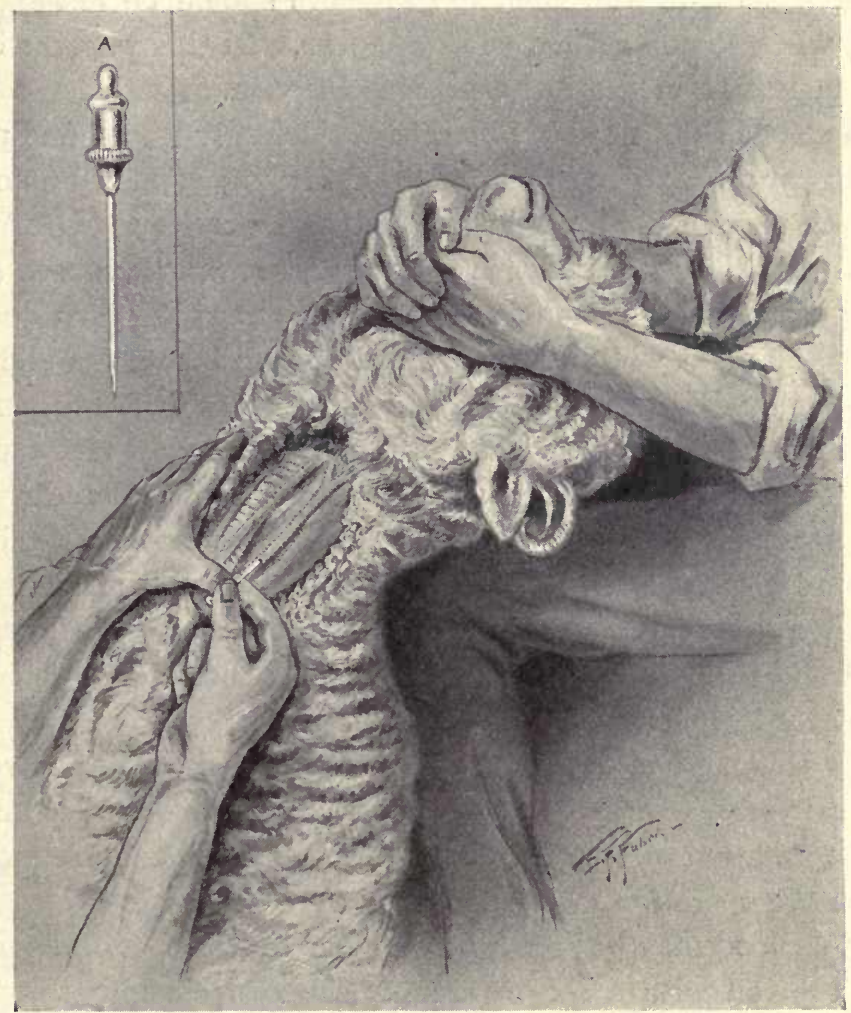

Fig. 25.-Method of Bleeding a Sheep from the External Jügular Vein.

The operator is distending the vein by pressure over the base of the neck with the left hand. When distended, the vein can usually be felt beneath the skin. The needle here shown is reduced to a little more than half the actual size.

1. One may do the bleeding alone, although the aid of an assistant is usually necessary, especially if the animal is large and vicious.

2. The sheep is thrown on its back, and the head is held on the knees of an assistant seated on a low box or stool.

3. The operator may straddle the animal to hold down the fore feet, although this is not necessary unless the animal is vicious. 
4. The wool on the left side of the neck is clipped closely with scissors and alcohol applied.

5. The operator then grasps the neck low down with the left hand, and by means of the thumb exerts pressure over the base of the neck. The external jugular vein will be found in a groove between the omohyoid and sternomastoid muscles (Fig. 24). Firm pressure over the base of the neck usually distends the vein, which may be seen or easily felt. After locating the vein, the pressure should be released for an instant, when the distention will disappear. In this way the operator may be more certain that he has located the vein.

6. A sterile stout needle, at least two inches in length and provided with a trocar and special shank for firm grasping, is passed quickly into the distended vein in an upward and inward direction (Fig. 25). It is essential that the needle be sharp, otherwise it will be turned aside by the wall of the vein. The end of the needle must not have too long a bevel, or the point will pierce the opposite wall before the body of the needle is well within the vein. The trocar is now removed, and blood collected in a flask or bottle and defibrinated with glass beads and rods. A short piece of rubber tubing may be attached to the needle. A suction apparatus is not needed because the flow of blood is good so long as pressure is preserved over the vein at the base of the neck.

7. When the required amount of blood has been secured, pressure is released and the needle quickly withdrawn. Bleeding ceases at once, and the neck is then washed with alcohol.

8. By this method the same vein may be used over and over again for several years. I have never known infection to occur, although the gradual formation of scar tissue about the site of puncture may interfere with the operation.

Hog.-Blood may be secured from hogs by clipping off a small portion of the tail with a sharp razor or scissors, beginning at the tip. Bleeding is usually quite free, but is easily controlled by a tourniquet and bandage. The serum of hogs immunized against hog cholera is secured in this manner.

Monkey.-1. Small quantities of blood-up to 10 or 20 c.c.-may readily be obtained from a small vein just beneath the skin which crosses over the inner malleolus at the ankle. When a tourniquet is applied, the vein becomes prominent; the hair is clipped, and tincture of iodin applied over the skin; a small needle is passed into the vein, and the blood collected in a centrifuge tube.

2. Large quantities of blood may be obtained from the femoral or external jugular vein under light ether anesthesia. 
Dog.-1. Small quantities of blood may be obtained in the following manner: Apply a tourniquet just above the knee; clip the hair over the anterior surface of the leg, and cleanse with tincture of iodin and alcohol; make a small incision in the long axis, exactly in the median line; a fairly large vein appears at once just beneath the skin; by inserting an appropriately sized needle, several cubic centimeters of blood are quickly and easily secured. The wound should be very small, and usually requires no treatment other than an application of collodion and cotton.

2. Large quantities of blood are obtained from the external jugular vein under ether anesthesia; the neck is shaved and cleansed; the skin is incised over the vein, which is just beneath the skin, and blood removed with a sterile needle and syringe. Pressure over the base of the neck renders the vein more prominent. In the case of large dogs, incision is not necessary, as it is easy to enter the vein directly through the skin, as in bleeding the sheep from the external jugular vein or the human from a vein at the elbow. Blood may also be secured from the femoral vein under ether anesthesia.

Horse.-1. Small quantities of blood for making agglutination and complement fixation tests may readily be secured from a superficial vein about the leg. The hair is clipped over the selected area, and the skin sterilized with tincture of iodin. A tourniquet is applied to render the vein prominent, the vessel is steadied between forefinger and thumb, and a needle quickly inserted.

2. Larger quantities of blood are secured from the external jugular vein. This operation is easily conducted in an aseptic manner and blood collected in sterile jars. The neck about the region of the vein, usually on the left side, is clipped, and a large area washed with hot lysol solution. A sterile sheet may be thrown about the shoulders. The animal is held or placed in specially constructed stalls that prevent him from backing away or causing mischief. In large antitoxin laboratories bleeding is conducted in special rooms, where a careful aseptic operating-room technic may be observed.

The external jugular vein is rendered prominent by exerting pressure at the base of the neck by the application of a special tourniquet or by the thumb and fingers of the left hand, the thumb being placed just above the vein. A small incision is made through the skin, directly above the vessel.

A large needle is passed under the skin for a distance of an inch or two and then thrust into the vein. Direct puncture into the vein is 
avoided, as the needle-track under the skin closes after the needle is withdrawn and serves to seal the puncture. The needle is attached to

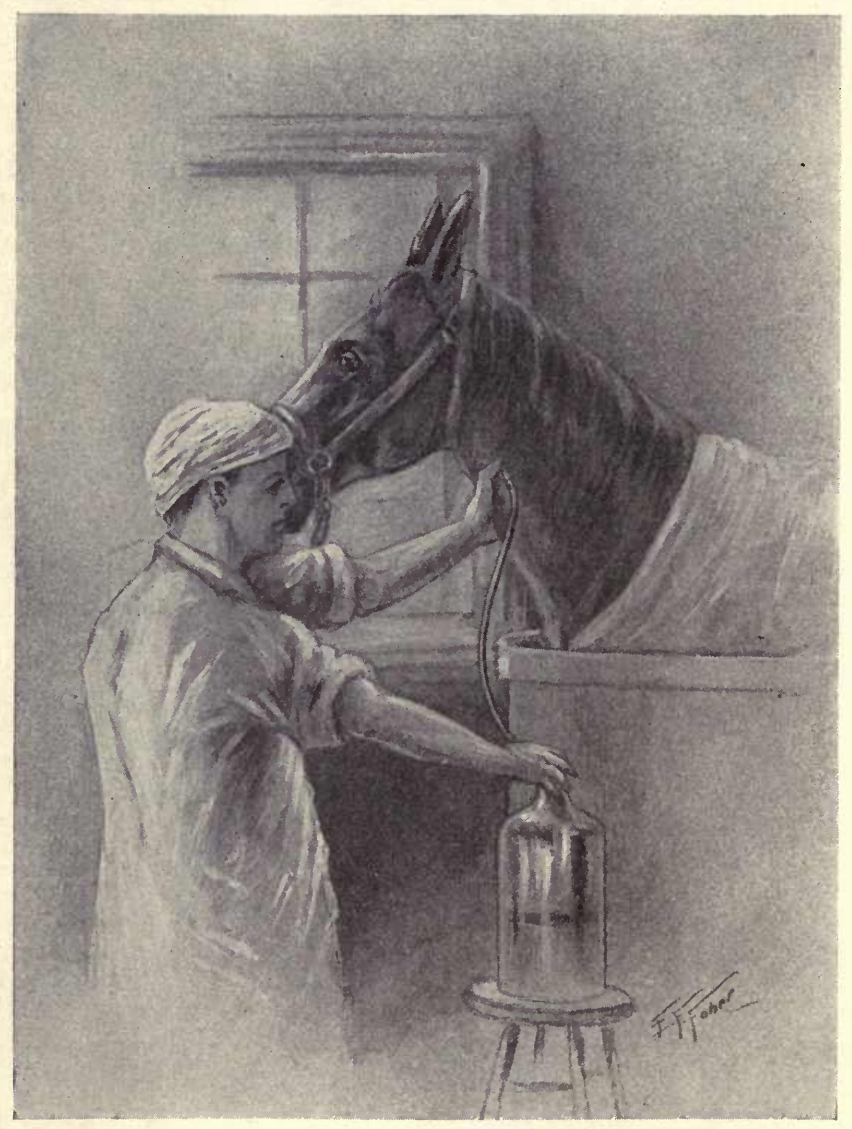

Fig. 26.-Bleeding a Horse from the Jugular Vein.

sterile rubber tubing that conducts the blood into special jars (Fig. 26). In this manner from six to twelve liters of blood are easily obtained. 


\section{CHAPTER III \\ TECHINIC OF ANIMAL INOCULATION}

THIs is a highly important part of immunologic work, as both for serum diagnosis and for serum therapy the serum must be secured from animals that have been artificially immunized. Successful inoculation requires unremitting care and thoroughness, as the toxic effects of the proteins in general may kill an animal before immunization has been completed. No hard and fast rules can be laid down-the weight of an animal and the reaction to an injection should decide the frequency and the size of subsequent injections. It is better to proceed slowly and gradually, than to give too large a dose at once and at too frequent intervals.

\section{GENERAL RULES}

1. Select an appropriately sized syringe that does not leak upon being tested with water. As has been stated elsewhere, nothing is more unsatisfactory than a leaking syringe, for not only may the hand become soiled, but an unknown quantity of inoculum is lost.

2. The inoculum should be sterile. This is especially desirable when giving intravenous and intraperitoneal injections. When living cultures of bacteria are to be injected, the syringe and the needle should be sterilized in order to avoid the introduction of contaminating organisms.

3. Remove the plunger from the barrel, and sterilize all the parts by boiling for at least one minute. As previously stated, an all-glass syringe or a glass barrel and metal plunger is the most satisfactory. (See. Fig. 8.) The old-fashioned syringe with washers and rubbertipped plunger should find no place in a laboratory.

4. After cooling, expel the water and load the syringe. This may be done by drawing the fluid directly into the syringe and measuring the dose by its markings or by pipeting the exact dose into a sterile Petri dish or capsule and drawing up in the syringe.

5. The animal should be fastened or held firmly and in an easy position. Everything should be in readiness, so that the injections may be given thoroughly and carefully. 
6. In preparing the inoculum care should be exercised that no solid particles enter the syringe. Aside from possibly blocking the needle and interfering with the injection, the subcutaneous injection of small fragments may do no particular harm, but in intravenous inoculation they may cause fatal embolism. To obviate this danger the inoculum should, if possible, be filtered through sterile filter-paper before the syringe is filled.

7. Air-bubbles should be removed. The injection of small bubbles of air into subcutaneous tissues may cause no harm, but when injected into veins they may cause serious disturbances or immediate death. To avoid this the syringe, after being filled, should be held vertically, with the needle uppermost. The needle should be wrapped in cotton soaked in alcohol, and the piston of the syringe pressed upward until all the air is expelled from the barrel and the needle. If a drop of inoculum is forced out, it will be collected on the cotton, which should immediately be burned.

8. Injections should be given slowly.

9. The animal is then tagged or marked, or its coloring recorded. In the case of rabbits, the metal ear tag is best. All data, e. g., the date, size of dose, preparation and kind of inoculum, etc., should be recorded in writing.

10. When it is necessary to incise the skin in order to reach a vein an anesthetic may be given. With superficial veins, and in subcutaneous inoculations, the injections may be given so readily and easily that no more pain can be felt than that which accompanies similar injections in human beings.

Animals may be actively immunized in a variety of ways and in different locations in the animal body. For a particular antibody, a certain method may be found especially efficacious, and this is dealt with in a subsequent chapter. In serologic work immunization may be performed by subcutaneous, intramuscular, intravenous, intracardial, and intraperitoneal injections.

\section{METHOD OF PERFORMING SUBCUTANEOUS INOCULATION}

Fluid Inoculum.-1. Injections are usually given in the median line of the abdominal wall.

2. Have the animal (a rabbit or a guinea-pig) held firmly by an assistant or secured to the operating-table.

3. Clip the hair where injection is to be made-it is not always 
necessary to shave the area. Apply a 2 per cent. iodin in alcohol solution.

4. Pinch up a fold of skin between the forefinger and the thumb of the left hand; hold the syringe in the right hand, and insert the needle into the ridge of skin between the finger and thumb, and push it steadily onward until the needle has been inserted about an inch (Fig. 27). Care must be exercised not to enter the peritoneal cavity. Relax the

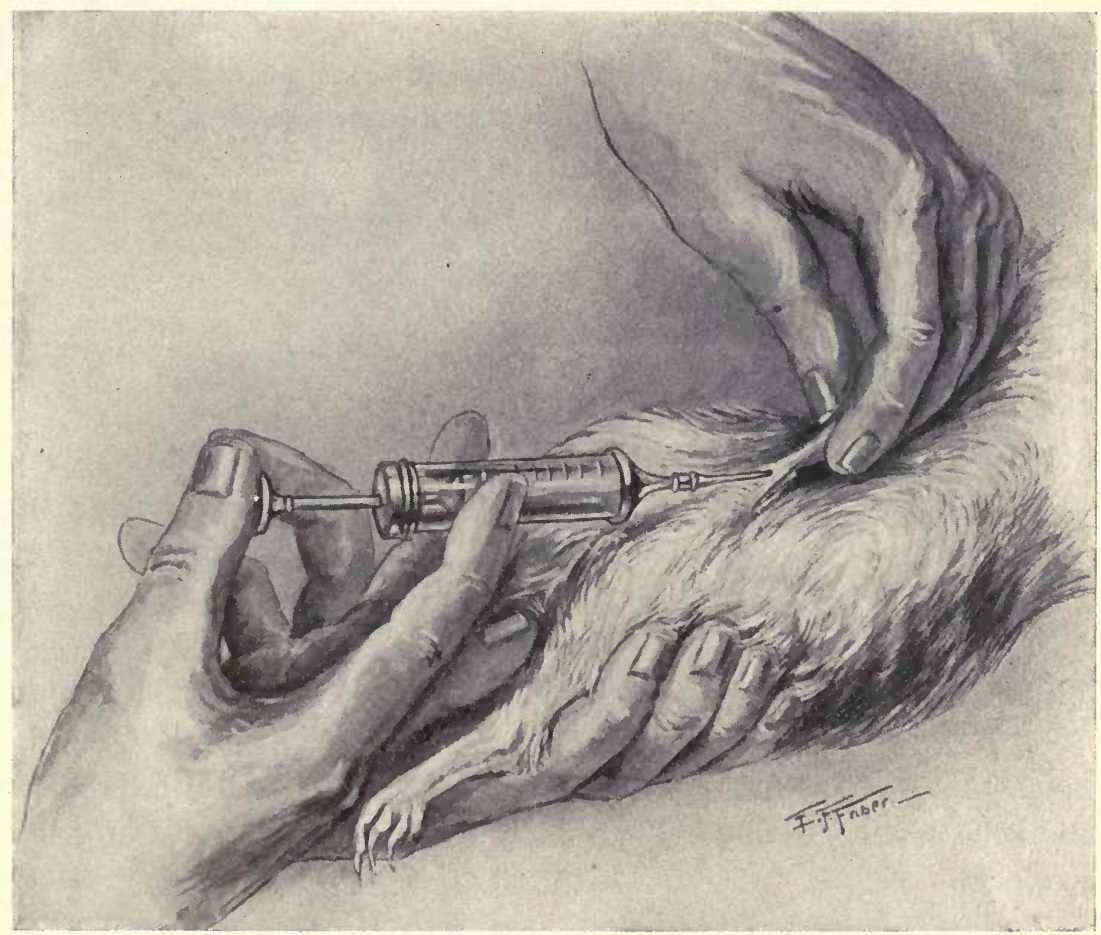

Fig. 27.-Subcutaneous Inoculation of a Guinea-pig.

A fold of skin is pinched up and the needle entered into the fold. The skin is then released, and the injection slowly given. A swelling indicates that the injection is subcutaneous.

grasp of the left hand and slowly inject the fluid. If the skin is raised, this shows that the injection is subcutaneous. If it is not, the needle should be slightly withdrawn and inserted.

5. Withdraw the needle, and at the same time cover the puncture with a wad of cotton wet with alcohol. A touch of flexible collodion over the puncture completes the operation.

Solid Inoculum.- Steps 1 to 3 are the same as in the preceding. 
4. Raise a small fold of skin with a pair of forceps, and make a tiny incision through the skin with a pair of sharp-pointed scissors.

5. With a probe, separate the skin from the underlying muscles to form a funnel-shaped pocket.

6. By means of a fine-pointed forceps or a glass tube syringe introduce the inoculum into this pocket and deposit it as far as possible from the point of entrance of the instrument.

7. Close the wound with collodion and cotton. A single stitch with fine thread may be necessary.

\section{METHOD OF MAKING INTRAMUSCULAR INOCULATION}

1. These injections are usually made into the posterior muscles of the thigh or into the lateral thoracic or abdominal muscles.

2. Clip away the hair over the selected area, cleanse, etc., as for subcutaneous injection.

3. Steady the skin over the selected muscles with the slightly separated left forefinger and thumb.

4. Thrust the needle of the syringe quickly into the muscular tissue, and slowly inject the fluid.

\section{METHOD OF MAKING INTRAVENOUS INOCULATION}

Rabbit. - 1. The posterior auricular vein along the outer margin of the ear is better adapted than a median vein for this purpose.

2. If a number of injections are to be made, commence as near the tip of the ear as possible, as the vein may become occluded with thrombi, and subsequent inoculations may then be given nearer and nearer the root of the ear.

3. The animal should be held firmly, as the slightest movement may result in piercing the vein through and through and require reinsertion of the needle. This is accomplished satisfactorily by placing the rabbit upon the edge of the table and holding it firmly there by grasping the neck and front quarters, the assistant at the same time compressing the root of the ear with the thumb and forefinger.

4. If the hair is long, clip it.

5. The ear is struck gently with the fingers and washed with alcohol and xylol; the friction will render the vein more conspicuous.

6. The ear is grasped at its tip and stretched toward the operator, or the vein may be steadied by rolling the ear gently over the left index-finger and holding it between the finger and thumb. 
7. The inoculum should be free from solid particles, and all the air excluded from the syringe. As a general rule, the amount injected should be as small as possible, and the temperature of the inoculum be near that of the body. If the syringe is filled shortly after sterilization, when it has cooled enough to be comfortably hot to the touch the heat will warm the injection fluid and not be hot enough to cause coagulation.

8. Hold the syringe as one would hold a pen, and thrust the point of the needle through the skin and the wall of the vein until it enters the lumen of the vein (Fig. 28).

9. Direct the assistant to release the pressure at the root of the ear, and slowly inject the inoculum. If the fluid is being forced into the

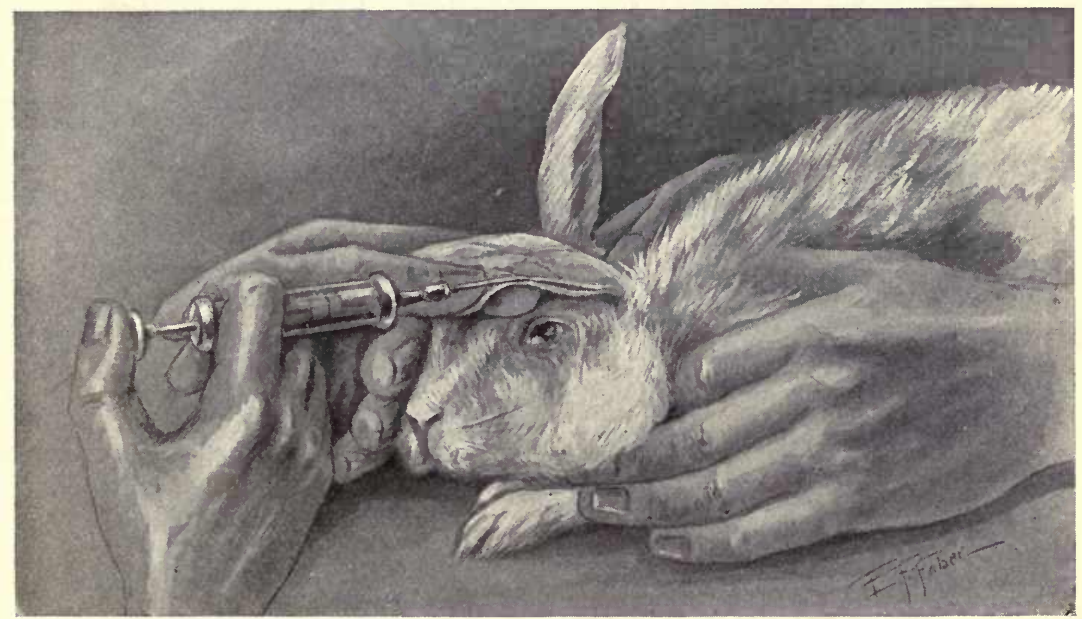

Fig. 28.-Intravenous Inoculation of a RabBit.

subcutaneous tissue, which will be evident at once by the swelling which occurs, the injection must cease and another attempt be made.

10. The needle is quickly withdrawn, a small piece of cotton moistened with alcohol placed upon the puncture wound, and firm compression applied. Wash the ear thoroughly with alcohol and water to remove xylol, otherwise a low-grade inflammation which will render subsequent injections more difficult will follow.

Guinea-pig.-1. Since the superficial veins are quite small, it is necessary to make the injection into the external jugular vein.

2. The animal is tied to the operating-table and the hair clipped away about the neck and shoulder on the right side, and 2 per cent. iodin in alcohol applied. 
3. A small roll is placed under the neck of the animal, to render the operative area tenser and more easily accessible.

4. A few drops of ether may be given by an assistant, although one soon learns to expose the vein quickly and there is practically no pain

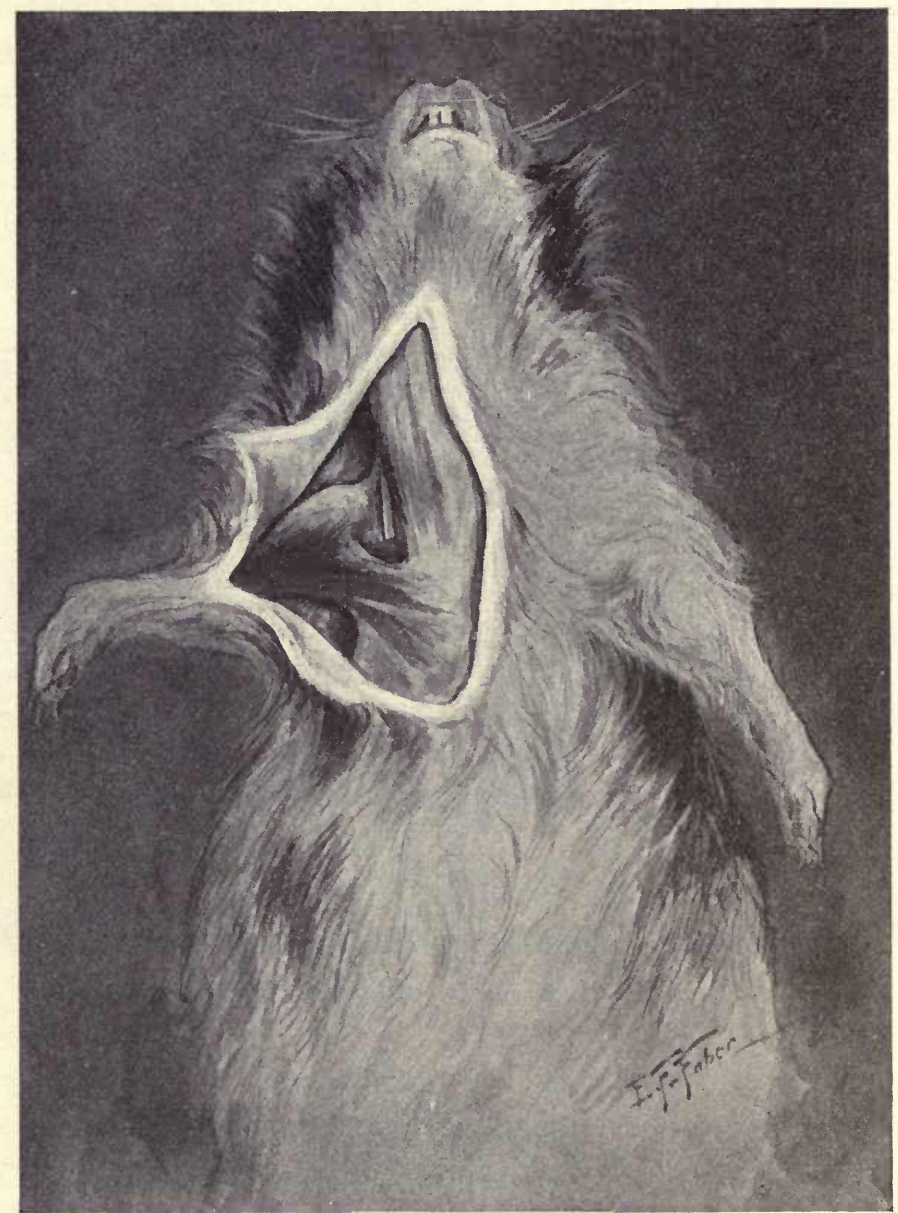

Fig. 29.-A Dissection of the Neck of a Guinea-pig to Show the Relations of the External Jugular Vein.

The skin has been turned aside and the superficial fascia and fat removed; the position of the vein is well shown and is readily exposed by a small and superficial incision.

after the skin has been incised. If anesthesia is employed, it should be just sufficient to overcome the struggles of the animal.

5. The assistant is directed to hold the head backward in the median line. 
6. Pick up the skin just above and in the middle of the space between the shoulder and the tip of the upper end of the sternum-just above and about in the center of the area where a clavicle in the human would be situated. With sharp small scissors incise the skin for about one-third of an inch. Separate the subcutaneous tissue gently with forceps; a large vein at once comes in view (Fig. 29). Gently dissect it free for about one-fourth of an inch.

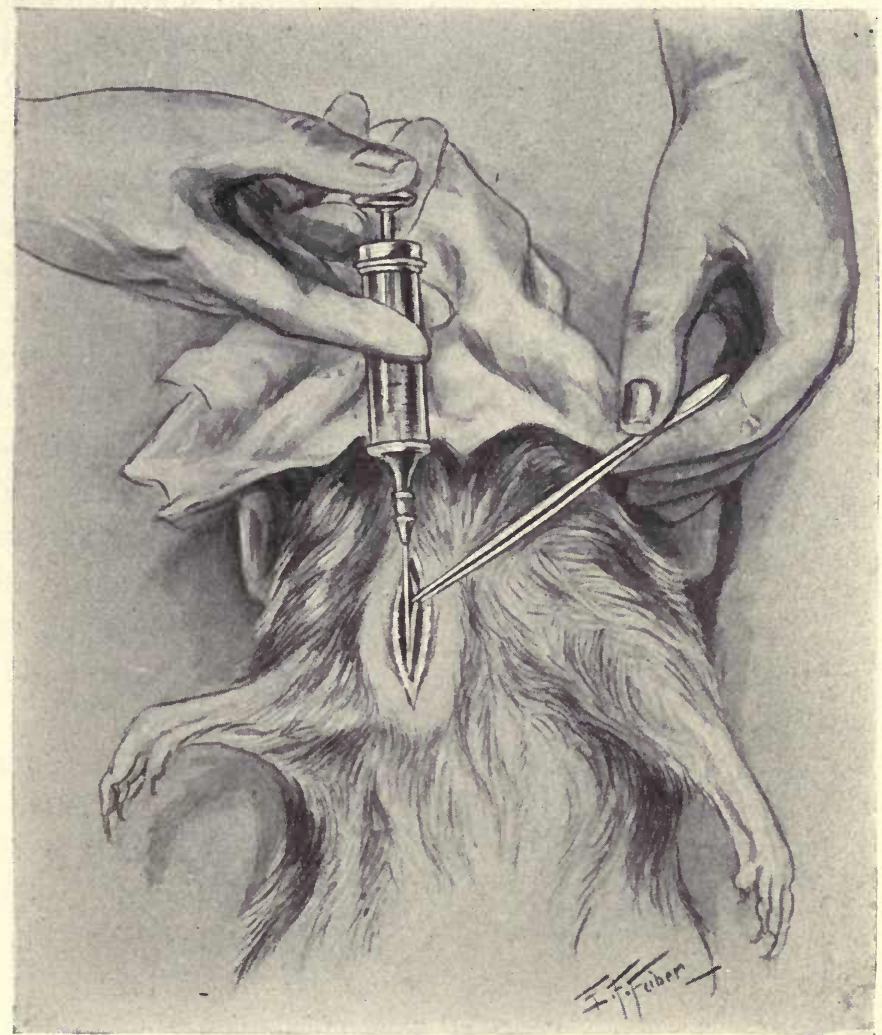

Fig. 30.-Intravenous Inoculation of a Guinea-pig.

The vein is steadied by a pair of fine forceps and the injection given through a small needle. The incision here shown is larger than actually required in practice; the vein is also smaller than normal, as the animal was dead for a few hours prior to making the illustration.

7. Pick up the vein with a pair of fine forceps, insert the needle of the syringe gently in the long axis of the vein and slowly inject the fluid (Fig. 30).

8. Withdraw the needle and apply firm pressure with a wad of clean gauze or cotton. It is not necessary to tie off the vein. A stitch may be inserted to close the skin wound and flexible collodion applied. 
Mice and Rats.-1. Mice and rats may be injected through a caudal vein of the tail. These veins are quite small, and the injection requires a fine needle and some experience in the manipulations.

2. Fasten the mouse

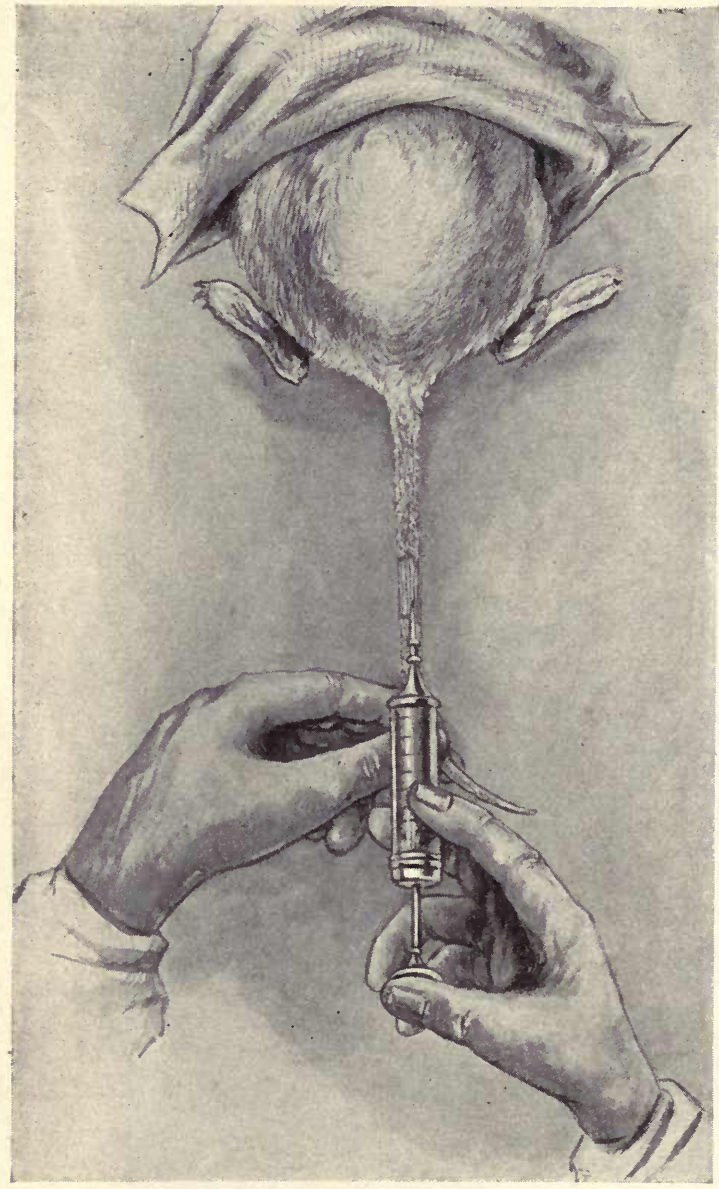

Fig. 31.-Method of Intravenous Inoculation of a RAT.

The hairs and superficial layers of the skin have been scraped away with a scalpel. The vein on each side of the middle line appears as a bluish line in the subcutaneous tissues.

5. Rats may also be injected through the external jugular vein, in exactly the same manner as a guinea-pig is inoculated. (See Fig. 30.) The animal is fastened to a small operating board, and an assistant holds the head to the left, which stretches the tissues of the right shoulder 
and side of the neck. A small incision is made midway between the middle line of the neck and the tip of the fore-shoulder. With superficial dissection a prominent vein appears; this vein is picked up with fine forceps and the injection is readily given through a fine needle.

Horse.-1. Horses are usually injected in the external jugular vein.

2. The hair of the neck in the region of the site of inoculation should be clipped and thoroughly scrubbed with a hot solution of lysol.

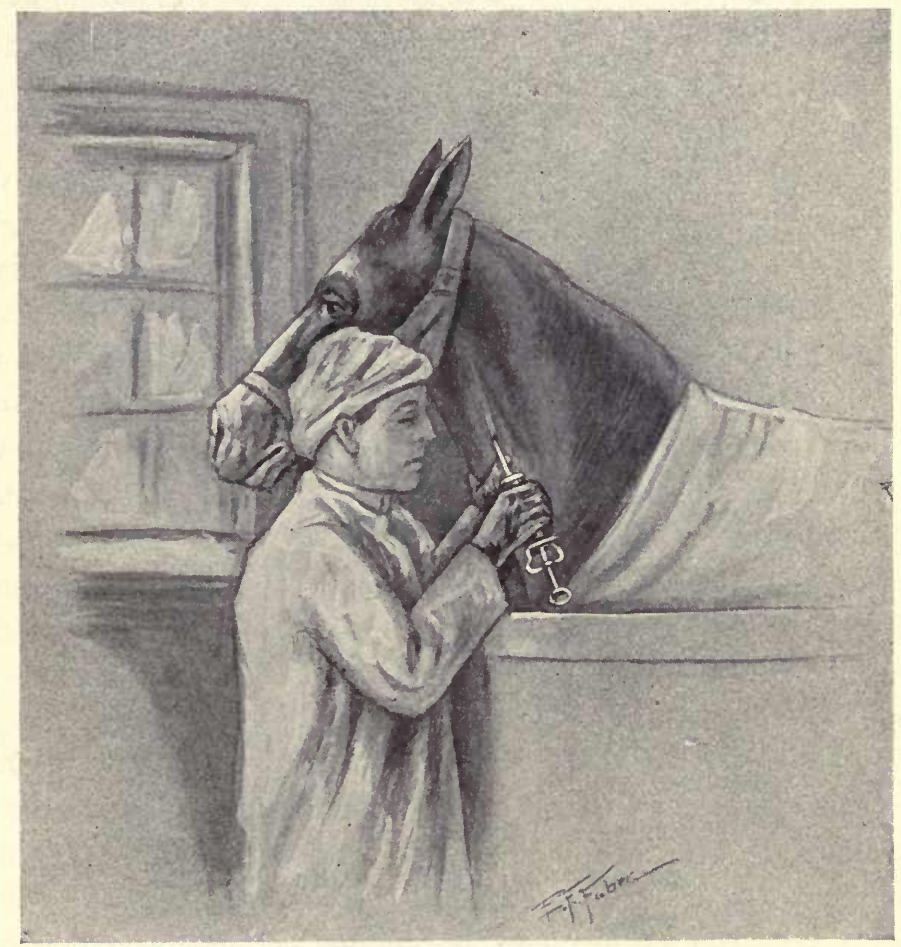

Fig. 32.-Intravenous Inoculation of Horse.

The operator causes the vein to distend and become prominent by pressure with the left hand. The needle is entered beneath the skin and is pushed upward for an inch or more before the vein is entered. When withdrawn, this tunneled passage closes and prevents bleeding. Larger injections may be given in the same manner by gravity.

3. The horse should be held by an assistant; if the animal is vicious, the injections should be given in a specially constructed stall.

4. The vein is distended by the operator, who grasps the region with his left hand, the thumb being directly over the vein (Fig. 32).

5. The needle is inserted beneath the skin, and passed upward for a short distance, and then thrust into the vein. 
6. After the injection has been given, either with a syringe or, when the inoculum is large in amount by gravity from a large jar, the needle is quickly withdrawn. Bleeding ceases as soon as pressure over the vein is removed.

Sheep and Goats.-In sheep and goats the intravenous injection is given into the external jugular vein, directly through the skin. The hair is clipped, and the part shaved and disinfected. Compression by the finger at the root of the neck renders the vein more prominent. Injections are also readily given through a popliteal or a femoral vein. If necessary, a small incision may be made through the skin in order to expose the vein chosen for the injection.

Dog.-Dogs may be injected through the external jugular or popliteal veins. The animal should be fastened to the operating-table.

2. There is a small vein just beneath the skin, in the median line, along the anterior surface of the leg, which is readily accessible. Clip away the hair, and disinfect with iodin and alcohol. Direct the assistant to grasp the thigh just above the knee, to distend the vein and prevent movement, and make a small incision directly in the median line. A small vein is seen at once. Dissect free or pick up gently with fine forceps and insert a small sharp needle. The injection can thus be readily given. Withdraw the needle, apply firm pressure, and insert a single stitch. Bind the wound with a few turns of a gauze bandage or seal with collodion and cotton.

\section{METHOD OF MAKING INTRACARDIAL INOCULATION}

1. Guinea-pigs may be injected by the intracardial route instead of intravenously. The technic is not, as a rule, more difficult, and no ill effects are noticed. Not infrequently, however, attempts to inject in the heart fail, and frequent trials are not permissible on account of the danger of injuring the organ.

2. The animal is tied to the operating board, or held firmly by an assistant; an anesthetic may be given.

3. Determine the point of maximum pulsation to the left of the sternum by palpation, and quickly insert a thin, sharp needle at the selected area. A flow of blood indicates that the needle has entered the heart. Attach the previously filled syringe and slowly inject the contents.

4. Detach the syringe in order to make sure that the injection was intracardial, as intended, which is indicated by a flow of blood; then quickly withdraw the needle. The puncture wound may be sealed with collodion. 


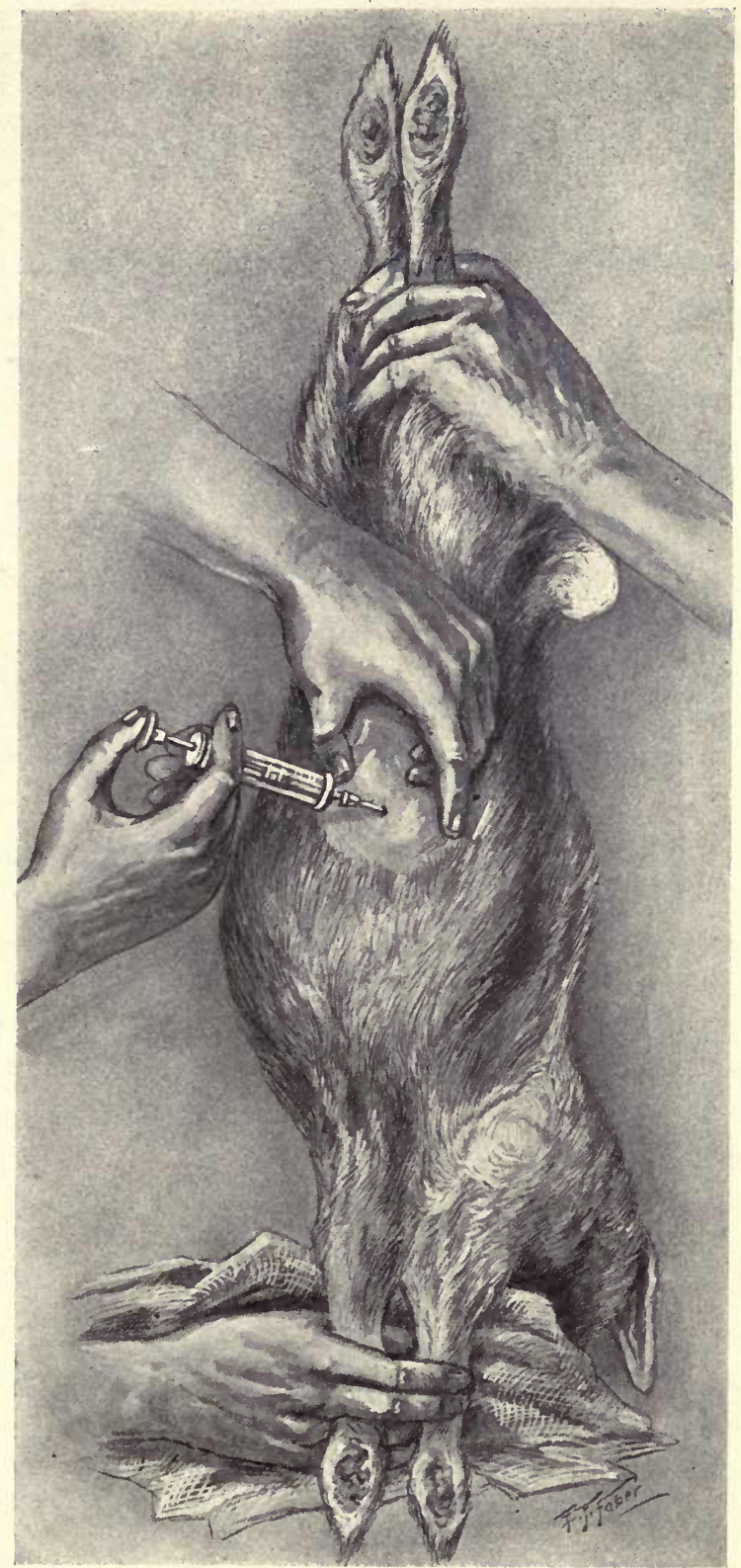

Fig. 33.-Method of Performing Intraperitoneal Inoculation of a Rabbit. The head is held downward; the intestines gravitate toward the diaphragm (note distention); this leaves an area between the umbilicus and pelvis relatively free of intestines and lessens the danger of puncturing the intestines. 


\section{METHOD OF MAKING INTRAPERITONEAL INOCULATION}

Rabbit.-1. Clip the hair and shave an area about two inches in diameter in the median abdominal line, just below the umbilicus. Apply 2 per cent. iodin in alcohol.

2. Direct an assistant to hold the animal firmly, head down. With the animal in this position the loops of intestine tend to sink toward the diaphragm, leaving an area above the bladder which is sometimes free of intestines (Fig. 33).

3. The syringe is grasped firmly, and the needle inserted beneath the skin for a short distance, in the direction of the head in the long axis of the animal when the hand is raised and the needle forced forward through the peritoneum. When the peritoneum has been entered, this is evidenced by a relaxation of the abdominal muscles. The needle is then withdrawn slightly and the injection made.

Guinea-pig.-1. Direct an assistant to hold the animal firmly upon its back. This is better than fastening it to an operating-table, for it permits relaxation of the abdominal wall when the injection is to be made.

2. Clip the hair close to the skin in the median abdominal line. A small area may be shaved although this is not necessary. Disinfect with an application of iodin in alcohol.

3. With the left forefinger and thumb pinch up the entire thickness of the abdominal parietes in a triangular fold, and slip the peritoneal surfaces over each other to ascertain that no coils of intestine are included.

4. Grasp the syringe in the right hand, and insert the needle into the fold near its base.

5. Release the fold and inject the fluid. If a swelling forms, this shows that the needle is in the subcutaneous tissues, and another attempt should be made to enter the peritoneum.

6. It may be difficult to pinch up the parietes without including the intestine. In such case straighten out the animal and stretch the skin between the left forefinger and thumb. Insert the needle obliquely until it is beneath the skin. A slight thrust suffices to pierce the peritoneum, when the abdominal muscles will be felt to relax. Withdraw the needle slightly and inject the fluid.

7. Seal the wound with a touch of collodion. 


\section{CHAPTER IV}

\section{METHODS FOR EFFECTING ACTIVE IMMUNIZATION OF ANIMALS}

IN overcoming an infection, acquired either naturally or artificially, the macroörganism develops antibodies, or protective substances, against the infecting agent. Possessed of these antibodies, the animal can subsequently withstand a more severe attack of the same infection. Thus, the body-cells of the animal itself are actively concerned in producing these antibodies, and the resulting protection or immunity is therefore called "active immunity."

The general term "antigen" has been applied to any substance that can stimulate the formation of an antibody. The immunity following scarlet fever is an example of active acquired immunity, although the antigen is unknown. In order to acquire immunity it is not always necessary for a person to have had the disease. Thus, a severe infection with the antigen of typhoid fever-Bacillus typhosus-results in a general reaction, exhibiting symptoms and course known clinically as typhoid or enteric fever; whereas, if the antigen is attenuated and injected artificially in small doses in the form of a vaccine, the body-cells react, producing antibodies and a resulting immunity against typhoid fever, without discomfort or danger to life. This process is known as vaccination, the term being first applied to a similar procedure employed in inducing an immunity against smallpox by the inoculation of a small dose of the antigen attenuated or modified by passage through the cow (cow-pox virus).

This process of stimulating the body-cells to produce antibodies is called active immunization, and, while the immunity following disease is an example, the term is generally applied to artificial immunization, as in vaccination, which serves in medicine, therefore, the primary purpose of effecting prophylaxis. In laboratories active immunization of animals is generally undertaken with a view to obtaining serums to be used for diagnostic or therapeutic purposes.

Practically, any protein may serve as an antigen. Thus, animals may be immunized not only with various bacteria, but with serum albumins and globulins, milk, egg-albumen, epithelial cells, etc. It must 
be remembered that these substances - the antigens-are toxic, and that the process of immunization may evoke a marked disturbance in the general health of the animal. Special attention should be given to feeding and the general care of the animal, the temperature, weight, the presence of diarrhea, and the occurrence of abscesses, edema, paralysis, etc.

If the animal dies, a careful postmortem and bacteriologic examination should be made, in order to study the changes produced by the inoculated antigen, and to ascertain if death was induced by the antigen or by contamination and secondary infection.

In the manufacture of serums on a large scale, especially for therapeutic use, horses are used almost exclusively. For diagnostic purposes and especially in the study of immunity, smaller animals, such as rabbits, guinea-pigs, white mice, and rats, and occasionally goats or sheep, are employed.

So far as their power of producing antibodies is concerned, there are individual differences among the same species of animals; thus, horses immunized against diphtheria differ in the quantity of antitoxin produced. Similar differences are observed in the smaller animals.

Immunization with a single antigen usually produces several different antibodies, although for diagnostic or therapeutic purposes one usually predominates. Thus immunization of a rabbit with dead typhoid bacilli produces agglutinin, opsonin, bacteriolysin, and complement-fixing bodies, although the agglutinin is probably the most prominent and is used in diagnosis. Immunization with the diphtheria bacillus and its toxin leads to the formation of an antitoxin, opsonin, and complement-fixing body, although antitoxin is by far the most prominent and is used therapeutically.

We give here methods for making various immune serums from small animals, to be used for the purpose of study and for aiding diagnosis. Curative serums, such as diphtheria and tetanus antitoxin, antimeningococcus serum, etc., are made on a larger scale by immunizing horses.

\section{- GENERAL TECHNIC}

1. The antigens are usually injected subcutaneously, intramuscularly, intraperitoneally, or intravenously. As a rule, the sooner the antigen comes in relation with the body-cells, the more rapid is the immunity gained, and for this reason the intravenous and intraperitoneal routes are frequently chosen. 
2. No fixed rules as to the amount to be injected can be given. Experience may show a general method to be the most successful, but as previously mentioned, the general condition and reaction of the animal will be the main guide as to the amount and frequency of the injections. Severe reactions may yield unsuccessful results, and doses so small as apparently to give no reaction may lead to a high-grade immunity.

3. A single injection seldom yields a highly valent serum. Repeated inoculations are usually necessary, and may be given in the following way:

(a) A small dose of antigen is injected. If a reaction sets in, wait until this has subsided, and then, after the fifth day, make a second injection of a somewhat larger dose. After another interval of from five to seven days a third injection of a still larger dosage is administered, and so on for two or more injections.

(b) Same as preceding method, except that the first dose is the maximum one; subsequent injections are of decreasing amounts.

(c) Same as preceding, with a constant dose of antigen at each injection which does not produce a severe reaction.

(d) For several successive days a small or medium-sized dose of antigen is injected (Gay).

The first three methods give excellent results, and the third is especially useful when a serum is needed as soon as possible.

4. It must be emphasized that good results are largely dependent on the care with which the animals are injected. The operator should work as aseptically as possible, especially when giving intraperitoneal and intravenous injections, and avoid the production of embolism by the injection of air or solid particles. Give the injections slowly, and take particular care of the animals. However carefully the injections may be given, unsatisfactory results not infrequently occur. Either the animal refuses to react with the production of antibodies, or dies just when immunization is about completed. It is, therefore, good practice to immunize more than one rabbit at one sitting, in order that immune serum may be had at the time planned.

Antigens.-Animals may be actively immunized with the following substances:

1. With soluble bacterial toxins, as in the manufacture of antitoxins.

2. With bacteria themselves; whether living, attenuated, or dead, as in making bacterial agglutinins, precipitins, immune opsonins, and lysins (bacteriolysins). 
3. With soluble alien proteins, as serum, milk, egg-albumen, etc., as in the manufacture of precipitins.

4. With various alien cells, as erythrocytes, kidney cells, spermatozoa, etc., in the manufacture of cytotoxic serums, such as hemolysins, nephrotoxin, spermatotoxin, etc.

\section{PRODUCTION OF ANTITOXIN}

Diphtheria and tetanus antitoxins are prepared on a large scale by the inoculation of horses with increasing doses of the respective toxins.

The methods for preparing these antitoxins, and also of antimeningococcus, antistreptococcus, antipneumococcus, and other curative serums are given in the chapter on Antitoxins and Passive Immunization.

\section{PRODUCTION OF AGGLUTININS}

Agglutinating serums are frequently of much value in making a bacteriologic diagnosis of typhoid fever and cholera. For this purpose unknown microörganisms are mixed with proper dilutions of known immune serum, and the presence or the absence of agglutination noted. As a rule, rabbits are used in the preparation of these serums; for the production of larger quantities of serum, goats and horses are occasionally employed.

The injections may be given intravenously or intraperitoneally; occasionally the first injections are given subcutaneously, to be followed later by intraperitoneal injections. By heating the cultures at a temperature not exceeding $60^{\circ} \mathrm{C}$. for from one-half to one hour, there is less danger in the subsequent handling of the cultures and agglutinins are readily produced.

1. Use forty-eight-hour agar cultures of the organism, such as Bacillus typhosus, Spirillum choleræ, etc. Bouillon cultures may be used, but are not recommended on account of the various other constituents present in the medium.

2. With a sterilized three-millimeter platinum loop remove one loopful of culture and rub up in 2 c.c. of sterile salt solution in a small test-tube until a homogeneous emulsion is secured.

3. Heat the emulsion for thirty minutes at $60^{\circ} \mathrm{C}$. in a water-bath.

4. Inject intravenously.

5. Give four more injections at intervals of a week as follows:

Second dose: 2 loopfuls in 2 c.c. $\mathrm{NaCl}$ solution, heated.

Third dose: 4 loopfuls in 2 c.c. $\mathrm{NaCl}$ solution, heated.

Fourth dose: 6 loopfuls in 2 c.c. $\mathrm{NaCl}$ solution, heated.

Fifth dose: 1 agar slant in 4 c.c. $\mathrm{NaCl}$ solution, heated. 
6. One week after the last injection has been made the blood is tested, and if found of satisfactory titer, the animal is killed and the serum secured.

Intraperitoneal Method (Rabbit).-1. Same as the preceding, excepting that larger doses are given.

First dose: 2 loopfuls in 4 c.c. $\mathrm{NaCl}$ solution, heated.

Second dose: 4 loopfuls in 4 c.c. $\mathrm{NaCl}$ solution, heated.

Third dose: 6 loopfuls in 4 c.c. $\mathrm{NaCl}$ solution, heated.

Fourth dose: 1 agar slant in 5 c.c. $\mathrm{NaCl}$ solution, heated.

Fifth dose: 1 agar slant in 5 c.c. $\mathrm{NaCl}$ solution, heated.

2. The blood is tested one week after the last injection has been made.

\section{PRODUCTION OF IMMUNE OPSONINS}

1. These may be produced in the same manner as the agglutinating serums, immune opsonins being readily demonstrated in the same serums. For actual diagnostic work, artificial immune opsonins are seldom required, but to secure an immune serum for experimental studies on opsonins a culture of Staphylococcus pyogenes aureus may be used in immunizing a guinea-pig as follows:

First dose: 1 loopful of twenty-four-hour agar culture in 2 c.c. $\mathrm{NaCl}$ solution heated for one-half hour at $58^{\circ} \mathrm{C}$. and given subcutaneously.

Second dose: 1 loopful in 2 c.c. $\mathrm{NaCl}$, heated; intraperitoneally.

Third dose: 2 loopfuls in 2 c.c. $\mathrm{NaCl}$, heated; intraperitoneally. Fourth dose: 3 loopfuls in 2 c.c. $\mathrm{NaCl}$, heated; intraperitoneally.

Fifth dose: 6 loopfuls in 2 c.c. $\mathrm{NaCl}$, heated; intraperitoneally.

2. Bleed the animals one week after the last injection has been made.

3. Owing to its large size, Bacillus anthracis may be substituted. This is a spore-forming organism, and since it is dangerous unless scrupulous care in handling is exercised, it is not usually wise to employ it in experimental work.

\section{PRODUCTION OF BACTERIOLYSINS (BACTERIOLYTIC SERUM)}

1. These are prepared in exactly the same manner as agglutinins. In practical diagnostic work the Spirillum choleræ is most frequently used. In experimental studies of bacteriolysis the typhoid bacillus and its immune serum may be employed with equal success. 


\section{PRODUCTION OF PRECIPITINS}

Precipitin immune serums are frequently of value in making a differentiation of the proteids, as in the examination of blood-stains, meat, milk, cheese, etc. They are usually prepared by immunizing large rabbits with injections of the sterile antigen. Injections may be given intravenously or intraperitoneally, the former usually yielding the more potent serums.

Any foreign serum may be used in the preparation of precipitins, such as that of the human, horse, ox, dog, cat, guinea-pig, etc. To produce an antirabbit precipitin a guinea-pig is immunized by intraperitoneal injections of rabbit serum. An antihuman precipitin serum of high titer is usually obtained with difficulty. It is good practice to immunize a number of rabbits with each antigen, as some animals will produce no precipitin whatever the method used.

In preparing precipitins for the purpose of identifying blood-stains whole blood may be injected. It is better, however, to use serum only, as the immune serum may be used in diagnosis, according to the method of complement fixation, when the presence of hemolysin is not advisable.

Serum Precipitins (Intravenous Method).-First Method.-Three injections are given - of 5,10 , and 15 c.c.- on each of three successive days, and the animals are bled twelve days after the last injection has been made.

Second Method.-One injection of 30 c.c. of serum may be given, and followed twelve days later by bleeding.

Third Method.-A slower method consists in giving the injections at intervals of a week. After the third dose a few cubic centimeters of blood are withdrawn from the ear, and the serum titrated, as rabbits are most prone to succumb after the third dose, and in many instances the serum is of such strength as to require no further immunization. The animals are bled one week after the last injection has been given.

Doses may be given as follows:

First dose: 10 c.c. serum intravenously.

Second dose: 8 c.c. serum intravenously.

Third dose: 5 c.c. serum intravenously.

Fourth dose: 5 c.c. serum intravenously.

Fifth dose: 3 c.c. serum intravenously.

Fourth Method.-Rabbits may be immunized by making intraperitoneal injections after any of the foregoing methods, and with the same or slightly larger doses. 
Milk Precipitins (Lactoserums).-These are prepared by immunizing large rabbits with intravenous or intraperitoneal injections of milk, that of either the human or the lower animals. Rabbits should be immunized with at least two kinds of milk in order to obtain different lactoserums for the study of specificity. The milk used for the injections should be as sterile as possible, and if heated to $56^{\circ} \mathrm{C}$. for one-half hour before the injections are made, the protein remains unchanged and the rabbits are less likely to succumb. Animals may be immunized in the same manner and with the same sized doses as were directed for the preparation of serum precipitins.

Bacterial Precipitins.- It is usually customary to differentiate between the bacterial and the protein precipitins, but for practical purposes this division is superfluous, as the bacterial precipitins are simply antiserums prepared by immunization with bacterial protein.

\section{PRODUCTION OF HEMOLYSINS}

Because of their use in the serum diagnosis of syphilis and other infections, hemolysins possess great practical value. They are best produced by injecting rabbits with washed human or sheep erythrocytes, or with those of some animal of another species. Rabbits differ considerably in their power to form hemolysins, and for some unknown reason hemolysins are more readily produced with some erythrocytes than with others. It is not possible, however, to immunize every kind of animal against every type of erythrocyte. As a general rule, an animal produces a better hemolysin the more remote its relationship is to the animal from which the erythrocytes for making the injection are taken.

Hemolysins may be prepared as the result either of intraperitoneal or of intravenous injections of erythrocytes washed at least three or four times to remove all traces of serum. Unless an antihuman hemolysin is required within a short space of time, better results are obtained, as a rule, by using the slower, intraperitoneal method.

In immunizing rabbits it must be remembered that the quantity of amboceptor produced bears no direct relation to the size of the doses given. Thus, a highly potent hemolytic serum may be prepared by three intravenous injections of from 3 to 5 c.c. of a 10 per cent. suspension of washed sheep cells.

It must be emphasized that as far as possible an aseptic technic should be employed, and that corpuscles used for making intravenous 
injections should be filtered to remove small particles of fibrin, and preferably washed four times with sterile salt solution. The method of preparing corpuscles for injection has been given in a preceding chapter. After the third or fourth injection the animal should be bled from the ear and the serum tested, as animals not infrequently succumb after the fourth injection, and many possess serums of high potency after receiving three injections. By this method success is better assured.

Intravenous Method.-For the preparation of most hemolysins the following methods yield very good results. Antihuman hemolysin is more difficult to prepare, and I have generally found that a slower, intraperitoneal method will yield better results.

First Method.-Three injections of 5 c.c. each of a 10 per cent. suspension of washed cells at intervals of three days. The animal is bled three or four days after receiving the last injection.

Second Method.-Three injections of 10 c.c. each of a 10 per cent. suspension of washed cells on each of three successive days. The rabbit is bled four days after receiving the last injection (after the method of Gay). Instead of these large injections, 1 c.c. of corpuscles, removed after thorough centrifugalization and diluted with sufficient sterile salt solution, may be given on each of three successive days.

Third Method.-A slower method consists in making weekly injections of a suspension of corpuscles. The cells must be thoroughly washed to free them from all traces of serum; if this is not done, the animals may die of anaphylaxis during the course of immunization; animals should be tested after the third dose has been injected:

First dose: 3 c.c. of a 10 per cent. suspension of corpuscles.

Second dose: 5 c.c. of a 10 per cent. suspension of corpuscles. Third dose: 10 c.c. of a 10 per cent. suspension of corpuscles.

Fourth dose: 15 c.c. of a 10 per cent. suspension of corpuscles.

Fifth dose: 20 c.c. of a 10 per cent. suspension of corpuscles.

Fourth Method.-In the preparation of antihuman amboceptor, Noguchi advises giving four intravenous injections, -4 c.c., 3 c.c., 4 c.c., 3 c.c.,-and possibly another- 4 c.c.-after an interval of four or five days. Rabbits are bled one week after the last injection is given.

Intraperitoneal Method.-In this method the most careful aseptic precautions should be observed in washing the cells and in giving the injections, or the animals are likely to succumb from peritonitis just about the time they are fully immunized and ready for bleeding. Injections may be given every four or five days, and one week after receiving the last injection the rabbits are to be bled. 
This method is especially serviceable in preparing antihuman amboceptor. Whenever possible, blood should be collected aseptically, and the corpuscles washed just before the injections are given. Placental blood is likely to be infected and hemolyzed, and frequently yields unsatisfactory results. In preparing the antihuman amboceptor several rabbits should be immunized at the same time, the doses being:

First dose: $\mathbf{5}$ c.c. of the washed corpuscles.

Second dose: 8 c.c. of the washed corpuscles.

Third dose: 12 c.c. of the washed corpuscles.

Fourth dose: 15 c.c. of the washed corpuscles.

Fifth dose: 20 c.c. of the washed corpuscles.

\section{PRODUCTION OF CYTOTOXINS}

The term "cytotoxins" is usually applied to cell toxins other than hemolysins, such as nephrotoxins, spermatotoxins, etc., and although "lysin" is frequently used, the term "toxin" is better, being descriptive of the changes produced by all cell toxins except the hemolysins and the bacteriolysins.

Cytotoxic serums can be made theoretically for any cell, but only the hemolysins possess much practical value. The cytotoxins are prepared with some difficulty by injecting emulsions of cells from one animal into another. Immunization should always be conducted by means of intraperitoneal or subcutaneous injection.

For the purpose of studying the action of cytotoxic serum, nephrotoxic serum is preferably to be used, as the effects, e.g., the production of albuminuria, may be observed. A series of two or three animals should be carried along at the same time, as many die after the third injection. So far as possible an aseptic technic should be carried out.

Practically all cytotoxic serums are hemolytic partly because of the great difficulty of removing all traces of blood from the organ used in preparing the emulsion. This difficulty may be reduced to a minimum by thoroughly washing the organ or tissue prior to preparing an emulsion for injection.

The following method, after Pearce, illustrates the mode of preparing a nephrotoxic serum by immunizing rabbits with dog kidney.

1. Anesthetize a dog with ether, open the abdomen, wash the blood out of the kidneys by inserting a cannula high up in the abdominal aorta, and flushing with from six to ten liters of salt solution; open the vena cava. 
2. Remove the kidney as aseptically as possible, and grind it in a meat-grinder; rub through fine-meshed wire gauze, and wash the residue in several changes of sterile salt solution. The fat should be rejected and only the cortex used.

3. Weigh and suspend 10 grams of the substance in 30 c.c. of sterile salt solution.

4. Inject the rabbit intraperitoneally with 10 c.c. of the emulsion every seven days.

5. After the third dose has been given test the serum, as subsequent doses increase the danger of losing the animal.

6. The animal is bled one week after receiving the last injection.

7. The injection of this serum into dogs is usually followed by albuminuria and possibly hemoglobinuria. This subject is further considered under the head of Practical Exercises with Cytotoxins.

Some investigators have asserted that by effecting immunization with the nucleoproteids of an organ more specific cytotoxic serums are secured. These claims have not been confirmed by Pearce, Wells, and others. (See Chapter XXV.)

Nucleoproteins may be secured as follows: Grind the organ or tissue in a meatgrinder, and finally rub up with sand with a pestle in a mortar; add two volumes of normal salt solution, and pass through a meat press; collect the effluent, place in a refrigerator for twenty-four hours and then filter through gauze and centrifuge the filtrate; to the supernatant fluid add acetic acid to remove the nucleoproteins. Place in the refrigerator for eighteen hours and centrifuge. Collect the sediment and wash several times with normal salt solution. Dissolve the sediment in normal salt solution containing 0.5 per cent. sodium carbonate. Reprecipitate with acetic acid, wash, and redissolve in the alkaline solution. 


\section{CHAPTER V}

\section{THE PRESERVATION OF SERUMS-METHODS}

IT is well to remember that serum collected shortly after a meal is likely to be cloudy or opalescent; it is therefore advisable that blood be collected several hours after eating or during a period of fasting.

After securing a specimen of blood, the container should be set aside and kept at room temperature until the serum separates. If the serum is to be used at once, blood may be collected in centrifuge tubes, allowed to coagulate, and then broken up, as gently as possible, with a sterile glass rod and thoroughly centrifuged. On account of the mechanical rupture of erythrocytes, such serums are usually tinged with hemoglobin. After serum has separated from the clot it should be transferred to another tube, or, if this is not immediately possible, the container should be placed in the refrigerator, to retard hemolysis, which may soon occur and render the serum unfit for many purposes.

Small amounts of serum are best removed from the clot with a capillary pipet and teat, or with an ordinary graduated pipet with rubber tubing and mouth-piece, in order that one may see exactly what he is doing and not disturb the clot. As a perfectly clear serum is always to be desired, serums mixed with corpuscles should be centrifuged.

It may be stated, as a general rule, that all normal and immune serums should be collected as aseptically as possible, and handled in a careful and aseptic manner, so as to insure a clear and sterile product. Notwithstanding the method of preservation all serums should be kept in a refrigerator or ice-chest at a low temperature.

\section{PRESERVATION OF NORMAL SERUMS}

Normal serums that are to be used for purposes of immunization are best preserved in small amounts in separate ampules, or in a large stock bottle holding from 100 to 200 c.c. and well stoppered. In the production of precipitin-serum, for example, sufficient serum of an animal may be obtained at a single sitting for the whole course of injections, and this serum is best preserved in separate ampules. Each ampule should contain sufficient serum for one injection, and be sealed and marked. 
In this manner the risk of contaminating a stock bottle is obviated. In the preservation of normal serum or the serum of luetics to be used as controls for the Wassermann reaction, it is better to store them in small amounts in sterile ampules.

As a rule, it is best not to add a preservative to serums that are to be used for purposes of immunization, for if the dose of serum is large, enough preservative may be injected to place the health of the animal in jeopardy. However, chloroform may be added in proportion of 1:10 or $1: 20$, provided the serum is placed in the incubator or heated in the water-bath at $40^{\circ} \mathrm{C}$. for fifteen minutes in order to drive off the chloroform previous to injection.

\section{PRESERVATION OF IMMUNE SERUMS}

Immune serums may be preserved either in the fluid or in the dry form.

Preservation in Fluid Form with Antiseptics.-Practically any immune serum may be preserved in the fluid state by adding a suitable preservative in the proper dose without exerting any deleterious influence on the antibody content. The exceptions to this general rule are the precipitin-serums, because these should be crystal clear, and a preservative may render the serum slightly cloudy. According to Uhlenhuth, Weidanz, and Wedemann, such serums should be filtered through a sterile Berkefeld filter and then stored without adding an antiseptic.

Various antiseptics have been advocated for the preservation of serums. Hemolytic serum is well preserved by adding an equal amount of chemically pure glycerin to the serum after it has been inactivated by heating at $55^{\circ} \mathrm{C}$. for a half-hour in a water-bath. The addition of 0.1 c.c. of a 1 per cent. solution of phenol in salt solution to each cubic centimeter of immune serum usually suffices to keep the fluid free from contamination, and produces only very slight, if any, clouding. Likewise, the addition of 2 per cent. formalin in a 5 per cent. solution of glycerin in normal salt solution, in the proportion of $1: 10$, makes a very useful antiseptic. Neither lysol nor trikresol should be used in the preservation of a serum, as they are more likely to produce clouding than does phenol.

Preservation in Fluid Form, by Bacteria-free Filtration. - If serums are to be preserved in fluid form without the addition of an antiseptic, special precautions in bleeding, collecting, and separating should be 
observed. If contamination has probably occurred, the serum should be filtered through a sterile Berkefeld filter (Fig. 34). The apparatus devised by Uhlenhuth (see Fig. 88) is especially useful, as the serum is collected at once in a sterile container, which is then plugged with sterile cotton and placed in the incubator for twenty-four hours. If the serum

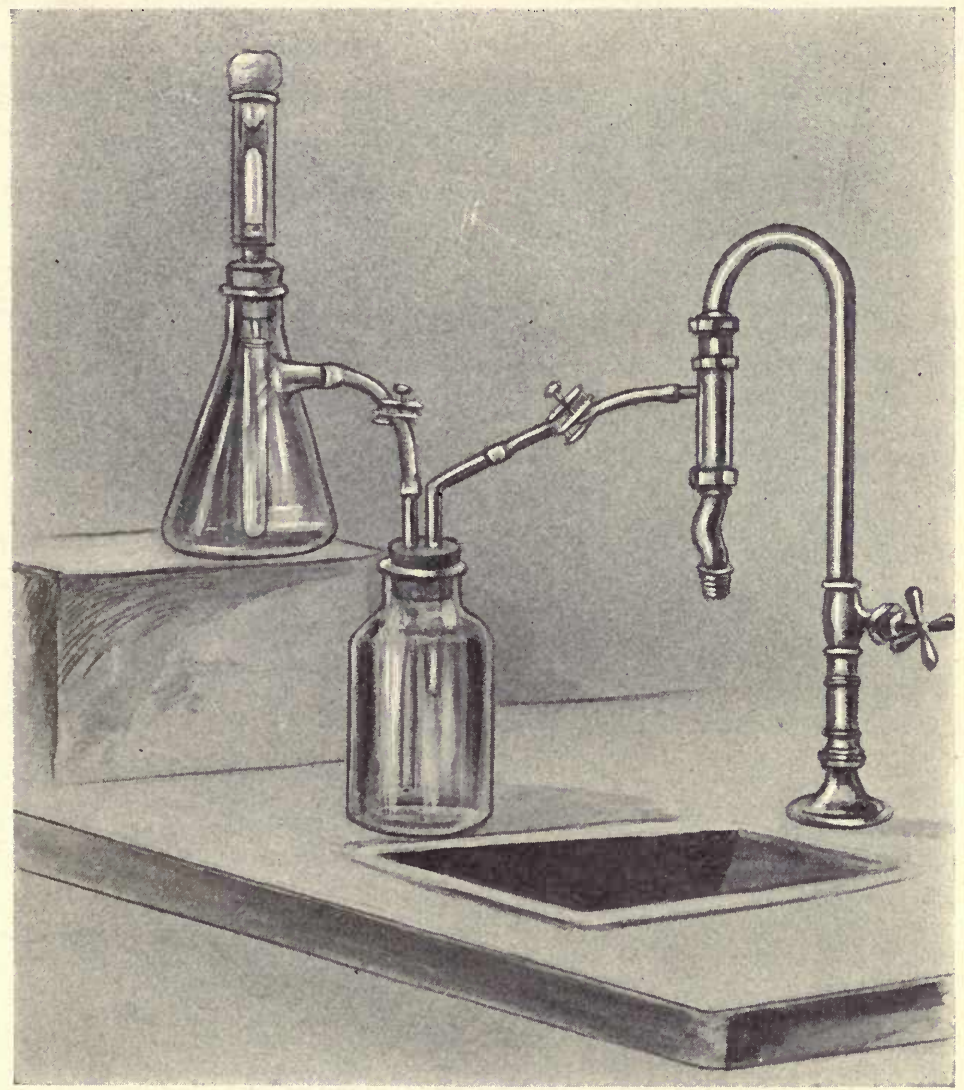

Fig. 34.-A Small Berkefeld Filter.

The fluid to be filtered is poured into the glass cylinder surrounding the earthen or porcelain "candle." Negative pressure within the candle is produced by the water-pump, which exhausts the air from the flask. The filtrate is collected in the test-tube within the filter flask. All parts are readily sterilized in an Arnold sterilizer or autoclave. The sterile cotton plug prevents air contamination.

proves to be sterile, it is transferred, with the aid of a sterile pipet, into ampules of 1 c.c. capacity. These are sealed hermetically and kept in the refrigerator.

Small amounts of serum may be lost in a large filter, and a smaller apparatus should therefore be used. The filter shown in the accom- 
panying illustration (Fig. 35) is quite serviceable, as the flask and earthen candle-filter may be wrapped in a towel and sterilized in the autoclave. The apparatus may carefully be attached to a suction pump, and the serum pipeted off into the hollow of the candle and filtered, the filtrate being removed, at the completion of the process, by another sterile pipet.

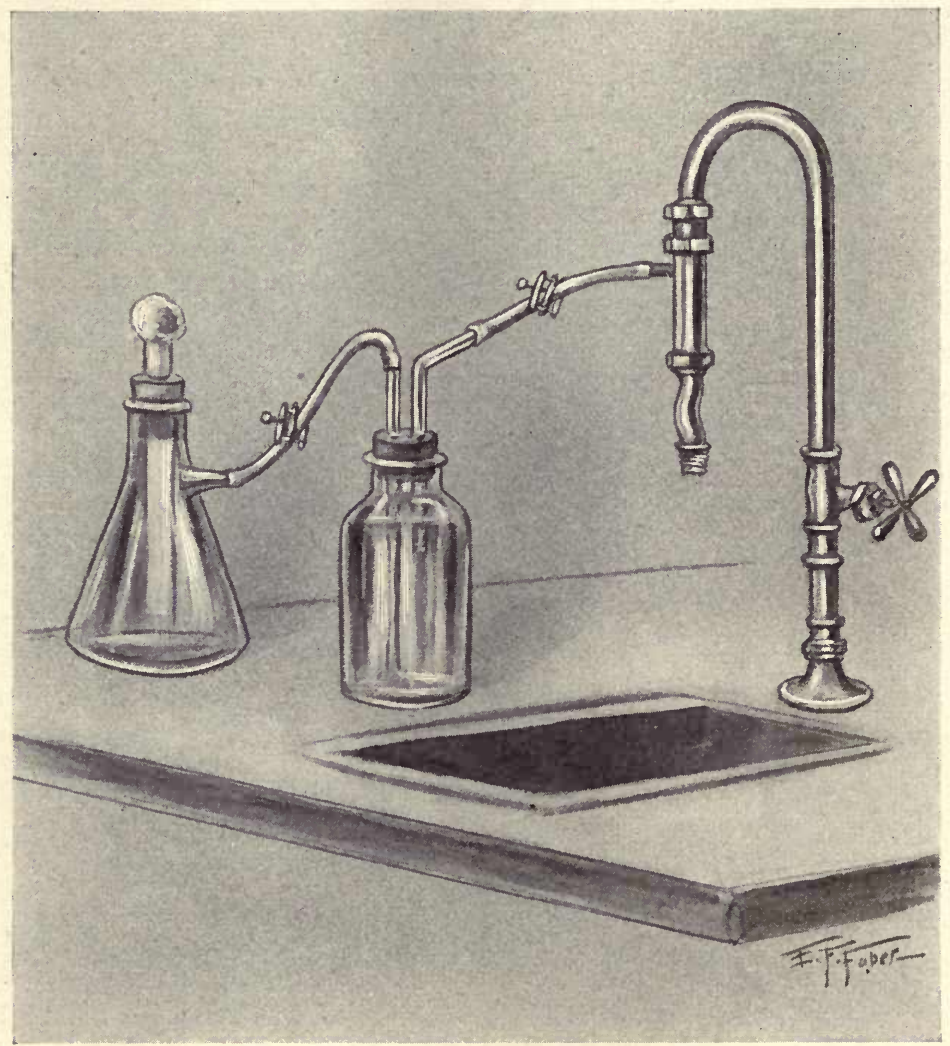

Fig. 35.-A Filter.

The cotton plug in the "candle" is removed and the fluid poured within the candle (hollow). The water is then turned on and the stop-cocks are opened; a vacuum is produced within the flasks, which draws the fluid through the candle. The filter is readily cleansed and sterilized (autoclave) and is quite efficient.

Preservation in Fluid Form, by Freezing.-Freezing a serum often renders it cloudy or causes a precipitate to be deposited, and interferes with the usefulness of a serum that should be absolutely clear. Freezing is the only practicable method so far devised for the preservation of thermolabile substances, such as complement. A small apparatus, 
named the "Frigo," has been devised for this purpose by Morgenroth. A satisfactory apparatus may be made by constructing a wooden box with a smaller sheet-metal-covered inner compartment, the space between them being well packed with sawdust. This inner box is then filled with crushed ice, and the whole is covered with a lid lined with several layers of felt.

Preservation in Powder Form.-When serum is poured out in thin layers and dried, it forms yellowish, amorphous masses, that may be collected and ground into a powder, which keeps well and forms an excellent medium for the preservation of many immune serums, especially those of the agglutinating type. Various toxins, such as tetanus toxin and cobra venom, may also be preserved in this form.

The serum or toxin may be spread out in thin layers on large glass plates, or placed in shallow dishes and dried in the incubator. After a few hours the dried serum, which adheres only slightly to the dish, can be removed with a spatula and placed in a mortar, and ground and stored in sealed tubes.

The drying process is better carried out in vacuo, and the large serum institutes are provided with these special drying apparatus. A simple form may be prepared after the method of Taeze, as follows: Place a large glass bell-jar with a ground base and a large opening at the top on a polished iron plate. Set this on a large tripod, as this will facilitate heating with a Bunsen burner. The serum is placed within the jar in a shallow dish, and the jar fastened to the iron plate with hot paraffin or wax. The opening at the top is closed with a three-holed rubber stopper: one hole carries a thermometer; a second is connected with a manometer (not absolutely necessary), and the third carries a bent glass tube which is connected, by means of thick-walled rubber tubing, to a suction pump. A low flame is kept burning so as to keep the temperature at about $35^{\circ} \mathrm{C}$. The degree of vacuum secured makes little difference, and usually that obtained with an ordinary water-suction pump, allowing for leaks in the tubing, is sufficient, rendering manometric measurements unnecessary.

The dried serum should be dissolved in sterile normal salt solution before it is used.

Preservation in Dried Paper Form.-This is a very serviceable method for preserving hemolysins, and to a lesser extent, agglutinins. In the preservation of hemolytic amboceptor Noguchi advises the use of Schleich and Schull's paper No. 597. The paper is cut into squares about 10 by $10 \mathrm{~cm}$., and saturated with the serum which, after prelimi- 
nary titration, has been found satisfactory. Sufficient serum is added to wet the sheets evenly, any excess of serum being absorbed with other sheets of paper. Each square is placed separately upon a clean sheet of unbleached muslin and dried at room temperature. When thoroughly dry, the squares are carefully ruled off with a hard pencil into widths of about $5 \mathrm{~mm}$., and cut into strips. The paper is then standardized and preserved in dark glass vials in a cool, dark place.

Preservation in the Living Animal.- In the living animal an immune serum may be preserved by removing a small amount of blood from time to time as needed, the titer being preserved or raised by occasional injections. This method, however, may be unsatisfactory and expensive, especially with the smaller animals, as they frequently show a marked tendency to sicken and die, or may succumb to anaphylaxis. After a time, too, they fail to respond to injections with the formation of antibodies, a condition ascribed to atrophy of the cellreceptors (receptoric atrophy). 


\section{PART II \\ CHAPTER VI}

\section{INFECTION}

Infection is the successful invasion and growth of microörganisms in the tissues of the body.

The skin and adjacent mucous membranes contain numerous microorganisms, and under normal conditions these may invade the tissues, but they are usually quickly destroyed and unable to proliferate, so that mere invasion does not necessarily constitute infection.

Unfortunately, custom has sanctioned the use of the term infection as synonymous with contamination. The bacteriologist may speak of the air, water, or his culture medium as being infected when they contain microörganisms, or, in other words, are not sterile; similarly the surgeon may speak of a knife or splinter of wood as being infected whereas, while these may be infective or capable of producing infection, it is more accurate to speak of them as being contaminated. In the early days of bacteriology, the mere presence of microörganisms in or on the skin and mucous membranes was regarded as equivalent to infection. It is now well known that a person may harbor various microorganisms, such as staphylococci, streptococci, and pneumococci, without apparent injury to the host, and this surface contamination, or even occasional invasion of the tissues, does not necessarily indicate that the host has been, is, or will be ill.

Definition.-When, however, microörganisms have passed the normal barriers of the skin or mucous membranes and have invaded and proliferated in the deeper tissues, the process is spoken of as an infection.

By common consent, the term infestation, or infestment, is being applied in a similar manner to the presence and growth of animal parasites; thus the intestine may be infected with Bacillus typhosus and infested by Tænia saginata.

A microörganism may be intimately associated with and have its normal habitat in a certain part of the body and do no harm until special conditions arise, when it may rapidly invade the tissues and produce infection; this condition has been described by Adami as a subinfection, and is illustrated by the constant presence of staphylococci and strepto- 
cocci in the tonsils of most persons, usually harmless, but capable, under special conditions, of producing severe and even fatal infection.

The abnormal state resulting from the deleterious local and general interaction between a host and an invading bacterium, with consequent tissue changes and symptoms, constitutes an infectious disease.

As has previously been stated, not every invasion of the deeper tissues by microörganisms results in injury or disease. A certain number of bacteria are constantly gaining admission to the deeper tissues of the alimentary and the respiratory tracts, without producing apparent injury to the host, as they tend to be destroyed very soon after they gain entrance. The terms invasion and infection are not, therefore, synonymous. Every true infection is accompanied by local changes, although these may be so slight as to escape notice; an infectious disease is practically made up of similar phenomena, but these are of an exaggerated or marked degree.

The hygienist distinguishes between-(1) Sporadic, or isolated, cases of infection; (2) endemic, in which a certain microbic disease affects the inhabitants of a given area year after year; and (3) epidemic, in which a disease appears suddenly and affects a large number of inhabitants, the number of cases rapidly increasing and decreasing. Among the lower animals equivalent terms for the types just described are sporadic, enzoötic, and epizoötic. A pandemic disease is one that is epidemic over a large territory.

In all infections there are two inseparable factors to be considered:

1. The offensive forces of the infecting agent, dependent upon its pathogenic or disease-producing nature and its power of defending itself against the antagonistic forces of the host and of thriving under these conditions.

2. The resistance offered by the host, and mainly dependent upon certain physical or non-specific local factors or specific antibodies, which constitute the defensive mechanism, or immunologic factors.

The former is concerned with the general subject of infection, and the latter with that of immunity.

Microörganisms and host may live together in apparent harmony, owing to the ability of the host to restrain the activity of the microorganism and neutralize its injurious effects or to an absence of infectivity on the part of the microörganism until the vital resistance of the host is diminished or the pathogenicity of the microörganism is increased, when the neutral relations are disturbed and infection occurs. 


\section{RELATION OF INFECTION TO IMMUNITY}

From what has been said, it is apparent that the subject of infection forms the basis for the study of immunology, for, paradoxic as it would at first appear to be, infection must usually have occurred in order that immunity may be acquired. This relation is not always apparent; for instance, man and some of the lower animals may possess a natural immunity to a certain parasite because of the presence of various physical or non-specific defensive factors, or to specific antibodies produced as the result of an earlier and unrecognized infection, or even one that has been inherited; under any circumstances, however, natural immunity is usually relative and seldom absolute. In passive immunity the same conditions are generally operative, and the antibodies present in the serum used to confer a passive immunity are produced in some other animal as the result of an active infection.

It may be stated, therefore, that specific antibodies are produced only by stimulation of the body-cells, and that this stimulation is furnished by the infecting agent either in living, disease-producing form, or in a modified and attenuated state, $i . e$. , in the form of a vaccine; thus it will be seen that infection and immunity are intimately associated, and that, generally speaking, there can be no pronounced protection unless infection has taken place.

\section{SOURCES OF INFECTION}

Bacteria are to be found everywhere. For general purposes they may be roughly divided into two classes-saprophytes and parasites. The saprophytes are those bacteria which thrive best in dead organic matter, and perform the very important function of reducing, by their physiologic activities, highly organized material into those simple chemical substances that may again be utilized by the plants in their constructive processes, and in this manner maintain the important chemical relation between the animal and the plant kingdom. Parasites, on the other hand, find the most favorable conditions for growth and activity upon the living tissues of higher forms of animal life. They include most of the so-called pathogenic or disease-producing bacteria.

No marked separation between these two divisions can be made, as numerous species occupy a transition point between the two. The terms are merely relative, and bacteria ordinarily saprophytic may develop parasitic and pathogenic powers when the resistance of the host is sufficiently reduced by another infection, fatigue, exposure, or other 
deleterious influence. In other words, a pathogenic microörganism is one that can grow in the living tissues because the immunologic defenses of the host are not sufficiently strong to resist it; in most cases, however, as will be pointed out further on, a higher degree of immunity can be produced artificially, rendering the bacterium in question relatively harmless for that particular animal. Similarly, under certain circumstances, the resistance of the body or of a part of it may be broken down to such an extent that microörganisms ordinarily regarded as saprophytes may gain access to the deeper tissues, flourish, and produce disease.

Accordingly, no fundamental distinction between pathogenic and non-pathogenic bacteria can be made. Any apparent differences are due not only to various degrees of pathogenicity possessed by the microorganism, but also to the different degrees of resistance against their attacks, since a microparasite that is highly pathogenic toward one animal, may be quite harmless to another.

\section{CONTAGIOUS AND INFECTIOUS DISEASES}

Just as all pathogenic bacteria do not possess the same habits of growth, so, likewise, they vary in their vitality and in their ability to proliferate under various conditions when removed from the animal body. Some are strictly parasitic, and are able to grow only at body temperature, or, indeed, only in the human body itself; when removed from these conditions, they may retain their vitality for a short period of time, but are unable to proliferate: from this it follows that communication of these bacteria and their disease must be direct or immediate, $i$. e., from person to person, or almost direct, by the conveyance of the infecting agent in the form of fomites, such as dust, epidermal scales, or discharges, or as the result of bites of suctorial insects. This form of infection, which requires such direct means of transmission, and of which gonorrhea is an example, constitutes what are known as contagious diseases.

Other microparasites are not so strictly parasitic; they may be able to preserve their pathogenic powers and proliferate outside of the body at ordinary temperatures, and may even withstand great extremes of heat or cold and various nutritional deficiencies; they may exist thus for weeks, and carry the disease to a second individual through contaminated material. Infections the result of indirect transmission are known as infectious diseases. 
There are no hard-and-fast rules that can be set down in classifying bacterial infections; bacteria that are commonly transmitted by one means may, under slightly altered conditions, be transmitted by another. The usual classification, by which certain diseases are classified as contagious and others as infectious, should be abolished, and all should be grouped under the term infectious, there being a definite understanding of those cultural characteristics that render infection more likely to occur by direct and immediate contact, and those that may occur in an indirect or roundabout manner. It may therefore be stated that all bacterial diseases are infectious; the term contagious may be reserved for those spread or contracted as the result of direct contact.

\section{EXOGENOUS AND ENDOGENOUS INFECTIONS}

Infection may occur as the result of the admission of microparasites to the tissues from sources entirely apart from the individual infected (exogenous infection), or from the admission of some of those microparasites living normally and harmlessly on the skin and adjacent mucous membranes, and which, under special conditions, have assumed pathogenic properties (endogenous infections).

Exogenous infections are the more usual form, and result from contact with infective material outside the body.

1. Microörganisms, such as typhoid and cholera bacilli, which can live for varying periods of time in water and foods, are particularly likely to gain entrance through the gastro-intestinal tract. Microorganisms may be present in milk derived directly from diseased animals or tissues, and when ingested, may produce disease. Thus, for example, the germs of tuberculosis may be conveyed in either milk or flesh, young children being particularly exposed to this method of infection.

2. The atmosphere may be laden with microörganisms, which, whether or not capable of proliferating outside of the body, are prone to gain entrance through the respiratory tract, especially through the upper air-passages, the pharynx and tonsils being often the seat of the infection.

3. Microörganisms capable of existing on the skin may gain entrance to the deeper tissues as the result of wounds. Under these conditions of lowered vitality of the local tissues microörganisms that would otherwise be harmless may become pathogenic and morbidly affect the host, either locally or generally. As the skin is brought so freely in contact with external objects, various microörganisms, and particularly 
the pathogenic cocci, may gain entrance to the dermis. Wounds may be infected by the teeth and secretions of animals, or by various weapons and implements contaminated with infective material, as, $e . g$. , the virus in the saliva of rabid dogs, or the spores of the tetanus bacillus on rusty nails.

Contact with unclean objects of various kinds-eating utensils, catheters, syringes, dental instruments, etc.-may serve to transfer pathogenic bacteria from one person to another. This is especially likely to occur if the skin or mucous membrane is abraded, the infecting parasites thus gaining ready access to the deeper tissues. In some infections, however, even this local injury is unnecessary, as the bacterium may be able to proliferate and produce lesions on an intact surface, as, for instance, the diphtheria bacillus in the pharynx, and various fungi, such as Achorion, Trichophyton, etc., on the scalp and skin in general.

Microparasites affecting the genital organs are likely to be conveyed directly from one sex to the other in conjugation, or to the child during parturition.

4. Suctorial insects may serve as the medium by which microorganisms are transmitted from person to person. In most instances the transmission is a purely mechanical process, as witness the transmissions of the plague bacillus in the intestinal contents of the rat flea; in the case of malaria, on the other hand, the interposition of the mosquito is essential to complete the life cycle of the protozoön.

5. Microörganisms infecting the placenta may pass to the fetus by way of the umbilical vein.

Endogenous infections arise as the result of the activity of microorganisms having their normal or customary habitat in the body. Such infections do not represent so much an assumption of pathogenic power on the part of the microörganism, as they do a disturbance of the defensive mechanism of the host, whereby the normal relations are disturbed, and microörganisms that normally are harmless, become infective and disease-producing. While the disturbance of the defensive mechanism may be general, it is far more likely to be local; an example is that of appendicitis the result of Bacillus coli infection following passive congestion due to fecal impaction of the colon.

\section{AVENUES OF INFECTION}

Local infection may occur in any portion of the body, and any part may prove the point of entrance of bacteria to the body fluids, the result 
being a general infection. Owing, however, to the peculiar pathogenic properties of different bacteria and their affinity for the cells of certain tissues, coupled with a peculiar tissue susceptibility for certain bacteria or their products, we find that many diseases have regular avenues of infection, and, indeed, in a few instances infection of the human body may be possible only through a particular and definite route. Infections of the gastro-intestinal, respiratory, and genito-urinary tracts and various sinuses with external openings must be considered as being potentially surface infections. The outer layers do not consist merely of the skin and adjacent mucous membranes, but are made up of all layers covering surfaces and channels, which, however indirectly, communicate with the exterior. In the higher animals there is only one direct channel of communication between the actual interior and the exterior of the body, this being through the Fallopian tube of the female, which normally has so fine a lumen and is so well protected that to all intents and purposes it may be regarded as closed. In certain inflammatory conditions of the genital organs, and particularly after parturition, the Fallopian tube may be open, and afford a direct route for the transmission of infection from the external parts to the peritoneal cavity.

Living in and on the actual and potential external surfaces are countless microörganisms, which are for the most part harmless, a few being, however, actually or potentially dangerous.

1. The skin and adjacent mucous membranes, particularly in those portions where warmth and moisture abound, are well adapted to bacterial growth, and their contact with surrounding objects causes a large variety of microörganisms to adhere to them.

As a result, the bacteriology of the skin is quite complex, since it may lodge microörganisms from the air, from water, and from soil. A group of cocci and diplococci, particularly the Staphylococcus epidermidis albus of Welch, and the various pseudodiphtheria bacilli, are habitually present upon the human skin. When local injury occurs, they may produce minor suppurative lesions, and may be concerned in the production of certain skin diseases, such as eczema, impetigo contagiosa, the pustules of variola, etc.

Other microörganisms may find temporary lodgment upon the skin, and are in no sense regular inhabitants. For example, the fingers and hands may become contaminated with colon, typhoid, and tubercle bacilli, pneumococci, etc.

The skin forms a very important barrier against the entrance of 
bacteria into the deeper tissues. The greater number of local surgical infections result from the entrance of bacteria into lesions of the skin, although these lesions may be so small as to escape notice.

Certain parasites are capable of producing direct action on the skin without previous existing injury, and especially upon the mucous membranes, where moisture and higher temperature are more favorable to bacterial growth. For example, a few of the higher fungi, such as Microsporon, Achorion, and Trichophyton, seem able to establish themselves in the superficial cells and invade the deeper tissues through the hair-follicles; staphylococci may reach the roots of hair-follicles and sweat-glands and set up suppurative conditions; diphtheria bacilli may lodge directly on the intact mucosa of the upper air-passages and cause local necrosis and general intoxication; cholera bacilli may have a similar effect upon the intestinal mucosa; the Koch-Weeks' bacillus and the gonococcus may produce severe inflammation of an intact. conjunctiva, etc.

2. The respiratory organs commonly afford admission to certain microörganisms. The nose may be the seat of local infection with Bacillus influenzæ, Micrococcus catarrhalis, Bacillus diphtheriæ, and other bacteria; it may be the entrance point for meningococci and the virus of anterior poliomyelitis. Similarly, the entrance of such unknown infectious agents as those of scarlet fever, measles, and smallpox can best be accounted for by assuming that they were inhaled and later entered the blood; there is much clinical evidence to support the belief that the contagium of scarlet fever is present in the discharges of the upper air-passages of persons suffering from that infection.

Whether or not tuberculosis of the lungs is the result of the inhalation of tubercle bacilli is a much-disputed point, but it cannot be denied that this theory most readily accounts for the far greater frequency with which tuberculosis affects the lungs than it does other organs of the body.

Pneumonia, caused by the pneumococcus of Weichselbaum, probably results from the direct inhalation of one of the various types of pneumococci, and bronchopneumonia of children is certainly chiefly inspiratory in origin.

3. The digestive tract may be the portal of entrance of many infections. The mouth usually harbors various fungi and bacteria, which may produce local infections, and either directly or indirectly cause caries of the teeth. The putrefactive changes they may produce is being generally recognized as having an important bearing on the causation and symptomatology of several infections, and a carious tooth has been 
found the portal of entry of microörganisms causing a general infection. 'The tonsils are well known to be the breeding- and lodging-place of various microparasites causing many general infections, such as acute rheumatic fever, tuberculosis, and possibly typhoid fever. The pharynx may harbor the microörganisms of diphtheria, pneumococcous angina, etc.

Normally, except for the presence of a few sarcinæ, the stomach is practically sterile. Under special conditions, however, typhoid, dysentery, cholera, tubercle, and other infectious bacteria may escape the germicidal effects of the hydrochloric acid, and, reaching the alkaline intestinal contents, which are rich in soluble proteins and carbohydrates, are rendered capable of producing their respective infections.

Although these conditions are primarily of the nature of local infection, there is much experimental evidence to show that bacilli, and particularly tubercle bacilli, may pass through a practically intact intestinal wall and find their way to the lymph-glands or to the bloodstream itself.

Aside from these direct and specific infections, various other microorganisms, by fermentative action, may alter the intestinal contents and produce toxic products capable of exciting acute and severe toxemias. Some authorities as, e.g., Metchnikoff, regard the various types of colon bacilli as producing toxic products responsible for chronic degenerative lesions of the cardiovascular and other organs. The digestive tract is therefore regarded by some pathologists as a constant menace to health, in that it permits bacteria to enter the lymphatic and blood-streams, or to produce toxic substances detrimental to health and longevity. Adami has drawn particular attention to a condition which he terms subinfection, and which is dependent upon the constant entrance of colon bacilli into the blood, whence they enter the liver, where their final dissolution takes place, appearing as fine, dumb-bell-like granules inclosed in the cells.

4. The genital organs are the seat of various local infections that may become wide-spread and general. Normally, the urethra may contain a few cocci which lodge about the meatus; the acid secretions of the vagina are generally inimical to bacterial growth, and the uterus and bladder are usually sterile. But three microörganisms-the gonococcus, Treponema pallidum, and the bacillus of Ducrey, here find favorable conditions for growth, and are usually transmitted from person to person by means of sexual congress. The local gonococcal lesion may be the portal of entry of gonococci into the blood-stream, resulting in 
wide-spread metastases in the heart valves and joints. The local syphilitic lesion is quickly followed by general infection. Chancroids alone remain localized, although the initial lesion frequently spreads quite rapidly by continuity of tissues. In rarer instances other microörganisms, such as the ordinary pyogenic cocci, tubercle bacillus, and diphtheria bacillus, may infect these organs and be transmitted by sexual conjugation.

5. There is considerable controversy of opinion regarding the susceptibility of the placenta and the filtering properties it possesses for various infectious agents. A study of this subject by Neelow ${ }^{1}$ would indicate that the non-pathogenic bacteria do not pass from the mother through the placenta to the fetus. Other pathogenic agents may, however, pass through quite readily, for example, pregnant women suffering from smallpox may be delivered of infants showing active lesions of prenatal infection, and syphilitic infection of the fetus is a well-known condition. Most controversy centers around congenital tuberculosis, and directly opposing views for and against prenatal infections are held by several authorities. Baumgarten is of the opinion that many children are subject to antenatal infection, though the disease infrequently develops in a few of them.

The general subject of antenatal infection and pathology is a field requiring considerable investigation and research.

\section{NORMAL DEFENSES AGAINST BACTERIAL INVASION}

When the large area of the body that is subject to traumatic injury and accidental infection is considered, it is remarkable that, considering the enormous numbers of various bacteria, infection does not occur more frequently.

Bacterial invasion of the tissues is of frequent occurrence, but in health they do not usually cause infection, and tend to be destroyed very soon after they enter the tissues.

It may be well to discuss at this point the factors tending to prevent invasion, and leave the consideration of the defensive mechanism, whereby the body destroys bacteria after successful invasion and thus prevents infection, for the chapter on Natural Immunity.

Of the factors preventing bacterial invasion, the following are recognized:

1. The structure of the surface layer of epithelium. The epidermal ${ }^{1}$ Centralb. f. Bakt., August, 1902, 1. Abt., Bd. xxxi, orig., 691. 
cells offer a mechanical obstacle to invasion. This resistance is naturally more complete where the cells are thickened and most compact. In the depths of glands and in mucous membranes, where numerous glands are present, and where the layers are thinner and moisture exists, the barrier is less complete.

2. Surface discharges are potent factors in preventing bacterial invasions by-(1) Washing away the bacteria mechanically; (2) by germicidal activity through the presence of various chemical agents, such as acids, which they may contain, and (3) by antiseptic and even bactericialal substances that may be present in the form of antibodies.

The saliva, with its antiseptic and germicidal properties, is potent in preventing infections of the mouth and upper air-passages; when this secretion is diminished, as during the course of high fever, bacterial activity is enhanced, which is evidenced by the development of fetid sordes about the teeth and on the lips.

The acidity of the gastric juice and its germicidal powers are well known and appreciated; similarly the urine, the milk, and to a slight extent, the bile, have been demonstrated by 'Adami to exert a distinct antiseptic effect upon certain bacteria, such as the Bacillus coli.

Surface moisture and discharges about the nose and throat are also potent factors in mechanically removing bacteria from inspired air, and no doubt frequently prevent bacterial invasion of the lower respiratory tract, where more mischief may be done.

3. The cells of certain excreting glands may possess bactericidal and excretory powers of value in preventing bacterial invasion (Adami).

\section{MECHANISM OF BACTERIAL INVASION}

We will now consider the method by which invasion, the first step of what may be an infection, is brought about. In brief, one or all of the normal defenses just described must be overcome; in some instances the microörganisms, by their inherent disease-producing powers, may accomplish this unaided; in other instances the resistance is overcome by a general lowering of the vitality of the body defenses.

1. Traumatic solution of the surface layers of epithelial cells is a very important factor in the production of infection, as the invading microparasites are thus given easier access to the deeper and less resistant tissues. The pathologist or surgeon may, in the course of his work, contaminate his hands with secretions containing virulent microörgan- 
isms, and may yet escape infection, unless a small break in the surface epithelium, in the form of a scratch or a needle-prick, is present.

2. As has been previously stated, certain bacteria, notably the diphtheria bacillus, by concentrating at one point, may lower the vitality and cause necrosis of superficial cells of the mucosa lining the upper airpassages, and in this manner induce a local break in the continuity of the epithelial covering. Staphylococci may exert a similar action in the depths of sweat and sebaceous glands, and, indeed, certain fungi, such as the Trichophyton, Microsporon, and Achorion, may attack the intact skin. While, therefore, solution of the surface coverings is a very important source of many infections, it is not essential for the production of all.

3. Alterations of the surface discharges, either in quantity or in quality, may permit bacteria to proliferate freely and produce sufficient toxic matter to affect the surface cells, lower their vitality, and destroy them, with the result that they may gain entrance to the deeper tissues. When the secretions are diminished or altered, as, for example, the saliva during a fever, unless the mouth is carefully and frequently cleansed, it becomes putrescent with bacterial growth. Similarly catarrhal gastritis, or any other factor tending to lower acidity of the gastric juice, favors infection by this route.

4. Not infrequently bacteria may gain access to the deeper tissues or to an internal organ, and infection may occur without any recognizable solution of continuity of the surface epithelium. In these hidden or "cryptogenic infections" the entrance point of the parasites may be healed over, or the infecting microörganisms may have been carried to the circulating body fluids by the wandering cells.

Not infrequently, in cases of tuberculosis of the cervical and mesenteric glands in children, there may be no signs whatever of local irritation in the fauces or in the intestine to explain the source of infection. The tonsils are now strongly suspected, and indeed known to be, the source of entry of bacteria causing several acute and chronic infections.

The leukocytes, in their phagocytic activities, no doubt, play an important rôle in the production of cryptogenic infections, especially when an excessive number of pathogenic bacteria have congregated at one point, and congestion, increased leukocytic infiltration, and a lowered vitality of the tissues have occurred prior to the invasions of microörganisms. Wandering cells are commonly found on mucous membranes, gathering up various bacterial and cellular débris. They may carry a virulent microörganism into the deeper tissues, and, al- 
though this may not produce an infection, a large number of bacteria so transported may be able to resist destruction and prove capable of causing infection.

5. Aside from the question of local conditions in the process of infection, other factors may exert an influence. The temperature of the host may be unsuitable for the growth of a certain parasite, even though it has gained entrance to the deeper tissues; a particular route for the introduction of the infecting agents may be necessary, as in typhoid fever and cholera, which are probably always intestinal infections, and, finally, even after the infecting agent has reached the deeper tissues, extension is prevented by a local inflammatory reaction. In many such instances the question of natural immunity is brought into intimate relation with the subject of infection.

After invasion has occurred, some bacteria can best sustain themselves against the defenses of the host at the local point of entry. Such microörganisms may, however, possess unusual vitality, and indirectly, through the lymphatics, find their way to the blood-stream, producing a bacteremia. This is a morbid condition characterized by the presence of microörganisms in the circulating blood.

Some microörganisms may gain entrance to the general circulation more readily than others, and their mode and route of entry vary in the different infections. It is essential that they possess an unusual degree of invasive power, and be capable of protecting themselves against the manifold defensive factors contained in the blood. Kruse believes that in local infections the high pressure of an inflammatory exudate may force bacteria into the adjacent vessels; that they may sometimes be carried into the deeper tissues, and even into the blood-stream, by leukocytes is not to be denied.

When bacteria have entered the circulation, they may act as emboli in the finer capillaries, or, being unable to remain in the circulation, may collect in the capillaries of less resistant tissues, proliferating and producing local metastatic lesions, usually purulent in character. The condition thus produced is known as pyemia.

Saprophytic bacteria or pathogenic bacteria of feeble invasive powers may be able to grow in diseased tissues, such as gangrenous areas, and may assist in effecting morbid changes, producing toxic products of decomposition, which when absorbed into the body, give rise to a series of toxic phenomena, such as fever, rapid pulse, malaise, etc. This condition is known as sapremia, a term that has also been applied to the decomposition of relatively sterile organic material and absorption of 
the toxic products, as when portions of placenta or fetal membranes are retained in the uterus after childbirth.

The term toxemia is employed rather loosely to mean the presence of any toxic material. Its use should be limited to the condition resulting from the absorption of the poisonous substances produced by the non-invasive bacteria themselves, as in diphtheria and tetanus. Septicemia is the term applied to the presence in the body-fluids of toxic products generated by the pyogenic microörganisms.

\section{MECHANISM OF INFECTION}

Since bacterial invasion is of frequent occurrence, the question naturally arises, Why are not infections, both local and general, more frequent? Thus abrasions of the surface epithelium are not uncommon in the presence of active microörganisms; tubercle bacilli may be inspired, and typhoid bacilli may be swallowed, the altered local conditions affording opportunity for producing infection, and yet the host may escape.

Bacterial invasion, therefore, does not necessarily mean infection, and it may be stated that infection can only take place when-

(1) The microörganisms are sufficiently virulent.

(2) When they invade the body by appropriate avenues and reach susceptible tissues.

(3) When they are present in sufficient numbers.

(4) When the host is generally susceptible to their action.

(5) When the microörganisms are able to resist the defensive forces of the host through special agencies aside from their offensive forces.

Not all these factors must necessarily be present before infection may occur. A microörganism may be particularly virulent, so that numbers are relatively unimportant; a host or a portion of the host may be so susceptible or vulnerable to infection that a microörganism of low virulence, which, under normal conditions, would be totally unable to produce infection, may now prove pathogenic.

\section{VIRULENCE}

Virulence refers to the disease-producing power of a microörganism, and is dependent upon two variable factors: (1) Toxicity, and (2) aggressiveness, or the invasive power of the bacteria. In most infections usually both factors are operative.

Toxicity is the term applied to the kind and amount of poison or toxin produced. This poison may be readily soluble, or exogenous, diffusing 
into the surrounding tissues and being readily absorbable; or it may be endogenous, and contained chiefly within the microörganisms, and be liberated only upon the dissolution of the bacterial cell.

Aggressiveness is a term applied to the invasive powers of a microorganism to enter, live, and multiply in the body-fluids, or, in other words, to the aggressive or progressive forces of the microörganism in its new environment.

Toxicity is generally confused with aggressiveness, a highly toxic microörganism being regarded as an aggressive one. For example, the bacillus of tetanus is highly toxic because of the production of a potent soluble poison which gives rise to the symptoms of tetanus, although it is only slightly aggressive, being almost unable to multiply in the tissues. The anthrax bacillus, on the other hand, is highly aggressive, owing to the fact that it usually multiplies to such an extent that it can be found in each drop of blood and in every organ of an infected animal; nevertheless it is but slightly toxic, the animals frequently showing few or no symptoms until shortly before death. The toxicity of a microorganism should, therefore, be regarded separate from its aggressiveness, although in many infections both factors are so intimately concerned that the term virulence may be used to express the degree of pathogenicity or the total disease-producing power.

The virulence of a microörganism is more or less specific, $i$. e., the toxin produced by one species is different from that produced by another in the kind of disease produced and the species of animal infected. Some toxins are active for certain animals only and not for others. Microörganisms of one group may possess general and common pathogenic properties differing only in degree; those of different morphologic and cultural characters may possess totally different powers.

The virulence of a given species is subject to great variation. A few bacteria almost constantly retain their virulence, even when kept for years under artificial conditions; as an example may be mentioned the diphtheria bacillus; others quickly lose their virulence as soon as they are grown artificially, as, e.g., the influenza bacillus; in others-and probably the larger class - the virulence may be raised or lowered according to the experimental manipulations to which they may be subjected. Variations may also be observed among members of the same group of microörganisms, and even among individual microörganisms of the same strain.

Decrease of virulence of a microörganism may be brought about artificially by repeated growth in or upon culture-media, especially 
when transfers to fresh media are made at prolonged intervals. This decrease probably depends upon an actual decrease in virulence, and particularly upon the selection, in artificial growth, of the less virulent or vegetative forms which grow actively and soon exceed in number their more pathogenic fellows. Each time the culture is transplanted more of the vegetative and fewer of the pathogenic microörganisms are carried over, until finally the pathogenic bacteria are entirely eliminated, or their virulence totally destroyed, and the entire culture is composed only of vegetative or harmless forms of bacteria.

Various other agencies lead to artificial lessening of virulence, such as exposure, for short periods of time, to a temperature just under the thermal death-point; exposure to sunlight; exposure to small quantities of antiseptic or germicidal substances; the action of desiccation; subjection to increased atmospheric pressure, etc., these methods being commonly employed in the preparations of vaccines to be used for purposes of active immunization.

The passage of a microörganism or virus through animals usually increases its virulence, but may modify or attenuate it, as in the case of the passage of smallpox virus through the calf, when it loses forever its power of producing smallpox.

Increase in virulence can best be secured by passing the microörganism through animals. It is practically impossible, by any means, to make a known non-virulent microörganism virulent, although it is comparatively easy to increase the virulence of a culture that has become well-nigh non-virulent on account of prolonged artificial cultivation. This fact is worthy of emphasis, and is well illustrated by the large amount of work that has been done in fruitless attempts to render non-virulent, diphtheria-like bacilli virulent by passage through various animals or growth on special culture-media.

In cases where the virulence is slight or absent, experimental manipulations of the culture are directed toward gradual immunization of the microörganisms to the defensive mechanism of the body of the animal for which the organism is to be made virulent. This is well explained according to the hypothesis of Welch, and will be referred to again in the latter part of this chapter. A number of methods are made use of for this purpose:

(a) Passage through animals, which enables the microörganisms gradually to immunize themselves or adopt certain morphologic and biologic changes enabling them best to resist the defensive forces of the host. Since these defensive forces vary with different animals, and 
indeed with the various organs of the same animals, it is usual to find that virulence raised by animal passage affects only the animal or the particular organ of a certain animal, and not all animals in general. Thus, in general, the passage of bacteria through rabbits increases their virulence for rabbits and not for mice, dogs, pigeons, etc.; passage through mice may increase their virulence for mice, but not for rabbits, guinea-pigs, etc.

(b) The use of collodion sacs for increasing virulence has been advocated, especially by French investigators. When microörganisms are inclosed in a collodion capsule of the proper thickness and placed within the abdominal cavity of a suitable animal, the slightly modified body-juices are able to transfuse through the sac, impeding the development of such microörganisms as are unable to immunize themselves or withstand the injurious influences. In this manner a race of virulent bacteria are artificially selected which can endure the defensive agencies of those juices with which they have come into contact.

(c) The addition of animal fluids to the culture-medium may enable the bacteriologist to maintain or even to increase the virulence of a microörganism according to the principles of artificial selection. The fluid, either a serum or whole blood, is secured in a sterile manner and added to the medium in a raw or unheated condition. In this manner the microörganisms are exposed to some of the defensive agencies contained in the juices under these conditions, and this tends to destroy the less resistant bacteria, encourage the more resistant, and at least maintain, for a longer or a shorter time, the virulence of a culture freshly isolated from a lesion or cultivated by animal passage.

\section{THE AVENUE OF INFECTION AND TISSUE SUSCEPTIBIIITY}

Successful infection of the body by certain bacteria can be accomplished only when invasion takes place through appropriate avenues. Thus typhoid, cholera, and dysentery infection seems to take place through the gastro-intestinal tract, and doubtfully by inhalation, and not at all through the skin or urogenital system; gonococci usually enter the body through the genital organs or the eye, and not through the respiratory apparatus or through the skin. The route of infection is less important with microörganisms characterized by great aggressiveness and producing general, rather than local, infections. For example, in most animals anthrax is a general bacteremia, regardless of the route of invasion; plague rapidly becomes a bacteremia, whether 
the bacilli are inhaled, rubbed into the skin, or reach the lymphatics through superficial abrasions; similarly, local staphylococcus and streptococcus infection may become general, regardless of the route of invasion or the location of the local lesion.

The avenue of invasion is also of importance in determining the form, nature, and virulence of an infection. . Thus virulent pneumococci lodging in the pharynx may produce a pseudomembranous angina; in the eye, a severe conjunctivitis; and in the lungs, a pneumonia. When tubercle bacilli gain admission through the skin, they may produce lupus, or a low-grade inflammatory disease rarely terminating fatally. When inhaled, they may produce tuberculosis of the lungs; in the throat they may reach the tonsils and later the local lymphatic glands, etc. When swallowed, they may produce ulceration of the intestines, or pass through the intestinal walls and involve the mesenteric glands, and later the lungs or other organs.

Just as general susceptibility of the host renders infection more likely to occur, so local susceptibility may be induced by injury and fundamental disorders. These changes may not only furnish pabulum for the invading bacteria, but more especially reduce the local resistance of the body defenses.

Even more important, however, is the predisposition of some pathogenic microörganisms to attack certain tissues or organs, and the fact that these tissues are particularly weak in defensive power, so that the bacteria naturally lodge where conditions are most favorable for their growth.

While the primary focus of infection is determined largely by the route of invasion, the selective affinity of microörganisms or their toxins for certain tissues and the inherent tissue susceptibility to the toxins are best in evidence in the location of secondary foci or localization of the infection in general bacteremias. Thus the seat of the principal local lesions in pneumonia is the lungs, and in typhoid fever the lymphoid tissues, especially that of the spleen, and Peyer's patches in the intestine. It is true that mechanical factors may aid in this selection, as, e. g., the occlusion by emboli of microörganisms caught in the capillaries of organs; but, in general, we must conclude that either-(1) Microörganisms tend to be destroyed in every tissue or organ except those that are poor in defensive forces and are susceptible, or (2) that microörganisms or their products circulate passively through a tissue and do not lodge because they possess no affinity for these cells. In many infections both processes are probably operative, and at least we 
are led to the very important conclusion, laid down by Adami, that "in infections the body is never involved as a whole. Coincidentally with the growth of the specific germs in individual organs there tends to be a reaction to, and destruction of, the same in other parts."

The numeric relationship of bacteria to infection is very important, and the number alone may determine whether or not it shall occur. Usually the normal defensive factors of the body are sufficient to overwhelm one or a few bacteria unless they are especially virulent. When an intercurrent or chronic disease, malnutrition, or injury renders the host more susceptible than normal, fewer bacteria than would otherwise be required may successfully infect the body. Also with true parasites, or those with well-marked aggressiveness, such as the anthrax bacillus, a few may be sufficient, if they reach the circulating fluids, to produce infection. Thus Webb, Williams, and Barbor ${ }^{1}$ have found that one anthrax bacillus was sufficient to infect a white mouse, and as few as 20 tubercle bacilli were sufficient in one instance to infect a guinea-pig.

Park has directed attention to the fact that when bacteria are transplanted from culture to culture, under supposedly favorable conditions, many of them die; it is highly probable that when they are transplanted to an environment that is likely to be unfavorable, as are the body tissues with various defensive mechanisms, many more must die. This is an important point to bear in mind in attempting to correlate experimental results with the natural cause of an infectious disease. In the laboratory we reproduce disease experimentally by the immediate injection of millions of bacteria, whereas in nature there is rarely any such immediate overwhelming of the tissues. For example, pneumonia may be produced experimentally in dogs by the injection of a large number of virulent pneumococci directly and at once into the bronchi, yielding a positive result with a microörganism which, under natural conditions and in smaller numbers, would be relatively innocuous for the animal under observation.

\section{GENERAL SUSCEPTIBIIITY IN RELATION TO INFECTION}

Under normal conditions the body-cells of a host will invariably offer some resistance to invasion and infection by pathogenic microorganisms. When, however, any condition that depresses or diminishes general physiologic activity and vitality exists, the host may be unable to master these defensive forces, and accordingly becomes predisposed or more susceptible to infection.

${ }^{1}$ Transactions Sixth International Congress on Tuberculosis, 1908, p. 194. 
Predisposition may be inherited or acquired.

Inherited predisposition may be-(a) Specific, or species susceptibility, as, e.g., dogs to distemper; cattle to contagious pleuropneumonia; hogs to hog cholera; man to gonorrhea; chancroids, acute exanthemata, typhoid fever, etc. (b) Racial, as Eskimos to measles and syphilis, ordinary sheep to anthrax, whereas Algerian sheep are immune, etc. Racial susceptibility is frequently but a lack of acquired immunity; for instance, measles, syphilis, gonorrhea, and other diseases brought by settlers to foreign peoples among whom these diseases were previously unknown, find them peculiarly susceptible and the diseases unusually virulent. (c) Familial, $i$. e., members of a family may, through generations, be unusually susceptible to scarlet fever, tuberculosis, rheumatism, rheumatoid arthritis, metabolic disturbances, etc. (d) Individual predisposition, which depends principally upon sex, age, and peculiar tissue susceptibility. Thus infants are especially prone to contract certain infections on account of the immature development of the body-cells, and this susceptibility to infection is further influenced by acquired factors, chiefly malnutrition. On the other hand, very young children enjoy an immunity to several infections, such as typhoid fever, scarlet fever, and even diphtheria, probably due, as Abbott has suggested, to the fact that pathogenic substances that may set up molecular and destructive disturbances in the poorly developed cell have but little effect upon the more inert protoplasm of the immature cell, and that if certain bacteria gain admission to the tissues, the cells may destroy them, their toxins not combining with the molecular side-chains, and, as a consequence, not injuring or interfering with the cell functions.

Acquired susceptibility bears a more important relation to infection, and may be due to various factors, most of which lead to a state of reduced vitality, normal physiologic processes being impaired to a greater or less degree.

(a) Overwork or overstrain leads to general or local predisposition to disease. Those engaged in hard labor, mental or physical, which involves late hours and inadequate periods of rest and recreation, frequently associated with inadequate nutrition and foul air, are likely to succumb to tuberculosis, typhoid fever, pneumonia, etc.

The influence of overstrain on acute infections has been shown experimentally by Charrin and Roger, ${ }^{1}$ who found that white rats naturally immune to anthrax became quite susceptible after being compelled to turn a revolving wheel until exhausted before they were inoculated;

${ }^{1}$ Compt. rend. Soc. de Biol. de Paris, January 24, 1890. 
similarly of four guinea-pigs who were placed in a cage so constructed that they were forced to keep moving for one or two days three died in from two to nine days after the experiment. Smears and cultures made from the livers, spleens, and blood gave positive results.

(b) Previous infection with the same or another infectious disease may predispose the individual to renewed infection. Thus some infections, such as erysipelas, furunculosis, acute rheumatism, pneumonia, and influenza, not only fail to leave the body-cells immine, but actually predispose to second attacks. Whether the microörganisms of these diseases are not all destroyed, but are retained in the system and become active when the general vitality is lowered, or whether a new infection occurs, is not definitely known, and probably either may occur.

One attack of an infectious disease may weaken the tissues and render them susceptible to an infection of a different nature. Thus the acute exanthemata may follow one another, and tuberculosis may supervene upon any of them.

(c) Malnutrition exerts some effect on the resistance to infection. Thus the terrible epidemics of plague, cholera, typhus fever, and typhoid fever which have followed in the wake of famines in Europe and Asia during the past centuries are examples of the influence of malnutrition as a factor in predisposing to disease. The tendency of marasmatic infants to develop enterocolitis, thrush, bronchopneumonia, and other infections, and of scorbutics to local infections of the mouth, illustrates the influence of insufficient food in decreasing the resistance to disease. Here may also be included local malnutrition, such as loss of nerve or blood supply, predisposing to local infection, especially with pyogenic microörganisms.

(d) Diet produces some variation in the resisting powers to infection. For example, the ordinary wild rat is not susceptible to anthrax unless it is fed for a week or more on coarse dry food, when it become susceptible. Here, of course, malnutrition may come in intimate relationship with diet, as an inefficient diet may greatly lower the general resistance. The influence of diet is particularly noticeable from the fact that the diseases of carnivorous animals are not the same as those that affect herbivorous animals, and that each class is frequently immune to some of the diseases that attack the other.

(e) Intoxications of various kinds predispose to infections. Thus it is a common clinical observation that excessive indulgence in alcoholic beverages predisposes to infections, notably pneumonia. Abbott ${ }^{1}$ has

${ }^{1}$ Jour, Exper. Med., 1896, 1, No. 3. 
demonstrated experimentally that the daily administration, to rabbits, of 5 to 10 c.c. of alcohol introduced into the stomach by a tube, renders these animals more susceptible to infection with Streptococcus pyogenes and Bacillus coli. Wagner, Leo, and Platania have also found animals that under the influence of chloral, phloridzin, alcohol, and curare are more susceptible to infection.

(f) Exposure to cold and wet frequently lowers the resistance of man and other warm-blooded animals to infection. The influence of these factors, well illustrated in the etiology of "colds" and pneumonia, is not without experimental foundation. Thus Pasteur found that fowls, which are naturally immune to anthrax, are readily infected if they are inoculated after their body temperature has been reduced by a cold bath. Conversely, Gibier ${ }^{1}$ has shown that frogs, which are also naturally immune to anthrax, are readily infected if their temperature is previously elevated and maintained at $37^{\circ} \mathrm{C}$.

(g) Trauma and morbid conditions in general may predispose to infection. Thus injuries reduce the local resistance and facilitate local infections that vary with the severity and extent of the trauma. The increased susceptibility of injured joints and pneumonic lungs to tuberculosis; the frequent and oftentimes extensive streptococcus infection accompanying scarlet fever and smallpox; the increased susceptibility of diabetics to furunculosis and local gangrenous lesions of the skinall show the increased susceptibility of individuals already injured or diseased to infection.

\section{THE DEFENSIVE MECHANISM OF THE MICROÖRGANISM IN RELATION TO INFECTION}

After bacterial invasion has occurred, the question of whether or not the microörganism can overcome the defensive forces of the host and prove pathogenic may depend to some extent upon the peculiar defensive factors of the invading bacteria against the offensive mechanism of the host, aside from their toxins or other distinctly offensive forces.

Morphologic and Physiologic Changes of the Microörganisms.-For example, capsule formation or thickening of the ectoplasm of certain bacteria is evidence of their increased powers of resistance against the opposing forces of the host. The capsule may be quickly lost when the microörganism is cultivated on artificial media, and its virulence be correspondingly lowered, but by repeated animal inoculations a race of

${ }^{1}$ Compt. rend. Acad. de Sci. de Paris, 1882, xcix, 1605. 
capsulated organisms with increased virulence is produced, explaining in a way the mechanism of animal passage in raising the virulence of a given organism. This, however, is not invariable, and, indeed, may act in a contrary manner, as the passage of smallpox virus through heifers attenuates and modifies instead of increasing its virulence.

Aggressins.-The microörganism may actively secrete a material that overwhelms the defensive forces of the host. This phase of the subject has been studied exclusively by Bail, who sought to prove that the question of pathogenicity of a microörganism is dependent upon its ability to secrete substances that are able to paralyze the protective forces of the host, especially the leukocytes. These substances are called "aggressins," and they were distinguished by the fact that they were formed by living bacteria and only in the living body. In support of this theory Bail was able to show that substances are present in the exudates of fatal infections, which, when injected in small quantities into another animal with sublethal doses of the microörganism, would cause a rapidly fatal infection. Later Wassermann and Citron showed that "artificial aggressins" could be prepared by autolyzing bacteria in water or serum. While the subject of aggressins is still unsettled, there is strong evidence to show that they are the endotoxins liberated by the breaking-up of the microörganism.

The well-known statement of Metchnikoff, that a particular virulent microörganism is not so readily taken up by leukocytes as is an avirulent strain, may be explained by the fact that the microörganism, in its virulent parasitic state, secretes substances that repel the phagocytes, neutralize the opsonins, or form actual leukocytic toxins. This action may be due to liberated endotoxins, or, as Bail claims, to specific secretory substances of the bacterium - the aggressins-specifically formed and liberated by the microörganism for protection against the host.

Hypothesis of Welch.-Not entirely foreign to this subject is the very interesting hypothesis of Welch. A bacterium may not only produce substances directly inimical to the defensive forces of the host, but it may actually immunize itself against these defensive powers. "Looked at from the point of view of the bacterium, as well as from that of the animal host, according to the hypothesis advanced, the struggle between the bacteria and the body-cells in infections may be concerned as an immunizing contest in which each participant is stimulated by its opponent to the production of cytotoxins hostile to each other, and thereby endeavors to make itself immune against its antagonist."

It is well known that, when freshly isolated from a patient having 
typhoid fever, the typhoid bacillus resists agglutination, whereas it becomes easily agglutinable after a period of artificial cultivation. It may be assumed that, when active, the bacillus as an infecting agent gradually became more resistant against the agglutinating properties of the patient's serum, and that, when grown on artificial media, it loses this resistance by being removed from the stimulating influence of the infected body.

This hypothesis, however, would go a step further in assuming the possibility of the receptors of the invading bacteria anchoring certain constituents of our body-fluids, and being stimulated to the production of various cytotoxins, which attack the leukocytes, erythrocytes, nervecells, liver, kidney, etc. In other words, each bacterium may be conceived as being composed of a central atom group with numerous sidechains, just as Ehrlich conceived the hypothetic structure of body-cells, and that these side-chains, primarily present for the purpose of anchoring food material, may likewise anchor various pathogenic animal substances, with the production of substances acting as antibodies to the opposing forces of the host. Welch assumed that these bodies were of the nature of amboceptors, which may become complemented by bacterial complement or by endocomplements of the tissue-cells; this is of secondary importance, and there is no reason why they may not be of different structure, and similar to all three orders of antibodies produced by body-cells according to Ehrlich's side-chain theory of immunity.

This hypothesis may possibly explain certain instances of so-called species and organ virulence, whereby the virulence of an organism artificially increased by repeated passage through animals of the same species, does not manifest this increased virulence for animals of different species. If, for example, the virulence of the chicken cholera bacillus is increased by repeated passage through the chicken, the increase of virulence affects this animal, but does not affect the guinea-pig. Certain organs may likewise be subject to a similar selective virulence, if the increase in virulence has been induced by the specific intervention of those organs, and this selective virulence shows itself, irrespective of the manner in which the infection was produced.

That virulence of this order is playing an important rôle in the processes of infection is a theory supported by the discovery that different strains of the same species of bacteria are found to produce characteristic lesions, and while this affinity for a certain organ may be natural and inherent, there can be no doubt that it may also be experimentally induced and acquired. For example, according to Rosenow, a certain 
strain of streptococcus will produce arthritis; another, endocarditis; another, gastric ulcer, etc.

This remarkable species and organ specificity may be due to the fact that the bacteria of a particular culture have been immunized against defensive forces of a particular animal host or a certain organ of the host, so that, when introduced, they thrive as a result of their special and acquired offensive forces. On the other hand, the specificity may be due to the fact that the bacteria have been accustomed to a certain nutriment furnished by a particular species or organ, and that they cannot thrive unless they receive this special nutriment, and, as a result, the species or organ fulfilling this requirement will become the special seat of infection (Simon).

\section{MIXED INFECTION}

Several different microörganisms may produce infection at the same time, or one may follow the other or others and produce secondary infection. The combined effects, upon the tissues of the host, of the products and action of two or more varieties of pathogenic bacteria, and also of the influence of these different forms on each other, are of great importance in the production of disease. The metabolic products of one bacteria may neutralize or accelerate the action of an associated species, or combine to form a new substance entirely different from its antecedents.

Thus pyogenic cocci affect anthrax bacilli in an injurious manner; on the other hand, aërobic bacteria accelerate or make possible the growth of anaërobes by absorbing uncombined oxygen. Tetanus bacilli will not grow outside of the body in the presence of oxygen unless aërobic bacteria are associated with them; not infrequently tetanus bacilli and their spores would not develop in wounds were it not for the presence of the aërobic bacteria introduced with them; this factor is of much importance, especially in tetanus produced by cowpox vaccine, where, through careless treatment of the lesion, both tetanus bacilli and pyogenic cocci are admitted to the wound.

Again, it may be found that one microörganism increases the virulence of another; thus the scarlet-fever virus is favorable to the development of streptococci.

Generally all infections of mucous membranes are mixed infections. Numerous bacteria are present upon the mucosa of the air-passages and gastro-intestinal tract; these are usually harmless, unless the resistance of the host is lowered in some manner, in which case not only one 
but several varieties of these bacteria invade the tissues and cause infection. When one pathogenic microörganism, such as the typhoid bacillus, has caused the primary infection, because of the local and general conditions of lowered vitality of the tissues, these otherwise saprophytic bacilli tend to intensify the infection. Blood infections, on the other hand, are usually due to one form of bacteria, and even when two or more varieties are introduced, only one, as a rule, is capable of surviving and developing. The products of certain bacteria, on the other hand, may immunize the host against infection with other bacteria, for, as shown by Pasteur, attenuated chicken-cholera cultures may produce immunity against anthrax. In the intestine harmless varieties of bacteria may be made to crowd out more dangerous ones; this is exemplified by the ingestion of soured milk which contains lactic-acid bacteria, as advocated by Metchnikoff.

\section{SUMMARY}

From what has been said it is clear that infection differs from mere surface contamination, and cannot be said to occur until the invading bacteria have reached the deeper tissues, or a point where they may grow and multiply. The surface epithelium and various secretions offer the most potent local obstacles to infection, but even when these barriers are broken down, the invaders may not survive the onslaughts of various protective agencies of the host. In order to withstand and overcome these attacks, the bacterium may undergo certain morphologic and physiologic changes, and actively secrete a substance that is inimical to the defensive forces of the host, or immunize itself against these forces. Thus a certain species of bacteria may become selectively fortified or immunized against a certain host or organ of that host, and show a specific affinity for producing infection of a certain animal or a particular organ. When the bacterium has overcome the defensive forces of a host, it may, by the formation and.action of exogenous and endogenous toxins, bacterial proteins, mechanical blocking of vessels, or formation of ptomains, produce disease. These various factors will be considered in greater detail in the following chapter. 


\section{CHAPTER VII \\ INFECTION (Continued) \\ PRODUCTION OF DISEASE}

When pathogenic microörganisms have reached the deeper tissues and multiplied, infection has occurred, but, as previously stated, tissue changes of sufficient extent to produce definite lesions and symptoms of disease may or may not result, depending upon whether or not the defensive forces of the host are able to overcome the invaders or are overcome by them. If the latter has occurred, and the invading bacterium is firmly established in its host, the question of how the bacterium and its products cause disease, that is, the mechanism of the production of an infectious disease, arises for consideration.

The subject is, indeed, quite complex. Although the etiologic relationship of a large number of pathogenic bacteria to definite pathologic changes and conditions has been proved by the regularity with which they are found in the diseased tissues, and in many instances has been corroborated by animal experimentation, yet the ways and means by which these bacteria produce disease are quite varied, and are seldom dependent upon one product of bacterial activity. For example, diphtheria and tetanus are apparently simple infections, being caused by soluble toxins secreted by the respective bacilli. There are many factors concerning the action of these toxins, however, which are not as yet understood. Again, the lesions of staphylococcus and other pyogenic infections are probably due to the activities of soluble toxins, endotoxins, and the protein of the bacterial bodies. All three of these factors are probably concerned in the production of typhoid fever and cholera, whereas the symptoms of sleeping sickness are due in part to blocking of a small but physiologically important vessel in the brain by trypanosomes, with the absorption, at the same time, of toxins and disintegration products. To these may be added the effects of other biologic activities of the bacteria in living or dead tissues, such as the production of gas from carbohydrates, proteins, etc., in Bacillus aërogenes capsulatus infection. Each infection, therefore, must be regarded as largely a law unto itself, so that all that will be included within the scope of this book will be the mention and illustration of what are 
generally accepted as the ways and means by which bacteria and protozoa produce disease, omitting a description of each disease in detail.

In the great majority of instances disease is produced as the result of chemical substances generated by the metabolic processes of bacteria. Animal parasites and certain bacteria, such as that of anthrax, may do harm mechanically by forming capillary emboli; but, as stated, bacteria, as a rule, produce their effects chiefly through chemical means. Accordingly, bacteria may give rise to infection and disease through the following agencies:

1. Soluble or extracellular toxins, which are poisons generated by bacterial cells and discharged into their surrounding media.

2. Intracellular toxins or endotoxins, which are specific poisonous products of bacterial activity, and are contained within the cells.

3. Aggressins, or substances secreted by bacteria, that neutralize opsonins and prevent phagocytosis.

4. Bacterial proteins, which are poisonous protein constituents of the bacterial cells and are responsible for certain general and non-specific lesions.

5. Ptomains, which are the secondary products of decomposition of the media upon which the bacteria are growing; these may be absorbed and produce symptoms of intoxication.

6. Mechanical action of bacteria, whereby certain symptoms or lesions may be due to the blocking of small but physiologically important vessels with emboli of bacteria, in addition to the effects of mechanical irritation.

\section{TOXINS}

Nomenclature.- Of all the various means whereby bacteria produce disease, none possesses so much importance as the poisonous substances, known as toxins, elaborated by the metabolic activities of the microorganisms. A few classes of bacteria secrete this poisonous principle directly into the tissues or artificial culture-media in which they are growing, and hence are known as soluble, exogenous, extracellular, or true toxins. Other bacteria retain most of their toxins within the bacterial cell, and for this reason are called endotoxins, or intracellular toxins; these are liberated upon the disintegration of the bacteria by various mechanical, physical, or chemical means.

By common consent the term "toxin" is applied to the soluble or true toxins, such as those of diphtheria and tetanus, and hence the term, when used without further qualifications, may be considered to refer to toxins of this class. 
Aside from bacterial toxins, characteristic poisons are also produced by certain of the higher plants (phytotoxins) and animals (zoötoxins), and although few are of medical interest, their study has thrown considerable light on the phenomena of toxin-antitoxin immunity.

Extracellular Bacterial Toxins.-Definition.-Bacterial toxins may be defined as poisonous products produced by bacteria in both living tissues and artificial culture-media. The symptoms resulting from their activity appear after a certain period of incubation, and all are capable of stimulating the production of specific antitoxins. They represent the chief poisonous product of bacteria, and are mainly responsible for the symptoms of infection caused by the specific bacteria that have produced them.

The true toxins causing infection in man are chiefly:

1. Diphtheria toxin.

2. Tetanus toxin.

3. Botulism toxin (a form of meat poisoning).

4. Dysentery toxin (Kruse-Shiga).

5. Staphylolysin and other bacterial toxins.

General Properties of Soluble Toxins.-Many of the true toxins are extremely labile, and susceptible to the action of heat, light, age, etc.; consequently an absolutely pure toxin is practically unknown. Oxygen, even as it occurs in the air, is harmful; all oxidizing agents, including the oxidizing enzymes, quickly destroy them, and Pitini ${ }^{1}$ has ascribed the harmful effects of toxins to their power of reducing the oxidizing capacity of the tissues. Some substances seem to attack only the toxophore portion of the toxin molecule, e.g., iodin and carbon disulphid (Ehrlich). In the preparation of antitoxin, the first doses of toxin are frequently modified by adding a chemical of this nature. According to Gerhartz $^{2} x$-rays tend to weaken the toxins.

Because of their great lability, the toxins do not lend themselves to accurate chemical analysis. Our knowledge of them has been gained largely through a study of the lesions and symptoms produced by injecting the toxins into susceptible animals.

The toxins are all poisonous, but in order to exert their toxic effect they must enter into chemical combination with cells; hence there is a necessary period of incubation before symptoms of their activity appear. Most bacterial toxins are not absorbed from the intestine (botulinus toxin excepted), and when introduced into the gastro-intestinal tract, they are usually unable to produce symptoms and are quickly destroyed.

${ }^{1}$ Biochem. Zeit., 1910, 25, 257.

${ }^{2}$ Berl. klin. Woch., 1909, 46, 1800. 
An essential property of a toxin lies in the fact that we can immunize a subject against it, and are able to demonstrate the presence of antitoxin within the serum of the immunized animal.

Chemical Properties of Soluble Toxins.-As has just been stated, the exact chemical nature of toxins is unknown. This is due principally to the fact that pure toxins of bacteria are rarely obtainable, except in conjunction with their associated products, such as lysins, pigments, acids, etc., as well as to the great lability of the toxins. A summary of the results of researches into the chemical nature of toxins would indicate that they are toxalbumins, or allied to proteins. Certain investigators have reported that very active toxins obtained by purification processes did not give the protein reactions, yet toxins are digested by proteolytic ferments, and, like proteins, are precipitated by nucleic acid (Kossel). According to Field and Teague, ${ }^{1}$ the toxins act like electropositive colloids, but diffuse faster than do proteins. Our present knowledge of the chemistry of the true toxins has been expressed thus by Oppenheimer: "We must be contented to assume that they are large molecular complexes, probably related to the proteins, corresponding to them in certain properties, but standing even nearer to the equally mysterious enzymes with whose properties they show the most extended analogies both in their reactions and in their activities."

Structure of Toxins.-According to Ehrlich, the toxin molecule consists of a main central atom or radical, with a large number of organic side-chains grouped, as in other organic compounds, about this main radical. Each of the side or lateral arms is composed of two portionsone, the haptophore group, which has a chemical affinity for certain chemical constituents of the tissues of susceptible animals, and the other, the injury-producing portion, called the toxophore group. (See Fig. 40.) An animal is susceptible to a toxin only when its cells contain substances that possess a chemical affinity for the haptophore group of the toxin, and also substances susceptible to the toxic action of the toxophore group.

The toxophore group is far more unstable and susceptible to deleterious influences than is the haptophore portion. When the molecule has lost the toxophore radical, it is known as a toxoid, which is still capable of uniting with the side arms of cells but is devoid of toxic action.

Nature of Toxins.-It has been abundantly demonstrated that toxins are colloids, and in many respects bear a close resemblance to enzymes. (See p. 244.) The toxins are synthetic products of bacterial activity. They are of absolutely specific nature, and in this manner ${ }^{1}$ Jour. Exper. Med., 1907, 9, 86. 
differ from ptomains, which are cleavage products from the medium upon which the bacteria have been grown. Furthermore, ptomains of similar properties may be produced by several different kinds of bacteria, and accordingly are non-specific in nature. Toxins, like ferments, can give rise to antibodies, whereas ptomains cannot produce them.

The extracellular or soluble toxins differ from the intracellular toxins in that they are more easily diffused throughout the animal juices, and that their diffusion occurs independently of the invasiveness of the bacteria, so that comparatively few microörganisms growing at some unimportant focus, and causing but slight local lesions, may be able to give rise to profound general intoxication. This is well illustrated in diphtheria, where the local lesion in the throat may be quite small, and in tetanus, where it may indeed be undiscoverable-yet either, through the action of their toxins on special tissues, may cause profound intoxication and death.

Selective Action of Toxins.-Extensive studies of the toxins of diphtheria and tetanus and of cobra venom have shown that they are quite complex, and are usually composed of two or more distinct and separate toxins possessing different pathogenic properties, although one of these may predominate in producing symptoms.

All infections with the group of true toxin-producing bacteria manifest certain non-specific symptoms of general intoxication, namely, fever, headache, malaise, prostration, etc.; but the typical symptoms of these diseases are due to the remarkable selective action of the toxins upon certain cells or organs, dependent upon the ability, chemical, physical, or both, of the toxin to combine with these specific cells. For example, tetanus toxin contains tetanospasmin, that has a special affinity for nervous tissue; and tetanolysin, a poison that has a selective affinity for erythrocytes and is hemotoxic. Ehrlich has shown that these are really different toxins, and not one toxin with a two-fold function, even the antitoxins of the two being different. Similarly, the general symptoms and necroses of diphtheria are attributed to the main toxin of the bacillus, and the nerve lesions and paralyses to a secondary but distinct secretory product known as toxon. This latter view of Ehrlich's, however, is much disputed, many investigators believing that toxon represents a degenerated or modified form of the one toxin.

The special affinities of toxins for certain tissues have analogies among the poisons of higher plant life, as, for example, strychnin has a similar selective affinity and is said to be specific in its action upon the motor cells. 
The venom of various serpents, especially that of the cobra, has specific action: the erythrocytes of various animals are readily attacked by it, and the cells of the respiratory center are apparently profoundly affected.

Aside from the special effects of the toxins upon certain cells and tissues, it must be remembered that toxins may involve the body-cells in general, and particularly those of the parenchymatous organs, such as the kidneys, heart, and liver, causing coagulation of the protoplasm (cloudy swelling) and final dissolution. The harm brought about by the toxins or toxic products of the pyogenic group of microörganisms, for instance, acts mainly in this manner.

\section{SPECIAL PROPERTIES OF THE PRINCIPAL TOXINS}

1. Diphtheria Toxin.-Diphtheria bacilli vary considerably, both in tissues and in artificial culture media, in the quantity of toxin secreted; thus in bouillon large amounts are seldom found in less than from seven to fourteen days.

The action of the toxin is dependent upon the dosage, and a certain period of time must always elapse before the symptoms appear, the minimum being about one day. Large doses may shorten this period of incubation, but cannot diminish it below a certain limit.

The lesion of diphtheria is practically always local, and is usually situated on the mucous membrane of the upper air-passages. It is characterized by the formation of a pearly white membrane that is adherent to the underlying edematous tissues. The toxin produces necrosis of the surface epithelium, and the product, together with fibrin and leukocytes, constitutes the membranous exudate. From this focus toxin is absorbed by the lymphatics and blood-stream, and distributed throughout the body, the bacilli being rarely found in the blood or internal organs. Later the effects of toxin intoxication are shown by paralyses of certain motor nerves and ganglia, particularly those of the palate and heart.

When a guinea-pig receives a subcutaneous inoculation with diphtheria toxin, a typical hemorrhagic gelatinous edema develops at the site of inoculation (Fig. 36). Upon opening the abdominal cavity one finds but little peritoneal exudate, but the vessels of the mesentery are injected and the adrenal glands show characteristic acute hyperemia (Figs. 37 and 38). Bloody perieardial and pleural exudates will be found in the thorax, and solidified areas in the lungs. Guinea-pigs surviving a dose of toxin may, after two or four weeks, begin to show paralysis of the hind 


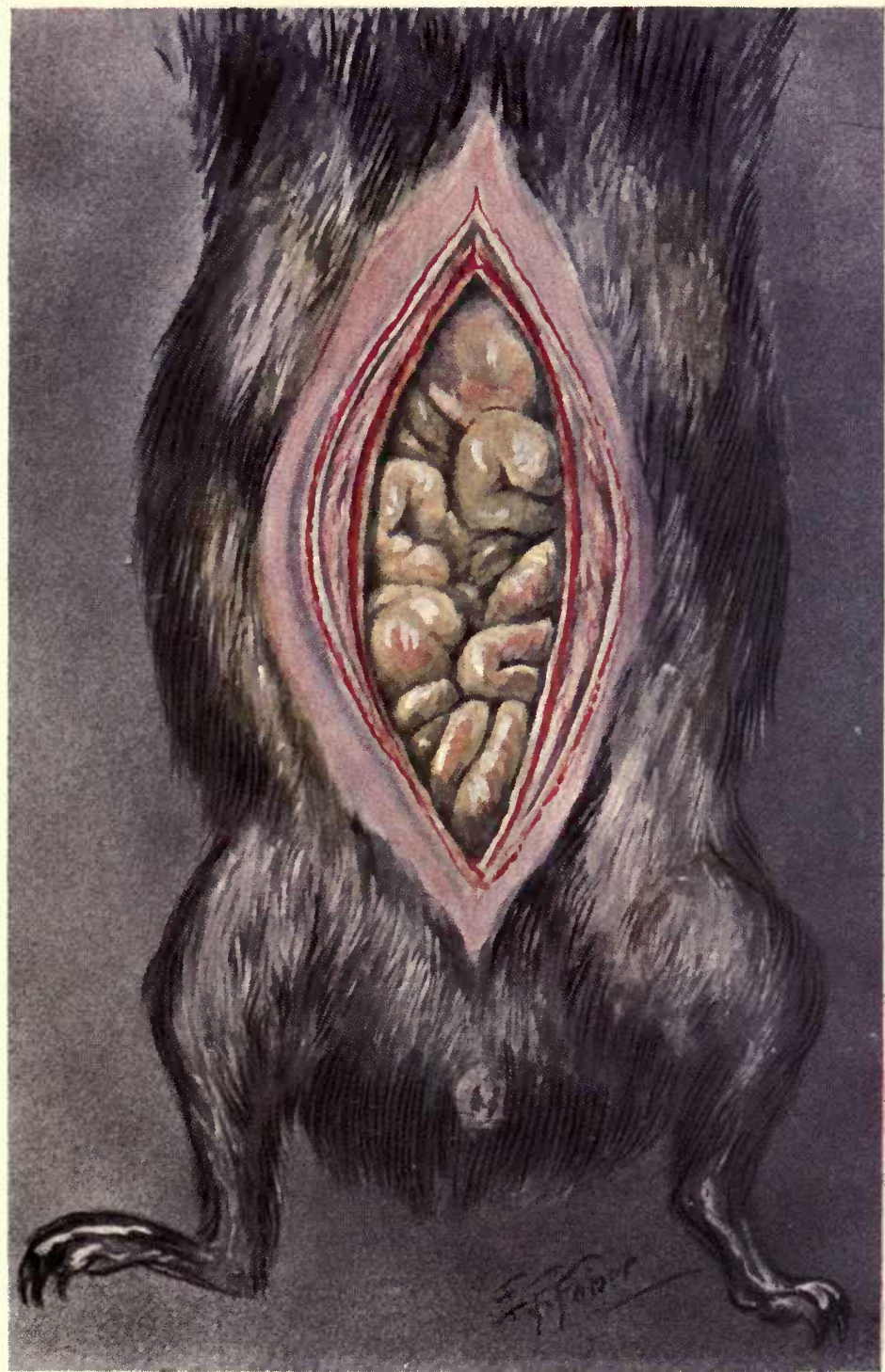

Fig. 36.-Abdominal Wall of Guinea-pig Showing Diphtheric Edema.

Shows abdominal wall of a guinea-pig forty-eight hours after subcutaneous injection with 2 c.c. of a seventy-two-hour bouillon culture of a diphtheria bacillus isolated from the throat of a diphtheria convalescent. 
and then of the fore extremities, a condition analogous to the post-diphtheric paralysis occurring in man and ascribed to the effects of toxon.

Method of Testing the Virulence and Toxicity of Diphtheria Bacilli.Young guinea-pigs weighing from 250 to 300 grams are quite susceptible to diphtheria toxin, and are used in determining the strength of a toxin and in standardizing antitoxin. The test may be of great value in the management of convalescent and "carrier" cases of diphtheria, harboring bacilli in the upper air-passages, in determining whether the microörganisms are dangerous or merely harmless non-pathogenic saprophytes. It is practically impossible, from the morphology of the organism alone, to decide whether or not a given culture is dangerous, and prolonged quarantine may not only be irksome and inconvenient, but, if the organisms are proved to be harmless, it is unnecessary as well.

To be reliable, however, such a test must be carried out very carefully. In the case of a highly virulent culture, the mere introduction of a few organisms beneath the skin will suffice to demonstrate their dangerous character, but with cultures only slightly virulent, more care is necessary, for although the patient may show no ill effects as a result of the presence of the bacilli, in the throat of another and less immune individual they may be highly dangerous.

The following method has been used by the author in many hundreds of such tests in the Philadelphia Hospital for Contagious Diseases, and has proved of distinct value:

1. Make a culture of the part harboring the bacilli on a tube of Löfler serum medium. Incubate at $35^{\circ} \mathrm{C}$. for from eighteen to twenty-four hours; prepare a smear and stain with Löffler's methylene-blue. If diphtheria bacilli are present, they must be isolated in pure culture. Never attempt a guinea-pig test with an impure culture!

2. Isolate by the "streak" method, on plates of blood-serum.

3. Inoculate a tube of 1 per cent. glucose bouillon, which is neutral or slightly alkaline, with several different colonies.

4. Incubate at $35^{\circ} \mathrm{C}$. for three days, keeping the tube in a slanted position in order to give the culture as much oxygen as possible. If a good growth does not appear in twenty-four hours, transplant to another tube of bouillon until the bacilli have been "educated" to grow on the medium.

5. Examine for purity. Select a 250- to 300-gram guinea-pig and inject 2 c.c. of the unfiltered culture in the median abdominal line. Animals over the weight specified are more resistant and less reliable for the test. The unfiltered culture is used, since toxin is but one element of the disease-producing power of diphtheria bacilli, and toxin production in bouillon may not be a true index of the toxin production in mucous membranes.

6. Carefully observe the animal for at least four days. Even slight toxemia, especially if accompanied by edema at the site of injection, should be regarded as a positive result (Fig. 37). 
7. After death perform a careful autopsy. Make cultures of the edematous area, peritoneum, and heart blood. Diphtheria bacilli may be found in the edematous fluid, but will rarely be found in the peritoneum or in the blood. Observe whether acute hyperemia of the suprarenal glands is present (Figs. 37 and 38).

8. Not infrequently animals showing mild or even an absence of the symptoms of toxemia develop paralysis of the hind quarters two or three weeks later. According to Ehrlich, this paralysis is due to the action of "toxon," a toxic substance secreted by the bacillus or, as believed by others, a modified form of toxin.

9. To prove that diphtheria was the cause of the toxemia or death mix 2 c.c. of the culture in a test-tube with 1 c.c. of diphtheria antitoxin (500 units). After standing aside for an hour at room temperature, inject the mixture subcutaneously in the median abdominal line of a 250 to 300 gram guinea-pig. Symptoms of toxemia do not develop.

Standardizing Diphtheria Toxin.-The strength of a diphtheria toxin is estimated by injecting subcutaneously a series of guinea-pigs weighing approximately 250 grams, with decreasing amounts of toxin. How many dilutions will be necessary it is impossible to state; for exact results several pigs of the same weight should be inoculated with the same dose, and the effects should show various gradations, dependent upon the size of the successive doses. In order to obtain a uniform method for estimating the strength of a diphtheria toxin and thus obtain comparative values, a standard unit has been adopted, consisting of the smallest amount of toxin that will kill a healthy guinea-pig weighing about 250 grams in from four to five days. This is known as the minimum lethal dose, or dosis lethalis minimus. The technic used for determining this dose is given in the chapter on Antitoxins, p. 232.

A quick and accurate method for estimating the amount of diphtheria toxin present in the body-fluids of a diphtheric patient would be of value in controlling the antitoxin treatment of this infection. At present the amount of antitoxin administered is regulated according to the clinical condition of the patient. Uffenheimer has used a method for determining the presence of toxin, consisting in injecting intraperitoneally a 250 -gram guinea-pig with 0.1 to 0.4 c.c. of the patient's serum, diluted with 2 to 4 c.c. of salt solution. The presence of a distinct doughy edema of the abdominal cavity after seventeen to twentyfour hours indicates the presence of diphtheria toxin, an observation that may be confirmed by making an autopsy at the end of forty-eight hours. The diagnostic value of this method has not been adequately established: it is doubtful if it yields any information other than is more readily gained by making a good cultural examination of the patient, and it does not aid in the estimation of the quantity of toxin, which is the result most desired. 


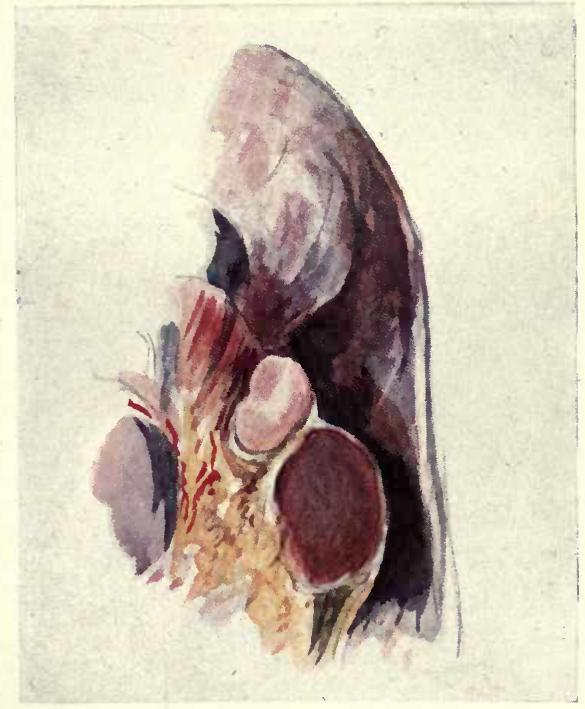

Fig. 37.-Normal Adrenal Gland of a Guinea-pig.

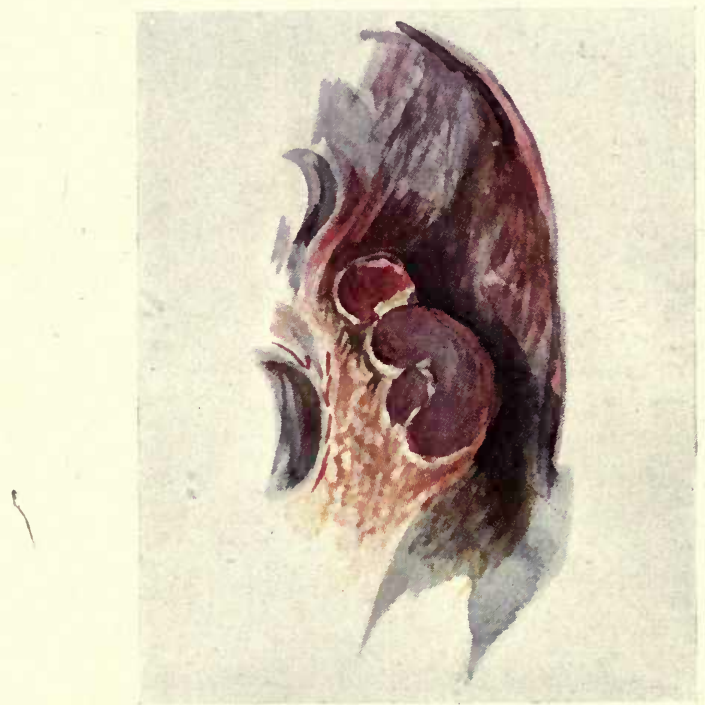

Fig. 38.-Adrenal Gland of a Guinea-pig after Fatal Diphtheric INTOXICATION. 

Diphtheria toxins have been classified into three groups, depending upon the degree of avidity for antitoxin they display, viz., prototoxin, deuterotoxin, and tritotoxin. Each of these toxin groups may, in whole or in part, be converted into toxoids. The prototoxin has a greater affinity for the antitoxin than has the deuterotoxin, and the deuterotoxin has a greater affinity for the antitoxin than has the tritotoxin. The same relation is apparent with the three toxoids, which are not poisonous, but which have the same power of combining with antitoxin as have the toxins from which they take their origin.

In standardizing antitoxin, it is found in general that with a perfectly fresh toxin a certain amount of antitoxin will just neutralize a definite amount of toxin. If older toxin is used, it is found that the toxin has lost about one-half its toxic power, but retains its initial power for neutralizing antitoxin. Ehrlich explained this by showing that the diphtheria toxin molecule is composed of two groups-one the carrier of the toxic qualities, the toxophore group, which is quite labile; the other uniting the whole molecule with antitoxin, being capable of neutralizing it, and characterized by its stability. The toxophore group being destroyed as in old toxin, the poison loses its toxic qualities, but retains its power to bind antitoxin. This modified toxin or non-poisonous diphtheria toxin has been designated by Ehrlich "diphtheria toxoid."

2. Tetanus Toxin.- Of all bacteria classed as true toxin producers, none possesses greater toxicity than does the tetanus bacillus. The number of organisms producing sufficient toxin to cause a fatal infection may be so small that careful anaërobic cultures made from the local lesion of infection, together with injection of the wound secretions into white mice, may fail to disclose the presence of tetanus bacilli.

According to Ehrlich, tetanus toxin is composed of two separate and distinct substances-(1) Tetanospasmin, a neurotoxin, which is very labile and responsible for the severe symptoms of the infection; tetanolysin, a hemotoxin, which is more stable and destructive for ery throcytes.

Tetanus toxin is prepared by cultivating the bacillus in bouillon under strict anaërobic conditions. Since tetanospasmin is so susceptible to the influence of heat, age, and even light, the toxin is best preserved in a dry form. The standard of tetanus toxin consists of 100 minimal lethal doses of a precipitated and dried toxin, preserved at the Hygienic Laboratory of the Public Health and Marine-Hospital Service.

If susceptible animals, such as mice or guinea-pigs, are injected subcutaneously or intravenously with tetanus toxin, they begin to manifest 
symptoms after a certain period; these are due to the action of tetanospasmin upon motor nerve-cells, and are characterized by hypersensitiveness, clonic convulsions, and rigidity of the muscles. In man the symptoms of tetanus are similar to those in the animal, the spasm starting quite regularly in the muscles of the lower jaw.

Experiments by Wassermann and Takaki have demonstrated that an especially close affinity exists between tetanus toxin and certain structures, particularly that of the central nervous system. Most writers agree that the toxin reaches these tissues largely by way of the nerve-paths.

3. Botulism Toxin.-This poison is generated by the Bacillus botulinus, first isolated, by Van Ermengem in 1896, from a ham during an epidemic of meat poisoning. It is the cause of a type of meat and sausage poisoning called botulism, more frequent in those countries where raw meat is eaten, and frequently confused with "ptomain poisoning."

The bacillus is a motile, spore-forming, anaërobic bacterium, which grows at room temperature and causes marked gas formation in glucose media.

The toxin is readily produced in anaërobic alkaline bouillon cultures. It is quite labile.

Symptoms of botulism appear only after a definite period of incubation, which varies from twenty-four to forty-eight hours. In contradistinction to the meat poisonings produced by other organisms, those due to Bacillus botulinus may show few or no symptoms directly referable to the intestinal tract, the chief symptoms being due to toxic interference with the cranial nerves: loss of accommodation, ptosis, dilated pupils, aphonia, dysphagia, and hypersecretion of mucus from the mouth and nose.

Guinea-pigs are quite susceptible, and may be infected by way of the mouth. The symptoms of intoxication usually follow in twenty-four hours, and are characterized by motor paralysis, dyspnea, and hypersecretion of mucus from the nose and mouth.

4. Dysentery Toxin. - The distinct types of dysentery bacilli vary exceedingly in their powers to produce toxins, the strongest poisons being produced with bacilli of the Shiga-Kruse variety, less regularly active ones, with bacilli of the Flexner type.

Investigations have shown quite conclusively that dysentery itself is a true toxemia, its symptoms being referable to the absorption of the toxins of the bacillus from the intestine. Flexner, who has studied this subject with great care, believes it probable that most of the pathologic 
lesions occurring in the intestinal canal are referable to the excretion of dysentery toxin, rather than to the direct local action of the bacilli. The action of the dysentery toxin upon animals is very characteristic, and throws much light upon the disease in man. Intravenous injection of the toxin in rabbits is followed by marked diarrhea, rapid fall in temperature, respiratory embarrassment, and terminal paralysis. Upon autopsy the intestinal mucosa, especially that of the cecum and colon, shows marked inflammatory involvement, supporting Flexner's observation of the necrotic action of excreted toxin.

Dysentery bacilli also produce an endotoxin, and poisonous substances are easily obtained by extracting the bacilli themselves or by filtration of properly prepared bouillon cultures. The toxin is fairly stable, and well preserved under toluol in the refrigerator.

5. Staphylolysin.-Two definite toxins have been isolated from cultures of Staphylococcus pyogenes aureus and albus, one of which exerts a destructive action on erythrocytes (hemotoxin), and the other on leukocytes (leukocidin).

An anti-hemotoxin that counteracts the effects of the toxin may be produced experimentally, and in human staphylococcus infections the demonstration of such antihemotoxic substances in the blood-serum may be of aid in making the diagnosis of staphylococcus infections. This antistaphylolysin may be found normally in small amounts in the serum of man and horse, and when anti-hemotoxic tests with human serum are made, a normal control should always be included. Antileukocidins have also been produced, but are not of practical importance.

The hemotoxin is readily formed in cultures of staphylococci; roughly, the amount produced depends upon the virulence of the culture. In human cases of staphylococcus infections this toxin produces hemolysis in vivo, and is partly responsible for the grave anemia that is frequently present.

6. Streptolysin.-The grave systemic symptoms that so frequently accompany slight streptococcus lesions are strong indications that these microörganisms produce a powerful diffusible poison, although extensive researches into the nature of these poisons have not given us any clear understanding of the subject.

Streptococci may yield soluble toxins that, when administered to guinea-pigs, produce rapid collapse and death. While these toxins are not comparable in potency to the soluble toxins of diphtheria and tetanus, they have, nevertheless, been differentiated from the endo- 
toxins contained within the cell-bodies, and have been found to possess less toxicity.

Beside these toxins, some streptococci produce a hemolysin which may be conveniently observed by cultivation of the organisms upon blood-agar plates. This hemotoxin is partly responsible for the sanguineous character of a streptococcus exudate.

\section{TOXINS OF THE HIGHER PLANTS AND ANIMALS}

\section{Phytotoxins}

As has previously been mentioned, the power of forming toxins is not confined to bacteria alone. There is a class of higher plants and animals that produces characteristic poisons against which immunization can be undertaken and an antitoxic serum obtained. Those of most interest medically are pollen toxin and snake poison.

The most important plant toxins (phytotoxins) are ricin, abrin, crotin, and pollen. All possess more or less toxic qualities; the first three either agglutinate or hemolyze the corpuscles of certain animals. Antitoxic serums have been prepared that will neutralize the respective toxins, and this factor constitutes the most important evidence of their toxin-like character.

General Properties. - These toxins resemble proteins in many respects. Jacoby, however, has placed them in the same class as bacterial toxins and enzymes, $i$. e., large molecular colloids closely resembling the proteins, but still not giving the usual protein reaction. More recent work by Osborne, Mendel, and Harris, ${ }^{1}$ however, does not support Jacoby's view. These observers found the toxic properties of ricin inseparably associated with the coagulable albumin, and were able to isolate it in such strength that $\frac{1}{1000}$ milligram was fatal per kilo of rabbit, and solutions of 0.001 per cent. would agglutinate red corpuscles.

Relation to Immunity. - The phytotoxins, since they obey the same laws as bacterial toxins, have been very serviceable in the study of immunity; they are more stable than the latter, and can be handled in more exact and definite quantities. They have apparently the same haptophore and toxophore structure as bacterial toxins; antitoxins are readily produced by immunizing animals, and seem to be specific for the toxins; in fact, Ehrlich made his earliest observations on the specificity and quantitative factors in toxin-antitoxin immunity from a study of these plant toxins.

${ }^{1}$ Amer. Jour. Physiol., 1905, 14, 259. 
The Toxin of Hay-fever.-Pollen toxin has been described by Dunbar $^{1}$ as the etiologic factor in the production of hay-fever. In all, the pollen of 25 varieties of grass and seven varieties of plants have been found capable of producing attacks of hay-fever in susceptible persons. This susceptibility to pollen intoxication is, fortunately, limited, and the sudden onset of an attack and the characteristic symptoms indicate an anaphylactic reaction due to sensitization with pollen protein. Dunbar has succeeded in producing a pollen antitoxin, which will be described in the chapter on Passive Immunization; the reports of various observers are, however, at variance in regard to its therapeutic value.

\section{ZoöToxıNS}

The most important animal toxins (zoötoxins) are those of the toad, spider, snake, scorpion, and bee. The most striking characteristic of these toxins is that an immunity against them can be established; in this respect they resemble true toxins. All are quite complex in structure and properties, and all are more or less hemotoxic.

Snake Venoms. ${ }^{2}$ - Medically, these are of particular interest. They were first thoroughly investigated by S. Weir Mitchell (1860) and Mitchell and Reichert (1883), and have aroused considerable attention because of their similarity to bacterial toxins and the aid their study has been in the elucidation of immunologic problems.

Properties of Venom.-In 1883 Mitchell and Reichert described two poisonous proteins, constituents of venom, one of which seemed to be a globulin and the other a proteose or "peptone." Faust ${ }^{3}$ believes that the poisons are not proteins, but glucosids free from nitrogen, and that they belong to the saponin group of hemotoxic agents. It may be that these glucosids are bound to proteins, and can be removed with the globulin in fractional separation, or that they may come down, at least in part, with the albumoses of the venom.

Various enzymes have been found in venoms; e.g., proteases (Flexner and Noguchi) and lipases (Noguchi); the latter probably have a definite relation to many of the effects of venom intoxication, especially hemolysis and fatty degeneration of the tissues.

The poisons, as a rule, produce both local and severe general disturbances, the rapidity of the onset of the symptoms and the prognosis

${ }^{1}$ For full review of this subject see Glegg: Jour. Hygiene, 1904, 4; Liefman: Zeit. f. Hygiene, 1904, 47, 153; Wolff-Eisner, Deut. med. Woch., 1906, 32, 138.

${ }^{2}$ See Faust: "Die tierischen Gifte," Braunschweig, 1906; Noguchi: Carnegie Institution Publications, 1909, No. 111; Calmette: "Les venins," etc., Paris, Masson, 1907.

${ }^{3}$ Arch. exp. Path. u. Pharm., 1907, 56, 236; 1911, 64, 244. 
in a given case depending largely on the situation of the bite. Most of these poisons exert their effect primarily upon the nervous and vascular systems, besides exhibiting other toxic properties.

Nature of Venoms.-All snake venoms possess a hemolytic power, and venom hemolysis is one of the most interesting of biologic phenomena. Flexner and Noguchi ${ }^{1}$ have distinguished and classified the various elements as hemotoxins, hemagglutinins, neurotoxins, leukotoxins, and endotheliotoxins (hemorrhagin). The endotheliolytic action of the toxins is shown in the glomerular capillaries, where it causes hemorrhage and hematuria (Pearce ${ }^{2}$ ).

Cobra hemotoxin is especially characterized by its power of dissolving the corpuscles of certain species (man, dog, guinea-pig, rabbit) without the presence of serum. The explanation of this interesting phenomenon has excited extensive discussion. It is probable that the hemotoxin is in the nature of an amboceptor (Flexner and Noguchi), which is activated, in the absence of serum, by complementing substances (chiefly lecithin) present in the red cells, and in this manner producing hemolysis of these cells. In syphilis the quantity of red-cell lecithin is probably diminished after the primary stage, so that when using definite dilutions of venom that are known to hemolyze a certain quantity of normal erythrocytes, an absence of hemolysis of the red corpuscles of a given patient would infer a decrease in complementing lecithin in these corpuscles and indicate the presence of syphilis. The technic of this reaction and its value as a diagnostic procedure will be discussed further on under the head of Venom Hemolysis.

\section{ENDOTOXINS}

There are a large number of microörganisms, notably the cholera spirillum, the typhoid bacillus, the pneumococcus, and other pyogenic cocci, which, when cultivated and separated from the culture-fluid by filtration, are found to be highly poisonous, whereas the filtrate itself is practically devoid of toxicity except for the soluble hemotoxic substances. In other words, we are dealing with endotoxins, or poisons that are not secreted into the medium in which the bacteria are growing, but are contained more or less firmly within the bacterial body, from which they are separable by some method of extraction or by autolysis, only after death.

${ }^{1}$ Jour. Exp. Med., 1903, 9, 257; Univ. of Penna. Med. Bull., 1902, 15, 345.

2 Jour. Exp. Med., 1909, 11, 532. 
Method of Obtaining Endotoxic Substances.-Endotoxic substances are obtained from bacteria by thorough disintegration of the bodies. This is accomplished by various methods: (a) The substances may be found in old cultures as a result of death and disintegration of numbers of bacteria; (b) they may be obtained by suspending the microörganisms in distilled water and shaking in a machine, much as Wassermann and Citron's artificial "aggressins" are prepared; (c) the bacteria may be dried and ground to a fine powder, as in the preparation of Koch's "bacillen emulsion" of tubercle bacilli; $(d)$ MacFadyen freezes masses of bacteria with liquid air, and then grinds them into a fine powder; $(e)$ Conradi recommends autolyzing the bacterial cells in non-nutrient fluids; $(f)$ Rosenau has studied the endotoxins of pneumococci obtained by alternate freezing and thawing of suspensions in distilled water; $(g)$ Vaughan has devised a method of growing massive cultures on solid media several square yards in extent, removing the bacteria with sterile salt solution, and digesting the bacterial masses with an excess of a 2 per cent. solution of caustic alkali in absolute alcohol.

In the body, the endotoxins are probably liberated either by autolysis or by disintegration through the bacteriolysins, complements, or enzymes of the body-cells and fluids.

Nature of Endotoxins.-Owing to their insoluble nature, endotoxins in pure form and free from other products of bacterial activity, cannot be obtained. As a result, their chemical nature and structure are unknown. Tuberculin, which was formerly believed to be an albumose, may be produced in a protein-free medium; it seems probable that this substance is of the nature of a polypeptid, giving no biuret reaction, but being destroyed by pepsin and trypsin (Laevenstein and Pick ${ }^{1}$ ). Whether or not tuberculin is an endotoxin liberated upon the disintegration of the bacilli is unknown. Pick regards it as a secretory product closely related to the true toxins. It is probable that some toxin is actually secreted into the culture-medium, and that the major portion, which is of a somewhat different nature, is intimately related to the constituents of the bacterial cells.

There is little doubt but that endotoxic substances are highly poisonous, and that they are chiefly responsible for the characteristic symptoms of diseases produced by the bacteria that contain them. Whether, however, they are actually preformed definite and specific constituents of bacteria, or merely the poisonous products of disintegration of the bacterial proteins, is still undecided. It would appear that bacteria

${ }^{1}$ Biochem. Zeit., 1911, 31, 142. 
produce and contain toxic substances. When, owing to the peculiar structure of the bacterial protoplasm or nature of the toxic substance itself, the toxin can diffuse readily into a surrounding medium, the toxic substance is known as a true, soluble, or extracellular toxin; when the toxin enters into combination with the bacterial protoplasm, it becomes known as an endotoxin. This union of toxin and bacterial protoplasm may be so firm as to render the toxic substance inseparable from the bacterial protein. The various toxic substances or toxins differ, therefore, according to their diffusibility through the membrane of bacterial protoplasm, or their power of combining with these protein substances, or both factors may be operative.

Satisfactory antitoxins for endotoxins have not been produced, and this is an important point in differentiating between a true toxin or an endotoxin of any particular microörganism. Animals immunized against endotoxin develop substances in their serum that are bactericidal, bacteriotropic, and agglutinative to the bacteria from which the poisons were derived, but the serum itself is not antitoxic for the endotoxins. Therapeutic serums for use against infections caused by the endotoxin class of bacteria are largely bacteriolytic and bacteriotropic in action. The endotoxins of some bacteria, and particularly those of streptococci, seem to repel the leukocytes, or exert a negative chemotactic influence, which may effectually retard or entirely prevent phagocytosis; in this respect they resemble the aggressins of Bail. Immune serums owe a portion, at least, of their therapeutic value to the power they possess of overcoming this influence and facilitating phagocytosis. These serums, however, have not proved of as much value as have the diphtheria and tetanus antitoxins in the treatment of the respective infections mentioned, and have proved a check to the progress of serum therapy. It is probable that the endotoxins are more specific for the various strains of the same species than are the true toxins, as indicated by the results of Cole in the treatment of pneumonia with an anti-pneumococcus serum corresponding to the type of microörganism responsible for the individual infection, as determined by a rapid method of diagnosis previous to the administration of serum.

\section{AGGRESSINS}

In an attempt to explain certain observations of Koch to the effect that when a tuberculous animal is injected intraperitoneally with a fresh culture of tubercle bacilli it succumbs quickly to an acute attack of the 
disease, the resulting exudate being composed almost exclusively of lymphocytes, Bail ${ }^{1}$ has advanced the hypothesis that bacteria may secrete aggressins, or substances that aim to protect the microörganism by either neutralizing the action of opsonins or directly repelling the body-cells and preventing phagocytosis. Bail found that if he removed a tuberculous exudate, sterilized it, and injected it into healthy animals, it had practically no effect. If tubercle bacilli were injected alone, lesions would develop in the usual number of weeks; but if sterile exudate and tubercle bacilli were injected together, death would follow in about twenty-four hours, indicating that the exudate contained a substance that acutely paralyzed the defensive forces of the animal, and thus greatly increased the virulence of the bacilli. That this effect was not the summation of endotoxins in the exudate plus living microörganisms was shown by Bail, who found that when large quantities of exudate alone were injected no untoward effects resulted, whereas the injection of a small amount of exudate, plus a sublethal dose of bacteria, would regularly produce acute infection and death. Bail therefore concluded that the exudate contained a substance that allowed the bacilli to become more aggressive, and for this reason he called this hypothetic substance "aggressin." He assumes that in a tuberculous animal the tissues are permeated with the aggressin, and that when fluid collects in the bodycavities after the injection of tubercle bacilli, this fluid contains large quantities of aggressin. This prevents migration and collection of polynuclear leukocytes, but not of lymphocytes, and hence allows the bacilli to develop rapidly, producing acute symptoms. On the other hand, when tubercle bacilli are injected into the peritoneal cavity of a healthy guinea-pig, polynuclear leukocytes which engulf the bacilli are attracted, thus inhibiting their rapid development, there being no aggressin to prevent phagocytosis.

Similar results were obtained with other microörganisms. Bail inoculated cholera and typhoid bacilli into the pleural and peritoneal cavities of animals, and an acute local infection occurred. From the exudates so produced he removed the bacteria by centrifugalization, and completed the sterilization with antiseptics or with heat at $44^{\circ} \mathrm{C}$. The clear fluid obtained was found to possess but mild toxic properties, and large amounts could be injected into animals of the same species without producing any marked effects; when, however, it was injected into an animal together with a sublethal dose of the particular micro-

${ }^{1}$ Wien. klin. Woch., 1905, 8, 14, 16, and 17; Berl. klin. Woch., 1905, 15; Zeit. f. Hyg., 1905, vol. i, 3; Arch. f. Hyg., 1905, 52, 272, and 411. 
organism, an acute and fatal infection followed. Similar results were secured with the bacilli of dysentery, chicken cholera, pneumonia, and other diseases.

Bail's Classification of Bacteria.-Bail found that bacteria differed in their power of forming aggressins; he therefore used this principle in making a division of bacteria into three classes, according to their disease-producing power, as dependent largely upon whether or not the microörganism can produce an aggressin that is active against the protective forces of the host, particularly against opsonins and leukocytes.

1. Saprophytes, or those bacteria that, when injected even in large doses, do not produce any characteristic disease.

2. True parasites, or those bacteria that, when injected even in the smallest amounts, will produce disease and death. These are truly virulent, and the number of bacteria increase so rapidly as to be demonstrable in every drop of blood and in all the organs. Examples of true parasites are the bacilli of anthrax and of chicken cholera, the tubercle bacillus for guinea-pigs, and the bacilli of the group of hemorrhagic septicemia for rabbits.

3. Half or partial parasites are those bacteria the infectious nature of which depends upon the number of bacteria injected. The smaller the number, the milder the symptoms, until a dose is reached below which no disturbances are produced. Organisms of this class possess some virulence and toxicity, examples being the Bacillus typhosus and the Spirillum choleræ.

It is to be remembered, however, that these effects are but relative, and dependent upon the organism, the species of animal, and the mode of infection. For example, the bacillus of anthrax is saprophytic for the frog and hen unless the temperature of these animals is brought to the body temperature of the human; a bacillus of the group of hemorrhagic septicemia of rabbits is saprophytic for human beings, a half parasite for the guinea-pig if injected subcutaneously, and a true parasite for the same animal if injected intraperitoneally.

Nature of Aggressins. - The aggressins in inflammatory exudates are presumably substances capable of paralyzing the protective agencies of the body. Bail regards the aggressins as of the nature of endotoxins liberated from the bacteria as a result of bacteriolysis, and believes that they act by paralyzing the polynuclear leukocytes, thereby preventing phagocytosis. In general, the production of these aggressins goes on more actively the greater the resistance to the bacteria; they are produced in greater quantities during the struggle between the bacteria and 
the body-cells, although they may be produced artificially in the testtube with large numbers of bacteria and a non-poisonous agent (serum or distilled water) which can disintegrate the cells. In this manner Wassermann and Citron have produced "artificial aggressins," which act in the same general manner as the "natural aggressins" of Bail.

By many the aggressins are regarded as endotoxins, and while they may possess the nature of endotoxic substances, it is to be remembered that there is no definite relation between the poisonous qualities of the aggressins and their power to increase the virulence of an infection. It is probable, as has been shown by Wassermann and Citron, that pathogenic bacteria contain small amounts of natural aggressin. This aggressin may be regarded as a normal antibody of the bacterium against the defensive forces of the body-cells of a host. During infection these aggressins or antibodies are naturally greatly increased, as the bacteria require more and more protection. Being contained to some extent within the bacterial cells, the antibodies are somewhat similar to endotoxins: while endotoxins may be regarded as offensive agents of bacteria, aggressins may be their defensive agents. This belief is in keeping with the hypothesis of Welch $^{1}$ and also of Walker ${ }^{2}$, according to which it may be presumed that bacteria, as living cells, when so placed that they are exposed to the defensive forces of their host, are, under favorable conditions stimulated to produce reciprocal antibodies for their protection, and to generate them in increasing amounts as may be necessary. I regard aggressins as antibodies of this nature, and consider that they are produced according to the conditions laid down in Professor Welch's hypothesis.

Bail regards the aggressins as new substances; as already stated others regard them as simple endotoxins; still others believe them to be free bacterial receptors, and that these receptors may combine with bacteriolytic amboceptors, producing, as it were, a deflection of the amboceptors, so that the bacteria themselves are not attacked, and thus continue to proliferate. The action of aggressins is not dependent upon the toxicity of the endotoxins, for the fluid containing them is devoid of toxic effects; at most, therefore, if they are of the nature of receptors, they possess no toxophorous portion.

Whatever aggressins may be, and we regard them as antibodies of bacteria, just as bacteriolysins are antibodies of tissue-cells, they appear to be especially directed against opsonins, neutralizing these, paralyzing leukocytes, and thus inhibiting or entirely preventing phagocytosis.

${ }^{1}$ Brit. Med. Jour., 1902, 2, 1105.

${ }^{2}$ Jour. of Path., 1902, 8, 34. 
Anti-aggressins may be produced experimentally by gradually immunizing animals with sterile exudates, and this immunity may be transferred passively from one animal to the other by inoculation of its immune serum. These anti-aggressins are quite specific, and neutralize the aggressins in an exudate.

\section{BACTERIAL PROTEINS}

In practically all bacterial bodies, after removal of toxins and endotoxins, a certain proteid residue remains, which, when injected into animals, is able to produce various grades of inflammatory reaction leading to tissue necrosis and abscess formation. This substance was first thoroughly studied by Buchner, who named it bacterial protein, and regarded it as identical in all bacteria, and having no specific toxic action, but characterized in general by its power of exerting a positive chemotactic influence on leukocytes, and thereby favoring the formation of pus. For example, in the development of an ordinary staphylococcus abscess it is probable that the proteins of the cocci, aside from their toxins, aid in producing tissue necrosis and in attracting leukocytes to the infected area. Similarly, an extract of dead tubercle bacilli may produce a tuberculoma or the tissue changes incident to tuberculosis, differing, however, from true tubercle in that they do not contain living bacilli and consequently are not infectious. When cultures of diphtheria bacilli are filtered and the residue washed, it is found that extracts of the bacterial substances or the bodies of the dead bacilli themselves are quite free from the typical toxin; but the bacterial substances or the proteins isolated from them, when injected into the subcutaneous tissues of animals, are found to produce a strong inflammatory reaction and necrosis of the tissue-cells.

Bacterial Split Proteins. - These have been studied extensively by Vaughan and his coworkers, who have ascribed to them the chief rôle in and a very important relation to the processes of infection and immunity.

Massive cultures of colon, typhoid, pneumonia, and diphtheria microörganisms are grown in special large tanks containing agar; anthrax is grown in Roux flasks, and tubercle bacilli in glycerin beef-tea cultures. After removal of the growths the bacterial cellular substances are washed once or twice with sterile salt solution by decantation, and then repeatedly washed with alcohol, beginning with 50 per cent. and increasing the strength to 95 per cent. The substance is then placed in large Soxhelet's flasks and extracted first for one or two days with absolute alcohol, and then for three or four days with ether. These extractions should be thorough in order to remove all traces of fats and waxes. 
After extraction the cellular substance is ground, first in porcelain, then in agate mortars, and passed through the finest meshed sieves to remove bits of agar. The person grinding the cellular substance should wear a mask in order to protect himself against poisoning. Vaughan reports that, despite this precaution, several workers have been acutely poisoned, especially with the typhoid bacillus. Of course, there is no danger of infection, as the bacteria are killed during the treatment. If the finely ground cellular substance, in the form of an impalpable powder, is kept in widemouthed bottles in a dark place, it will retain its toxicity for years. This powder constitutes the bacterial protein substance, which may be split up by various means. Vaughan found digestion with 2 per cent. caustic soda in absolute alcohol especially satisfactory for extracting the poisonous group from bacterial or any other protein.

Nature of Bacterial Proteins.-Vaughan and his coworkers regard bacteria as essentially particulate, specific proteins. He has not been able to demonstrate the presence of cellulose and carbohydrates; fats and waxes that may be present are somewhat secondary and less essential constituents or stored food material. The sum total of the work of these observers would indicate that the greater part of bacteria are made up of true proteins, especially nucleoproteins or glyconucleoproteins, and although they may be simple in structure, they are chemically complex-quite as much so as many of the tissues of the higher plants and animals.

When bacterial cellular substances are split up with mineral acids or alkalis they yield ammonia, mono-amino- and diamino-nitrogen, one or more carbohydrate groups, and humin substances. These protein substances are the same as those obtained by the hydrolysis of vegetable and animal proteins.

By digestion with dilute acids or alkalis, especially the latter, in the form of a 2 per cent. solution of sodium hydroxid in absolute alcohol, a soluble split product is obtained that resembles in some respects the protamins, although they do not all give a satisfactory biuret reaction. This product is highly toxic, but shows no specificity in its action, being the same whether derived from pathogenic or from non-pathogenic bacteria, or from egg albumin or other protein substance. All that is definitely known regarding it is that it is toxic, protein in nature, but simpler in structure than the complex proteins of the bacterial cells themselves.

This soluble toxic portion as obtained in vitro is regarded by Vaughan as the main factor in the production of the general symptoms of infection, the special and distinctive lesions being due to the location of the infection. During the infective process the body-cells produce an antiferment which, when it reaches a certain concentration or power, begins to split the protein of the microörganism and new bacterial tissue, with 
the liberation of this toxic moiety, in a manner similar to the splitting observed in vitro by dilute alkalis or acids.

The insoluble and non-poisonous portion of the cellular proteins shows most of the color reactions for proteins, and contains all the carbohydrate of the unsplit molecule and most of the phosphorus.

Action of Bacterial Proteins. - The effects produced by bacterial proteins are not specific; the protein substance of non-pathogenic bacteria and, indeed, many proteins derived from vegetable and animal sources, have equally marked pyogenic properties. All foreign proteins introduced into the circulation of animals are more or less toxic, and the toxic effects of all bacterial proteins are, in general, quite similar and non-specific.

Bacterial protein substances may be responsible for certain minor anaphylactic reactions, as has been observed occasionally in the administration of ordinary bacterial vaccines. They may bear an important relation to the development of the state of hypersensitiveness of a tuberculous person in the course of a series of tuberculin injections.

Theory of Vaughan.-According to Vaughan and his coworkers, all true proteins contain a common and non-specific poisonous group. This group may be regarded as the central or key-stone portion of every protein molecule, with secondary and possibly tertiary subgroups, in which the specific property of different proteins is inherent. When the main or primary group is detached from its subsidiary group, it manifests its poisonous action by the avidity with which it attacks the secondary group of other proteins. These are detached from their normal positions, and consequently deprive the living protein of its power of functionating normally. When proteins are split, the chemical nucleus or non-specific toxic portion is more or less completely set free, and its toxicity varies according to the thoroughness with which the secondary groups have been removed.

The pathogenicity of a bacterium is determined not by its capability of forming a poison, but by the ability it possesses to grow and multiply in the animal body. When, during an infection, a pathogenic microorganism reaches the deeper tissues, it is not immediately killed by the defensive ferments of the host, but continues to grow and multiply, throwing out a ferment that feeds upon the native proteins of the bodycells, tearing them down and building up a specific bacterial protein that may select a certain point of predilection in which it is most prone to accumulate. Thus the typhoid bacillus accumulates in the adenoid tissue of Peyer's patches on the intestine, the spleen, and the mesenteric 
glands; the pneumococcus tends to lodge in the lungs; the smallpox virus selects the skin, etc.

The bacterial toxins and viruses, as, e. g., diphtheria toxin and the virus of smallpox, are regarded as ferments of protein nature, capable of attacking native body protein and building up a specific foreign protein. This foreign bacterial protein is formed during the period of incubation of disease when there is no effective resistance on the part of the body-cells to its growth and multiplication. During this time the infected person is not ill, so that the foreign protein in itself cannot be toxic, and the body-cells are busy preparing and elaborating a new and specific ferment that will digest and destroy the foreign protein. When this new ferment becomes active, the first symptoms of disease appear, and the active stage of the disease marks the period over which the parenteral digestion of the foreign protein extends. These specific ferments split up the foreign protein and liberate the toxic portion or the protein poison; this poison is not a toxin and is not specific, but occurs commonly in all proteins.

The characteristic symptoms and lesions caused by the various infectious processes are determined largely by the location of the foreign protein. The poison elaborated is the same in all infectious diseases, and it is the location of the infection, rather than the exact nature of the infecting agent, which gives rise to the more or less characteristic symptoms and lesions of the several infectious diseases.

Death may be produced by the too rapid breaking-up of the foreign protein, and the consequent liberation of a fatal dose of the protein poison, or it may result from a lesion induced by the products of this disruption, such as perforation of the intestine and hemorrhage in typhoid fever, or it may follow from chronic intoxication and consequent exhaustion. If recovery takes place, the individual enjoys an immunity of variable duration, owing to the presence of specific ferments capable of destroying the particular substrata if infection should occur.

It is this power of body-cells, when permeated by a foreign protein, to elaborate a specific antiferment by which the protein is destroyed, that, in the opinion of Vaughan, forms the basis of a correct understanding of infection and immunity.

\section{PTOMAINS}

It was at one time believed that the symptoms of many diseases were due to the absorption of soluble basic nitrogenous substances pro- 
duced by bacterial action upon various albumens, these toxic, alkaloidlike substances being known as ptomains. It was soon found, however, that the ptomains produced by pathogenic bacteria were insufficient of themselves to cause the symptoms and lesions characteristic of the respective microörganisms; that they were in general less toxic than the cultures themselves; that the majority of ptomains are not very poisonous; and that they are not specific, since equally potent ptomains are produced by non-pathogenic bacteria. This lack of specificity is in sharp contrast to the toxins. No matter upon what medium a true toxin producer is grown, the toxin is qualitatively the same, whereas the nature and toxicity of ptomains depend upon the microörganism, the culture-medium used, the duration of growth, and the quantity of oxygen furnished. The same microörganism, when grown on different media or under different conditions, may produce totally different ptomains.

Ptomains may, however, produce disease, and even death, when they are ingested with food that has undergone bacterial decomposition. In most instances of meat-poisoning, however, which are frequently ascribed to the presence of ptomains, a specific microörganism, the Bacillus botulinus, or a member of the Bacillus enteritidis group of Gärtner, is usually responsible. The commonest sources of ptomain poisoning are improperly preserved meats, fish, sausages, cheese, icecream, and milk. This subject received full consideration in Vaughan and Novy's "Cellular Toxins."

Besides occurring in food-poisoning, ptomains may be formed as the result of putrefactive processes going on in abscesses, gangrenous areas, and within the gastro-intestinal canal, and enough of these may be absorbed to produce symptoms of intoxication. Under these conditions it is possible for bacteria to produce ptomains that may be absorbed and produce symptoms of intoxication without the bacteria themselves actually gaining entrance to the tissues, and therefore not constituting, according to our definition, a true infection. Pernicious anemia, chlorosis, and allied conditions have been ascribed to the absorption of such ptomains from the intestinal canal. Obviously it is difficult or impossible to always differentiate between bacterial toxins and bacterial ptomains, or the products of protein decomposition dependent upon bacterial activity, and we can but admit the possibility of the production and absorption of both bacterial toxins and ptomains under certain pathologic conditions. Most ptomains probably are produced as the result of decomposition of the dead protein medium upon which the bacteria grow, and to a lesser extent by the destruction of the bacterial cells them- 
selves. It is extremely doubtful if ptomains are produced in important quantities by pathogenic bacteria infecting living tissues.

\section{MECHANICAL ACTION OF BACTERIA}

In former years the theory as to the influence of mechanical blocking of vessels with masses of bacteria was regarded with much favor in the etiology of certain infections, particularly anthrax. At the present time this factor has not the same importance, for while it is true that bacterial emboli may occasion harm by blocking important vessels, further researches have shown that it is doubtful if any pathogenic microörganisms are totally devoid of toxic action, and that their toxins are largely responsible for the tissue changes and symptoms of infections.

It can readily be understood that emboli of microörganisms may produce metastatic lesions; thus when staphylococci are injected into the ear vein of a rabbit they produce abscesses in the kidney and heart, and masses of bacteria from an ulcerative endocarditis, when carried to different portions of the body, will cause abscess formation; but the question in hand, however, deals with the effects of mechanical blocking itself.

Investigations with anthrax bacilli have shown that they are remarkably free from soluble toxins and endotoxins, although the local lesion develops so rapidly and becomes so quickly necrotic as to suggest very strongly the action of some local toxic substance. Cases of human anthrax seldom prove fatal if the lesion is removed and the blood-stream remains free from the bacilli. Vaughan has shown that anthrax protein possesses toxic qualities, and since the majority of fatal cases of anthrax show enormous numbers of bacilli in the blood-stream and internal organs, it may be that this bacteremia produces an accumulation of toxins which is greatly augmented when the body-cells of the host have produced an antiferment that splits up the protein of the bacilli, the combined toxic substances being responsible for the severe symptoms and death.

With protozoan disease, the possibility of serious symptoms following blocking of vessels is far greater, and, indeed, the cerebral symptoms of malignant malaria and sleeping sickness may be due in part to the blocking of small, but physiologically important, vessels with masses of plasmodia and Trypanosoma gambiensi, together with the absorption of toxic agents and the products of disintegration. Thus Bass, who has successfully cultivated the malarial plasmodium outside of the 
body, believes that the parasites, after attaining sufficient size, lodge in the capillaries of the body, especially where the blood-current is weakest, and where slight obstruction occurs as the result of the protruding inward of nuclei of the endothelial cells. Here they remain and develop until segmentation takes place. In the meantime other red corpuscles are forced against them, and if the opening in the infected cell is in a favorable location, one or more merozoites pass directly into another cell; if it is not, the merozoites are discharged into the blood-stream and are speedily killed.

\section{INFECTION WITH ANIMAL PARASITES}

Infection with animal parasites is similar in many respects to infection with bacteria. Owing to the difficulty of isolating and cultivating these parasites in vitro, our knowledge of their toxic properties is somewhat meager. Most attention has been given to a study of their life history and the modes of transmission.

Modes of Infection.-Primary infection with animal parasites is often facilitated by, or in some instances only rendered possible through, the intervention of special carriers, usually various species of the Arthropoda. Thus we now know that malaria is transmitted through the bite of infected mosquitos; African relapsing fever and Texas cattle fever, through the bite of certain infected ticks; trypanosomiasis, through biting flies. The ova of various intestinal parasites may require residence in certain of the lower animals before they can infect man.

Infection may occur along the same routes as bacterial infection, and is governed in general by the same factors of local selection, tissue susceptibility, etc. Biting insects usually deposit the parasite directly in the subcutaneous tissues or in the circulatory fluids. Abrasion of the epithelium may be necessary in order to produce infection with Treponema pallidum, as in the majority of the bacterial infections. The ova or larva of other parasites may be swallowed or find lodgment in the upper or lower air-passages or accessory sinuses.

It would appear that our natural defenses against infection with animal parasites are much weaker than those against bacteria; this is probably due to the greater resistance offered by animal parasites to such physical destructive influences of the host, as the acidity and germicidal activity of the secretions, temperature, etc., as well as to a general lack of natural antibodies in the body-fluids of the host, and inability of leukocytes and other phagocytic cells to deal successfully 
with the invaders. That natural immunity against infection with certain animal parasites may exist is shown by the prevalence of certain infections among man, and their absence among lower animals, or vice versâ.

The aggressiveness of animal parasites is in general probably even greater than that of most bacteria, and a more or less extensive infection apparently occurs in all cases in which the parasite had made successful invasion, some multiplying in the blood-stream (malaria, relapsing fever, trypanosomiasis, Texas fever, filariasis), others in the lymphstream (filariasis), and others in the tissues (syphilis, trichiniasis, amebiasis) without much opposition on the part of the host. Whether these factors are due to the aggressive forces of the parasites which neutralize the defenses of the host, or whether they are due to the hardiness of the parasites and a lack of defense on the part of the host, is not known, but probably the latter is generally the case.

As with bacteria, animal parasites show a well-marked selective affinity for certain tissues, as the malarial plasmodium for red bloodcorpuscles, trypanosomes and spirochetes for blood plasma, trichina for voluntary muscle, various parasites for the intestinal canal and even for certain portions of the intestinal tract, others for the lung, etc.

Production of Disease.-Comparatively little is known regarding the formation of toxic products on the part of the animal parasite. Some, as, e. $g$., the Treponema pallidum and spirochete of relapsing fever, probably cause disease largely through the production of toxins, especially of the intracellular variety. The chill, fever, and sweat of malaria suggest the liberation of toxic products coincident, or nearly so, with segmentation and rupture of the plasmodium. The late symptoms of sleeping sickness and the whole course of relapsing fever are strikingly similar to the bacterial toxemias. The metabolic products of all animal parasites are probably injurious in some manner and to some degree. The pathogenicity of others is due, in part at least, to mechanical blocking of vessels, as with the filaria, trypanosomes, and malarial organisms; others (hookworm) abstract blood or consume food material in the intestine, as the intestinal parasites; and others, as migrating foreign particles with irritating secretions, produce local inflammatory changes.

Nevertheless, we know comparatively little of the offensive factors, and still less of the immunologic defensive factors, operative during the course of infections with animal parasites. With the development of a technic for the cultivation of animal parasites in vitro similar to that 
devised for the ameba, certain trypanosomes, spirochetes, and malarial plasmodia, we will be enabled to study the products of their growth or of disintegration, and the immunologic agencies concerned in infection and recovery; this offers a very important and fruitful field for research.

\section{THE COURSE OF INFECTION}

In conclusion, we may briefly consider the results of infection or the general symptoms following bacterial growth and the manner in which these are produced.

The Stages of Infection.-Practically all infections pass through the following stages:

1. The period of incubation, which begins at the time of infection and ends with the development of the earliest general symptoms, during which time the invading bacteria are multiplying in the tissues of the host. During this stage no symptoms, or only those of a purely local nature, are present. This period varies considerably in different infections, and to a lesser extent in different individuals having the same infection. Some bacteria may be so virulent as to overwhelm the bodycells, thus making the period of incubation very short or entirely unobservable. On the other hand, as, e. g., in rabies, the period may be of several weeks' and, indeed, of several months' duration. In tuberculosis there is usually a primary local growth, which develops so gradually and the toxins are so slowly diffused that it is difficult or, indeed, impossible, to estimate the length of the period of incubation.

According to Vaughan, during the period of incubation the bacteria or their toxins or the viruses are actively engaged in changing the natural body proteins into new and specific bacterial proteins, and since this stage is constructive, there are no symptoms and the host is not ill. Even with the experimental administration of the most poisonous of toxins a definite period of incubation is usually to be observed, which cannot be reduced below a certain minimum, independent of the size of the dose injected; in general, however, a large dose of bacteria or of toxin is likely to be followed by a shorter period of incubation than if a smaller dose were administered.

In a given case the period of incubation may be determined by several factors:

(a). The number of bacteria gaining entrance, and especially their toxicity and aggressiveness. The primary factors are the degree of toxicity and the amount of toxic substances produced and absorbed. 
(b) Upon the site of infection. Thus the introduction of rabies virus or of tetanus bacilli into the tissues of the face or into a deep wound is likely to be followed by a shorter period of incubation than when these are introduced into the foot or in superficial wounds.

(c) Upon the degree of resistance offered by the host. For instance, one individual may contain more antitoxin or bacteriolysin for a certain bacterium than another, and consequently a longer period of incubation is required, during which these substances are neutralized and an excess of toxic bacterial substance is produced. In fact, these may offer such resistance to the bacterium that the process of infection is inhibited, or but slight and evanescent disturbances appear.

(d) Upon the general susceptibility of the host and the route of invasion.

2. The period of prodromal symptoms, characterized by systemic disturbances of a relatively mild type, due to diffusion of the bacteria and their products into the general circulation and their wide-spread effect upon the body-cells in general. If the bacteria select a special tissue or organ for attack, as the typhoid bacillus for lymphoid tissue, and pneumococci for the lungs, definite symptoms develop later, their nature depending on the special tissue or organ involved. The prodromata, however, are more marked, and indicate a wide-spread but mild action upon the body-cells in general. Vaughan believes that these symptoms mark the time when sufficient proteolytic ferments have been generated by the body-cells against the new bacterial protein of the invading bacteria to attack the latter, splitting the molecule and liberating a toxic moiety responsible for the general symptoms of intoxication.

3. The period of fastigium, or of high fever, during which the disease is at the height of its severity. Special and distinctive symptoms and lesions, according to the organ or organs especially involved, are present; the struggle between the offensive and defensive forces of parasite and host is at its height, with remissions or exacerbations dependent upon the supremacy of any one of these, and the general stability of the bodycells in withstanding the wear and tear. During this time the protective proteolytic ferments of Vaughan are most active in disrupting the newly formed bacterial protein, with the liberation of the toxic portion. This process may be so active as to overwhelm the host with the toxic split product, or lead to grave secondary lesions, such as extensive necrosis, perforation of a viscus, or hemorrhage.

4. The period of decline, during which the patient is gradually overcoming the infection, and amelioration of the symptoms takes place. 
5. The period of convalescence is now ushered in, during which the host gradually overcomes the effects of disease and returns to health.

During this entire time the emaciation and tissue exhaustion leave the patient quite weak, and undue exertion, errors in diet, or reinfection may lead to a relapse, or a reactivation of the disease. Certain sequeloe or morbid conditions may follow a disease, and are due to the same original cause; e.g., in typhoid fever the development of cholecystitis; at any time during the disease complications, or morbid conditions due to some other microparasite, as the development of pneumonia during the course of typhoid fever, may seriously jeopardize the life of the patient.

Grades of Infection.-According to the manner in which a parasite and its products act upon the cell of a host and the power of the host to neutralize or overcome these the following various grades and types of infection are encountered:

(a) Malignant or fulminating infection, during which there is no fever, but, on the contrary, a subnormal temperature, with rapid prostration of the patient and death within a brief period. The cells of the body are overwhelmed and paralyzed by the toxic substances; metabolism is arrested, and the heat centers are exhausted with the fall of the temperature, an indication of the intense and overwhelming intoxication.

(b) Acute infection, which is the ordinary type of an infectious disease as previously described, and having a definite incubation period, prodromal symptoms, fastigium, defervescence, and convalescence.

(c) Chronic infection, or a prolonged process characterized by insidious onset and symptoms of relatively mild or moderate severity, and terminating either in death, after months or years, or in gradual recovery. A chronic infection may be remittent, as may be observed in the rheumatic group of disorders; during the remission with defervescence the infecting bacterium is not totally destroyed, and subsequently lights up, producing an acute exacerbation of the disease.

In chronic infections it would appear that the parasites develop and produce their toxins slowly, or that these are slowly and imperfectly absorbed on account of the sluggish local circulation and the presence of scar tissue. The body-cells become accustomed, as it were, to these toxic products, and produce only sufficient antibodies to effect their immediate neutralization. The bacteria themselves become distinctly resistant to the action of the tissues and the defensive forces, and there is neither the same degree of intoxication nor reaction as are seen in acute infections. Gradually, however, the body-cells become exhausted, and 
unless the cells are aroused and stimulated, by judicious administration of bacterial vaccines, to produce an oversupply of antibodies, the host shows progressive emaciation and weakness.

The Systemic Reaction to Infection.-It is not within the scope of this book to discuss the various symptoms of infection, and we will limit ourselves to a brief discussion of the most important, namely, the febrile reaction.

According to Vaughan, the fever of infection is due mainly to the toxic split protein resulting from the action of the protective proteolytic ferments upon the new bacterial protein. This observer and his associates were able, by the injection of multiple doses of protein derived not only from the typhoid bacillus but from various vegetable and animal proteins, to reproduce experimentally in rabbits a febrile reaction known as protein fever, and which is not unlike typhoid fever. This induced fever may continue for weeks, and is accompanied by increased nitrogen elimination and gradual wasting; it is followed by immunity, and the serum of immunized animals digests the homologous protein in vitro. As has repeatedly been stated, Vaughan regards the split toxic product as the cause of the general symptoms of infection, the special and characteristic symptoms and lesions of the different diseases depending upon the site where the bacterial proteins have been deposited, and where they are, in large part at least, digested.

In addition to this toxic action of split protein, fever may be due(a) to the unusual activity of the cells supplying the proteolytic enzymes; and $(b)$ to the cleavage of the foreign bacterial protein by these ferments.

The fever of infection, therefore, is caused by the toxic action of pathogenic parasites, both bacterial and animal forms, upon the bodycells and heat-regulating centers. It must be regarded by itself as a beneficent phenomenon, inasmuch as it marks a reaction of the bodycells to toxic agents, for the purpose of neutralizing these and, by the development of antibodies, ridding the body of foreign substances. 


\section{PART III}

\section{CHAPTER VIII}

\section{IMMUNITY.-THEORIES OF IMMUNITY}

IN the preceding chapter on the mechanism of infection and the production of an infectious disease the statement was frequently made that the microparasites of disease are required to overcome the defensive forces of a host which are ever on guard to protect the organism against parasitic invasion and infection. Certain of these defenses are natural to the host, and in a great majority of instances suffice to protect the body against invasion and infection with bacteria, animal parasites, and various inanimate and injurious substances. When, however, these natural defenses are broken down and infection has occurred, the bodycells are not usually rendered powerless and completely overcome, for the products of infection serve as a stimulus to the body-cells, calling forth renewed cellular activity and the production of various specific defensive weapons, termed antibodies, which maintain an incessant struggle against the invading pathogenic agents in an effort to rid the body of them and to neutralize their products.

Just as microparasites have various offensive weapons, consisting chiefly of their toxins, so, in like manner, the defensive forces of the host are numerous and even more complex. If the toxin of a microörganism is its chief pathogenic weapon, as, e. g., the soluble and extracellular toxin of the diphtheria or the tetanus bacillus, then the body-cells produce an antitoxin as their chief defensive force. If the offensive weapon is largely in the nature of an endotoxin, as, for example, the endotoxins of the typhoid or the cholera bacillus, then a chief antibody is in the nature of a bacteriolysin, whicb endeavors to dissolve the bacillus in an effort, as it were, to attack the enemy in his stronghold. In other infections, especially those due to the pyogenic cocci, certain of the bodycells, and chiefly the polynuclear leukocytes, are observed in the tissues to have engulfed the invaders bodily (phagocytes) in an endeavor to digest them and neutralize their products. In addition to these chief antibodies, there are others that appear to aid them in their work.

That one attack of many of the infectious diseases may protect the individual against subsequent attacks, or at least render subsequent attacks mild and harmless, is well known. In India and the East for 
centuries practical advantage has been taken of this observation in the management of smallpox. In order to protect persons against a severe attack of variola they were deliberately brought in contact with a person suffering with a particularly mild form, in the hope that, by inducing a mild attack of short duration, they would thus obtain protection against the severe, disfiguring, and fatal form of the disease.

The practice, however, was not without danger to the individual and to the public at large, as the induced disease would at times become malignant, and constitute a focus of infection for an entire community, When Edward Jenner discovered that inoculation with cowpox virus could not produce smallpox, but would, nevertheless, stimulate the production of specific antibodies and confer immunity against it, an enormous forward stride was taken that has since proved a priceless boon in helping to rid the world of the dreadful scourge of smallpox.

The object of all these procedures has been to secure a resistance or immunity to smallpox, either by inducing a mild form of the disease or by protecting the individual by means of inoculation with a virus that has been so changed in its passage through a cow as to render it unable to produce smallpox, but yet is capable of stimulating the body-cells to produce antibodies that will neutralize the effect of the true virus. This induced resistance to a given infection constitutes immunity or resistance, and since the body was purposely inoculated and the body-cells rendered active in producing the antibodies, this form of resistance is known as active acquired immunity.

Many persons recover from an infection that may have been unusually severe not because the infecting agent became exhausted or died for want of pabulum, but because it had been gradually worsted in the battle with the defensive forces of the host. In many such instances the host is now immune to this infection for a longer or shorter time, because the body-cells have been so profoundly impressed that they continue generating defensive weapons or antibodies for some time after the last vestige of the infecting agent has disappeared. Or, on the other hand, the quantity of antibodies may be so great that they may persist for varying periods of time, even for the remainder of life, ever on guard, and ready to overwhelm their specific enemy should it ever again gain access to the tissues.

Here, then, arises the question concerning the mechanism of recovery from an infection, and since this is so intimately concerned with the general subject of resistance to disease, it is considered under the general head of immunity. 
Even superficial observation shows that not all persons are equally susceptible to a given disease, and during the course of epidemics it will be seen that some individuals, although freely exposed, escape infection. Certain species of animals may likewise display a uniform resistance to an infection that will readily enough attack another species of the same general family. It has been demonstrated experimentally that a certain pathogenic bacterium will produce a severe infection in one species of animals and not in another. It may frequently be noticed that even though an infection occurs, it is readily overcome by the natural resources of the host, the latter escaping with slight or no symptoms of disease. In other words, certain persons and animals apparently possess a natural resistance or immunity to disease, which may be general, non-specific, or due to specific antibodies, this type of immunity being frequently relative and seldom absolute.

Definition.-Immunity, therefore, in a broad sense, is the effective resistance of the organism against any deleterious influence; in the usual and more restricted meaning the term is applied to resistance against infection with vegetable and animal parasites and their products, which are pathogenic for other animals of the same or of different species.

It should be remembered that immunity means not only the ability to resist an infection or successful invasion of the tissues by microparasites, but also the continual resistance offered as long as the infection lasts; that is, immunity implies not only resistance to the onset of infection, but also to the course and progress of the resulting infectious disease. The science of immunity has, therefore, for its object the study of the mechanism of resistance to and recovery from an infection.

\section{HISTORIC}

The development of the science of immunity forms one of the most interesting chapters in the history of medicine. Even in ancient history we can trace the conception of our modern ideas on immunization.

Hippocrates taught that the factor that causes a disease is also capable of curing it-practically the same theory as the more modern homeopathic doctrine of "similia similibus curantur." Pliny the Elder recommended the livers of mad dogs as a cure for hydrophobia, thus coming very near to the basis of the Pasteur discovery. As was pointed out by Elizabeth Fraser, the same idea is expounded in the mythologic tale of Telephus, who cured his wound by applying rust from the sword which inflicted it, and in the story of Mithridates, King of Pontus (B. 
C. 120), who immunized himself against poisons by drinking the blood of ducks that had been treated with the corresponding toxic substances.

Immunization against various venoms has been practised by many of the savage tribes of Africa since earliest times. Mention has previously been made of the method of preventive inoculation against smallpox practised in Asia and other Oriental countries for several centuries by exposing the subjects to mild cases of the disease.

A very definite step in progress must ever be associated with the name of Edward Jenner, who first demonstrated experimentally, and in a scientific manner, that cowpox conveyed to man protected him against smallpox. Jenner was not the first person to make this observation, as many of the Gloucestershire farmers knew that cowpox protected them against smallpox; nor was he the first deliberately to inoculate persons with cowpox virus, as this method had been practised sporadically before his time. Jenner was, however, the first medical man to give the matter serious thought and consideration, and to test the method as thoroughly and scientifically as it was possible to do at that period. Thus he inoculated with smallpox virus those whom he had previously vaccinated with cowpox virus, and found them immune to smallpox. These experiments were courageously repeated, until a great truth was established, which has resulted in almost completely eradicating the disease from those countries or communities where vaccination is thoroughly carried out. As was to be expected, Jenner met with considerable opposition, and this is readily understood when it is remembered that even today-one hundred and eighteen years laterthere are those who refuse to accept, or are unable to realize, the great benefits of this pioneer work. Jenner could not explain his results; he maintained that he was dealing with a modified form of smallpox. We of today have no better means of establishing the truth of the efficiency of cowpox vaccination, nor have we improved any on his method. The specific germ of smallpox is still undiscovered, and we must agree with Jenner that cowpox is probably a modified form of smallpox and practically harmless, the virus of cowpox being the virus of smallpox modified, attenuated, and rendered practically innocuous by passage through a lower animal.

Nothing further of importance was accomplished during the following eighty years, until the next and even greater epoch ushered in the discoveries in bacteriology and the first immunization by Pasteur based on scientific reasoning. The chickens around Paris were being destroyed by a virulent intestinal infection, and Pasteur first isolated the causative 
microörganism, a minute bacillus, which he found was capable of producing the disease experimentally in healthy chickens. Quite by accident, so it seemed, he discovered that cultures of this bacillus could, by prolonged cultivation be attenuated, for when these cultures were inoculated into chickens, the fowls did not die or suffer any ill consequences; further, and what was of the utmost importance, when these same chickens were inoculated with virulent cultures, they were found to be immune to chicken cholera. Here, then, was the key to active immunization in the prevention of disease, and Pasteur possessed the genius to realize the full significance of his discovery.

Armed with this knowledge Pasteur, and his assistants, Roux and Chamberland, next studied anthrax, an infectious disease of cattle that was causing a great annual loss to the farmers of France, and the bacillus of which was among the first pathogenic microörganisms to be discovered. It was found that prolonged cultivation of this bacillus was insufficient to attenuate the cultures, as the spores were highly resistant and retained their pathogenicity under extreme circumstances and over prolonged periods of time.

In 1880 Touissant published a method of attenuating the bacilli by heating the blood of an infected animal to $55^{\circ} \mathrm{C}$. for a few minutes; later, Chauveau secured similar results by heating fresh cultures for a few minutes at $80^{\circ} \mathrm{C}$. Both methods were uncertain, and neither safe nor practical. After prolonged experimentation Pasteur found that cultivating the bacilli at the relatively high temperature of from $42^{\circ}$ to $43^{\circ} \mathrm{C}$. resulted in gradual attentuation, and if this cultivation was continued, the cultures were robbed entirely of their disease-producing power. Further, subcultures of these growths when kept at $37^{\circ} \mathrm{C}$. did not regain their original virulence, but maintained for generations the grade of attenuation reached in the original culture the result of cultivation for a certain number of days at the higher temperature. In this manner Pasteur was able to control to some extent the degree of attenuation, and by inoculating first a highly attenuated and later a less markedly attenuated culture he could immunize animals against anthrax. This discovery was soon amply verified. The original method is practically the one employed today, and is proving of considerable economic value.

Pasteur's next great experiment was undertaken for the relief of rabies, a condition in which, for the first time, he came to deal with a disease that not infrequently affects man. His success and the results of his discovery of an effective means of immunization against hydro- 
phobia were even greater than in previous experiments. Here he was dealing with a disease of unknown etiology, the causative agent of which he could not cultivate artificially, but which he sought to attenuate by a new process - that of drying.

Pasteur first established that the virus of rabies is contained within the tissues of the brain and spinal cords of infected animals.

He then invented a method of inoculating animals by making subdural injections of an emulsion of these tissues. By repeated passage of a virus through a number of rabbits a virus of fixed pathogenic power (virus fixe) was obtained. By inoculating rabbits with this virus and removing their spinal cords immediately after death and drying these over a desiccating agent at room temperature, he found that he could modify the virulence of the virus at will, depending on the length of the period of drying. By emulsifying small portions of attenuated spinal cord in salt solutions and injecting these he was able gradually to immunize animals against rabies, and finally he applied the treatment successfully to the prevention of rabies in the human being.

Antirabic vaccination is largely responsible for extending our knowledge of the possibility of securing immunization. Pasteur has taught us at least three different methods for modifying a virus in the preparation of a vaccine, and that each disease, being itself a special entity, having its own characteristics, must be dealt with along special lines.

These discoveries were largely empirical, and the explanations of their mechanism are now only of historic interest. It was not until 1883, when Metchnikoff shed light upon the problems of immunity by making a series of remarkable studies on the rôle played by certain of the body-cells in overcoming infection, and the part they played in the processes of immunity in general, that the world was given a glimpse into the dark problems of immunity. These observations were soon followed by investigations showing the importance of the body fluids, and since that time a great deal of work has been done upon these subjects. As a consequence, a large amount of data of a wholly new order has accumulated, accompanied by the introduction of a host of new terms expressing diverse views and theories advanced by individual workers. Of the many theories advanced from time to time to explain the phenomenon of immunity, two have claimed the most attention: one ascribes protection and cure to the activity of certain body-cells; this is known as the cellular theory; and the other attributes these qualities to the bodyfluids - the humoral theory. The chief exponent of the former is Metch- 
nikoff, with his theory of phagocytosis, whereas Ehrlich is the father and leader of the latter, with that marvelous invention of human ingenuity, the side-chain theory.

\section{THEORIES OF IMMUNITY}

The earlier hypotheses advanced by various investigators are now only of historic interest, as in the light of subsequent discoveries and observations they have failed to offer adequate explanations.

Pasteur's own theory and explanation of the mechanism of acquired immunity sought to show that the microörganisms living in the infected animal used up some substance essential to their existence, so that, for lack of proper nourishment, the microörganisms were eventually forced to retire, the soil being unfit for further occupation. This was known as the "exhaustion theory."

Chauveau considered it more probable that the microörganisms, after having lived in the body of an infected animal, produced substances that, accumulating in the blood, had an inhibitory action on the bacteria and were inimical to their further existence. This was known as the "retention theory," and in some particulars was just the opposite of the exhaustion theory.

\section{The TheORy of Phagocytosis}

In 1883, when Metchnikoff showed that certain of the body-cells, and, particularly, the polynuclear leukocytes, were active in the defense of the human body against invasion by microparasites, real light was thrown upon the unknown problems of immunity. Although he has since amplified his theory, as new theories were adduced to describe the part played by the body-fluids and the organisms themselves, yet his theory of phagocytosis remains a demonstrable fact, and establishes the important rôle of cells in the processes of immunity.

According to this theory, certain of the body-cells are able to ingest an infecting parasite, a red corpuscle, or other cell in the same manner as an ameba ingests a food-particle, and to dispose of it by intracellular digestion through the agency of ferments known as "cytases." To such cells Metchnikoff applied the name phagocyte, as he likened them to scavengers, $i$.e., they were concerned in picking up and disposing of offensive material, both living and dead.

Various body-cells are capable of becoming phagocytes. The polynuclear leukocytes are particularly active in acute infections, and have been called microphages. Endothelial cells, mononuclear leukocytes, 
and embryonic connective-tissue cells are more active in chronic infections and have been designated macrophages.

Although the original explanation of phagocytosis was quite plain and far reaching, subsequent discoveries by the adherents of the "humoral theory" showed the potent influence of the body-fluids upon the process. It was demonstrated that without the aid of these fluids phagocytosis is almost negligible. Metchnikoff early recognized this fact, and sought to explain the influence of the body-fluids by assuming that they contained "stimulins," or substances that stimulated phagocytosis. It was likewise shown that the body-fluids contained antibodies and were antibacterial, independent of cells. Metchnikoff recognizes the existence of these conditions, but claims that they are due to the soluble products of phagocytic cells, and thus in a broader sense would maintain the importance of his phagocytes.

It was soon observed that in certain infections leukocytes or other cells, instead of being attracted toward the seat of infection by some unknown chemical stimulus, (positive chemotaxis), were repelled, or at least the attraction was counterbalanced or did not exist (negative chemotaxis). While a satisfactory explanation of these phenomena is still lacking, it may be stated that, in general, the degree of negative chemotaxis is in proportion to the virulence of the microparasite.

In 1903 Wright and Douglas, and Neufeld and Rimpau threw considerable light upon this subject. They demonstrated experimentally that one action of the body-fluids was directed against the microörganisms, lowering their resistance, and, making them, as it were, more attractive to the phagocytes, the process of phagocytosis was facilitated. To these substances the name of "opsonins" (from opsono, I prepare for) was applied by Wright, while Neufeld called them. "bacteriotropins."

The leukocytes are not, however, entirely passive and willing to wait until their prey is weakened and fully prepared for their attack. In the presence of an infection they are found to increase, and this leukocytosis is known to be a valuable addition to the defensive forces. They probably also undergo qualitative changes, which increase their antibacterial power. It has been shown that opsonized bacteria attach themselves to the protoplasm of the leukocytes, a physiochemical phenomenon that occurs regardless of whether the leukocyte is dead or alive, although, of course, only the living leukocyte is able to ingest them.

That phagocytosis is a potent and very important factor in the mechanism of recovery from certain infections is generally admitted, and although it probably has not the far-reaching significance originally 
attributed to it, yet, throughout the discussion of immunity the importance of the phagocyte itself is emphasized. This theory of Metchnikoff is treated more fully in the chapter on Phagocytosis.

\section{SIDE-CHAIN THEORY}

The humoral theory of immunity, which would ascribe the power to resist infection to the body-fluids, may be said to have had its origin in 1896, when Fodor discovered that the blood of the rabbit will kill anthrax bacilli in the test-tube, independent of cells and phagocytosis. Later Buchner adopted this theory, and sought to explain the bactericidal action of blood-serum as dependent upon a special constituent which he called alexin.

With the discovery, in 1890, of antitoxins by von Behring and Kitasato, the theory received fresh support, and while an effort was made to demonstrate that antitoxins were of paramount importance in acquired immunity, evidence soon accumulated to show that this antitoxic power is operative only in a few diseases, chiefly in diphtheria and tetanus.

Fresh support to the "humoral" as against the "cellular" explanation of immunity was given by Pfeiffer in 1894, with the discovery that cholera vibrios introduced into the peritoneal cavity of a guinea-pig previously immunized against cholera became transformed into granules, and ultimately passed into complete solution (bacteriolysis), apparently without the aid of cells. Bordet then showed that this phenomenon was due to two distinct substances-one, the "sensitizing substance," which is specific and exists only in the immune serum, acting only on the bacteria against which the animal was immunized, and the other a nonspecific substance, found in the fresh serum of practically all animals, and to which he gave the name "alexin," and which was later renamed by Ehrlich and called "complement."

Of the various theories offered in explanation of these observations, the suggestive, fascinating, though highly hypothetic theory of Ehrlich, known as the side-chain theory, has been most widely accepted and adopted to explain new discoveries as they were made. The theory has, indeed, aided investigators in making new discoveries. Nevertheless the contention of Bordet, that its too ready acceptance without sufficient convincing proof has retarded investigation, should not be ignored.

The basis of this theory, as originally proposed, bore no relation to the subject of immunity, but was advanced in 1885 to explain the processes of nutrition. 
Ehrlich asserts that a cell has two important functions: The first is the special physiologic function, as that of a nerve-cell to conduct; of a gland-cell, to secrete, etc. The second function is that of nutrition, and presides over the processes of waste and repair. Furthermore, each of the molecules composing the complex cell is believed to possess these two functions, $i$. e., one is concerned with the special function of the molecule, and the other, the more important functional portion, is concerned in the nourishment of the molecule.

The second portion, or that concerned with nutrition, is of more importance in relation to the problems of immunity. Ehrlich conceives this as consisting of a special executive center or main portion ("Leistenkern"), in connection with which there are nutritive sidechains, receptors, or haptines ("Leitenketter"), which "seize," or rather enter into chemical combination with, suitable food atoms, which is followed by a sort of digestive or absorptive process, whereby the food material is incorporated in the molecule.

The function of "seizing" molecules of food from the surrounding tissues implies a selective action or chemical affinity between food atoms and the portion of a cell or side-arm for which it has a chemical affinity, for we cannot conceive that all atoms that circulate in the blood and lymph are suitable for all cells at all times.

The food molecule in the fluid surrounding the cell is conceived as possessing a special or haptophore portion for union with the side-arm of a cell molecule, and when brought into relation with one of the sidearms or receptors of the cell molecule, the two are "anchored," or unite, just as a key fits a lock. The second stage involves a process that may be compared to digestion, by which the food material is prepared and absorbed, in whole or in part, into the molecule of protoplasm.

These processes, therefore, are conceived as being chemical rather than physical, and our diagrammatic representations of them have no necessary or actual morphologic basis. One is quite likely to regard the main central portion as the nucleus of a cell, and the side-arms as small morphologic projections resembling the prickles of certain epidermal cells. These processes are concerned with each molecule of a cell, the main portion, or "Leistungskern," being conceived as diffusing through the nutritive part of the molecule, and the side-arm receptors, or "Leitenketter," as numerous atoms or groups of atoms, each of which has a chemical affinity for some particular food-substance circulating in the body-fluids, and necessary for the life of the molecule in question.

Later this theory was amplified by Ehrlich to explain the action of 
toxins and the production of antitoxins. It assumes that the side-arms to a cell molecule are exceedingly numerous, not only because nutritive substances are varied, but because special cells also possess different and special side-chains, which anchor pathologic material. When infection occurs, and in addition to toxins the physiologically normal substances are brought to the cells, they likewise find suitable receptors in practically all or certain cell groups, and become anchored, causing more or less damage to the cells.

Having combined with the side-arms or receptors of a cell, the toxin may be sufficiently potent to kill the cell, and if a large number of cells are so injured, symptoms of disease present themselves and death of the infected host may follow. On the other hand, although the cell has lost, one or more of its side-arms, it may not be dead, and it proceeds at once to repair the damage done. According to Weigert's "overproduction theory," nature is lavish in its processes of repair, and the cell not only replaces the lost receptors, but produces them in numbers; the excess receptors, having no space for attachment to the cell, are thrown off into the blood-stream. Each of these cast-off receptors or haptines possesses the same structure as the original receptor. These free receptors, then, are capable of combining chemically with their antigen, neutralizing the antigen and rendering it innocuous. In diphtheria and tetanus the antigen is largely the soluble toxin of the bacilli, and the antitoxins are these cast-off receptors produced as a result of the stimulating action of the toxins upon the cells. This excess of receptors is made by repeatedly injecting a horse with increasing doses of diphtheria toxin. By injecting this receptor-laden (antitoxin) serum into one suffering from diphtheria, the receptors unite with free diphtheria toxin and thus protect the body-cells.

For the production of these receptors, or antibodies, as they are now called, it is necessary, as previously stated, that the antigen enter into chemical combination with the cell, so that the usual illustrations showing the theoretic union of antigen and side-arm by physical contact alone probably do not correctly portray what actually occurs. As Adami points out, the antigen probably enters into intimate relationship with the cell, and the continued stimulation of its presence is responsible for the production of an excess of receptors, in addition to the overproductive tendencies of nature's repair.

It is also necessary that the antigen possess sufficient toxic power at least to stimulate the cell, for otherwise antibodies may not be produced. Food material, for instance, being physiologic, is assimilated by the 
cells without stimulating the production of antibodies, as otherwise the food would be attacked by cast-off receptors and rendered useless before it reaches cells, the process ending in starvation and death.

The host in whom certain cells with special receptors for a given poison are present will make use of these, no matter how the pathologic agent is introduced. This affinity is well illustrated in tetanus, where the effects produced are dependent to a large extent upon the selective affinity of the toxin for nerve-cells.

The Three Orders of Receptors and Corresponding Antibodies

First Order: Antitoxin and Simple Antiferments.-The simplest receptor of the cell molecule is composed of a single arm or haptophore, for union with the haptophore portion of a food molecule. As previously stated, a molecule of toxin is conceived as being composed of two portions-one, the haptophore, for union with the side-arm or receptor of a cell molecule, and the second, the toxophore, in which its toxic action resides.

The first stage of intoxication of a cell produced by a true toxin consists in the union of the haptophore portion of the toxin molecule to a receptor or side-arm of the cell molecule, this receptor being one that fits the toxin molecule "like a key fits a lock." Each molecule of the body-cell has innumerable receptors, of which only a certain number are suitable for a particular toxin. The toxin molecule is now anchored to the living cell, and, as animal experiments with a great number of toxins show, this union is a firm and enduring one (Fig. 39).

So long as the union lasts the side-chain involved cannot exercise its normal nutritive physiologic function-the taking up of food-stuffs. Furthermore, the toxophore group of the toxin molecule may now exert an injurious, enzyme-like action on the protoplasm of the cell, with the result that the protoplasm is poisoned. If only a few of the cell receptors are united with toxin molecules, or if the toxin is of low toxicity, the effects on the cell may be slight. If more are joined to the molecule or the toxin is highly poisonous, the whole molecule, and finally the cell itself, may be greatly disturbed, and produce marked symptoms, or the host may be destroyed.

Since the receptors joined to the toxin molecules are incapacitated or destroyed, the damage is repaired by the regeneration of new receptors. According to the reparative principles worked out by Weigert, the repair is not a simple replacement of the defect-the compensation proceeds far beyond the necessary limit; indeed, overcompensation is 
the rule, and this forms the basis of Ehrlich's theory. If, after repair has taken place, new quantities of toxin are administered at proper intervals and in suitable quantities, the side-chains that have been produced by the regenerative process are taken up anew in combination with the toxin, and so again the process of regeneration gives rise to the formation of fresh side-chains. "The lasting and ever-increasing regeneration must finally reach a stage at which such an excess of side-

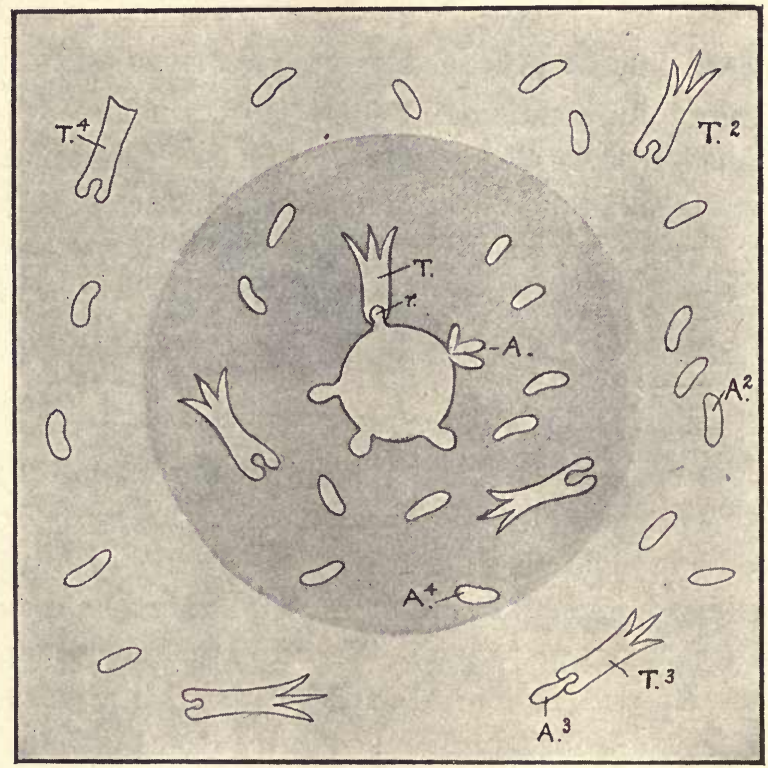

Fig. 39.-Formation of Antitoxins.

The central white area represents a molecule of a cell; the shaded portion represents the cell itself; the surrounding area represents the body-fluids about the cell.

r, A receptor of the molecule (first order); A, overproduction of receptors, which are being cast off; $A^{2}$, a cast-off receptor free in the body-fluids-now an antitoxin; $\mathrm{A}^{3}$, a molecule of antitoxin combination with a toxin molecule $\mathrm{T}^{3}$. $\mathrm{A}^{3}$, a cast-off receptor still within the parent cell; $T$, a toxin molecule in combination with the receptor of a cell molecule; $\mathrm{T}^{2}$, a toxin molecule free in the body-fluids; $\mathrm{T}^{3}$, a toxin molecule in combination with antitoxin; $\mathrm{T}^{4}$, a molecule of toxoid (toxophore group lost).

chains is produced that, to use a trivial expression, the side-chains are present in too great a quantity for the cell to carry, and are, after the manner of a secretion, handed over as a needless ballast to the blood. Regarded in accordance with this conception, the antitoxins represent nothing more than side-chains reproduced in excess during regeneration, and therefore pushed off from the protoplasm and so coming to exist in the free state" (Ehrlich). 
This theory explains the specificity of the antitoxins for a given toxin; thus the latter causes specific chemical stimulation of the cell, which induces the formation of specific side-chains,- the cast-off receptors, - which are capable of uniting with the toxin molecules free in the body-fluids and thus neutralizing them; they are, therefore, called antitoxins.

This theory also explains why a minute quantity of toxin is capable of stimulating the production of a large amount of antitoxin, and why the production of antitoxin persists for some time. The toxin molecule must be conceived as entering into the protoplasm of a body molecule and residing there for some time, acting as a stimulus to the cell, with consequent production of antitoxin. Diagrammatic representations of this process would seem to show that a physical union exists between toxin and cell receptors, resulting in the destruction of receptor, which drops off and is replaced by a number of receptors that, for lack of space for attachment to the cell, are thrown off into the blood-stream. In reality, by the act of immunization certain cells of the body become converted into cells that secrete specific antitoxin, and, as shown by Salmonson and Madsen, the administration of pilocarpin, which augments the secretion of most glands, also produces in immunized animals a rapid increase in the antitoxin content of the serum. The formation of antitoxin is constantly going on, and so throughout a long period the antitoxin content of the serum remains nearly constant.

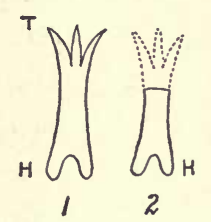

Fig. 40.-TheоRETIC STRUCTURE OF A MOLECULE OF TOXIN AND ToxoID.

1, Toxin: $\mathrm{H}$, haptophore group for union with the receptors of cells or antitoxin; T, toxophore group.

2, Toxoid: Same structure as toxin molecule except that the toxophore group is lost.

In the production of antitoxin the haptophore group is the essential and important portion of the toxin molecule. Even though the toxophore group is lost,- -and when this occurs the toxin is called toxoid (Fig. 40),- - the haptophore group is capable of uniting with receptors and stimulates the production of antitoxin. In fact, in effecting immunization with powerful toxins it may be necessary, in the first few injections that are given, to convert artificially all or a portion of the toxin into toxoid, so that antitoxins will be produced that will protect the animal against subsequent overdoses of toxin.

The production of antitoxins must, in keeping with this theory, be regarded as a function of the haptophore group of the toxin. It is easy, therefore, to understand why, out of the great number of alkaloids, 
none is in a position to cause the production of antitoxins, for alkaloids possess no haptophore group that anchors them to the cells of organs. As has been stated, in the formation of antitoxin the haptophore group of the toxin molecule is the essential portion; the toxophore group is much less important, and during immunization the symptoms of illness due to the action of the latter group are not essential to and play no part in the production of antitoxin. It must be said, however, that a toxin molecule with an intact toxophore group is more stimulating than a toxoid in which this group is absent; therefore, in artificially immunizing horses for the production of antitoxin, after the first few injections increasing amounts of toxin are administered.

Antibodies of the Second Order (Agglutinins and Precipitins).As new discoveries were made, Ehrlich amplified his theory of the formation of antibodies, but always upon the original and basic conceptions as just set forth.

We have seen that the simplest molecules of food substances, toxins, and ferments, substances really in solution, are anchored to molecules of cell protoplasm by means of the simple side-arms of the latter. When this chemical union has taken place, the food or toxin may be assimilated without undergoing any further change. With more complex food substances, however, some preparatory treatment is necessary before they become available for final assimilation. The large molecule may readily enough be anchored to the molecule of the cell, but it probably requires some preparation before it becomes available for the nutrition of the cell.

Accordingly, Ehrlich assumed that the body-cells are furnished with another order of side-chains or receptors composed of two portions; one part or group for union with the food substance, and called the haptophore group; and the second portion, called the toxophore or zymophore group, in which the special function of the receptor resides.

Similarly, certain pathogenic agents that are more complex than soluble toxins or ferments combine with receptors of this kind. One arm, the haptophore group of the receptor, combines with the haptophore portion of the pathogenic molecule, and then the second or toxophore portion of the receptor exerts some special action upon the attached molecule. Receptors or haptines of this nature are known as receptors of the second order; antibodies of the same structure, produced and cast off into the blood-stream as the result of toxic injury and stimulation of body-cells, are known as antibodies of the second order. 
Two such antibodies are well known. In one we find that the toxophore group of the antibody causes clumping or agglutination of its antigen, or the agent that caused its production, and hence this antibody is called an agglutinin. In typhoid fever, for example, the bacillus or one of its more complex products causes the production of an antibody of this nature, so that when the serum of a typhoid fever patient is mixed with the bacilli, the latter lose their motility and form clumps or agglutinated masses. This phenomenon was first observed by Gruber and Durham, and was applied in a practical way to the diagnosis of

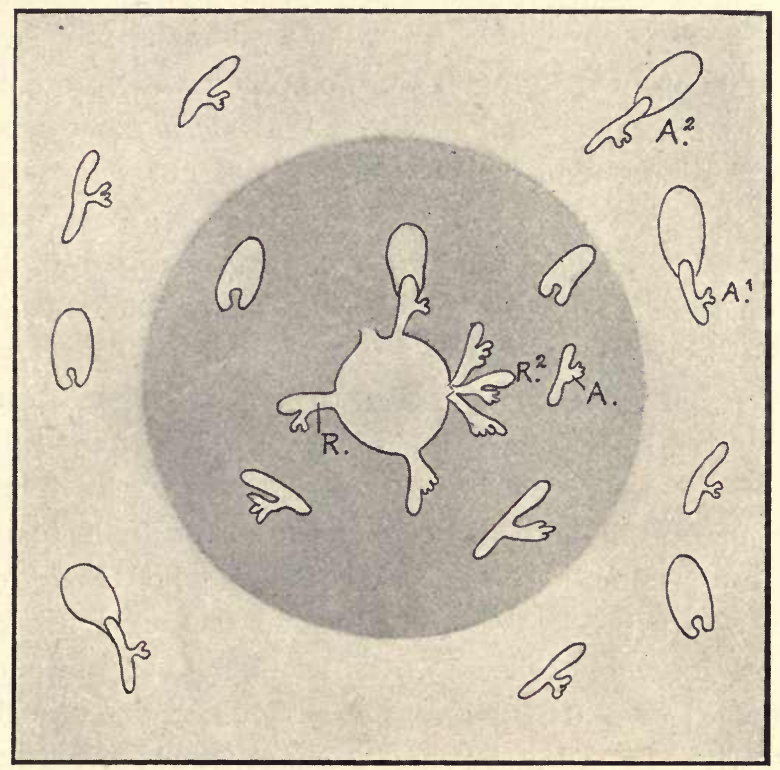

Fig. 41.-Formation of Agglutinins and Precipitins.

The central white area represents a molecule of a cell; the shaded portion represents the cell itself; the surrounding area represents the body-fluids about the cell.

$\mathrm{R}$, Receptor of the molecule (second order); $\mathrm{R}^{2}$, overproduction of receptors, which are being cast off; A, a cast-off receptor which now constitutes the antibody; $\mathrm{A}, \mathrm{A}^{2}$, agglutinins in combination with the antigen (bacilli).

typhoid fever by Widal and Grünbaum. The second antibody of this class, the precipitins, resemble the agglutinins quite closely (Fig. 41).

Kraus discovered that if a bouillon culture of the typhoid bacillus is filtered through porcelain, and a few drops of serum from a typhoid fever patient or from an animal immunized by injections of typhoid bacilli are added to a small quantity of the bacilli-free filtrate, a faint cloud will appear resembling in some respects that observed at the line of contact between nitric acid and urine that contains a trace of albumin. 
The toxophore portion of this antibody, therefore, appears to coagulate or precipitate soluble substances, and, accordingly, the antibody is known as a precipitin. As will be pointed out later, various protein substances, such as blood-serum, milk, egg-albumin, etc., may cause the production of specific precipitins.

Antibodies of the Third Order (Hemolysins, Bacteriolysins, Cytotoxins).- Still more complex molecules of food material require conversion into simpler substances before they may be assimilated by the molecules of the cell. It is essential that they undergo a sort of digestion, and accordingly Ehrlich has conceived that special side-arms or receptors exist for this purpose, these being composed of two grasping portions, or haptophore groups, one for union with the complex food molecule, the second for union with a special, ferment-like substance present in the blood and called complement. The receptor, therefore, acts simply as a connecting link or interbody between food molecule and complement, bringing the two into relation with each other when the food molecule is rendered soluble, $i$. e., undergoes lysis.

With highly organized cell material, such as red blood-corpuscles or bacteria, it is found that receptors of this nature bring about their destruction by lysis by attaching them to a suitable complement. During infections with various bacteria, therefore, we find that numerous antibodies are produced. If the bacteria produce soluble toxins, specific antitoxins are produced to counteract the effects of these; other products stimulate the production of agglutinins and precipitins; still other products or the whole cell cause the production of antibodies, which are not in themselves destructive, but which have the specific power of combining with the cell and bringing about its lysis or destruction by bringing it into relation with the ferment-like complement. It is only by means of a special antibody of this nature that a complement may be united with the pathogenic agent, $i$. $e$, the complement itself cannot act directly upon the cell, but must be united by means of the antibody.

Ehrlich has termed an antibody of this nature an amboceptor, or interbody. In structure, amboceptors are believed to possess two combining or grasping portions: one, the haptophore or antigenophore group, for union with the cell; the other the complementophile group, for union with a complement (Fig. 42).

The lysins (bacteriolysins, hemolysins, and other cytolysins) are antibodies of this order. If, for example, the erythrocytes of one animal are injected into an animal of a different species, hemolysins will be produced, the hemolysin being a specific hemolytic amboceptor that will 
unite corpuscles of the animal used in the injection and only these cells, with a complement, and thus bring about their solution or lysis. If certain bacteria (e. g., the cholera bacillus) are injected into an animal, specific bacteriolysins (bacteriolytic amboceptors) will be produced. Similarly, specific amboceptors are produced during the course of infections with typhoid bacilli, and are largely instrumental in combating and overcoming this infection. It is important to remember, however, that although these amboceptors probably prepare their antigens for

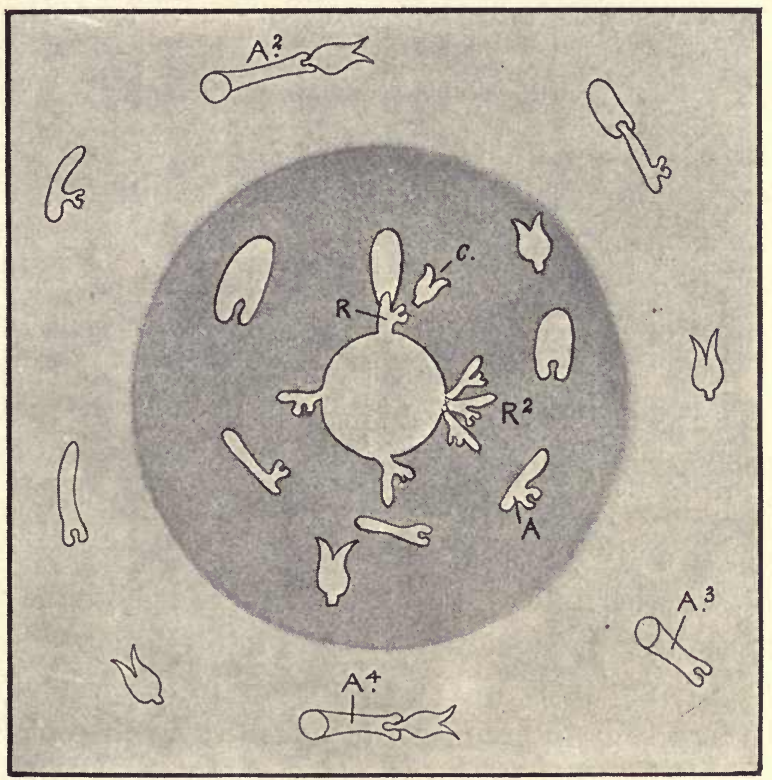

Fig. 42.-Formation of Cytolysins (Hemolysins, Bacteriolysins, Cytotoxins).

The central white area represents a molecule of a cell; the shaded portion represents the cell itself; the surrounding area represents the body-fluids about the cell.

$\mathrm{R}$, Receptor of the molecule (third order); $\mathrm{R}^{2}$, overproduction of receptors, which are being cast off; A, a cast-off receptor which now constitutes the antibody or amboceptor; C, molecule of complement free in the body-cells and body-fluids; $\mathrm{A}^{2} \mathrm{~A}^{4}$, amboceptors in combination with molecules of a cell (antigen) and a complement; $\mathrm{A}^{3}$, an amboceptor in combination with a molecule of a cell. The cell (antigen) is now said to be sensitized. Lysis does not occur because a complement is not united.

lysis, or, in the meaning of Bordet, "sensitize" them, they are not in themselves lytic, final solution of the antigen being accomplished by the ferment-like substance-the complement.

\section{COMPATIBILITY OF THE PHAGOCYTIC AND SIDE-CHAIN THEORIES}

When we seek to compare the theory of Metchnikoff with that of Ehrlich, we find that they differ only in minor details, the fundamental 
principles not being contradictory; they may, rather, be regarded as one set of phenomena viewed from different aspects.

Since its original announcement, Metchnikoff has, on different occasions, enlarged upon his theory to meet certain discoveries, made chiefly by adherents of Ehrlich's theory, showing the presence of substances in the blood-serum and other body-fluids that are potent in the processes of immunity independent of cells. Metchnikoff claims, however, that these antibodies are derived from the group of cells classified as phagocytes, and thus would preserve the primary importance of his theory. Ehrlich, on the other hand, while not denying that these cells may be a source of their formation, points out that they are not necessarily the sole or supreme source, but may ive formed by the general body-cells or by special groups of cells possessing a selective affinity for the pathogenic agent.

The theory of Ehrlich is essentially a chemical one, and maintains that the union of food or pathologic material with cells is a chemical union; his views, therefore, possess that degree of definiteness necessary to constitute a plausible chemical theory. The theory of Metchnikoff would explain processes of nutrition and immunity as largely founded on a physical basis, and is therefore, necessarily more general, being largely biologic and vitalistic.

The two theories differ in two more or less hypothetic points: (1) In the manner by which material enters into relation with cells, and (2) the relative importance of certain cells in the formation of antibodies. Otherwise both are intimately related, in that phagocytosis is unimportant if removed from the influence of antibodies in the bodyfluids, and these same antibodies, although probably formed according to Ehrlich's theory, are derived in part from Metchnikoff's phagocytes.

Phagocytosis, whether by leukocytes, endothelial cells, or by newly developed connective-tissue cells, is very common, and is obviously a most important factor in the destruction of pathogenic bacteria and in the cure of infectious disease. In virulent infections, however, phagocytosis may not be apparent; the leukocytes are not attracted, and those in the vicinity undergo dissolution. Later in these infections, however, phagocytosis may become apparent, due, according to Metchnikoff, to the "adaptation" of the cells to the products of the invading microorganism, whereby the weak or negative chemotaxis is converted into an active positive chemotaxis with vigorous digestion. This, however, is not primarily due to increased digestive capacity of the phagocytes, 
but to an increase of opsonins in the body-fluids; these opsonins prepare the bacteria for digestion.

The original phagocytic theory did not explain the destruction of bacteria within the living tissues without the intervention of leukocytes, and, what is even more striking, a similar destruction occurring in vitro by serum and other body-fluids totally devoid of cells. Bacteriolysis has been shown to be due to two different substances-one, a thermolabile, ferment-like body called "cytase" by Metchnikoff and "complement" by Ehrlich, and the other a more specific thermostabile body called "fixateur" by Metchnikoff and "amboceptor" by Ehrlich. These substances appear to play an important rôle in certain infections, as, for example, in typhoid fever and cholera, and were studied mainly by the adherents of the side-chain theory. Metchnikoff recognized their existence and significance, but endeavored to preserve the primary importance of the phagocytic theory by claiming that they are products of the group of cells classified as phagocytes. Ehrlich, however, while not denying that these cells may be one source, holds that they are not necessarily the sole source, but that they are products of general cellular activity or of special groups of cells that have shown a combining affinity for the antigens.

For example, Metchnikoff holds that there are but two complements,- macrocytase and microcytase,- and that these are formed by destruction or solution of macrophages and microphages. Ehrlich has shown quite conclusively that there are many complements, and that these are the excretory products of leukocytes, and probably of other cells as well. Ehrlich teaches also that specific amboceptors or fixateurs may be products of various body-cells other than those classified as phagocytes, and Metchnikoff recognizes their existence, but holds that they are formed and discharged solely by the leukocytes or other phagocytic cells. Ehrlich has shown the manner in which complement and amboceptor produce bacteriolysis, and Metchnikoff has amplified his theory to meet these observations, to the extent that destruction of bacteria is recognized as being brought about either intracellularly, by the digestive action of the leukocytes, or extracellularly, by the enzymelike action of the cytase, or complement, working through the intermediation of the fixateur or amboceptor, and that cells that are potentially phagocytic give origin to these antibodies.

Regarding the structure of toxins and the action of antitoxins the two theories are divergent, and whereas Metchnikoff is inconclusive, Ehrlich presents definite conceptions that are well supported by experi- 
mental data. Metchnikoff maintains that it is the cells that absorb the "toxin" that furnish the antitoxin. In other words, the enzymes, as microcytase and macrocytase, exert their action not only upon the more complex molecules of microörganisms, but also upon their simpler toxins, fixing or otherwise altering them until they can finally be destroyed. This explanation would lead us to conclude that the nervecells which bind the tetanotoxin are capable of furnishing antitoxin, whereas experimental observations are absolutely opposed to this narrower view. Metchnikoff also maintains that antitoxin acts by stimulating the leukocytes to absorb and destroy toxin, whereas Ehrlich has clearly shown that antitoxin, by combining chemically with the toxin, neutralizes it, a process that may be shown in vitro entirely independent of cells.

From what has been said it will be seen that the two theories are not essentially divergent, and that we are unwarranted in clinging to one view to the absolute exclusion of the other. The question rests largely on which of the body-cells are most active in forming antibodies, and also on a recognition of the rôle played by phagocytosis in certain infections, such as staphylococcus, streptococcus, and pneumococcus infections. Ehrlich has attempted an explanation of the method by which body-cells form antibodies, and the manner in which these antibodies overcome their antigens; he has placed both processes upon a chemical basis, involving no one particular group or class of cells. Metchnikoff, on the other hand, has shown the important rôle played by phagocytosis in many infections, and claims that the antibodies in the circulating fluids are the products of these phagocytes; he places immunity more largely upon a physical basis.

The various phenomena of immunity cannot be ascribed either to the activity of the body-cells or to the body fluids alone, to the total exclusion of the other-both are intimately concerned in the various phases of immunity.

It is, moreover, becoming more obvious that too little attention has been paid to the influence of the microörganism in the phenomena of immunity reactions. It is important to recognize that some bacteria are apparently able to immunize themselves against the combative forces of their hosts, as is demonstrated by the manner in which streptococci and pneumococci protect themselves with a capsule and resist phagocytosis. Virulent strains and "resistant races" may be evolved in this manner. This has been demonstrated by Ehrlich with regard to the action of various arsenical compounds on protozoa, work that 
finally culminated in the brilliant discovery of salvarsan. Thus atoxyl may not kill all the trypanosomes in an infected animal, those escaping acquiring a new power of resistance to the poison and become atoxylresistant. The production of "resistant races," not only among the protozoa, but also in the class of bacteria, complicates enormously the practical problems of immunity.

\section{ANTIGENS}

Briefly defined, antigens are substances that can cause the formation and appearance of antibodies in the body-fluids.

So far as is now known, antigens are colloids, and are usually protein in nature. Every known soluble protein may in some degree act as an antigen, and recent investigations would seem to show, although they do not definitely prove, that toxic glucosids and various lipoids may to some extent act in this same capacity The protein antigens may be quite varied: thus antibodies are produced not only by the injection of bacteria or their toxins, but also by erythrocytes, serums of different animals, egg-albumen, milk, etc.

Of the cleavage products of proteins, it is certain that none of the amino-acids and simple polypeptids can act as antigens; there is, however, some evidence to show that the proteoses possess antigenic properties. It has been shown by Gay and Robertson ${ }^{1}$ that if the antigenic cleavage products of casein are resynthesized by the reverse action of pepsin into a protein resembling paranuclein, this synthetic protein is capable of acting as an antigen. Protamins and globin were found to be non-antigenic, although globin combined with casein formed a compound of antigenic power in that it produced an antibody yielding complement-fixation reactions with globin.

Whether the entire protein molecule, or only groups thereof, determine the characteristics of the antigen and the antibody is not definitely known. Wells and Osborne ${ }^{2}$ have recently submitted evidence showing that a single protein molecule can act as an antigen and produce more than one antibody.

Non-protein Antigens.-Ford ${ }^{3}$ was able to immunize rabbits by injecting a toxic glucosid contained in extracts of Amanita phalloides, producing an antibody antihemolytic for the hemolysin of Amanita when diluted 1:1000. Abderhalden and others have found that specific enzymotic substances appear in the blood of animals injected with car-

${ }^{1}$ Jour. Biol. Chem., 1912, 12, 233; Jour. Exp. Med., 1912, 16, 479; 1913, 17, 535.

2 Jour. Infect. Dis., 1913, 12, 341.

3 Jour. Infect. Dis., 1907, 4, 541. 
bohydrates and fats. Recent developments in immunologic research would indicate, therefore, that a complex toxic glucosid that can be hydrolyzed by enzymes may act as an antigen.

The intimate relationship of lipoids to complement fixation reactions, especially in syphilis, has naturally led to investigations regarding the possibility of lipoids acting as true antigens. In testing for the Wassermann reaction the use of lipoids in the form of tissue extracts to serve as an antigen does not mean that it is a true antigen; in fact, experimental work indicates quite strongly that these lipoidal substances are incapable of producing antibodies when injected into animals.

Much and others have worked with lipoids secured from a streptothrix, and which is called "nastin," and they assert that this substance may be used in immunizing animals with the production of an antibody yielding complement fixation, with nastin as the antigen. Similar results have been described for the fatty substances from tubercle bacilli ("tuberculonastin"). Kleinschmidt 1 accepts the antigenic nature of nastin in reactions, but was unable to secure antibodies by immunizing rabbits with this substance.

Ritchie and Miller ${ }^{2}$ could demonstrate no antigenic activity in the lipoids of serum or corpuscles. Thiele ${ }^{3}$ calls attention to the fact that lipoids possess no specificity, and that they cannot act as antigens with the production of antibodies. On the other hand, Meyer ${ }^{4}$ has reported the production of specific complement-fixation antibodies by immunizing rabbits with acetone-insoluble lipoidal substances obtained from various teniæ. He has also found the acetone-insoluble fraction of tubercle bacilli to serve as antigens in complement-fixation reactions with antibodies of the tubercle bacillus, and much more effectively than with the protein residue of the bacilli. Beigel ${ }^{5}$ has observed that after injecting lecithin in rabbits an increase occurs in the lipase content of the blood and tissues, with the presence of complement-fixing antibodies, and Jobling and Bull ${ }^{6}$ have found an increase in serum lipase after immunizing with red corpuscles.

It will be noticed, therefore, that the results of various investigations regarding the true antigenic properties of lipoids are not in accord. It should be emphasized that the complement-fixation reaction does not constitute a reliable index to the study of this problem, as so little is

${ }^{1}$ Berl. klin. Wochschr., 1910, 47, 57. 2 Jour. Path. and Bact., 1913, 17, 427.

3 Zeit. f. Immunität., 1913, 16, 160.

4 Zeit. f. Immunitat., 1910, 7, 732; 1911, 9, 530; 1912, 16, 355.

5 Deut. Arch. f. klin. Med., 1912, 106, 47.

6 Jour. Exper. Med., 1912, 16, 483. 
understood of the actual nature of this reaction itself. That lipoids serve a very important purpose in the absorption or fixation of complement in vitro, as is so well demonstrated in Wassermann's reaction for syphilis, is undoubtedly true, but this does not indicate that the antibody in the blood-serum of syphilitics is in the nature of a true lipoid antibody, and, indeed, investigation on this subject would seem to indicate that it is not. ${ }^{1}$

It will be understood, therefore, that the question of substances other than proteins acting as true antigens must be regarded as an open one, requiring further investigation. The relation of proteins, however, to the production of antibodies has been fully established, and is at present receiving renewed attention through the researches of Vaughan and his coworkers and Abderhalden. As has been stated in a previous chapter, Vaughan regards the protein constituents of bacterial and other cells as the main antigenic principle capable of causing the production of specific proteolytic ferments, which split the new bacterial protein, releasing a toxic product responsible for the symptoms and lesions of the infection. Abderhalden has also demonstrated the presence of proteolytic ferments in the blood-serum after experimental immunization with proteins, and in the serum of pregnant women, due to the antigenic stimulation of syncytial cells, capable of splitting their substrata in vitro into aminoacids and other simple cleavage-products. These investigations serve to show the intimate relation that proteins bear to the problems of infection and immunity, and demonstrate that antibodies may be largely in the nature of ferments, and that immunologic reactions, both in the living tissues and in the test-tube, are largely in the nature of disintegrative enzymic processes.

\section{ANTIBODIES}

The term antibody is used to designate the specific bodies produced by the cells of a host in reaction against an antigen, as an infecting microparasite and its products or other foreign protein.

Various kinds of antibodies may be produced by the same antigen and by different antigens. Some neutralize the soluble toxin of the antigen (antitoxin); others agglutinate or precipitate their antigens (agglutinins and precipitins); still others cause complete dissolution of the antigen (hemolysins, bacteriolysins, etc.), and others again may so alter the antigen and lower its resistance as to render it more easily phagocytable by the body-cells (opsonins or bacteriotropins).

${ }^{1}$ Further discussion on the question of lipoids acting as antigens will be found in Chapter XXVII in a consideration of anaphylactogens. 
Specificity of Antibodies.-Antibodies are usually specific for their antigen, and it is upon this general law that the reactions of immunity are based. It should be remembered, however, that not all antibodies are protective; the agglutinins, for instance, apparently do not injure their antigen. On the other hand, an animal may enjoy an immunity without demonstrating the presence of any antibody in the body-fluids, and another animal may show antibodies generally considered as possessing protective powers, as, for example, the bacteriolysins, without necessarily being immune.

Upon what does the specificity of antibodies and immunologic reactions depend? Specificity was at first believed to depend solely upon some peculiar biologic relationship of the antigens, for it was found comparatively easy to differentiate the serum of animals of dissimilar nature by means of the precipitin and other reactions, and, as serum proteins, which seemed to be quite similar chemically, but which were obtained from unrelated species, were sharply differentiated by the biologic reactions, it was considered that the specificity must be dependent upon some principle quite apart from the ordinary chemical substances.

With the use of proteins other than serums, and especially when more or less purified proteins were employed, it has been quite firmly established that specificity depends upon chemical composition, and that differences in species, as exhibited by their biologic reactions, depend upon distinct differences in the chemistry of their proteins (Wells).

Pick and his coworkers have shown that two kinds of specificity exist in each protein molecule: (1) One of these is easily changed by various physical agents, such as heat, cold, and partial coagulation. When an antigen is altered by heat, it produces an antibody that reacts best with the heated antigen; heating does not, however, destroy the characteristics of the antigen of this species, as its antibody will not react with the heated antigen of another species.

(2) The second alteration involves a profound chemical change of the antigen, whereby it is so altered that it loses the characteristics peculiar to the species, and produces an antibody that will react with the altered antigen, but not with the unaltered antigen, even from the same animal. For example, it is possible so to alter the serum protein of a rabbit by treatment with nitric acid that the nitroprotein injected back into the same rabbit will produce an antibody specific for the nitroprotein, but which does not react with the unchanged serum protein. These changes are apparently closely related to the aromatic radicals of the protein antigen, for they 
are effected by introducing into the protein molecules substances that are known to combine with the benzine ring, e.g., iodin, diazo- and nitrogroups. Pick, appreciating the fact that the number of different aromatic radicals in the protein molecule are limited, interprets the significance of these radicals as depending upon their arrangement, rather than upon their number, in the protein molecule. Granting the number of possible variations in the arrangement of the aminoacids in a protein molecule which the great number of these radicals provides, there is no difficulty in understanding the possibility of an almost limitless number of specific distinctions between proteins.

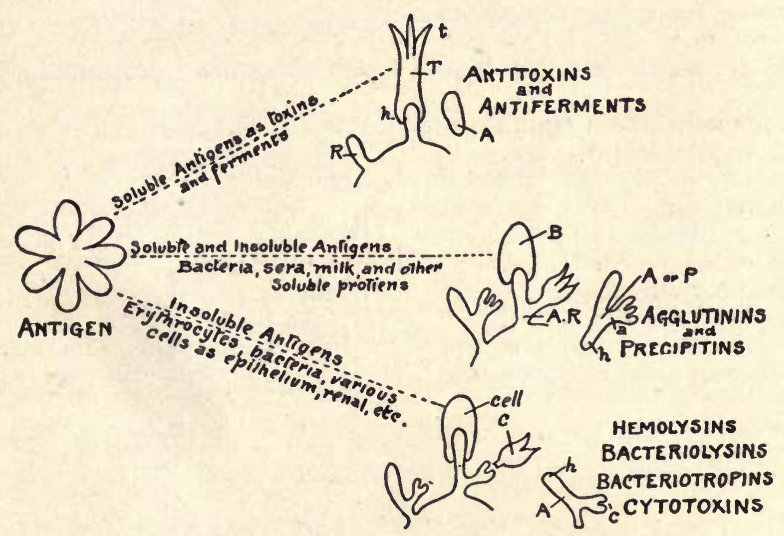

Fig. 43.-General Scheme of Antigens and Antibodies.

Antitoxins and antiferments: R, Receptor of a molecule of a cell; $T$, a toxin molecule; $t$, toxophore group of the toxin molecule; $h$, haptophore group of the toxin molecule; A, cast-off receptor and constitutes antitoxin.

Agglutinins and precipitins: A. R, Receptor of cell with antigen attached; B, a bacterial molecule (antigen) attached to a receptor; A or P, an agglutinin or precipitin; $h$, haptophore group of the antibody; a, agglutinophore group of an agglutinin.

Hemolysins, etc.: A, Cast-off amboceptor (hemolysins, bacteriolysin, etc.); $h$, haptophore group of amboceptor; $c$, complementophile group; C, molecule of complement.

It may be stated, however, in general, that immunologic reactions, such as that of anaphylaxis, are as delicate in distinguishing between proteins as are chemical analyses. Distinctions may be made by these reactions with quantities too small for making accurate chemical determinations.

In succeeding chapters we shall consider, first, the different kinds of immunity, as dependent upon the presence of various factors, and then the rôle played by the phagocytes and the body-fluids in immunity, with a more detailed consideration of the various antibodies. 
It may be useful here to draw up in tabular form a list of the various antigens and antibodies with which we are mainly interested in that portion of immunity involving infection with vegetable or animal parasites, and the products of their metabolism or degeneration (Fig. 43).

Toxins:

\section{Antigens}

1. Soluble bacterial toxins (diphtheria and tetanus toxins, etc.).

2. Phyto- (vegetable) toxins (ricin, abrin, etc.).

3. Simple zoö- (animal) toxins; (snake, spider, toad venoms).

4. Complex zoötoxins, as snake venom, requiring complement for action.

Enzymes or ferments (rennin, lipase, etc.).

Precipitogenous substances (soluble animal and vegetable proteins).

Agglutinogenous substances (bacteria, erythrocytes, etc.).

Opsonigenous substances (bacterial endotoxins or aggressins?).

Cytoligneous substances:

1. Vegetable cells (bacteria).

2. Animal cells (erythrocytes, spermatozoa, kidney tissue, etc.).
ANTIBodres

1. Antitoxins (diphtheria and tetanus antitoxins, etc.).

2. Anti- (phyto-) toxins (antiricin, antiabrin, etc.).

3. Anti- (zoö) toxins (antivenins).

4. Antihemolysins, etc.

Anti-enzymes (antirennin, antilipase, etc.).

Precipitins.

Agglutinins.

Opsonins (acting singly or with complement).

Cytolysins:

1. Bacterî́lysins.

2. Hemolysins, spermatolysins, nephrolysins, etc. 


\section{THE VARIOUS TYPES OF IMMUNITY}

As has been stated in the preceding chapter, it is generally agreed that various antibodies and other protective agencies exist, although opinions differ as to the source and relative importance of these to resistance to and recovery from various infections. Whether or not a particular antibody is derived from a certain group of cells is largely a matter of individual opinion, because of the difficulty of deciding the point by actual experimental evidence. Of far more importance is a knowledge of the properties of antibodies and of the rôle they may play in the processes of immunity. It is seldom that resistance to, or recovery from, an infection is dependent upon one defensive factor: usually several agencies are operative, although one factor may predominate. For example, antitoxins are known to neutralize their respective toxins, and are of most value in combating the toxemias, such as diphtheria and tetanus; bacteriolysins cause the death of and may totally destroy their antigens, and play an important part in the recovery from infections with bacilli of the typhoid-colon and cholera groups; phagocytosis in itself is of importance in staphylococcus infections, and is of primary importance, in conjunction with the opsonins, in recovery from pyogenic infections in general; agglutinins and precipitins do not appear to have a direct inimical influence on their antigens, but are probably secondary factors, and contribute in some manner toward their destruction. Along with important non-specific factors, these various antibodies are responsible for the different forms of immunity, which may now be considered in their more general aspects.

There are two forms of immunity—natural and acquired.

\section{NATURAL IMMUNITY}

Natural immunity is the resistance to infection normally possessed, usually as the result of inheritance, by certain individuals or species under natural conditions.

The mechanism of this type of immunity is very complex, and bears an intimate relation to the subject of infection, both local and general, 
the nature of the infecting parasite, and the presence or absence of specific antibodies in the body-fluids. In many instances this type of immunity is dependent upon non-specific causes - is frequently relative and seldom absolute. For example, forwls are immune to what may be called an ordinary dose of tetanus toxin, but succumb readily to larger doses; rats are highly immune to diphtheria toxin, and readily withstand the effects of an amount equaling 1000 lethal doses for a guinea-pig, but still larger doses may prove fatal; hedgehogs possess complete or almost complete immunity for the amount of snake venom deposited in an ordinary strike, but if the venoms of several snakes are collected and injected at one time, the result is fatal.

Species immunity is a type of natural immunity, best illustrated by the immunity of man to certain diseases of the lower animals, such as fowl cholera, swine-plague, distemper, Texas cattle fever, mouse septicemia, etc.; and, conversely, by the immunity of animals to diseases common to man, such as measles, cholera, typhoid fever, scarlet fever, chicken-pox, etc. Although the close relation of man to the domestic animals furnishes ample opportunity for infection, yet a complete immunity is frequently observed.

Racial immunity is that type of natural immunity existing among members of the same species. For example, negroes are believed to enjoy immunity to yellow fever and Mongolians to scarlet fever. As a matter of fact, well-marked examples of racial immunity are extremely rare, as not infrequently the disease in question may have been acquired in early infancy in a clinically unrecognized form.

Similarly, close biologic relationship is no guarantee that animals will behave alike toward infection. For example, the white mouse is immune to glanders, the house mouse is somewhat susceptible, and the field mouse is highly susceptible. The rabbit, guinea-pig, and rat are rodents, but though the rabbit and the guinea-pig are susceptible to anthrax, the rat is largely immune. Mosquitos, though closely related, behave differently toward, the malarial parasite. The Culex does not carry the parasite at all, and of the Anopheles, one species, Anopheles maculipennis, is quite susceptible and well recognized as a carrier of the parasite, whereas Anopheles punctipennis, though closely related, is not susceptible to it.

Examples of individual immunity, while not infrequent, are not constant and seldom absolute. Certain persons appear to possess a definite immunity to scarlet fever and diphtheria, although they may be freely exposed; others may pass through various epidemics of other 
infectious diseases, such as measles, pertussis, etc., without becoming infected. I have noticed, on several occasions, that resident physicians, on service in scarlet-fever wards for many months or years, having escaped infection though brought in intimate contact with severe forms of the disease, finally contracted the disease upon returning from a short vacation.

Natural immunity may be due to the following causes:

1. Various non-specific factors may prevent infection; among these may be mentioned-(a) The integrity of the epithelium of the skin and mucous membranes, and $(b)$ the chemical and physical action of various secretions, such as the gastric fluid, the intestinal juices, and the saliva.

2. A particular route for the introduction of infecting microparasites may be necessary. For example, intestinal diseases, such as typhoid fever and cholera, are usually due directly to swallowing of the infecting microörganisms, infection in this type of disease seldom, if ever, occurring through the skin. This is probably due in part to the lowered vitality of the intestinal mucosa, together with a peculiar selective affinity of the bacteria for the cells of these tissues, aided by- $(a)$ the biologic nature of the invading bacterium, which grows best under the more favorable cultural conditions of the intestinal canal. This selective action is further illustrated by the tendency of dysentery toxin to attack the intestinal mucosa when the bacilli or toxin is administered intravenously.

3. Certain tissues appear to possess a marked local immunity to certain bacteria. In considering examples of local immunity, various factors, such as the question of exposure, the thickness of the epidermis, and the kind and quantity of the local secretions must be borne in mind. For example, Trichina spiralis affects the muscles, never the bones, and but rarely any other tissue. Likewise, although diphtheria in the throat may spread in many directions, it seldom passes down the esophagus.

Some differences are known to exist in regard to local immunity as observed in the child and in the adult. For example, ring-worm of the scalp is practically unknown among adults, whereas children under seven years of age are quite susceptible to the disease. These differences may be due to the greater susceptibility in general of young tissues to infection, and the local immunity constitutes but an index to the general rise in resisting power accompanying improvement in strength and vitality. In some cases this may be due perhaps to an actual strengthening of local tissues, as in the case of the adult vaginal mucosa, which is 
immune to the gonococcus, whereas the thin and immature infantile membrane is peculiarly susceptible.

In general, our knowledge of local immunity is quite incomplete. The subject is a difficult one, hence most attention has been given to the study of general immunity. A striking example of acquired local immunity may be seen in a patch of psoriasis, where the center is observed to be largely free from scales, whereas the margins are quite active.

The question of local immunity may be largely determined by various local non-specific factors, such as loss of blood supply due to traumatism, thrombosis, tight bandaging, etc., and the action of severe irritants, tending to produce necrosis of the tissues.

4. The importance of phagocytosis in natural immunity must be emphasized. Microörganisms are constantly gaining entrance to the tissues through numerous small abrasions of the skin and along the intestinal and respiratory tracts, and investigations have shown how important the wandering cells are in preventing infection, being ever on guard and ready to pick up and dispose of any injurious material. Even after mild infection has occurred, the local inflammatory reaction in which the phagocyte is a prominent factor may be so prompt in overcoming the invaders that the host will escape serious infection.

The natural immunity of the frog to anthrax has been shown to be partly dependent upon the activity of the leukocytes in engulfing and disposing the bacilli.

Similarly a mild irritant may produce hyperemia and exudation or local accumulation of leukocytes, which aid in establishing a local immunity largely dependent upon phagocytosis. In this manner the intraperitoneal injection of sterile bouillon or even of salt solution may produce exudation and increase the immunity to infection.

5. It may be that even after the introduction of a microörganism or its toxin no harm results because of a lack of suitable receptors on the part of the body-cells of the host for union with the pathogenic agent. For example, tetanus toxin, being unbound by the cells, produces no effect on the turtle, and antitoxin is not produced. On the other hand, suitable receptors may be present that will bind the toxin, but produce no harmful effects because the body-cells are not susceptible to the action of the microparasite or its products. Thus it is asserted that tetanus toxin has no effect upon the alligator, although the toxin is bound and antitoxin is produced by its body-cells.

In other instances, a host may escape infection owing to the fact that there is a lowered affinity between a pathogenic agent and the 
body-cells, so that but a small amount of harmful substances are bound to the body-cells, and no particular harm results, whereas a larger dose, uniting with a greater number of cells, is capable of producing some disturbance.

6. A natural antitoxin immunity may exist, as the immunity of the alligator to tetanus toxin, just mentioned. Similarly natural diphtheria antitoxin may prevent infection, especially in those persons known to harbor virulent bacilli in the faces. In such instances, however, it is difficult to exclude entirely the possibility that a previous minor infection has occurred, as natural antitoxin immunity persists much longer than the passive immunity resulting from the administration of an antitoxin serum.

Otto, who has recently investigated the content of diphtheria antitoxin in the blood of normal persons, found more than $\frac{1}{100}$ of a unit of antitoxin in each cubic centimeter of the blood of those who had been in close contact with cases of diphtheria without having been sick themselves; others usually had much less. Observations would tend to show that this quantity of antitoxin is generally sufficient to confer immunity to diphtheria, and the object of von Behring's method of active immunization is to induce the production of at least that much antitoxin by the body itself. Otto found that diphtheria carriers, both those who had had the disease and those who had not, contained more antitoxin in their blood than did patients who had just recovered from an attack. This shows that the mere presence of bacilli in the throat is sufficient to stimulate the production of antitoxin, on which the immunity of the carrier himself would seem to depend. Undoubtedly physicians and nurses who are freely exposed to diphtheria and yet escape infection owe their safety rather to an acquired immunity the result of repeated contact with the bacilli, than to a natural antitoxin immunity.

7. In some instances a natural immunity may be dependent, at least in part, upon antibacterial activity, due to the presence of bacteriolysins and bacteriotropins in the body-fluids, as, for example, that shown by the dog and the rat to anthrax. In other instances, however, a similar immunity may be observed that cannot be ascribed to the presence of antitoxins or bacteriolysins. In this type of immunity microparasites are apparently unable to sustain themselves, and proliferate in one animal, although aggressive enough in another of the same species.

8. An immunity to infection, especially with such microörganisms as the anthrax bacillus, which is markedly aggressive and but slightly toxic, 
may be due to the presence or production of anti-aggressins. This immunity would seem to depend not upon the bactericidal properties of the serum or leukocytes nor upon the antitoxins, but on the presence of substances that prevent the microörganisms from exercising their special aggressive forces.

9. Finally, an immunity may exist because the parasite or other foreign cell does not obtain suitable nutrition in a host and thus fails to grow. This condition of athrepsia, is responsible for what has been calledathreptic immunity. It has been more recently studied by Ehrlich, who found that upon transferring mouse cancer to the rat, the tumor grew for a short time only, or presumably until the special nutriment carried over with the tumor was consumed. While there is no experimental basis for assuming that a similar condition may be present in bacterial life, yet such a cause may be operative and should be kept in mind.

\section{ACQUIRED IMMUNITY}

Acquired immunity occurs in two distinct forms: (1) Active and (2) Passive. A mixed form may exist, brought about by a combination of factors necessary for the development of the other two.

Active Acquired Immunity.-Active acquired immunity is that form of resistance to infection brought about by the activity of the cells of a person or animal as a result of having had the actual disease in question, or as a result of artificial inoculation with a modified or attenuated form of the causitive microparasite.

The essential feature of this immunity is that the cells and tissues of persons or animals should, by their own efforts, and as a result of their own active struggle against the action of a microparasite or its products, overcome these and become less susceptible to them than they were before.

This form of immunity is gained, therefore, only as the result of an active struggle between body-cells and infecting agent, and this battle may be of any degree of severity, ranging from an attack of the disease itself that may threaten life, down to the most transitory and trivial reaction due to the injection of a minute dose of a mild vaccine.

Active acquired immunity may be gained-(1) By accidental infection, which is the most familiar form of acquired immunity, and follows an attack of an infectious disease, such as scarlet fever, measles, varicella, variola, or typhus fever; (2) by inducing an attack of the disease by artificial inoculation. This latter method of producing an active acquired 
immunity was illustrated by the ancient, obsolete, and discarded practice of smallpox inoculation, by which healthy persons were inoculated with the virus of a mild case of smallpox, at a time when no epidemics existed and the person was in good general health and able to secure proper attention from the outset.

This process of immunization is used much more extensively in veterinary practice, where an occasional untoward or fatal result is of comparatively little importance if by its means an outbreak can be controlled or the great majority of the animals saved. As a rule, an attempt is made to render the induced disease as mild as possible by- $(a)$ Using a small amount of infective material; $(b)$ by inoculating it through an unusual avenue; or $(c)$ by inoculating it at a time when the animals are naturally less susceptible, or $(d)$ by a combination of these methods. For example, Texas cattle fever, which is due to a protozoan (Piroplasma bigeminum) conveyed by the bites of infected ticks, may be combated by exposing calves while still milk fed to the bites of a few infected ticks. Another method consists in injecting a small amount of blood from an infected animal directly into the jugular vein. The object is to induce a mild attack of the disease. Occasionally a severe or fatal reaction occurs, but the number of these untoward results is much lower than the mortality among untreated animals.

(3) Active immunity may also be gained by vaccination, $i$. e., by inoculation with a virus or microparasite or its products, modified and attenuated by passage through a lower animal (Jennerian vaccination) or by various other means, as age, unfavorable cultural conditions, heat, germicides, etc. (Pasteurian vaccination or bacterination). These subjects are considered more fully in the chapter on Active Immunization.

Active immunity, whether induced accidentally or artificially, may be antitoxic, as after recovery from diphtheria or as the result of active immunization with diphtheria toxin, as by von Behring's method; or antibacterial, as the immunity following typhoid fever or induced by typhoid vaccination, and largely dependent upon the presence-of bacteriolysins in the circulating fluids.

c During the process of active immunization an animal not infrequently fails to react to relatively large doses of toxin, and at the same time the quantity of antibody in the body-fluid may decrease. This phenomenon has been explained as being due to atrophy of the receptors of the body-cells (receptoric atrophy), whereby the toxin fails to exert its deleterious influence because it fails to unite with the body-cells. It is curious, however, that the toxin is innocuous when present in a 
free state within the body-fluids, even though unbound to the bodycells; this condition is not well understood, and may be dependent upon other factors. A rest may restore the activity of the receptors and cells, a fact that is well recognized in the immunization of horses for the preparation of antitoxin. Not infrequently a rabbit fails to produce hemolytic amboceptor if the injections of erythrocytes are too frequent. After a rest, however, the animal may react promptly with much smaller doses.

Passive Acquired Immunity.-As the name indicates, this is a form of immunity that depends upon defensive factors not originating in the person or animal protected, but is passively acquired by the injection of serum from one that has acquired an active immunity to the disease in question.

This is a sort of secondary immunity, acquired by virtue of having received antibodies actively formed by another animal that has had to resist the infecting agent in order to produce them. Two well-known examples of this type of serums are the diphtheria and tetanus antitoxins. These are produced by injecting horses with successive doses of the respective toxins. The horses are required to combat the effects of the toxins, and acquire an active immunity of increasing grade, due to the production of antitoxins. When the animals are bled, the antitoxinladen serum, separated from the corpuscular elements, may be used for conferring an immunity in a person or another animal simply by injecting the serum, the latter receiving and enjoying an immunity in a passive manner.

Passive immunity is specific, that is, the serum of an animal immunized against one microörganism will protect a second animal against that and against no other. This type of immunity is acquired just as soon as the immune serum has become mixed with the blood of the person or animal injected, and there is no negative phase. Hence in severe infections our hopes of specific therapy rest on the production of passive immunity. No matter how sick the recipient may be, under ordinary circumstances the immune serum produces no further disturbance than would be expected from the injection of a normal serum. The recipient's body-cells have no additional burdens, or very slight ones only, to bear and these are more than counterbalanced by the release from combat with toxic substances neutralized by the antibodies in the immune serum. Unfortunately, this field of therapy is limited, although recent discoveries are indicating the reasons for failure, and when these are eliminated, the field of usefulness will be much extended. 
Passive immunity is of shorter duration than active immunity, and the former is especially indicated in prophylaxis for warding off an acute infection that has a relatively short incubation period. The degree of passive immunity is also seldom equal to that of an active immunity. The antibodies produced by our own cells are more lasting and possess higher protective value. This is an important factor in von Behring's method of immunization in diphtheria, when a small amount of toxin loosely bound to antitoxin is injected in the belief that the toxin becomes dissociated and serves to stimulate our body-cells into producing our own antitoxin.

Passive acquired immunity is usually antitoxic, as, for example, that induced by the administration to man of diphtheria antitoxin prepared by the body-cells of the horse. Antibacterial serums may likewise induce a passive immunity, as, for instance, that used in immunization against plague.

It is evident, therefore, that the processes whereby infections are overcome and immunity is conferred, and the general reactions that follow the introduction into the body of modified antigens in the practice of immunization, are complex processes, and in none is one antibody produced or solely responsible for the resulting immunity. The properties and action of the known antibodies are considered in subsequent chapters, particular attention being given to methods for determining their presence in the body-fluids, which serve as an aid to the diagnosis of infection as based upon the general law that the antibody is specific for its antigen, and so, when the presence of an antibody is demonstrated, it may be assumed that the antigen is or has been present.

Nothing is known concerning the nature of the immunity that is acquired against several infections, such as scarlet fever, measles, smallpox, etc., nor will much be known until the causes that give rise to these conditions have been discovered.

Theory of Vaughan.-According to Vaughan, the inability of a bacterial cell to grow in the animal body either because it cannot feed upon the protein of the body or because it is itself destroyed by the ferments elaborated by the body-cells explains all forms of bacterial immunity, either natural or acquired. Thus in antitoxin immunity the toxin is regarded as a ferment that splits up the proteins of the body-cells, setting the protein poison free. The body-cells react with the formation of an antiferment or antitoxin, which neutralizes the toxin and prevents cleavage. The toxin itself is regarded as harmful only in so far as it is 
able to set free the protein poison responsible for the symptoms of the infection.

Natural immunity to any infection, according to Vaughan's theory, is explained as being due to an inability of the infecting agent to grow in the animal body.

Acquired immunity, due to recovery from an infection or occurring as a result of vaccination, is regarded as the outcome of the development in the body, during the course of the infective process, of a specific ferment that, on renewed exposure, immediately destroys the infection. The vaccine is the same protein that causes the disease, so modified that it will not produce the disease, but yet so little altered that it will stimulate the body-cells to form a specific ferment that will promptly and quickly destroy the infecting agent on exposure.

The various kinds of immunity and the factors probably concerned in their production, may be summarized as follows:

Natural Immunity

Acquired Immunity
1. Due to non-specific factors:

1. Barrier of epithelium.

2. Various secretions.

3. A particular route of infection may be necessary, aided by the biologic nature of the invading bacterium.

2. Due to local tissue immunity and selective action of micro-parasites for certain tissues.

3. Due to phagocytosis.

4. Due to lack of suitable receptors of body-cells for a particular bacterium.

5. Due to natural antitoxins.

6. Due to natural bacteriolysins.

7. Due to anti-aggressins.

8. Due to lack of suitable food material-athrepsia.

Active (accidentally or 1 . Antitoxic. artificially acquired) $\}$. Antibacterial.

Passive $\left\{\begin{array}{l}\text { 1. } \text { Antitoxic. } \\ \text { 2. } \text { Antibacterial. }\end{array}\right.$ 


\section{CHAPTER X}

\section{PHAGOCYTOSIS}

Historic.-As Lord Lister stated in 1896, "If ever there was a romantic chapter in pathology, it has surely been that of the story of phagocytosis." The author of this "story" is Elie Metchnikoff. His early researches on phagocytosis in the lowly organized forms of life constituted the starting-point for an entirely new series of researches on the subject of Immunity, and his treatise on the "Comparative Pathology of Inflammation" must ever remain a most entertaining work and a medical classic.

Several observers before Metchnikoff had considered that leukocytes might assist in bringing about the destruction of microparasites. Panum (1874) suggested that the bacilli of putrefaction might, by making their way into the blood-corpuscles and being carried off to the lymph-glands, spleen, etc., thus disappear from the body-fluids. Carl Roser (1881) had also observed the ability of certain "contractile cells" to ingest the enemy that enters the animal body. These statements, however, were poorly supported by scientific data, and the subject was not followed in subsequent research. As the result of zoölogic studies, Metchnikoff was led to discover the part played by body-cells in the processes of immunity. He observed that when a food-particle arrives in the vicinity of a simple unicellular organism as an ameba, the latter, by reason of its "irritability," moves forward and sends out processes of its protoplasm (pseudopodia) that flow around the particle and finally gather it into the interior of the cell. The particle then undergoes a process of intracellular digestion, losing its sharp outline and clear appearance, becoming granular, and disappearing within the protoplasm of its host. Similar studies were made of the processes of nutrition in many unicellular animals, then through actininas, sponges, and similar animals of transparent and simple organization.

Metchnikoff was primarily a biologist, and up to this time was mainly interested in the processes of nutrition in the simple forms of animal life. At this stage, however, he became greatly impressed by Cohnheim's description of the phenomena of inflammation. The diapedesis of leukocytes through the walls of the blood-vessels in an 
inflammatory reaction impressed him as significant and similar to the movements of the ameba in its process of feeding, and led to comparative studies in inflammation in the lower forms of life, where processes were much simpler to watch and may indicate what occurs in the higher vertebrates.

He found that when daphnias are invaded by the spores of a yeast,the monospora,- - they may multiply in the body of the host and bring about its destruction. When, however, a few spores gained access, he found that the daphnia's leukocytes approached them, formed a wall around them, and finally brought about their destruction by a process of digestion. He also observed that if rose prickles were stuck into large bipinnaria larvæ of star fish, these were soon enveloped in a mass of ameboid cells. From this he concluded that, in inflammation, the exudation of leukocytes may be regarded as a reaction against any sort of injury, whether mechanical or due to bacterial invasion.

Metchnikoff traced this defensive reaction against an invading microörganism from small invertebrates up to man, and showed that instead of bacteria attacking leukocytes and forcing a passage into them, as was then believed, they were indeed pursued and engulfed by the leukocytes. Connecting his various discoveries, he was able to formulate the idea that "the intracellular digestion of unicellular organisms and of many invertebrates had been hereditarily transmitted to the higher animals, and retained in them by the ameboid cells of mesodermic origin. These cells, being capable of ingesting and digesting all kinds of histologic elements, may apply the same power to the destruction of microörganisms." To a cell, and especially to a leukocyte, possessing this activity and power he applied the name phagocyte, because of its ability to act as a scavenger, gathering up the living and dead material.

\section{THE ORIGINAL THEORY OF PHAGOCYTOSIS}

The theory as originally adduced by Metchnikoff regarded the leukocytes and certain other cells as specific fighting cells, able to engulf and consume living as well as dead bacteria and cellular débris. The outcome of any infection would depend upon the success or failure of the phagocytes to overcome the invaders: if they were successful in ingesting all the bacteria, their victory meant recovery; if, on the other hand, they were destroyed by the invaders, the host was overcome by the infection.

Phagocytes may ingest not only living and dead bacteria, but also 


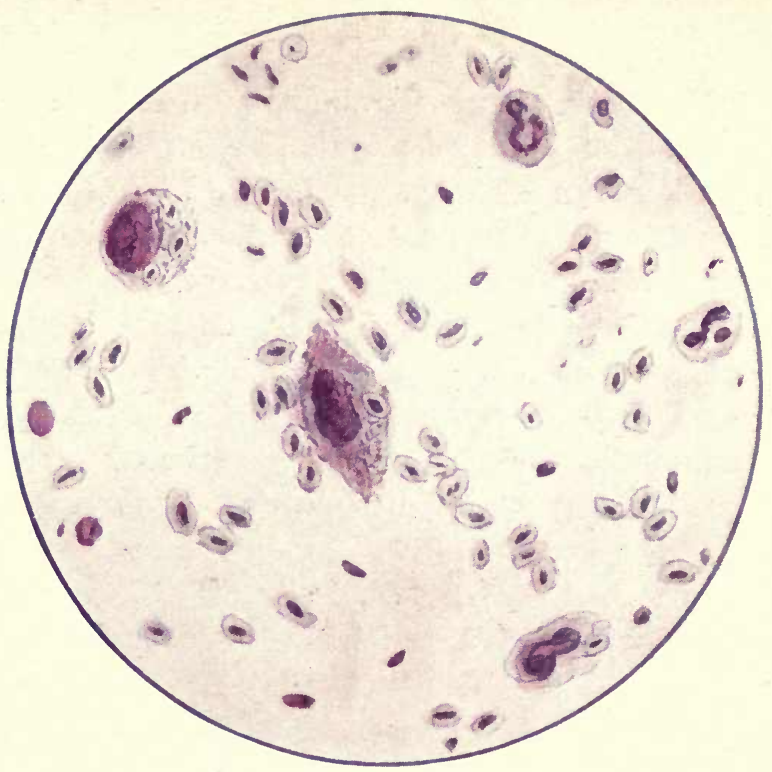

Fig. 44.-Phagocytosis-Macrophages.

A smear of peritoneal exudate from a guinea-pig twenty-four hours after injection with 3 c.c. of a 5 per cent. suspension of pigeon's blood. Note that the corpuscles (nucleated) are being ingested by the large endothelial cells of the peritoneum.

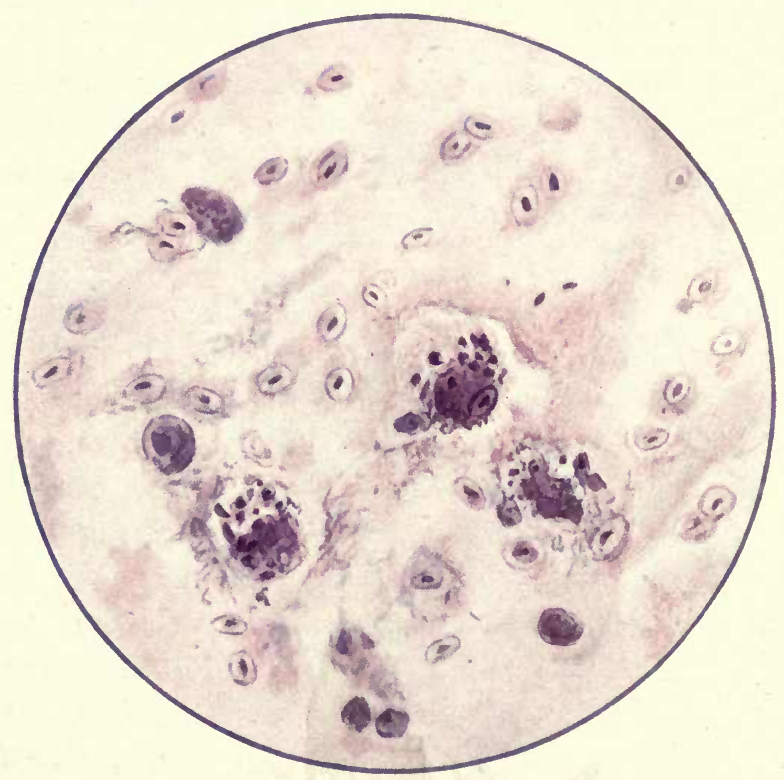

Fig. 45.-Phagocytosis-Macrophages.

A smear of peritoneal exudate from the same guinea-pig forty-eight hours after injection with pigeon's blood. Note the large numbers of corpuscles ingested by the endothelial cells. In most instances the corpuscles have undergone digestion, the nuclei being more resistant. These nuclei are shrunken, and in some instances are broken up. The extracellular red blood-corpuscles are swollen and stain lightly. 
red corpuscles, cellular débris, inorganic particles, such as coal-dust, and even soluble substances, such as bacterial toxins.

Subsequent discoveries have shown that many other factors are present that considerably modify the workings of so simple a process. Metchnikoff has, therefore, modified his theory from time to time as new discoveries were made, but has always preserved the primary importance of the phagocyte itself.

Before stating the revised theory as it stands today, we will describe the kind of cells that may act as phagocytes, and consider the methods and reasons why these cells assume the functions of phagocytes.

\section{VARIETIES OF PHAGOCYTES}

Not only leukocytes, but other body-cells, have been found active in the processes of phagocytosis. Metchnikoff has divided the phagocytes into two great classes:

1. Microphages-principally the polynuclear neutrophilic leukocytes. (See Fig. 46.) The eosinophilic leukocytes are also included in this group, but are of doubtful importance and weak in phagocytic powers. The small lymphocyte may be included in this class, although it is usually considered with the second group.

2. Macrophages, principally the large mononuclear leukocytes; ameboid cells of the spleen and lymphatic glands; alveolar cells of the lung; endothelial cells of the serous cavities and lymph-spaces; bonecorpuscles and giant-cells of bone-marrow and embryonic connectivetissue cells (Figs. 44 and 45).

The most important are the leukocytes, especially the polynuclear leukocytes and the large lymphocytes of the blood. All the leukocytes, however, have phagocytic powers, as is well seen in opsonic determinations. Eosinophiles are seldom known to ingest bacteria, but in infections with animal parasites, or after the injection of extracts of animal parasites, both a local and a general increase in eosinophilous forms may be observed.

Small lymphocytes are much less active than the large, presumably because they contain less of the mobile cytoplasm, and consist chiefly of the structurally fixed nuclear substance, and while they take up but a small number of bacteria, they may be observed to contain various other cells, such as red corpuscles and cellular débris.

Besides the leukocytes, some of the tissue-cells which are free or have the power of becoming so are actively phagocytic. Endothelial 
cells of the lymph-spaces and serous cavities are especially active, not only in the phagocytosis of other cells and cellular débris, but also of various bacteria. In exceptional instances epithelial cells may act as phagocytes. In the presence of an irritant these cells may become detached and act as phagocytes; this is exemplified in the case of chronic passive congestion of the lungs, in which the alveolar cells of the lung ingest the hemosiderin formed and deposited (heart failure cells).

Relation of the Cell Types to Infection.-The kind of cells that take part in phagocytosis is determined to some extent by the nature of the irritant. Thus in acute pyogenic infections the polynuclear cell is found to be most active. (See Fig. 46.) It is extremely rare to find these cells containing bacilli in the tissues, although they will take them up readily enough under the artificial conditions of an opsonic determination. (See Fig. 53.) In chronic bacterial infection, such as tuberculosis and syphilis, and in infections with various fungi, the small lymphocyte and macrophages are the types most concerned.

Experimental evidence regarding lymphocytic activity is quite contradictory. Undoubtedly many of the cells in the lymphocytic accumulations seen in such conditions as tuberculosis and syphilis are not really lymphocytes from the blood, but are newly formed cells of the tissues. There is no direct means of inducing experimentally a local accumulation of lymphocytes similar to that induced by amost any irritant, resulting in an outpouring of polynuclear cells. Long-continued intoxication of animals may result in increasing the numbers of lymphocytes, but the local introduction of the toxin leads to an accumulation of polynuclear cells, rather than lymphocytes. Reckzeli ${ }^{1}$ found that in lymphatic leukemia, in which the lymphocytes greatly exceed the polynuclears, the pus from an acute lesion or the fluid from the vesicles produced by cantharides, contained practically no lymphocytes, but was composed of the usual polynuclear cell forms. Wlassow and Sepp ${ }^{2}$ state that lymphocytes are not capable of ameboid movement or phagocytosis at ordinary body temperature; Wolff, ${ }^{3}$ on the other hand, claims that tetanus and diphtheria toxins produce lymphocytosis in experimental animals, and Zieler ${ }^{4}$ claims that in the skin of rabbits exposed to the Finsen light active migration of lymphocytes takes place during the reaction. General lymphocytosis may be produced experimentally by the injection of pilocarpin and muscarin, but these bear no relation to the vital process of phagocytosis, as they are apparently ex-

1 Zeit. f. klin. Med., 1903, 50, 51.

${ }^{3}$ Berl. klin. Woch., 1904, 41, 1273.
2 Virchow's Archives, 1904, 176, 185.

4 Central.f. Pathol., 1907, 18, 289. 

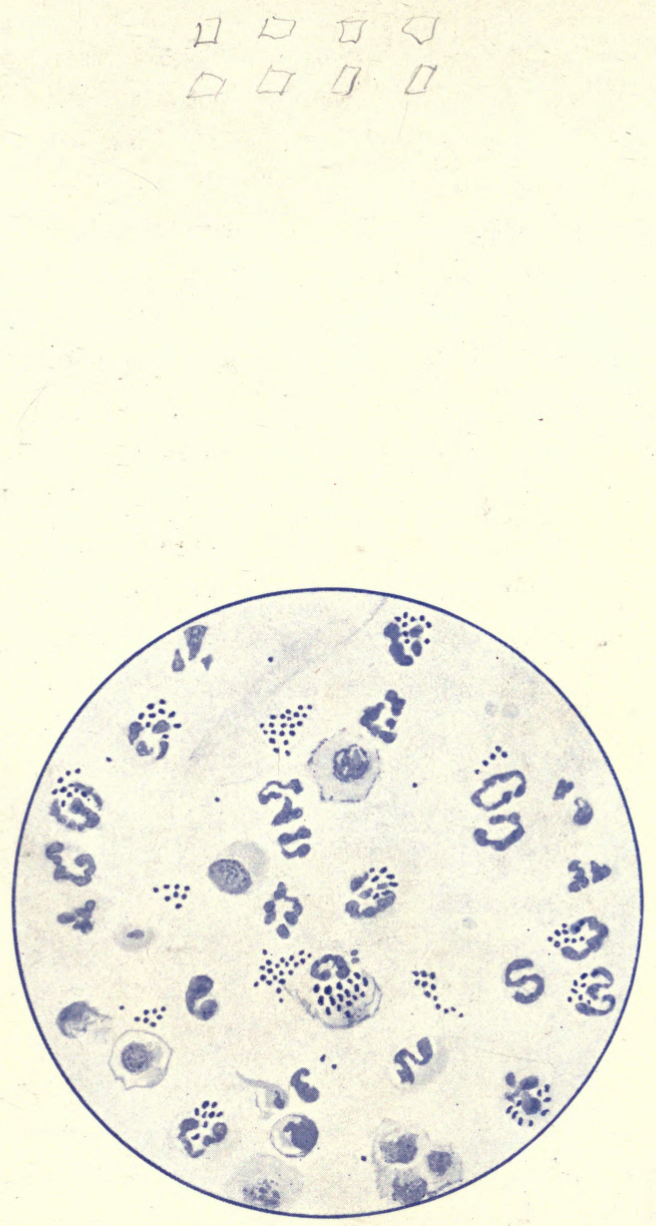

Fig. 46.-Positive Chemotaxis. Phagocytosis of Staphylococci. 

truded from the lymphoid organs by contraction of the smooth muscles $\left(\right.$ Harvey $^{1}$ ).

As previously stated, the eosinophiles undergo a marked increase during infections with various animal parasites.

The typical macrophages, such as the endothelial cells of serous cavities (Figs. 44 and 45) and the lymph-spaces, are mostly concerned in the phagocytosis of other cells and inorganic material. Brodie $^{2}$ considers the phagocytosis of leukocytes and red corpuscles by the endothelial cells of the lymph-glands and the spleen as the normal end of these cells. It is a mistake to believe, however, that they do not ingest bacteria, since endothelial cells are extremely active phagocytically for bacteria, and, on the other hand, polynuclear leukocytes may be observed to contain red corpuscles, especially when aided by a suitable opsonin.

\section{CHEMOTAXIS}

An important question in the study of the phenomena of phagocytosis is the manner in which the various leukocytes and other bodycells are attracted to a focus of infection and brought into contact with the microparasites or other foreign substances. It must be assumed that some means of communication must exist between this point and the leukocytes in the circulating blood. Since there is no direct communication by way of the nervous system or other structural route, it would appear that the only mode of communication is through the bodyfluids. Chemical agencies, produced either directly by the bacteria or other foreign substarice, or indirectly by their action upon cells at the site of residence in the tissues, are regarded as furnishing the attractive forces that are transmitted through the body-fluids and exert what has been called chemotaxis.

The movement of a cell in response to a chemical stimulus is a phenomenon that is displayed by almost all motile and unicellular organisms, whether animal or vegetable, and by the leukocytes and other unfixed cells of the higher animals. As a rule, chemical stimuli serve to attract cells to the site of infection, thus constituting what is known as positive chemotaxis; on the other hand, the stimuli may fail to attract or actually repel the cells, or be so powerful as to paralyze them en route, this constituting negative chemotaxis.

Positive Chemotaxis.-That leukocytes reach the site of an infection because of chemical substances produced by bacteria at this point

${ }^{1}$ Jour. Physiol., 1906, 35, $115 . \quad 2$ Jour. Anat. and Physiol., 1901, 35, 142. 
was first clearly demonstrated by Leber ${ }^{1}$ in 1879 . This writer observed that in keratitis leukocytes invaded the avascular cornea from the distant vessels, not in an irregular manner, but direct to the point of infection, where they accumulated. As dead cultures of staphylococci produced a similar although a less pronounced accumulation of leukocytes, Leber sought the chemotactic substance in their bodies, and isolated a crystalline, heat-resisting substance-phogosinwhich attracted leukocytes in the tissues.

Since these fundamental studies were made many other investigations, with various chemical substances of many different origins, have been undertaken upon leukocytes, amebæ, ciliata, and plasmodial forms, indicating that chemical substances are mainly concerned in exerting either a positive or a negative chemotactic influence.

Experimental evidence tends to show that cells respond to stimuli of various kinds chiefly through the effect of these stimuli upon surface tension: if they decrease the surface tension, the cell goes forward; if they increase the tension, the cell recedes.

The behavior of leukocytes in inflammation may be explained on these purely physical grounds. At the site of cell injury or infection chemical substances are produced that tend to lower the surface tension of leukocytes and thus exert a positive chemotactic influence. These chemical stimuli are transmitted by the body-fluids to the nearest capillaries, where they enter through the vessel-wall and come in relation with the slowly moving peripheral leukocytes. The leukocytes will be brought into touch by the chemotactic substances most largely on the side from which the substances diffuse; accordingly, the surface tension being least nearest the stomata in the capillary wall, this results in the formation of pseudopodia, and motion in this direction, dragging the nucleus along in an apparently passive manner. Those cells, therefore, containing most of the mobile cytoplasm, such as the polynuclear leukocytes, are chiefly affected in these processes; those containing little cytoplasm and a relatively large and dense nucleus, such as the lymphocytes, are affected primarily to a much less extent.

Once outside of the vessel-wall, the leukocytes tend to move toward the focus from which the chemotactic substance comes. If the leukocyte meets a substance that greatly lowers its surface tension, it will flow around the object and inclose it, this constituting phagocytosis. The toxins of the ingested bacteria may kill the cell, or so equalize surface tension that movement ceases. Otherwise the leukocytes tend to move

${ }^{1}$ Fortschritte der Med., 1888, 6, 460. 
forward until checked by any one of several influences, as pointed out by Wells, - as (1) Until the chemotactic substance has been used up or removed, or from any of the causes that terminate inflammation; (2) the leukocytes may reach a point where the chemical stimulant is so generally diffused that surface tension is decreased equally in all directions and motility stops; (3) the leukocytes may reach a place where toxins or other chemical substances coagulate their cytoplasm or ferments cause their solution; (4) they may be blocked by a dense wall of leukocytes and other cells while being held fixed by the chemical attraction that diffuses through this wall. These factors would explain the formation of the wall of leukocytes about an area of infection. When, for example, the abscess has ruptured or has been incised, with removal of the chemotactic substances, there may be less chemotactic substances in the center of the inflamed area than there is further out; hence the leukocytes will move away from the center toward the periphery, following the chemotactic substances back into the blood-vessel and lymphstream. This would explain the dispersion of living leukocytes at the close of an inflammatory process.

General leukocytosis can be explained equally well by assuming that the chemotactic substances from the area of inflammation, reaching the blood-stream, pass through the bone-marrow, lowering surface tension and attracting leukocytes into the blood-stream as long as it contains more chemotactic substances than the marrow.

The exact chemical nature of chemotactic substances is unknown. In bacterial infection the toxins, and especially the protein of dead microorganisms, are regarded as mainly responsible for the occurrence of positive chemotaxis. Chemotaxis and phagocytosis of chemically inert particles, such as coal-dust, stone-dust, and pigments, are more difficult to explain on this physical basis of alteration in surface tension. It is probable that the death of tissue-cells, brought about by these materials, may produce the chemical stimulant responsible for a mild but definite chemotactic influence. Although the movement of amebæ and similar higher animals cannot be fully explained on this physical basis, the surface tension theory best explains leukocytic movement. Although the ameba may possess some special property that endows it with the power of selecting and engulfing a food particle, it would appear to be entirely unreasonable to assume that a simple, undifferentiated, and naked leukocyte possesses similar powers. The physical theory, therefore, appears to be the most reasonable offered in explanation of the ameboid movements of these simple cells. 
Negative Chemotaxis.-In nearly all infections we find that leukocytes are attracted in large numbers into the involved area, $i . e$. , nearly all bacteria give off substances that are positively chemotactic. In certain infections, however, we may find the tissues poor in leukocytes, as exemplified in infections due to the presence of virulent streptococci. This negative chemotaxis is more difficult of explanation. Kantlack doubts the existence of really negative chemotactic action upon leukocytes. Verigo ${ }^{1}$ also considers that as yet no actual negative chemotactic substances have been satisfactorily demonstrated; certainly no marked example of negative chemotaxis has been shown since methods involving the study of phagocytosis in vitro have been devised. It is true that virulent bacteria appear to repel the leukocytes, but, as Kantlack has pointed out, these are not necessarily examples of negative chemotaxis, and it is probable that the paucity in numbers of the leukocytes about such an area of inflammation is due to their overstimulation or paralysis and destruction of the powerful ferments that are given off by the bacteria. Thus Metchnikoff has asserted that leukocytes might, after a time, be attracted toward substances that would kill them. Therefore, while leukocytes will migrate freely toward substances that would kill them, they may be destroyed before they reach the inflammatory area, or, having reached there, are promptly destroyed and pass into solution (Fig. 47).

While it is doubtful, therefore, whether substances are produced by bacteria that actually repel leukocytes, the point has not been definitely settled. If such substances exist, it would appear that they are closely identified with either the endotoxins or the aggressins, the latter being definite secretory products of bacteria that neutralize opsonins and retard phagocytosis. In many instances it is probable that the same substances that exert a positive chemotaxis are, when concentrated, negatively chemotactic, through overstimulation and paralysis of the leukocytes. With diminution in the numbers or vitality of bacteria and dilution of their chemotactic substances, this inhibiting influence is removed and the leukocytes are attracted to the focus of infection, thus explaining in a way those instances in which positive chemotaxis is observed to follow a primary period of negative chemotaxis.

\section{RESULTS OF PHAGOCYTOSIS}

After phagocytosis has been accomplished, the fate of the engulfed objects depends upon their nature. In general they undergo a process

1 Arch. d. med. Exper., 1901, 13, 585. 


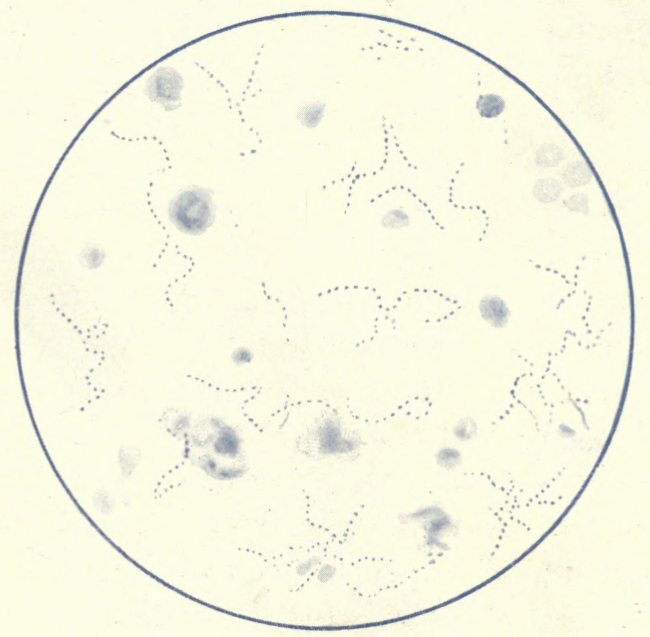

Fig. 47.-Negative Chemotaxis.

A smear of exudate from the peritoneal cavity of a guinea-pig twenty-four hours after injection with virulent streptococci. The exudate was thin, serous, and tinged with hemoglobin. Note the large numbers of streptococci and relatively few leukocytes. 
of digestion. The ameba, for example, is able to kill and digest engulfed material through an intracellular ferment regarded as a form of trypsin, demonstrated by Mouton $^{1}$ and called amebadiastase. According to Metchnikoff, the digestion of erythrocytes and tissue fragments is accomplished through an enzyme of the macrophages called macrocytase; that of bacteria or other substances engulfed by microphages by a similar enzyme called microcytase.

Following the general law that living protoplasm cannot be digested, we are confronted with the very important question as to whether living bacteria are engulfed by phagocytes or whether they are first destroyed by extracellular agencies before they undergo phagocytosis.

Endolysins. - It seems positively established at the present time that leukocytes do take up living bacteria, which may either grow inside the leukocyte or be destroyed by intracellular substances called endolysins. On the other hand, leukocytes do not take up extremely virulent bacteria, hence the question arises as to the importance of substances in the body-fluids which neutralize the repelling substances of bacteria and facilitate phagocytosis. This subject, which has considerably modified Metchnikoff's views of phagocytosis, will be considered in a succeeding chapter. It will suffice here to state that leukocytes may engulf living bacteria possessing some virulence, for not infrequently an infection may be spread by bacteria transported into deeper tissues by phagocytes, when they resist the germicidal activity of the endolysins, bring about the death of the phagocyte, and are thus liberated into new tissues.

Death of the engulfed bacteria is, therefore, brought about by endolysins $^{2}$ that are probably different from the digestive enzymes. These substances are strongly bactericidal, and have a complex structure resembling bacteriolysins. According to Weil, ${ }^{3}$ they are not specific. They are resistant to $65^{\circ} \mathrm{C}$. or even higher, do not readily pass through porcelain filters, are precipitated by saturation with ammonium sulphate, and resemble the enzymes in many respects (Manwaring ${ }^{4}$ ). It is probable that the endolysins act not only upon bacteria that have been phagocytosed, but also upon free bacteria when liberated through disintegration of the leukocytes. In this manner the endolysins would closely resemble the normal opsonins or bacteriolysins, and support the contention of Metchnikoff that these important substances contained

${ }^{1}$ Compt. rend. de l'Acad. Sci. de Paris, 1901, exxxiii, 244.

${ }^{2}$ For general review, see Kling: Zeit. Immunitäte, 1910, 7, 1.

${ }^{3}$ Arch. f. Hyg., 1911, 74, 289.

${ }^{4}$ Jour. Exper. Med., 1912, 16, 250. 
in the body-fluids are derived primarily from the cells which he has classed as phagocytes. According to Schneider, ${ }^{1}$ lymphocytes and macrophages seem to contain little or no endolysin, and these cells are not so active in the phagocytosis of virulent bacteria as are the microphages.

Indigestible substances, if chemically inert, may remain in cells, particularly in fixed tissue-cells, for variable periods of time. The leukocytes seem to transfer such particles to other tissues, particularly to the lymph-glands. It is probable that these phagocytes are in turn engulfed by the endothelial cells. Macrophages of the lymph-sinuses or the leukocytes may be destroyed in the glands, and their contents rephagocyted by these cells. In just what manner these insoluble particles reach the gland stroma or perilymphangeal tissues is unknown; it is probable that they are liberated from the lining endothelial cells, and are again seized by the young connective-tissue cells.

\section{THE RELATION OF THE BODY-FLUIDS TO PHAGOCYTOSIS}

Important and far-reaching as were Metchnikoff's researches and conclusions, they were not allowed to pass unchallenged, especially by the adherents of the humoral school, who were able to show the potent influences of the body-fluids in the mechanism of recovery from infections where phagocytosis. was little in evidence, or, indeed, where phagocytosis was impossible. It was shown that Metchnikoff's original theory was untenable, and that the leukocyte is almost impotent if removed from the influence of the body-fluids.

As demonstrated by Denys, Leclef, Flügge, Nuttall, Pfeiffer, and others, bacteria may be killed, $i$. e., may undergo a process of lysis or disintegration, by means of substances in the blood-serum entirely independent of phagocytosis Later researches by Wright, Neufeld, and their coworkers demonstrated most clearly that even in those infections in which phagocytosis was observed and known to be of great importance, the bacteria are first prepared for phagocytosis by substances in the body-fluids, and that without this preliminary preparation of the bacteria phagocytosis was slight and of little consequence.

Metchnikoff corroborated most of these discoveries, and modified his theory from time to time to meet the developments and keep them within the limits of the phagocytic theory. For example, when bacteriolysis was shown by Bordet to be due to two separate substances in

$$
1 \text { Arch. f. Hyg., 1909, 70, } 40 .
$$


the body-fluids, which he called substance sensibilisatrice and alexin (later renamed by Ehrlich amboceptor and complement respectively), Metchnikoff claimed that this phenomenon was extracellular digestion, similar to the intracellular digestion that occurs within the phagocyte, and brought about by ferments secreted and liberated from leukocytes or other cells classed as phagocytes. He regards alexin as a cytase secreted by leukocytes, or liberated upon their disintegration; similarly the substance sensibilisatrice is regarded as a free ferment (fixateur), derived principally from leukocytes, and concerned in preparing the bacterial or other cell for the digestive-like action of the cytase.

Aside from these free ferments that are capable of producing extracellular lysis, Metchnikoff has long known that other substances that aid phagocytosis itself may be present in the body-fluids; he regards these as of the nature of stimulins, or substances that stimulate leukocytes to become more actively phagocytic. On the other hand, Leishman, Wright and Douglas, Neufeld, and Rimpau, Hektoen, and others have clearly demonstrated that they facilitate phagocytosis, not by stimulating the leukocytes, but rather by lowering the resistance of bacteria or in some way rendering them more vulnerable to phagocytosis (opsonins, bacteriotropins).

Thus the gap between the original cellular theory, which ascribed protection and cure to phagocytosis pure and simple, and the humoral theory (finally summed up by Ehrlich in his side-chain theory), which ascribed the chief and primary rôles to substances in the body-fluids, and relegated phagocytes to a position of secondary importance, regarding them only as scavengers that remove dead or disabled microorganisms, has been filled with discoveries correlating both processes.

The vitality of the leukocyte is to be regarded as important in the consideration of phagocytosis as a means of defense. While the bodyfluids are acting upon the invaders, the leukocytes themselves are probably undergoing quantitative and qualitative changes. They are increasing in numbers, and, as Rosenow has shown, are undergoing more specific changes. Thus, for instance, the leukocytes from a pneumonia patient were found more vigorous against invasion of the pneumococcus than are those from a normal person, regardless of the influence of serum.

When a microparasite is ingested, the process has only begun. Unless suitable endolysins are present and the endotoxin is absorbed or otherwise dealt with, and unless suitable digestive enzymes are secreted and the bacterium is dissolved, the process is useless, or, indeed, if viable bacteria are transported to other parts of the body, it may be dangerous. 


\section{THE REVISED THEORY OF PHAGOCYTOSIS}

As previously stated, Metchnikoff has revised his theory from time to time, as these discoveries were made on the influence of substances in the body-fluids, not only upon phagocytosis itself, but also upon the processes of immunity in general. He would regard extracellular cytolysis (bacteriolysis, hemolysis, etc.) as due to the same ferments that bring about the destruction and solution of the ingested bacterium or other cell within the phagocyte, and, further, these extracellular ferments are derived from the cells that are classed as phagocytes. By this method of reasoning he would preserve the importance of the phagocytic theory.

In local infections phagocytosis is usually well marked, and no doubt plays an important part in resistance to and recovery from these conditions. In general infections, however, as in bacteremias due to staphylococci, streptococci, and particularly the typhoid and allied bacilli, it is rare indeed to find evidence of a leukocyte in the bloodstream becoming phagocytic. Here extracellular substances or antibodies are chiefly operative in affording protection or in overcoming infection. Even in local infections, where phagocytosis along the more simple lines originally described by Metchnikoff is well marked, the resistance of the bacteria is first attenuated or modified by the opsonins of the body-fluids before the phagocytic process becomes well marked.

The question, then, of the relative importance of the cellular and humoral theories of immunity resolves itself to a consideration of the origin of the substances in the body-fluids so potent in both processes. If they are derived solely from the cells known to act as phagocytes, then the cellular theory of phagocytosis, in its broader meaning, is the one explanation of the processes of immunity as they are now understood. This, however, has never been proved, and it is entirely likely that these substances are products of a general, rather than of a more restricted, cellular activity, so that ultimately all immunologic processes are cellular in origin. For this reason we prefer to speak of the phagocytic cell in its relation to immunity when dealing with the relation and activity of microphages and macrophages in a limited sense in the process of phagocytosis. 


\section{CHAPTER XI}

\section{OPSONINS}

Historic.-Although there can be no doubt as to the importance of phagocytosis in the mechanism of recovery from infection, yet it was shown by Metchnikoff, as early as 1893, that the body-fluids contained substances that greatly facilitated the phagocytic process, and that leukocytes removed from this influence were practically powerless to engulf and destroy the invading bacterium. In other words, if leukocytes and bacteria are washed free from all traces of serum and then mixed, very few of the leukocytes will be found capable of phagocytizing the bacteria, which means that spontaneous phagocytosis is feeble and hence of slight importance. When, however, fresh serum is added, especially the serum of an animal immunized against the microörganism used in the experiment, phagocytosis is marked, and, indeed, most impressive. Metchnikoff attributed this difference to the influence of a substance in the serum that stimulated (stimulins) the leukocytes to become phagocytes, but later researches have shown that this is probably erroneous, and that the serum facilitates phagocytosis not by exerting a stimulating influence upon the leukocytes, but by preparing the bacteria for the process by making them, as it were, more attractive to the leukocytes.

Denys and Leclef, in 1895, were among the first to demonstrate the effect of serum on bacteria in the process of phagocytosis, and the fact that the active substance was not bactericidal in action, but in the nature of a new antibody. Since Metchnikoff had shown that freshly isolated or virulent strains of bacteria were not readily phagocytized, but seemed to resist or repel the leukocytes, it was natural for these observers to suggest that the action of this substance in serum was to neutralize the exotoxins and endotoxins of microörganisms that were regarded as responsible for negative chemotactic influences, and thus, by robbing them of at least two defensive weapons, prepare them for phagocytosis.

The subject remained in an uncertain state until 1903, when Wright, and later Wright and Douglas, demonstrated more clearly this action of serum upon bacteria in aiding phagocytosis. Using their own modification of the technic devised by Leishman for measuring the phagocytic 
power of the blood, these observers first determined the direct dependence of phagocytosis upon some ingredient of the blood-serum, and further proved that this substance acts directly upon bacteria, is bound by the bacteria, and renders them more easily ingested by the leukocytes, i. e., more readily phagocytable. To this substance they gave the name opsonin (from opsono, I prepare food for). At the same time, and independently of Wright, Neufeld and Rimpau conducted similar investigations with immune serum and reached similar conclusions, but called the substance bacteriotropin. Since then both terms have been used,the former more frequently in English literature,-and this is permissible, providing that it is understood that both are practically the same antibody, and not distinct and separate from each other.

As will readily be understood, the bacterial opsonins have been studied most extensively, but opsonins may be present in normal and immune serums for other cells, such as erythrocytes, and these hemopsonins are regarded as separate antibodies, distinct from hemagglutinins and hemolysins.

Definition.-Opsonins are substances in normal and immune serums which act upon bacteria and other cells in such a manner as to prepare them for more ready ingestion by the phagocytes.

Properties and Nature of Opsonins.-There is considerable difference of opinion regarding the identity and probable structure of opsonins in normal and immune serums. Just as agglutinin for a bacterium, such as Bacillus typhosus, may be found in varying amounts in normal serum, so various opsonins for different bacteria may be found in normal serums. These normal opsonins appear more or less specific for a given bacterium, and in immune serum the specific opsonic substance for the particular bacterium or cell with which immunization has been produced is developed to a high degree. Both owe their full effect to the interaction of two substances. One of these, the common substance, is thermolabile, and destroyed by heating the serum to from $56^{\circ}$ to $58^{\circ} \mathrm{C}$. for half an hour, whereas the other more specific substance remains unaffected. The latter, in both normal and immune serums, is opsonic by itself, although in the absence of the common thermolabile substance, to a less degree, and is produced anew and specifically by artificial immunization or as the outcome of spontaneous infections.

The true nature of opsonins is, therefore, difficult to understand. They have been compared to receptors of the second order, with a haptophore and a toxophore or opsoniferous group. Receptors of this order, however, are active and independent of the presence or absence 
of complement, whereas the opsonins, although active to some extent in the absence of complement, are far more so if a complement is present. In this respect they resemble amboceptors, or receptors of the third order, opsonins in normal and immune serums representing respectively normal and immune bacterial amboceptors. One objection to this view of their structure is their activity, however slight, when the thermolabile substance is removed by heating, unless the amboceptors are complemented by an endocomplement, as from the bacteria themselves.

At the present time, therefore, not a few observers doubt that opsonins exist as true and separate antibodies, and are inclined to regard thermolabile opsonin (largely the opsonin in fresh normal serum) as a complement, and thermostabile opsonin (largely immune opsonin or bacteriotropin) as an amboceptor; it would appear that either alone, but more especially the latter, may facilitate phagocytosis to some extent. This process is, however, much more marked when both substances are acting in unison. While it is true that the bacteriolysin and opsonin content of a serum do not run parallel, our methods for measuring these are not entirely satisfactory; both intracellular and extracellular lysis may be mere differences in degree, depending upon the nature of the bacterium or the concentration of the antibodies, rather than upon separate and distinct antibodies.

Source of Opsonins.-Little is definitely known regarding the source of opsonin. Thermostabile opsonin-that which is increased by artificial immunization or during disease, and is largely in the nature of an amboceptor-is probably a product of general cellular activity, and especially of the local cells at the site of infection. Thermolabile opsonin - largely the opsonin occurring in normal serum, and in the nature of a complement-is probably a product of the leukocytes and other cells as well, as it has never been proved that the leukocytes are the sole source of the complements, as Metchnikoff would have us believe.

Susceptibility to Opsonification.-As previously stated in the chapter on infection, not all bacteria are equally susceptible to opsonification. As a general rule, recently isolated and virulent microörganisms resist the influence of opsonins until they have undergone culture several times. This resistance may be due to capsule formation, thickening of the ectoplasm, actual self-immunization of the bacterium, or the influence of endotoxins as a protective means against the antibodies of a host, all of these being weakened or lost upon artificial culture-media.

Effect of Opsonins on Bacteria.-We know nothing definite regarding the manner in which opsonins prepare bacteria for phagocytosis 
except that opsonification in itself apparently does not impair the vitality of the bacterium, in so far, at least, its viability is concerned.

Rôle of Opsonins in Immunity.-Although the exact identity of normal and immune opsonins and their relation to other antibodies is as yet unsettled, the important relation they bear to processes of immunity is generally recognized, especially their ability in aiding resistance to infection by facilitating phagocytosis. They are operative in some infections more than in others, and they are especially active in those conditions in which phagocytosis is recognized as the chief defensive force, as, for example, in pyogenic infections. In these conditions their presence has been taken as a measure (opsonic index of the resistance of the host) and, largely through the researches of Wright and Douglas, a technic for detecting their presence, kind, and quantity in the bodyfluids has been devised, the method and information it yields being of value under certain limitations and in some infections. (See next chapter.)

If experiments in vitro may be taken as an example of what occurs in vivo, it must be true that leukocytes are capable of consuming an enormous number of bacteria. Experiments with washed leukocytes - those removed from the influence of serum-show that spontaneous phagocytosis is very slight. Metchnikoff declared these experiments to be untrustworthy for the reason that the various manipulations of washing injures the vitality of the leukocytes. When, however, bacteria are opsonized, that is, are exposed to a serum containing opsonins, and then are thoroughly washed, it is found that the washed leukocytes engulf enormous numbers of bacteria, showing that Metchnikoff's objection to these experiments is unwarranted. Granting, then, that what we call opsonins are substances that facilitate phagocytosis, and that phagocytosis is a process of great importance, especially in certain infections, we must conclude that opsonins play a very important rôle in immunity; in fact, they constitute the very basis of the phenomenon of phagocytosis in the broader meaning of the term. 


\section{CHAPTER XII OPSONIC INDEX}

WHETHER opsonins are regarded as separate antibodies or as being identical with complements and amboceptors, a measure of their quantity and power may be of aid in formulating a diagnosis, as a guide to active immunization, and as one means of determining the potency of various immune serums used for therapeutic purposes, such as antimeningococcus and antipneumococcus serums. We are mainly indebted to Leishman, Wright and Douglas, Neufeld and Rimpau, and their coworkers for devising a technic that, however imperfect it may be according to the results obtained, has opened a new and important field for the study of immunologic processes.

Principle.-This is based upon the method devised by Wright and Douglas, whereby it was sought to determine the amount and kind of opsonin in a patient's serum by comparing the degree of phagocytosis with that occurring when normal serum was used.

Definition.-The opsonic index is the ratio of the number of bacteria ingested by a given number of phagocytes in the presence of a patient's serum, to the number ingested by the same number of phagocytes in the presence of normal serum.

"An equal volume of the patient's serum, measured in a capillary pipet, is mixed with an equal volume of a suspension of washed leukocytes derived from a normal blood. After this 'phagocytic mixture' has been digested for a suitable period at $37^{\circ} \mathrm{C}$., film preparations are made and stained.

"A 'phagocytic count' is then undertaken, i.e., the average bacterial ingest of the leukocytes in the phagocytic mixture is determined, and this is compared with the average ingest of the leukocytes in a phagocytic mixture made with normal blood.

"The expression thus obtained,

Average ingest of the individual phagocyte in the mixture containing the patient's serum.

Average ingest of the individual phagocyte in the mixture containing normal serum is denoted the opsonic index" (Wright). 
Purpose of the Method.-The opsonic index aims to serve as a guide:

1. In diagnosing the presence of bacterial infection, or rather in discovering whether the natural protective powers of the patient's blood have been diminished or increased as the result of the immunizing influence of the infection.

2. In connection with vaccine therapy, to guard against diminishing the opsonin content of the patient's blood; to assure ourselves that our efforts to increase them have been successful, and occasionally to ascertain how long the store of opsonin that has been obtained for the patient remains in the blood.

Limitation of the Method.-In ascertaining the opsonic index of a patient's serum, we must take it for granted-although it has not been proved:

1. That the bacteria act the same in the body as they do in the testtube. This is known not to be the case, for virulent organisms resist phagocytosis, whereas a non-virulent strain of the same bacterium is easily phagocyted. If, therefore, a laboratory culture of attenuated organisms is used in making the opsonic index, the result can hardly be accepted as a criterion of the power of the patient to overcome the "resistant" or more virulent organism as it occurs in the body. This source of error can be overcome in a manner if the microörganism is isolated and used at once before attenuation occurs.

2. That the leukocytes are a constant factor, and need not be taken into account. Investigation has shown that, as a result of infection, the leukocytes probably undergo qualitative changes and it is hardly fair to accept phagocytosis by normal leukocytes as a criterion of phagocytosis with the patient's own leukocytes, as it occurs in the body during the infection.

3. The method assumes that phagocytosis by the polynuclear leukocyte plays a large part in overcoming the infection. In many cases, however, this is by no means proved. For example, in tuberculosis it is not this form of leukocyte, but the mononuclear form or the lymphocyte, which seems to be more important, and hence it is difficult to understand how the index with the polynuclear leukocyte can aid the question of diagnosis or treatment.

4. The chances for error are considerable. To be of any value, the work requires experience and painstaking care. The results obtained by competent workers with the same blood may show variation, but it must be said that, with strict attention to technic and insistence upon perfect preparations, the worker may usually obtain valuable results. 
An index taken at one time by one person and later by another, and so on, will not be of as much value as when all are taken by the same worker, who brings practice, skill, and conscientious care to his aid.

Precautions in Technic.-1. Proper controls should be used. When dealing with the tubercle bacillus, the staphylococcus, or any other saprophyte of the external surfaces, or with any pathogenic organism with which we have normally no relations, the serum of a normal individual or the mixed serum of a number of normal persons will furnish the standard of comparison. When, on the other hand, we are dealing with intestinal bacteria or with a saprophyte of the mucous membrane, where, as a rule, any relation with them will be denied, it is difficult to establish a standard of health. Pooled serum is, therefore, necessary, and will furnish a standard for comparison for the purpose of measuring the fluctuations that may occur in the patient's blood.

2. A reasonable degree of phagocytosis should occur in the control serum. This is one of the main drawbacks to the value of the method for certain pathogenic organisms, as the pneumococcus, meningococcus, streptococcus, etc., may resist phagocytosis in normal serum, and thereby show abnormally high indices with immune serum.

3. Efforts at spontaneous phagocytosis should be suppressed in order to measure more accurately the opsonin, as shown by the degree of phagocytosis independent of the inherent activities of the cell itself. Spontaneous phagocytosis can largely be overcome by using 1 per cent. solution of sodium citrate such as is used for the collection of leukocytes.

4. The ingest of a sufficiently large number of phagocytes should be counted. As a general rule, 100 cells should represent the minimum.

\section{TECHINIC}

The necessary constituents for making the test are as follows:

1. The patient's serum and normal serums to serve for the control.

2. A bacterial emulsion.

3. A suspension of washed human leukocytes in normal salt solution.

Collection of Patient's and Control Serum.-1. The blood is collected in a Wright capsule, as described in Chapter II.

2. Care must be taken not to heat the blood when sealing the tube. In drawing off the serum, avoid an admixture of corpuscles, as these may lower the opsonic index.

3. If coagulation is incomplete or the serum has not been well separated, the clot may be broken up gently with a platinum wire and the 
tubes centrifuged. Slight discoloration of the serum from mechanically breaking-up a few erythrocytes will not interfere with the test.

4. If gross contamination is avoided, blood may be kept for one or two days in a dark place without much deterioration of its opsonin content.

5. It is always well to collect the control bloods at the same time the patient's blood is taken, or, if this cannot be done, to place them in an ice-chest as soon after collection as possible. When conducting the test, the control serums are pooled and mixed in a clean watch-glass.

The Bacterial Emulsion.-1. This must be perfectly uniform, free from clumps, and must not undergo agglutination, either spontaneous or with the serum to be tested.

With many bacteria, especially the motile ones, such as Bacillus coli and Bacillus typhosus, it is comparatively easy to secure a uniform emulsion. Staphylococci, streptococei, and pneumococci, as a rule, present no difficulties. After growing the culture for from eighteen to twenty-four hours on slants of a suitable medium, add three cubic centimeters of sterile 1 per cent. salt solution, and gently remove the bacterial growth with a platinum loop. The mixture is then pipeted into a separate flask or thick glass test-tube containing glass beads, and shaken by machine or by hand until it is thoroughly emulsified. If necessary, the emulsion may be centrifugalized to remove clumps and is then ready for use.

2. It must consist of bacteria that stain evenly and well.

Only young cultures should be used; for example, an eighteen to twenty-four-hour culture of freely growing organisms and a seven days' growth of tubercle bacillus.

3. It must be of such strength as to give a leukocytic ingest that will enable adequate differentiation to be made of the opsonin content of the various serums.

An emulsion that does not yield a count of at least 250 bacteria within 100 leukocytes is too weak to yield a satisfactory differential count. If the emulsion is too thick, bacteria overlie the leukocytes and introduce error. As a general rule, a suspension containing 500,000,000 bacteria per cubic centimeter is satisfactory. Experience will teach the right density to be used, and frequent trials may be necessary before the right one is secured.

4. The bacteria must be such as will not prove seriously dangerous. In order to obviate any danger attending work with such organisms as the glanders and the tubercle bacillus, first kill the culture by pouring. 
on a 10 to 40 per cent. solution of formalin, mix the culture, shake, transfer to a centrifuge tube, and centrifugalize until the bacteria have been carried to the bottom of the tube. Pipet off the supernatant formalin, wash in normal salt solution, centrifuge, pipet off again, and finally mix the sediment in sufficient salt solution to make a satisfactory suspension.

The Washed Leukocytes. - These should consist mainly of the polynuclear leukocytes of a healthy person washed free from any admixture with serum. As usually obtained, the leukocytes are mixed with red corpuscles. It is necessary to collect blood for the leukocytic mixture from a person whose corpuscles are known to be insensitive to agglutination, as otherwise there is an undue lowering of the opsonic effect.

1. Place 4 c.c. of sterile 2 per cent. solution of sodium citrate in distilled water in sterile 10 c.c. centrifuge tubes.

2. Prick the finger, and add 1 c.c. of blood. Agitate well to insure thorough mixing.

3. Centrifuge at a sufficiently high speed to mix the red corpuscles and leukocytes at the bottom of the tube and avoid clumping of the leukocytes.

4. Draw off the supernatant fluid, add 5 c.c. of 1 per cent. salt solution, mix, and centrifuge.

5. Wash once more. Draw off the supernatant fluid.

6. Add sufficient salt solution to bring the total volume up to that of the blood originally taken-i. e., 1 c.c. Mix well.

The Test.-1. Prepare capillary pipets of approximately the same caliber. These are made by taking 6 -inch lengths of soft clean glass tubing having an external diameter of $\frac{5}{16}$ inch, heating them in the middle in the tip of the blow-pipe or the Bunsen flame until about $1 / 2$ inch length of tubing is quite soft. Remove from the flame, and by rapidly separating the two hands draw out the molten glass to a length of from 18 to 20 inches. After cooling, the eapillary thread is cut across with a small file, so that from 6 to 8 inches is left attached to each piece of tubing. The ends must be cut square, as ragged and uneven ends are difficult to handle. By means of a wax-pencil make a fine mark at a point about an inch from the free end of each capillary thread. This indicates the unit volume (Fig. 48).

2. Adjust a well-fitting rubber teat, and draw up the unit volume of blood-cells. A tiny bubble of air is now allowed to enter the thread, and then one volume of the bacterial emulsion is added; another air-bubble is allowed to enter, and finally one volume of serum, so that we have 


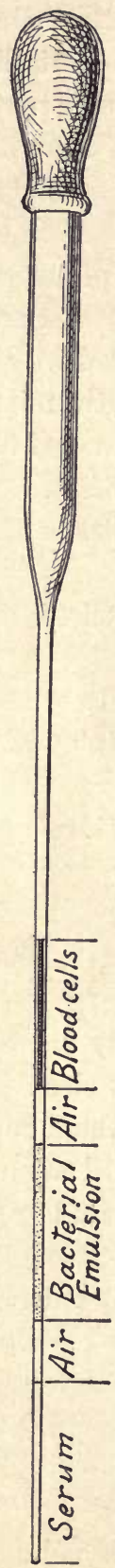

FIG. 48.-CAPILLARY PIPET FOR OPSONIC INDEX DETERMINATION. named in their order in the capillary tube from above downward, one volume of blood-cells, an air-bubble, one volume of bacterial emulsion, an air-bubble, and one volume of serum (Fig. 48).

3. By making gentle pressure on the teat these are then blown out on the surface of a clean glass slide, and perfect mixture effected by making alternate aspiration and expulsion from the capillary tube at least six times (Fig. 49).

4. Carefully reaspirate into the capillary thread, so that the mixture occupies about the middle, and seal the tip in a low Bunsen flame (Fig. 50).

5. Remove the teat, and with the wax-pencil mark the tube with the name or number of the serum.

6. A similar preparation is prepared with the pooled serum (control).

7. The phagocytic mixtures are then placed in an incubator at $37^{\circ} \mathrm{C}$. for fifteen minutes, except in the case of such bacteria as the Bacillus typhosus and the Bacillus coli, as lysin and agglutinin may be present in the serums of such bacteria when the period is reduced to ten minutes.

8. The tubes are then removed from the incubator, the teats readjusted, the tip of the capillary threads scratched with a file, and evenly broken off. The phagocytic mixture is carefully expelled on a clean glass slide, and a perfect mixture made by alternate aspiration and expulsion. Avoid airbubbles. The whole is then reaspirated, and a small drop of the mixture placed on each of two clean slides that have been roughened with emery paper about $3 / 4$ inch from one extremity. With the edge of a spreader slide held at an angle of about 30 degrees, and with moderate pressure, the drop is distributed evenly along about $1 \frac{1}{2}$ inches of the surface of the slide (Fig. 51). The smears are made in duplicate, because one may be more nearly perfect (Fig. 52) than the other, or one may be spoiled in the staining, when the second may be utilized. Each slide is then labeled at one end. 
9. After drying in the air, the slides must be fixed and stained.

(a) For Ordinary Bacteria.-1. Fix by covering the slide with a saturated aqueous solution of mercuric chlorid for one minute. Wash in water.

2. Cover with carbolthionin and stain for one or two minutes. Wash in water.

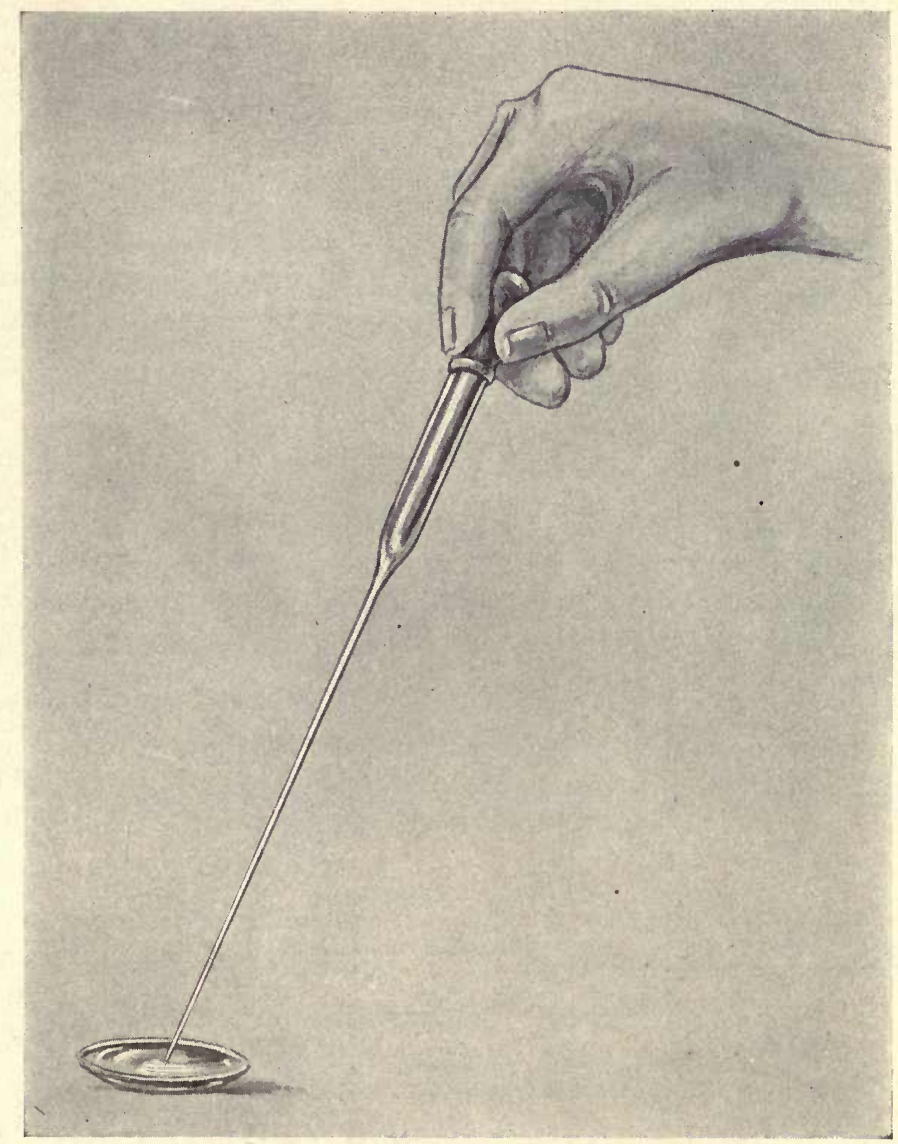

Fig. 49.--Mixing the Contents of a Capillary Pipet.

Due precautions must be exercised to avoid the formation of bubbles.

3. Dry thoroughly.

(b) For Acid-fast Bacilli.-1. Fix by inverting the films for thirty seconds over a watch-crystal or jar containing formalin, being careful that there are no drops of formalin on the edge of the vessel that might come in contact with the preparation. The films may be fixed also 
with a saturated solution of mercuric chlorid or with pure methyl alcohol for two minutes. Wash in water and dry.

2. Heat a portion of carbolfuchsin almost to boiling in a test-tube, and pour the hot stain over the films. Allow to remain for at least fifteen minutes. Wash under the tap and dry.

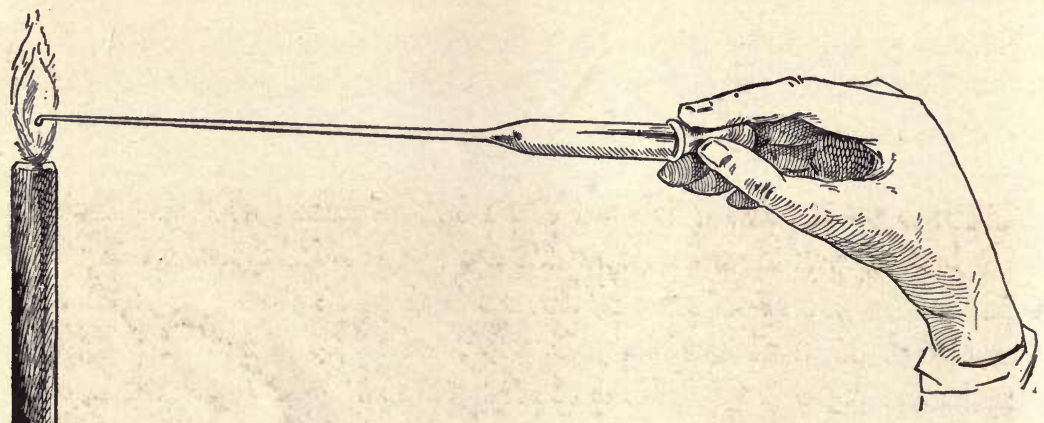

Fig. 50.-Method of Sealing a Capillary Pipet.

The tip of the pipet is placed in the edge of a flame. The teat is held in the same position until the tip has been sealed, when it may be removed without disturbing the contents of the pipet.

3. Cover with a 5 per cent. solution of nitric or sulphuric acid for half a minute or longer if necessary, until decolorization is complete. Wash thoroughly under the tap.

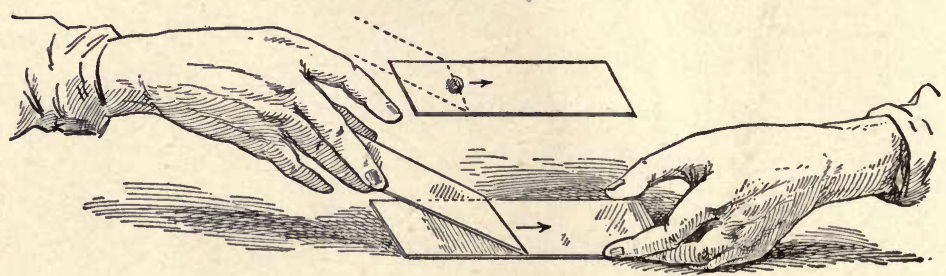

Fig. 51.-Method of Preparing a Blood Film.

The slide is laid on a flat surface; the drop of blood is placed near one end; the spreader is held between the thumb and middle finger of the left hand, at an angle of about 30 degrees, and quickly pushed to the opposite end of the slide.

4. Cover with 4 per cent. aqueous solution of acetic acid for one to two minutes to remove the hemoglobin from the red cells. Wash and blot lightly.

5. Cover with Löffler's methylene-blue for two minutes. Wash in water and dry thoroughly (Fig. 53). 
10. Examination of the stained films with the oil-immersion objective of the microscope will show that polynuclear leukocytes have collected more toward the edges and the end at which the spreading was completed. The individual leukocytes, however, should be separated from one another (Figs. 54 and 55).

11. The edge of the film is examined, and the number of bacteria found in each series of five consecutive phagocytes is noted. If the technic has been satisfactory, no great divergence should be found in the count of each set of five cells.

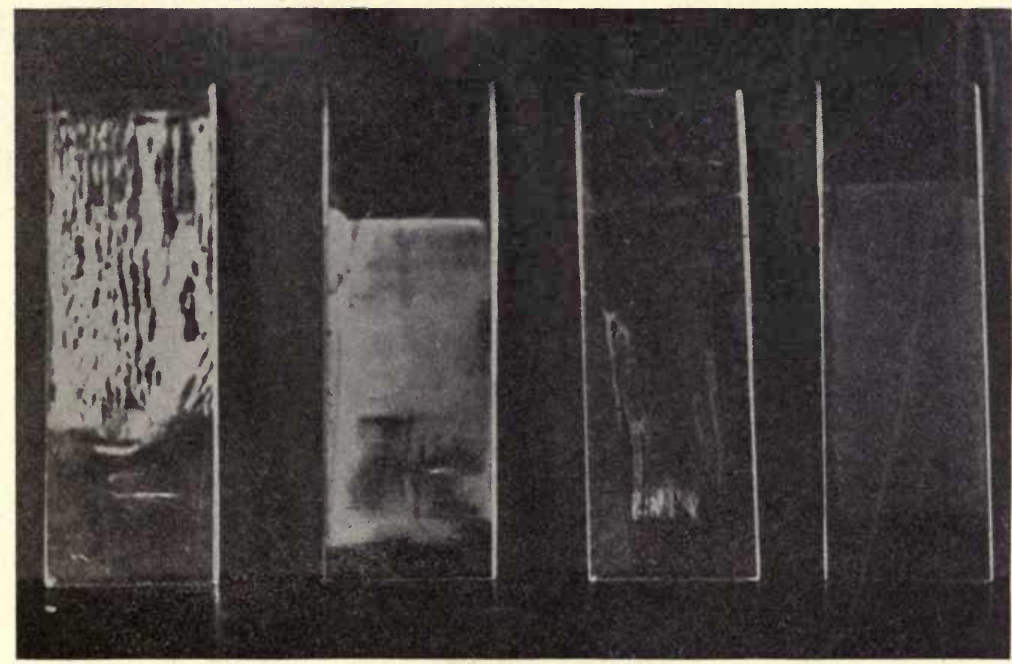

Fig. 52.-Blood Films for Phagocytic Counts.

The first slide (on the extreme left) is too thick and honeycombed, due to a greasy slide and large drop of blood; the second is likewise thick and uneven; the third is too thin, and was spread with too small an amount of blood and with the spreader held too upright; the fourth (extreme right) is a satisfactory film; it was spread on a clean slide, is even, smooth, and of the proper thickness.

12. If the films are satisfactory, divide 100 phagocytes into groups of 20. The average ingest of each group should not show a difference of over 10 per cent., otherwise the technic has been faulty and it is necessary to count 250 phagocytes or to repeat the test. If divergence is due to the fact that every now and then one cell has a considerable higher ingest than others and the bacteria are well separated, hyperactivity of the cell is probably the cause. If the bacteria are all clumped together, it must be assumed that there has been a lack of care in preparing the bacterial emulsion or that agglutinin is present in the serum, and the test must be repeated with fresh precautions. 
13. In opsonic work the question as to how a certain element ought to be counted becomes quite evident and important. The proper method of procedure is to determine definitely how they may best be dealt with, and then to follow the rule adopted consistently. If an organism rests on the border of a cell, it will be better to consider it as within the cell and count it. Diplococci or division forms may be counted as one or as two, provided we are consistent in our method. Individual organisms, as distinguished from zoögleic masses, which may be lying on the top of the cell, are counted as if they were within the cell; for we have no means of determining definitely whether or not our suspicions are well grounded. In the case of a beaded or vacuolated bacillus, it is always better to count the whole element as a unit.

14. The phagocytic index is the average number of bacteria or other cells ingested per leukocyte after counting at least from 50 to 100 cells. The total number of bacteria ingested is divided by the total number of phagocytes, the result being the average number of bacteria ingested per leukocyte-i.e., the phagocytic index.

15. The opsonic index is then given by the ratio-

Patient's phagocytic index

Control phagocytic index

For example, with patient's serum, 100 phagocytes contain 300 bacteria, the phagocytic index being $\frac{300}{100}=3$. With the control serum, 100 phagocytes contain 500 bacteria, the phagocytic index being $\frac{500}{100}=5$. The opsonic index is:

$$
\frac{3 \text { Patient's phagocytic index }}{5 \text { Control phagocytic index }}=0.6
$$

16. Simon and Lamar have suggested a modification of Wright's method that has been adopted by many laboratories. It consists in diluting the patient's and control serums up to $1: 10$ or $1: 100$, and preparing mixtures of various dilutions with leukocytes and thinner bacterial emulsions. The percentage of phagocytic cells in the mixtures containing the serum to be tested is compared with the mixtures containing normal serum. It is, therefore, a method of comparative phagocytic indices.

\section{QUANTITATIVE ESTIMATION OF BACTERIOTROPINS (NEUFELD)}

Of the various methods for standardizing an immune serum, particularly antimeningococcus serum, and of obtaining some idea of its 


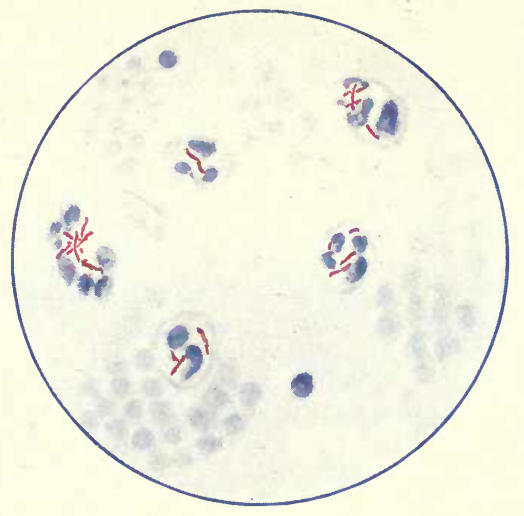

Fig. 53.-Tubercle Opsonic Index.

A smear, stained after the method given in the text. Case IX, C. M., aged twentytwo years; active pulmonary tuberculosis; opsonic index, +1.6.

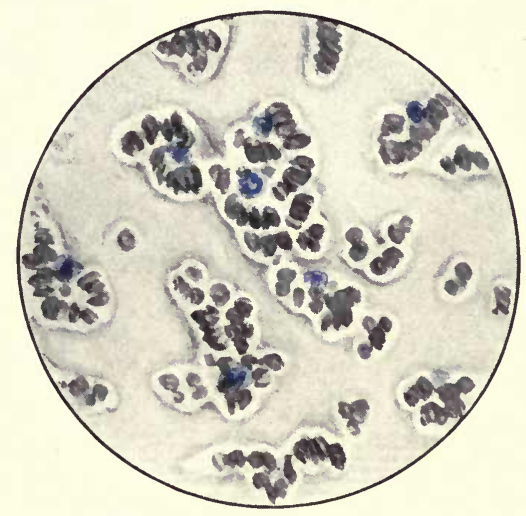

Fig. 54.-An Unsatisfactory Film for a Phagocytic Count.

Note that the leukocytes are collected in masses of erythrocytes. The slide was greasy and the smear too thick.

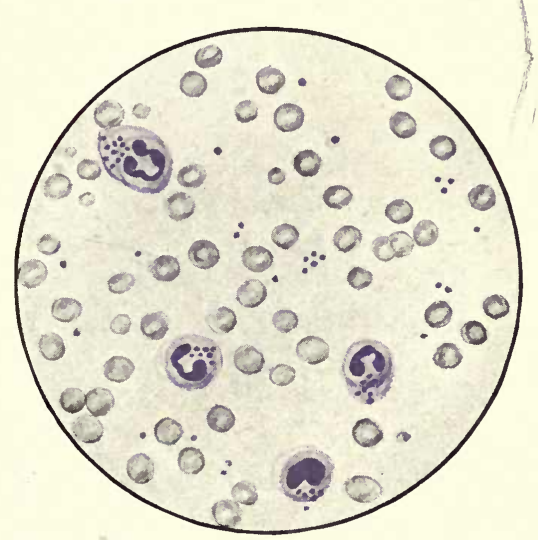

Fig. 55.-A Satisfactory Film for a Phagocytic Codnt. 

potency, that of determining the bacteriotropic or opsonic index of the serum is in most general use.

Neufeld's technic is that generally employed, and is similar to the serum dilution method employed by Simon. It varies from the technic of Wright in several particulars:

1. The immune serum is free from complement (thermolabile opsonin).

2. The actual number of bacteria within the leukocytes are not counted. Various dilutions of serum are used, and the highest dilution in which the bacteria are ingested in great numbers is compared with a normal serum in similar dilution as a control. The highest dilution that still favors phagocytosis is then taken as the bacteriotropic titer of the serum.

Serum.-The serum is inactivated by heating it to $55^{\circ} \mathrm{C}$. for onehalf hour. In old or carbolized serums this may be omitted, as they are usually free from complement. Tuberculous serums also should not be heated, as their bacteriotropins are very susceptible to heat.

Normal serum from an animal of the same species as was used in the preparation of the immune serum should be used as the normal control.

An exactly parallel series of dilutions with normal salt solution are made of the immune serum and pooled normal serums in a series of small test-tubes. At least 0.5 c.c. of each dilution should be available for the test; the following dilutions may be used: 1:10, 1:20, 1:50, $1: 100,1: 200,1: 400,1: 600,1: 800,1: 1000,1: 2000$, etc. After working for some time with normal serums one soon learns the dilution in which the normal bacteriotropins are attenuated. It may not be necessary, therefore, to use the whole series of dilutions with the normal serum.

Leukocytes.-Leukocytes may be obtained in several different ways: (1) By injecting a guinea-pig intraperitoneally sixteen to twenty-four hours previously with from 5 to 10 c.c. of sterile aleuronat solution (for method of preparation see p. 339). Pipet the peritoneal exudate into about 20 c.c. of sterile 1 per cent. sodium citrate in normal salt solution in centrifuge tubes. Centrifugalize, and wash the leukocytes again three times with sterile normal salt solution. The sodium citrate solution prevents the coagulation and formation of clumps of leukocytes.

2. Instead of aleuronat, a sterile saturated solution of peptone may be injected.

3. If rabbit's leukocytes are preferred, 10 c.c. of aleuronat should be injected into each pleural sac or 20 c.c. intraperitoneally. For mice, an injection of 1 c.c. of aleuronat intraperitoneally is sufficient; human leukocytes may be obtained after the method of Wright. 
4. After the final washing, the leukocytes are suspended in sufficient normal salt solution until an opacity equal to a 0.3 per cent. lecithin emulsion in salt solution is attained.

Culture.-Cultures should be selected with great care, in order to avoid using one that displays a well-marked tendency to undergo "spontaneous phagocytosis," or, on the other hand, one unduly resistant to phagocytosis. Usually an old strain of meningococci is serviceable; it is generally necessary to try out a number of strains and select the best.

Meningococci are grown for twenty-four hours on slants of glucose agar. To each slant add 0.5 c.c. each of bouillon and of normal salt solution, and emulsify the growth. Or the bacteria may be employed in the form of a sixteen to twenty-four hour homogeneous broth culture. Tubercle bacilli may either be triturated in an agate mortar with 1.5 per cent. salt solution added slowly drop by drop, or the tubercle powder of Koch may be employed in an emulsion prepared in the same manner. The resultant emulsion should be freed from coarser clumps by brief centrifugalization, but, as a general rule, it is very difficult to secure a uniform emulsion of tubercle bacilli by any method.

The Test.-1. The mixtures are made preferably in a series of small test-tubes about $5 \mathrm{~cm}$. long and $1 \mathrm{~cm}$. wide.

2. Mix 0.1 c.c. of each dilution of immune serum with 0.1 c.c. of bacterial emulsion. Plug each tube with cotton.

3. Mix and incubate for one hour.

4. Add 0.1 c.c. of leukocytic emulsion to each tube. Double this quantity may be used if the emulsion is weak.

5. Mix and incubate for from one-quarter to two hours, depending upon the variety of microörganism.

6. At the end of the second incubation the leukocytes will have settled to the bottom of the tubes. Carefully remove the supernatant fluid from each tube; mix the sediment well with a loop, and make smears on slides. Label each slide carefully to correspond to its serum dilution.

7. Dry the smears in the air, fix with methyl alcohol, and stain with carbolthionin, as previously described.

The Controls.-1. A series of the lower dilutions of pooled normal serums are set up in exactly the same manner.

2. A tube containing bacteria and leukocytes without serum-to detect the extent of spontaneous phagocytosis.

Readings.-A great number of fields are examined microscopically, and a note is made of the weakest dilution that still favors phagocytosis. 
No attempt is made to count the phagocyted bacteria. The relative amount of phagocytosis with the immune serum in various dilutions is compared with the normal controls, and the result is expressed as the bacteriotropic titer.

Simon's Method.-This method of counting the number of empty leukocytes with a given dilution of serum is followed; a similar count is made of the normal serum control in the same dilution; thus, if in the control film 25 per cent. of leukocytes were empty, and in the patient's film, 50 per cent., the index would be $\frac{25}{50}=0.5$. A study of the results obtained by this method, and by careful counting after Wright's method, shows that they are fairly comparable, and the method may be used where it is only necessary to determine whether the index is high or low.

Precautions.- 1 . If phagocytosis is entirely absent, one should not conclude that bacteriotropins are not present. The leukocytes may have been injured, especially if heterologous leukocytes have been present; control examinations with homologous leukocytes $(i . e$., from the same animal) as the serum should result in phagocytosis.

2. The time during which the tubes were in the incubator may have been too short or too long. Most microörganisms require from onehalf to two hours-meningococci require about one-half hour; pneumococci usually need at least two hours; typhoid and cholera bacilli about fifteen minutes to thirty minutes, as they undergo extracellular or even intracellular lysis quite readily.

3. If the control of bacteria and leukocytes alone shows well-marked phagocytosis the test should be repeated with another strain.

\section{Practical Value of THE Opsonic INDEX}

1. In competent hands, the opsonic index of normal persons to most pathogenic organisms has been found to vary from 0.8 to 1.2. As previously mentioned, it is difficult to find a perfectly normal serum for such microörganisms as the Bacillus coli, the staphylococci, Bacillus tuberculosis, etc., as it is unlikely that any individual can altogether escape active infection at some period of his life. As menstruation approaches, even wider fluctuations occur, the normal index being reestablished by the second or third day. During the first year of life the opsonic index varies to such a degree that it has little or no practical value.

2. Although a large amount of work has been done with the opsonins in disease, it is the consensus of opinion that the determination of the opsonic index has less practical significance than was originally believed. One point is clear, however, that the work of Wright and others has broadened the field of vaccine therapy and placed it upon a firmer 
foundation. Aside from the 10 per cent. of chances of technical error in making an opsonin measurement, other factors may be present that are entirely beyond control and cannot be measured by the immunisator, and that may seriously affect the value of the opsonic index.

3. As a diagnostic procedure, Wright has shown that the opsonins possess a certain specificity, and that in a given infection a low index

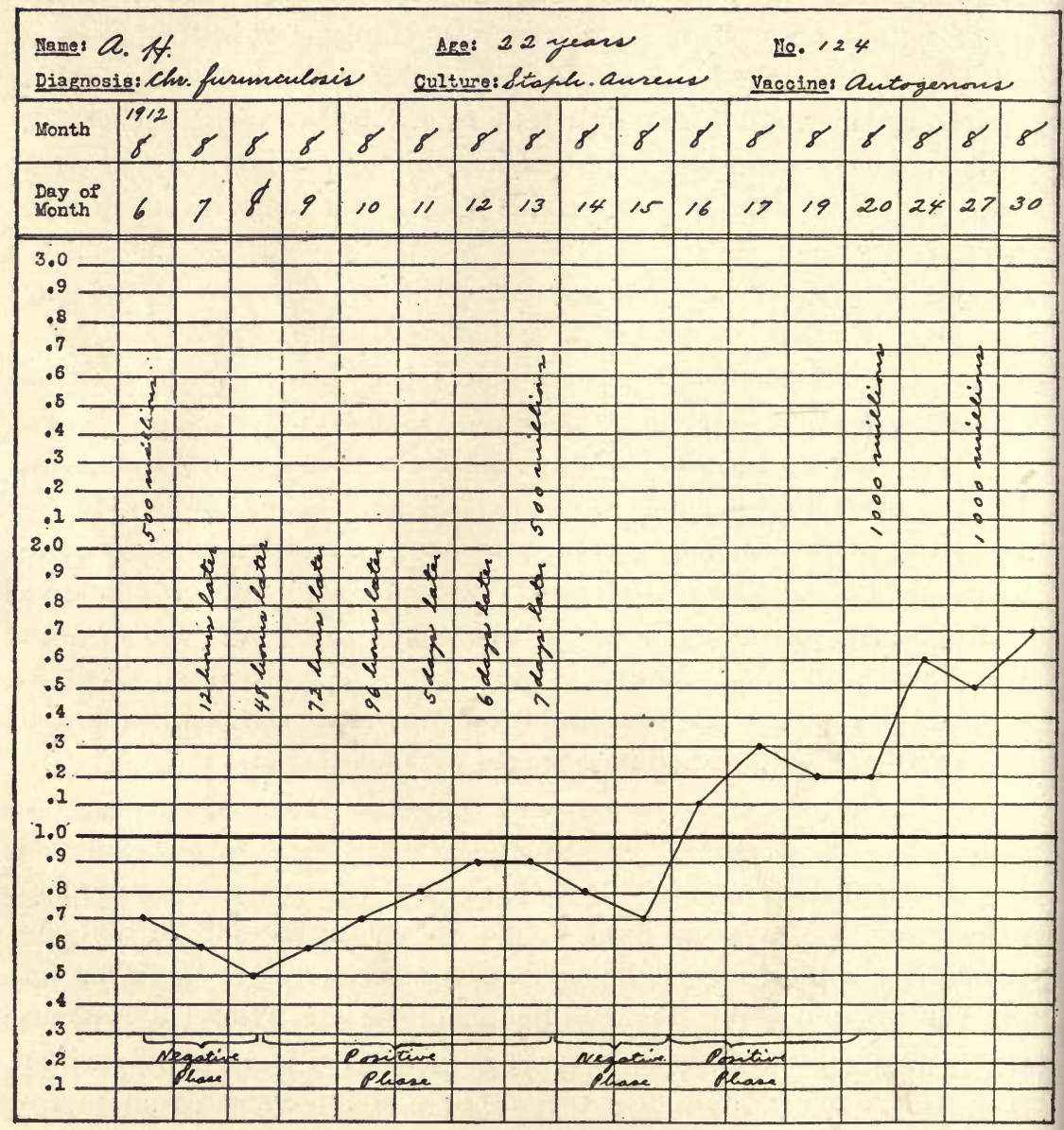

Fig. 56.-An Opsonic Index Chart.

for a certain microörganism indicates that this organism is probably the etiologic factor. This possibility is strengthened if the opsonic index for this microörganism is increased by careful manipulation or exercise of the diseased part, when auto-inoculation occurs, with consequent increase of opsonin.

4. In prognosis the opsonic index may have some value in deciding 
whether an infection has been entirely overcome or is still active. An attempt is made to induce auto-inoculation, as by gentle massage of a knee-joint or a hip; active exercise; deep breathing, etc., and the index is made before, and at frequent intervals after, such attempts. If the index remains unchanged within the normal limits, the assumption is that the infection has been overcome; if, on the other hand, an increase in opsonin occurs, this indicates that an active focus remains.

5. Most value was placed by Wright upon the opsonic index as a guide to the size and frequency of doses of bacterial vaccines in the treatment of disease. A large number of careful determinations showed that an injection of vaccine is followed by a decrease of the opsonins (negative phase), which is of variable degree and duration, according to the amount injected (Fig. 55). This is followed by an increase (positive phase), and coincidentally there is a corresponding improvement in the patient's condition. This subject is discussed more fully in the chapter on Active Immunization.

The purpose of proper vaccination, therefore, is so to gage and time the different doses that a pronounced or prolonged negative phase is prevented, as far as possible, and a high positive phase secured and maintained. It is obvious that the technic of opsonic measurement consumes much time, and that the immunisator cannot mark the index at the time a dose of vaccine is given. However, the determination of the opsonic index at proper intervals after the first dose of vaccine may give valuable information as regards the reaction of the patient, and serve as a guide to the size and frequency of subsequent doses.

As a routine measure, the opsonic index has fallen into disuse, vaccine therapy being largely guided by the clinical evidences of reaction and the condition of the patient. That it has distinct value, particularly in scientific investigation, is generally admitted, and it is well to remember that in the early years following Wright's investigations the practice of vaccine therapy was limited to those skilled in determining the index, preparing the vaccine, and carefully guiding and guarding its administration. It is to be regretted that the wholesale and indiscriminate manufacture and use of vaccines have brought this valuable field of therapy inevitably into disrepute. This is being realized more and more, and the effort is being made to restore the value of this form of therapy. This effort consists in recognizing the possibilities and limitations of the method, and confining its practice to those who possess, at least, sufficient knowledge of bacteriology to prepare a vaccine and make an opsonic measurement, the best results being secured by cooperation between bacteriologist and clinician. 


\section{CHAPTER XIII}

\section{BACTERIAL VACCINES}

IN this chapter a method for preparing bacterial vaccines will be described, the general discussion of vaccine therapy, with the special technic for preparing cow-pox vaccine, rabies vaccine, tuberculin, and other special vaccines, being taken up in Chapter XXIX.

Definition.-Bacterial vaccines are "sterilized and enumerated suspensions of bacteria which furnish, when they dissolve in the body, substances which stimulate the healthy tissues to a production of specific bacteriotropic substances which fasten upon and directly or indirectly contribute to the destruction of the corresponding bacteria" (Wright).

\section{TECHNIC FOR PREPARING BACTERIAL VACCINES}

Bacterial vaccines are made-(1) By procuring the infected material; (2) by preparing pure cultures of the bacteria that are to be attacked; (3) by making suspensions of these in saline solution, adding a preservative, and placing in proper containers.

1. Procuring Infected Material.-Various precautions, according to existing circumstances, should be taken to avoid contamination and to secure material that is truly representative of the focal secretions. For instance, pus should be collected from an abscess cavity or sinus after the surrounding tissues have been cleansed with dilute tincture of iodin, for if we secured a culture of the relatively harmless Staphylococcus epidermidis albus from the skin instead of the Staphylococcus aureus, which may be the cause of infection, our vaccine will have little or no value.

Nasal secretion may be secured after cleansing the nasal orifice with soap and warm water, passing a sterile cotton swab through a nasal speculum, and rubbing the surfaces of the lower turbinates and septum lightly.

An ear should be cleansed, the excess of secretions removed with sterile swabs, and the culture be made of pus from the infected tissues. Various saprophytes quickly gain admission and grow in the necrotic pus, whereas the infecting bacterium is more likely to be found in the tissues. 
In the collection of sputum special care is required: the patient should be instructed to brush the teeth with a sterilized brush, rinse the mouth several times with boiled water, and after swallowing several mouthfuls of water, to cough and expectorate into a wide-mouthed sterilized bottle. The sputum may be plated at once, or washed several times in sterile Petri dishes with sterile water and then cultured.

Lung Puncture may occasionally be required in infective lung conditions in which sputum is not obtainable or is too badly contaminated. A 5 to 10 c.c. all-glass syringe with a strong needle is sterilized by boiling, and 2 or 3 c.c. of peptone broth introduced. The skin of the chest-wall over the site of infection, as shown by clinical evidence, is sterilized with tincture of iodin, and puncture made into the pulmonary tissues. When the desired depth has been reached, 1 c.c. of the broth is injected gently into the tissues, and after the lapse of a few seconds reaspirated as far as possible into the syringe. During the operation the patient should refrain from respiratory movements, in order to minimize any risk of lacerating the pulmonary tissues (Allen).

Urine should always be withdrawn with a sterile catheter after thoroughly cleansing the meatus. This last is especially important, for the infection may be due to a certain strain of Bacillus coli, and unless we are successful in obtaining a culture of this particular strain, the vaccine will have little value.

Blood specimens are taken with a sterile syringe from a prominent vein at the elbow after the skin has been cleansed and sterilized with tincture of iodin, and cultured in large amounts on proper culture-media.

2. Preparing Pure Cultures.-This is frequently the most difficult step in the whole technic, for some microörganisms, as, for example, the gonococcus and Bacillus influenzæ, grow slowly, require special culturemedia, and their colonies may easily be overlooked. To secure pure cultures, and especially to select one or at most two organisms that may be the chief offenders, considerable bacteriologic knowledge is necessary, and no simple rules or directions can be given in the limited space of this volume.

1. Stained smears of the secretions of a lesion may indicate the nature of the infection and the best culture-medium and technic to use for purposes of isolation.

2. Cultures of the lesion may be made upon solid media, and isolation carried out after a primary growth has been secured. With proper care, primary cultures may be grown, such as staphylococci from the pus of a freshly incised abscess, or the microörganism of a case of cystitis 
or pyelitis by securing urine with the aid of a sterilized catheter. If slowly growing organisms, such as Bacillus influenzæ, gonococcus, pneumococcus, etc., are to be cultured, "streak" plates are usually satisfactory, and as a routine the best culture-media are, as a rule, those containing serum or blood.

3. The cultures that are to be worked up into a vaccine are usually best made on solid media.

4. In making a bacterial vaccine of a freely growing microörganism for an individual patient, it will suffice to plant two agar slant tubes; when dealing with bacteria that grow much less luxuriantly, such as streptococci and pneumococci, four to six tubes should be used.

5. Cultures are usually grown for twenty-four hours at $37^{\circ} \mathrm{C}$., but

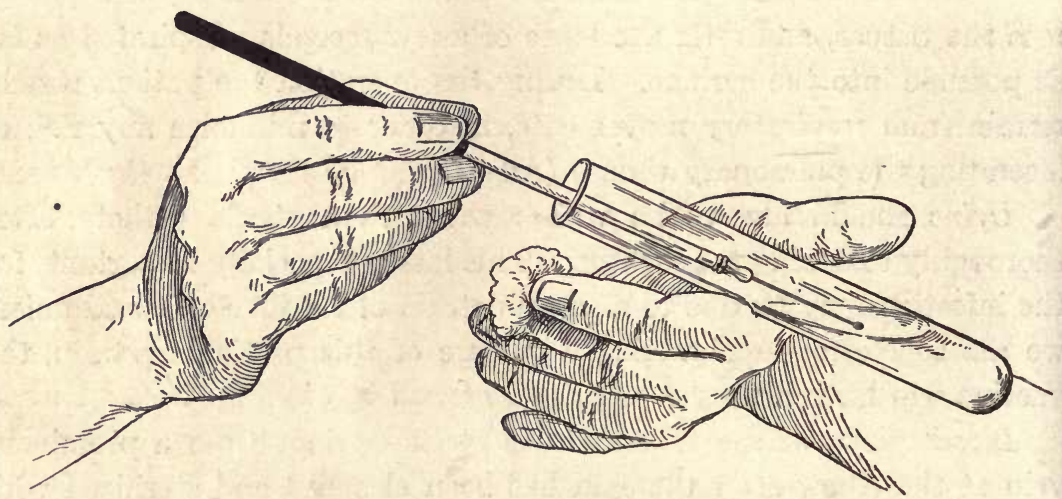

Fig. 57.-Preparation of a Bacterial Vaccine.

Removing the culture of bacteria by gently rubbing over the medium (agar-agar) with a sterilized platinum loop.

in the case of rapidly growing organisms a shorter period in the incubator will suffice.

6. When the cultures are ready, a smear of each growth is made and stained in order to see that pure cultures of the right microörganism were made.

7. Inasmuch as the immunizing power of a vaccine is in most cases a factor of the virulence of the organism, this being especially true of such organisms as the pneumococcus, streptococcus, and influenza bacillus, it is well, whenever possible, to employ the first pure subculture for the preparation of the vaccine.

3. Preparation of the Emulsion.-Carefully observing aseptic precaution throughout, pour a portion of a test-tube of a sterile normal salt solution over the surface of the first culture, shaking the fluid in such a 
way as to bring the microörganisms into suspension. If the culture is not easily washed from the medium, a sterile platinum loop may be used to remove the growth, care being taken not to cut into the medium and mix the fragments with the bacterial suspension (Fig. 57).

The bacterial suspension thus obtained is poured on the surface of the second culture, bringing this into suspension, and repeating the process until the whole series of cultures have been suspended, adding more salt solution if necessary.

The final suspension is transferred to a sterile, thick-walled flask containing glass beads, and shaken by hand or in a mechanical shaker until the bacterial masses are broken up (Fig. 58). This may be especially difficult with diphtheria bacilli and streptococci. Unless the

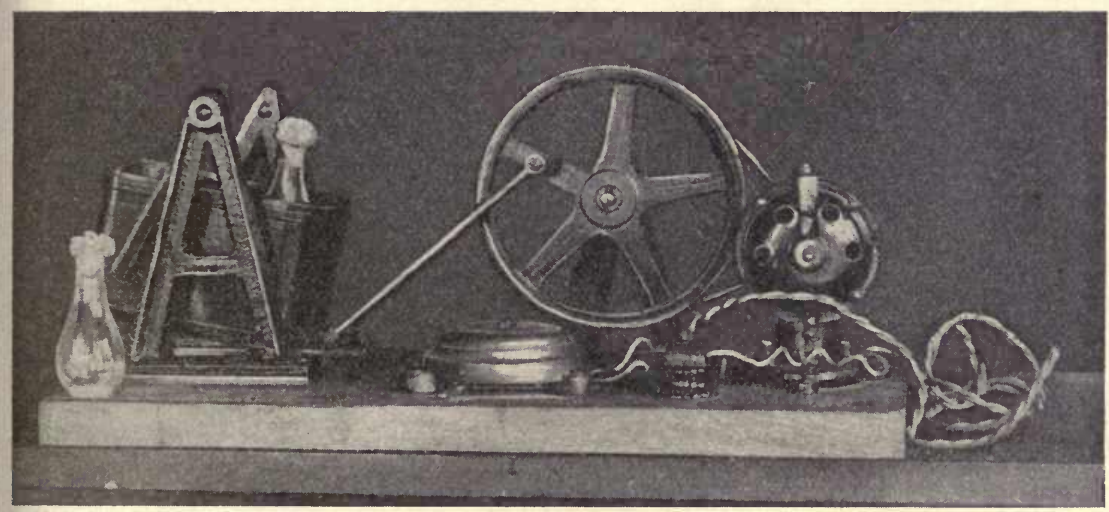

Fig. 58.-A Shaking Apparatus (Electric, 110 volts, direct).

A satisfactory machine for shaking vaccines, preparing antigens, making emulsions, etc.

emulsion is perfectly homogeneous, the larger particles may be removed by brief centrifugalization or, better, by filtering through a sterile filter.

There is evidence to show that bacteria grown on culture-media containing peptone may produce objectionable toxic substances capable of producing anaphylactic phenomena (Reichel and Harkins ${ }^{1}$ ). In addition, when, in the preparation of a vaccine, bacteria grown on a serum medium are washed off with normal salt solution, a portion of the serum may be removed and this may be capable of producing disagreeable local and general reactions. For these reasons it is advisable to wash all suspensions by repeated centrifugalization until the supernatant fluid reacts negatively to the biuret or ninhydrin reaction (Willard Stone).

${ }^{1}$ Centralb. f. Bakteriolog., etc., 1913, 69. 
4. Counting of the Bacterial Suspension.-Standardization, best accomplished by counting the bacterial elements con-

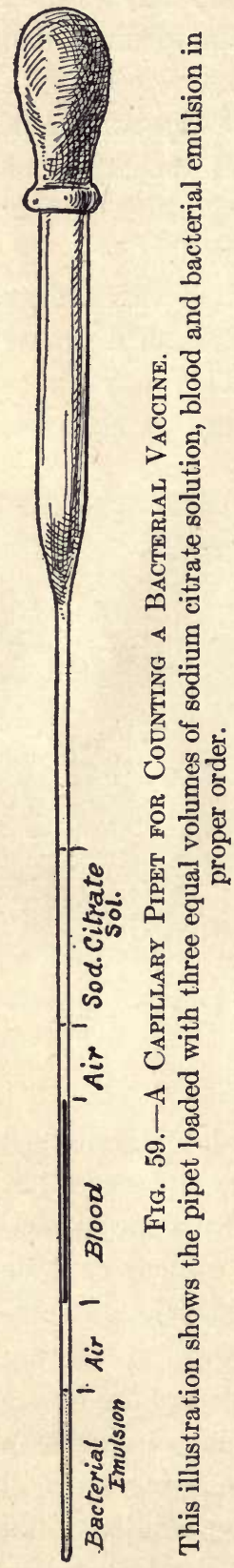
tained in a unit volume of the suspension, is necessary in order to adjust our initial dose as experience will dictate and for guidance in making subsequent injections.

In dealing with a vaccine we have to count both the dead and the living bacteria, making no distinction, for both furnish the chemical agent that calls forth the elaboration of bacteriotropic substances. Inasmuch as sharp definition and the staining properties of bacteria may be lost in the process of sterilization by heat, the specimen of vaccine to be examined should be secured before sterilization is undertaken.

The counting or standardization may be done in several ways: (a) By mixing equal portions of normal blood and bacterial emulsion and counting the proportion of corpuscles to bacteria in our mixture (Wright); (b) by diluting, staining, and counting with the hemo cytometer, as in the enumeration of red blood-corpuscles; (c) for standardizing large quantities of bacterial vaccine the method of Kolle or $(d)$ that of Hopkins may be used.

(a) Method of Wright.-Prepare a simple capillary pipet, making a mark on its stem about an inch from the tip, and fit a teat to its barrel (Fig. 59). Cleanse and prick the finger, press out a drop of blood, take up the pipet and draw up into it first one volume of sodium citrate solution, one of blood and then either one volume of bacterial suspension or two or more volumes, if it appears on inspection to contain much fewer than $500,000,000$ of bacteria to the cubic centimeter. To guard against crimping of the corpuscles in drying the films, Wright advocates aspirating one or two volumes of distilled water after the blood and bacterial suspension.

Now expel from the pipet first only the distilled water and bacterial emulsion, and mix these, so that there may be no danger of the redd corpuscles becoming hemolyzed, and 



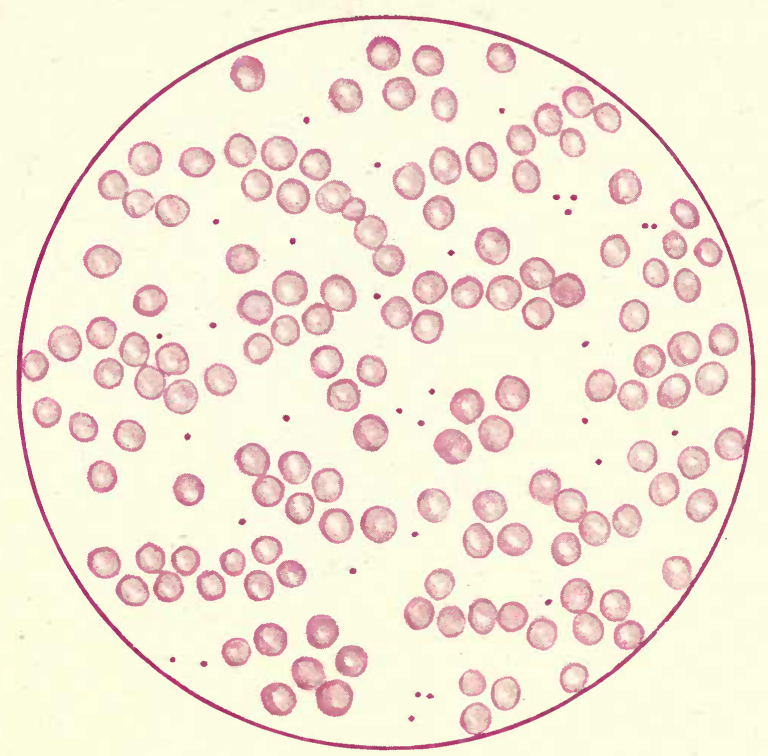

Fig. 60.-A Satisfactory Preparation for Counting a Bacterial Vaccine.

The microörganisms are well separated and evenly distributed among the corpuscles; the spread is even and regular, and the corpuscles are not gathered in rouleau formation or irregular clumps.

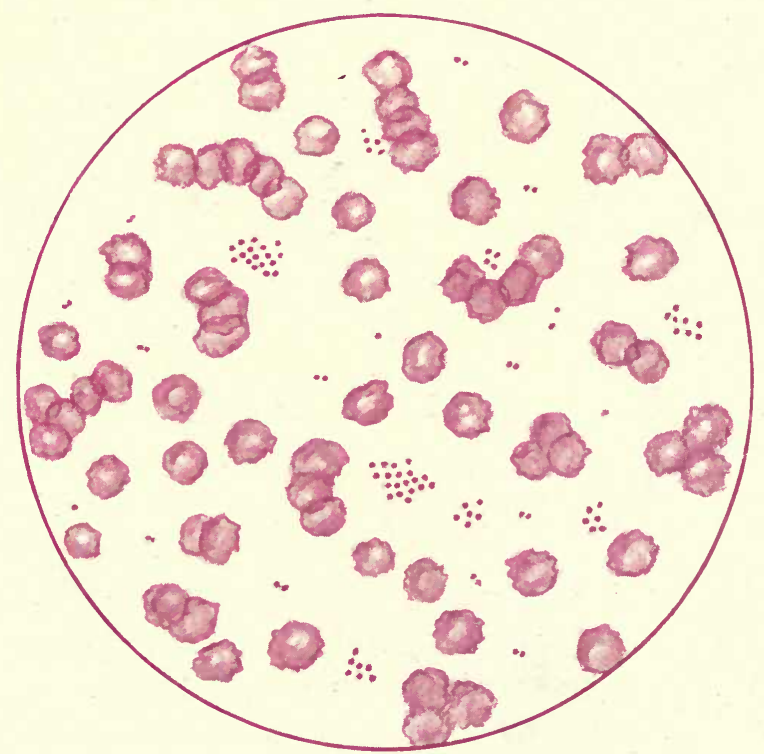

Fig. 61.-An Unsatisfactory Preparation for Counting a Bacterial Vaccine.

The microörganisms are mostly gathered in irregular masses instead of being separated and evenly distributed; the corpuscles are not separated and evenly distributed, but show a tendency to gather in clumps; both factors render a count difficult, inaccurate, and unsatisfactory. 
then proceed to mix together the whole contents of the pipet, aspirating and reëxpelling these a dozen times. Then make two or three microscopic films from the mixture, spreading these out on slides that have been roughened with emery.

The films are dried in the air, fixed by immersing them for two minutes in a saturated solution of corrosive sublimate, washed thoroughly, and stained for a minute with carbolfuchsin diluted 1:10 or carbolthionin for two to five minutes, and then washed and dried.

The films are now given a preliminary examination. If red corpuscles and bacteria are found in approximately the same numbers and the suspension is free from bacterial aggregates, the count may be made (Fig. 60). If either the bacteria or the corpuscles are largely in excess, new mixtures and new films must be made. In case the bacteria are gathered in clumps, the suspension should be shaken again and new films prepared (Fig. 61).

When satisfactory films have been obtained, the actual counting may be done. This is carried out with an oil-immersion lens, and in order to secure accuracy, it is necessary to restrict or divide the field by a small square diaphragm made of paper or cardboard, or by inscribing cross lines on a small clean cover-glass and dropping them on the diaphragm of the eye-piece.

A field is now chosen at random, and the corpuscles and bacteria are counted, the results being jotted down on a sheet of paper, keeping each enumeration separate and writing the numbers in two columns. Proceed at random from field to field, traversing every part of the slide. Establish a rule for counting corpuscles that transgress or touch the edge of the field. Eliminate from consideration any parts of the films in which the preparation is unsatisfactory as regards the staining, or with respect to the integrity of the red corpuscles. The examination is continued until at least 500 corpuscles have been counted, half of the count being made from the second slide. The number of microörganisms counted is now totaled, and the approximate number per cubic centimeter estimated. Let us assume, for example, that 600 red cells and 1200 bacteria have been counted. Now, a cubic millimeter of blood contains 5,500,000 red corpuscles, and equal volumes of blood and emulsion were taken. A cubic millimeter of the emulsion, therefore, contains $\frac{5,500,000 \times 1200}{600}=11,000,000$ organisms per cubic millimeter, or $11,000,000,000$ per cubic centimeter.

(b) The second method of counting is precisely similar to that em- 
ployed for the enumeration of blood-corpuscles, the diluting and staining fluid being made by adding to a 1 per cent. solution of sodium chlorid in distilled water sufficient formalin to make 2 per cent., and alcoholic gentian-violet, 5 per cent. The emulsion is drawn up in a white corpuscle pipet to the mark 0.5 , and with diluting fluid to the mark 11 . The contents are then mixed thoroughly for several minutes, several drops expelled, a drop placed in the counting chamber and properly covered with a special thin cover-glass. The bacteria are allowed to remain in the counting cell for

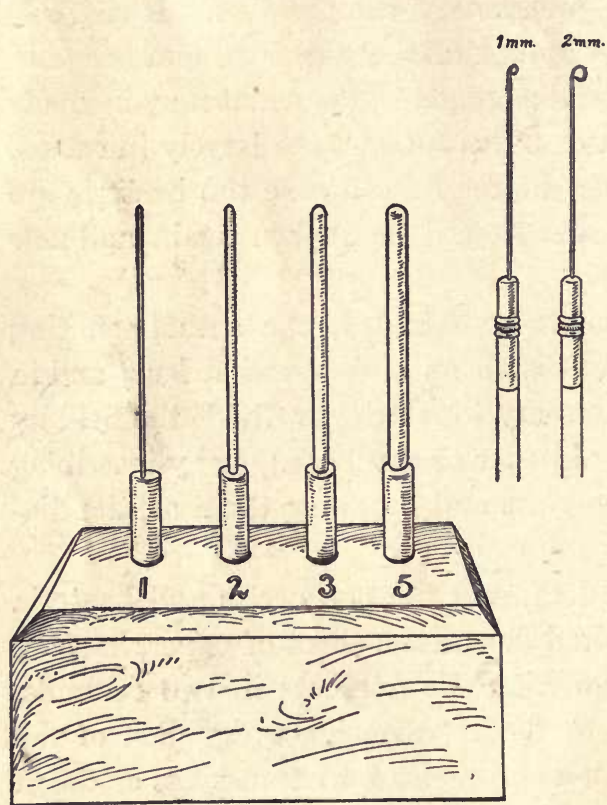

Fig. 62.-Instrument For the Standardization of Platinum Loops.

The $2 \mathrm{~mm}$. loop holds approximately $2,500,000$,000 bacteria (as, Bacillus typhosus).

at least half an hour prior to enumeration. A large number of small squares are counted, and the average of one square obtained. By multiplying this figure by 4000 and then by 20 , the number of bacteria per cubic millimeter is obtained, and 1000 times this figure gives the number of bacteria contained in one cubic centimeter of the vaccine. If the emulsion is highly concentrated, the red cell pipet may be used and the calculations made accordingly.

(c) Method of Kolle.-A platform loop adjusted to fit No. 2 of a Lautenschläger wire gage (Fig. 62) measures about $4 \mathrm{~mm}$., and holds approximately $2 \mathrm{mg}$., or about $2,500,000,000$ organisms. By growing an organism on slants of agar and emulsifying a certain number of loopfuls in a measured quantity of saline solution, an approximate method of standardization is obtained. According to Kolle, ordinary slants of agar will hold about 15 loopfuls of staphylococci, Bacillus typhosus, or Bacillus coli, and about 5 loopfuls of streptococci and gonococci.

(d) Method of Hopkins. - This is based upon concentrating a bacterial suspension by centrifugalization and the preparation of standard emulsions from the sediment. The emulsion is filtered through a

1 Jour. Amer. Med. Assoc., 1913, xl, 1615. 
small cotton filter to remove larger clump of bacteria and particles of agar, and is then placed in specially constructed centrifuge tubes (International Centrifuge Company, see Fig. 63), covered with rubber caps, and centrifugalized for half an hour at a speed of approximately 2,800 revolutions a minute. The salt solution and bacteria above the 0.05 mark are then removed, and 5 c.c. saline solution is measured into the tube, so as to make a 1 per cent. emulsion. If the sediment does not reach the 0.05 mark, its volume is read on the scale, and a corresponding quantity of saline is added to make the emulsion 1 per cent. in strength. The bacteria are forced into suspension, the vaccine transferred to a sterile tube, and the microörganisms killed in the usual manner.

Estimations of carefully counted suspensions obtained by centrifugalization in the foregoing manner gave the following results:

\begin{tabular}{|c|c|c|}
\hline & $\underset{\text { CENT }}{\text { PER }}$ & $\begin{array}{c}\text { BILlioN } \\
\text { PER C.C. }\end{array}$ \\
\hline lococcus aureus a & $\ldots 1$ & 10.0 \\
\hline coccus hæmolyticus... & 1 & 8.0 \\
\hline Gonococcus $\ldots \ldots \ldots \ldots \ldots$ & .1 & 8.0 \\
\hline Pneumococcus . . . . . . . . . & .1 & 2.5 \\
\hline lus typhosus... & .1 & 8.0 \\
\hline acillus coli $\ldots \ldots \ldots \ldots \ldots \ldots$ & .1 & 4.0 \\
\hline
\end{tabular}

5. Sterilizing the Vaccine and Testing its Sterility.-When, after the preliminary examination, the films for counting have been found satisfactory, a pause is made to start the process of sterilization, which may continue while the count is being made. Either heat or a germicide may be used for sterilizing vaccine, preferably the former.

The vaccine may be placed in a test-tube, which is then sealed (Fig. 64), and the whole immersed in the water-bath; a simpler method, and

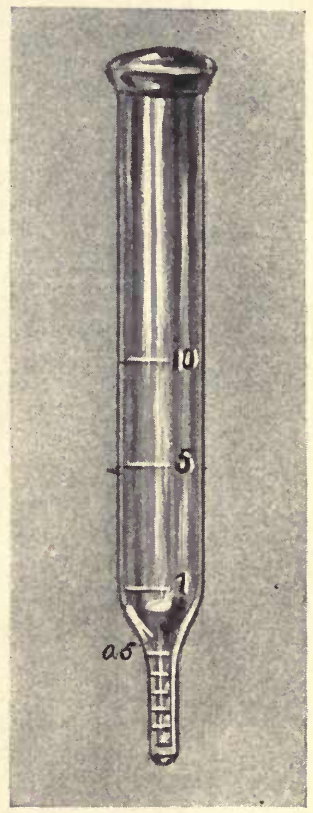

Fig. 63. - Hopkins' TUBE For STANDARDIZING A BACTERIAL VACCINE. one just as good, is to place the flask or tube of vaccine in the bath, observing special care to see that the water is above the level of the vaccine.

Efficient sterilization is dependent upon permitting the process to continue at the minimum temperature for the minimum length of time. With the water-bath at $56^{\circ}$ to $60^{\circ} \mathrm{C}$. sterilization is nearly always complete in an hour.

The vaccine should be now cultured to test its sterility. At least a dozen platinum loopfuls are transferred, under strict aseptic precautions, 
to a slant of a suitable culture-medium, such as Löffler's blood-serum or blood-agar; this is incubated at least twenty-four hours, or longer if the organism is a slowly growing one. It is then examined, and if found sterile, the preparation of the vaccine may be completed. If not, the vaccine is heated for another hour, or, preferably; a new vaccine is prepared.

6. Diluting the Vaccine and Adding a Preservative.-Having made the count and sterilized the vaccine, it is next diluted with sterile saline

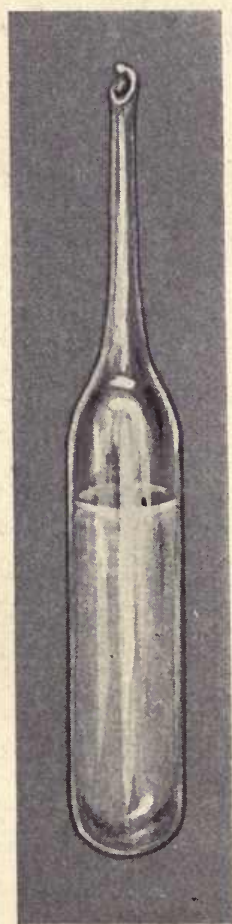

Fig. 64A.-A Stock Ampule of
VACCiNe. solution so that each cubic centimeter contains the dose decided upon. If

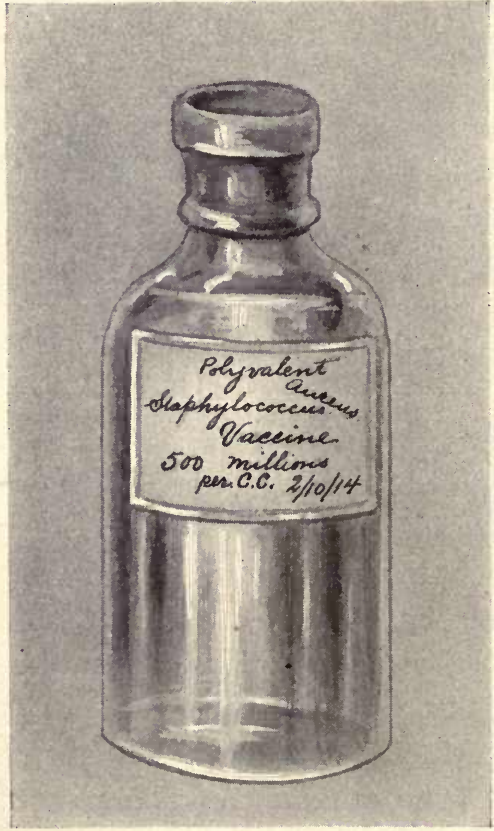

Fig. 64B.-Stock Bottle of BaCterial VACCINE.

the treatment is likely to be prolonged, a sufficient number of doses should be provided for. It is a good plan not to dilute all the vaccine, but to preserve the remainder undiluted in case larger doses are subsequently needed. If, for instance, a vaccine of Staphylococcus aureus contains $1,500,000,000$ organisms per cubic centimeter and the dose decided upon is 500,000,000 per cubic centimeter, sufficient vaccine for 30 doses is prepared by withdrawing 10 c.c. of vaccine in a sterile container and adding 20 c.c. of sterile salt solution. This mixture is agitated, 
to insure thorough mixing, and 0.1 c.c. of a $1: 100$ dilution of phenol is added to each cubic centimeter of vaccine as a preservative against chance contamination. Thus, in the foregoing example, 3 c.c. of the diluted phenol would be added. The amount of stock vaccine is estimated or measured, 0.5 per cent. phenol is added, and the vaccine stored in a sterile container in the refrigerator, being first properly labeled with the patient's name, the date, and the number of bacteria per cubic centimeter.

The vaccine may now be placed in a sterile vaccine bottle, fitted with a sterile rubber cap, and properly labeled (Fig. 64). When it is to be

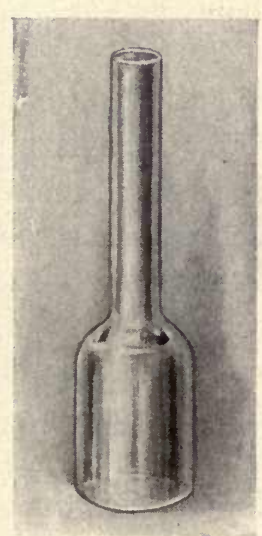

Fig. 65.-A SMALL Vaccine Ampule.

Capacity 1 c.c. administered, the cap is touched with tincture of iodin, the needle plunged through the cap, and a dose withdrawn with a sterile syringe. The puncture in the cap is then sealed with a drop of flexible collodion. This method is inexpensive, and with proper care is quite satisfactory, especially for stock vaccines.

Another and probably better method, especially for autogenous vaccines, consists in tubing each dose in separate sterile ampules (Fig. 65), which are then sealed in the flame. When the vaccine is to be administered, the ampule is well shaken, the neck broken in a towel, and the contents aspirated into a sterile syringe. These ampules may be purchased ready for use or be made in the laboratory, using $6 \mathrm{~mm}$. soft glass tubing (Fig. 6). For pipeting a vaccine into ampules the special automatic pipet shown in the illustration

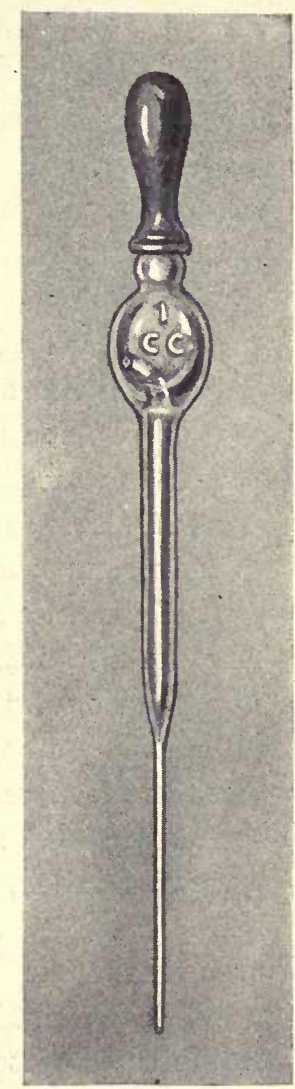

FIG. 66. - CoMER's Automatic Pipet. (Steele Glass Co., Philadelphia.)

The inner tube to the tip of the pipet holds exactly 1 c.c. If the rubber teat raises too much fluid, the excess is received in the glass reservoir; when too much fluid accumulates in this, it may be emptied by turning the point of the inner tube downward and ejecting the fluid by pressure on the teat. (Fig. 66) is quite convenient. As a rule, vaccines should be preserved in a cool place, such as a refrigerator. 


\section{PREPARATION OF SENSITIZED BACTERIAL VACCINES}

A highly immune serum is prepared by immunizing a series of rabbits or a goat with the microörganism to be used in preparing the vaccine. The first injections consist of heat-killed emulsions, administered subcutaneously. After the first or second dose the period of heating is gradually reduced, and the dose increased, until finally the injections may be given intravenously and with living microörganisms. From time to time a small amount of serum should be examined for immune bodies: with the typhoid-cholera group, by testing for bacteriolysin and agglutinins; with staphylococci and streptococci, by agglutination, bacteriotropic, and complement-fixation tests; with pneumococci, gonococci, and meningococci, by bacteriolytic, agglutination, and bacteriotropic tests. When a highly immune serum is secured, the animal is bled, the serum isolated, heated to $56^{\circ} \mathrm{C}$. for half an hour, and stored in a strictly aseptic manner.

To "sensitize" the bacteria, thick, even emulsions of young cultures in normal salt solution are treated with one-half to an equal bulk of inactivated immune serum, and the mixture gently agitated at room temperature for from six to twelve hours. The emulsion is then thoroughly centrifuged, and the residue of bacteria washed three times with sterile salt solution, after the manner in which the red corpuscles are washed. After the final washing the bacteria are resuspended in salt solution, shaken for a time to insure breaking up of agglutinated clumps, counted, heated at $60^{\circ} \mathrm{C}$. for an hour, cultured as a test for sterility, and then diluted so that the emulsion will contain slightly larger doses than a corresponding dose of ordinary vaccine prepared for administration.

"Sensitization" probably consists in the union of bacteriolytic amboceptor with its antigen, and when injected, serves, with the patient's complement, to hasten solution or lysis of the bacteria (antigen), thereby liberating quickly the chemical substances required for the stimulation of antibodies.

Metchnikoff and Besredka are using sensitized living bacteria, and their work is being followed with much interest. In this country strict legal restrictions and regulations exist regarding the sending of living cultures through the mails. If, therefore, the method should fulfil the high claims and expectations made for it, there may be considerable difficulty in bringing it into general use. 


\section{THE ADMINISTRATION OF A BACTERIAL VACCINE}

Syringe.-Vaccines are best administered with the aid of a 1 c.c. all-glass syringe, furnished with a sharp platinum iridium or steel needle. These may be sterilized in boiling water for a minute or longer. After sterilization, the parts should be carefully adjusted and the syringe loaded.

According to Wright, time and trouble may be saved by sterilizing in oil at a temperature of $125^{\circ}$ to $140^{\circ} \mathrm{C}$. If this temperature is not exceeded, the oil can be drawn into the syringe without danger of breaking the glass. The procedure is as follows: Partly fill a tablespoon with any vegetable oil, and into this place a bread-crumb about the size of a large hemp-seed. Then heat over a spirit-lamp until bubbles of steam begin to appear about the bread-crumb. The temperature of the oil is now about that of boiling water. After bubbles have ceased to form about the bread-crumb-meanwhile drawing up the oil once or twice into the syringe-reapply the heat very cautiously until the breadcrumb shows the first sign of turning brown (at about $140^{\circ} \mathrm{C}$.). Then, without allowing time for the oil to cool, draw it up two or three times into the syringe, being careful to see that it comes into contact with every part of the interior.

If it is desired, so as to improve the appearance, to get rid of all remaining traces of oil from the syringe, this can be easily effected by drawing up into the syringe a very weak ( 0.25 to 0.50 per cent.) solution of sterilized sodium carbonate.

Method of Making the Inoculation.-As the administration of a vaccine is frequently followed by a temporary depression of the resisting powers of the individual and a feeling of lassitude, the injections are, as a rule, best given during the afternoon and evening, the night's rest aiding in overcoming the depression. Since the determination of proper dosage rests mainly on the observation of such clinical signs and symptoms as temperature, pulse, and local reaction at the site of the lesion, the patient should be watched during the following twenty-four to forty-eight hours.

The injections should be given at a point where the tissues are loose, where muscular action is not much in evidence, and where pressure by clothing or weight is not made. The most suitable localities are in front of the shoulder, at a site about $1 \frac{1}{2}$ inches below the center of the clavicle; high up in the buttock, or in the side of the abdomen, about two or three inches inside the anterior-superior spine of the ilium. The skin at the point of injection should be touched with tincture of iodin, 
which is removed after the injection has been given by washing with alcohol.

Both convenience and experimental work to test the comparative efficacy of inoculation into different tissues point to the subcutaneous tissues as the most suitable site for inoculation. The best method of procedure is to pick up a fold of skin between the finger and thumb, and then to push the needle well down into the middle of the fold, and slowly inject the fluid.

Since it is known that the power of response of the tissues to the stimulus of a vaccine is somewhat limited, it would seem advisable to choose a new site for each successive inoculation.

The Effects of Inoculation.-The local effects produced at the site of inoculation vary considerably, being influenced by the nature of the individual, the variety and amount of the inoculum, and the sensitiveness of the patient's tissues to stimulation. In the majority of cases the local reaction is limited to a very slight reddening of the skin around the puncture for an area of about one inch. In some instances, occasionally encountered where a large number of typhoid inoculations have been made, the reaction after the first dose is more severe than after subsequent doses, and is accompanied by considerable edema, hyperemia, and pain.

The focal effects about the lesion are exceedingly important in determining the reaction of the patient, and serve as a guide to the adjustment of dosage and intervals. Where, in a case of furuncle, an appropriate dose of staphylococcus vaccine is administered, within a few hours increased hyperemia is seen around the focus, and there is a slight increase in the swelling. When very small doses are given, these focal symptoms may practically be absent, but, as a rule, a slight reaction does no harm, but serves rather to show that the vaccine possesses some degree of potency and may aid in the curative process.

The constitutional effects may also vary within wide limits. An adequate, but not excessive, dose may, within a few hours, produce a feeling of lassitude, headache, slight rise in temperature, and acceleration of the pulse-rate. Severe constitutional reactions are generally due to excessive dosage, but may occur in some persons after doses that were previously well borne.

Frequency and Dosage of Inoculation.-No definite rules can be laid down, each patient being a law unto himself. The opsonic index has been largely abandoned as a guide to the administration of vaccine, the reaction and condition of the patient now governing the dosage. 
In more acute infections, and in delicate persons, smaller doses are usually indicated. It is well to make the first dose small, and if no reaction occurs within forty-eight hours, a second and a larger dose may be given. If, however, the patient presents other symptoms of a general reaction, the dose given was large enough, and may be repeated, as necessary, at intervals of from five to seven days. It should be carefully borne in mind that an increase in dosage is contraindicated so long as any sign of general or focal reaction is produced and steady progress is maintained. One should always be on guard to detect any signs of fresh infection by some other organism, and if a given vaccine is failing to exert a beneficial effect, additional cultures should be made, instead of continuing to administer dose after dose of the same vaccine.

The intervals at which injections are to be made are of some importance. It is certainly better to wait too long than to inoculate prematurely, but the ghost of the "negative phase" is always too prominent in the minds of the inexperienced. The inoculations may be given while improvement is still in progress or convalescence well established, in the endeavor to secure a summation of positive phases of clinical improvement; or one may wait for the first signs of retrogression before administering another dose. The former method is the preferable procedure, but is difficult to accomplish; the latter is less ideal, but is easier to perform and more devoid of risk.

The dosage varies according to whether the infection is acute or chronic, the nature of the microörganism, and the age of the patient. No fixed rules can be given. In acute infections the dose should be small and may frequently be repeated; in chronic infections larger doses may be given at longer intervals. If in doubt as to the size of the dose to be given, it is better to give a small dose, and carefully observe the effect on the patient, letting this serve as an index to subsequent doses. Children tolerate relatively large doses of bacterial vaccines, but the dosage should depend on the weight and not on the age of the child.

The following is a list of the ordinary doses for adults of various bacterins:

Staphylococcus aureus. .........100,000,000 to $1,000,000,000$

Staphylococcus albus and citreus. . .200,000,000 to $1,000,000,000$

Streptococcus pyogenes . . . . . . . 25,000,000 to 200,000,000

Gonococcus. . . . . . . . . . . . 25,000,000 to 200,000,000

Typhoid bacillus . . . . . . . . . 250,000,000 to $1,000,000,000$

Colon bacillus. . . . . . . . . . . $1,000,000,000$ 


\section{CHAPTER XIV}

\section{ANTITOXINS}

For general purposes the antibodies produced during infection may be divided into two groups, the first consisting of those antibodies that are truly antagonistic to the bacterium or its products responsible for their production, and the second those that are not in themselves destructive, but that probably prepare the bacterium for the action of a more powerful antibody of the first group.

To the first group belong the antitoxins, which neutralize the toxins of a bacterium without being directly destructive to the microörganism itself; and the bacteriolysins, which are truly destructive, causing the bacterium to break up and finally disappear.

To the second group belong the opsonins, which, as we have seen, prepare the bacterium for phagocytosis; and the agglutinins and precipitins, which, while not in themselves destructive, probably in some manner prepare their antigen for the action of bacteriolysins, just as opsonins prepare them for phagocytosis.

Definition.-Antitoxins are antibodies in the blood that are capable of directly and specifically neutralizing the dissolved toxins that caused their production.

Historic.-Bacteriolysins were discovered before antitoxins. Their discovery is due to the researches of Nuttall, Fodor, Buchner, and others, who showed that normal serum, and especially the serum of animals artificially immunized against a certain bacterium, was able to exert a destructive action on the microörganism, causing its dissolution and final disappearance. This property of the blood-serum was found to diminish with age, and to disappear completely when the serum was heated to $56^{\circ} \mathrm{C}$. Buchner laid greatest stress upon the importance of the thermolabile substance which he called alexin, but later researches have shown that the main factors are the specific bacteriolysins, which, however, are practically powerless to destroy their antigen without the coöperation of alexin (later renamed "complement" by Ehrlich).

While these studies were being made, in the hope of thus explaining all phases of immunity, Behring discovered that in diphtheria infections 
induced experimentally, while the animals became more and more immune, virulent bacilli may, nevertheless, be present at the site of injection. Here, then, was an example of immunity that could not be explained on the basis of bacteriolysis. Later, in 1890 and 1892, Behring, in collaboration with Kitasato and Wernicke, made further important discoveries, showing that the blood-serum of animals actively immunized against diphtheria and tetanus would protect normal animals against these diseases, and, furthermore, that the blood-serum of the immune animals did not possess bactericidal properties. These observers also demonstrated that such serum could be used therapeutically for the cure of an infection already in progress.

Soon after these discoveries Ehrlich showed that specific antitoxins (antiricin, antiabrin, etc.) could also be produced against the poisons of some plants, and Calmette produced a similar antitoxin (antivenin) against snake poison. Other observers since then have increased the list of poisons against which antitoxins can be produced; as, for example, Kempner has produced an antitoxin against the poison of Bacillus botulinus, and Wassermann one against that of Bacillus pyocyaneus.

Formation of Antitoxins.--It was formerly believed that there was a direct conversion of toxin into antitoxin, but this certainly is not the case, for the amount of antitoxin produced is altogether out of proportion to the amount of toxin injected.

Antitoxins are formed by those cells that anchor the toxins. In order to produce them it is necessary that the toxin enter into direct union with the cells and exert a stimulating influence on them, for where a loose union occurs, as between cells and alkaloids, antibodies are not formed.

Having entered into chemical union with the side-arms of cells, a toxin may destroy the entire cell, and if a sufficient number of these are destroyed, the host will show symptoms of infection and may succumb. If, however, the cell itself is not destroyed, but only one or more of the side-arms injured, the damage is repaired by the cell forming new side-arms that have a specific affinity for the toxin responsible for their production. According to Weigert's overproduction theory, a cell once stimulated to produce these side-arms or receptors continues to produce them for some time, even after the stimulus has been removed. In this manner the specific receptors are produced in excess, and since all cannot remain attached to the parent cell, the excess is discharged into the blood-stream. Each of these cast-off receptors is capable of uniting with toxin, thus neutralizing the poisonous principles 
the toxin, and rendering it practically harmless. Antitoxins, therefore, are nothing more than these cast-off receptors, which have a specific affinity for their toxins (Fig. 67).

As Adami has pointed out, it is probable that the toxins exist for some time within the cell, not as part and parcel of the cell, but as a stimulating agent that causes the cell to develop the habit of producing the specific receptors. The mere union of toxin with a receptor, causing it to fall off, and being followed by nature's mode of repair, with the formation of an excess of receptors and no further stimulation, is hardly sufficient to explain the enormous activity of the cells.

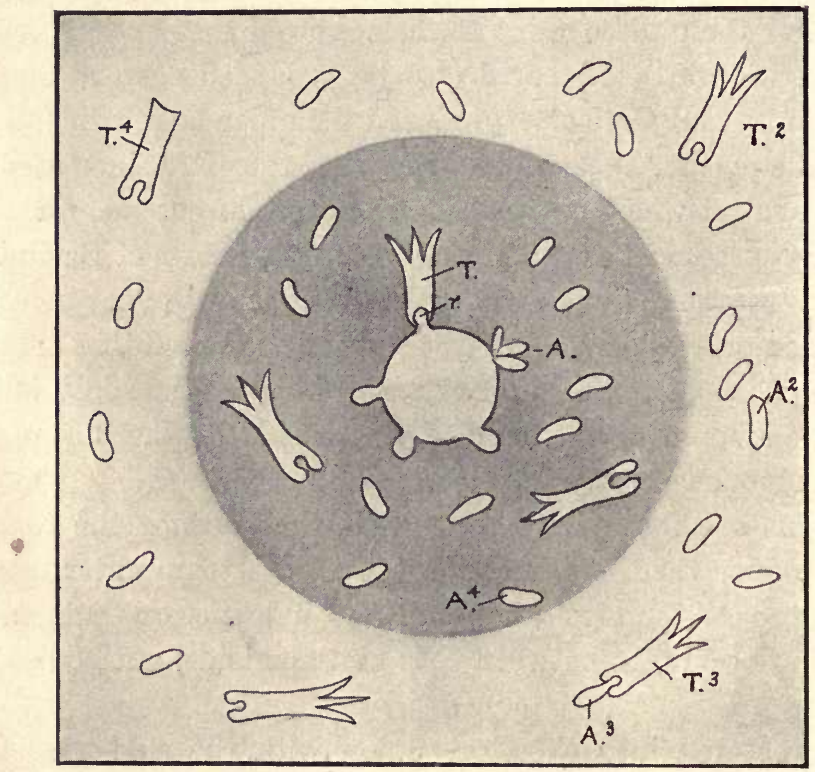

Fig. 67.-Theoretic Formation of Antitoxins.

That antitoxins may be produced locally was illustrated by the experiment of Romer with abrin. This substance has a peculiarly powerful effect upon the conjunctiva. By gradually immunizing the right conjunctiva of a rabbit with increasing doses, it was shown that, after killing the animal and triturating the conjunctiva with a fatal dose of abrin, an injection of the emulsion of the right or immunized conjunctiva was without effect, whereas the emulsion from the left proved fatal. Thus it will clearly be seen that the cells that had absorbed the abrin had developed and contained antiabrin in sufficient amounts to neutralize the poison. 
While leukocytes, such as Metchnikoff's macrophages, are likewise active in the formation of antitoxins, it is certain that they are not the only cells involved. Metchnikoff claims that antitoxins are merely toxins altered by leukocytic activity, rather than constituents of tissuecells; this explanation is, however, inadequate, and it has been shown experimentally that the quantity of antitoxin produced is so far in excess of the amount of toxin injected as to render this view untenable.

Structure of Antitoxins.-According to the side-chain theory, antitoxins are the simplest of antibodies, being composed of a single arm or haptophore group for union with the toxin, and called receptors of the first order. While illustrations of this theoretic structure will convey the impression of mere physical contact or union with toxin, it is to be remembered that experimental data indicate that the union and consequent neutralization of the toxin are chemical processes.

Properties of Antitoxins.-While chemical analyses to determine the nature of antitoxin serums were made as early as 1897, little is known regarding it because it is impossible to secure the antitoxic element free from serum and serum constituents. Belfanti and Carbone found that most of the antitoxin in a serum is precipitated with the globulins by saturation with magnesium sulphate. This work, which has been verified by Atkinson and Pick, shows that the antitoxin is carried down with the globulin precipitates, but does not necessarily prove that it is itself a globulin. Later Gibson and Banzhaf showed that the portions of the globulin precipitate soluble in saturated sodium chlorid solution carried most of the antitoxin, and with this discovery a practical method of eliminating much of the non-antitoxic portion of the serum was perfected.

The relation of antitoxins to proteids has also been studied, digestive ferments being permitted to act on antitoxic serum. It has been shown that antitoxin resists tryptic digestion to a well-marked degree; in this respect it resembles the serum globulin. All the evidence obtained indicates that a closer relation of antitoxins to proteids exists than has been shown for the toxins, although all attempts to separate antitoxins from proteids have thus far failed.

Antitoxins are fairly resistant bodies, and a properly prepared antitoxic serum, when kept in a cool place and protected from light and air, may be preserved for a year or more with very little deterioration in strength. At times, however, for unknown reasons, antitoxins gradually deteriorate, losing about 2 per cent. in strength a month. Manufacturers have endeavored to calculate this loss in strength, and have 
placed a label on each package of antitoxin, bearing a date beyond which the serum is not guaranteed to contain the amount of antitoxin present at the time it was put up.

The antitoxins, with few exceptions, are far more stable than the toxins, resisting heating up to $62^{\circ} \mathrm{C}$., but gradually deteriorating with higher temperatures. Boiling destroys them completely. They are readily preserved with small amounts of chloroform, phenol, tricresol, etc., although strong solutions of these produce destructive changes. Putrefaction of the serum destroys the antitoxin content. Ehrlich has devised the best method for their preservation, which consists in drying the serum in vacuo and preserving it in the dark, at a low temperature, in the presence of anhydrous phosphoric acid. So preserved, antitoxin retains its strength for prolonged periods and is used in standardizing toxins.

Natural Antitoxins.-The appearance of so-called natural antitoxins can be explained on the basis of Ehrlich's theory. Since the antitoxin is composed of receptors that are not new bodies, but simply normal receptors produced in excess, it is reasonable to assume that a few may be thrown off occasionally, constituting the natural antitoxin.

Small amounts of natural diphtheria antitoxin may be found in certain individuals and lower animals. Since the diphtheria bacillus is so wide-spread in its distribution, it is possible that minor subinfections may be responsible for antitoxin production, and this is probably always the case when large amounts are found.

Information regarding natural antitoxins for other members of the toxin-producing group of microörganisms is less complete, although it is highly probable that natural antitoxins for these exist.

Specificity of Antitoxins.-Antitoxins well illustrate the law of specificity that exists between antigen and antibody, since they are strictly specific for their toxins. Diphtheria antitoxin will neutralize only diphtheria toxin; tetanus antitoxin, only tetanus toxin, and so on through the list. This specificity is not confined to the particular toxin-producing organism that generates the antitoxin; for example, there are various kinds of diphtheria bacilli, differing as regards morphology and toxicity, although one antitoxin appears to act the same with their various toxins.

Nature of the Toxin-Antitoxin Reaction.-While the injection of toxinantitoxin mixtures into the lower animals is the only practical method of testing and standardizing the curative and prophylactic powers of their serums, this method does not throw much light upon the nature of the toxin-antitoxin reaction, or show how antitoxin overcomes the toxin. 
Antitoxin is protective and curative, in that it actually destroys the toxin, in a manner similar to the dissolution of a bacterium caused by a specific bacteriolysin; or it may influence the tissue-cells in some way and render them more resistant to the toxins, a view that was held by Roux, and particularly by Buchner; or the antitoxin may form a direct chemical union with the toxin, similar to the chemical neutralization of an acid by a base- an opinion early held by Behring and elaborated later by Ehrlich.

Experimental data support the view of chemical union with the toxin. In the test-tube some time is required for the union of toxin and antitoxin to occur; this union is hastened by heat and retarded by cold; it is more rapid in concentrated than in dilute solutions, and in general takes place in accordance with the law of multiple proportions-all of which tends to show the close similarity of the toxin-antitoxin reaction to a chemical process.

It is generally conceded that antitoxin does not directly destroy the toxin, for when neutral mixtures of toxin and antitoxin are injected into animals, portions of toxin may become dissociated and unite with tissuecells possessing greater affinity for the toxin, and symptoms of infection may result. It is probable that toxin and antitoxin form a distinct compound, and this action requires time for its consummation. For example, Martin and Cherry, by filtering mixtures of toxin and antitoxin through fine filters that would permit the toxin molecule to pass through but restrain the larger antitoxin molecule, found that, if filtered immediately, all the toxin in the mixtures was extruded, but that, as the interval between mixing and filtration was prolonged, less and less toxin appeared in the filtrate, until finally, two hours after mixing, no toxin whatever passed through the filter.

This element of time in support of the chemical nature of the reaction is further strengthened by the experiments of Calmette with snake venom and antivenin, and likewise serves to demonstrate that the antitoxin apparently does not directly destroy the toxin. Although most toxins are thermolabile, Calmette found that snake venom is rendered inert by heating to $68^{\circ} \mathrm{C}$., whereas the antivenin remains uninfluenced by a temperature of $80^{\circ} \mathrm{C}$. When neutral mixtures of venom-antivenin were heated to $70^{\circ} \mathrm{C}$., they were found to become toxic again, presumably on account of the destruction of the antivenin, the venom itself not being destroyed. Similar experiments were carried out by Wassermann with mixtures of pyocyaneus toxin-antitoxin, with similar results. In both instances, however, as developed later, if the mixtures had been 
allowed to stand longer, these results would not have been secured. Although performed originally to show that an antitoxin does not act by actually destroying its toxin, these experiments simply demonstrate the importance of the element of time in the reaction, without throwing any real light upon the nature of the new toxin-antitoxin compound, if such exists.

That toxin is counteracted by antitoxin, independent of the participation of living tissue-cells, has been quite conclusively proved by experiments in vitro. Ehrlich showed that the agglutinating qualities of ricin - a vegetable toxin-may be overcome in the test-tube by adding antiricin, the corresponding antitoxin. Similar results were obtained by Ehrlich with tetanolysin and tetanus antitoxin, and by Stephens and Myers with cobra venom and its antivenin.

It is probable that antitoxin has a similar action when injected for therapeutic purposes, as for curing an infection. The longer the interval that has elapsed between the time of infection and the administration of antitoxin, the less satisfactory will be the result, as antitoxin becomes less powerful when toxins have formed a firm union with the body-cells. This is especially true in tetanus, where even very large doses of antitoxin may be incapable of dissociating the toxin molecule from the nerve-cells, the serum, therefore, being of greatest value in prophylaxis. In diphtheria, however, the union between toxin and cells is less firm, and the antitoxin is probably capable of neutralizing the toxin already present in the cells, and especially any toxin that may become dissociated from the cell or is freshly prepared by the diphtheria bacillus at the site of infection. The indication, therefore, in giving antitoxin, is to give a dose large enough to neutralize all free and loosely bound toxin, with an excess to neutralize dissociated toxin and that prepared by the bacillus during the course of the infection.

The introduction of the test-tube experiment into the investigation of these reactions permitted more exact observations to be made, and the evidence secured by this means, as well as by carefully graded quantitative animal experiments, would seem to indicate that we should accept, for the present at least, the conception of the chemical nature of the process.

\section{PRODUCTION OF ANTITOXINS FOR THERAPEUTIC PURPOSES}

Diphtheria and tetanus antitoxins are manufactured on a large scale, and are used extensively in the prevention and cure of these in- 
fections. They are prepared by immunizing horses with carefully graded and increasing doses of the respective toxins until the serum of the animals shows a sufficiently high antitoxin content, after preliminary trials, to warrant more extensive bleeding. Large quantities of blood are then collected aseptically by puncturing the jugular vein. The serum is carefully separated and standardized according to an accepted technic, in order to determine the antitoxin content in units. A small amount of preservative is added, and the serum is finally dispensed in special containers or syringes ready for administration. In some laboratories it is customary to precipitate the globulin fraction of the serum with magnesium or ammonium sulphate, and redissolve the portion containing most of the antitoxin in saturated sodium chlorid solution. The bulk of the serum is thus greatly decreased, and objectionable constituents largely eliminated, to the obvious advantage of the preparation for therapeutic purposes.

Antitoxins have also been prepared for other bacterial toxins, as those of the dysentery bacillus (Kruse-Shiga) and Bacillus botulinus, for the vegetable toxins in pollen, and for the animal toxins in snake venoms.

There are other serums for the treatment of certain infections, which depend for their effects chiefly upon the presence of bacteriolysins and immune opsonins, and these are described in a subsequent chapter.

\section{THE PRoduction of DiPHTHERIA ANTITOXIN}

The following, taken largely from Park, is a widely used and accepted technic for the production of diphtheria antitoxin:

Production of the Diphtheria Toxin.-A strong diphtheria toxin should be obtained by growing a virulent culture in a 2 per cent. nutrient peptone bouillon made from "bob" veal, of an alkalinity that should be about 9 c.c. of normal soda solution per liter above the neutral point to litmus, and prepared from a suitable peptone (Witte). The broth should be poured into large-necked Erlenmeyer flasks in comparatively shallow layers, so as to allow of the free access of air, and maintained at a temperature of about $35^{\circ}$ to $36^{\circ} \mathrm{C}$. (Fig. 68).

In the Hygienic Laboratory of the Public Health and Marine-Hospital Service "Smith's bouillon" is used for preparing the toxin. This is made of fresh lean beef, after the muscle sugar and all other sugars have been removed by fermentation with a good culture of Bacillus coli. The reaction is adjusted until 0.5 per cent. acid to phenolphthalein, that is still distinctly alkaline to litmus, and 1 per cent. peptone, 0.5 per cent. 
sodium chlorid, and 0.1 per cent. dextrose are added. The reaction is again noted, and adjusted to +0.5 per cent. The broth is then filtered through filter-paper into flasks and test-tubes and sterilized in the autoclave at a temperature of $120^{\circ} \mathrm{C}$. for twenty minutes.

After incubating for from seven to ten days the culture is removed, and its purity having been tested by microscopic and cultural methods, it is rendered sterile by the addition of 10 per cent. of a 5 per cent. solution of carbolic acid. After forty-eight hours the dead bacilli have settled on the bottom of the jar, and the clear fluid above is siphoned off, filtered, and stored in full bottles in a cold place until needed (Fig. $69)$.

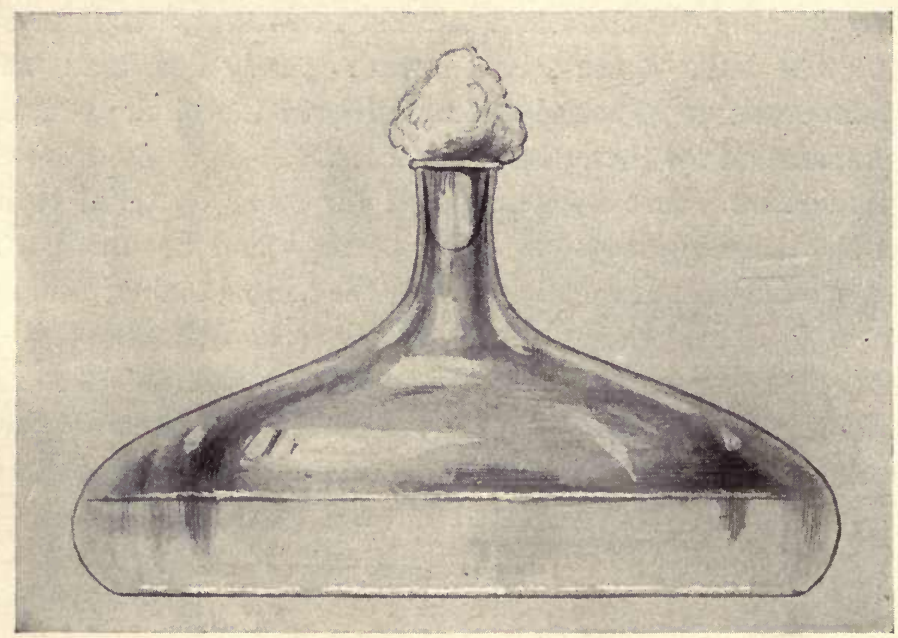

Fig. 68.-A Flask of Diphtheria Culture.

The bacilli grow on the surface and form a scum. As the culture grows older, the bacilli die and sink to the bottom of the flask. A flask of this shape affords a large surface of culture-medium in contact with oxygen and facilitates toxin production.

Testing the Toxin.-The strength of the toxin is then tested by injecting a series of guinea-pigs with carefully measured amounts. When injected hypodermically, less than 0.005 c.c. should kill a 250 gram guinea-pig, and a toxin requiring more than 0.01 c.c. to kill a pig of this weight is too weak for present purposes. This preliminary titration of the toxin will suffice for determining the dosage for horses, but in standardizing antitoxin, the technic must necessarily be more accurate.

Immunizing the Animals.-The horses used should be young, vigorous, of fair size, and absolutely healthy. They should be severally 
injected with 10,000 units of antitoxin and with 5000 units of toxin, an amount sufficient to kill 5000 guinea-pigs each weighing 250 grams. If

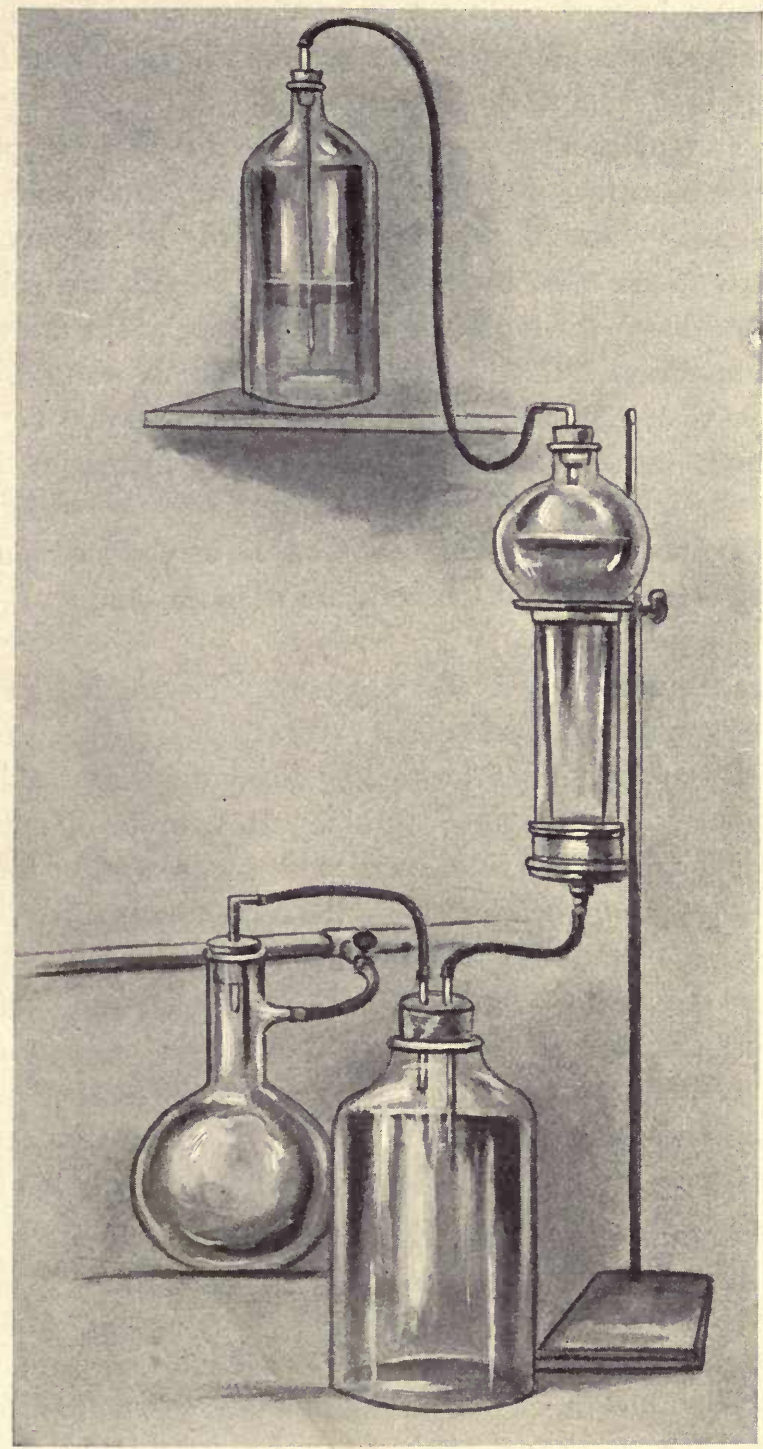

Fig. 69.-A Large Toxin Filter.

The culture is contained in the large bottle on the shelf, and drains into the flask, which in turn empties into the earthen "candle." By means of a vacuum the culture is filtered through the "candle" and collects in the large bottle at the base of the stand.

antitoxin is not given with the first doses of toxin, only one-tenth of the dose advised is to be given. After from three to four days, or as soon as 
the temperature reaction has subsided, a second subcutaneous injection of a slightly larger dose is given, the amount of toxin increasing about 10 to 15 c.c. per dose, until, six weeks later, the animal is receiving from 20 to 30 times the amount originally given. At the end of this time a trial bleeding is made and the serum tested.

There is absolutely no way of judging which horses will produce the highest grades of antitoxin. Roughly estimated, those horses that are extremely sensitive and those that react feebly are the poorest, but there are exceptions even in these cases. The only reliable method, therefore, is to bleed the horses at the end of six weeks or two months and test their serum. If only high-grade serum is wanted, all horses that give less than 150 units per cubic centimeter should be discarded. The remaining horses should receive steadily increasing doses, the rápidity of the increase and the interval of time between the doses (three days to one week) depending somewhat on the reaction following the injection, an elevation of temperature of more than $3^{\circ} \mathrm{F}$. being undesirable.

For example, according to Park, a horse that yielded an unusually high grade of serum was started on 12 c.c. of toxin $\left(\frac{1}{400}\right.$ c.c. fatal dose), together with 10,000 units of antitoxin. Sixty days later a dose of 675 c.c. was given, and the serum contained 1000 units of antitoxin per cubic centimeter. Regular bleedings were made weekly for the next four months, at the end of which time the serum had fallen to 500 units in spite of weekly gradually increasing doses of toxin. At the end of three months the antitoxic serum of all the horses should contain over 300 units, and in about 10 per cent. as much as 800 units in each cubic centimeter. Not more than 1 per cent. give above 1000 units, and, according to Park, so far none has given him as much as 2000 units per cubic centimeter. The very best horses, if pushed to their limit, continue to furnish blood containing the maximum amount of antitoxin for several months, and then, in spite of increasing injections of toxin, begin to furnish blood of gradually decreasing strength. If an interval of three months' freedom from inoculation is allowed once every nine months, the best horses will furnish high-grade serum for from two to four years.

Collecting the Serum.--In order to obtain the serum, the neck of the horse should be cleansed thoroughly as for an aseptic operation, and a special tourniquet applied to distend the jugular vein. A small slit is made through the skin over the vein, and a special sharp-pointed cannula is passed upward under the skin for two inches or more and 


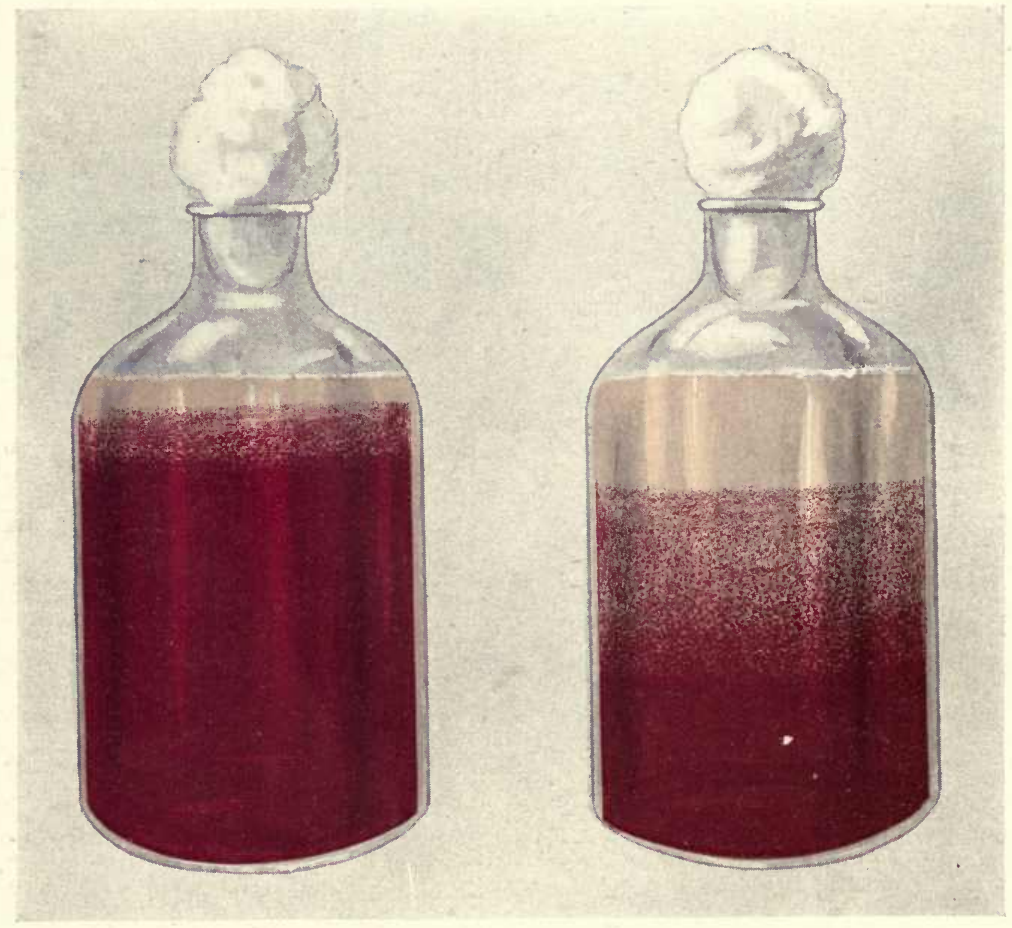

Fig. 70.-Preparation of Diphtheria Antitoxin. Separation of BloodSERUM.

The bottle on the left shows blood after standing about an hour; the bottle on the right shows the separation of serum about twelve hours after bleeding. 
then plunged into the vein. From 6 to 12 liters of blood are collected by a rubber tube into cylindric jars provided with special tops, facilitating filling with blood and subsequent withdrawal of the serum. The cannula, tubing, jars, and everything used in collecting the blood and serum should be carefully sterilized, and the whole operation should be conducted with scrupulous aseptic care in order to avoid contamination. (See Fig. 26.)

The jars are set aside (Fig. 70) for three or four days, and the serum is drawn off by means of sterile glass and rubber tubing and stored in large sterile bottles. When the globulins are to be separated, the blood may be added directly to one-tenth of its volume of a 10 per cent. solution of sodium citrate, which prevents clotting of the blood.

The serum should be clear and free from blood, and its sterility should be proved by culture tests. An antiseptic, such as 0.4 per cent. tricresol, 0.5 per cent. phenol, or chloroform, may be added, but this is not necessary unless it is desired to keep the serum for some time. The serum is poured into small bottles fitted with rubber stoppers, or placed in special syringes labeled with the number of units contained. The whole process should be conducted with scrupulous aseptic technic. Diphtheria toxin varies too much to be used as a standard in determining the antitoxin content of a serum; hence a dried antitoxin is prepared by the Hygienic Laboratory and is distributed for this purpose. The serum is evaporated and dried in vacuo by passing dry sterile air heated to $35^{\circ} \mathrm{C}$. through it, and when perfectly dry, is preserved in special containers over anhydrous phosphoric acid at a constant temperature of $5^{\circ} \mathrm{C}$. Preserved in this manner, the antitoxin is quite stable. Just before use it is dissolved in the required amount of sterile normal salt solution.

Standardizing the Serum.-During the earlier investigations it was believed that toxin was quite stable, and that it possessed a definite toxicity with a constant value in neutralizing antitoxin. Upon these suppositions the original Behring-Ehrlich antitoxin unit was based, consisting of 10 times the amount of antitoxin that neutralized 10 fatal doses of toxin. For example, if the minimal lethal dose (M. L. D.) of toxin was 0.001 c.c., and 0.01 c.c. was neutralized by 0.01 c.c. of serum, then 0.1 c.c. of serum equaled one unit, or 10 units in a cubic centimeter. Later stronger serums were found, and von Behring and Ehrlich modified the unit, which they now call the immunity unit, to be that quantity of antitoxin which will neutralize 100 times the minimal fatal dose for a 250-gram guinea-pig. 
It was soon discovered that toxins are unstable compounds, and that, almost immediately after their production, they begin to change into toxoids, which are not acutely poisonous, but which retain their power to neutralize antitoxin.

In order to standardize a serum it is necessary that the strength of the toxin be known, and since this is so variable, a standard antitoxin is supplied by the Hygienic Laboratory, by which the various antitoxin plants may measure the strength of their toxins. By mixing varying quantities of toxin with one unit of this standard antitoxin and injecting these into 250-gram guinea-pigs, the $\mathrm{L}_{+}$(limes death) dose is obtained, which is the dose of toxin required to kill a pig in four days with the one unit of antitoxin. In order accurately to determine this dose many pigs may be required, but this method of titration is the key-note to successful standardization.

Such a titration for instance, has shown a toxin to react as follows:

\section{TABLE 1.-METHOD OF DETERMINING THE L+ DOSE OF DIPH- THERIA TOXIN}

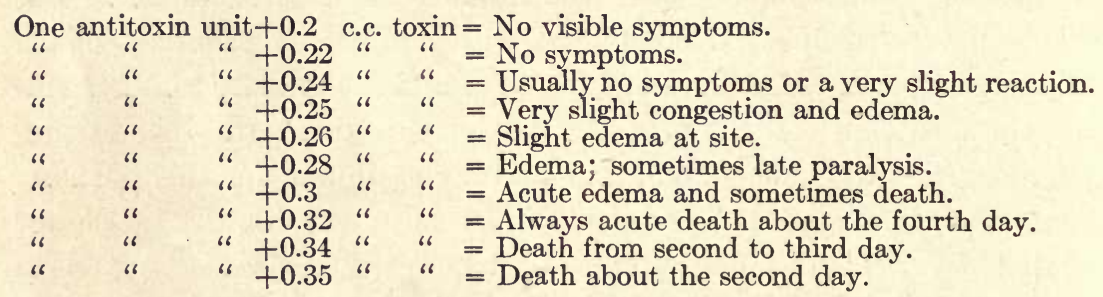

Here the $\mathrm{L}_{+}$dose is 0.32 c.c. The dose of toxin that just neutralizes the antitoxin without causing symptoms has been called by Ehrlich the Lô (limes zero) dose, and in this instance it is about 0.24 c.c. This determination, however, has not the same practical value as the $\mathrm{L}_{+}$dose.

Having determined the $\mathrm{L}_{+}$dose of the toxin, a series of six to eight guinea-pigs are injected with this constant dose of toxin and increasing amounts of the corresponding antitoxin serum; for instance, No. 1 would receive 0.001 c.c. of serum; No. $2,0.002$ c.c.; No. $3,0.003$ c.c.; No. $4,0.004$ c.c.; No. $5,0.005$ c.c.; No. $6,0.006$ c.c., etc. If at the end of the fourth day Nos. 1, 2, 3, and 4 were dead and Nos. 5 and 6 were alive, the serum would contain 200 units of antitoxin in a cubic centimeter. These injections are best given with precision syringes, the one devised by Hitchens being particularly serviceable (Fig. 71). The syringes are sterilized, and the needles are dipped in sterile vaselin to 
plug them. The mixtures are made in the barrel of the syringe, and sufficient sterile salt solution is placed in the side-arm to bring the total volume of the injection up to 4 c.c., and to wash in all traces of toxin and

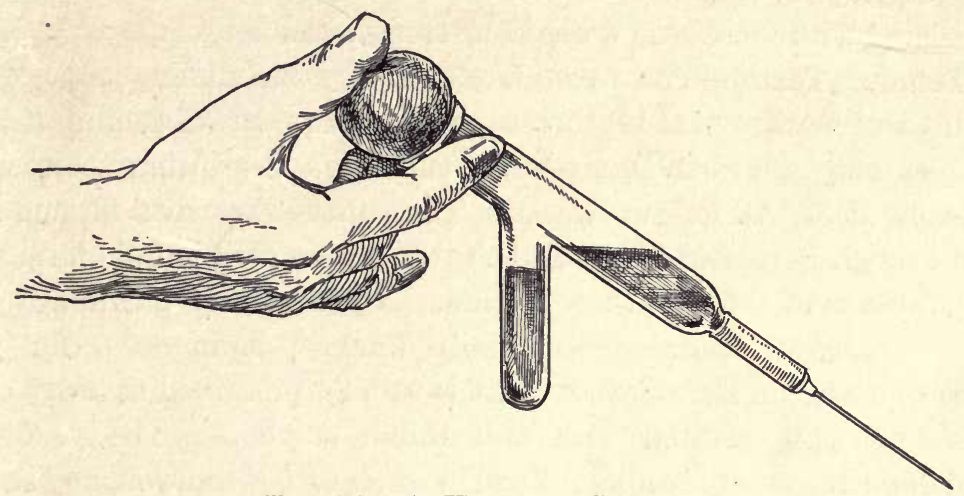

Fig. 71.-A Hitchens Syringe.

The needle is plugged by dipping the tip in carbolized vaselin. The side arm holds sterile salt solution; when the needle has been entered, the injection is given by pressure on the bulb; the side arm is then turned upward, when the contents flow into the main barrel, and injected in this manner insures accuracy in dosage and uniform bulk of inoculum.

antitoxin. The mixtures are allowed to stand for at least fifteen minutes (Park) before being injected (Fig. 72). The pigs must be of proper weight-i. e., about 250 to 300 grams; the abdominal wall is shaved,

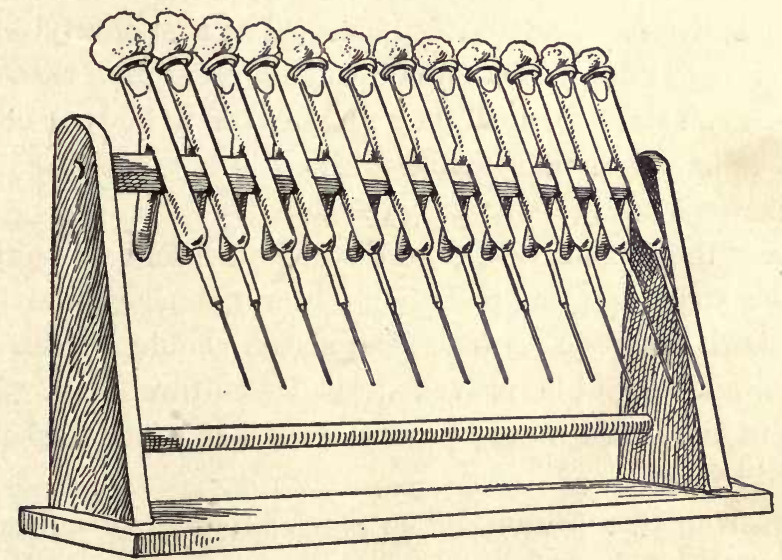

Fig. 72.-A Battery of Hitchens Syringes.

and the injection given directly in the median abdominal line. The animals are placed two in a cage, and carefully observed for four or five days for symptoms of toxemia and edema about the site of injection. 


\section{PRODUCTION OF TETANUS ANTITOXIN}

The method used in the production of tetanus antitoxin is similar to that employed in producing diphtheria-antitoxin, the horses being inoculated with increasing doses of a strong tetanus toxin.

Tetanus Toxin.-The toxin is secured by inoculating large flasks or tubes of neutral veal broth containing 1 per cent. of sodium chlorid and peptone with abundant tetanus culture, and growing these anaërobically at $37^{\circ} \mathrm{C}$. for two weeks. The cultures are then filtered rapidly through Berkefeld filters, and the toxin preserved in fluid form with the addition of 0.5 per cent. phenol. As previously mentioned, the toxin rapidly deteriorates - especially tetanospasmin-and for purposes of antitoxin standardization it is usually preserved in a dry state after being precipitated with ammonium sulphate. The yellowish, crystalline masses are readily soluble in water or salt solution, and should be used immediately after solution takes place. The strength of the toxin is determined by injecting increasing amounts into white mice or 350-gram guinea-pigs.

Immunizing the Animals.-According to Park, the "horses receive 5 c.c. as the initial dose of a toxin, of which 1 c.c. kills 250,000 grams of guinea-pig, and along with this twice the amount of antitoxin required to neutralize.it. In five days this dose is doubled, and then every five to seven days larger amounts are given. After the third injection the antitoxin is omitted. The dose is increased at first slowly until appreciable amounts of antitoxin are found to be present, and then as rapidly as the horses can stand it, until they support 700 to 800 c.c. or more at a time. This amount should not be injected in a single place, or severe local and perhaps fatal tetanus may develop."

Collecting the Serum.-The horses are bled, and the serum is collected under strict aseptic precautions, in a manner similar to the collection of antidiphtheric serum. The serum should be clear and free from blood, and should be proved sterile by cultural tests. It may be preserved in the liquid state by adding 0.5 per cent. of phenol or 0.4 per cent. of tricresol.

Standardizing the Serum.-The official immunity unit of tetanus antitoxin of the United States Government is based largely upon the work of Rosenau and Anderson. These investigators, together with a Committee of the Society of American Bacteriologists, have defined the unit of tetanus antitoxin to be "ten times the least amount of serum necessary to save the life of a 350-gram guinea-pig for ninety-six hours against 
the official test dose of a standard toxin. This test dose consists of 100 minimal lethal doses of a precipitated and dried toxin, tested out against 350-gram pigs, and preserved in the Hygienic Laboratory, from where it is sent to various antitoxin plants for the purpose of securing a uniform method and unit of standardization.

In standardizing tetanus antitoxin, the $\mathrm{L}_{+}$dose of toxin is employed. A standard toxin and an antitoxin, arbitrary in their first establishment, are preserved in the Hygienic Laboratory, and are kept constant by making frequent tests one against the other. In determining the $\mathrm{L}_{+}$dose, increasing amounts of toxin are mixed with a constant amount of antitoxin equal to one-tenth of an immunity unit, and injected into 350 -gram pigs. The $\mathrm{L}_{+}$dose must contain just enough toxin to neutralize this amount of antitoxin and kill a pig in four days. This $\mathrm{L}_{+}$dose of toxin is sent out by the Hygienic Laboratory to those interested, commercially or otherwise, in the manufacture of antitoxin for purposes of standardization.

For determining the strength of an unknown serum a large number of mixtures are made, each containing the $\mathrm{L}_{+}$doses of the toxin and increasing quantities of antitoxin. The measurements are made with accurate volumetric pipets, and the total volume brought up to 4 c.c. with sterile salt solution in order to equalize concentration and pressure. The mixtures are allowed to stand at room temperature for an hour, and are then injected subcutaneously into 350-gram pigs. This method of titrating the antitoxin is shown in the following example from Rosenau and Anderson:

TABLE 2.-METHOD OF TITRATING TETANUS ANTITOXIN

\begin{tabular}{|c|c|c|c|c|}
\hline \multirow{2}{*}{$\underset{\text { PIG }}{\text { No. or }}$} & \multirow{2}{*}{$\begin{array}{l}\text { WEIGHT OF } \\
\text { PIG IN GRAMS }\end{array}$} & \multicolumn{2}{|c|}{$\begin{array}{l}\text { Subcutaneous Injection of } \\
\text { a Mixture of- }\end{array}$} & \multirow{2}{*}{ Time of Death } \\
\hline & & $\begin{array}{l}\text { Toxin (Test } \\
\text { Dose) }\end{array}$ & Antitoxin & \\
\hline $\begin{array}{l}1 . \\
2 . \\
3 . \\
4 . \\
5 .\end{array}$ & $\begin{array}{l}360 \\
350 \\
350 \\
360 \\
350\end{array}$ & $\begin{array}{c}\text { Gram } \\
0.0006 \\
0.0006 \\
0.0006 \\
0.0006 \\
0.0006\end{array}$ & \begin{tabular}{l}
\multicolumn{1}{c}{ C.c. } \\
0.001 \\
0.0015 \\
0.002 \\
0.0025 \\
0.003
\end{tabular} & $\begin{array}{l}\text { Two days four hours } \\
\text { Four days one hour } \\
\text { Symptoms } \\
\text { Slight symptoms } \\
\text { No symptoms }\end{array}$ \\
\hline
\end{tabular}

In this series the animal receiving 0.0015 c.c. of antitoxin died in approximately four days; this amount of serum, therefore, represents $\frac{1}{10}$ of one unit. 


\section{BOTULINUS ANTITOXIN}

The nature of the botulinus poison has previously been described. Wassermann has recently immunized horses against this toxin, and the antitoxin shows unmistakable value in animal experiments, although it has not been employed frequently enough in this form of poisoning in human beings to prove its value.

\section{ANTIDYSENTERIC SERUM}

The Kruse-Shiga type of dysentery bacillus has been shown to produce varying amounts of a soluble toxin; and antiserums, which are partly antitoxic and partly bactericidal in nature, have been prepared, and have apparently yielded good therapeutic results in the hands of several observers. Potent antiserums for the Flexner type of bacillus and for various strains isolated from the feces of cases of infantile ileocolitis have not been produced. Even a virulent strain of the dysentery bacillus does not produce true soluble toxins in a manner comparable to those produced by tetanus and diphtheria. Potent toxins are seldom secured with less than two to three weeks' incubation, and fresh cultures of whole or autolyzed bacilli are likewise quite too toxic, indicating that although a soluble toxin may be produced, considerable endotoxin is also present in the bacilli.

Antidysenteric serum has very little prophylactic value, but in individual cases it frequently exerts a curative action, and should be available for use in institutions and armies when dysenteric infection is prevalent.

The older investigators, such as Kruse and Shiga, produced antiserums by immunization with whole bacilli. Later Kraus and Doerr prepared antitoxic serums with the toxin alone. At the present time the evidence would seem to indicate that the best serums are prepared by injecting both toxins and bacilli, producing a serum that is essentially antitoxic and bactericidal in action.

Culture.-Young and healthy horses are best adapted for immunization. Two methods may be followed: (1) Immunization with toxin or (2) with young cultures of whole bacilli. As previously mentioned, investigations have tended to show that the most potent serums are secured by using mixtures of both toxin and microörganisms.

Several strains of dysentery bacilli should be used, in order that a polyvalent serum may be prepared. Cultures should be grown for two 
weeks at $37^{\circ} \mathrm{C}$, in alkaline broth similar to that used for preparing diphtheria toxin; this should be neutralized to phenolphthalein, and 7 c.c. normal soda solution to a liter added. The minimal lethal dose of the mixed unfiltered cultures is determined by giving young rabbits increasing doses intravenously, in order to obtain a guide as to the proper dose for immunization. Fatal doses produce severe diarrhea and paralysis of the extremities, with rapid loss in weight. Rabbits and horses are quite susceptible to the toxin; guinea-pigs and mice are more resistant.

TABLE 3.-METHOD OF DETERMINING THE MINIMAL LETHAL DOSE OF DYSENTERY CULTURE

\begin{tabular}{|c|c|c|c|}
\hline No. & Weight, Grams & Dose in C.c. & Result \\
\hline $\begin{array}{l}1 \ldots \ldots \cdots \\
2 \ldots \ldots \cdots \\
3 \ldots \ldots \cdots \\
5 \ldots \ldots \cdots\end{array}$ & $\begin{array}{l}710 \\
690 \\
695 \\
690 \\
700\end{array}$ & $\begin{array}{l}0.025 \\
0.05 \\
0.1 \\
0.2 \\
0.3\end{array}$ & $\begin{array}{l}\text { No symptoms } \\
\text { No symptoms } \\
\text { Diarrhea. Recovered } \\
\text { Death third day } \\
\text { Death second to third day }\end{array}$ \\
\hline
\end{tabular}

In this instance the minimal lethal dose was 0.2 c.c. and subsequent cultures of the same strains, grown under similar conditions, showed this dose to remain quite constant.

It is good practice to keep the cultures growing during the entire time of immunization. Cultures may, however, be grown for three weeks, filtered through porcelain, and with the addition of 0.5 per cent. phenol, the toxin preserved for long periods of time. The minimal lethal dose of such a toxin is determined in the manner directed above.

Immunizing the Animals.- Since horses are quite susceptible, the initial dose of unfiltered and unheated culture should not be larger than the minimal lethal dose for a young rabbit. The dosage is gradually increased, and the injections are given subcutaneously for from four to six months, after which several injections of from 300 to 350 c.c. may be given intravenously at one time. If at any time diarrhea and other symptoms of toxemia are well marked, subsequent doses should be smaller and should be given at longer intervals until a higher immu-' nity is produced.

Instead of using bouillon cultures, young agar cultures may be used, the bacilli being grown for seventy-two hours, and one-tenth of an ordinary slant being given as the first dose. The early doses are heated to $60^{\circ} \mathrm{C}$. for an hour and injected subcutaneously; the later doses consist of cultures washed from 30 to 40 tubes, and are given intravenously. 
Collecting and Testing the Serum.-After three or four months a trial bleeding should be made and the serum tested as follows: the minimal lethal dose of a culture is determined and ten times this amount placed in a series of tubes or syringes with increasing doses of serum; the total quantity of injection is made up to 4 c.c. with sterile salt solution. The mixtures are set aside for one hour at $35^{\circ} \mathrm{C}$. and injected intravenously in young rabbits. The animals are to be observed for at least five days for diarrhea, paralysis and loss in weight.

TABLE 4.-METHOD OF TESTING ANTIDYSENTERIC SERUM (KRUSESHIGA)

\begin{tabular}{|c|c|c|c|c|}
\hline No. & $\begin{array}{l}\text { WEIGHT, } \\
\text { GRAMS }\end{array}$ & $\mid \begin{array}{l}\text { CULTURE, } \\
0.2 \text { C.c. M. L. D. } \\
\text { C.c. }\end{array}$ & SERUM, C.c. & RESULT \\
\hline $\begin{array}{l}1 \ldots \ldots \\
2 \ldots \ldots \\
3 \ldots \ldots \\
4 \ldots \ldots \\
5 \ldots \ldots \\
6 \ldots \ldots\end{array}$ & $\begin{array}{l}600 \\
610 \\
615 \\
590 \\
600 \\
590\end{array}$ & $\begin{array}{l}2.0 \\
2.0 \\
2.0 \\
2.0 \\
2.0 \\
2.0\end{array}$ & $\begin{array}{l}0.00025 \\
0.0005 \\
0.001 \\
0.002 \\
0.004 \\
0.006\end{array}$ & $\begin{array}{l}\text { Died second day } \\
\text { Died third day } \\
\text { Diarrhea, recovered } \\
\text { Diarrhea, paralysis } \\
\text { No symptoms } \\
\text { No symptoms }\end{array}$ \\
\hline
\end{tabular}

In this instance 0.004 c.c. of serum was sufficient to protect young rabbits against 10 fatal doses of culture, and demonstrated that it is possible to secure a fairly potent serum against the toxins of the KruseShiga microörganism.

According to Todd, if the antiserum is given at least one-half hour after administering the culture, it will protect the rabbit. If given twenty-four hours later, it affords no protection. Similarly, the mixtures of culture and serum must not be injected immediately after mixing, as the results are more irregular than if they are allowed to stand for one-half to one hour before injecting.

If the trial bleeding shows a satisfactory serum, the horse is bled aseptically, as was previously described, and the serum is separated and preserved with 0.5 per cent. phenol in quantities of 10 c.c. in sterile containers. As there is no official immunity unit, the serum is administered in doses of from 5 to 10 c.c. until a therapeutic effect is secured.

\section{ANTISTAPHYLOCOCCUS SERUM}

Both Staphylococcus pyogenes aureus and S. pyogenes albus have been shown to produce certain soluble toxins, such as a leukocidin and a hemolysin, which are partly responsible for the tissue destruction and symptoms that accompany these infections. Severe staphylococcus 
infection is probably due in part to the paralyzing effect and actual destructive action of the leukocidin upon the leukocytes, preventing, for the time being, the walling-off of the lesion and effectual phagocytosis. Antistaphylococcus serums have been shown to counteract the action of the leukocidin and the hemolysin, and may be useful in the treatment of severe, spreading, or metastatic staphylococcus infections.

According to Neisser and Wechsberg, during staphylococcus disease an antihemotoxin is produced against the hemotoxin of the cocci; later Bruck, Michaëlis, and Schulze attempted to show that a demonstration of this antistaphylolysin in the serum may be regarded as evidence of a staphylococcus infection.

Preparation of Antistaphylococcus Serum.-For immunization purposes several different cultures of the Staphylococcus aureus should be used, in order that the antiserums may be polyvalent. Goats or horses may be employed. Cultures may be grown on neutral agar for fortyeight hours, and an emulsion, equivalent to half an agar slant, heated to $60^{\circ} \mathrm{C}$. for one hour and injected subcutaneously in an adult goat. If 10 different strains are used, a four-millimeter loopful from each culture, emulsified in 5 c.c. of sterile salt solution, will be about the proper dose for the first injection. Subsequent doses are given at intervals of a week, and are rapidly increased in size until full, living, unheated cultures are injected intravenously without harm to the animal. The serum may be tested by determining its content of antilysin or of bacteriotropin. Complement-fixation tests are occasionally useful for obtaining an insight into the quantity of bacteriolysin present.

Technic of the Antilysin Test.-The object of this test is to determine the amount of antihemolysin present in a serum, which is dependent on the amount of serum necessary to protect the red blood-cells of rabbits against a solution of the staphylolysin.

(a) Staphylolysin.-This is prepared by growing a known hemolysinproducing staphylococcus in slightly alkaline broth for three weeks, filtering through a Berkefeld filter, and preserving the filtrate with 0.5 per cent. phenol in the refrigerator.

(b) Rabbit Blood.-Remove 2 or 3 c.c. of blood from the ear of a rabbit and place in 5 c.c. of a 1 per cent. sodium citrate in normal salt solution. Wash the corpuscles three times, and make up in a 1 per cent. suspension (dose 1 c.c.) or up to the original volume of blood (dose, 1 drop).

(c) Patient's Serum.-The serum is inactivated by heating to $56^{\circ} \mathrm{C}$. for half an hour. 
(d) Control Serum.-As every normal serum contains a certain amount of antilysin, it is necessary to use a normal control serum. Normal horse serum, dried in vacuo to prevent deterioration, and freshly dissolved for each test in 10 volumes of sterile distilled water or salt solution, has been advocated by Bruck, Michaëlis, and Schulze.

(e) The Test.-It is first necessary to titrate the staphylococcus filtrate to ascertain the amount of lysin present. This is accomplished according to the following scheme:

TABLE 5.-METHOD OF TITRATING STAPHYLOLYSIN

\begin{tabular}{|c|c|c|c|c|}
\hline & $\begin{array}{l}\text { AMOUNT OF } \\
\text { STAPHYLOLYSIN } \\
\text { FILTRATE }\end{array}$ & $\begin{array}{c}\text { RABBit } \\
\text { BLOOD } \\
\text { ONE PER CENT. }\end{array}$ & $\begin{array}{c}\text { Normal } \\
\text { Salt Solution }\end{array}$ & 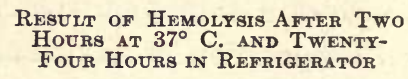 \\
\hline $\begin{array}{l}0.005 \\
0.01 \\
0.02 \\
0.05 \\
0.1 \\
0.2 \\
0.5 \\
1.0\end{array}$ & $\begin{array}{l}5 \text { c.c. } \ldots \ldots \ldots \\
\text { c.c. } \ldots \ldots \\
\text { c.c. } \ldots \ldots \\
\text { c.c. } \ldots \ldots \\
\text { c.c. } \ldots \ldots \\
\text { c.c. } \ldots \ldots \\
\text { c.c. } \ldots \ldots \\
\text { c.c. } \ldots \ldots \\
\end{array}$ & $\begin{array}{ll}1 & \text { c.c. } \\
1 & \text { c.c. } \\
1 & \text { c.c. } \\
1 & \text { c.c. } \\
1 & \text { c.c. } \\
1 & \text { c.c. } \\
1 & \text { c.c. } \\
1 & \text { c.c. }\end{array}$ & $\begin{array}{l}\text { q. s. } 2 \text { c.c. } \\
\text { q. s. } 2 \text { c.c. } \\
\text { q. s. } 2 \text { c.c. } \\
\text { q. s. } 2 \text { c.c. } \\
\text { q. s. } 2 \text { c.c. } \\
\text { q. s. } 2 \text { c.c. } \\
\text { q. s. } 2 \text { c.c. }\end{array}$ & $\begin{array}{l}\text { No hemolysis } \\
\text { No hemolysis } \\
\text { Slight hemolysis } \\
\text { Marked hemolysis } \\
\text { Complete hemolysis } \\
\text { Complete hemolysis } \\
\text { Complete hemolysis } \\
\text { Complete hemolysis }\end{array}$ \\
\hline
\end{tabular}

In this test 0.1 c.c. is the smallest amount of lysin that can completely hemolyze the given quantity of erythrocytes, and is taken as the unit for the second part of the test.

The lytic dose of filtrate just determined is now placed in a series of small test-tubes, with increasing doses of serum to be tested and a constant dose of corpuscles.

TABLE 6.-METHOD OF TITRATING ANTISTAPHYLOLYSIN IN A SERUM

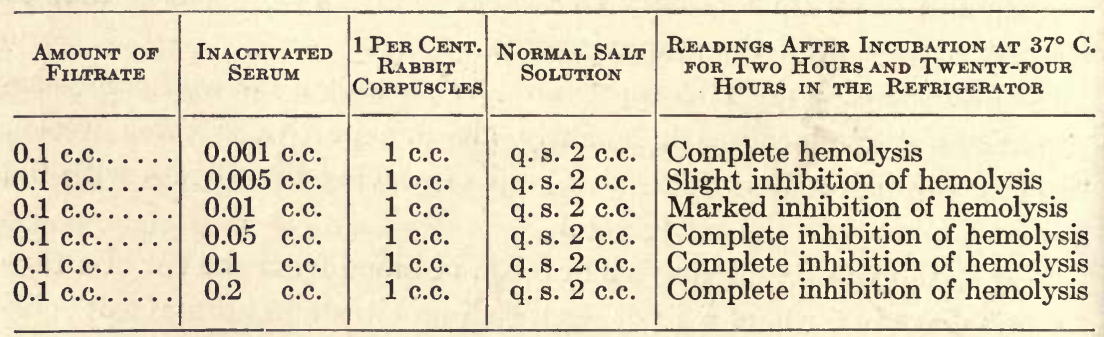

In this instance 0.05 c.c. of the patient's serum was sufficient completely to neutralize the lysin.

A similar test is carried out with normal horse serum. The antilytic dose of this serum is taken as 1 , and the patient's serum is compared 
with this unit. For example, if 0.1 c.c. of normal horse serum was sufficient to neutralize the lysin in this experiment, then the antilysin value of the patient's serum is 2 .

According to Arndt and others, a high antilysin content of a serum is to be regarded as indicating a staphylococcic infection, even if it is impossible to establish fixed limits for the values.

\section{PRODUCTION OF ANTIVENIN}

Snake venom contains two toxins, one being largely neurotoxic and producing paralysis of the respiratory centers, and the other being hemotoxic and irritant, and producing local necrosis of tissues, hemolysis, etc. In venom poisoning the neurotoxic effect is most dangerous. Largely as the result of the work of Calmette and Fraser an antivenin has been prepared that is capable of counteracting the neurotoxic action not only of cobra venom, but to a lesser extent of other venoms as well. These serums, however, appear to have no effect or but very little upon the irritant toxins. In the poisonous American snakes, such as the rattler, moccasin, and copperhead, the effects of the irritant toxins are largely in evidence, and satisfactory antiserums for these venoms have not been prepared (McFarland).

In preparing antivenins the toxins, since they are thermolabile, must be used unheated; subcutaneous injections are usually followed by extensive sloughing, and although a certain amount of immunity may be induced in the horse by intravenous injection, there is apparently no protection against the local action of the toxins.

Preparation of Antivenin.-According to Calmette, horses may be immunized by giving them weekly subcutaneous injections of gradually increasing doses of cobra venom, heated to $70^{\circ} \mathrm{C}$., for an hour, which precipitates the irritant toxins without injuring the neurotoxin. The initial dose is usually 0.01 gram, gradually increased until, by the end of four months, 4 grams may be given at a single dose. The serum is then tested by mixing increasing doses with the minimal lethal dose for a young rabbit, and injecting the mixtures intravenously into a series of rabbits.

Since the neurotoxin may prove dangerous in any case of snake-bite, antivenin may be given to advantage, although the local pain and necrosis are not relieved by the serum. 


\section{PRODUCTION OF POLLEN ANTITOXIN}

The pollen of certain plants is markedly toxic for susceptible individuals. In America the pollen of the golden-rod and of rag weed frequently produce a syndrome of distressing symptoms known as "autumnal catarrh." The onset and character of the symptoms of pollen intoxication are strongly suggestive of an anaphylactic reaction. Dunbar has studied pollen toxins quite extensively, and considers them the etiologic factor in the production of hay-fever.

Pollen antitoxin has been prepared by immunizing susceptible horses, the toxin being isolated by mixing the ground pollen with 5 per cent. sodium chlorid solution and 0.5 per cent. phenol at $37^{\circ} \mathrm{C}$. for ten hours. In the form of a proteid, it is then precipitated by adding eight to ten volumes of 96 per cent. alcohol, dissolving the resultant white precipitate in physiologic salt solution (Citron).

\section{THE MEASURE OF ANTITOXINS}

Antitoxin Unit. $-A$ unit is the definite measure of antitoxin in any serum or solution that will neutralize a certain amount of toxin. As previously stated, the United States Government has established a definite unit for the standardization of diphtheria and tetanus antitoxins, and frequently examines the serums made by various licensed manufacturers. Officers of the Public Health and Marine-Hospital Service purchase from reliable pharmacists several grades of antitoxins made by each manufacturer, which are then sent to the Hygienic Laboratory at Washington, where they are tested for potency, freedom from contamination by bacteria, chemical poisons, especially tetanus toxin, and for excessive amounts of preservative. Delinquencies are reported immediately, and steps are taken to withdraw that particular lot of serum from the market.

$A$ unit of diphtheria antitoxin may be defined as the "amount of antitoxin that will just neutralize 100 minimal fatal doses of toxin for a 250gram guinea-pig."

$A$ unit of tetanus antitoxin may be defined as the "amount of antitoxin which will just neutralize 1000 minimal fatal doses of toxin for a 350gram guinea-pig."

The standardization of these serums is useful as a guide to their administration, especially when given for prophylactic purposes, where experience has taught that so many units usually confer protection; it also serves for purposes of record. In the treatment of diphtheria and 
tetanus, however, the serums are usually given until a therapeutic effect is noted, regardless of the number of units administered. If it were possible to determine quickly and accurately the amount of toxin in a given patient, then neutralization could be accomplished along the same lines that make this possible in the test-tube. The indications are to administer at once sufficient antitoxin to neutralize all the toxin, giving subsequent doses large enough to overcome the toxin as it is produced until the focus of infection is removed.

Antitoxin should be kept in a cold place and protected from air and light. When this is done, they usually do not deteriorate more than 30 per cent. of their original strength, and often much less, within a year. All manufacturers place a larger number of units in the container than the label calls for, in this way allowing for the gradual loss in strength up to the date specified on the label. According to Park, the antitoxin in old serum is just as effective as that in fresh serum, except that there is less of it.

\section{PRACTICAL APPLICATION}

The employment of antitoxic serums both in prophylaxis and in the treatment of infection, is considered in greater detail in the chapter on Passive Immunization and Serum Therapy.

Römer's Method of Determining Small Amounts of Diphtheria Antitoxin.-The principle of this method is based upon the observation that, when very small amounts of diphtheria toxin are injected intracutaneously into the abdominal skin of guineapigs, small areas of edema and necrosis result in about forty-eight hours. When such injections are made with mixtures of toxin and antitoxin, the presence of free toxin is indicated by such tissue changes. It is chiefly used in determining the antitoxin content of human serums after active immunization with the toxin-antitoxin mixtures of von Behring. (See p. 718.)

Technic.-I conduct this test in the following manner: The "limes-necrosis" $\left(\mathrm{L}_{\mathrm{n}}\right)$ dose of a toxin is first determined, which is the amount of toxin which, together with $\frac{1}{1000}$ of a unit of standard antitoxin, will still produce a minimal amount of necrosis in forty-eight hours after intracutaneous injection into guinea-pigs. A series of dilutions of the $\mathrm{L}_{+}$dose of a toxin is made, ranging from $1: 5$ to $1: 100$, and 0.2 c.c. of each mixed with 0.2 c.c. of antitoxin so diluted that each 0.1 c.c. contains $\frac{1}{1000}$ of a unit. These mixtures are made in small test-tubes, the cotton stoppers paraffined, and the tubes incubated for three hours and placed in the refrigerator for twenty-one hours, after which 0.2 c.c. of each is injected into guinea-pigs (prepared by pulling out the hairs); several injections may be made in each pig.

When the $L_{n}$ dose of the toxin has been determined this amount is mixed in a similar manner with varying amounts of the patient's serum being tested. The amount of serum just neutralizing the toxin contains $\frac{1}{1000}$ of a unit of antitoxin from which the amount of antitoxin per cubic centimeter of serum may be computed. For example I have found that 0.003 c.c. of serum of a person reacting negatively to the Schick test (p. 719) neutralized this amount of toxin; therefore each cubic centimeter of this person's serum contained 0.33 unit of antitoxin. 


\section{CHAPTER XV}

\section{FERIENTS AND ANTIFERMENTS}

Bacterial Ferments.-In addition to the toxins, most bacteria possess certain ferments or enzymes that may play an important rôle in the processes of disease. After toxins have destroyed body-cells, proteolytic ferments, partly derived from the bacteria, aid in their digestion and produce a homogeneous puriform substance. A similar condition may be demonstrated in vitro in cultures of Bacillus subtilis on coagulated blood-serum media when the entire tube of medium. is quickly liquefied into a creamy fluid resembling pus. The action of the proteolytic ferment is also demonstrated in the liquefaction of gelatin.

Pyocyanase is a ferment that is capable of digesting other bacteria, such as Bacillus anthrax, Bacillus diphtheriæ, staphylococci, and streptococci. It has been asserted that the injection of this ferment is followed by an increased resistance to infection. Some years ago pyocyanase was manufactured extensively and its use advocated in the treatment of local infections, the purpose being to effect digestion of the disease-producing microörganisms. These claims have not, however, been substantiated, and the treatment has been generally abandoned.

In addition to this proteolytic ferment, bacteria may possess diastatic ferments capable of converting starches into sugars; inverting ferments, which may change polysaccharids into monosaccharids; rennin-like ferments capable of coagulating milk, etc. Not all bacteria possess all these ferments, but a study of them may aid greatly in the identification of the various bacterial species.

Similarity Between Toxins and Ferments.-Aside from the definite ferments that are in the nature of secretory products of the bacteria, there are many points of resemblance between the toxins, both exotoxins and endotoxins, and the ferments or enzymes. It would seem that the whole subject of infection and immunity is becoming more and more closely identified with physiologic chemistry, especially with the lipoids and lipolytic ferments. This subject constitutes today a most important field for investigation. 
1. Both toxins and ferments are products of the metabolism of living animal and vegetable cells, and may be extracellular (free enzymes and soluble toxins) or intracellular (intracellular enzymes and endotoxins).

2. Both exhibit a latent period before manifesting their individual activities; in general, the effect of each is more rapid the larger the amount present.

3. Both substances represent a method or means by which the organism attempts to modify its environment and render the surroundings suitable for its nutrition and growth.

4. Both show a strong affinity for their substratum, and first manifest their activity by combining with it. For example, fibrin placed in gastric juice at $0^{\circ} \mathrm{C}$. and then repeatedly washed in cold water to remove all traces of pepsin will undergo digestion when raised to body temperature. Similarly, if red corpuscles are placed in fresh tetanus toxin at $0^{\circ} \mathrm{C}$. for an hour, washed repeatedly with cold normal saline solution, and then raised to $37^{\circ} \mathrm{C}$, , hemolysis will take place, indicating the primary union of the bacterial hemolysin or tetanolysin with the corpuscles. In a similar manner toxins probably unite chemically with tissue-cells, as the toxin quickly disappears from the blood following its injection and but a small fraction can be recovered from the excretions. Furthermore, the injection of an emulsion of these cells into other animals may be followed by specific symptoms of intoxication.

5. The activities of both toxins and ferments seem to depend largely upon the temperature to which they are exposed. For instance, in the example previously cited, tetanus toxin is harmless for the frog until the temperature of the animal is raised to about $37^{\circ} \mathrm{C}$.

6. Both are usually affected by temperatures above $70^{\circ} \mathrm{C}$.

7. The one great difference, however, between toxins and enzymes is the greater activity of the latter, even very minute amounts of an enzyme having the power to split up or dècompose large quantities of complex organic compounds. An enzyme attaches itself to a substance and absorbs water; the molecule breaks down, the enzyme is liberated, and then attacks another molecule, this process being repeated until large amounts of fermentable substances have been attacked. When, however, a toxin has united with a substance it loses its identity, and in this manner it follows the law of multiple proportions. This has been discussed as it relates to the soluble toxins of diphtheria and tetanus, and is likewise easily demonstrable in the action of tetanolysin upon erythrocytes of the rabbit. It is true that a toxin may become dissociated and attack another molecule, but this action is different from that 
of an enzyme, because the molecule first attacked is not injured. However, as Adami points out, the toxins may be equally active in the body until arrested by antitoxins, although experiments in vitro clearly demonstrate the greater activity of the ferments.

8. Even in serum hemolysis it would appear that a lipolytic ferment is vitally concerned. According to Jobling and Bull, ${ }^{1}$ the end-piece of split complement contains a lipolytic ferment. These observers believe that a parallelism exists between lipase content and complement activity of the serums of various animals. As will be pointed out further on, the complements possess many properties resembling those of the ferments, and many factors are being established to show the important relation that lipoids and lipases bear to immunologic processes.

\section{ANTIFERMENTS}

According to some investigators, antiferments are to be found in large amounts in all normal serums, and are probably vitally concerned in the processes of life in preventing autodigestion. That they may be increased in number artificially by immunization up to a certain limit has been disputed; it is certain that they never attain the extreme amounts possible with the injection of toxins. This may be due to the formation of anti-anti-enzymes, produced by a regulating mechanism that prevents anti-enzymes from accumulating beyond a certain point and interfering with nutrition. It is possible that the body mechanism exerts a strict regulating effect between the formation of enzymes and anti-enzymes. Furthermore, when free receptors, such as normal anti-enzymes, are present in the body-fluids, the body-cells are not stimulated to produce these anti-enzymes in excess, nor does the presence of the free receptors stimulate the cells to produce anti-bodies against their normal side-chains.

Many investigators claim to have produced antiferments experimentally. Morgenroth ${ }^{2}$ believed that he obtained a specific antirennin by inoculating goats with rennin. Sachs ${ }^{3}$ and Achaline ${ }^{4}$ assert that they have produced specific antipepsin or antitrypsin by inoculating animals with these ferments. Antisteapsin and antilactase have been prepared by Schutze, ${ }^{5}$ antityrosinase by Gessard, ${ }^{6}$ and antiurease by Moll. ${ }^{7}$

On the other hand, these observations have not been generally con-

${ }_{1}^{1}$ Jour. Exper. Med., 1913, xvii, 61. $\quad{ }^{2}$ Centralbl. f. Bakteriol., 1899, xxvi, 349.

${ }^{3}$ Fortschr. d. Med., 1902, 20, $425 . \quad{ }^{4}$ Ann. de l'Inst. Pasteur, 1901, xv, 737.

${ }^{5}$ Zeitschr. f. Hygiene, 1904, 48, 457; Deutsch. med. Wochen., 1904, 30, 308.

${ }^{6}$ Ann. de l'Inst. Pasteur, 1901, $15 . \quad{ }^{7}$ Hofmeister's Beitr., 1902, 2. 
firmed. The inhibitory substances on trypsin in blood-serum, for instance, have been ascribed by Jobling and Petersen ${ }^{1}$ to the presence of compounds of the unsaturated fatty acids.

Antibodies and Antiferments.-Elsewhere has been discussed more fully the intimate parallelism that exists between bacterial antibodies and antiferments. One is impressed with the similarity of the processes concerned in the breaking-down of food-stuffs into simple substances for assimilation by ferments and the destruction of bacteria and their products by antibodies. The processes that occur when a cell digests an ingested microbe by a cytase must be similar to that which occurs in the digestion of any other foreign matter, and it is but a short step to conceive that bacteriolysis in the body-fluids is similar to the processes concerned in the digestion of fibrin in the gastric juice. The close similarity that exists between the toxins and the ferments, the antitoxins and the antiferments, complements and kinases, and the quantitative relations existing between a toxin and its antitoxin, between a ferment and its antiferment-all these indicate, as Adami has pointed out, the close parallelism that exists between toxins and cytolysins and ferments of different orders and grades. They would seem to indicate, moreover, that we are probably dealing with one common group of enzymic substances that act not by physical contact, but by chemical combination.

Antiferments in Disease.-In this connection a subject of considerable interest is the probable nature of the syphilitic antibody so vitally concerned in the Wassermann reaction. Although the true nature of this reaction is unknown, there can be no doubt as to the intimate relation of lipoidal substances to the processes concerned. It is probable that the Treponema pallidum produces a true antibody, and a second body, in the nature of a cellular reactionary substance, which has a marked affinity for lipoids. When the two substances are mixed and complement added, the latter is adsorbed or inactivated to a greater or less degree, so that upon the subsequent addition of washed erythrocytes and its corresponding hemolytic amboceptor hemolysis either does not occur at all or is more or less incomplete. A similar "reagin" is present in the blood-serum of persons suffering with yaws and tuberculous leprosy, and although its exact nature is unknown, its peculiar behavior toward the lipoids suggests strongly that it is a product of the toxic action of the causal parasite upon the lipoids of the body-cells and is in the nature of an antilipoid. (See Wassermann reaction.)

${ }^{1}$ Jour. Exper. Med., 1914, xix, 459. 
Jochmann and Müller have demonstrated the presence of an antiferment in the serum used against leukocytic ferments in diseases associated with great destruction of the leukocytes. Following these observers, Marcus Brieger and Trebing ${ }^{1}$ found that 90 per cent. of the patients suffering from carcinoma or sarcoma examined by them showed an increase of antitrypsin in the blood. Von Bergmann and Meyer ${ }^{2}$ confirmed this observation, although they found that a similar increase also occurred in 24 per cent. of non-cancerous patients. More recent work would indicate that the antitrypsin may be present in acute infections, such as pneumonia, typhoid fever, etc., in chronic infections, such as tuberculosis and syphilis, in exophthalmic goiter, and in severe anemias. As previously mentioned, Schwartz, ${ }^{3}$ Suginoto, ${ }^{4}$ and Jobling and Petersen ${ }^{5}$ believe that the antitryptic influence of blood-serum is due to the lipoids, and especially to the compounds of the unsaturated fatty acids.

The tryptic ferment liberated by disintegrating leukocytes and connective-tissue cells is largely responsible for the liquefaction of these cells and the formation of pus, as in abscess formation and autodigestion of infected surface wounds. On the other hand, an antitrypsin-like substance tends to limit the activities of the ferment and protect the surrounding tissues from progressive destruction. A deficiency of this substance may account for the rapid breaking-down of infected glands and of a walled-off tuberculous lesion, the development of carbuncles, etc. A study of the antitryptic power of the blood may, therefore, prove of value in suppurative processes and in malignant disease, and considerably influence a prognosis.

Ferments in Pregnancy and Disease.-It is largely to the researches of Abderhalden and his associates that we owe our knowledge of the fact that when food-stuffs are introduced into the body parenterally, i.e., by subcutaneous or intravenous injection, ferments are produced that, by process of cleavage and reduction, deprive them of their individuality.

For example, normal dog serum cannot reduce cane-sugar, whereas the serum of a dog immunized by several injections of this sugar is able to reduce it in vitro. Similarly, normal serum is unable to cleave edestin (vegetable albumin), whereas the serum of an immunized dog will split this protein into simpler substances.

1 Berl. klin. Wochschr., 1908, xlv, 1349.

2 Ibid., 1908, xlv, 1673.

${ }^{3}$ Wien. klin. Wochschr., 1909, xxii, 1151.

${ }^{4}$ Arch. f. exper. Path. u. Pharmakol., 1913, lxxii, 374.

5 Jour. exper. Med., 1914, xix, 239 and 459. 
After he had proved experimentally that the animal organism is able to defend itself against foreign substances by mobilization of ferments, Abderhalden next took up the question whether protective ferments are produced when substances native to the body but foreign to the blood are introduced into the circulation. Having learned from the researches of Veit, Schmorl, Weichard, and others, that during pregnancy syncytial cells frequently enter the maternal circulation, Abderhalden used the serums of pregnant animals, and found that they contained a ferment capable of splitting placental peptone into amino-acids and coagulated placenta into peptones, polypeptids, and amino-acids.

It was thus established that the body-cells are harmonically attuned to one another, and if new or modified cells or their products are brought into relation with other cells, they are received as foreign invaders, and their entrance is followed by the production of protective ferments ("Abwehrfermente") capable of bringing about their cleavage into simpler products. In this manner the presence in the circulation of some of the body-cells may give rise to the production of these ferments if the cells in question are really foreign to the blood-plasma and other cells.

Abderhalden is careful to note that although he was led to make these investigations on the supposition that syncytial elements were present in the blood of pregnant women, it is not necessary that they be constantly in the blood, for every case of pregnancy has a complicated protein metabolism and there is a general exchange of substances between the placenta and the maternal blood that permits the entrance into the latter of protein products that have not been broken down completely into amino-acids, and that cause the organism to produce defensive proteolytic ferments.

In cancer, where the production of new cells is so marked, some of these cells or their products may easily be swept into the general circulation, where they act as foreign invaders and cause the formation of protective proteolytic ferments. It is a noteworthy fact, moreover, , that the serum of carcinoma cases reacts best with carcinoma cells and that of sarcoma with sarcoma cells.

Similar ferments have been described in other conditions. Fauser has demonstrated that the blood-serum of dementia præcox patients contains ferments that act on the reproduction glands, so that the serum of males reacts with testicular extracts and that of females with ovarian extracts. These serums were, however, also found to react with thyroid tissue and brain cortex. In general paresis reactions were obtained with brain cortex and liver, also at times with thyroid gland, reproductive glands, and more rarely with kidney. 
Abderhalden has found ferments for the tubercle bacillus in the bloodserum of tuberculous persons, and they have also been found in the bloodserum of syphilitics for the Treponema pallidum, either in pure culture or in organs containing large numbers of the parasites.

Although these protective ferments are in general similar to the cytolysins or antibodies produced during bacterial and protozoan infections capable of lysing or digesting their antigens, their exact nature and relation to the cytolysins have not been determined. From the evidence at hand it would appear that the ferment-like cytolysin is specifically directed against the toxic portion of a bacterial cell, and the proteolytic ferment against the bacterial cell itself. Recent work by Pearce and Williams ${ }^{1}$ would seem to indicate that the cytolysins and protective ferments are separate substances, but considerable additional experimental investigation is required to clear up the point.

Although recent investigations would tend to show that these proteolytic ferments are not so specific as Abderhalden believes, the subject is one of the greatest importance, and its elucidation may possibly throw much light upon the nature of immune bodies in general. The technic, specificity, and practical value of the methods devised by Abderhalden for detecting the proteolytic ferments in the serum of cases of pregnancy, cancer, etc., are considered in a later portion of this chapter.

\section{FERMENT REACTIONS}

\section{ANTITRYPSIN TEST}

Bergmann and Meyer ${ }^{2}$ have devised a test for estimating the titer of antitrypsin that possesses value in determining the presence of tryptic ferment in the blood-serum and in the intestinal and stomach contents. The test may also prove of value in making a functional study of the pancreas. At present it is regarded by some as an aid to the diagnosis of cancer, and it may also be of service in establishing the diagnosis and prognosis of suppurative processes.

Solution of Trypsin.-This is made by dissolving $0.5 \mathrm{gm}$. of pure trypsin (Grübler) in 50 c.c. of $\mathrm{NaCl}$ solution and adding 0.5 c.c. of normal soda solution; make up to 500 c.c. with physiologic salt solution.

Casein Solution.-Dissolve one gram of pure casein in 100 c.c. of sodium hydroxid solution with the aid of gentle heat. Neutralize to litmus with $\frac{\mathrm{n}}{10}$ hydrochloric acid solution and dilute with physiologic

1 Jour.' Infect. Dis., 1914, xiv, 351.

${ }^{2}$ Berl. klin. Wochenschr., 1908, No. 37. 
salt solution up to 500 c.c. Filter and sterilize in an Arnold sterilizer. Preserve in the refrigerator.

Acetic Acid Solution.-To 5 c.c. of acetic acid (c. p.), add 45 c.c. of absolute alcohol and 50 c.c. of distilled water.

The patient's serum must be fresh, and should be diluted 20 times with salt solution. Dose, 0.2 c.c.

Technic.-A titration of the trypsin solution must precede the test proper. Into each of several small test-tubes place increasing amounts of trypsin solution, as, for example, $0.1,0.2,0.4,0.6,0.8$, and 1.0 c.c. Add 2 c.c. of the casein solution to each tube; shake carefully and place in an incubator or water-bath for half an hour at $50^{\circ} \mathrm{C}$. Then add three or four drops of the acetic acid solution to each tube, and observe which tube first shows cloudiness after a few minutes. The tube containing the smallest amount of trypsin and which remains perfectly clear contains enough trypsin fully to digest the 2 c.c. of casein solution.

Into each of six small test-tubes now place 0.2 c.c. of the 1 : 20 dilution of the patient's serum, and increasing amounts of the trypsin solution, beginning with the completely digesting dose, as determined above, and increasing by 0.1 c.c. Add 2 c.c. of casein solution to each tube, and bring all tubes to a like volume by the addition of normal salt solution. Shake gently, and incubate at $50^{\circ} \mathrm{C}$. for half an hour. Add several drops of acetic acid solution to each tube, and again observe the tube containing the smallest amount of trypsin in which cloudiness can be seen. In this way the amount of trypsin neutralized by the antitrypsin of the serum is determined.

For example, in an experiment the preliminary titration showed that 0.5 c.c. of trypsin completely digested the casein. In the second part of the test the lower limit of trypsin was this 0.5 c.c. increased by 0.1 c.c. in successive tubes up to 1 c.c. It is now found that 1 c.c. of the trypsin solution is required to bring about the complete digestion of the casein in the presence of the serum, or 1 c.c. -0.5 c.c. $=0.5$ c.c., which is the amount of trypsin neutralized by 0.01 c.c. of undiluted serum.

A control experiment is conducted with the pooled serum of several normal persons, and a comparison of the value thus obtained shows whether the antitryptic power of the serum tested is altered.

The method of Marcus, ${ }^{1}$ which is a modification of the method of Müller and Jochmann, ${ }^{2}$ is described in the laboratory exercises on Experimental Infection and Immunity.

${ }^{1}$ Berl. klin. Wochschr., 1908, No. 4; 1909.

2 Münch. med. Wochschr., 1909, Nos. 29 and 31. 


\section{ABDERHALDEN'S SERODIAGNOSIS OF PREGNANCY ${ }^{1}$}

Principles.-As previously stated, this test aims to discover in the blood-serum of pregnant women, the presence of a proteolytic ferment capable of splitting coagulated placenta or placental peptone into simple substances, such as amino-acids. The presence of this ferment indicates that its antigen is present, $i$. e., the woman is pregnant, and syncytial cells or their products have gained access to the maternal circulation with the result that a protective ferment has been produced. According to Abderhalden, while this ferment is not absolutely specific for the placenta of the same species, it is incapable of cleaving any other protein. For example, the serum of a pregnant cow contains a ferment capable of splitting the protein of human placenta, but does this less satisfactorily than does the ferment in human serum. The ferment of pregnancy, however, will not split the protein of cancer-cells or of normal tissues.

Although all investigators are in accord regarding the presence of a proteolytic ferment in the blood-serum of pregnancy, the specificity of the ferment has been questioned. To all such queries Abderhalden has usually replied by calling attention to errors in technic, and, indeed, while the dialysis method is easy of manipulation, opportunities for technical error are so numerous that the test is far from simple.

Methods. - Two methods have been devised by Abderhalden for the demonstration of the protective ferments in the blood-serum of pregnancy:

1. The Dialyzation Method.-Specially prepared and coagulated placenta and fresh serum are placed in a dialyzing capsule so prepared that it will permit the passage of peptones and amino-acids only. The filled capsule is placed in sterile distilled water, and incubated for from sixteen to twenty-four hours, when the dialysate is tested by the biuret or ninhydrin test for peptones and amino-acids. Under proper conditions the presence of these substances indicates that the placental tissue has been digested by a ferment in the serum, and, if this ferment is specific for placental cells, this indicates that the serum tested is from a pregnant woman. This is the method usually employed.

2. The Optical Method.-This method is based upon the same principle as the dialyzation method. Into the tube of a polariscope place a solution of placental peptone and the serum to be tested. Warm the

${ }^{1}$ Abderhalden: Abwehrfermente des tierischen Organisims, Julius Springer, third edition, 1913. 
mixture to $37^{\circ} \mathrm{C}$., and after an hour note the degree of rotation and record it; repeat this at intervals during the following twenty-four to forty-eight hours. If the serum contains a proteolytic ferment, the peptone is split into amino-acids and the degree of rotation increased from $0.05^{\circ}$ to $0.5^{\circ}$ and higher. This method requires an expensive polariscope, considerable practice in making the observations and readings, and is only reliable in skilful hands.

\section{The Dialyzation Method}

Testing the Dialyzing Shell.-The quality of the dialyzing shell largely determines the success of this method. It must fulfil two requirements:

1. It must be absolutely non-permeable for albumin.

2. It must be evenly permeable for the protein cleavage products, such as peptones, polypeptids, and amino-acids.

Special shells are made by Schleichter and Schull, No. 579a being recommended at the present time. The shells must be of correct size, and every one must be tested before being used. If a shell allows uncleaved protein to pass through, then all reactions would react positively regardless of the presence or the absence of the specific ferment. If the shell is too thick and too tight, and prevents the passage of peptones and amino-acids, then all reactions would be negative, even though the ferment were present in the serum and had digested the placental protein. Accordingly, each shell must be tested and standardized, and only those employed that have proved satisfactory.

Glassware.-It is highly important that all glassware should be free from clinging particles or traces of albumin, acids, and alkalis. Pipets and dialyzing cylinders should be washed in water, alcohol, ether, and finally in distilled water, and sterilized by dry heat. Boiling rods of solid glass $(10 \mathrm{~cm}$. by $0.5 \mathrm{~cm}$.) should be washed in alcohol, ether, and distilled water, wrapped in bundles of six in newspaper, and sterilized by dry heat.

A very convenient dialyzing cylinder is shown in the accompanying illustration (Fig. 73). This cylinder measures 8 by $3 \mathrm{~cm}$. It should be plugged with cotton and sterilized. When the shell is loaded with coagulated placenta and serum and covered with toluol, it will rest well beneath the surface of the outside distilled water. The wide mouth of the cylinder facilitates all manipulations and the shell cannot upset. The apparatus is easily sterilized, and the cotton plug prevents bacterial contamination and undue evaporation of the contents. 
General Precautions.-According to Abderhalden, the work should be conducted in a special room, where there is no dust or fumes of acids, and where no bacteriologic work is in progress. This observer also recommends that a special incubator be used for this work. If, however,

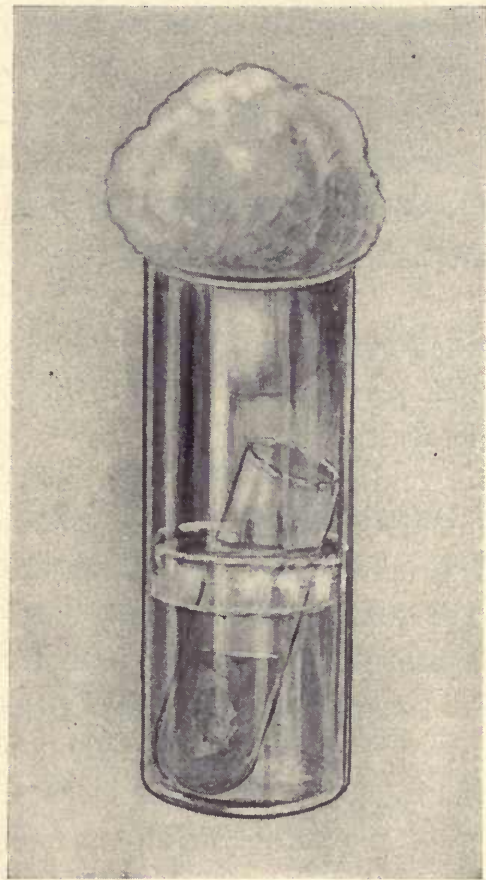

Fig. 73.-A Dialyzing Cylinder FOR THE ABDERHALDEN FERMENT TEST.

The shell contains placental tissue and fresh serum; it is surrounded with 20 c.c. of distilled water and covered with toluol. The cotton plug prevents contamination. The cylinder is readily sterilized in a hotair oven and affords a simple and efficient means for conducting the test by the dialyzation method. the working table is scrupulously clean and the glassware is clean and sterile, and if the shells are handled with sterile forceps and the dialyzing cylinder is stoppered with a plug of sterile cotton, all requirements are practically fulfilled.

Reagents. - The presence of albumin or its split products may be detected by two color reactions: (1) the biuret reaction, and (2) the ninhydrin reaction. The first is especially delicate for uncleaved albumin, and the latter for peptones and aminoacids. The technic of the biuret test is described with the technic of testing shells for permeability to albumins.

Ninhydrin. - This is the trade name for triketohydrindenhydrate. It is a whitish yellow, readily soluble powder, dispensed in brown glass vials containing 0.1 gram of the drug. A circular describing its method of use accompanies each package. As 0.2 c.c. of a 1 per cent. watery solution is the amount necessary for a test, the contents of the vial are dissolved in 10 c.c. of distilled water, and the vial rinsed with a portion of the solvent. This solution should

be preserved in a brown bottle in a cold place, and precautions taken to prevent infection. Triketohydrindenhydrate has been described by Ruheman, ${ }^{1}$ who gives its formula as follows:<smiles>CCCCC(=O)OC(=O)c1ccccc1</smiles>

${ }^{1}$ Jour. Chem. Soc., London, 1910, xcvii, 2025. 

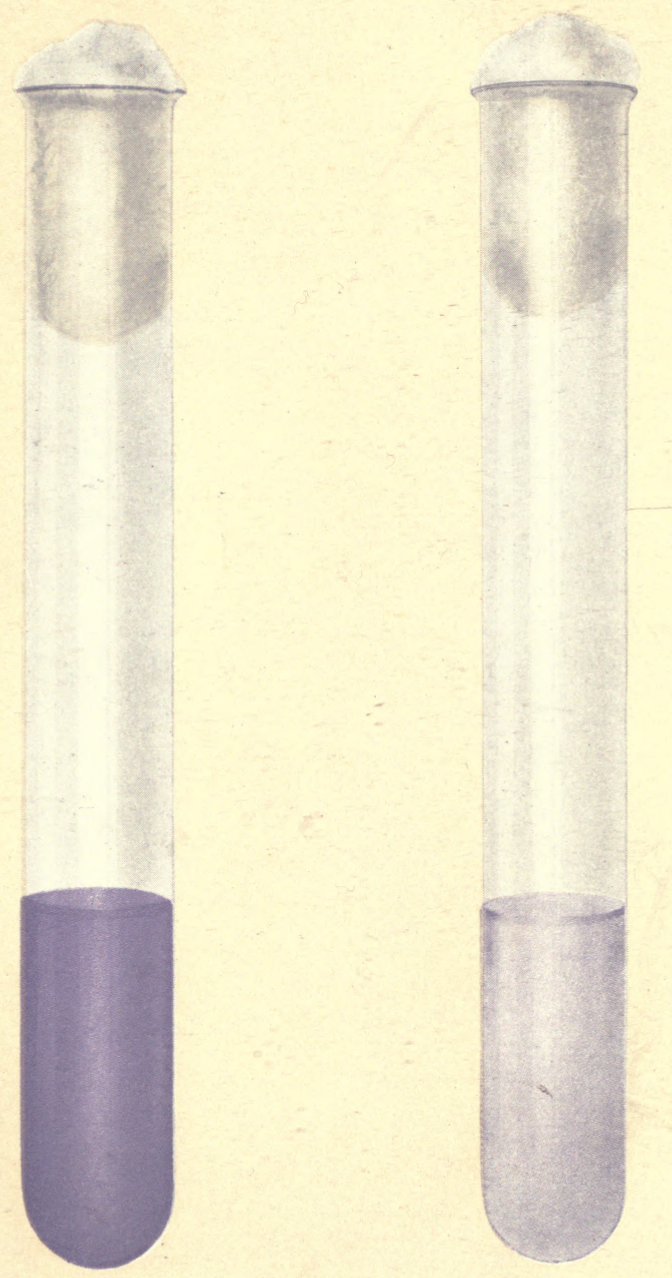

Fig. 74.-Ninhydrin Reaction (Abderhalden Ferment Test). The tube on the left shows a positive reaction with the serum of a pregnant woman; the tube on the right is the serum control and shows a faint violet color, due, presumably, to the passage of dialyzable substances in this serum. 
Owing to the fact that it gives a blue color in the presence of any compound that possesses an amino-group in the alpha position of the carboxyl group, it is of great value as an aid in recognizing the products of protein digestion (Fig. 74).

Testing the Shell for Non-permeability to Albumin.-1. New shells should be softened by soaking them for half an hour in sterile distilled water. A dozen or more may be tested at one time.

2. The albumin solution is prepared by placing 5 c.c. of the albumin of fresh eggs in a mixing cylinder, and adding distilled water to make 100 c.c. Mix well. There must be no flakes. Instead, a clear, hemoglobin-free serum may be used in doses of 2 c.c. for each shell.

3. Carefully pipet 2.5 c.c. of the albumin solution into each shell. Great care should be exercised that none of the solution contaminates the outside of the shell. The preferable method is to hold the shell with a pair of broad-toothed sterilized forceps and carry the pipet to the bottom, in order that none of the albumin should contaminate the upper portion of the inside of the shell. The pipet may easily touch the edge of the shell and thus contaminate the dialysate. If in doubt, cover the upper end of the shell with the forceps or with a clean thumb and forefinger and wash the outside with running water.

4. The loaded shell is now placed in a sterile dialyzing cylinder containing 20 c.c. of sterile distilled water. Never load the shell in this cylinder, for some of the albumin may fall into the distilled water.

5. Cover the contents of the shell and the surrounding distilled water with a layer of toluol about $1 / 4$ inch in depth. Replace the cotton plug in the cylinder.

6. Incubate at $37^{\circ} \mathrm{C}$. for sixteen hours.

7. Pass a sterile pipet quickly through the layer of toluol and remove 10 c.c. of the dialysate to a clean sterlle test-tube, and test for albumin by the biuret reaction. Add 2.5 c.c. of a 33 per cent. solution of sodium hydroxid; shake gently, but remove the thumb from the top of the tube. The solution may become slightly cloudy. Carefully overlay with 1 c.c. of a 0.2 per cent. solution of copper sulphate in such manner that a sharp line of demarcation separates the alkaline dialysate from the copper sulphate solution. A delicate violet tint at this line indicates that albumin is present and that the shell is useless. If one cannot see this color or is in doubt, it is well to make the ninhydrin test. To do this dialysis should be continued for twenty-four hours; ninhydrin reacts with albumin in addition to peptones and amino-acids, but, according to Abderhalden, this test is less sensitive than the biuret test. 
8. All shells should react negatively, $-i$. e., they should not permit the passage of unchanged albumin. If the ninhydrin test is used, the tubes should be inspected one-half hour after boiling, and the contents should be as clear as water or show but the faintest blue tint. If this is not the case, shells should be discarded as being permeable to albumin. Those that are satisfactory in this respect should be tested further as follows:

Testing the Shell for Permeability to Peptone.-1. The shells should now be thoroughly cleansed, but not with a stiff brush, washed in running water, and boiled for thirty seconds.

2. Prepare a 1 per cent. solution of silk peptone (Höchst) in distilled water, and carefully pipet 2.5 c.c. into each shell, using every precaution against contaminating the upper portion on the inside, and especially of the outside, of the shell.

3. Place the loaded shell in a sterile dialyzing cylinder containing 20 c.c. of sterile distilled water, and cover the contents of the shell and water with toluol. Replace the cotton plug and incubate at $37^{\circ} \mathrm{C}$. for twenty-four hours.

4. Remove 10 c.c. of the dialysate (avoid removing toluol) to a clean, sterile, thin-walled test-tube, and add 0.2 c.c. of the 1 per cent. ninhydrin solution. Insert a sterile boiling rod and boil for exactly one minute.

5. The boiling process is quite an important feature of this test. Always boil in precisely the same manner. A high Bunsen flame should be used, and about one minute after air-bubbles first appear on the sides of the tube lively boiling commences. The flame should then be turned down and the boiling continued for exactly one minute.

6. Place the tube in a rack. With a fresh sterile pipet remove 10 c.c. of dialysate from the next cylinder and test in the same manner, and repeat until the entire series have been finished.

7. After half an hour inspect all the tubes; they should show a deep blue color; if they do not do so they are impermeable or partly permeable to peptone and should be discarded. There is usually a difference in the degree of color reaction among a number of shells, as their permeability varies.

8. Those shells that have withstood both tests are now thoroughly washed in running water, boiled for from thirty seconds to one minute, placed in a jar of sterile distilled water containing a few drops of chloroform, and covered with toluol. From this time on they should not be handled with the fingers, but only with forceps that have been sterilized 
by boiling. Of the entire number of shells, usually from 20 to 30 per cent. or more are found to be unsatisfactory.

Preparation of the Placental Tissue.-This is the substratum, and should consist of coagulated placental protein free from dialyzable substances that react with ninhydrin.

1. A fresh normal placenta should be prepared soon after delivery. It is highly important to wash it free from all blood, Abderhalden having laid considerable stress upon this point. He explains that in the blood of all animals there is always a specific ferment for the red blood-corpuscles, as even the smallest hemorrhage into the tissue calls forth a protective ferment. For this reason all organs that contain blood may contain the substratum and ferment, and yield false positive reactions.

2. The placenta should be placed in warm water and freed as far as possible of clots. The membranes and cord are removed, and the placental tissue cut into pieces about the size of a dime. These are placed in a sieve under running water, and each piece squeezed with the hand. From time to time the entire mass is thoroughly squeezed out in a towel. Tissues that cannot be freed from clots should be discarded. The tissues are now crushed in a mortar, connective-tissue strands removed, and the washing continued until the tissue is snow white. Decolorizing substances, such as $\mathrm{H}_{2} \mathrm{O}_{2}$, should not be used. If the tissue is not white and free from blood it should not be employed. Liver, spleen, and kidney tissue cannot be made perfectly white, although all traces of blood have been removed.

3. Add 100 times as much distilled water as there is tissue, and to each liter add five drops of glacial acetic acid and boil for ten minutes.

4. Wash the coagulated tissue with distilled water, and boil again without the addition of acid. This should be repeated six times in succession. If an interruption occurs, cover the tissue and water with a layer of toluol.

5. After the sixth boiling add a small quantity of water to the tissues - just sufficient to enable it to boil for about five minutes without burning, for the water is now to be tested with the ninhydrin reaction and it is important that this be as concentrated as possible. Filter the water, and to 5 c.c. in a sterile test-tube add 1 c.c. of the ninhydrin solution. Boil vigorously for one minute. If there is the slightest discoloration within half an hour, the tissues must be boiled again, but with only five volumes of water and no longer than five ninutes each time. These boilings should be repeated as often as is necessary until the ninhydrin reaction remains water clear for at least one-half hour. 
6. The tissues are again gone over with a sterile forceps, and a search made for brown masses resembling blood-clots. These are to be discarded.

7. The tissue is now preserved in a sterile jar containing sufficient sterile water and chloroform and covered with toluol. All tissue should be handled with sterile forceps, and when once removed from the jar, they should never be returned. The whole operation requires several hours and it should be conducted without interruption. If the process is interrupted, the tissue should be covered with a layer of toluol.

8. It is well to try out the tissue with a known serum of pregnancy to make certain that it is a suitable substratum.

9. Only normal placenta should be used, as in certain instances a normal organ may be satisfactory, whereas a diseased organ would be unsuitable.

10. Animal placenta may be substituted for human placenta and vice versâ, but Abderhalden cautions against this substitution until further work has been done.

The Blood-serum.-The serum to be tested must fulfil three conditions:

(1) It must contain the smallest amount of dialyzable substances that would react with ninhydrin. Blood is best drawn in the morning before breakfast. In all diseases accompanied by marked protein disintegration, such as cancer, the blood-serum may contain large amounts of dialyzable substances.

(2) It must be absolutely free from hemoglobin and clear.

(3) It must be free from cells. Even an apparently clear serum may contain millions of erythrocytes.

1. From 10 to 20 c.c. of blood are withdrawn from a vein at the elbow with a dry sterile needle into a sterile centrifuge tube. This is placed aside at room temperature for several hours, when sufficient serum has usually separated out; if this has not occurred, centrifuge for several minutes. The serum is removed to a second sterile centrifuge tube, and centrifuged at high speed for several minutes until all corpuscles have been precipitated to the bottom of the tube.

2. The serum should be used within twelve hours after the blood has been withdrawn. Abderhalden claims that heating a serum to $60^{\circ} \mathrm{C}$. robs it of its digesting powers. Pearce and Williams have found that inactivation considerably weakens the reaction, but does not abolish it altogether.

3. Specimens of blood sent through the mails are really unsatisfac- 
tory, for even if they are delivered within twelve hours after bleeding, the amount of handling has usually resulted in the breaking up of a number of corpuscles and the tinging of the serum with hemoglobin.

The Test.-1. Absolute cleanliness should be employed. The glassware should be sterile and dry, and everything should be in readiness. The technic should be aseptic and thoroughly understood.

2. Remove a portion of the prepared placenta with sterile forceps and wash in a dish of sterile distilled water to remove toluol and chloroform. Place on sterile filter-paper, and squeeze to remove any excess of water. Weigh and place 0.5 gram in each of two shells (one for a control).

3. Holding each shell with a second pair of boiled forceps, pipet 1.5 c.c. of the patient's serum into one shell containing placenta, and the same amount into a third shell which is to serve as a control on the serum. Place 1.5 c.c. of sterile distilled water in the placental tissue control shell.

4. Unless one is absolutely sure that neither the tissue nor the serum has touched the outside of the shells, they should be held shut and washed with sterile distilled water.

5. Each of the three shells is now placed in cylinders containing 20 c.c. of sterile distilled water. Under no circumstances are the shells to be loaded while they are in the dialyzing cylinders.

6 . The contents of each shell and the water surrounding them are covered with a layer of toluol about $1 / 4$ inch in depth, and the cylinders plugged with cotton to prevent evaporation and contamination. The shell should be at least $1 / 4$ to $1 / 2$ inch above the level of the outside fluids, and due care must be exercised in carrying the cylinder back and forth from the incubator that the contents of the shell and the surrounding water do not become mixed.

7. If it is at all possible, it is well to set up two more shells as controls, each containing placenta and normal serum and the serum of pregnancy respectively.

8. All the cylinders are incubated at $37^{\circ} \mathrm{C}$. for twenty-four hours. Ten c.c. of the dialysate are then removed from each tube with a separate sterile pipet and placed in sterile test-tubes of the same size and boiled with 0.2 c.c. of the 1 per cent. ninhydrin solution for exactly one minute. After standing for half an hour, the readings are made.

Reading the Reaction.-The dialysate of the serum alone should be clear as water or show but the faintest blue tinge. The dialysate of the placenta alone should be clear; the dialysate of the patient's serum 
plus that of the placenta may show a deep violet-blue color when the reaction is strongly positive, or a fainter blue when it is weakly positive. If this dialysate is water clear or has a faint blue color, comparable to the controls, the result is negative. If there is any doubt, the test should be repeated. The negative control should be water clear or have a faint tinge comparable to its control. The positive control should show a deep violet-blue color.

I generally control the result given by the shell containing tissue and patient's serum by cleansing it thoroughly, boiling for a minute, and testing it with egg-albumen solution or a serum, in case the reaction was positive, to make sure that the shell has not allowed the passage of serum, or with peptone solution, in case the reaction was negative, to make sure that it was not thick enough to block the passage of peptones and amino-acids. This procedure delays the report on a serum for another twenty-four hours, but the greater accuracy obtained warrants the delay.

Readings should never be made by artificial light. Tubes should be held against a white background the better to appreciate the color changes.

A pinkish or brownish yellow discoloration has nothing to do with the ninhydrin reaction.

Sources of Error in the Dialyzation Method.-There are many sources of error, and until the technic has been improved sufficiently to eliminate these, Abderhalden's directions should be followed minutely.

1. The shells may become spoiled in time. They should not be cleansed with rough brushes or boiled too long. They should be cleansed at once after using, and tested every four weeks. If a wrong diagnosis results, the shell should be retested at once.

2. The placental tissue is an important source of error, due to the fact that it contains blood.

3. The serum should be fresh and free from hemoglobin and corpuscles.

4. The controls on placenta alone and each serum alone are absolutely necessary, as both may contain various substances capable of reacting with ninhydrin and thus yielding false positive reactions.

5. The water used should be distilled and sterile: The glassware should be chemically clean and sterile, and the laboratory free from the fumes of acids and alkalis. It is very important that absolutely the same conditions should exist for the control tests as for the main test itself. 


\section{THE OPTICAL MethoD}

In the dialyzation method, we establish the transformation of a colloid into a diffusible crystalloid; in the optical method we start, for purely technical reasons, not with the whole protein molecule, but with a peptone prepared of placental protein. The unsplit protein itself cannot be used, as this will interfere with the determination of the rotation of the mixture of substratum plus serum. Further, in such mixtures precipitation may occur and render the readings difficuit. Instead, we transform the protein into peptone, and observe the final changes in the tube of the polariscope.

Placental Peptone.-This requires considerable care in its preparation. Placental peptone may be purchased of the Höchst Farbwerke, and is expensive. Each specimen should be tested and its rotation determined, as otherwise uncertain and unreliable results may be secured. According to Abderhalden, a peptone may be prepared as follows:

The tissues are first cut into small pieces and thoroughly washed until they are white, as in the preparation of tissues for the dialyzation method.

The tissue is freed of any excess of water by pressing it through several layers of filter-paper, and the process of hydrolysis is begun. If a larger quantity of the same tissue is to be collected, the pieces are prepared as they are secured by washing them free from blood, boiling in water for ten minutes, and preserving in a stock jar containing sterile water and chloroform, and covered with toluol. The tissues are boiled in order to destroy the cell ferments and to prevent autolysis.

The tissues are now weighed, placed in a large flask, and treated with three volumes of cold 70 per cent. sulphuric acid. The flask is well shaken and carefully stoppered, and placed aside at room temperature (not higher than $20^{\circ} \mathrm{C}$.) until the tissues have gone into solution. The flask is shaken occasionally. Solution is usually completed within three days. The flask is now placed in iced water and treated with 10 volumes of distilled water added slowly so that the temperature does not rise above $20^{\circ} \mathrm{C}$.

The sulphuric acid is now precipitated with pure crystallized barium hydroxid until the solution gives no precipitate with either the hydroxid or sulphuric acid. A precipitate of a barium salt of peptones may appear in spite of the fact that no sulphuric acid is present. This precipitate is soluble in nitric acid, whereas barium sulphate is not soluble. The neutralization point is controlled with litmus paper. Small portions are then filtered through filter-paper and tested, first with barium hydroxid and then with sulphuric acid. If a turbidity develops on testing with the barium hydroxid, nitric acid is added and the whole gently warmed. If the precipitate persists, it is evident that more barium sulphate should be added. The final neutralization is effected with dilute sulphuric acid and barium hydroxid. When the solution is free from sulphuric acid, the precipitate of barium sulphate is secured by filtering through filter-paper or by centrifugalization. It is then worked up in a mortar with distilled water and again filtered. It is well to repeat this washing once more.

The peptone solution is now concentrated in a special apparatus that permits 
evaporation of the solution under diminished pressure and at $40^{\circ} \mathrm{C}$. A special drop funnel delivers the peptone solution drop by drop and thus prevents foaming.

The yellowish, syrupy residue that remains is covered with 100 volumes of methyl alcohol and boiled. The boiling hot solution is then filtered through five thicknesses of filter-paper into five volumes of cold ethyl alcohol, which is kept in ice water. Precipitation may be accomplished by the addition of ether. Just as soon as the precipitate has formed it is filtered out, preferably through a porcelain filter. The filter is then placed in a vacuum desiccator, and after one or two days the peptone is dry and easily removed and weighed.

A 10 per cent. solution in 0.9 per cent. salt solution is prepared, and the rotation determined. If the rotation is more than $1^{\circ}$, the solution is diluted until the rotation is $0.75^{\circ}$.

Testing the Peptone.-One cubic centimeter of fresh, clear, hemoglobin- and corpuscle-free serum from a man and an equal amount of a 5 to 10 per cent. solution of the peptone are placed in a sterile polariscope tube, warmed to $37^{\circ} \mathrm{C}$., and the rotation read. If marked changes occur, the peptone is not free from sulphuric acid or barium hydroxid. With the serum of pregnancy, readings should be taken each hour for six hours, and then at intervals of from thirty-six to forty-eight hours. It is well to test a number of pregnancy serums until a normal curve can be charted.

Peptones of other tissues and bacteria may also be prepared.

The Polariscope.-A perfect and delicate instrument is necessary, that of Schmidt and Hansch, Berlin, being recommended by Abderhalden. The instrument must be delicate enough to record differences in rotation of $0.01^{\circ}$, and be furnished with an electric incubator attachment for keeping the tube at a constant temperature of $37^{\circ} \mathrm{C}$. The tubes may, however, be kept in a bacteriological incubator, removed, quickly read, and then returned.

The Test.-One cubic centimeter of fresh and absolutely hemoglobin- and corpuscle-free serum is placed in the polarization tube with 1 c.c. of a 10 per cent. solution of standardized placental peptone; sufficient sterile saline solution is added to fill the tube. The tube is then placed in an incubator at $37^{\circ} \mathrm{C}$. for an hour, and a reading made. Another reading is taken an hour later, and the two readings should not show more than a minute difference in rotation. Another reading is made at the end of six hours, and others at intervals during the next thirty-six or forty-eight hours.

Reading the Reaction.-In serums of pregnancy cleavage is usually apparent at the end of six hours, and rotation may amount to $0.05^{\circ}$ to $0.2^{\circ}$ in thirty-six hours. With non-pregnant serums the rotation is seldom more than $0.03^{\circ}$. 
Considerable experience is required in making these readings, and the novice should practise well before conducting diagnostic tests.

Individual readings by different persons may vary $0.02^{\circ}$, and in order to secure absolute certainty Abderhalden gives $0.04^{\circ}$ as the limit for error.

A large margin for error will result if the primary reading is taken when the tube is cold, as it is immediately after being filled. Only that reading taken after the tube has been in the incubator for at least one or two hours is to be taken as the guide for determining the amount of digestion according to the degree of rotation.

The greatest source of error lies in the observer himself. One must be trained to make the readings in about thirty seconds; the eye soon grows tired, and the readings are then unreliable.

\section{Practical Value of Abderhalden's Pregnancy Test}

1. It is too soon to express a definite opinion of the specificity and diagnostic value of this reaction. Most reports have been based upon the dialyzation method. According to Abderhalden, ${ }^{1}$ Veit, ${ }^{2}$ Frank and Hermann, ${ }^{3}$ Franz and Jarisch, ${ }^{4}$ Petri, ${ }^{5}$ Judd, ${ }^{6}$ Schwartz, ${ }^{7}$ and others the ferment is highly specific, and the test is of considerable value in the diagnosis of pregnancy.

2. The reaction appears in the middle of the second month, and disappears in from ten to fifteen days after pregnancy has been interrupted, regardless of whether the fetus is born before, at, or after the normal period of gestation. Nursing has no effect upon the reaction.

3 . The reaction has been recommended in making an early diagnosis of pregnancy, when the symptoms and physical signs are indefinite; also in making the differential diagnosis between pregnancy and tumors in the pelvis.

4. The reaction is likely to be positive in hydatidiform disease and in chorio-epithelioma.

5. In acute febrile and cachectic diseases the serum may contain relatively large amounts of dialyzable compounds; positive reactions have occurred in tuberculosis of the female generative organs.

${ }^{1}$ Münch. med. Wochenschr., 1912, lix, 1305; 1912, lix, 1939 and 2172. Berl. tierarzt. Wochenschr., 1912, xxvii, 446; 1912, xxviii, 685 and 774 . Zeitschr. f. physiol. Chem., 1912, lxxvii, 249. Deutsch. med. Wochenschr., 1912, xxxviii, 2160 and 2252. Prakt. Ergebn. d. Geburtsh. u. Gynak., 1910, ii, 367.

${ }^{2}$ Zeitschr. f. Geburt. u. Gyn. 1913, 77, 463.

${ }^{3}$ Berl. klin. Wochenschr., 1912, No. 36.

4 Wien. klin. Wochenschr., 1914, 44, 144.

${ }^{5}$ Centralbl. f. Gyn., 1913, No. 7. ${ }^{6}$ Jour. Amer. Med. Assoc., 1913, lx, 1947.

7 Interstate Med. Jour., St. Louis, 1913, xx, 195. 
6. All investigators in this field are in general accord regarding the constant presence of the reaction in the serums of pregnancy, but there is a growing tendency to regard the ferment as non-specific and capable of splitting the coagulated protein of other organs, and, indeed, of organs from the lower animals. For example, Pearce and Williams ${ }^{1}$ have found positive reactions with pregnancy serums, liver, and kidney; normal male serums and those of various diseases have reacted with coagulated placenta. Williams and Ingraham $^{2}$ have had positive reactions with definitely non-pregnant persons. Michaëlis and Lugermark $^{3}$ have likewise found non-specific reactions. Pearce is inclined to believe that there is a general proteolytic ferment that is not specific for any one protein. He is careful to add that this opinion is based entirely upon the results of the dialyzation method. Abderhalden has usually replied to these adverse criticisms by calling attention to possible errors in technic, and at the present time all that one can do is to follow his directions closely and preserve a mind receptive to results until the question of specificity is settled definitely.

\section{SERO-ENZYMES IN DISEASE}

Cancer.-Freund and Abderhalden ${ }^{4}$ claim to have found protective ferments in the serum of cancer that will digest coagulated cancer protein in the same manner as the ferments in pregnancy digest placental protein. Frank and Heiman ${ }^{5}$ reported positive results in 53 of 54 cases of cancer; Markins and Munze, ${ }^{6}$ Epstein, ${ }^{7}$ Gambaroff, ${ }^{8}$ Erpicum, ${ }^{9}$ Brockman, ${ }^{10}$ Lampe, ${ }^{11}$ Lowy, ${ }^{12}$ Ball, ${ }^{13}$ and others have reported highly favorable results. Frankle ${ }^{14}$ and Lindig ${ }^{15}$ have found the reactions generally non-specific in character.

${ }^{1}$ Surg., Gyn., and Obst., April, 1913, 411.

${ }^{2}$ Colorado Med., Denver, 1913, x, 367.

${ }^{3}$ Deut. med. Wochenschr., 1914, No. 7, 316.

4 Münch. med. Wochenschr., 1913, 14, 763.

5 Berl. klin. Wochenschr., 1913, 1, No. 14.

- Berl. klin. Wochenschr., 1913, 1, No. 17.

7 Wien. klin. Wochenschr., 1913, xxvi, No. 17.

${ }^{8}$ Berl. klin. Wochenschr., 1913, l, No. 17.

9 Bull. de l'Acad. Roy. de Belg., 1913, xxvii, 624.

${ }^{10}$ Lancet, London, November 15, 1913.

11 Münch. med. Wochenschr., 1914, lxi, No. 9.

12 Jour. Amer. Med. Assoc., 1914, lxii, 437.

${ }_{13}$ Jour. Amer. Med. Assoc., 1914, Ixii, 599.

14 Deutsch. med. Wochenschr., xl, No. 12.

15 Münch. med. Wochenschr., 1913, 60, 288. 
Either the dialyzation or the optical method may be used in conducting these tests, precisely the same technic being followed as in making the pregnancy test, except that several different cancer tissues should be used with each serum. It is well to make the experiment with a number of cancer tissues taken from various parts, also with sarcoma tissue and that of various benign tumors.

Mental Diseases.-Fauser ${ }^{1}$ has studied the serums of 88 cases of dementia præcox and other mental diseases with various antigens composed of the ductless glands, testicles, ovaries, etc., and attained interesting results, tending to show that in many of these brain affections there may be associated lesions in other organs, and that the symptoms may be due to perverted functions of certain ductless glands. Munzer, ${ }^{2}$ Bundschue and Roener, ${ }^{3}$ and Fisher ${ }^{4}$ have also found in the serums of mental and nervous diseases ferments for the protein of the ductless and generative glands, tending to show that lesions of these organs may be operative in the symptomatology of these conditions.

Syphilis.-Baeslack ${ }^{5}$ has reported having had exceptionally good results with the serums of syphilitics and a substratum composed of coagulated syphilitic lesions of rabbit's testicle. Using the dialyzation method, he found the sero-enzyme test more constant and earlier than the Wassermann reaction.

Tuberculosis and Acute Infections.-Abderhalden and Andryewsky ${ }^{6}$ have suggested the use of the dialyzation or the optic method in the diagnosis of acute infections. The peptone may either be prepared of the bacilli, or the boiled organisms used in the dialyzing shell. In preparing a bacterial substratum, the material must be carefully centrifuged in order to facilitate washing. The tubercle bacilli are degreased by extraction in fat solvents. According to Abderhalden, the presence of ferments in acute infections indicates that the animal is defending itself. Abderhalden and Andryewsky found ferments present in the serum of cattle receiving injections of suspensions of dead tubercle bacilli and in experimental infections, and suggest that the test may prove efficacious in testing cattle. This work should receive further study in human infections.

${ }^{1}$ Deutsch. med. Wochenschr., 1913, xxxix, No. 7.

2 Berl. klin. Wochenschr., 1913, 1, No. 5.

${ }^{3}$ Deutsch. med. Wochenschr., 1913, No. 42, 2069.

${ }^{4}$ Deutsch. med. Wochenschr., 1913, No. 44, 2138.

5 Jour. Amer. Med. Assoc., 1914, lxii, 1002; ibid, lxiii, 559.

${ }^{6}$ Münch. med. Wochenschr., 1913, lxi, 1641. 


\section{CHAPTER XVI}

\section{AGGLUTININS}

As previously stated, given any infection, several antibodies of different properties may be produced. If the infecting microörganism produces characteristically an exogenous toxin, as, for example, that produced by the diphtheria bacillus, an antitoxin is produced as the most prominent of several antibodies. With other pathogenic bacteria that produce mainly an endogenous toxin various antibodies are formed, and one or more may play a prominent rôle in protecting the host, such as opsonins, agglutinins, precipitins, bacteriolysins, etc.

If typhoid immune serum from an immunized animal or a patient: suffering from typhoid fever is added to an emulsion of typhoid bacilli in a test-tube and the mixture placed in an incubator, the following phenomenon will be observed: the bacteria, which previously formed a uniform emulsion, clump together into little masses, settle at the sides of the test-tube, and gradually fall to the bottom, the fluid becoming almost clear. In a control test to which no active serum is added, the fluid remains uniformly cloudy. If the reaction is observed microscopically in a hanging drop, it is noted that with the addition of the serum the bacilli move nearer and nearer one another, this process being followed by a gradual loss in motility and the formation of clumps. The substance in the serum causing this phenomenon is called agglutinin, and the reaction is known as agglutination.

Definition.-Agglutinins are antibodies that possess the power of causing bacteria, red blood-corpuscles, and some protozoa (trypanosomes) suspended in a fluid to adhere and form clumps.

Historic.-Although Metchnikoff, Charrin, and Roger had noticed peculiarities in the growth of Bacillus pyocyaneus when cultivated in immune serum which we now believe were due to agglutinins, Gruber and Durham and Bordet (1894-1896) were the first to recognize that the agglutination reaction was a separate function of immune serum. While investigating the Pfeiffer phenomenon of bacteriolysis with Bacillus coli and the cholera vibrio, these investigators found that if the respective immune serums were added to bouillon cultures of these two 
species, the cultures would lose their turbidity, flake-like clumps would form and sink to the bottom of the tube, and the supernatant fluid would become clear. Gruber at the same time called attention to the fact that agglutinins were not absolutely specific for their own antigen, but would agglutinate, to a lesser extent, closely allied species of bacteria.

In 1896 Pfaundler drew attention to a peculiar phenomenon observed when bacteria were grown in an immune serum. Long and more or less interlaced threads of bacteria developed, which were regarded as due to agglutinins. At that time considerable emphasis was laid upon the importance of Pfaundler's reaction, but at present the ordinary

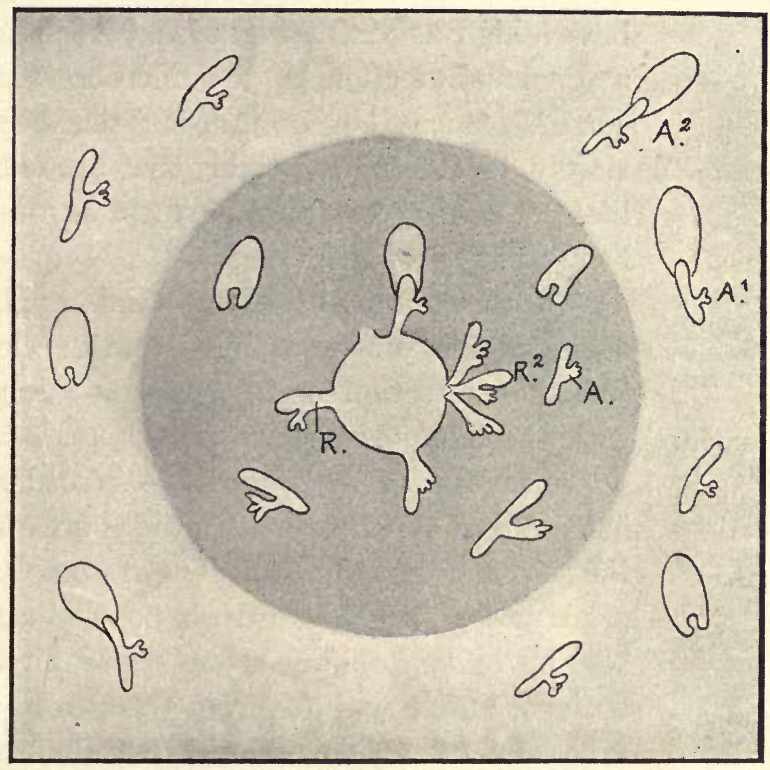

Fig. 75.-Theoretic Formation of Agglutinins.

agglutination tests have superseded this reaction as a practical diagnostic procedure.

In 1896 Widal and Grünbaum first turned these facts to practical use in the diagnosis of typhoid fever. These investigators found that the serum of patients suffering from typhoid fever acquires a high agglutinating power for Bacillus typhosus, and since this phenomenon generally manifests itself comparatively early in the disease, its recognition has considerable diagnostic importance. It is purely accidental that we speak of the "Widal reaction" in typhoid fever, rather than of the "Grünbaum reaction," for the latter observer conducted similar studies 
independent of Widal, but, owing to a lack of patients, Widal preceded him in the publication of a more extensive work.

At the present time this diagnostic reaction is known as the GrüblerWidal reaction. It has proved of great value to a large number of different investigators, not only in making the serum diagnosis of typhoid fever, but in other infections as well.

Normal and Immune Agglutinins.-Normal serums are frequently capable of agglutinating bacteria, such as the typhoid, colon, pyocyaneus, and dysentery bacilli. In some cases the typhoid bacillus may be agglutinated in dilutions as high as $1: 30$, a point of practical importance in the clinical use of the test. When a normal serum is found to have a high agglutinating power, it is probable that

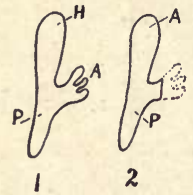

Fig. 76.-THeоRETIC STRUCTURE OF AGGLUTININ AND AGGLUTINOID.

1, Agglutinin: $H$, Haptophore group for union with antigen; $A$, the agglutinophore or zymophore group.

2, Agglutinoid. Same structure as agglutinin, except that the agglutinophore or zymophore group is lost. a previous infection by the microörganism has occurred. Since the serum of a new-born child is largely devoid of agglutinins that are found in later life, the so-called normal agglutinins may, after all, be acquired properties.

The term immune agglutinin is applied to the agglutinating substance in a serum developed as the result of infection or of systematic immunization with the microörganism.

Formation of Agglutinins. - According to Ehrlich's side-chain theory, agglutinins are antibodies of the second order (Fig. 75). They resemble antitoxins or receptors of the first order in possessing an affinitybearing or haptophore group that unites with the antigen, but they differ from them in having also a functional or agglutinophore group that agglutinates the antigen when this union has occurred (Fig. 76).

Agglutinins that have lost their zymophore or agglutinophore group through the action of heat, age, acids, etc., but that still possess their haptophore group, are called agglutinoids, just as toxins that have lost their toxophore group are called toxoids. Such agglutinoids, then, may still combine with the bacteria or blood-cells without being able, however, to produce agglutination (Fig. 77).

It is found, at times, that even a fresh serum, when concentrated, will cause less agglutination than when it is diluted. This is ascribed to the presence of agglutinoids, which have a stronger affinity for agglutinogen than has the agglutinin. When producing a reaction of this character they are called pro-agglutinoids. When the serum is diluted, the 
pro-agglutinoids become less concentrated, and finally, when they are diluted as to have no influence on the reaction, the agglutinins are still present in sufficient quantity to bring about agglutination. As a practical fact, in agglutination reactions the action of pro-agglutinoids is of much importance, for the inexperienced may be misled by the absence of or by poor agglutination in lower dilutions to neglect the use of higher dilutions (see Fig. 83).

The substance in bacteria or other cells that produces agglutinin is called agglutinogen. It appears to be formed in the cell, and in some cases may be excreted into the surrounding medium. Certainly when bacteria die and become disintegrated, agglutinogen is liberated and the filtrates (entirely free from bacterial cells), when injected into animals, will cause the formation of agglutinins.

Agglutinogen must be considered as having a simple haptophorous group, through which it may unite with the receptors of the tissue-cells. This haptophore comes into play again in the union between agglutinogen and agglutinin, which precedes agglutination.
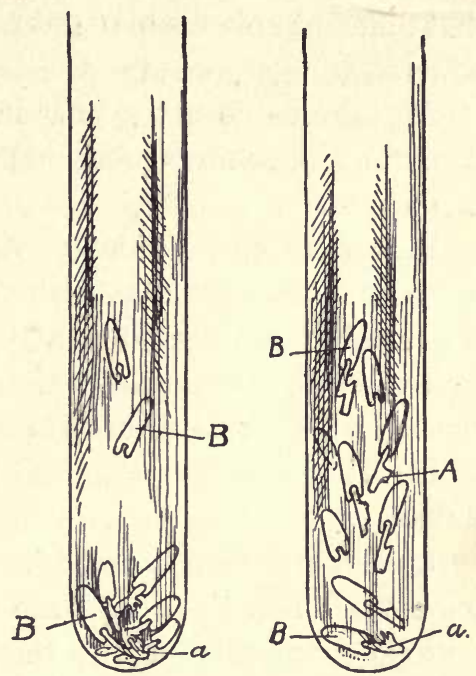

Fig. 77.-A Diagrammatic IllustraTION OF THE ACtion of AgGLUTININS AND AGGLUTINoIDs.

In the first tube (left) most of the bacilli $(B)$ have been agglutinated and massed in the bottom of the tube by the agglutinins $(a)$.

In the second tube (right) the bacilli $(B)$ are in combination with the agglutinoids $(A)$, but agglutination does not occur because the agglutinophore groups are lost. A few bacilli have been agglutinated by the agglutinins $(a)$.

It is a passive body, similar to the haptophore of antitoxin, and has no other function than that of uniting either with cell or with agglutinin.

Origin of Agglutinins.-The investigations that have been carried out for the purpose of determining the site of formation of agglutinins have not thus far yielded conclusive results. The lymphoid tissues appear especially concerned, agglutinins being found early in the bonemarrow and the spleen (Pfeiffer and Marx). Metchnikoff believes that agglutinins may be derived from leukocytes and endothelial cells. It is more probable, however, that the formation is general, and is the result of wide-spread cellular activity. 
In accordance with the side-chain theory, the ability of an animal to form agglutinins for a certain microörganism would depend on its possession of receptors of the second order, which are able to unite with the agglutinogenic receptors of the microörganism. It has been well established that the number of such suitable receptors vary in animals, and that different animals may not produce serums with equal agglutinating powers.

Agglutinins do not appear in the serum immediately after inoculation, but require an incubation period of from two to four days for their production.

Properties and Nature of Agglutinins.-(1) Agglutinins are fairly resistant substances that withstand heating to $60^{\circ} \mathrm{C}$. and lose their power only when heated to higher temperatures. It is possible, therefore, to make a serum bacteriolytically inactive by destroying complement at $55^{\circ} \mathrm{C}$., and still retain its agglutinating power.

(2) They resist drying, and their activity is best preserved in this state.

(3) They are precipitated from a serum by magnesium or ammonium sulphates, when these salts are used in proper concentration, and are thus closely associated with the globulin fraction of serum.

(4) They are separate and distinct antibodies, and are not associated with bacteriolysins. Thus the agglutinins of an immune serum may be lost, destroyed, or absorbed and the bacteriolysins retained. As previously mentioned, the bacteriolytic power of a serum may be inhibited by heating it to $55^{\circ} \mathrm{C}$. for one-half hour without influencing the agglutinin content, and during disease processes the formation of agglutinins and that of bacteriolysins are apparently not parallel processes.

Mechanism of Agglutination.-The true nature of the phenomenon of agglutination is unknown, as is shown by the number of theories advanced. Thus-

1. Gruber's idea of the mechanism of this phenomenon was that the agglutinin so changed the bacterial membrane as to render it more viscous, and that this increased viscosity caused the bacteria to adhere and form clumps. No visible changes in the organisms or red corpuscles can, however, be seen.

2. Paltauf's theory is somewhat similar, he believing that the agglutinogen is precipitated on the surface of the bacteria by union with the agglutinin, with the formation of a sticky substance. He cites evidence that tends to show that such substances are actually thrown out from the bacteria during agglutination, as may be seen in a properly stained 
preparation in the form of a precipitate surrounding the bacterial cells.

3. The presence of some salt is necessary for the occurrence of agglutination. Bordet found that if the salts were removed from the serum and from the suspension of bacteria by dialysis and that the two were then mixed, agglutination did not occur, but that if a small amount of sodium chlorid was added, agglutination promptly took place. According to this view, therefore, agglutination is a phenomenon of molecular physics - the agglutinin acts on the bacteria or other cells and prepares them for agglutination by altering the relations of molecular attraction between them and the surrounding fluid, the second phase, the loss of motility, clumping, etc., being brought about by the presence of salt. This second phase, therefore, would be a purely physical phenomenon, the salts altering the electric conditions of the colloidallike agglutinin-bacterium combination, so that their surface tension is increased. To overcome this the particles adhere together, presenting in a clump less surface tension than if they remained as individual particles. Bordet cites the precipitation of clay as an analogous case: if a little salt is added to a fine emulsion of potters' clay in distilled water, the clay immediately clumps and falls to the bottom, the resemblance between these flakes and the clump of agglutinated bacteria being very striking.

Specificity of Agglutinins.-For a time after their discovery the agglutinins were regarded as strictly specific, $i$.e., a typhoid-immune serum would agglutinate only typhoid bacilli and no others. Gruber early pointed out that an immune serum will frequently agglutinate other closely related organisms, although not usually to so high a degree.

Group or partial agglutinins, therefore, refer to the presence in a serum of certain agglutinins that agglutinate certain other microörganisms that are morphologically, biologically, and often pathogenetically closely related to the homologous microörganism (the bacterium causing the infection or used in artificial immunization). For example, a typhoid-immune serum possesses, besides its greatly increased agglutinating power for Bacillus typhosus, some agglutinin for Bacillus paratyphosus, notably above that of normal serum. This is explained by the very close biologic relationship of these bacteria, together with the fact that the agglutinin-producing substance (agglutinogen) is a complex and not a single chemical substance. This has been explained by Durham in the following example: If the typhoid agglutinogen is composed of various elements, A, B, C, D, it is conceivable that the closely related 
paratyphoids might contain one or more of these four agglutinogens, and, therefore, the agglutinating power of the typhoid serum for a paratyphoid bacillus, though not so great as on the typhoid bacillus, is still considerable. Accordingly, in an infection with one microörganism a specific agglutinin will be formed for that microörganism, and group agglutinins for other more or less allied microörganisms, and consequently the specificity of the agglutinating reaction depends upon the principle of dilution, the specific agglutinin being present in largest amount and operative in dilutions above the range of the group agglutinins.

Absorption Methods for Differentiating Between a Mixed and a Single Infection.-In 1902 Castellani discovered that if the serum of an animal immunized against a certain microörganism contains that microörganism in large numbers, the serum will lose its agglutinating power not only for that microörganism, but also for all other varieties on which it formerly acted. If, however, the serum contains the organism corresponding to the group agglutinins, the agglutinating power of the serum for the homologous organism is reduced but little or not at all.

In a mixed infection due to two or more varieties of bacteria there will be specific agglutinins for each of the microörganisms, and group agglutinins for each of them as well. If the immune serum is saturated with one of these varieties its chief or major agglutinins and some or all of the group agglutinins will be removed, but the major agglutinin of the second species will remain. On the addition of the second bacterium to the immune serum agglutination occurs and its agglutinin is absorbed. Park, who has carefully investigated this subject, finds that the absorption method proves that when one variety of bacteria removes all agglutinins for a second, the agglutinins in question were not produced by the second variety.

Hemagglutinins.-Agglutination, like other immunity reactions, is a manifestation of broad biologic laws and is not limited to bacteria. As hemolysins are produced by the injection of an animal with red bloodcorpuscles from another speries, so agglutinins that agglutinate the red blood-corpuscles may be developed at the same time. When a serum containing hemagglutinin is added to a suspension of the corresponding red blood-corpuscles contained in a test-tube, it causes these to collect into clumps and flakes and sink to the bottom, just as a typhoid immune serum agglutinates typhoid bacilli. These clumps are broken up with some difficulty, and may interfere with hemolytic reactions. They are especially to be observed in antihuman hemolytic serums when agglu- 
tination may be so marked as to prevent hemolysis unless the tubes are frequently and vigorously shaken.

Small amounts of normal hemagglutinins may be found. Of particular practical importance are those for animals of the same species, the so-called isohemagglutinins.

Isohemagglutinins were discovered independently by Landsteiner and Shattuck in 1900, and were studied quite extensively by Hektoen and Gay. At first the occurrence of isoagglutination was regarded as of pathologic significance, but later researches showed that they may be found in a large percentage of normal bloods. According to Landsteiner and Hektoen, human bloods may be divided into four groups, as follows:

Group 1: Here the corpuscles are not agglutinated by any human serum, whereas the serums agglutinate the corpuscles of the other groups. This group includes about 50 per cent. of all persons.

Group 2: In this group the corpuscles are agglutinated by the serums of other groups, whereas the serums agglutinate the corpuscles of Group 3 but not of Group 1.

Group 3: The corpuscles are agglutinated by all other serums, and the serums agglutinate the corpuscles of Group 2 but not of Group 1.

Group 4: The corpuscles are agglutinated by all other groups, but the serums are unable to agglutinate any human corpuscles. 'These are quite rare.

The group characteristics are hereditary, and permanent throughout life.

With the increasing number of blood transfusions, the phenomena of isoagglutination and isohemolysis-the two being closely related-are of considerable practical importance, especially if the patient is suffering with cancer, when the serum is likely to be actively hemolytic for the donor's corpuscles.

In selecting the donor for a transfusion; agglutination and hemolysis tests should always be made before operation if time permits. The tests made in vitro are usually safe guides as to conditions existing in vivo, and such preliminary tests may prevent the occurrence of untoward symptoms associated with intravascular hemolysis or agglutination, such as fever, dyspnea, edema, and hemoglobinuria. As a rule, the donor selected should be a near relative, and whenever time permits, a Wassermann reaction and the isoagglutination and isohemolysin tests should be made. That donor should be chosen whose blood shows no inter-agglutination or hemolysis with the patient's serum and corpuscles. 
If such a donor cannot be obtained, it is safer to use a person whose serum is agglutinative toward the patient's cells than one whose cells are agglutinated by the patient's serum.

The technic of these transfusion tests is given at the end of this chapter.

Non-agglutinable Species of Bacteria.-Certain species of bacteria, especially when freshly isolated from the animal body, may prove themselves immune to the action of agglutinins; this is true especially of the bacillus of Friedländer. As a rule, this resistance is lost when the microörganism is grown for some time in artificial media. In some instances the typhoid bacillus, when freshly isolated from a patient, may resist agglutination until after it has passed a period of existence on artificial media. This variability is probably due to some change taking place in the agglutinable substance of the agglutinins during the sojourn of the bacilli in the animal body, and possess such an excess of agglutinogenic receptors as to require a much larger amount of agglutinin to cause agglutination.

It should be remembered that agglutinins act on dead as well as on living bacteria, those killed by heat, formalin, phenol, etc., being similarly agglutinable. In making the microscopic test the use of dead bacteria is not so satisfactory as when the test is made with living motile bacteria, for the influence of the serum on motility alone is of value in interpreting a reaction.

Variation in Agglutinating Strength of a Serum.-In a given infection, such as typhoid fever, there is usually a continued increase in the amount of agglutinin in the blood from the fourth day until convalescence is established, and then a decrease occurs. It is a fact of practical importance that the agglutinating power of a serum may vary from day to day, so that it is very strong one day and may become weak or disappear entirely on the next day or two. Hence the importance of making more than one test in a suspicious case when the first trial has been doubtful or negative. There is no satisfactory explanation for this variation, mixed infection, intestinal hemorrhage, etc., being regarded by some as responsible for it.

Rôle of Agglutinins in Immunity.-The agglutinins were formerly regarded as possessing a true protective and curative power. It has previously been mentioned that bacteria may be grown in a specific agglutinating serum, and cultures made of agglutinated bacteria show them to be fully alive and as virulent as before agglutination took place. In certain cases agglutinins for a microörganism may be entirely absent, 
and yet the animal enjoy an immunity. Bacteria that have been acted upon by an agglutinin are apparently not altered in appearance, viability, or virulence.

Many observations tend to show that the agglutinating power of a serum gives no indication of the degree of immunity that exists. For instance, relapses may occur in typhoid fever at a time when the agglutinating power of the patient's blood is at its highest.

At present agglutinins are regarded as playing a subsidiary rôle in immunity, their presence being of diagnostic value, and an indication of the presence of more important factors, and as an aid to bacteriolysis and phagocytosis.

\section{PRACTICAL APPLICATIONS}

The agglutination reaction is used for the following purposes:

1. For the diagnosis of disease, by identifying the bacterial infection from which the patient is suffering. To do this satisfactorily we must have on hand stock cultures of bacteria, and test the patient's serum for agglutinins for these bacteria. For instance, if a patient presents symptoms of typhoid fever, the serum is tested for typhoid agglutinins; if the reaction is very weak or negative and continues so, the serum is further tested for agglutinins for Bacillus paratyphosus A and B.

In typhoid fever the Gruber-Widal reaction may be positive as early as the third day; usually, however, the positive reaction is obtained somewhat later-about the seventh or the eighth day. A day or so earlier the bacilli used in making the test may be seen to lose their motility, and two or three may form a loose clump. This is the doubtful reaction, and it is well to test every day or every other day until a decisive reaction is obtained.

According to Park, "about 20 per cent. of typhoid infections give positive reactions in the first week; about 60 per cent. in the second week; about 80 per cent. in the third week; about 90 per cent. in the fourth week, and about 75 per cent. in the second month of the disease." In about 90 to 95 per cent. of cases in which repeated examinations are made a positive reaction is to be found at some time during the patient's illness.

Occasionally the reaction appears first during the stage of convalescence, and at times it may even be absent, the diagnosis being confirmed by cultivating typhoid bacilli from the blood. The possibility of a given case reacting strongly one day and weakly or entirely negative a day or so later has been emphasized elsewhere. 
Usually the reaction is strongest during convalescence, remains positive for several weeks, and then gradually returns to the normal. Occasionally the reaction remains positive for months or even years after the attack of typhoid fever-many such cases are "carriers" and harbor the bacilli in the gall-bladder, although the person appears to be quite well.

Only very rarely does normal serum immediately agglutinate typhoid bacilli in a dilution higher than 1:10; where a time limit of one to two hours is given, a few may show some agglutination in dilutions up to $1: 30$.

If the typhoid bacillus is agglutinated by the patient's serum in a dilution of $1: 100$, or at least $1: 40$, the Widal reaction may be regarded as positive. It is not safe to use lower dilutions, as occasionally the serum of healthy persons may agglutinate Bacillus typhosus in dilutions up to $1: 30$. Due care must be exercised not to mistake a pseudoreaction about detritus for true agglutination.

Positive reactions are occasionally obtained in other diseases-acute miliary tuberculosis, malaria, malignant endocarditis, and pneumonia. It is also well to bear in mind the possibility of a patient having been vaccinated against typhoid at some early date, with resulting agglutinin formation.

Owing to the similarity of symptoms an infection with Bacillus paratyphosus A and B may be difficult to distinguish from typhoid fever. This difficulty is increased by the confusion of the Widal reaction owing to the presence of group agglutinins in the serum if proper dilution is not practised. Bacillus A and Bacillus B are not identical in their agglutinable properties, the latter being more closely related to the typhoid bacillus than the former. In this country Bacillus paratyphosus is usually held responsible for paratyphoid fever. Conclusions should not be drawn until tests have been made with both strains of the paratyphoid bacillus and with the typhoid bacillus. In case of mixed infection the absorption method of Castellani will serve to clear up the diagnosis.

In dysentery the agglutination reaction with the serum of patients shows great variability. In spite of the presence of bacilli in the feces the reaction is sometimes absent, often disappears rapidly during convalescence, and rarely is as high as in typhoid fever. The tests should always be performed with both the "Flexner" and "Shiga" types of bacilli, as the two do not possess identical agglutinable properties, and either may be the cause of infection in a given case. The absence of the reaction does not exclude a dysenteric infection. 
In cholera the agglutination test has so far proved of doubtful aid in establishing a diagnosis of the disease. However, for the purpose of recognizing bacilli isolated from the feces of suspicious cases the reaction with known immune serum is of great value.

In cerebrospinal meningitis the agglutination occurs within an hour in dilutions of $1: 10$. It is seldom that the patient's serum agglutinates in a dilution higher than 1:50.

In tuberculosis the agglutination reaction has no value as a diagnostic procedure. Koch recommended the agglutination test for the estimation of the degree of immunity conferred by tuberculin treatment. As pointed out elsewhere, agglutinins have apparently no antimicrobic influence, but, as with typhoid vaccination, may indicate the degree of reaction and the presence of other antibodies.

Many strains of tubercle bacilli are almost non-agglutinable. The preparation of a homogeneous emulsion is not easily made, and the results are likely to be confusing and contradictory.

In plague the agglutination reaction becomes quite marked about the ninth day of the disease - too late, however, to be of much practical value in diagnosis. It is occasionally useful, however, for deciding whether a patient in the convalescent stage has really suffered from the disease.

In Malta fever the agglutination reaction is of considerable value in making the diagnosis.

In pneumonia the reaction is of value in rapidly differentiating pneumococci and as an aid in specific serum therapy, and it may also be of aid in making the diagnosis of infection with Bacillus enteritidis and Bacillus botulinus.

In veterinary practice agglutination reactions are of value in the diagnosis of glanders, infected horses reacting in some instances to dilutions as high as 1:2000. For diagnostic purposes the agglutination test in glanders must be in dilutions higher than 1:800. A positive reaction in dilutions of $1: 1000$ is regarded as suggestive, and is controlled by a complement-fixation test; agglutination in dilutions of 1:1500 practically always indicates an infection. The complement-fixation test, however, is a better diagnostic reaction.

2. Agglutination reactions are also of value as an aid to the identification of a microörganism that has been cultivated from a patient. For this purpose we must have on hand various standard immune serums. For example, if a bacillus resembling the typhoid bacillus is isolated from the feces of a patient, the diagnosis may be aided by a positive agglutination reaction with typhoid immune serum. 
The "group agglutinins" constitute a source of difficulty in making the differentiation among the numerous members of a group of microorganisms, but if a highly potent agglutinating serum is used and the test is carried to the point of determining the highest dilution that will agglutinate the bacteria, it will in most cases be possible to differentiate the variously allied microörganisms by this test.

In conducting these reactions it is best to use a macroscopic method, and the agglutinating serum used must have been previously titrated against an easily agglutinable and known strain of the microörganism in question

In this connection it is well to remember that freshly isolated cultures of a microörganism may be not at all or but very slightly agglutinable. Thus colonies of typhoid bacilli found in feces or in an abscess may, if picked from a plate, resist agglutination until subcultured several times in artificial media.

The agglutination test has great value as a mode of differentiating among the members of the typhoid-colon group of bacilli. In the diagnosis of cholera, suspicious bacilli isolated from the feces may be tested with a known cholera immune serum, and the bacteriologic diagnosis thus be greatly facilitated. The test also has some value in making a biologic differentiation between meningococci and gonococci, and also between other groups of bacteria.

3. Agglutination tests are of value in determining whether, in a case in which more than one microörganism has been cultivated, the condition at hand is a single or a mixed infection. The absorption agglutinin test is made with the patient's serum, and the cultures are isolated from the patient.

4. Agglutination tests are also of value for measuring the immunizing response that a patient is making to his infection or to artificial immunization. Thus the test is of some value in determining the response to inoculation with typhoid vaccine, although it is probable that the agglutinin itself does not possess true antimicrobic properties.

\section{THE AGGLUTINATION REACTION}

The value of the agglutination test in the diagnosis of disease is limited chiefly to typhoid and paratyphoid fevers; and, in a less degree, to cerebrospinal meningitis and bacillary dysentery. It is especially useful as a practical test in the diagnosis of obscure and atypical cases of typhoid fever and also in the diagnosis of "typhoid carriers." 
Two methods may be employed:

1. The microscopic method which is generally employed where the Gruber-Widal reaction for typhoid fever has been employed, as the reaction is quickly done and requires but a small amount of blood.

This test is usually performed with serum separated from the clot and in various dilutions (wet method). The test may also be performed with dried blood (dry method), the agglutinins being preserved and redissolved with a diluent. The technic of the latter method is very simple. The blood is easily collected and may be sent for long distances, and for these reasons the method has been adopted by many boards of health.

2. The macroscopic method is that generally preferred if sufficient blood is on hand, and is the method of choice in scientific research. Absorption tests must be performed with the macroscopic technic.

Requisites for Conducting Agglutination Reactions.-(a) Bacterial Emulsion.-This may be prepared by growing the microörganism in broth. Solid media may be used, and the culture washed off with sterile salt solution and emulsified.

(1) The bacterial emulsion should be prepared of young cultures, should be homogeneous and free from clumps, and of such density as to furnish a sufficient number of microörganisms to give the reaction (Fig. $78)$.

(2) For the ordinary microscopic Widal test, eighteen to twentyfour hour bouillon cultures of Bacillus typhosus, Bacillus coli, and Bacillus paratyphosus yield uniform and satisfactory results. An old laboratory culture - one that is known to be agglutinable - should be used. Broth cultures should be cultivated at a temperature lower than body heat, in order that long motile forms may be secured. During the summer and early autumn months the culture can be grown at room temperature; during the winter, on top of the incubator.

Thick cultures are unsatisfactory for making the microscopic test, as there is always some false clumping and motility is not well marked (Fig. 79).

When these tests are done routinely, it is good practice to subculture in broth every day in order that a satisfactory culture may always be on hand. When performed at irregular intervals, a broth culture can be prepared from a stock agar culture and the test performed twentyfour hours later.

(3) Emulsions may be prepared of young cultures on solid media by removing portions of the growth with a platinum loop and emulsifying 
in a diluent, such as normal salt solution or broth. This may be performed by placing the diluent in a test-tube and rubbing the loop over the glass just at the margin of the fluid, the bacteria being gradually emulsified and floated into the diluent. When larger quantities of emulsion are desired, as for making the macroscopic test, 5 c.c. of diluent may be poured upon the culture and the growth washed off with the aid of the platinum loop. The emulsion is gently shaken and removed to a second tube, when unresolved bacterial clumps will sink to the bottom. In other cases the emulsion may be centrifuged for a short time or filtered through sterile filter-paper. Sufficient salt solution is added to

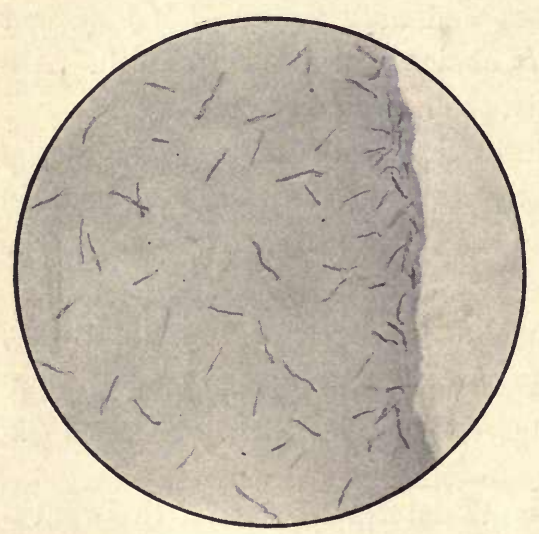

Fig. 78.-A Satisfactory Culture for the Microscopic AgGlutination REACtion. $\times 430$.

This shows a satisfactory culture of the proper density and free of clumps of bacilli. (Twenty-four hour culture of Bacillus typhosus grown at room temperature.)

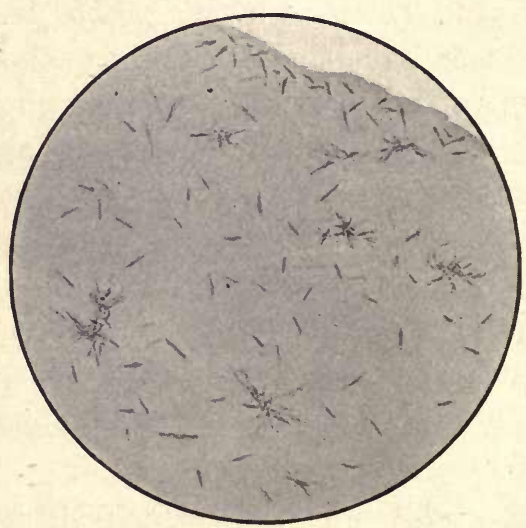

Fig. 79.-An Unsatisfactory Culture FOR THE Microscopic AgglutinaTION REACtion. $\times 430$.

The culture is rather too dense and shows considerable spontaneous or false agglutination of the bacilli. (Twentyfour hour culture of Bacillus typhosus grown at $37^{\circ} \mathrm{C}$.)

give the emulsion a density equal to that of a rich twenty-four-hour bouillon culture.

(4) To emulsify a culture of the plague bacillus or any other microorganism that displays a strong tendency to undergo "spontaneous" agglutination, distilled water or 1:1000 salt solution should be used.

(5) In the case of a culture of tubercle bacillus, the growth can be resolved into its elements by prolonged trituration in normal salt solution, and any residue or unresolved clumps removed by centrifugalization. A less laborious and dangerous method is to use the tubercle powder of Koch, which is obtained by reducing dried tubercle cultures to a fine 
powder by machinery. The powder may be made up into a suitable suspension by rubbing it in a mortar with normal salt solution.

(6) When it is necessary to work with highly dangerous microörganisms, or to operate from day to day with the same bacterial suspension, one may employ suspensions that have been heated for one hour to $60^{\circ}$ C., or suspensions in salt solution to which 1 per cent. formalin has been added. These will keep well in the refrigerator, but the sediment of dead bacteria must be well shaken before it is used.

(b) Serum.-Serum should preferably be fresh, clear, and free from corpuscles.

(1) For the microscopic "wet" method, ample serum is furnished from the blood contained in the ordinary Wright capsule (see p. 33).

(2) For the microscopic "dry method," blood is secured by pricking the finger or lobe of the ear and collecting a few drops of blood upon aluminum foil, on a clean glass slide, or on partially glazed paper. The blood must not be heated to hasten drying, or agglutinins may be destroyed. Smears on aluminum foil and on glass slides are to be preferred to those on paper, as the blood can be moistened and portions removed without the likelihood of transferring extraneous material, such as paper fiber. While there are certain objections to this method to be pointed out later, yet practical experience has demonstrated its value, as the serum does not readily deteriorate or become contaminated with bacteria, and the ease with which blood may be collected and mailed recommends the process for board of health laboratories.

(3) For the macroscopic test larger amounts of serum are needed. Sufficient blood is easily obtained by pricking the finger deeply and collecting several cubic centimeters in a small test-tube.

(4) Because normal agglutinins may be active in dilutions as high as $1: 30$, for diagnostic tests in typhoid fever the serum should not be diluted lower than 1:40. For routine work dilutions of $1: 50$ and $1: 100$ are well adapted for the microscopic test. Dilutions of $1: 40$ and 1:80 are readily made with the white corpuscle pipet and are equally useful.

With the macroscopic technic, dilutions of from 1:20 up to any dilution are readily made in appropriate test-tubes.

Precautions.-In bacteriologic technic due care should be observed to avoid contamination and possible infection when working with living cultures.

(a) Agglutinated bacteria are not necessarily dead, and hanging-drop preparations, test-tubes, etc., should be immersed in 1 per cent. formalin before cleansing. 
(b) The working table or desk and the hands should be washed with a solution of lysol or 1 per cent. formalin after the reactions have been made and the work completed.

(c) Early in typhoid fever the bacillus may be present in the blood, and consequently due care should be exercised in handling it, in diluting the serum, and in the disposal of the clot.

\section{TeChNiC OF the Microscopic AgGlutination Test (Wet Method),-The WIDAL REACTION IN TYPHOID FEVER}

(1) Dilute the patient's serum by placing one drop from a capillary pipet in a small watch-glass and adding 19 drops of normal salt solution. This gives a dilution of $1: 20$. Mix thoroughly.

(2) With a 3 or $4 \mathrm{~mm}$. platinum loop place a drop on a clean coverglass that is sufficiently thin to permit the use of an oil-immersion lens. The loop is better than a capillary pipet because the drop it gives is smaller, and when it is later diluted with an equal quantity of bacterial emulsion, it is not too large and is easily manipulated.

(3) With the same sterilized platinum loop add one loopful of a twenty-four-hour broth culture of Bacillus typhosus to the drop of diluted serum on the cover-glass. Mix gently and without spreading the drop. This gives a final dilution of $1: 40$.

(4) Edge a hanging-drop slide with vaselin, and invert the coverglass slide over the hollow portion in such a manner that the drop will be suspended in its center. Care must be exercised not to spread the drop, for if this occurs and the fluid flows around the margins of the chamber a new preparation must be made. Inspect the slide, and add vaselin, if necessary, until it is sealed tightly. By means of a grease pencil label the slide with the name of the patient, the dilution, and the time when the preparation was made.

(5) Place 2 to 5 drops of serum dilution $1: 20$ in a second watchglass, and add an equal quantity of normal salt solution. Mix well. This gives a dilution of $1: 40$.

(6) Prepare a second slide by mixing a loopful of this dilution with an equal sized loopful of culture. Mix gently. This gives a final dilution of $1: 80$. Mark the slide with the name, dilution, and the time.

(7) Prepare a third slide by placing a loopful of culture on a coverglass and invert over a concave slide to which vaselin has been applied in the usual manner. This is the culture control. Label the slide.

(8) Place the slides in a dark place at room temperature and examine at the end of an hour with the $1 / 6$ or oil-immersion lens. 
(a) First inspect the control. The bacilli should not be clumped, but should be motile, and preferably in the form of long slender rods. (See Fig. 78.)

(b) Examine the 1:40 and 1:80 dilution preparations: a positive reaction is indicated by loss of motility and definite clumping (Fig. 80). A few free motile bacilli may be seen, or a clump may be seen to move, owing to the efforts of the bacilli to break away. A doubtful reaction is indicated by a partial loss of motility and a few indefinite clumps. A negative reaction is indicated when there is no loss in motility or no clumping, or when the reactions resemble the control to which no serum has been added. In reporting upon agglutination tests always state the time at which the test was made and the dilution used.

A $1: 20$ and a $1: 40$ dilution may be prepared and examined at the end of half an hour. Prompt agglutination is found practically only in typhoid fever.

Dilutions may be conveniently prepared by drawing the serum up to the mark 0.5 in the white corpuscle pipet, and the distilled water up to the mark 11. Mix well. This gives a dilution of $1: 20$. One loopful of this diluted serum and one loopful of bouillon culture

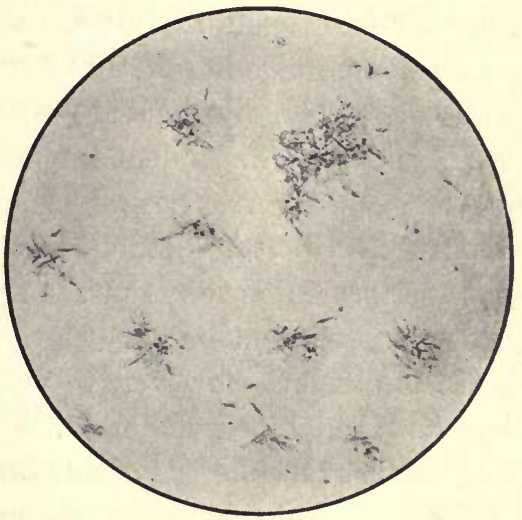

Fig. 80.-A Positive Agglutination (Widal) Reaction in Typhoid FeVER. $\times 430$.

Serum from a patient ill about twenty-two days; a $1: 100$ dilution at the end of one hour. of the microörganism to be tested give a dilution of $1: 40$. One loopful of the $1: 20$ diluted serum and three loopfuls of the culture give a dilution of $1: 80$. Having mixed the diluted serum and the bacterial suspension on a cover-glass, prepare the cultures on the vaselined concave slides in the usual manner.

\section{The Microscopic Agglutination TeSt (DRY Method)}

(1) Place a loopful of a twenty-four-hour bouillon culture of Bacillus typhosus in the center of a clean cover-glass.

(2) Moisten the dried blood which has been collected on aluminum foil, glass slide, or paper with a loopful of normal salt solution. (A second and smaller loop may be used for this purpose.) Gently rub up the dried blood and transfer a sufficient amount to the drop of culture 
on the cover-glass until, when thoroughly mixed, it presents a delicate orange tint (Fig. 81). Avoid transferring too much débris with the solution of blood, especially if the blood has been collected on paper. It is good practice to mix the culture and solution of blood with the cover-glass held over a white surface, in order that the color may readily be observed.

(3) Having made the mixture on the cover-glass, invert it over a vaselined concave slide, label, and stand aside for an hour.

(4) Prepare the culture control in the usual manner and label.

(5) Examine at the end of an hour with the $1 / 6$ or oil-immersion lens. If minute fragments of fiber, etc., have been transferred, due allowance for false agglutination for these should be made. Otherwise the readings are made in exactly the same manner as in the "wet" method.

(6) Accurate dilutions are not possible with this technic. Satisfactory results are dependent largely upon the color; a faint orange tint of the suspension is desirable, and probably represents a dilution of about $1: 40$. This method, however, is very simple, and when carefully performed, yields results in the practical serum diagnosis of typhoid fever almost as satisfactory as the serum-dilution method.

It is possible, however, to work with known approximate dilutions by the dried blood method if a good chemical balance is available. Blood must be collected on aluminum foil or glass, and is then scraped off and weighed. To each five milligrams of dried blood 0.5 c.c. of salt solution is added which equals a dilution of $1: 25$ of whole blood or 1:100 of dried blood (Wesbrook). After permitting the mixture to stand for half an hour it is centrifuged for a short time. To one drop of the dilution thus obtained one drop of culture is added, which gives a final dilution of about $1: 50$. At the end of an hour it is examined. Higher dilutions can be prepared from this stock dilution at the will of the operator.

\section{MACROSCOPIC AgGLUTINATION TEST}

This is frequently the method of choice in conducting agglutination tests, especially in investigation and research work, where a high degree of accuracy is desirable. All dilutions and measurements are to be made with accurate volumetric pipets.

1. Place a row of seven small test-tubes $(10 \times 1 \mathrm{~cm}$.) in a test-tube rack and add 1 c.c. of normal salt solution to each.

2. Dilute the serum $1: 5$ in the first tube, as follows: 0.2 c.c. serum plus 0.8 c.c. salt solution. This now gives in this tube 2 c.c. of a dilution of $1: 10$. Mix well with the pipet. 


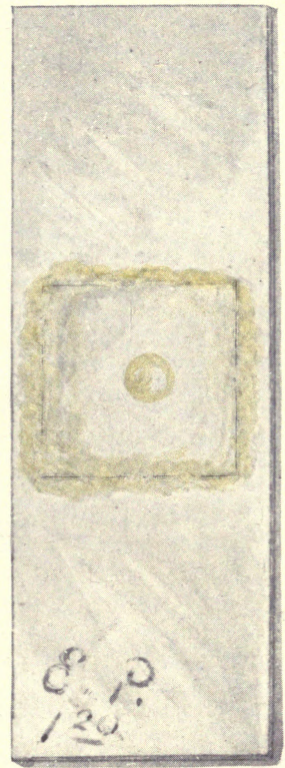

Fig. 81.-Microscopic Agglutination Test with Dried Blood.

Shows the proper color of the suspended drop of typhoid culture when the solution of dried blood has been added. The tinge should be light orange or yellow, and a shade lighter than ordinary vaselin used in sealing the preparation. 

3. Place 1 c.c. of the serum from tube 1 into tube 2. Mix well, and place 1 c.c. of the mixture from tube 2 into tube 3 , and so on. When the sixth tube has been reached, discard 1 c.c., as no serum is to be added to the seventh tube, which is the culture control; $i$. e., it will contain salt solution plus bacterial emulsion.

4. Add 1 c.c. of bacterial emulsion to each tube, which doubles the serum dilution in each. Tube 1 now contains a serum in a dilution of

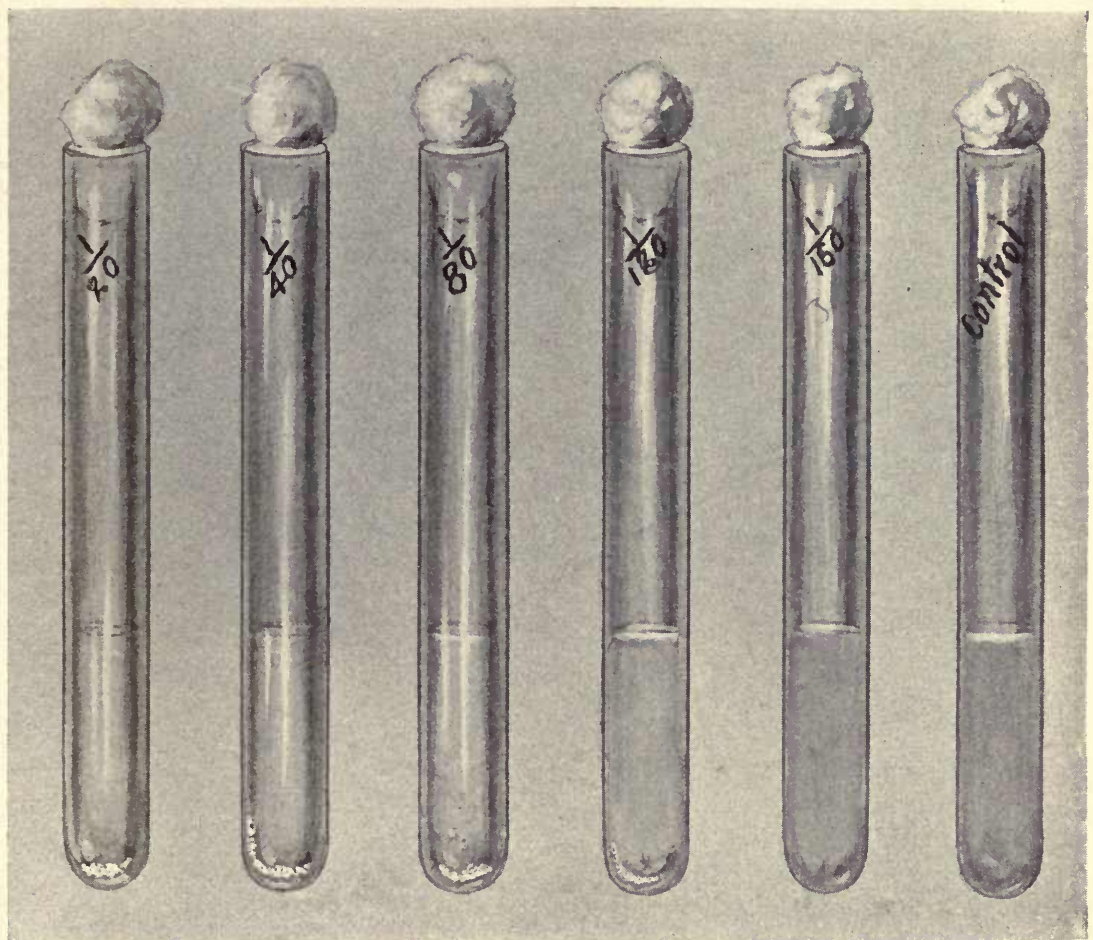

Fig. 82.-Macroscopic Agglutination Reaction.

Serum of a person who had received three injections of typhoid vaccine. This drawing was made twenty-four hours after the test was set up. The dilutions are marked on the tubes.

$1: 20$, acting on the bacteria; tube 2 , one of $1: 40$; tube 3 , one of $1: 80$; tube 4 , one of $1: 160$; tube 5 , one of $1: 320$; tube 5 , one of $1: 640$. Tube 7 , as just stated, contains the bacterial emulsion in salt solution and is the culture control.

In determining the agglutination titer of a highly immune serum, these dilutions may be continued to any degree.

5. On each tube the final dilution is marked with a wax pencil. 


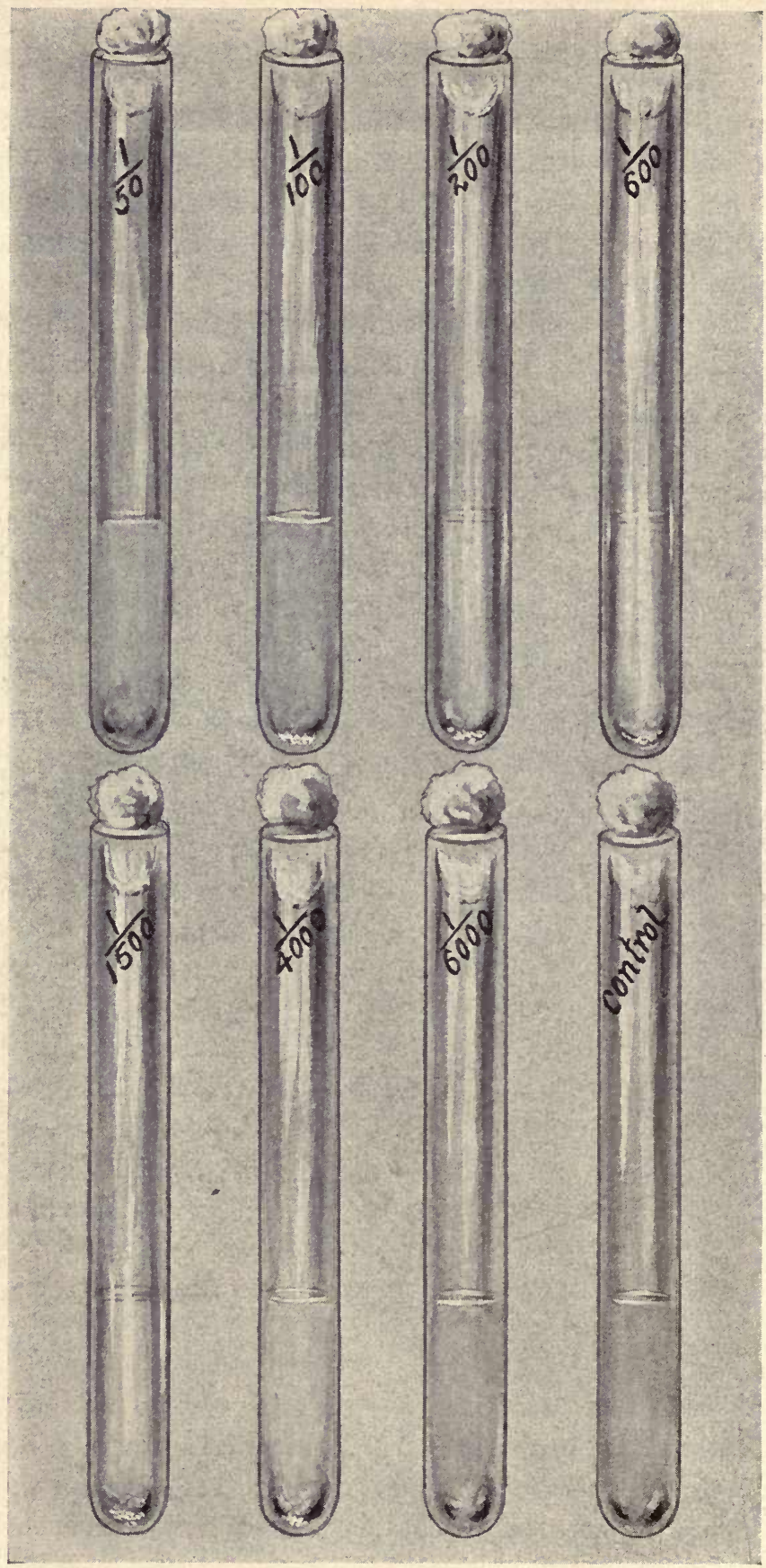

Fig. 83.-Macroscopic Agglutination Reaction. Shows Action of Agglutinoids (Pro-AgGLUtination).

Note absence of agglutination in dilution 1:50; agglutination beginning in 1:100, and fairly well marked to 1:4000 inclusive. Note uniform cloudiness of control. This reaction was set up with a typhoid immune serum over six months of age; the drawing was made after the tubes had been incubated two hours and placed in a refrigerator overnight. 
The tubes are then shaken gently, stoppered with cotton plugs, and placed in the incubator at $37^{\circ} \mathrm{C}$. for two hours. The tubes are then allowed to remain at room temperature for six hours, or in the refrigerator for twenty-four hours, after which readings are made.

6. The culture control should show a uniform cloudiness, with no sediment or flakes, or at most a very slight precipitate that is readily

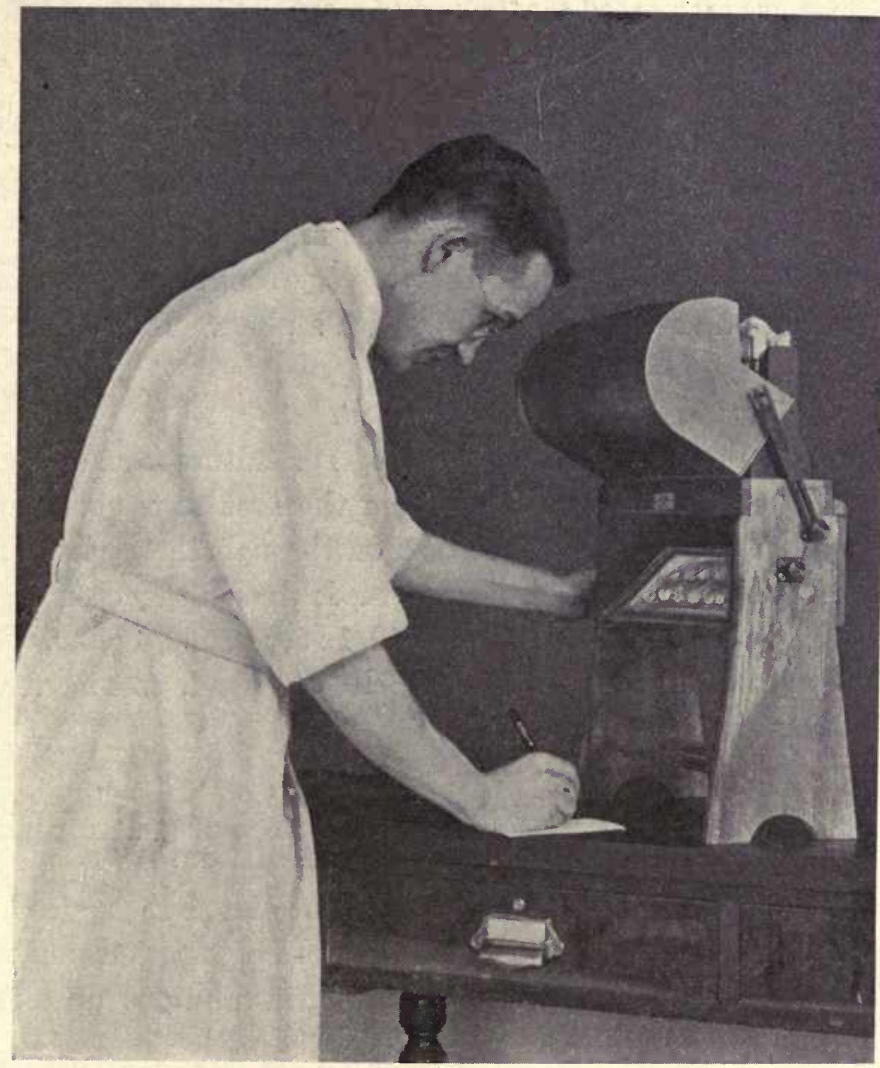

Fig. 84.-AgGlutinoscope (Altman).

The test-tubes are arranged in the rack and viewed from below in the mirror. In this manner the smallest deposits are easily seen and compared with the control.

broken up by gentle agitation. A positive reaction shows masses and clumps of bacteria adhering to the sides and bottom of the tube, which are broken up with some difficulty (Fig. 82). The supernatant fluid should be clear. As dilutions become higher and the amount of contained agglutinin correspondingly less, agglutination becomes less and less complete. There is less sediment, and the turbidity of the supernatant fluid is greater, until the negative tube closely resembles the cul- 
ture control. A microscopic examination of a deposit will show that the bacilli point in all directions, whereas in a deposit of unagglutinated bacilli they lie horizontally side by side.

When agglutinoids are present, agglutination is absent or incomplete in the lower dilutions of serum, and complete in the tubes containing the higher dilutions. This is called pro-agglutination (Fig. 83).

Readings are facilitated by the use of a special instrument known as the agglutinoscope (Fig. 84). The tubes are placed in a rack having numbered holes, and are viewed from beneath with the aid of a mirror. In this way one looks upward through the column of fluid, and secures a combined view of sediment and turbidity, and when examined with the culture control, fine and accurate readings may be made.

The method of Kolle and Pfeiffer is very convenient, and may be safer than that of adding live cultures with a pipet. It is conducted as follows:

1. Make dilutions of serum as described.

2. Emulsify thoroughly a loopful ( $2 \mathrm{mg}$.) of culture from an eighteento twenty-four-hours-old agar culture in the first test-tube, repeating the process in the second tube, and so on through the series. In this method the serum dilutions are not doubled; thus in the foregoing series the dilutions would be $1: 10,1: 20,1: 40,1: 80,1: 160,1: 320$.

3. The tubes are gently shaken, labeled, plugged, and incubated as directed in the preceding method.

\section{TECHNIC OF THE ABSORPTION Agglutination TeSt IN MIXed INFECTION (THE SATURATION TEST OF Castellani)}

The practical importance of partial agglutinins is recognized in the diagnosis of mixed infections. Thus the serum of a patient may agglutinate typhoid as well as paratyphoid bacilli in dilutions up to $1: 100$. This may indicate one of three possibilities:

1. The patient may be infected with typhoid, but has formed an exceptionally large quantity of group agglutinins for paratyphoid bacilli. Saturation of this serum with typhoid bacilli will remove all the typhoid and a portion, if not all, of the group agglutinins. Saturation with paratyphoid bacilli will remove the group agglutinins, but not the main or typhoid agglutinin.

2. The patient may be infected with paratyphoid bacilli, but has formed, at the same time, many partial agglutinins for typhoid bacilli. Saturation of the serum with paratyphoid bacilli will remove all the paratyphoid and a large portion of the typhoid agglutinin. 
3. The patient may have a mixed infection of typhoid and paratyphoid, and therefore agglutinin for both may be present. Saturation of the serum with typhoid bacilli will remove the typhoid and probably a small portion of the paratyphoid agglutinin. After this reaction the serum will still show the presence of a decided quantity of paratyphoid agglutinin.

In selecting the most likely one of these hypotheses a decision may be reached by adopting the method of Castellani (Citron), which is as follows:

1. Four rows of test-tubes are arranged, each row being made up of four small tubes each containing 1 c.c. of serum dilutions $1: 20,1: 40$, $1: 80$, and $1: 160$ respectively.

2. In each of the tubes of the first and second rows five loopfuls of typhoid bacilli are emulsified. An extra tube containing 1 c.c. of normal salt solution receives a similar amount of bacteria, and serves as the typhoid control.

3. In each tube of the third and fourth rows five loopfuls of paratyphoid bacilli are emulsified. Arrange the paratyphoid culture control.

4. Mix gently and incubate for four hours. Carefully record the presence or absence of agglutination in each test-tube. Centrifuge all the tubes excepting the two controls, and transfer the supernatant fluid of each to other test-tubes arranged in the same order.

5. To each tube of the first and third rows add five loopfuls of typhoid bacilli; to each of the second and fourth rows, five loopfuls of paratyphoid bacilli. Mix well and incubate for four hours.

(a) If typhoid is present, the agglutination titer in the first part of the test will be strong in the tubes of the first and second rows, and weak in those of the second and third rows. In the second part of the test the titer for typhoid will be weak or nil in the first, second, and fourth rows, whereas in the third row it will remain practically the same.

(b) If paratyphoid exists, the agglutination titer in the first part of the test will be strong in the tubes of the third and fourth rows, and weak in those of the first and second rows. In the second part of the test the titer for paratyphoid will be less or nil in the fourth row, and strong or unchanged in the second row.

(c) If a mixed infection exists, the agglutination titer in the first part of the test will be strong in the tubes of all four rows. In the second part of the test the titer in the first and fourth rows is much weaker or nil, and in the second and third rows it will remain the same. 


\section{TESTS BEFORE TRANSFUSION FOR ISOHEMAGGLUTININS AND ISOHEMOLYSINS}

1. Two or three c.c. of blood are obtained from each donor from a vein at the elbow and 0.5 c.c. is placed at once in a centrifuge tube containing 5 c.c. of a 1 per cent. sodium citrate in normal salt solution. The remainder is placed in a small, dry test-tube until coagulation has occurred and the serum has separated.

2. From the recipient, 3 to 4 c.c. of blood are necessary; 0.5 c.c. is placed in sodium citrate solution, and the remainder is allowed to coagulate and the serum collected.

3. The sodium citrate tubes are centrifuged; the supernatant fluid is pipeted off, and the cells are washed again with normal salt solution. After the final washing enough normal salt solution is added to the sediment of cells to bring the total volume up to 5 c.c.

4. The serum tubes are also centrifuged, so that clear serums are obtained. These should preferably be free from hemoglobin stain.

5. The following mixtures should be set up within twenty-four hours of the time of collecting blood, in order that native complements may not have undergone deterioration. Measurement may be made according to a drop from an ordinary 1 c.c. graduated pipet held vertically. Small sterile test-tubes ( 8 by $1 \mathrm{~cm}$.) are to be used.

Tube 1: 4 drops of donor's serum +1 drop of recipient's red-cell emulsion.

Tube 2: 4 drops of recipient's serum +1 drop of donor's red-cell emulsion.

Tube 3: Control: 4 drops of donor's serum +1 drop of donor's red-cell emulsion. Should show no agglutination and no hemolysis.

Tube 4: Control: 4 drops of recipient's serum +1 drop of recipient's red-cell emulsion. Should show no agglutination or hemolysis.

Tube 5: Control: 1 drop of donor's red-cell emulsion +4 drops of normal salt solution. This serves as a control on the toxicity of the corpuscles and isotonicity of the salt solution.

Tube 6: Control: 1 drop of recipient's red-cell emulsion +4 drops of saline solution.

One cubic centimeter of salt solution is added to each tube and the tubes are gently shaken and placed in the incubator for two hours. They are to be inspected every half hour. Agglutination is recognized 
macroscopically by the clumping of the red blood-cells into small masses that later sink to the bottom of the tube as a small clot. Hemolysis is likewise easily detected, as corpuscles tend to become precipitated within two hours. If doubt exists, the finer grades of hemolysis may be detected after the tubes have been allowed to stand overnight in an icechest, or at once by thorough centrifugalization.

In cases where, for any reason, the quantities of blood previously named cannot be secured, the whole operation may be conducted with smaller amounts, using Wright's capillary pipets (Epstein and Ottenbarg). This method is as follows:

Blood is secured from both recipient and donor by pricking the finger of each and allowing five or six drops of blood to flow into 5 c.c. of sodium citrate solution, and collecting a good-sized Wright capsule full for securing the serum. The corpuscles are washed twice and enough normal salt solution added to make up approximately a 10 per cent. suspension. The capsules are centrifuged if necessary, filed, opened, and the serum pipeted off.

The mixtures are prepared in Wright's capillary pipets (see Fig. 48) fitted with rubber nipples. The unit volume is marked off by a blue pencil on the stem about an inch from the tip. Four volumes of serum, one volume of cell emulsion, and four or five volumes of salt solution are drawn up into the pipet and then mixed gently, running them out on a watch-glass. The entire mixture is then drawn into the barrel of the pipet and the tip sealed. The pipets are carefully labeled, incubated for two hours, and examined for agglutination and hemolysis.

The results are somewhat more difficult to read, but the method is of value when many donors are to be examined or when the supplies of serum and corpuscles are limited. 


\section{CHAPTER XVII}

\section{PRECIPITINS}

Closely allied to the agglutinins are antibodies known as precipitins. They act on dissolved albuminous bodies in a manner quite similar to the action of agglutinins upon formed cellular elements. For example: (1) If typhoid immune serum is added to a bouillon culture of typhoid bacilli, agglutination occurs; (2) if the culture is filtered and the immune serum is added to the clear sterile filtrate, cloudiness appears and finally a precipitate forms. The first is an example of the action of agglutinins upon the formed bacilli, and the second illustrates the action of precipitins upon the albumins of dead and dissolved bacilli.

Precipitins are formed not only for bacterial albumins, but for most any soluble animal (zoöprecipitin) and vegetable protein (phytoprecipitin) as well. If the serum of a rabbit immunized with horse serum is added to horse serum, a precipitate forms, owing to the presence of a specific precipitin in the immune serum. Normal rabbit serum does not possess this power.

Definition.-The precipitins are specific antibodies that develop in the serum of animals inoculated with bacteria or with solutions of animal or vegetable albumins, which possess the power of producing a precipitate in a clear solution of the particular albumin or culture filtrate against which the animal has been immunized.

Historic.-Kraus (1897) was the first to study and describe the bacterial precipitins. He observed that when the serums of animals that have been immunized against cholera, typhoid, or plague are added to a clear filtrate of the respective bouillon cultures of their bacteria, instead of to the bacteria themselves, the clear solution becomes turbid and a precipitate forms.

This reaction was found to be quite specific, $i$. e., it occurs best with the filtrate of the homologous bacteria, and to a much less extent with closely allied species. For example, the typhoid immune serum does not produce a precipitate with a filtrate of Spirillum choleræ, and similarly a cholera immune serum does not produce a precipitate with the filtrate of Bacillus typhosus. Kraus advocated the precipitin reaction 
as a means of identifying and differentiating certain species of bacteria, but the test possesses no advantage for these purposes over agglutination reactions and is not generally employed.

Tchistovitch was the first to call attention to the non-bacterial precipitins. This observer found that the serum of rabbits inoculated with eel serum, when mixed with a small quantity of the eel serum, caused a precipitate to form.

About the same time (1899) Bordet found that the serum of rabbits inoculated with the serum of chickens, when mixed with the chicken serum, gave a specific precipitate. A little later Bordet produced an anti-milk immune serum (lactoserum) by inoculating rabbits intraperitoneally with milk partially sterilized by heating to $65^{\circ} \mathrm{C}$. When this immune serum was mixed with the homologous milk, small particles appeared, which gradually formed larger flakes and sank to the bottom of the fluid. It was found that the lactoserums were specific-i.e., cow lactoserum would precipitate only cow casein, human serum only human casein, etc.

Wladimiroff was the first to use the bacterial precipitin reaction as a practical diagnostic test. He showed that the serum of a horse suffering from glanders would, when added to a clear filtrate of a culture of Bacillus mallei, produce a precipitate. The technic of these reactions is, however, more difficult than with the agglutination tests, and as the reactions are usually not more delicate or more advantageous than the latter, they are seldom employed.

Following Wladimiroff, Uhlenhuth and Wassermann made a very . important practical demonstration of the value of serum precipitins in differentiating the blood and secretions of man and animals. For example, the serum of rabbits immunized with various bloods would react with solutions of old and dried specimens of their respective bloods, and although "group" precipitins were found present in the tests with the blood of closely allied species, yet the value of the reaction was not impaired to any extent when a proper technic, with correct dilutions, was employed. These discoveries were found to possess considerable value in forensic medicine, particularly in the recognition of the source of blood-stains.

Nuttall, in a thorough and painstaking research with the blood from 500 animals, was able to study the "blood relationship" of various animals as based upon group precipitins. For example, the serum of a rabbit immunized with human blood will react best with human serum, then with the serums of the higher apes, and finally with the lower orders 
of monkeys. Similar reactions were found to occur among the lower animals.

Nomenclature.-The antibody in an immune serum responsible for the phenomenon of precipitation is called precipitin; the substance or antigen responsible for the production of this antibody is known as the precipitinogen; the precipitate is the end-product of the reaction between precipitinogen and precipitin. Just as toxoids and agglutinoids may be formed, so precipitin may be modified to precipitoid.

Although, since bacterial precipitins are produced by the protein constituents of bacteria, the custom of differentiating between bacterial and protein precipitins is superfluous, nevertheless, when the meaning is clearly understood, the term bacterial precipitin is convenient and may be employed.

The precipitins derive their names from their precipitinogens, as, for examplé, a precipitin produced by injecting rabbits with ox serum is designated anti-ox precipitin.

Normal Precipitins.-Although agglutinins may be found in normal serum, it is decidedly uncommon to find normal precipitins. Extracts of organs have been known to contain normal precipitins for certain albumins, although at the same time they were absent from the serum of the animal. In this case the active bodies exist in the cells as "sessile receptors," and by the process of extraction they are brought into solution. During immunization these same receptors are stimulated to overproduction and are thrown into the circulation as free precipitin receptors.

Immune precipitins are antibodies produced by immunization with a foreign albumin, either during the course of a bacterial infection or as the result of artificial inoculation.

Structure and Properties of Precipitins.-According to the side-chain theory, precipitins are antibodies or receptors of the second order, composed of a combining arm or haptophore group for the precipitinogen, and a zymophore or precipitinophore group that precipitates the antigen. Their structure is, therefore, seen to be quite similar to that of agglutinin, the difference being largely due to the different functions of the zymophore group.

The properties of precipitins are quite similar to those of agglutinins. They are fairly resistant bodies, resist the effect of drying for prolonged periods, but are gradually destroyed by heating to $60^{\circ}$ to $70^{\circ} \mathrm{C}$. When inactivated by exposure or heat, they cannot be reactivated by the addition of fresh normal serum, and therefore they bear no relation to the 
complements. As with agglutinins, the presence of a salt is necessary to secure the reaction.

The haptophore or combining arm is quite stable; the precipitophore group is more labile, and is affected by heat, and when this less resistant arm is lost, the receptor is called a precipitoid. Like agglutinoids, the precipitoids are of practical interest from the fact that their haptophore arm will not only combine with precipitinogen, but displays a greater activity in this direction than the whole receptor or precipitin itself, and when union between precipitinogen and precipitoid has occurred, precipitation does not result. Hence in low dilutions of a precipitin serum the phenomenon of precipitation is slight or altogether absent, whereas in higher dilutions the reaction becomes evident.

Group precipitins are not so prominent as group agglutinins, yet they are formed to a certain degree and are of much practical importance in attempting to differentiate bacteria and serums by the precipitation method. Although precipitins are highly specific, the principle of serum dilution, as emphasized under Agglutination, must be closely observed in order to dilute the group precipitins to such small amounts as to prevent them from interfering with the chief precipitin. This principle is of particular importance in differentiating the bloods of various animals, and especially in medicolegal cases, where the precipitin reactions are employed for the diagnosis of blood-stains.

Formation of Precipitins.-Immune serums for diagnostic purposes are produced by injecting the precipitinogenous fluid into the veins, peritoneal cavity, or subcutaneous tissues of animals, usually rabbits. The power of forming precipitins is probably disseminated among the organs and general body tissues. Kraus and Levaditi assign the leukocytes as the chief source of precipitin formation.

As in the case of agglutinin formation, not all animals possess equally the power of forming a precipitin for a given albumin. While this point is of general interest with the bacterioprecipitins, it becomes of particular importance in relation to serum precipitins. For example, an animal will not form a precipitin active against its own serum. If formed, it would be an autoprecipitin, or isoprecipitin, and, as a rule, animals do not form antibodies for their own tissue constituents. Furthermore, animals are unlikely to form precipitins for the proteins of other members of the same species, or if precipitins are produced, they are usually the result of prolonged immunization of a number of animals. Precipitin formation is also slight for the proteins of other animals that are closely related either zoölogically or biologically. For example, attempts at 
immunization of a guinea-pig with the serum of a rabbit, a pigeon with that of a hen, or a monkey with human serum, are procedures that do not usually yield good precipitating serums.

Attempts have been made to produce antiprecipitin by effecting immunization with immune precipitating serums. Such attempts have been reported as partially successful with serum and milk, but not with bacterial precipitins. Antiprecipitins possess no practical value.

Mechanism of Precipitation.-Of the various theories advanced to explain the phenomenon of precipitation, none has received so much support experimentally as that advanced by Bordet in explanation of agglutination.

Colloids may be precipitated by salts, and probably the salts so alter the electric state of colloidal particles that their surface tension is decreased, and, as a result of this change, neighboring particles coalesce in such quantities as to produce a visible precipitate. Salts are likewise necessary for serum precipitation, and there is a close analogy between serum and colloidal precipitation.

The origin of the precipitate formed during the reaction is of interest. When a very potent immune serum is employed, the precipitinogen is so highly diluted that it no longer gives any of the chemical reactions for proteins, but when the precipitating serum is added, it may yield, nevertheless, a heavy precipitate. The precipitate can, therefore, hardly be regarded as due to the slight trace of albumin in the precipitinogen, and, furthermore, if the precipitating serum is diluted, the precipitate becomes smaller and smaller, and, if the dilution is increased, it finally disappears altogether. For this reason the precipitate is generally considered as originating in the immune serum.

Specificity of Precipitins.-Precipitins react but feebly on closely related albumins of the same species, but are specific against those of unrelated species. In other words, the precipitation test merely determines the animal species from which the proteid originates, but cannot demonstrate positively whether it comes from the blood, the semen, milk, or other albuminous body. For medicolegal purposes, therefore, a diagnosis of "human blood-stain" cannot be made without chemical evidence to prove that the stain actually consists of blood.

An immune serum prepared by the injection of the serum of a certain animal gives a precipitate also with the juices of the various organs of that animal. The only exception to this rule is the protein of the crystalline lens of the eye, which gives no precipitate with the antiblood immune serum. The same albumin exists in the crystalline lenses of all 
animals,-from fishes to man,- - and a serum produced by immunization with lens substance will react with the protein derived from the lens of any animal, but with no other animal proteid. This theory of species specificity may, however, be carried a little further, for by carefully freeing the organs from all blood and then using organic extracts for inoculation, it is possible to produce serums that will yield a precipitate best with the particular variety of organic extract (liver, kidney, etc.), and a weak or no precipitate with extracts of other organs of the same animal.

Besides this animal specificity, precipitin reactions also demonstrate the "constitutional specificity" of proteins. If, instead of using a pure animal or plant albumin for immunization, variously altered albumins are used (heated albumins, acid albumin, formaldehyd albumin, and the like), the organism reacts by producing antibodies of a characteristic nature, differing from those developed after inoculation with pure albumin. For example, if a rabbit is immunized with normal horse serum, the resulting immune serum will produce a precipitate when added to pure horse serum, but not when added to horse serum that has been heated. On the other hand, if a rabbit is inoculated with horse serum that has been diluted and boiled for a short time, the resulting immune serum will react not only with normal horse serum, but also with heated serum and a group of its decomposition products with which the normal immune serum ordinarily never produces a precipitate.

This observation is of practical importance in detecting meat substitution by precipitin reactions. In order to render the detection difficult, the meat is commonly boiled; with the aid of precipitins produced by immunization with heated proteins, this fraud is more easily detected than if a normal immune serum were used.

Obermeyer and Pick have demonstrated that while animal specificity is not destroyed when the albumins are modified by heat, tryptic digestion, or oxidation, their specificity is lost when an iodin, nitro- or diazo-group is inserted into the protein molecule. Immunization with such transformed proteins, e. g., xanthoprotein, can produce a precipitating serum that will react with every xanthoprotein, even that of different animals. These investigators conclude that species specificity is probably dependent upon a certain aromatic group of the protein molecule.

Rôle of Precipitins in.Immunity.-Precipitins are probably not truly protective antibodies, like antitoxin and the lysins, but they are quite similar to the agglutinins in being secondary products of cellular activ- 
ity, and they are of value chiefly as indicators of this general antibody formation. They may, however, be concerned in preparing their antigens for destruction and solution, just as opsonins prepare cells for phagocytosis, but precipitins themselves possess no appreciable curative or protective virtues, and are of value chiefly in diagnostic procedures.

\section{PRACTICAL APPLICATIONS}

\section{BACTERIAL PRECIPITINS}

Bacterial precipitins have no clinical diagnostic value. Their reactions have no advantage over the agglutination test, they are more difficult of execution than the latter, the sources of error are greater.

In scientific research they may be of value in differentiating microorganisms from closely allied species, but even here agglutination reactions serve the purpose equally well and are less difficult of execution.

Occasionally bacterial precipitins are of service in demonstrating the presence of soluble bacterial substances within exudates or organic fluids. For example, Vincent and Bellot recommend the reaction as being of considerable value in the diagnosis of cerebrospinal meningitis. The cerebrospinal fluid is centrifugalized until it is clear; 2 c.c. of this clear fluid is then placed in one tube and 4 c.c. into another. One-tenth cubic centimeter of a standard antimeningococcic serum is added to each tube. The tubes are kept for from twelve to fifteen hours at $37^{\circ} \mathrm{C}$. In the presence of cerebrospinal meningitis a precipitate forms. If the fluid is normal or if it was derived from some other form of meningitis, no cloudiness results. This reaction is said to occur within the first twenty-four hours of the illness and to persist until the twelfth to the twentieth day.

Similar reactions have been advocated in the diagnosis of other infections, particularly syphilis. Fornet believed that the presence of typhoid antigen (precipitinogen) ought to be capable of demonstration in the blood-serum of typhoid fever patients long before antibodies themselves are in evidence. One cubic centimeter of potent immune serum in concentrated and diluted form (1:5 and $1: 10$ with normal salt solution) is placed into small test-tubes, and an equal amount of the serum for examination, also in concentrated and similar dilutions, is carefully floated on top of the immune serum. Control tests with normal and immune serum and normal with unknown serum are made. The mixtures are allowed to stand undisturbed at room temperature for 
two hours, and if the reaction is positive, a whitish ring makes its appearance at the point of contact of the two serums, the controls remaining negative. According to Citron, this ring test is also evident in the presence of scarlet fever, measles, and syphilis.

\section{FLOCCULE-FORMING REACTIONS}

Fornet Ring Test.-Owing to the wonderful activity that has marked the research work of syphilis several precipitation tests for diagnostic purposes were devised. These have all been overshadowed and forsaken for the Wassermann complement-fixation test. Fornet applied his ring test, using the serum of patients with manifest luetic symptoms as the precipitinogen, and the serum of paretics as the precipitating or immune serum. Klausner advocated a simple test consisting of mixing in a small test-tube 0.2 c.c. of fresh, active, and absolutely clear serum, with 0.6 c.c. of distilled water. This serum and the control mixtures are allowed to stand at room temperature for from seven to fifteen hours, when a thick, flocculent precipitate of fibrin globulin will appear at the bottom of the tube.

Porges-Meier Reaction.-Porges and Meier observed that luetic serums are capable of producing flocculent precipitates from solutions of lecithin and similar salts. Two-tenths of a cubic centimeter of a 1 per cent. solution of Merck's sodium glycocholate in distilled water is placed in narrow test-tubes, and an equal amount of the patient's serum, which must be absolutely clear and inactivated by heating at $56^{\circ} \mathrm{C}$. for thirty minutes, is added. This mixture and the known normal and luetic controls are kept at room temperature for from eighteen to twenty-four hours. A positive reaction is marked by the appearance of distinct coarse flocculi, mere turbidity or faint precipitation being regarded as negative.

Herman-Perutz Reaction.--More recently Herman and Perutz have devised a similar test requiring the following two solutions: Solution 1 (stock solution, diluted 1:20 with distilled water before use) consists of : Sodium glycocholate, 2 gm., cholesterol, 0.4 gm.; 95 per cent. alcohol, 100 c.c. Solution 2 (freshly prepared before use) is a 2 per cent. solution of sodium glycocholate in distilled water. The test is performed by adding to 0.4 c.c. of clear inactive serum (heated at $56^{\circ} \mathrm{C}$. for half an hour) in a small test-tube 0.2 c.c. of solution 1 and 0.2 c.c. of solution 2 . The tubes are tightly plugged with cotton and set aside at room temperature for twenty-four hours, after which the presence or absence of precipitation is noted. It is well in this test, as in all immunologic re- 
actions, to prepare controls with known normal and luetic serums and with distilled water.

None of these reactions has been found specific, and none has been generally adopted, the far greater accuracy of the Wassermann reaction having made this method more valuable.

Noguchi Butyric-acid Test.-Noguchi has devised a very useful test for the detection of an increased amount of protein, particularly globulin, in cerebrospinal and other body-fluids. In my experience this test has proved of particular value in establishing the differential diagnosis between serous and tuberculous meningitis, being negative in the former and positive in the latter, whereas in both the fluid may be clear, the cytology may be indefinite, and tubercle bacilli may escape detection. Serous meningitis is not a true infection, but a reflex vasomotor disturbance of the cerebral vessels, causing an outpouring of serum that leads to various pressure symptoms closely resembling those of a true meningitis. This condition is particularly common during childhood, and the general symptoms, the increased pressure of the cerebrospinal fluid, and its clear, watery character, are features that resemble those of tuberculous meningitis. It is just in such cases - and they are frequent-that I have found this protein reaction of considerable value. A positive reaction practically always means a true meningitis; a negative reaction usually means "serous meningitis," with a much better prognosis if the underlying cause is corrected.

Noguchi has found the test positive in about 90 per cent. of cases of general paralysis and in 60 per cent. of cases of locomotor ataxia or cerebral or spinal syphilis. In the diagnosis of syphilis the Wassermann reaction with cerebrospinal fluid has greater value than the protein reaction. However, the best results in diagnosis are usually secured by a Wassermann test, butyric-acid test, and total and differential cell-counts. In a case where the diagnosis rests between tuberculous meningitis and syphilis, a positive butyric-acid test and a negative Wassermann reaction would decide in favor of the former.

The test is extremely simple. Into a small, thin-walled test-tube place 0.2 c.c. of cerebrospinal fluid (which must be clear and free from blood); add 1 c.c. of a 10 per cent. solution of butyric acid in normal salt solution; heat over a low flame and boil for a short period. Then add quickly 0.2 c.c. of a normal solution of sodium hydroxid and boil once more for a few seconds. The presence of an increased content of protein is indicated by the appearance of a granular or flocculent precipitate, 
which gradually settles to the bottom of the tube, under a clear supernatant fluid (Fig. 85).

The velocity and intensity of the reaction vary with the quantity of the protein contained in a given specimen. The granular precipitate appears within a few minutes in a specimen containing a considerable increase in protein, whereas one hour may be required to obtain a distinct reaction in specimens weaker in protein. In obtaining the reaction, the time period should not be greater than two hours. A faint opalescence without the formation of a distinct precipitate is to be regarded as within the limits of the normal.

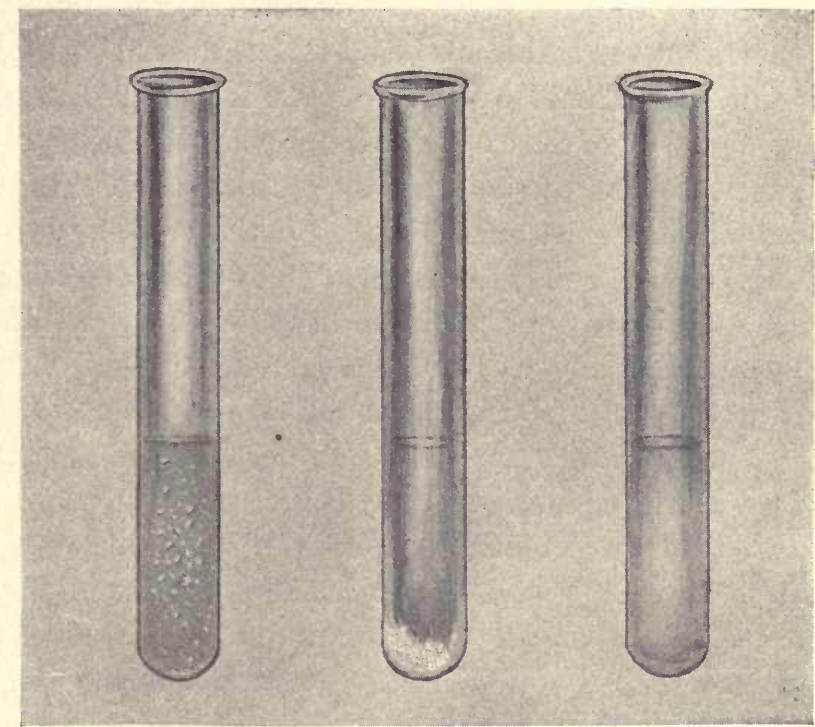

Fig. 85.-The Noguchi Butyric-acid Test for Globulins.

The tube on the extreme left shows the formation of flocculi within a few minutes after adding $\mathrm{NaOH}$; the middle tube shows a strongly positive reaction after standing several hours (supernatant fluid quite clear); the tube on the extreme right shows a very slight opalescence, but no flocculi (within the limits of normal).

\section{PROTEIN PRECIPITINS}

The protein precipitins have a larger range of value and represent one of the most important practical aids in forensic medicine. As mentioned elsewhere, a precipitating immune serum reacts only, or at least best, with its homologous serum. The precipitin reaction is, therefore, highly specific, and offers a method whereby proteins can be easily and definitely determined-a problem that could not be solved by chemistry.

By means of lactoserums various milks and cheeses may be recognized and their source determined. 
By using appropriate immune serums seminal fluids and stains may be detected, and in medicolegal cases, where the question is one of rape, this reaction possesses considerable value in differentiating seminal from leukorrheal stains.

The precipitin reaction is likewise of great value in medicolegal cases in determining the source of blood-stains, the original application and technic having been largely worked out by Wassermann, Schutze, Uhlenhuth, and Weidanz. Thus in a case where, for example, a bloody towel is found in the possession of a man charged with murder, the prosecution may see in this a proof of crime, whereas the defendant may claim that the stains are those of dog's blood. Microscopic and chemical tests may show that the stains are blood-stains, but they cannot determine their source. The blood-stained towel is placed in water or salt solution, and a portion of the extract is mixed with the serum of a rabbit immunized against human serum, and another portion with the serum of a rabbit immunized against dog's serum. If the first mixture shows a precipitate, the stain was made by blood from a human being; if, on the other hand, this mixture remains clear and the second shows a precipitate, this is strongly indicative of the presence of dog's blood.

This method has also cleared up a number of scientific problems, especially that of showing the blood relationship of man and the lower animals. Just as a group aggluination demonstrates the close relationship existing between various bacteria, so, also, serum precipitins prove that a distinct relationship exists between the different species of animals.

For example, an anthuman serum in low dilution will precipitate the serum of monkeys. The differentiation between human and monkey serum can be accomplisherri' $h$ 'hever, by immunizing the monkey with human serum, when a precipitin is formed that reacts with human serum alone, an isoprecipitin, or one active against the monkey's own serum, not being developed as a general rule.

The precipitin tests are likewise of value in food inspection, as, for instance, to determine the nature of meats. For example, in order to detect the presence of dog or horse flesh in sausage, extracts of the sausage are made and tested with anti-dog and anti-horse serum, the presence of precipitates indicating strongly the presence of the meat of these animals. With an appropriate technic even salted and cooked flesh may be recognized, although when the meat has been cooked it is necessary to prepare immune serums by immunizing rabbits with extracts of cooked meats.

In this connection it may be stated that specific organic reactions have been secured by various investigators by prolonged immunization 
of rabbits with certain organ extracts. Thus it is possible to differentiate between the liver and kidney of the same animals; such tests have, however, but limited practical value. Maragliano attempted to apply this test of organic specificity to the serodiagnosis of malignant tumors by preparing immune serums by the injection of tumor juices, securing a serum that yielded a precipitate with the albumins of a cancerous tumor. The test is not absolutely specific, and its practical value in diagnosis requires confirmation.

\section{TECHNIC OF PRECIPITIN REACTIONS}

\section{Differentiation of Human and Animal Blood-Biologic Blood Test}

Unless a stain is definitely known to be a blood-stain it is necessary to establish its identity by making a chemical test before proceeding with the precipitin reactions. For example, old stains upon clothing may be due to substances other than blood, such as coffee and fruit-juices. Blood-stains upon clothing, metal, wood, or glass may be used for making these reactions and their source determined.

To identify the stain as one of blood, a portion may be taken into solution in distilled water, rendered slightly acid with dilute acetic acid, filtered until clear, and examined spectroscopically. Or the Teichmann hemin crystal test may be applied to the stain by transferring to a clean slide a small amount of material scraped from the stain; add a few small crystals of sodium chlorid, crush the crystals, and mix the powder with the dry material. Place a clean cover-glass over the stained material and run a small amount of glacial acetic acid under the cover-glass. Heat the preparation to just about the boil ;-point for a minute, replenishing the acid as may be necessary. The fluid turns brown. The specimen is allowed to cool a few minutes, and is then examined microscopically for the presence of brown rhombic crystals of hemin (Fig. 86). It may be necessary to reheat the specimen several times before the crystals are obtained.

Having shown that a given stain is actually a blood-stain, the source of the blood may be determined as the result of the precipitin reaction, which consists in extracting the stain in normal salt solution and mixing with antiserums prepared by immunizing rabbits with human and various animal serums. Since the antiserums are known, a precipitate with any one of the extracts indicates that the blood in the stain was derived from the same species of animal. The reaction is based upon the principle of the specificity of antigen and its antibody. Here the antibody is known, and is used in the test to detect the antigen. 
As mentioned elsewhere, because of the presence of group precipitins the reaction is fraught with certain technical difficulties of importance, especially in medicolegal cases. In most instances it may suffice to show that a stain is of human blood, as will be indicated by a strong reaction with human blood and negative reactions with the bloods of lower animals. If the reaction is negative with antihuman serum, the antiserums of the domestic animals, such as that of the dog, cat, hog, ox, horse, etc., are tried. Although the blood of the higher apes, and even of the lower orders of monkeys, may react slightly with human blood, this factor may be determined by observing a proper technic of dilution, or the possibility of a given stain being one of monkey, blood being definitely ruled out.

The reaction can be obtained from blood in an advanced state of putrefaction, or from a stain that has been dried for a year or more. Tests with mummies, however, have reacted negatively, and stains or clots altered by heat may not react unless the antiserum has been prepared with a similar antigen.

This same technic may be employed for the recognition of seminal stains, especially in cases of rape. The stain is taken into solution in exactly the same manner as the blood-stain, and tested with an antihuman semen serum prepared by immunizing rabbits with human semen.

Preparation of the Extract of Stain (the Precipitinogen).-If the stain is on clothing, a portion three inches square should be carefully torn into shreds with forceps and scissors, and not with the fingers, and placed in 40 c.c. of normal salt solution. If the stain is upon wood, glass, or metal, the staining substance should be carefully scraped off with a knife and placed in the salt solution. As a further control on the technic an unstained portion of the clothing should be extracted in the same manner, in order to show that the latter alone does not give the reaction. The mixtures must not be shaken, but should be stood aside for from two to twenty-four hours, depending upon the rapidity of extraction. The extract should preferably not be stronger than 1:1000. The strength may be estimated by removing 1 c.c. of the extract into another tube, diluting with from 10 to 20 c.c. of normal salt solution, and gently shaking. If a persistent froth appears upon the surface of the fluid, sufficient extraction has occurred. Place 2 c.c. in a test-tube, heat to bo:ling, and add a drop of a 25 per cent. solution of nitric acid. A faint opalescence indicates that the strength of the extract is about 1:1000 (Fig. 87). If a heavy precipitate forms, the amount of normal salt solution that must be added to a portion of the extract to reduce it to a 


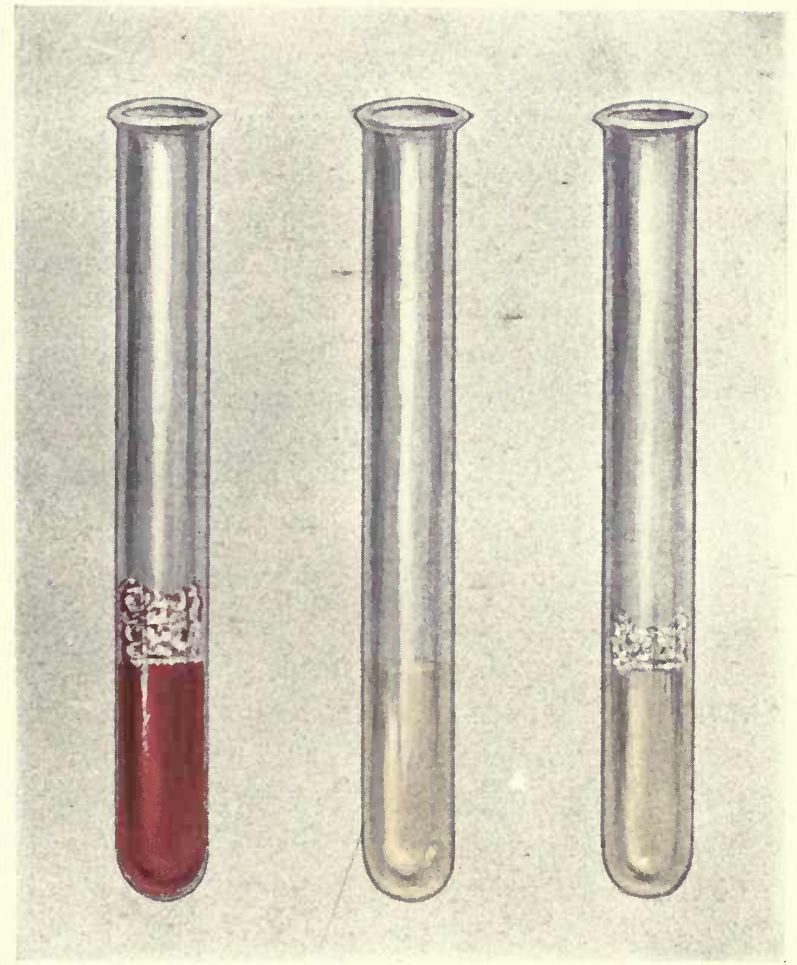

Fig. 87.-Precipitin Reaction. Preparation of Extract of Blood-stain.

The tube on the left shows the color of an extract of a blood-stain; the middle tube shows this extract so diluted as to yield a faint albumin reaction with nitric acid; the tube on the right shows the foam test with the same diluted extract (about 1:1000). 

dilution of 1: 1000 should be determined. The extract should be almost colorless by transmitted light, and must be crystal clear; this may be accomplished by filtering it through a clean sterile Berkefeld filter. It is highly desirable that the extract be sterile, as the reaction may require

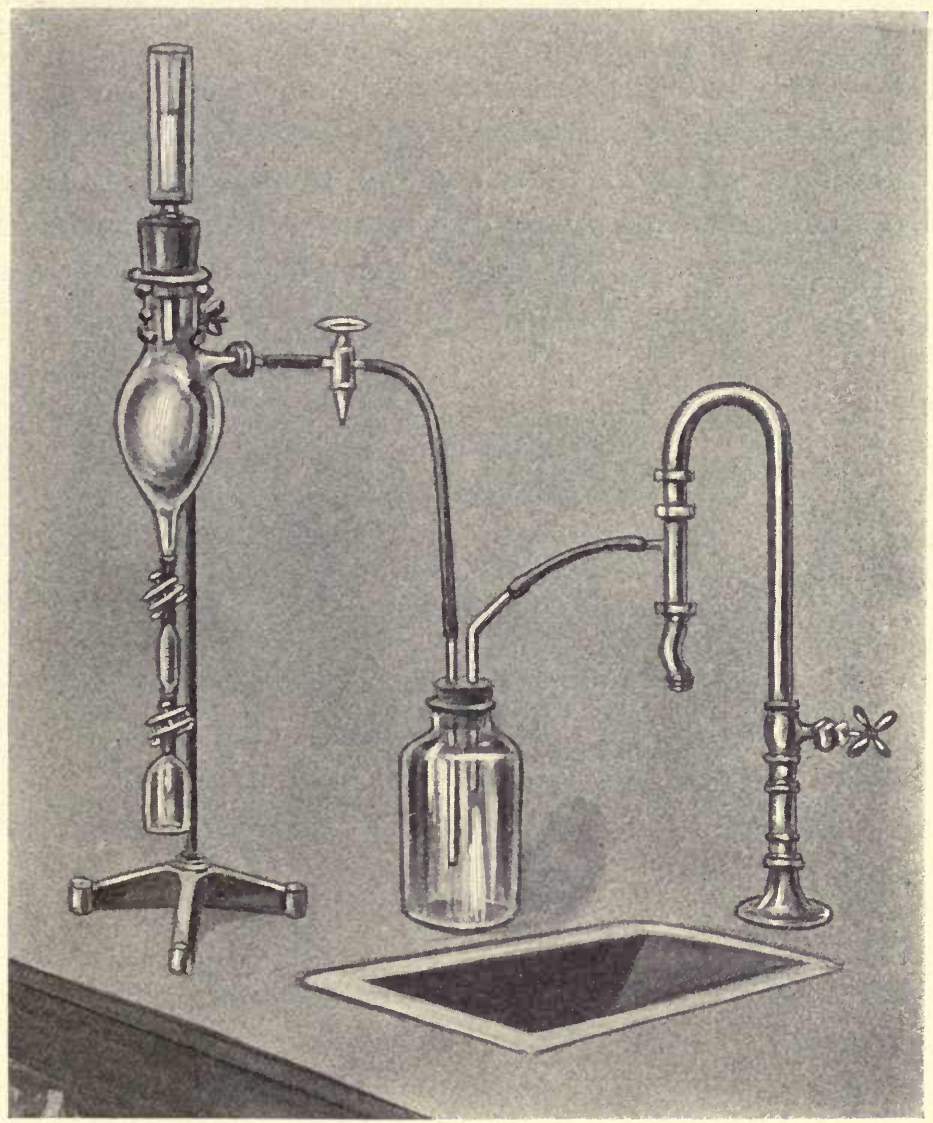

Fig. 88.-An Uhlenhuth Filter.

Serum is poured into the glass cylinder surrounding the "candle"; a vacuum is produced by the water-pump; the filtrate is collected in the small bottle attached by rubber tubing to the graduated cylinder at the lower portion of the apparatus. This filter is especially adapted for filtering small amounts of serum or other fluid.

several hours, and cloudiness due to bacterial growth may interfere with the result.

The Immune Serum.-A highly potent, sterile, and absolutely crystal-clear serum immune against the protein to be recognized must be prepared. The method of preparation is given in the chapter on Active Immunization of Animals. For the recognition of blood-stains it is not 
necessary that whole blood be injected, as the serum alone will suffice. It is better to inject a number of rabbits with each serum and to give all injections intravenously. From the third injection on, preliminary titrations are made according to the technic to be described later, as many animals succumb after a large number of injections have been given them.

The serum must be absolutely clear. Animals should be bled after a period of fasting, as the opalescence of the serum following feeding cannot be removed by filtration and will interfere with the reaction. Precipitin immune serum should be collected with a scrupulous aseptic technic, and stored in ampules holding 1 c.c. Although it is best not to add a preservative, the addition of 0.1 c.c. of a 1 per cent. solution of

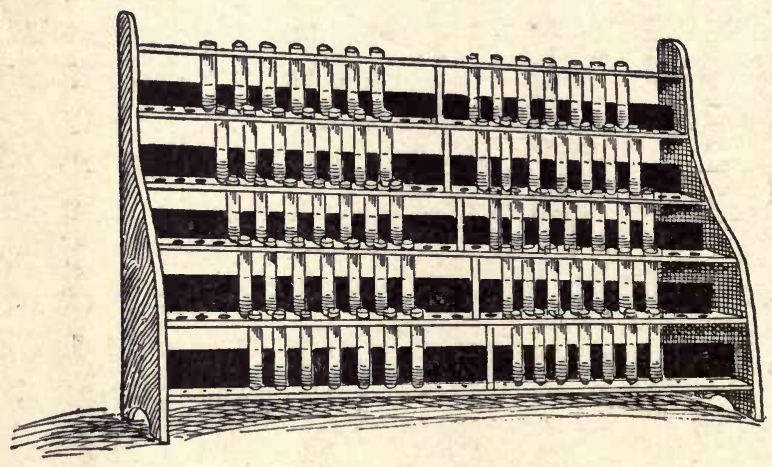

Fig. 89.-Test-tube Rack for Precipitin and Agglutination Reactions. The strips of black material in the rear of the tubes facilitate reading the reactions.

phenol to each cubic centimeter of serum does not render the fluid cloudy and aids greatly in its preservation.

If, after long standing, a precipitate has become deposited in an antiserum, this should not be shaken up, but the ampule should be carefully opened and the clear supernatant serum drawn off with a capillary pipet. A serum that has become cloudy may be cleared partially or entirely by filtering it through a small candle filter, although even an infected and offensive serum will give the reaction (Fig. 88).

Apparatus.-Long and narrow test-tubes, $10 \mathrm{~cm}$. by $0.8 \mathrm{~cm}$., are used: These must be absolutely clean, and preferably sterile.

The test-tube rack devised by Uhlenhuth, in which the tubes hang suspended in beveled holes, is quite satisfactory. Where a test is carried out with many controls, a rack similar to the one shown in the illustration (Fig. 89) is quite serviceable. A strip of black material placed be- 
hind the tubes aids greatly in the detection of the finer degrees of opalescence or precipitation.

Preliminary Titrations. - The precipitin content of an immune serum is titrated frequently during the process of immunization and after the animal has been bled. For medicolegal purposes, Uhlenhuth advises the use of only highly valent serums. He considers an antiserum ef-

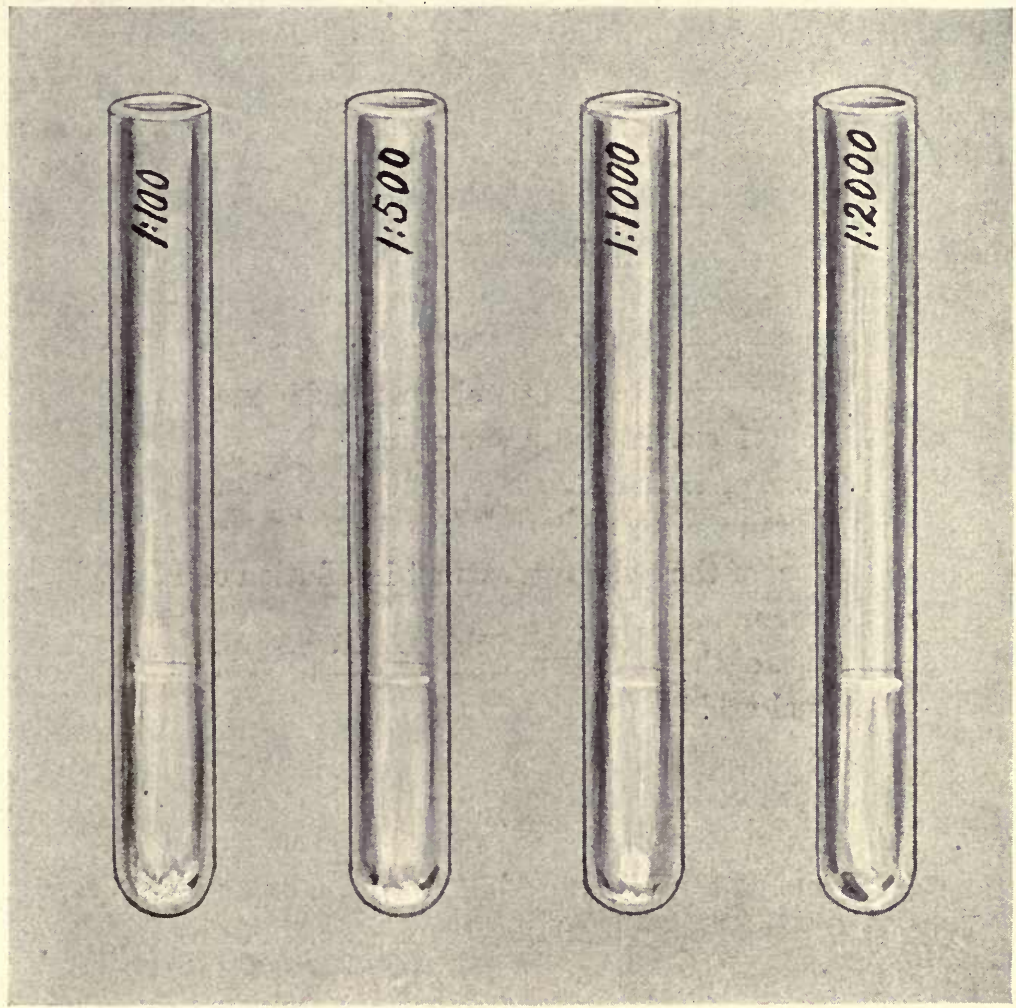

Fig. 90.-Titration of a Precipitin (Serum).

Not all tubes of the series are here shown. Note the well-marked precipitate in the bottom of the first two tubes $(1: 100$ and $1: 500)$; the third tube $(1: 1000)$ shows less precipitate; the fourth tube $(1: 2000)$ is negative (clear and no precipitate). The titer of this serum was recorded as $1: 1000$.

ficient if 0.1 c.c. of it, when added to its respective serum-antigen diluted 1:1000, produces a distinct turbidity, either at once or in from one to five minutes at the latest.

Into a series of six test-tubes place 2.0 c.c. of the following dilutions of serum-antigen, prepared with normal salt solution: 1:100, 1:500, $1: 1000,1: 2000,1: 5000$, and $1: 10,000$. To each tube add 0.1 c.c. of 
clear immune serum. The tubes must not be shaken. Within from one to five minutes a faint, misty cloud appears at the bottom of the tubes reacting positively, and this becomes a distinct precipitate within onehalf to one hour (Fig. 90).

Before performing the actual test with the unknown blood-stain it is advisable to test the entire reaction with a similar known blood-stain in order to make sure that all ingredients are in good working order. In laboratories equipped for medicolegal examinations stains upon linen, as by the blood of man, dog, cat, ox, horse, etc., and their respective antiserums are always kept in readiness for making the preliminary and actual tests.

Technic of the Test..-The following mixtures are set up in a series of test-tubes. Fresh sterile pipets should be used in handling the various solutions. The immune serum should be added slowly, and in such a way that it will flow down the side of the tube and collect at the bottom.

Tube 1: 2 c.c. of extract of unknown substance in dilution of $1: 1000+0.1$ c.c. of immune serum.

Tube 2: 2 c.c. of the unknown extract in dilution of $1: 5000+0.1$ c.c. of immune serum.

Tube 3: 2 c.c. of the unknown extract in dilution of $1: 10,000+0.1$ c.c. of immune serum.

Tube 4: 2 c.c. of the unknown extract in dilution of $1: 100+0.1$ c.c. of normal rabbit serum (control).

Tube 5: 2 c.c. of a 1:1000 dilution of the serum of that species of animal whose blood is suspected to be present in the unknown solution +0.1 c.c. of immune serum (control).

Tube 6: 2 c.c. of the extract of unknown substance alone (control). Tube 7: 0.1 c.c. of the immune serum +2 c.c. of normal salt solution (control).

Tube 8: 2 c.c. of the extract of the unstained portion of clothing + 0.1 c.c. of immune serum (control).

The tubes are not shaken, are kept at room temperature, and the results are read after from ten to twenty minutes. Exposure to incubator temperature facilitates the reaction. With proper immune serums, and especially in medicolegal work, a positive reaction should appear within two to five minutes as a faint, misty cloud at the bottom of the test-tube. Within five minutes this becomes more definite, and in from ten to twenty minutes the precipitate is seen (Fig. 91). Any cloudiness that develops later than tweinty minutes after the beginning of the reaction has no significance. 
In tests other than those employed in medicolegal work, especially if the antiserums are weaker than desired, the reaction may be read after one to two hours.

In the foregoing test, if positive results are obtained in tubes 1,2 , or

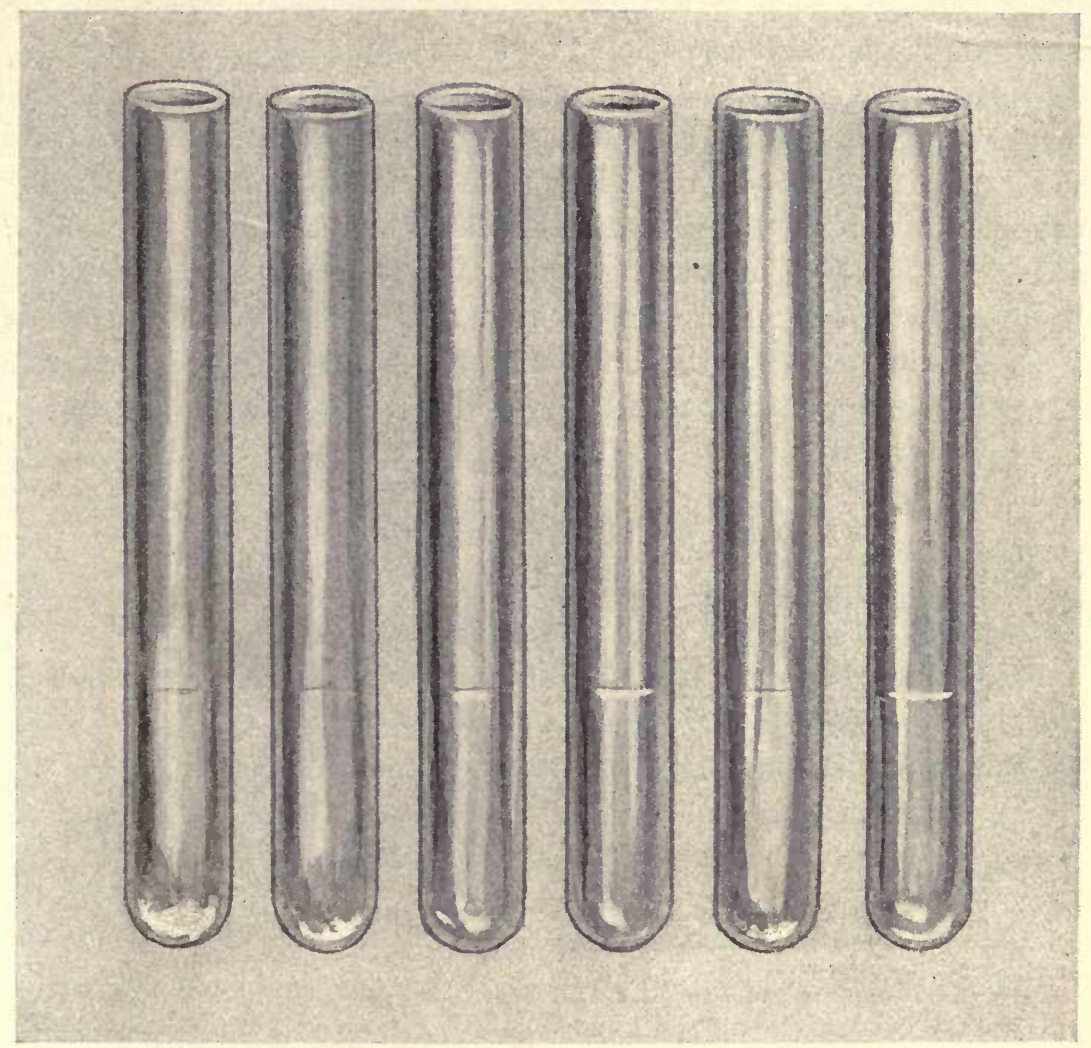

Fig. 91.-A Precipitin Reaction-Biologic Blood Test.

This reaction was set up with an extract of a stain of sheep blood on a towel, over three months of age, with an anti-sheep immune serum.

The first tube, containing a 1:1000 dilution of the blood (extreme left), shows a well-marked precipitate; in the second $(1: 2000)$ the reaction is also well marked; the third $(1: 5000)$ shows no precipitate; the fourth contains a $1: 1000$ dilution of blood-stain, with normal rabbit serum as a control, and shows no precipitate; the fifth is the positive control with known sheep blood extract and immune serum; the last tube is No. 8 of the series, and contains an extract of the non-bloody portion of the towel with immune serum; no precipitate.

3 and tube 5 , and all the others react negatively, the presence of the blood or protein of the species suspected in the unknown extract is established. If the entire test proves negative, the species to which the unknown specimen belongs must be determined with new antiserums prepared for each species, and the tests conducted in the manner described. 
Partial reactions between closely related species due to group precipitins seldom occur, and are easily detected when the technic described is employed. The precipitin test, as determined by the extensive experience of Nuttall, is highly specific, and it is only between very closely related animals, such as the hare and the rabbit, the horse and the mule, the sheep and the goat, etc., that any doubt can arise.

When only very limited amounts of the unknown stain are available, the test, according to Hauser, can be carried out in slender, clean, and sterile capillary tubes. The piece of stained clothing is torn into shreds, extracted, and filtered until clear. The tests are performed by drawing a small amount of the unknown solution into a capillary tube, and underlying this with a small amount of immune serum. As many controls as possible are put up in the same manner. A distinct whitish ring will form in the positive tubes at the line of contact between the two fluids; this is best seen by holding the tube against a black background.

\section{Detection of Meat AdULTERATION}

The principle of this method is the same as that in the foregoing test. An extract of a meat will yield a precipitate when mixed with its antiserum, prepared by immunizing rabbits with an extract of the flesh or with the blood-serum of some other animal.

The method is especially serviceable in food inspection for the detection of horse, dog, or other foreign flesh in meat mixtures, such as sausage and the like. Even salted and cooked meats may be used in the test, although the latter may require the use of antiserums prepared by immunizing with heated or cooked antigen.

Preparation of the Meat Extract.-To prepare this, about 50 grams of flesh are removed from the deeper parts of the specimen by means of a sterile knife, and through a fresh opening, as this portion has been least exposed to the methods of preservation, especially at the high temperatures to which the meat may have been exposed. It should contain as little fat as possible. It is then placed on a clean sterile tile and cut into smaller pieces, and finally minced by passing it through a perfectly clean meat-grinder or chopping it with a sterile chopping knife. After being finely minced the meat is placed in a sterile Erlenmeyer flask, and covered with 100 c.c. of sterile normal salt solution. The mixture of meat and salt solution is kept for about six hours at room temperature, or overnight in the refrigerator, the flask being gently shaken from time to time.

Salted meat should be ground and freshened by placing it in a large 
sterile Erlenmeyer flask and covering it with sterile distilled water, renewed several times in the course of fifteen minutes without shaking the flask.

Since the presence of a great deal of fat interferes with the reaction, it is advisable to remove it beforehand by extracting it with ether and chloroform for from twelve to twenty-four hours (Miessner and Herbst). Pork sausages are usually quite fatty, and may require this preliminary treatment. To make the extraction, take about 75 to 100 grams of the minced meat or sausage and place it in a large Erlenmeyer flask and cover with equal parts of ether and chloroform. After twenty hours the ether and chloroform are poured off, the meat is washed once or twice with sterile normal salt solution, and then extracted in 100 c.c. of salt solution, as described elsewhere.

To determine whether a sufficient quantity of protein substances has passed into solution place 2 c.c. in a test-tube and shake vigorously. If a foam develops and persists for some time, the extraction may be said to be complete. It must then be filtered until it becomes perfectly clear. With extracts of fresh lean meat this is usually accomplished by filtering through a hard filter-paper moistened with salt solution. If it is not crystal clear, and especially if the meat to be examined is fat or salt, it may be necessary to filter through a sterile Berkefeld filter.

To make the test the extract should contain about one part of protein in 500 parts of salt solution. To determine this, 2 c.c. of the clear filtrate are placed in a test-tube and heated, a drop of dilute nitric acid being added. If a marked cloudiness and a flocculent precipitate develops, the extract is too concentrated and must be diluted with clear normal salt solution until the heat and acid test causes only a diffuse, opalescent cloudiness that settles at the bottom of the tube after five minutes as a slight precipitate.

Before proceeding with the experiment the reaction of the solution should be tested with litmus paper, and if it is found to be acid, it should be neutralized very carefully with $\frac{n}{10}$ sodium hydroxid.

Extracts of the meats that are known or likely to be present, such as extracts of pork and beef, should be prepared as controls.

Preparation of Immune Serum.-An immune serum against that variety of flesh that is to be determined in the unknown specimen is prepared by injecting rabbits intravenously with the serum of an animal of that species. For example, if the object is to test for dog meat, an anti-dog serum is prepared by immunizing rabbits with sterile dog serum. 
As has repeatedly been mentioned, it is advisable to immunize a number of rabbits at the same time, for only a small number will yield a satisfactory serum after the third injection.

Immunization may be performed with extracts of flesh that have been filtered and heated at $56^{\circ} \mathrm{C}$. for an hour to secure partial sterilization. Such injections, when given subcutaneously, are likely to produce extensive sloughing, and with any method of immunization the mortality is high.

After the third inoculation it is well to remove a small amount of blcod from the ear and make a preliminary titration. This is performed in exactly the same manner as in making the forensic blood test previously described. An antiserum is satisfactory if 0.1 c.c. produces a well-marked cloudiness and a precipitate in ten minutes with 2 c.c. of a $1: 1000$ dilution of the serum or extract of flesh.

In addition to being highly potent, the immune serum must be crystal clear and sterile. To avoid opalescence the animal should be bled only after a period of fasting.

Technic.-If, for example, the object is to determine whether a piece of meat is horse flesh or, if sausage, contains the meat of this animal the test is conducted as follows:

Tube 1: 2 c.c. of unknown extract, $1: 500+0.1$ c.c. of antihorse serum.

Tube 2: 2 c.c. of unknown extract, $1: 1000+0.1$ c.c of antihorse serum.

Tube 3: 2 c.c. of unknown extract, $1: 5000+0.1$ c.c. of antihorse serum.

Tube 4: 2 c.c. of horse flesh extract, $1: 1000+0.1$ c.c. of antihorse serum (positive control).

Tube 5: 2 c.c. of unknown extract, $1: 500+0.1$ c.c. of normal rabbit serum.

Tube 6: 2 c.c. of pork extract, $1: 500+0.1$ c.c. of antihorse serum.

Tube 7: 2 c.c. of beef extract, $1: 500+0.1$ c.c. of antihorse serum.

Tube 8: 2 c.c. of unknown extract.

Tube 9: 2 c.c. of sterile salt solution +0.1 c.c. of antihorse serum.

The immune serum is added to each tube very carefully and run down the sides of the tube, rather than dropped into them. The tubes. should not be shaken.

If the preliminary titration of the immune serum fulfils the ideal requirement of yielding a well-marked cloudiness within five to ten minutes with a $1: 1000$ extract, the foregoing test should be recorded at 
the end of half an hour at room temperature. If in tubes $1,2,4$, and possibly 3 a misty cloudiness should appear within five minutes, the extract is very probably one of horse flesh. If a definite precipitate forms within thirty minutes, the other tubes remaining slear, horse flesh or the flesh of some other single-toed animal is present.

If the preliminary titration does not show a precipitate with the immune serum until at the end of one or two hours, this interval may be utilized for conducting the test.

In a similar manner tests may be made for the meat of dogs, cats, or any other animals if the respective immune serums are used with the extract.

\section{BACTERIAL PRECIPITINS}

As has previously been stated, these precipitins have slight diagnostic significance, as the information they yield in the diagnosis of an infection or in the differentiation of bacterial species may be gained much more easily with the agglutinin test.

Bacterial precipitinogens are prepared by filtering ten to twenty-one day bouillon cultures through Berkefeld filters. The filtrates must be absolutely clear and sterile, for the reaction frequently requires a number of hours, and if bacteria are present, they may grow quickly, produce turbidity, and mask a reaction.

Immune Serum.-This is prepared according to the technic described under Active Immunization. Rabbits are given intravenous injections of increasing doses of cultures of the bacteria themselves or of filtrates, the inoculum being heated at $60^{\circ} \mathrm{C}$. for an hour previous to making the injection. After the third dose the serum is titrated and the injections continued unless the serum is satisfactory.

Technic.-A known quantity of precipitinogen and varying amounts of immune serum are employed. If too much precipitinogen is furnished, the precipitate will not form, and one that has already formed may dissolve on the addition of more precipitinogen.

If, for example, one desires to determine if typhoid precipitin is present in a given serum, the test is conducted as follows:

Tube 1: 2 c.c. of typhoid bouillon filtrate +0.05 c.c. of unknown serum +0.9 c.c. of normal salt solution

Tube $2: 2$ c.c. of typhoid bouillon filtrate +0.1 c.c. of unknown serum +0.9 c.c. of normal salt solution.

Tube 3: 2 c.c. of typhoid bouillon filtrate +0.5 c.c. of unknown serum +0.5 c.c. of normal salt solution. 
Tube 4: 2 c.c. of typhoid bouillon filtrate +1 c.c. of unknown serum.

Tube 5: 2 c.c. of typhoid bouillon filtrate+1 c.c. of typhoid immune serum (positive control).

Tube 6: 2 c.c. of typhoid bouillon filtrate +1 c.c. of normal salt solution.

Tube 7: 2 c.c. of typhoid bouillon filtrate+1 c.c. of normal serum.

Tube 8: 1 c.c. of serum +1 c.c. of normal salt solution.

The tubes are not shaken, and are kept at room temperature for from one to six hours. If the unknown serum contains considerable typhoid precipitins, a positive reaction will be noticed in the first four tubes in a short time- often within from ten to fifteen minutes. Tube 5 should show a strong reaction and the other tubes should remain clear.

In studying the biologic relationship of an organism to others of the same group its immune serum may be used in amounts of 1 c.c. of varying dilutions, as 1:50, 1:100,1:500,1:1000, 1:2000, 1:4000, and so on, with a constant dose of 1 or 2 c.c. of the bouillon filtrates of the various organisms studied. A comparison of the precipitates in the respective dilutions of the different filtrates indicates the relationship, according to the amount of group precipitins present in the immune serum.

\section{PRECIPITIN TEST IN CANCER}

Freund and Kaminer have devised a precipitin test to be used in conjunction with their cytolytic reaction in the diagnosis of cancer. The extract of cancer-cells is prepared by treating fresh tissue or tissues preserved in alcohol, finely minced with 10 volumes of 0.6 per cent. acid sodium phosphate solution. After agitating the mixture the cells are allowed to settle, and the extract is decanted off and preserved in an icechest, a few crystals of thymol being added as a preservative. For use add 5 c.c. of a 5 per cent. acetic acid solution to 100 c.c. of the fluid; heat the mixture in a water-bath for fifteen minutes at $80^{\circ} \mathrm{C}$., and filter. Cool, and neutralize to litmus with sodium carbonate. Heat again as previously directed, cool, and filter. The extract may now be tested undiluted, and diluted 10, 50, and 100 times with 10 drops of known normal and cancerous serums, the latter yielding a precipitate that is plainly visible in test-tubes held against the light. The extract keeps for only a few days.

The dilution of extract that yields a precipitate with known cancerous serum, but not with a normal serum, is used in testing unknown serums. Place 10 drops of the patient's serum in a small test-tube and 
add 2 c.c. of the properly diluted extract. Controls should be made up with normal serums, and also the extract with a fluid corresponding to that with which the tissue extract was prepared; that is, by adding to 100 c.c. of a 1 per cent. solution of acid sodium phosphate 5 c.c. of 5 per cent. acetic acid and neutralizing with sodium carbonate.

The precipitate forms at once, and must be viewed by transmitted and not by reflected light. The practical value of the test, however, has not been established. 


\section{CHAPTER XVIII}

\section{CYTOLYSINS}

\section{Amboceptors and Complements}

The cytolysins include a number of antibodies of considerable diagnostic and therapeutic importance, for example, the hemolysins and the bacteriolysins. It will be remembered that the various antibodies act differently upon their antigens, and that, according to the side-chain theory, as their antigens become more highly organized, their structure becomes more complicated. For example, the molecule of a soluble toxin may be considered as simple in structure, and accordingly its antibody has been conceived as being likewise simple, and composed of a plain cast-off receptor or side-arm that unites directly with the toxin and neutralizes it without further aid. Antitoxins and antiferments are antibodies of this nature. For more highly organized antigens, however, so simple an antibody will not suffice, and we now find a more complicated antibody, composed of a portion that unites with the antigen and another portion, an integral part of the antibody, that exerts a special selective action upon the antigen, and either neutralizes its activity or prepares it for ultimate destruction. To this class of antibodies belong the agglutinins and precipitins, which agglutinate or precipitate their antigens preparatory, in a sense, to their final disintegration. For still more complex antigens nature has provided special ferments, always present in varying proportions in the blood, which, when united with the antigen, cause its disintegration and solution in a manner similar to the process of digestion as it takes place in the intestinal canal. These ferments are, however, powerless unless united with the antigens, and here we find that the antibody serves as the connecting link, binding antigen with ferment, which results in a form of digestion and final lysis or solution. The antibody is, therefore, simple in structure, and is composed of two binding or grasping arms-one for the antigen and one for a ferment. This interbody, or amboceptor, is specific for the antigen, and will act only and specifically with this antigen. It is important to remember that the ferment or complement is not an integral part of the antibody, but is free in the blood-stream; that the antibody is only a connecting link, but preserves its importance by being specific for its 
antigen; that the primary function of this antibody is to unite antigen and complement, and that the latter then causes the lysis or solution of the antigen. The connecting link or antibody of this nature is known as an antibody or receptor of the third order.

Different cells produce their own and specific interbodies or amboceptors. Thus bacteria or vegetable cells, blood-corpuscles, and various other cells, such as ciliated epithelium, spermatozoa, renal epithelium, etc., when present in the form of an infection or when injected into an animal, generate different and specific amboceptors, which bring about their solution by binding them with a ferment or complement. One ferment or complement does not serve for all; there are various ferments, which act with the different amboceptors, but all have properties so nearly alike that many believe, with Bordet, that but a single complement exists.

Definition.-This special digestive and lytic process is known to occur with cells, and hence the antibodies capable of bringing about this action are called cytolysins, or substances that cause lysis or solution of the various cells that may be their antigens.

It is well to remember that, according to Ehrlich, the three orders of antibodies each have their counterpart, both in structure and in effect, in the receptors serving for the normal nutrition of cells. For the simplest molecule of food that is in solution the cell is provided with a simple receptor for union with the molecule, which is then directly assimilated. This receptor is similar to an antitoxin, or an antibody of the first order, which destroys its toxin directly and without further ado. More complex food material must first undergo some preparation by the cell before it can be assimilated, and accordingly we find receptors provided with a more complex structure which have their counterpart in the antibodies of the second order, or those possessing a special toxic portion that agglutinates or precipitates their antigen or prepares it for phagocytosis. It is possible that with physiologic substances this is all that the cell requires of its receptor, but so far as is known, it would appear that for antibodies this action does not in itself injure the antigen, but is rather one step toward preparation for its further destruction. Organized and complex food substances must be digested before assimilation can occur, and here we find that the receptor acts as a link in binding the food molecule to a ferment, with resulting dissolution and assimilation of the products of solution. These are called receptors of the third order, and have their counterpart in similar antibodies,- the cytolysins,--which act as links or interbodies between antigen and a comple- 
ment, the latter being entirely free and separate, and independent of the receptor or antibody (interbody) itself (Fig. 92).

Varieties of Cytolysins.-The cytolysins produced by bacteria are known as bacteriolysins, $i$. e., antibodies producing disintegration and lysis of bacteria. The cytolysins known as hemolysins cause lysis or hemolysis of the erythrocytes. Similar cytolysins may be formed for practically all cells, such as leukocytes, epithelium, liver, kidney, spleen, etc., and to these the general name cytotoxin has been given; thus we have leukotoxin, hepatotoxin, nephrotoxin, neurotoxin, etc., these

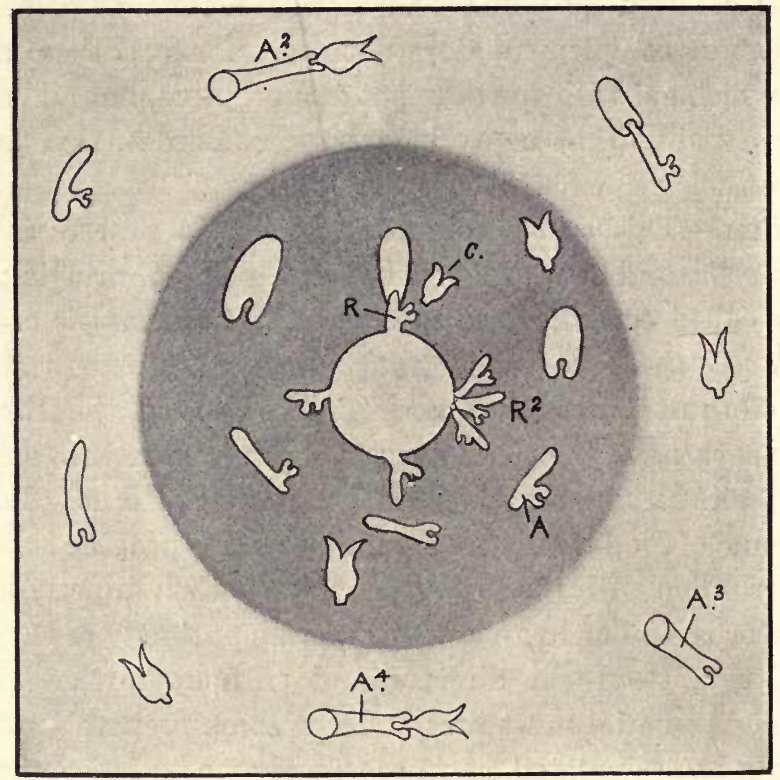

Fig. 92.-Theoretic Formation of Cytolysins.

terms being more nearly correct and expressive of the actual mechanism by which their action is produced.

Nomenclature.-In no other field of immunity have so many different names been applied to the same substances as have been applied to this order of antibodies. This confusion of terms, added to the various interpretations placed upon their significance, has rendered the subject incomprehensible to those not specially interested.

The ferment-like and thermolabile substances present in all serums and actively concerned in lytic processes have been given the name of alexin by Bordet; Metchnikoff called it cytase, and Ehrlich designated it as complement because in the conception of the side-chain theory it 
completes the reaction after being linked with the antigen. The term "alexin" was first applied by Buchner to the germicidal substance found in normal serum. We now know that Buchner was working with both amboceptor and complement, although Bordet was the first to discover the former, Buchner having been unconsciously most interested in the thermolabile complement.

To the antibody itself the term substance sensibilisatrice has been applied by Bordet, for he believes that this antibody sensitizes or prepares the cell for the action of the alexin or complement. The following names have been applied to the antibody by various observers: fixateur, by Metchnikoff; preparator, by Müller; and amboceptor, interbody, and immune body by Ehrlich. Of these, the term "amboceptor" is in most general use, signifying a two-armed body that unites antigen on the one hand, with a complement on the other.

When using the term amboceptor, care should be used to designate its specific character; thus, for example, a hemolytic amboceptor and a bacteriolytic amboceptor mean respectively a hemolysin and a bacteriolysin.

It is common practice to designate an amboceptor according to the cell for which it has a special affinity; thus antisheep amboceptor or hemolysin means an amboceptor for sheep cells, the prefix "anti" being affixed because it is destructive for those cells.

\section{AMBOCEPTORS}

Although antitoxins have received considerable study from a therapeutic standpoint, probably no order of antibodies has been given more attention than the cytolysins have received, not only because of theiz vast therapeutic possibilities, but also from their value as an aid to diagnosis. The hemolysins especially have been utilized-in making the Wassermann test for syphilis and similar reactions, the very nature of the phenomenon offering a visible and fascinating method of study.

Since the general structure, formation, and "action of the various amboceptors, such as the bacteriolysins, hemolysins, and other cytolysins, are essentially similar, the general character of amboceptors may be here considered, a study of the special characteristics of each being reserved for subsequent chapters on the more important members of the group.

Historic.-The alexins or complements were first discovered through the researches of Nuttall and Buchner in 1889. The amboceptors were, of course, present in the various serums with which these observers were 
working, but it was not until 1895 that Bordet showed quite clearly that two substances were concerned in the phenomena of bacteriolysis and hemolysis. At this time he demonstrated that the alexin or complement may be removed from a serum by heating it to $55^{\circ}$ to $56^{\circ} \mathrm{C}$., and that it may be reactivated by the addition of fresh serum from another animal; that an old bacteriolytic serum cannot produce bacteriolysis unless it is reactivated by a fresh normal serum or is placed in the peritoneal cavity of a living animal, from which it may derive the thermolabile alexin. In other words, the amboceptors in these serums withstood the effects of heating and age, but were unable to produce lysis without the aid of an alexin furnished by a fresh normal serum.

Structure of Amboceptors.-According to the theory of Ehrlich, an amboceptor is but a simple interbody furnished with two haptophore or grasping portions. One haptophore group attaches the antibody to its antigen, whatever that may happen to be-bacterium, erythrocyte, epithelial cell, etc., while the other attaches a suitable complement (Fig. 92). The first is called the cytophile or antigentophile group, and the second, the complementophile group. The amboceptor is specific in the sense that it will unite only with its antigen or other very closely related body. For example, when a rabbit is injected with sheep corpuscles an amboceptor is formed that will unite only with sheep, and not with human, dog, ox, or other cells.

As will be shown further on, Ehrlich believes that many different complements may be present in a serum, whereas Bordet believes that one complement exists that will act with the amboceptor, whether this is bacteriolysin or hemolysin. This view is based mainly upon the observation that the complement in a serum may be absorbed out by furnishing an excess of either bacteriolytic or hemolytic amboceptors, the one variety of amboceptor removing all the complement for the other. Although the results of experimental work would seem to indicate that Ehrlich's belief in the plurality of complements is correct, and while this view is quite generally held, conclusive proof regarding this has not as yet been furnished. An amboceptor may have more than one complementophile group, and may bind a number of different complements simultaneously (polyceptor) (Fig. 93). Ehrlich and Morgenroth called attention to this possibility when they stated: "Finally, it is possible that an immune body, besides one particular cytophile group, contains two, three, or more complementophile groups." Later Ehrlich and Marshall showed that, in order to get a specific lytic effect, it was not necessary for all complements to become active, but that only a few 
are necessary in any single instance to bring about effect. These complements are termed "dominant complements," the remainder being known as "non-dominant complements."

Whether amboceptors can undergo degenerative changes and lose their cytophile or complementophile groups and become amboceptoids, just as toxoids and agglutinoids are formed, is still doubtful. Reasoning from analogy to the toxins and agglutinins; it is probable that amboceptoids may be produced by a loss of the complementophile group, the cytophile portion of all antibodies being more stable; such amboceptoids, by uniting with their antigens, may effectually block the action of an amboceptor, just as agglutinoids prevent agglutination.

General Properties of Amboceptors.-Amboceptors are fairly resistant bodies, withstanding to a well-marked degree the effects of heat, acids, alkalis, exposure, and drying. A hemolytic serum, for instance, may be preserved in a sterile condition for many months and show but slight deterioration in its activity. Such a serum may be dried in vacuo or on suitable filter-paper, and preserve its activity for remarkable intervals of time, with but slight and gradual deterioration. While a temperature of $55^{\circ}$ C. will inactivate complement in from

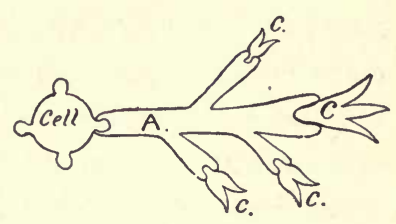

Fig. 93.-Theoretic StrucTURE OF A POLYCEPTOR.

$A$, Main portion of amboceptor in combination with a cell; $C$, dominant complement; $c$, lesser complements. fifteen to thirty minutes, amboceptors can tolerate from $60^{\circ}$ to $65^{\circ} \mathrm{C}$. for an hour and show but slight depreciation in activity.

Mechanism of the Action of Amboceptors.-An amboceptor or antibody of the third order is believed to act as a simple chemical interbody between antigen and complement- $i$. e., it is a connecting link between the two. Complement is, therefore, the active agent in lytic processes, but is practically powerless to act upon the antigen until brought into chemical union through the intervention of an amboceptor. Complement is present in normal serums, and its quantity cannot be increased by immunization. . Amboceptors are specific for their antigen, may be present in small amounts in a normal serum, but may be greatly increased during infection or as the result of artificial immunization. A hemolytic amboceptor for sheep corpuscles is specific for these, and will not unite with the corpuscles of any other animal. A bacteriolytic amboceptor as for Bacillus typhosus will unite only with those bacilli, and to a lesser extent with closely related bacilli, such as the paratyphoids 
but not with other bacterial species or other cells. As to the specificity of complement, opinions differ. Ehrlich believes that there are many complements, as, e. g., one for a particular hemolytic amboceptor, another for a bacteriolytic amboceptor, and so on through the extensive list of known amboceptors. Bordet, on the other hand, believes that there is but one complement, which will act with any amboceptor.

Bordet's conception of the mechanism of this order of antibodies is also different from that of Ehrlich. Both agree that the antibody prepares the cell for the lytic action of a complement, but Ehrlich holds that the antibody acts as a simple link between antigen and complement, whereas Bordet believes that the antibody acts as a mordant or dye, penetrating its antigen, and sensitizing or preparing it for the action of the complement. For this reason Bordet calls these antibodies "sensitizers," or substances sensibilisatrice. The term "sensitizing" is now in general use, and is employed especially in hemolytic tests when corpuscles or bacteria are mixed with their amboceptors to effect their union. It is expressive and satisfactory, and may be used without necessarily subscribing to Bordet's view.

Metchnikoff believes that amboceptor or his "fixator" is found in the leukocytes. He asserts that the amount of fixator or amboceptor produced is proportional to the amount of phagocytosis and phagolysis that occur during the absorption of the antigen. Metchnikoff considers the fixators as analogous to enterokinase, and he believes that, like the latter, the fixator acts as an accessory digestive ferment, having for its object the linking of the more potent ferment, as a cytase or complement, to the cell or molecule to be digested.

Formation of Amboceptors.-While experimental data are at hand to show that amboceptors may be produced by local tissues, it is entirely probable that in wide-spread infection or as the result of artificial immunization there is general cellular activity with extensive antibody formation. The spleen and hematopoietic tissues in general and the mononuclear leukocytes are regarded by many as being particularly active in the formation of hemolysins and bacteriolysins (Pfeiffer and Marx, Deutsch, Wassermann).

As has been stated, Metchnikoff believes that antibodies of the class under consideration are the products of the leukocytes, thus tending to preserve the importance of the phagocytic theory. While there is little doubt that the various leukocytes, endothelial cells, and other phagocytic cells are sources of amboceptor production, there is no reason for accepting the belief that their formation is confined strictly to these cells. 
Specificity of Amboceptors.-It has been stated elsewhere in this volume that amboceptors are highly specific bodies. This specificity is not, however, absolute, for just as group agglutinins are produced by one bacterium for closely allied species, so in like manner experimental investigation by Ehrlich and Morgenroth, von Dungern, and others has shown that immunization of an animal with the erythrocytes of another animal would produce one chief hemolysin for these cells and a secondary hemolysin for the cells of another animal. For example, on immunizing a rabbit with ox blood a hemolytic serum was obtained that was hemolytic not only for goat blood, but also for ox blood. These secondary amboceptors are known as group or partial immune bodies. Their production may readily be understood when it is remembered that the body-cells are conceived as being provided with various side-arms for many different blood-cells, bacteria, etc. Now, if the erythrocytes of the goat possess receptors not only for the particular goat-blood sidearms of the body-cells, but also for the ox-blood side-arms, both sets of side-arms will be attacked and consequently two amboceptors are formed-one, the main one, for goat corpuscles, and a secondary one for ox corpuscles. Ehrlich and Morgenroth, therefore, claim that the immune body of a hemolytic serum is composed of the sum of the partial immune bodies, which correspond to the individual receptors used to confer the immunity. Since the cells of various animals of the same and of different species vary in the number and variety of side-arms or receptors, which are not present in another, the different combining group possessed by a blood-cell or a bacterium will not, therefore, find fitting receptors in every animal, and thus there may be a different variety of partial immune bodies in two animals. This would lead to the possibility of the occurrence of antibodies for the same blood-cell or bacterium, differing from one another in the partial immune bodies of which they are composed, depending on the variety of the animals used in preparing the serum.

This view is directly opposed to that of Metchnikoff and Besredka, who believe that a certain immune body is always the same, no matter what species of animal was used in preparing the serum. As will be pointed out further on, in addition to theoretic interest, the subject possesses great practical importance, for, as is well known, most curative serums are best prepared with many different strains of a particular microörganism because of certain differences in their antigenic properties, and if, in addition, the value of a bacteriolytic serum depends upon the sum-total of the immune bodies, it may be advisable to secure 
as many of these as possible by preparing the serum from various animals of the same and of different species.

It will be understood, therefore, that the specific action of antibodies of this order is not limited to the cells used in the immunizing process, but extends to other cells that have receptors in common with these, a condition that is analogous to group agglutinins and precipitins for closely allied cells and bacteria or dissolved albumins.

\section{NATURAL OR NATIVE AMBOCEPTORS}

Difference Between a Normal and a Specific Immune Serum.-Just as small and varying amounts of native agglutinins and antitoxins may be found in normal serums, so, in like manner, various native bacteriolytic and hemolytic amboceptors may be found. According to the sidechain theory, these various amboceptors are normally attached to bodycells, hence it is probable that a few are being continually swept off into the blood-stream. In some instances the amount of a natural amboceptor may be quite high; thus, for example, many human serums contain relatively large amounts of antisheep hemolytic amboceptor. These natural amboceptors will be considered more fully in the chapters on Hemolysins and Bacteriolysins.

The difference between a normal and an immune serum lies in the fact that the normal serum contains a number of amboceptors in small amounts, whereas the immune serum contains a greatly increased amount of at least one amboceptor for a particular cell. As has been shown by numerous investigators, this difference is not due to the complements, as these are not increased during the process of immunization. Since the presence of an amboceptor cannot be demonstrated unless complement is present, in testing a serum for an amboceptor we must furnish sufficient complement to bring out the maximum activity of the amboceptor. If the serum of a rabbit before and after immunization is titrated with sheep erythrocytes, it may be found that the immune serum contains from a hundred to many thousand times the normal quantity of antisheep amboceptor.

These facts bear a further practical relation to the treatment of infectious diseases with bacteriolytic serums. Ordinarily, when we inject an immune serum we furnish but one bactericidal substance, namely, the bacteriolytic amboceptor, and no complement at all. If the patient's complement is decreased or at least insufficient to activate the amboceptor furnished, lysis will not occur, and accordingly an increased therapeutic effect may be secured by the injection simultaneously of 
an immune serum and a fresh normal serum. This procedure presents certain difficulties, and the subject is considered more fully in the chapter on Passive Immunization.

Anti-amboceptors.-Just as anti-agglutinins and antiprecipitins may be formed, so anti-amboceptors may be produced experimentally by immunizing an animal with an amboceptor-laden serum. An antiamboceptor is specific for the amboceptor that caused its production, and when these are mixed, the activity of the amboceptor is impeded by the anti-amboceptor, which unites with its cytophilic group. It is possible that old erythrocytes are destroyed by an autohemolysin present in the blood-stream under normal conditions, and that a physiologic equilibrium is maintained through the production of an anti-amboceptor.

\section{QUANTITATIVE ESTIMATION OF AMBOCEPTORS}

Titration of a Hemolytic Amboceptor.-The quantity of amboceptor in a given amount of serum may be determined by titration. If, for instance, a rabbit is injected with sheep corpuscles, the amount of hemolytic amboceptor for these cells in the rabbit serum may be determined by the following method of titration: To a series of test-tubes increasing amounts of the rabbit immune serum (heated to remove complement) are added, with a constant dose of sheep-cells and a constant dose of fresh guinea-pig serum to furnish complement. After a suitable period of incubation the tube showing complete hemolysis would contain sufficient hemolytic amboceptor, $i$. e., just one unit. The value of the immune serum may then be expressed by stating that so much serum, as, e. g., 0.001 c.c., contains one unit of amboceptor. Of course, if the amounts of complement or corpuscles are varied the unit will likewise vary. In order to establish or measure the content of hemolytic amboceptor a constant amount of corpuscles and complement must be used. The details of this amboceptor titration are given in the chapter on Hemolysins.

Titration of a Bacteriolytic Amboceptor.-A bacteriolytic amboceptor may be measured in much the same manner as a hemolytic amboceptor, although less accurately, because of technical difficulties. If a standard and fixed dose of an emulsion of living bacteria and a fixed dose of complement are mixed with varying amounts of inactivated immune serum containing amboceptors for these bacteria, the amount of amboceptors present may be determined by plating the mixture and estimating the number of bacteria that have been killed. Or we may use fixed doses of immune serum and complement with varying amounts of bacteria and 
obtain an approximate estimate of the content in bacteriolytic amboceptors after the same manner.

The technic of these tests is given in the chapter on Bacteriolysins.

\section{COMPLEMENTS}

Historic.-As early as 1876 Landois described the hemolytic action of fresh blood-serum upon the blood-corpuscles of animals of certain species. Traube and others observed that animals could withstand the injections of relatively large amounts of septic material, but it was not until 1886-90 that Fodor, Nuttall, Buchner, and others fully established the bactericidal properties of fresh blood-serum. Buchner demonstrated the fact that the active principle causing bacteriolysis or hemolysis is very labile, and can be inactivated by a temperature of $55^{\circ} \mathrm{C}$., by dialysis, or by dilution with distilled water. He designated the active principle "alexin."

Subsequently, in 1899, Bordet found that the alexin of Buchner was composed of two distinct substances-one a sensitizing substance, which is thermostabile, and a second, the thermolabile substance. Somewhat later (1899) Ehrlich and Morgenroth confirmed these observations, but applied the name "amboceptor" to the sensitizing substance and "complement" to the alexin. These terms are most widely employed at the present time. Bordet adheres to the term alexin, meaning thereby the thermolabile principle, and does not use it in the original sense of Buchner, which included both the sensitizing substance and the alexin. Metchnikoff's cytases are practically the same as Ehrlich's complement and Bordet's alexin.

Definition.-Complement [Lat., complementum, that which completes] is the substance, present alike in normal and in immune serum, which is destroyed by heating to $55^{\circ} \mathrm{C}$., and which acts with an amboceptor to produce lysis.

As mentioned in the discussion on amboceptors, the complement is the active lytic substance concerned in the phenomenon of cytolysis, but is powerless until united with the cell, corpuscle, or bacterium by means of the interbody or amboceptor.

Structure and General Properties of Complement.-Complement is ordinarily not attached to the body-cells and is free in the blood-serum. According to Ehrlich, complement is simple in structure, and is composed of a haptophore portion for union with the complementophile haptophore of an amboceptor, and a second toxic or lytic portion, called the 
cytophile group. In other words, the theoretic structure is similar to that of a toxin, although the function and action of the two are quite different.

By heating a complement serum to $55^{\circ} \mathrm{C}$. for half an hour the cytophile portion is destroyed, and the serum is now said to be inactivated, as the complement is no longer active. If the complement serum is allowed to stand at ordinary room temperature for forty-eight hours or longer, the same change will take place. The cytophilic or active portion of the complement is, therefore, quite unstable. When this portion is altered or lost, the substance is called complementoid, and this is analogous to toxoids and agglutinoids.

Since complementoids have their haptophore groups intact, they will unite with amboceptors and to some extent prevent lysis by blocking the active complement, just as toxoids unite with antitoxin and agglutinoids with their antigens.

Anticomplements may be obtained by immunizing suitable animals with serums that contain complement or complementoid. When an inactivated anticomplement serum is mixed with the homologous complement, the haptophores of the latter are bound by means of the haptophores of the anticomplements. A proof of this union lies in the fact that a complement serum that has been treated with its specific anticomplement is no longer able to activate an appropriate amboceptor.

According to Gay, the production of anticomplements is only apparent; he explains the loss of complement activity when a fresh serum and its antiserum are mixed as due to the absorption of complement in the precipitate which forms, although the latter may be invisible.

Anticomplements may be of practical importance owing to the formation of auto-anticomplements. The complements must exercise an important function, not only in the destruction of bacteria, but also in the digestion and solution of all kinds of foreign albuminous bodies that enter the organism. As was shown by Wassermann, anticomplements may so bind up their complements as to render their host much less resistant to certain infectious diseases. The spontaneous development of auto-anticomplement in an animal has never been demonstrated, as there are no receptors in an organism of the complements of the same organism. The injection of the serum of another animal containing complements that are almost identical may, however, lead to the formation of an auto-anticomplement in the serum of the immunized animal.

The extreme lability or sensitivity of complements to heat, exposure, acids, alkalis, etc., is their most prominent general characteristic. An 
active serum is one containing complement, and this must, under ordinary circumstances, be a fresh serum. On heating or exposure the serum becomes inactive. An inactivated serum may be reactivated by the addition of fresh complement serum. These terms are in general use, especially in testing for hemolytic and bacteriolytic reactions.

Origin of Complement.-Buchner regarded complement as a true secretory product of the leukocytes, and Metchnikoff also maintains that leukocytes are the main source, the complement being liberated upon disintegration of the white cells. There is considerable experimental evidence both for and against these views, but at the present time the consensus of opinion would tend to regard the leukocytes as an important, but not necessarily the sole, source of complement formation.

Complement has been demonstrated in plasma, where its presence is probably due to continual disintegration of leukocytes and liberation of complement during life. It is probably increased to a slight extent as serum is left in contact with the blood-clot, indicating that disintegration of leukocytes may augment the complement supply.

The liver (Müller and Dick), pancreas, and other organs have been regarded as sources of complement formation, but in general the evidence points to the leukocytc ${ }^{\sim}$ as the chief source of supply, the liver being probably concerned through its activity in blood destruction.

Multiplicity of Complements.-Ordinarily, a fresh serum, such as that of the guinea-pig, will furnish complement for either bacteriolytic or hemolytic amboceptors, and the question arises as to whether one complement unites equally well with all amboceptors, or whether several complements are present in one serum that act more or less specifically with different amboceptors. .

Bordet believes that only one complement is present, and bases this opinion mainly on the fact that a complement that can be shown to activate either a hemolytic or a bacteriolytic amboceptor may be absorbed out of a serum by furnishing an excess of either amboceptor.

Metchnikoff maintains that there are two cytases or complements, one being derived from macrophages and mainly hemolytic, and the second derived from microphages and chiefly bacteriolytic.

Ehrlich and Morgenroth, Sachs, Wassermann, Wechsberg, and the German school in general believe that many different complements are present in amounts varying with the different serums. These observers have sought to prove this experimentally, and while the evidence is not absolutely convincing, because of the difficulty of working with sub- 
stances that are so labile, yet the doctrine of the multiplicity of complements is quite generally accepted.

(a) By digesting 20 c.c. of fresh goat serum that was found to activate different hemolytic amboceptors with 3 c.c. of a 10 per cent. solution of papain in the incubator for from thirty to forty-five minutes, it was found that the complement for one amboceptor was destroyed, whereas those remaining were left intact or but slightly impaired.

(b) By treating 10 c.c. of this goat serum with 1 c.c. of a 7 per cent. solution of soda for an hour it was found that some complements were destroyed and others were weakened.

(c) By sensitizing different blood-cells with homologous amboceptors and adding these to a fresh serum for short and varying periods of time, some complements could be destroyed, whereas others would be left behind with undiminished or but slightly decreased activity. Prolonged exposure would remove all complements.

(d) As was previously stated, anticomplements may be produced by immunizing an animal with the complement of an animal of a different species. The anticomplements appear to be specific for the complements responsible for their production, and by means of these anticomplements different complements may be demonstrated in one serum. Since the formation of anticomplements would depend upon whether or not the body-cells of the immunized animal possess suitable receptors for the various complements, in a series of animals it may be found that one does not produce anticomplements for all the complements injected, a finding that would tend to support the theory of the multiplicity of complements. In addition, Marshall and Morgenroth actually found in ascitic fluid an anticomplement for at least one of two complements present in guinea-pig serum.

These experiments go to show that complements differ in this respect at least: that not all have identical haptophores. Whatever differences between complements exist must be slight; probably the cytophilic group of all are alike. At present the subject has more theoretic than practical importance. In the various diagnostic reactions guinea-pig serum ordinarily furnishes the complement for hemolysin, bacteriolysin, or other cytolysins, and in the therapeutic administration of bacteriolytic serums we are compelled in any case to depend for activation of the amboceptor upon the natural complement in the patient's serum.

The Nature and Action of Complement.-The true nature of the complements is unknown. In many respects they bear a resemblance to ferments, and certainly the part they play in the processes of cytolysis 
suggests a ferment-like activity. They differ from true proteolytic ferments, such as trypsin, in not digesting the stroma of corpuscles, although recent work by Dick would seem to indicate that proteolysis actually occurs, a process that increases the permeability of the cell and permits the escape of hemoglobin.

On the other hand, it is possible that the nature and action of complements may be placed upon a chemical basis. Following the discovery of the hemolytic power of cobra venom by Flexner and Noguchi, a power they ascribed to the presence of an amboceptor in the venom acting with serum complement, Kyes found that the amboceptor may be activated not only by a complement in the blood-serum, but also by some constituent of the red blood-corpuscles themselves. This last observer speaks of the latter as endocomplement, $i$. e., endocellular complement.

In attempting to discover the nature of this endocomplement various substances existing normally in the erythrocytes, such as cholesterin and lecithin, were obtained in a pure state and their activating powers for cobra amboceptors tested. These investigations showed that lecithin has an activating power, whereas cholesterin is antihemolytic. Although all erythrocytes contain lecithin, yet all are not equally susceptible to the action of venom amboceptors, which is probably due to the fact that the lecithin in the cells of some animals is bound to other cell constituents in a loose way and is thus available as complement. In syphilitic infection the lecithin content of the erythrocytes is actually diminished or in some manner rendered less available, so that the inhibition or absence of venom hemolysis is diagnostic of this infection.

Kyes was able to obtain the union of cobra amboceptor and lecithin, forming what is known as cobra lecithid. Although lecithin is an unstable substance and is difficult to obtain free from fatty acids and soap, there is little doubt but that Kyes' lecithid is a phosphatid compound and is actively hemolytic after all traces of fatty acids have been removed.

The next important observations were made by Noguchi, ${ }^{1}$ who found that soap isolated from blood and various tissues possessed active hemolytic properties. The salts of the fatty acids, and particularly of oleic acid, were found to possess similar hemolytic properties. Pure soluble oleates mixed with serum were found to produce compounds possessing many of the characteristics of true complements: - (1) They are inactivated by heating to $56^{\circ} \mathrm{C}$. for half an hour; (2) they are inactive at $0^{\circ}$ C.; (3) the addition of acids, alkalis, and yeast renders them inactive.

${ }^{1}$ Proc. Soc. Exper. Biol. and Med., 1907, 4, 107; Biochem. Zeitschr., 1907, 6. 
Von Liebermann, ${ }^{1}$ as the result of his own experiments, came to practically the same conclusions, and advanced the hypothesis that the complements of the blood are to be sought for in the soaps of the serum; that these soaps are united with serum albumin, and are inactive until liberated by the amboceptors, when they become actively hemolytic.

Further than this, it was shown that oleic acid may act as an amboceptor, and when added to an inactive soap-albumin combination, it would render this actively hemolytic. Von Liebermann and Fenyvessy ${ }^{2}$ have shown that a mixture of soap, serum, and oleic acid possesses a striking resemblance to complements and amboceptors, and that the amboceptor-complement action is much more than a mere linkage of complement to antigen by means of an amboceptor. These observers suggest that an amboceptor may have an affinity for certain constituents of the cell or bacterial body, and, on the other hand, act upon the complement and separate one of its constituents, which then breaks up the cell. These artificial hemolysins, however, completely dissolve the stroma of the corpuscles, whereas the immune hemolysins appear to dissolve out the hemoglobin, leaving the stroma undissolved. As has been mentioned elsewhere, recent work would tend to show that the stroma is also dissolved, at least in part, in specific hemolysis, so that the difference in action between the two is not quite so apparent.

While the simplicity of the substances concerned in these observations does not harmonize with the great variety and complexity of the immune bodies, nevertheless, as Adami has pointed out, the points of resemblance between artificial and natural complements and amboceptor are so striking that material advances in our knowledge of their nature and action may be gained by further researches into the chemistry of immunity.

Complement Splitting.-During recent years considerable attention has been directed toward a phenomenon known as the splitting of complement. It was generally conceded that when treated with hydrochloric acid (Sachs), carbon dioxid gas (Liefmann), or acid and alkaline phosphates (Michaëlis and Skwissky), or when dialyzed against distilled water (Ferrata), complement may be split into two parts, known as a mid-piece and an end-piece. According to certain investigators, these two components of the complement differ in their behavior in hemolytic processes: one, the mid-piece, is bound by the sensitized cells, while the other, or end-piece, possesses the lytic action. Speaking in terms of the side-chain theory, it is just as if the haptophore and cytophilic

${ }^{1}$ Biochem. Zeitschr., 1907, 4.

2 Biochem. Zeitschr., 1907, 5. 
portions of the complement were separated: the haptophore portion corresponding to the mid-piece (the cell or bacterium being the first piece), and the lytic cytophilic portion corresponding to the end-piece. In successful complement splitting the mid-piece is believed to be in the globulin fraction and the end-piece in the albumin fraction. Noguchi and Bronfenbrenner ${ }^{1}$ have cast considerable doubt upon these views, and have shown that what is known as complement splitting is really nothing more than an inactivation of the active principle of complement, since both globulin and albumin fractions contain a part of the complement, a fact that can be demonstrated by the removal of the inhibiting action of the acid or alkali used in the process.

\section{The Bordet-Gengou Phenomenon of Complement Fixation}

In the endeavor to demonstrate the unity of complement Bordet and Gengou devised an experiment that has proved of great practical value in the serum diagnosis of syphilis and other infectious diseases. By mixing bacteria and their amboceptors with a little fresh serum containing complement and letting the mixture stand aside for an hour or so it was found that, upon the addition of corpuscles and their amboceptor, hemolysis did not occur, although the serum that had been used as complement was capable, in its original condition, of producing hemolysis of these corpuscles. Bordet advanced this experiment to show that the complement concerned in bacteriolysis is the same as that at work in hemolysis, and consequently concluded that there is but one single complement.

This experiment of Bordet is usually spoken of as the "BordetGengou phenomenon," and is now used extensively in determining whether or not a given serum contains certain amboceptors. The serum to be tested is first inactivated, treated with the antigen composed of an emulsion of the bacterium whose amboceptor it is desired to discover, and then mixed with a small quantity of a fresh normal complement serum. The mixture is placed in the incubator for an hour, during which time the bacterial antigen unites with its amboceptor and the complement, i. e., fixes the complement, so that when red blood-cells previously sensitized with heated hemolytic serum are added, hemolysis does not occur because the complement in the fresh serum, which was suitable for lysis of the sensitized corpuscles, has been "fixed" by the bacteria by reason of the presence of specific amboceptors in the serum tested

1 Jour. Exper. Med., 1912, 15, 598 and 625. 
(Fig. 94). If these amboceptors were not present, then the complement would remain unfixed and be free to hemolyze the sensitized corpuscles, a negative reaction being indicated, therefore, by hemolysis, whereas the absence or inhibition of hemolysis indicates a positive reaction.
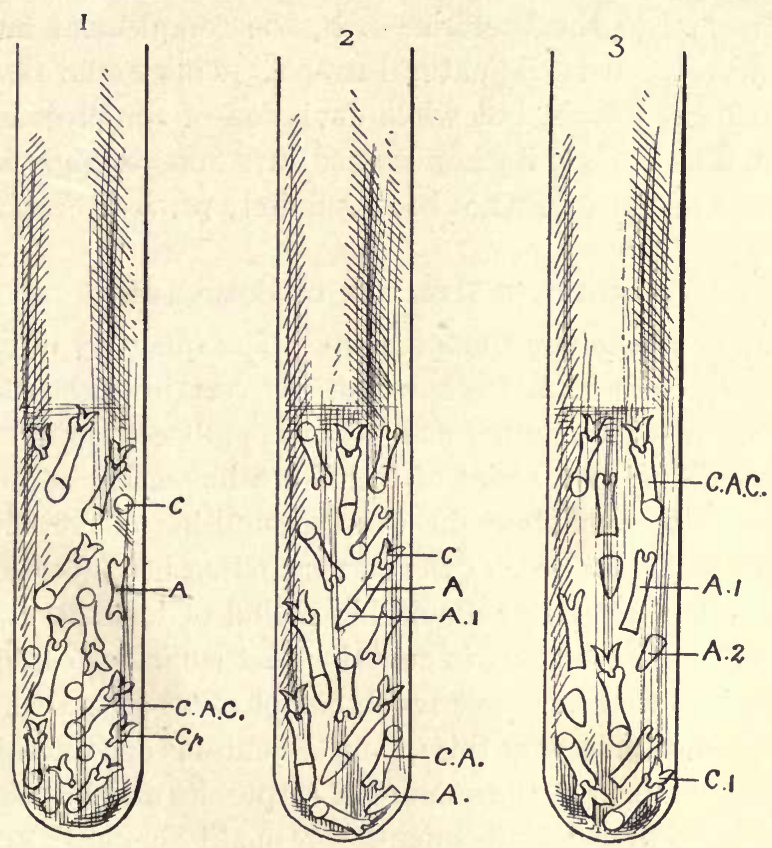

Fig. 94.-Mechanism of Complement Fixation.

Tube 1 shows the hemolytic system; $C$, a red blood-corpuscle; $A$, a hemolytic amboceptor; $C p$, complement; $C . A . C$., complement united to a corpuscle by means of the specific amboceptor. Hemolysis results.

Tube 2 shows complement fixation by bacterial antigen and amboceptor; $A_{1}$ antigen; $C$, complement united to the antigen $A_{1}$ by the amboceptor $A$. When hemolytic amboceptors are added hemolysis does not occur because the complement has been previously fixed by the bacterial antigen and amboceptor.

Tube 3 shows absence of complement fixation because the bacterial amboceptor $A_{1}$ is not specific for the bacterial antigen $A_{2}$ and hence complement is not fixed; when hemolytic amboceptor and the corresponding corpuscles are added complement unites with these, C.A.C. and hemolysis occurs.

Wassermann, Neisser, and Bruch have applied this test to the serum diagnosis of syphilis, the technic of the test being considered in a subsequent chapter.

Deviation or Deflection of Complement.-While large doses of antitoxin are indicated in the treatment of diphtheria and tetanus, theoretically the administration of too large an amount of a bacteriolytic serum 
may result in more harm than good. As has been pointed out by Neisser and Wechsberg, more amboceptors may be introduced than can be taken up by the bacteria causing the infection, and those that remain free are capable of combining with some of the complement that is present, and thus prevent a portion of the complement from acting with the amboceptors attached to the bacteria- $i$. e., the complement has been deviated or deflected from its natural course. This would really mean a decrease in bacteriolysis, but while deviation of complement has been demonstrated as a fact, its importance in serum therapy is probably overrated and has certainly not been definitely proved.

\section{Quantitative Titration of Complements}

Titration of Hemolytic Complement.-The quantity of a hemolytic complement present in a fresh serum for certain corpuscles may be measured in the same manner as hemolytic amboceptors are measured, namely, by adding to a series of test-tubes increasing amounts of the fresh serum with a constant dose of an emulsion of corpuscles and a constant and sufficient dose of the corresponding hemolytic amboceptor for these corpuscles. After a suitable period of incubation, that tube that shows complete hemolysis contains just sufficient complement, or one unit. Since we know how much complement serum was placed in this tube, we now know that this quantity contains one unit of hemolytic complement. Of course, the amount of corpuscles and amboceptor must be constant in all tubes; if the quantity or quality of one or both of these is altered, the unit of complement will vary.

Titration of a Bacteriolytic Complement.-The quantity of bacteriolytic complement in a given amount of fresh serum may be determined in a similar manner, although far less accurately, for instead of viewing the results of lysis in the test-tube as we can do in hemolysis, the degree of lysis must be determined by plating out the mixtures to determine the relative numbers of living and dead bacteria. The degree of bacteriolysis may, however, be viewed after a manner with the aid of the microscope.

Gay and Ayer employ a direct method, which consists in adding varying amounts of the serum to be tested to a definite volume ( 0.5 c.c.) of a suspension of cholera vibrios, prepared by emulsifying a twentyfour-hour agar culture in 10 c.c. of normal salt solution and adding a constant and sufficient dose of serum from a rabbit immunized against cholera. The mixtures are placed in small test-tubes and incubated for one and one-half hours at $37^{\circ} \mathrm{C}$. Films are then prepared, stained, and 
examined to ascertain the degree of changes undergone by the vibrios. Or the changes may be observed in hanging-drop preparations. In either case a control is prepared of the culture which has also been incubated along with the mixtures. Gay and Ayer found that 0.02 c.c. of normal human serum contained sufficient complement to effect complete lysis of this dose of vibrios-even 0.001 c.c. produced distinct changes. 


\section{CHAPTER XIX}

\section{BACTERIOLYSINS}

HAving considered the general nature and properties of amboceptors and complements and the mechanism of their action in producing solution or lysis of cells, we will now study more closely the bacteriolysins, which are antibodies belonging to this group and possessing diagnostic and considerable therapeutic importance.

Historic.-The early history of the discovery of the bacteriolysins is closely associated with the history of immunity in general, for with the discoveries in bacteriology and the establishment of the relation of bacteria to disease, it followed as a matter of course that investigations should be undertaken to ascertain the mechanism of resistance to and of recovery from an infection.

In 1874 Traube showed that septic material may be destroyed in the blood of living animals, and in 1881 Lister demonstrated the same phenomenon in extravascular blood. These experiments were naturally somewhat crude, as they antedated the period in which the pyogenic microörganisms were isolated and studied in pure culture, but they served, nevertheless, to demonstrate the germicidal powers of the blood.

In 1886 Fodor demonstrated the germicidal action of blood-serum upon anthrax bacilli. This work was followed shortly after by that of Flügge and Nuttall, who showed the germicidal powers of the body-fluids in general independent of cells. The controversy between the adherents of the cellular and humoral theories of immunity now began, as Metchnikoff was actively engaged in stüdying phagocytes and in formulating his phagocytic theory.

Buchner and others took up the subject, emphasizing the important germicidal powers of the body-fluids and ascribing this function to the presence of "alexins" (substances that ward off disease). Buchner showed that if the serum was heated this germicidal power was lost; hence it followed that the active bacteriolytic agent was considered very unstable and was quickly destroyed outside of the body.

In 1894 Pfeiffer demonstrated most clearly the phenomenon of bacteriolysis, which gave great encouragement and impetus to studies in 
immunity, and incidentally strengthened the claims of the humoral theory. He showed that cholera vibrios introduced into the peritoneal cavity of a guinea-pig that had been immunized against cholera lost their motility and finally became disintegrated and passed into solution regardless of the presence of cells.

It remained for Bordet, however, to show the mechanism of this interesting phenomenon. This observer demonstrated the fact that the thermolabile body was but one substance concerned in the reaction, and that the specific substance was thermostabile and the actual product of immunization, results that were later corroborated and elucidated by his researches upon hemolysis, and by those of Ehrlich and his pupils on cytolytic phenomena in general. As previously mentioned, Bordet retained the name "alexin" for the thermolabile substance and applied the new term, "substance sensibilisatrice," to the specific thermostabile antibody. Later, both substances were renamed by Ehrlich, and called "complement" and "amboceptor" respectively.

As will be pointed out further on, as the result of these observations Metchnikoff modified his phagocytic theory, and recognized the existence of both substances, which he named "cytases" and "fixateurs," believing that both were derived from cells classed as phagocytes.

All are agreed as to the presence of two different bodies in the bodyfluids concerned in bacteriolysis, although opinions vary as regards their origin and mechanism of action. The side-chain theory has been widely accepted in explanation of their action, and the terms applied by Ehrlich to the two substances concerned, namely, complements and amboceptors, are in general use.

Definition.-Bacteriolysins are substances present in the serum and other body-fluids that kill bacteria with or without lysis.

The term itself would infer that solution or lysis of the bacterium is an essential property of an antibody of this order. Bactericidins are substances that kill bacteria without lysis, and, strictly speaking, an effort should be made to differentiate between the terms, although from a practical standpoint this is not important. Certain microörganisms may be killed and resist solution or digestion for a comparatively long time, whereas, on the other hand, the same bacteria, under different circumstances, may readily be lysed.

Although the endotoxins liberated from the lysed bacteria may produce symptoms of disease, followed by death, yet the bacterium itself is usually destroyed and unable to proliferate. A bacteriolysin is, therefore, always bactericidal, although the converse is not necessarily true. 
Custom, however, has never strictly differentiated between the two terms, and bacteriolysis appears to be but a continuation of and a more nearly complete bactericidal process. Hence the definition just given covers both terms-bacteriolysins and bactericidins.

Origin of Bacteriolysins. - Our knowledge regarding the origin of the bacteriolysins is quite fragmentary. The investigations of Pfeiffer and Marx in cholera, and Wassermann in typhoid, have shown that the spleen and hematopoietic organs in general may be especially concerned.

According to Metchnikoff, the "bacterial fixateurs," which are practically the bacteriolysins, are secretory or excretory products of phagocytic cells, especially the polynuclear leukocytes or microphages. It is commonly believed that during infection the bacteria cause phagolysis or disintegration of these cells, with liberation of both complements (cytases) and amboceptors (fixateurs), which produce extracellular lysis of the invading bacterium (bacteriolysis). If, on the other hand, the phagocytes are fortified and phagolysis is prevented, the bacteria are phagocytized and undergo intracellular lysis, a condition that, according to Metchnikoff, may be induced experimentally by giving an animal an intraperitoneal injection of sterile bouillon twenty-four hours before bacteria or other cells are injected.

That leukocytes afford a bacteriolytic substance is supported by observations showing that leukocytic exudates, secured by the injection of a sterile aleuronat suspension, possess a well-marked germicidal activity. Issæff found that the intraperitoneal injection of sterile bouillon and other mild irritants, by producing a leukocytic exudate that supplied certain bactericidal substances and facilitated phagocytosis, increased the resistance of animals to bacterial infection. At one time surgeons made practical use of this observation by injecting nucleinic acid and other substances into the peritoneal cavity before performing laparotomy, in order to induce a local resistance to a possible infection.

\section{LEUKINS AND LEUKOCYTIC EXTRACTS}

The bactericidal substance contained within leukocytes has been extensively studied by Schattenfroh, Schneider, Peterson, Hiss and Zinsser, Manwaring, and others. It has been observed that when leukocytes are suspended in diluted blood-serum, the bactericidal properties of the serum are increased without coincident destruction of the cells, showing that the leukocytes may secrete germicidal substances into the fluid. The same observation has been made with Bier's congestive 
lymph, indicating that this activity takes place both in the test-tube and in living tissues.

Hiss, and later Hiss and Zinsser, found that autolyzed leukocytic exudates possess some bactericidal activity, and that they may profoundly modify experimentally induced infection of rabbits and guineapigs with the pneumococcus, staphylococcus, streptococcus, and other bacteria. In applying this method of treatment to man by means of subcutaneous injections, these investigators observed distinctly beneficial results in cases of epidemic cerebrospinal meningitis, lobar pneumonia, staphylococcus infections, and erysipelas.

Preparation of Leukocytic Extracts.-Hiss and Zinsser have prepared leukocytic extract by giving rabbits intrapleural injections of aleuronat suspension. Manwaring has secured much larger quantities by making his injections into the horse.

The aleuronat is prepared by making a 3 per cent. solution of starch in bouillon without heating, and adding 5 per cent. of powdered aleuronat to this emulsion. The starch helps to keep the aleuronat in suspension. The mixture is boiled for five minutes and placed in large sterile testtubes, 20 c.c. being placed in each tube. Final sterilization is done in an autoclave.

For making the injections large rabbits are selected. The hair over both sides of the thorax is removed, the skin is sterilized with tincture of iodin, and 10 c.c. of the aleuronat suspension are injected into each pleural cavity at a point in the anterior axillary line, at the level of the sternum, great care being taken to avoid puncturing the lungs. After twenty-four hours the animals are chloroformed and the pleural cavities carefully and aseptically opened. The cellular exudate is pipeted into centrifuge tubes containing at least 10 c.c. of sterile 1 per cent. sodium citrate in normal salt solution. One or more cubic centimeters of exudate may be obtained from each cavity. The exudate is usually tinged with blood. It is then centrifuged and the supernatant fluid removed. The sediment is broken up with a platinum spatula, and 20 volumes of sterile distilled water are added. The tubes are set aside in the incubator for twenty-four hours, after which cultures are made to insure sterility. A small amount of preservative may be added, and the extract placed in bottles or ampules ready for administration. It is given subcutaneously in doses of from 5 to 10 c.c. every four to six hours.

The endocellular bactericidal substances, or endolysins, mentioned in a previous chapter, which can be extracted from leukocytes, are not in the nature of complements, as they are not rendered inactive by tem- 
peratures below $80^{\circ} \mathrm{C}$. They cannot apparently be increased by immunization, the quantity present in each leukocyte being probably at all times just sufficient for the digestion of a limited number of bacteria, which can be ingested at one time by the individual leukocyte. It is probable that the excess of bactericidal substance is thrown off into the blood-stream, representing the serum bacteriolysins, and at least indicating that the leukocytes are one source for the production of bacteriolytic amboceptors.

Mechanism of Bacteriolysis.-According to the side-chain theory of Ehrlich a bacteriolysin is an antibody of the third order, or an amboceptor furnished with two haptophore or grasping arms for uniting the bacterium on one side with a suitable complement on the other. The antibody, therefore, acts simply as an interbody or connecting link; it is specific for the bacterium causing its production, but is unable itself directly to injure the bacterium, lysis being brought about by an at-

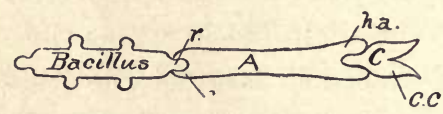

Fig. 95.-Theoretic Structure OF A BACTERIOLYTIC AMBOCEPTOR.

$A$, Amboceptor; $C$, complement; $r$, receptor of bacillus; $h$, haptophore group of the amboceptor; h.a., complementophile group of the amboceptor. tached complement. Bacteriolysis is, therefore, an interaction of amboceptor and complement upon the bacterial body (Fig. 95).

While bacteriolytic amboceptors will unite with their antigens under practically all conditions, nevertheless a suitable complement may not be present, and hence a bacteriolytic serum may not be active in all animals.

The influence of bacteriolysins upon endotoxins is a question of considerable interest and importance. As the result of convincing experiments performed, especially by Pfeiffer, it is evident that a bacteriolytic serum does not neutralize the endotoxin at the time the bacterium undergoes disintegration. Highly immune serums appear to be unable to protect an animal against the endotoxins, and, indeed, may even increase the intoxication and, by liberating an excess of endotoxin, kill the animal.

The bactericidal substances derived from leukocytes are, however, apparently capable of neutralizing endotoxins, to some extent at least, as Hiss and Zinsser were unable to ascribe the beneficial effects of leukocytic extracts to the bacteriolytic action alone.

As mentioned elsewhere, both Metchnikoff and Bordet maintain that the bacteriolysin is in the nature of a "sensitizer," preparing the bacterium for the action of the alexin or cytase, just as a mordant aids in the penetration of a dye-stuff. 
General Properties of Bacteriolysins.-As with other cytolysins, the bacteriolysins are thermostabile and resist heating to $60^{\circ} \mathrm{C}$., being gradually destroyed at temperatures ranging from $70^{\circ}$ to $80^{\circ} \mathrm{C}$. They are likewise highly resistant to acids and alkalis, and when preserved in a sterile condition with the addition of small quantities of a preservative bacteriolytic serums for therapeutic and diagnostic purposes may remain active for long periods of time. Cholera immune serum as a diagnostic aid in making the agglutination and Pfeiffer bacteriolytic reactions is best preserved in dry form, the serum retaining its activity under these conditions for considerable periods of time.

Normal Bacteriolysins.-As a result of the contention of Metchnikoff that bacteriolysins (fixateurs) are produced only upon the disintegration of leukocytes, and that the plasma is accordingly free from these antibodies, much experimental work has been done. The weight of evidence is against this view, as both amboceptors and complements have been demonstrated in plasma, although the quantity of bacterial amboceptors normally present in the body-fluids is quite small. It is quite natural to expect that under normal conditions small amounts will be present, as receptors are being constantly thrown off into the blood, and leukocytes are, of course, being constantly formed and destroyed.

Specificity of Bacteriolysins.-The bacteriolysins are highly specific antibodies, and are useful in making the differentiation of bacterial species. Group bacteriolytic reactions are less common as compared to group agglutination, as was shown by Kolmer, Williams, and Raiziss with the typhoid-colon group of bacilli. As a practical procedure, however, the agglutination reaction is so easily secured as to be the test of choice in making a differentiation between closely allied organisms. As with the agglutinins, the influence of partial bacteriolysins may be removed by using highly immune serums in high dilutions.

Practical Applications.-The bacteriolysins have considerable value in the following procedures:

1. In making a differentiation of bacteria, especially when the presence of cholera vibrios is suspected.

2. In the diagnosis of certain infections, such as cholera and typhoid fever.

3. In the treatment of some infections with specific bacteriolytic serums. 


\section{TECHNIC OF BACTERIOLYTIC TESTS}

\section{THE PFEIFFER EXPERIMENTS}

The essentials of this important test have been described at the beginning of this chapter. Briefly, it consists in making intraperitoneal injections of a bacteriolytic serum mixed with living bacteria into a normal guinea-pig. The resulting bacteriolysis is studied microscopically by withdrawing small amounts of peritoneal exudate at varying intervals. By performing the experiment with varying dilutions of serum, the bacteriolytic titer may be determined by noting the dilution in which bacteriolysis just fails to occur in a specified time.

Pfeiffer also showed that the phenomenon could be produced by injecting a mixture of serum from an immunized animal and the culture of cholera into the peritoneum of a normal guinea-pig. This phenomenon appeared when an old specimen of serum was used, as well as when a fresh specimen that had been heated to $60^{\circ} \mathrm{C}$. was employed. Later, this observer found that if an old immune serum was injected into the peritoneal cavity and allowed to remain for a time, it regained its bactericidal powers.

Pfeiffer believed that the bacteriolytic substance may exist in the serum of an immunized animal either in an active or in an inert state. In the blood-serum or peritoneal fluid of the living animal it occurs as an active substance, but when kept for a few days or when heated rapidly to $60^{\circ} \mathrm{C}$. it becomes inert; it may be rendered active again by coming in contact with the lining endothelial cells of the peritoneum.

The foregoing constitutes the classic Pfeiffer experiment. The bacteriolytic amboceptor present in the immune serum is activated by the complement furnished by the guinea-pig. The same serum will not produce bacteriolysis in the test-tube in case it has been heated or the complement is lost through age unless fresh normal serum or peritoneal exudate is added. By immunizing guinea-pigs with gradually increasing doses of cholera serum and then introducing fatal doses of cholera culture intraperitoneally, the same phenomenon of bacteriolysis is observed. In this instance the guinea-pig furnishes both amboceptor and complement.

By these and similar experiments and observations Bordet was able to show the rôle played by the two bodies concerned in cytolysis in general, namely, the thermolabile alexin or complement and the specific sensitizer or bacteriolytic amboceptor. 
Bacteriolytic Test in vivo for the Identification of Bacteria Recovered from Feces, Water-supplies, etc.-This method is employed chiefly in the identification of suspected cholera cultures. According to Citron, in Germany, the Pfeiffer test, made with microörganisms obtained in pure culture from suspected patients, is required for the official diagnosis of the first cases of cholera.

As a rule, the agglutination test is first applied in making the diagnosis of a suspected microörganism, as the technic of this test is more easily carried out.

This bacteriolytic test may also be employed in the study of typhoid and paratyphoid bacilli, although bacteriolysis of these microörganisms is less complete than that observed with cholera, and agglutination tests answer all practical requirements.

The test consists in mixing varying dilutions of a known and highly immune serum with a constant dose of unknown microörganisms, and injecting the mixtures intraperitoneally into guinea-pigs. After twenty minutes small amounts of exudate are withdrawn by means of fine capillary pipets, and studied in hanging-drop preparations. In the presence of a positive reaction the bacilli are observed to lose motility, become swollen and coccoid in shape, and gradually form granules, ultimately disappearing in complete solution.

Preparing the Immune Serum.-A highly immune serum is required. This may readily be prepared by giving rabbits a series of intravenous injections of a known culture, according to the technic described in the chapter on Active Immunization of Animals. The official test in Germany demands that the serum be of such strength that 0.0002 gram of dried serum will suffice to disintegrate completely within one hour one loopful (2 mg.) of an eighteen-hour-old culture of virulent cholera in 1 c.c. of nutrient bouillon when injected into the peritoneal cavity of a guinea-pig. The Hygienic Laboratory ${ }^{1}$ in Washington is prepared to furnish board of health laboratories with a dried serum of high titer for diagnostic purposes.

In testing an immune serum to determine its bacteriolytic titer the dose of microörganisms should not be larger than one loopful, so that if any particular strain of cholera or typhoid is not sufficiently virulent, necessitating the use of larger doses, the virulence should be increased by passing the organism through guinea-pigs.

Method of Testing the Virulence of a Culture.-The unit of measurement is a 2 millimeter platinum loop, which, when loaded, will usually

${ }^{1}$ Personal communication from Dr. John F. Anderson, Director of the Laboratory. 
hold about 2 milligrams of microörganisms. (See p. 212.) All dilutions are made with sterile neutral broth, and not with salt solution. Mixtures are injected intraperitoneally into 250-gram guinea-pigs to determine the dose that will be fatal within twenty-four hours.

Great care should be exercised in all manipulations to avoid accidental infection. The mouth-ends of pipets should be plugged with cotton. Sufficient assistants should be on hand to facilitate the making of injections and the examination of peritoneal exudates with ease, caution, and certainty. All pipets, measuring glasses, test-tubes, and hanging-drop preparations should be immersed after using in 1 per cent. formalin solution before cleaning. In other words, every precaution should be taken to carry out a thorough and conscientious bacteriologic technic.

Guinea-pig No. 1: Four loopfuls of agar culture emulsified in 4 c.c. of bouillon; inject 1 c.c. intraperitoneally (=1 loopful).

Guinea-pig No. 2: 1 c.c. of foregoing emulsion +1 c.c. of bouillon; inject. 1 c.c. intraperitoneally (=1/2 loopful).

Guinea-pig No. 3: 1 c.c. of first emulsion +4 c.c. of bouillon; inject 1 c.c. intraperitoneally ( $=\frac{1}{5}$ loopful).

Guinea-pig No. 4: 1 c.c. of emulsion No. $3+1$ c.c. of bouillon; inject 1 c.c. intraperitoneally ( $=\frac{1}{10}$ loopful).

A satisfactory culture is one in which a dose of $\frac{1}{5}$ loopful will prove fatal within twenty-four hours. The immune serum is then titrated with five times this amount of culture, or one loopful.

Method of Titrating a Bacteriolytic Serum.-The serum is inactivated by heating to $56^{\circ} \mathrm{C}$. for half an hour, and dilutions are made with bouillon in sterile shallow glasses or test-tubes. One loopful of an eighteen-hour agar culture of the microörganism is thoroughly emulsified in the diluted serum, and the mixtures are injected intraperitoneally in 250-gram guinea-pigs. Higher dilutions than those given here may be employed until the limit of bacteriolytic activity is reached.

1. Mix 0.5 c.c. of inactivated serum with 4.5 c.c. of bouillon $(1: 10)$. Place 2 c.c. of this mixture in a separate test-tube, and add 2 loopfuls of culture. Inject 1 c.c. $(=0.1$ c.c. immune serum).

2. Mix 2 c.c. of the first dilution $(1: 10)$ with 18 c.c. of bouillon (= $1: 100)$. Place 2 c.c. in a separate tube and add 2 loopfuls of culture. Inject 1 c.c. $(=0.01$ c.c. immune serum).

3. Mix 1 c.c. of the second dilution $(1: 100)$ with 4 c.c. of bouillon $(=1: 500)$. Place 2 c.c. in a separate tube and add 2 loopfuls of culture. Inject 1 c.c. $(=0.05$ c.c. immune serum). 
4. Mix 2 c.c. of the third dilution $(1: 500)$ with 2 c.c. of bouillon $(=1: 1000)$. Place 2 c.c. in a separate tube and add 2 loopfuls of culture. Inject 1 c.c. $(=0.001$ c.c. immune serum).

5. To 1 c.c. of the fourth dilution $(1: 1000)$ add 9 c.c. of bouillon $(=1: 10,000)$. Place 2 c.c. in a separate tube, and add 2 loopfuls of culture. Inject 1 c.c. $(=0.0001$ c.c. immune serum).

6. Control: Emulsify 2 loopfuls of culture in 2 c.c. of bouillon. Inject 1 c.c. This animal will probably succumb within twenty-four hours.

7. Control: To 2 c.c. of a $1: 10$ dilution of inactivated normal rabbit serum add 2 loopfuls of culture and inject 1 c.c. intraperitoneally. If goats or horses are used in preparing the immune serum, this control should be conducted with normal goat or horse serum. According to Kolle, one loopful of virulent cholera culture is destroyed in the peritoneal cavity of a guinea-pig by 0.1 to 0.3 c.c. of normal rabbit's serum; 0.02 to 0.03 c.c. of normal goat's serum; 0.005 to 0.01 c.c. of normal horse's serum.

8. Control: A pig may be injected with 1 c.c. of a $1: 100$ dilution of the immune serum, and with a loopful of some other microörganism, such as Bacillus coli. This control, however, is not absolutely necessary.

An area of the abdominal wall of the guinea-pig about one inch in diameter is shaved and cleansed with alcohol. After the injections have been made the bacteriolytic phenomena are observed.

In making this test fine capillary pipets are prepared and used for withdrawing the peritoneal exudate.

After permitting the animal to inhale a few drops of ether, to make sure that it will not suffer, a small incision is made through the skin of the abdomen. The capillary pipet, the large end of which is kept closed with the index finger, is then quickly passed into the abdominal cavity. The index-finger is released, and the tube is gently moved about and withdrawn. As the result of capillary attraction sufficient exudate usually passes into the tube without the aid of suction (Fig. 96). The tube may be fitted with a rubber teat in case suction should be necessary. Hanging-drop and smear preparations are made and studied microscopically (Fig. 97). Stained smears are, however, less reliable and not so useful in determining the degree of bacteriolysis (Fig. 99).

It is best to withdraw the exudate immediately after injection, and then at intervals of ten, twenty, thirty, forty, and sixty minutes.

A hanging-drop preparation, made from the culture by emulsifying a minute quantity in bouillon, should be on hand as a control in studying 
bacteriolysis in the exudate (Fig. 98). A smear of the culture stained with dilute carbolfuchsin should also be on hand for making comparison with smears of the exudate (Fig. 100).

Bacteriolysis is first manifested by loss of motility. As the process progresses many of the bacilli become swollen and distorted, and later irregular and broken fragments or granules become apparent. Finally, at the end of an hour, the exudate is practically sterile. In those cases in which bacteriolysis is complete in an hour the animal generally sur-

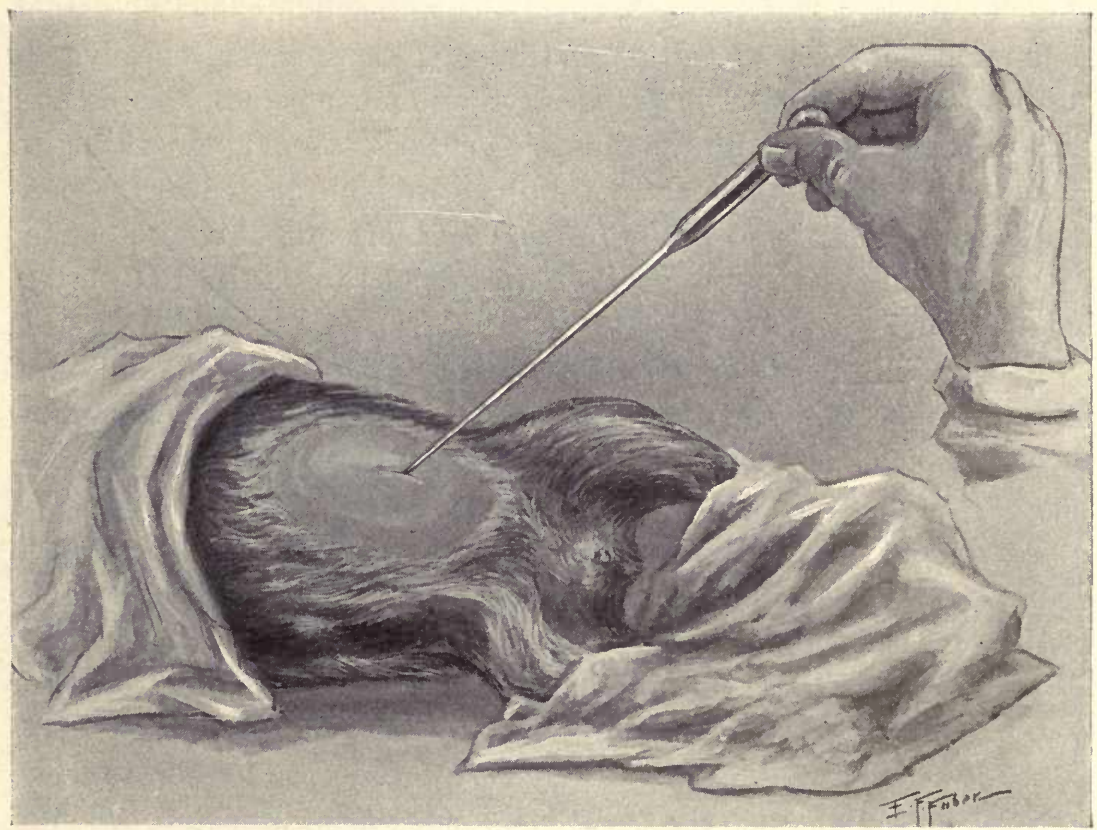

Fig. 96.-Removing Exudate from the Peritoneal Cavity of a Guinea-pig (PFeiffer Bacteriolytic Test).

A small incision is made through the skin, and the exudate is withdrawn by means of a pipet.

vives. In the higher dilutions of serum bacteriolysis is incomplete, and the animal becomes toxic and may succumb after twenty-four hours, double the virulent dose being used in all injections.

In the foregoing titration a serum that is bacteriolytic in dilutions of $1: 1000$ or over will be satisfactory for purposes of diagnosis. When preserved in a sterile condition in separate ampules in a dark cold place the titer usually remains unaltered for several months.

Dried Serum.- - If the serum is dried in vacuo, it will be necessary to titrate with dilutions of the dried products in bouillon, as during the 



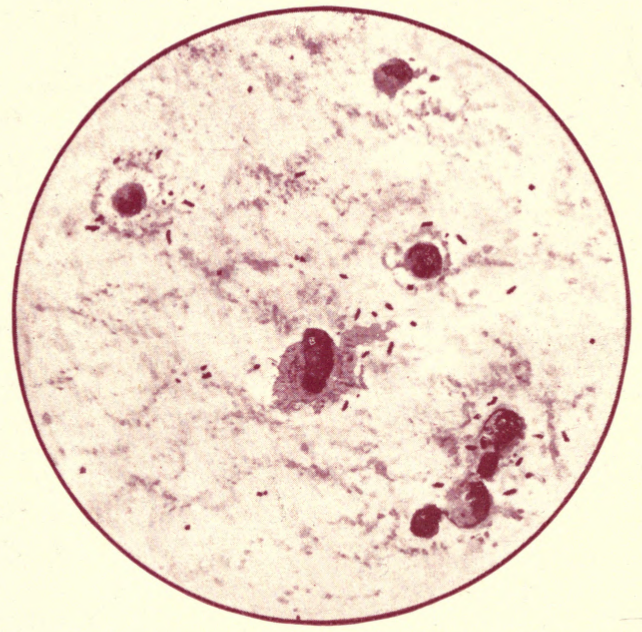

Fig. 99.-A Smear of Peritoneal Exudate Removed Twenty Minutes after Injection of Culture of Cholera in Fig. 100. Stained with 1:10 carbolfuchsin.

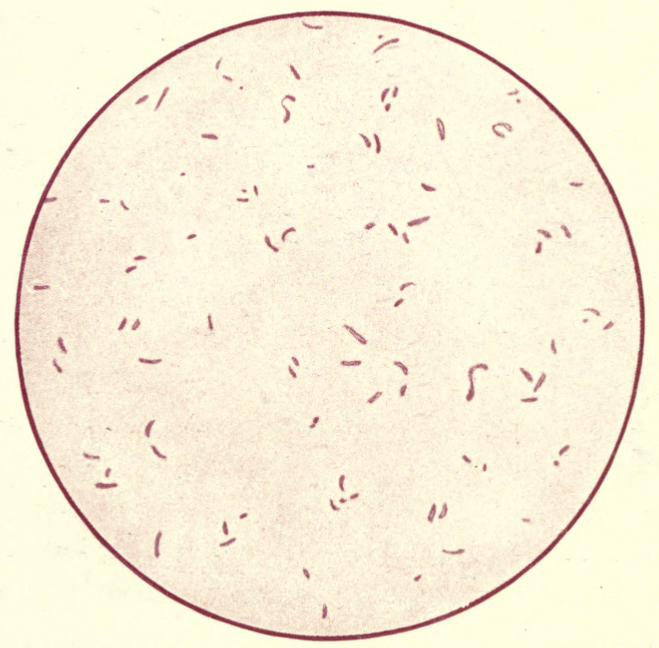

Fig. 100.-Stained Preparation of Cholera before Bacteriolysis. 
process of drying the amboceptor content may be decreased. Dried serum is to be preferred, however, as it keeps much longer and there is no danger of rendering it worthless by contamination. After drying, the product is ground in a mortar and stored in amounts of 0.1 or 0.2 gram in separate ampules.

In determining the bacteriolytic titer of dried serum 0.1 gram is carefully weighed out and dissolved in 9.9 c.c. of sterile bouillon. From this stock dilution other dilutions may be prepared, in the manner previously described, and injected with a loopful of the culture.

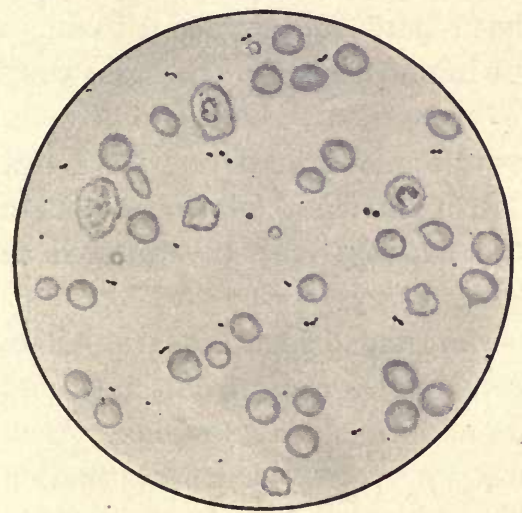

Fig. 97.-CUlture of Cholera Undergoing Bacteriolysis. A Positive Pfeiffer Reaction.

A hanging drop of peritoneal exudate removed from a guinea-pig one-half hour after injection with 1 c.c. of the suspension shown in Fig. 99 with 1 c.c. of $1: 1000$ dilution of cholera immune serum. Note that the bacilli are now quite short and coccoid in shape. At the end of seventy minutes the exudate was sterile. What appears to be two or three coccoid forms in apposition is really one bacillus undergoing lysis.

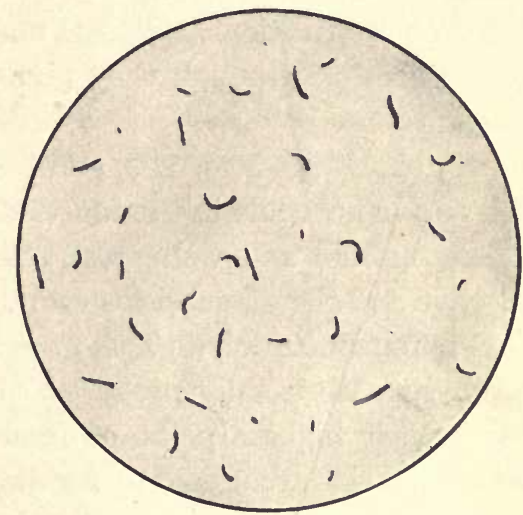

Fig. 98.-Culture of Cholera Before BaCteriolysis. $\times 720$.

A hanging drop of cholera in normal salt solution prepared from a twentyfour-hour culture of cholera on agaragar.

After securing an immune serum of satisfactory potency the main test may be conducted. While the technic is more difficult than that of agglutination tests, the results, under proper conditions, are more conclusive, for there is less likelihood of group or partial amboceptor activity for closely allied bacteria taking place.

Technic of the Pfeiffer Test.-The suspected culture to be tested should be grown on agar for from eighteen to twenty-four hours, and used in doses of one loopful (2 $\mathrm{mg}$.).

If cholera is suspected, cholera immune serum of known titer should be used. Dilutions of serum are made with sterile bouillon, and 2 c.c. 
of each dilution, representing 2, 5, and 10 times the titer dose, are placed in separate glasses or small test-tubes. A fourth tube should contain 2 c.c. of a $1: 100$ dilution of normal serum, according to the animal used in producing the immune serum; a fifth tube should contain 2 c.c. of sterile bouillon (culture control).

Two loopfuls of suspected culture are thoroughly emulsified in each of the five tubes of the series, and 1 c.c. of each is injected intraperitoneally in five guinea-pigs weighing about 250 grams each.

At intervals of ten, twenty, forty, and sixty minutes the peritoneal exudate should be removed with capillary pipets and examined in hanging-drop preparations. Smears may be prepared and stained with dilute carbolfuchsin, although they give less information than hanging-drop preparations.

If guinea-pigs Nos. 1, 2, and 3 show granule formation at the latest after an hour, while in the fourth and fifth animals the bacteria remain whole, motile, and well preserved, the reaction may be regarded as positive and the diagnosis as established.

Pfeiffer Bacteriolytic Test in vivo in the Diagnosis of Disease.-Bacteriolysins are usually produced somewhat later than agglutinins, and reach their highest point of production during convalescence. Bacteriolytic tests are used only for diagnostic purposes, when agglutination reactions are negative or doubtful. The most satisfactory results from these tests are obtained in cholera. Bacteriolysis with typhoid bacilli is less typical and more incomplete; with the paratyphoid and dysentery bacilli it is even more unsatisfactory, and in anthrax, pest, and the various diseases due to cocci it does not occur.

The test is conducted in a manner similar to the preceding test, except that instead of an immune serum the patient's serum is used, with a known culture of typhoid, cholera, paratyphoid, etc., according to the infection suspected to be present.

One cubic centimeter of the patient's serum is secured in a sterile manner, inactivated by heating to $55^{\circ} \mathrm{C}$. for one-half hour, and dilutions of $1: 20,1: 100,1: 250$, and $1: 500$ prepared with sterile bouillon. Two cubic centimeters of these dilutions are placed in separate tubes, and two loopfuls of an eighteen-hour culture of the test organism emulsified in each. A fifth tube contains 2 c.c. of bouillon with two loopfuls of culture, and serves as the culture control.

One cubic centimeter of each dilution and of the culture control is injected intraperitoneally into five guinea-pigs, and the exudate examined after twenty, forty, and sixty minutes. 
If granule formation occurs in the first two or three animals, but is absent in the culture control, the reaction may be regarded as positive, and the diagnosis of typhoid, cholera, etc., considered as established.

If granule formation occurs to any extent in the fifth animal (culture control), the culture is to be regarded as unsuitable and the test repeated.

Measuring the Bactericidal Power of the Blood in vitro by the Plate Culture Method (Stern and Körte).- Since the earliest days of bacteriology the aim and purpose have been to devise some means by which the bactericidal power of the blood could be measured, just as is done in testing an ordinary germicidal substance, namely, by adding a bacterial suspension to the serum and observing the effect on the bacterial content. This is shown by counting the bacteria in a loopful immediately after adding the bacterial suspension, and repeating this at regular intervals, the counting being done by the method of plate culture.

The use of the platinum loop in these tests is objectionable, since the dose is quite variable, depending upon whether the loopful is removed from the fluid edgewise, or with the loop held flat, like a spoon in use. If the serum contains agglutinins, the results with any form of technic are likely to be irregular, and the number of colonies upon the plate stand in no relation to the actual number of microörganisms present. The results may be masked by a rapid multiplication of the survivors, and accordingly several controls are necessary with any technic.

Neisser and Wechsberg have recommended the so-called bactericidal plate culture method. By this method the patient's serum is inactivated, and varying amounts mixed with definite and constant quantities of bacteria. To this a constant quantity of active normal serum is added as complement, to reactivate the bacteriolytic amboceptors. These mixtures are then incubated for several hours. To determine whether and in what proportion death of bacteria has resulted from the effect of the bacteriolysins, agar is added, and the mixtures are then plated and incubated for twenty-four hours or longer. The number of colonies are then counted and the results compared with control plates of the culture alone.

Stern and Körte have modified this technic slightly, and recommend the procedure as a substitute for the Pfeiffer test in the clinical diagnosis of typhoid fever. The method spares a certain number of animals, but it is somewhat more complicated than the Pfeiffer reaction, and its results are less trustworthy. As a clinical test, therefore, it is to be recommended only in cases in which the agglutination reactions have yielded uncertain results, although with practice and care the test oftentimes 
yields uniform and satisfactory results, and may be employed in making special investigations for determining the bactericidal power of the blood, as after typhoid immunization.

Technic of the Test.-The requisites for success are that all vessels and diluting fluids, as well as the serums employed, should be absolutely sterile. To secure uniform and reliable results it is necessary to familiarize oneself with the technic by repeated practice. In order to carry out the steps in the technic according to strict aseptic bacteriologic principles the services of an assistant are required.

Sterile bouillon is largely used throughout, to maintain proper osmotic conditions.

With so many tubes and controls, it is important that all tubes and Petri dishes be properly labeled with a wax-pencil to avoid confusion.

One cubic centimeter of sterile patient's serum and an equal amount from a normal and healthy person to serve as a control are inactivated by heating to $55^{\circ}$ or $56^{\circ} \mathrm{C}$. for half an hour. These serums are then diluted $1: 50$ by adding 49 c.c. of sterile salt solution to each and mixing thoroughly.

Complement is prepared by securing 4 to 5 c.c. of sterile rabbit's blood and separating the serum. This serum is chosen because it should be from the same species of animal as that used in producing the immune serum to be tested. In determining the bactericidal titer of human serum either guinea-pig or rabbit complement serum may be used. Dilute 2 c.c. of this fresh serum (not over eighteen hours old) with 18 c.c. of sterile normal salt solution ( $1: 10)$ : Dose, 0.5 c.c.

Secure a good twenty-four-hour bouillon culture of typhoid bacilli (grown in the incubator) and dilute $1: 500$ by thoroughly mixing 0.1 c.c. of the culture in a flask containing 50 c.c. of sterile normal salt solution. This dilution of culture, when used in constant doses of 0.5 c.c., generally yields satisfactory results, but it may be necessary to try it out beforehand, for the control plates must regularly show a uniformly good growth and contain many thousands of colonies before uniform results can be expected. The bactericidal effect will then be distinctly shown by the reduction, in the proper plates, of this large number of colonies to zero or near it.

Place 1 c.c. of sterile neutral bouillon in each of 12 test-tubes which have been plugged with cotton, sterilized, and large enough to hold at least from 12 to 15 c.c. Place in the first of these tubes 1 c.c. of the diluted patient's serum and mix thoroughly by alternate sucking up and forcing out of the fluid; then, with the same pipet, draw up 1 c.c. and 
transfer it to the second tube of the series; mix as before, and transfer 1 c.c. to the third tube; continue in this manner to the last tube, from which, finally, 1 c.c. is discarded.

Each tube now contains 1 c.c. of fluid, representing dilutions of $1: 100$, $1: 200,1: 400,1: 800,1: 1600,1: 3200$, and so on up to $1: 204,800$ of the patient's serum. Higher or lower dilutions than these may be employed. It is very desirable that the dilutions be high enough to secure the limit of bactericidal activity, so that the last plates will show an increase in the number of colonies.

Arrange four tubes with the normal control serum, each containing 1 c.c. of fluid and representing the first four dilutions, viz., $1: 100$, $1: 200,1: 400$, and $1: 800$.

Label each tube carefully with the initials of the patient and the dilution it contains. Add 0.5 c.c. of the diluted bacterial emulsion, and finally 0.5 c.c. of the diluted complement serum, to each tube.

All manipulations should be made with strict bacteriologic care and with the aid of an assistant, as the introduction of contaminating microorganisms that may cause spore-formation will considerably vitiate the value of the test.

The following controls are necessary:

1. 0.5 c.c. culture directly in a sterile Petri dish +5 to 10 c.c. of neutral agar cooled to $42^{\circ} \mathrm{C}$. and thoroughly mixed. This control will show the original number of bacteria contained in the emulsion. Mark as control No. 1.

2. 0.5 c.c. culture +1.5 c.c. bouillon. Mark as control No. 2 . After three hours' incubation this tube receives the usual amount of agar and is plated. It will show to what degree the culture has multiplied in the incubator.

3. Since the complement serum frequently contains bacteriolysin, it is necessary to control this element carefully. To a series of four tubes add the following doses of the serum, diluted $1: 10$ : 1 c.c., 0.5 c.c., 0.2 c.c., 0.1 c.c. Add 0.5 c.c. culture to each tube, and sufficient bouillon to make the total volume in each tube 2 c.c.

4. 0.5 c.c. complement +1.5 c.c. bouillon. To control the sterility of the complement serum.

5. 1 c.c. of patient's serum $(1: 100)+1$ c.c. bouillon. To control the sterility of this serum.

6. 1 c.c. of the control serum $(1: 100)+1$ c.c. of salt solution. To control the sterility of this serum. 
7. 1 c.c. of the patient's serum in dilution of $1: 100+0.5$ c.c. culture +0.5 c.c. bouillon. A control on the possible presence of complement in this serum.

8. 1 c.c. of the control serum in dilution of $1: 100+0.5$ c.c. culture +0.5 c.c. bouillon. A control on the possible presence of complement.

All tubes with the exception of the first control which has been plated are placed in an incubator at $37^{\circ} \mathrm{C}$. for three hours. At the end of this time the contents of each tube are plated in neutral agar. Sterile Petri dishes should be properly and plainly labeled with a wax-pencil and arranged in order. A flask of plain neutral agar is melted in boiling water and cooled to $42^{\circ} \mathrm{C}$. The tubes are removed from the incubator and shaken gently and with the aid of an assistant from 5 to 8 c.c. of agar are added carefully to each tube with a sterile 10 c.c. pipet. The contents are mixed by gentle rotation of the tube and then poured in the corresponding Petri dish, followed by an additional mixing according to the usual bacteriologic procedure. With ordinary speed the whole set of tubes may be poured in a satisfactory manner before the flask of agar has had time to harden.

Another method of plating consists in pipeting the contents of each tube into its corresponding dish, and then washing the tube with an additional 1 c.c. of sterile salt solution, to remove all traces of serum and culture. Or the end of each tube may be flamed and the contents poured directly into a dish. If this method is adopted, small test-tubes should be used. While the method is more convenient, it is usually not so accurate as the first two methods.

Neisser plates but 5 or 10 drops from each tube. The dose decided upon is the one employed throughout. For example, it would be erroneous to take 5 drops from one tube and 10 from another. Neisser, however, uses much smaller amounts of the serum, as $1,0.3,0.1,0.03$, and 0.01 c.c., instead of the much higher dilutions given in this technic. These differences must be remembered and the proper dilutions employed, and but 5 to 10 drops should be plated in performing the bactericidal plate test according to Neisser's technic.

Töpfer and Jaffe pour a thin layer of agar into a Petri dish and allow it to harden. Upon this they pour the culture-serum agar mixture, which, after settling, is covered with another thin layer of agar. In this way a culture in the water of condensation is avoided. In the usual technic this may be avoided by turning the plates over soon after hardening occurs so that the water of condensation collects on the cover. 
All plates are incubated at $37^{\circ} \mathrm{C}$, for from twenty-four to thirty-six hours and the colonies then counted. In some plates the number may be so large that counting will be inaccurate and unnecessary. Significance can be attached only to marked and easily recognizable differences. According to Neisser, the growth is best and most rapidly described by means of approximate estimates, using a scheme somewhat like the following: 0 or almost none; about 100; several hundreds; thousands; very many thousands; infinite numbers. A distinct bactericidal action is then present only if the controls react normally, and if a reduction of colonies from an infinite number or many thousands to 0 or very few has occurred. As previously stated, the test can then be regarded as satisfactory only if the lower limits of bactericidal activity have been reached and the last plates show an increase in the number of colonies. Examination of these plates is very much facilitated by using a colony counter, that of Stewart being particularly serviceable with agar plates.

The controls are first examined and the results recorded, and finally those of the patient's serum are set down.

As a practical illustration, the results of a test with a rabbit typhoid immune serum are given, because this is in general an average example of those usually obtained:

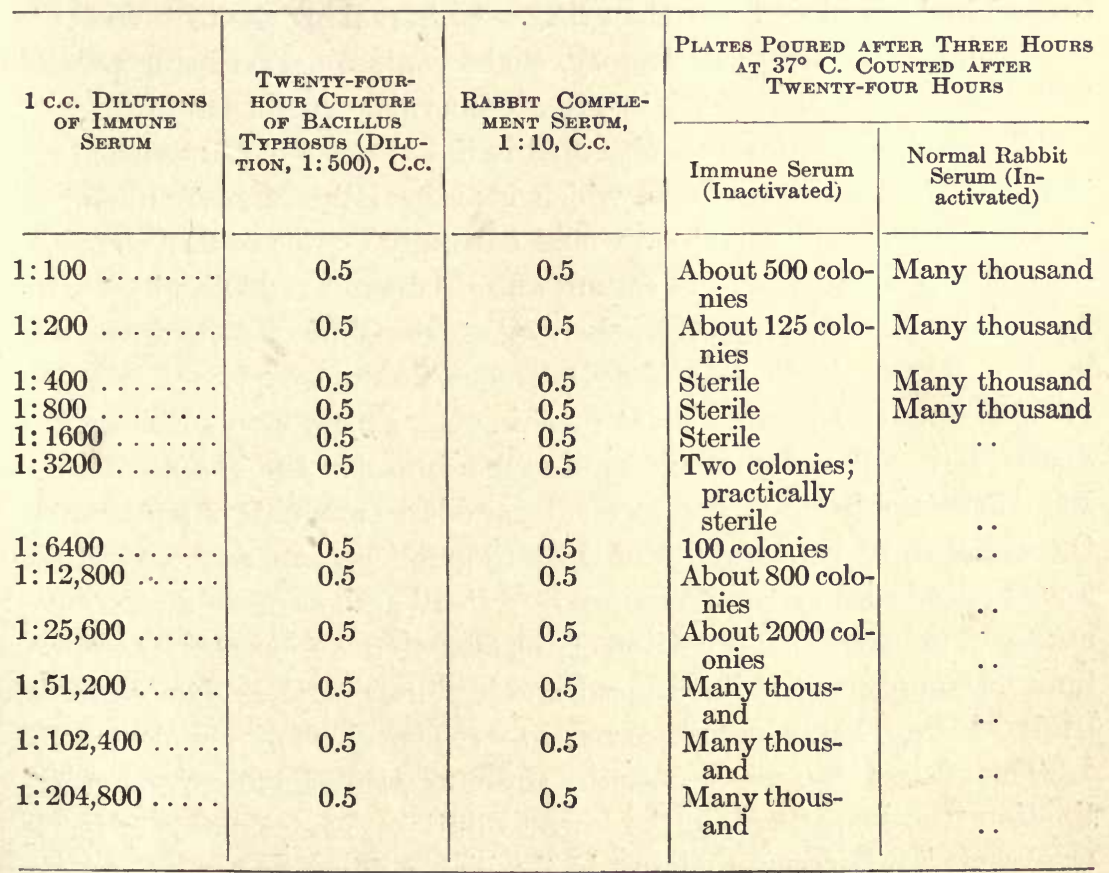




\section{Controls}

Control 1: 0.5 c.c. culture plated immediately: many thousands of colonies.

Control 2: 0.5 c.c. culture plated after being incubated three hours: plate very crowded; number of bacteria increased.

Control 3: Varying amounts of normal rabbit serum used as complement. The first plate containing 0.1 c.c. serum showed a slight bactericidal action; the remaining plates showed many thousand of colonies and were comparable to control No. 1.

Control 4: 0.5 c.c. complement serum: sterile.

Control 5: 0.01 c.c. inactivated immune serum: sterile.

Control 6: 0.01 c.c. inactivated normal control serum: plate shows several colonies of contaminating bacteria.

Control 7: 0.01 c.c. inactivated immune serum plus culture: plate shows many thousands of colonies.

Control 8: 0.01 c.c. of inactivated normal serum plus culture: many thousands of colonies.

An examination of this experiment shows that the dose of culture was satisfactory, that the culture increased during the three hours of primary incubation, that the complement serum was very slightly bactericidal in a dose lower than that used in making the test, and that the technic was fairly satisfactory, slight contamination being present in but one plate - that of the inactivated normal control serum.

The most striking result observed is the absence of bactericidal activity in the first two plates, which contained the largest amount of immune serum and where one would naturally expect to find complete sterility. The titer of this serum was between $1: 3200$ and $1: 6400$. According to Neisser and Wechsberg, these paradoxic results are caused by deviation or "deflection of complement," as was explained in a previous chapter. In bactericidal experiments, according to Neisser, the deflection is caused by an excess of amboceptors in the immune serum. In a mixture of bacteria, complements, and large amounts of amboceptor the complement is bound not only by the amboceptors anchored to the bacteria, but also in large measure by "free" amboceptors that are not anchored to bacteria. A portion of the anchored amboceptor, therefore, finds no complement at its disposal, and is, therefore, unable to exert any bactericidal action, which gives rise to a relative lack of complement.

This phenomenon resembles the action of agglutinoids in the agglutination reaction, where, in the lowest dilutions, agglutination is feeble or absent, but becomes manifest in the higher dilutions. 
In the foregoing experiment the immune serum used was several weeks old; perfectly fresh serums are not so likely to show this so-called "deflection of complement." In many instances, and especially if a fresh serum is used, one cannot help thinking that agglutinins may be responsible for the absence or diminution of bactericidal activity in the lower dilutions. It is certainly true that hemagglutinins considerably inhibit hemolysis, and this is especially the case with serums of low hemolytic activity. With more potent serums the agglutinins are diluted until activity ceases and hemolysis is ready and complete. Reasoning from analogy, therefore, the absence or diminution of bactericidal activity may be due to agglutinins, and the theory of "deflection of complements" may be emphasized a little too strongly.

According to Halm, normal human serums show bactericidal activity in only about one-third of the cases, and the titer is only very exceptionally demonstrable in dilutions higher than $1: 500$. The serums of advanced cases of typhoid fever or of those but recently recovered are bactericidal in dilutions greater than $1: 1000$, and may reach $1: 50,000$ or higher. Similarly after typhoid immunization the patient's serum may show a high bactericidal titer. Weston has found such serums active in dilutions of $1: 20,000$, higher dilutions not being used.

Besides being used in typhoid, the plate culture method has been employed for experimental purposes in cholera, dysentery, paratyphoid, and other infections with bacilli of the typhoid-colon group. With these, however, the test possesses but little diagnostic significance.

The bactericidal titer does not run strictly parallel with the agglutinins or complement-fixing bodies.

Measuring the Bactericidal Power of the Blood by Capillary Pipet Method (After Wright). ${ }^{1}-$ By this technie it is sought to overcome the fallacies of the "loopful" method of measurement and those due to agglutination of the test organism.

The native complements of the patient's own serum are used; hence the serums used in this technic must be fresh. Quantitative titration is accomplished by furnishing varying dilutions of culture, with a constant quantity of serum. A series of volumes of serum is taken, and to these are added equal quantities of progressively increasing dilutions of a counted bacterial culture. The mixtures are kept at $37^{\circ} \mathrm{C}$., for twentyfour hours, after which each is introduced into nutrient broth and cultivated to see whether a complete bactericidal effect has been exerted.

${ }^{1}$ Wright, A. E.: Technique of the Teat and Capillary Glass Tube, 1912, London, Constable \& Co. 
The largest number of bacteria that a constant quantity of serum has been able to kill furnishes a measure as to its bactericidal power.

After a few technical details have been mastered, this method is quickly performed and yields fairly constant and reliable results. It is not adapted for the titration of old immune serums, but is a ready clinical test, finding a special field of usefulness in determining the bactericidal powers of the blood after typhoid immunization.

Requisites for Carrying out the Test.-1. A specimen of the patient's blood is collected aseptically, a process that may be accomplished by thoroughly cleansing the finger with alcohol and collecting blood in a sterile Wright capsule, or better, perhaps, by means of venipuncture, when from 2 to 5 c.c. may be collected aseptically in a sterile centrifuge tube. The control blood from a healthy individual may be collected in a capsule. The serums are carefully separated and pipeted into small sterile test-tubes; 1 c.c. of each is ample for the test.

2. A twenty-four-hour-old broth culture of the test organism (a young culture is required, because such a culture contains a few dead microörganisms and the absorption of bactericidal elements by dead organisms is thus avoided).

3. About two dozen "looped pipets," made according to the directions given in Chapter I.

4. Sterile neutral broth for making dilutions and cultivations. This is prepared and sterilized in the usual manner, from 5 to 10 c.c. being placed in test-tubes. When working with the typhoid bacillus a special broth containing 1 per cent. of mannite and sufficient litmus to color it a deep blue (Smallman) should be on hand.

5. Two dozen small test-tubes, plugged and sterilized, for making dilutions of the culture.

Preparation and Enumeration of the Bacterial Culture.-As the principle of the test depends upon measuring the bactericidal activity of the blood according to the number of organisms that are killed, it is necessary to prepare somewhat high dilutions and count the number of organisms in a unit volume in each dilution.

For this purpose place 10 sterile test-tubes in a rack, and arrange 10 sterile Petri dishes on the table to correspond to these. To the first tube add 4.9 c.c. of plain sterile broth; to the second, fourth, sixth, eighth, and tenth tubes add 1 c.c., to the third, fifth, seventh, and ninth tubes, add 4 c.c.

With a sterile graduated 1 c.c. pipet add 0.1 c.c. culture to the first tube and then discard the pipet by placing it in a cylinder of disinfecting 



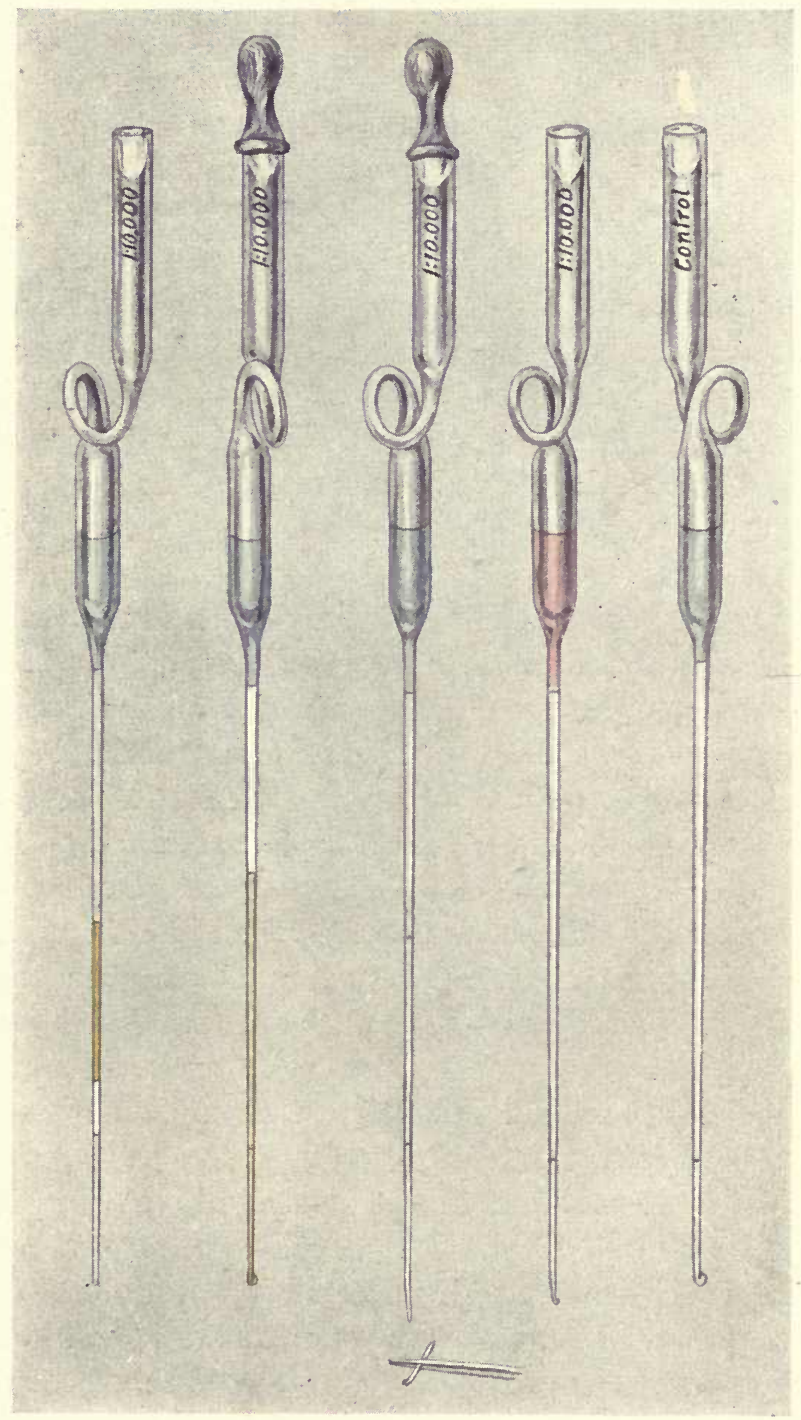

Fig. 101.-Bactericidal Test (Looped Pipet Method of Wright).

The pipet shown on the extreme left contains the mannite-litmus broth and an equal volume of patient's serum and emulsion of typhoid bacilli; the second pipet shows the serum and bacillary emulsion mixed; the middle pipet shows the serumbacillary mixture cultured in the broth; the fourth pipet shows acid production in the broth by living bacilli which escaped destruction after twenty-four hours' incubation; the fifth pipet (extreme right) is the serum control. The broth, being clear and unchanged, shows that the serum was sterile. 
solution or hot water. With a second sterile pipet mix the contents of tube No. 1 by carefully sucking it in and forcing it out of the pipet several times, and place 0.1 c.c. in Petri dish No. 1 . Then, with the same pipet, transfer 1 c.c. to the second tube, mix well, and place 0.1 c.c. in Petri dish No. 2; next transfer 1 c.c. to the third tube, mix, and place 0.1 c.c. in the third Petri dish. Continue in this way until the last tube is reached, when 1 c.c. is discarded into a germicidal solution and 0.1 c.c. placed in the tenth Petri dish. To each Petri dish now add from 8 to 10 c.c. of neutral agar at $41^{\circ} \mathrm{C}$., and mix thoroughly. After the plates have hardened they are turned over in order that the water of condensation may collect on the cover. They are then incubated for twenty-four hours, and the colonies carefully counted.

We now have the following dilutions of culture: $1: 50,1: 100$, $1: 500,1: 1000,1: 5000,1: 10,000,1: 50,000,1: 100,000,1: 500,000$, and $1: 1,000,000$. Since but 0.1 c.c. of these dilutions were plated, the total number of colonies in each plate must be multiplied by 10 in order to obtain the approximate number per cubic centimeter of the various dilutions.

To secure fairly uniform results, the various dilutions must be thoroughly mixed and the pipeting be accurately performed. We have found that this method usually gives better results than are obtained by plating but one or two of the higher dilutions, which are used as a basis for calculating the number of bacteria in the other dilutions.

Filling the Pipets. -With a wax pencil make a mark upon the capillary stem of a sterile looped pipet at a point $2 \mathrm{~cm}$. from the lower end, and fit a rubber teat to the barrel. The point of the capillary stem is now broken off between the finger and thumb, the lower portion is sterilized in the flame, and the air is expelled from the teat.

Mannite broth is then aspirated into the pipet until the bulb is about two-thirds full and the capillary portion contains an air column rising to at least $5 \mathrm{~cm}$. from the end (Fig. 101).

The end of the capillary stem is now inserted into the tube containing the patient's serum, and the serum is allowed to flow in until it reaches the pencil mark.

The orifice of the pipet is now raised above the surface of the serum, and a small bubble of air is admitted into the tube, to serve as an index for the measurement. The end of the capillary stem is now carried into the tube containing the highest dilution of the organism, and the culture is allowed to flow in until the bubble of air has been carried just past the pencil mark. 
The serum and culture must now be mixed, being careful not to contaminate the broth. This is effected by blowing these two volumes out into a sterile mixing tube and drawing up and blowing out the fluid several times in succession. By starting with the highest dilution, the same mixing tube may be used throughout the series. With an airbubble of at least $5 \mathrm{~cm}$. between serum and broth, this can be quite easily achieved without driving the sterile broth down from the bulb of the pipet into the lower part of the capillary stem and contaminating it there.

The column of mixed serum and culture is now drawn up into the middle region of the capillary stem and the lower end of the tube is sealed. The teat is then removed, and the dilution that has been used is written on the barrel of the pipet.

The series of pipets are now filled with the nine measuring dilutions of the culture.

Controls.-The serum from a healthy individual, or, better still, the pooled serums of several persons, may be used in precisely the same way, with at least the second, fourth, sixth, and eighth dilutions of culture.

Several culture controls are advisable. The pipets are filled with mannite broth in the usual manner, and then with one volume of at least four different dilutions, usually $1: 50,1: 5000,1: 100,000$, and $1: 1,000$,000 . The tubes are sealed, labeled, and incubated together with those concerned in the test proper.

One pipet is to contain the usual quantity of broth and one volume of the patient's serum. This is a control on the sterility of the patient's serum. A similar preparation is made with the control serum to serve as a control on its sterility.

When the whole series of tubes have been filled, these are placed upright in a large test-tube or cylinder labeled with the date and the source of the serum, and incubated at $37^{\circ} \mathrm{C}$. for from eighteen to twentyfour hours.

Test for the Germicidal Activity of the Serum.-The serum and culture in the capillary portion of the tube must now be mixed with the broth in the barrel. This is accomplished by taking each pipet in hand singly, and heating the lower portion of the capillary stem in a "peep-flame" and drawing out into a small thread with a pair of forceps.

A collapsed teat is now fitted over the barrel, and the negative pressure carefully regulated by keeping the finger and thumb in position on the teat, and the finely drawn out end of the capillary stem gently snapped across. The column of serum and culture will then be carried up into the bulb of the pipet. The end of the tube is now sealed. 
When the whole series of pipets has been dealt with in similar fashion they are returned to the incubator for another twenty-four hours.

Reading the Result.-The continued sterility of the broth may be determined from direct naked-eye inspection.

When a complete bactericidal effect has been secured, the broth will have undergone no color change, but will still be clear.

When a growth of the typhoid bacillus has occurred, a perceptible cloudiness will be apparent in the broth, and the color will have changed from blue to reddish-blue, indicating that acid has been formed from the mannite.

Turbidity of the broth without a change of color would indicate the admission of contaminating microbes that were unable to split mannite with the formation of acid.

The controls are first examined and recorded. All the culture controls should show a growth and change of color. The serum controls should be sterile; the normal serum controls may or may not be sterile, depending upon the amount of natural bactericidal amboceptors which it contains for the test organism.

The pipets containing the patient's serum are now examined, and a simple numeric expression for the result is obtained by referring to the result of the enumeration of the various dilutions, as determined by the counting of the plates. In this manner the number of bacteria contained in 1 c.c. of the lowest dilution of the bacterial culture that has been completely sterilized is calculated. This will give the number of microorganisms which 1 c.c. of serum would be capable of killing.

Although this test affords a convenient basis for the comparison of serums, it must be understood that the expression is entirely arbitrary, and will vary according to the culture employed; this is true of any technic for determining the bactericidal titer of a serum.

\section{BACTERIOLYTIC SERUMS IN THE TREATMENT OF DISEASE}

While bacteriolysis is readily demonstrated with some bacteria both in vivo and in vitro, nevertheless, when such serums are used therapeutically, beneficial results are not dependent solely upon lysis of the infecting bacterium, but are usually the result of a combination of lysis with increased phagocytosis, due to the simultaneous presence of bacteriotropins (immune opsonins). When one recalls the close similarity in general properties that exists between bacteriolysins and bacteriotropins, the difference between extracellular and intracellular lysis is 
relatively slight, and tends to strengthen Metchnikoff's views regarding the close relationship of the bacteriolysins to leukocytic products and phagocytosis. While extracellular lysis can occur in the absence of cells, especially in vitro, yet in the administration of antibacterial serums the activities of the leukocytes are much in evidence, extracellular lysis or bacteriolysis proper being rather subsidiary to intracellular lysis or phagocytosis.

The preparation and methods of standardization of antibacterial serums are given in the chapter on Passive Immunization. Most effort in this direction has been expended on the preparation of antiserums for the treatment of meningococcic, streptococcic, pneumococcic, and gonococcic infections, and the greatest success has been attained with the various antimeningococcic serums. 


\section{CHAPTER XX}

\section{HEMOLYSINS}

Hemolysis. is the term applied to the solution or lysis of red bloodcorpuscles. Strictly speaking, it would include the disintegration and solution of the stroma, although in practice the term is applied to any process in which the cells are so injured as to liberate hemoglobin into the surrounding fluids, with or without solution of the stroma.

Hemolysis may be caused by various physical, chemical, and specific agencies. The prolonged agitation of blood with glass beads, for instance, may result in the mechanical rupture of erythrocytes. The addition of blood to hypotonic solutions of sodium chlorid or to plain water, results in ready and complete hemolysis, the fluid being transformed from an opaque red suspension of erythrocytes to a clear, transparent red fluid. Various chemicals, such as acids and alkalis, may also produce hemolysis. As previously stated, a few bacterial toxins, such as tetanolysin and staphylolysin, are known to be hemolytic; the same is true of certain vegetable toxins, such as abrin and ricin, and of some animal toxins, such as cobra, toad, and scorpion venoms.

Just as bacteria may be killed and possibly broken up by specific bacteriolytic amboceptors and complements, so, in like manner, hemolysis may be caused by specific hemolytic antibodies known as hemolysins. Working in unison with complements, the mechanism of both bacteriolysis and serum hemolysis is probably identical. The simplicity of hemolytic experiments, the rapidity with which they may be performed and terminated, and the ease with which hemolysis may be observed by the naked eye have rendered the specific serum hemolysins particularly useful in the study of amboceptors and of complements, and of cytolytic phenomena in general. In fact, bacteriolysis was not thoroughly understood until Bordet discovered the hemolysins, and demonstrated the analogy that exists between bacteriolysis and hemolysis, a discovery that led to a vast amount of research work and controversy, to many important discoveries, and to the final evolvement of diagnostic reactions of great value.

Historic.-For many years physiologists were aware that the bloods of various animals transfused into man or animals of a different species 
were more or less directly injurious, and incapable of replacing human blood. In 1875 Landois demonstrated experimentally that while transfusion of blood from one animal to another of a different species may prove injurious and even fatal, transfusion to an animal of the same or of very closely related species produced no ill effects. The explanations offered were inadequate, until later researches on the hemolysins showed that the normal blood-serum of one animal may contain hemolysins for the erythrocytes of other animals, and, consequently, upon transfusing this blood to another animal the hemolysin acting with the complement present produced hemolysis in vitro, thereby explaining in part the toxicity of the alien blood.

In 1898 Belfanti and Carbone made the observation that the serum of a horse receiving several injections of rabbit blood was toxic for rabbits, whereas normal horse serum was without injurious effects.

At about the same time Bordet published his epoch-making discoveries. He observed that while normal guinea-pig serum had little or no hemolytic action on rabbit erythrocytes, the serum of a pig that had received a few intraperitoneal injections of rabbit blood was able quickly and completely to hemolyze rabbit blood, just as an animal may acquire, through immunization with cholera, the property of dissolving cholera vibrios. He demonstrated further that this acquired hemolytic activity was highly specific, for when animal A was immunized with the corpuscles of animal $\mathrm{B}$ the serum of $\mathrm{A}$ acquired the power of hemolyzing only the erythrocytes of B, and possibly of other animals closely related zoölogically. It was found also that the hemolytic activity of an immune serum was lost by age or could be removed by heating; that in either case the serum could be reactivated by the addition of a little normal serum or peritoneal exudate-phenomena closely resembling that observed in bacteriolysis, and due to the action of a thermolabile body or alexin and a second and specific thermostabile antibody named by Bordet the "substance sensibilisatrice."

These observations were soon confirmed by Landsteiner and von Dungern, and were followed by very extensive studies by Ehrlich and Morgenroth, who likewise confirmed Bordet's experiments, but offered a different explanation for the mechanism of the phenomenon, according to the side-chain theory, and renamed the alexin and sensitizing substance concerned in the process "complement" and "hemolytic amboceptor" or "immune body," respectively.

Definition.-Hemolysins [Gr., aija = blood $+\lambda \boldsymbol{v}_{\epsilon \iota \nu}=$ to dissolve] are antibodies in a serum that, when acting with complement, have the 
power of "lysing" or breaking up red blood-corpuscles, or so altering their envelop as to allow the hemoglobin to escape.

Nomenclature.-Normal hemolysins are those found in normal serums; specific or immune hemolysins are those produced as the result of the injection of blood-corpuscles from an animal of a different species. Heterolysins are the hemolysins formed by immunization with corpuscles of a different species (the immune hemolysins). Isolysins are hemolysins for the corpuscles of animals of the same species. Autolysins are hemolysins that act upon the corpuscles of the same animal and are quite rare. It should be remarked that isolysin and autolysin are not strictly synonymous terms, as the former does not act upon the corpuscles of the animal producing the hemolysin, but may hemolyze the corpuscles of other animals of the same species. For example, Ehrlich has shown that the serum of a goat that had received several injections of blood from other goats, although actively hemolytic for the corpuscles of goats 1, 2, $4,5,6,9$, and less so for goats 3 and 8 , was not able to hemolyze those of goat 7 or of itself at all. This immunity of the corpuscles of an animal to its own isolysin was subsequently shown to be due to a complete absence of suitable receptors in its corpuscles. Therefore in cases in which a large internal hemorrhage occurs and the blood is absorbed an autohemolysin is not produced, or produced only in small amounts, and likely to be followed by the formation of an anti-autolysin which regulates the process of blood destruction within physiologic limits. Although little is known concerning the processes of normal blood destruction, as in the disposal of old erythrocytes, it is possible that an autohemolysin is being produced, and that its activity is held within normal limits by an anti-autolysin. A disturbance of this physiologic equilibrium may then be the basis of certain types of primary anemia characterized by excessive blood destruction.

Nature of Hemolysins.-According to the side-chain theory, hemolysins are amboceptors or antibodies of the third order, requiring the action of a complement before hemolysis can be produced (Fig. 102). Bordet showed that two substances were concerned in the phenomenon of serum hemolysis, although his views on the mechanism of the process differ from those advanced by Ehrlich and Morgenroth.

Ehrlich argued that the hemolytic amboceptor or hemolysin must be an antibody to the receptors of the red blood-corpuscles used in the process of immunization, and if this is true, it ought to unite with the corpuscles. Taking the serum of a goat that had been injected with and was hemolytic for the erythrocytes of a sheep, he destroyed the comple- 
ment by heating the serum to $56^{\circ} \mathrm{C}$. To this he added some sheep's corpuscles, and allowed the mixture to stand for a short time at room temperature, after which it was centrifuged and the supernatant fluid pipeted off in another test-tube. No hemolysis had occurred, and the corpuscles were to all appearances unaltered, but it was now found that if a small amount of normal goat serum, as complement, was added to the corpuscles and the mixture placed in the incubator, hemolysis occurred. By adding sheep's corpuscles and normal goat serum (complement) to the supernatant fluid that had been removed to a separate testtube, hemolysis did not occur. This experiment indicated, therefore, that the red blood-corpuscles had combined with all the antibody. That the action was specific was shown by the fact that the corpuscles of other animals, such as rabbits or goats, for example, exerted no combining power when used instead of the sheep's cells. The union between cell and antibody was considered as

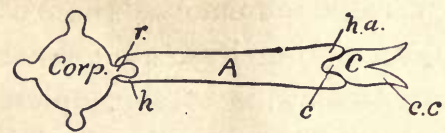

Fig. 102.-Theoretic Structure of a Hemolytic Amboceptor (Hemolysin).

$A$, Amboceptor; $C$, complement; $r$, receptor of the corpuscle; $h$, haptophore group of the amboceptor; h.a., complementophile group of the amboceptor.

being in the nature of a chemical combination and quite firm, as repeated washing of the cells with normal salt solution did not break it up.

Having shown that the antibody had a combining affinity for the cells, it was important to solve the question of the relation of alexin or complement to the process. In other words, does this substance unite directly with the cell or does it unite with the antibody and thus indirectly with the cell?

Ehrlich and Morgenroth studied this by means of a similar experiment. Sheep's corpuscles were mixed with normal goat serum (complement), and after a time the mixture was centrifuged and the two portions tested separately. To the corpuscles, heated immune serum was added, but hemolysis did not result. To the supernatant fluid corpuscles and heated immune serum were added and hemolysis occurred. This indicated that the alexin or complement did not unite with the corpuscles, as did the antibody in the first experiment, but remained free in the supernatant fluid.

By mixing corpuscles, immune serum, and complement and keeping the mixture at $0^{\circ}-3^{\circ} \mathrm{C}$. for several hours, hemolysis did not take place. By centrifuging the mixture and separating the supernatant fluid from the corpuscles, a similar test showed that the red cells had combined with all the antibody, but had left the alexin practically undisturbed. 
At higher temperatures these relations were more difficult to demonstrate, as hemolysis occurs rapidly, but by leaving the cells and serum in contact for short periods of time, centrifuging rapidly, and testing the corpuscles and supernatant fluid in the same manner, similar relations were found to exist.

These experiments led Ehrlich to formulate the theory that complement will unite only with the antibody and not with the red corpuscles, but that it acts upon the corpuscles when united.indirectly by means of the antibody. As will be pointed out further on, this view is in direct opposition to that of Bordet, who does not accept this interpretation, but believes that the complement acts directly upon the corpuscles.

Ehrlich, therefore, conceived the antibody as being in the nature of an amboceptor or of an interbody between an antigen and complement, with two combining arms-one the cytophile haptophore for union with the cell, and the second, the complementophile haptophore for union with complement. The amboceptor is unable in itself to injure the cell, but preserves its importance in being the only and specific means by which the ferment or complement can attack the cell and cause its destruction.

The process of specific serum hemolysis is therefore supposed to be as follows: In fresh immune serum containing both amboceptors and complement, or in a mixture of old or heated immune serum and fresh normal serum (i.e., of amboceptor and complement), the two substances occur independently of each other. When the corpuscles corresponding to the amboceptor are added, the amboceptor unites with these and the complement unites with the amboceptor, the amboceptor, therefore, standing midway between corpuscles and complement. When these unions have taken place, hemolysis will result. The amboceptor has a greater affinity for the corpuscles than the complement has for the amboceptor, and will unite with the cells at a low temperature, whereas the complement unites with the amboceptor only very slowly at low temperatures. Body temperature favors a quicker union of both, and especially that of complement with amboceptor. Hemolysis, therefore, may occur at low temperatures, but is hastened by higher temperatures, and occurs best at $37^{\circ} \mathrm{C}$.

As stated in a previous chapter, Ehrlich believes that a great many complements exist in normal serums, which view is in direct opposition to the "unitarian theory" of Bordet, which holds that the one alexin or complement will act with any sensitizer or amboceptor. Of the large number of complements, each is especially adapted for the solution of one or more varieties of cells, which it can dissolve in conjunction with a 
suitable amboceptor. This is known as the main or dominant complement; other complements that may aid in the process are termed nondominant. In general, it is held that those complements that are especially active in hemolysis are but slightly active in bacteriolysis, and vice versâ. Although the amboceptor is depicted as having but one haptophore arm for the dominant complement, it is really a polyceptor, and is so constituted that it combines with the cell to be dissolved, on the one hand, and with a number of complements, on the other.

Bordet has never accepted these views. He holds that the antibody is not an amboceptor for uniting cell and complement, but that it sensitizes the cell and renders it susceptible to the direct lytic action of the alexin or complement. According to his views, both antibody and complement may unite directly with the cell, and he has borne out this belief by making experiments almost exactly similar to those made by Ehrlich.

In accepting Ehrlich's view, it is a question of considerable practical importance whether the complement may unite directly with free amboceptor. Ehrlich maintains that the two may enter into a loose and easily dissociated chemical combination, which is hastened by heat and retarded by cold. The union of hemolytic amboceptor in cobra venom with the lecithin of corpuscles (Kyes' cobra lecithid), which acts as complement, while it tends to strengthen this view can hardly be accepted as direct proof, as lecithin differs markedly from the ordinary complements found free in serum. Likewise the theory of Neisser and Wechsberg regarding complement deviation, whereby it appears that an excess of amboceptor may combine directly with complement and in this manner rob those amboceptors that are attached to cells of the complement necessary to produce lysis, is quite complicated, and is not universally accepted, the evidence of direct union of complement and amboceptor having not been proved beyond the peradventure of a doubt. It follows, then, as Emery has stated, that we must either assume that the complementophile haptophore of an amboceptor united with its antigen has an increased affinity for complement over and above that of free amboceptors, or we must agree with Bordet that cell and complement unite directly after the former has been sensitized by the action of the antibody. This latter view of the separate union of cell with antibody and complement is supported by the observations of Muir, who found, upon saturating red blood-corpuscles with antibody and then with complement, that some of the former but none of the latter may be dissociated from the combination and become free in the fluid. However, 
the dissociated amboceptors found free of complement may be those that did not have time to unite with complement, and there does not appear to be any direct and positive experimental evidence to permit one to decide between Ehrlich's and Bordet's views:

While Ehrlich believes that the union between cell and amboceptor is a chemical one and follows ordinary chemical laws, obeying the law of multiple proportions, Bordet holds that the antibody acts as a mordant and sensitizes the cell, comparing the process to the staining of filter-paper when immersed in a dye, or to the use of mordants preparatory to the staining of flagella of certain bacteria. For example, 0.4 c.c. of a hemolytic serum, if added at once, was found to dissolve $\mathbf{0 . 5}$ c.c. of corpuscles. If 0.2 c.c. of corpuscles were first added, and amounts of 0.1 c.c. were added subsequently, no lysis took place after that of the first portion added. Bordet cited this as an example of a physical process of the nature of absorption, just as filter-paper when added at once to a dye will be stained a uniform color, whereas if it be added a little at a time, the first pieces inserted will be stained deeply, the subsequent ones less and less so, until the dye is completely absorbed. Recent researches on the colloidal theory of antibodies would indicate that the hemolysins are governed by very complex chemicophysical laws, not as yet fully understood, which regulate the action of colloids on one another and are probably intimately concerned in the processes under discussion.

As was stated in a previous chapter, Metchnikoff maintains that both substances concerned in hemolysis are ferments, and that both are adapted for intracellular digestion. He regards complement or his cytase as a digestive ferment derived from leukocytes, and believes that it is set free only when leukocytes are dissolved (phagolysis), either as the result of the injection of a foreign substance or during the process of coagulation. Amboceptor or his "fixateur" is likened to enterokinase, and like it acts as an accessory ferment that unites the more potent ferment (cytase) to the particle to be digested. He also regards it as being derived from leukocytes, and considers that the amount formed depends upon the degree of phagocytosis that occurs during the absorption of the antigen. In the conception of immunity as being fundamentally a process of nutrition, and in the belief of the existence of more than one complement, the similarity between the views of Ehrlich and those of Metchnikoff is indeed striking.

Analogy between Bacteriolysis and Hemolysis.-Studies in hemolysis aided greatly in a correct understanding of the mechanism of bacteriolysis. It became apparent that two substances were concerned in 
bacteriolysis - one, the thermostabile amboceptor present in the immune serum, and the second, thermolabile alexin or complement, furnished by the peritoneal exudate in the Pfeiffer test, or by any fresh normal serum in the test-tube bacteriolytic reaction. The discovery and study of the specific serum hemolysins aided greatly in a better understanding of bacteriolysis and cytolysis in general.

Specificity of Hemolysins.-The hemolysins are highly specific antibodies, and although partial or group hemolysins may be formed and act upon the corpuscles of closely related species, as antihuman hemolysin on the corpuscles of the higher apes, yet the main hemolysin may be so potent that high dilution of the immune serum practically rules out the activities of group hemolysins, the hemolytic amboceptor proving highly specific for the alien corpuscles responsible for its production.

\section{NORMAL HEMOLYSINS}

Just as small amounts of antitoxins, agglutinins, and opsonins may be found in normal serums, so, also, normal hemolysins may be present. Their most important practical significance is concerned with complement-fixation reactions, where a close and intimate quantitative relation exists between the complement and hemolytic amboceptor used. As an excess of hemolytic amboceptor may produce hemolysis with a decreased amount of complement, in a given test for free complement, as in Wassermann's syphilis reaction, the patient's serum may contain so much natural antisheep amboceptor as to make up for slight binding of complement and give undue hemolysis or even a false negative result.

Hemolysis of alien corpuscles by a normal serum is found to depend upon the same mechanism of amboceptor and complement as in the artificial immune serums. The amboceptors are easily removed by adding the corresponding corpuscles to the cold serum and centrifuging the mixture after allowing it to stand at $0^{\circ}-5^{\circ} \mathrm{C}$. for an hour or two. The supernatant fluid will now be found to be free from amboceptors, whereas by adding a little normal complement serum to the corpuscles hemolysis results, indicating that the amboceptors had been bound to these. If a normal serum contains several different amboceptors for as many different bloods, all may be removed at the one time by adding the respective corpuscles to the serum and allowing sufficient time to elapse for the amboceptors to become linked to their corpuscles.

Normal serum, therefore, probably contains numerous antibodies of the amboceptor type, adapted to dissolve various foreign substances 
when they gain access to the blood. It is probable that, aside from hemolysins, various normal and immune cytolysins play an important part in the processes of immunity.

While the normal hemolysins that may be found in the serums of various animals have not as yet been fully worked out, the following table, compiled by Sachs ${ }^{1}$ is a résumé of the work reported in the literature on the subject:

TABLE 7.-NATURAL HEMOLYSINS

\begin{tabular}{|c|c|c|c|c|c|c|c|c|c|c|c|c|c|}
\hline \multirow[b]{2}{*}{$\begin{array}{l}\text { HEMOLYZE THE } \\
\text { ERYTHROCYTES } \\
\text { OF THE- }\end{array}$} & \multicolumn{13}{|c|}{ The Serum of } \\
\hline & 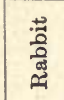 & : & $\stackrel{\infty}{\circ}$ & 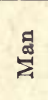 & ర్ల & $0^{\star}$ & 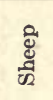 & $\stackrel{\infty}{\stackrel{4}{*}}$ & 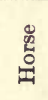 & $\begin{array}{l}\text { के } \\
\text { ర் }\end{array}$ & 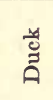 & 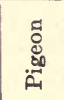 & 竎 \\
\hline Rabbit. & 0 & $t$ & + . & + & + & .t & + . & + & $\dot{ \pm}$ & + & + & + & t. \\
\hline Guinea-pig. & + & 0 & + & + & + & + & + & + & $\neq$ & + & + & \pm & + \\
\hline $\operatorname{Dog} \ldots \ldots$ & $(+)$ & \pm & 0 & & & & & & $\ldots$ & & & & \\
\hline Man.. & \pm & + & + & 0 & + & + & \pm & \pm & - & + & + & $( \pm)$ & + \\
\hline Goat.......... & + & . & + & + & 0 & & .. & t & - & + & $\cdots$ & . & . \\
\hline $\mathrm{Ox} \ldots \ldots \ldots \ldots$ & \pm & \pm & $( \pm)$ & \pm & 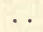 & 0 & - & \pm & - & + & + & - & - \\
\hline Sheep. . . . . . . . & \pm & + & + & + & \pm & - & 0 & \pm & - & - & - & - & - \\
\hline $\operatorname{Hog} \ldots \ldots \ldots \ldots$ & \pm & & $\cdots$ & \pm & . & - & - & 0 & - & + & + & - & + \\
\hline Horse. . . . . . . . & \pm & + & + & + & $\ldots$ & + & \pm & + & 0 & - & 一 & 一 & 一 \\
\hline Goose. . . . . . . . & + & .. & .. & . & . & 一 & - & - & - & 0 & - & - & - \\
\hline Duck.... . & + & .. & . & $\because$ & . & - & - & - & - & - & 0 & - & 一 \\
\hline Pigeon..... & + & . & . & + & . & \pm & - & - & 一 & 一 & - & 0 & - \\
\hline Hen.......... & + & . & .. & . & . & + & - & - & 一 & - & - & 一 & 0 \\
\hline
\end{tabular}

$+=$ Well-marked hemolysis.

$(+)=$ Questionable or feeble hemolysis.

$\pm=$ Doubtful.

$-=$ No hemolysis.

Kolmer and Casselman have titrated the natural hemolysins for the corpuscles of various vertebrates in a large number of human serums. Most interest centers about the occurrence of natural antisheep hemolysin, because of the wide-spread use of an antisheep hemolytic system in complement-fixation reactions, as, for example, the Wassermann syphilis reaction. In over 80 per cent. of human serums there is present sufficient natural amboceptor for sheep's cells to give well-marked or complete hemolysis. Although this factor must be considered in using an antisheep hemolytic system in complement-fixation reactions, yet with a proper understanding of principles and the employment of a satisfactory technic the danger of error is reduced to a minimum. In the following table the percentages of serums showing $100,75,25$, and 0 per

${ }^{1}$ Sachs, H.: Handbuch der pathogenen Mikroörganismen, Kolle and Wassermann, 2. Auflage, 2, p. 799. 
cent. hemolysis, with the maximum dose of serum ( 0.2 c.c.) used in the method of titration, are shown:

TABLE 8.-SUMMARY OF NATURAL HEMOLYSINS IN HUMAN SERUM

\begin{tabular}{|c|c|c|c|c|c|}
\hline BLOOD & $\begin{array}{l}\text { NUMBER OF } \\
\text { SERUMS } \\
\text { TESTED }\end{array}$ & $\begin{array}{c}\text { PER CENT. } \\
\text { SHOWING } \\
100 \text { PER CENT. } \\
\text { HEMOLYSIS }\end{array}$ & $\begin{array}{c}\text { PER CENT. } \\
\text { SHOWING } \\
75 \text { PER CENT. } \\
\text { HEMOLYSIS }\end{array}$ & $\begin{array}{c}\text { PER CENT. } \\
\text { SHOWING } \\
25 \text { PER CENT. } \\
\text { HEMOLYSIS }\end{array}$ & $\begin{array}{l}\text { Per Cent. } \\
\text { Showing No } \\
\text { Hemolysis }\end{array}$ \\
\hline $\begin{array}{l}\text { Sheep....... } \\
\text { Dog ....... } \\
\text { Ox........ } \\
\text { Goat....... } \\
\text { Hog ....... } \\
\text { Rat....... } \\
\text { Chicken.... } \\
\text { Horse...... } \\
\text { Rabbit..... } \\
\text { Guinea-pig . }\end{array}$ & $\begin{array}{r}125 \\
25 \\
85 \\
25 \\
40 \\
25 \\
25 \\
25 \\
25 \\
50\end{array}$ & $\begin{array}{r}64 \\
16 \\
6 \\
0 \\
0 \\
0 \\
0 \\
0 \\
0 \\
0\end{array}$ & $\begin{array}{r}20 \\
36 \\
20 \\
8 \\
1 \\
0 \\
0 \\
0 \\
0 \\
0\end{array}$ & $\begin{array}{r}9 \\
30 \\
24 \\
16 \\
3 \\
12 \\
8 \\
4 \\
4 \\
2\end{array}$ & $\begin{array}{l}7.5 \\
18 \\
50 \\
76 \\
96 \\
88 \\
92 \\
96 \\
96 \\
98\end{array}$ \\
\hline
\end{tabular}

Kolmer and Williams ${ }^{1}$ have likewise studied the hemolysins found in normal rabbit serum, as preliminary to some work concerning the site of formation of immune hemolysins. The results obtained with the maximum dose of serum ( 0.2 c.c.) are given in the following table:

TABLE 9.-SUMMARY OF NATURAL HEMOLYSINS IN NORMAL RABBIT SERUM

\begin{tabular}{|c|c|c|c|c|c|}
\hline BLOOD & $\begin{array}{l}\text { NUMBER oF } \\
\text { SERUMS } \\
\text { TESTED }\end{array}$ & $\begin{array}{c}\text { PER CENT. } \\
\text { SHOWING } \\
\text { 100 PER CENT. } \\
\text { HEMOLYSIS }\end{array}$ & $\begin{array}{c}\text { PER CENT. } \\
\text { SHOWING } \\
75 \text { PER CENT. } \\
\text { HEMOLYSIS }\end{array}$ & $\begin{array}{c}\text { PER CENT. } \\
\text { SHOWING } \\
\text { 25 PER CENT. } \\
\text { HEMOLYSIS }\end{array}$ & $\begin{array}{l}\text { Per Cent. } \\
\text { Showing No } \\
\text { HeMolysis }\end{array}$ \\
\hline $\begin{array}{l}\text { Goat....... } \\
\text { Sheep...... } \\
\text { Dog....... } \\
\text { Human ... } \\
\text { Hog ...... } \\
\text { Ox....... } \\
\text { Chicken ... } \\
\text { Guinea-pig } \\
\text { Rat, white. }\end{array}$ & $\begin{array}{l}25 \\
50 \\
25 \\
50 \\
25 \\
25 \\
25 \\
25 \\
25\end{array}$ & $\begin{array}{r}4 \\
18 \\
32 \\
0 \\
0 \\
0 \\
0 \\
0 \\
0\end{array}$ & $\begin{array}{r}52 \\
56 \\
40 \\
4 \\
4 \\
4 \\
4 \\
0 \\
0\end{array}$ & $\begin{array}{r}88 \\
76 \\
72 \\
20 \\
20 \\
20 \\
8 \\
4 \\
0\end{array}$ & $\begin{array}{r}12 \\
24 \\
28 \\
80 \\
80 \\
80 \\
92 \\
96 \\
100\end{array}$ \\
\hline
\end{tabular}

\section{ISOHEMOLYSINS}

In 1892 Maragliano $^{2}$ directed attention to the fact that the bloodserum of patients afflicted with various diseases exerted a hemolytic influence on the blood-corpuscles of healthy persons. Later Ascoli ${ }^{3}$

1 Jour. Infect. Dis., 1913, xiii, No. 1, 96.

2 Deutsch. med. Wochenschr., 1892, xviii, 411.

${ }^{3}$ Münch. med. Wochenschr., 1901, xlviii, 1239. 
found the serums of cancer, pneumonia, and Addison's disease to be actively hemolytic for normal corpuscles: Weil, Crile, Blumgarten and Whittemore, and others have found a large proportion of the serums of lower animals and man suffering with malignant tumors to possess hemolytic activity for normal erythrocytes, and that a reaction based upon this property may have diagnostic value. While isohemolysins are to be especially found in the serums of cancerous persons, and the corpuscles of tuberculous persons are hypersensitive and readily hemolyzed, reactions based upon these observations are not specific, although they may have some diagnostic value in relation to other symptoms. It is well to remember this property of cancer serum, especially in making blood transfusions.

Production of Immune Hemolysins.-Hemolysins are readily produced by injecting suitable animals with several doses of red bloodcorpuscles. For this purpose rabbits are commonly employed. Some hemolysins are more readily produced than others; for example, antisheep hemolysin is easily prepared, whereas it is far more difficult to secure a potent antihuman hemolysin. The various methods employed in preparing hemolytic serums have been described in the chapter on Active Immunization of Animals. Antisheep hemolysin for conducting the Wassermann reaction is readily prepared by giving a rabbit three or four intravenous injections of 5 c.c. of a 10 per cent. suspension of washed sheep's cells in sterile normal salt solution at intervals of three days. The blood-cells should always be washed three or four times with an excess of salt solution to remove all traces of serum, in order that precipitins may not be produced and anaphylactic shock of the inoculated animal avoided.

The portion of the erythrocyte that is responsible for the production of hemolysins is a moot question. Bordet and von Dungern maintain that the stroma is the exciting agent; Nolf and others believe that the stromata produce hemagglutinins, and that the hemoglobin is chiefly concerned in the production of the hemolysin.

General Properties of Hemolysins.-Hemolysins are highly resistant antibodies, and are easily preserved. Sterile immune serum may be inactivated by heating in a water-bath for half an hour at $56^{\circ} \mathrm{C}$., and may be preserved for many months if small amounts are placed in ampules and kept in a cold place. If an equal quantity of neutral glycerin is added to the clear inactivated serum it will aid greatly in its preservation. The amboceptors resist drying to a well-marked degree, and filterpaper saturated with the immune serum and dried, after the method of 
Noguchi, preserves the hemolytic activity in a remarkable degree. Heating an immune serum at $56^{\circ} \mathrm{C}$., for half an hour, as in the process of inactivating complement, does not materially injure the hemolytic activity of a potent serum. A temperature of $70^{\circ} \mathrm{C}$. or above may cause deterioration and finally destroy the amboceptors.

As was previously mentioned, hemolytic amboceptors possess a great affinity for the receptors of their homologous corpuscles, and will readily unite with them at a low temperature. At incubator temperature the union is quite rapid, so that corpuscles may be "sensitized" within half an hour.

Source of Hemolysins.-As has been stated elsewhere, Metchnikoff regards the leukocytes as the source of fixateur or amboceptor formation. Bulloch found that the amount of hemolytic amboceptor in a serum runs parallel with the number of mononuclear leukocytes, and he regards this as an indication of the activity of the lymphoid tissue in general, which he considers as the main source of amboceptor formation. While it is probable that endothelial cells and mononuclear leukocytes are especially concerned in the process, our own investigations in this field would indicate that the process is more general, being participated in by cells of other tissues which possess suitable combining affinities for the alien corpuscles.

Antihemolysins.-A further step in the study of hemolysins, but one more of theoretic than of practical interest, was the discovery of antihemolysins. By injecting guinea-pigs with normal rabbit serum containing amboceptors for ox blood, Bordet secured a serum that inhibited the action of anti-ox immune serum. Ehrlich and Sacks, by injecting a goat with normal rabbit serum, likewise secured a serum that acted as an anti-amboceptor against immune hemolytic amboceptors for ox blood. Ehrlich argued that the anti-amboceptor acted against the complementophile group of the amboceptor, which prevented union with a complement from taking place. This view was advanced in support of his theory concerning the two-armed character of the amboceptor and that an anti-amboceptor may be produced against either the cytophile or the complementophile group or both. In these particular serums, however, the investigators may have been working with an anticomplement instead of an anti-amboceptor.

A specific hemolysin-one, for example, specific for dog blood, derived by treating a rabbit with dog cells - is highly toxic for dogs, being capable of producing hemolysis in vitro and a clinical condition known as hemolytic jaundice. It is possible, however, gradually to immunize 
a dog against this amboceptor for his own cells by starting with very small doses and gradually increasing these until it is found that the animal tolerates amounts that would be fatal to non-immunized animals. If a portion of this serum is now added to the specific hemolytic serum, it will be found that the power of the latter is inhibited. Although this action may likewise be due to anticomplement, it is probable that an anti-amboceptor against the cytophile group of the amboceptor is also formed, which prevents the amboceptor from uniting with the red bloodcells, although conclusive experimental evidence of this has not been adduced.

\section{PRACTICAL APPLICATIONS}

There is probably no other group of antibodies that possesses greater diagnostic value than do the hemolysins, a fact that was demonstrated in the practical application of the Bordet-Gengou phenomenon of complement fixation in the diagnosis of syphilis and other infections. By adding sensitized corpuscles-i.e., corpuscles with their homologous amboceptors - to a fluid, the presence or absence of complement may be determined. If complement is present, hemolysis will occur and be complete or partial, depending upon the amount of complement available; if hemolysis does not occur, it may be concluded that free complement is absent. This is the basis of the complement-fixation diagnosis of syphilis, gonorrhea, glanders, differentiation of proteins, etc. When a proper amount of complement is added to a mixture of antigen and its immune serum (containing amboceptors), it is bound to these amboceptors, so that when corpuscles and hemolytic amboceptor are subsequently added, hemolysis does not occur, since there is no available complement, it having been "fixed" by the first amboceptors. If, however, amboceptors for the antigen in the first instance are not present, as where a normal serum is used, the complement remains free and acts with the hemolytic amboceptor to produce hemolysis of the test corpuscles. In this manner the hemolysins and their corresponding corpuscles are employed as indicators or tests for the presence of free complement, so that if an antigen is known, the antibody may be determined; or vice versâ, by using a known antibody, the antigen may be determined, the criterion in each instance being whether complement is or is not bound or "fixed," a fact that is determined by the subsequent addition of a hemolysin and its homologous corpuscles.

The practical applications and technic of these reactions are given in subsequent chapters. 
The hemolysins have no therapeutic application or value. They have their chief value in diagnostic reactions and in the study of cytolytic phenomena in general.

Quantitative Relationship between Hemolytic Amboceptor and Complement.-From a practical as well as a theoretic standpoint an important property of amboceptor and of complement is the quantitative relationship that each bears to the other. This is especially important in hemolytic reactions, where an excess of either may compensate for a decrease of the other and yield fallacious results. If a certain amount of guinea-pig's complement is necessary to lyse 1 c.c. of a 2.5 per cent. suspension of sheep's cells, along with hemolytic amboceptor, then double this amount of complement will be required to lyse 2 c.c. of the same blood, and so on. If a constant quantity of corpuscles and hemolysin are added to a series of test-tubes, and increasing amounts of complement after incubating the mixtures for an hour the smallest amount of complement that produces complete hemolysis is called a unit, and in this manner the strength or activity of a serum complement is measured or titrated. In a similar manner the hemolytic activity of a serum or its measure of hemolysin may be determined by placing in a series of tubes, as previously directed, a definite and equal amount of corpuscle suspension, and to each tube is then added an amount, also definite and equal, of a normal serum as complement, which is known to be incapable of causing hemolysis. There are next added decreasing and graduated amounts of the immune serum whose native complement has been destroyed by inactivation. After incubating the mixtures for an hour, the smallest amount of inactivated immune serum that will just produce complete hemolysis is known as the amboceptor unit of the serum. In other words, there are three substances concerned in serum hemolysis: the amboceptor, the corpuscles, and the complement. By taking two of these as constants, e. g., the corpuscles and the complement, the unit of amboceptor may be determined; or by taking the corpuscles and amboceptor as constants the unit of complement may be determined. Since the corpuscles and amboceptor are most stable, these may be used as constants, and the unit of complement determined under these conditions as preliminary to complement-fixation reactions.

It is important to bear in mind, in this connection, that the titer of an immune hemolytic serum will vary with the complement used. For example, an antisheep amboceptor is much more active when guinea-pig serum is used as complement than it is when tested with the same quantity of rabbit serum as complement. 
After determining the unit of amboceptor or complement-that is, after adjusting the hemolytic system to exact proportions - the results that follow the varying quantities of complement and amboceptor require the most careful consideration. Less than one unit of amboceptor with one unit of complement cannot yield complete hemolysis; likewise if with one unit of amboceptor less than a unit of complement is combined hemolysis is incomplete; with one unit of amboceptor and one unit of complement and a double dose of corpuscles hemolysis will also be incomplete. With less than a unit of complement and an excess of amboceptor, however, hemolysis may be complete. The complement may be reduced to so small an amount that hemolysis is incomplete no matter how much amboceptor is used, but the important fact to be borne in mind is that a slight decrease in complement may be compensated for by the presence of many units of amboceptor, so that complete hemolysis results and a false reaction is secured. The converse of this is true to a less marked extent-i.e., an excess of complement may compensate for a decrease in amboceptor, but is less capable of doing so.

These facts are of the utmost importance in making hemolytic experiments, as in complement-fixation reactions, where the entire test depends upon demonstrating whether or not a portion or the whole of the complement used has been fixed. Unless, in a series of hemolytic reactions, the amount of amboceptor employed is the same throughout, the amount of complement acting in these cannot be determined by comparing the degree of hemolysis. This is true especially in cases where a small amount of complement is fixed, as in the Wassermann reaction, with a serum containing a small amount of syphilitic antibody, when the presence of an excess of hemolytic amboceptor may give complete hemolysis and overshadow the fact that a small amount of complement has been actually fixed by syphilitic antibody and antigen.

Method of Titration of Immune Hemolysin.-Various methods have been employed by different workers in this field, but all are based upon the same principles as have been here outlined.

A small amount of immune serum is inactivated by heating in a waterbath at $56^{\circ} \mathrm{C}$. for half an hour. In testing the serum of a rabbit during the process of immunization 2 or 3 c.c. of blood are easily secured from the ear, and the serum is separated. After it has been inactivated, the serum is diluted to $1: 100$ ( 1 c.c. of serum to 99 c.c. of salt solution, or 0.1 c.c. serum +9.9 c.c. of salt solution).

Fresh guinea-pig serum is secured for complement by bleeding a 
healthy pig under ether anesthesia into a Petri dish or centrifuge tube. This serum is diluted $1: 20$, making a 5 per cent. solution, by adding 1 c.c. of serum to 19 c.c. of salt solution. Each cubic centimeter of this dilution contains 0.05 c.c. of undiluted serum, which experience has shown is a satisfactory amount to use.

The corpuscle suspension is then prepared. The blood used depends upon the kind of amboceptor that is to be titrated. With sheep and ox blood, a 2.5 per cent. suspension of washed corpuscles may be employed. With antihuman amboceptor, the corpuscles are usually used in 1 per cent. suspension. (See Noguchi Modification of Wassermann Reaction.) After the corpuscles have been washed three times, 1 c.c. is placed in 39 c.c. of salt solution, or sufficient salt solution is added to 2.5 c.c. of the corpuscles to make the total volume equal 100 c.c.

To a series of six sterile test-tubes increasing doses of the diluted immune serum are now added, together with 1 c.c. of complement dilution, 1 c.c. of corpuscles suspension, and sufficient normal salt solution to make the total volume in each tube about 3 or 4 c.c. The following table shows the method of preliminary titration of a hemolytic serum:

TABLE 10.-PRELIMINARY TITRATION OF A HEMOLYSIN

\begin{tabular}{|c|c|c|c|c|c|}
\hline TUBE & $\begin{array}{c}\text { AMOUNT OF INACTIVATED } \\
\text { IMMUNE SERUM IN C.c. } \\
(1: 100)\end{array}$ & $\begin{array}{l}\text { DOSE OF } \\
\text { COMPLE- } \\
\text { MENT, C. } \\
\text { c. }(1: 20)\end{array}$ & $\mid \begin{array}{c}\text { DOSE OF } \\
\text { CORPUS- } \\
\text { CLES, C.C. } \\
\text { (2.5 PER } \\
\text { CENT.) }\end{array}$ & $\begin{array}{l}\text { Normal } \\
\text { SALT } \\
\text { SolUtion }\end{array}$ & $\begin{array}{l}\text { RESULT OF HEMOLYSIS } \\
\text { AFTER ONE HOUR IN THE } \\
\text { INCUBATOR }\left(37^{\circ} \mathrm{C} \text {.) }\right.\end{array}$ \\
\hline $\begin{array}{l}1 \ldots \\
2 \ldots \\
3 \ldots \\
4 \ldots \\
5 \ldots \\
6 \ldots\end{array}$ & $\begin{array}{l}0.1 \text { (0.001 c.c. undiluted) } \\
0.2(0.002 \text { c.c. } \text { undiluted) } \\
0.4 \text { (0.004 c.c. undiluted) } \\
0.6(0.006 \text { c.c. } \text { undiluted }) \\
0.8 \text { (0.008 c.c. undiluted) } \\
1.0(0.01 \text { c.c. } \text { undiluted })\end{array}$ & $\begin{array}{l}1 \\
1 \\
1 \\
1 \\
1\end{array}$ & $\begin{array}{l}1 \\
1 \\
1 \\
1 \\
1\end{array}$ & $\begin{array}{l}\text { q. } s .4 \text { c.c. } \\
\text { q. } s .4 \text { c.c. } \\
\text { q. } s .4 \text { c.c. } \\
\text { q. } s .4 \text { c.c. } \\
\text { q. } s .4 \text { c.c. } \\
\text { q. } s .4 \text { c.c. }\end{array}$ & $\begin{array}{l}\text { No hemolysis } \\
\text { Partial hemolysis } \\
\text { Complete hemolysis } \\
\text { Complete hemolysis } \\
\text { Complete hemolysis } \\
\text { Complete hemolysis }\end{array}$ \\
\hline
\end{tabular}

In this instance the unit of amboceptor is about $1: 500$, which is too low for a satisfactory antisheep serum. The rabbit should, therefore, receive another dose or two of corpuscles, and the serum be titrated again in from four to seven days after the last injection has been given. In this titration it will be well to use a higher dilution of the inactivated immune serum, as $1: 1000$. This may be prepared by adding 1 c.c. of a dilution of $1: 100$ with 9 c.c. of normal salt solution and mixing well. The titration is then proceeded with as follows: 



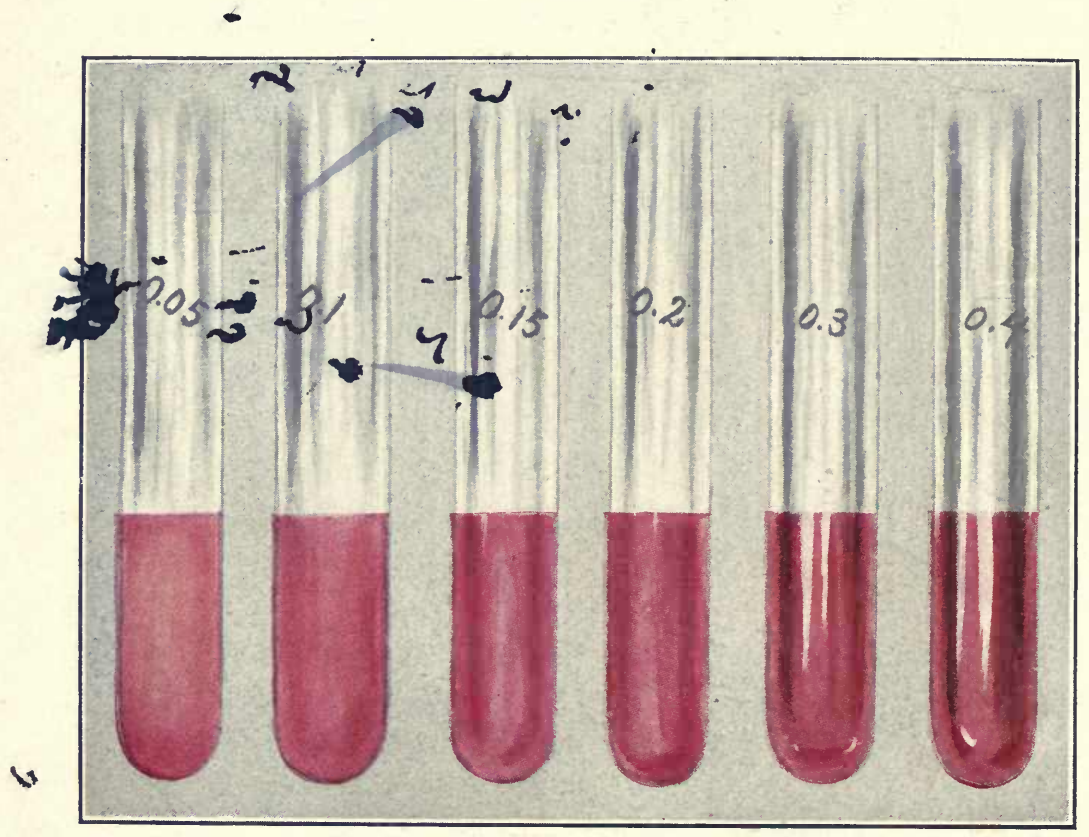

Fig. 103.-Titration of Hemolytic Amboceptor. 
TABLE 11.-PRELIMINARY TITRATION OF HEMOLYSIN

\begin{tabular}{|c|c|c|c|c|c|}
\hline TUBE & $\begin{array}{c}\text { AMOUNT OF INACTIVATEd } \\
\text { IMMUNE SERUM IN C.C. } \\
(1: 1000)\end{array}$ & $\begin{array}{c}\text { Dose } \\
\text { OF } \\
\text { CoM- } \\
\text { PLE- } \\
\text { MENT } \\
\text { IN C.c. } \\
(1: 20)\end{array}$ & $\begin{array}{c}\text { Dose } \\
\text { OF } \\
\text { Cor- } \\
\text { PUS- } \\
\text { CLES, } \\
\text { C.C. } \\
\text { (2.5 } \\
\text { PER } \\
\text { CENT.) }\end{array}$ & $\begin{array}{c}\text { NoRMaL } \\
\text { SALT } \\
\text { SoLUTION }\end{array}$ & $\begin{array}{l}\text { RESULT OF HEMOLYSIS } \\
\text { AFTER ONE HOUR IN THE } \\
\text { INCUBATOR AT } 37^{\circ} \text { C. }\end{array}$ \\
\hline $\begin{array}{r}1 \\
2 \\
3 \\
4 \\
5 \\
5 \\
6 \\
7 \\
8 \\
8 \\
9 \\
9 \\
10\end{array}$ & 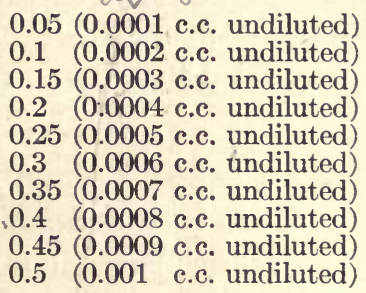 & $\begin{array}{l}1 \\
1 \\
1\end{array}$ & $\begin{array}{l}1 \\
1 \\
1\end{array}$ & $\begin{array}{l}\text { q. } s .4 \text { c.c. } \\
\text { q. } s .4 \text { c.c. } \\
\text { q. } s .4 \text { c.c. } \\
\text { q. } s .4 \text { c.c. } \\
\text { q. } s .4 \text { c.c. } \\
\text { q. } s .4 \text { c.c. } \\
\text { q. } s .4 \text { c.c. } \\
\text { q. } . \text { } 4 \text { c.c. } \\
\text { q. s. } 4 \text { c.c. } \\
\text { q. } s .4 \text { c.c. }\end{array}$ & $\begin{array}{l}\text { No hemolysis } \\
\text { No hemolysis } \\
\text { Beginning hemolysis } \\
\text { Partial hemolysis } \\
\text { Just complete hemolysis } \\
\text { Complete hemolysis } \\
\text { Complete hemolysis } \\
\text { Complete hemolysis } \\
\text { Complete hemolysis } \\
\text { Complete hemolysis }\end{array}$ \\
\hline
\end{tabular}

The following controls should be set up at the same time:

1. 1 c.c. of corpuscles in 1 c.c. of amboceptor dilution. This tube should show no hemolysis, as the serum has been inactivated and is too highly diluted for complement activity, even though native complement were present.

2. 1 c.c. of corpuscles in 1 c.c. of complement dilution. This tube may show a trace of hemolysis, due to the presence of a small amount of natural amboceptor for the corpuscles used. As a general rule, guinea-pig serum is free from natural antisheep amboceptor, or the amount is so small under these conditions that it is not necessary to remove it.

3. 1 c.c. of corpuscles in 3 c.c. of salt solution. This tube should show no hemolysis, and serves to show that the diluent was isotonic.

In the foregoing titration it is found that 0.25 c.c. of $1: 1000$ dilution of amboceptor is the unit, or the titer is $1: 4000$. This method is less difficult, and probably more accurate, than preparing a series of dilutions of amboceptor as a $1: 100,1: 200,1: 500,1: 1000,1: 2000$, etc., using a cubic centimeter of each dilution. The method requires accurate pipets and careful work, but yields uniform and satisfactory results (Fig. 103).

The rabbit may now be bled under anesthesia. The serum is sep arated and inactivated and again titrated, as the final titration, for some unknown reason, is likely to be a little lower than in the primary tests.

When suitably preserved, a hemolytic serum will maintain its activity for long periods of time; it should always, however, be titrated before complement-fixation tests are undertaken. 
Methods for Removing Hemolysins from a Serum.-In general, these aim to remove the natural hemolysins, such as natural antisheep hemolysin, from human serums preliminary to making the Wassermann test, or from a guinea-pig serum that is to be used as complement.

The method of removal consists simply in adding corpuscles to the serum, and allowing sufficient time for the corresponding hemolytic amboceptor to become attached and then removing both by centrifuging the mixture. If the serum is fresh, it should be cooled to $0^{\circ}$ to $3^{\circ} \mathrm{C}$., in order to inhibit complement activity, which would hemolyze a portion of the corpuscles.

To remove natural antisheep hemolysin from a patient's serum place a measured quantity of cold serum in a centrifuge tube and add four volumes of a 2.5 per cent. suspension of sheep's cells. This will make a dilution of $1: 5$, so that 0.5 c.c. of the dilution is equivalent to 0.1 c.c. of undiluted serum. After placing the tube in ice-water for from fifteen to thirty minutes centrifuge thoroughly to remove the corpuscles. The process may be carried out after the serum has been inactivated, in which case it is not necessary to work with cold serum.

In removing 'a natural hemolytic amboceptor from a guinea-pig serum that is to be used as complement a measured amount of serum is first removed to a separate tube and thoroughly chilled in a glass of cracked ice. If a large amount of serum is to be used, for example, 5 c.c., it is well to place about 0.1 to 0.2 c.c. of pure undiluted corpuscles, after their last washing, in the bottom of a centrifuge tube. This quantity of corpuscles does not materially affect the dilution of the serum. If a smaller amount of serum is used, such as 1 c.c., it is well to add 8 c.c. of a 2.5 per cent. suspension of corpuscles, and after centrifuging the mixture the final dilution of $1: 20$ is secured by adding 10 c.c. of salt solution to the supernatant diluted serum.

Method of Determining Natural Hemolysins in Serum.-To ascertain whether or not a certain natural amboceptor is present in a serum it is merely necessary to inactivate the serum, and to a measured amount, for example, 0.2 c.c., add 1 c.c. of complement serum $(1: 20)$ that is known to be free of the particular amboceptor in question, and 1 c.c. of a 2.5 per cent. suspension of the corresponding corpuscles. Sufficient salt solution is added to bring the total volume to 3 or 4 c.c. The mixture is then incubated at $37^{\circ} \mathrm{C}$. for one or two hours, when the occurrence of hemolysis indicates the presence of the amboceptor for the corpuscles employed.

To determine the amount of natural amboceptor by titration dilute 
the serum with nine parts of normal salt solution, and to a series of testtubes add increasing amounts of 0.05 c.c., 0.1 c.c., 0.2 c.c., 0.4 c.c., 0.8 c.c., 1 c.c., and 2 c.c., corresponding respectively to $0.005,0.01,0.02$, $0.04,0.08,0.1$, and 0.2 c.c. of the undiluted serum. Add 1 c.c. of a 5 per cent. dilution of fresh amboceptor-free guinea-pig serum as complement, and 1 c.c. of a 2.5 per cent. suspension of the corpuscles; sufficient salt solution is added to make the total volume about 4 c.c. After shaking, the tubes are placed in the incubator at $37^{\circ} \mathrm{C}$. for two hours, removed, and the results read, or the tubes may be placed in a refrigerator overnight and the results read in the morning.

\section{SERUM DIAGNOSIS OF PAROXYSMAL HEMOGLOBINURIA}

A hemolytic substance may be demonstrated in the blood-serum of most cases of paroxysmal hemoglobinuria at certain periods. Although the exact nature of this substance is unknown, it has many of the properties of isohemolysins, being capable of sensitizing the red corpuscles of the patient or those of a normal person at a low temperature, hemolysis being effected in the presence of fresh serum, presumably with complement, and best at body temperature.

According to Cook, about 90 per cent. of hemoglobinurics show a positive Wassermann reaction. Landsteiner found that about 10 per cent. of paretics showed similar reactions, and other observers have reported finding isohemolysins in epileptics and idiots. Malaria and trypanosomiasis have also been regarded as causes of this condition, the most evidence, however, indicating that the etiology has a luetic origin. It is possible that the hemotoxin is similar to the hemolysin of cobra venom, being in the nature of an amboceptor complemented by the fatty acids or lecithin of red corpuscles (endocomplement) or by a serum complement.

First Method.-According to Ehrlich, a small tourniquet should be applied about the base of one of the patient's fingers, and this is then kept immersed in ice-cold water for half an hour. Blood from the finger thus constricted is then collected in a Wright capsule, and blood from a finger of the other hand is used as a control. Both are allowed to clot and are then centrifugalized. The serum from the finger held in iced water is tinged red from dissolved hemoglobin, whereas the control serum is not tinged or at least not tinged so deeply.

Second Method.-Donath and Landsteiner have applied Ehrlich's method in vitro. Their method consists of collecting blood in a small 
test-tube, cooling to $0^{\circ} \mathrm{C}$. for half an hour, heating subsequently to $37^{\circ} \mathrm{C}$. for three hours. The presence or absence of hemolysis is observed, and the results compared with those obtained from normal blood treated in the same manner and at the same time.

Third Method.-This technic is carried out in vitro in the following manner: Pipet 2 c.c. of the patient's blood in a small test-tube and separate the serum. At the same time place 1 c.c. of blood in a centrifuge tube containing 9 c.c. of a 1 per cent. solution of sodium citrate in normal salt solution. Wash the corpuscles twice and suspend the sediment in 10 c.c. of normal salt solution. Then, secure a cubic centimeter of a fresh serum from a normal person. Proceed to make the following mixtures:

Tube 1: 0.2 c.c. patient's serum +1 c.c. corpuscle suspension.

Tube $2: 0.1$ c.c. patient's serum +1 c.c. corpuscle suspension.

Tube 3: 0.2 c.c. normal serum +1 c.c. corpuscle suspension.

Tube 4: 0.1 c.c. normal serum +1 c.c. corpuscle suspension.

Tube 5: 1.0 c.c. corpuscle suspension.

Add sufficient normal salt solution to each tube to make the total volume measure 2 c.c. Shake gently, and place in the refrigerator at a low temperature (not higher than $4^{\circ} \mathrm{C}$.) for an hour. Shake each tube gently and place them in the incubator at $37^{\circ} \mathrm{C}$. for two hours. The tubes are then centrifuged and the presence or absence of hemolysis is noted. Usually the patient's serum shows hemolysis of greater or less degree.

Similar mixtures may be made with the patient's serum and the corpuscles of a normal person. The hemolytic substance is capable of lysing these to the same degree that it does the patient's own cells.

\section{Method of Determining tHe Resistance of Red Blood-Corpuscles. NON-SPECIFIC HEMOLYSIS}

Various substances have been employed to test the resistance of the red blood-corpuscles. Of these, the hypotonic solutions of sodium chlorid, of varying strength, have yielded results of clinical importance, especially in the study of paroxysmal hemoglobinuria, the primary anemias, etc.

The following technic, slightly modified after the methods used by Smith and Brown, Gay, Moss, Karsner, and Pearce, is a ready means for determining the resistance of human corpuscles to salt selutions of different tonicities. Chemically pure sodium chlorid is dried for two hours at $170^{\circ} \mathrm{C}$., and immediately weighed in amounts necessary to 
make 500 c.c. of salt solution, ranging from 0.1 to 0.6 per cent. in gradations of 0.02 per cent. This means the preparation of twenty-six different solutions, which should be preserved in proper-sized bottles fitted with tight rubber stoppers.

When the test is needed only occasionally, these solutions are readily prepared by filling a 50 c.c. buret, graduated in one-tenths, with distilled water, and another with a 1 per cent. solution of pure dried sodium chlorid. From these, the various solutions are readily prepared after the following manner:

10 c.c. of 0.6 per cent. sodium chlorid $=6$ c.c. of 1 per cent. salt solution +4 c.c. of distilled water.

10 c.c. of 0.58 per cent. sodium chlorid $=5.8$ c.c. of 1 per cent. salt solution +4.2 c.c. of distilled water.

10 c.c. of 0.56 per cent. sodium chlorid $=5.6$ c.c. of 1 per cent. salt solution +4.4 c.c. of distilled water.

10 c.c. of 0.54 per cent. sodium chlorid $=5.4$ c.c. of 1 per cent. salt solution +4.6 c.c. of distilled water.

10 c.c. of 0.52 per cent. sodium chlorid $=5.2$ c.c. of 1 per cent. salt solution +4.8 c.c. of distilled water.

10 c.c. of 0.5 per cent. sodium chlorid $=5$ c.c. of 1 per cent. salt solution +5 c.c. of distilled water.

Similar dilutions are made, until the final dilution is reached. In many instances it may not be necessary to use so large a number of dilutions, as from 0.5 to 0.2 per cent. may be sufficient range to indicate the tonicity.

Five cubic centimeters of blood are aspirated, under aseptic precautions, from an arm vein of the patient, and immediately placed in 25 c.c. of sterile 1 per cent. sodium citrate in 0.85 per cent. sodium chlorid to prevent coagulation. The flask or large centrifuge tube is well shaken, and the mixture is centrifuged at sufficient speed to throw down the corpuscles. The supernatant fluid is drawn off, and the corpuscles are washed once or twice more with sterile normal salt solution. After the last washing the supernatant fluid is removed, leaving the erythrocytes at the bottom of the tube. 
A series of small test-tubes (10 by $1 \mathrm{~cm}$.) are marked appropriately and placed in a rack. To each tube 3 c.c. of the various hypotonic salt solutions and 0.05 c.c. of the red blood-corpuscles (about 1 drop) are added. The salt solution and corpuscles in each tube are well mixed, and the whole series is placed in the refrigerator for from eighteen to twenty-four hours, after which the readings are made.

The tube of lowest dilution-even if it shows but a trace of hemolysis-represents the point of minimal resistance. The strength of salt solution in which all the corpuscles are hemolyzed represents the maximal resistance.

Normally, the minimal resistance is about 0.47 , and the maximal resistance about 0.3 (Morris). 


\section{CHAPTER XXI \\ VENOM HEMOLYSIS}

Nature of Venom Hemolysis. - In a previous chapter the statement was made that certain snake poisons, and especially cobra venom, are actively hemolytic. Flexner and Noguchi ${ }^{1}$ first demonstrated that the blood-corpuscles of certain species of animals undergo hemolysis when a suitable serum is present, and believed that the venom contained an amboceptor that was active with serum complement.

Shortly afterward Kyes ${ }^{2}$ discovered that venom may hemolyze the corpuscles of certain animals without the presence of serum, and believed that the complement-like activator was contained within the corpuscles, to which he accordingly applied the name endocomplement.

Later Kyes ${ }^{2}$ confirmed Calmette's observation that practically any serum, when heated to $65^{\circ} \mathrm{C}$. and higher, showed an increased activity in the process of venom hemolysis. Kyes and Sachs ${ }^{3}$ then concluded that endocomplement was not of the nature of a thermolabile complement, but was, rather, a combination of lecithin and the stromata of erythrocytes.

Kyes later succeeded in combining cobra venom and lecithin by shaking a watery solution of venom with a solution of lecithin in ether, forming cobra-lecithid, which was found to be actively hemolytic.

The erythrocytes of various animals differ in their susceptibility to venom hemolysis. For instance, those of the dog and guinea-pig are most susceptible to the process; those of the ox, goat and sheep are entirely refractory, whereas those of the horse, rabbit, rat, pig, and man occupy an intermediate position. Sacks suggested that the variation in hemolytic resistance of red blood-cells from these species of animals was dependent on the amount of lecithin contained in the cells. Kyes, on the other hand, believes that since all erythrocytes contain sufficient lecithin to activate cobra venom, the varying susceptibility depends rather on the availability of the intracellular lecithin for the reaction, $i$. e., whether the lecithin in the cell is available in a free state.

1 Jour. Exper. Med., 1902, vi, 277.

${ }^{2}$ Berl. klin. Wochenschr., 1902, xxxix, 886; ibid., 1903, xlii, 21; ibid., 1903; xlii, 956; Biochem. Zeitschr., 1907, iv, 109; Jour. Infect. Dis., 1910, vii, 181.

${ }^{3}$ Berl. klin. Wochenschr., 1903, xliii, 21, 57, 82. 
According to this theory, therefore, any factor that modifies the availability of the cell lecithin may modify the susceptibility of the cells for hemolysis with cobra venom.

Noguchi ${ }^{1}$ has questioned the correctness of this view. He holds that although lecithin exists in the stroma of all kinds of corpuscles, it is not present in a form available for venom activation, and that the degree of susceptibility to hemolysis depends chiefly upon the amount of ether-soluble activators present in the cells, as, for example, fatty acids, particularly oleinic acid, and their soluble soaps. In his opinion heating an inactive serum to $65^{\circ} \mathrm{C}$. and higher renders it active with venom, owing to the presence of a protein compound of lecithin.

A normal serum may, therefore, contain two activators, one being thermolabile and resembling complement (inactivated by calcium chlorid), and the other being thermostabile and a protein lecithin. By adding oleinic acid or its soluble soap to a non-activating serum the latter is rendered highly active so far as venom hemolysis is concerned. Hence while Kyes regards lecithin as the chief component of endocellular complement, Noguchi regards the fatty acids, neutral fats, and soluble soaps as the active agents.

Other observers consider the fatty acids and soap as indirect activating agents in venom hemolysis, in that they possess the power of modifying the cell and rendering the intracellular lecithin available for the formation of complete hemolysin.

On the other hand, in susceptible cells the union of cobra venom and lecithin occurs directly with the formation of the complete hemolysin, Kyes' cobra-lecithid, due to the splitting of the fatty acid radical from the lecithin. Ludecke, von Dungern, and Coca and Manwaring regard this product as a venom-free lecithin derivative, and not as a lecithin. They prefer to call the active principle "cobralecithinase," and the complete hemolysin" mono-fatty-acid-lecithin."

According to Kyes, the relative amounts of lecithin and venom amboceptor show quantitative relationship comparable to serum amboceptors and complements, namely, that, within certain limits, the larger the amount of venom, the smaller the amount of lecithin necessary to effect hemolysis; and, conversely, the larger the amount of lecithin, the smaller the amount of venom required.

Further reference to the intimate relationship that exists between lipoids and complements and hemolysis is also made in the discussion on the nature of complements, on p. 329. 


\section{VENOM HEMOLYSIS IN SYPHILIS}

The first application of venom hemolysis was made by Weil, ${ }^{1}$ who found, in testing the hemolytic powers of cobra venom with cells derived from persons suffering from different diseases, that the red cells of syphilitic individuals offered a characteristic resistance. Various explanations have been offered for this phenomenon:

1. It was argued that the quantity of red-cell lecithin is actually diminished in syphilis after the primary stage because pallidum toxin attacks the same lipoidal substances of tissue cells as does cobra venom, in this way accounting for the diminished amount of lecithin that can be extracted in syphilis, as compared with that obtained from normal tissues. Accordingly, the increase in resistance is only apparent, and is due rather to the fact that there is insufficient endocomplement for the venom amboceptor.

2. Another explanation offered was that the increased resistance of the red cells of syphilitic persons to venom hemolysis is due to the fact that pallidum toxin attacks endocomplement, and that the cells become specifically immunized to this deleterious influence in much the same way that repeated injections of such a hemolytic agent as saponin leads in rabbits to the production of red cells, which show a marked resistance to saponin hemolysis but not to any other hemolytic agent.

3. Pallidum toxin was believed to so affect the lecithin content of red cells as to render a smaller quantity of it available in a free state for union with the venom amboceptor to form the hemolysin.

4. Another theory advanced was that pallidum toxin effects a dissociation of red cells between lecithin and cholesterin, the latter substance causing inhibition of hemolysis.

Whatever may be the true explanation, the fact has been quite well attested that the red cells of a large percentage of persons in the tertiary stage of syphilis exhibit a characteristic increased resistance to venom hemolysis, and while the cobra hemolysis test in this disease is of secondary importance to the Wassermann reaction as a diagnostic procedure, yet it represents one of the most interesting of biologic phenomena, and may possibly be employed in other clinical methods.

\section{Technic of the Cobra Venom Tests}

Preparation of Venom Solution.-A $1: 1000$ stock solution of dried cobra venom is prepared by accurately weighing out 0.01 gram of dried

${ }^{1}$ Proc. Soc. Exper. Biol. and Med., 1909, vi, 49; ibid., 1909, vii, 2; Jour. Infect. Diseases, 1909, vi, 688. 
pulverized venom and dissolving this in 10 c.c. of normal saline solution $(1: 1000)$. This stock dilution is best preserved in amounts of 1 c.c. in sealed ampules, kept in the frozen state in the ice chest in a wide-mouthed well-stoppered vacuum bottle containing salt and ice (Schwartz).

Each cubic centimeter is sufficient for making three tests, so that the 10 ampules will be enough for 30 tests, or 1 gram of venom for 3000 reactions. Or the dried venom may be weighed out in amounts of 0.0005 gram in test-tubes, and diluted, just before being used, with 1.c.c. of normal salt solution $(1: 2000)$. Immediately before the tests are conducted subdilutions are prepared of the stock dilution $(1: 1000)$, using separate pipets for each, as follows:

Solution A: $1: 10,000=1$ c.c. stock solution +9 c.c. normal saline solution.

Solution B: $1: 15,000=2$ c.c. solution $A+1$ c.c. normal saline solution.

Solution C: $1: 20,000=1$ c.c. solution A +1 c.c. normal saline solution.

Solution D: $1: 30,000=1$ c.c. solution $B+1$ c.c. normal saline solution.

Solution E: $1: 40,000=1$ c.c. solution C +1 c.c. normal saline solution.

These amounts are sufficient for making three tests; if more tests are to be made, larger amounts of the various dilutions will keep fairly well in a good refrigerator for several days, but it is always well to plan the work so that the exact amount will be prepared and no waste occur.

Each lot of stock solution should be tested occasionally with the cells of known normal and positive persons, to make certain that the venom is active in these dilutions. These titrations are conducted in the same manner as the test.

Preparation of Blood-cells.-With a sterile syringe blood is drawn from a vein at the elbow and 2 c.c. placed in an accurately graduated centrifuge tube containing 5 c.c. of a 2 per cent. solution of sodium citrate in normal saline solution. The suspension should be shaken gently to insure mixing and the prevention of coagulation, but defibrination by means of whipping should never be practised. The cells may be prepared at once or placed in the ice-chest overnight. Sufficient normal saline solution is added to bring the total volume to 15 c.c. Mix gently and centrifuge at low speed until the supernatant fluid is clear. Draw off the fluid, add more normal saline solution, mix up the cells, and centrifuge again until clear. Repeat this process once more so that all traces 



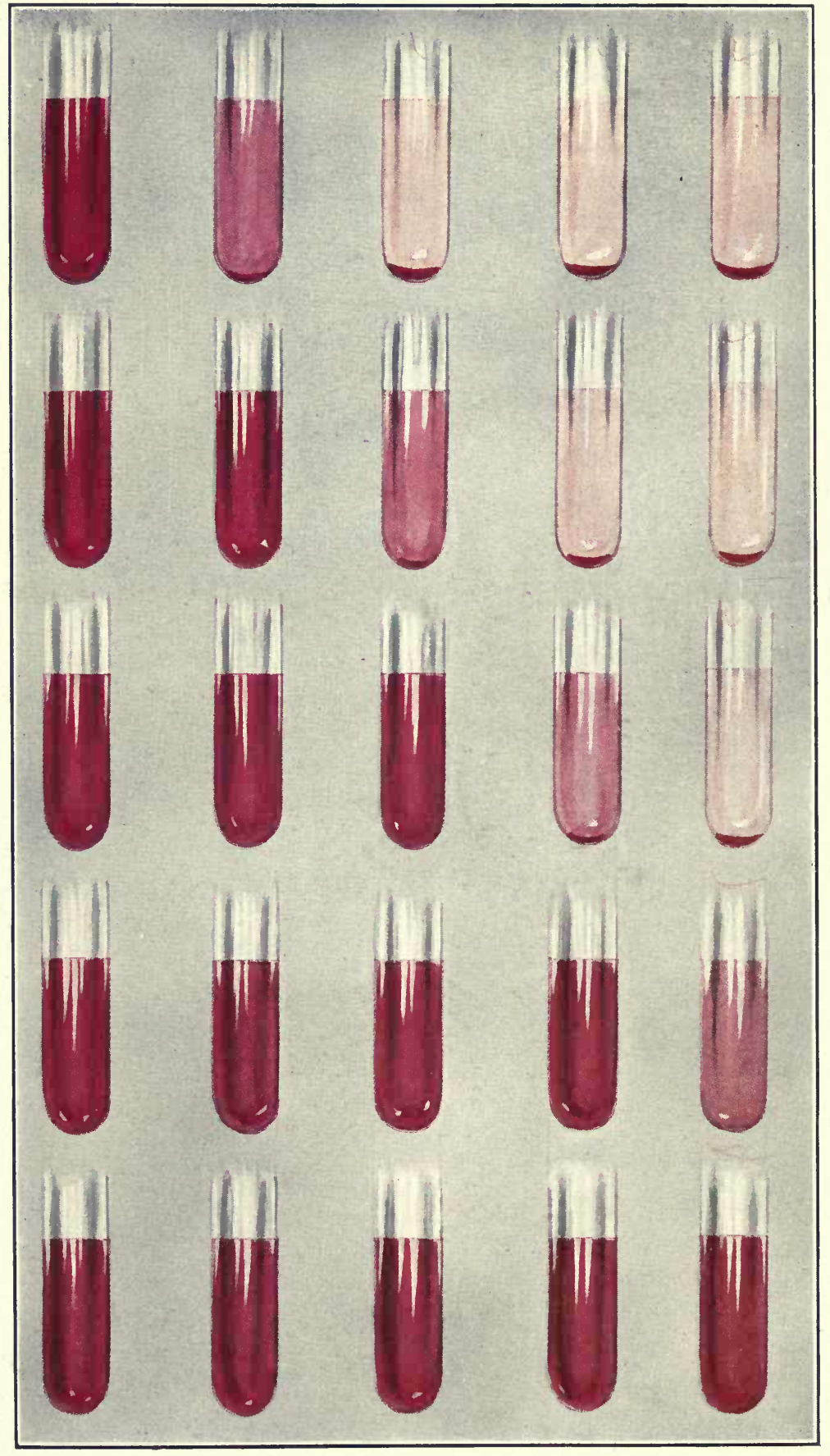

Fig. 104.-Venom Hemolysis. 
of serum will be removed. After completing the last centrifugalization, which should be thorough (ten minutes), the cells are diluted with 25 volumes of normal saline solution, which makes a 4 per cent. suspension-for instance, 0.8 c.c. of corpuscles would require 20 c.c. of diluent.

Just what influence sodium citrate has upon the process is not known, but it is certain that satisfactory results are seldom obtained with blood defibrinated by whipping with rods of glass beads. Similarly, if centrifuged too rapidly, cells are broken up or rendered more susceptible to the venom ambocepter.

The Test.-Into a series of five test-tubes ( 4 by $1 / 2$ inches) place 1 c.c. of each dilution of venom, and label each tube correctly; add 1 c.c. of the 4 per cent. suspension of cells to each tube, shake gently, and incubate for one hour at $37^{\circ} \mathrm{C}$. Except in cases where the venom dilutions are being frequently used and are known to be reliable, controls should be included. I usually add a normal control of cells from a healthy person with the $1: 30,000$ or $1: 40,000$ dilution and expect complete hemolysis to occur. A preliminary reading is made at the end of an hour; the tubes are shaken gently and placed in the refrigerator overnight; the final reading is made next morning, and should tally quite closely with the preliminary reading.

Reading the Results. - Unless the cells are derived from a very strongly reacting case of syphilis, the 1:10,000 dilution will be hemolyzed. The normal control tube should show complete hemolysis. If the $1: 10,000$ tube is not hemolyzed and some of the higher dilutions show hemolysis, an error in technic has occurred, and the test should be repeated with fresh dilutions.

The reactions are read and recorded as follows (Fig. 104):
A B
$\mathrm{C}$
D
E
H. M. H. - -
- $=$ strongly positive.
H. H.
M. H. -
- $\quad$ moderately positive
H. H.
H.
S. H.
- = weakly positive.
H. H.
H.
H.
M. H. = negative.
H. H.
H.
$\mathrm{H}$.
H. = certainly negative.

$\mathrm{H} .=$ complete hemolysis; M. H. = marked hemolysis; S. H.=slight hemolysis; the dash $(-)=$ no hemolysis.

If complete hemolysis has occurred in all tubes after an hour's incubation, the cells are regarded as being hypersensitive to cobra venom. This occurs as a rule in primary syphilis and in active tuberculosis. 


\section{Practical Value of the Venom Test in Syphilis}

1. While the test is much simpler than the Wassermann reaction and there is less possibility for errors in technic to creep in, it possesses but two other advantages, namely: (1) It may react positively in latent or tertiary syphilis when the Wassermann reaction may be negative, and (2) it may react positively in treated syphilitic cases when the Wassermann reaction is negative, and thus point to a continuation of treatment. Corson-White and Ludlum ${ }^{1}$ found 94 per cent., Schwartz ${ }^{2} 69.3$ per cent., and Stone and Schottstaedt ${ }^{3} 90.9$ per cent. of positive reactions in the active stages of syphilis.

2. The test is positive in but about 20 per cent. of cases of tabes dorsalis and general paralysis (White and Ludlum), a finding obviously inferior to the Wassermann reaction.

3. During primary syphilis the cells are hypersensitive and positive reactions are but occasionally obtained.

4. Positive reactions may occur in cancer, but otherwise the test is quite specific, and may, in selected cases, prove a valuable adjunct to the Wassermann reaction. However, with a more improved technic in performing the Wassermann reaction, and especially if antigens reenforced with cholesterin are used, the venom test is inferior to the Wassermann. In cases where syphilis or tuberculosis of the lungs is to be differentiated, a negative venom test would indicate tuberculosis, as in this disease the cells are hypersensitive.

\section{THE PSYCHO-REACTION OF MUCH}

Normal serum, when added to a lytic dose of cobra venom and human red blood-cells, will not interfere with hemolysis. According to Much and Holzman, ${ }^{4}$ however, if the serum obtained from a patient suffering from depressive mania or dementia præcox is added to the mixture of venom and human red blood-cells, the expected hemolysis does not take place.

Technic.-A 1:5000 dilution of cobra venom is prepared by diluting 1 c.c. of the stock dilution (p. 385) with 4 c.c. of normal saline solution. Enough of the patient's blood is collected from a vein at the elbow to yield at least 1.5 c.c. of serum; heat the serum to $55^{\circ} \mathrm{C}$. for an hour. Prepare a 5 per cent. suspension of washed human blood-cells. An effort

1 Jour. Nervous and Mental Dis̀eases, 1910, xxxvii, 721.

${ }^{2}$ New York Medical Journal, 1912, xcv, 23.

${ }^{3}$ Archiv. of Int. Med., 1912, x, 8. $\quad{ }^{4}$ Münch. med. Wochenschr., 1909, 20. 
should be made, if possible, to use as a control the cells of a person which are known to be readily hemolyzed by 1 c.c. of the $1: 5000$ dilution of venom in half an hour.

Into a series of three small test-tubes place 0.4 c.c. of the patient's serum and decreasing amounts of venom $-1,0.8$, and 0.5 c.c. respectively. Next add to each tube 1 c.c. of the blood-corpuscle suspension. The total volume in each tube is brought up to 2.5 c.c. by the addition of normal saline solution.

A similar series of tubes should be set up as controls, the patient's serum being omitted. The following table shows the arrangement:

Tube 1: 0.4 c.c. serum +1 c.c. venom +1 c.c. blood.

Tube $2: 0.4$ c.c. serum +0.8 c.c. venom +1 c.c. blood.

Tube 3: 0.4 c.c. serum +0.5 c.c. venom +1 c.c. blood.

Tube 4: 1 c.c. venom +1 c.c. blood.

Tube 5: 0.8 c.c. venom +1 c.c. blood.

Tube $6: 0.5$ c.c. venom +1 c.c. blood.

Tube 7:0 1 c.c. blood.

The tubes are shaken gently and incubated for an hour at $37^{\circ} \mathrm{C}$., when a preliminary reading is made. If the control tubes 4,5 and 6 are hemolyzed, a positive reaction would be indicated by the inhibition of hemolysis in the first three tubes, 1,2 and 3. Control tube 4 at least should be completely hemolyzed at the end of half an hour, and in a positive reaction tube 1 , containing the same amount of venom with the patient's serum, will show inhibition of hemolysis.

Practical Value.-Corson-White and Ludlum ${ }^{1}$ have found the reaction positive in 80 per cent. of cases of the catatonic form of dementia præcox and in 62 per cent. of the hebephrenic type. Only 3 out of 37 cases of manic-depressive insanity reacted positively. Among controls with serums of other diseases positive reactions were secured in one case each of cerebrospinal syphilis, tertiary lues, exophthalmic goiter, and confusional insanity, and in two cases each of general paralysis and epilepsy. The afore-mentioned observers claim, however, that if the venom is carefully standardized with blood-cells that are completely hemolyzed in 1:5000 dilution of venom in thirty minutes, the reaction possesses some diagnostic value, having been found, under these conditions, to yield positive reactions in 87 per cent. of cases of dementia præcox and in 100 per cent. of catatonics.

According to Citron, the reaction is probably due to interference with hemolysis by an increase in the cholesterin of the serum-a pos-

${ }^{1}$ Jour. Nerv. and Mental Diseases, 1910, xxxvii, 721. 
sibility more apt to occur in diseases of the central nervous system than in any physiologic or other pathologic condition.

\section{VENOM HEMOLYSIS IN TUBERCULOSIS}

Calmette found that the blood of tuberculous patients may activate cobra hemolysin in very small doses, and upon this observation he devised a test that yielded about 65 per cent. of positive reactions in tuberculosis. Positive reactions have, however, been found in other diseases, and the practical value of the test has not been established.

\section{VENOM HEMOLYSIS IN CANCER}

Although the red blood-corpuscles of the horse may be hemolyzed by venom without the aid of serum, Kraus, Graff and Ranzi ${ }^{1,2}$ found that about 70 per cent. of cancer serums considerably hastened and aided the hemolytic process.

A 1:5000 dilution of venom is used. In two series of four tubes each place respectively $0.1,0.2,0.3$, and 0.5 c.c. of the patient's serum (heated); to the first series add 0.3 c.c. of the venom solution, and to the second series 0.15 c.c. of the same. To each of the tubes in the series add 5 drops of a 10 per cent. suspension of washed horse corpuscles; shake thoroughly and incubate at $37^{\circ} \mathrm{C}$. Inspect the tubes at the end of fifteen and thirty minutes, and then after one, two, and three hours.

Positive reactions have also been found in pregnancy after the fourth month, in icterus, advanced tuberculosis, and other diseases.

1 Wien. klin. Wochenschr., 1911, No. 28.

2 Münch. med. Wochenschr., 1912, No. 59, 574. 


\section{CHAPTER XXII}

\section{PRINCIPLES OF THE PHENOMENON OF COMPLEMENT FIXATION}

Historic.-With Bordet's discovery of the hemolysins in 1898, and his demonstration of the rôle of the antibody or sensitizer and alexin in the process, new light was thrown upon the bacteriolysins, and the close analogy between hemolysis and bacteriolysis soon became apparent. Bordet's discoveries were quickly verified by Ehrlich and Morgenroth and the German school in general, although his views regarding the mechanism of the processes were questioned. The controversy soon centered upon the question of the unity or the multiplicity of alexins or complements. Bordet at this time advanced his belief in the existence of one alexin or complement that would act with any sensitizer or amboceptor, and he still maintains this view. One of the experiments conducted by him, and later made in conjunction with his pupil, Gengou, in support of his theory, is now known as the Bordet-Gengou phenomenon of complement fixation. This has become widely known as the precursor of all complement-fixation tests, and is the basis of the well-known and invaluable Wassermann reaction for the diagnosis of syphilis.

In devising the technic of this important method, Bordet's main object was to show that the complement in a normal serum would unite with either a bacteriolytic or a hemolytic amboceptor, and that, by furnishing sufficient of either amboceptor, all the complement may be "fixed." He argued that if two or more complements existed in the same serum, as was held by Ehrlich, they would demonstrate their presence by exhibiting different affinities for these widely varying amboceptors.

Prior to this Bordet had shown that the addition of a small amount of normal serum to an immune hemolysin would result in lysis of the homologous corpuscles, and that the process could not take place without the alexin. He then mixed an emulsion of pest bacilli with antipest serum and added a small amount of normal, unheated guinea-pig serum to supply the alexin or complement. After allowing the mixture to stand for four hours at room temperature, it was sought to determine whether the alexin had been fixed by pest antigen and pest amboceptor, or whether 
it was free in the fluid. Bordet knew that the normal alexin serum used in the experiment could produce hemolysis of corpuscles with a homologous amboceptor, so he tested for free alexin by subsequently adding to the mixture anti-rabbit hemolysin and rabbit corpuscles. Hemolysis did not occur, because the alexin or complement had been bound by the pest antigen and amboceptor. When a normal serum was substituted for the antipest serum, hemolysis occurred, because the normal serum contained no sensitizers or amboceptors that could unite with the pest bacilli and "fix" the alexin, which, therefore, remained free, and when the hemolysin and red corpuscles were subsequently added, united with them to lyse the red cells. In this way the corpuscles and hemolysin served as indicators for free or unfixed alexin or complement, just as litmus or phenolphthalein may be used as a test for the presence of an acid or an alkali.

By showing, in this manner, that the complement of a serum could be fixed by either bacteriolytic or hemolytic amboceptors, Bordet endeavored to support his views on the unity of complement. Ehrlich and Morgenroth later verified his findings, and in addition, by more delicate and complicated experiments, showed that many complements may be present in a serum, a fact manifested by a different rate of absorption, by the action of specific anticomplements, etc.; as mentioned in a previous chapter.

While Ehrlich's theory as to the multiplicity of complements has been widely accepted, the subject really possesses greater academic than practical interest, for experience has shown that the results are the same in complement-fixation tests at least, regardless of whether we believe in the unity or in the multiplicity of complement, as under ordinary conditions the complement or complements in a given quantity of serum are capable of being absorbed by bacteriolytic, hemolytic, or other amboceptors.

Gengou showed later that not only cellular antigens, such as bacteria and red blood-cells, are capable of stimulating the production of amboceptors, but that the proteins in solution, such as serum and milk, may produce complement-binding amboceptors in addition to precipitins. This subject was later studied more extensively by Moreschi, whose interest became aroused as the result of his theoretic studies upon anticomplements. This investigator observed that, upon mixing a soluble protein with its antiserum precipitation occurred and the existing complement disappeared, a coincidence that led him to assert that the complement disappeared because it was carried down mechanically in 
the precipitate. This explanation naturally had the effect of leading many to assume that the Bordet-Gengou phenomenon of complement fixation may be the result of a similar precipitation process, and led to many interesting and valuable investigations, especially those made by Gay. It is now generally agreed, however, that protein amboceptors are formed, and that actual complement fixation occurs independently of precipitation. Later Neisser and Sachs elaborated on Gengou's studies, and perfected a complement-fixation technic for the differentiation of proteins that is much more delicate than the precipitin test, and serves to demonstrate and differentiate traces of protein, as in blood-stains, so minute in quantity as not to be appreciable by the precipitin test.

Widal and Lesourd applied the Bordet-Gengou reaction to the diagnosis of typhoid fever, using an emulsion of typhoid bacilli and the serum of a typhoid-fever patient, and found that a positive reaction occurred more frequently and earlier than the agglutination test. These observations were made soon after Bordet and Gengou's discovery, and were probably the first direct and practical application of a complementfixation technic in diagnosis. It was not until several years later, however, that the possibilities of the method were seriously considered.

Hitherto most experiments were conducted with known antigens and their antibodies. It was shown, especially in the work of Neisser and Sachs on protein differentiation, that when an antigen and its specific antibody are present complement is absorbed, and the specific relation existing between these bodies was again emphasized. Hence in a complement-fixation test, if the antibody is known the antigen may be found, or if the antigen is known the antibody may be found, the detection in either instance depending upon whether or not complement is absorbed, this being decided by adding corpuscles and their amboceptors to the mixture, the absence or the occurrence of hemolysis determining this point. In this manner Neisser and Sachs were able to diagnose the nature of bloodstains by using solutions of the suspected stains as antigen, and adding, in different experiments, known antiserums secured by injecting rabbits with various bloods. When a positive complement-fixation reaction occurred, they concluded that the antigen of the blood corresponded to the known antibody, and they were thus able to identify the species of animal from which the blood in the stain was derived.

Wassermann and Sachs, encouraged by these results, endeavored, by complement-fixation tests, to show the existence of antigens in diseased organs, using tuberculous glands and lungs with an antituberculous 
serum and the serums of tuberculous persons. Complement fixation occurred under certain circumstances, and these are discussed more fully in Chapter XXIV. These investigations finally led to the serum diagnosis of syphilis, in which the antigen was supplied by tissues containing large numbers of the Spirocheta pallida. By furnishing the antigen it was hoped that the luetic antibody could be detected in the body-fluids through the absorption of complement, by the union of the antigen and its antibody in the complement-fixation test. Although the primary results were somewhat discouraging, the possibility was shown to exist, and while the original theories regarding the specific nature of the antigen-antibody reaction have been modified by subsequent discoveries, nevertheless this reaction of Wassermann, Neisser and Bruck, and Detre has proved itself one of the most valuable diagnostic procedures known.

\section{THE ORIGINAL COMPLEMENT-FIXATION METHOD OF BORDET}

In a paper published in 1901 Bordet $^{1}$ gives the results attained with three different antigens and their respective antiserums-pest, typhoid, and Proteus vulgaris. The details of the technic employed with an antigen of pest bacilli and an antipest horse serum are given.

(a) The antigen consisted of twenty-four-hour cultures of pest bacilli in normal salt solution, making a somewhat concentrated emulsion.

(b) The antipest horse serum was heated for half an hour at $56^{\circ} \mathrm{C}$., to remove the alexin or complement. Normal horse serum (heated) was also employed as a negative control.

(c) As alexin, the fresh serum of a normal guinea-pig was used.

(d) The substance sensibilisatrice or hemolysin consisted of the inactivated serum of a guinea-pig injected with rabbit's red blood-cells.

(e) The corpuscles of the rabbit were washed, to free them of alexin, and were used as the indicatory antigen.

To 0.4 c.c. of the pest emulsion 1.2 c.c. of inactivated antipest serum and 0.2 . c.c. of guinea-pig alexin were added. This mixture was allowed to remain at the ordinary laboratory temperature $\left(18^{\circ}-20^{\circ} \mathrm{C}\right.$.) for several hours. In order to ascertain whether or not the alexin had been absorbed, hemolysin and erythrocytes were added to the mixture. This was accomplished by sensitizing about 20 drops of washed rabbit's cells with 2 c.c. of inactivated hemolysin for about fifteen minutes, and adding two drops to each of the test-tubes. Hemolysis did not occur

${ }^{1}$ Bordet: Ann. de l'Inst. Pasteur, 1901, xv, 19. 
because the alexin had been fixed by the pest antigen and antibody. A similar test, conducted with normal serum, hemolyzed in a few minutes because the complement or alexin remained free in the mixture. Even at this early stage Bordet included the important controls on his antigen and serums that are so necessary in all complement-fixation tests.

The following table gives the original details and results of the first complement-fixation experiment with pest antigens and antipest serum:

TABLE 12.-THE ORIGINAL BORDET-GENGOU COMPLEMENTFIXATION REACTION

\begin{tabular}{|c|c|c|c|c|c|}
\hline TUBE & $\begin{array}{l}\text { ANTIGEN, } \\
\text { C.c. }\end{array}$ & SERUM & $\begin{array}{l}\text { Auexin, } \\
\text { C.c. }\end{array}$ & $\begin{array}{l}\text { HEMOLYSIN AND } \\
\text { ERYTHROCYTES }\end{array}$ & Results \\
\hline$(a)$. & 0.4 & 1.2 c.c. inactivated & 0.2 & 2 drops sensitized & No hemol- \\
\hline & 0.4 & $\begin{array}{l}\text { antipest serum } \\
12 \text { c.c. inactivated } \\
\text { normal serum }\end{array}$ & 0.2 & $\begin{array}{l}\text { rabbit's blood } \\
2 \text { drops sensitized } \\
\text { rabbit's blood }\end{array}$ & Complete \\
\hline & & 1.2 c.c. inactivated & 0.2 & 2 drops sensitized & Complete \\
\hline & .. & 1.2 c.c. inactivated & 0.2 & 2 drops sensitized & Complete \\
\hline & 0.4 & $\begin{array}{l}1.2 \text { c.c. inactivated } \\
\text { antipest serum }\end{array}$ & & $\begin{array}{l}2 \text { drops sensitized } \\
\text { rabbit's blood }\end{array}$ & $\begin{array}{l}\text { No hemol- } \\
\text { ysis }\end{array}$ \\
\hline ( & 0.4 & $\begin{array}{l}1.2 \text { c.c. inactivated } \\
\text { normal serum }\end{array}$ & . & $\begin{array}{l}2 \text { drops sensitized } \\
\text { rabbit's blood }\end{array}$ & $\begin{array}{l}\text { No hemol- } \\
\text { ysis }\end{array}$ \\
\hline
\end{tabular}

Mechanism of Complement Fixation.-The divergent views of Bordet and Ehrlich on the mechanism of antigen-amboceptor action have been given elsewhere. Bordet believes that the antibody unites directly with the antigen, and serves to sensitize and prepare it for direct union with the alexin or complement, in a manner similar to that of using a mordant in aiding the penetration of a dye-stuff. In the absence of the homologous and specific antibody (sensitizer), the antigen is incapable of absorbing more than very small amounts of complement or none at all. In the absence of antigen, the sensitizer and complement do not unite, or unite to but a very slight degree. I'The important requirement for complement fixation is, therefore, an antigen that has been sensitized by the antibody, and in this manner has an increased combining affinity for complement.

According to Ehrlich and Morgenroth, however, the complement does not unite directly with the antigen, but only indirectly through the antibody, which acts as a connecting link or amboceptor between antigen and complement. Antigen alone, or even amboceptor alone, binds the complement only very slightly or not at all. The important requirement for complement fixation is an amboceptor attached to its homol- 
ogous or suitable antigen, which increases the affinity and fixing power of the amboceptor for the complement. (See Fig. 82.)

Complement-fixation tests also serve to demonstrate that absorption of complement is not necessarily followed by lysis of the antigen. For example, anthrax and pest bacilli, when mixed with their homologous amboceptors and complement, show no bacteriolysis or but a very slight reaction. The erroneous conclusion thus reached, that these serums contained no amboceptors, was disproved by Bordet, who demonstrated that they contained amboceptors and that complement was absorbed or fixed although bacteriolysis had not taken place. Whether the difference here depends upon variations in the nature of lytic and non-lytic amboceptors or whether it is due to the relative amounts of an amboceptor or the construction and constitution of the antigen is not known. It would appear, however, that the last two possibilities are largely concerned, although, so far as complement fixation is concerned, it is immaterial whether or not bacteriolysis occurs.

Complement-fixing antibodies, therefore, are probably all in the nature of amboceptors, and these are to be found in varying amounts in practically all immune serums, including antitoxic, agglutinating, and precipitating serums.

\section{NON-SPECIFIC COMPLEMENT FIXATION}

The importance of having proper controls in practically all tests is especially to be emphasized in complement-fixation work. While the underlying principles are readily understood and the technic is comparatively simple, there are, however, many sources of error that require a thorough understanding in order that an intelligent and reliable complement-fixation reaction may be secured. These refer mainly to nonspecific fixation of complement and to quantitative factors governing complement-fixation technic.

Formed elements, such as bacteria and tissue-cells, as well as various organic and inorganic material, may fix complement by themselves, i.e., in a non-specific manner, and chemicals, such as acids and alkalis, may destroy it.

1. An antigen alone in certain amounts may absorb complement. This anticomplementary dose of an antigen, as it is called, must be determined beforehand by a process of titration when increasing amounts of antigen are mixed with a constant dose of complement, hemolysin; and corpuscles and the anticomplementary action of the antigen noted by the results 
of hemolysis, $i$. e., if a small amount of complement is absorbed, hemolysis will be correspondingly incomplete, if all complement is absorbed, hemolysis does not occur at all. If, therefore, in any complement-fixation test the antigen is used in an amount that will give this non-specific absorption, even to a slight degree, a grave source of error is introduced.

As a general rule for all complement-fixation tests, the dose of antigen employed should never be more than one-fourth or one-half of its anticomplementary dose (that amount which of itself is capable of non-specific complement-fixation).

2. A serum may of itself absorb a small amount of complement, especially if it is old or infected with bacteria. This is known popularly as the anticomplementary action of a serum, and in every complementfixation test in order to detect this condition a proper control, consisting of the dose of serum used plus complement, hemolysin, and corpuscles is required, the non-specific absorption of complement being determined by the results of hemolysis.

Moreover, perfectly fresh serums may show this non-specific absorption of complement to a slight degree, especially in the presence of the lipoidal extracts used as "antigens" in the Wassermann reaction.

Heating a serum to $56^{\circ} \mathrm{C}$. for half an hour largely removes this anticomplementary effect of serums, unless they are quite old and infected; accordingly, heated serums are used almost exclusively in complement-fixation tests. This is usually called inactivation, or the removal of native complement from a serum, but in the majority of instances the complement of a serum generally deteriorates rapidly, and the serum is heated mainly for the purpose of removing its anticomplementary action, $i . e$. , its ability to effect non-specific absorption of complement.

By referring to the original Bordet experiment, it will be observed that this investigator controlled any non-specific absorption of complement by both the immune and the normal serum in tubes $\mathrm{C}$ and $\mathrm{D}$ of the series by using the full dose of these serums, with a similar amount of complement, and noting that hemolysis was complete. His controls, $\mathrm{E}$ and $\mathrm{F}$, were to determine if the process of inactivation or removal of native complement from the two serums was complete, and the total absence of hemolysis showed that it was. His control on the anticomplementary action of the antigen was also included in tube $\mathrm{D}$, for if the emulsion alone had absorbed complement to any degree, hemolysis would have been incomplete.

Quantitative Factors in Complement-fixation Tests.-From what has been said it will, therefore, readily be appreciated that complement- 
fixation tests are largely quantitative. Equally fallacious results may be obtained by using too large or too small amounts of the various ingredients.

While it is possible to use too large quantities of antigen, so that non-specific absorption of complement occurs, leading to false positive reactions, it is also possible to use an amount so small that any specific absorption of complement by antigen and antibody cannot readily be detected.

The same is true, but to a much less extent, of the immune serum, for while too large amounts of serum may lead to non-specific fixation of complement, surprisingly small amounts may give well-marked specific fixation, this factor depending, of course, upon the quantity of antibodies contained in the serum.

Of even greater importance are the quantity of complement employed and the proper adjustment of the hemolytic system, composed of complement, hemolysin, and corpuscles.

Too large an amount of complement may furnish sufficient to satisfy the amboceptors of an immune serum united with the antigen, with enough free complement left over to produce partial or complete hemolysis when corpuscles and hemolysin are subsequently added. In this manner specific complement fixation would be overlooked and a false negative reaction secured.

It is also possible to use too small an amount of complement, with relatively large doses of serum and antigen, so that the complement becomes unduly susceptible to non-specific fixation and consequently false positive reactions may be secured.

It has previously been explained that an excess of hemolysin may offset any slight deficiency in the amount of complement. For instance, if a small amount of complement is specifically fixed by an antigen and its amboceptor, the addition of too large an amount of hemolysin may result in complete hemolysis of the corpuscle, and thus overshadow the slight but specific fixation of complement.

On the other hand, hemolysis cannot be complete if the dose of amboceptor is too small. With a given dose of corpuscles and complement a certain amount of hemolysin is necessary to produce hemolysis, this dose being determined by a process of titration, as described in a previous chapter. If less than this dose is used, but the amounts of corpuscles and complement remain the same, hemolysis will be correspondingly incomplete and lead to false positive reactions.

A very important feature of all complement-fixation tests will be 
seen to be a proper and accurate adjustment of the hemolytic system. Taking arbitrary amounts of corpuscles and hemolysin as constants, the quantity of complement necessary to produce hemolysis may be determined (titration of complement); or, taking corpuscles and complement as constants, the amount of hemolysin necessary to effect complete hemolysis may be determined. One or the other or both titrations should be made before the main test is attempted, in order to avoid using an excess or too little of either ingredient. If the exact unit of complement and hemolysin are used, the results must be very carefully guarded, because in a general way all antigens and serums exert a slight anticomplementary action that may yield results that will be interpreted as weak positive reactions. For this reason the original complement-fixation tests invariably called for a slight excess of complement or hemolysin or both, to allow for possible non-specific complement fixation, and this is a good general rule that makes the reaction somewhat less delicate, but more reliable in the long run, especially for inexperienced workers.

Complements of different species of animals act differently in activating a hemolytic amboceptor and toward fixation by antigen-antibody combinations. For instance, a complement from one animal may readily enough combine with a hemolytic amboceptor to produce hemolysis, but will not lend itself for fixation, and is, therefore, unfit for complement-fixation tests. Noguchi and Bronfenbrenner have found guineapig serum most suitable from all standpoints, but it is important to remember that the complementary activity of the serums from different guinea-pigs varies, and, therefore, it is necessary to titrate each complement serum or hemolysin, $i$. e., adjust the hemolytic system, before the main test is conducted.

These quantitative factors are of great importance, and complicate any complement-fixation method, but efforts to circumvent or ignore them are likely to lead to errors in technic. A proper understanding and appreciation of these factors constitutes the basis for reliable work, whereas less essential details may be altered to conform to the ideas and convenience of the individual worker.

\section{PRACTICAL APPLICATIONS}

It will be understood, therefore, that complement-fixation reactions may serve two primary purposes:

1. With a known antigen, the antibody may be found. This is the 
usual order in diagnostic tests. For example, in the reaction for syphilis the antigen is furnished and the antibody sought for in the body-fluids. So specific has this test proved in the diagnosis of this disease that a positive reaction secured with a proper technic is regarded as strong evidence of the existence of lues, even though the primary lesion had occurred years before and the person is at the time in apparent good health. In the gonococcus fixation test and other tests of a similar nature the antigen is known and is furnished, and the antibody is tested for in the serum.

2. With a known antibody the corresponding antigen may be found. This order of events has less practical application, and is used principally in the diagnosis of blood-stains and in the differentiation of proteins in general. It is also used in making special bacteriologic investigations, when an organism may be identified by specific complement fixation with its known antibody serum. In these instances the antibody serum is secured by immunizing rabbits with a known antigen, the immune serum then being used for selecting the antigen in unknown substances and mixtures.

Complement-fixation methods have their greatest value, and are probably best known, in the serum diagnosis of syphilis-the biologic syphilitic reaction of Wassermann, Neisser and Bruck, and Detre. Although originally believed to be a direct application of the specific Bordet-Gengou phenomenon of complement fixation, subsequent investigations have shown that the antigen need not be specific, in the sense of containing the Spirocheta pallida, but that lipoidal substances in general may serve as "antigen," the peculiar and specific character of the reaction depending upon the nature of the antibody, which has a strong affinity for lipoids, and in such a mixture is capable of absorbing or fixing a considerable amount of complement.

In no other disease has the method been so widely employed as in syphilis, although it possesses value in the serum diagnosis of various bacterial infections, such as gonorrhea, glanders, typhoid fever, echinococcus disease, etc., and in the diagnosis of blood-stains and in the differentiation of proteins in general.

In the following chapter the Wassermann syphilitic reaction will be considered in some detail, as a thorough working knowledge of this test is of great value, and serves as the foundation of complement-fixation technic in general. 


\section{CHAPTER XXIII}

\section{THE TECHNIC OF COMPLEMENT-FIXATION REACTIONS}

\section{THE WASSERMANN REACTION IN SYPHILIS}

Historic.-Following Bordet's important discovery of complement fixation no practical applications were made for several years until Neisser and Sachs continued Gengou's studies on protein antigens and amboceptors, and advocated complement fixation as a fine and delicate method of control on the precipitin test in the detection and differentiation of minute traces of proteins, as in the recognition and diagnosis of blood-stains.

Encouraged by these results, Wassermann used the method in an attempt to discover in the blood-serum, during the course of an infection, the bacterial proteins derived from a microörganism. Practical application proved, however, that enough of these proteins did not exist free in the blood to give definite complement fixation.

In 1905 Wassermann and Bruck found that bacterial extracts may be substituted for emulsions of bacteria as antigen in performing the Bordet-Gengou test, and that extracts of diseased organs containing large numbers of bacteria or their products may be employed. Accordingly, these observers prepared aqueous extracts of tuberculous lungs and glands and used them as antigens in the study of complement fixation in tuberculosis. Positive reactions were secured with an antituberculous serum and with the serums of persons who had received injections of tuberculin.

At this time Schaudinn and Hofmann discovered the spirochete of syphilis, a finding that served to focus the attention of the medical world upon this disease. In coöperation with Neisser, who was conducting extensive researches on experimental syphilis in monkeys, Wassermann and Bruck applied the complement-fixation method to the study of these experimental infections, and published a report of their work on May 10, 1906.

At first monkeys were immunized with aqueous extracts of human chancre, condylomata, syphilitic placenta, etc., and their serums, mixed in vitro with these extracts, were found to give the complement- 
fixation reaction. Since these results may have been due to protein amboceptors or precipitins produced simultaneously by the injection of human serum contained in the extracts, the experiment was carried out with extracts of bone-marrow and other organs of syphilitic monkeys used to obviate this error. It was found, however, that the inactivated serums of syphilitic monkeys reacted positively with antigens of either human or monkey lesions, and regardless of whether the monkeys had been injected with human extracts, since, after ordinary cutaneous infection, their serum would show complement fixation. These early reports also showed a high specificity for complement fixation, as monkey immune serum did not react with extracts of normal organs or normal monkey serum with extracts of syphilitic organs.

Just fourteen days after Wassermann, Neisser, and Bruck published their report, a second paper on the same subject appeared, showing the work of Detre. Using aqueous extracts of luetic papules, liver, pancreas, and tonsillar exudate as antigens, Detre performed the complement-fixation method with the serums of six syphilitic and four normal persons, finding positive reactions with two of the six luetic serums.

In 1906 Wassermann and Plaut studied the cerebrospinal fluids of 41 cases of paresis, and found positive reactions in 32,4 cases reacting doubtfully and 5 negatively. In the following year Levaditi and Marie and Schutze observed positive reactions with the cerebrospinal fluid of tabetics, whereas Morgenroth and Stertz confirmed the previous finding in paresis. Since then numerous investigators have corroborated these observations, and while all the evidence tended to strengthen the belief in the luetic origin of general paralysis and tabes, decisive confirmation was lacking until Noguchi and Moore, in 1913, demonstrated the presence of the Treponema pallidum in sections of the cerebral cortex.

In 1906 Wassermann, Neisser, Bruck, and Schucht applied the complement-fixation test to a large number of cases of syphilis in Neisser's clinic. Aqueous extracts of luetic liver, placenta, glands, chancres, and gummata were used as antigens. Of 257 cases in all stages of the disease, only 49 reacted positively. With but 19 per cent. positive reactions, the method did not appear to have a promising future, although at the present time, with a better understanding of the technic and of the importance of quantitative factors that greatly influence the results, the value of the test has been greatly enhanced.

As it appeared that, after all, no method of diagnosis was to be secured as the result of the demonstration of the syphilitic antibody in the body-fluids, Neisser and Bruck determined to return to earlier 
methods and attempt to discover if luetic antigen could be demonstrated in the serums of luetics through complement fixation. Antigens prepared of the red corpuscles of syphilitic persons gave positive reactions with the serums of highly immunized monkeys. Of 160 luetic patients, in 70 per cent. either antigen or antibody was found. Later, however, Citron showed that the extracts of corpuscles of normal persons yielded similar results, which, in the light of subsequent discoveries, was due to their content in lipoidal substances.

Up to this time the syphilitic reaction was considered as but a simple and direct application of Bordet's phenomenon, requiring a specific syphilitic antigen before complement could be fixed with the syphilis antibody. In January, 1907, Weygandt reported that he had obtained a positive reaction in tabes with an extract of normal spleen. Marie and Levaditi, using an aqueous extract of normal fetal liver, secured positive reactions with the cerebrospinal fluid of paretics, but observed that it was necessary to use larger doses than when extracts of syphilitic organs were used. Subsequently other investigators, as, for example, Fleischmann, Michaëlis, Landsteiner, and Plaut, found that watery extracts of normal organs served to fix the complement with luetic antibody. Finally, in December, 1907, a profound impression was created by the discovery made by Landsteiner, Müller, and Pötzl, that an alcoholic extract of guinea-pig heart yielded results equal to those obtained with an aqueous extract of syphilitic liver. These results indicated that the antigenic principle was soluble in alcohol, and a prolonged series of investigations on the various lipoids and their relation to the reaction was begun. These included the employment of lecithin by Porges and Meier; sodium taurocholate and glycocholate by Levaditi and Yamonouchi; cholesterin and vaselin by Fleischmann; oleic acid by Sachs and Altman; acetone-insoluble fractions of alcoholic extracts by Noguchi; and many other combinations of various lipoidal substances by different investigators.

These dealt a blow to the theory of the Wassermann reaction, which, taken in conjunction with the wide-spread use of the test by inexperienced and unskilful persons and the many sources of error, tended to retard an earlier appreciation of the great value of the test, and served to swing the pendulum of medical opinion so far in the wrong direction that it is only now, with a better understanding of its possibilities and limitations, that the method is being established in its proper sphere. Positive reactions were said to have occurred in frambesia, leprosy, malaria, pellagra, pneumonia, scarlet fever, typhoid fever, malignant tumors, and 
practically every other disease liable to afflict humanity. The careful work of Citron was largely instrumental in preserving the importance of the reaction until a better understanding of the technic resulted in improved and more careful work, with a greater respect for the real value of the Wassermann reaction.

Although the true explanation of the mechanism of complement fixation in syphilis is still lacking, sufficient work has been done to show that the specific nature of the reaction is dependent upon a peculiar luetic antibody, and that the older belief in the specificity of antigen, in so far as it insisted upon the presence of the Treponema pallidum in the tissues extracted for "antigen," is largely disproved. While the method cannot be said to be absolutely diagnostic of syphilis, since positive reactions were had in frambesia (yaws) and leprosy, yet it is practically so, especially in those countries where these two infections are unknown or are relatively infrequent.

Principles and Theories of the Syphilitic Reaction.-The discovery that the antigen in the Wassermann reaction is not necessarily biologically specific, but may be furnished by a variety of different lipoids ${ }^{1}$ from normal or syphilitic tissues, opened up an entirely new phase of the well-known theory of complement fixation, and separated the syphilitic reaction for the classic Bordet-Gengou phenomenon, as based upon the absorption of fixation of complement by a specific antigen and its antibody.

As is now well known, the lipoids have always been chiefly concerned in the reaction, although Wassermann and Detre and their coworkers naturally ascribed the complement-fixing powers of their extracts to the presence of the Treponema pallidum. It is, indeed, fortunate that pure cultures of the treponema were not available at the time the original studies were made, for these would naturally have been employed as antigen, and as subsequent work with pallidum antigens has shown complement fixation to be quite irregular and less reliable than when lipoidal extracts are used, this result, coupled with the imperfect understanding and faulty technic of the earlier investigations, would probably have yielded results so discouraging as to constitute weighty drawbacks to the full development of the reaction.

Notwithstanding the large amount of work that has been done in an effort to ascertain the true nature of the syphilitic reaction, a correct

1 The term "lipoid" ("fat-like") is applied to compounds that are soluble in ether, alcohol, chloroform, and benzol, but every lipoid is not soluble in all these reagents. 
explanation of its mechanism is still lacking, as the large number of theories advanced tend to show.

While lipoidal extracts, as well as normal and luetic serums, may separately absorb or fix small amounts of complement, a mixture of a suitable extract and syphilitic serum is capable of fixing large amounts of complement, and this constitutes the main principle and all that is definitely known of the syphilitic reaction.

The serum of a syphilitic is characterized, therefore, by the presence of this lipodotropic, antibody-like substance, which has a great affinity for lipoids and in mixture with them will cause the absorption or fixation of complement to a well-marked degree. Instead of being an example of complement fixation in a mixture of specific antigen with specific antibody, as originally believed, it is technically a non-specific reaction, but practically it is highly specific, (since this peculiar antibody is found in largest amount and most constantly in syphilis, and to a lesser extent in practically only two other diseases, namely, leprosy and frambesia.) In countries and districts where these diseases are infrequent or unknown with proper technic the reaction for syphilis is highly specific.

As will be shown further on, the presence of this lipodotropic substance is dependent upon the activities of the Treponema pallidum, and when repeated tests continue to show its presence, there is every reason to believe that a cure has not been effected, but that the patient still harbors the living parasite.

Citron has advanced the hypothesis that the antibody-producing antigen is a toxolipoid, which would explain the fact that while pure lipoids, such as lecithin, cannot stimulate antibodies (Bruck), as the toxolipoid does, they can, nevertheless, react with the lipodotropic antibodies in vitro, with fixation or absorption of complement. As Sachs and Altman point out, an equally tenable theory would be that in syphilis the tissues undergo such alterations that they can produce antibodies to the lipoid substances as may be contained in the spirochetes themselves.

While the production of this lipodotropic antibody is still unexplained, the fact remains, nevertheless, that it forms the basis of the biologic syphilitic reaction, and in a mixture with a suitable lipoid is capable of absorbing or inactivating complement to a marked degree. Whether or not it is a true antibody in the sense that it is inimical to the spirochete is doubtful; by many it is regarded as a secondary product of cellular activity, and has been called syphilis "reagin."

Since similar "reagins" are to be found in other infections, notably 
in frambesia and leprosy, investigators in this field anxiously awaited the isolation of the Treponema pallidum in pure culture, believing that if this result were secured it would be possible to work with a specific antigen, determine the nature of the true syphilis antibody, and possibly establish a complement-fixation test specific for syphilis.

In 1909 Schereschewsky, using an antigen of an impure and nonpathogenic culture of a spirochete regarded as the Treponema pallidum, reported positive complement-fixation reactions with the majority of serums tested.

In 1912 Noguchi, having undoubtedly isolated the spirochete in pure culture, prepared antigens and found that, whereas certain longstanding or treated cases of syphilis yielded positive reactions with the pallidum antigens, the reactions were uniformly negative when the lipoidal extracts were used. In primary and secondary syphilis the reactions with pallidum antigens were uniformly negative, whereas with the lipoidal extracts they were uniformly positive. As a result of his experiments Noguchi concluded that in syphilis there is produced a true antibody that reacts specifically with pallidum antigen, in addition to the lipodotropic "reagin," which reacts with lipoidal extracts, and whereas the latter indicates activity of the infecting agent, the former is a gage of the defensive activity of the infected host.

Craig and Nichols, using alcoholic extracts of pure cultures in ascites kidney agar of Treponema pallidum, Spirochete pertenuis, and Spirochete microdentium, found similar positive reactions in all stages of syphilis with the. three antigens, but the reactions were weaker and less constant as compared with those obtained with a stock of lipoidal extract.

Similar studies conducted by Kolmer, Williams, and Laubaugh with aqueous and alcoholic extracts of pallidum cultures showed positive reactions in secondary, tertiary, and congenital syphilis. The aqueous extracts yielded better reactions than the alcoholic extracts; in practically all instances, however, the reactions were weaker than those obtained with the ordinary lipoidal extracts. Control antigens of typhoid and cholera bacilli and sterile culture mediums demonstrated that all contained lipoidal substances that may give weak reactions with the lipodophilic "reagin." This may explain Schereschewsky's positive reactions with an antigen of a spirochete that in all probability was Spirochete microdentium (Noguchi).

The true nature of the Wassermann-Detre reaction, therefore, cannot be said to have been determined. It is probable that the ordinary syphilis reaction is in itself not dependent upon a true antibody, and that 
the reaction is not an immunity reaction, but due rather to the presence of peculiar tissue products (reagins) altered by the presence and activities of the spirochetes themselves, and that the Wassermann reaction is an expression of this active injury to tissue-cells. In addition to this secondary product there is probably a true syphilis antibody that may yield specific complement fixation with pallidum antigens.)

In so far as the Wassermann reaction is concerned, the true antibody is entirely secondary in importance, and the whole question is intimately concerned with the chemistry of lipoids. While future researches in immunochemistry may reveal the mechanism of the reaction, the principles are at least well understood at present, so that the syphilis reaction is proving of great diagnostic and practical value.

\section{TECHNIC OF THE WASSERMANN REACTION}

Glassware for Complement-fixation Reactions. - Test-tubes should be of convenient sizes, -12 by $1.5 \mathrm{~cm}$,-- perfectly clean, free from acids and alkalis, and preferably sterile. They need not be plugged with cotton as it suffices to sterilize them in a wire basket with their mouthends downward. Smaller test-tubes, as those used in the Noguchi modification of the Wassermann reaction ( 8 by $1 \mathrm{~cm}$.), are wrapped in newspaper in bundles of 25 and sterilized.

Pipets should be perfectly clean and preferably sterile. Three kinds are required: The ordinary 1 c.c. pipet, graduated to 0.01 c.c. and calibrated to the tip; a number of special pipets for the fourth method and the gonococcus fixation test, of about the same length and external diameter as an ordinary 1 c.c. pipet, but of much smaller caliber, so that the pipet will hold 0.2 c.c.; it should be graduated to 0.01 c.c. and calibrated to the tip; 5 c.c. pipets divided into 0.1 c.c. Care should be exercised in handling pipets to avoid breaking the tips. After use they should be washed free from blood, serum, etc.

\section{GENERAL TECHINIC}

For testing for the Wassermann syphilis reaction five reagents are used:

1. The fluid to be tested.

2. Complement.

3. Hemolytic amboceptor.

4. Blood-corpuscles.

5. Antigen (organic extract). 
I. The Fluid to be Tested.-(a) Serum.-As a general rule, all specimens of blood submitted for complement-fixation tests should be collected aseptically in sterile containers. This is especially necessary when there has been delay in transmitting the fluid to the laboratory, as when sent through the mails from distant points. When the reactions are to be conducted on the same or on the following day, the specimen of blood may be collected in chemically clean but not necessarily sterile containers. Bacterial contamination renders a fluid anticomplementary and unfit for complement-fixation tests. Specimens should be kept on ice until used, and the serum promptly separated from the clot.

Collecting Blood for the Wassermann Reaction.-In collecting blood for the Wassermann reaction the following points should be remembered:

1. That during active antisyphilitic treatment the blood may react negatively, whereas at a later period a true positive reaction is observed. It is well, therefore, not to collect blood until all specific treatment has been suspended for at least two weeks.)

2. That blood collected during or immediately after an alcoholic debauch may yield a false negative reaction (Craig and Nichols).)

3. That blood should not be collected just after anesthesia or while the patient has a high temperature. )

As a general rule, at least 1 c.c. of serum and 2 c.c. of cerebrospinal fluid are required for making the syphilitic reaction. From 2 to 3 c.c. of blood are needed, these amounts being easily collected from adult persons by pricking the finger deeply and filling a small test-tube or vial, as shown on p. 32 . This method is very convenient, especially for physicians, hospitals, and dispensaries where direct access to a laboratory can be had. When the treatment is to be guided by the Wassermann reaction, a number of tests are required, and patients may object to repeated venipuncture, whereas no objections will be raised to simple puncture of the finger.

Larger amounts of blood are collected from a vein at the elbow under aseptic precautions, as described on p. 33 . As a rule, it is well to collect at least 5 c.c. of blood, especially if the specimen is shipped from a distant point (Fig. 105). An excess of serum permits the technician to repeat a test when necessary, or to apply more than one method, and thus at times both the physician and the patient are saved the time and annoyance incident to collecting another specimen (Fig. 106).

The Keidel tube, which is sterilized and ready for use, is quite a convenience (p. 36). However, a test-tube or a centrifuge tube may be used, or, when a specimen is to be mailed, a 5 or 10 c.c. vial of thick glass, 
stoppered with a cork or a rubber stopper is quite satisfactory (Fig. 105). Vial, stopper, and needle are readily sterilized in boiling water, drained, and cooled, the specimen collected, the vial tightly stoppered, and the whole sent at once to the laboratory. Cotton stoppers are unsatisfactory, as unless the tube or vial is maintained in an upright position, the fluid may be absorbed. When specimens of blood are to be mailed, it is better to fill a small vial than to place the same amount in a large container, for in the latter case agitation through handling may result in so much mechanical hemolysis taking place as to render the serum unsatisfactory for use. Specimens so collected may be sent for long distances, even in warm weather, and undergo no change.

In collecting blood from children, or where the veins are small, a proportionately smaller needle may be used. In infants the cupping apparatus of Blackfan is quite satisfactory ( $p$. $36)$; frequently sufficient blood may be obtained from a great toe.

The specimen of blood should be kept in a cold place, and the serum removed at the end of twenty-four hours. Serum that is allowed to remain with the clot for longer periods is more likely to become anticomplementary, especially if it becomes deeply tinged with hemoglobin. In cases where the serum does not separate the clot may be broken up gently with a sterile glass rod and centrifugalized. The serum should be clear and free from corpuscles. Opalescent and milky serums, obtained during the period of digestion and from nursing women, usually do not interfere with

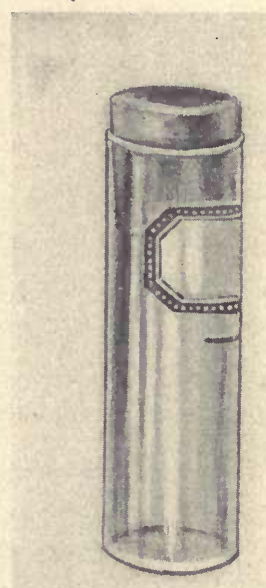

Fig. 105.-A Vial to CONTAIN BLOOD FOR the Wassermann ReACTION.

This is an ordinary glass vial fitted with a rubber stopper. It holds 5 c.c. to the mark, and is readily packed for mailing. Never stopper with cotton. A good cork stopper may be used. the reaction; bile-stained serum may at times give marked non-specific fixation of complement.

It is essential that all serums be heated at $55^{\circ} \mathrm{C}$. for half an hour immediately before the test is made.) This exposure to heat somewhat diminishes the reacting power of a syphilitic serum, but, as shown by Seligman and Pinkus, it is a necessary procedure, for a considerable proportion of normal serums or those from diseases other than syphilis will react positively when unheated, whereas when heated, they will give a negative reaction. 
Furthermore, serums that are sterile and are kept for some time at room temperature or even in an ice-chest acquire an anticomplementary power that is destroyed by heating at $55^{\circ} \mathrm{C}$. for half an hour. Therefore when serums are preserved for a number of days they are heated not so much for the purpose of removing native hemolytic complement, which has probably already deteriorated, but to remove thermolabile anticomplement. Serums that are old or contaminated with bacteria are likely to be highly anticomplementary, and this property cannot be destroyed

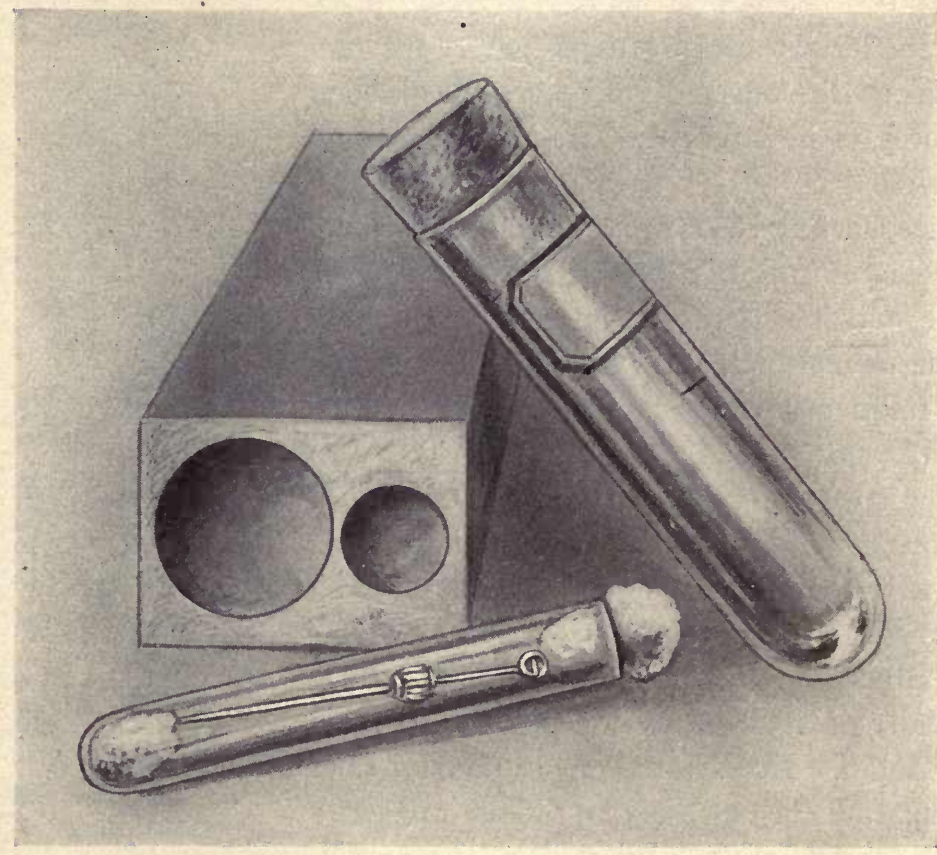

Fig. 106.-OUtfit for Collecting Blood for the Wassermann Reaction (New YORK BOARD OF HeALTH).

The container is a centrifuge tube which holds about 15 to 18 c.c. of blood to the mark. The needle is furnished in a separate tube, sterilized and ready for use. This outfit is not adapted for mailing.

by heating at $55^{\circ} \mathrm{C}$. or filtration through a Berkefeld filter. In order to conduct a reliable test it is usually necessary to secure fresh serum. This anticomplementary action of serums is so important that in every complement-fixation test there is a serum control tube containing all the ingredients except antigen, the object being io discover any inhibitory action of the serum itself upon the complement.

Wechselmann's method of converting syphilitic serums showing a negative or weakly positive reaction to those exhibiting a marked positive 
reaction depends upon removing the excess of indifferent and inhibiting serum components and upon the destruction or diminution of the natural antisheep amboceptor present in so large a percentage of human serums. As Noguchi and Bronfenbrenner have pointed out, this method may likewise remove the antibodies concerned in the reaction. To 1 c.c. of heated serum add 3.5 c.c. of saline solution and 0.5 c.c. of a 7 per cent. suspension of freshly precipitated barium sulphate; shake, and let it stand for one hour at $37^{\circ} \mathrm{C}$; ; centrifugalize, and pipet off the diluted serum, which is now ready to be tested $(1$ c.c. $=0.2$ c.c. of undiluted serum).

Cadaver serums are likely to be highly discolored with hemoglobin and quite anticomplementary. Such serums may be tested in half the usual dose, and while the results are quite specific, they are not so reliable or constant as those obtained from the living.

The doses of serum used in testing for the syphilis reaction are given with each method. In the original Wassermann test 0.2 c.c. was used. As a rule, from 0.05 c.c. to 0.2 c.c. of serum are satisfactory; higher doses may occasionally show a stronger positive reaction, but the serum must be perfectly fresh to avoid non-specific complement fixation, and the natural antisheep amboceptor should first be removed.

(b) Cerebrospinal Fluid. - In certain nervous diseases the cerebrospinal fluid is examined for the syphilis reaction.) Fluid is secured by lumbar puncture, according to the method described on p. 37 . If the specimen contains blood, it should be centrifuged until it is clear. It should not be heated before use, as it does not contain hemolytic complement, and fresh ftuids from cases other than syphilitics do not react positively. Cerebrêspinal fluids, as a rule, possess weaker deviating powers than the corresponding blood-serum, and hence it is necessary to use larger doses - at least 0.5 to 1 c.c., instead of 0.05 to 0.2 c.c., as in the case of blood-serum.

(c) Other Fluids.-Positive syphilitic reactions have been described as occurring with milk, pleural and peritoneal exudates, and albuminous urine (Bauer and Hirsh) from luetic cases. The reactions with these substances are conducted in the same manner as with cerebrospinal fluid. The material should be perfectly fresh, as anticomplementary action is likely to occur.

II. Complement.-While complement is to be found in the fresh normal serum of practically all warm-blooded animals, (not all are suitable for complement-fixation tests.) A suitable complement must possess two important properties: (1) Complementary activities, or 
the power of activating a hemolytic amboceptor, and (2) fixability, or the power of being "fixed" by antigen and antibody. Noguchi and Bronfenbrenner have studied the complements of the dog, sheep, hog, ox, rabbit, and other animals, and found that the complement of the guinea-pig was best adapted, from all standpoints, for the complementfixation test. The complements of pigs and sheep are quite fixable, but their weak hemolytic action and rapid deterioration render them unsuitable for fixation purposes. Rabbit complement is quite active, but is not easily fixable. Kolmer, Yui, and Tyau found rat complement fairly well suited for making the syphilitic reaction with an antihuman hemolytic system.

(The hemolytic power of guinea-pig complement is not constant.) In unhealthy animals it is likely to be low, and even among normal animals it may show some variation. For this reason the hemolytic power of each serum is determined by a method of titration before complement-fixation reactions are conducted. Fixed doses of hemolysin and corpuscles may be used, and the amount of complement necessary for effecting complete hemolysis may be determined, or a fixed dose of complement and corpuscles may be used with different amounts of hemolysin, the chief object being to adjust all three factors of the hemolytic system, namely, complement, corpuscles, and hemolysin, to exact and known proportions.

The complement in the serums of different guinea-pigs may show considerable variation in fixability. The amount of complement inhibited by serum alone and organic extract alone, or by given constant quantities of serum and extract, may vary more markedly than their complementary activity. To reduce this error to a minimum it is advisable, whenever possible, to use the pooled serums of two or more pigs for making complement-fixation tests.

Guinea-pig complement serum is collected by bleeding, the animal, under ether anesthesia, into a Petri dish or centrifuge tube. The large vessels on both sides of the neck are quickly severed with a pair of sharp-pointed scissors or a scalpel, care being exercised not to sever the trachea and esophagus. A funnel is used for collecting blood in centrifuge tubes. It is well finally to sever the spinal cord, in order that the animal may not recover from the anesthetic and thus insure a painless operation throughout. (See p. 46.)

It is best to keep the blood at room temperature for a few hours until coagulation has occurred and the serum has separated; then place the whole in the ice-chest until needed. Blood may be collected in 



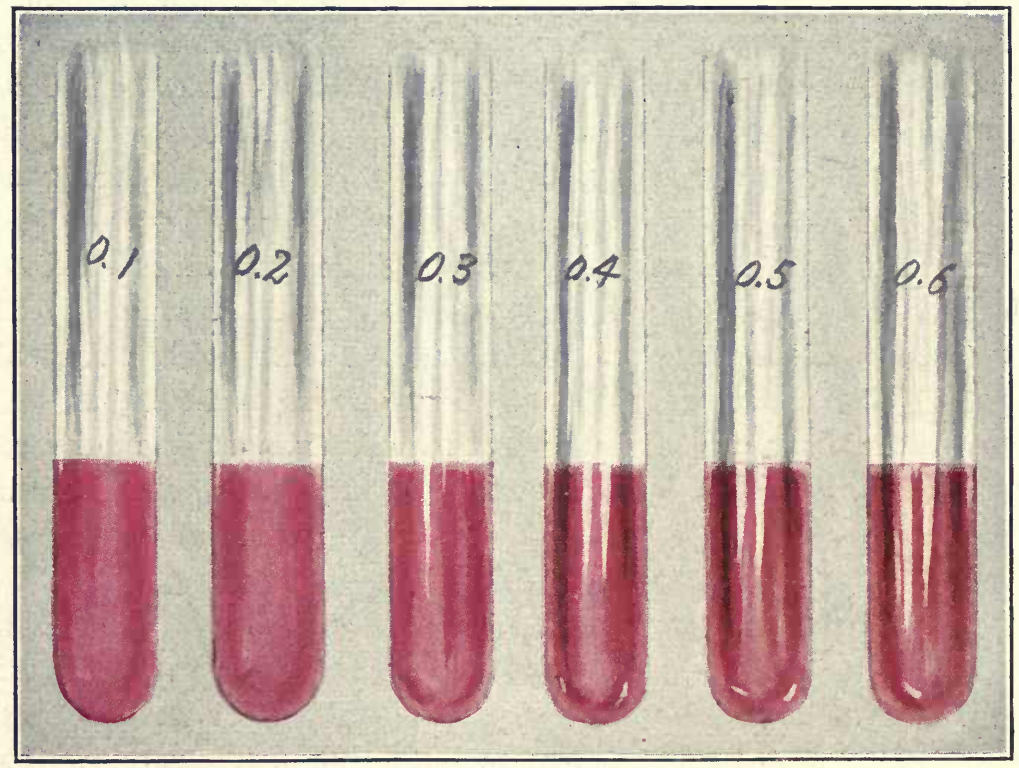

Fig. 107.-Titration of Hemolytic Complement. 
centrifuge tubes and allowed to coagulate; it is then broken up with a glass rod, centrifuged, and the serum secured at once; such complement, however, is likely to be unduly hypersensitive to the anticomplement action of organic extract and of mixtures of extract with normal serums. As a general rule, therefore, it is good practice to bleed the animal late in the afternoon preceding the day on which the experiment is to be made, or at least some hours before the regular work of the day begins. The serum should be clear and contain no corpuscles. )

Titration of Complement.-In the original Wassermann reaction fresh guinea-pig serum is used in a constant dose of 0.1 c.c. I have used for several years just half the amount of corpuscles and complement directed in the original technic, and find that the reactions are somewhat sharper and clearer, besides being more economic. Using the corpuscles and amboceptor as constants, the amount of complement necessary to produce hemolysis may be determined after the manner described in a previous chapter. To a series of test-tubes add increasing amounts of complement serum diluted $1: 20$ as follows: $0.2,0.3,0.4,0.5,0.6,0.7$, $0.8,0.9,1$, and 1.2 c.c. Add 1 c.c. of a 2.5 per cent. suspension of sheep's cells and an amount of hemolytic amboceptor equal to two units. To this add sufficient salt solution to bring the total volume of each tube up to about 3 c.c.; shake gently and incubate for one hour at $37^{\circ} \mathrm{C}$. That tube showing just complete hemolysis contains one unit of complement (Fig. 107).

And now we come to a very important question, namely, the amount of complement that is to be used in conducting complement-fixation tests. Many present-day observers use exactly one unit of complement and one unit of amboceptor. This is permissible, providing the complement is titrated in the presence of a constant dose of antigen and a constant dose of serum, in order that due allowance for the anticomplementary action of these may be made in the titration. Under these circumstances, however, it is necessary to titrate each patient's serum with the complement, because one serum or even the pooled serums of different persons should not be taken as a standard in the titration, for two important reasons: (1) The patient's serum which we are about to test may be more anticomplementary than the serum used in the complement titration, and hence when used in the main test, with exactly one unit of complement, mild degrees of inhibition of hemolysis will be secured that may be interpreted as slightly positive reactions; or (2) the serum used in the complement titration may contain more or less natural hemolytic amboceptor than the patient's serum, and this factor exerts 
an influence on the titration, so that the unit varies with different serums. For these reasons I have included here a fourth method for using a single unit of complement under conditions where the anticomplementary action of each serum and the antigen are determined, in preference to titrating the complement with a serum and using this unit for a number of other serums that are sure to differ from each other.

In this titration, therefore, we determine the amount or unit of complement necessary to produce hemolysis with fixed amounts of amboceptor and corpuscles. It will be observed that I have used two units of amboceptor, as determined by a previous titration. These two units are equivalent to one dose, and the same would be true whether three, four, five, or more units were used, because in this titration the corpuscles and amboceptor are arbitrary and fixed constants, and are used for determining the amount of complement necessary to bring about complete hemolysis.

In conducting the main tests the dose of corpuscles and that of amboceptor are the same as those used in the complement titration, but instead of using exactly one unit of complement, it is necessary to use one and one-half or two units, to allow for the anticomplementary action of antigen and patient's serum. I have long used the former dose when testing serums not more than three days old, as the extra half-unit is all that need be allowed for these anticomplementary influences. With older serums it is well to use two units, and this is true also when using antigens reënforced with cholesterin, as the latter is well known for its antihemolytic properties.

As the result of a large number of comparative titrations and tests I have found that 0.05 c.c. of complement serum (=1 c.c. of a 1:20 dilution) is a safe amount to use with 2.5 per cent. corpuscle suspension, and equally good results are secured by adopting this amount as a fixed dose in titrating the amboceptor before each day's work. If the pig serum happens to be weaker or stronger in complement than it is expected to be, the differences are adjusted in the amboceptor titration. Following the same rule, one and one-half or two units of amboceptor are used in conducting the main test, the extra half, or one unit, overcoming the anticomplementary action of antigen and patient's serum.

It is important to remember that, in conducting this titration, amboceptor, complement, and corpuscles are to be mixed at once; if amboceptor and corpuscles are mixed and allowed to stand for ten minutes or more before receiving the complement the corpuscles become "sensitized," and the amount of complement required for effecting 
hemolysis will be less than if all three are mixed one after another.) If this rule is not adhered to, an error in technic may result. On p. 447 another method of complement titration is given, using corpuscles previously sensitized for at least half an hour at room temperature; this method is to be preferred, and is the one I usually employ when titrating complement.

III. Hemolytic Amboceptor.-Since the original work of Wassermann appeared, the antisheep hemolytic system has been most widely used in experimental investigations.

Antisheep amboceptor is readily prepared by immunizing rabbits with washed sheep's corpuscles. A simple and efficient method is to give intravenous injections of four doses of 5 c.c. each of a 10 per cent. suspension every three or four days. Other methods and the details of the preparation and preservation of amboceptor are given in Chapters IV and V.

The one objection to the use of the antisheep hemolytic system is the presence, in a large proportion of human serums, of variable amounts of natural amboceptor for sheep's cells. In about 70 per cent. of fresh inactivated human serums sufficient amboceptor is present partially or completely to hemolyze the usual dose of sheep-cell emulsion with the customary amount of guinea-pig complement. In fact, the Bauer and Hecht modifications of the Wassermann reaction utilize this natural amboceptor, but, as will be pointed out further on, this factor is too variable to be employed in conducting the reaction, as non-specific or false positive results are quite likely to occur.

As has been stated in the preceding chapter, the delicacy and accuracy of any complement-fixation test depend to a large extent upon proper adjustment of the hemolytic system.) It will readily be understood that the presence of an unknown quantity of natural amboceptor in a serum is a drawback to accurate quantitative estimations. The importance of this lies in the fact that for some unknown reason an excess of amboceptor may completely hemolyze the corpuscles, even though a small amount of the necessary complement has been specifically fixed by antigen and syphilis antibody. In this manner negative reactions may result with serums that would otherwise show a slight positive reaction. To remove this source of error Noguchi has advocated the use of an antihuman hemolytic system, which renders the reaction more delicate. However, comparative studies between antisheep and other hemolytic systems demonstrate that, with proper technic, the influenee of natural amboceptors may be reduced to a minimum and rendered almost neg- 
ligible. At any rate it is very simple and but little trouble to remove the antisheep amboceptor routinely from human serums previous to making the tests) (for technic, see p. 378).

A method for titrating immune amboceptor has been given on p. 375 . This important feature will be dealt with again in giving a detailed description of the various methods that follow.

IV. Red Blood Corpuscles.-Defibrinated sheep blood is washed three times with an excess of sterile normal salt solution to remove all traces of serum (p. 28).

In the original Wassermann reaction a 5 per cent. suspension in salt solution is used in doses of 1 c.c. This emulsion is quite heavy, and sharper and clear results are secured by using just half this amount and at the same time sufficient cells are used to make the readings easy and distinct. Either 0.5 c.c. of a 5 per cent. or 1 c.c. of a 2.5 per cent. suspension may be used. I have used the latter with entire satisfaction for several years.

The emulsion of cells is prepared with sterile 0.85 per cent. sodium chlorid solution. To 2.5 c.c. of corpuscles sufficient salt solution is added to bring the total volume of the emulsion up to 100 c.c., or smaller amounts may be prepared by suspending 1 c.c. of corpuscles in 39 c.c. of salt solution.

Before each day's work the amount of corpuscle suspension needed should be calculated, and sufficient for the day prepared at one time, for if a fresh emulsion is prepared later, titration with the complement and amboceptor would be required. Attempts to count the corpuscles in suspension can only be regarded as approximate and are unreliable. By titrating each emulsion with the complement and amboceptor to be used, that particular emulsion is thereby adjusted, so that it is immaterial whether a few more or a few less corpuscles are present.

Sheep's blood is obtained either from an abattoir or by bleeding an animal from the external jugular vein (p. 48). The latter method is preferable, but due care must be exercised not to bleed too frequently or in excessive amounts, as if anemia occurs the corpuscles become unduly fragile.

Sheep's cells are easily preserved in a satisfactory condition for forty-eight hours by first washing them and then storing the sedimented corpuscles in a good ice-chest. Suspensions are less easily preserved. It is best to use fresh corpuscles, and those that are several days old and unduly fragile should never be used. Attempts to preserve corpuscles with the aid of mercury bichlorid, formalin, etc., have not yielded satisfactory results. 
V. Antigen.-As was previously stated, the ordinary alcoholic extracts of syphilitic livers used as "antigens" in conducting the Wassermann reaction are not biologically specific. It is generally accepted that even in watery extracts of syphilitic livers the main antigenic principles are lipoidal substances, independent of the Treponema pallidum itself. Next to pure cultures of pallida, these aqueous extracts of luetic livers come closest to being a specific biologic antigen. Although alcoholic extracts of luetic liver may contain special lipoidal substances that enhance their efficacy as antigens, yet, as shown by Noguchi, and as confirmed later by us, alcohol does not serve well to extract pure cultures of pallida, and therefore these extracts can hardly be regarded as specific, in the sense that they contain antigenic principles of the spirochetes themselves. The only specific biologic antigen is an aqueous extract of a pure culture of pallida; this antigen is, however, much less serviceable than an ordinary organic extract, because the Wassermann reaction depends upon the peculiar lipodophilic "reagin," which absorbs complement with lipoids in a characteristic but biologically non-specific manner.

The term "antigen," as ordinarily used in the Wassermann reaction, must therefore be regarded as a misnomer.) It is, however, so generally used that it may be retained, with a distinct understanding as to its actual meaning.

With the discovery that alcoholic extracts of normal organs may serve as antigen and that the chief antigenic principles reside in the lipoids, it followed that extensive researches were undertaken in the hope of discovering a lipoid, or a combination of lipoids, that would prove sufficiently delicate to act specifically in the serum diagnosis of syphilis, and not with normal serums or those of persons suffering from other diseases. As a result, a large number of different extracts are in use. Each has its own advocates, so that the general subject of antigens is the most complicated one with which we have to deal in performing the Wassermann reaction.

While various organic extracts may be used, practical experience has shown that some are better than others. It is important to remember:

1. That all antigens are capable in themselves of absorbing a certain amount of complement. This is due to the presence of undesirable extractives, some preparations containing more than others. In certain doses, however, all antigens are capable of exerting this anticomplementary action, and, other things being equal, that antigen is best which shows this non-specification in the smallest degree. Before an antigen 
may be used in conducting any complement-fixation test it is necessary to ascertain its anticomplementary dose, for if this dose were used, a portion of or all the complement would be fixed in a non-specific manner, so that hemolysis, being partial or absent, yields false positive reactions.

2. Most antigens, when in sufficiently large amounts, are hemolytic, ) $i$. e., they may hemolyze corpuscles in a non-specific manner.) This hemolytic action is usually due to the presence of undesirable extractives, and extracts of organs that have undergone advanced autolysis or fatty degeneration are known to contain more of these hemolytic substances than do extracts of normal organs. As a general rule, a highly anticomplementary antigen is likely to be correspondingly highly hemolytic. The hemolysis may be due to the presence of lipoidal substances or to the alcohol used in preparing the extract. If an antigen were used in an amount equal to its hemolytic dose, partial or complete hemolysis would occur in all tubes, so that a false negative result would be secured. As a rule, the hemolytic dose of an antigen as determined by titration in the presence of serum is larger than the anticomplementary dose, so that if the latter is known, it is not always necessary to determine the former.

3. Practically every alcoholic organic extract will serve, in certain amounts, to absorb complement in the presence of the serum of a syphilitic person.) Some extracts, however, will do this better than others. The Wassermann reaction depends upon the fact that a larger amount of complement is fixed by the syphilis antibody and extract than is fixed by normal serum or the serum of a person with some disease other than syphilis and this same extract. The only two notable exceptions to this general rule are to be found with the serum of tuberous leprosy and that of frambesia. (The amount of antigen that is found, by a process of titration, to fix a large amount of complement with a constant dose of syphilitic serum is known as its antigenic dose.) Not every lipoid serves equally well as antigen, and therefore considerable research work has been done in the hope of discovering an extract or a combination of lipoidal substances that would show a constant reaction and would react only with the syphilis antibody. Thus far this has not been accomplished; unfortunately, pure pallida antigens are not entirely specific or serviceable. and if the specific and ideal antigen is discovered in the future it will probably be of the nature of a lipoidal substance, altered or produced in a specific manner by the Treponema pallidum itself. In the meantime we have antigens sufficiently delicate and specific, when properly used, to render the Wassermann reaction of great value in the - diagnosis of syphilis and to serve as a guide to its treatment. 
From a practical standpoint, therefore, to be suitable for use as antigen in the syphilitic reaction any extract or preparation must fulfill the following requirements:

1. It should be largely free from anticomplementary action.

2. It should likewise be free from hemolytic action, in small doses at least.

3. It should possess a high degree of sensitiveness for the syphilitic antibody, $i$. e., be capable of absorbing relatively large amounts of complement in the presence of syphilitic serum. A good antigen is one that, in small amounts, is perfectly antigenic, and that does not become anticomplementary or hemolytic until from four to ten times this amount is used.

4. It should be quite stable and not difficult to prepare, and different preparations should bear a certain relationship to one another in their properties - that is they should keep well, and different extracts prepared in the same manner should show fairly constant antigenic, anticomplementary, and hemolytic doses.

Preparation of Antigens. - The following antigens have been most widely used and recommended:

1. Aqueous extract of syphilitic livers.

2. Alcoholic extracts of syphilitic liver.

3. Alcoholic extracts of normal organs.

4. Alcoholic extracts of normal organs reënforced with cholesterin.

5. Acetone-insoluble lipoids.

6. Lecithin and cholesterin.

7. Aqueous extracts of pallidum culture.

1. Aqueous Extracts of Syphilitic Livers.-This is the original antigen, as employed by Wassermann, Neisser, and Bruck; Wassermann still uses these extracts in preference to others. They may contain spirochetes or their direct derivatives, and, as shown originally by Wassermann and Neisser, may be true biologic antigens, for when injected into monkeys, antibodies are formed.

No satisfactory analyses of these extracts have been made. Chemically they differ in no essential respect from the liver of acute yellow atrophy (Ehrmann and Stern; Seligman and Pinkus). As antigen they are more efficient than similar extracts of normal liver. The nature of the specific factor has not yet been demonstrated with certainty. They react with the serums of leprosy and yaws, and, as in the case of other antigens, their main antigenic principle is apparently due to the presence of lipoids. 
They are less stable than alcoholic extracts, and are likely to become highly anticomplementary and lose their power of reacting with syphilitic serums. Citron is convinced that these changes are brought about by careless handling of the extract, or its exposure to the light. $\mathrm{He}$ recommends that the extract be kept constantly in the ice-chest, and that it be kept out only long enough to remove sufficient for the day's work.

Preparation.-The fresh liver taken from a syphilitic fetus, and showing the presence of spirochetes by dark-ground illumination, is* weighed and cut into fine pieces. Four times its weight of 0.5 per cent. phenol in normal salt solution is added. The mixture is placed in a brown bottle and shaken mechanically at room temperature for twenty-four hours. It is then filtered through gauze, to remove the larger particles, and stored in a brown bottle in an ice-chest. After several days of sedimentation the fluid assumes a yellowish-brown copalescence and is ready for the preliminary titration to determine its anticomplementary and hemolytic doses. The sediment should not be disturbed, but the supernatant fluid should be carefully removed by means of a pipet. According to Citron, extracts that must be used in quantities of less than 0.1 c.c. are, as a general rule, unsatisfactory. Only such extracts should be used as in doses of 0.4 c.c., will not interfere with hemolysis. The method of making these titrations is given on page 428 .

\section{Alcoholic Extracts of Syphilitic Livers.-These antigens are} extensively used. They are not true biologic antigens, for they do not give rise to antibodies (Schatilof and Isabolinsky; Seligman and Pinkus); they are, however, usually better antigens than similar extracts of normal liver, a fact that may be explained, in part at least, by chemical changes, namely, fatty changes, autolysis, soaps (Beueker), excess of cholesterin (Piglini), etc., which, while not specifically syphilitic in nature, are often produced to a striking degree in congenital syphilis.

Preparation.-Fetal liver known to contain numerous spirochetes is used in preparing this extract. Fresh organs may be examined at once by dark-field illumination, or if this is impossible and the fetus shows signs of syphilis, the liver may be cut into large pieces and preserved in 70 per cent. alcohol. After a few days a section is removed and stained by the Levaditi method for spirochetes. If these microörganisms are numerous, the liver is suitable for preparing the antigen; otherwise it should be discarded. Very fatty livers are to be avoided, and those of still-born fetuses are to be preferred.

Ten grams of liver are minced, ground with quartz sand, and treated with 100 c.c. of absolute ethyl alcohol. The mixture is shaken mechanically with glass beads for twenty-four hours, and extracted in the incubator for ten days. The containing flask or bottle should be well stoppered to prevent undue evaporation, and should be shaken up at least once a day. The extract is then filtered through fat-free paper or paper washed with ether and alcohol to remove the hemolytic substances that may be present. The filtrate is measured, and the loss by evaporation is made up by the addition of more alcohol. If a shaking apparatus is not at hand, extractions may be left in the incubator a few days longer. After standing a few days a sediment forms, which should not be removed or disturbed.

3. Alcoholic Extracts of Normal Organs. - These are used extensively, at present, and apparently yield results equal to those obtained with extracts of luetic liver. It is certainly true that a good extract of a normal organ is superior to a poor one prepared from luetic liver. Many, with 
the idea of specificity uppermost in their mind, adhere to the use of the latter, whereas the results of research and of practical work shows that lipoids from normal organs serve equally well as antigen in making the syphilitic reaction, and, indeed, may prove superior if luetic liver is used that has undergone advanced fatty changes or autolysis, when undesirable hemolytic and anticomplementary derivatives are extracted in excess.

Human, guinea-pig, and beef-heart muscle are usually employed. The first is especially efficient and is to be preferred.

Preparation.-The organ is obtained fresh from the autopsy room. It is freed from fat, and to each 10 grams of minced muscle 100 c.c. of absolute ethyl alcohol are added. Extraction is conducted in exactly the same manner as was described in the preparation of alcoholic extract of luetic liver.

If guinea-pig heart is employed, as much of the fat as possible should be removed, otherwise the extract may be quite anticomplementary.

Boas prepared an extract of human heart by treating the ground muscle with nine parts of absolute alcohol, shaking for an hour at room temperature, filtering, and storing away in a stoppered bottle. He found that different extracts so prepared are remarkably constant in their properties, although they deteriorate rapidly and should be prepared freshly every few weeks.

\section{Alcoholic Extracts of Normal Organs Reënforced with Choles-} terin.- Sachs advocated the addition of pure cholesterin to alcoholic extracts of normal heart as a means of rendering these antigens more delicate, without materially increasing their anticomplementary and hemolytic properties. He found that such preparations possess properties equal to the best syphilitic extracts. This work has been confirmed by Hemlein, Altman, McIntosh and Fieldes, Desmonliere, Walker, and Swift. We have studied the subject with much interest, comparing the results with those secured from other antigens, as alcoholic extracts of syphilitic liver and acetone-insoluble lipoids. It is true that these preparations are highly sensitive-so much so that I never employ them alone in making diagnostic reactions, but always in conjunction with other extracts as controls, in order to detect and avoid non-specific reactions with non-luetic serums. We have found that they occasionally give faint positive reactions with normal serums; on the other hand, not infrequently, they react strongly positive in cases where, with other extracts, the reactions are negative; in the majority of such cases the serum is from a long-standing or a treated case of lues that needs further treatment until the reaction with a cholesterin extract becomes negative. These alcoholic extracts of normal organs have their greatest value, therefore, with known syphilitic serums when the reaction is conducted as a guide to treatment. In diagniostic reactions, however, it is my opinion that they should not be used alone, but together with less 
sensitive antigens. In other words, one should use every precaution and exercise great care before making a diagnosis; when lues is known to be present, however, the treatment should be thorough, and there would seem to be no better criterion for judging the state of the infection than repeated negative reactions with cholesterin extracts.

Preparation.-These extracts are prepared of human, ox, and guinea-pig heart. Human heart usually yields the best extract. Care should be taken to use only muscle and to avoid fat. To 10 grams of minced muscle add 100 c.c. of absolute ethyl alcohol. Shake in a mechanical shaker for twenty-four hours, and continue the extraction in the incubator for ten days or two weeks. Then filter through fatfree filter-paper, and add absolute alcohol to make up for the loss through evaporation. Add 0.4 gram of Kahlbaum's cholesterin ( 0.4 per cent.); shake well and stand aside in the refrigerator for a few days. The cholesterin goes into solution slowly in cold alcohol, and 0.4 per cent. of cholesterin usually saturates the solution. After a week the extract may be again filtered and stored in a tightly stoppered bottle. The slight sediment that may form should not be disturbed.

These extracts keep fairly well. Different preparations are quite similar in their properties; they are usually found to be highly antigenic and no more anticomplementary than crude alcoholic extracts. They constitute, therefore, inexpensive and very sensitive antigens.

5. Acetone-insoluble Lipoids.-As previously stated, crude alcoholic extracts may contain an excess of undesirable constituents, such as neutral fats, fatty acids, soaps, and certain protein materials, which are responsible for the untoward anticomplementary and hemolytic effects. To eliminate these Noguchi advised the exclusive use of the acetone-insoluble fraction instead of the entire unfractionated alcoholic extract, especially if unheated human serums are used in conducting the syphilis reaction. These extracts are composed essentially of lecithins, which, when prepared from any one source, consist of a mixture of analogous bodies; lecithins from different sources vary in their composition. In speaking of lecithins, one is prone to regard them as chemicals, and to overlook their biologic properties. Noguchi no longer employs the term lecithin to designate the acetone-insoluble fraction of tissue lipoids.

These antigens are readily prepared of ox-heart or of human liver, the former being preferable for use. Their main disadvantage is the expense of preparation, for it may be necessary to prepare several extracts before one that is satisfactory is secured. A good extract will, however, keep well, and is a reliable and valuable antigen for the testing for the syphilitic reaction.

Preparation.-A mashed paste of the muscle of ox-heart is extracted with 10 parts of absolute alcohol at $37^{\circ} \mathrm{C}$. for four days. It is then filtered through filterpaper and the filtrate collected and brought to a state of dryness by evaporation. The use of the electric fan is not necessary, for if poured into large flat dishes, the filtrate will evaporate in from twelve to twenty-four hours. The residue is then taken up with a sufficient quantity of ether, and the turbid ethereal solution is allowed 
to stand for a few hours in a cool place until cleared. The clear ethereal portion is then carefully decanted off into another clean evaporating dish, and then allowed to become concentrated by evaporating the ether off. The concentrated ethereal solution is now mixed with about 10 volumes of pure acetone. A light yellow precipitate forms, which is allowed to settle, and the supernatant fluid is decanted off. Dissolve each $0.3 \mathrm{gm}$. of this substance in 1 c.c. of ether and add 9 c.c. of pure methyl alcohol. As a rule, the greater part of the substance goes into solution. This alcoholic solution remains unaltered for a long time, and is kept as a stock solution from which the emulsion for immediate use may be prepared at any time by mixing 1 c.c. with 19 c.c. of saline solution. This solution is then titrated for antigenic, anticomplementary, and hemolytic action.

According to Noguchi, if the extract is anticomplementary or hemolytic in doses of 0.4 c.c. of a $1: 10$ dilution, it is unsuitable. If it produces complete inhibition of hemolysis with 0.1 c.c. of syphilitic serum in doses of 0.02 c.c. or less of the same dilution ( $=0.2$ c.c. of a $1: 100$ dilution), it is suitable. In making the fixation test, 0.1 c.c. of a $1: 10$ emulsion is to be used, thus employing five times the minimal antigenic dose which does not cause non-specific fixation and is not unduly sensitive.

6. Lecithin and Cholesterin.-Browning, Cruickshank, and McKenzie advocate the use of a mixture of ox-liver lecithin and cholesterin as antigen in testing for the syphilitic reaction. They find that the amount of complement fixed by lecithin alone with syphilitic serums is very much increased by the addition of cholesterin; that cholesterin does not increase the anticomplementary action of the antigen; that the binding power or antigenic value of a mixture of lecithin and cholesterin is equal in most cases to crude alcoholic extracts, but is less anticomplementary. These observers use this mixture in their modified method, which consists in the accurate estimation of the amount of complement fixed by extract and syphilitic serum. It would seem that this extract should, in the ordinary methods, be controlled with less sensitive antigens, and I have found that ordinary cholesterinized alcoholic extracts of human heart are equally efficient and less difficult to prepare in performing a quantitative Wassermann reaction after the method just outlined.

Preparation.-Minced ox-liver, obtained within three or four hours after death, is digested with four parts of 95 per cent. alcohol for three or four days at room temperature, during which time the mixture is stirred up at least once a day. The filtrate is evaporated in an open flat porcelain dish on the water-bath at $60^{\circ} \mathrm{C}$. for four or five hours until a syrupy mass remains. This is rubbed up with washed and dried quartz sand until a firm mass results. The mixture of dried extract and sand (about 50 grams of sand to the residue of 1000 c.c. of extract) is placed in a spheric flask, closed with a perforated rubber stopper through which runs a short piece of quill tubing drawn out to a capillary point at the end. This tube serves to prevent the vapor in the flask from forcing out the stopper. Ethyl acetate is placed in the flask, which is then stoppered and immersed up to its neck in water at $60^{\circ} \mathrm{C}$. The flask is shaken repeatedly, and after ten minutes the ethyl acetate is poured off into a hot-water filter, the funnel jacket being kept at $60^{\circ} \mathrm{C}$., and the solution filtered through fat-free filter-paper. Another portion of ethyl-acetate is added to the sand, and the extraction repeated. After a third treatment practically all the soluble matter will have been extracted. In all, about 170 c.c. of ethyl acetate should be used to 
extract the residue of $1000 \mathrm{c}$ c. of crude extract. The portion that is precipitated when the ethyl acetate solution is placed in the ice-chest overnight is again dissolved in ethyl acetate at $60^{\circ} \mathrm{C}$. and the solution again placed in the ice-chest overnight. Finally the portion insoluble in ethyl acetate in the cold is dissolved in water-free ether (sp. gr., 0.717) at room temperature. To the ethereal solution in a glass cylinder four volumes of acetone are added, causing a precipitate to be deposited. Precipitation is aided by shaking the mixture for several minutes. Separation is complete in ten minutes. The supernatant fluid is poured off, and the crude precipitate of lecithin is redissolved in ether and the precipitation with acetone repeated twice. The precipitate is finally rubbed up with sand and the soluble portion taken up by extraction with absolute ethyl alcohol for twenty-four hours at room temperature. The last traces of lecithin may be removed by extracting the residue with an additional small quantity of alcohol. The ordinary commercially "pure" reagents are satisfactory for this method of preparation of lecithin.

The lecithin of 1000 c.c. of crude extract should be extracted with about 100 c.c. of alcohol. The strength of the solution is estimated by evaporating 10 c.c. at $57^{\circ}$ C. and weighing. The alcoholic lecithin solution is kept in a stoppered bottle at room temperature in the dark. After allowing it to stand for a week a 0.75 per cent. solution in alcohol is diluted 1:7 with normal salt solution, to secure the maximum turbidity, and titrated. Browning and McKenzie use 0.6 c.c. of this emulsion as the antigenic dose.

7. Aqueous Extract of Pallidum Culture. This antigen is prepared by Noguchi, who uses pure cultures of Treponema pallidum in ascites kidney agar. Preferably several strains should be used in the preparation, which corresponds quite closely to luetin. Cultures grown seven, fourteen, twenty-one, twenty-eight, thirty-five, and forty-two days are chosen and examined, and those that show the best growths in the agar columns are selected. The oil is poured off, the tubes cut just above the kidney, and the column of ascites agar between the piece of kidney and the oil removed with particular care, so as not to include the kidney or the oil. This substance is ground in a mill until the spirochetes show disintegration. The thick emulsion is then diluted with normal salt solution and heated to $60^{\circ} \mathrm{C}$. for one-half hour; 0.4 per cent. phenol is added as a preservative, and the emulsion titrated for its anticomplementary dose. In conducting complement-fixation reactions with pallidum antigen one-half of the anticomplementary dose is used, and the serum must be inactivated.

\section{Comparative antigenic Values of Various Extracts}

For several years past I have been particularly interested in studying, from a practical standpoint, antigenic values of the extracts most commonly employed in the serum diagnosis of syphilis, and comparing them with suitable alcoholic extracts of syphilitic liver as a standard antigen.

For this purpose antigens were carefully chosen after titration, and only those were employed that were safely free from anticomplementary action and whose antigenic dose was known. A large number of serums and cerebrospinal fluids from syphilitic and non-syphilitic persons were 
tested with numerous different extracts at the same time, and under similar conditions. A suitable alcoholic extract of syphilitic liver was always included among the antigens in testing each serum or fluid, and the other extracts compared with it in determining their antigenic value.

In the following table the results of such studies, covering a period of two years, are given:

\section{TABLE 12.-COMPARATIVE ANTIGENIC VALUES OF VARIOUS TISSUE} EXTRACTS

\begin{tabular}{|c|c|c|c|c|c|}
\hline \multirow[b]{2}{*}{ Extracts } & \multicolumn{5}{|c|}{$\begin{array}{c}\text { Antigenio Properties as Compared to Alcoholic Ex- } \\
\text { tracts of SYPHILITIC Liver }\end{array}$} \\
\hline & Equal & Stronger & Weaker & $\begin{array}{c}\text { Negative } \\
\text { (Positive } \\
\text { with Alco- } \\
\text { holic Extract } \\
\text { of Syphilitic } \\
\text { Liver) }\end{array}$ & $\begin{array}{c}\text { Positive } \\
\text { (Negative } \\
\text { with Alco- } \\
\text { holic Extract } \\
\text { of Syphilitic } \\
\text { Liver) }\end{array}$ \\
\hline \multirow{2}{*}{\multicolumn{6}{|c|}{$\begin{array}{l}\text { Cholesterinized alcoholic ex- } \\
\text { tracts of human, pig, and } \\
\text { beef heart............ }\end{array}$}} \\
\hline & 50.0 & 30.0 & & & 20.0 \\
\hline $\begin{array}{l}\text { Acetone-insoluble lipoids... } \\
\text { Alcoholic extract of pig and }\end{array}$ & 73.0 & 10.8 & 9.7 & 3.2 & 1.8 \\
\hline beef heart .............. & 71.1 & 1.9 & 13.4 & 5.7 & 7.6 \\
\hline $\begin{array}{l}\text { Acetone extract of syphilitic } \\
\text { liver } \ldots \ldots \ldots \ldots \ldots \ldots\end{array}$ & 68.7 & 4.4 & 18.9 & 6.6 & 1.3 \\
\hline $\begin{array}{l}\text { Alcoholic extract of normal } \\
\text { liver } \ldots \ldots \ldots \ldots \ldots\end{array}$ & 73.2 & & 23.5 & 3.5 & \\
\hline
\end{tabular}

The results of these studies have shown:

1. That cholesterinized alcoholic extracts of human, beef, and guineapig heart are far more sensitive than simple alcoholic extracts of syphilitic liver. Another peculiar feature of these antigens is the fact that in syphilis, if they react at all, they usually do so quite strongly.

2. That the addition of cholesterin to crude alcoholic extracts of syphilitic liver and of normal liver doubles their antigenic sensitiveness without materially increasing their anticomplementary and hemolytic action.

3. We have practically never found a serum that reacted negatively with a cholesterin extract and positively with an alcoholic extract of syphilitic liver. On the other hand, in about 20 per cent. of cases the cholesterinized antigens will react positively, whereas with the plain antigen of syphilitic liver the reactions are negative. In the majority of such instances the person was known to be luetic, but had received treatment and was regarded clinically as cured, or the serum was that of a long-standing and unrecognized case of lues. Unfortunately, slight 
reactions may be secured with about 5 per cent. of normal serums. For this reason, when conducting tests for diagnostic purposes, I control the cholesterinized extracts with less sensitive antigens, such as alcoholic extract of syphilitic liver and acetone-insoluble lipoids.

4. It is highly important that these extracts be carefully standardized and that any serum, even if but slightly anticomplementary, be discarded.

5. In our experience, repeated negative reactions with satisfactory cholesterinized antigens constitute the best evidences of the absence of lues or testify to the recovery from a luetic infection. The treatment of syphilis should be continued until the patient's serum reacts negatively with alcoholic extract of syphilitic liver, and finally with cholesterinized extracts. The disease cannot be regarded as cured until the reaction has remained negative for a year or two at least, and treatment must not be discontinued until this result is secured, or it is shown that the serum is "Wassermann fast" and that it is impossible to secure a negative reaction.

6. For the less experienced worker, or when but one antigen is being used in conducting the reaction, a properly prepared alcoholic extract of syphilitic liver is to be recommended. One drawback to the use of this extract is the difficulty of obtaining suitable tissues for the preparation of the antigen. It has been my practice for many years to preserve the livers of as many still-born fetuses as I could obtain in 70 per cent. alcohol, and to discard them later unless on section they showed the presence of numerous spirochetes. These antigens are usually more sensitive than similar extracts of normal liver, but it is important to remember that not every extract is satisfactory simply because it is prepared of syphilitic tissues.

7. A suitable preparation of acetone-insoluble lipoids, prepared after the method of Noguchi, constitutes a sensitive, reliable, and satisfactory antigen. When properly titrated and standardized and used with inactivated serums, this antigen may prove quite sensitive and safe. Noguchi, in his efforts to simplify the technic of the syphilis reaction, impregnated filter-paper with this antigen and allowed it to dry. This preparation is unstable and generally unsatisfactory. The antigen is best preserved in a stock bottle or in ampules, and is diluted with salt solution just before being used in the test. Under these conditions we have found these extracts to be quite stable.

4. Plain alcoholic extract of human, guinea-pig, and beef heart are easily prepared, are quite inexpensive, and when properly standardized 
serve as satisfactory antigens. Boas uses alcoholic extracts of human heart (Michaëlis) exclusively, and has found that they yield better results than alcoholic extracts of syphilitic liver. Garbat and others use and recommend similar extracts of guinea-pig heart.

8. Aqueous extracts of pure cultures of pallida have not thus far yielded results equal or superior to ordinary non-specific antigens. As compared with lipoidal extracts, they have generally yielded reactions that are much weaker, and in primary and secondary syphilis may react entirely negatively. Much is yet to be learned, however, of bacterial antigens in general, and the subject must be regarded as still in the experimental stage.

It may be said to be well proved that extract antigens in the syphilis reaction are not biologically specific, and need not be extracts of syphilitic tissues. An antigen cannot give reliable or satisfactory results unless it is carefully titrated and its properties determined. Antigens may serve as a frequent source of error when the complement-fixation reactions are conducted by those possessing insufficient knowledge of their good and bad properties. The test for the syphilitic reaction should not be undertaken by any one not competent to titrate and judge of the qualities of the antigen to be employed.

Method of Diluting Antigens. As a general rule, all organic extracts must be diluted with normal salt solution before being used.

If extracts and diluent are mixed quickly, the emulsion is clear or slightly opalescent. If the diluent is added slowly to the organic extract, the resulting mixture becomes quite turbid and milky. As shown by Sachs and Roudoni, the antigenic power of the extract is more marked with the turbid than when the clear or opalescent emulsion is used. For this reason, in testing for the syphilis reaction the emulsion of organic extract should be made so as to secure the maximum amount of turbidity. The required amount of antigen is placed in a test-tube, and the salt solution is added slowly with a pipet; or the salt solution may be placed in a tube and the extract floated on it and gradually mixed.

Although with each new extract it may be necessary to titrate with various dilutions before one that is satisfactory is reached, experience has shown in the majority of instances that the following dilutions are usually correct:

1. Alcoholic extract of syphilitic liver: 1 part with 9 parts of salt solution. Extracts of German manufacture are usually diluted six or seven times.

2. Cholesterinized extracts and acetone-insoluble lipoids in methyl 
alcohol require higher dilution, as 1 part of extract with 19 parts of salt solution.

3. Plain alcoholic extracts of human, beef and guinea-pig heart likewise require high dilution, as, for example, extract, 1 part and salt solution, 19 parts.

4. Alcoholic extract of human heart, prepared after the method of Boas (quick method), is used in lower dilution, as 1 part of extract with 9 parts of salt solution.

5. The solution of lecithin and cholesterin used by Browning, Cruickshank, and Mackenzie is diluted with seven parts of salt solution.

6. Aqueous extracts of syphilitic liver and extracts of pallida culture are used undiluted, or may require dilution with four parts of salt solution.

Although these emulsions will keep for a few days if placed in the refrigerator, it is advisable to make up only the amount required for immediate use, as freshly prepared emulsions are better than older ones.

Method of Titrating Antigens. Three values are to be determined:

1. The anticomplementary dose, or that amount of antigen that in itself is capable of fixing or inactivating the complement.

2. The hemolytic dose, or that amount of antigen that in itself is capable of lysing red blood-cells. This action is probably due to the presence of certain lipoids and alcohol.

3. The antigenic dose, or that amount of antigen that serves to absorb or fix a certain and constant dose of complement with a definite amount of syphilitic serum.

- 1. Anticomplementary Titration. The determination of the anticomplementary dose is probably the most important, for if an extract were used in an amount that was anticomplementary or capable of fixing complement in a non-specific manner, all the tests would show false positive reactions, regardless of whether the serum was from a normal or from a luetic person.

After determining this anticomplementary dose, in conducting the main test the antigen may be used in one-fourth the amount.

All tests are conducted with chemically clean and preferably sterile test-tubes $(12 \mathrm{~cm}$. by $13 \mathrm{~mm}$.) and graduated pipets. Accuracy in measurements is very essential in performing all complement-fixation work.

A preliminary titration of the hemolysin is made, with the complement and corpuscle suspension to be used in titrating the antigen, in order to determine its hemolytic dose, $i$. e., to adjust the hemolytic system. If, 
for instance, the hemolytic serum is known to have a titer of about $1: 2000$ (see p. 375), a stock solution is made by diluting 1 c.c. of the serum with sterile salt solution to make a dilution of $1: 200$. In a series of six test-tubes increasing amounts of this diluted amboceptor are placed: 0.05 c.c., 0.1 c.c., 0.15 c.c., 0.2 c.c., 0.3 c.c., and 0.4 c.c.; to each tube add 1 c.c. of complement serum diluted $1: 20$ ( $=0.05$ c.c. undiluted serum) and 1 c.c. of a 2.5 per cent. suspension of sheep's cells and sufficient salt solution to bring the total contents up to 3 or 4 c.c. Each tube is shaken gently and incubated at $37^{\circ} \mathrm{C}$. for an hour. At the end of this time that amount of amboceptor that just completely hemolyses the corpuscles is taken as the hemolytic unit (Fig. 103). In conducting the antigen titrations one and one-half or twice this amount is used as the dose.

The antigen extract is diluted $1: 10$ by placing 1 c.c. in a test-tube and slowly adding 9 c.c. of normal salt solution.

Increasing amounts of this emulsion are placed in a series of testtubes: $0.2,0.4,0.6,0.8,1,1.2,1.5$, and 2 c.c. To each tube are now added 1 c.c. of the diluted complement serum $(=0.05$ c.c. undiluted serum) and sufficient normal salt solution to bring the total volume up to 3 or 4 c.c. Shake each tube gently and incubate for one hour at $37^{\circ} \mathrm{C}$. Then add to each tube 1 c.c. of the corpuscle suspension and a dose of amboceptor equal to $1 \frac{1}{2}$ units, as just determined by previous titration. Shake gently and reincubate for another hour and a half, when a preliminary reading of the results may be made. That amount of antigen that shows beginning inhibition of hemolysis is regarded as the anticomplementary unit. The final readings are made after the tubes have stood overnight in a refrigerator at low temperature (Fig. 108).

This titration may also be made in the presence of normal serum, although this is not absolutely necessary. The serum must be perfectly fresh, and must be that from a person known to be free from lues. It is inactivated by heating to $55^{\circ} \mathrm{C}$. for half an hour, and 0.1 c.c. is added to each tube. Complement and salt solution are now added, and the titration conducted in the manner just described. Normal serum may absorb a small amount of complement in itself, and hence a titration conducted with serum may show a slightly lower anticomplementary dose.

The following controls are included:

1. A hemolytic system control, containing the complement, corpuscles, and amboceptor in the same amounts as were used in conducting the titration. This control should show complete hemolysis.

2. A serum control, which is the same as the hemolytic system control 
plus 0.1 c.c. of the serum. This should show complete hemolysis, and indicates that the serum was not anticomplementary. This control test should never be omitted.

3. A corpuscle control, including 1 c.c. of the corpuscles in salt solution. This tube should show no hemolysis.

The following table gives the results of a titration with an alcoholic extract of syphilitic liver diluted 1:10 (see Fig. 108):

TABLE 13.-ANTICOMPLEMENTARY TITRATION OF AN ORGANIC EXTRACT

\begin{tabular}{|c|c|c|c|c|c|c|}
\hline TUBE & $\begin{array}{l}\text { ANTIGEN } \\
(1: 10) \\
\text { C.c. }\end{array}$ & $\begin{array}{c}\text { CoMPLE- } \\
\text { MENT } \\
(1: 20) \\
\text { C.C. }\end{array}$ & $\begin{array}{l}\text { NORMal } \\
\text { SERUM } \\
\text { C.c. }\end{array}$ & $\begin{array}{c}\text { ANTISHEEP } \\
\text { HEMOLYSIN } \\
\text { UNITS }\end{array}$ & $\begin{array}{c}\text { SHEEP's } \\
\text { CORPUS- } \\
\text { CLES } \\
2.5 \text { PER } \\
\text { CENT., C.C. }\end{array}$ & REsULts \\
\hline 1. & 0.2 & 1 & 0.1 & $11 / 2$ & 1 & Hemolysis \\
\hline 2. & 0.4 & 1 & 0.1 & $11 / 2$ & 1 & Hemolysis \\
\hline 3. & 0.6 & 1 & 0.1 & $11 / 2$ & 1 & Hemolysis \\
\hline & 0.8 & 1 & 0.1 & $11 / 2$ & 1 & Hemolysis \\
\hline 5 & 1.0 & 1 & 0.1 & $11 / 2$ & 1 & Hemolysis \\
\hline 6 . & 1.2 & 1 & 0.1 & 11/? & 1 & $\begin{array}{l}\text { Slight inhibition of } \\
\text { hemolysis }\end{array}$ \\
\hline 7. & 1.5 & 1 & 0.1 & $11 / 2$ & 1 & Marked inhibition of \\
\hline 8. & 2.0 & 1 & 0.1 & $11 / 2$ & 1 & Complete inhibition \\
\hline 9. & Control & 1 & 一 & $11 / 2$ & 1 & Hemolytic control: \\
\hline 10. & Control & 1 & 0.1 & $1 \frac{1}{2}$ & 1 & $\begin{array}{l}\text { Serum control: com- } \\
\text { plete hemolysis }\end{array}$ \\
\hline
\end{tabular}

In this titration tube No. 6 , containing 1.2 c.c. of the antigen emulsion showed beginning inhibition of hemolysis and was recorded as the anticomplementary dose.

2. Hemolytic Titration. As previously mentioned, organic extracts are capable in themselves of hemolyzing red cells; this is due to the hemotoxic action of lipoids and alcohol. Extracts of organs that have undergone advanced autolysis and decomposition are very likely to be hemolytic.

Serum exerts an inhibiting influence on the lytic action of an organic extract. Hence the hemolytic dose of an extract depends largely on whether or not complement serum is used in the titration.

When an organic extract is titrated in the presence of complement, the hemolytic dose is higher than the anticomplementary dose. In the foregoing titration 3 c.c. of the extract emulsion showed beginning hemolysis, and when 4 c.c. was used, hemolysis was complete. These large amounts of emulsion give the tube contents quite a milky appearance, but close inspection shows that all the cells are broken up. 
$+$

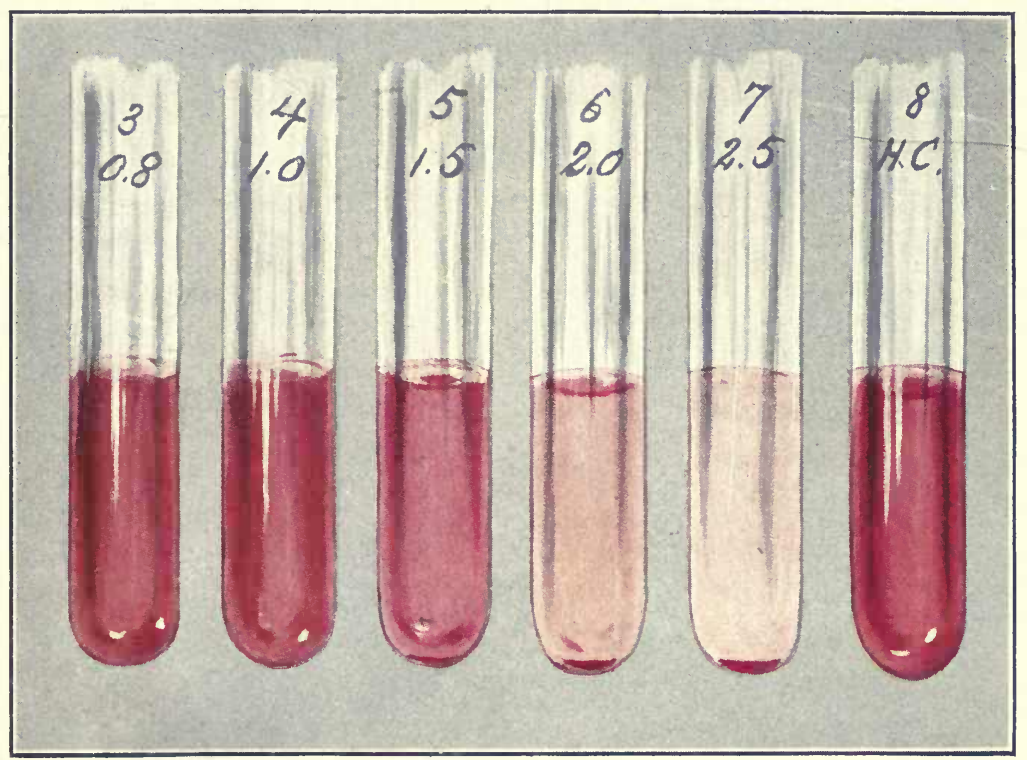

Fig. 108. - Titration of Antigen for Anticomplementary Unit.

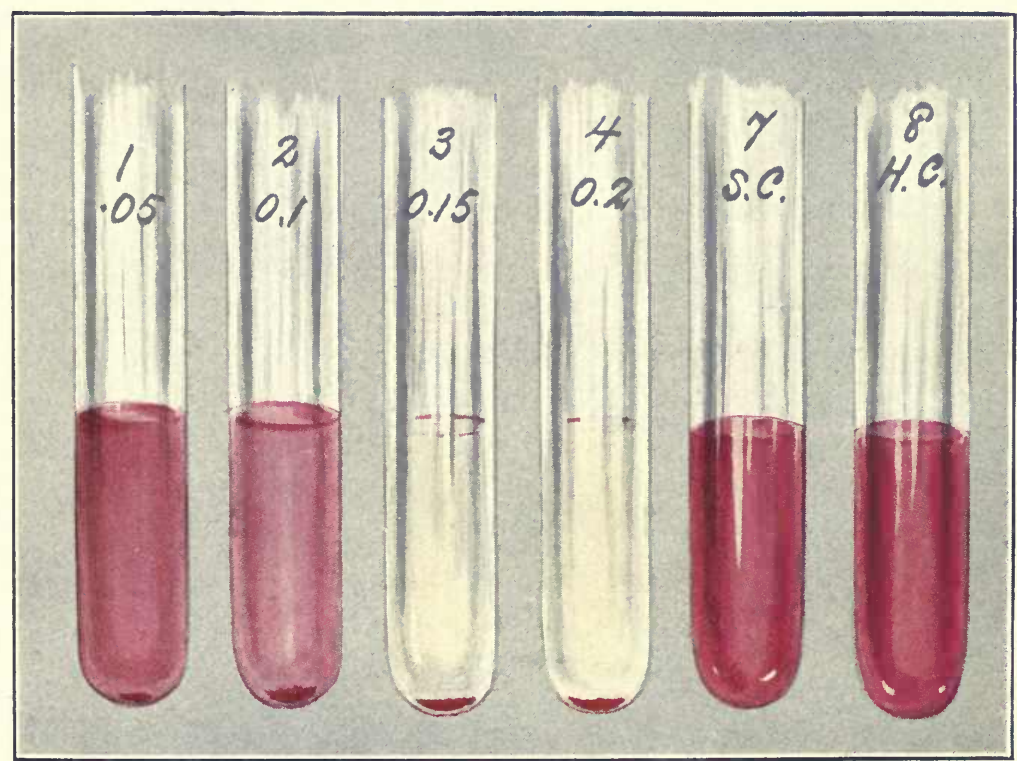

Fig. 109.-Titration of Antigen for Antigenic Unit. 

As a general rule, the hemolytic titration is not absolutely necessary. It may be conducted with the anticomplementary titration by adding another tube or two to the foregoing series, with higher doses of extract; or this titration may be conducted separately, and without complement and hemolysin, by using the same doses of antigen with 1 c.c. of corpuscle suspension and sufficient salt solution to bring the total volume in each tube up to 3 or 4 c.c.

3. Antigenic Titration.-As previously stated, this titration is not absolutely necessary, as one-fourth the anticomplementary dose of an extract may be used in the main test. For instance, in the foregoing titration 0.3 or 0.4 c.c. may safely be used in making the test for the syphilitic reaction. Different extracts vary, however, in their antigenic value. Some may be highly anticomplementary and have a comparatively low antigenic value; purer extracts, such as acetoneinsoluble lipoids or cholesterinized alcoholic extracts of heart, are largely free from anticomplementary action, and at the same time possess a high antigenic value. It is advisable, therefore, to use an antigen whose full antigenic as well as anticomplementary doses are known, for, while it is necessary to use sufficient antigen, it is not advisable to use a larger amount than is necessary.

For this titration all antigens except alcoholic extracts of syphilitic liver, should be diluted 1:20 with normal salt solution. Usually the antigenic unit is so much lower than the anticomplementary unit that it is best determined with a more dilute antigen.

The titration is conducted in a manner similar to the anticomplementary titration, except that 0.1 c.c. of fresh and inactivated serum from a known and untreated syphilitic person is added to each tube. Increasing doses of antigen, patient's serum, and complement are mixed, shaken, and incubated for one hour. One and one-half doses of hemolysin and corpuscles are then added, the tubes shaken and incubated for another hour, after which the preliminary reading is made. The final reading is taken after the tubes have been placed overnight in a refrigerator at low temperature. That amount of antigen that shows just complete inhibition of hemolysis is taken as the antigenic unit (Fig. 109). In conducting the syphilis reaction two to four times this unit is used, providing that these amounts are at least four or five times less than the anticomplementary dose. This larger antigenic dose is advisable, because the exact unit may not be sufficient with serums containing but small amounts of syphilis antibody such as those of treated or long-standing cases of lues. 
The following table illustrates this titration with the same alcoholic extract of syphilitic liver (see Fig. 109):

TABLE 14.-ANTIGENIC TITRATION OF AN ORGANIC EXTRACT

\begin{tabular}{|c|c|c|c|c|c|c|c|c|}
\hline TUBE & $\begin{array}{l}\text { ANTI- } \\
\text { GEN } \\
1: 10, \\
\text { C.c. }\end{array}$ & $\begin{array}{c}\text { SYPHI- } \\
\text { LITIC } \\
\text { SERUM } \\
\text { (INACTIVE) } \\
\text { C.c. }\end{array}$ & $\begin{array}{l}\text { COM- } \\
\text { PLE- } \\
\text { MENT } \\
1: 20 \text {, } \\
\text { C.c. }\end{array}$ & 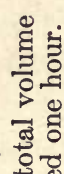 & $\begin{array}{c}\text { ANTI- } \\
\text { SHEEP } \\
\text { HEM- } \\
\text { OLYSIN, } \\
\text { DOSES }\end{array}$ & $\begin{array}{c}\text { SHEEP } \\
\text { COR- } \\
\text { PUSCLES } \\
2.5 \\
\text { PER } \\
\text { CENT., } \\
\text { C.c. }\end{array}$ & 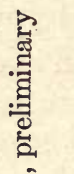 & Results \\
\hline 1.. & 0.05 & 0.1 & 1 & $=$ & $11 / 2$ & 1 & రై & Slight inhibition \\
\hline 2. & 0.1 & 0.1 & 1 & . & $11 / 2$ & 1 & ¿. & $\begin{array}{l}\text { Marked inhibi- } \\
\text { tion of hemoly- } \\
\text { sis }\end{array}$ \\
\hline 3 & 0.15 & 0.1 & 1 & 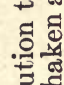 & $11 / 2$ & 1 & 疍 & $\begin{array}{l}\text { Complete inhi- } \\
\text { bition of hemol- } \\
\text { ysis }\end{array}$ \\
\hline & 0.2 & 0.1 & 1 & & $11 / 2$ & 1 & $\bar{z}$ & No hernolysis \\
\hline & 0.25 & 0.1 & 1 & & $11 / 2$ & 1 & สี & No hemolysis \\
\hline & 0.3 & 0.1 & 1 & 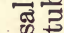 & $11 / 2$ & 1 & శี & No hemolysis \\
\hline & Control & 0.1 & 1 & $\exists$ & $11 / 2$ & 1 & ฮี & Serum control: \\
\hline 8 & Control & 0 & 1 & 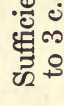 & $11 / 2$ & 1 & $\begin{array}{l}0 \\
0 \\
0 \\
0 \\
3 \\
1\end{array}$ & $\begin{array}{l}\text { Hemolytic con- } \\
\text { trol: Hemoly- } \\
\text { sis }\end{array}$ \\
\hline
\end{tabular}

In this instance 0.15 c.c. of the emulsion represents the antigenic unit. In performing the Wassermann reaction 0.3 or 0.4 c.c. was used, and these amounts were about one-fourth the anticomplementary dose.

It is not unusual to find cholesterinized alcoholic extract and acetone-insoluble lipoids perfectly antigenic in 0.05 c.c. of a 1:20 dilution, and not anticomplementary under 1 or 2 c.c. of a 1:10 dilution. In these instances four times the antigenic dose, or 0.2 c.c. can be used, and yet this amount is at least 10 times smaller than the anticomplementary dose-a condition of affairs that constitutes a safe and desirable antigen.

Each new antigen should be tested with a number of serums and controlled by an older antigen of known value before being finally accepted as satisfactory.

Antigen containers should be well stoppered and kept in the refrigerator. Deterioration may set in suddenly, and they should, therefore, be retitrated every few weeks.

\section{VARIOUS METHODS FOR CONDUCTING THE SYPHIIIS REACTION}

First Method.-The simplest technic, and the one best adapted for inexperienced workers, is the original Wassermann reaction, performed 


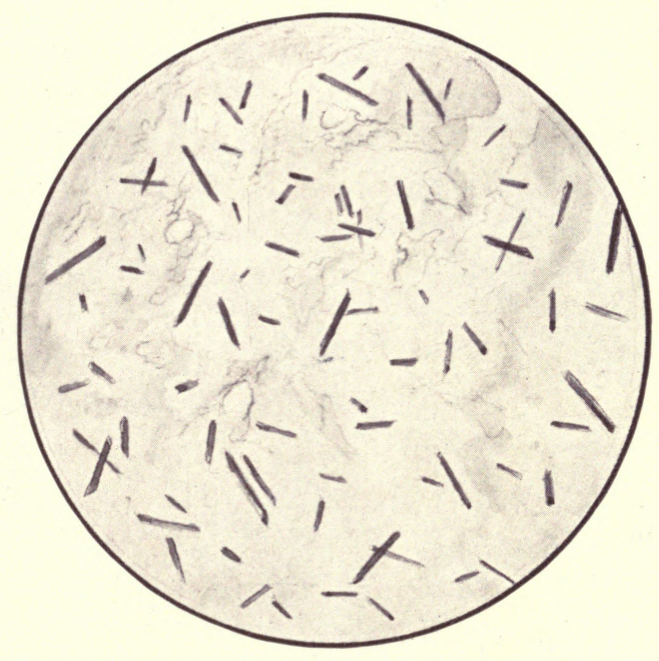

Fig. 86.-Hemin Crystals.

Prepared after the method described in the text. From a stain (over two months old) of sheep blood on a towel. 

with alcoholic instead of aqueous extracts of syphilitic liver as antigen. It is true that this method is not an exact quantitative reaction, and that it is probably less delicate than some of the modified methods, but its advantages are that it is easy of manipulation, is readily learned, and is especially recommended for persons who perform these tests at irregular intervals, as false positive reactions are less likely to occur than when the more delicate methods are used.

Second Method.-In this method the technic is essentially the same as in the first method, except that three different antigens are used instead of one, namely, cholesterinized extract of normal heart, alcoholic extract of syphilitic liver, and acetone-insoluble lipoids. This method has three advantages: (1) It permits the use of a cholesterinized extract under conditions where any tendency to non-specific fixation is to be controlled; (2) an antigen may at any time suddenly become anticomplementary and yield false results, whereas by this method the source of error is detected and may be avoided, since it is not dependent upon any one extract; (3) an extensive study of the comparative values of antigens has led to the distinct impression that the lipodophilic antibody in different syphilitic serums frequently shows a special affinity for the lipoids in a certain plain antigen more than it does for those in another antigen; in fact, I have not infrequently found that, with weakly positive serums, if one antigen had been employed, a false negative report would have been rendered, the true reaction being given by the other two antigens. These results could not be ascribed to faulty antigen, for with other weakly positive serums the extract would be found to react satisfactorily.

As previously mentioned, cholesterinized alcoholic extracts are very sensitive, so that from this standpoint additional antigens would appear to be superfluous. This very property, however, in my opinion, renders it advisable to control them with less sensitive extracts. In this way all the advantages of a very sensitive antigen may be secured, and the disadvantages avoided until more extended use demonstrates whether or not it is entirely safe to use these extracts alone.

With strongly reacting serums all antigens possess equal antigenic power. With the serums of long-standing or treated cases of syphilis the cholesterinized extracts may react strongly positive, whereas with the aqueous and the alcoholic extracts the reactions are weakly positive, or negative with one and positive with the other. In cases of syphilis that have received considerable treatment the reaction 
may be negative at first with the aqueous and the alcoholic extracts, and as treatment is continued it may finally be negative with the cholesterinized extracts. In a certain percentage of cases, especially those of old infections of the central nervous system, the reaction is positive with the cholesterinized extract and negative with the other extracts; strong reactions of this character usually indicate syphilitic infection. Occasionally a weak (10 per cent. or less, inhibition of hemolysis) reaction may be had with the serum of a person who denies syphilis.

Third Method.-One disadvantage of the regular Wassermann technic is that it may not readily show improvement of the patient while the treatment is going on. For instance, if complete fixation of complement occurs with 0.2 c.c. of serum, one does not know whether this is the smallest fixing dose, or whether there might be a fixation even with much smaller quantities of serum. This is very important in examining cases during the course of the treatment, as otherwise improvement in the condition may be overlooked. Just as soon, however, as the reaction with 0.2 c.c. of serum is a degree less than absolutely positive, then the various steps, down to complete negative reactions, are readily observed and recorded by the usual Wassermann technic. This disadvantage may be overcome by using at least six different doses of serum: $0.01,0.02,0.04,0.06$, $0.08,0.1$ c.c. In this way a means is afforded for judging of the strength of the reaction, and the effect of anti-syphilitic treatment is readily observed.

Fourth Method.- It has previously been pointed out that the syphilis reaction is dependent upon the fact that while hemolytic complement may be rendered inactive or fixed by serum alone and organic extract alone, it is characteristic of syphilis that a mixture of serum and extract will absorb or fix more complement than the sum of the amounts absorbed by these two substances alone. In the foregoing methods no attempt has been made to measure the amount of complement absorbed by serum and antigen alone, but sufficient complement has been furnished to allow for this non-specific fixation, and we are content to show that the serum and antigen alone do not absorb enough complement to interfere with hemolysis, so that any inhibition of hemolysis may be interpreted as specific complement fixation.

Browning and Mackenzie have devised a technic whereby it is possible to estimate the actual amounts of complement absorbed, 
first, by the serum and antigen alone, and second by these two substances combined. The complement absorbed is measured in terms of hemolytic doses. This method consumes a little more time and more of the various reagents is required. It is, nevertheless, the best quantitative method we have, and shows exactly the degree of complement fixation in each case.

In conducting any complement-fixation test the following are essential factors if success is to be achieved: (1) Reliable reagents, particularly a good antigen must be had, for no matter how much care is exercised, good results cannot be secured with indifferent reagents; (2) the observer must possess a thorough working understanding of the underlying principles and particularly of the quantitative relations of the various reagents; (3) there must be an accurate adjustment of the hemolytic system; (4) he must have a careful, painstaking and accurate habit of pipeting small amounts. Accuracy should never be sacrificed for speed, as the latter is properly acquired only with experience.

\section{TECHNIC OF THE FIRST MeThod}

This is the original Wassermann reaction, except that an alcoholic, instead of an aqueous extract of syphilitic liver, is used as antigen, and the amounts of each reagent are just one-half those originally employed. This is the simplest of all technics and, when properly performed, constitutes, in the final analysis, a reliable test and one especially adapted for those not constantly engaged in this work.

1. Complement.-Fresh clear serum (not over twenty-four hours old) of a healthy guinea-pig. Dilute $1: 20$ by adding 19 c.c. of sterile normal saline solution to each 1 c.c. of serum. Dose, 1 c.c. $(=0.05$ c.c. of undiluted serum).

2. Corpuscles.-Sheep's blood washed three times and diluted to make a 2.5 per cent. suspension. For example, 1 c.c. of corpuscles in 39 c.c. of salt solution makes up sufficient for a number of tests.

3. Hemolytic Amboceptor.-Serum of a rabbit immunized with washed sheep's corpuscles. As stated elsewhere, this serum is heated to $55^{\circ} \mathrm{C}$. for half an hour, and an equal part of chemically pure glycerine is added. Mix well and preserve in sterile 1 c.c. ampules. Each ampule will, therefore, contain 0.5 c.c. of serum. One stock dilution is prepared in such manner that about 0.2 c.c. represents one hemolytic dose. It is usually well to prepare a whole series of flasks with various dilutions, and in making a titration to use 1 c.c. of each 
dilution; I have found it much more accurate, simple, and economical, however, to prepare one stock dilution, which is titrated with each complement and corpuscle suspension before each day's work. For example, if a serum is known to have a titer of $1: 2000$, an ampule ( 0.5 c.c. of serum) is diluted with 200 c.c. of salt solution; this gives a dilution of serum approximately $1: 400$, of which 0.2 represents one hemolytic dose. The titration must be repeated each time to make sure of this, because the complement of different pigs may vary in activity, and the chief object is to adjust the amboceptor and complement to each other.

Titration of Amboceptor.-Into a series of six test-tubes place increasing amounts of the amboceptor dilution: 0.05 c.c., 0.1 c.c., 0.15 c.c., 0.2 c.c., 0.25 c.c., and 0.3 c.c. Add 1 c.c. of complement $(1: 20)$ and 1 c.c. of corpuscle suspension to each tube, and sufficient salt solution to make the total volume in each tube about 4 c.c. Shake gently and incubate for one hour at $37^{\circ} \mathrm{C}$. At the end of this time the tube showing just complete hemolysis contains one hemolytic dose, or unit of amboceptor. In the tests double this amount, or two units, is used.

The amboceptor titration is very important. Under no circumstances should the same dose be used day after day without titration, because the complement of different guinea-pigs may vary in its activity, and these variations would be detected and would be adjusted in this titration. For example, with a weaker complement the dose of amboceptor required to effect complete hemolysis becomes higher; each new corpuscle suspension may also vary slightly in the actual number of cells contained in 1 c.c., but this makes no difference when each suspension is titrated with the complement and amboceptor to be used in the day's work. This titration is set up first, and while it is in the incubator, the main tests are arranged.

4. Antigen.-Alcoholic extract of syphilitic liver or acetoneinsoluble lipoids of proved value may be used. It is well to estimate just how much antigen will be required for the tests on hand, so that no waste will occur, as fresh emulsions are better than old ones carried over from day to day. The dose should be at least double the titrated antigenic unit, or one-fourth of the anticomplementary dose. For instance, if an alcoholic extract of syphilitic liver diluted $1: 10$ is found on titration to be perfectly antigenic in doses of 0.2 c.c., and not anticomplementary in amounts under 2 c.c., then 0.4 c.c. may be used in making the tests, as this amount is still about five times less 
than the anticomplementary dose, and well within the range of safety against non-specific complement fixation. If 10 tests are to be made, then at least 4.4 c.c. of diluted antigen are required, including sufficient for the antigen control, or in round numbers, 0.5 c.c. of antigen plus 4.5 c.c. of salt solution in order to secure the maximum turbidity slowly added.

5. Serum.- Serum should be fresh and clear and heated in a waterbath to $55^{\circ} \mathrm{C}$. for half an hour before using. The temperature should not go above $56^{\circ} \mathrm{C}$. nor below $55^{\circ} \mathrm{C}$. The complement in perfectly fresh serum is usually inactivated at this temperature within fifteen minutes, but it is better to adopt the period of one-half hour as a routine, especially in removing heat-sensitive anticomplementary substances that develop in serums more than a day old. Dose, 0.1 c.c., or if the serum is perfectly fresh, 0.2 c.c. may be used.

6. Cerebrospinal Fluid.-This should be fresh and free from blood. It is used unheated, as spinal fluid contains little or no hemolytic complement. The dose should be at least four times that of the serum, or from 0.4 to 1 c.c.

The Test.-A front and a rear tube for each serum are placed in a rack. Each tube is marked plainly with the patient's name or initials, and in addition the front tube is marked with the number of the antigen or with the letter " $\mathrm{A}$," or the word "antigen" is written on it, the rear tube being marked "control" (serum control). The necessity for carefully marking each tube is nowhere more important than in conducting Wassermann reactions with a number of serums, as the slightest error or lapse of memory may result in confusion and prove to be quite a serious matter.

In each series of reactions the serum from a known case of syphilis that has given a positive reaction and the serum of a known nonsyphilitic person are included as positive and negative controls respectively.

Into each front tube the proper dose of antigen is placed; to the front and rear tubes 0.1 c.c. of the patient's serum is added. (If 0.2 c.c. is being used as the dose, this amount should be placed in both tubes.) To all tubes 1 c.c. of the complement $(1: 20)$ and sufficient normal salt solution are then added to bring the total volume in each to about 3 c.c.

The rear tube of each set is the serum control; the positive and negative serums are treated in just the same manner as the patient's serum. In addition to these there are three other important controls that should not be omitted: 
1. The antigen control: Dose of antigen plus 1 c.c. of complement $(1: 20)$ and a sufficient quantity of salt solution.

2. Hemolytic system control: 1 c.c. of complement $(1: 20)$ and 2 c.c. of salt solution.

3. Corpuscle control: 1 c.c. of corpuscle suspension plus 3 c.c. of salt solution.

Each tube is gently shaken and incubated at $37^{\circ} \mathrm{C}$. for an hour, when two hemolytic doses of amboceptor and 1 c.c. of corpuscle suspension (2.5 per cent.) are added to each tube except the corpuscle control. Tubes are shaken and reincubated for an hour or an hour and a half, depending upon the hemolysis of the serum controls, after which a preliminary reading is made and recorded. With partially positive reactions the tubes may be centrifuged in order to read the relative amounts of hemolysis, and the final reading made at.once, or the tubes may be placed in the refrigerator (just above freezingpoint) and the final readings made the next morning.

\section{TABLE 15.-SCHEME FOR CONDUCTING A WASSERMANN REACTION} (FIRST METHOD) (SEE FIG. 110)

\begin{tabular}{|c|c|c|c|c|}
\hline $\begin{array}{l}\text { UNKNOWN SERUM, } \\
\text { MR. B. }\end{array}$ & $\begin{array}{c}\text { UNKNOWN CERE- } \\
\text { BROSPINAL FLUID, } \\
\text { Mr. C. }\end{array}$ & $\begin{array}{c}\text { Known Positive } \\
\text { SYPHILITIC } \\
\text { SERUM }\end{array}$ & $\begin{array}{c}\text { KNOWN NEGATIVE } \\
\text { NORMAL SERUM }\end{array}$ & Controls \\
\hline $\begin{array}{c}2 . \\
\text { Serum, } 0.1 \text { c.c. } \\
+ \\
\text { Complement }(1 \\
\text { c.c. of } 1: 20)+ \\
\text { Salt solution } \\
\text { (q. s. } 3 \text { c.c.) }\end{array}$ & $\begin{array}{c}4 . \\
\text { Cerebrospinal } \\
\text { fluid, } 0.8 \text { c.c. }+ \\
\text { Complement }(1 \\
\text { c.c. of } 1: 20)+ \\
\text { Salt solution } \\
\text { (q. s. } 3 \text { c.c.) }\end{array}$ & $\begin{array}{l}6 . \\
\text { Serum, } 0.1 \text { c.c. } \\
+ \\
\text { Complement }(1 \\
\text { c.c. of } 1: 20)+ \\
\text { Salt solution } \\
\text { (q. s. } 3 \text { c.c.) }\end{array}$ & $\begin{array}{c}8 . \\
\text { Serum, } 0.1 \text { c.c. } \\
+ \\
\text { Complement }(1 \\
\text { c.c. of } 1: 20)+ \\
\text { Salt solution } \\
\text { (q. s. } 3 \text { c.c.) }\end{array}$ & $\begin{array}{l}10 . \\
\text { Antigen control: } \\
\text { Antigen, } 0.4 \\
\text { c.c. }+ \\
\text { Complement }(1 \\
\text { c.c. of } 1: 20)+ \\
\text { Salt solution } \\
\text { (q. s. } 3 \text { c.c.) }\end{array}$ \\
\hline $\begin{array}{l}1 . \\
\text { Antigen, } 0.4 \text { c.c. } \\
+ \\
\text { Serum, } 0.1 \text { c.c. }+ \\
\text { Complement }(1 \\
\text { c.c. of } 1: 20)+ \\
\text { Salt solution } \\
\text { (q. s. } 3 \text { c.c.) }\end{array}$ & $\begin{array}{l}3 . \\
\text { Antigen, } 0.4 \\
\text { c.c. }+ \\
\text { Cerebrospinal } \\
\text { fluid, } 0.8 \text { c.c. } \\
+ \\
\text { Complement (1 } \\
\text { c.c. of } 1: 20)+ \\
\text { Salt solution } \\
\text { (q. s. } 3 \text { c.c.) }\end{array}$ & $\begin{array}{l}5 . \\
\text { Antigen, } 0.4 \\
\text { c.c. }+ \\
\text { Serum. } 0.1 \text { c.c. } \\
+ \\
\text { Complement ( } 1 \\
\text { c.c. of } 1: 20)+ \\
\text { Salt solution } \\
\text { (q. s. } 3 \text { c.c.) }\end{array}$ & $\begin{array}{c}7 . \\
\text { Antigen } 0.4 \text { c.c. } \\
+ \\
\text { Serum, } 0.1 \text { c.c. }+ \\
\text { Complement }(1 \\
\text { c.c. of } 1: 20)+ \\
\text { Salt solution } \\
\text { (q. s. } 3 \text { c.c.) }\end{array}$ & $\begin{array}{l}9 . \\
\text { Hemolytic con- } \\
\text { trol: } \\
\text { Complement }(1 \\
\text { c.c. of } 1: 20)+ \\
\text { Salt solution } \\
\text { (q. s. } 3 \text { c.c.) }\end{array}$ \\
\hline
\end{tabular}

Tubes are shaken gently and incubated at $37^{\circ} \mathrm{C}$. for an hour, after which two hemolytic doses of amboceptor and 1 c.c. of corpuscle suspension are added to each. They are then gently shaken and reincubated for an hour or an hour and a half, after which a preliminary reading is made.

All the tubes in the rear row (upper row in table) (serum controls), the antigen and hemolytic system controls, and the front tube with the negative normal serum, are completely hemolyzed. The front tubes with the unknown serum and cerebrospinal fluid and the positive serum control show inhibition of hemolysis or positive reactions. 


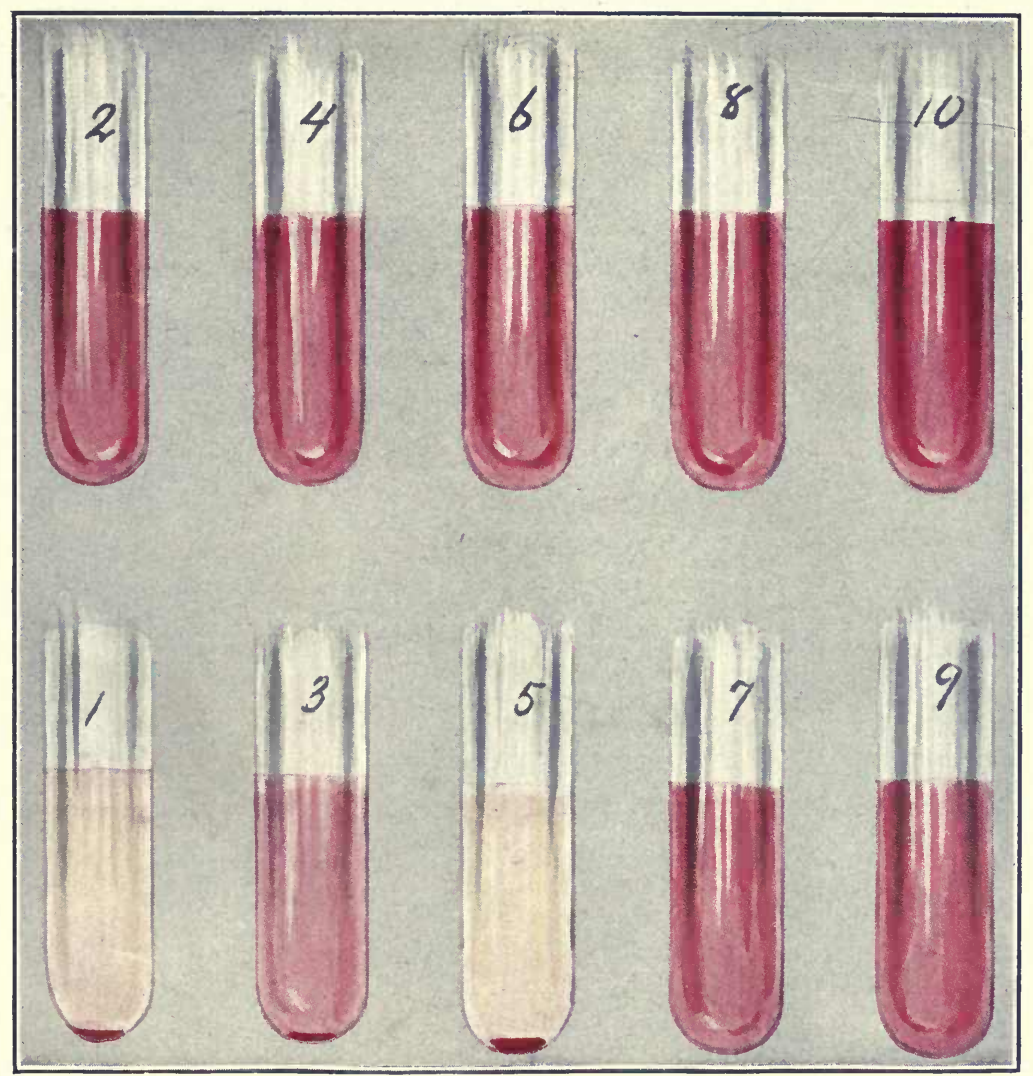

Fig. 110.-Wassermann Reaction (First Method).

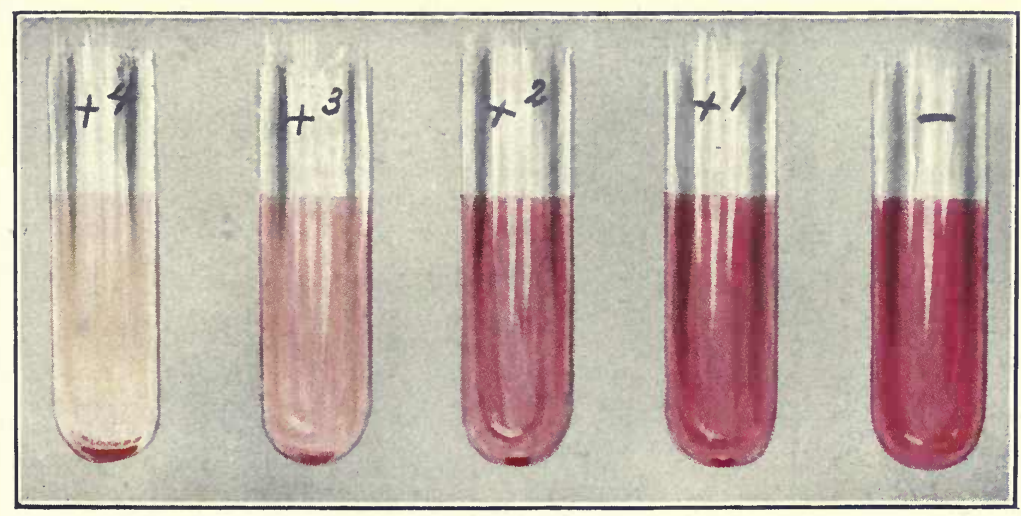

Fig. 111.-Reading the Wassermann Reaction. 

This scheme illustrates the technic employed with an unknown serum and cerebrospinal fluid. The proper dose of diluted antigen is taken as 0.4 c.c., and two doses of hemolytic amboceptor determined by titration as equivalent to 0.4 c.c. of the stock dilution.

\section{READING AND RECORDING THE WASSERMANN REACTION}

1. The hemolytic system control is inspected first. It should show complete hemolysis, indicating that the complement and amboceptor were active and have been used in sufficient amounts. If a few corpuscles are found in the bottom of the tube, some error in pipeting has probably occurred, too many corpuscles or too little complement or amboceptor having been introduced.

2. The corpuscle control should show no hemolysis, indicating that the solution is isotonic and that the corpuscles are not unduly fragile.

3 . The antigen control should show complete hemolysis, indicating that the dose used was not anticomplementary. If this tube shows incomplete hemolysis, due to the anticomplementary action of the antigen, all the front two tubes will also show some inhibition of hemolysis, due to this non-specific complement fixation, and it is necessary to repeat the tests with another extract.

4. The rear tubes of all serums should be completely hemolyzed, indicating that the serums were practically free from anticomplementary action as previously stated, most antigens and serums are usually very slightly anticomplementary if small amounts of complement are used with a close single unit of amboceptor, but in this technic the complement and two units of amboceptor are sufficient, under ordinary circumstances, to offset this influence. If, however, a serum is more than normally anticomplementary, the rear tube will show some inhibition of hemolysis, and, of course, in the front tube a similar inhibition, and probably to a greater degree, will be seen. If the serum is very slightly anticomplementary and the front tube shows complete inhibition of hemolysis, the reaction is in all probability positive. If the rear tube, however, shows marked inhibition of hemolysis, indicating that it is highly anticomplementary, the result cannot be determined, but a retest with fresh serum must be made. This indicates the great importance of the "serum control," and it may be stated that a test should never be made without it.

5. The front tube containing the known syphilitic serum should show inhibition of hemolysis, indicating that the extract possesses antigenic properties. 
As the complement is "fixed" by the syphilis antibody and extract, hemolysis could not occur when the corpuscles and amboceptor were added. If a portion of the complement is fixed by antibody and extract, then the unfixed portion will hemolyze some of the corpuscles, the reaction being moderately positive, slightly positive, etc., depending upon the degree of hemolysis that takes place. This illustrates the importance of observing exactness in pipeting, and the great influence of quantitative factors in testing for the Wassermann reaction, for if an excess of complement is used, there may be sufficient for all the syphilis antibody, and enough unbound complement to hemolyze all the corpuscles. In this manner a false negative reaction will result. Corpuscles and sufficient hemolytic amboceptor are added merely in order to test for any free complement. Under proper conditions a total lack of hemolysis indicates that there is no free complement, but that it has been fixed by syphilis antibody and extract, constituting a positive reaction $(++++)$. Complete hemolysis indicates that complement was not bound and that syphilis antibody was, therefore, absent from the fluid tested-a negative reaction (-). Partial hemolysis indicates that a portion of the complement has been fixed by smaller amounts of syphilis antibody and of the extract, yielding partially positive reactions $(+++$; $++;+; \pm)$.

6. The front tube containing the known normal serum should show complete hemolysis because, in the absence of syphilis antibody, the complement remains free to hemolyze the corpuscles with the hemolytic amboceptor.

7. Various methods have been proposed for recording the results of hemolytic tests. The following scheme, after Citron, is widely used (Fig. 111):

$++++=$ complete inhibition of hemolysis = strongly positive.

$+++=75$ per cent. inhibition of hemolysis $=$ moderately positive.

$++=50$ per cent. inhibition of hemolysis $=$ weakly positive.

$+=25$ per cent. inhibition of hemolysis $=$ very weakly positive.

$\pm=$ less than 25 per cent. inhibition of hemolysis = delayed hemolysis or doubtful reaction.

- = complete hemolysis $=$ negative reaction .

Under the third method a scale is given that is easily prepared for making these readings. However, after some experience they are readily made, and at first should be attempted only after the nonhemolyzed corpuscles have been centrifuged or allowed to settle to the 
bottom of the tube. As stated elsewhere, this method is not an accurate measure of the amount of syphilis antibody, but constitutes a relative and convenient gage of value within certain limits. In reporting reactions to the clinician, the plus signs should not be used, or if used, should be interpreted by the terms "strongly positive," "weakly positive," etc.

\section{TECHNIC OF THE SECOND METHOD}

Practically the same technic is used in this as in the first method, except that three different antigens, instead of one, are used with each serum, for the reasons previously stated.

This method is to be strongly recommended, as it is simple, accurate, and reliable. Although a little more work is demanded and a larger quantity of the various reagents is required, the results warrant the expenditure of a little more labor, and the second objection is readily overcome by using half the quantities prescribed in the original Wassermann technic, as given in the first method.

1. I generally use the following three antigens: (1) A cholesterinized alcoholic extract of human heart; (2) alcoholic extract of syphilitic liver; (3) acetone-insoluble lipoids.

As previously stated, these extracts are used in amounts equal to from two to four times their titrated antigenic unit, providing these doses are at least four times smaller than the anticomplementary units. The amount of each antigen required for the work at hand is calculated, placed in test-tubes, and slowly diluted with the requisite amount of salt solution to secure maximum turbidity of the emulsions.

2. The complement is diluted $1: 20$ and is used in doses of 1 c.c.; sheep's corpuscles are made up into a 2.5 per cent. suspension, and used in doses of 1 c.c.; antisheep amboceptor is titrated as in the first method, and used in doses equal to $11 / 2$ units; serums are heated to $55^{\circ} \mathrm{C}$. for half an hour, and used in doses of 0.1 to 0.2 c.c.; cerebrospinal fluid is used unheated in amounts of 0.8 c.c.

The Test.-For each serum four test-tubes are arranged in a row and marked with the patient's name or initials. The first tube is marked "C. H.," and receives the cholesterinized heart extract; the second tube is marked " $\mathrm{S}$ " for the alcoholic extract of syphilitic liver; the third is marked "A" for acetone-insoluble lipoids, and the fourth is not marked at all or simply marked with the letters "S. C." (serum control). 
To each of the four tubes 0.1 or 0.2 c.c. of the patient's serum is added, or 0.8 c.c. of cerebrospinal fluid.

To each tube 1 c.c. of the diluted complement $(1: 20)$ and suffcient salt solution to bring the total volume in each up to 3 c.c. are now added.

Controls.-A known positive and negative serum should be included, unless one is performing a large number of tests with reliable antigens every week, in which case, among many serums, a few at least are likely to be positive. Under these circumstances these controls may be omitted; as a general rule, however, they should be included.

To the hemolytic system control tube 1 c.c. of complement dilution and 2 c.c. of salt solution are now added. Three antigen control tubes are set up for each antigen with the dose employed, plus 1 c.c. of complement dilution and sufficient salt solution to make the total volume about 3 c.c. The corpuscle control receives 1 c.c. of the suspension plus 3 c.c. of salt solution.

All the tubes are shaken gently and placed in the incubator for an hour at $37^{\circ} \mathrm{C}$. At the end of this time the amboceptor and corpuscles are added to each tube except that containing the corpuscle control. Each tube is shaken gently and reincubated for an hour or longer, depending upon the hemolysis of the serum controls. A preliminary reading is now made, and the tubes set aside in the refrigerator at low temperature until the corpuscles have settled. As a general rule, the two readings coincide quite closely. Occasionally, with serums of vigorously treated cases of syphilis, at the preliminary reading the antigen tubes will show very slight inhibition or delay in hemoly is, whereas if allowed to settle overnight this may not be noticeable.

Reading the Results.- The readings are made in the same manner as described in the first method, the controls always being inspected first. The hemolytic, antigen, and serum controls and known negative serum tubes should all be hemolyzed. The antigen tubes containing the positive syphilitic serum should not be hemolyzed. Results with the unknown serums are dependent upon whether or not the serums are luetic, and if they are, upon the quantity of syphilis antibody present.

With strongly positive serums there is complete inhibition of hemolysis with all three antigens. With serums of long-standing or treated cases of syphilis containing smaller amounts of antibody the reaction with the cholesterinized extract is usually strongly positive, whereas with the other two antigens the degree of inhibition of hemol- 
ysis is less marked and variable (see Fig. 112). In from 15 to 20 per cent. of cases the cholesterinized extract shows a 50 per cent. or more inhibition of hemolysis, whereas with the other two antigens the reactions are negative. In our experience the majority of such serums were taken from patients giving a frank history of syphilis of many years' standing and from known cases undergoing treatment, further therapy being indicated until the reaction finally becomes negative when cholesternized extracts are used. In a small proportion of cases a feebly positive reaction of 25 per cent. or less inhibition of hemolysis may be found with the cholesterinized extract alone. Many of these reactions occur with serums of treated cases of syphilis; on the other hand, a similar reaction may occur with about 5 per cent. of normal serums, so that if the history and clinical conditions are clearly negative, a slight degree of inhibition of hemolysis (5 to 10 per cent.) with the cholesterinized extract and marked hemolysis with the other two antigens may be interpreted as a negative reaction.

After a new antigen has been prepared and titrated, it should be tested out in this manner by placing it in the series along with at least two other older antigens of proved value, and used in the examination of a large number of serums before it is finally accepted as reliable.

\section{TECHNIC OF THE THIRD METHOD}

As previously stated, it may be desirable to measure the quantity of syphilis antibody in a patient's serum more accurately, especially when observing the effect of treatment. This is readily accomplished by using decreasing doses of serum with constant doses of antigen and complement. In those tubes showing partial inhibition of hemolysis the degree of hemolysis is determined by comparison with a hemoglobin scale (Boas).

The technic is quite similar to that followed in the second method.

1. One antigen is employed, either an alcoholic extract of syphilitic liver or acetone-insoluble lipoids. The same general rule as to dosage is employed, namely, from two to four times the titrated antigenic unit, providing these amounts are not more than one-fourth the anticomplementary dose.

2. 0.5 c.c. of each serum is heated to $55^{\circ} \mathrm{C}$. for half an hour and diluted $1: 10$ by adding 4.5 c.c. of salt solution.

3. Fresh guinea-pig serum complement is diluted 1:20 as usual, and used in doses of 1 c.c.; sheep corpuscles are made up in a 2.5 per 
cent. suspension and used in doses of 1 c.c.; antisheep amboceptor is adjusted to the complement and corpuscles by a method of titration, as given with the first method, and used in a dose equal to $11 / 2$ hemolytic units.

The Test.-Seven test-tubes are arranged in a row and marked with the patient's name; in each of the first six tubes the dose of antigen is placed. The following amounts of diluted serum (1:10) are then added:

First tube: $\quad 0.1$ c.c. serum diluted $1: 10=0.01$ c.c. serum.

Second tube: 0.2 c.c. serum diluted $1: 10=0.02$ c.c. serum.

Third tube: 0.4 c.c. serum diluted $1: 10=0.04$ c.c. serum.

Fourth tube: 0.6 c.c. serum diluted $1: 10=0.06$ c.c. serum.

Fifth tube: 0.8 c.c. serum diluted $1: 10=0.08$ c.c. serum.

Sixth tube: 1.0 c.c. serum diluted $1: 10=0.1$ c.c. serum.

Seventh tube: 1.0 c.c. serum diluted $1: 10=0.1$ c.c. serum (control).

The seventh tube contains no antigen, and is the serum control with the maximum dose employed.

In testing cerebrospinal fluid the following doses may be used (undiluted): 0.1 c.c., 0.2 c.c., 0.4 c.c., 0.6 c.c., 0.8 c.c., 1 c.c., and 1 c.c. for the seventh or control tube.

To each tube 1 c.c. of the diluted complement $(1: 20)$ is now added, and sufficient salt solution to bring the total volume in each up to 3 c.c.

Controls.-Unless one is examining a large number of serums and is sure that the antigen is satisfactory, known positive and negative serum controls may be included in the series. As a general rule, however, they should not be omitted.

The hemolytic system control receives at this time 1 c.c. of the complement dilution and 2 c.c. of salt solution. The antigen control receives the dose employed plus 1 c.c. of complement and sufficient salt solution to bring the total volume up to 3 c.c. The corpuscle control receives 1 c.c. of the suspension and 3 c.c. of salt solution, and the tube is plugged with cotton to show that it is finished.

All tubes are shaken gently and incubated for one hour at $37^{\circ} \mathrm{C}$., at the end of which time $1 \frac{1}{2}$ units of amboceptor and 1 c.c. of the corpuscle suspension are added to all except the corpuscle control. Each tube is shaken and all are reincubated for an hour or an hour and a half, the length of time depending upon the hemolysis of the serum controls when all are placed in the refrigerator at a low temperature overnight, readings being made the next morning.

Reading the Results.-As is usual, the controls are examined first. 


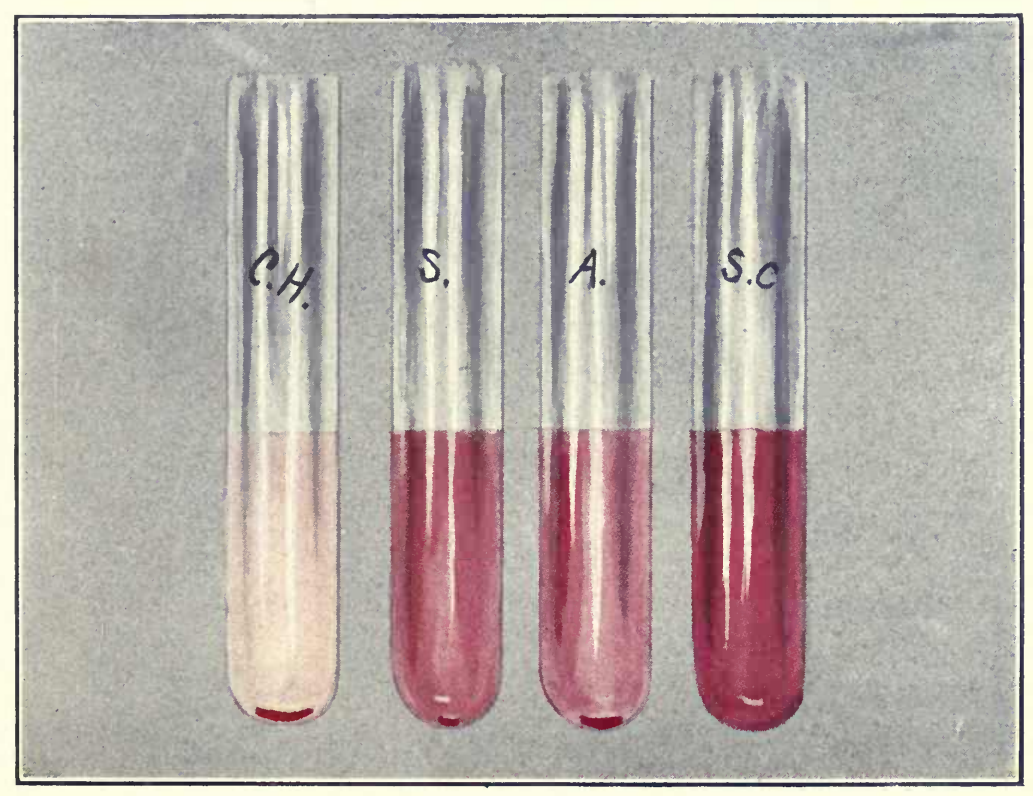

Fig. 112.-Wassermann Reaction (Second Method). 


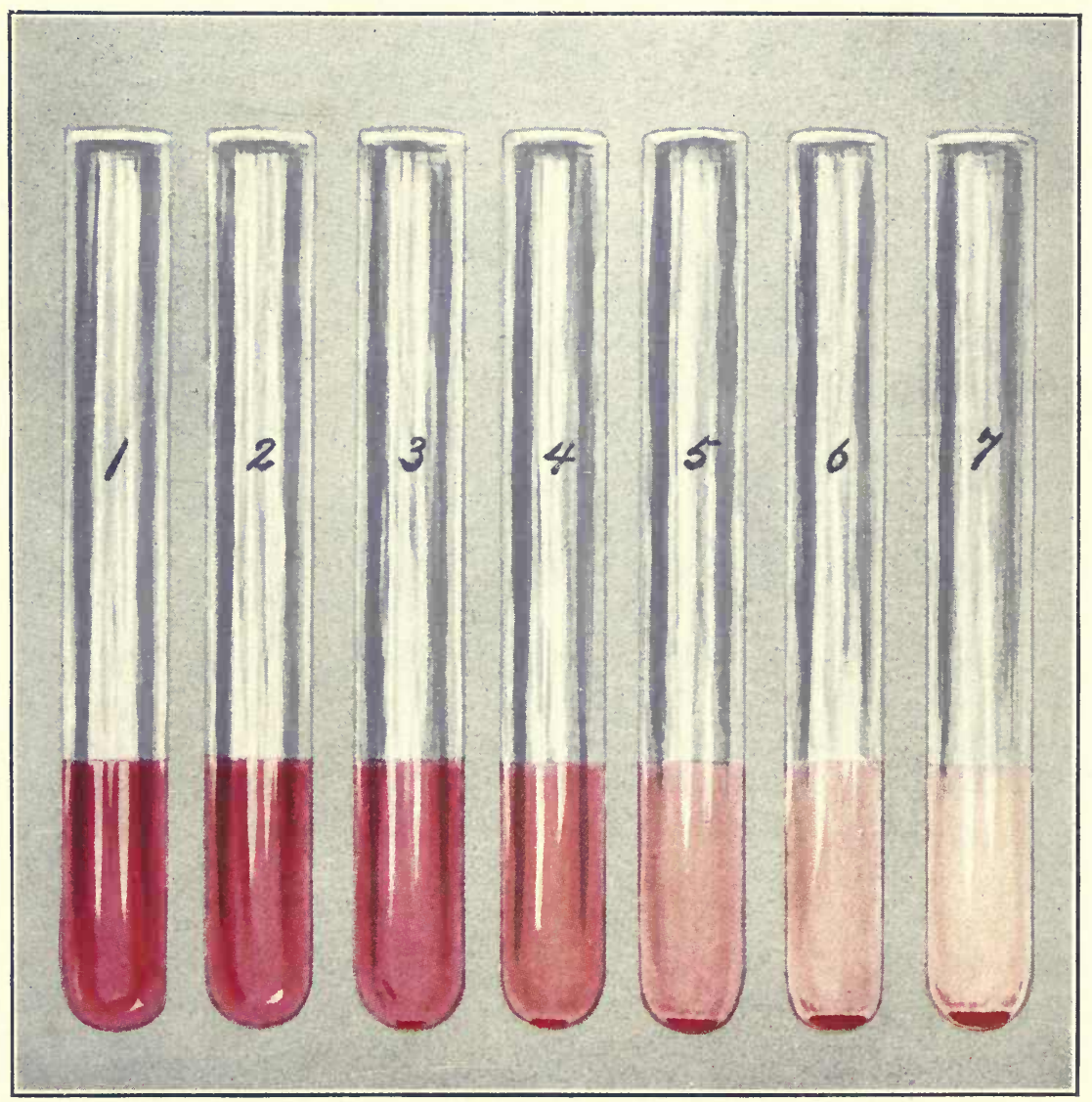

Fig. 113.-Wassermann Reaction (Third Method). 
The hemolytic, antigen, negative serum, and all serum controls should be completely hemolyzed. The corpuscle control should show no hemolysis, indicating that the salt solution was isotonic and the corpuscles free from undue fragility.

A 1 per cent. hemoglobin solution in distilled water is prepared by mixing 1 c.c. of the washed corpuscles used in preparing the suspension with 99 c.c. of distilled water. Into a series of tubes dilutions of this solution are placed as follows:

Tube 1: 10.0 c.c. of hemoglobin solution............ $=100$

Tube 2: 9.0 c.c. " " " 1.0 c.c. distilled water $=90$

Tube 3: 8.0 c.c. " " " " " $" 2.0$ c.c. " $"=80$

Tube 4: 7.0 c.c. " " " $"$ " 3.0 c.c. " $"=70$

Tube 5: 6.0 c.c. " " " " $" 4.0$ c.c. " " $"=60$

Tube 6: 5.0 c.c. " " " $" ~ " 5.0$ c.c. " " $"=50$

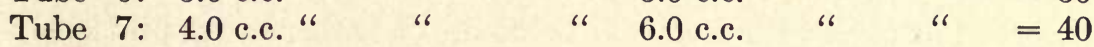

Tube 8: 3.0 c.c. " " " " $" 7.0$ c.c. " " $"=30$

Tube 9: 2.0 c.c. " " " " $" 8.0$ c.c. " " $"=20$

Tube 10 : 1.0 c.c. " " " $" ~ " 9.0$ c.c. " " $"=10$

Tube 11: 0.4 c.c. " " $"$ " $" 9.6$ c.c. " $"=4$

Tube $12: \ldots \ldots \ldots \ldots \ldots \ldots \ldots \ldots \ldots 10.0$ c.c. " " $"=0$

This scale is not permanent, and must be prepared anew for each set of reactions.

A negative reaction is indicated in the sixth tube, which contains the maximum dose of serum ( 0.1 c.c.), by complete hemolysis or by hemolysis ranging from 80 to 100 , according to the scale. Inhibition of hemolysis in this tube giving a hemoglobin scale ranging from 70 to 4 (inclusive) is regarded as a positive reaction. Absolute lack of hemolysis in this tube is 0 according to the scale, but usually the patient's serum or complement serum is sufficiently tinged with hemoglobin to give a slight color to the supernatant fluid, so that an absolute positive reaction may range from 0 to 4 . For instance, the serum of an untreated case of secondary syphilis gave the following reading (see Fig. 113).

Tube 1: 0.01 c.c. serum..................100

Tube $2: 0.02$ c.c. serum................. 100

Tube 3: 0.04 c.c. serum............... 30

Tube 4: 0.06 c.c. serum.............. 10

Tube 5: 0.08 c.c. serum.............. 4

Tube $6: 0.1$ c.c. serum............... 4

Tube 7: 0.1 c.c. serum (control) ........ 2 
Although these figures suffice for recording purposes, they are not adapted, without some qualifying phrase, for rendering reports to clinicians. According to the inhibition of hemolysis or the degree of hemolysis in the sixth tube containing the maximum quantity of serum the following scheme may be used in reporting the results:

$$
\begin{aligned}
0 \text { to } 10 & =\text { strongly positive }=(++++) \\
10 \text { to } 30 & =\text { moderately positive }=(+++) \\
30 \text { to } 50 & =\text { slightly positive }=(++) \\
50 \text { to } 80 & =\text { very weakly positive }=(+) \\
80 \text { to } 90 & =\text { doubtful or delayed hemolysis }=( \pm) \\
100 & =\text { negative }=(-)
\end{aligned}
$$

\section{TECHNIC OF THE FOURTH METHOD}

In this method the amount of syphilis antibody in a serum is measured according to the number of hemolytic doses of complement absorbed or fixed with a constant amount of antigen. As previously stated, any organic extract used as antigen may of itself fix a certain amount of complement; a non-syphilitic serum may do the same, and a mixture of the two may fix still more, though the amounts may be relatively small. A peculiarity possessed by a syphilitic serum is that it fixes a large amount of complement when mixed with antigen; as a result, the test becomes a quantitative and not a qualitative reaction. The only practical means we possess of estimating the amount of complement in a fresh serum is to ascertain the hemolytic dose, $i$. e., to find the smallest quantity of serum that is just sufficient completely to lyse the test amount of sensitized corpuscles. When this has been done, the quantity of complement used in the reaction may be expressed in terms of hemolytic doses fixed, and not in terms of the amount of fresh serum.

When properly performed according to this method, which has been modified after the technic of Browning and Mackenzie, the syphilis reaction becomes quite delicate and accurate, but is more complicated than the other methods, and should not be attempted until one is accustomed to the simpler test and thoroughly understands the underlying principles of the syphilis reaction and knows the many sources of fallacy. The greater amount of work that it entails and the larger quantities of complement-serum and amboceptor that are required may serve as factors against its adoption as a routine method.

1. Hemolytic Amboceptor and Corpuscles.-A stock dilution of antisheep amboceptor is titrated according to the technic given in the 
first method. It is well to prepare this dilution in such a manner that the hemolytic dose will not be more than 0.1 c.c. If the amboceptor is being constantly used, so that its titer is well known, it will not be necessary to titrate, but 5 times this unit is used in sensitizing the corpuscles. Otherwise small amounts of complement and corpuscle suspension must be prepared, and the amboceptor titrated as follows:

Sufficient complement is prepared by diluting 0.5 c.c. of fresh guinea-pig serum with 9.5 c.c. of salt solution $(1: 20)$, each cubic centimeter of which contains 0.05 c.c. undiluted serum. It is well at this time to prepare only sufficient 2.5 per cent. suspension of sheep's cells for the titration, which is then conducted after the technic given in the first method. For instance, if 0.1 c.c. of amboceptor dilution represents one hemolytic unit, 0.5 c.c. would be used in sensitizing each dose of corpuscles. If this quantity of amboceptor dilution is added to a 2.5 per cent. suspension of cells, the emulsion would be considerably reduced, which would not be advisable. It is well, therefore, to calculate the amount of amboceptor and corpuscle suspension required for the work on hand; measure out the amboceptor dilution in a flask, and add the corpuscles and then sufficient salt solution to bring the total volume to the required amount to make a 2.5 per cent. suspension of cells. To continue the foregoing example: five hemolytic doses of amboceptor and sufficient corpuscular suspension are required for 100 tubes; this requires 50 c.c. of amboceptor dilution, $21 / 2$ c.c. of washed corpuscles, and sufficient sterile normal salt solution to bring the total volume up to 100 c.c. Permit the mixture to remain for at least half an hour at room temperature for the process of sensitization to take place before the complement titration is performed.

2. Complement.-Fresh clear guinea-pig serum is diluted with four parts of normal salt solution and titrated to determine the hemolytic unit.

Complement Titration.-Into a series of eight test-tubes place increasing amounts of the diluted complement serum (using the special 0.2 c.c. pipets graduated in 0.01 c.c.), as follows: $0.01,0.02$, $0.03,0.04,0.05,0.06,0.08,0.1$ c.c.; add 1 c.c. of sensitized corpuscles and sufficient salt solution to make the total volume about 3 c.c. After incubating for one hour at $37^{\circ} \mathrm{C}$., the smallest amount of complement giving complete hemolysis represents one hemolytic unit.

3. Antigen.-Browning and Mackenzie use a 0.75 per cent. dilution of lecithin and cholesterin in alcohol, prepared according to their 
method. This is diluted 1:7 with normal salt solution to secure the maximum turbidity, and is used in a constant dose of 0.6 c.c.

A cholesterinized alcholic extract of human heart, acetone-insoluble lipoids, or plain alcoholic extract of syphilitic liver may be used in four times their titrated antigenic dose, with satisfactory results.

4. Fluid to be Tested.-Serum is heated at $55^{\circ} \mathrm{C}$. for half an hour and used in dose of 0.1 c.c. Cerebrospinal fluid should be fresh, and is used unheated in dose of 0.8 or 1 c.c.

\section{The Test.-Two series of tubes are required.}

Series A: These tubes should contain the dose of antigen employed with one, two and three hemolytic doses of complement respectively, and sufficient salt solution to bring the total volume in each up to 3 c.c. These are the antigen controls to determine how much complement may be fixed by antigen alone. Usually but one or at most a portion of two doses are fixed.

Series B: For each serum 12 test-tubes are arranged in a row. They are labeled with the initial of the patient's name and numbered. Into each is placed 0.1 c.c. of the patient's serum. Into each of the first nine tubes are placed the dose of antigen and $2,4,6,8,10,12,15$, 18, 20 hemolytic doses of complement respectively. The last three tubes are the serum controls, to determine the amount of complement fixed by serum alone, and receive 1,2 and 3 hemolytic units of complement respectively. Sufficient salt solution is added to each tube to bring the total volume up to 3 c.c., and all are incubated for an hour at $37^{\circ} \mathrm{C}$.

Controls.-Series "A" contains the antigen controls, and these suffice for making any number of tests with the same complement and sensitized corpuscles.

(2) Each serum is controlled in the last three tubes of series B.

(3) A known positive syphilitic serum may be included and tested in the usual manner.

(4) A known negative normal serum may be included, but it is not necessary to use more than 2 to 4 and 6 hemolytic units of complement instead of running out to 18 or 20 units, as is done with an unknown serum or with cerebrospinal fluid.

(5) A hemolytic control is set up with one dose of complement and sufficient salt solution to make 3 c.c.

(6) A corpuscle control may be included, containing 1 c.c. of sensitized corpuscles and 3 c.c. of salt solution. This tube is plugged with cotton to indicate that it is finished. It controls the tonicity of the salt solution and should show no hemolysis. 



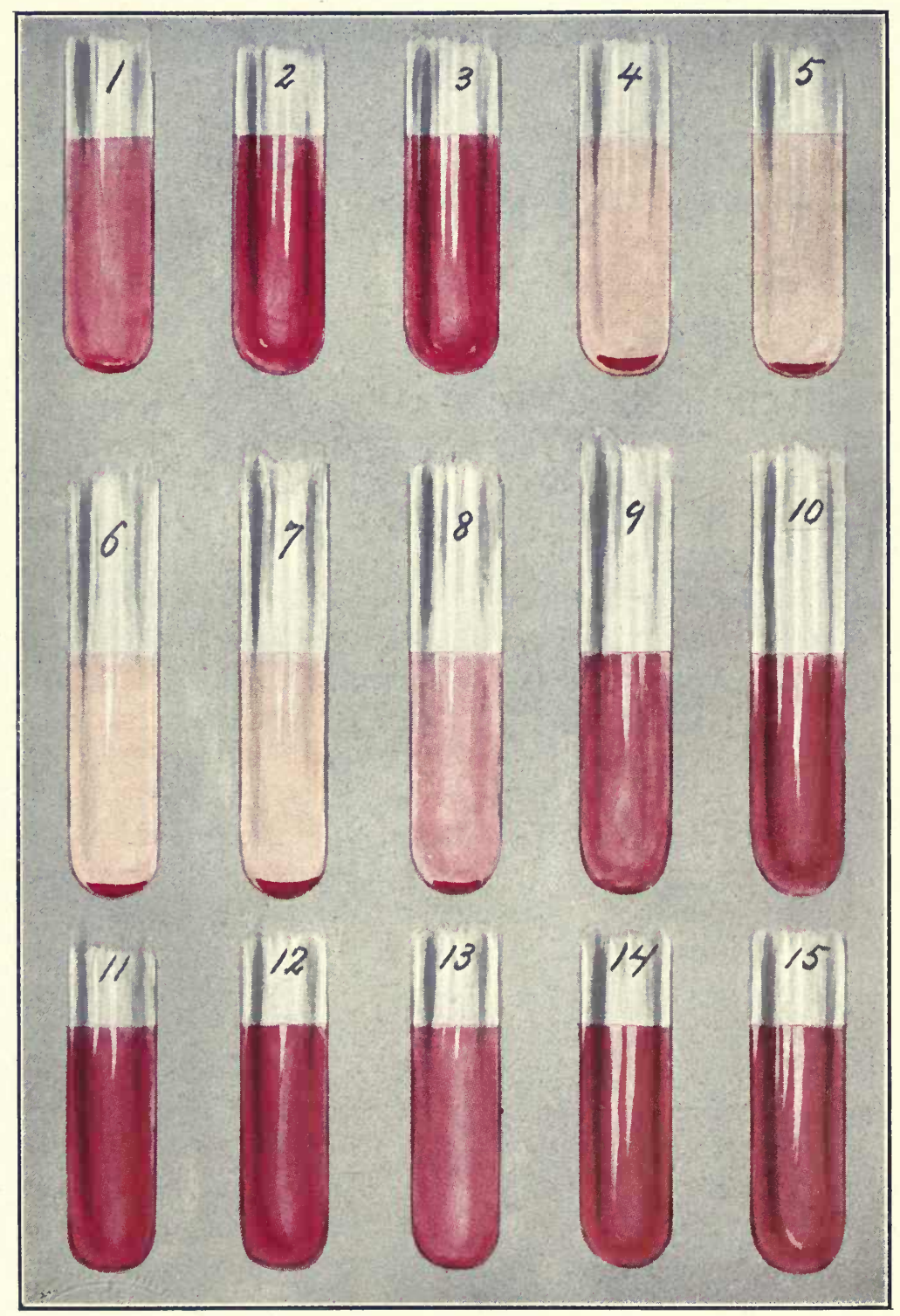

Fig. 114.-Wassermann Reaction (Fodrth Method). 
At the end of an hour 1 c.c. of sensitized corpuscles is added to all the tubes. These are then gently shaken and reincubated for an hour and a half, after which time the results are read. A final reading made after the tubes have been allowed to stand overnight, usually tallies very closely with this reading.

Reading the Results.-The controls are first inspected. The corpuscle control should show no hemolysis, and the hemolytic control be just hemolyzed. In series $\mathrm{A}$ of the antigen controls it is usually found that in the first tube hemolysis is incomplete, whereas the second and third tubes show complete hemolysis, indicating that the antigen alone fixed a unit or one hemolytic dose of complement. The last three tubes of series B are the serum control tubes, and the first tube usually shows incomplete hemolysis, its degree depending upon the age of the serum. Occasionally the second tube is also incompletely hemolyzed, indicating that about two units of complement have been absorbed. Unless the serum is quite anticomplementary, the third tube is completely hemolyzed.

The first nine tubes of series B are now examined. Browning and Mackenzie have made an arbitrary rule to regard the reaction as positive when lysis is incomplete with five hemolytic doses of complement, in addition to the sum of the amounts inhibited by serum and by antigen alone. When an alcoholic extract of syphilitic liver is used as antigen, I have found this rule to be too lax, and I regard the reaction as slightly positive when lysis is incomplete with two or three doses of complement, in addition to the sum of the amounts inhibited by serum and by antigen alone. For example, if a given serum and antigen each show fixation of but one dose of complement, and hemolysis is slight or entirely inhibited in the second tube of the series $\mathrm{B}$ containing serum and antigen with four doses of complement, the reaction is usually weakly positive. More strongly reacting serums will absorb from six to ten doses of complement, and not infrequently the serum of an active case of syphilis will fix more than 20 hemolytic doses of complement (see Fig. 114).

\section{MODIFICATIONS OF THE WASSERMANN REACTION}

\section{MODIFICATION OF NoGUCHI}

Among the large number of modifications of the original syphilis leaction that have been devised, that of Noguchi has proved of distinct value. In this method an antihuman hemolytic system is 
employed that eliminates one possible source of error, due to the natural antisheep amboceptor in human serum.

Noguchi advocated the use of active serum for this test, with an antigen of acetone-insoluble lipoids. Active serum yields a more delicate reaction, but may give false or proteotropic complement fixation, especially when crude alcoholic extracts are used as antigens. Before I began using cholesterinized alcoholic extracts in making the Wassermann reaction I not infrequently found that the Noguchi test with active serum was more delicate than the Wassermann reaction, but with cholesterinized extracts the results ran closely parallel. It is a good practice to conduct both a Wassermann and a Noguchi test with each serum, as a negative Noguchi test with active serum is a better indication of the absence of syphilis than is a negative Wassermann reaction. A positive Wassermann reaction, however, is better evidence of the presence of syphilis than is a positive Noguchi reaction, because of the possibility of false complement fixation occurring in the latter when active serums are used. I may state, however, that when a good antigen of acetone-insoluble lipoids is used, the percentage of false reactions is relatively small, being less than 2 per cent. The Noguchi test, on the other hand, may be conducted with inactivated serums, when the danger of false reactions is removed, but the delicacy of the test is likewise diminished, so that it more closely approaches the Wassermann reaction.

Noguchi endeavored to simplify the technic of the syphilis reaction by preparing complement, antigen, and amboceptor dried on filterpaper. These were titrated and so adjusted that a certain measure of paper represented the required amount of each reagent. In this manner it would be possible for a large central laboratory to prepare and standardize these reagents, putting them up ready for use in the simplest possible form, and thus making them available for the practising physician. Complement, however, deteriorates so rapidly that the paper is useless unless it is freshly prepared. The antigen slips likewise deteriorate, but not so rapidly as the complement; the amboceptor, however, is well preserved by this method, and forms a simple method for titrating and handling this important reagent.

Technic of the Noguchi Modification.-(1) Complement is furnished by the fresh, clear serum of the guinea-pig, put up in 40 per cent. solution, prepared by diluting 1 part of serum with $1 \frac{1}{2}$ parts of normal salt solution. Dose 0.1 c.c. ( 5 drops from a capillary pipet). Whenever possible it is always best to use a mixture of the serums from two or more guinea-pigs. 
2. Human Corpuscles.-These are washed three times with normal salt solution, and used in dose of 1 c.c. of a 1 per cent. suspension. To a graduated centrifuge tube containing 9 c.c. of sterile 2 per cent. sodium citrate in normal salt solution add 1 c.c. of blood and shake gently. This amount of blood is easily secured by pricking the finger with a lancet and collecting the blood in the centrifuge tube up to the mark 10. This is centrifuged thoroughly, and the supernatant fluid drawn off. More saline solution is then added to the corpuscles stirred up and the mixture centrifuged. The washing is repeated once more, and the supernatant fluid discarded. The corpuscles are then suspended in sufficient salt solution to make a total volume of 100 c.c.

3. Hemolytic Amboceptor.-Antihuman hemolysin is prepared by immunizing rabbits with human cells, as described on p. 72. It is a difficult matter to secure a potent amboceptor; human erythrocytes are more toxic than sheep's cells for rabbits, and most animals produce but small amounts of the amboceptor. Hemagglutinins are produced in large amounts, and when using a low titer hemolytic serum, the testcorpuscles are quickly agglutinated in small dense masses that are broken up with difficulty and that interfere greatly with hemolysis. With serums having a titer of 1:1000 or over, the agglutinins are not so much in evidence; a satisfactory reaction is best observed, therefore, with a potent amboceptor $(1: 1000)$ serum.

The hemolytic serum may be preserved in 1 c.c. ampules after adding an equal part of glycerin, and a stock dilution prepared and titrated in the usual manner. The serum is also well preserved dried on filter-paper, as described on p. 79. A trial titration should always be made to determine the potency of the serum before the paper slips are prepared.

If paper amboceptor is used, the uniform rule of titrating it with the complement and corpuscles on hand should be observed before the actual tests are made. This is done chiefly because, where one guinea-pig serum is used for complement, it may occasionally happen that the serum is less active than usual, so that if fixed doses of complement and amboceptor are used, the reactions may at times prove to be incomplete and inaccurate. The process of titration is so simple that any one may readily conduct it, and thus fulfil the most important requirement of any complement-fixation test, namely, adjustment of the complement, amboceptor, and corpuscles to one another.

Titration of Serum Hemolysin.-Prepare a $1: 100$ dilution by mixing 0.1 c.c. of immune serum (inactivated) with 9.9 c.c. of saline solution. 
To a series of six small test-tubes add increasing amounts of this diluted serum as follows:

Tube 1: 0.1 c.c. amboceptor serum $(1: 100)+0.1$ c.c. complement (40 per cent.) +1 c.c. corpuscle suspension ( 1 per cent.).

Tube $2: 0.2$ c.c. amboceptor serum $(1: 100)+0.1$ c.c. complement (40 per cent.) +1 c.c. corpuscle suspension (1 per cent.).

Tube 3: 0.4 c.c. amboceptor serum $(1: 100)+0.1$ c.c. complement (40 per cent.) +1 c.c. corpuscle suspension ( 1 per cent.).

Tube $4: 0.5$ c.c. amboceptor serum $(1: 100)+0.1$ c.c. complement (40 per cent.) +1 c.c. corpuscle suspension ( 1 per cent.).

Tube 5: 0.8 c.c. amboceptor serum $(1: 100)+0.1$ c.c. complement $(40$ per cent. $)+1$ c.c. corpuscle suspension (1 per cent.).

Tube $6: 1$ c.c. amboceptor serum $(1: 100)+0.1$ c.c. complement (40 per cent.) +1 c.c. corpuscle suspension ( 1 per cent.).

Sufficient saline solution is added to the first tubes of the series to bring the total volume up to 2 c.c. The tubes are then shaken gently and placed in the incubator at $37^{\circ} \mathrm{C}$. for two hours (or one hour in water-bath at the same temperature), during which time they should be inspected and shaken gently several times. At the end of the period of incubation that tube which shows just complete hemolysis contains one amboceptor unit, and double this amount is used in making the main tests. If the serum has a titer of less than $1: 500$, it should not be used either in preparing the amboceptor slips or in-conducting the reaction.

Titration of Dried Amboceptor Paper.-After the paper (S. \& S. No. 597 ) has been evenly saturated with immune serum and dried, the sheets are cut into $5 \mathrm{~mm}$. strips and standardized by placing increasing lengths of paper into a series of tubes as follows:

Tube 1: $1 \mathrm{~mm}$. paper +0.1 c.c. complement (40 per cent.) (5 drops) +1 c.c. corpuscle suspension ( 1 per cent.).

Tube 2: $2 \mathrm{~mm}$. paper +0.1 c.c. complement ( 40 per cent.) (5 drops) +1 c.c. corpuscle suspension ( 1 per cent.).

Tube $3: 3 \mathrm{~mm}$. paper +0.1 c.c. complement ( 40 per cent.) ( 5 drops) +1 c.c. corpuscle suspension (1 per cent.).

Tube 4: $4 \mathrm{~mm}$. paper +0.1 c.c. complement (40 per cent.) (5 drops) +1 c.c. corpuscle suspension (1 per cent.).

Tube 5: $5 \mathrm{~mm}$. paper +0.1 c.c. complement ( 40 per cent.) ( 5 drops) +1 c.c. corpuscle suspension ( 1 per cent.).

Tube 6: $6 \mathrm{~mm}$. paper +0.1 c.c. complement (40 per cent.) (5 drops) +1 c.c. corpuscle suspension ( 1 per cent.).

One cubic centimeter of saline solution is added to each tube, and the mixture shaken gently and incubated at $37^{\circ} \mathrm{C}$. for two hours or one hour in a water-bath. At the end of this time the tube just completely 
hemolyzed contains one amboceptor unit, and in performing the test double this amount is used. (See Fig. 115.)

This titration should always be conducted before the actual tests are set up, as is the rule in conducting the Wassermann reaction. When the titer of the paper is known, it may not be necessary to set up all the tubes of the foregoing series, as a few only are necessary to determine if the same amount of paper as was used in the previous tests will suffice with the new complement and corpuscle suspension at hand.

All titrations and the main tests may be conducted in a water-bath $\left(37^{\circ}\right.$ C.). With the aid of a good thermometer a satisfactory bath is easily improvised. In fact, I believe that better results are secured in the water-bath than in the incubator. It is possible, therefore, to conduct these reactions in a perfectly satisfactory manner without the aid of an expensive incubator.

4. Antigen.-Acetone-insoluble lipoids (Noguchi) are to be used exclusively if the tests are conducted with active serums. When heated serums are used, any extract may be employed, as in making the Wassermann reaction, but the same antigen gives excellent results, and I use it exclusively in conducting the Noguchi reaction with both active and inactivated serums.

The antigen must be titrated as usual, and its anticomplementary hemolytic and antigenic properties determined. According to Noguchi, an extract is satisfactory if it is antigenic in 0.02 c.c. of a $1: 10$ dilution, and not anticomplementary or hemolytic in amounts under 0.4 c.c. $(1: 10)$. In conducting the tests five times the antigenic unit, or 0.1 c.c., is employed; this dose is at least four times smaller than the anticomplementary unit, and is therefore safe and satisfactory.

The antigen is best preserved in methyl alcohol, as described on p. 422. Dried on paper and properly preserved in sealed tubes in a cold place it will retain its activity for several months, but as a general rule it is best to use fresh emulsions of the alcoholic solution.

Titration of Antigen.-The anticomplementary, hemolytic, and antigenic doses of an extract are determined in the same general manner as was described under the Wassermann reaction.

1. Anticomplementary Titration.-A portion of the stock alcoholic solution of acetone-insoluble lipoids is diluted with 9 parts of saline solution. This is the emulsion that is employed in conducting the Noguchi reaction, and contains 0.3 per cent. of the original lipoidal substances. 
Sufficient emulsion for these titrations is prepared by diluting 0.4 c.c. of the alcoholic solution with 3.6 c.c. of saline solution.

Into a series of seven small test-tubes place increasing amounts of this emulsion as follows: $0.1,0.2,0.3,0.4,0.6,0.8,1.0$ c.c., add 0.1 c.c. ( 5 capillary drops) complement (40 per cent.) to each; also 1 c.c. of a 1 per cent. suspension of corpuscles and sufficient saline solution to make the total volume in each tube about 2 c.c. Incubate at $37^{\circ} \mathrm{C}$. for one hour (one-half hour in water-bath), and add two units of amboceptor. Shake the tubes gently and reincubate for two hours (one hour on water-bath). That tube showing beginning inhibition of hemolysis contains the anticomplementary dose, which should not be under 0.4 c.c. In the tubes containing the larger doses slight hemolysis may be noticed, which is evidence of the hemolytic action of the extract.

An eighth tube should be included, containing 0.1 c.c. diluted complement, two units of amboceptor, and 1 c.c. of the corpuscle suspension. This is the hemolytic control and should show complete hemolysis.

2. Antigenic Titration.- Since the extract is likely to have a high antigenic value, it is necessary to dilute the antigen still further by placing 0.5 c.c. of the foregoing emulsion in a test-tube and adding 4.5 c.c. of saline solution (1:100 dilution of the antigen). Into a series of six test-tubes place increasing amounts of this emulsion as follows: 0.05 , $0.1,0.2,0.3,0.4,0.6$ c.c. To each tube add four drops $(0.08$ c.c.) of inactivated or one drop ( 0.02 c.c.) of fresh active syphilitic serum; also 0.1 c.c. (five capillary drops) of complement ( 40 per cent.) and 1 c.c. of 1 per cent. corpuscle suspension. Then add sufficient salt solution to bring the total up to 2 c.c.

Two controls should be included: (1) The serum control, containing the dose of serum, 0.1 c.c. of the complement, 1 c.c. of corpuscle suspension, and saline solution; (2) the hemolytic control, containing at this time 0.1 c.c. of complement, 1 c.c. of corpuscle suspension, and sufficient saline solution.

All tubes are incubated for one hour at $37^{\circ} \mathrm{C}$. (one-half hour in water-bath), after which two units of amboceptor are added to each tube. The tubes are then shaken gently and reincubated for two hours (one hour in water-bath). At the end of this time the two controls should be completely hemolyzed, and in the series proper that tube showing just complete inhibition of hemolysis contains one antigenic unit. Usually the first and second tubes show some inhibition of hemolysis, and in the third and other tubes hemolysis is completely inhibited. In this case 0.2 c.c. of this emulsion would be one anti- 


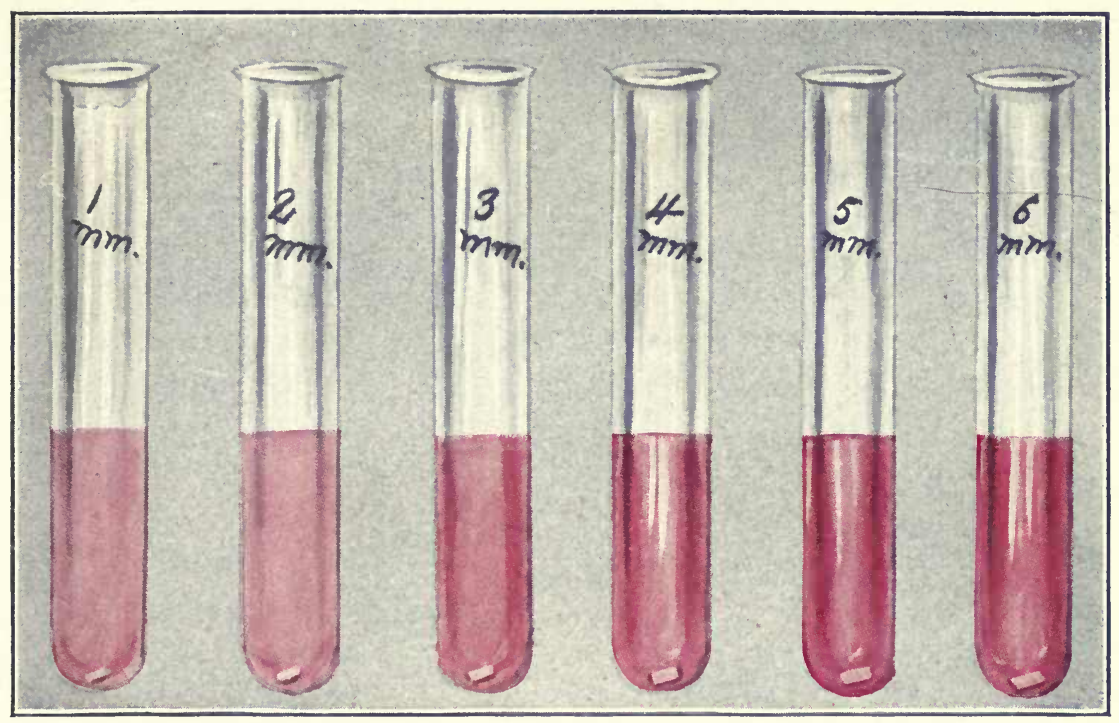

Fig. 115.-Titration of Antihuman Hemolytic Amboceptor.

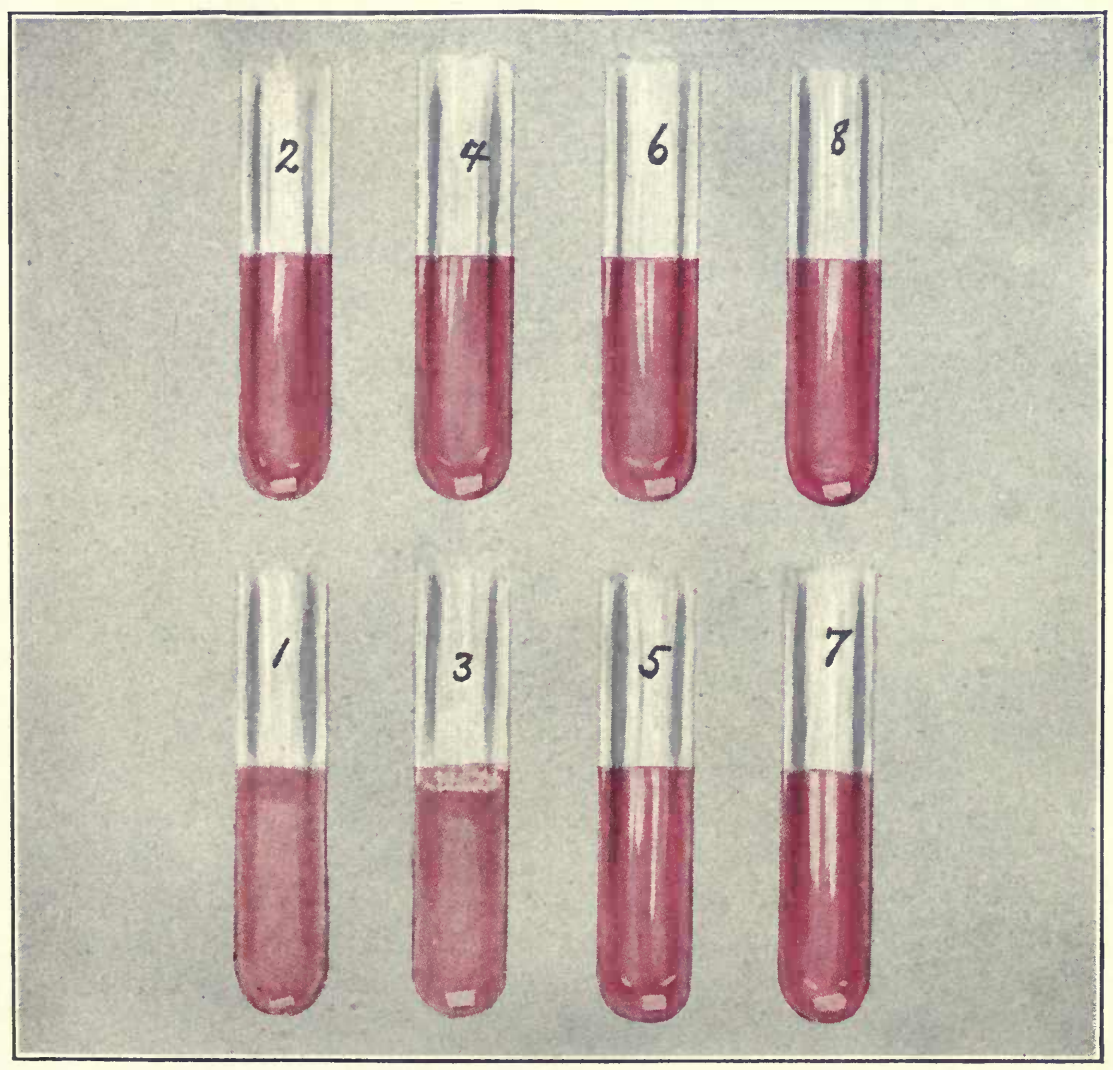

Fig. 116.-Noguchi Modification of the Wassermann Reaction. 

genic unit ( $=0.02$ c.c. undiluted antigen); five times this amount equals 0.1 c.c. of the first emulsion $(1: 10)$, which is the amount to be used in making the main tests.

Unless the antigen shows signs of deterioration, these titrations need be made only about once a month.

If paper antigen is employed, both titrations are conducted in exactly the same manner by adding increasing lengths of a strip of dried paper $5 \mathrm{~mm}$. in width, impregnated with the antigen.

5. Fluid to be Tested.-If active serum is used, it should be fresh, free from hemoglobin, and preferably not over twenty-four hours old. The dose is 0.02 c.c., or one capillary drop; inactivated serums are used in doses of 0.08 c.c., or four capillary drops. Cerebrospinal fluid is used unheated in doses of 0.2 c.c., or 10 capillary drops. Sufficient blood for this test may be collected in a Wright capsule. (See p. 32.)

6. The Test.-The complement, amboceptor, antigen, and serums may be conveniently measured by drops from a capillary pipet (Fig. 2). In placing a drop the pipet should be held uniformly at an angle of 45 degrees, or else the size of the drop will differ, depending on whether the pipet is held vertically or horizontally.

Arrange four pairs of small test-tubes (10 by $1 \mathrm{~cm}$.) in a rack containing two rows of holes. Into each of the tubes on the front row place five drops ( 0.1 c.c.) of antigen emulsion (alcoholic solution, 1 part, with saline solution, 9 parts); then add five drops ( 0.1 c.c.) of complement (40 per cent.) to all the tubes. Into each of the first pair of tubes place one drop (0.02 c.c.) of active or four drops $(0.08$ c.c.) of inactivated patient's serum, and mark the front tube with the patient's name. To each of the second pair of tubes add an equal amount of syphilitic serum known to give a positive reaction (positive control), and to each of the third pair add normal serum known to give a negative reaction (negative control). Mark the tubes in the front row of each pair respectively. The front tube of the fourth pair is the antigen control, and the rear tube the hemolytic control, and each should be so labeled. Into each tube place 1 c.c. of the 1 per cent. corpuscle suspension and 1 c.c. of saline solution, making the total volume in each tube about 2 c.c. Shake each tube and incubate at $37^{\circ} \mathrm{C}$. for one hour (half an hour in the water-bath). At the end of this time add two units of amboceptor to each tube, shake gently, and reincubate for two hours (one hour in the water-bath). During this time the tubes should be shaken gently once or twice to break up any masses of agglutinated corpuscles.

The following chart, after Noguchi, illustrates the various steps to 
be taken in making the test with one patient's serum. Of course, any number of serums may be examined with the same controls (Fig. 116).

TABLE 16.-NOGUCHI MODIFICATION OF THE WASSERMANN REACTION

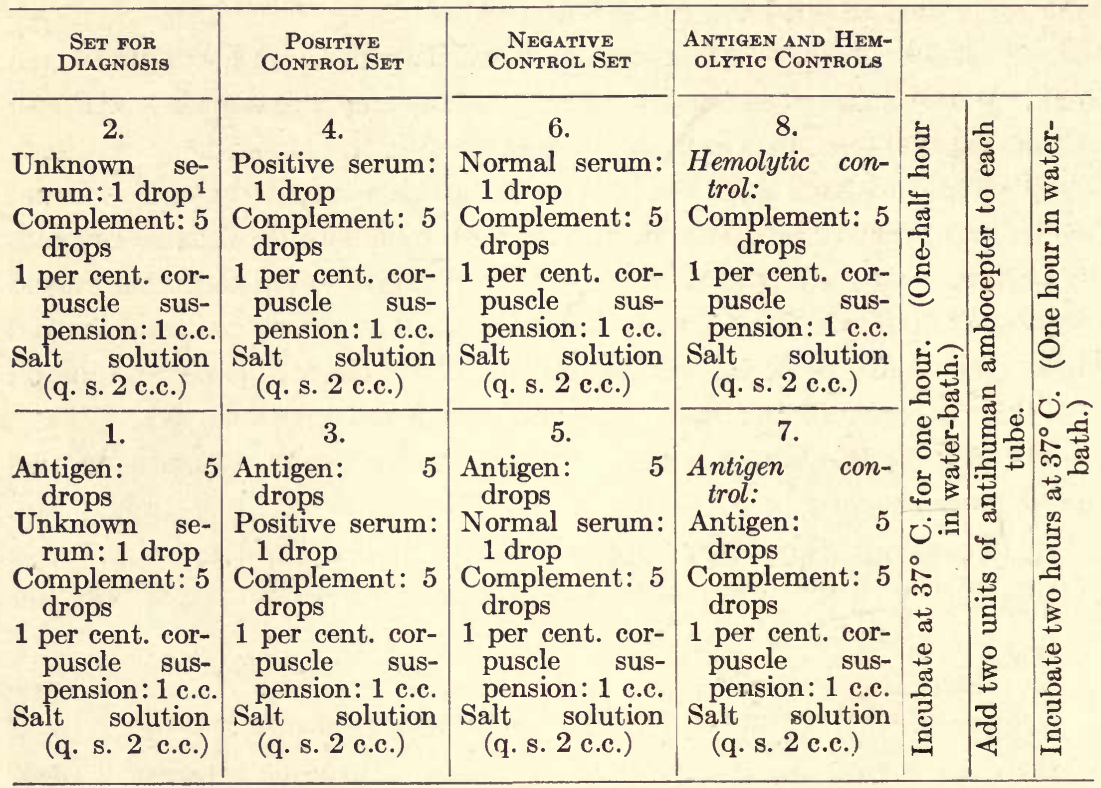

At the end of the second incubation, or after two hours more at room temperature, the tubes are inspected. The antigen and hemolytic system controls, as well as all the rear tubes or serum controls, should be completely hemolyzed. The first tube containing a known syphilitic serum shows inhibition of hemolysis; the front tube containing normal serum is completely hemolyzed; the front tube containing the patient's serum shows complete inhibition of hemolysis (strong positive), varying degrees of inhibition (moderately or weakly positive), or is completely hemolyzed (negative). The results may be recorded and reported after the same manner described on p. 440.

\section{MODIFICATION OF BAUER}

Bauer does not use rabbit-sheep amboceptor, but takes advantage of the antisheep amboceptor normally present in variable amounts in a large proportion of human serums. Although this test is quite delicate, the quantity of natural amboceptor in human serums is too variable a factor to be depended upon, and the modification is not, therefore, in general use.

${ }^{1}$ Four drops if serum is inactivated. 


\section{MODIFICATION OF HECHT-WEINBERG}

In conducting the syphilitic reaction Hecht $^{1}$ utilizes not only the natural antisheep amboceptor in human serum, but also the native hemolytic complement. The serum must be perfectly fresh, and, of course, is used unheated. This modification has been said to be more delicate than the Wassermann reaction, because none of the syphilis antibody is destroyed or complementoids produced, as, presumably, will occur during inactivation (heating) of a serum. In my experience, this test has proved quite delicate, but is open to the same error that may occur whenever an active serum is used, with a crude alcoholic organic extract as antigen-i.e., the appearance of false positive or proteotropic reactions. As with the Noguchi reaction, using active serum, a negative Hecht-Weinberg test has considerable diagnostic value in excluding syphilis; a positive reaction must be, however, carefully controlled. In performing the test I always use an extract of acetone-insoluble lipoids as antigen.

The Hecht test is performed as follows: An alcoholic extract of human heart is used as antigen. Each serum is tested in four small tubes. The serum must be fresh (not over twenty-four hours old) and unheated.

Tube 1: 0.02 c.c. serum ( 1 drop) and 0.08 c.c. ( 4 drops) of $1: 50$ dilution of heart extract.

Tube 2: 0.02 c.c. serum and 0.08 c.c. of $1: 100$ dilution of heart extract.

Tube 3: 0.02 c.c. serum and 0.08 c.c. of $1: 200$ dilution of heart extract.

Tube 4: 0.02 c.c. serum and 0.08 c.c. of normal salt solution. This is the one control of the test and should show complete hemolysis.

The tubes are placed in the incubator for half an hour, or into a water-bath at a temperature of $37^{\circ} \mathrm{C}$. for ten minutes. One cubic centimeter of a 1 per cent. suspension of sheep's cells is added to each tube, and the tube shaken gently and reincubated for half an hour or ten minutes, as the case may be.

Tube 4 must show complete hemolysis; if it does not do so, the test is worthless. In the test of a strongly positive acting serum all the other tubes show no hemolysis, whereas a weakly acting serum shows lysis in all tubes but Tube 1 .

This test is exceedingly simple, but is somewhat crude and unreliable. If employed at all, the results should always be controlled by the Wassermann reaction.

It is necessary to avoid using too large doses of sheep's cells. Since in the test just described, there is no way of determining beforehand the amount of corpuscles a serum may hemolyze, Gradwohl ${ }^{2}$ has modified the technic so that, the hemolytic index of each serum is determined before corpuscles are added to the main tubes. His method is as follows:

"Place in a rack 14 small test-tubes. The first 10 of these tubes are used to determine the hemolytic index of the suspected blood. By this we mean the exact amount of hemolytic amboceptor present in the given blood-serum. The last

${ }^{1}$ Wien. klin. Wochenschr., 1909, xxii, 265.

2 Jour. Amer. Med. Assoc., 1914, lxiii, 240. 
four tubes are used in the actual test. Add 0.1 c.c. of fresh unheated patient's blood-serum to each of the first 10 tubes. Then add decreasing amounts of normal salt solution to these tubes, beginning with 1 c.c., then $0.9,0.8,0.7,0.6$, $0.5,0.4,0.3,0.2,0.1$ c.c. to the succeeding nine tubes. Next add increasing amounts of fresh 5 per cent. suspension of sheep's blood, starting with 0.1 c.c. and ending with 1 c.c. Place the rack in the water-bath for one-half hour. The tube which last shows complete hemolysis constitutes our "hemolytic index"; if it is Tube 4 , our index is 4 , because this tube had received 0.4 c.c. of sheep's corpuscles. The index determines the amount of sheep's corpuscles to be added to the last four tubes. The first three tubes $(11,12$, and 13) constitute the tubes for the actual test, while the last tube in the rack (Tube 14) serves as our serum control tube. Tubes 11,12 , and 13 receive, therefore, the patient's serum, the proper amount of sheep's corpuscles (dependent on the hemolytic index), rising strengths of antigen, but no complement and no amboceptor. Tube 14 receives only sheep's corpuscles but no antigen. In our technic we use 0.1 c.c. of a diluted antigen, determined by titration in Tube $11,0.15$ c.c. antigen in Tube 12 , and 0.2 c.c. in Tube 13 . In order to equalize the volume of fluid in all these tubes we add 0.2 c.c. normal saline to Tube $11,0.15$ c.c. to Tube 12 , and 0.1 c.c. to Tube 13 , and 0.3 c.c. to Tube 14 . The tubes are then agitated and placed in the water-bath for half an hour. These last four tubes are filled at the time we make the additions to the first 10 and are left with them in the water-bath for half an hour for fixation of complement; the rack is then taken out and the hemolytic index computed. If the index is low, say from 1 to 4 , we add 0.1 c.c. of sheep's blood to the last four tubes. If the index is between 5 and 7 , we use 0.15 c.c. sheep's blood, and if it is between 7 and 10 , we use 0.2 c.c. In our experience in this country we have never found an index above 10, although in France it is not uncommon to obtain an index of 15 or 17.

"If the patient's serum has an index below 3, we regard the reaction as of doubtful value. If it is above 3 , we regard it as absolute. The reaction is read off exactly as is the Wassermann, that is, inhibition or non-inhibition of hemolysis."

\section{MODIFICATION OF STERN}

Margaretta Stern devised a modification of the Wassermann reaction, using fresh active serum and the patient's complement, and overcoming non-specific reactions by using $\frac{2}{5}$ to $\frac{1}{5}$ of the usual dose of extract, and three or four times the amboceptor unit. This method is open to defects inherent in the use of variable amounts of complement and excessive amounts of hemolytic amboceptor, which makes it impossible to test a specimen a few days after it has been collected.

\section{MODIFICATION OF TCHERNOGUBOU}

Tchernogubou proposed an antihuman hemolytic system with active serum. Blood is collected in sodium citrate, and therefore contains erythrocytes, complement, and syphilis antibody if the patient is luetic. Antigen is added, and after sufficient time has elapsed for fixation of complement to take place, antihuman amboceptor is added to test for free complement. There are many objections to this method, the chief ones being the variable amount of complement present in human serum, the large amount of hemolytic amboceptor required, the absence of a suitable control on the antigen, and the fact that old blood is entirely unsuited for making the test.

Tchernogubou has also proposed a system in which the natural amboceptor and complement of human serum are utilized against 
guinea-pig corpuscles. These factors are so variable that this modified test has been largely abandoned.

\section{MODIFICATION OF DETRE AND BREZOVSKY}

In this modification an antihorse hemolytic system is used with rabbit's complement. The chief objections are the variations in the activity and fixability of rabbit complement, and the difficulty of obtaining horse blood. In addition to these, this method possesses no advantages over Wassermann's antisheep system.

\section{MOdIFICATION OF BROWNING AND MACRENZIE}

These investigators use an antiox hemolytic system, and have modified the original Wassermann technic so as to make the method an accurate quantitative test. (See p. 446.) They claim that with their modified technic the results secured are practically the same with the antiox and antisheep systems, although the human serums contain much less natural antiox amboceptor and, theoretically, therefore this system is better.

\section{MODIFICATION OF VON DUNGERN}

More recently von Dungern has proposed a modification similar to that of Noguchi. Like Tchernogubou, he uses active serum only, and utilizes the patient's own blood-cells. The blood is defibrinated and used in doses of 0.1 c.c. Complement in the human blood is disregarded, and is furnished by guinea-pig serum in dried-paper form. Alcoholic extract of syphilitic liver is used as antigen, and this opens up an avenue for false positive or proteotropic reactions to creep in. Antihuman amboceptor is prepared by immunizing goats, but, as Noguchi has shown, this amboceptor gives a much weaker hemolytic reaction than that derived from the rabbit. Von Dungern generally omits the important control with known syphilitic serum; there is no direct control on the antigen, and old bloods cannot be used.

\section{THE WASSERMANN REACTION IN THE VARIOUS STAGES OF SYPHILIS}

1. In Primary Syphilis.-As would be expected, a certain degree of tissue change mist occur before syphilis reagin appears in the blood. Even with the best technic there is a limit to the sensitiveness of the Wassermann reaction, so that while the reagin may be produced at the very onset of an infection, time and further tissue changes are required before sufficient reagin is produced to yield a complement-fixation reaction.

Since, therefore, the results of the Wassermann reaction in primary 
syphilis are dependent upon the virulence of the infection, the time at which the reaction is made, and the delicacy of the technic, it is not surprising that the results of different investigators vary in the proportion of positive reactions obtained. While positive reactions have been said to have been secured before the appearance of the initial lesion, these are rare, and there is always the likelihood that an earlier infection was overlooked. A careful review of our own work and the literature upon this subject establishes the following:

(a) A positive reaction may be secured as early as from four to five weeks after infection has occurred.) In such cases, however, it is oftentimes probable that the time of infection in reality antedates the time given by the patient. Craig has reported a positive reaction occurring five days after the appearance of the initial lesion. Levaditi, Laroche and Yamanouchi, and others have recorded many positive reactions occurring in ten days or more after the chancre made its appearance.

(b) As a general rule, the Wassermann reaction becomes positive during about the seventh to the eighth week after infection, or just a week or two before the onset of the secondary eruption. In other words, the reaction is usually secured first late in the primary stage, and in a large proportion of cases before the secondary symptoms appear.

(c) In general, in primary syphilis the Wassermann reaction will be positive in about 80 per cent. of cases; where cholesterinized extracts are used as antigens, or with the Noguchi system, using active serum, the reactions are secured earlier and in a larger percentage of cases.

(d) It is generally agreed that a diagnosis should be made as early as possible, and vigorous treatment instituted. A Wassermann reaction may be performed, and if it shows a positive result, this indicates the presence of syphilis, even if the lesion under suspicion is not specific, the reaction being due to a previous infection. A negative reaction, however, does not exclude syphilis, and if it is at all possible, a microscopic examination, using the dark-ground illuminator, should be made for the treponema. In primary syphilis a microscopic examination of the secretions of the lesion by a competent person is usually more valuable than the serum test; as a general rule, both examinations should be made, especially with patients in whom the chancre is almost healed or atypical.

(e) The cerebrospinal fluid of persons in the primary stage of syphilis has always reacted negatively (Boas).

2. In Secondary Syphilis.-It is in untreated cases of secondary syphilis that the remarkable specificity of the Wassermann reaction 
is so well demonstrated. The initial lesion may have been inconspicuous and hence have been overlooked, and the secondary lesions may be quite mild and inconclusive; in either case the Wassermann reaction will usually establish the diagnosis.

(a) In untreated secondary syphilis the reaction is positive in from 92 to 100 per cent. of cases. In the examination of 437 serums from untreated cases Boas has never had a negative reaction, and my own experience has been the same.

(b) With the serums of patients who have received some treatment, the percentage of positive reactions will be slightly lower. Of 310 such cases examined by Boas, 97.6 reacted positively. The influence of treatment upon the reaction is to be remembered, and a single negative reaction does not by any means exclude the possibility of syphilis.

(c) The intensity of the reaction does not bear any direct relation to the severity of the infection: a mild infection with indefinite signs may react quite strongly and absorb a large number of units of complement, whereas a severe case may react quite mildly.

(d) In secondary syphilis without cerebral symptoms the cerebrospinal fluid is practically always negative (Plaut, Boas and Lind); conversely, cases showing cerebral involvement usually react positively. More recent work has shown that the cerebrospinal system is involved early and in a relatively large number of cases (Craig and Collins ${ }^{1}$ ). Udo J. Wile has found that about 30 per cent. of secondary syphilitics give a positive reaction with cerebrospinal fluid.

3. In Tertiary Syphilis. - It is probably in tertiary syphilis that the Wassermann reaction has its greatest value. Lues is so diverse in character, and may be responsible for so many diverse clinical conditions, that the reaction has become well-nigh indispensable as a diagnostic aid. There is no limit to the time following infection in which positive reactions may not be found.

(a) In cases of untreated and active tertiary syphilis the reaction is positive in about 96 per cent. of cases.

(b) In cases receiving more or less antispecific treatment the reactions are positive in about 75 per cent. In general, therefore, a positive reaction in tertiary syphilis may be expected in from 80 to 85 per cent. of cases.

(c) In a large percentage of cases of syphilitic aortitis, aortic aneurysm, aortic insufficiency, gummas of various organs, etc., the reaction is positive and possesses great diagnostic value.

1 Jour. Amer. Med. Assoc., 1914, 1xii, 1955. 
(d) The Wassermann reaction has been especially valuable in the study of the so-called parasyphilitic diseases. In general paralysis or paralytic dementia the serum reacts positively in about 100 per cent. of cases, and the cerebrospinal fluid reacts positively in about 92 per cent. The final and conclusive proof of the syphilitic nature of this disease has been furnished by Noguchi and Moore, who found the Treponema pallidum in sections of the brain. In certain cases of general paralysis the blood-serum may react negatively, whereas with the cerebrospinal fluid the reaction is positive.

The fact that the blood-serum of a patient with a nervous disease reacts positively does not necessarily indicate that the nervous disease is of syphilitic origin, as the reaction may be due to specific infection of some other structure; if, however, the cerebrospinal fluid also reacts positively, then it is almost certain that syphilitic infection of the central nervous system is present.

In untreated and active cases of tabes dorsalis the blood-serum reacts positively in from 96 to 100 per cent. of cases. In treated cases the number of positive reactions drops to about 40 to 50 per cent; in general, therefore, a positive reaction with the serums of tabetics may be expected in 73 per cent. of cases. With the cerebrospinal fluid the percentage of positive reactions is somewhat lower, being about 60 per cent. The positive Wassermann reaction is less constant in locomotor ataxia than in general paralysis, due probably to the fact that the former is more chronic and that intercurrent periods of arrest are more prone to occur.

In cerebral syphilis the blood-serum, and particularly the cerebrospinal fluid, will react positively less frequently than in general paralysis. In some instances a positive reaction is found with the cerebrospinal fluid and not with the serum, a matter difficult to explain and believed to be due to the confining of the reacting substances in the subarachnoid space. On the other hand, the lesions are probably not brought in direct contact with the spinal fluid.

There is much evidence to indicate that localization of syphilis in the nervous system is dependent upon a particular strain of Treponema pallidum; other strains appear to possess a special affinity for the visceral organs, bones, etc.

(e) In tertiary syphilis not accompanied by lesions of the central nervous system the Wassermann reaction with cerebrospinal fluid may be positive in a relatively large percentage of cases.

4. In Latent Syphilis.-In cases of latent syphilis the Wassermann 
reaction may constitute the only evidence of the existence of the disease, and prompt institution of treatment may prevent the development of tertiary lesions, which are so likely to follow. When the spirochetes are few in number and are dormant, there is little tissue destruction or alteration, and, as a result, so little reagin is frequently present in the body-fluids that the Wassermann reaction will fail to detect the disease.

(a) In 363 cases of early latent syphilis, or those included within a period of three years after infection, Boas found positive reactions in about 40 per cent.; in latent cases of long standing, or in those following manifest tertiary lesions, the same investigator found 22 per cent. of positive reactions among those who had received proper treatment; of those receiving indifferent treatment, 74 per cent. reacted positively, giving a general average of about 48 per cent.

(b) The reaction with cerebrospinal fluid depends upon whether or not the central nervous system is involved in the syphilitic process. Of 104 latent cases of syphilis in whom the spinal fluid was examined by Altman and Dreyfus, positive reactions were found in about 10 per cent.

5. Congenital Syphilis.-The Wassermann reaction has thrown considerable light upon the subject of congenital syphilis. While, in general, the majority of cases react positively, the results are largely dependent upon the time when the examinations are made, a fact brought out by the highly instructive and systematic investigations of Boas and Thomsen. These investigators divided their cases into three groups: (1) Newly born children and their mothers; (2) two-year-old children; (3) older children with congenital syphilis.

(a) Of 88 children born of syphilitic mothers and examined at birth, the reaction was positive in 31 and negative in 57 cases. Of the 31 positive cases, 4 showed no symptoms of syphilis for a period of observation covering from three to nine months, and it is possible that the syphilis reagin, and not the spirochetes, from the blood of the mother, passed into the circulation of the child; on the other hand, all four cases may have been examples of retarded congenital syphilis. The remaining 27 cases either developed symptoms of syphilis or died later with syphilitic manifestations in various organs.

Of the 57 children reacting negatively at birth, 42 showed no symptoms of syphilis during a period of three months of observation; 2 died with evidences of syphilis in the internal organs; 13 developed symptoms after birth and gave positive reactions.

It may therefore be stated that a Wassermann reaction of the mother and of the child at the time of birth in cases where syphilis of the mother 
is suspected has considerable prognostic value. A large majority of children reacting positively develop symptoms of syphilis; on the other hand, the majority reacting negatively remain healthy. While an examination of the mother alone does not warrant an absolutely definite prognosis for the child, in general it may be said that a positive reaction does not constitute a favorable prognostic sign for the child.

(b) The Wassermann reaction has also shed new light upon the interpretation of Colles' law. Since the "apparently healthy mother of a syphilitic child could suckle the child without being infected, whereas the child is capable of giving syphilis to others," the most logical conclusion to draw is that the mother was gradually immunized against syphilis during pregnancy, whereas we now know that the majority of mothers show positive serum reactions and are really latent syphilitics; in not a few such instances tertiary lesions have developed at a later date.

It is possible, however, for a syphilitic mother showing a positive Wassermann reaction to give birth to a healthy child. Of 46 mothers whose children showed no evidences of syphilis over a period of observation of three months, 17 reacted positively. Of 81 mothers giving birth to syphilitic children, 61 reacted positively, and many of these would naturally, in former years, have been regarded as examples of Colles' immunity and considered free of syphilis. In many instances the apparently healthy child of a syphilitic mother that could not be infected by the mother (Profeta's law) has been shown by the Wassermann reaction to be in reality a case of retarded congenital syphilis, and that such children are not immunized, during intra-uterine life, either passively or by means of pallidum toxins, against syphilis, as has been so generally believed in past years. In other words, there appears to be no lasting passive immunity in syphilis; it is doubtful if the toxins of pallidum can pass between mother and child and immunize one or the other without actual infection with the spirochetes themselves taking place; that most examples of so-called immunity in syphilis in both the mother (Colles' law) and the child (Profeta's law) are due to the actual presence of pallidum in the tissues and are really latent infections.

(c) In manifest untreated congenital syphilis of children one year or over in age the Wassermann reaction is positive in from 97 to 100 per cent. of cases. The clinical manifestations may be quite varied and clinically ill defined, so that the serum reaction possesses considerable diagnostic value. In most instances the reactions are quite strong, and while active treatment may improve local lesions, it is very difficult, indeed, to secure negative reactions. 
(d) In congenital mental deficiency the Wassermann reaction shows that syphilis plays a larger part in the etiology of this condition than is generally supposed. A not inconsiderable proportion of cases are of infectious origin, and that infection is syphilis. In Little's disease, which is regarded as due to meningeal hemorrhage incidental to injury received during labor, the serum reactions have shown that not infrequently the hemorrhage has a syphilitic origin.

\section{THE SPECIFICITY OF THE WASSERMANN REACTION}

The highly specific nature of the syphilis reaction has been proved by very extensive investigations with the serums of normal persons and of persons afflicted with diseases other than syphilis. Unfortunately, the reaction is beset by so many technical errors that a review of the literature, and especially of the early literature, shows results that are quite confusing and contradictory. Following the original communications of Wassermann and Detre, and especially after it was demonstrated that the antigen need not be biologically specific, the subject was extensively investigated by various observers, who reported securing positive reactions in many different diseases, results that we now know must have been due largely to technical errors. At present it is known that positive Wassermann reactions may occur in a few diseases other than syphilis, but not to the extent that earlier investigators would have us believe. In most of the diseases yielding positive reactions the clinical symptoms are so marked that they may readily be differentiated from syphilis, and accordingly the Wassermann reaction is of unequaled and incalculable diagnostic value.

Positive reactions have been reported in frambesia (yaws), in which the causal microörganism, the Spirochæta pertenuis, is morphologically almost indistinguishable from Spirochæta pallidum. In leprosy of the tuberous type positive reactions are frequently found, but cases of anesthetic leprosy usually react negatively. Positive reactions have been reported in cases of malaria during the febrile stage, when parasites are present, although the majority of cases react negatively. In my own series of 11 cases all the reactions were negative. Positive reactions have also been found in some cases of relapsing fever.

In scarlet fever the Wassermann reaction is uniformly negative. Owing to the original communication of Much and Eichelberg, however, in the minds of many this disease is prominently associated with a positive reaction. While it is true that a positive reaction is very rarely found, it is almost impossible entirely to exclude a diagnosis of con- 
genital lues, at least in some of these cases. In my own series of 250 cases examined by the Wassermann and Noguchi methods, with antigens of alcoholic extract of syphilitic liver and acetone-insoluble lipoids, the reactions were positive in 5 cases, or 2 per cent. Similar results have been secured by Boas, Browning and Mackenzie, and others, so that it may be said that the reaction in scarlet fever is uniformly negative. It is also to be remembered that occasionally, or in about 1 to 2 per cent. of cases, a positive reaction may follow ether or chloroform anesthesia, but that this will later disappear. In pellagra Fox, and later Bass, have found occasional positive reactions.

Normal cerebrospinal fluid or the fluid from persons with ordinary non-syphilitic diseases reacts negatively. Positive reactions have been reported in leprosy and in frambesia.

\section{THE EFFECT OF TREATMENT UPON THE WASSERMANN REACTION}

Citron originally observed that during the mercurial treatment of syphilis the Wassermann reaction gradually became weaker, and finally disappeared. He also found that treatment was best governed by the serum reaction, and that it should be persisted in until a negative reaction was secured. His observations have in the main been abundantly confirmed by various observers the world over, although the extensive series of observations now on record have given us a fuller understanding of its principles.

The Wassermann reaction is the most constant and delicate single symptom of syphilis, and whenever a serum is found to react positively, antisyphilitic treatment is indicated, and should be persisted in until the reaction becomes negative and remains so for a sufficiently prolonged period of observation. It is now quite generally believed that a persistently positive reaction indicates the presence of living spirochetes, and that treatment should be continued until the blood reacts negatively. The reports of observers from all parts of the world indicate quite clearly and conclusively that the schematic, symptomatic, intermittent, and hard and fast rules of treatment of former days are not sufficient. They would also tend to show that the Wassermann reaction is the most delicate symptom and the last to disappear, and that treatment should be continued until this reaction disappears entirely and permanently. (It has been abundantly proved, however, that in syphilis a single negative reaction is not sufficient or definite evidence that a cure has been effected, for the disease may recur after treatment is discontinued, at least to the extent that the Wassermann reaction reappears, 
followed by clinical manifestations. It is necessary, therefore, that successive examinations be made during a period of at least two years, and off and on during the remainder of life. Recent work indicates that certain strains of Spirochæta pallida have an apparent selective affinity for the tissues of the central nervous system; the Wassermann reaction with blood-serum may be negative, whereas with the cerebrospinal fluid it may be positive. In cases, therefore, of tertiary syphilis, at least, it is advisable to examine the spinal fluid and continue treatment in case it shows a positive Wassermann reaction.

It should be the object of treatment, in every case, not only to dissipate the external and obvious lesions of the disease, but to produce a condition of the blood in which the Wassermann reaction is permanently negative. It is quite generally agreed that the older methods of treatment, consisting of the administration of mercury and the iodids over fixed and arbitrary periods of time, or until all manifest symptoms have disappeared, are insufficient, and that the criteria by which the effects of treatment can best be judged are: (1) Continued absence of symptoms, and (2) permanent negative Wassermann reactions.

It is to be remembered, therefore, that while a single negative reaction is a satisfactory indication of the progress of treatment, it does not signify that a permanent cure has been effected. The Wassermann reaction cannot be regarded as sufficiently delicate to indicate that a single negative reaction means that a patient is totally free from all spirochetes, for in some instances the reaction and the clinical symptoms may recur after the treatment has been suspended, but the reaction is the first symptom to reappear and the earliest indication of an impending lesion. For all practical purposes the occurrence of a negative reaction after treatment indicates either complete destruction of all the spirochetes, or at least that the parasites are being held in abeyance and rendered potentially harmless.

It is, accordingly, reasonable to regard the Wassermann reaction as the most delicate indicator of generalized spirochetal infection or the assumption of spirochetal activity. A positive reaction indicates that serious effects and gross local lesions are likely to occur at any time, and that treatment should be continued. For all practical purposes a con. tinued absence of symptoms and a permanently negative reaction are strong presumptive evidences that a cure has been effected.

The serum should be tested every six months during the treatment, and at periods of at least six months to a year after treatment has been discontinued for several years. Persistently positive reactions during 
treatment would indicate that more active measures or a change in therapy are needed. The occurrence of a positive reaction after treatment has been discontinued is an indication for its resumption.

For a control on treatment the Wassermann reaction should be made as delicate as possible, for while more prolonged treatment may be somewhat irksome to the patient, it is clearly indicated as a preventive of serious after-effects, especially of involvement of the central nervous system. It is in this branch of the work I have found that the use of sensitive cholesterinized extracts as antigens in making the Wassermann reaction or the Noguchi modification with the use of active serum, of great value as the most delicate indicators.

One fact is to be clearly emphasized, namely, that the earlier energetic treatment is begun, the more likely it is that a permanent cure will be effected. Energetic treatment with mercurials or salvarsan, or, better, with a combination of both, begun early and continued long, will in the majority of cases restore the serum to its normal condition. In general, the greater the interval of time allowed to elapse between infection and institution of treatment, the more difficult it is to restore the serum to normal. Tertiary cases are cured only as the result of most persistent treatment, and not infrequently in congenital syphilis, locomotor ataxia, and general paralysis all one can hope to accomplish is to check the progress of the disease. The most favorable cases are those in which early diagnosis is made possible by clinical manifestations, preferably confirmed by a demonstration of pallidum, and in which treatment is undertaken before the serum has begun to react positively, and in which the reaction remains negative throughout.

Treatment will, however, at least influence the Wassermann reaction in practically all stages of syphilis. In a series of 435 cases of syphilis in all stages reported by Boas, a negative Wassermann reaction was secured in no less than 80 per cent., and all but one of the remaining cases showed a weaker reaction. The figures of different observers are not all so favorable as these, a factor dependent to some extent, at least, upon differences in the technic of the reaction. In general, however, Boas' observations have been confirmed by other competent workers.

The effect of any treatment is greatly influenced by the individuality of the host, certain persons possessing tissues more amenable to the effects of the therapeutic agent than those of others. The therapeutic effect is also dependent upon the virulence of the parasite and the apparent selective affinity of certain strains of pallidum for particular organs, and upon the method of treatment selected. 
The influence of salvarsan and neosalvarsan as agents in the treatment of syphilis is considered in more detail in the chapter on Chemotherapy. My experience has shown that the earlier belief in the complete sterilization of the human patient by a single dose was generally unfounded, and that repeated smaller doses of the drug, used in conjunction with mercurials, are necessary. Potassium iodid alone may favorably influence the clinical symptoms and weaken the Wassermann reaction in a small percentage of cases, and the same result has been observed with such arsenical preparations as Fowler's solution, atoxyl, arsacetin, and arsenophenylglycin.

It is to be remembered that, during or immediately after active treatment with salvarsan or mercury, the Wassermann reaction may be negative, even though the patient is not cured. As a general rule, a negative reaction under these conditions should not be considered of value unless all treatment has been omitted for at least two weeks; even then the test, if negative, should be repeated a month or so later. Craig has recently drawn attention to the fact that in frank untreated cases the degree of the reaction may vary within wide limits, and this is especially true if the patients are receiving active treatment.

Provocatory Stimulation.-Paradoxic as it would at first appear, antisyphilitic treatment may convert a negatively reacting serum into a positive one. In not a few cases of latent syphilis reacting negatively the administration of a specific spirillicidal agent, such as mercury or salvarsan, is followed by positive reactions, due probably to the liberation of endotoxins from destroyed spirochetes or to a stimulation of the spirochetes by a dose of drug that did not suffice to kill them. This condition is analogous to the Herxheimer reaction, or the aggravation of skin lesions sometimes observed to follow the administration of mercury or salvarsan. The fact possesses practical value, for in cases where lues is known to have been present or is strongly suspected, and the Wassermann reaction is indefinite or negative, the administration of salvarsan or mercury, either internally or by inunction for a period of from ten days to two weeks, followed a week after the last dose by a Wassermann reaction, may now show a positive reaction and thus indicate a latent syphilis requiring further treatment.

\section{PRACTICAL VALUE OF THE WASSERMANN REACTION}

As previously stated, the Wassermann reaction serves two important purposes: (1) As an invaluable aid in the diagnosis and (2) as a guide in the treatment of syphilis. 
The reaction may be of great value in determining the diagnosis of extragenital sores and of atypical lesions in all stages of syphilis. A negative reaction, however, has less value than a positive one, and whenever possible, a microscopic examination of the secretions with the dark-field illuminator should be made in order to confirm the diagnosis. In early latent syphilis, after the initial lesion has healed, and before the secondary eruption appears, the Wassermann reaction is frequently the only means of making the diagnosis, especially if the chancre has been small, atypical, and practically neglected.

Indefinite symptoms and clinical unrecognizable cases constitute a considerable proportion of cases of syphilis, and, as is true in all other infections, this class constitutes the greatest menace to public health. Many patients are sincere in denying knowledge of infection and early symptoms may be overlooked, the Wassermann reaction being the sole means of diagnosis and serving in this connection as an invaluable aid.

Usually the symptoms of syphilis are so well marked in the secondary stage that the reaction is in most instances but confirmatory evidence. However, in doubtful cases a negative reaction excludes syphilis with almost absolute certainty, especially if the reaction is repeatedly negative.

In the late latent and tertiary stages of syphilis the Wassermann reaction may be the only available basis on which to establish a diagnosis. When one remembers how varied are the clinical manifestations of chronic syphilis, how wide-spread is the disease, and how frequently the reaction establishes the true diagnosis, the reaction must be regarded as being of great value and as an indispensable diagnostic aid. (It must not be forgotten that patients showing an early involvement of the central nervous system, and even those showing no such symptoms, may react negatively with blood-serum and positively with spinal fluid; in all such cases the spinal fluid should be examined whenever possible.

A positive reaction occurring in aborting women is an indication for treatment and may protect the fetus. Similarly a positive reaction in either parent of a seemingly healthy infant is an indication for treatment of the child especially if the mother reacts positively.

In this connection, however, one point is worthy of special emphasis, namely, that although a positive reaction indicates that the patient is luetic, it does not necessarily mean that a particular lesion is syphilitic. For example, a person may be luetic and yet have a cancerous ulceration of the larynx. The mere fact that the lesion does not improve under antisyphilitic treatment does not detract from the value of the Wasser- 
mann reaction, but is a warning that more care is required in making the clinical examination. I have seen a number of such cases in which a positive Wassermann reaction was held a priori as evidence of the syphilitic nature of a lesion that later proved to be either malignant or tuberculous. A weak positive reaction, associated with an active ulcerating lesion, very frequently indicates that the lesion is not syphilitic, for active lesions usually yield strongly positive reactions.

In this connection may also be mentioned the growing importance the Wassermann reaction has assumed in life-insurance examinations. Statistics show that from one-tenth to one-third of all persons infected with syphilis die as the results of the disease, and the death-rate among 5000 syphilitics accepted for insurance was one-third over expectation (Brockbank).

An important question, especially from the standpoint of therapeutics is: Does a positive reaction invariably indicate the presence of living spirochetes? May the reaction remain positive for an indefinite time after the patient has been cured, just as agglutinins and antitoxins may persist in the blood for some time after recovery from typhoid fever and diphtheria has taken place? The sum total of the experience of investigators from all parts of the world would indicate that a persistently positive reaction means the presence of/living spirochetes somewhere in the body. The lesions may not be active; the patient, while clinically healthy, may be infective, and is always subject to possible recurrences of clinical syphilis.

Although gummas are slightly infectious, it is now known that they contain living spirochetes, and the former view, which regarded them as sequels, rather than as actual active lesions of syphilis, is no longer tenable.

Just how long the reaction may remain positive after the patient is actually cured and all spirochetes are dead is, of course, difficult to state, but experimental studies on the lower animals has shown that the reagin disappears somewhat quickly under these conditions.

Although a persistently negative reaction is of good prognostic importance, it is not so conclusive in the information it yields as is a positive reaction. In other words, an occasional active lues may react negatively, and not infrequently active syphilitic lesions are found at autopsy in persons whose blood reacted negatively during life. While it is true that great harm may result from a false positive diagnosis due to faulty technic, yet it must be admitted that the Wassermann reaction is not too delicate, and that we are just as prone to err on the side 
of securing too many negative reactions. Every effort should be made to render the test as delicate as is possible with specificity.

While the value and dependability of the Wassermann reaction are based upon skilful technic that will eventually limit the performance of the test to specially trained persons in central laboratories, every effort should be made to render accessible to all persons this valuable diagnostic test of a disease that has such great social and economic importance. At present many persons are unable to afford the expense of a number of tests, or even of one test, as required in the modern treatment of this disease. This deficiency should be corrected, and the test made available in all free dispensaries, especially those under the supervision of a Social Service Department. 


\section{CHAPTER XXIV}

\section{THE TECHNIC OF COMPLEMENT-FIXATION REACTIONS}

(Continued)

Specific Complement Fixation in Bacterial Diseases.-As has been stated elsewhere, the first complement-fixation tests were performed by Bordet with bacterial antigens and antiserums (pest and typhoid). Following the application of the principles of complement fixation in the serum diagnosis of syphilis, it was but natural that the possibilities of this method as a general means of diagnosis soon became appreciated, and in a short time numerous infections were studied.

Probably in no disease has complement fixation proved so constant or so valuable a diagnostic procedure as in syphilis. In this condition the peculiar lipodophilic reagin is largely responsible for the marked fixation of complement, and from our present knowledge on the subject we learn that this phenomenon has practically no analogy in any other disease except frambesia.

With few exceptions bacterial antigens are likely to yield weaker and more inconstant reactions. This is due to the fact either that our antigen lacks a more available and specific antigenic principle, or that the amount of complement-fixing bodies is small and variable. For these reasons it becomes apparent that the preparation of antigen and delicacy of technic are highly important factors.

Preparation of Bacterial Antigens.-Either the endotoxins or whole bacterial body may constitute the main portion of an antigen. Most recent efforts have aimed thoroughly to disorganize the bacterial cell in order to liberate the endotoxic substances that pass into solution and constitute the antigen. Experience has frequently shown, however, that the protein substances of the bacterial cell itself possess antigenic properties, and accordingly I have generally found that antigens composed of cells and the products of cellular activity are usually more satisfactory than those prepared of the endotoxic substances alone.

As a general rule, bacterial antigens should be polyvalent -i. e., made up of a number of different strains of the same microörganism. Recent researches in bacteriology tend to show that different strains of the same microörganism have particular and more or less individual pathogenic and sometimes biologic characteristics, and it is reasonable to 
assume that the antibody will likewise show individual properties and a special affinity for its particular antigen. When, therefore, one antigen is being used in complement-fixation work, the results are more likely to be satisfactory if a large number of different strains are included in the antigen, with the hope that at least one of them will show a particular affinity for the antibody in the patient's serum.

Bacterial antigens may be prepared in various ways.

First Method.-Cultures are grown in a suitable fluid medium, such as plain bouillon, for forty-eight hours, or upon a solid medium, and washed off with a suitable quantity of normal saline solution. The culture or emulsion is shaken for an hour or so to break up the clumps, and then heated to $60^{\circ} \mathrm{C}$. for an hour. It is preserved by the addition of 1 per cent of glycerin and 0.5 per cent. of phenol.

This constitutes the simplest bacterial antigen. It is composed of both bacterial cells and the products of bacterial activity, and frequently yields uniform and satisfactory results.

Second Method.-Cultures are grown on a suitable solid medium for from twenty-four to forty-eight hours. Growths are removed by adding sufficient distilled water or normal saline solution to yield a milky suspension. The emulsion is heated to $60^{\circ} \mathrm{C}$. for two hours, and shaken mechanically with glass beads for twenty-four hours, to facilitate disintegration. It is then filtered through a sterile Berkefeld filter or thoroughly centrifugalized; the filtrate is preserved with 0.5 per cent. phenol and used as antigen.

This antigen is composed essentially of endotoxic substances, and is the one usually employed in the preparation of gonococcus antigen.

Third Method.-Cultures are grown on a solid medium, washed off with normal saline solution, and the emulsion centrifuged thoroughly. The sediment is dried over sulphuric acid or calcium chlorid, and the dried material thoroughly ground with crystals of sodium chlorid. Sufficient distilled water is then added to render the solution isotonic, and so that it will contain about 0.05 gram of dried material in each cubic centimeter. This emulsion is then shaken for twenty-four hours, filtered or centrifuged, the filtrate preserved with 0.5 per cent. of phenol, and used as antigen.

Fourth Method.-Cultures are grown on a solid medium and washed off with normal saline solution. Saline suspension is then precipitated with an equal quantity of absolute alcohol and centrifugalized. The sediment is dried in vacuo over sulphuric acid, weighed, and ground into a fine powder with sufficient crystals of sodium chlorid to make a 2 per 
cent suspension of dried material in isotonic saline solution. . This stock suspension is not filtered or centrifuged, but is further diluted with saline solution, and constitutes the antigen (method of Besredka, modified by Gay). The actual amounts of dry antigenic substance contained in 1 c.c. of various dilutions are as follows:

$$
\begin{aligned}
& 1 \text { c.c. of } 1: 40 \text { dilution }=0.5 \mathrm{mg} . \\
& 1 \text { c.c. of } 1: 80 \text { dilution }=0.25 \mathrm{mg} . \\
& 1 \text { c.c. of } 1: 160 \text { dilution }=0.125 \mathrm{mg} . \\
& 1 \text { c.c. of } 1: 320 \text { dilution }=0.062 \mathrm{mg} . \\
& 1 \text { c.c. of } 1: 640 \text { dilution }=0.031 \mathrm{mg} \text {. } \\
& 1 \text { c.c. of } 1: 1280 \text { dilution }=1.0155 \mathrm{mg} \text {., etc. }
\end{aligned}
$$

Standardizing Bacterial Antigens.-After an antigen has been prepared it is standardized by determining the anticomplementary dosei.e., the amount of antigen that just begins to show inhibition of hemolysis due to non-specific complement fixation. This dose is easily determined by adding increasing amounts of antigen to a series of testtubes with a constant dose of complement in each. As a general rule, it is well to add to each tube a constant dose of fresh normal inactivated serum, e. g., as 0.1 to 0.2 c.c., when the anticomplementary action of serum alone is allowed for. I would emphasize the necessity of doing this in experimental work with rabbit, dog, or any other animal serum. After incubating for one hour, one and a half units of hemolytic amboceptor and 1 c.c. of corpuscle suspension are added to each tube, and the tubes are reincubated for an hour or two and the reading made. In the main test, one-quarter to one-half the anticomplementary unit may be used, as this amount is known to be free from any power of non-specific complement fixation. The former dose is, of course, safer than the latter.

The standardization may be completed by determining the antigenic dose of the antigen by titrating with a suitable and constant dose of specific immune serum. This titration is conducted by placing in a series of test-tubes increasing doses of antigen with a constant dose of heated immune serum (usually 0.1 c.c.) and a constant dose of complement. After an hour the proper dose of hemolytic amboceptor and corpuscles is added. The readings may be made an hour or two later, or after the tubes have been allowed to settle in a refrigerator. That tube showing just complete inhibition of hemolysis contains the antigenic dose. For the main test, it is well to use double this amount, providing this dose is not more, and preferably less, than half the anticomplementary dose.

The antigenic titration is not always satisfactory, for when an artificial immune serum is used, the concentration of antibodies may yield 
a much stronger reaction than one would expect in testing human serums. Further than this, the antibody content in antiserums varies considerably so that the antigenic unit fluctuates according to the particular serum used in making the titration. In general, therefore, it is sufficient to determine the anticomplementary dose and to use half or quarter this amount in performing the main test.

After antigens are prepared they may require further dilution with saline solution. This can be determined only by experience and as the result of a trial titration.

As watery extracts are prone to deteriorate, it should be made a rule that the anticomplementary dose be determined each time before the main test is conducted.

It has quite generally been proved that alcoholic extracts of bacteria do not yield satisfactory antigens, despite the advantage to be gained because of their stability.

Principles of Complement Fixation with Bacterial Antigens.-The principles of complement fixation in general should be thoroughly understood.

After making considerable comparative studies with various methods, I am convinced that, in the final analysis, a simple technic is best. I use a relatively small but safe dose of complement,-1 c.c. of a $1: 20$ dilution ( $=0.05$ c.c. undiluted serum), - and titrate the hemolytic amboceptor with this constant dose or unit of complement and the corpuscle suspension. This titration is made each time the reactions are performed, and with each and every complement serum and corpuscle suspension. In this way differences in the activity of different guinea-pig serums are readily detected and adjusted in the amboceptor titration. If exactly one unit of amboceptor is used in conducting the main test, the controls are not likely to be completely hemolyzed unless the serum contains natural antisheep amboceptor, for the serum alone will probably be slightly anticomplementary. For this reason I am accustomed to use $1 \frac{1}{2}$ doses of amboceptor, and I secure reactions that are very delicate and yet sharp and clear cut. When testing an old serum,-one that is likely to contain considerable thermostabile anticomplementary bodies,-I use two hemolytic doses of amboceptor. If one wishes to use exactly one unit of complement and one unit of amboceptor, the serum and antigen alone should be controlled, as in the fourth method of performing the Wassermann reaction (p. 446). I frequently use this technic in the gonococcus-fixation test, but as a rule I have found the simpler technic herein given equally sensitive and reliable. 
In this chapter are considered the main bacterial infections in which complement fixation has been shown to possess value as a means of diagnosis. Complement fixation has also proved of value in the diagnosis of animal parasitic diseases, such as ecchinococcus infection, and in the differentiation of the proteins. With each of these the special methods for preparing the antigen, titrating the antigen, and conducting the test are given.

\section{COMPLEMENT FIXATION IN GONOCOCCUS INFECTIONS}

This was one of the first infections to be studied by means of the complement-fixation technic, but the results secured were not generally satisfactory until it was shown that the antigen must be polyvalent.

Historic-In 1906 Muller and Oppenheim ${ }^{1}$ applied the complement-fixation test to the diagnosis of gonorrheal arthritis, using a culture of the gonococcus as antigen. To these observers, therefore, belongs the credit of being the first to record a complement-fixation test in a gonococcus infection. A little later in the same year Carl Bruch $^{2}$ applied the reaction to three cases of gonorrhea, using the serum of immunized rabbits, and reported favorable results. In 1907 Meakins ${ }^{3}$ reported having secured positive reactions in three cases of gonorrheal arthritis, which was the first report in America published on this subject. Th. Vannod ${ }^{4}$ studied the specificity of the reaction with the serums of rabbits immunized with gonococcus protein and one of a meningococcus, and reported that the meningococcus immune serum did not show complement fixation with gonococcus antigen, and, vice versa, that gonococcus amboceptor was not bound by meningococcus antigen. Wollstein ${ }^{5}$ (1907), in a study of the biological relationship of the gonococcus and meningococcus, reported findings differing from those of Vannod. The former observer found that bacteriolytic amboceptors in the serums of rabbits immunized with these cultures were closely related and yielded fixation of complement with either antigen. Teaque and Torrey, ${ }^{6}$ in 1907, issued a very important communication showing that the differences in results of previous investigators were probably due in part to the use of single strains of the organisms in the preparation of antigens and immune serums. They emphasized the fact that the gonococcus belongs to a heterogeneous family, and that in attempting to formulate a diagnosis of gonorrheal infection by the complement-fixation method, the extracts of several different strains should be used. Naz Vannod and later Watabiki found that the gonococcus and meningococcus antibodies were quite specific for their homologous antigens in complement-fixation reactions.

Particular attention was drawn to the gonococcus complement-fixation test by the work of Schwartz and McNeal. ${ }^{8}$. These investigators emphasized the necessity of using polyvalent antigens, and their encouraging reports have stimulated renewed interest in this subject. They found that if the infection is confined to the anterior urethra, a positive reaction is not obtained; that a strong reaction is not to be expected before the fourth week of the infection, and then only in acute cases with complications. They regard a positive reaction as indicating the presence or recent activity in the body of a focus of living gonococci, although a negative reaction does not exclude gonococcus infection. The test, therefore, has a more positive than a negative value. With Flexner's antimeningococcus serum positive reactions re-

${ }^{1}$ Wien. klin. Wochenschr., 1906, 19, 894.

${ }^{2}$ Deutsch. med. Wochenschr., 1906, 70, 36.

${ }^{3}$ Johns Hopkins Medical Bull., 1907, 18, 255.

${ }^{4}$ Zeitschr. f. Bakter., 1907, 44, 10.

${ }^{5}$ Jour. Exp. Med., 1907, 9, 588.

${ }^{6}$ Jour. Med. Research, 1907, 17, 223.

7 Jour. Infect. Diseases, 1910, 7, 159. 1912.

${ }^{8}$ Amer. Jour. Med. Sci., May, 1911; ibid., September, 1912; ibid., December, 
sulted with their gonococcus antigen; with serums from cases of cerebrospinal meningitis (meningococcic) the results were negative.

In the succeeding years numerous investigators, including Swinburne, Gradwohl, O'Neil, Gardner and Clowes, Thomas and Ivy, Kolmer and Brown, have reported favorably upon the practical value of the gonococcus complement-fixation test, particularly as an aid in determining whether or not a patient is cured of the infection.

Technic.-Since, because of the comparatively slight cellular involvement, the quantity of antibody produced in a localized gonococcus infection is probably small, the complement-fixation reactions are generally weak, and consequently require the closest technical attention, especially as regards the preparation of antigen and accurate adjustment of the hemolytic system.

Hemolytic System.-As a rule, the antisheep hemolytic system is employed; the various ingredients may be used in one-half the quantity employed in the original Wassermann reaction, as given in the preceding chapter, with the technic of the syphilis reaction, or one-tenth the quantity employed in the original Wassermann technic may be employed. I prefer to employ the larger amounts because the readings are usually easier to interpret.

Fresh guinea-pig complement serum is diluted $1: 20$ and used in dose of 1 c.c. ( $=0.05$ c.c. serum); sheep's corpuscles are made up in a $21 / 2$ per cent. suspension and used in dose of 1 c.c.; antisheep amboceptor is titrated (see p. 377) and used in an amount equal to $11 / 2$ hemolytic doses in conducting the antigen titration and in the test proper.

Kolmer and Brown have compared the practical value of the antisheep and antihuman hemolytic systems in the examination of a number of serums. When the latter were used, some of the reactions were somewhat stronger and yielded slightly better results, showing the influence, probably, of natural antisheep amboceptor present in a large proportion of human serums.

Antigen.-This constitutes the most important ingredient of the test. As Teague and Torrey and Schwartz and McNeal have emphasized, the antigen should be prepared of many different strains of gonococci. The difficulty of isolating this organism and the constant care required in subculturing and keeping a large number of strains alive render it practically impossible for many persons to prepare a gonococcus antigen. Therefore until simpler methods are devised, this antigen is best prepared in large central laboratories, where the cultures are handled and preserved by specially trained persons.

The gonococci are well grown on a salt-free veal agar, neutral in 



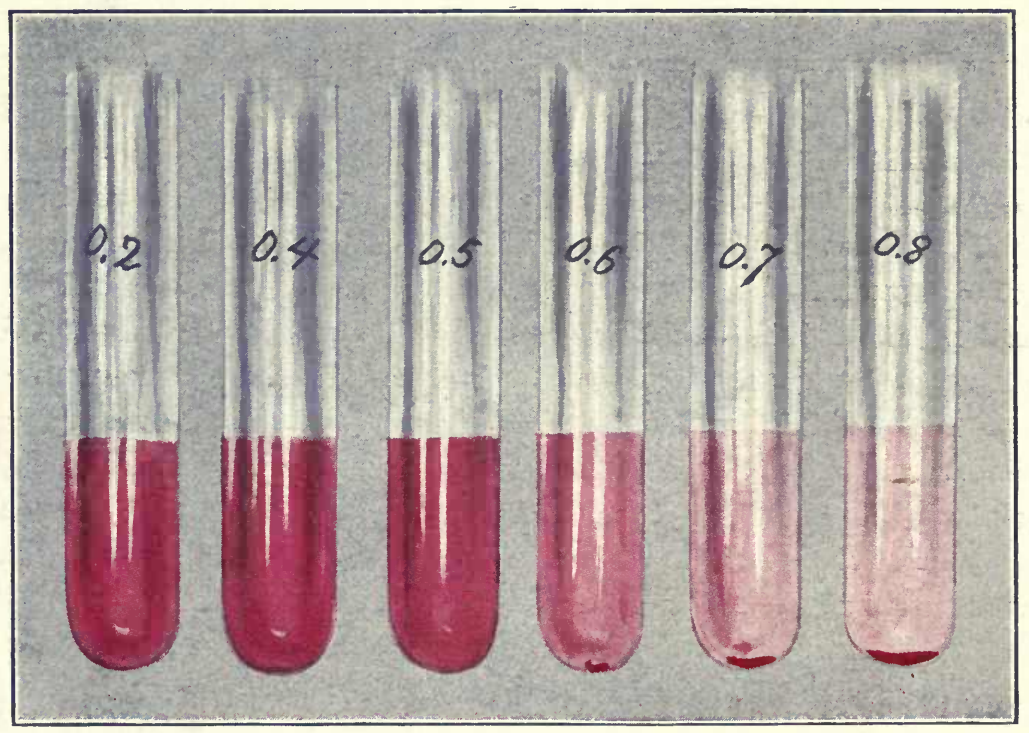

Fig. 117.-Anticomplementary Titration of a Gonococcus Antigen. 
reaction to phenolphthalein, and to which a few drops of sterile hydrocele fluid may be added. After culturing for from twenty-four to fortyeight hours, the growths are washed off with distilled water, and the emulsion is heated in a water-bath for two hours at $56^{\circ} \mathrm{C}$. It is then centrifugalized and passed through a Berkefeld filter. A small amount of preservative, as, e. g., 0.1 c.c. of a $1: 100$ dilution of phenol to each cubic centimeter of antigen, may be added. The antigen is then well preserved in small amounts in ampules that are sealed and heated to $56^{\circ} \mathrm{C}$. for half an hour on three successive days. Just before being used the antigen is made isotonic by adding 1 part of a 10 per cent. salt solution to 9 parts of antigen. I preserve the antigen in ampules containing 1 c.c., and after removing the antigen from the ampule to a large test-tube, add 1 c.c. of 10 per cent. salt solution, and dilute the whole 1: 10 with the addition of 8 c.c. of normal salt solution, after which the anticomplementary titration is made.

In this method of preparing antigen the endotoxins constitute the main antigenic principle. Kolmer and Brown, after an experimental study of the various antigens, found that a simple suspension of gonococci in salt solution yielded slightly better results. The various strains are grown for from forty-eight to seventy-two hours, and are then washed off with sterile saline solution, observing particular care not to include portions of the culture-medium. The suspension is then shaken to break up clumps, and heated to $56^{\circ} \mathrm{C}$. for an hour. A small amount of preservative is now added, and the antigen stored in 1 c.c. ampules. Before using it is diluted $1: 10$ or $1: 20$, and titrated for the anticomplementary dose.

Alcoholic extracts of gonococci have very little practical value, as alcohol is not satisfactory for extracting the antigenic principles of bacteria.

The anticomplementary dose of the antigen should be determined, and one-half or one-quarter this amount should be used in conducting the main test. An antigenic titration may also be conducted with an antigonococcus serum, to determine the antigenic value of the antigen, but in practice it is sufficient to use one-half the anticomplementary dose. This titration should be conducted and the antigen standardized before the main tests are adjusted.

In the following table the results of an anticomplementary titration of a gonococcus antigen are given, the approximate dose having been ascertáined in previous titrations (Fig. 117). 
TABLE 17.-ANTICOMPLEMENTARY TITRATION OF A GONOCOCCUS ANTIGEN

\begin{tabular}{|c|c|c|c|c|c|c|c|}
\hline TUBE & $\begin{array}{l}\text { ANTIGEN, } \\
\text { 1: 10, C.c. }\end{array}$ & $\begin{array}{l}\text { COM- } \\
\text { PLE- } \\
\text { MENT, } \\
1: 20 \text {, } \\
\text { C.c. }\end{array}$ & 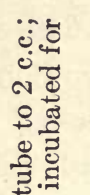 & $\begin{array}{c}\text { ANTI- } \\
\text { SHEEP } \\
\text { AMBO- } \\
\text { CEPTOR, } \\
\text { UNITS }\end{array}$ & $\begin{array}{c}\text { SHEEP'S } \\
\text { COR- } \\
\text { PUS- } \\
\text { CLES } \\
\text { (2.5 } \\
\text { PER } \\
\text { CENT), } \\
\text { C.C. }\end{array}$ & 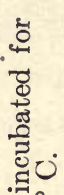 & Results \\
\hline & 0.2 & 1 & $\mathscr{E}$ & $11 / 2$ & 1 & $\varpi$ & Complete hemolysis \\
\hline & 0.4 & 1 & घ0 & $11 / 2$ & 1 & $E_{0}^{\infty}$ & Complete hemolysis \\
\hline & 0.6 & 1 & ป త్తై & $11 / 2$ & 1 & बै & Complete hemolysis \\
\hline & 0.8 & 1 & .0 & $11 / 2$ & 1 & क्षै- & Slight inhibition of \\
\hline 5 . & 1.0 & 1 & 死 & $11 / 2$ & 1 & 离 & Marked inhibition of \\
\hline 6 . & 0 & $1^{\circ}$ & 承 & $11 / 2$ & 1 & $\frac{2}{2}$ & $\begin{array}{l}\text { Hemolytic control } \\
\text { Complete hemolysis }\end{array}$ \\
\hline
\end{tabular}

If the antigen is new and the anticomplementary dose is entirely unknown, it may be necessary, in making this titration, to use a different dilution, with higher and lower doses. In conducting the main test the foregoing antigen could be used in dose of 0.2 or 0.4 c.c. of this dilution.

The Test.-The serums should be fresh and clear, and heated to $56^{\circ} \mathrm{C}$. for one-half hour. For each serum use four test-tubes $(12$ by $1 \mathrm{~cm}$.), arranged in a row. Into each of the first three place the dose of antigen and increasing doses of serum- 0.05 c.c., 0.1 c.c., 0.2 c.c.; the fourth tube is the serum control, and into this is placed the maximum dose of serum ( 0.2 c.c.) but no antigen; 1 c.c. of complement diluted $1: 20$ is added to each tube. The following controls are included:

1. A positive control with an antigonococcus serum or with the serum of a patient who reacted positively on a former occasion.

2. A negative control with the serum of a healthy person.

Both of these controls may be set up with but the maximum dose of serum $(0.2$ c.c.).

3. The serum control of each serum is conducted in the fourth tube of each series. At the completion of the test this tube should show complete hemolysis and thereby indicate that the serum was not anticomplementary.

4. The antigen control at this time includes the dose of antigen and complement.

5. The hemolytic system control at this time receives the dose of complement.

6. The corpuscle control receives 1 c.c. of the corpuscle suspension. To each tube sufficient saline solution is added to bring the total 



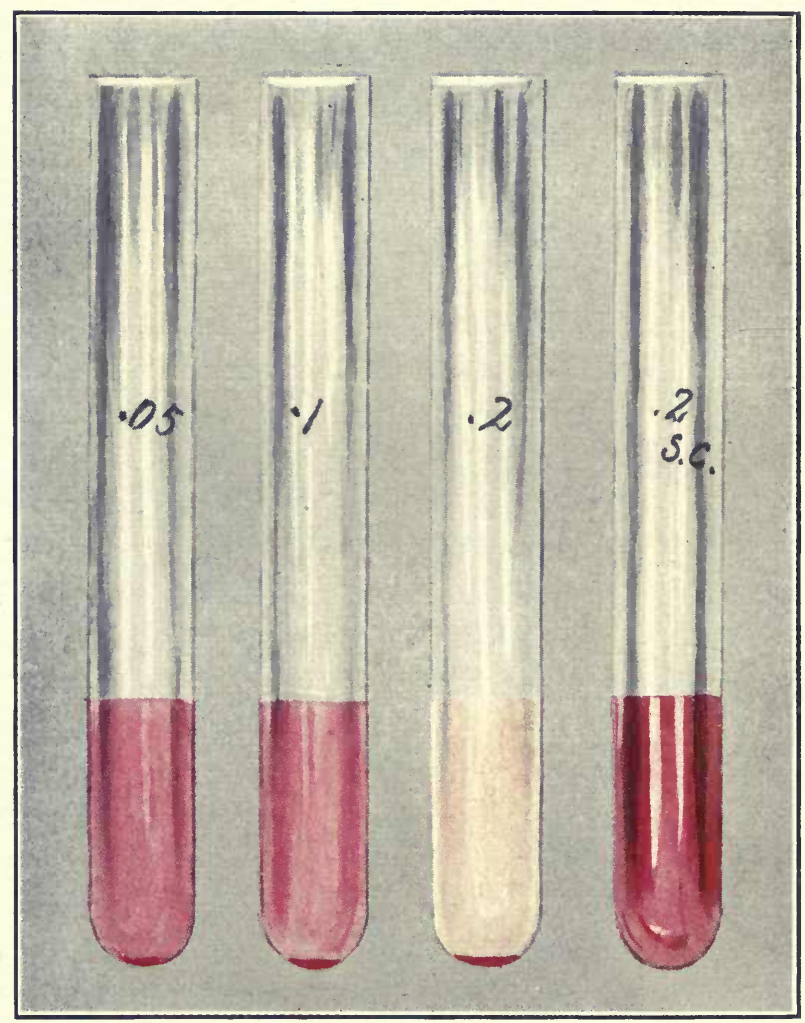

Fig. 118.-Gonococcus Complement-fixation Reaction. 
volume up to about 2 c.c. The tubes are shaken and incubated for one hour at $37^{\circ} \mathrm{C}$., when $1 \frac{1}{2}$ units of antisheep amboceptor and 1 c.c. of sheep corpuscle suspension are added to each tube except the corpuscle control. The tubes are gently shaken again and reincubated for an hour or longer, depending upon the hemolysis of the controls, after which the results are recorded. This secondary incubation may be omitted and the tubes placed in a refrigerator overnight and the results read the next morning. Under these conditions hemolysis occurs slowly, and according to some workers in this field the reaction becomes more delicate.

The following table is an example of a gonococcus fixation test with the serum of a case of gonorrheal arthritis (Fig. 118).

TABLE 18.-GONOCOCCUS COMPLEMENT-FIXATION TEST

\begin{tabular}{|c|c|c|c|c|c|c|}
\hline $\begin{array}{l}\text { Patient's Serdu, } \\
\text { C.c. }\end{array}$ & $\begin{array}{l}\text { ANTIGEN, } \\
1: 10 \text {, C.c. }\end{array}$ & $\begin{array}{l}\text { CoM- } \\
\text { PLE- } \\
\text { MENT, } \\
1: 20 \text {, } \\
\text { C.c. }\end{array}$ & 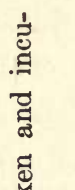 & $\begin{array}{l}\text { ANTI- } \\
\text { SHEEP } \\
\text { AMBO- } \\
\text { CEPTOR, } \\
\text { UNITS }\end{array}$ & $\begin{array}{c}\text { SHEEP } \\
\text { COR- } \\
\text { PUS- } \\
\text { CLES } \\
(2.5 \\
\text { PER } \\
\text { CENT), } \\
\text { C.C. }\end{array}$ & $\begin{array}{l}\text { RESULTS AFTER ONE AND A } \\
\text { HALF HOURS INCUBATION }\end{array}$ \\
\hline 0.05 & 0.2 & 1 & .ै匕 & $11 / 2$ & 1 & Slight inhibition of hem- \\
\hline 0.1 & 0.2 & 1 & 羊识 & $11 / 2$ & 1 & Marked inhibition of \\
\hline 0.2 & 0.2 & 1 & 表 & $11 / 2$ & 1 & $\begin{array}{l}\text { Complete inhibition of } \\
\text { hemolysis }\end{array}$ \\
\hline 0.2 & 0 & 1 & है & $11 / 2$ & 1 & $\begin{array}{l}\text { Serum control: hemoly- } \\
\text { sis }\end{array}$ \\
\hline $\begin{array}{c}\text { Positive serum, } \\
0.2\end{array}$ & 0.2 & 1 & 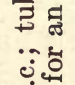 & $11 / 2$ & 1 & $\begin{array}{l}\text { Complete inhibition of } \\
\text { hemolysis }\end{array}$ \\
\hline 0.2 & 0 & 1 & ن. & $11 / 2$ & 1 & $\begin{array}{l}\text { Serum control: hemoly- } \\
\text { sis }\end{array}$ \\
\hline $\begin{array}{c}\text { Negative serum, } \\
0.2\end{array}$ & 0.2 & 1 & $\dot{\sigma}$ & $11 / 2$ & 1 & Hemolysis \\
\hline $\begin{array}{c}0.2 \\
0\end{array}$ & $\begin{array}{c}0 \\
0.2\end{array}$ & $\begin{array}{l}1 \\
1\end{array}$ & 急 & $\begin{array}{l}11 / 2 \\
11 / 2\end{array}$ & $\begin{array}{l}1 \\
1\end{array}$ & $\begin{array}{l}\text { Hemolysis } \\
\text { Antigen control: hem- } \\
\text { olysis }\end{array}$ \\
\hline 0 & 0 & 1 & $\nsubseteq$ & $11 / 2$ & 1 & $\begin{array}{l}\text { Hemolytic control: } \\
\text { hemolysis }\end{array}$ \\
\hline 0 & 0 & 0 & हึ & 0 & 1 & $\begin{array}{l}\text { Corpuscle control: no } \\
\text { hemolysis }\end{array}$ \\
\hline
\end{tabular}

In reading the results the controls are first examined and the test reported as negative, weakly positive, moderately positive, or strongly positive, as the case may be.

\section{Gonococcus Fixation TeSt, Using ONE-TENTH the UsUal AMOUNTS}

This technic is employed for purposes of economy, especially since the antigen is likely to be expensive. Otherwise the method is less 
desirable than the preceding one, as the results are more difficult to read.

Complement serum is diluted $1: 10$ and used in dose of 0.1 c.c.; corpuscles are made up in a 10 per cent. suspension and used in dose of 0.1 c.c., the amboceptor is titrated with these amounts of complement and corpuscles, and used in dose equal to one and one-half units. Each day, before the main tests are undertaken, the anticomplementary dose of antigen is determined by placing increasing doses of diluted antigen with complement and salt solution in a series of tubes, incubating for an hour, adding one and one-half units of amboceptor and the corpuscles, followed by incubation for another hour. One-half or one-quarter of the anticomplementary dose is used in making the main test. The serums are inactivated and used in three ascending doses, $-0.005,0.01$, and 0.02 c.c.,- - equivalent respectively to $0.5,1$, and 2 c.c. of a $1: 100$ dilution ( 0.1 c.c. serum, 9.9 c.c. salt solution). The fourth tube of each series contains the maximum dose of serum without antigen, and is the serum control. The other controls, general technic, and readings of the reaction are the same as those previously described.

\section{SPECIFICITY OF THE GONOCOCCUS COMPLEMENT-FIXATION TEST}

Viewed from a practical standpoint, the reaction is highly specific. While complement-fixation experiments with antigens of gonococci and meningococci and their respective immune serums have demonstrated a biologic relationship between these microörganisms, yet practically with human serums an antigen of pure cultures of gonococci will fix complement only with the gonococcus antibody (amboceptor). In this technic a specific antigen is employed, and it is, therefore, a true application of the Bordet-Gengou reaction of complement fixation by specific antigen and specific antibody (amboceptor). Obtained under proper technical conditions, a positive reaction is invariably reliable, and indicates the presence of a focus of living gonococci.

\section{Practical Value of the Gonococcus Complement-Fixation Test}

From our present knowledge of this reaction, it may be stated:

1. That the difficulty of isolating and preserving a sufficient number of cultures of true gonococci in order to prepare a satisfactory polyvalent antigen constitutes a weighty drawback to the practical use of the test.

2. Because the gonococcus antibody, unless complicated by widespread gonococcal metastases, is produced in small amount in the 
majority of cases, the degree of complement fixation is usually much less than that which occurs in the syphilis reaction, and accordingly the reactions are usually weaker and often indefinite.

3. The reaction is seldom positive during the first four to six weeks of an acute anterior or posterior urethritis, in the absence of complications. In acute exacerbations of a chronic urethritis the reaction is positive in about 80 per cent. of cases. In ordinary chronic urethritis with mild infection of the prostate gland the reaction is positive in from 30 to 40 per cent. of cases. In chronic urethritis complicated by marked involvement of the prostate gland and epididymitis the reactions are frequently positive, occurring in from 50 to over 80 per cent. of cases.

The test possesses considerable value in determining the fitness of an applicant for a marriage license, and will, no doubt, be employed for this purpose quite extensively, as a positive reaction is now generally regarded as indicating the presence of a focus of active gonococcal infection.

4. During the course of an acute or a subacute urethritis the occurrence of an acute complication, such as prostatitis, epididymitis, etc., is likely to result in a positive fixation test.

5. A positive reaction may persist for several weeks after the patient is clinically cured. Torrey ${ }^{1}$ has shown experimentally that the antibody persists in the blood of rabbits artifically immunized for from ten days to six or seven weeks. Usually, under proper treatment, the reaction in ordinary cases of urethritis disappears in from two to three weeks; if, however, a positive reaction persists, a focus of infection is probably present, and the patient should be kept under further observation and the treatment persisted in.

6. In women the reaction is seldom positive until the infection has reached the cervical canal. In the case of little children, however, we have known positive reactions to occur in acute and chronic vulvovaginitis, indicating either that the disease is more severe in children, with more antibody formation, or that it may reach the cervical canal.

The reaction is positive in about 60 per cent. of cases of pyosalpingitis, and the test may prove of value in making the differentiation of inflammatory lesions from certain cystic and neoplastic conditions, and in establishing the gonorrheal basis of many of these infections.

7. Cases of gonorrheal urethritis yield from 80 to 100 per cent. of positive reactions, and the complement-fixation test has considerable value in establishing the diagnosis of these infections.

1 Jour. Med. Research, 1910, i, 95. 
8. The administration of gonococcus bacterin and antigonococcus serum is likely to be followed by positive reactions. Just how long the antibodies may persist in the blood after a clinical cure has been effected it is difficult to state; at least from six to twelve weeks' time should be given for them to disappear.

9. In medico-legal cases the courts may not accept the usual evidence offered by a bacteriologic diagnosis based upon stained smears of a secretion, and cultures are frequently differentiated from other Gramnegative diplococci, only with difficulty. Conducted with the proper technic, the gonococcus fixation test is highly specific and much less difficult to perform.

10. Finally, it must be emphasized that the reaction has a far more positive than negative value. The reaction is highly specific, but there is a limit to its delicacy, so that a negative reaction in urethritis does not exclude the possibility of gonococcal infection.

\section{COMPLEMENT-FIXATION TEST IN GLANDERS}

The complement-fixation test is used extensively by veterinarians in making a laboratory diagnosis of glanders. The test has been found very reliable, and is usually more delicate than the agglutination test and the Strauss guinea-pig test. It has also been used successfully in the diagnosis of human glanders.

Preparation and Standardization of Antigen.-The antigen should be polyvalent, and composed of at least several different strains. Cultures of Bacillus mallei are grown on slants of glycerin agar (1 per cent. acid) for from forty-eight to seventy-two hours. The growths are then removed, and sufficient distilled water added to give a milky suspension. This suspension is sterilized by heating the tubes to $60^{\circ} \mathrm{C}$. for two hours. They are then shaken mechanically with glass beads for a few hours on two successive days. Enough sodium chlorid is added to make the solution isotonic, and the whole is preserved with 0.5 per cent. phenol and stored in a dark, cold place, where it will keep for many months.

A simpler antigen is prepared by growing the bacillus in glycerin bouillon for seventy-two hours, sterilizing by heating to $60^{\circ} \mathrm{C}$. for two hours, and preserving with the addition of 0.5 per cent. of phenol.

The anticomplementary dose is then determined by titration. The antigen is diluted $1: 20$ by mixing 1 c.c. with 19 c.c. of normal saline solution. To a series of seven test-tubes add increasing amounts of diluted antigen as follows: $0.1,0.2,0.4,0.6,0.8,1$, and 2 c.c. Add 1 c.c. 
of complement serum $(1: 20)$ to each tube, and sufficient salt solution to bring the total volume in each up to 3 c.c. Incubate for an hour at $37^{\circ} \mathrm{C}$., and add $11 / 2$ units of antisheep amboceptor titrated just previous to making the test (see p. 377 ) and 1 c.c. of sheep corpuscle suspension. Reincubate for an hour or an hour and a half. At the end of this time that tube showing beginning inhibition of hemolysis contains the anticomplementary dose, and one-fourth to one-half this amount is used in making the main test.

An antigenic titration may also be made, but this is not absolutely necessary. To a series of tubes containing $0.05,0.1,0.15,0.2,0.25$, and 0.3 c.c. of diluted antigen add 0.1 c.c. of fresh heated glanders serum (known to yield a positive reaction) and 1 c.c. of complement serum $(1: 20)$ and sufficient salt solution. Incubate for one hour and add $1 \frac{1}{2}$ units of hemolytic amboceptor and corpuscles. After a second incubation of from one to two hours, that tube showing just complete inhibition of hemolysis contains the antigenic dose, and double this amount may be used in performing the main test, providing that it is still one-half or, better, but one-quarter the anticomplementary dose.

A hemolytic system control is included in both titrations, and in the antigenic titration an additional control on the serum, to determine whether it is free from anticomplementary action.

The dilution here advised may be too low; if this is the case, the titrations must be repeated with the antigen diluted $1: 50$ or $1: 100$.

The Test.-The external jugular vein of the horse is punctured with a sterile needle, and from 5 to 10 c.c. of blood is collected in a centrifuge tube or other glass container, which preferably should be sterile. The clear serum is heated to $55^{\circ} \mathrm{C}$. for one-half hour just before the tests are conducted.

Into a series of four small test-tubes place the following doses of serum: $0.05,0.1,0.2$, and 0.2 c.c. To the first three tubes add the proper dose of antigen; to all tubes add 1 c.c. of complement $(1: 20)$ and sufficient salt solution to bring the total volume up to 3 c.c.

The following controls should be included:

1. The serum control on each serum is conducted in the fourth tube of each set.

2. A known positive serum should be tested in the same manner.

3. A known negative serum should be tested in the same manner.

4. The antigen control, which at this stage contains the dose of antigen, complement, and saline solution.

5. The hemolytic control, which at this time contains but 1 c.c. of complement plus saline solution. 
6. The corpuscle control, containing 1 c.c. of corpuscle suspension and 3 c.c. of saline solution. This tube should be plugged with cotton.

All tubes are gently shaken and incubated for an hour, after which $11 / 2$ units of hemolytic amboceptor and 1 c.c. of corpuscle suspension are added to all except the corpuscle control. The tubes are gently shaken and reincubated for an hour or two, depending upon the hemolysis of the controls.

The controls are first inspected. They should all show complete hemolysis, except the first three tubes of the positive serum series and the corpuscle control. Inhibition of hemolysis in the first three tubes of the series containing the unknown serum indicates a strong positive reaction. Complete hemolysis in all tubes indicates a negative reaction. Partial hemolysis in the first three tubes indicates a partially positive reaction. If the serum control or antigen control tubes should show inhibition of hemolysis, these were probably anticomplementary and the test should be repeated.

\section{COMPLEMENT-FIXATION TEST IN CONTAGIOUS ABORTION}

It is now generally conceded among veterinarians that the Bacillus abortus of Bang is the specific cause of contagious abortion of cows.

Evidence is gradually accumulating to show that an organism belonging to the paratyphoid group is frequently the cause of a similar condition among mares (Kilbourne and Smith, ${ }^{1}$ Liguierer, ${ }^{2}$ Liguierer and Zabala; Good; ${ }^{3}$ Van Neelsbergen; ${ }^{4}$ de Jong; ${ }^{5}$ Meyer and Boerner ${ }^{6}$. Meyer and Boerner, who have studied this bacillus with particular care, classify it with the paratyphoid-enteritidis group (Bacillus aborti equi).

Veterinarians are generally agreed that in contagious abortion of cows the complement-fixation test is highly specific, and is frequently of considerable value in establishing a diagnosis (Meyer and Hardenburgh and others).

Meyer and Boerner have found fixation of complement to occur in contagious abortion of mares with an antigen of Bacillus abortus equi, and recommend the test as diagnostic aid in this infection.

1 Kilbourne and Smith: United States Department of Agriculture, Bulletin No. 3, 1893, 49 and 53 .

${ }^{2}$ Liguierer: Rec. Med. veterinaire, lxxxii, 1905.

${ }^{3}$ Good: Kentucky Agriculture Exper. Station Bulletin No. 165, 1912

${ }^{4}$ Van Neelsbergen: Tijdschrift. v. Veeartsenijk., xxiv, 1912.

${ }^{5}$ de Jong: Archiv. f. Wissenshaftl. u. prak. Tierheilkunde, xxv, 1900.

${ }^{6}$ Meyer and Boerner: Jour. Med. Research, 1913, xxix, No. 2, 325. 
Preparation and Standardization of Antigens.-The antigen of Bacillus abortus (Bang) for use in the complement-fixation test in contagious abortion of cows is prepared by cultivating a number of strains of the bacillus, which have been trained to grow aërobically, upon slants of glycerin agar for seventy-two hours. The growths are then washed off with sufficient normal saline solution containing 2 per cent. phenol to yield a cloudy emulsion. Shake briskly in order to break up the clumps of bacilli, and filter through paper. Place in a refrigerator for several days to complete the sterilization, and titrate the anticomplementary dose each time before the main test is conducted.

The antigen may also be prepared by cultivating a number of strains in glycerin-serum bouillon for five or six weeks. Centrifuge thoroughly and wash the bacilli once or twice with normal saline solution, to remove all traces of serum. Dilute the washed bacilli with sufficient normal saline solution to give an emulsion equal in density to a twenty-fourhour bouillon culture of Bacillus coli, and add 0.4 per cent. of phenol as a preservative.

The antigen of Bacillus abortus equi for making the complementfixation diagnosis of contagious abortion of mares is prepared of eighteen- to twenty-hour-old glycerin bouillon cultures, with an addition of 0.5 per cent. of phenol. These antigens are less anticomplementary than shake extracts, and keep their titer unaltered for many weeks (Meyer and Boerner). They may also be used for making the macroscopic agglutination test.

The anticomplementary dose is determined each time, and one-half this amount is used in performing the main test. The technic is the same as that employed in the titration of glanders antigen.

The tests and controls are conducted with descending doses of fresh inactivated serum $(0.05,0.1$, and 0.2 c.c.), in exactly the same manner as the glanders reaction is performed.

\section{COMPLEMENT FIXATION IN DOURINE}

Dourine, or horse syphilis, is a specific infectious disease of the horse and ass, transmitted from animal to animal by the act of copulation, and caused by the Trypanosoma equiperdum. It is characterized by an irregular incubation period, the localization of the early symptoms to the genital organs, and, finally, by complete paralysis of the posterior extremities, a fatal termination ensuing in from six months to two years.

The disease is especially prevalent among horses in the northwestern 
states, and may occur in such various and atypical forms as to render clinical diagnosis difficult.

Complement-fixation methods of diagnosis have been tried by Pavlosvici, Winkler and Wyschelersky, Moller, Watson, Brown, and in a large series of cases with good results by Moller, Eichhorn, and Buck. ${ }^{1}$ These last-named investigators examined 8657 specimens of blood from horses in Montana and North and South Dakota, and of these, 1076 yielded positive reactions.

In most of these experiments the results were corroborated by clinical and pathologic findings, and the investigators conclude that the complement-fixation test is of great value, especially in countries where only one of these protozoan diseases exists.

Preparation and Standardization of Antigen.-This is the most difficult part of the technic, because the trypanosome is not readily grown on artificial culture-media. Watery, alcoholic, and acetone extracts of various organs of horses dead of the disease do not yield satisfactory antigens. Since the reaction is a group reaction, and dourine is the only trypanosome infection in this country, Moller, Eichhorn; and Buck selected the surra organism for the preparation of antigen. After infecting a dog and at the height of infection withdrawing 200 c.c. of blood into potassium citrate and hemolyzing with 0.5 gram of saponin, the trypanosomes were secured after thorough centrifugalization and washed three times. After the last washing the trypanosomes were emulsified in 50 c.c. of salt solution and preserved with phenol. This antigen yielded highly satisfactory results, but the difficulty of preparing it, and the small quantity secured, made it necessary that another method be used.

An extract of the spleen of a rat just dead of surra was found to yield a satisfactory antigen. The extract does not keep well, and must be prepared freshly every few days and carefully standardized. Gray or white rats are infected with surra by injecting 0.2 c.c. of blood from a rabbit with this disease. If a large number of tests are to be made, the rats should be so infected that one or two are available each day for the preparation of the antigen.

The spleen from a rat with a small amount of salt solution added is ground in a mortar until a pulpy mass results. More of the salt solution is added from time to time, and the suspension thus obtained is filtered twice through a double layer of gauze and diluted with salt solution to 40 c.c.

${ }^{1}$ Amer. Jour. Veter. Med., 1913, viii, 581. 
The anticomplementary and antigenic doses are then determined, and the extract used in double the antigenic unit, providing that this amount is not more than half the anticomplementary dose. If a positive serum from an infected horse is not available, the anticomplementary dose may be determined and half this amount used in conducting the main test.

Anticomplementary Titration.-Into a series of six test-tubes place increasing amounts of antigen-0.1, 0.2, 0.3, 0.4, 0.5, and 0.6 c.c.; add 1 c.c. of complement $(1: 20)$ and sufficient salt solution to bring the total volume in each tube up to 2 c.c. Incubate for one hour. Add $1 \frac{1}{2}$ or 2 units of antisheep amboceptor (determined by preliminary titration) and 1 c.c. of 2.5 per cent. sheep's corpuscles. Incubate for one hour, after which the reading is made.

The Test.-The serum is inactivated and used in dose of 0.15 c.c., since it has been found that fixation in this quantity is obtained only with serums of horses affected with dourine. In some instances the serum of horses has reacted in doses as small as 0.02 c.c., and the reaction may be conducted with increasing doses of serum- $-0.05,0.1$, and 0.15 c.c.-in exactly the same manner as when the glanders reaction is performed. The same Controls are included.

\section{COMPLEMENT-FIXATION TEST IN TYPHOID FEVER}

This was one of the original diseases in which Bordet and Gengou first demonstrated the occurrence of complement fixation. Widal and Lesourd attempted to make practical application of this method in the diagnosis of the disease, but their results were indifferent, and since then numerous writers have expressed various opinions as to the value of the test. Garbat has secured uniform and reliable reactions with a polyvalent antigen, and emphasizes the importance of this factor.

The antigen is prepared of numerous strains of typhoid bacilli-the more the better. Cultures are grown on slants of agar for forty-eight hours, washed off with small quantities of sterile distilled water, heated to $60^{\circ} \mathrm{C}$. for two hours, shaken mechanically for twenty-four hours, and either filtered through a sterile Berkefeld filter or thoroughly centrifugalized. The filtrate is preserved with 0.5 per cent. phenol and used as antigen.

I have secured good results by removing the growths with small amounts of normal salt solution, and placing them in a shaking flask, and shaking for an hour to break up the clumps. After heating to $60^{\circ} \mathrm{C}$. 
for an hour, 1 per cent. glycerin and 0.5 phenol are added as preservatives, and the mixture stored away in ampules containing 1 c.c. each. The emulsion should be slightly milky in appearance.

The antigen is diluted $1: 10$ or $1: 20$, and the anticomplementary dose determined by titration before the main tests are conducted. The technic of the reaction is exactly similar to the gonococcus fixation test.

The ease with which the Widal reaction is performed renders it the method of choice. Nevertheless the complement-fixation test is quite delicate, and will frequently aid, where the agglutination test is negative or absent and in making the differential diagnosis from paratyphoid fever. The strongest reactions are secured late in the disease.

\section{COMPLEMENT-FIXATION TEST IN TUBERCULOSIS}

It was the original studies in complement fixation in tuberculosis made by Wassermann and Bruch that later induced these workers, in coöperation with Neisser, to apply the method to the diagnosis of syphilis.

Antigens were prepared of tuberculous glands and lungs, and complement fixation was found to occur with an artificial immune serum (Höchst) and with the serums of persons who had received injections of tuberculin, but not the serums of other tuberculous persons who had not received tuberculin.

It would appear, therefore, that tuberculin may stimulate the production of tuberculin antibodies in the nature of amboceptors (Citron). These amboceptors will frequently fix complement in vitro with a suitable tuberculin antigen.

According to Citron, a tuberculous focus may contain tuberculin, and antituberculin is probably produced by healthy cells in or about the focus, which are capable of reaction. The production of antituberculin, however, is a transitory process, arising only when tuberculin has reached the circulation, either spontaneously or artificially. During this stage the serum of the patient may yield a positive complement-fixation test. Following this stage of activity there comes a period of quiescence during which no free antituberculin can be demonstrated in the blood-serum. The cells, however, are sensitized, and possess many sessile receptors that possess a high affinity for tuberculin and produce antituberculin much more readily than do normal cells. Hence when a small amount of tuberculin is injected it is bound by the sensitized cells in the zone surrounding the tuberculous focus, and thus explains the heightened action at this point, with the production of antituberculin. 
Further discussion on Citron's tuberculin theory is reserved for the chapter devoted to this subject. Evidence indicates, however, that immunity in tuberculosis is not dependent solely upon the development and presence of antituberculin, but that other antibodies are likewise concerned.

As a practical test, complement fixation has not been successful in tuberculosis. As previously stated, this may be due to the fact that antituberculin production is transitory and variable, and hence if the amboceptors are absent or present in but very minute amounts, complement fixation in vitro cannot occur.

Considerable attention has also been given the preparation of the antigen, in the belief that a special endotoxic substance or part of the bacillus is required. Various antigens have been used, such as Bacillen emulsion, old tuberculin, tuberculin filtrate, and a watery emulsion of tubercle bacilli. A mixture of Koch's old and new tuberculins has also been used. Antigen may also be prepared after the method of Besredka (p. 474), in which the bacilli are dried, ground, and used as a fine suspension of the bacterial substance.

More recently Hammer ${ }^{1}$ has reported favorably upon an antigen composed of an alcoholic extract of tuberculous tissue and old tuberculin. The tissue is extracted for five days with four parts alcohol and filtered. To each cubic centimeter of extract, 0.1 c.c. of old tuberculin is added; the anticomplementary dose is determined, and one-half this amount is used in conducting the main test.

At present, however, complement-fixation tests have yielded indifferent results, although a test sufficiently delicate and constant to permit early infections to be detected would be of inestimable value. If the failures of the past have been due rather to faulty antigen than to absence of tuberculin amboceptors, researches in the future will probably solve the problem.

\section{THE COMPLEMENT-FIXATION TEST IN THE STANDARDIZATION OF IMMUNE SERUMS}

The technic of complement fixation has also been employed as one means in effecting the standardization of antimeningococci and antigonococcic serums. Since, however, the amount of complement-fixing amboceptors in a serum is no index to its therapeutic and prophylactic value, a measure of this one factor is not a reliable standard.

${ }^{1}$ Munch. med. Wochenschr., 1912, 59, 1750. 
The technic consists in preparing the antigen and in determining its anticomplementary dose. Whatever this is, one-half to one-quarter this amount is added to increasing quantities of heated immune serum, ranging from 0.001 to 0.1 c.c. Complement and saline solution are added, and after incubating one hour at $37^{\circ} \mathrm{C}$., the amount of complement fixation is determined by adding hemolytic amboceptors and corpuscles.

While this titration is one measure of the reaction of the animal used in the immunization, better evidence of the therapeutic value of the serum is obtained by determining the content in bacteriotropins, by testing the serum with the antigen in susceptible animals, or by a combination of all methods.

\section{THE COMPLEMENT-FIXATION TEST IN ECHINOCOCCUS DISEASE}

Complement-fixation tests have been advocated as aiding the diagnosis of echinococcus disease. In making the tests, hydatid cyst fluid of the human or sheep is used as antigen (Ghedini). Reports upon the specificity and usefulness of this reaction are somewhat contradictory, although, as a rule, they are generally favorable. Cases of recent infection with fresh active lesions may react negatively, a result that is dependent presumably upon non-absorption of antigen and slight antibody formation (Gaetano, ${ }^{1}$ Weinberg and Bordin, ${ }^{2}$ Kurt Meyer ${ }^{3}$ ).

Thomsen and Magnussen, ${ }^{4}$ in a recent study of 12 cases, found that 10 reacted positively. Of 55 control cases (32 of whom reacted positively to the Wassermann reaction), all were negative except one. Kurt Meyer found the serums of echinococcus infected persons to react positively with antigens of other tænia (Tænia solium and Tænia saginata), and, conversely, the serums of persons infected with Tænia saginata and Tænia solium to react with an echinococcus antigen. Thomsen and Magnussen, however, could not support these findings, and report most favorably upon the specificity of the reaction. Of 10 persons infected with Tænia saginata, 2 with Tænia solium, and 1 with Bothriocephalus latus, all reacted negatively with echinococcus antigen.

The antigen is best prepared of the fresh fluid of an echinococcus cyst of man or sheep. It should be filtered, if necessary, preserved with

1 Gaetano: Riforma Medica, 1910, Nos. 39 and 40. Reference in Deut. med. Wochenschr., 1910, No. 44.

${ }^{2}$ Weinberg and Bordin: Compt. rend. soc. biol., 1909, Bd. 66.

${ }^{3}$ Meyer (K): Berl. klin. Wochenschr., 1910, No. 28.

4 Thomsen (O.), and Magnussen (G.): Berl. klin. Wochenschr., 1912, No. 25. 
0.5 per cent. phenol, and kept constantly at a low temperature. It is highly important not to use the antigen in an anticomplementary dose, hence it should be titrated before each test is made.

Anticomplementary Titration.-Dilute the fluid $1: 10$ with normal saline solution, and into a series of eight small test-tubes place increasing amounts as follows: $0.1,0.2,0.4,0.6,0.8,1,2$, and 3 c.c. Add 1 c.c. of complement $(1: 20)$ and sufficient saline solution to bring the total volume in each tube up to 4 c.c. Shake gently, incubate for one hour, and then add $11 / 2$ units of antisheep amboceptor (previously titrated) and corpuscles. Reincubate for one to two hours; the tube showing beginning inhibition of hemolysis contains the anticomplementary dose, and in performing the main test, one-half to one-quarter this amount may be used.

If titration with diluted fluid does not give the anticomplementary dose, the titration should be repeated with undiluted fluid.

Alcoholic extracts of daughter cysts, the hydatid wall, or the fluid have been found to give positive reactions in syphilis (Israel, Brauer, Henins), and are, therefore, unsatisfactory.

Thomsen and Magnussen speak favorably of antigen paper infiltrated with cyst fluid and titrated.

The Test.-The patient's serum should be fresh, and should be heated to $55^{\circ} \mathrm{C}$. for half an hour. Into a series of five test-tubes place 0.025 , $0.05,0.1,0.2$, and 0.2 c.c. of serum. To each of the first four tubes add one-half the anticomplementary dose of antigen; to all the tubes add 1 c.c. of complement $(1: 20)$ and sufficient saline solution to bring the total volume up to 3 c.c.

The fifth tube is the serum control; an antigen, hemolytic system, and corpuscle control should be included, as is usual in complementfixation tests. A normal serum may be included, and, if possible, a known positive serum.

After incubating for an hour at $37^{\circ} \mathrm{C}$, add $1 \frac{1}{2}$ units of hemolytic amboceptor and 1 c.c. of the corpuscle suspension to each tube. Reincubate for an hour or longer, depending upon the hemolysis of the controls. Marked or complete inhibition of hemolysis in the fourth tube ( 0.2 c.c. patient's serum), with lesser degrees of inhibition in the other tubes of the series, indicates a positive reaction. Larger doses of patient's serum should not be used on account of the probability of nonspecific complement fixation. 


\section{PROTEIN DIFFERENTIATION BY COMPLEMENT FIXATION}

The Determination of an Antigen by Complement Fixation.-In the tests hitherto considered the antigens were known, and the suspected antibodies sought for in the blood-serum or other body-fluid. In making the reactions it was necessary to bring the serum to be tested into contact with the antigen specific for the suspected antibody, in the presence of complement, and at a suitable temperature. At the end of an hour the mixture was tested for free complement by adding hemolytic amboceptor and red blood-corpuscles. This order may be reversed, and with a known antibody the suspected antigen may be detected. The antigen to be detected, as in a solution of blood or bacterial extract, is brought into contact with its specific antibody in the presence of complement. At the end of an hour, at a suitable temperature, the mixture is tested as previously for free complement, by adding corpuscles and hemolytic amboceptors. Under proper conditions complement fixation would indicate a specific reaction between the antibody and its antigen, and thus serve to identify the latter.

This method has clinical applications similar to those in which the precipitin reaction is used:

1. In the differentiation of blood-stains a solution of the stain constitutes the unknown antigen. By furnishing a known antiserum the antigen is detected, $i$. e., the animal from which the blood was derived is ascertained.

2. In the recognition and differentiation of meats.

3. In the detection of bacterial antigens in the blood-serum of patients, or with highly immune serums an unknown bacterial antigen may be identified and the test employed as a means of differentiation among bacterial species.

4. Similar applications of the test may be made in the differentiation of milks, seminal stains, and other albuminous substances.

As compared with precipitin reactions, the complement-fixation test is probably more delicate and reliable and easier of interpretation. The technic of the latter method is, however, more complicated, and the liability to error is greater unless the principles of complement fixation in general are thoroughly understood and the importance of quantitative factors is appreciated.

1. Complement Fixation for the Identification of Blood-stains. - The application of the technic of complement fixation to the determination of specific protein antigen, such as human or animal blood, was demon- 
strated by Gengou in 1902 . The principles worked out by him were extensively studied and practically applied by Neisser and Sachs in the forensic differentiation of animal proteins.

Hemolytic System.-Complement is furnished by the fresh serum of a guinea-pig diluted $1: 20$ and used in dose of 1 c.c. $(=0.05$ c.c. serum); washed sheep's corpuscles are made up in a 2.5 per cent. suspension and used in dose of 1 c.c.; antisheep amboceptor should be highly potent, and is titrated after the method previously given (p. 377). In the following titrations and in conducting the main test the hemolytic amboceptor is used in an amount equal to $1 \frac{1}{2}$ or 2 units.

Specific Antiserum.-This is obtained from a rabbit immunized with the protein for which the test is to be made, namely, human or animal blood-serum. In forensic tests it may be necessary to prepare a number of these antiserums with the serums of man and the ordinary domestic animals. The technic of mmunization is the same as that employed for the preparation of precipitins (p. 70). An antiserum for forensic tests must be sufficiently potent to fix complement with 0.0001 c.c. of its antigen. This is determined by a process of titration. If, for example, an antihuman serum is to be titrated, the method of procedure is as follows:

Secure 0.1 c.c. of fresh human serum and dilute $1: 1000$ by adding 99.9 c.c. of normal saline solution. Of this dilution, 0.1 c.c. is equivalent to the standard dose of 0.0001 c.c. of undiluted serum. The antiserum is heated to $55^{\circ} \mathrm{C}$. for half an hour and diluted $1: 10$ (1 c.c. immune serum plus 9 c.c. of saline solution). Decreasing doses of immune serum are mixed with a constant dose of antigen and complement. At the same time the anticomplementary titration of the immune serum is made by substituting salt solution for antigen. The doses to employ and the results of an actual titration are shown in Table 19:

Tube 16 is the hemolytic system control, and shows complete hemolysis; tube 15 is the antigen control, and shows complete hemolysis, as the quantity of serum is too small to exert an anticomplementary influence; tubes 11 to 14 are the tests for anticomplementary action of the antiserum. In the present instance the serum was several months old and the maximum dose of 1 c.c. $(=0.1$ c.c. undiluted serum) was very slightly anticomplementary. A fresh serum is practically never anticomplementary in this dosage, but these tubes should; nevertheless, be included in each titration. Tubes 1 to 10 include the antigenic titration, and show that the antiserum is perfectly antigenic in dose of 
0.3 c.c. of this dilution ( $=0.03$ c.c. undiluted serum). In performing the main test double this quantity, or 0.6 c.c., would be used.

TABLE 19.-TITRATION OF AN IMMUNE SERUM

\begin{tabular}{|c|c|c|c|c|c|c|c|}
\hline TUBE & $\begin{array}{c}\text { ANTI- } \\
\text { AERUM, } \\
\text { 1:10, C.C. }\end{array}$ & $\begin{array}{c}\text { SERUM } \\
\text { ANTIGEN, } \\
\text { 1: 10.00, } \\
\text { C.C. }\end{array}$ & $\begin{array}{l}\text { CoM- } \\
\text { PLE- } \\
\text { MENT, } \\
1: 20 \\
\text { C.c. }\end{array}$ & 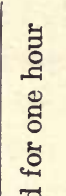 & $\begin{array}{l}\text { ANTT- } \\
\text { ANEER } \\
\text { AMBPO- } \\
\text { CEPTOR, } \\
\text { UNITS }\end{array}$ & $\mid \begin{array}{c}\text { SHEEP's } \\
\text { CoR- } \\
\text { PUS- } \\
\text { CELE, } \\
\text { (2.5 } \\
\text { PER } \\
\text { CENT.), } \\
\text { C.c. }\end{array}$ & $\begin{array}{l}\text { RESEULTS AFTER INCU- } \\
\text { BATION FOR ONE AND } \\
\text { ONE-HALF HOURB }\end{array}$ \\
\hline 1. & 1.0 & 0.1 & 1 & 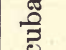 & $11 / 2$ & 1 & Inhibition of hem- \\
\hline 2. & 0.9 & 0.1 & 1 & 군 & $11 / 2$ & 1 & Inhibition of hem- \\
\hline 3. & 0.8 & 0.1 & 1 & ₹ & $11 / 2$ & 1 & Inhibition of hem- \\
\hline 4. & 0.7 & 0.1 & 1 & 幽. & $11 / 2$ & 1 & Inhibition of hem- \\
\hline 5. & 0.6 & 0.1 & 1 & $\begin{array}{l}\vec{v} 0 \\
\lambda_{0}\end{array}$ & $11 / 2$ & 1 & Inhibition of hem- \\
\hline 6. & 0.5 & 0.1 & 1 & 造 & $11 / 2$ & 1 & Inhibition of hem- \\
\hline 7. & 0.4 & 0.1 & 1 & $\tilde{\Xi}_{\pi}^{2}$ & $11 / 2$ & 1 & Inhibition of hem- \\
\hline 8 . & 0.3 & 0.1 & 1 & & $11 / 2$ & 1 & Inhibition of hem- \\
\hline 9. & 0.2 & 0.1 & 1 & N & $11 / 2$ & 1 & Partial inhibition of \\
\hline 10. & 0.1 & 0.1 . & 1 & $\dot{\dot{x}}$ & $11 / 2$ & 1 & Slight inhibition of \\
\hline 11. & 1.0 & 0 & 1 & छี & $11 / 2$ & 1 & Very slight inhibi- \\
\hline $\begin{array}{l}14 . . \\
15 . . \\
16 . .\end{array}$ & $\begin{array}{c}0.8 \\
0.4 \\
0.2 \\
0 \\
0\end{array}$ & $\begin{array}{c}0 \\
0 \\
0 \\
0.1 \\
0\end{array}$ & $\begin{array}{l}1 \\
1 \\
1 \\
1 \\
1\end{array}$ & 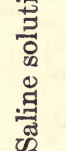 & $\begin{array}{l}11 / 2 \\
11 / 2 \\
11 / 2 \\
11 / 2 \\
11 / 2\end{array}$ & $\begin{array}{l}1 \\
1 \\
1 \\
1 \\
1\end{array}$ & $\begin{array}{l}\text { Complete hemolysis } \\
\text { Complete hemolysis } \\
\text { Complete hemolysis } \\
\text { Complete hemolysis } \\
\text { Complete hemolysis }\end{array}$ \\
\hline
\end{tabular}

Each antiserum is tested in a similar manner. In forensic blood tests an antihuman serum is, of course, employed first; if this is negative and it is desirable to determine the source of the blood, other antiserums, so that the ox, horse, dog, etc., are prepared, titrated, and tested with a solution of the blood-stain.

The Blood-stain. - It is first necessary to ascertain that the stain is a blood-stain; this is done by performing the hemin crystal test (p. 303). The stain is then extracted in normal saline solution, as described on p. 304. A 1:1000 dilution is made approximately by so diluting the extract that it just gives a slight opalescence when boiled with a few drops of acetic acid, and a slight foam persists after shaking. Unless it is perfectly clear, it should be filtered. 
The Test.-Into a series of six small test-tubes place increasing doses of extract of the blood-stain (antigen), as follows: 0.1 c.c., 0.2 c.c., 0.4 c.c., 0.6 c.c., 0.8 c.c., 1 c.c.; add double the titrated dose of antiserum and 1 c.c. of complement $(1: 20)$, with sufficient salt solution to bring the total volume in each tube up to 3 c.c.

The following controls are included:

1. Antigen control; 1.0 c.c. of the blood extract plus 1 c.c. of diluted complement and salt solution.

2. Antiserum control: double the titrated dose plus 1 c.c. of diluted complement and salt solution.

3. Hemolytic control; at this time, 1 c.c. of diluted complement and salt solution.

4. Corpuscle control: 1 c.c. of corpuscle suspension and salt solution. The tube should be plugged with cotton.

Shake all the tubes gently and incubate for an hour at $37^{\circ} \mathrm{C}$. Add $11 / 2$ units of hemolytic amboceptor and 1 c.c. of corpuscle suspension to each tube except the corpuscle control. Shake gently and reincubate for from one to two hours, depending upon the degree of hemolysis present in the controls.

The readings are made at once, and again after the tubes have been allowed to settle in the refrigerator overnight. Inhibition of hemolysis with the smallest dose of blood extract -0.1 c.c. (= approximately 0.0001 c.c. of blood)-indicates that the blood extract is most certainly the antigen for the antiserum employed. Even with the maximum dose of extract-1 c.c. (=approximately 0.001 c.c. of blood)-inhibition of hemolysis serves to show the nature of the blood. With an antihuman serum, for instance, a similar specific reaction would be possible only with the bloods of the higher apes.

In making blood tests for medicolegal purposes the antiserum should not only be standardized with a definite dilution of human serum, but the whole test should first be conducted with a known dried human blood-stain, and it must be borne in mind that extreme accuracy in all manipulations is essential.

In Table 20 are shown the method and the results of an actual test, using a dried human blood-stain and the same antiserum as previously directed.

I prefer this complement-fixation test to the precipitin reaction in the differentiation of proteins, as the readings are sharper and more definite. This test is fully as reliable as the precipitin test, and there is less danger of group reaction. 
TABLE 20.-FORENSIC BLOOD TEST

\begin{tabular}{|c|c|c|c|c|c|c|c|}
\hline TUBE & $\begin{array}{c}\text { EXTRACT } \\
\text { OF } \\
\text { BLOOD- } \\
\text { STAIN, } \\
\text { 1:1000 } \\
\text { C.c. }\end{array}$ & $\begin{array}{l}\text { ANTI- } \\
\text { SERUM } \\
1: 10, \\
\text { C.C. }\end{array}$ & $\begin{array}{c}\text { CoM- } \\
\text { PLE- } \\
\text { MENT, } \\
1: 20, \\
\text { C.c. }\end{array}$ & 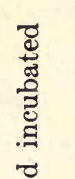 & $\begin{array}{c}\text { ANTI- } \\
\text { SHEEP } \\
\text { AMBO- } \\
\text { CEPTOR, } \\
\text { UNITS }\end{array}$ & $\begin{array}{c}\text { COR- } \\
\text { PUS- } \\
\text { CLES } \\
(2.5 \\
\text { PER } \\
\text { CENT.). } \\
\text { C.C. }\end{array}$ & $\begin{array}{l}\text { Results AFTER ONE } \\
\text { AND ONE-HALF HoUR' } \\
\text { INCUBATION }\end{array}$ \\
\hline $1 \ldots$ & 0.1 & 0.6 & 1 & व & $11 / 2$ & 1 & Marked inhibition \\
\hline 2. & 0.2 & 0.6 & 1 & 氙 & $11 / 2$ & 1 & $\begin{array}{l}\text { Marked inhibition } \\
\text { of hemolysis }\end{array}$ \\
\hline 3. & 0.4 & 0.6 & 1 & छి & $11 / 2$ & 1 & $\begin{array}{l}\text { Complete inhibition } \\
\text { of hemolysis }\end{array}$ \\
\hline 4. & 0.6 & 0.6 & 1 & F。 & $11 / 2$ & 1 & Complete inhibition \\
\hline 5. & 0.8 & 0.6 & 1 & نं & $11 / 2$ & 1 & $\begin{array}{l}\text { Complete inhibition } \\
\text { of hemolysis }\end{array}$ \\
\hline 6. & 1.0 & 0.6 & 1 & $\begin{array}{l}N \\
0\end{array}$ & $11 / 2$ & 1 & $\begin{array}{l}\text { Complete inhibition } \\
\text { of hemolysis }\end{array}$ \\
\hline 7. & 1.0 & 0 & 1 & ๑ี & $11 / 2$ & 1 & $\begin{array}{l}\text { Antigen control: } \\
\text { hemolysis }\end{array}$ \\
\hline 8. & 0 & 0.6 & 1 & $\stackrel{\square}{\underline{Z}}$ & $11 / 2$ & 1 & $\begin{array}{l}\text { Antiserum control: } \\
\text { hemolysis }\end{array}$ \\
\hline 9. & 0 & 0 & 1 & & $11 / 2$ & 1 & $\begin{array}{l}\text { Hemolytic control: } \\
\text { hemolysis }\end{array}$ \\
\hline 10. & 0 & 0 & 0 & $\stackrel{\Xi}{\overparen{\Xi}}$ & 0 & 1 & $\begin{array}{l}\text { Corpuscle control: } \\
\text { no hemolysis }\end{array}$ \\
\hline
\end{tabular}

2. Complement-Fixation Method for the Identification of Meats. The technic is essentially similar to that used in the foregoing test. Antiserums are prepared by immunizing rabbits with the serums of various animals, as the ox, horse, dog, cat, or any other animal the presence of whose flesh is to be identified in sausages, bologna, etc. It is not necessary to immunize with an extract of these meats themselves, as the blood or blood-serums will suffice. The technic of immunization is the same as that employed in the preparation of precipitin serums. Each antiserum is titrated with its antigen, as previously described, and is used in double the titrated dose in conducting the main test.

An extract of the flesh to be examined is prepared as described on p. 310 .

The test is then conducted in exactly the same manner as previously described.

3. Complement-Fixation Method for the Identification of Bacterial Antigens.-As a means of diagnosis, this test has very limited practical value. It aims to detect, by means of complement fixation with a known antiserum, a soluble bacterial antigen in the blood-serum of a patient. For example, in typhoid fever the patient's serum is mixed with a potent antityphoid serum in the presence of complement. After 
incubating for an hour at $37^{\circ} \mathrm{C}$., amboceptor and corpuscles are added to test for free complement. An absence of hemolysis indicates that complement has been fixed by the antiserum and soluble typhoid antigen in the serum of the patient. As a rule, and for purposes of diagnosis, this order of procedure is reversed: the antigen is furnished and then sought for in the patient's serum (p. 473), as in the gonococcus fixation test, syphilis reaction, etc.

An immune serum is prepared by immunizing rabbits with increasing doses of an emulsion of the bacteria which we wish to test for in the patient's serum.

The bacterial extract is prepared as follows: Make cultures of the bacteria on slants of agar; wash off a sufficient number with normal saline solution until 20 or 30 c.c. of a heavy emulsion are secured; add 0.4 per cent. of phenol, and shake mechanically with glass beads for twenty-four hours; then heat to $60^{\circ} \mathrm{C}$. for an hour, and either centrifuge thoroughly or filter through a Berkefeld filter. The clear filtrate should be preserved in a tightly stoppered bottle in an ice-chest. It is well to titrate this extract for its anticomplementary dose. As a rule, these extracts are free from anticomplementary action until relatively large doses are employed.

The antiserum is heated to $55^{\circ} \mathrm{C}$. for half an hour and titrated with 0.01 c.c. of the bacterial extract ( 0.1 c.c. of a $1: 10$ dilution) in 10 doses, ranging from 0.1 c.c. to 1.0 c.c. Double the dose giving complete fixation of complement is used in testing for the bacterial antigen in human serum.

In conducting this test the patient's serum is heated to $55^{\circ} \mathrm{C}$. for half an hour, and decreasing doses, ranging from 0.5 c.c. to 0.01 c.c., are placed in a series of test-tubes together with double the titrated dose of antiserum. Complement and salt solution are now added, and after incubating for an hour at $37^{\circ} \mathrm{C}$., amboceptor and corpuscles are added and the tubes reincubated. The general technic and controls are the same as those previously described.

The test has some value in special research work, but for practical use it has given way to the agglutination reactions and complementfixation tests for the detection of antibody with a known antigen.

\section{COMPLEMENT-FIXATION TEST IN CANCER}

None of the various complement-fixation methods that have been advocated from time to time in the diagnosis of cancer have proved of 
practical value. More recently von Dungern ${ }^{1}$ has advocated a method that yielded 90 per cent. of positive reactions in known cases of cancer. Positive reactions have also occurred in tuberculosis and syphilis, and the reports of various other investigators are somewhat contradictory. Whitman, in a study of 30 cases, found the method highly satisfactory.

Preparation of Antigen.-Two different antigens may be employed. Of these, von Dungen prefers the first.

First Method.-Place 10 c.c. of blood (preferably obtained from a patient suffering from general paralysis) in a centrifuge tube containing 0.1 c.c. of a 20 per cent. solution of sodium oxalate. Wash the cells three times with normal saline solution. After the last washing, measure the corpuscles and add 20 parts by volume of chemically pure acetone. Let this stand for three days at room temperature, giving it an occasional shaking. Filter and evaporate the filtrate to dryness in the incubator, and take up the residue at once with enough 96 per cent. alcohol to form a 1 per cent. solution. This usually requires about 10 times as much alcohol as the amount of acetone used. Before using, the alcoholic solution is mixed slowly with four parts of normal saline solution and titrated for its anticomplementary dose. Half this amount is used in making the main test.

Second Method.-The tumor tissue, freed as much as possible of fat and degenerated portions, is minced and treated with 20 volumes of acetone and extraction continued for one or two weeks at room temperature with an occasional shaking. The extract is then filtered, evaporated to dryness at $37^{\circ} \mathrm{C}$., and the residue taken up with half the amount of absolute alcohol. This extract is then diluted $1: 10$ or $1: 20$ with salt solution, and titrated for its anticomplementary dose, one-half this amount being used in making the main test.

The Test. - The serum should be fresh. Add two volumes of $\frac{\mathrm{n}}{50}$ caustic soda $\left(\frac{\mathrm{n}}{10}\right.$ soda diluted with four volumes of normal saline solution), and inactivate the mixture for one-half hour at $54^{\circ} \mathrm{C}$. (not $56^{\circ} \mathrm{C}$.). The soda solution must be prepared accurately from chemically pure substances, must be free from carbonate, and must be protected from the air.

Into a series of five test-tubes place $0.075,0.15,0.3,0.6$, and 0.6 c.c. of the serum-soda mixture. To the first four tubes add the proper amount of antigen. To all tubes add 1 c.c. of complement diluted $1: 20$ and sufficient salt solution to bring the total volume up to 3 or 4 c.c.

${ }^{1}$ Münch. med. Wochenschr., 1912, 59, 65, 1098, 2854. 
The following controls are included:

1. The fifth tube is the serum control.

2. A known positive serum should be included and set up in the same manner.

3. A known normal serum should be used.

4. The antigen control, containing at this time antigen, complement, and salt solution.

5. A hemolytic control, containing at this time complement and salt solution.

Each tube is gently shaken and incubated for an hour, after which $11 / 2$ to 2 units of amboceptor and 1 c.c. of corpuscles are added to each tube. After one to two hours' incubation, the results are read, inhibition of hemolysis with satisfactory controls indicates a positive reaction. With these antigens, it is not strange that positive reactions may occur in syphilis. 


\section{CHAPTER XXV}

\section{CYTOTOXINS}

IN Chapter XXIII the cytolysins in general were considered, especially their theoretic structure and the mechanism of their action.

It will be remembered that the general name "cytolysin" is applied to an amboceptor or antibody of the third order of receptors that is capable of preparing its antigen for the disintegrative or lytic action of a complement. The two best known and most important members of this group of antibodies have been considered, namely, the hemolysins and the bacteriolysins.

Following the discovery of the hemolysins and the bacteriolysins and of the mechanism of their action, it was not long before similar studies were undertaken with other cells, with the result that attempts have been made to prepare immune cytolytic serums for practically every organ of the body. This outcome was but natural, in view of the enormous theoretic importance of specific cytolysins, not only from the additional light that may be thrown upon physiologic and pathologic processes in general, but also from the standpoint of specific therapeutics.

Nomenclature.-While actual lysis or solution of erythrocytes and bacteria may be brought about by antibodies of this order, yet actual solution is not apparent with most other body-cells, although a distinct toxic action may be observed. For instance, an antispermatozoa serum will cause these cells to lose their motility, but does not actually dissolve them. Hence the name cytotoxin has been applied to these immune serums. This is probably a better term than cytolysin; but it is to be remembered that, so far as is now known, both cytolysins and cytotoxins are antibodies that possess the same nature and structure, except that in the former group the process is complete and ends in actual lysis of the cell. The term cytolysin is, therefore, more appropriately applied to the bacteriolysin and hemolysins; whereas the term cytotoxin is reserved for those immune serums that injure their cells without complete lysis (a toxic action), such as nephrotoxin, hepatotoxin, etc. This chapter is mainly concerned with the latter group.

Nature and General Properties of Cytotoxins.-As previously stated, cytotoxins are amboceptors or antibodies of the third order, and 
possess the same general properties as the bacteriolysins and hemolysins, except that, as will be pointed out later, they do not possess the same specificity. Without the presence of a complement they are inactive. They are thermostabile, possess the same general affinity for their antigen, and may be removed from a serum by saturation with the antigen in a manner similar to that used for the removal of a hemolysin.

Preparation of Cytotoxins.-Cytotoxic serums are prepared by immunizing an alien animal with fine suspensions of cells of the particular organ being studied. (See p. 73.) Every effort should be made to remove all traces of blood, and to secure as pure an emulsion of the same cells and to work as aseptically as possible. Injections are best given intraperitoneally, rabbits being well adapted for the preparation of these serums.

Beebe ${ }^{1}$ has made the statement that more specific serums are obtained if the nucleoproteins are isolated and used in the process of immunization, than if the cells themselves are used. Wells, ${ }^{2}$ however, believes that the nucleoproteins of cells are not specific in character, and Pearce, Karsner, and Eisenbrey ${ }^{3}$ found that nephrotoxic and hepatoxic serums prepared by the injection of the nucleoproteins of these cells, were no more toxic than serums prepared from the globulins and albumins of the same organs.

Methods of Studying Cytotoxins. - While the phenomena of hemolysis and bacteriolysis may readily be observed in experiments in vitro, the influence of other cytotoxins on the particular cells used as antigens is more difficult to determine. The methods of exposing cell emulsions to the action of the serum have not been found satisfactory for testing the specificity of cytotoxins.

The technic generally employed consists in making subcutaneous, intraperitoneal, or intravenous injections of the immune serum into the animal, or into the arteries leading to particular organs. Functional disturbances and delicate histologic changes in various organs have served as criteria for determining the degree of specificity that exists. The loss of some manifestation of vitality on the part of the cell, as a loss of motility (spermatozoa) or an inability to proliferate, may aid in studying the effect of these serums.

More recently several other methods have been used, especially the

1 Jour. Exper. Med., 1905, vii, 733.

${ }^{2}$ Chemical Pathology, 1914, Second Edition, W. B. Saunders Co.

${ }^{3}$ Jour. Exper. Med., 1911, xiv, 44. 
complement-fixation and the epiphanin reactions and that of observing the influence of cytotoxic serums upon cells grown in vitro (Lambert).

Specificity of Cytotoxins.-As has been stated elsewhere, the hemolysins and the bacteriolysins are highly specific, especially the former group. With the cytotoxins, however, this specificity is not observed. Most cytotoxic serums are also hemolytic, notwithstanding the fact that careful precautions have been taken to remove, so far as possible, all traces of blood from the inoculum during the process of immunization. Metchnikoff found a spermatotoxic serum to be also hemolytic, but he believed that this property could be removed by treating the immune serum with the corresponding corpuscles, and in this manner dissolve out the hemolysin. Numerous other investigators have found, however, that cytotoxic serums may attack the cells of other organs, as well as those that have been used as their antigens.

The subject has been very carefully investigated by Pearce. ${ }^{1}$ The injection of an antidog nephrotoxic serum prepared by immunizing rabbits with washed dog kidney is followed by the development of a tubular nephritis, with albuminuria and occasionally hemoglobinuria, and accompanied by granular degeneration of the liver. These serums are usually hemolytic in vitro. Similarly, in a study of hepatotoxic serums, Pearce found that the most striking lesions were referable to the hemagglutinating and hemolytic properties of the serum, causing thrombosis, embolism, and hemorrhages, whereas secondary necroses may be caused by a direct toxic action of the serum on certain parenchymatous cells.

Pearce, Karsner, and Eisenbrey found that the serums of rabbits injected repeatedly with the nucleoproteins, globulins, and albumins of the liver and kidney of the dog, gave no evidence of organ specificity in vitro or in vivo experiments. These investigators were not able to support the view put forward that nucleoproteins play an important part in the production of cytotoxic immune serums.

Lambert ${ }^{2}$ has recently studied the subject with cultures of rat sarcoma and rat embryo skin and their immune serums, and found that these cytotoxins were not specific for the tissue injected.

These results are not'surprising when it is remembered that all the body-cells have a common origin, and that, although the cells of various organs may differ considerably in morphologic and functional characters, they have certain receptors in common, and, as Pearce originally main-

1 Jour. Exper. Med., 1914, xix, 277.

${ }^{2}$ Univ. Penna. Med. Bull., 1903, xvi, 217; Jour. Med. Research, 1904, xii, 1 
tained, it is hardly conceivable that specific somatogenic cytotoxins can be produced.

Autocytotoxins.-While these toxins possess some theoretic interest, they are of very rare occurrence in experimental work. Certainly cells of the kidney, liver, and other organs are constantly dying and being replaced by new cells; the receptors of these cells are thereby set free, and are capable of forming a union with the receptors of other cells, and the possibility for the formation of autocytotoxins is established. According to Ehrlich, however, the side-arms anchoring the receptors of the dead cells are sessile in nature, and are unlikely to cause overproduction, as in the case of antibodies for bacterial substances or for the cells of other species. On the other hand, a simultaneous production of antiautotoxins that counteract the autotoxins and preserve a delicate physiologic equilibrium may occur, the whole subject being, however, still in the experimental stage.

Of most interest in this connection are the theoretic autonephrotoxins. These may be produced when part of a kidney becomes disorganized in the living body, as by means of a toxin. Theoretic autotoxins may then be produced, which, acting upon other kidney cells, institute a vicious cycle. Acting upon these assumptions, Ascoli and Figari ${ }^{1}$ and Lindeman ${ }^{2}$ have proposed a new theory as to the pathogenesis of certain of the nephritides. These observers would account for the cardiac hypertrophy of nephritis by attributing it to the action of the nephrotoxic serum in causing contraction of the peripheral vessels, with consequent increase of blood-pressure; the nephritic nervous symptoms, they believe, are due to the fact that the serum contains a neurotoxic constituent.

Lindeman has produced a toxic nephritis in dogs by giving them injections of potassium bichromate, and found that the serum, although free from the chromate, was toxic to other dogs, a finding he believed due to the presence of antonephrotoxins produced in the first dog as a result of the destruction of kidney cells. According to this view, the original toxic cause of a degenerative nephritis would be less responsible for the continuance of the process than would the formation of an autonephrotoxin. While these conclusions are somewhat far-reaching, they serve to indicate that the same processes operative in bacterial infection and immunity may have an important relation to other pathologic conditions.

${ }^{1}$ Berl. klin. Wochenschr., 1902, xxxix, 634.

${ }^{2}$ Ann. de l'Inst. Pasteur, 1900, xiv, 49. 
It is not rarely observed that in large tumors and similar lesions certain groups of cells may undergo digestion, but in these the lysis is commonly ascribed to the action of ferments liberated upon the death of cells.

Isocytotoxins have been produced experimentally, as, for example, by Ehrlich, who produced isohemolysins by injecting goats with goat blood, and by Metchnikoff, who prepared isospermatoxic serums.

Anticytotoxic serums have likewise been prepared by careful immunization with cytotoxic serums.

Varieties of Cytotoxins.-As previously stated, attempts have been made to prepare cytotoxic serums for practically all the organs and tissues. Since none of these has been found to be absolutely specific, and hence since they possess little or no practical value, they will receive but brief consideration here.

1. Spermatotoxin.-This serum was prepared simultaneously by Metchnikoff ${ }^{1}$ and Landsteiner in 1899, and was one of the earliest cytotoxins to be studied. It is a hemolytic serum, and causes spermatozoa to lose their motility. It would seem to affect also the vitality of the spermatozoa in vivo, inasmuch as De Lester, by the injection of this serum, rendered male mice sterile for from sixteen to twenty days.

2. Epitheliotoxin.-A cytotoxic serum for the ciliated epithelium of the trachea was prepared by von Dungern. ${ }^{2}$ The cells became disintegrated in the peritoneal cavity of the immunized animal, but not in that of the normal animal. This serum also proved to be hemolytic.

Similar serums have been prepared with cancer cells, in the hope of establishing a specific serum therapy, but all efforts have thus far proved futile.

3. Leukotoxins.-This serum was first prepared by Metchnikoff ${ }^{3}$ and Besredka ${ }^{4}$ by injecting the spleen of rats into guinea-pigs. The serums have also been prepared by effecting immunization with exudates rich in leukocytes or with the emulsion of lymphoid organs (Flexner and Ricketts). They are usually hemolytic, and also attack endothelial cells. Their action may be observed in vitro when the leukocytes lose their ameboid motility and the protoplasm swells, clears, and may disintegrate, leaving the nucleus.

4. Nephrotoxin.-This serum is best adapted for experimental studies

${ }^{1}$ Ann. de l'Inst. Pasteur, 1900, xiv, 369.

${ }^{2}$ Münch. med. Wochenschr., 1899, xlvi, 1228.

${ }^{3}$ Ann. de l'Inst. Pasteur, 1899, xiii, 737.

4 Ann. de l'Inst. Pasteur, 1900, xiv, 402. 
of the cytotoxins. As shown by Pearce, with the aid of this serum physiologic and anatomic alterations and lesions are readily studied. The injection of a nephrotoxic serum produces a tubular nephritis, with albuminuria and possibly hemoglobinuria. The serums are usually hemolytic, and frequently cause degenerative lesions in the liver, due in part to hemolysis and hemagglutination of red corpuscles.

5. Hepatotoxin.-Delezenne ${ }^{1}$ was the first to work with the so-called hepatotoxin, which, he claimed, possessed absolute organ specificity. Subsequent investigations, however, have brought forth contradictory findings. Pearce found that hyaline thrombi, formed of agglutinated red corpuscles, are primarily responsible for the areas of necrosis and hemorrhage, with secondary effects, which may be ascribed to a cytotoxin liberated chiefly by the cells in the thrombus, and acting on the livercells. These findings and views have recently received support from the investigations of Karsner and Aub. ${ }^{2}$

6. Gastrotoxin.-Gastrotoxic serums have been studied by Bolton, ${ }^{3}$ who immunized rabbits with emulsions of the mucosa of the stomach of the guinea-pig. The injection of this serum into guinea-pigs was followed by the development of areas of hemorrhage, necrosis, and ulcer formation that resembled peptic ulcers. According to Bolton, if the gastric secretions were neutralized with large quantities of alkali, the ulcers did not develop, indicating that the peptic ferments may be operative in the digestion of the cells after their destruction by the immune serum. Gastrotoxic serums were found to produce precipitates with clear filtrates of gastric cells, and were also shown to be hemolytic.

7. Synocytotoxin.-This serum has been produced experimentally by immunization with an emulsion of placental cells. According to Liepmann, ${ }^{4}$ it produces a precipitate with a filtrate of placenta cells, and at one time it was believed that it might constitute a diagnostic test for pregnancy.

Syncytotoxins are interesting as considered in reference to eclampsia and other toxemias of pregnancy. As is well known, placental cells may become detached and, gaining entrance to the circulation, become lodged in remote organs (Schmorl). This has given rise to the theory that a placentotoxin is developed that produces the nephritis of pregnancy and necrotic lesions in the liver. Weichardt asserts that, by digesting placenta in vitro with an active placentotoxic serum and in-

${ }^{1}$ Semaine Med., 1900, xx, 290.

2 Jour. Med. Research, 1913, xxviii, 377.

${ }^{3}$ Proc. Roy. Soc., lxxvii, 426, and lxxix, 533.

${ }^{4}$ Deutsch. med. Wochenschr., 1902, xxviii, 911. 
jecting the digestate, he produced symptoms resembling eclampsia in the lower animals. It was hoped that an anticytotoxic serum might be prepared to combat the effects of the placentotoxin, but this hope has not been realized. Renewed interest in this particular subject has been manifested by the recent studies of Abderhalden in ferments as applied to the diagnosis of pregnancy (p. 248).

8. Neurotoxin.-This toxin has been prepared and studied by Delezenne, Centanni, Delille, and others, by immunization experiments with emulsions of cerebrum, cerebellum, and spinal cord. When injected into the brain direct, these serums may cause profound intoxication of the nerve-centers, with torpor or convulsions, subnormal temperature, and death. When injected directly into the veins, they are usually without effect. In addition to their neurotoxic action, they are generally hemolytic, and frequently endotheliotoxic and leukotoxic.

9. Thyrotoxins.-This serum is prepared by immunizing animals with emulsions of thyroid gland. Thyrotoxins were quite prominently before the profession a few years ago, owing to the work of Beebe, who advocated their use in the treatment of various goiters. They have not fulfilled their expectations, however, since they may also produce degenerative changes in the various organs, as the liver, spleen, and kidneys.

\section{RÔLE OF CYTOTOXINS IN IMMUNITY}

It is apparent that, according to our present knowledge, the cytotoxins proper, although they possess great theoretic importance from their possible relationship to the removal and disposal of enfeebled and dead cells, occupy a subsidiary place in the processes of immunity. The processes governing these changes are finally and delicately balanced, and although obscure, they offer an intricate but fascinating field for research.

If the ferments concerned in Abderhalden's studies are related in any way to the cytolysins, the subject becomes of great interest, and a new field, with immense possibilities, is opened for further study.

Practical Applications.-(1) In therapeutics the cytotoxins have not established a place for themselves and their use has been disappointing. As previously stated, the use of thyrotoxic serums has not met with considerable success; epitheliotoxic serums have likewise not been efficient in the treatment of cancer. They possess theoretic interest, however, from the possibility of their so injuring glands that their functions may be studied; theoretically, the use of minute and carefully 
graded doses of hemolytic serums may effect the production of antihemolysins and aid in the treatment of certain anemias.

(2) In diagnosis cytotoxic reactions have been employed by Freund and Kaminer. These observers used the cytotoxins as a diagnostic aid in cancer, but with indifferent success. Abderhalden's pregnancy test possesses some practical value, and a similar technic has been successfully employed in the diagnosis of cancer. The work of Abderhalden has served to open up a comparatively new and intensely interesting field, which, when fully developed, as the result of overcoming difficult technical procedures, may offer additional means in the diagnosis of various diseases. This test has been described elsewhere under the head of Ferments (p. 252).

\section{CYTOTOXIC REACTIONS}

Cytolytic Cancer Diagnosis of Freund and Kaminer. ${ }^{-}-$This reaction is based upon the observation that while normal serum has the power to dissolve cancer cells, the serum of cancerous persons lacks this property, and has the power to inhibit the destruction of such cells by normal serum.

The same authors have also observed that when cancer serum is mixed with an extract of cancer cells a precipitate forms. (See p. 314.) They claim to have secured 88 per cent. of positive reactions in 113 cases examined, and believe that the reaction occurs early enough and is sufficiently specific to render it of practical value. These observations, however, have not been sufficiently confirmed.

An emulsion of cancer cells is prepared by grinding the undegenerated portions of a tumor, freed as much as possible from fat and fibrous tissue, in a mortar and adding about five volumes of 1 per cent. sodium biphosphate. The suspension is filtered through several layers of gauze, and after the cells have become precipitated, the supernatant fluid is decanted. The residue of cells is washed with 0.6 per cent. sodium chlorid and allowed to settle again, the supernatant fluid is decanted, and the residue covered with 1 per cent. sodium fluorid. The lastnamed fluid must first be neutralized against alizarin until only a trace of the violet color remains. The emulsion will keep for several weeks in an ice-chest.

Serum.-The patient's serum should be collected just a few hours (not over twenty-four) before the test is to be made, and must be clear and free from cellular elements.

${ }^{1}$ Biochem. Zeitschr., 1910, 26, 312; Wien. klin. Wochenschr., 1910, 23, 378, and 1221; ibid., 1911, 24, 1759. 
The Test.-To 10 drops of the patient's serum add one drop of 0.5 per cent. solution of sodium fluorid. Then add one drop of the cancercell emulsion so diluted that when one drop of the mixture is placed in a blood-counting chamber, about 10 to 20 cancer cells will be found in a field of four large squares. Close the counting chamber carefully, ring with vaselin to prevent evaporation, and place in the incubator for twenty-four hours.

A second slide is prepared from a mixture composed of one volume each of normal serum, cancer serum, and 0.6 per cent. sodium chlorid and sufficient cell emulsion.

A third slide is prepared with a fresh normal serum in the same manner as when the patient's serum is used.

All slides are incubated for twenty-four hours at $37^{\circ} \mathrm{C}$. and the cells counted. A material reduction in the number of cells with the normal serum will be noted; if the patient has carcinoma, the first and second slides will not show this reduction, whereas if the patient is free from cancer, similar reductions will be found in all three slides.

The authors recommend that both the cytolytic and the precipitin test be conducted when enough serum is available for both. 


\section{CHAPTER XXVI}

\section{THE RELATION OF COLLOIDS AND LIPOIDS TO IMMUNITY}

WHILE at the present time Ehrlich's side-chain theory best explains the specificity and mode of action of various antibodies, there is a growing tendency to explain many of these reactions on a physicochemical and colloidal basis.

From the fact that, without exception, antigens are colloids, and that antibodies also are colloid in their chemical characters, it is advisable to review briefly some of the main facts and theories concerning these bodies and their reactions.

Varieties of Colloids. - Colloids may be composed of two different classes of substances:

1. Organic substances, as, e. g., all forms of proteins and also gums, starch, glycogen, tannin, chondrin, the greater number of organic dyes, and probably the enzymes.

2. Inorganic substances, as, for example, the inorganic colloids, such as silicic acid, ferric hydroxid, arsenic sulphid, and many other similar compounds.

Since the living tissues and fluids are, without exception, colloids and colloidal solutions, the properties of the cells are largely the properties of colloids.

Nature and Properties of Colloids.-Since Graham, in 1861, studied the differences between the substances that did or did not diffuse readily through animal or parchment membranes, soluble substances have been classified in two main groups: (a) Colloids, or those substances that were dissolved to the extent of showing no visible particles in suspension, but that did not pass through diffusion membranes at all, or did so very slowly indeed, and (b) crystalloids, or solutions that diffuse through membranes quite readily.

On the other hand, we may have substances that are quite insoluble when aggregated in masses, but when derived in pure form by mechanical means, can be suspended and uniformly distributed through a fluid without showing any marked tendency to precipitate. Such suspensions or emulsions contain particles that are visible under the microscope; they usually appear turbid, do not transmit electricity, and 
are not diffusible. Colloids occupy a place between the true solutions of crystalloids and the emulsions. Sharp boundaries cannot usually be drawn between any of the members of the series. They differ quantitatively in some manner from the true solutions and the emulsions, but may approach them closely, and sometimes resemble them so strongly as to be almost indistinguishable from them. For the most part, however, they show decided characteristics that will differentiate them from the crystalloids, on the one hand, and the suspensions, on the other.

Those colloids that closely resemble the true solution have been designated "colloidal solutions," and those resembling more closely the suspensions, "colloidal suspensions." Of the two types, the colloidal solutions are far more important biologically, since the colloidal suspensions are usually prepared artificially and seldom occur in nature.

Colloids, therefore, appear to be suspensions of masses of molecules, or perhaps of very large single molecules. When these aggregations are sufficiently large, we have an ordinary suspension.

1. Colloids are usually amorphous in character, and with few exceptions do not present a typical structure; they are not crystalline under any visible condition. This, however, is not invariably the case, for we may have a protein, like hemoglobin, which resembles a typical colloid in every respect, and may yet form crystals readily and abundantly.

2. Colloids do not form true solutions, but the solvent is probably an important factor in determining whether or not a substance is colloidal in nature; e.g., soaps form true solutions in alcohol and colloidal solutions in water; rubber forms colloidal solutions in ether, but not in water. The term colloidal solution does not, therefore, refer to a true solution in the sense of a crystalloid, but to a colloidal state of suspension (the so-called colloidal solution).

3. Colloids are non-diffusible, or lack the power of passing through animal and parchment membranes. Not all colloids possess the same rate of diffusion, this property being relative, rather than absolute; however, solutions of salts (crystalloids) pass through so readily that they are easily separated from proteins (colloids) by dialyzation, a process that is in constant practical use.

4. Colloids have an extremely small osomotic pressure. They may, to a very slight degree, exert some influence upon osmotic pressure, the freezing and boiling-points of fluids, but in all cellular processes in which manifestations of osmotic pressure or diffusion are present the crystalloids may be considered as almost entirely responsible for these.

5. The colloids exhibit surface tension to a high degree - in other words, 
colloid fluids possess the force that strives to reduce its free surface to a minimum. As partial expressions of this force, the formation of emulsions when oil and water are mixed and the ameboid movements of the ameba and leukocytes may be mentioned as examples.

6. Colloids do not separate freely into ions when dissolved, and accordingly do not conduct electricity to an appreciable extent. When an electric current is passed through a colloidal fluid, most of the colloids move toward the anode; this phenomenon, known as cataphoresis, is also generally exhibited by suspensions, and in this particular the colloids resemble suspensions.

7. Colloids are usually easily precipitable and coagulable, and this is readily understood when the slender margin that exists between many of the colloids and the suspensions is borne in mind. Relatively slight changes, such as exposure, gentle heat, the presence of large quantities of crystalloids, the action of enzymes, etc., may throw an organic colloid out of solution, and when once precipitated, it is often incapable of again dissolving in the same solvent. Colloids are also precipitated by many electrolytes, apparently through the formation of true ion compounds.

8. The physical structure of colloids. This subject has been studied extensively by Hardy. ${ }^{1}$ Cells contain but one type of colloids, the proteins that form non-reversible coagula. So long as a colloid is in solution it is structureless; but such solutions may become solid as the result of changes of temperature and other physical means and from admixture with certain chemical fixing agents. The structure of the coagula varies according to the concentration of the colloidal solution and the nature of the coagulant, but in general the figures obtained in the solidification of protein solutions by such fixing agents as mercury bichlorid and formalin bear a striking resemblance to the finer structure of protoplasm as described by cytologists. These facts, no doubt, have an important bearing upon the various "foam," "reticular," and "pseudo-alveolar" structures of the protoplasm of cells described by Bütschli, Fromann, Arnold, Reinke, and others, and may indicate the effect of fixatives upon colloid solutions, explaining the usual time-worn objections to theories of protoplasmic structure as based upon artificial conditions not present in the normal living cell, and variously interpreted according to the fixative employed.

9. Colloids may be precipitated by electrolytes of opposite sign, as well

${ }^{1}$ A good general outline of the subject of colloids may be found in Pauli's "Physical Chemistry in the Service of Medicine," 1907, translated by Fischer (Chapman and Hall). 
as by colloids. In a colloidal solution surface tension constantly tends to make the particles of colloid approach one another, so that the surface may become as small as possible, and in this manner brings about precipitation or coagulation.

In a stable solution this action is counterbalanced by a force of electric repulsion. Pure colloids do not carry an electric charge and are not conveyed by an electric current; their apparent charge depends upon the nature of electrolytes that may be present. Traces of acid and of acid salts give it a positive charge, whereas alkalis and alkaline salts do the opposite (Pauli).

The process of coagulation of proteins, therefore, must depend upon the neutralization of their electric charge, and, as previously stated, this can be accomplished either by electrolytes or by colloids:

(a) Precipitation by electrolytes is best illustrated by the action of a strong acid on an albuminous solution. The negatively charged particles attract to themselves the positively charged hydrogen ions; their charge is now neutralized, and the force of attraction due to their surface tension is no longer counterbalanced by an electric repulsion. The particles are drawn together, form larger and larger masses, which finally come under the influence of gravity and precipitation takes place.

(b) Precipitation of colloids by colloids is illustrated by the precipitation of albumin by acetic and ferrocyanic acids. The colloid must be of opposite sign. As a result of the acid the particles acquire a positive charge, if they are not so charged already. This charge is then neutralized by the colloidal ferrocyanic acid of negative sign; the surface tension is no longer neutralized by an electric repulsion, and particles come together to form larger masses that are finally deposited as a precipitate.

Instead of precipitating the other, an excess of one colloid may act in a reverse manner. For example, as Neisser and Friedmann have shown, a suspension of particles of mastic in water (made by dropping an alcoholic solution in water) takes on a negative charge, and can be precipitated by positive colloids or ions, such as ferric chlorid. If the dose of ferric chlorid is increased gradually, the precipitate becomes more and more abundant, until an excess of ferric chlorid is present, when the reaction ceases and the precipitate may be redissolved. This has been explained on the assumption that when two colloids of opposite sign are mixed, they tend to fuse and form masses; the addition of an excess of either colloid tends to electrify the masses, causing mutual repulsion and possibly resolution of the masses. Hence the precipitate is 
soluble in an excess of both substances, just as a precipitate is soluble in an excess either of precipitin or of its antigen.

10. Absorption is the taking up of dissolved or volatile substances by finely divided or colloidal bodies. It is a combination between two substances dependent on physical attraction rather than on chemical affinity, and taking place in variable ratios, rather than in simple and constant ones, as occur in a true chemical union. It is believed by many that the two substances entering into the phenomenon of absorption exist as such side by side in the compound, which is to be regarded as an intimate admixture of the two, rather than as a new compound.

The lack of definite ratios by which colloids are absorbed has been shown by Bordet in the amount of hemolytic immune body that can be taken up by a given volume of corpuscles-i. e., the amount varies according to whether the corpuscles are added at once or in successive small portions. Thus, in one example, 0.4 c.c. of a hemolytic serum dissolved 0.5 c.c. of corpuscles if added at once; but if 0.2 c.c. of corpuscles was added first and successive amounts of 0.1 c.c. then put in, no solution took place after the one that followed the addition of the first portion. This was explained by Bordet according to the principles of absorption, this observer comparing it with the absorption of a dye by filter-paper. While other explanations are possible, yet exactly analogous phenomena may be seen in the mutual absorption of colloids of opposite sign. Thus, as we have previously stated, the addition of a solution of an electropositive colloid to a solution of an electronegative one tends to repel the particles, with the formation of masses for the purpose of self-protection, and in this manner the process of agglutination and precipitation is begun. But if a small amount of a second colloid is added to the same volume of the others, new aggregates of the two are formed that are less favorable to precipitation and require more of the second colloid to bring about complete precipitation.

\section{ANALOGY BETWEEN THE REACTIONS OF IMMUNITY AND COLLOIDAL CHEMISTRY}

With these few brief remarks on the properties and nature of colloids and the close resemblance of cellular protoplasm and fluids to colloids, we may consider briefly the apparent similarity that exists between the colloidal reactions and some of the reactions of immunity. This is especially pertinent for several reasons: it has been shown that cellular protoplasm is colloidal in nature; that antigens are certainly colloidal, and that antibodies, while they may or may not be solutions of colloids, 
are, in the final analysis, products of cellular activity, and therefore derived from colloidal solutions.

1. Antitoxins.-The side-chain theory of Ehrlich was first applied in explanation of the principles of immunity as affording an explanation of the action of toxins, the formation of antitoxin, and the interaction between these. Ehrlich has placed the various phenomena of immunity upon a chemical basis, bringing forward new theories to explain the various discrepancies that were found. For example, it was soon found that both toxin and antitoxin were unstable, and that neutralization of a toxin by the addition of antitoxin was not a simple process, like the neutralization of an acid by an alkali, but, on the contrary, was likely to be exceedingly complicated. This was explained as being due to the degeneration of toxin into various toxoids, which were able to neutralize antitoxin without being in themselves toxic when in a free state. They were likewise found to have a greater affinity for antitoxin than the toxin itself, so that when a toxin was tested and its toxicity determined, it was discovered that more antitoxin was needed to neutralize the mixture than was originally calculated, because the toxoids took no part in testing the toxin, but were active in uniting with antitoxin, and in this manner leaving true toxin unneutralized, and therefore toxic, unless an excess of antitoxin was used.

Analogous conditions may be observed among colloidal solutions. Thus, Danysz has shown that more toxin is neutralized if antitoxin is added at once than when it is added in successive doses. As stated elsewhere, this is explained by Ehrlich upon the assumption that time is allowed for the degeneration of toxin into toxoids to take place, the latter having a greater affinity for the antitoxin. It has been shown, however, that in some cases the addition of a small amount of a second colloid of opposite sign to a colloidal solution may render the solution more stable and protect it from precipitation by an excess of the second substance. Similarly, the amount of colloid necessary to precipitate a constant amount of another colloid is reduced to a minimum if the addition is made at once, and is rendered much greater if the colloid added is made slowly in small amounts, an interval being allowed to elapse after each addition. This is closely analogous to the Danysz reaction, and explains the latter as being due, when antitoxin is added slowly to toxin, to the formation of transitional compounds of toxin and antitoxin of diverse nature, requiring more antitoxin for complete neutralization of the toxin than if the antitoxin were added at once and in one dose. 
The difference between the $\mathrm{L}_{0}$ and $\mathrm{L}_{+}$dose of a toxin also has an analogy in the reaction of simple colloidal substances. Thus, Bilty has used ferric hydroxid, which neutralizes arsenic trioxid (the antidote for acute arsenical poisoning), and found that the addition of one lethal dose of arsenic to a neutral mixture of the two did not render the mixture toxic, but that several lethal doses were required, just as it is necessary to add several instead of one lethal dose of diphtheria toxin to the $\mathrm{L}_{0}$ dose.

Thus it would appear that the neutralization of a toxin by an antotoxin has analogies among the known and simple colloidal reactions. One objection to placing the toxin-antitoxin reaction upon a colloidal basis is that both have the same electric charge, $i$.e., both move toward the cathode, and, as we have seen, for the neutralization and precipitation of colloids the solution of colloids should be of opposite sign. It must be remembered, however, that toxins and antitoxins react in very complex fluids containing other substances consisting of both colloids and electrolytes, and until the electric charge of these in pure form is determined, the apparent similar electric charge of toxin and antitoxin can hardly outweigh the otherwise remarkable analogy it bears to colloidal reactions.

2. Agglutinins and Precipitins.-Various theories in explanation of the phenomenon of agglutination have been described in a previous chapter. The theory of Bordet appears to be best, and is based upon certain principles of colloidal chemistry. When bacteria are suspended in a fluid free from salt, agglutination does not take place because the bacteria carry a similar negative charge of electricity. When, however, ions of positive charge are added, as, e. g., sodium chlorid, the bacteria or other cells are repelled and coalesce to form masses, according to the laws of surface tension, in an effort to protect themselves. Larger masses may be formed that finally come within the influence of gravity and are deposited at the bottom of the test-tube. According to the same laws, the addition of agglutinin removes the negative charge of bacteria or other cells, with the consequent formation of clumps and masses. Similar phenomena may be observed in the precipitation of colloidal suspensions of clay in distilled water by the addition of a salt.

Solutions of inorganic colloids, as, for example, that of silicic acid, may agglutinate red corpuscles; bacteria, such as suspensions of typhoid and colon bacilli, may be agglutinated by solutions of the ferric salts.

Just as an excess of one colloid solution will charge masses of the other, resulting in a repelling action and breaking up of the agglutinated clumps, so the addition of an excess of agglutinin is found to prevent 
agglutination or to give but a slight reaction. This phenomenon has been explained, according to Ehrlich's side-chain theory, as due to the presence of agglutinoids that have a great affinity for the bacteria and unite with them without being active in the free state, owing to a loss of the agglutinophore portion of the molecule. Each cell united with an agglutinoid is one cell less to undergo agglutination by agglutinin, and accordingly in weak dilutions of serum agglutination is feeble or absent whereas in higher dilutions the phenomenon may be clearly observed.

Other explanations of the action of agglutinins and precipitins, based upon colloidal reactions, have been advanced. Thus Neisser and Friedmann have shown that suspensions of mastic may be "protected" against the precipitating action of ferric hydroxid by the addition of a small amount of organic colloid, such as serum, leech extract, or extract of typhoid bacilli, regardless of whether this colloid is charged positively or negatively or is neutral. The aforenamed observers believe that normal bacteria may be surrounded by a similar protective envelop that prevents the agglutinating action of substances of opposite sign. The action of agglutinin, therefore, would be to-remove this layer, so that the ions of opposite electric charge can unite with the bacteria and bring about their agglutination. This may be an explanation of the rôle of salts in the phenomenon of agglutination, the agglutinins removing the protecting envelops and the salt furnishing the ions of opposite charge that bring about agglutination.

Owing to the fact that a discrepancy arises here for the reason that emulsions of red corpuscles are agglutinated by both positive and negative colloids (ferric hydroxid and cuprum ferrocyanid), Girard, Mangin and Henri have given the following explanation of agglutination: When a red corpuscle is suspended in a fluid, various salts, especially the sulphates of magnesium and calcium, are diffused, which tends to facilitate the precipitation of negative and positive colloids, so that each corpuscle comes to be surrounded by a layer of precipitated colloid material. This zone of precipitated colloids of either negative or positive charge determines agglutination in the presence of a colloid solution of opposite charge, such as agglutinin or inorganic colloids (silicic acid, etc.).

3. Hemolysins.-Reference has been made elsewhere to the original observations of Bordet, showing that red corpuscles may absorb much more hemolytic antibody than is necessary to bring about their lysis, and that this absorption is analogous to colloidal absorption. 
Inorganic colloidal solutions, such as that of silicic acid, may produce hemolysis of red blood-corpuscles, e. g., those of the rabbit. Its action is manifested in extremely small doses. It is rendered inert by heat, and gradually deteriorates at room temperature. Furthermore, this inorganic colloid possesses some of the properties of a serum hemolysin; thus mice red corpuscles that have been agglutinated by colloidal silicic acid are dissolved by traces of lecithin or of fresh serum, but not by serum that has been heated to $60^{\circ} \mathrm{C}$. (inactivated). An excess of silicic acid tends to prevent hemolysis, which is another example of the action of an excess of one colloidal solution upon another of opposite sign.

Probably saponin hemolysis and the influence of fatty substances, such as lecithin and the fatty acids, upon the phenomenon of hemolysis, are closely related to, or to be explained by, the action of organic colloidal solutions.

\section{THE COMPLEMENT-FIXATION TEST AS A COLLOIDAL REACTION}

Many observations support the view that complement fixation by a specific antigen and its antibody is really complement absorption by a precipitate that forms when antigen and antibody are mixed. As previously stated, all antigens are protein in character. While there is some evidence to show that lipoids, and even carbohydrates, may act as antigens, there is no doubt but that the chief antigenic principles of any antigen are of protein structure; hence when mixed with an immune serum containing specific antibodies, it is believed that an invisible precipitate is formed that absorbs the complement. With serum antigens the quantity of protein is so large that a precipitate can readily be seen (the precipitin test). A serum antigen and its antibody may, however, be so highly diluted that, when mixed, a precipitate is not visible, although complement may be fixed (complement-fixation test for the differentiation of proteins). Moreschi and Gay have contended for many years that complements may become entangled and absorbed in such precipitates. Reasoning on the basis of the colloidal theory, it is possible that transition compounds of very diverse nature are formed when antigen, antibody, and a complement are mixed. This view on the action of complements and anti-complements is supported by numerous investigators who have examined the question from the standpoint of colloidal reactions. Thus in a complement-fixation test a mixture of antigen, antibody, and a complement in definite proportions results in the formation of new compounds of opposite electric charge, 
which tend to aggregate in masses (although these may be so small as to be invisible) and reduce their surface tension in just the same manner as agglutination and precipitation are brought about after the colloidal theories. When corpuscles and hemolytic antibody are subsequently added, hemolysis does not occur because free complement is absent.

A process similar to complement absorption by a specific antigen and its antibody is the Wassermann reaction. According to the colloidal theories, this reaction may be explained as due to the formation of an invisible precipitate by interaction between some substance in the serum of a luetic person (probably in the nature of an altered globulin), complement, and lipoidal substances contained in an alcoholic or watery extract of a normal or a diseased organ. This view is supported by the fact that euglobulin is known to be generally increased in the body fluids of syphilitics, and by analogy with the various precipitin tests that have been devised for the diagnosis of syphilis, as, for example, the reaction of Porges and Meier, which is dependent upon the appearance of a precipitate when luetic serum is mixed with an emulsion of lecithin or sodium glycocholate, etc. The exact nature of the antibody in syphilitic serums that forms these new compounds with lipoids and complement, resulting probably in the absorption of complement, is unknown. It is most likely in the nature of a globulin, its main characteristic being the power it possesses of reacting with lipoids. Schmidt ${ }^{1}$ ascribes the reaction to the physicochemical properties of the globulins of the syphilitic serum, which he believes possess a greater affinity for the colloids of the antigen than do normal globulins. This view is supported by the common observation that the turbidity of the antigen emulsion is closely related to its efficiency, since clear solutions are less active. Since various lipoidal substances may be employed, the Wassermann reaction can not be regarded as specific in the immunologic sense, although practically it is highly specific, as similar conditions are to be found in only two other diseases with any degree of regularity, namely, frambesia and tuberous leprosy.

\section{THE RELATION OF LIPOIDS TO IMMUNITY}

It is becoming more and more evident that lipoids bear an important relation to various immunologic processes, especially to certain cytolytic phenomena.

As stated in Chapter VIII, the results of some researches that go to

${ }^{1}$ Zeit. f. Hygiene, 1911, 69, 513. 
show certain lipoids and lipoidal substances may act as true antigens and produce antibodies. ${ }^{1}$ This, however, has not been definitely proved, and while it is of great importance, is not necessarily pertinent to the subject in hand. As the relation of lipoids to various immunologic processes has frequently been described in earlier chapters, as, e. g., where the rôle of lipoids in venom hemolysis, in the Wassermann syphilis reaction, and in the various precipitin reactions in syphilis were considered, a brief résumé may be of service in directing attention to this important and particular phase of immunity.

Relation of Lipoids to Hemolysis.- (a) From the standpoint of immunity, venom hemolysis is of peculiar interest as indicating the possible important relation of lipoids to hemolytic complement. Granting that venom contains a hemolytic amboceptor (Flexner and Noguchi), the complementing substance must be derived from the corpuscles, and, according to Kyes, this complementary agent is represented in lecithin. Kyes was able to produce what he considers are compounds of the hemolysin with lecithin, namely, "lecithids." Whether these "lecithids" are true compounds of hemolysins and corpuscular lecithin or simply the active hemolytic products of the cleavage of lecithin by ferments contained in the venom, is at present unknown. Noguchi and Lieberman have shown that not only lecithin, but soap as well, especially unsaturated fatty acids, and probably protein compounds of soaps and lecithin, may act as the hemolytic complement and activate the hemolysin of the venom. Lipoids from bacteria and trypanosomes have been found to possess similar properties. Hemolytic lipoids have been secured from serum, and the complementary activity of a fresh normal serum may be destroyed by fat solvents, e. g., ether. While other investigators have not been able to confirm Noguchi's attempts to produce an artificial complement of fatty substances of exactly the same properties as serum complement, this work indicates most strongly the close relation of serum complement to lipoids.

(b) The hemotoxic activity of various texins is probably dependent largely upon their action on the lipoids of red corpuscles. The saponin substances, ${ }^{2}$ a group closely related to glucosids, and found in at least 46 different families of plants, are strongly hemolytic. Ransom ${ }^{3}$ has

${ }^{1}$ Bibliography on Lipoids and Immunity given by Landsteiner, Kolle and Wassermann's Handbuch, 1913, 2, 1240. Also review of literature by Landsteiner, Jabresb. Immunität, 1910, 6, 209. gart, 1904.

${ }^{2}$ Complete literature on saponin, see Kobert "Die Saponinsubstanzen," Stutt-

${ }^{3}$ Deut. med. Wochenschr., 1901, 27, 194. 
found that an ethereal extract of red corpuscles contains a substance that inhibits saponin hemolysis. This substance consists largely of cholesterin, and it is the presence of cholesterin in normal serum that inhibits saponin hemolysis. This may be demonstrated experimentally by adding cholesterin to a solution of a saponin. Noguchi ${ }^{1}$ has shown that lecithin does not possess the same antihemolytic action on saponin. It would appear, therefore, that saponin causes hemolysis by combining with, altering, or dissolving the lipoids of the stroma of corpuscles. The resistance of corpuscles to saponin hemolysis varies in certain diseases, being especially low in jaundice (McNeil) ${ }^{2}$.

While saponins, solanins, phallin, and other vegetable poisons are of relatively simple chemical composition and quite unlike proteins, enzymes, or toxins, it is possible that bacterial and vegetable hemotoxins, such as tetanolysin, abrin, ricin, crotin, and robin, may produce their effects by a similar action on the lipoids of the erythrocytes. Noguchi has shown that cholesterin inhibits the action of tetanolysin. Landsteiner and Bottori have found that protagon, a brain lipoid, possesses the property of binding tetanus toxin, which indicates that this toxin may produce its effects by some action upon the lipoids of nerve-cells.

(c) The important relation of lipoids to the Wassermann reaction and certain precipitin or floccule-forming reactions (Klausner, Porges-Meier, Hermann-Perutz) has been mentioned repeatedly. Just what rôle the lipoids play in these phenomena is not known. While the globulins of syphilitic serums are strongly suspected of being concerned in these processes, their relation is not clear. Klausner ${ }^{3}$ now believes that the precipitate that forms when distilled water is added to syphilitic serum is due to the highrlipoid content.

\section{THE EPIPHANIN REACTION}

Principle.-This reaction is based upon the observation made by Weichardt ${ }^{4}$ in 1908; he found that diffusion is accelerated when differently colored solutions of antigen and its specific antibody are brought together. Changes in diffusion are associated with changes in the surface tension, both of which depend on a change in the osmotic pressure. This is the principle made use of by Ascoli in his miostagmin reaction, which will be described further on.

${ }^{1}$ Univ. of Penna. Med. Bull., 1902, 15, 327.

2 Jour. Path. and Bact., 1910, 15, 56.

${ }^{3}$ Biochem. Zeit., '1912, 47, 36.

${ }^{4}$ Berl. klin. Wochenschr., 1908, No. 20; Centralbl. f. Bakteriol., xliii, 143; ibid., xlvii, 39; Zeitschr. f. Immunitätsforsch., 1910, vi, 651; Deutsch. med. Wochenschr., 1911, No. 4, 154. 
Later Weichardt made the reaction more accessible to practical use by introducing into the solution of serums and antigen a system composed of sulphuric acid and barium hydroxid, together with certain catalytic agents. Using phenolphthalein as an indicator, he could show that fresh serums in high dilutions alter the surface tension of the finely divided barium sulphate particles by their colloidal action, so as to increase the absorption of $\mathrm{H}$-ions, thus rendering the solution more alkaline.

This phenomenon has been utilized by Weichardt, under the name of "epiphanin reaction," to determine the occurrence of such interaction of antigen and antibody. The reaction probably depends upon physicochemical principles of absorption, but the exact nature of the change is not yet understood. The reaction is based upon the following generalizations:

1. Solutions containing colloids-i.e., antigen alone, antiserum alone, or antigen plus non-specific antiserum in certain dilutions-act in the foregoing system by shifting the phenolphthalein end-point (the point of neutralization when acid and alkali are brought together in the presence of this indicator) in the sense of increased $\mathrm{OH}$-ions (pink color).

2. Specific antigens can inhibit the activity of their specific antiserums, the specific antigen-antibody combination then becoming evident in vitro by a shift of the end-point in the sense of increased $\mathrm{H}$-ion concentration (light color).

Specificity.-The specificity of the reaction has been confirmed by a number of investigators who used the test for the identification of a host of antigen-antibody combinations in vitro. The underlying principles have been confirmed by Kraus and Amiradzibi, ${ }^{1}$ Schroen, ${ }^{2}$ Seifert, ${ }^{3}$ Mosbacher, ${ }^{4}$ and others. The reaction has been applied to a study of various antigens and their antibodies, such as diphtheria toxin, tetanus toxin, typhoid and tubercle bacilli, tumor extracts and placenta extracts by Weichardt; extracts of syphilitic livers and serums of syphilitic patients by Seifert, Keidel and Hurwitz. ${ }^{5}$

Technic.-The technic of this reaction has been modified from time to time. The method here given is essentially the latest given by Weichardt, ${ }^{6}$ slightly modified by Keidel and Hurwitz.

1 Zeitschr. f. Immunitätsforsch., 1910, vi, 16.

${ }^{2}$ Münch. med. Wochenschr., 1910, 38, 1981.

${ }^{3}$ Deutsch. med. Wochenschr., 1910, 50, 2333.

${ }^{4}$ Deutsch. med. Wochenschr., 1911, 22, 1021.

5 Jour. Amer. Med. Assoc., 1912, lix, 1257.

' Berl. klin. Wochenschr., 1911, 43, 1935. 
Five constituents enter into the test:

1. The Antigen.-This is an alcoholic extract of syphilitic liver, prepared in exactly the same manner as for performing the Wassermann reaction. High dilutions of the antigen, ranging from $1: 100$ to $1: 10,000$, are prepared with normal salt solution. As in the Wassermann reaction, not every antigen is satisfactory, a point that can be determined only by making preliminary tests.

2. The patient's serum should be fresh, unheated, and highly diluted, the dilutions ranging from $1: 100$ to $1: 10,000,000$. Usually it is better to use higher than lower dilutions. When too concentrated solutions of serums and of antigen are used, erroneous results are likely to be obtained.

3. A normal solution of sulphuric acid.

4. A saturated solution of barium hydroxid made equivalent to the normal solution of sulphuric acid. In the use of the barium hydroxid it is imperative to prevent its exposure to the air. A solution that has become cloudy, owing to the entrance of carbon dioxid, should not be used. In carrying out the test it is best to pour out the amount of barium hydroxid needed for the test into a rubber-stoppered bottle or test-tube, so as not to contaminate the stock solution.

5. A 1 per cent. alcoholic solution of phenolphthalein containing 1 per cent. of a 10 per cent. solution of strontium chlorid. The strontium chlorid has been found to catalyze the reaction.

The Test.-The test is conducted as follows: A number of clean beakers of about 50 c.c. capacity are used. For each dilution of the serum a separate beaker is required. One beaker is used for an antigen control, and another to control the system of barium hydroxid and sulphuric acid. Five beakers may be used, Nos. 1, 2, and 3 constituting the main test, No. 4 the antigen control, and No. 5 the system control.

The reagents are added by means of overflow pipets. To each of the first four beakers is added 1 c.c. of the dilute antigen to be used in the test (about $1: 10,000$ ). To beaker 5 is added 1 c.c. of the salt solution used in making the dilutions of the antigen and the serums. Now 0.1 c.c. of the dilute serum to be tested is added to each of the first three beakers, each beaker, however, containing the same serum in a different dilution. To beaker 5 the same quantity of salt solution is added, but to beaker 4-the antigen control-no serum or salt solution is added.

To each of the five beakers the system of sulphuric acid and barium hydroxid and phenolphthalein is now added carefully. First, 2 c.c. of the normal suphuric acid solution are added to each; then 2 c.c. of the 
barium hydroxid, and finally 0.1 c.c. of the phenolphthalein strontium chlorid mixture.

It will be seen that beaker 4 -the antigen control-contains all the constituents of the test-beakers 1 to 3 except serum. To make beaker 4 qualitatively as well as quantitatively equal to beakers 1 to $3,0.1$ c.c. of the dilute serum (the average of the dilutions of serum which are used in the test) is now added to beaker 4 , the reaction having already taken place.

The addition of the sulphuric acid and barium hydroxid requires great care. Since the reaction depends on small differences in acidity or alkalinity, it is obvious that slight errors will vitiate the results. For the acid and the alkali separate pipets are used. After emptying the pipet of its content of acid or alkali, the last traces adhering to the inside of the pipet are removed by washing. These washings are later added to the beakers to which they belong. In filling the pipets with acid or alkali, the latter should first be drawn up into the pipet at least once, and then emptied again before the pipet is finally filled for delivery into the next test-beaker. Only by careful attention to these points in the technic can reliable results be obtained.

Reading the Results.- If beakers 1 to 3 contained the antiserum to the antigen used, a positive epiphanin reaction will be obtained, and if the barium hydroxid and sulphuric acid were previously carefully adjusted to each other, it will be found that beakers 1 to 3 will be lighter than the antigen control, beaker 4 . The presence of a specific antigen-antibody combination has shifted the phenolphthalein end-point in the sense of increased $\mathrm{H}$-ion concentration. The exact differences in the alkalinity between beakers 1 to 3 and beaker 4 can be quantitatively determined by titration with $\frac{\mathrm{n}}{100}$ suphuric acid, and the results expressed as a curve.

If the antigen and antibody were not specific, the epiphanin reaction will be negative. Beakers 1 to 3 will be more alkaline than the antigen control, beaker 4 , because, as previously pointed out, serums alone or antigen with non-specific serums shift the phenolphthalein end-point in the sense of increased $\mathrm{OH}$-ion concentration.

The results may be plotted as curves. The titration values in $\frac{n}{100}$ sulphuric acid are placed on the ordinates, and the serum dilutions on the abscissæ. The positive values are plotted above the line and the negative values below the line. No reaction is regarded as positive unless it gives a titration value of at least 0.05 c.c. $\frac{\mathrm{n}}{100}$ sulphuric acid. Values below 0.05 c.c. are easily within the limits of error. 
The following method, employed by Seifert, is much simpler, but is open to the error on account of using the antigen and serum in too concentrated a state. In a small test-tube place 0.1 c.c. of a $1: 10$ solution of the serum in normal salt solution, and add 0.1 c.c. of an alcoholic extract of syphilitic liver. To this slowly add 1 c.c. of decinormal sulphuric acid and 1 c.c. of a solution of barium hydroxid of the exact concentration needed to neutralize the sulphuric acid solution. On the addition of the drop of the phenolphthalein solution the fluid turns red when the serum is from a syphilitic, whereas no change in tint occurs with non-syphilitic serum.

Practical Value.-The reaction appears to be of considerable value in the diagnosis of syphilis. With serums and antigen in proper dilutions, the results closely parallel those secured by the Wassermann reaction. Keidel and Hurwitz report positive reactions with luetic serums in about 75 per cent of their cases. The reaction was found highly specific in that syphilitic extracts gave negative reactions with serums of non-syphilitic persons and patients suffering from malignant disease. Extracts of normal fetal liver and beef heart gave negative reactions with serums of syphilitic persons.

Positive reactions have also been found in malignant disease, as with the antigens of carcinoma and sarcoma. Keidel and Hurwitz obtained 16 positive reactions in a series of 24 serums of persons suffering with definite or suspected malignant disease. Burmeister did not find the reaction of value in cancer.

The epiphanin reaction has also been used in the diagnosis of pregnancy, but sufficient work has not been done to render an expression as to its merits of value at this time.

\section{The Mrostagmin Reaction}

Among the very large number of immunity reactions employed in attempts to secure a diagnostic test for cancer, the "miostagmin reaction" of Ascoli and Izar ${ }^{1}$ is the only one thus far devised that claims the serious attention of the clinician.

Principles. - This reaction is founded on the fact, noted by Ascoli, that by the mixing of an antigen and its corresponding antibody there results a reduction of the surface tension of the liquid containing these, which may be demonstrated by counting the number of drops of the fluid in a given volume (usually 1 c.c.), under constant conditions. Normal serum diluted with salt solution is first tested, and the number of

${ }^{1}$ Münch. med. Wochenschr., 1910, 57, 62, 182, and 403. 
drops found in a cubic centimeter determined with a specially devised instrument known as Traube's stalagmometer. The antigen is so diluted that when mixed with this normal serum it does not increase the number of drops more than one in a cubic centimeter. When properly diluted patient's serum and antigen are mixed, it may be found that the number of drops is increased from 2 to 8 in a cubic centimeter. This constitutes a positive reaction. The reaction is apparently due to the lowering of surface tension, so that more and smaller drops are found; hence the term "miostagmin" has been applied to the test, the word being devised from the Greek, meaning "small drop."

The reaction is said to be sharply specific and very delicate, so that antigens diluted up to $1: 100,000,000$ or higher may be detected. The technic requires considerable practice and experience or erroneous results are quite likely to occur.

The exact nature of the reaction is not known. The antigens are soluble in alcohol, but their nature is obscure. The antibody involved in the reaction is referred to as the miostagmin, but its relation to other antibodies is also unknown. It is probably a physicochemical or colloidal reaction, and for this reason it has been placed in this chapter.

Technic.-The antigen is most difficult to prepare. A recent method described by Ascoli is as follows:

1. Cut non-degenerated portions of malignant tumor (cancer or sarcoma) into small pieces and dry in vacuo or spread out in a thin layer on clean glass plates and keep at a temperature of $37^{\circ} \mathrm{C}$.

2. Pulverize the dried substance and extract with pure methyl alcohol (in the proportion of $5 \mathrm{gm}$. to 25 c.c.) for twenty-four hours at $50^{\circ} \mathrm{C}$. in closed vessels, and shake occasionally.

3. Filter while still hot, and allow the filtrate to cool, and then filter again through Schleicher and Schull's filter-paper No. 590.

4. It is now necessary to titrate the antigen and to determine in what dilution it should be employed. Various dilutions of the antigen are made with distilled water, as, e. g., $1: 10,1: 25,1: 50,1: 100,1: 150$, $1: 200$, etc. A fresh normal serum is diluted $1: 20$ with normal salt solution, and 9 c.c. of this are mixed with 1 c.c. of the various antigen dilution. Into another tube place 9 c.c. of the diluted serum and 1 c.c. of distilled water. All test-tubes, pipets, and other glassware used must be perfectly dry.

The tubes are gently shaken and placed in an incubator at $37^{\circ} \mathrm{C}$. for two hours. The drop number for each fluid is then estimated by Traube's stalagmometer. This instrument is merely a finely and 
elaborately graduated pipet with a central bulbous reservoir. The dropping end of the instrument ends in a flattened ground base, thus insuring uniformity in the size of the drops. The instrument is so graduated that a fraction of a drop can be estimated. That antigen is to be chosen that does not alter the drop number for normal serum by more than one drop in a cubic centimeter-the strongest dilution that fulfils this condition being chosen.

The Test.-The patient's serum is diluted 1:20 with normal salt solution and its drop number determined. Then take two tubes, and into one place 9 c.c. of diluted serum plus 1 c.c. of antigen dilution; into the other place 9 c.c. of diluted serum plus 1 c.c. of distilled water. A third tube may be prepared, which should contain 9 c.c. of normal serum $(1: 20)$ plus 1 c.c. of the same antigen dilution. A fourth tube contains 9 c.c. of a known positive serum $(1: 20)$ from a case of cancer and 1 c.c. of the antigen dilution.

All tubes should be carefully labeled, their drop numbers determined, and then placed in an incubator at $37^{\circ} \mathrm{C}$. for two hours or in the waterbath at $50^{\circ} \mathrm{C}$. for one hour. At the end of this time they are removed, allowed to cool, and the drop number of each is determined.

The controls are first examined to show that the antigen has not undergone any change. Variations of the number above one and a half or two drops (as compared with the control containing distilled water instead of antigen) are regarded as positive reactions. The increase in drops is seldom greater than eight.

\section{TABLE 21.-MIOSTAGMIN REACTION IN CANCER}

\begin{tabular}{|c|c|c|c|}
\hline 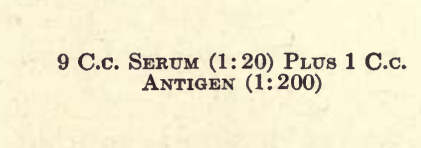 & $\begin{array}{l}\text { Drops PER C.C. } \\
\text { AFTER INCUBA- } \\
\text { TION AT } 37^{\circ} \text { C. } \\
\text { For TWO HoURS }\end{array}$ & $\begin{array}{l}\text { DROPS PER C.c. } \\
\text { CONTROLS 9 C.c. } \\
\text { SERUM (1:20) PLUS } \\
1 \text { C.c. DISTILLED } \\
\text { WATER AFTER } \\
\text { INCUBATION }\end{array}$ & Results \\
\hline $\begin{array}{l}\text { Normal serum ................. } \\
\text { Known cancer serum . . . . . . } \\
\text { Unknown serum for diagnosis . . } \\
\text { Unknown serum for diagnosis. } \\
\text { Unknown serum for diagnosis... }\end{array}$ & $\begin{array}{l}56.0 \\
62.4 \\
61.2 \\
57.2 \\
62.4\end{array}$ & $\begin{array}{l}56.4 \\
59.2 \\
60.0 \\
57.0 \\
59.8\end{array}$ & $\begin{array}{l}\text { Negative } \\
\text { Positive } \\
\text { Positive } \\
\text { Negative } \\
\text { Positive }\end{array}$ \\
\hline
\end{tabular}

Other Methods for Preparing Antigens.-Various methods for preparing antigen and conducting the test are to be found in the literature, and it is extremely difficult to arrive at a correct conclusion as to which is the best method for preparing antigen. Among these methods for 
the preparation of antigen other than those previously described are the following:

1. After securing the alcoholic extract described elsewhere evaporate it to dryness. Again extract in methyl alcohol and evaporate. Extract with warm ether, renewed several times during the course of twentyfour hours. Dry, and repeat the extraction several times until the alcohol remains colorless. Evaporate the alcoholic and ethereal extracts at $50^{\circ}$ and $37^{\circ} \mathrm{C}$. respectively. A yellowish-red, sticky mass results. Dissolve this in a large amount of water-free ether. Filter, and evaporate at room temperature until a slight powdery precipitate is deposited. This solution constitutes the stock antigen, which, however, may require still further concentration.

2. A synthetic cancer antigen may be prepared by grinding up 0.5 gram of lecithin (ovolecithin Merck, or lecithin Richter), and extract it with 50 c.c. of acetone for twenty-four hours at $50^{\circ} \mathrm{C}$. Filter through Scheicher and Schull's filter-paper No. 590, until clear. Just before it is to be used it should be diluted with water in such amount that 1 c.c. will contain the largest amount that does not cause a marked reduction of surface tension in normal serum. As a rule, this dilution is between $1: 50$ and $1: 100$ (Köhler and Luger) ${ }^{1}$.

3. A syphilis antigen may be prepared by extracting 0.5 gram of dried and powdered syphilitic liver with 50 c.c. of absolute alcohol for two hours at $37^{\circ} \mathrm{C}$. with frequent shaking. Filter, and concentrate to 10 c.c.

4. A bacterial antigen, as e.g., one of typhoid bacilli, may be prepared as follows: Wash off five forty-eight-hour agar cultures of typhoid bacilli with 5 c.c. of normal salt solution for each tube. Cover the emulsion with toluol, and shake vigorously for several hours. Place in an incubator at $37^{\circ} \mathrm{C}$. for forty-eight hours, and filter through a sterile Berkefeld filter. This filtrate may be used as antigen, or it may be used in preparing an alcoholic extract in the following manner: To the original aqueous filtrate add 50 c.c. of absolute alcohol. Allow the mixture to stand for one-half hour, shake, centrifugate, and then mix the sediment with 20 c.c. of absolute alcohol. Shake thoroughly once more, and again centrifugate. Combine the two extracts, and concentrate on the water-bath to about 20 c.c.

Practical Value.-This test is quite delicate, and errors due to faulty technic are quite likely to creep in. Unless all precautions are rigidly observed, the results are worthless. Although an extensive literature

${ }^{1}$ Wien. klin. Wochenschr., 1912, 25, 1114. 
has accumulated bearing evidence as to the value of the test as a diagnostic procedure, the method has not, however, come into general use.

Ascoli and Izar especially have advocated the test in the diagnosis of cancer. In 100 cases of malignant tumors, they obtained 93 positive reactions; in 103 cases of other diseases they obtained only one positive reaction. Tedesko, Stabilini, Leitch, Kelling, and others have reported favorably upon the practical value of the test in the diagnosis of cancer. Burmeister ${ }^{1}$ has found that a negative reaction has some value in excluding cancer, and is of more value in arriving at a diagnosis than a positive reaction, $i$. e., it has a higher negative than a positive value.

The test has also been used in the diagnosis of typhoid fever, paratyphoid fever, syphilis, tuberculosis (positive only in active cases) echinococcus disease, etc. Obviously, other methods of diagnosis, such as the agglutination reaction and the Wassermann reaction, have superseded this test in practical diagnosis. The method possesses, however, considerable theoretic interest and is worthy of further investigation.

1 Jour. Infect. Dis., 1913, 12, 459. 


\section{CHAPTER XXVII}

\section{ANAPHYLAXIS}

$\Lambda_{\mathrm{T}}$ is generally believed that when an animal previously injected with an antigenic substance is subsequently reinjected with the same substance, the antibodies induced by the first injection are reënforced, and that a continuation of the process of immunization will eventually lead to a high degree of immunity. Under certain circumstances, however, this is not the case, because severe and even fatal symptoms, as well as other manifestations, may set in after the second injection, indicating that, instead of being immune, the animal is indeed hypersusceptible or hypersensitive to the affects of the antigenic substance.

- Similarly, it is a common observation that whereas certain infections, such as smallpox, scarlet fever, and measles, confer a state of immunity, others, as, for example, pneumonia, erysipelas, and influenza, not only are not followed by immunity, but, indeed, that a decreased resistance or predisposition to subsequent infection by the same microörganism may be induced.

Before experimental investigation of this subject was undertaken, not a few observations were made and described by the early workers in the fields of bacteriology and immunity that correspond exactly with the phenomenon of hypersensitiveness, as we understand it today, although the true explanation of their unexpected results was not suspected, and they were modestly ascribed to faulty technic, embolism, toxicity of the inoculum, etc. From this it followed that the discovery that the experimental injection of such ordinary innocuous substances as normal serum and milk may produce violent symptoms and death gave rise to much surprise and incredulity, since scientists had long been accustomed to regard the reaction of an animal to an injection as a process of immunization, or diminished sensitiveness, instead of one of increased sensitiveness. Here, as Besredka remarked, the rules of immunity are "standing on their heads."

To this state of hypersensitiveness Richet, one of the earliest investigators in this field, applied the term "anaphylaxis," meaning "without protection." While it appears to be the exact antithesis of "immunity," which means "with resistance to infection," recent re- 
searches would tend to indicate that the two subjects are indeed closely related. An enormous amount of work has already been done on anaphylaxis, the subject being of the greatest importance, not only on account of its practical bearing on serum therapy, but because of its intimate relationship to the subjects of infection and immunity, and the new light that these studies may throw upon the nature and mechanism of these processes. Although the phenomena of anaphylaxis are now known to be due to the proteins, and while the symptoms and lesions of the condition are fairly well understood, the exact nature and mechanism involved in the process are not established, and the entire subject is fraught with so much interest as regards infection and immunity that it affords a fruitful field for further research.

In this chapter will be presented the known facts regarding anaphylaxis and the theories that have been advanced in explanation of its nature and mechanism, the consideration of anaphylaxis in its practical application to medicine being left for the following chapter.

Historic. - The first observation of anaphylaxis as it occurs in an infectious disease was probably made by Jenner in 1798. This investigator observed the sudden appearance of an "efflorescence of a palish red color" about the parts where variolous matter had been injected into a woman who had had cowpox thirty-one years before.

In 1839 Magendi found that rabbits that had been injected with eggalbumin died after a repetition of the injection, a phenomenon strikingly similar to that observed sixty-five years later by Theobald Smith following injections of horse serum. This phenomenon was subsequently studied thoroughly by Rosenau and Anderson and Ot`s.

While the effects of diphtheria and tetanus antitoxins were being studied, peculiar and apparently. paradoxic results were occasionally observed during immunization of animals with the bacterial toxins. Thus in 1895 Brieger $^{1}$ reported the case of a goat that was highly immunized against tetanus and yet was subject to tetanus. In 1901 von Behring and Kitashima ${ }^{2}$ reported similar findings with diphtheria in horse immunized against that infection. At this time it was shown that the results could not be due to the cumulative effect of the toxin, and the explanation offered aimed to show that the process was purely histogenetic, and based upon the assumption that receptors attached to the body-cells had a closer affinity for toxin than the free (antitoxin) receptors in the blood-stream. At the present time toxin hypersusceptibility is held by some to be a true anaphylactic reaction brought about

${ }^{1}$ Zeitschr. f. Hyg., 1895, 101.

2 Berl. klin. Wochenschr., 1901, xxxviii, 157. 
by protein substances in the toxin filtrate; others regard von Behring's explanation as satisfactory. Further reference to this subject will be made later on in this chapter.

Richet's Studies. - The fundamental observations upon which our present knowledge of anaphylaxis is based were made in 1898 by Hericourt and Richet. ${ }^{1}$ These observers found that repeated injections of eel serum into dogs gave rise to an increased susceptibility to this substance, instead of immunizing the dogs against the serum. These studies were continued by Richet ${ }^{2}$ and his assistants with extracts of the tentacles of certain sea anemones. These studies showed that a second injection of the poison into dogs, given after an interval of several days, is followed by greater and more intense activity than marked the first injection. If the animal survives, however, the disease is conquered more readily after the second than after the first injection. As previously stated, Richet coined the word "anaphylaxis," meaning "without protection," and indicating that the first injection destroyed any natural resistance that the animal might possess against the poison (actionocongestin). From these studies he concluded that two different substances are contained in eel serum and in the tentacles of actiniens, one concerned in establishing an immunity, and the other in calling forth a hypersensitiveness; thus far, however, the separate existence of these two hypothetic substances has not been proved.

Arthus Phenomenon.--In 1903 Arthus, ${ }^{3}$ at the instigation of Richet, showed that similar results may be obtained with non-toxic substances, like serum and milk. On injecting rabbits at definite intervals with normal horse serum, he found that the first two or three doses were absorbed, whereas subsequent injections, given subcutaneously, led to increasingly severe local reactions (Arthus phenomenon). If the animals, however, were first injected subcutaneously and later intravenously, or intraperitoneally, serious symptoms of dyspnea, convulsions, and diarrhea, and even death resulted.

Von Pirquet's Early Studies.-For a long time urticarial eruptions were occasionally observed to follow transfusion of blood from lambs and other lower animals to persons suffering from anemia and similar conditions. Soon after diphtheria antitoxin was discovered the medical

1 Compt. rend. Soc. de Biol., 1898, 53.

2 Compt. rend. Soc. de Biol., 1902, liv, 170; 1903, 1v, 246; 1904, 1vi, 302; 1905, lviii, 112; 1907, lxii, 358, 643; 1909, lxvi, 763; 1909, lxvi, 810; 1909, lxvi, 1005. Ann. de l'Inst. Pasteur, 1907, xxi, 497, 1908, xxii, 465.

${ }^{3}$ Compt. rend. Soc. de Biol. 1903, lv, 817; 1906, lx, 1143. Archiv. internat. de Physiol., 1908-09, vii, 472. 
profession was shocked to learn of the sudden death of the healthy child of an eminent German professor following a prophylactic injection of the serum. In 1902 von Pirquet began the study of these clinical manifestations with a child in Escherich's clinic who, after receiving a second dose of horse serum ten days after the first, on the same day developed symptoms of fever and a rash. On the basis of this observation von Pirquet ${ }^{1}$ reached the conclusion that the prevailing views regarding the length of the incubation period of an infectious disease could not be correct. He therefore propounded the theory that the organism concerned in the etiology of disease calls forth symptoms only when it has been altered by antibodies, the period of incubation representing the interval necessary for the formation of these antibodies.

In conjunction with Schick, von Pirquet endeavored to study all infectious diseases from the same point of view, especially smallpox, measles, recurrent fever, streptococcus infections, and the reactions to cowpox virus, tuberculin, and mallein. Later these same observers ${ }^{2}$ studied the symptoms following injection and reinjection of horse serum, designating the train of symptoms "serum sickness." They emphasized that a single injection of serum may suffice to bring about the symptoms, and that this immediate reactivity possesses diagnostic value in so far as it enables us to decide whether a previous infection has occurred. How near the astute Jenner came to reaching the same conclusion is shown in the following abstract from his report in 1798:

"It is remarkable that variolous matter, when the system is disposed to reject it, should excite inflammation on the part to which it is applied more speedily than when it produces the smallpox. Indeed, it becomes almost a criterion by which we can determine whether the infection will be received or not (italics ours). It seems as if a change, which endures through life, had been produced in the action, or disposition to action, in the vessels of the skin; and it is remarkable, too, that whether this change has been effected by the smallpox or the cowpox, that the disposition to sudden cuticular inflammation is the same on the application of variolous matter."

Von Pirquet at this time proposed the term "allergy," from ergeia, reactivity, and allos, altered, meaning altered energy or a changed reactivity, as a clinical conception expressing a truth without binding

1 Zur Theorie der Infectionskrankheiten (Vorläufige Mitteilung), April 2, 1903. "Zur Theorie der Vaksination," Verhandl. d. Gesellsch. f. Kinderh., Kassel, 1903.

${ }^{2}$ Wien. klin. Wochenschr., 1903, xvi, 758, 1244; 1905, xviii, 531. Die Serumkrankheit, Leipsic, Deuticke, 1905; Münch. med. Wochenschr., 1906, liii, 66. For a full bibliography on this subject of allergy up to 1910 see von Pirquet, Archiv. Int. Med., 1911, vii, 259 and 383. 
any one to a theory based upon bacteriologic, pathologic, or biologic findings.

Theobald Smith Phenomenon.-While von Pirquet was making these studies, great impetus was given the experimental study of anaphylaxis by the observation of Theobald Smith, who found that guineapigs that were used for standardizing the strength of diphtheria antitoxin after a second injection of serum frequently presented symptoms of a serious character, such as great restlessness, dyspnea, itching of the skin, and violent convulsive seizures. In fully 50 per cent of the animals death occurred within half an hour.

Simultaneously Rosenau and Anderson ${ }^{1}$ in this country and Otto ${ }^{2}$ in Germany undertook the study of this phenomenon. The first-named investigators showed most conclusively, by a thorough series of experiments, the action of horse serum and other substances in guinea-pigs, and proved that serum sickness was due to some constituent of the serum independent of the antitoxic antibodies, as normal horse serum yielded exactly similar results.

Among the earlier studies of anaphylaxis of importance were those of Weichardt. ${ }^{3}$ These were made with extracts of placental cells, and later with the proteins of pollen, in relation to hay-fever. Wolff-Eisner ${ }^{4}$ wrote a treatise that had as its fundamental idea the belief that hypersensibility was due to endotoxins liberated by a lysin formed as a result of the first injection. Also among the earliest and most valuable studies upon the nature of anaphylaxis, and showing the important relation of proteins to the process, are those of Vaughan ${ }^{5}$ and his coworkers; indeed the studies of Smith, Rosenau and Anderson, Vaughan and Wheeler, Gay and Southard, Auer and Lewis, and others have gained for America a prominent part in the development of this important subject.

Definition.-By anaphylaxis, in the limited meaning of the term, as, e. g., in that following the injection of horse serum in man or following the experimental administration of practically any protein in the lower animals, is understood the following train of phenomena. When a foreign protein is introduced into the animal body, usually parenterally,

${ }_{1}^{1}$ Bull. 29, Hyg. Lab., U. S. P. H. and M. H. S., 1906; Bull. 36, Hyg. Lab., April, 1907; Jour. Infect. Dis., 1907, iv, 552; Bull. 45, Hyg. Lab., June, 1908; Bull. 50, Hyg. Lab., 1909; Jour. Amer. Med. Assoc., 1906, xlvii, 1007; Archiv. Int. Med., 1909, iii, 519 .

${ }^{2}$ von Leuthold Gedenkschrift, 1905, i; Münch. med. Wochenschr., 1907, liv, 1665; Kolle and Wassermann, 1908, ii, 255.

${ }^{3}$ Berl. klin. Wochenschr., 1903, No. 1.

${ }^{4}$ Zeitschr. f. Bakteriol., 1904; Berl. klin. Wochenschr., 1904, xli, 1105, 1131, 1156, 1273; Münch. med. Wochenschr., 1906, liii, 217.

"Summarized in "Protein Split Products," Lea and Febiger, 1913. 
after a time a specific hypersensitiveness of the animal for this protein will appear. After a definite interval, a second injection of the same substance, harmless in itself, may produce an itching rash and fever or violent symptoms of illness, and rapid death may even occur in an animal so inoculated. In other words, the first injection of the protein (serum, milk, egg-albumen, etc.) produces no symptoms, but serves to alter the power of reaction on the part of the body cells by rendering them unusually sensitive or susceptible to the same or to closely related foreign protein. Therefore, as defined by Rosenau, anaphylaxis may be considered as "a condition of unusual or exaggerated susceptibility of the organism to foreign proteins."

Terminology.-As previously stated, the word "anaphylaxis"(ana, against, and phylax, guard, or phylaxis, protection) was given to the condition by Richet, because he considered it one "without protection," or a state just the opposite to immunity, or prophylaxis. In the sense in which the phenomenon is now regarded the word is a misnomer, for we look upon the condition of hypersusceptibility as a step toward the attainment of a state of immunity, and as a distinct benefit and advantage to the organism. The term "allergy," introduced by von Pirquet, is more appropriate, as it expresses the condition of the bodycells, $i$. e., their hypersensitiveness or altered reactivity, regardless of any theories we may entertain as to the manner in which this change is brought about or manifested. - The word anaphylaxis has, however, come into general use, and with this explanation, we may continue to so use it.

The term anaphylactogen is applied to the protein, as serum, milk, egg-albumen, etc., which sensitizes the body-cells; sentizer is also a good word, and the process of rendering body-cells hypersensitive by administering a foreign protein has been called sensitization. In von Pirquet's nomenclature the protein would be called an allergen.

The term anaphylatoxin is applied to the toxic substance believed to be formed at the time of reinjection of the protein, and is regarded as responsible for the lesions and symptoms of anaphylaxis. In the belief that anaphylaxis resembles an intoxication, the second inoculation of protein is frequently spoken of as the intoxicating dose.

\section{PHENOMENA OF ANAPHYLAXIS}

The essential symptoms and lesions of anaphylaxis vary in the different animals, and, indeed, they have been found to vary among animals of the same species under different experimental conditions. 
Man.-When it is remembered that anaphylaxis and immunity are closely interwoven, and that anaphylaxis may be but a step toward securing prophylaxis and immunity, it will readily be understood that under the varying conditions of different injections the phenomena may be quite dissimilar. One of the best known examples of a general anaphylactic phenomenon in man is that following the injection of a foreign serum, as, e. g., horse serum (diphtheria antitoxin), which is characterized by an itching urticarial eruption, fever, and joint pains, and which is commonly known as "serum sickness." Fortunately, the severer and fatal forms of anaphylaxis in man are extremely rare, most cases having occurred in persons known to be hypersensitive to horse protein or in those suffering from the condition known as status lymphaticus. Familiar local anaphylactic reactions are the tuberculin, mallein, and luetin reactions. With this brief statement we shall pass to a consideration of anaphylaxis in the lower animals, experimentation having given us some insight into the mechanism of the process. Anaphylaxis in man and the relation it bears to immunity and disease, will be discussed again in Chapter XXVIII.

Guinea-pig.-This animal gives the most constant and the most intense symptoms. According to Doerrr, guinea-pigs are four hundred times as sensitive an anaphylactic reagent as the rabbit.

Horse serum; when injected into normal guinea-pigs, gives rise to no symptoms. As much as 20 c.c. may be injected into the peritoneal cavity, and small amounts may even be injected into the brain without causing any untoward symptoms.

When a small dose of serum is injected intravenously, intraperitoneally, or subcutaneously, and ten days later a second injection is made, the animal develops symptoms of acute anaphylactic asphyxia, which, in the majority of instances, terminates fatally. "In five or ten minutes after injection the pig becomes restless and then manifests indications of respiratory embarrassment by scratching at the mouth, coughing, and sometimes of spasmodic, rapid, or irregular breathing; the pig becomes agitated, and there is a discharge of urine and feces. This stage of exhilaration is soon followed by one of paresis or complete paralysis, with arrest of breathing. The pig is unable to stand, or if it attempts to move, falls upon its side; when taken up it is limp; spasmodic, jerky and convulsive movements now supervene. This chain of symptoms is very characteristic, although they do not always follow in the order given. Pigs in the state of complete paralysis may fully recover, but usually convulsions appear, and are almost invariably a 
forerunner of death. Symptoms appear about ten minutes after the injection has been given; occasionally in pigs not very susceptible they are delayed thirty to forty-five minutes. Animals developing late symptoms are not very susceptible and do not die. Death usually occurs within an hour, and frequently in less than thirty minutes. If the second injection be made directly into the brain or circulation, the symptoms are manifested with explosive violence, the animal frequently dying within two or three minutes" (Rosenau).

H. Pfeiffer has shown that a depression of the temperature is a constant finding in the severer forms of anaphylaxis in the guinea-pig. In fatal cases this decrease may be as much as from $7^{\circ}$ to $13^{\circ} \mathrm{C}$. Some relation exists between the extent and the duration of the fall of temperature and the severity of the symptoms. During acute anaphylaxis the blood shows a leukopenia,- - a diminution in complement,- -and, as shown by Friedberger, ${ }^{1}$ a delay in or a loss of coagulability. The most striking - change observed after death is permanent distention of the lungs, resembling emphysema, described by Gay and Southard, and particularly by Auer and Lewis. ${ }^{2}$ The lungs do not collapse, but remain fully distended, forming a cast of the pleural cavities. The alveoli are distended, and in some instances the walls may be ruptured. The walls of the secondary and tertiary bronchi are contracted, with infoldings of the normally thick mucosa, due to contraction of the smooth muscle by peripheral action, death really resulting from inspiratory immobilization of the lungs. The heart continues to beat long after respiration has ceased. Rosenau ${ }^{3}$ and Gay and Southard ${ }^{4}$ have also described minute hemorrhages in various organs and mucous membranes.

The amount of serum necessary to sensitize a guinea-pig is surprisingly small. Rosenau and Anderson found one guinea-pig that was sensitized by $0.000,001$ c.c. As a rule they used less than 0.004 c.c. in their experiments. Besredka places the minimum amount necessary to secure uniform results at 0.001 c.c., whereas 0.0001 c.c. proved sufficient in a considerable percentage of animals. The sensitizing dose of horse serum ordinarily employed in experiments upon guinea-pigs is 0.01 c.c.; amounts ranging from 0.001 c.c. to 1 c.c. are ordinarily followed by an incubation period of from ten to sixteen days. Large doses also sensitize, but a longer incubation period is required. In order to produce a fatal result, the second or intoxicating dose must be considerably

1 Zeitschr. f. Immunitätsf., 1 orig., 1909-1910, 8, 636.

${ }^{2}$ Jour. Exp. Med., 1910, xii, $172 . \quad{ }^{3}$ Bull. No. 32 of the Hyg. Lab., 1906.

4 Jour. Med. Research, 1908, xix, 1, 5, 17. 
larger than the minimum sensitizing dose, the proportion between the two having been placed by Doerr and Russ as $1: 1000, i$. e., if 0.001 c.c. of serum is injected intraperitoneally in order to effect sensitization, 1 c.c. injected by the same route ten or twelve days later would in all probability kill a half-grown guinea-pig, whereas 0.1 c.c. subcutaneously would be followed by serious symptoms.

Rabbit.-Reference has been made elsewhere to the pioneer work of Arthus, who first described the local anaphylactic reaction about the site of subcutaneous injection. He also described objectively the most important symptoms of acute anaphylactic death in the rabbit, as well as the more ordinary type, which ends in recovery.

In acute and fatal anaphylactic shock in the rabbit Auer ${ }^{1}$ found slow respiration, weak or absent heart action, with fall in blood-pressure, general prostration, the sudden falling of the animal on its side, a short clonic convulsion, increased peristalsis, and expulsion in feces and urine. Death is ascribed to a vascular or cardiac shock or to a failure of the heart action of peripheral origin, mostly affecting the right side, and due to a form of chemical vigor. The muscle may be gray, stiff, very tough to the finger-nail, and non-irritable. Further evidence of the importance of heart failure in anaphylaxis in the rabbit is furnished by the electrocardiographic study of Auer and Robinson. ${ }^{2}$ Blood coagulability is delayed.

Rabbits are by no means so easily sensitized nor to so high a degree as guinea-pigs. Non-fatal anaphylaxis accompanied by fall in bloodpressure, increased heart-rate, and active intestinal peristalsis is readily produced, but there is considerable uncertainty in inducing acute anaphylactic death. Sensitization is usually effected by two or three intravenous injections of 1 c.c. of serum at intervals of three days. Intoxication generally follows an intravenous injection of 1 to 5 c.c. of serum about four to six weeks later. Much smaller doses than these may, however, be used, as was occasionally shown during immunization of rabbits with erythrocytes for the production of hemolytic amboceptor, when minute traces of serum, escaping the washing process, served to sensitize, and at a later injection produced acute anaphylactic shock and death within a very few minutes.

Cats.-Anaphylaxis in the cat has been studied especially by Schultz, ${ }^{3}$ who observed that cardiac disturbances followed. Horse serum, however, was found markedly toxic in effect, even in the un-

1 Jour. Exp. Med., 1911, xiv, 476.

2 Jour. Exp. Med., 1913, xviii, 450.

${ }^{3}$ Jour. Phar. and Exper. Therap., 1911-12, 3, 302. 
sensitized animal, as little as 0.25 c.c. per kilo killing young, normal cats, and 0.1 c.c. per kilo causing a fall in blood-pressure both in the normal and in the sensitized animals.

Dogs.- In these animals the most constant symptom of anaphylaxis is an initial and transitory rise in blood-pressure, followed by a prompt fall of from 80 to $100 \mathrm{~mm}$. of mercury. This was first described by Biedl and Kraus, ${ }^{1}$ and subsequently by Eisenbrey and Pearce, ${ }^{2}$ Robinson and Auer. ${ }^{3}$ The general symptoms are not so violent as are those that occur in the guinea-pig and death is infrequent. Following intravenous injection of the intoxicating dose of serum there may be great restlessness, marked prostration, and vomiting, tenesmus, and involuntary discharge of feces and urine. If death does not occur, a condition of hemorrhagic inflammation in both the large and the small intestine may develop, called by Richet "chronic anaphylaxis," and by Schittenhelm and Weichardt, "enteritis anaphylactica." . Robinson and Auer, by an electrocardiographic study, detected cardiac changes consisting of disturbance of the heart impulses, abnormalities in ventricular contractions, and other interferences with the mechanism of the heart, due probably to the effect of horse serum on the peripheral cardiac tissue, and independent of the drop in blood-pressure or any effect upon the central nervous system. The heart changes do not appear to exert a primary influence on the blood-pressure, which is due to an effect upon the splanchnics, and is probably a secondary factor in anaphylaxis or vascular shock of the dog. In many instances there is leukopenia, with loss of mononuclear cells. Coagulation of the blood is delayed, a condition first described by Biedl and Kraus, ${ }^{4}$ who believed it to be due, probably, to a decrease in thromboplastin or an excess of antithrombin. Pepper and Krumbharr ${ }^{5}$ have shown that, by adding small amounts of thromboplastin to the non-coagulating, post-anaphylactic, oxalated plasma, the coagulability of the blood will be restored.

As stated elsewhere, it may be difficult to produce anaphylaxis in a dog. Usually a subcutaneous injection of 10 c.c. of horse serum, followed in from three to six weeks by 5 c.c. intravenously, will at least cause a marked fall in blood-pressure or fatal anaphylaxis.

Other Animals. - White mice and rats, while they may not develop acute anaphylactic asphyxia, such as is observed in guinea-pigs, do react

${ }^{1}$ Wien. klin. Wochenschr., 1909, xxii, 365.

2 Jour. Pharmacol. and Exper. Therap., 1912, iv, 27.

${ }^{3}$ Jour. Exper. Med., 1913, xviii, 556.

${ }^{4}$ Wien. klin. Wochenschr., 1909, ii, $363 . \quad{ }^{5}$ Jour. Infect. Dis., 1914, xiv, 476. 
to horse serum, as was shown by Schultz and Jordan. This reaction is evidenced by restlessness, marked irritability of the skin, involuntary passage of urine and feces, and temperature and blood-pressure changes. Anaphylactic reactions have also been observed to occur in numerous other animals, e.g., in cows, sheep, horses, hens, pigeons, and in certain cold-blooded animals, the symptoms varying according to the species. These reactions have not as yet been carefully studied.

\section{MECHANISM OF ANAPHYLAXIS}

Whereas the lesions and symptoms of anaphylactic shock here described in different species of animals are those commonly observed with serum proteins, they vary in no essential when any protein agent is used when the conditions of dosage and administration are the same. It is evident, however, that no one symptom, or group of symptoms, can be regarded as characteristic of anaphylaxis in all animals. The various species present widely differing pictures with the. same protein substance, and these differences are best explained on the ground of changes in the anatomic structure and physiologic reaction of different animals. Thus, Schultz has shown that serum anaphylaxis is essentially a matter of hypersensitization of smooth muscle in general, and that, during anaphylactic shock, all smooth muscle contracts. In the guineapig this effect is most evident in the bronchi, owing to the peculiar, though normal, anatomic structure of the mucosa, which is relatively thick as compared with the lumen, so that contraction of the smooth muscle throws it into folds that completely occlude the bronchi causing death from inspiratory asphyxia. The bronchial mucosa of dogs, rabbits, and rats, however, is relatively thin and poor in smooth muscle tissue, which may account for an entire absence of transitory respiratory difficulties during anaphylactic shock in these animals. In the dog the most marked effect is apparent upon the smooth muscle of the gastrointestinal tract, contraction resulting in setting up vigorous intestinal peristalsis, vomiting, and involuntary emptying of the urinary bladder. The characteristic initial rise in blood-pressure may be due to constriction of the splanchnic, pulmonary, coronary, and systemic arteries, followed by a condition of paresis and a fall in blood-pressure. The cardiac muscle is also involved, particularly on the right side, as shown by Robinson and Auer, and this favors a venous accumulation of blood. In the rabbit a similar effect is noted upon the smooth muscle of the blood-vessels, and particularly on the heart, as well as upon the gastro- 
intestinal tract. Our present knowledge would ascribe these effects, therefore, to a local or peripheral action of the protein upon smooth muscle, and not primarily on the central nervous tissues, as was originally believed.

The fall in blood-pressure, therefore, appears to be a most constant and primary factor. So far as I am aware, no blood-pressure studies on the anaphylactic guinea-pig have been made. In this animal the heart continues to beat after respiration ceases, but this phenomenon may be due to mechanical and other factors dependent upon the extreme pulmonary emphysema. Fall in blood-pressure and congestion of the splanchnic area may produce cerebral anemia, and be responsible in some measure for the respiratory disturbances, the retching, the involuntary expulsion of urine and feces, the great depression and muscular weakness, and the speedy recovery when death does not result.

In man the marked urticarial and other rashes and the inspiratory asthma of those peculiarly sensitive to a protein due to a narrowing of the bronchi, the latter being analogous to the condition observed in the guinea-pig, the diarrhea, and the secondary drop in blood-pressure, all indicate a similar action on smooth muscle. This also provides an adequate pharmacologic explanation of the action of atropin, sedatives, and anesthetics in alleviating or masking the symptoms of acute anaphylaxis. (See Chapter XXVIII.)

Aside from the severe fall in blood-pressure and temperature, other effects of the anaphylactic poison are leukopenia, local and general eosinophilia (Vaughan, ${ }^{1}$ Moschowitz, ${ }^{2}$ Schlecht and Schwenket ${ }^{3}$ ), and reduced coagulability of the blood. Pfeiffer ${ }^{4}$ found poisonous substances in the urine during anaphylactic intoxication, and Hirschfeld ${ }^{5}$ detected a pressor substance in the serum of intoxicated guinea-pigs.

A more critical study of the nature and varieties of anaphylactogens, or substances capable of producing anaphylactic sensitization, will now be made. This will include also a consideration of the nature of the substances directly responsible for the anaphylactic intoxication, commonly known as anaphylactotoxins, and of the question as to whether anaphylactic intoxication is the result of an interaction in the bloodstream (humoral) or in the cells (histogenetic) or in both.

1 Zeitschr. f. Immunitätsf., 1911, 9, 458.

${ }^{2}$ New York Med. Jour., January 7, 1911.

3 Arch. exp. Path. u. Pharm., 1912, 68, 163.

4 Zeitschr. f. Immunitätsf., 1911, 10, 550.

5 Zeitschr. f. Immunitätsf., 1912, 14, 466. 


\section{ANAPHYLACTOGENS, OR ALLERGENS}

So far as is now known, only proteins may become anaphylactogens, and with the exception of gelatin and a few other proteins, practically any soluble protein will produce sensitization and intoxication of susceptible animals. Bacterial substances, extracts of plant tissues, purified vegetable proteins, and proteins derived from invertebrates and cold-blooded vertebrates have all been found capable of acting as anaphylactogens when introduced in a soluble and unaltered condition into an animal.

The proteins concerned must be foreign to the circulating blood of the injected animal, but they may be tissue proteins of the same animale.g., syncytial cells - that are not normally present in the blood. Indeed, Uhlenhuth and Haendel ${ }^{1}$ claimed to have sensitized a guinea-pig with the dissolved lens of one eye so that it reacted to a subsequent injection of the lens of the other eye. Proteins in solution are more active than those in suspension or in partial solution, and in general tissue proteins are less active than proteins in the blood, lymph, and secretions, but even keratins may produce anaphylaxis when dissolved (Krusins ${ }^{2}$ ), Uhlenhuth $^{3}$ has obtained positive results with proteins from mummies. As previously stated, the altered protein of an animal may be reinjected again into the animal and induce an anaphylactic reaction. Recently Richet ${ }^{4}$ has directed attention to this phenomenon, which he calls "indirect anaphylaxis," through observing an intense leukocytosis in a dog which reached the maximum on the eighth day following a second chloroformization.

Non-protein Anaphylactogens.-As with other immunologic reactions, observations have been made that are interpreted as indicating that non-protein substances are capable of producing anaphylaxis; thus Pick and Yamanouchi ${ }^{5}$ sought to demonstrate the antigenic properties of alcohol-soluble constituents of horse and beef serum, but conservatively concluded that their results may have been due to a combined action of protein and fat combinations. Similar conclusions were also drawn by Uhlenhuth and Haendel ${ }^{6}$ in their study of animal and

1 Zeitschr. f. Immunitätsf., 1910, 4, 761.

2 Arch. f. Augenheilk., Suppl., 1910, 47, 47.

3 Zeitschr. f. Immunitätsf., 1910, 4, 774.

4 Quoted in Jour. Amer. Med. Assoc., 1914, lxii, 711.

5 Zeitschr. f. Immunitätsf., 1909, 5, 676.

6. Zeitschr. f. Immunitätsf., 1910, iv, 761. 
vegetable oils and fats. Bogomolex ${ }^{1}$ is less conservative, and believes that he has succeeded in producing lipoid anaphylaxis; these claims, however, could not be confirmed by Thiele and Embleton. ${ }^{2}$ Meyer $^{3}$ was able to sensitize pigs with pure lipoids extracted from tape-worms, but was unable to intoxicate them with the same extracts, results that may be understood, since White and Avery ${ }^{4}$ have shown that as little as 0.0001 milligram of edestin will serve to sensitize a pig, whereas larger amounts of protein-more than is contained in Meyer's preparations-are necessary to produce intoxication. Finally, the studies of Wilson ${ }^{5}$ and White ${ }^{6}$ leave no doubt as to the fact that pure lipoids cannot produce anaphylaxis.

It is possible, however, for non-protein substances to combine with or alter the proteins of an animal and thus cause anaphylaxis. In this way can be explained apparent anaphylactic reactions to iron, salvarsan, iodin, arsenic compounds, and other medicinal agents.

Chemistry of Protein Anaphylactogens.-The purest known proteins act as anaphylactogens or sensitizers; in fact, the purer the protein, the more thoroughly it sensitizes the animal and the smaller is the dose necessary to produce intoxication. The crystallized proteins of hemoglobin, egg-albumen, and such pure vegetable proteins as edestin and excelsin, are powerful sensitizers. According to Wells, ${ }^{7}$ nothing less than an entire protein molecule will suffice to produce anaphylaxis, although $\mathrm{Zunz}^{8}$ claims to have observed typical reactions with the proteoses of fibrin, and Abderhalden ${ }^{9}$ obtained one with a synthetic polypeptid. It is not necessary, however, for a protein, in order to be active, to contain all the known amino-acids of proteins, for certain vegetable proteins, e.g., hordein and gliadin, which lack one or more amino-acids, such as glycocoll or tryptophane, may produce typical reactions. Presumably, the inability of pseudoproteins, such as gelatin, to act as anaphylactogens depends upon their deficiency in aromatic radicals.

Wells has obtained negative results with purified nucleoproteins, as well as with the isolated components of nucleins, such as histon and nucleic acid.

1 Zeitschr. f. Immunitätsf., 1910, v, 121, ibid, 1910, vi, 332.

2 Zeitschr. f. Immunitätsf., 1913, xvi, 160.

${ }^{3}$ Folia Serologica, 1911, vii, 771; Zeitschr. f. Immunitätsf., 1914, xxi, 654.

4 Jour. Infect. Dis., 1913, xiii, 103.

5 Jour. Path. and Bact., 1913, xviii, 163.

6 Jour. Med. Res., 1914, xxx, 383.

7 Jour. Infect., Dis. 1913, xii, 341.

8 Zeitschr. f. Immunitatsf., 1913, 60, 580.

๑ Zeitschr. physiol. Chem., 1912, 81, 314. 
While, therefore, it is probable, although it has not been definitely proved, that nothing less than the entire protein molecule is capable of producing the typical reaction, the questions arise whether the whole molecule, or only a certain group thereof, determines the specificity, and whether the whole molecule, or only a portion, is concerned as the sensitizing agent. It is now generally accepted that both the sensitizing and the intoxicating agents are one and the same protein, and the older view, which held that in a mixed protein substance, such as blood-serum, corpuscles, egg-albumen, etc., one protein is present that sensitizes and another that intoxicates, is probably erroneous. Besredka, for instance, finds that when a protein used to produce intoxication is heated it is less likely to prove fatal, and he concludes that proteins contain a thermostabile sensitizing and a thermolabile intoxicating portion. Doerr and Russ, however, have shown by carefully conducted experiments that heat affects both properties of proteins to the same degree. Since pure proteins, as, e. g., highly purified edestin, which is believed to be a chemical unit, act as exquisite sensitizers and intoxicants, it seems reasonable to believe that the sensitizing and poisonous group are constituents of the same protein substance. Whether or not both sensitizing and intoxicating groups are contained in each single molecule of a pure protein is a question that cannot be answered until we can be certain that absolutely pure proteins are secured to start with, and until our methods of effecting its cleavage have been perfected. Vaughan and his coworkers have long maintained that a sensitizing non-poisonous and a nonsensitizing toxic portion are groups of the same molecule, which they are able to obtain in vitro from animal, bacterial, and vegetable proteins by a method of splitting with sodium hydroxid in absolute alcohol, as described in the chapter on Infection. The toxic intramolecular group is regarded as non-specific, and the same for all proteins, which explains the identity of the symptoms of anaphylactic shock whatever the protein by which it is induced. The non-toxic sensitizing group, however, is specific, although it may not itself be a protein, or at least a biuret body. Whether or not all proteins contain a sensitizing group has not been determined. In keeping with his theory of the rôle of the toxic moiety of a split protein molecule in the production of disease, Vaughan believes that when proteins are introduced parenterally into animals, the non-toxic portion stimulates the body-cells to elaborate specific ferments, constituting the phase of sensitization, so that when this protein is subsequently introduced, digestion rapidly takes place with the liberation of the toxic substance responsible for the characteristic symp- 
toms, which may terminate in death. This interesting and plausible theory will be referred to again. It has received further experimental support from the work of White and Avery ${ }^{1}$ with split edestin and split tubercle-cell substance; ${ }^{2}$ and from that of Zunz ${ }^{3}$ and Wells and Osborne ${ }^{4}$ the last-named observers working with vegetable proteins, and concluding that although it is probable that the entire protein molecule is involved in the anaphylactic reaction, only certain groups are specifically concerned in the process. In other words, it would appear that anaphylaxis,-for example, serum anaphylaxis-is not due to one protein substance in the serum that sensitizes and another that intoxicates, both properties residing in the same protein molecule. Whether they are the same intramolecular substances existing side by side, - - one the sensitizer and the other the intoxicator,- - as is believed by Vaughan, cannot be definitely decided, although experimental work would tend to indicate that the latter may be the true explanation.

Physical State of Anaphylactogens.-The results of experiments all tend to support the theory that proteins in solution are most powerful in producing anaphylaxis, because they are able to come into intimate contact with body-cells, and cell permeation is probably necessary for the most complete sensitization. This explains in part the conflicting statements concerning the effect of heat on the sensitizing properties of blood-serum. Rosenau and Anderson found that animals could not be sensitized with serum that has been heated at $100^{\circ} \mathrm{C}$., whereas Doerr and Russ placed the point at $80^{\circ} \mathrm{C}$. Besredka showed that the sensitizing properties are in part at least, dependent upon the physical condition of the protein, and that heating undiluted blood-serum coagulates the protein and leads to a decrease of its anaphylactogenic properties. Similarly, Vaughan found that proteins that were insoluble in water, as, for example, edestin, sensitize more readily when dissolved in salt solution. The same factors are operative with the protein used for intoxication, the physical state of the protein substance having a direct bearing on the rapidity with which shock is produced. Temperatures high enough to disrupt and destroy proteins are, however, equally destructive to their sensitizing properties.

An interesting question in this connection is whether sensitization and intoxication may occur with the parenteral introduction of protein as with the food. In the great majority of instances the gastro-intestinal enzymes so completely disrupt the protein molecule that sen-

1 Jour. Inf. Dis., 1913, xiii, 103.

${ }^{2}$ Jour. Med. Res., 1912, xxvi, 317.

${ }^{3}$ Zeitschr. f. Immunitätsf., 1913, xvi, 580.

4 Jour. Inf. Dis., 1913, xii, 341. 
sitization and intoxication do not occur. Guinea-pigs have, however, been sensitized by feeding them meat or serum, and instances of buckwheat, fish, and egg idiosyncrasies would tend to indicate that intoxication may result from the ingestion of these substances in sensitive persons.

Rosenau and $\mathrm{Amos}^{1}$ have demonstrated that proteins in a volatile state, as in the exhaled breath of men, when condensed and injected into guinea-pigs will sensitize these animals to subsequent injections of human serum. While it is doubtful if the complex molecule possesses the power of passing into the air in a gaseous form, it may probably exist in colloidal solution. Rosenau was also able, by keeping guinea-pigs in stables together with horses, to sensitize them to horse serum. These experiments are of fundamental importance in explaining instances of human anaphylactic phenomena among those sensitive to horse protein, -as, e. g., persons seized with sneezing and asthma when they come near horses, - and also tend to show how minute may be the quantity of protein capable of sensitizing and intoxicating body-cells.

Bacterial Anaphylactogens.-All bacterial proteins are anaphylactogens, although, on account of the physical state of the bacteria, they yield reactions more irregular and weaker than those observed with proteins in solution. The tuberculin, luetin, mallein, and similar reactions are true anaphylactic phenomena. Rosenau and Anderson, Vaughan and Wheeler, Kraus, and others have observed anaphylactic reactions with various bacteria, such as Bacillus subtilis and colon, typhoid, anthrax, and tubercle bacilli. Not infrequently reactions occur during the therapeutic administration of tuberculin and bacterial vaccines.

This brings up the interesting question as to whether toxins are anaphylactogens, a subject previously mentioned in the historic review of this subject. Instances of hypersensitiveness to diphtheria and tetanus toxins were early observed in attempts to immunize horses in the production of antitoxins. As it is extremely difficult, if not impossible, to isolate a toxin free from other constituents of the medium into which it was excreted by microörganisms, this question cannot be answered in a definite manner. There is no direct proof, however, that toxins sensitize, although the protein in the toxin filtrate may serve to do so. In 1902 Vaughan and Gelston ${ }^{2}$ showed that the poison contained in the cellular substance of the diphtheria bacillus is an entirely different one

1 Jour. Med. Research, 1911, xxv, 35.

2 Trans. Assoc. Amer. Phys., 1902, 17, 308. 
from the toxin elaborated by the same microörganism, results that were confirmed in 1911 by Friedberger and Reiter, ${ }^{1}$ working with the dysentery bacillus. Thus hypersensitiveness to toxins is probably not an anaphylactic phenomenon, but is due to a greater affinity of the bodycells for the toxin. This explains the so-called paradox of Kretz, who found that while the injection of an accurately neutralized toxin-antitoxin mixture produces no bad results in a normal animal, in one that has been previously actively immunized with toxin, the reverse occurs. Apparently the sessile receptors have a stronger affinity for toxin than have the free receptors, and accordingly the toxin becomes dissociated and combines with the cells.

This view is also substantiated by the observation that the symptoms of intoxication caused by the toxin used for immunization are not those of anaphylaxis, which, for a certain animal, are the same regardless of the source of the protein. Toxin hypersensitiveness does not seem to be transmissible to normal animals, whereas in anaphylaxis the condition may be transmitted (passive anaphylaxis).

Whether or not endotoxins act as anaphylactogens cannot be definitely stated. If they do, their action and effects are intimately connected with those ascribed to the protein contained in the bacterial cell. It is unlikely, however, that they play any rôle in inducing hypersusceptibility, as their toxicity is usually apparent soon after injection, and before sensitization has occurred.

The necessary period of incubation between sensitization and intoxication, the symptoms, and the fact that in some cases an immunity is induced, are results that strengthen the belief that the phenomenon of hypersensitiveness has a practical significance in the prevention and cure of certain infectious diseases.

\section{ANAPHYLATOXIN (PROTEIN POISON)}

The symptoms of anaphylaxis that follow injection of the protein into an animal previously sensitized with the same protein are such as to leave no doubt that a poison is the etiologic factor, although as yet this poison has not been isolated in a pure state,

Vaughan and Wheeler were the first to demonstrate this poison in vitro by splitting proteins with a solution of $\mathrm{NaOH}$ in absolute alcohol. Friedmann was first to produce it in vitro through the action of ferments contained in immune and normal rabbit serum upon ox corpuscles and

${ }^{1}$ Zeitschr. f. Immunitatsf., 1911, 11, 493. 
serum precipitates. Weichardt also produced it by digesting placental protein with the serum of rabbits immunized with placental cells. Friedberger ${ }^{1}$ studied the subject more extensively by observing the action of normal guinea-pig serum upon serum precipitates, and was first to apply the term "anaphylatoxin" to the poison. At the time he regarded it as a true toxin, similar to diphtheria and tetanus toxins, but at present we know that this poison is not a true toxin, because it cannot produce an antitoxin, is thermostabile in acid solution, and is not a single specific substance, but a mixture of more or less closely related substances in the nature of protein cleavage products, as first shown by Vaughan and Wheeler, and since accepted by Friedberger himself and a number of other investigators. In a strict sense, therefore, this term is a misnomer, but it is in such general use that it need not be discarded if we have a clear understanding that the sum total of independent researches by numerous investigators shows that it is not a true toxin, as tetanus toxin, for instance, but a protein poison.

As the symptoms of anaphylaxis are always the same in the same animal, no matter what protein is used, it would appear that the protein poison is either always the same or composed of a group of very closely allied products, and, indeed, this seems to have been proved by an extended series of researches with the most diverse proteins of animal, bacterial, and vegetable origin.

It may be stated, therefore, that anaphylatoxins may be regarded as protein poisons composed of protein cleavage products, and that these are responsible for the lesions and symptoms of anaphylaxis. There is some difference of opinion regarding the source of the protein matrix and the mechanism of its cleavage with the production of the poison in anaphylaxis, and I shall consider this phase of the subject later. There is, however, a striking uniformity of experimental evidence and opinion regarding the rôle and primary importance of the protein cleavage poisons in the anaphylactic process. Briefly summarized, the evidence on this point is as follows:

1. As was just stated, the first to advance the theory regarding the rôle of protein-split products in anaphylaxis, as well as in infection and immunity in general, were Vaughan and Wheeler. In 1907 these observers showed that proteins may be split by boiling with alcoholic sodium hydroxid solution into two fractions-one non-toxic and alcohol soluble and the other toxic and alcohol insoluble. The toxic fraction, when injected into normal guinea-pigs in doses of from 8 to $100 \mathrm{mg}$.,

1 Zeitschr. f. Immunitätsf., 1910, 4, 636. 
kills the animals, death being preceded by all the symptoms of acute anaphylactic intoxication. This toxic moiety has been obtained by this method from the most diverse proteins, and seems to be the same for all, the specificity residing in the non-toxic attached group. This and other observations led Vaughan and his collaborators to formulate the hypothesis that the non-toxic and specific intramolecular group of a protein serves to stimulate the body-cells to produce specific proteolytic enzymes, and that upon the injection of a second dose of the same protein, these enzymes at once disintegrate it, setting free the toxic group that produces the lesions and symptoms of acute anaphylactic intoxication. This protein cleavage in vivo is entirely analogous to the cleavage process occurring in vitro with the alcoholic sodium hydroxid solution, and the poison in both instances is regarded as the same.

Many of the later studies in this field, especially those of Friedberger and the investigations of Abderhalden on "protective ferments," have given support to this hypothesis, so that in its fundamental principles it constitutes the most plausible and generally accepted explanation of the processes involved in anaphylaxis.

2. Mention has previously been made of the protein poison obtained by Friedmann by digesting ox corpuscles with immune and normal rabbit serum, and by Weichardt as the result of the digestion of placental protein with immune rabbit serum. In 1910 Friedberger, ${ }^{1}$ by digesting a serum precipitate with normal guinea-pig serum, obtained a similar toxic substance; by means of ferments he secured a protein cleavage poison that, when injected into normal guinea-pigs, produced the symptoms of acute anaphylaxis, the process being entirely analogous to that obtained by Vaughan and Wheeler by protein splitting with alcoholic sodium hydroxid solution. Subsequent studies by Friedberger and his collaborators ${ }^{2}$ showed that similar protein poisons could be obtained by digesting microörganisms, as, e. g., Bacillus prodigiosus, Bacillus typhosus, and Bacillus tuberculosis, with normal guinea-pig serum. Friedberger and Nathan ${ }^{3}$ obtained the poison by digesting normal horse serum with fresh guinea-pig serum; Bordet, ${ }^{4}$ by digesting agar with fresh serum; Dold and Aoki, ${ }^{5}$ by digesting meningococci, streptococci, pneumococci, gonococci, and various other microörganisms with fresh normal gunea-pig serum. As was expected, cleavage could be facili-

1 Zeitschr. f. Immunitätsf., 1910, 4, 636.

2 Zeitschr. f. Immunitätsf., 1911, 9, 369.

3 Zeitschr. f. Immunitätsf., 1911, 9, 567.

${ }^{4}$ Compt. rend de Soc. de Biol., 1913, lxxiv, No. 5.

5 Zeitschr. f. Immunitätsf., 1912, 12, 200. 
tated and hastened by using immune serum specific for any particular bacterial or other protein.

These results indicate that fresh normal guinea-pig serum contains a normal thermolabile non-specific proteolytic ferment capable of splitting some, through probably not all, proteins, a view advanced by Vaughan many years previously. After sensitization, a specific proteolytic ferment is produced for the particular protein injected, the presence of the specific in addition to the normal ferments constituting the difference between normal and sensitized animals.

Since these studies show that, when incubated. with normal serum, bacteria and certain other proteins yield a soluble and active poison, the question naturally arises why this reaction does not occur when these proteins are first injected directly into the blood? Vaughan has answered this question by assuming that the ferment is in a more available form in the serum than it is in the blood, since the ferment is probably largely a leukoprotease mainly derived from the disintegration of leukocytes. Or it may be that the cleavage is carried on in the circulating blood beyond the point of the products constituting the protein poison, or that the inclusion of the foreign protein by the phagocytes may delay the disruption of the former.

While there is this consensus of opinion regarding the rôle frnrotein poison in the production of anaphylaxis, there is some diversit of mpinion regarding the source of the protein matrix, $i$. e., the protes that is broken down, especially in view of the fact that mixtures of kaolin and normal guinea-pig serum produce the poison. It has been suggested(a) That the kaolin, agar, bacteria, etc., absorb the complement from the serum, and that this renders the serum poisonous; $(b)$ that the poison is preformed in the serum, but that its action is neutralized by some other constituent of the serum that is absorbed; $(c)$ that the absorption of some constituent of the serum leads to a breaking-up of serum proteins, with liberation of the poison. The latter view, as shown by Jobling and Petersen, ${ }^{1}$ appears to be the most plausible. These writers believe that the normal tryptic or proteolytic ferment of the blood is held in check by an antiferment of the nature of unsaturated fatty acids, and that laokin, bacteria, agar, etc., remove this antitryptic influence by absorbing the lipoidal antiferment, setting the ferment free, which then acts upon the serum protein, producing the toxic protein poison (serotoxin). While Vaughan, Friedberger, and their collaborators believe that the protein poison is derived from the injected protein,

1 Jour. Exper. Med., 1914, xix, 459 and 480. 
Jobling and Petersen believe that the matrix is, indeed, the protein of the animal's own serum. Doerr ${ }^{1}$ likewise regards it as highly improbable that the poison is generated by the bacteria or other foreign protein, but believe that it is derived from the serum through the absorption of inhibitory antibodies by the bacteria, precipitates, etc. There can be no doubt, however, of the high specificity of the anaphylactic reaction, which indicates that specific ferments are produced for the protein injected, and while the discussion cannot be considered closed, it would appear that, for the present, it would be best to accept the theory that anaphylaxis is due to the cleavage of the injected anaphylactogen by normal and specific proteolytic ferments.

3. Further evidence of the rôle played by protein cleavage products in anaphylaxis is furnished by the action of $\beta$-imidazolyethylamin ("histamin"), an amin produced by splitting off carbon dioxid from histidin by chemical or bacterial agencies. This substance, first prepared synthetically by Windam and Vogt, ${ }^{2}$ and studied by Ackermann, ${ }^{3}$ Harger and Dale, ${ }^{4}$ and especially Dale and Laidlow, ${ }^{5}$ produces, in doses of about $0.5 \mathrm{mg}$., effects resembling acute anaphylactic intoxication. Heyde ${ }^{6}$ has described similar effects with methylguanidin, and other amins may possibly be involved.

\section{ANAPHYLACTIN (ALLERGIN)}

There is a diversity of opinion concerning the nature of the substance developed in the body under the influence of the anaphylactogen, which is capable of cleaving the latter and producing the anaphylactic poison. That such a body exists in the blood of the sensitized animal is shown by the production of passive anaphylaxis in normal animals by injecting into them a few cubic centimeters of blood or serum from a sensitized animal. The animal so sensitized becomes at once or within a few hours sensitive to the specific anaphylactogen, regardless of the species of animal furnishing the immune serum.

Terminology.-Various names have been applied to this body. Vaughan objects to the term "antibody," although this designation would seem to be appropriate if we consider that it is a reactionary product or antibody to the protein responsible for its production, al-

${ }^{1}$ Handb. d. path. Mikroörgan., Kolle and Wassermann, second edition, ii, 947.

2 Berichte, 1907, xl, 3691.

3 Zeitschr.f. physiol.Chem., 1910, xlv, 504.

${ }^{4}$ Proc. Chem. Soc., 1910, xxvi, 128. $\quad 5$ Jour. Physiol., 1911, lxi, 318.

6 Cent. f. Physiolog., 1911, 25, 441; 1912, 26, 401. 
though, instead of being protective to the animal, its host, it is just the reverse. Richet named it toxogen, because it is responsible for the production of a poison. Otto speaks of it as "reaction body," Nicolle as an albuminolysin, Besredka as sensibilisin, and von Pirquet as allergin.

Nature of Anaphylactin.-A full discussion of the nature of this body, which is believed to cleave the anaphylactogen and set free the anaphylatoxin or protein poison, would involve a review of the entire subject of antibodies and immunity in general. Vaughan regards it as a ferment, evidently meaning that an amboceptor and a complement act together and produce lysis or disruption of the protein molecule. It is difficult, and indeed impossible, at this time to discuss with any degree of definiteness the propriety of regarding an immune and cytolytic amboceptor as a ferment, just as we regard trypsin as a ferment. If the ferments are to be considered as identical with amboceptors, then we must regard Abderhalden's protective ferments as cytolysins, but certainly, in the light of our present knowledge, we are hardly justified in drawing hard and fast lines. Therefore while I have confined the discussion of the protective ferments to a separate chapter (Chapter XV), and have not considered it under the general head of the cytolysins, it is to be remembered that the ferments possess many of the properties of lytic amboceptors, and they may be identical with them although apparently different owing to the application of chemical methods, especially by Abderhalden, in their study.

Most observers regard the anaphylactic antibody, toxogen or allergin, as an amboceptor and a complement. The actual antibody then must be considered as an immune albuminolysin, for complement is present in normal serum, and is not necessarily increased during the process of sensitization. As in other lytic processes, however, complement or alexin is of great importance, constituting, as it does, the actual lytic agent, after the antigen, or, in this instance, the anaphylactogen of the second injection, has been sensitized by the amboceptor. Some observers believe that the complement is decreased during anaphylaxis, presumably being used up in effecting lysis of the protein. Friedmann claims that in allergy to red corpuscles there is a close parallelism between the anaphylactic bodies and the hemolytic amboceptor.

With the more recent work of Abderhalden on protective ferments and the development of a dialysis and optical method of detecting the products of protein cleavage, it was hoped that the true and exact nature of anaphylaxis would finally be established. It would appear possible to determine the presence, in the blood-serum of sensitized animals, of specific proteolytic ferments capable of demonstrating their presence 
in vitro, and to show the products of protein cleavage just after anaphylactic shock and a corresponding decrease or total absence of the ferments as a result of their participation in the albuminolysis. Indeed, Abderhalden ${ }^{1}$ has recently claimed that all these conditions have been found to exist: (1) The serums from 12 guinea-pigs sensitized to eggalbumen, when mixed with antigen, showed digestive power by both optic and dialysis (biuret) methods; (2) similar serums, dialyzed alone, showed digestive products in only one of six serums tested: (3) the serum of six guinea-pigs taken at intervals of from five minutes, to one and onehalf hours after the second injection (egg-albumen) and dialyzed, gave negative results after from five and fifteen minutes, whereas four taken after thirty, forty-five, sixty, and ninety minutes respectively were positive. In each test the serum (10 c.c.) was dialyzed against distilled water for sixteen hours at $37^{\circ} \mathrm{C}$., and the presence of the products of digestion determined by the biuret reaction. In this manner the final evidence of the rôle played by the protein split products in the production of acute anaphylaxis has apparently been furnished. These results, however, cannot at the present time be regarded as final. Pearce and Williams, ${ }^{2}$ in a similar study with horse serum, employing the dialysis technic and using ninhydrin as the indicator, were unable to demonstrate the presence of the ferments after sensitization, although by using large amounts of serum secured after anaphylactic shock had occurred they observed positive reactions, which may have been due, in part at least, to the presence of cleavage products. Zunz ${ }^{3}$ found that the proteinsplitting properties of blood-serum, as tested on the sensitizing protein, increased in the anaphylactic state from the fifth to the twentieth and sixtieth day, and were not recognizable in blood-serum taken during or soon after anaphylactic shock occurred. Pfeiffer and Mita and Vaughan have observed the apparent disappearance of the ferment from the blood, even though the animal is sensitized, and the last-named observer explains this on the assumption that the ferment comes from the fixed cells, which are stimulated to elaborate the ferment only when the specific protein is brought into contact with them.

One of the older theories of anaphylaxis regards the process as a precipitin reaction. It is apparent that, in the serum of an animal immunized with a soluble protein, such as a serum, a precipitin and complement-fixing body, presumably an albuminolysin or so-called "ferment," are produced, and exist together in the immune serum. While

1 Zeitschr.f. physiol. Chem., 1912, 82, $109 . \quad 2$ Jour. Infect. Dis., 1914, 14, 351. ${ }^{3}$ Zeitschr. f. Immunitätsf., 1913, 17, 241. 
the rôle of precipitins themselves appear to have been excluded as directly participating in the production of anaphylactic shock, recent experiments of Zinsser ${ }^{1}$ and others would tend to show that a precipitin possesses the nature of a protein sensitizer or antibody that sensitizes its antigen, just as a hemolytic amboceptor sensitizes its corpuscles, precipitation being a secondary phenomenon due to the colloidal nature of the reacting bodies under conditions of quantitative proportions and environment that favor precipitation. This would assign to the precipitins and agglutinins an active though secondary rôle in the processes of anaphylaxis and immunity.

At the present time, therefore, we may tentatively assume that the anaphylactin or allergin is of the nature of a specific lytic amboceptor or albuminolysin, which, in conjunction with non-specific complement, constitutes what is called a "ferment," and is capable of splitting a protein molecule with the liberation or formation of a toxic moiety responsible for the lesions and symptoms of anaphylaxis. Just as other normal amboceptors, such as hemolysins, are present in normal serum, so these protein amboceptors or albuminolysins may be present for various proteins, explaining the production of the anaphylaxis poison in vitro with a normal serum when the latter is fresh and active, $i$. e., when complement is present.

Theoretically, at least, it should be possible to detect the anaphylactic amboceptor in a serum by a method of complement fixation, although practically this is not the case. The whole subject of "ferments" requires further study, and, as a result, our knowledge and views of antibodies and the processes of immunity in general are likely to undergo some change.

The Cellular Theory of Anaphylaxis.-An interesting question in this connection is whether the anaphylactic reaction is humoral or occurs in the blood-stream, $i$. e., between the free anaphylactin or antibody and the antigen, or whether it is cellular and occurs between the attached or sessile antibodies and the antigen. Friedberger and Girgolaff ${ }^{2}$ now support the cellular theory, basing their belief on the results of their experiments, which consisted of thoroughly washing the organs of a sensitized animal with salt solution, and then transplanting them to a normal animal, when the latter became sensitized. The recent and brilliant studies of Dale, ${ }^{3}$ whose investigations were carried out by the

1 Jour. Exp. Medicine, 1913, 18, 219.

2 Zeitschr. f. Immunitätsf., 1911, 9, 575.

3 Jour. of Pharm. and Exper. Therap., 1913, 4, 167. 
graphic method, employing the excised uteri of sensitized pigs and applying the antigen direct, decided the question in favor of the cellular theory. In a similar study Weil ${ }^{1}$ reached the same conclusions, namely, that the anaphylactic condition is entirely dependent upon the sensitization of the cells of the body; that all conditions that in any way influence the degree of sensitiveness of the cells in the same degree alter the anaphylactic state, or sensitiveness, of the animal. It does not seem possible, however, entirely to exclude the agency of the antibodies in the blood, as Weil apparently does, or we would be at a loss to explain the mechanism of passive anaphylaxis. It would appear that both the attached and the free antibodies, particularly the former, contribute toward the production of the anaphylactic response.

\section{THEORIES OF ANAPHYLAXIS}

With the foregoing explanation of the most widely accepted theory of anaphylaxis, I may briefly summarize all the more important theories advanced from time to time in explanation of the process. These include the following.

1. Richet held that the sensitizer, or anaphylactogen, contains a substance which he called "congestion" (because he did his original work with extracts of the tentacles of sea anemones, which are toxic and produce congestion of the internal organs), and that this generates in the animal another substance, known as the "toxogenin." The reaction between the latter and the homologous protein on reinjection sets free a poison, "apotoxin," which, because of its effect on the nervous system, produces the symptoms of anaphylaxis. This theory is practically the same as that generally accepted to-day, except that the antigen is not of necessity primarily toxic for the animal.

2. Hamburger and Moro suggest that the first injection leads to the formation of precipitins, and that on reinjection precipitates are formed; these they contend, may, by the formation of capillary emboli, produce acute anaphylaxis, or at least that precipitin formation runs parallel with the antibody formation. The symptoms of anaphylaxis, however, are not those of embolism, and there is no evidence to show that precipitation occurs in vivo, although, as Zinsser points out, precipitins may play the rôle of sensitizers of the antigen, preparing them for final lysis or cleavage by a complement. In other words, the precipitin would act as an amboceptor, differing, however, from our general conception of ${ }^{1}$ Jour. Med. Research, 1914, 30, No. $2,87$. 
the nature of amboceptors by being active in the absence of complement, unless precipitation is a secondary physical phenomenon in the nature of a colloidal reaction.

3. Besredka taught that the sensitizer contains two substances"sensibilisinogen" and "antisensibilisin." When injected the first time, the former develops in the body a substance called "sensibilisin," and on reinjection the sensibilisin and antisensibilisin continue to form a poison that acts on the nervous system.

4. Gay and Southard believe that, as a result of the first injection of serum, there remains in the circulation a protein substance called "anaphylactin," which is slowly absorbed and continues to stimulate the cells, leading to an abnormal affinity for the homologous protein, which, on reinjection, leads to anaphylactic shock.

5. Vaughan and Wheeler are of the opinion that, with the parenteral introduction of a foreign protein, the body-cells are stimulated to produce a specific zymogen or ferment that digests it. The protein of the first injection is so slowly digested that the effects are not recognizable. After the protein of the first injection has been disposed of, the new ferment continues to be formed in the cells, and on the second injection, after the proper interval has been allowed to elapse, this zymogen is activated and splits up the protein, which promptly and abundantly results in the production of the symptoms of anaphylactic shock. Vaughan believes that there is a non-specific poisonous group or moiety in each protein molecule which, when liberated by the ferment, is responsible for anaphylaxis. This poisonous group is held as being the same in all proteins, and hence the similarity of lesions and symptoms of anaphylactic intoxication in animals regardless of whether the protein is of animal, vegetable, or bacterial origin. The nature of the ferment is not clear. In 1907 they regarded it as a zymogen - a theoretic labile chemical body resulting from intramolecular rearrangement in the protein molecules of the cell. Little is known of the action of these ferments except that in some manner they cause cleavage of the protein molecule and liberation of the toxic moiety. Later, Vaughan speaks of the ferment as consisting of an amboceptor and a complement, the ferment (presumably the complement portion) being inactivated by a temperature of $56^{\circ} \mathrm{C}$. and reactivated on the addition of serum and organic extracts. Although Vaughan's theory best explains the nature and source of the anaphylactic poison, that of Friedberger explains the production of the "ferment," or rather the protein sensitizer (amboceptor), which, with 
a complement, digests the protein and sets free or produces the protein poison.

6. Friedberger has attempted to explain anaphylaxis on the basis of Ehrlich's side-chain theory of the action of antigens and the production of antibodies similar to toxin-antitoxin immunity. This theory assumes that, on the first injection, the protein finds but few groups of cellular receptors with which it can combine, and for this reason it is not poisonous. During the period of incubation the animal cells develop receptors specific for the homologous protein; with a single small dose of protein most of these receptors remain attached to the cell (sessile); on repeated injections, the newly formed receptors are in large part cast off into the blood, and constitute the precipitins. In this manner an animal relatively insusceptible to a foreign protein is rendered highly susceptible, and on the second injection the protein is anchored firmly to the cell, just as the cells of an animal may anchor diphtheria toxin. One of the essential features of this theory is that it assumes that, ordinarily, the receptors are not preformed in sufficient numbers to anchor enough protein to injure the animal with the first injection, regardless of the size of the dose. On the other hand, tetanus and diphtheria toxins find large numbers of sessile or cellular receptors, and are highly toxic on the first injection. As originally evolved, the theory did not explain the nature of the toxic agent responsible for the lesions and symptoms of anaphylaxis, and made no mention of the protein poison. Nevertheless it affords the best explanation we have on the formation of the "ferment" or protein sensitizer (amboceptor). At one time Friedberger believed that anaphylaxis could be explained on the basis of a precipitin reaction. Anti-anaphylaxis was explained on the assumption that the protein of the reinjection uses up the sessile receptors already developed, and, accordingly, not enough are present at the end of the period of incubation to produce anaphylactic shock. Passive anaphylaxis was explained on the ground that the free receptors in the blood of a sensitized animal become, on injection into a fresh animal, anchored to the cells, thus forming fixed or sessile receptors that anchor the protein on reinjection and lead to anaphylaxis.

Nolf ${ }^{1}$ has proposed a theory of anaphylaxis that has come to be known as the "physical theory." It assumes that the active constituent of proteins is a thromboplastic substance that disturbs the colloidal equilibrium of the blood and leads to the deposition, on the surface of the leukocytes and the endothelial cells of capillaries, of a delicate film

1 Quoted by Vaughan, "Protein Split Products," 1913, 340. 
of fibrin. Thus stimulated, the cells pour out an unusual amount of antithrombin. On account of the consumption of a part of the fibrinogen and the increased formation of antithrombin the blood fails to coagulate after anaphylactic shock or peptone poisoning. Owing to the coagulation deposits on the endothelial cells, the viscidity is increased and the leukocytes adhere to the vessel-walls, thus accounting for the leukopenia observed after protein injection. The endothelial cells are injured, and the walls of the capillaries become more readily permeable, thus accounting for the local edema often seen in anaphylaxis. The fine capillaries of a given area may be occluded by thrombi, thus explaining the necrosis characteristic of the Arthus phenomenon. The irritation of the endothelial cells extends to the smooth muscle, leading to vasoparalysis and the characteristic fall in blood-pressure. The affinity of the endothelial cells for the protein is stimulated by the first injection, and acts in a fulminating way on reinjection, thus explaining the suddenness of anaphylactic shock.

The theory, therefore, also assumes the formation of a ferment that acts primarily upon the proteins of the blood, leading to the formation of fibrin, which, as it were, mechanically induces the lesions and symptoms of anaphylaxis. While it offers a plausible explanation, the theory is not well supported, and at best may be regarded as a modification of Vaughan's theory, demonstrating one way in which the protein poison may act.

\section{PASSIVE ANAPHYLAXIS}

Passive anaphylaxis is produced by the injection of normal animals with the blood or serum of animals or persons already sensitized. It is similar to passive immunization, and is specific for the anaphylactogen with which the donor is sensitized.

The second animal may be of the same or of another species. If it is of the same species, the condition induced by the transference of the serum is called homologous; if it is of a different species, it is known as heterologous, or passive anaphylaxis.

Passive anaphylaxis was discovered almost simultaneously and independently by Gay and Southard, ${ }^{1}$ working with guinea-pigs, by Nicolle, ${ }^{2}$ with rabbits, and by Otto, ${ }^{3}$ who, working with both guinea-pigs and rabbits, showed that a rabbit immune serum would passively sensitize a guinea-pig.

${ }^{1}$ Jour. Med. Research, 1907, xvi., $143 . \quad$ 2 Ann. de l'Inst. Pasteur, 1907, xxi, 128. ${ }^{3}$ Münch. med. Wochenschr., 1907, No. 39. 
At this place it may be mentioned that the new-born of a sensitized mother animal may be sensitive, and remain so for a longer or shorter period of time. This is an illustration of passive homologous anaphylaxis, a process that has been especially studied by Rosenau and Anderson, Gay and Southard, and Otto, the last observer finding that young guineapigs remained sensitive for as long as forty-five days after birth. This function of transmitting the condition of sensitization is solely maternal: the male takes no part whatever in the transmission of these acquired properties.

The Production of Passive Anaphylaxis.-An important phase of this subject over which there has been considerable difference of opinion refers to the question whether some time must elapse between the injection of immune serum and anaphylactogen before anaphylaxis is produced, or whether intoxication may follow the simultaneous injection of both antibody and antigen. Thus Gay and Southard, in their early studies, found their recipients first sensitized on the fourteenth day after injection of immune serum. Otto and Friedmann observed shock twenty-four hours after injecting the anaphylactic serum subcutaneously and antigen intraperitoneally. By injecting both serums intravenously and simultaneously, Doerr and Russ finally succeeded in producing acute anaphylaxis and almost immediate death. Weil, ${ }^{1}$ however, believes that the simultaneous injection of antigen and of antiserum into opposite jugular veins in the guinea-pig never produces an anaphylactic reaction, in spite of the use of wide quantitative variations in both substances. On the other hand, if the antigen were injected a few hours after the antiserum, in the same quantitative variations, the reaction occurred regularly. From this it was concluded that the body-cells anchor the antibody during the latent period, and that anaphylaxis is the result of an interaction between the cellular antibodies and the antigen.

It would appear that passive anaphylaxis is not wholly determined by the amounts of antigen or antibody, but by the proportion that exists between the two. For example, Friedmann, ${ }^{2}$ in his studies on passive homologous anaphylaxis in rabbits, found that, by employing 2.5 c.c. of antiserum with from 2.5 to 0.25 c.c. of antigen, no results were observed, whereas positive reactions were obtained when the amount of antigen was reduced from 0.025 to 0.0025 c.c.

Ordinarily, normal guinea-pigs may be passively sensitized by 0.1 to 0.5 c.c. of serum injected intraperitoneally, and anaphylactized one or

1 Jour. Med. Research, 1914, 30, No. 2, 87.

2 Jahr. u. d. Ergeb. d. Immunitätsf., 1910, vi, 67. 
two days later by an intravenous injection ( 0.1 to 0.5 c.c.) of the antigen. The immune serum may be prepared by injecting rabbits with horse serum, after the methods for the production of precipitins described in Chapter IV.

The duration of passive sensitization is quite variable. Weil ${ }^{1}$ found that guinea-pigs sensitized with a homologous serum, that is, with the serum of another guinea-pig sensitized with horse serum, remain typically anaphylactic as long as seventy days after the injection. With heterologous sensitization, however, as with the serum of a sensitized rabbit, hypersensitiveness is almost invariably lost by the tenth day. One explanation of this would be that a heterologous serum is excreted more rapidly than a homologous serum, a condition commonly observed in serum therapy with any heterologous serum.

The Mechanism of Passive Anaphylaxis.-Presumably the mechanism of passive anaphylaxis is relatively simple, and consists in the transfer of the specific protein sensitizer or amboceptor that unites the anaphylactogen or protein antigen with a complement, bringing about lysis or cleavage of the protein and liberation of the toxic moiety responsible for the lesions and symptoms of anaphylaxis. In other words, the mechanism of passive anaphylaxis may be likened to passive antibacterial immunization, with, however, one important clinical difference, namely, that whereas in the former the microörganisms are destroyed without apparent injury to the host, in anaphylaxis the body-cells are acutely poisoned. However, a similar phenomenon in the serum treatment of disease, with lysis of bacteria, may be overshadowed or possibly prevented by a condition of anti-anaphylaxis.

\section{ANTI-ANAPHYLAXIS}

The term anti-anaphylaxis was first applied by Besredka and Steinhardt to a condition of insensibility to further injection of the anaphylactogen that may follow recovery from anaphylaxis, or be induced artificially by a single or by repeated small injections of the anaphylactogen during the incubation period following the first injection, and before sensitization is completed. The state is usually only temporary, the animal gradually becomes sensitive again after three weeks.

Theobald Smith had observed that those guinea-pigs that had received the largest dose of diphtheria toxin-antitoxin mixture more frequently survived the second dose than did those that received smaller

1 Jour. Med. Research, 1913, 28, No. 2, 359. 
doses. Rosenau and Anderson found that animals reinjected before the end of the period of incubation did not become responsive until some time later. Otto has also made this observation, but the most thorough study of the subject has come as the result of the researches of Besredka and Steinhardt.

Experimental Production of Anti-anaphylaxis.-It has long been known that the larger the first or sensitizing injection of antigen, the greater must be the dose of the second or intoxicating injection, indicating that a large sensitizing injection introduces the factor tending to produce the condition of anti-anaphylaxis. Partial desensitization, or anti-anaphylaxis, may be produced by the injection of a sublethal intoxicating dose of anaphylactogen during the period of incubation or at its close. Quantitative relations between the size of the sensitizing, intoxicating, and desensitizing doses of antigen and the period of incubation have been worked out in a series of studies by Weil, ${ }^{1}$ both in the living guinea-pig and on the excised uterus, after the graphic method of Dale. ${ }^{2}$ Weil found that a small sensitizing dose of horse serum (0.01 c.c. subcutaneously) is followed by a relatively prolonged period of incubation (from fourteen to sixteen days); that the minimal anaphylactic or lethal dose is small ( 0.02 to 0.05 c.c.); that the blood, as a rule, does not contain more than one sensitizing unit; and that the minimal desensitizing dose is small (0.01 c.c.). Conversely, after repeated large sensitizing doses ( 2 c.c. of serum subcutaneously on each of three days in succession) the incubation period is shorter (about ten days after the last injection); the minimal anaphylactic lethal dose is larger ( 0.4 c.c.), and the minimal desensitizing dose is at least more than 0.2 c.c. of serum given intravenously.

Besredka and Steinhardt observed that the refractory state could easily be developed in sensitized guinea-pigs by one of the following methods: (1) The intracerebral injection of 0.25 c.c. of horse serum before the expiration of the period of incubation (twelve days). (2) The intracerebral injection of less than the fatal dose (from 0.002 to 0.025 c.c.) after the period of incubation. (3) Rectal injections of from 5 to 10 c.c. of serum. (4) By slowly reinjecting small amounts while the animal is deeply narcotized with ether or alcohol. As shown later by Rosenau and Anderson, a narcotic may mask but does not prevent the occurrence of severe or fatal symptoms. Of these methods, Besredka

1 Jour. Med. Research, 1913, 29, No. 2, 233; 1914, 30, No. 3, 299.

2 Jour. Pharm. and Exper. Ther., 1913, iv, 167. 
prefers the rectal injection, or, better still, the subcutaneous injection of less than a fatal dose.

The subject of anti-anaphylaxis is of great importance from its relation to serum therapy. No satisfactory method for producing this state in a sensitized person has as yet been devised, owing, probably, to the important quantitative factors shown, by the studies of Weil, to exist. This subject will be discussed again in the following chapter, under the head of Serum Sickness.

The Mechanism of Anti-anaphylaxis.-A true explanation of this phenomenon cannot as yet be given. In the first place, the term antianaphylaxis cannot be considered a proper one, as the animal is not entirely and permanently anti-anaphylactic, but subsequently becomes sensitive. The blood-serum of a refractory or anti-anaphylactic animal does not confer a similar condition on a second sensitized animal.

As previously stated, Friedberger believes that the refractory state is due to neutralization or absorption of the anaphylactic antibody by the antigen, but this explanation does not fit in with the facts, first, because the serum of an anti-anaphylactic animal will still passively sensitize a normal animal, and, secondly, as shown by Weil, passive anaphylaxis of a guinea-pig, such as that induced by the injection of a rabbit antihorse serum, may be prevented for at least eight days by a previous injection of normal rabbit or sheep serum. In other words, it would appear that normal rabbit and sheep serum may protect the body-cells of the guinea-pig against the anaphylatoxin produced by horse protein and horse anaphylactin or antibody. In explanation of this paradoxic and non-specific reaction Weil, ${ }^{1}$ who believes in the cellular theory of anaphylaxis, has tentatively advanced the hypothesis that the indifferent serum persists in the body-cells and markedly lowers the reactivity of the cellular antibodies.

\section{SPECIFICITY OF ANAPHYLAXIS}

The anaphylactin, or so-called anaphylaxis antibody, displays quite the same characteristics of specificity as do the other immune antibodies, in that proteins of closely related species tend to interact, whereas proteins of very distinct biologic or chemical nature are easily distinguished. In other words, the anaphylactic reaction is highly specific, and of considerable value in the study and identification of different proteins. For example, Dale has recommended the use of the graphic method in

${ }^{1}$ Jour. Med. Research, 1914, 30, No. 3, 299. 
vitro with excised muscle (uterus) for the identification of the protein substances, such as blood-stains. Guinea-pigs sensitized with human serum will react best with human serum, and to a lesser extent with that of the higher apes, but not at all with the serum of the dog, ox, or fowl. Wells and Osborne, ${ }^{1}$ working with purified vegetable protein, were able to demonstrate that a single isolated protein (hordein or gliadin) may contain more than one antigenic radical, and that not the whole protein molecule, but certain groups thereof, determine the specificity. Wells ${ }^{2}$ was able to distinguish in the hen's egg five distinctly different antigens, and these corresponded to five proteins that were isolated by chemical methods, so that it would appear that the specificity of the anaphylaxis reaction is determined by the chemical structure of the reacting proteins, rather than by their biologic origin. Whether the chemical differences that determine specificity are of quantitative nature, or whether they are sometimes dependent upon the number and relationship of the amino-radicals, as was suggested by Pick, remains to be determined.

1 Jour. Infect. Dis., 1913, 12, 341.

2 Jour. Infect. Dis., 1911, 9, 147. 


\section{PART IV \\ CHAPTER XXVIII \\ ANAPHYLAXIS IN ITS RELATION TO INFECTION AND IMMUNITY}

IN reviewing our knowledge of the nature and mechanism of anaphylaxis we found that ordinarily innocent substances, such as sterile normal serum and egg-albumen, when injected into animals, may give rise to severe and even fatal intoxication, not because these substances are poisonous in themselves, but because the antibodies which they stimulate the body-cells to produce react upon the innocent protein, causing its cleavage, with the liberation of a harmful poison. Here, indeed, we have an example of antibodies apparently injuring our body-cells instead of protecting them. And now the question very naturally arises, are the lesions of the many diseases caused in a similar manner, the antibodies splitting the bacterial cell and liberating the protein poison? In view of the fact they may do actual harm, in some instances at least, are antibodies really protective and beneficial, and if so, in what manner?

It is not my purpose to review here the general subjects of infection and immunity, but to discuss briefly the intimate and inseparable connection of what we call anaphylaxis or allergy with infection, and to show that anaphylaxis is really the first step in the process of immunity; indeed, that well-marked antibacterial immunity is an example of an early and efficient "anaphylactic" reaction. In other words, while the striking and severe symptoms of serum anaphylaxis in either man or lower animals give us the impression that we are here dealing with some new, distinct, and strange phenomenon, these are, in fact, but exaggerated and severe examples, largely dependent upon quantitative factors of what occurs during each infection. More than this, these symptoms are due in part to the action of a poison formed or released through the destruction of the antigen by the antibody; the antibody being, therefore, apparently imperfect in its action, as it does not neutralize the protein poison. Taking, as an example, a substance, such as sterile horse serum; while ordinarily harmless itself, bad effects may follow its injection at once if antibodies are present in our body-fluids, or later if some of the serum persists in the body until antibodies are 
produced. It would appear, therefore, that when the antibodies are produced for a given infectious agent, then the severity of the disease becomes apparent; that the lesions and symptoms are due not only to the infecting microörganism and its products, but to the results of their destruction. An invading army may do some pillaging, but the greatest injury is done when the defenders begin the attack, the resulting fire and destruction doing more harm than the invaders themselves.

While Vaughan and his coworkers were studying the protein poison in vitro, and Friedberger was investigating it in vivo, the former and then the latter aiming to show that the poison liberated from the protein molecule through the action of specific ferments is responsible for the lesions and symptoms of disease, von Pirquet was studying the question from the clinical aspect, formulating a working hypothesis on the nature of infection and immunity based upon the principles of anaphylaxis, a theory that has been supported by experimental data and has thrown a new light upon the nature and mechanism of these processes.

\section{RELATION OF ANAPHYLAXIS TO INFECTIOUS DISEASES}

Although we may not be in general accord regarding the mechanism of anaphylaxis, there is, however, general agreement as regards the nature of the anaphylatoxin responsible for the lesions and symptoms of anaphylactic intoxication, namely, that it is a protein cleavage product. In other words, a foreign protein and, indeed, the misplaced protein of our own body-cells, may be disrupted or digested by an antibody, and liberate or generate a poison that, being derived from protein, is known as the protein poison. In the chapter on Infection it was stated that Vaughan and his collaborators regard this protein poison as the same for all proteins, and as responsible for all infectious diseases, the particular lesions and symptoms of each disease being dependent upon the site of the infection and, accordingly, upon the location of the protein poison. Similarly, in the preceding chapter we asserted that anaphylaxis is ascribed to an exactly similar phenomenon, namely, the splitting of the foreign protein by an antibody (ferment) and the liberation of a protein poison. In studying serum sickness, an anaphylactic phenomenon frequently observed in man following the administration of horse serum, von Pirquet argued that the period of from eight to ten days usually following the injection before the appearance of symptoms was the time required for the production of the antibody, which then reacted upon the serum still remaining in the body-cells and fluids, and 
that the products of this interaction caused the lesions and symptoms of serum sickness. It was then but a short step to apply these principles to other infectious diseases. This "period of incubation" was formerly regarded as representing a stage during which the infecting microörganisms multiply in the body of the infected individual, to that point at which they could give rise to symptoms of disease through the agency of their toxins or through interference with the metabolism of the host in other ways. But, as von Pirquet has pointed out, this theory does not hold in serum sickness, as the serum may be sterile and no infecting microörganisms are at work. Instead, he and Vaughan would have us believe that during this period antibody formation is taking place, and that an antibody-antigen reaction will occur with the development of pathologic changes and symptoms just as soon as these changes have progressed to a certain point. The period of incubation will vary not only in point of time of reaction, but also qualitatively and quantitatively, and using this as a basis von Pirquet recognizes three main groups, depending upon whether the antibody is present in our body-fluids as the result of a previously acquired infection (accidental or by vaccination), or whether it must first be developed.

Group I: Reaction appears after eight to twelve days, as in measles, smallpox, whooping-cough, chickenpox, and other infectious diseases in which the antibodies must be developed before the symptoms are produced. This interval corresponds quite closely to that observed in serum sickness. If at this time the antigen, - $i$. e., either the albumins of the horse serum, if we are dealing with serum injections, or the bacteria in case of an infection-has disappeared from the body, no symptom will, of course, result; if, however, some of the material is still present, a reaction occurs, during which the protein poison (anaphylatoxin) is produced, and to which, in turn, the symptoms that then develop may logically be attributed.

Group II: The reaction appears after three to seven days. If, on the other hand, the secondary infection, as, e.g., pneumonia, erysipelas, etc., is acquired after a lapse of months or several years, or if the second injection of serum is given after this time, $i$. e., at a time when the antibodies called forth by the primary infection or first injection have disappeared, a certain interval of time will elapse before symptoms of sickness develop, as in the case of the first group. This interval, however, instead of being from eight to twelve days, is now from three to seven, a fact readily explained on the basis that a cell that has once been stimulated to active antibody formation will subsequently respond to 
the same stimulus with increased activity. This has been called by von Pirquet the accelerated reaction.

Group III: The reaction appears immediately. If the first injection of horse serum or infection is followed by actual disease or vaccination, the reinjection or reinfection is acquired at a time when the antibodies are present in the circulation in considerable amount, a reaction will occur either immediately or within the first twenty-four hours. This reaction may be quite virulent in intensity, although it is shorter in duration than when it occurs in the first group. von Pirquet speaks of this as the immediate reaction. It is to be observed in cases of serum sickness where the symptoms develop almost immediately following an injection of serum months and even years after a previous injection; it also occurs in cowpox vaccination, where a local reaction takes place very quickly and soon disappears after a previous attack of smallpox or vaccination.

If the antibodies are present in lesser amounts, the reaction may occur in from the second to the fourth day; this is called the torpid early reaction.

At the time of the second injection of serum or reinfection with bacteria a small amount of antibody may still be present; this will give an immediate though mild reaction, and is not enough to neutralize the total amount of foreign protein introduced. A portion of the latter, therefore, will result in the production of an additional amount of antibody, which occurs in an accelerated manner, and coming in contact with some of the free antigen, gives rise to the accelerated reaction. Hence we may have an immediate, followed by an accelerated, reaction.

To illustrate these principles, von Pirquet names vaccinia as an example of an acute infection in which the processes may be observed on the skin. As the result of vaccination a colony of microörganisms is formed on the skin. For the first two days the local response is evidently traumatic in character. After the third or fourth day the specific reaction sets in, in the form of a small papular elevation surrounded by a small areola due to the local action of toxins or protein poison from disintegrated microörganisms. By the eighth day a vesicle has formed, and from its contents new colonies can be grown on thousands of other arms. But one or two days later the ferment-like antibody appears. The colony is attacked, its contents are digested, a toxic substance is formed that diffuses into the neighboring tissues, and the intense local inflammation which we call the areola appears. In addition the toxin enters the general circulation and fever sets in. 
Simultaneously the microörganisms are destroyed, and we may no longer be able to vaccinate with the contents of the now yellow pustule. After two or three days the real struggle is ended, although the local lesion may be aggravated by secondary infection, and the body contains the new antibody for a long time.

If we now revaccinate, the antibody present will at once attack and digest the microörganisms introduced into the scarification, and, as these do not have time to multiply, only an extremely small amount of toxin is formed, which gives the "immediate or early reaction" in vaccinia. If a number of years have elapsed between the first and the second vaccination, antibodies may be absent or present in only small amount, but the body-cells have been "keyed up" by the first vaccination, and hence react more quickly to the second. The antibodies are produced in from three to five days, and attack the microörganisms before they have had time to multiply in sufficient numbers; the relatively small amount of digestion product produces a comparatively mild local inflammation and practically no general symptoms. This is sometimes known as the "immunity reaction," or vaccinoid, and is illustrated in Fig. 131.

It would, of course, lead us too far were we to analyze all the different infectious diseases along these lines; suffice it to say that the anaphylactic principle serves to explain many points in the clinical symptomatology of disease that were not explained heretofore, or were ascribed to the effects of the bacteria and the bacterial products. I am not among those who, with Vaughan, would ascribe all symptoms to the protein poison alone; nor do I regard the part played by the microörganism as simply dependent upon whether or not it can grow in the body and produce the ferment antibody. In the acute toxemias, for instance, such as tetanus and diphtheria, where the amount and toxicity of the toxin are out of all proportion to the number of bacteria, and where the symptoms develop after a very brief incubation period, tissue changes and symptoms are in all probability not primarily due to any antigenantibody reaction, with liberation of the protein poison, but are rather to be attributed to a direct action of the toxin upon the body-cells, especially upon those for which the toxin possesses a special affinity. When the antibodies appear, we may rightfully assume that a ferment, in the nature of a protein amboceptor or lysin, appears with the antitoxin, and theoretically we may expect a clinical reaction due to the protein poison or anaphylatoxin liberated from the bacilli. It may be that diphtheria antitoxin is in the nature of this ferment, or antitoxin and 
ferment may exist together and in this manner afford an explanation for the local destruction of the bacilli and the cleaning up of the membrane, the effects of the anaphylatoxin being overshadowed by the true toxin, or expressing itself in the paralyses and other symptoms commonly ascribed to the toxones. von Pirquet concedes that in scarlatina the primary symptoms of the malady, $i$.e., the eruption and angina, are due to a pure toxin effect,- - to the true scarlatinal virus,- - but that the sequelæ, and notably the nephritis, are the expression of the action of anaphylatoxins that are formed when the corresponding antibodies are produced.

Likewise in such infections as typhoid fever and pneumonia, I cannot eliminate the action of true toxins and endotoxins, and ascribe all the symptoms to the protein poison or anaphylatoxin. It is not always true that the incubation period is devoid of symptoms. Mild and evanescent symptoms are frequently present, and it is but natural to ascribe these to the effects of bacterial products themselves. After a time, when the bacteria have multiplied and reached special tissues, the symptoms become more intense and the typical lesions are produced. These may be ascribed to the result of the combined action of toxins themselves and the protein poison, rather than to the protein poison itself to the entire exclusion of the metabolic products of the bacteria. In the present state of our knowledge it is, of course, very difficult to decide which symptoms in a given disease are due to bacterial toxins, which to endotoxins, which to ptomains, which to a mechanical action of the bacteria, and which to the protein poison or anaphylatoxin. While it is perfectly true that we can now understand symptoms as due to protein poison that cannot be ascribed entirely to toxins and endotoxins, it seems to me unwise to ascribe all lesions and symptoms to the toxins, on one hand, or the protein poison, on the other. It is necessary for us to know the manner in which the protein poison is produced, how infection is so closely allied to what we know as anaphylaxis, and that in any one disease, such as diphtheria, tetanus, and pneumonia, the toxins and endotoxins are of primary and immediate importance, whereas in others, such as smallpox, chickenpox, and whooping-cough the protein poison itself is of primary importance and that in all disease all factors may be concerned to a more or less degree.

\section{RELATION OF ANAPHYLAXIS TO NON-INFECTIOUS DISEASES}

When we come to consider non-infectious diseases,- and by this I refer particularly to the symptom-complex of serum disease and those 
conditions commonly ascribed to idiosyncrasies toward certain substances, such as horse asthma, satinwood dermatitis, buckwheat poisoning, urticarias due to the ingestion of strawberries, pork, and the like,the relation of anaphylaxis to the processes involved is more intimate. Here, indeed, we may regard the symptoms as entirely anaphylactic in origin and character, as the substances in themselves, such as serum, egg-albumen, horse effluvia, etc., are not regarded as toxic and we have not the coincident effects of toxins, endotoxins, and ptomains, as in bacterial infections.

Here, indeed, we may accept Vaughan's and Friedberger's theory as to the action of the protein poison in its entirety, regarding it the same in all proteins, and set free from the protein molecule by the so-called "ferment," which is in the nature of a protein sensitizer or amboceptor, and which, with a complement, brings about lysis or cleavage of the molecule, just as a similar ferment or antibody, called bacteriolysin, disrupts a bacterial cell or its protein constituents. In short, we may say that the work of Vaughan and his collaborators has shown us the presence of this poison in all protein material with a method of extracting it in vitro. Friedberger and his collaborators have shown how this poison is set free in the body through the agency of the "ferment." von Pirquet has studied the question at the bedside, dispensing with the microscope and test-tube and depending solely upon the vital processes and reaction, skilfully combining laboratory findings with bedside observations-in fact, he built up his theory on the nature of infection and immunity before the rôle of the protein poison was discovered, and subsequent work has added support to his conceptions. Of clinical and practical importance in this connection are serum disease and hypersensitiveness or idiosyncrasies for other proteins and certain drugs.

\section{SERUM DISEASE}

This name was applied by von Pirquet and Schick ${ }^{1}$ to the various clinical manifestations, such as eruptions, fever, edema, and pain in the joints, following the injection of horse serum. These symptoms are due to the horse serum itself, for, as was early shown by Johannessen, ${ }^{2}$ Bokay, ${ }^{3}$ and others, they may manifest themselves after the injection of sterile normal horse serum. The serum, moreover, of certain horses

1 "Die Serumkrankheit," Leipsic, Deuticke, 1905.

${ }^{2}$ Deutsch. med. Wochenschr., 1895, 21, 855

3 Jahresb. f. Kindesh., 1897, xliv, 133. 
appears to be more likely than that of others to cause these symptoms, thus accounting for the fact that one lot of antitoxin will cause a higher percentage of serum sickness than will another. A concentrated serum is not so likely to produce serum sickness as whole serum, owing partly to the fact that smaller doses of it are given. According to Rolleston and Ker, the frequency of serum sickness is, as a rule, in direct proportion to the amount of serum given, and in inverse ratio to the severity of the attack; in other words, we may expect to encounter it most often in mild and moderately severe cases that have received very liberal dosages of serum.

The Nature of Serum Disease.-Serum sickness is a true anaphylactic phenomenon. We are prone to call the severe, fatal, and rare instances of death following serum injection examples of anaphylaxis, and to regard serum sickness as a different condition. Both are fundamentally the same, except that in the first instance the body-cells are, for some unknown reason, unduly and highly susceptible to the protein poison. Fortunately, this undue hypersensitiveness is frequently foreshadowed by the asthmatic or hay-fever-like attacks which the susceptible person may exhibit when he enters stables or is otherwise around horses. It goes without saying that horse serum should never be given to such persons.

While serum sickness is usually due to horse serum, for the reason that the horse is so commonly employed in the preparation of various curative serums, the serum of the ox, rabbit, and other animals may induce the same train of symptoms in addition, in some instances, to producing a direct toxic effect.

The foreign serum introduced into the human circulation acts as an antigen, and calls forth the production of an antibody, the so-called "ferment," which reacts with the antigen (remaining serum), causing its cleavage and liberating a protein poison that acts primarily upon smooth muscle and is responsible for the lesions and symptoms. An immediate reaction rarely follows the first injection of serum unless the patient is one of those unfortunate but rare persons who in some manner have been rendered highly sensitive to horse protein. In the majority of instances symptoms do not develop for from eight to twelve days, during which time the antibody is being produced. When antibody formation has reached a certain point, it reacts upon any of the horse serum that may persist in the circulation, producing the anaphylactic or protein poison. If the dose of serum has been small, antibody forma- 
tion goes on as usual, but the serum may not persist in the circulation. Hence symptoms do not develop in such a person, although if reinjected subsequently symptoms will appear. This is one reason why a concentrated serum is not so likely to produce serum sickness, since a smaller quantity of it is injected.

If, however, the patient has received an injection of serum some months previously, a reinjection is likely to be followed by an immediate reaction, that is, the symptoms appear within from twenty-four to fortyeight hours. If the first injection had been given a year or more previously, no antibody may be present in the blood, so that an immediate reaction does not occur. If, however, the cells once stimulated are "keyed up" indefinitely, and, accordingly, antibody formation is quite rapid, so that we find symptoms developing in from four to seven days after injection-the accelerated reaction. Or a small amount of antibody may be present which gives a mild reaction around the site of serum injection, followed in from four to seven days by a general reaction, this being an immediate followed by the accelerated reaction.

As was previously stated, anaphylaxis is specific - that is, a person receiving ox serum in the first injection would not be affected subsequently by an injection of horse serum, but only by ox serum. For this reason it has been recommended that diphtheria antitoxin to be used for prophylactic purposes should be prepared by immunizing cattle, reserving the horse serum for treatment if the disease should be contracted subsequently.

Symptoms of Serum Disease.-The most typical of these symptoms are rash, fever, and prostration, besides joint and muscle pains, edema, and adenitis.

The most obvious and important of the symptoms are undoubtedly the various forms of rash. In 1000 consecutive cases of diphtheria treated with antitoxin in the Philadelphia Hospital for Contagious Disease, a rash developed in 430 , or 43 per cent. The time of appearance of the eruption depends, as was just stated, upon whether or not the patient has been injected on a previous occasion, and if so, the length of the interval between the first and subsequent injection, and to a lesser extent upon the amount of the first injection, these factors influencing the quantity of antibody present at the time of reinjection. Of the 430 cases just mentioned, the time of appearance of the rash, in days, after subcutaneous injection of antitoxin, was as follows: 
TABLE 22.-TIME OF APPEARANCE OF SERUM RASH IN 430 CASES OF SERUM DISEASE

\begin{tabular}{|c|c|c|}
\hline $\begin{array}{l}\text { DAY UPON WHICH THE RASH APPEARED AFTER INJECTION } \\
\text { OF DIPHTHERIA ANTITOXIN }\end{array}$ & $\begin{array}{l}\text { TOTAL NUMBER } \\
\text { SHOWING RASH }\end{array}$ & Percentage \\
\hline 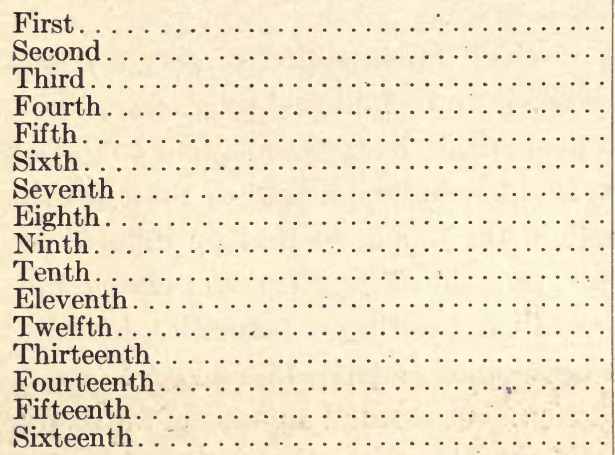 & $\begin{array}{r}4 \\
6 \\
7 \\
30 \\
35 \\
75 \\
65 \\
85 \\
44 \\
39 \\
22 \\
5 \\
6 \\
5 \\
1 \\
1\end{array}$ & $\begin{array}{r}0.9 \\
1.4 \\
1.6 \\
6.9 \\
8.1 \\
17.6 \\
15.1 \\
19.8 \\
10.2 \\
9.0 \\
5.1 \\
1.1 \\
1.4 \\
1.1 \\
0.2 \\
0.2\end{array}$ \\
\hline
\end{tabular}

In this table are included some cases of reinjection, as, $e . g$., scarletfever patients who received a routine immunizing dose of antitoxin upon admission, and another after having contracted diphtheria; also cases of diphtheria that became reinfected within a few months after their discharge from the hospital. As will be seen, about 63 per cent. of cases develop a rash between the sixth and the ninth day after the injection of antitoxin.

Three main types of rashes are generally recognized:

1. Urticarial Rashes. - If we include in this group all eruptions that present a resemblance to urticaria, these rashes are the most common, constituting from 70 to 90 per cent. of all eruptions. They usually appear after the seventh day, becoming manifest first about the site of injection. Large, irregularly shaped, and scattered blotches appear, frequently with true wheals in the center (Fig. 119), accompanied by intense itching and irritation. Sometimes true wheals do not appear. The rash is often very profuse, and fresh blotches may continue to appear for two or three days. Occasionally, the rash is quite sparse and mild, and may disappear within twenty-four hours.

2. Multiform Rashes.-This type of rash is quite common. It is often circinate in its arrangement, or occurs in large blotches mixed with a scattered morbilliform or measly form of rash (Fig. 120). Different parts of the body may present different appearances at the same time. This rash may occasionally closely simulate true measles, especially since it involves the face, and as the conjunctivæ are likely to be con- 


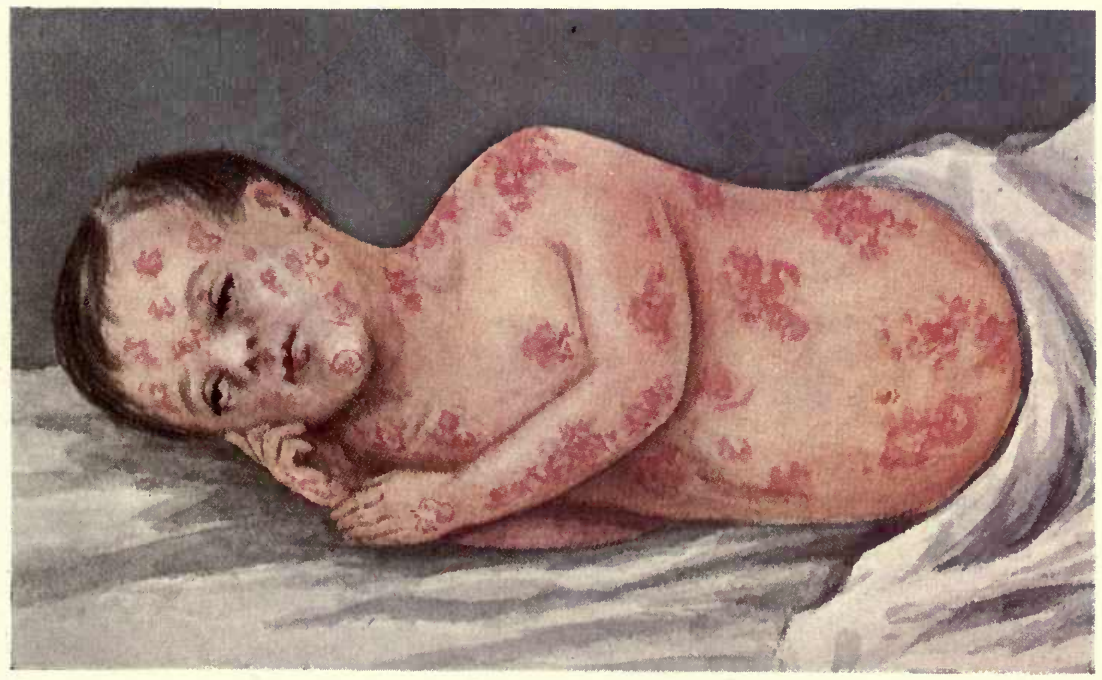

Fig. 119.-Urticarial Rash of Serum Sickness.

Case of laryngeal diphtheria; had received 40,000 units of antitoxin eight days previously; urticarial rash first appeared about thirty hours before this drawing was made. 



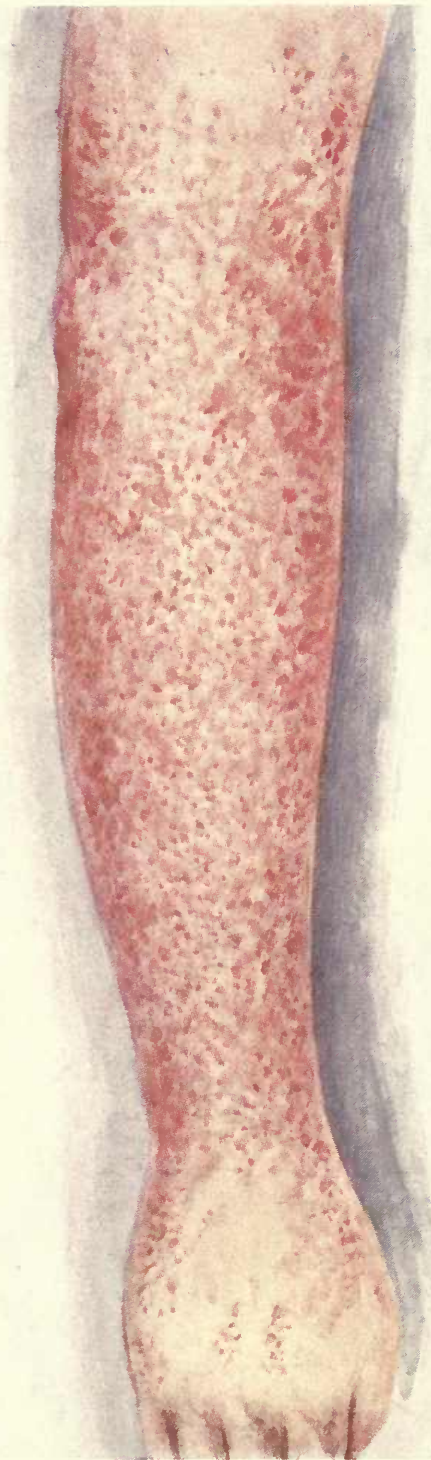

Fig. 120.-Multiform Rash of Serum Sickness.

Child with laryngeal diphtheria on the sixth day after receiving 65,000 units of antitoxin. 

gested in almost any variety of serum sickness. It is differentiated from measles by the fact that Koplik's spots are absent, there is no prodromal rise in temperature, the papules are not elevated above the skin as much as in measles, and that the eruption frequently starts from the site of injection, instead of on the face.

3. Scarlatiniform Rashes.-This type of rash occasionally occurs, and may bear so close a resemblance to true scarlet fever as to be indistinguishable from it. The eruption usually appears early, that is, in from the first to the sixth day after injection, and may vary from a uniform erythema which first appears about the site of injection, to a true punctate scarlatinal rash. The differentiation of these rashes from the true scarlet-fever eruption is one of the greatest sources of trouble in hospital practice, especially when they develop in the diphtheria wards, where occasional cases of true scarlet fever are always likely to appear from time to time. The absence of the following symptoms, or their occurrence only in mild degree, would favor a diagnosis of serum disease: Fever, or, at least, the presence of but a mild pyrexia, the vomiting, the typically furred tongue, the angina, or at least but a mild throat involvement, and the leukocytic inclusion bodies-all forming the symptomcomplex of true scarlatina. In many instances, however, it is necessary to isolate the patient, when speedy recovery, absence of complications, and less definite desquamation, which does not involve the palms and soles, indicate that the patient was suffering from serum disease and not from scarlet fever.

Severe Serum Disease.-The severer forms of serum disease, characterized by sudden onset,- often within a few minutes after the injection of serum,--extreme dyspnea, prostration, and death are, fortunately, rare, about 30 cases in all being now on record. While physicians are justified in exercising care and caution in the administration of serum, there are no absolute contraindications to its use except status lymphaticus and those instances where the person is known to be hypersensitive to horse serum. Occasionally sudden and severe dyspnea and prostration accompany an immediate reaction when a reinjection of serum is given within a few weeks after the first. Persons suffering with asthma are also bad risks, especially since the effects of serum disease upon the bronchial mucosa are likely to aggravate the already existing condition. This subject will be discussed in greater detail under the head of Contraindications to Passive Immunization in Chapter XXX. 
The Prevention of Serum Disease.-Anaphylactic phenomena can usually be expected to occur if serum is given within a year or so following a previous injection. Persons who experience discomfort when about horses should always be closely questioned. Fig. 121 shows the urticaria-like lesion that develops on the arm of one of my colleagues following scarification and the thorough application of a drop of horse serum. This man is also susceptible, to a lesser extent, to guinea-pig and rabbit serum, and is seized with sneezing and distressing dyspnea after entering a house where these animals are kept. Thayer has reported a case of buckwheat hypersensitiveness where vaccination with the flour resulted in a local reaction. It would be well for physicians to make this simple test whenever they suspect a patient of being hypersensitive to horse serum. All that is necessary is to cleanse the arm with alcohol, scarify, as when vaccinating with cowpox virus, and rub in a drop of the diphtheria antitoxin with a tooth-pick or some suitable instrument. The reaction usually appears within fifteen minutes, and there are usually no, or but very slight, general symptoms.

Various means have been tried and advocated to bring about a state of anti-anaphylaxis or desensitization of the patient:

1. A preliminary injection of 0.5 c.c. of serum as antitoxin may be given, followed in three or four hours by the regular injection. While it is difficult to produce anti-anaphylaxis (see Chapter XXIX), this method is in common use.

2. According to Auer and Lewis, ${ }^{1}$ Auer, ${ }^{2}$ Anderson and Schultz, ${ }^{3}$ Biedl and Kraus, ${ }^{4}$ and Karsner, ${ }^{5}$ atropin sulphate has a distinct protective action against the asphyxia of acute anaphylaxis in the guinea-pig. It may be well to administer from $\frac{1}{150}$ to $\frac{1}{100}$ grain of the drug hypodermically before injecting the serum, and once or twice subsequently, at intervals of twelve hours, in those cases in which hypersensitiveness is suspected, especially when serum has been injected within a year's time.

3. Rectal injections of serum have been advised by Besredka, who bases his recommendations upon the fact that by this route absorption takes place slowly and desensitization is gradual. While in the chapter on Serum Therapy I have frequently advised the intravenous injection of serum, it is to be understood that this applies to first doses. It is

1 Jour. Amer. Med. Assoc., 1909, liii, 458; Jour. Exper. Med., 1910, xii, 151.

${ }^{2}$ Amer. Jour. Physiol., 1910, xxvi, 439.

${ }^{3}$ Proc. Soc. Exper. Biol. and Med., 1910, vii, 32.

4 Wien. klin. Wochenschr., 1910, xxiii, 385.

6 Jour. Amer. Med. Assoc., 1911, lvii, 1023. 


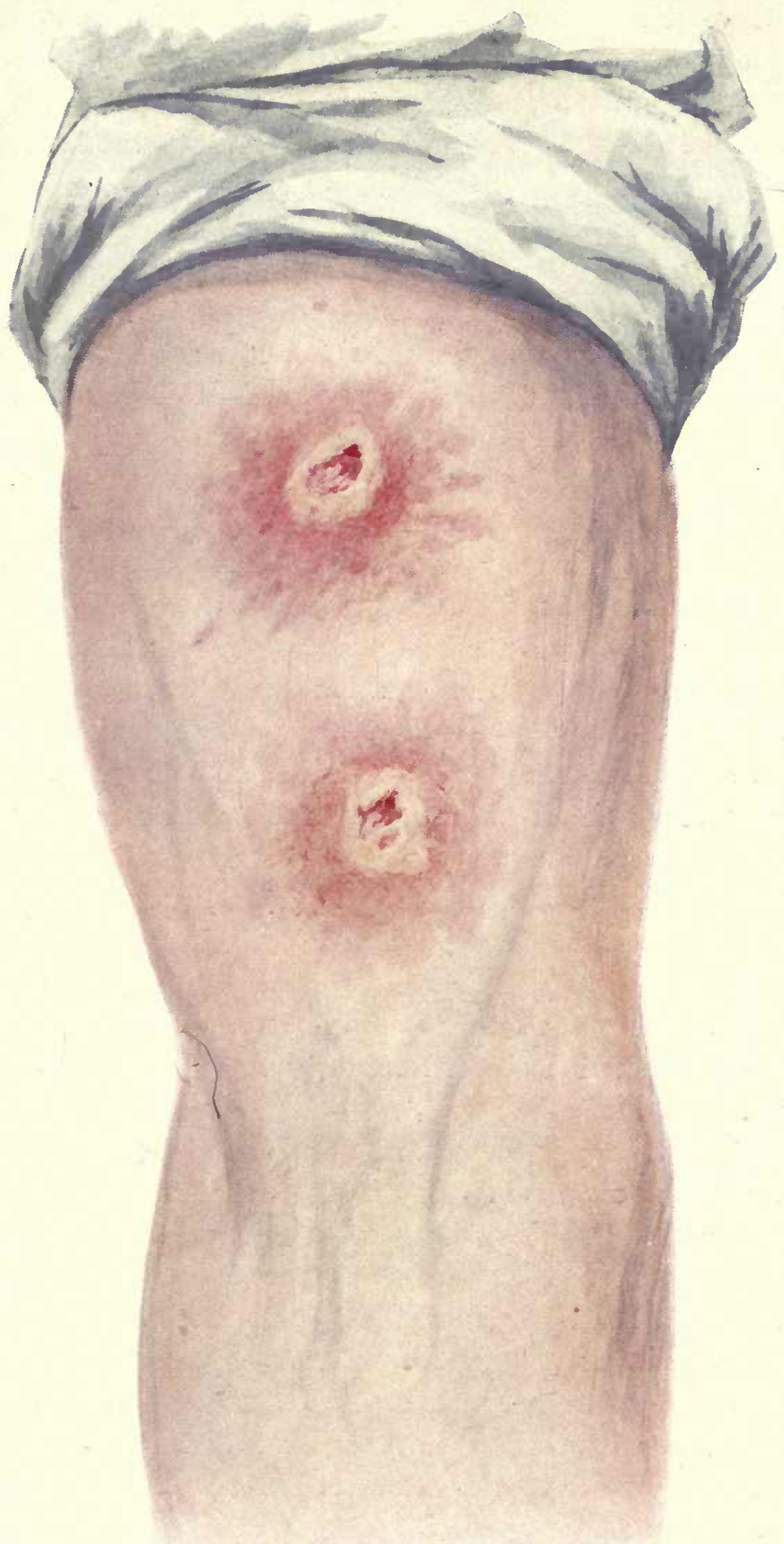

Fig. 121.-Local Serum Anaphylactic Reactions.

Dr. W. P., anaphylactic reactions fifteen minutes after application of serum; rabbit serum in the upper; horse serum in the lower. Subject to asthmatic attacks when in an animal house or stable where rabbits, horses, and guinea-pigs are kept. 

far more dangerous to give serum intravenously to those injected on a former occasion; in these instances the physician will do well to give his injections intramuscularly or subcutaneously, when, if symptoms develop, they will not be explosive nor so dangerous. Since rectal injections are usually refused, the serum may be administered very slowly subcutaneously. Friedberger and Mita have advocated the slow intravenous infusion of serum-a drop at a time.

The Treatment of Serum Disease.-In the majority of instances no treatment is necessary. Calcium chlorid and lactate, in doses of from 3 to 5 grains, have been advocated as a prophylactic and as a cure, but they are of doubtful utility. Soothing lotions, a brisk cathartic, and sedatives are indicated, and occasionally an opiate is advisable.

In the rare acute attacks marked by extreme dyspnea or rapid and shallow breathing with rapid and feeble pulse, atropin and caffein should be administered hypodermically.

\section{IDIOSYNCRASIES}

Studies in anaphylaxis have also offered an explanation of many, if not of all, of those peculiar instances in which the inhalation of some animal effluvium or of the pollen of certain plants or the ingestion of certain food-stuffs and drugs is followed by a train of symptoms, among which asthma and an urticarial rash are usually quite prominent. Hitherto these manifestations have not been understood, and were simply classed as idiosyncrasies - a term that is correct if we can make it mean hypersensitiveness, for experimental investigation leaves little doubt but that in these persons antibodies for the substances in question are present which attack the particular protein when it gains access to the body, liberating the poison responsible for the symptoms. One remarkable feature of these instances of idiosyncrasy, however, is the extreme hypersensitiveness of the body-cells, especially in those cases where the inhalation of such infinitesimal quantities of protein as are contained in the air will bring on a typical asthmatic attack in a person hypersensitive to horse protein.

Examples of idiosyncrasy are relatively common. Susceptible persons learn to know that the ingestion of this or that substance is sure to be followed by various distressing symptoms. How and when these persons became hypersensitive are usually not known. In some instances the condition is found in one or both parents and in several members of the same family, making it appear to be hereditary. It is 
well known that animals may be sensitized by feeding them proteins not usually present in their diet, as by giving guinea-pigs horse serum or the flesh of other animals.

Among the commoner examples of this form of allergy or anaphylaxis may be mentioned:

1. Horse asthma, observed among those persons who are seized with sneezing, cough, dyspnea, coryza, and prostration when they come near horses, as in a stable or when driving.

2. Hay-fever, first ascribed to the pollen of plants by Elliotson in 1831, and thoroughly studied by Dunbar. Wolff-Eisner was the first to regard the reaction as a phenomenon of hypersensitivity or anaphylaxis. Individuals subject to hay-fever show a uniform series of symptoms at certain definite seasons, either in the early summer or in autumn. These are a reddening, swelling, and watering of the eyes, sneezing, a sore feeling in the throat and larynx, and asthmatic disorders. The instillation into the eye of a 1 per cent. solution of pollen in physiologic salt solution is usually sufficient to elicit a typical attack. Certain persons are susceptible to various weeds, and each usually knows the particular weed to avoid. In hay-fever we have the most marked instances of extreme hypersensitiveness; persons may be seized with an attack when some distance from the particular weed in question. Similar phenomena are occasionally observed among workers in satinwood (satinwood dermatitis).

3. Certain foods, such as egg-albumen, buckwheat (phagopyrismus), pork, oysters, clams, lobster, cheese, and various fruits, such as strawberries, gooseberries, and even vegetables, may act as poisons when ingested by persons who are hypersensitive to them. The symptoms vary from a feeling of "indigestion" and "heartburn" to severe diarrhea, vomiting, asthma, and the development of an itching urticarial rash. In some instances it has been possible passively to sensitize guinea-pigs against the particular protein by injecting a few cubic centimeters of the patient's serum. Thus Bruck succeeded in doing this with the serum of a person hypersensitive to pork. In many instances it is possible to demonstrate the hypersensitive state by rubbing a small amount of the substance into a superficial abrasion of the arm, as in the example of horse serum anaphylaxis illustrated in Fig. 121, and, as shown by Thayer, in a case of buckwheat hypersensitiveness. It may also be demonstrated in hay-fever by making conjunctival instillations of the particular pollen.

4. Certain drugs, such as iodoform, iron citrate, and even atropin, 
strychnin, morphin, etc., appear to develop a state of hypersensitiveness. Klausner was able passively to sensitize guinea-pigs against iodoform by injecting the serum of a person sensitive to this drug. Examples of drug anaphylaxis or idiosyncrasy are more difficult to explain unless such drugs contain a protein substance. On the other hand, a drug may alter a body protein, rendering it really a foreign protein, and this may sensitize body-cells in the same manner as in the "indirect anaphylaxis" of Richet, referred to in the preceding chapter, following the second chloroforming of a dog.

As was previously stated, anaphylaxis, or rather the anaphylactic mechanism, may be considered one of the essential steps in affording resistance to disease or the state of immunity. Broadly speaking, the lesions and symptoms of infection may be ascribed to the effects of soluble toxins, endotoxins, and a protein poison. According to our present knowledge, the endotoxins are mainly liberated with lysis or disrupture of the bacterial cell. Similarly the protein poison is produced by cleavage of the bacterial protein substance. Whether the endotoxins and protein poison are identical it is impossible to state. For the present it may be well to consider them as separate entities. In certain infections, such as tetanus and diphtheria, the soluble toxins are chiefly concerned, and these are neutralized by specific antibodies, the antitoxins. The antitoxins are not similar to the "ferment" that splits protein, because they are able to neutralize their toxins without the aid of complement. In other infections, such as typhoid fever, cholera, and pneumonia, the endotoxins and protein poison may be considered the main etiologic factors. The chief antibodies are a cytolysin (bacteriolysin), which disrupts or kills the bacterial cells, and bacteriotropin, which brings about the same result by favoring phagocytosis. Apparently the cytolysins and the so-called "ferments" responsible for cleaving the protein substance are quite similar in their mechanism. The former are amboceptors, thermostabile and inactive without the presence of a complement. There is no doubt but that heating a serum containing a bacteriolysin and a complement will render the serum inactive through destruction of the complement. While the ferment concerned in splitting protein is regarded by many as an amboceptor and complement, there is no general agreement on this point. Some investigators, for example, believe that the ferment concerned in splitting placental protein, as in Abderhalden's pregnancy reaction, is rendered totally inactive by heating the serum. Others believe that heating diminishes the activity of the ferment, but does not destroy it altogether; 
the point cannot be decided at present, mainly because of technical difficulties, but, for the sake of simplicity at least, we may regard the protein-splitting ferments as quite similar to the cytolysins; indeed, they may be identical.

In anaphylaxis we recognize two primary factors: first, the antibody, which splits the protein substance, which may be either a harmless sterile protein, such as horse serum or pathogenic bacteria, with the liberation of a protein poison which intoxicates body-cells; second, a state of hypersensitiveness of the body-cells to this poison. While it is clearly apparent that the protein poison generated in the test-tube may intoxicate normal animals with the first injection, yet to understand the extreme sensitiveness of body-cells in persons susceptible to horse protein, where, for example, a few inspirations of stable air are sufficient to bring on an attack of asthma, we must recognize a peculiar hypersensitiveness of these cells, due probably to the fact that protein amboceptors are attached to the cells and unite with the inhaled protein with great avidity.

The relation of anaphylaxis to immunity consists, therefore, in the fact that the mechanism concerned in anaphylaxis is identical with that concerned in antibacterial immunity. Vaughan believes that the same mechanism is identical in all forms of immunity, but we cannot subscribe to this view, because the mechanism concerned in anaphylaxis does not explain antitoxin immunity, or at least the antibodies concerned in neutralizing diphtheria toxin are different from those digesting or splitting a bacterial protein, as, for example, typhoid bacilli. In antibacterial immunity, however, where the chief action lies in digesting the infecting cells, the mechanism may be regarded as identical with that concerned in producing the anaphylatoxin or protein poison. The effects are, however, different. In infection we have the combined action of toxins, endotoxins, and protein poison upon the body-cells; in serum anaphylaxis we have the effects of the protein poison alone. Lesions and symptoms of disease, therefore, may be regarded as the summation of the products of infection and anaphylaxis.

When we inject a bacterial vaccine we inject so much bacterial protein. This protein sensitizes body-cells and causes them to produce an amboceptor (sensitizer or the anaphylactic ferment); this antibody serves to bring about death by lysis of any corresponding bacteria in the body (therapeutic immunization), or of any that may subsequently gain access (prophylactic immunization). The effects, if apparent, 
may be ascribed to the protein poison liberated, also to the liberated endotoxins, if we consider these as separate from the protein poison.

In antibacterial immunity, therefore, we recognize lysis or digestion of the infecting bacteria by an antibody as the chief means of defense. This antibody is produced by previous injection of the bacterial protein in the form of a vaccine, or as the result of a previous infection. This is true also of serum anaphylaxis, or of egg, milk, pollen, or any other form of anaphylaxis, and in this way anaphylaxis is brought into relation with immunity.

With the antibody in our body-fluids, the corresponding bacterium is destroyed soon after it comes into contact with the antibody, but since the amounts of protein poison and endotoxin released are small and highly diluted, we experience none or but slight effects. If, however, the infection or entrance of bacterial protein is strictly localized to a small area, so that the liberated poisons are concentrated, a local reaction is produced, such as is seen, for example, in the cutaneous tuberculin, luetin, mallein, and similar reactions.

The question may now arise as to the manner in which the body cells dispose of, or become accustomed to, or are protected from the protein poison and endotoxin. There is no satisfactory answer to this question. We have seen that repeated injections of the same protein lead to a condition of decreased sensitiveness. The method by which endotoxins and the protein poison are neutralized, and whether antibodies for these are produced, are points that require further investigation. This subject has been discussed in the preceding chapter, under the head of Anti-anaphylaxis. It is true that our body-fluids may contain large amounts of the antibody; that protein, bacterial or other, may be vigorously split, but still we do not suffer from the effects of the protein poison or endotoxins. Whatever the mechanism, it in some manner concerns a neutralization or depression of the susceptibility or hypersensitiveness of the body-cells; either the protein antigen is stored in the cells and in some manner depresses cellular activity, as Weil suggested, or else the protein is split beyond the toxic moiety by the free amboceptor in the body-fluids, and in this manner prevents the protein poison from reaching the sessile receptors (those amboceptors still attached to the body-cells). This reasoning is based upon the assumption that symptoms are produced only in case the poison becomes attached to the cells, according to the cellular theory of anaphylaxis (see the preceding chapter). 


\section{ANAPHYLACTIC OR ALLERGIC REACTIONS}

As was previously stated, if a protein, such as tubercle protein (tuberculin), syphilis protein (luetin), glanders protein (mallein), etc., is concentrated and applied to the skin or mucous membrane in a local area, and if the corresponding antibody or "ferment" is present in the body-fluids, the protein will be digested or split and the liberated protein poison and endotoxin will diffuse into the local tissues and produce a local reaction, characterized chiefly by congestion and edema. This local reaction, marked by paralysis of the vessel-walls with dilatation, is due to the action of the protein poison on smooth muscle, and is analogous to the urticarial or other eruptions accompanying general serum anaphylaxis (serum disease). Since the antibody-protein reaction is highly specific, these tests possess considerable diagnostic value. The technic of application and the practical value of the more important tests will now be considered. The same underlying principle governs all. It would appear that these reactions should be obtained in all infections where we can secure and cultivate the causative microparasite. Theoretically, this is true, although practically the problem is greatly complicated, or indeed impossible, owing to technical difficulties and especially to the fact that the protein antibody for one strain of a particular microparasite may not be identical for all strains.

\section{TUBERCULIN REACTION}

An account of Koch's discovery of tuberculin, in 1891, is given in the chapter on Tuberculin Therapy. Suffice it to say here that Koch was most interested in the curative properties of tuberculin, and while he has accurately and clearly described the classic picture of the systematic tuberculin reaction, he failed to appreciate the true significance of the reaction at the site of injection, although its occurrence is carefully noted.

The Tuberculin Reaction.-The reaction to tuberculin is characterized by three essential features:

1. A constitutional reaction, consisting of fever and the accompanying general symptoms of lassitude, anorexia, and rapid pulse, varying in severity with the intensity of the reaction.

2. A local reaction at the site of administration, varying in intensity from slight tenderness and redness to severe inflammation with adenitis.

3. A focal reaction about the tuberculous lesion. 
These reactions do not by any means run parallel. An intense local reaction may occur, with no or but slight constitutional disturbance. Not infrequently, and particularly in slight pulmonary lesions, signs indicating a focal reaction may not be elicited.

Nature of the Tuberculin Reaction.-Koch believed that the tuberculin reaction was due to a summation of the effects of the injected toxin and the toxic bodies formed by the tubercle bacillus within the infected host. Koehler and Westphal, in 1891, suggested that, by a union of the tuberculin with the products of the tubercle bacillus, a third new body was formed in the tuberculous focus. Marmorek, in 1894, suggested that the tuberculin stimulated the tubercle bacilli to secrete a fever-producing substance.

Finally, in 1903, von Pirquet and Shick explained the reaction as due to a "vital antibody reaction," and this explanation is the one most generally accepted to-day. According to this conception, an antibodylike substance produced by the bacilli and diffused through the tissues enters into combination with the tuberculin, giving rise to the formation of a toxic substance in the general circulation, as well as at the point of inoculation of the tuberculin. von Pirquet's discovery of the cutaneous reaction, in 1907, was a result of this theory, and served to establish a further analogy between cowpox vaccination, tuberculosis, and the tuberculin reaction. According to the principles laid down in the earlier portion of this chapter, the tubercle bacilli are considered as stimulating the body-cells to produce an-antibody or a "ferment" in the nature of an amboceptor, which splits the tubercle protein contained in tuberculin, liberating a protein poison, which produces a general, local, and focal reaction.

The general reaction may be explained as due to a general effect of the poison on body-cells. The local reaction is caused by a concentration of the poison at the site of administration of the tuberculin, and the focal reaction is due to the fact that cells about the lesions are more sensitive to the effects of the poison than are other cells, probably because they are most concerned in antibody production and are supplied with a large number of sessile or attached receptors (amboceptors) for the tuberculin.

Specificity of the Tuberculin Reaction.-The tuberculin reaction is highly specific. This does not mean that every case of tuberculosis will give a tuberculin reaction, and positive reactions are occasionally found in apparently healthy persons and cattle. The conditions under which a negative reaction may occur. in the presence of tuberculosis 
are, however, fairly well understood, and physicians should be thoroughly acquainted with these. Likewise most instances in which a positive reaction was observed in the apparent absence of tuberculosis have usually narrowed down to the fact that the lesion was so small or so situated as to escape detection, and, indeed, this has been shown so conclusively by autopsies that, in the presence of a tuberculin reaction, on the autopsy must rest the burden of proof and blame. When we realize how small a lesion may produce hypersensitiveness, it will readily be understood how easily the clinician and pathologist may fail to detect the lesion.

A large part of our knowledge regarding the specificity of the tuberculin reaction has been gained from veterinary practice, as the results of a test in an animal could immediately be controlled by the autopsy findings. Thus Fraenkel ${ }^{1}$ collected from the literature 8000 carefully observed instances, and found only from 2 to 3 per cent. of differences between the result of the tuberculin test and of the autopsy. Voges, ${ }^{2}$ in 7327 instances, noted 2.7 per cent. of contradictions. Kuhnau, ${ }^{3}$ Bang, ${ }^{4}$ and von Behring ${ }^{5}$ speak of similar experiences.

It has long been known that the prevalence of tuberculous findings anatomically far exceeded the number of cases recognized clinically. Among cattle, anatomic tuberculosis is found in from 12 to 25 per cent., and about 80 per cent. or more react to tuberculin. In many of the latter, however, the disease does not progress, but, on the contrary, tends to recede.

Similar conditions exist in human pathology. That tuberculosis is very frequent among adults is now well known. The figures of Nageli ${ }^{6}$ and Burkhardt ${ }^{7}$ showed that the increasing frequency of tuberculous infection with advancing years reached over 90 per cent. among those past the eighteenth year; these figures are now well corroborated. Hamburger, ${ }^{8}$ in the published results of an analysis of 848 autopsies on children, showed that tuberculosis was in evidence in 40 per cent., increasing from 4 per cent. among infants under three months of age to 70 per cent. among children from eleven to fourteen years. This explains, in part

1 Zeitschr. f. Tuberk., 1900, i, 291.

2 "Tuberculin und Organismus," Jena, 1905, 77.

${ }^{3}$ Berl. tierarztl. Wochenschr., 1899, 78.

4 Sixth Internation. Congress on Tuberculosis, 1908, 211.

${ }^{5}$ Beit. z. exper. Therap., 1905, x, 1.

${ }^{6}$ Virch. Arch. f. path. Anat., 1900, clx, 426.

${ }^{7}$ Zeitschr. f. Hyg. u. Infectionsk., 1906, liii, 139.

${ }^{8}$ Wien. klin. Wochenschr., 1907, xx, 1070. 
at least, the relatively high resistance of children to tuberculin, the difficulty there is said to be in eliciting reactions, and the necessity that exists for using large doses. Usually, when children fail to react, it is because they are not tuberculous or because the lesion is too small, whereas in later years, until adult life is reached, the reaction is observed with increasing frequency and with smaller doses, because the incidence of infection increases progressively from 5 per cent. during infancy to 90 per cent. and over in adult life.

The prevalence of tuberculosis, however, by no means indicates that the infected individual suffers ill health or will succumb to the infection. An individual may be enjoying excellent health, and still harbor a tuberculous lesion, and display a marked degree of hypersensitiveness to tuberculin. Such a person is not usually regarded as tuberculous until there are tangible symptoms referable to its existence. It is important to remember that tuberculin is an index of tuberculous infection, and not of disease in a clinical sense. Numbers of persons and cattle reacting to tuberculin remain healthy and do not develop symptoms of disease, the autopsy disclosing the presence of inactive or regressing lesions.

In former years it was considered possible to obtain false positive reactions in convalescents and patients in an enfeebled condition who were non-tuberculous, and also in other diseases, such as syphilis, leprosy, and actinomycosis. More accurate anatomic statistics and careful studies of the tuberculin test administered to a large number of individuals, healthy, tuberculous, and sufferers from other diseases, have gradually changed the attitude of the profession and served to establish the high specificity of the tuberculin reaction.

Sources of Error in the Tuberculin Reaction.-From the foregoing it will readily be understood that most errors in the tuberculin reaction refer to false negative rather than to false positive reactions.

False positive reactions may be observed in leprosy, where the bacillus bears such close morphologic and biologic resemblance to the tubercle bacillus, and it is likewise true that massive doses of tuberculin injected subcutaneously may produce a toxic fever in debilitated individuals, but positive reactions in healthy individuals can usually be ascribed(a) to a small hidden tuberculous lesion or (b) to a healed tuberculous lesion. As just stated, tuberculin simply indicates hypersensitiveness to the tubercle protein, and this may exist with a very small unimportant lesion, or persist after a lesion has been "healed" to the extent of encapsulation.

False negative reactions are much more likely to occur, and the various 
conditions that may be responsible for these should be well understood and remembered.

1. In the final stage of tuberculosis, especially in miliary tuberculosis and in tuberculous cachexia, as in the third stage of pulmonary tuberculosis, the tuberculin reaction may be negative, or be attained only after the injection of very large doses. There is a lessened cutaneous reactivity (cachectic reaction), marked by the appearance of colorless or pinkish spots, instead of an intense papillary eruption. Koch and Ehrlich have explained this by assuming that the tissues had become too thoroughly saturated with tuberculin produced at the infected area to respond to further artificial additions. This condition may be regarded as analogous to a state of anti-anaphylaxis in which we may consider the free and sessile receptors united with the tubercle protein or the cells loaded with the antigen, with depression of cellular activity.

2. In the first stage of infection. At this period the antibody has not been formed in sufficient amounts, different authors obtaining such findings in nurslings.

3. In small, completely healed lesions, especially in those showing nothing but scar tissue, as in the apex of a lung. In these the antibody and cellular hypersensitiveness have disappeared, and while the lesion may have been tuberculous, one cannot tell anatomically, in a given instance, whether or not hypersensitiveness should have been present.

4. During continued treatment with tuberculin, when the reaction may be negative owing to a condition of anti-anaphylaxis.

5. During measles. As von Pirquet and Preisich have demonstrated, the cutaneous reactivity disappears during the first days after the eruption, reappearing after about a week. Greuner showed that the "Stichreaktion" which occurs after large doses of tuberculin did not disappear entirely, indicating that in measles there is a lessened activity, rather than a total disappearance.

6. Finally, according to von Pirquet, there are a few cases in which we have a minimal reactivity and to which none of the former explanations can be applied. Some cases of active tuberculosis may show only a slight hypersensitiveness, although they are not cachectic.

Of course, it can readily be understood that the use of weak or an otherwise unsatisfactory solution of tuberculin and an improper dosage and technic will greatly influence the results. Likewise errors in interpreting what constitutes a positive tuberculin reaction are to be guarded against. This applies especially to veterinary practice. For example, cattle brought from the fields and confined in a stall for the purpose of 
making a tuberculin test may exhibit a fever for several days without apparent cause. On the other hand, dishonest dealers may force cattle to drink cold water or have given cold water irrigations just before the temperature is taken, preventing the registration of a febrile reaction.

Methods of Conducting the Tuberculin Test.-The object of the tuberculin test is to introduce sufficient tubercle protein to react with the tubercle antibody, with the formation of the protein poison, which shows its presence and effects by a general, a local, or a focal reaction or by a combination of these. Various methods have been proposed, of which the following are best known:

1. The subcutaneous tuberculin test is the oldest test of its kind, having been discovered by $\mathrm{Koch}^{1}$ in 1891. It consists in the subcutaneous injection of old tuberculin. A positive reaction manifests itself in a constitutional disturbance, accompanied by fever, a local reaction at the site of injection ("Stichreaction"), and frequently a focal reaction at the site of tuberculous disease.

2. The cutaneous tuberculin test of von Pirquet, ${ }^{2}$ consisting in the local application of old tuberculin to a superficial abrasion of the skin. A positive reaction is indicated by redness, edema, and other inflammatory phenomena.

3. The conjunctival tuberculin test of Wolff-Eisner ${ }^{3}$ and Calmette, ${ }^{4}$ consisting in the local application to the conjunctiva of one eye of a drop of 1 per cent. solution of old tuberculin or purified tuberculin. A positive reaction is indicated by congestion and lacrimation.

4. The percutaneous tuberculin test of Moro and Doganoff, ${ }^{5}$ consisting in the application of tuberculin ointment prepared by mixing equal parts of old tuberculin and anhydrous lanolin and applying it to the skin over the upper portion of the abdomen or about the nipple. A positive reaction is indicated by an efflorescence of papules upon the anointed skin.

5. The intracutaneous tuberculin test of Mendel $^{6}$ and Mantoux, ${ }^{7}$ consisting in injecting into the superficial layers of the skin 0.05 c.c. of diluted old tuberculin. A positive reaction is denoted by infiltration and hyperemia about the site of injection, similar to the reaction to the cutaneous test.

Comparative Delicacy and Relation of the Various Tuberculin Tests. -In judging of the comparative delicacy of the various tuberculin

${ }^{1}$ Deut. med. Wochenschr., 1891, xvii, 101.

2 Berl. klin. Wochenschr., 1907, xliv, 699.

3 Discussion, Berl. klin. Wochenschr., 1907, xliv, 70.

4 Presse médicale, 1907, xv, 388.

${ }^{5}$ Wien. klin. Wochenschr., xx, 933.

${ }^{6}$ Med. Klin., 1908, iv, 402.

${ }^{7}$ Münch. med. Wochenschr., 1908, No. 40. 
tests by a review of the literature, it must be remembered that results will vary according to the portion of the body inoculated, as sensitiveness of the cells varies in different parts of the body, and there are individual differences in various persons that are difficult or impossible to explain.

By comparing the figures obtained as the result of different tests upon the same person with the relative frequency of individual tests, Hamman and Wolman ${ }^{1}$ have drawn the following conclusions:

"1. The intracutaneous and subcutaneous local tests are the most delicate we possess. They reveal practically the full percentage of tuberculosis-infected individuals.

" 2 . In the order of their sensitiveness, the tests arrange themselves as follows:

Intracutaneous. Test.

Subcutaneous Local Test.

Cutaneous Test.

Subcutaneous Test.

Percutaneous Test.

Conjunctival Test.

" 3 . There is a definite but not a constant relation between the various tests. An individual reacting to the conjunctival test will, as a rule, give all the others, but not always. The cutaneous or the subcutaneous tests may be negative when the conjunctival is positive. The subcutaneous positive when the cutaneous is negative, etc. Some of the unusual variations may, no doubt, depend upon faulty technic in performing the tests, but all can certainly not be thus explained. Local changes in sensitiveness and variation in the facility of absorption are probably factors, but the exact conditions are not understood.

" 4 . We have been unsuccessful in an attempt to make the cutaneous test with different strengths of tuberculin equivalent to the conjunctival test."

Although the subcutaneous test may be dangerous on account of the harm that may result from too severe focal reactions, yet, when carefully conducted, it is frequently the method of choice, especially in the diagnosis of an obscure pulmonary lesion, and in bone, joint, skin, and other local infections, where the focal reaction may be detected by direct examination. It is to be emphasized, however, that the absence of focal changes during a constitutional reaction does not exclude the tuberculous nature of a suspected lesion. In children, as shown by Hamill,

1 Tuberculin in Diagnosis and Treatment, 1912, Appleton, 167. 
Carpenter, and Cope, ${ }^{1}$ the various tuberculin reactions are likely to yield results that are quite similar.

The Value of Tuberculin in Diagnosis.-As previously stated, a reaction to tuberculin means essentially that the individual reacting has a tuberculous infection, and in itself means nothing more. Since tuberculin tests disclose inactive and relatively benign tuberculous infections, it has little value, in doubtful cases, in aiding us to decide whether or not the individual has active disease, which clinically is the type of infection about which we are most concerned. Lack of critical discernment in the interpretation of the reaction and its apparent indefiniteness have contributed toward diminishing its diagnostic value.

A positive tuberculin reaction is to be regarded as a symptom, or as another link in the chain of clinical evidence, but is not in itself indisputable evidence that a certain lesion is tuberculous, for it can never decide with certainty an otherwise doubtful diagnosis. A similar example is that of a positive Wassermann reaction in a patient with a lesion in the throat; such a reaction does not necessarily mean that the lesion is syphilitic, for the lesion itself may be cancerous, although, coincidentally, a latent syphilitic infection may be present.

If tuberculin could differentiate between active and inactive lesions according to the degree of reaction, its value would be greatly increased. While the studies of Krompecker and Romer upon animals indicate that the more virulent the infection the greater the degree of hypersensitiveness, no such fixed relation exists in man. All that may be said is that, in general, the severer the reaction, the more acute the infection. On the other hand, acute miliary tuberculosis or chronic cachectic cases may react negatively.

The conditions under which a negative reaction may be obtained in a tuberculous person are to be carefully borne in mind, for if these can be excluded, a negative tuberculin reaction precludes, in all probability, an active or clinically important tuberculous lesion. Tuberculin has, therefore, a higher negative than a positive value in diagnosis.

While it is obviously beyond the scope of this volume to discuss the diagnostic value of tuberculin in tuberculous infection of the different organs, I may briefly refer to a few of the more important conclusions reached by individual investigators of large experience in this particular field:

1. In the diagnosis of pulmonary tuberculosis, while a positive constitutional or local tuberculin reaction is never conclusive evidence that a ${ }^{1}$ Archiv. Int. Medicine, 1908, ii, 405. 
definite pulmonary lesion is tuberculous, a focal reaction, on the other hand, tells definitely of the presence of the disease, and shows, in some measure at least, its extent. In these questionable cases, therefore, the subcutaneous injection of tuberculin finds its most important application, since a definite focal is of more value than alocal reaction.

Tuberculin may also be of service when the symptoms suggest the presence of a pulmonary tuberculous lesion, but the physical signs are indefinite. Here the focal reaction is likely to be slight and to escape detection, so that one of the cutaneous tests are usually employed.

2. In the diagnosis of bone, joint, and glandular tuberculosis the subcutaneous test is likely to be most valuable, on account of the focal reaction of hyperemia, swelling, heat, and pain about the lesions. The cutaneous test is also valuable, but since this may react on account of the presence of a lesion situated elsewhere, the focal reaction is more conclusive. According to Hamman and Wolman, (a) a focal reaction confirms the diagnosis of tuberculous bone or joint disease; $(b)$ an absence of reaction to the subcutaneous test excludes, with the highest probability, the presence of tuberculosis.

3. In the diagnosis of genito-urinary and pelvic tuberculosis a positive tuberculin reaction is of little value unless the subcutaneous method is employed and the physician is sure of his ability to detect a focal reaction, a proceeding that may be very difficult or indeed impossible. A positive cutaneous test indicates the presence of a lesion somewhere in the body, without disclosing the nature of the renal or pelvic lesion. A negative reaction, however, is of more value, especially when the physician bears in mind the conditions under which a falsely negative result may occur.

4. In the diagnosis of tuberculosis of the eye, ear, and larynx, the tuberculin reaction usually has a limited value, because the nature of the disease can be so readily detected by direct inspection. In tuberculosis of the larynx a focal reaction may be dangerous on account of edema. Similarly in advanced tuberculosis of the ear a focal reaction may lead to extension of the process to the meninges. In these instances, therefore, a cutaneous test should first be made, and if it is found to be negative or inconclusive, the subcutaneous test should be applied with extra caution.

5. In the diagnosis of tuberculosis of the skin tuberculin may occasionally prove of value - especially the focal reaction following subcutaneous injection or a direct local application upon the lesion with a weak dilution, such as a 1 per cent. solution of old tuberculin. 
6. In the diagnosis of tuberculosis of a serous membrane tuberculin usually possesses a limited value. In tuberculous meningitis the subcutaneous test is contraindicated, as a focal reaction may do harm. Owing to the acute infection the cutaneous test may be negative, and even if positive, would not aid greatly in the diagnosis because the meningeal condition is always secondary to a primary focus. Tuberculous pleurises, dry or with effusion, and unaccompanied by evident pulmonary disease, are frequently associated with a low-grade tuberculin hypersensitiveness. According to Hamman and Wolman, in a large proportion of cases of pleurisy with effusion the conjunctival test is negative and the cutaneous test but mildly positive. Bandelier and Roepke assert that in a dry pleurisy increased pain and more pronounced and extensive friction may occur during a constitutional reaction to the subcutaneous test and indicate a focal reaction. In tuberculous peritonitis the tuberculin test is frequently negative. A positive reaction has far more value, especially in virulent types of the disease, which come on insidiously with little or no constitutional disturbance.

The Value of Tuberculin in Prognosis.-As previously stated, tuberculin may not react in the very early and very late cases of tuberculosis. In patients with rapidly advancing lesions the power to react tends to decrease and frequently is absent. But this condition is apparent without the aid of tuberculin. While Wolff-Eisner and Stadelmann ${ }^{1}$ laid some stress upon the conjunctival reaction in prognosis, others have been unable to confirm the results, and, as stated by Hamman and Wolman, ${ }^{2}$ tuberculin fails to yield us information of prognostic value that other methods of clinical observation do not bestow.

The Dangers of Tuberculin.-Practically, the only danger lies in the subcutaneous and conjunctival tests. With the subcutaneous test, the greatest danger in pulmonary tuberculosis is the possibility of overdosage, with the production of an extensive focal reaction which may bring on hemorrhage or lead to local extension of the lesion. Because of its very important bearing on tuberculin treatment, the subject will be discussed in the next chapter. The same danger of excessive focal reaction holds for tuberculous meningitis (increased intracranial pressure), in tuberculous laryngitis (edema), and in tuberculosis of the ear and nasal accessory sinuses (extension to meninges).

The cutaneous, intracutaneous, and percutaneous reactions are

${ }^{1}$ Deutsch. med. Wochenschr., 1908, xxxiv, 180.

2 "Tuberculin in Diagnosis and Treatment," 1912, 179. 
practically devoid of danger, providing that a careful technic is observed.

Regarding the dangers of the conjunctival test, opinions differ. There can be no doubt, however, but that distressing sequelæ, such as severe recurring conjunctivitis, phlyctenular conjunctivitis, and corneal ulcers with permanent opacities have resulted from the use of this test. Most of the unfavorable results have followed instillation in already diseased eyes, or of too strong solutions, but this is not true of all cases. As will be pointed out further on, in the first instillation not over 1 per cent. of old tuberculin should be used, and a second instillation should never be made in the same eye for at least several years.

Since old people are especially prone to conjunctival inflammation and corneal ulceration, it is probably better to exclude them from the test. Another drawback to this test is the possibility of a "flare up" in the eye following subsequent subcutaneous administration of tuberculin, either for diagnostic or therapeutic purposes. Hamman and Wolman, however, do not consider this dangerous, and have observed but two cases in an extensive experience.

\section{THE SUbCUtANEOUS TUbeRculin REACTION}

As has been stated repeatedly, the subcutaneous injection of tuberculin is resorted to at the present time mainly for the purpose of eliciting a focal reaction, and, as a rule, this is more easily appreciable when the general reaction is well marked. If tuberculin is used simply to establish whether or not a person is hypersensitive, this fact may be demonstrated by employing much smaller doses, as by the cutaneous, intracutaneous, or conjunctival tests, the patient being spared the discomfort of the constitutional symptoms.

Variety of Tuberculin.-Koch's old tuberculin is now used almost exclusively. The technic of its preparation is given in the chapter on Tuberculin Therapy.

Manufacturing chemists usually market this tuberculin in a series of dilutions, labeled and accompanied by explicit directions, so that the physician may administer practically any dose desired by injecting so many minims of such or such dilution. Otherwise a series of dilutions are readily prepared in the physician's office or dispensary, according to the method followed by Hamman and Wolman:

(a) Seven wide-mouthed bottles of about from 12 to 15 c.c. capacity, and fitted with ground-glass or rubber stoppers, are sterilized, labeled, and numbered from 2 to 8 . 
(b) The diluent is sterile 0.8 per cent. salt solution with 0.25 per cent. pure phenol. This is readily prepared by adding 8 grams of pure sodium chlorid and 2.5 c.c. of pure phenol to 1000 c.c. of distilled water. Dissolve, and filter into one large Erlenmeyer flask or, preferably, into ten smaller flasks. Sterilize in the Arnold sterilizer for one hour, or in the autoclave for twenty minutes, or by gently boiling for fifteen minutes on each of two consecutive days.

(c) Into each bottle place 9 c.c. of the diluent with a graduated and sterile pipet. Bottle 1 contains pure tuberculin. To bottle 2 add 1 c.c. of tuberculin and shake carefully; to bottle 3,1 c.c. from bottle 2 and shake; to bottle 4,1 c.c. from bottle 3 and shake; continue in this manner to bottle 8 , from which 1 c.c. is discarded.

(d) We now have the following dilutions:

No. 1-pure tuberculin.

No. $2-0.1 \quad$ c.c. tuberculin in each cubic centimeter.

No. $3-0.01$

c.c.

No. $4-0.001$

c.c.

No. $5-0.0001$

c.c.

No. 6- 0.00001 c.c.

No. $7-0.000001$ c.c.

No. $8-0.0000001$ c.c.

(e) These dilutions are usually prepared every two weeks. When not in use, the bottles are kept in a cool, dark place. It may not be necessary to prepare all dilutions. For example, dilutions No. 3 and No. 4 are sufficient for diagnostic purposes, as 0.1 c.c. of No. 4 equals $0.1 \mathrm{mg}$. of tuberculin and 1 c.c. of No. 3 equals $10 \mathrm{mg}$., thus affording an ample range of dosage.

Method of Conducting the Test.-1. The patient's temperature and pulse-rate should be taken every two hours for from four to seven days. This is easily accomplished in a hospital; ambulatory patients can usually be readily trained to take their own temperature. All observations should be recorded in writing, and preferably on a temperature chart. The patient's temperature must be constantly below $99^{\circ} \mathrm{F}$. before beginning the test, and, if necessary, prolonged rest in bed should be enforced to overcome any existing fever. The test may be given in spite of a daily rise of not over $100^{\circ} \mathrm{F}$., but tuberculin by subcutaneous injection should be given only exceptionally to febrile patients.

2. A very careful physical examination should be made, and the results recorded just before and just after the test in order to detect a focal reaction. This is extremely important, for it is our main justification for injecting the tuberculin. 
3. Injections are made subcutaneously in the region of the back, below the angle of the scapula, or in the arm. The skin needs no preparation other than to be rubbed with alcohol. The injections are best given during the late evening hours or early in the morning, in order that the temperature and pulse changes may be observed, especially twelve hours after the injection. Records of temperature and pulse should be made every two hours during the day and night for forty-eight hours following an injection. Hamman and Wolman recommend the "Tuberculin Sub 2 Syringe," made by the Randall, Faichney Co. Each syringe should be sterilized by boiling prior to use, and it is recommended that a separate syringe be provided for each dilution.

4. Considerable controversy has arisen over the size of the doses to be employed. Koch's directions called for one milligram at first, then for five, then for ten, and if no reaction followed this dose, it was repeated. There is a decided tendency, however, to use smaller doses. If the object is to establish the presence or absence of tuberculin hypersensitiveness, mild reactions will suffice, and for this purpose small doses repeated or in gradually increasing amounts may be given. If one aims to produce a focal reaction, larger doses and more rapid increase are desirable. Hamman and Wolman advise the following plan for adults: For the first dose, $\frac{1}{5} \mathrm{mg}$. is given. If there is a slight febrile reaction of about one-half a degree, and especially if this is accompanied by a local reaction at the point of injection, the second dose, which is the same as the first, is given at the end of forty-eight hours. The reaction will now most likely be more conclusive. If there is no appreciable reaction after the first dose, the second, consisting of one milligram, is given at the end of forty-eight hours, and the third dose, if one is necessary, consists of five milligrams. Occasionally the third dose must be repeated, or even ten milligrams given if the negative result is at variance with the clinical impressions.

If the temperature shows any irregularities, the intervals between injections should be prolonged to three or four days or more.

Roth-Schultz advise the following doses: $0.5 \mathrm{mg}$; $1.25 \mathrm{mg}$., and $2.5 \mathrm{mg}$. as the terminal dose.

For children under fifteen years of age smaller doses are indicatedan initial dose of one-tenth milligram and a terminal dose of one milligram, with one or two intervening doses. Baldwin has advised 0.05, $0.2,0.5$, and $1 \mathrm{mg}$.

The Reaction.-The constitutional reaction is quite variable. Fever 
is the most delicate indicator. A rise of $1^{\circ} \mathrm{F}$. or more above the previous maximum is considered positive, especially if it is accompanied by a local and a focal reaction. A definite febrile reaction due to tuberculin is rare without the presence of a local reaction to the same or the preceding doses. General symptoms of headache, muscle pains, anorexia, nausea, etc., may accompany the reactions. The local reaction consists of redness and pain at the site of injection, with tenderness of the neighboring lymph-glands, and is absolutely specific of tuberculin hypersensitiveness. The focal reaction consists of an inflammatory reaction with the production of râles, change in breath-sounds, etc.

\section{THE INTRACUTANEOUS TUBERCULIN REACTION}

Variety of Tuberculin.-Koch's old tuberculin is used either in one dose of $0.005 \mathrm{mg}$., or preferably in three different doses injected simultaneously; these will be described further on.

Method of Conducting the Test.-The skin of the forearm is cleansed with alcohol and then dried. A small glass syringe fitted with a fine needle is used. A separate syringe is used for the control fluid, consisting of sterile salt solution, and three others for each of the different dilutions used. In performing this test Hamman and Wolman make four simultaneous injections:

First: 0.05 c.c. of sterile normal salt solution (control).

Second: 0.05 c.c. of a $1: 1,000,000$ dilution of old tuberculin, which equals $0.00005 \mathrm{mg}$. This equals a dose of 0.05 c.c. of dilution No. 4 , just described in the preceding test.

Third: 0.05 c.c. of a $1: 100,000$ dilution, or 0.05 c.c. of dilution No. 3 , equivalent to $0.0005 \mathrm{mg}$.

Fourth: 0.05 c.c. of a $1: 10,000$ dilution, or 0.05 c.c. of dilution No. 2 , equivalent to $0.0005 \mathrm{mg}$.

Mantoux uses the last or fourth dose only. The injections are given with the skin held taut or pinched up in a fold between the index-finger and thumb. The needle is inserted superficially, with the aperture directed toward the outer surface of the skin. If the point of the needle is in the skin, a white elevation occurs immediately upon the introduction of the solution; if it is in the subcutaneous tissue, no infiltration is apparent.

The Reaction.-The reaction consists of infiltration and hyperemia about the site of injection, similar to the reaction in the cutaneous test. It appears in from six to eight hours, reaches its maximum intensity in from twenty-four to forty-eight hours, and usually disappears in 
from six to ten days. The salt solution generally produces a traumatic reaction, similar to a mild tuberculin reaction, which subsides in forty-eight hours.

The reactions are best recorded after twenty-four hours, and the simplest method of recording the results is to measure the width of the area of infiltration of each reacting point.

\section{The Cutaneous Tuberculin Reaction (VoN PIRQuet)}

Variety of Tuberculin.-Undiluted old tuberculin is now used almost exclusively in conducting the test.

Method of Conducting the Test.-1. The flexor surface of the forearm is chiefly used for making the applications, but it should be remembered that tests performed on different portions of the body are not strictly comparable.

2. The skin is cleansed lightly with alcohol and dried. Three abrasions are made, about $1 \frac{1}{2}$ or 2 inches apart, with a von Pirquet skin borer (Fig. 122) or with a needle, small lancet, or blood sticker. The object is to scarify the superficial layers of the skin, avoiding as much as possible bleeding, although a few small points of blood should appear. To the upper and lower abrasions add a drop of tuberculin; after ten minutes wipe away the excess with a bit of cotton. No shield or protective dressings are required. The middle abrasion is the control, and shows the amount of traumatic reaction following the scarifying process. Due precautions should, of course, be observed that none of the tuberculin flows down the arm and reaches this spot.

3. The tests are inspected at the end of twenty-four hours.

The Reaction.-The traumatic reaction as shown in the control area may present an inflammatory areola with, at times, slight infiltration. Before a test may be considered positive its areola should be at least five millimeters wider than the control area. The reactions are usually designated as follows:

1. Negative Reaction.-No appreciable difference between the tuberculin areas and the control.

2. Slight Reaction.-Definite but slight redness with some infiltration.

3. + Reaction.-A wider area of redness, with definitely raised centers.

4. ++ Reaction. -Wider area of redness, with more marked infiltration than + .

5 . +++ Reaction. - Unusual redness and a wide area of infiltration, all cases which go on to vesiculation. 
The usual or normal reaction begins to appear in from four to six hours, reaches its maximum intensity in from twenty-four to fortyeight hours, and then fades rapidly, although the infiltration may persist for some days. Special types of the reaction have been described as follows:

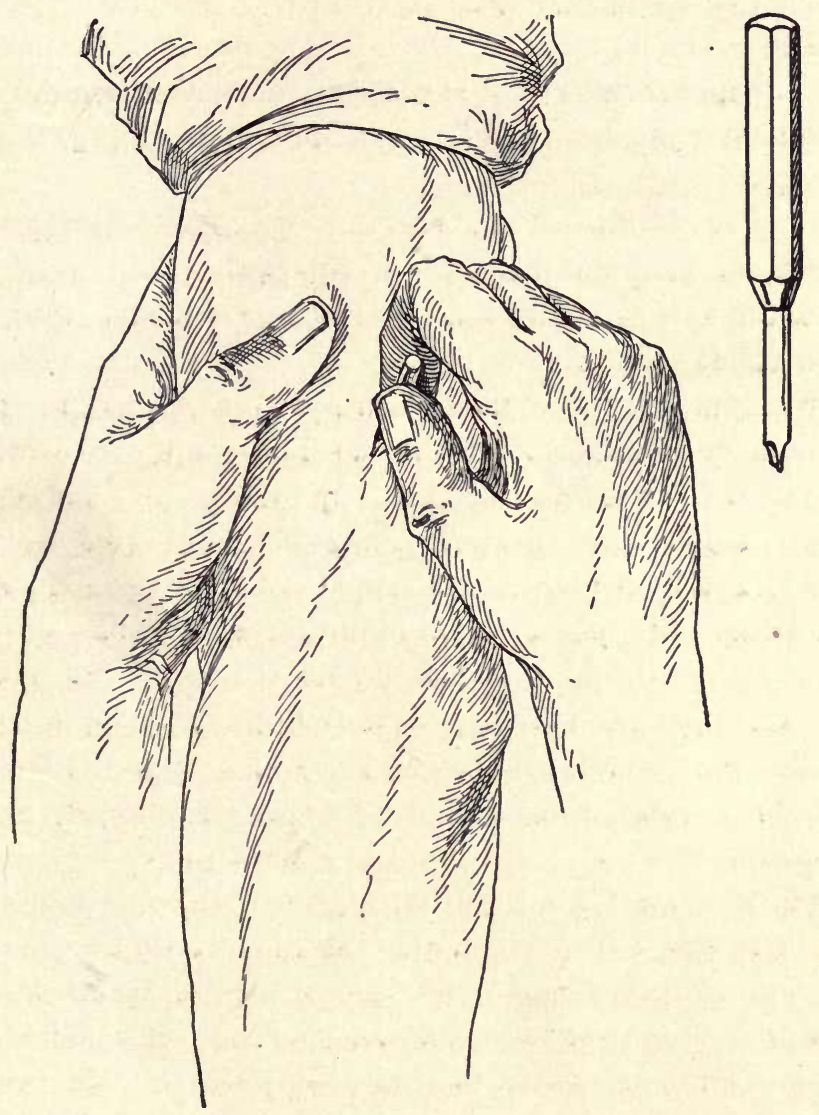

Fig. 122.-Method of Performing a von Pirquet Tuberculin Test.

The abrasion is being made over the insertion of the deltoid muscle. The borer is held firmly and perpendicular to the arm. A quick rotatory motion serves to remove a circular area of epidermis.

The borer is shown in the upper right-hand corner.

1. The premature reaction, characterized by a rapid course and slight intensity. This type is supposed to occur in patients with manifest tuberculosis who are not doing well.

2. The persisting reaction, which reaches its maximum intensity about the second day and persists for a week or longer. 
3. The late reaction, which appears after twenty-four hours and develops and recedes slowly. These last two types are believed to occur in patients having inactive lesions.

4. The cachectic reaction, which is characterized by infiltration with little or no redness. This type is common in the late stages of tuberculosis.

5. The scrofulous reaction, which is characterized by numerous small elevated nodules, which may also appear on the extremities and trunk. This reaction is peculiar to children and rare in adults.

\section{The Conjunctival Tuberculin Reaction (CALMETTE)}

Variety of Tuberculin Used.-A 1 per cent. solution of Koch's old tuberculin is now generally used, as it is quite reliable, least expensive, and the results that follow its use are more regular than those obtained with purified tuberculin ( 0.5 to 2 per cent. aqueous solution).

Method of Conducting the Test.-The conjunctivæ of both eyes are inspected to ascertain if there is any evidence of disease and if they are strictly comparable in color. The lower lid of one eye is drawn forward to form a little pouch, and the patient is directed to look upward; one drop of a 1 per cent. dilution of old tuberculin is then applied from an ordinary eye-dropper to the lid at the inner canthus. Profuse lacrimation impairs the test, and it is useless to attempt it with weeping or resisting children. If no reaction is apparent and it is still desired to further the test, a drop of a 5 per cent. dilution may be placed in the opposite eye.

The Reaction.-In a positive reaction the conjunctiva begins to redden in from six to eight hours, and reaches its maximum in from twenty-four to forty-eight hours, and then rapidly subsides and disappears in from four to six days. In mild reactions the inner canthus is the seat of the most marked changes. Positive reactions have been classified as follows:

+ Reaction: Definite palpebral redness.

++ Reaction: More marked palpebral redness with secretion.

+++ Reaction: Palpebral and bulbar redness with subjective symptoms and well-marked secretion. (See Fig. 124.)

Precautions.- On account of severe reactions and the danger of inflicting permanent injury on the cornea there is a growing tendency to regard this reaction with disfavor. Hamman and Wolman, however, believe that, with proper precautions, these risks may be minimized, if not completely avoided; they believe, moreover, that the risk in- 


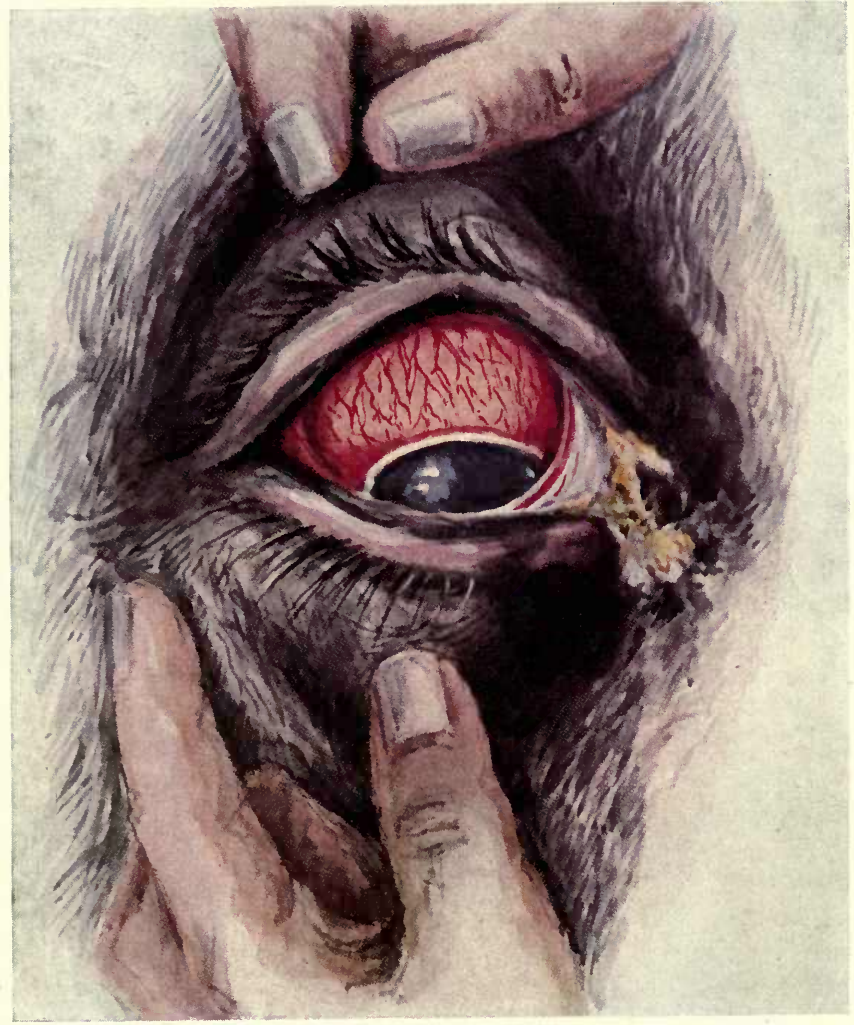

Fig. 124.-A Positive Conjunctival Tuberculin Reaction (Wolff-EisnerCalmette).

Severe reaction $(+++)$ in a tuberculous cow; appearance of the eye about fourteen hours after second instillation of tuberculin. 


Fig. 125.-A Positive Percutaneous Tuberculin Reaction (Moro).

E. McK., adult male with arrested early pulmonary tuberculosis. Lesions about sixty hours after application of tuberculin ointment. 
curred is not great enough to warrant the abandonment of a procedure that has proved itself of such great value in diagnosis. Nevertheless, they emphasize the necessity for observing proper precautions, and give the following rules to be adopted:

1. The conjunctival test should never be repeated in the same eye.

2. A solution stronger than 1 per cent. original tuberculin should never be used for making the first instillation.

3. Any existing inflammatory disease of the eye is an absolute contraindication to the test.

4. The test should not be given to manifestly scrofulous children.

5. Skin diseases in which the lesions are situated upon the face, near the eye, especially when these are suspected of being tuberculous, preclude the application of the test.

6. It is safest not to give the test to elderly persons, and particularly to arteriosclerotics, as they are unduly prone to develop corneal ulceration.

\section{The Percutaneous Tuberculin Reaction (MORO)}

Variety of Tuberculin Used.-This consists of 5 c.c. of old tuberculin and 5 grams of anhydrous lanolin thoroughly mixed. The ointment retains its potency for many months when preserved in a cold dark place. Manufacturers market the preparation in small collapsible tubes containing sufficient for at least one or two tests.

Method of Conducting the Test.-A piece of ointment about the size of a pea is thoroughly rubbed for at least a minute into an area of skin about two inches in diameter over the upper abdomen or near a nipple. The patient may be instructed how to apply this ointment, or the physician may do it himself, protecting the finger with a rubber cot.

The Reaction.-This may appear within twenty-four hours, or be delayed for as long as from four to six days. It consists of an eruption of slightly elevated papules situated upon a hyperemic base, which vary in size from a pinhead to large areas of infiltration (Fig. 125). It subsides in from three to ten days. Moro described these grades of reaction as follows:

1. Mild reactions: From 1 to 10 scattered papules.

2. Moderate reactions: About 50 or more papules, partly discrete, partly confluent, and with a general reddening of the skin. There may be well-marked itching.

3. Severe reactions: Numerous large, extremely red papules or vesicles up to $8 \mathrm{~mm}$. in diameter, having an intensely reddened base. 


\section{TUBERCULIN REACTIONS AMONG THE LOWER ANIMALS}

In veterinary practice tuberculin is used almost solely for diagnostic and only occasionally for therapeutic purposes.

Four reactions are in common use:

The Subcutaneous Test.

The Conjunctival Test.

The Cutaneous Test.

The Intracutaneous Test.

The technic for conducting these tests and the reactions secured are quite similar to those just described, and I would refer the veterinary surgeon to these respective descriptions. Differences in technic are confined principally to dosage.

The Subcutaneous Tuberculin Test.-This is conducted with Koch's old tuberculin. The method of preparation is given in the succeeding chapter, under Tuberculin Therapy. Concentrated tuberculin is prepared by concentrating the bouillon filtrate to one-tenth its original volume. Diluted tuberculin is the concentrated product diluted to its original volume by mixing 1 part of the dilution with 9 parts of 0.5 to 1 per cent. phenol in sterile normal salt solution or distilled water.

The injections are always given subcutaneously in some convenient area, preferably around the shoulder, which has been shaven and cleaned beforehand with a solution of creolin.

The first dose for horses and cattle is usually 0.4 c.c. of concentrated or 4 c.c. of diluted tuberculin; the second dose is usually 0.8 c.c. of concentrated or 9 c.c. of the diluted tuberculin.

The general local and focal reactions are similar to those previously described.

The Conjunctival Tuberculin Test.-For this test Koch's concentrated tuberculin may be used. Prefereace is usually given to the purified product, prepared as follows: Mix one part of Koch's concentrated tuberculin with 20 parts of absolute alcohol. The precipitate that forms is filtered off and dried over sulphuric acid. This powder is then made up into a 4 per cent. and 8 per cent. solution in sterile distilled water.

Two or three drops of the 4 per cent. dilution are placed in the inner canthus of one eye, to sensitize the tissues. After twenty-four hours, unless a positive reaction is present, two or three drops of the 8 per cent. solution are instilled in the same eye in the same manner. The reaction is usually apparent in from six to twelve hours (Fig. 124).

For the types of reaction and precautions to be observed see the previous description. 
One advantage of this test is that the animal will give a reaction in cases where, prior to the test, dishonest dealers have injected tuberculin.

The Cutaneous Tuberculin Test.-In making this test some convenient area is shaved and scraped slightly until serum exudes. A small amount of Koch's old tuberculin is applied to the prepared area. In a positive case a well-marked area of congestion and hyperemia appear at the end of twenty-four hours. This may also be accompanied by a rise in temperature.

The Intracutaneous Tuberculin Test.-In performing this test from 0.2 to 0.4 c.c. of Koch's concentrated tuberculin are injected into the skin through a fine needle. A white swelling should appear while the injection is being given; if it does not appear, the injection is subcutaneous and unsatisfactory for this test. The appearance of hyperemia and redness with a rise in temperature indicates a positive reaction.

\section{LUETIN REACTION}

The clinical course of syphilis indicates that the infecting microparasite, Treponema pallida, possesses all the qualities essential to the development of an anaphylactic condition in syphilitic patients. Thus the primary lesion appears after an incubation period of two or three weeks. The secondary stage is manifested by periodic waves of various general symptoms; the primary, secondary, and tertiary lesions show a qualitative difference. Stimulated by von Pirquet's discovery of a specific cutaneous reaction for tuberculosis, a number of investigators (Finger and Landsteiner, Wolff-Eisner, Nobe, Ciuffo, Nicolas, Favre, and Gauthier) attempted to obtain a specific reaction for syphilis by applying extracts of syphilitic tissues-prepared from syphilitic fetal liver or chancre-to the skin of syphilitic patients. In spite of some encouraging effects their results were, on the whole, contradictory. Further, Neisser and Bruck found that a reaction similar to that produced with syphilitic extract can be obtained also with a concentrated extract of normal liver. This peculiarity of the skin of syphilitics is ascribed by Neisser to what he calls the state of "Umstimmung" in the later stages of syphilis. Both Neisser and von Pirquet expressed the hope and belief that a reaction may be secured by employing an extract of pallida free from tissue constituents; this was first and finally accomplished by Noguchi, ${ }^{1}$ in 1911, first with syphilitic rabbits and then with human patients. Noguchi gave the appropriate name of "luetin"

1 Jour. Exper. Med., 1911, 14, 557; Jour. Amer. Med. Assoc., Iviii, 1163. 
to the extract of pallida. Theoretically, one should not expect to obtain an allergic reaction in syphilis so long as the activity of pallida is maintained at its maximum, or in the very early stages, before there is sufficient time for antibody formation. One can reasonably expect the appearance of the phenomenon when the activity of the microparasite begins to abate through a gradually acquired defensive power of the host, or under an effective therapeusis, as in the later stages of the disease and in hereditary syphilis. Practical results have borne out these theoretic expectations.

Preparation of Luetin.-At least six different strains of pallida in pure culture are being used by Noguchi in the preparation of luetin. These are cultivated in ascites-kidney agar for periods of six, twelve, twenty-four, and fifty days, at $37^{\circ}$ C., under anaërobic conditions. The tubes showing large numbers of spirochetes are then selected, the oil is poured off, the tube is cut, the agar column is removed, and the tissue then cut off. The ascites-agar cultures are then carefully ground in a sterile mortar, the resulting thick paste being gradually diluted with a fluid culture until a homogeneous liquid emulsion is secured. The preparation is next heated for an hour in a water-bath at $60^{\circ} \mathrm{C}$., and then tricresol or phenol added to make 0.5 per cent. Cultures are made from this suspension, and rabbits inoculated intratesticularly; both, after suitable intervals, must show an absolutely sterile preparation.

The luetin should be kept in the refrigerator when not in use. So far as I am aware, all "luetin" is prepared in the Rockefeller Institute, under Noguchi's own supervision. The isolation of Treponema pallidum in pure culture is a difficult procedure, and, obviously, a luetin must be prepared of pure cultures and from as many different strains as possible. While pallida quickly loses its pathogenicity in artificial culture-media and is also highly susceptible to the influence of germicides, its preparation, nevertheless, is an important matter requiring skilful supervision.

A control fluid prepared of sterile agar and bouillon in exactly the same manner as luetin was originally advised by Noguchi, but recently he claims that its use is not necessary.

Method of Application.-Luetin is not applied to an abrasion of the skin, but is injected intracutaneously with a very fine needle and a sterile syringe. According to directions from the Rockefeller Institute, the luetin is to be well shaken, diluted with an equal part of sterile salt solution, and 0.07 c.c. injected ( 0.035 c.c. undiluted). A slightly smaller dose, as, e. g., 0.05 c.c., may be used for children. The skin of the upper.arm is usually selected as the site for inoculation. If a control fluid is used, the luetin may be injected into the skin of the left and the control fluid into the skin of the right arm, or both injections 
may be given in the same arm, about two inches apart, the control being above the luetin.

After cleansing the skin with alcohol it is drawn taut or pinched up between forefinger and thumb and the needle introduced, with the aperture directed toward the outer surface of the skin. If the point of the needle is in the skin, a white elevation occurs immediately upon injecting the solution; if it is in the subcutaneous tissue, no infiltration is apparent.

A special tuberculin syringe, may be used, and the luetin drawn directly into the barrel from the stock bottle and diluted with sterile normal salt solution. To obviate waste and the likelihood of contamination, I dilute a portion of luetin (1 c.c.) with an equal amount of sterile salt solution (1 c.c.) in a sterile vessel, and then add 6 c.c. more of sterile salt solution, shaking thoroughly and placing 0.2 c.c. in each of 30 small sterile ampules, which are then sealed. Before using, the ampule is shaken thoroughly, opened, and the contents aspirated into the syringe; 0.1 c.c. is allowed for waste in loading the syringe and displacing air; 0.1 c.c. is injected, and this is practically equivalent to 0.035 c.c. of the undiluted luetin, the dose recommended. When so prepared, the dilution keeps well, is especially adapted for dispensary use, and the phenol is so diluted as to exclude any traumatic reaction.

Reactions.-Normal or Negative Reactions.-In the majority of normal persons the injection of luetin is followed by a very slight traumatic reaction, or a small erythematous area appears, after twenty-four hours, at and around the point of injection. No pain or itching sensation is experienced; the reaction recedes in forty-eight hours and leaves no induration. In certain individuals the reaction may reach a stage of small papule formation after from twenty-four to forty-eight hours, which subsides within seventy-two hours, leaving no induration.

Positive reactions have been classified by Noguchi into three main varieties:

(a) Papular Form.- "A large, raised, reddish, indurated papule, usually five to ten millimeters in diameter, makes its appearance in twenty-four to forty-eight hours. The papule may be surrounded by a diffuse zone of redness and show marked telangiectasis. The dimensions and the degree of induration slowly increase during the following three or four days, after which the inflammatory processes begin to recede. The color of the papule gradually becomes dark bluish red. The induration disappears within one week, except in certain instances in which a trace of the reaction may persist for a longer period. The 
latter effect is usually met with among cases of secondary syphilis under regular mercurial treatment in which there are no manifest lesions at the time of making the skin test. Cases of congenital syphilis also show this reaction.

"(b) Pustular Form.-The beginning and course of this reaction resemble the papular form until about the fourth or fifth day, when the inflammatory processes commence to progress. The surface of the indurated round papule becomes mildly edematous, and multiple miliary vesicles occasionally form. At the same time a beginning central softening of the papule can be seen. Within the next twentyfour hours the papule changes into a vesicle, filled at first with a semiopaque serum that later becomes definitely purulent. Soon after this the pustule ruptures spontaneously or after slight friction or pressure. The margin of the broken pustule remains indurated, while the defect caused by the escape of the pustular content becomes quickly covered by a crust that falls off within a few days. About this time the induration usually disappears, leaving almost no scar after healing. There is a wide range of variation in the degree of intensity of the reaction described in different cases, as some show rather small pustules, while in others the pustule is much larger. This reaction was found almost constantly in cases of tertiary syphilis, as well as in cases of secondary or hereditary syphilis which had been treated with salvarsan.

"(c) Torpid Form.-In rare instances the injection sites fade away almost to invisible points within three or four days, so that they may be passed over as negative reactions. But sometimes these spots suddenly light up again after ten days or even longer and progress to small pustular formation. The course of this pustule is similar to that described for the preceding form.

"This form of reaction has been observed in a case of primary syphilis, in one of hereditary syphilis, and in two cases of secondary syphilis, all being under mercurial treatment."

Aside from these three types of reactions, there have since been described several cases of the formation of a hemorrhagic exudate, the lesion usually rupturing spontaneously and not running a more severe or a longer course than the pustular. Two such reactions have been reported by Kilgore. ${ }^{1}$

"Neither in syphilitics nor in parasyphilitics did a marked constitutional effect follow the intradermic inoculation of luetin. In most positive cases a slight rise in temperature took place, lasting for one day. 


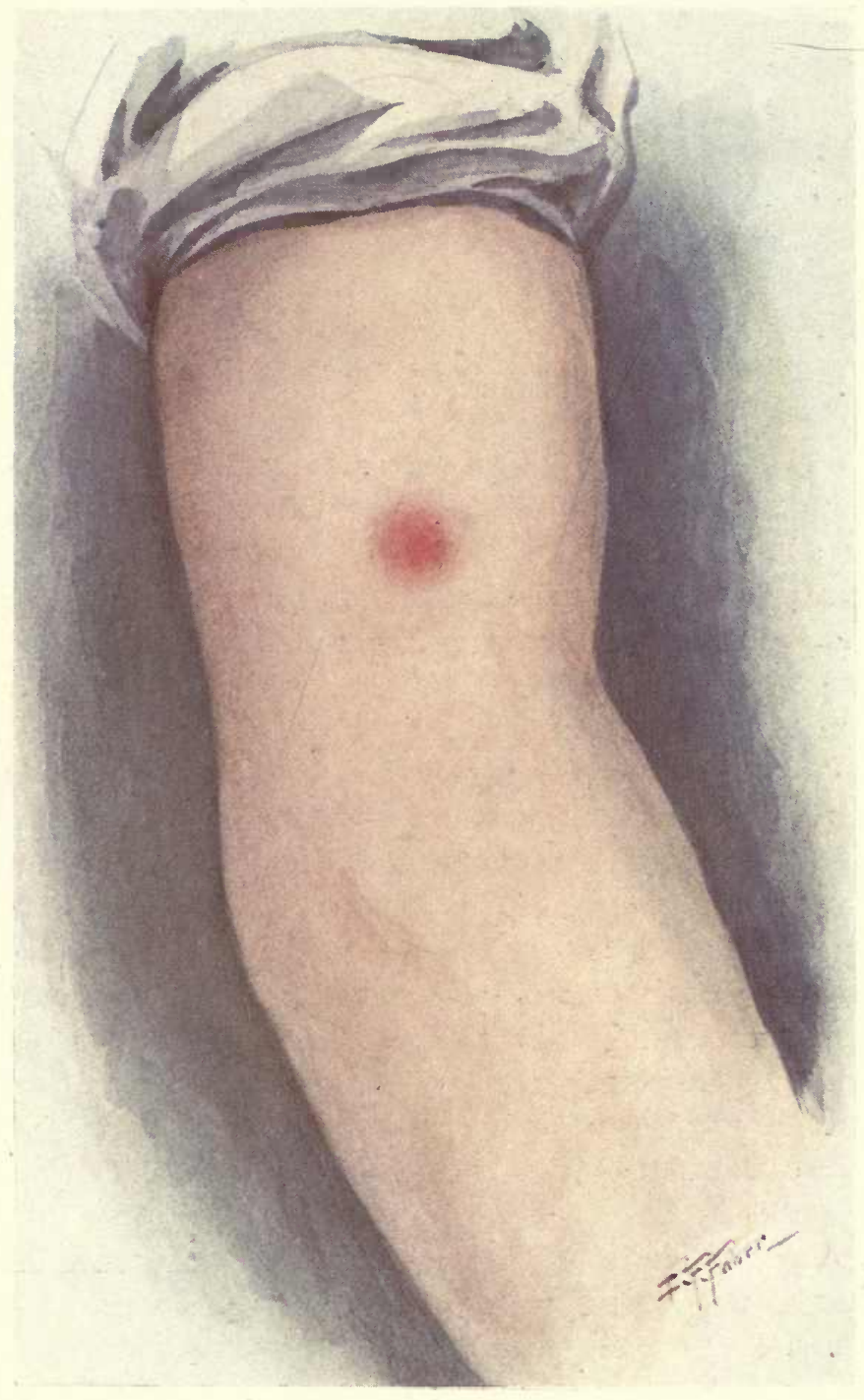

Fig. 126.-A Positive Luetin Reaction.

E. C., adult male; tertiary syphilis with detachment of the retina; papular lesion of moderate severity; about forty-eight hours after injection of 0.07 c.c. luetin. 

In three tertiary cases and in one hereditary case, however, general malaise, loss of appetite, and diarrhea were noted."

As was previously stated, Noguchi now asserts that the control fluid may be omitted. In syphilitic persons this fluid may give a reaction that is less intense and that is not followed by induration, but is due probably to a true allergic condition, whereas in normal persons none or but a slight traumatic reaction occurs. It may at times be difficult, however, to distinguish a slight luetin reaction from a wellmarked traumatic reaction, and in these instances an opinion may be withheld until controlled by a second injection in the other arm with control fluid. In other words, while the control fluid need not be used routinely, it is well to have it at hand to be used in these doubtful cases.

In view of the occasional instances in which the reactions are retarded a patient should be observed for two weeks before a reaction is regarded as negative.

Results.-1. The reports of Noguchi and of a number of different observers show that the luetin reaction is generally negative in the primary and secondary (untreated) stages of syphilis.

2. In latent and tertiary syphilis Noguchi has reported positive reactions in from 80 to 95 per cent. respectively, and the reports of others have showed from 64 to 100 per cent. of positive reactions.

3. In cerebrospinal syphilis positive reactions have been reported in from 42 to 80 per cent. of cases.

4. In congenital syphilis the results have varied within wide limits10 to 96 per cent. of positive reactions. In cases under one year of age Noguchi has reported about 23 per cent., and among later cases 96 per cent., of positive reactions.

5. While a few observers have reported positive results in diseases other than syphilis, it is frequently very difficult absolutely to exclude syphilis, and the general consensus of opinion is unmistakably to the effect that in this country, at least, the luetin reaction is specific for syphilis. Slight reactions may be obtained in frambesia or yaws and leprosy.

6. Second injections of luetin apparently do not give positive reactions in non-syphilitic cases.

Practical Value.- It may be stated in general that the chief value of the luetin reaction is in the diagnosis of those occasional cases of latent, tertiary, or congenital syphilis that fail to react positively with the Wassermann reaction. I am quite convinced that in the majority of cases a negative Wassermann reaction, carefully and skilfully per- 
formed, and especially with an antigen reënforced with cholesterin, is stronger evidence of the absence of syphilis then is a luetin test. On the other hand, a definitely positive luetin reaction may be regarded as indicating that the patient is or has been syphilitic, even though the Wassermann reaction is negative.

2. Results indicate that in syphilis the luetin reaction persists longer than does the Wassermann reaction. This is to be expected when we assume that the substance in the blood-serum of a syphilitic responsible for the Wassermann reaction, is a separate reactionary product of the body-cells to pallida toxin, the allergic luetin reaction being due to the true pallida antibody. The toxin is believed to disappear from the body-fluids within a few weeks after all the spirochetes have been killed, whereas the allergic antibody may persist for longer periods of time, as it does in other conditions and diseases. In a case of treated syphilis, therefore, showing a persistently negative Wassermann reaction with both serum and spinal fluid, a positive luetin reaction does not necessarily indicate that further treatment is required.

3. Briefly stated, to determine if a frankly syphilitic patient requires further treatment the Wassermann reaction with serum and cerebrospinal fluid is the better test. To determine more definitely whether a given person showing a negative Wassermann reaction has ever had syphilis the luetin reaction is the better and more conclusive test; or if in such a person the Wassermann reaction is positive, the luetin test may be used for control and as corroborative evidence.

\section{MALLEIN REACTION}

Mallein is a glycerin extract containing the toxic principles of the Bacillus mallei, the microörganism causing glanders. It is used entirely as a diagnostic agent in veterinary practice, but may also be used for the diagnosis of human glanders, the dosage being the same as that of old tuberculin.

Two methods are commonly employed: (1) The subcutaneous injection and (2) instillation into the eye (ophthalmic test).

Method of Preparing Mallein. ${ }^{1-A}$ pure culture of Bacillus mallei is usually obtained by injecting a male guinea-pig with infected material, and at the end of twenty-four or forty-eight hours, isolating a pure culture from the testicle.

The microörganism is grown for from six to eight weeks in special bouillon containing 5 per cent. glycerin, at $37^{\circ} \mathrm{C}$., similar to tuberculin. Unlike the tubercle

${ }^{1}$ Technic employed by the Penna. State Live Stock and Sanitary Board, Dr. A. B. Hardenburgh, Director. 
bacillus, glanders bacillus grows evenly through the bouillon instead of upon the surface.

At the end of six or eight weeks the flasks are removed from the incubator and placed in a sterilizer at $100^{\circ} \mathrm{C}$. for at least two hours. This process kills the bacilli and extracts the toxic principles. The entire solution is evaporated down to $\frac{1}{10}$ of its volume, filtered in small bottles, and sterilized. This is called concentrated mallein, and is kept in this form until ready for use.

Before using it is diluted to its original volume with 0.5 per cent. phenol solution, and passed through a porcelain filter. This process removes all the bacilli, and renders the solution aseptic and ready for use.

Ophthalmic mallein is prepared by taking one part of concentrated mallein and adding 20 parts of absolute alcohol. This forms a precipitate that is filtered and dried in a desiccator over sulphuric acid. A 5 per cent. sclution of the powder is made with sterile water.

Subcutaneous Mallein Reaction.-The dose of mallein for a horse is 0.4 c.c. of concentrated mallein, or 4 c.c. or 1 dram of the diluted product. The dose for a retest is 0.8 e.c. of concentrated or 8 c.c. or 2 drams of the diluted solution. Mallein is injected subcutaneously in some convenient area, such as around the shoulder, which has been shaved and cleaned.

A positive reaction is based on the same principle as tuberculin, that is, a rise of temperature within twenty-four hours following the injection, with a local inflammatory reaction at the site of injection.

Ophthalmic Mallein Reaction.-Two or three drops of the aqueous solution are dropped into the inner canthus of one eye. In case of a positive reaction this is followed by a marked conjunctivitis, associated with a purulent exudate extending from the inner canthus similar to the reaction shown in Fig. 124. In most cases there is also a rise of temperature. Only one dose is to be applied in the ophthalmic mallein test. It is not considered necessary to sensitize the eye, as in tuberculosis. The ophthalmic mallein test has the same advantages as the ophthalmic tuberculin test, that is, one can obtain a reaction when dishonest horse dealers have injected mallein prior to a subcutaneous mallein test, also in cases of far-advanced glanders, which at times give no reaction to a subcutaneous injection of mallein.

\section{ALIERGIC REACTIONS IN TYPHOID FEVER}

In 1907 Chantemesse $^{1}$ observed characteristic inflammatory symptoms follow the instillation of typhoid-bacilli extract into the eye of

1 Deut. med. Wochenschr., 1907, 33, 1572. 
patients suffering from typhoid fever. Kraus ${ }^{1}$ and his associates, repeating these experiments, could not convince themselves of the specificity of this reaction, stating that healthy individuals also give it to some extent, and that other bacterial extracts cause similar symptoms in typhoid-fever patients. In addition he tried a cutaneous reaction, but without result. Zupnik, ${ }^{2}$ on the contrary, states that a cutaneous reaction is useful, while the ophthalmic reaction is not useful. Deehan ${ }^{3}$ obtained a weak to moderate reaction in 12 cases of typhoid fever, whereas eight control cases showed none. Floyd and Barker ${ }^{4}$ obtained positive results in 19 out of 30 cases and none in 18 controls, including two cases of paratyphoid fever. Chaufford and Trosier $^{5}$ reported unfavorably on the reaction.

Austrian ${ }^{6}$ has reported very favorably upon an ophthalmic reaction in typhoid fever following the installation of "typho-protein" prepared by cultivating a large number of different strains of typhoid bacilli, precipitating the protein with alcohol, drying the precipitate, and redissolving in water so that from one-third to one-half milligram is contained in each drop. In typhoid-fever patients reaction of the palpebral conjunctiva of the lower lid and of the caruncle appears on an average of two and a half hours later, reaching the maximum about the sixth hour, and usually subsiding within forty-eight hours. 'In 75 cases of typhoid fever this test was found positive in 71 and negative in four. In three cases the eye test antedated the Widal reaction, and in only 23 per cent. was the Widal reaction positive at as early a date as the eye test. A study of 190 persons normal or ill with diseases other than typhoid has convinced Austrian of the specificity of the test, and he recommends it as an aid to diagnosis on account of its simplicity and the absence of any discomfort to the patient.

More recently, Gay and Force ${ }^{7}$ have reported favorably upon a cutaneous reaction indicative of immunity against typhoid fever. The preparation which they used, "typhoidin," is prepared in the same manner as Koch's old tuberculin: 250 c.c. of a 5 per cent. glycerin bouillon is inoculated with Bacillus typhosus and cultivated at $37^{\circ} \mathrm{C}$. for five days. It is then reduced, without filtration, to one-tenth of its original

1 Wien. klin. Wochenschr., 1907, 20, 1335.

${ }^{2}$ Münch. med. Wochenschr., 1908, 45, 148.

${ }^{3}$ Univ. Penn. Med. Bull., 1909, 22, 6.

4 Amer. Jour. Med. Sci., 1909, 38, 188.

6 Compt. rend. Soc. de Biol., 1909, lxvi, 519.

6 Bull. Johns Hopkins Hosp., 1912, 23, 1.

${ }^{7}$ Archiv. Int. Med., 1914, 13, 471. 
volume by evaporation over an acetone bath for about eight hours. A control solution of sterile 5 per cent. glycerin bouillon is prepared in the same manner.

The skin of the forearm is cleansed with alcohol, and two abrasions are made with the von Pirquet borer, as described under the cutaneous tuberculin test. The "typhoidin" is applied to one cut and the control fluid to the other. The reactions are observed six and twenty-four hours later. Occasionally there is a traumatic reaction in the control, but a positive reaction may be detected by a wider areola and increased induration.

Positive reactions were secured in 95 per cent. of cases that had recovered from typhoid fever, two of the cases having had the disease respectively forty-one and thirty-three years before. The reaction was found negative in 85 per cent. of individuals not having typhoid fever. Of 15 persons immunized by the army method, from four and threequarter years to eight months previously, nine gave a positive skin reaction. Twenty-four individuals immunized by a sensitized vaccine (Gay and Claypoole) for from one to eight months previously reacted positively. The test is advocated as a means of determining whether or not a person possesses immunity to typhoid fever, either acquired by recovery from the disease or by artificial immunization.

\section{ALLERGIC REACTIONS IN OTHER DISEASES}

Gonococcus Infections.--In 1908 Irons ${ }^{1}$ reported general and local reactions in persons suffering from gonococcus infections following the subcutaneous injection of gonococcal vaccines. This reaction has been observed by Bruck ${ }^{2}$ in epididymitis, by Reiter ${ }^{3}$ in pelvic infections in women, and also by other observers in other conditions.

Experiments with glycerin extracts of the gonococcus prepared from several strains, singly or combined in one preparation, have yielded Irons ${ }^{4}$ well-defined cutaneous reactions. These tests were conducted after the method of von Pirquet's tuberculin test.

Diphtheria.- Shick has advocated the intracutaneous injection of a minute dose of diphtheria toxin as a test for antitoxin in the serum of an individual. If sufficient antitoxin is present, the toxin is neutralized and no local disturbances are apparent; otherwise local inflammatory

1 Jour. Infect. Dis., 1908, v, 279. $\quad 2$ Deut. med. Wochenschr., 1909, xxxv, 470.

${ }^{3}$ Zeitschr. f. Geburtsh. u. Kinderh., 1911, Ixviii, 471.

${ }^{4}$ Jour. Amer. Med. Assoc., 1912, lviii, 931. 
areas may be observed. This test is not regarded as an allergic reaction; a further description of it will be found in Chapter XXX, under Diphtheria.

Allergic reactions have also been observed, mostly in experimental animals, in leprosy, sporotrichosis, in diseases due to hyphomycetes, and in pregnancy. Further investigations will, no doubt, disclose reactions of practical value in other diseases, such as rabies, scarlatina, measles, etc., following the successful isolation and cultivation of their respective causative microparasites.

\section{ALLERGIC REACTIONS AS A MEASURE OF IMMUNITY}

Based upon the assumption that the antibodies (anaphylactins) concerned in splitting their specific protein antigen at the local site of application (as tuberculin, mallein, luetin, and typhoidin), with the liberation or formation of the toxic material (anaphylatoxin) responsible for the local reaction of infiltration, edema, pain, and redness of the skin, are the same as those which bring about the destruction of this protein antigen in a living state as in the form of living microparasites, it is at once apparent that these reactions are of value not only in the diagnosis of various infections, but also as a measure of the defensive power or immunity of a person to a certain disease after recovery from this disease or after active immunization by means of a vaccine.

In other words, an allergic reaction may have a prognostic value. As pointed out by Gay and Force, in typhoid immunization the continued ability of a person to react positively to typhoidin may be accepted as an indication of the presence of antibodies and of sufficient immunity against typhoid fever; when, however, a person fails to react, this may be regarded as an indication for further immunization. Likewise in cowpox vaccination an immediate or "immunity reaction," consisting in the development of a small papule at the site of inoculation within twentyfour to forty-eight hours after vaccination, indicates that the person possesses the specific antibodies and does not "take" in the usual sense of the term, because the virus or antigen has been acted upon at once by the antibodies present. For this reason vaccinations should be inspected within a few days after inoculation, as after five days this "immunity reaction" disappears and the result may be interpreted as a failure to vaccinate successfully, whereas, on the contrary, it may actually indicate a state of immunity. 


\section{CHAPTER XXIX}

\section{ACTIVE IMMUNIZATION.-VACCINES IN THE PROPHYLAXIS AND TREATMENT OF DISEASE}

WhILE the importance of natural immunity must not be underrated in the protection it gives us after bacterial invasion has occurred, this immunity is, however, usually relative and seldom absolute, and may afford insufficient protection if the invading bacteria are numerous, or particularly virulent, or if the natural resistance of the organism is weakened by fatigue, disease, or injury.

Usually the best and most lasting immunity is that actively acquired, in which our own body-cells are stimulated or trained, as it were, to produce specific antibodies against the offensive forces of a particular bacterium or other pathogenic agent. A well-marked and lasting degree of this form of immunity usually follows recovery from many of the acute infections, particularly the acute exanthemata, such as smallpox, scarlatina, measles, typhoid fever, typhus fever, etc. In other infections, such as erysipelas, gonorrhea, and pneumonia, the immunity is less complete, of short duration, or entirely absent, and, indeed, a state of hypersusceptibility may actually follow.

It is very important, in this connection, to remember that the degree of immunity is not necessarily in proportion to the severity of the disease; thus a mild infection may be followed by the much-desired immunity, and while in general there is not considerable protection without infection, the latter does not necessarily imply that a virulent infection, or even the actual disease itself, is present, for discoveries have shown that an active immunity may be acquired by inoculation with the antigen so modified or attenuated that it can stimulate the production of specific antibodies without producing the disease or otherwise greatly disturbing the health of the individual.

Historic. - The facts here detailed are well illustrated in the history of vaccination in smallpox and the development of vaccine therapy in general. Hundreds of years ago the people of the eastern countries were accustomed to expose their children to a mild case of smallpox in order that a similar mild infection might be acquired and a lasting im- 
munity thus secured with the least danger to life. This practice, however, was not without risk, as the mild disease not infrequently became a virulent one. Later the dose of infectious agent was decreased by applying the virus to a small abrasion on the skin, and the resulting mild but genuine attack of smallpox usually conferred the much-desired immunity. But here again the severity of the disease was not under control, as the virus occasionally assumed increased virulence and induced severer infections than were desirable. Finally, Edward Jenner observed and showed experimentally (1796) that when cowpox virus is inoculated into the human being, a trivial infection, since called "vaccinia," is induced, and that this is followed by an absolute or nearly absolute immunity of many years' duration against smallpox. In other words, the virus, in its passage through the cow, becomes so modified that it can no longer produce smallpox, but is still able to stimulate the production of the specific antibodies against this disease. Jenner worked so hard to establish the truth of this finding that he had little time to devote to the mechanism involved in the process.

In other words, the work of Jenner was largely empirical, and the explanation was not forthcoming until many years later, when Pasteur laid the basis of scientific immunization by discovering that light, high and low temperature, and exposure could so reduce the virulence of a microörganism that while its injection into an animal was practically without danger or ill effect, it could still stimulate the protective mechanism of the host and induce a high degree of immunity.

That this could be done was a fact discovered accidentally by Pasteur in 1879 while working with the organism of chicken cholera. After an absence from home he found, on examining his cultures on his return, that they had become innocuous - that hens could bear without any ill effect inoculation of what would formerly have been a lethal dose. The prolonged cultivation of the microörganism had caused its attenuation and Pasteur immediately grasped the far-reaching importance of this discovery. He conjectured that it might be possible to produce a mild and modified form of chicken cholera with a vaccine of the attenuated microörganism which would afford protection to the fowl against the severe form of the disease. This proved to be the case, and established the possibility of so modifying or attenuating the virulence of a virus or germ that, while its administration is not followed by the actual disease, it ts capable of so stimulating the body-cells that the specific antibodies are produced. This discovery formed the basis of prophylactic immunization or bacterin therapy in general. 
Following this discovery, much work was done, for it appeared that the question of prevention of any bacterial disease simply depended upon whether the bacterium could be cultivated and so modified or attenuated that while its injection would not be followed by disease or other harmful effects, it would be capable of causing the production of specific antibodiess.

Naturally, most of the earlier work was done with the infections of the lower animals, and, consequently, most discoveries were directly beneficial to them. Pasteur soon devised a method of attenuating anthrax bacilli by exposing them to certain temperatures for varying lengths of time, so that a vaccine could be prepared that has proved of great value. Later the same observer discovered a method of attenuating the virus of hydrophobia by a process of drying, and devised a practical method of prophylactic immunization against this disease. In addition to these his vaccines against swine erysipelas, symptomatic anthrax, and rinderpest have become well known.

The knowledge gained from a study of the diseases of the lower animals and the aid given them has been applied to human medicine with considerable benefit, not only in prophylactic immunization, but also in therapeutics (bacterin therapy). The latter application is a more recent discovery, for which we are mainly indebted to the researches of Wright, Leishman, Douglas, and their colleagues.

Nomenclature.- The word vaccine is from the Latin vacca (a cow). Cowpox was called "vaccinia," or the cow disease, and Jenner designated protective inoculation against smallpox with cowpox virus as vaccination. With true courtesy Pasteur adhered to Jenner's nomenclature and applied the term vaccine to emulsions of dead or attenuated bacteria. This is unfortunate and tends to create confusion, as the term vaccine is inseparably associated with cowpox virus or lymph. The term bacterial vaccine has become widely known, and is used to designate bacterial suspensions prepared for purposes of immunization. There is no essential difference, however, between cowpox vaccine, which contains the modified germ or virus of smallpox in a diluent of lymph, and a bacterial vaccine containing the germ, modified by some physical or chemical agency in a diluent of saline solution or bouillon. It is, however, well to reserve the unqualified term "vaccine" for cowpox virus, and to retain the designation "bacterial vaccine" for suspensions of attenuated or dead bacteria. More recently the term bacterin has been applied to the latter, but this would imply an extract of bacteria, as, e. g., tuberculin, which is not always the case. 
Some confusion likewise exists as regards the terms serum and vaccine therapy. Serum therapy is a process of passive immunization induced for either protective or curative purposes by the injection of the bloodserum of another animal that has been actively immunized by inoculation with bacterial toxins or the bacteria themselves, as, for instance, the injection of diphtheria or tetanus antitoxins. Vaccine or bacterin therapy is a process of active immunization brought about by the injection of the bacteria or their products directly into a patient. Bacterial vaccines that are simple emulsions of dead or attenuated bacteria are not, therefore, serums, and the indiscriminate use of the two terms is much to be regretted.

Method of Preparing Vaccines.-It may be stated that, in general, the specific microörganism or virus used in a vaccine should be modified as little as possible, or just sufficient to rob it of its disease-producing power. For example, typhoid bacterial vaccine is prepared by suspending the bacilli in salt solution and exposing them to just enough heat to modify them so that they can no longer multiply. The less modification, the better the vaccine. If the exposure is too prolonged or the temperature too high, the vaccinogenic power of the bacilli is destroyed, and the suspension in salt solution is no more potent or of no greater value than the salt solution itself. Therefore the nearer the vaccine approaches the fully viable virus or microörganism, the more potent it will be. The proper preparation of a vaccine, therefore, is the first step to successful vaccine therapy.

Vaccination, using the term in its broadest sense, may be performed for prophylactic purposes and curative immunization in the following ways:

1. The living microörganism may be inoculated. This is the ideal method, but for obvious reasons has not been generally used and is still in the experimental stage. It is based upon experimental observations made on the lower animals that an organism may be so introduced as to render it incapable of producing disease, but may, however, stimulate the production of specific protective antibodies. Most work on typhoid fever is at present being done in the Pasteur Institute at Paris. Evidence thus far indicates quite conclusively that the typhoid bacillus is unable to produce typhoid fever unless it is introduced into the gastro-intestinal tract, and the subcutaneous injection of living bacilli, modified only to a slight extent by artificial cultivation, is not followed by ill effects and produces a high grade of immunity. The principle is a good one, i. e., in a vaccine the microörganism should be modified as little as possible. 
For obvious reasons this method is being extensively tried out on the lower animals, especially the chimpanzee, before it is applied to human medicine.

2. By inoculation with a modified virus or with microörganisms attenuated or modified according to various methods.

(a) By passing the virus through a lower animal, as the passage of smallpox through the heifer when the virus is incapable of producing smallpox, although vaccinia confers a specific immunity against smallpox. A vaccine for swine erysipelas is prepared in the same manner (Pasteur) by passing the bacillus through the rabbit several times, which increases its virulence for the rabbit but decreases it for swine.

(b) By exposing suspensions of microörganisms to heat. They are usually grown on a suitable solid medium, suspended in salt solution, and exposed to a temperature at or just above their thermal death-point for just sufficient time to kill or attenuate them in so far that they cannot multiply. The same result may be secured by longer exposure to a lower temperature. To secure a potent vaccine the principle of minimum exposure at the minimum temperature should be observed, the question of viability being controlled by culturing the vaccine. Most bacterial vaccines are prepared in this manner. Of course, the fact that a vaccine is sterile could be confirmed by exposing it to a very high temperature, but in this case the product may be of no more value than so much salt solution. Usually an exposure of $53^{\circ}$ to $60^{\circ} \mathrm{C}$. for from one-half to one hour is sufficient, and only exceptionally are these limits exceeded.

(c) By exposing the microörganism to air and light. The first bacterial vaccine (chicken cholera) was accidentally prepared by Pasteur in this manner.

(d) By desiccating or drying the virus. This is the method of vaccination in rabies, as the virus contained in the spinal cord of rabbits is dried for varying lengths of time, emulsified, and injected. The longer the period of drying, the greater the attenuation, and in this manner the strength of the vaccine and the progress of immunization are under control.

(e) By exposing the microörganism to a high temperature for varying lengths of time. Anthrax vaccine, for the immunization of lower animals, is prepared in several strengths by exposing suspensions of the bacilli to $42^{\circ} \mathrm{C}$. for varying periods of time.

$(f)$ By exposing the microörganisms or their products to certain chemical germicides, as in the preparation of anthrax vaccine by Roux, 
diphtheria and tetanus toxins (Behring), and in some preparations of tuberculin.

3. By inoculating with bacterial constituents, as the soluble toxins, bacterial extracts, and products of bacterial autolysis, as in the preparation of Koch's tuberculin T. R., Koch's old tuberculin, mallein, diphtheria and tetanus toxins, etc.

In Chapter XIII a method is given for preparing bacterial vaccines, of which typhoid vaccine is a type. Special methods of preparing certain bacterial vaccines and other vaccines, such as cowpox virus and rabies vaccine, are given in this chapter.

Mechanism of Active Immunization.-As was stated in the chapters on Immunity, in the presence of an infection the host endeavors to protect itself and overcome the invaders by various means, among which are phagocytosis and the production of more or less specific antibodies that may neutralize the poisons of the parasite (antitoxins), directly kill or destroy them (bactericidans), or so lower their vitality or resistance that they are more easily phagocyted (opsonins or bacteriotropins).

During an infection, one or more of these protective forces, or all of them, are brought into action. After the infection has been overcome, the antibodies do not always disappear at once, but remain for some time in the body-fluids and gradually diminish, so that if the host is reinfected with the same parasite, the antibodies are at hand immediately to overcome it and protect the host absolutely, or at least so to modify the pathogenicity of the parasite or neutralize its products that the host will suffer but mildly while the parasite is being finally destroyed. The concentration and duration of the various defensive forces or antibodies vary in different individuals and in different infections, so that the degree and duration of an active acquired immunity are variable factors. Nevertheless-and this is the basis of active immunization - an animal or a person may have specific antibodies for a certain parasite, produced by its own cells, without actually or necessarily suffering from the disease, due to the effects of inoculation with the germ or virus in a modified or attenuated form. The dose of vaccine may be so controlled that general symptoms the result of stimulation of the body-cells are slight or not at all apparent, and, by gradually increasing the dose, more and more antibodies may be produced until a high degree of immunity is secured.

Active immunization may be practised for two purposes:

(a) For prophylaxis, or the prevention of a disease, which is accom- 
plished by the production of antibodies so that they may be at hand to overcome an infection if it should occur. For example, the antibodies specific against the virus of smallpox may be produced by inoculation with cowpox virus, so that for years the system will be protected against smallpox. Even if vaccination has been delayed until smallpox has actually been contracted, inoculation with cowpox virus early in the period of incubation so stimulates the body-cells that sufficient antibodies are produced to modify and lessen considerably the virulence of the smallpox virus.

This is especially true in rabies, when the vaccine is given in such doses and at such intervals that sufficient antibodies are produced to neutralize the effects of rabic virus and actually to destroy it during the period of incubation or during the interval that elapses between the time of infection and the appearance of the symptoms. In this way the great majority of infected persons escape the sufferings of rabies by enduring the relatively slight discomfort consequent to a series of subcutaneous injections.

(b) For the treatment of disease. This is the bacterin or vaccine therapy, a method that owes its origin to the researches of Sir Almroth Wright and his colleagues. It was originally employed in the treatment of those infections that showed a tendency to chronicity in which true toxins played little or no part. Since recovery from an infection is in general dependent upon the mechanical removal of the infecting agent, aided by antibodies that facilitate phagocytosis or directly destroy the invading bacterium and neutralize its products, Wright believed that in chronic infections autovaccination, or stimulation of the body-cells to the production of antibodies, by reason of the fact that it is irregularly timed, is generally insufficient or altogether absent. For these reasons he believes that any stimulus that will arouse the body-cells to throwing into the circulation substances from the invading bacterium or diseased tissues, may result in increased antibody formation, followed eventually by clinical improvement or cure. In certain cases this stimulation may be secured by judicious massage or manipulation of the diseased part, by passive hyperemia (Bier), or by similar procedures.

However, if the microörganism is obtained and cultivated artificially, it is possible, in many instances, so to modify or attenuate the bacilli (usually by heat) that they may be reinjected into the patient in sufficient numbers to furnish the stimulus necessary for arousing dormant or uninvolved body-cells to produce the opsonins and other antibodies necessary for overcoming the infection. In other words, with each in- 
fection the host endeavors to protect itself by producing antibodies. When the protection is insufficient, the infection will spread; when the antibodies are in excess, the infection is overcome; when the forces are about equal, a stage of chronicity may result in which the host becomes accustomed, as it were, to the invaders, and, while the infection does not spread rapidly, it does not, on the other hand, recede. In cases of the latter type an extra dose of bacterial stimulant (a bacterin) may arouse dormant or inactive cells to furnish an extra quantity of antibodies and thus turn the tide. In acute infections indifferent groups of cells may likewise be brought into action, although it is more likely that they are already involved, so that the extra stimulation in the form of bacterin must be cautiously and carefully applied, if applied at all.

In therapeutic inoculation, therefore, the fundamental principle is to stimulate in the interest of the infected tissues the unexercised immunizing capacities of the uninfected tissues. This is especially true in chronic infections, when the use of a bacterial vaccine may be likened to the application of the whip to a lazy horse that is capable of further effort and work. In acute infections, however, while the cells are at work they may be capable of greater effort, but vaccines should be given cautiously, as they may, to use the same simile, act as a whip to a willing and well-worked horse that is unable to respond or does respond, with resulting disastrous overexertion.

It should be remembered, in this connection, that usual forms of treatment should be given while bacterin therapy is being instituted. For instance, it is useless to administer a vaccine to a patient with a suppurative fistula or sinus if an infected silk suture is directly responsible for the suppuration. The suture should be removed, if possible, and after this is done, a vaccine may be of considerable aid in overcoming the coincident infection.

In what manner can dead bacteria cause the production of antibodies? The mechanism is similar to that involved during infection with the living microörganism, and involves the first principles of immunity. The antigenic powers of a vaccine are probably always more or less inferior to the living antigen, as some principle may be lost during heating, drying, passage through animals, the action of germicides, etc. For this reason living vaccines are to be preferred, although, for obvious reasons, they cannot generally be employed in human practice.

Just what portion of the bacterial cells is mainly antigenic it is difficult to determine, for it probably varies with different species. With a true toxin, as, for example, the diphtheria bacillus, the toxin 
constitutes the main principle, and causes the production of an antitoxin as its main antibody; with other bacteria, such as the typhoid bacillus, a soluble toxin and an endotoxin in combination with the protein of the bacterial cell are probably the main antigenic factors responsible for the formation of a bacteriolysin, opsonin, antitoxin, agglutinin, etc.

In brief, the antigenic principles of a microörganism are mainly thermostabile and fairly resistant substances, so that a bacillus may be so attenuated or altered that it cannot multiply or produce disease, and yet is capable, through the agency of substances that have escaped destruction, of causing the production of specific antibodies.

As was previously stated, vaccines may cause the production of different antibodies. As curative agents, however, it would appear that they are most efficacious in those infections in which phagocytosis is known to be chiefly concerned in the defense of the host, e.g., in staphylococcus infections. As shown by Wright and Douglas, Neufeld and Rimpau, a bacterial vaccine facilitates phagocytosis, not so much qualitatively or quantitatively as through the production of specific substances that act directly and primarily upon the bacteria and render them more vulnerable to phagocytosis (opsonin or bacteriotropin). Wright has advised a method of opsonic measurement, previously described, for measuring the immunity response, but, as will be understood, while the opsonin may be the chief antibody, it is seldom if ever the only one, so that the opsonic index is but one measure of defensive power.

According to Vaughan, a microörganism is directly responsible for the production of a specific proteolytic ferment capable of causing the disintegration or destruction of the bacterial cell and its products. The ferment is the antibody, and is produced during an infection or by a vaccine in just the same manner as antibodies in general are produced. In other words, Vaughan regards antibodies as of the nature of proteolytic ferments; thus the protein of the microörganism composing a vaccine produces a specific proteolytic ferment capable of overcoming its substratum when it meets the latter in the form of the invading microörganism of an infection.

For example, the tissues affected may be unable to produce a sufficient quantity of the specific ferment necessary to overcome the infection. The injection of bacterial protein in another and healthier part of the body leads to the production, in this locality, of a specific ferment that is conveyed to the diseased area by way of the circulatory system, 
and aids in destroying the protein of the infecting microörganism and its tissues.

Living versus Dead Vaccines.-Barring accidents, the employment of a living virus is the most certain way of calling forth a maximum output of antibodies. There is at present no satisfactory explanation for this except that heat-labile substances destroyed in the ordinary preparation of bacterial vaccines have antigenic properties (Smith). Living vaccines are also capable of penetrating into deeper tissues, whereas dead vaccines may remain where they are deposited. Similarly living viruses are capable of exerting a continuous action and of delivering an infinite number of blows, whereas the injection of a dead virus produces an interrupted action and deals but a single blow. The actual dangers of using a living vaccine, as the possibility of it being too virulent and thus producing disease, or of regaining virulence or producing chronic "carriers" preclude their general employment in human practice.

Sensitized Vaccines.-Besredka and Metchnikoff ${ }^{1}$ have suggested a plan of injecting a vaccine composed of living bacteria that have been immersed in their specific immune serum or, in other words, have been sensitized (serobacterin). They believe that such vaccines produce practically no negative phase, but only slight local and general reaction, and that the general response with antibody formation is facilitated. This principle is supported by the observations of Theobald Smith, who found that the experimental injection of a toxin-antitoxin mixture aids the dissemination of the toxin through the body quite generally; whereas the pure toxin is chiefly held at or near the place of injection. This diffusion tends to cause maximum antibody formation over an entire portion of the body. by a relatively small amount of free or easily dissociated toxin in the toxin-antitoxin mixture. Smith inclines to the belief that a similar phenomenon of diffusion may occur with sensitized dead bacteria.

In addition, the specific immune serum may aid in the disintegration of the bacterial cell, either through the attachment of a bacteriolytic amboceptor that would tend to lyse the bacterium with a complement of the tissues, or through a preliminary action of opsonin which prepared the bacterium for ultimate destruction and liberation of antigenic principles.

Autogenous versus Stock Bacterial Vaccines.-It may be stated in general that autogenous vaccines, $i$. e., those prepared from the pa${ }^{1}$ Ann. de l'Inst. Pasteur, 1913, xxvii, 597. 
tient's own bacteria, should be used whenever possible, especially in the vaccine treatment of disease. To be successful, vaccine therapy demands that the bacteria be as little changed as possible. Before they are killed, the bacteria should be endowed with as many of the potencies as possible with which they maintain themselves in the body. As .these potencies do not remain unchanged during artificial life, as the loss of capsules, loss of virulence, etc., it is advisable to secure the organism causing the infection as quickly as possible and prepare a vaccine without undue delay.

Variants may occur among cultures of the same species, and the injection of one strain may not protect against another, as shown by Neufeld for the pneumococcus. In the use of an autogenous vaccine this risk of using an alien species or a different strain is reduced to a minimum.

In some cases the difficulty of securing and of identifying the infective agent may be so great that much time is lost in preparing autogenous vaccines, as, for instance, in gonorrheal and tuberculous infections, and in such cases it may be necessary to use a stock vaccine.

In protective immunization stock vaccines are used, as, for instance, in the preparation of typhoid vaccine. In certain instances, as in gonococcus and tuberculous infections, stock vaccines possess but slightly inferior therapeutic value as compared with autogenous vaccines, not to mention the delay and difficulty in cultivating and preparing autogenous vaccines. In many other infections, as with the Bacillus coli and streptococci, stock vaccines possess little or no value.

The wholesale manufacture of various bacterial vaccines and their indiscriminate use have brought disappointment to many. Rational and scientific vaccine therapy does not consist in the administration of ready-made, uncertain, and oftentimes hit or miss mixtures, recently so widely exploited. Especially is this true in the use of vaccines for therapeutic purposes. The bacteriotherapeutist must possess sufficient skill to enable him to make bacteriologic diagnoses, prepare autogenous vaccines, and skilfully guard their administration. In most instances these requirements are fulfilled by coöperation between the clinician and bacteriologist, or, better, by one who is especially trained in vaccine therapy.

The Negative Phase.-As has been stated in a previous chapter, certain local, constitutional, and focal disturbances may follow the injection of a bacterial vaccine. The first or local symptoms are not infrequently due to an excess of preservative in the fluid or to the presence 
of contaminating microörganisms, but abscess formation is distinctly rare. I, in common with others, prefer to find slight focal and constitutional symptoms following the first one or two doses of vaccine. In furunculosis, for instance, when the old lesions discharge a little more and one or two others threaten to develop during a day or so following the injection of vaccine, I feel assured that the ultimate result will be good. In tuberculin therapy, however, the trend of opinion is very much in favor of administering doses so small that no appreciable focal or constitutional lesions will follow. Both the pathologic and the immunologic process concerned in tuberculosis are somewhat different, and in ordinary bacterin therapy a slight focal disturbance is desirable and indicates that the vaccine in question possesses some potency. Allen, who has an exceptionally rich experience in vaccine therapy, frequently mentions the desirability of administering doses sufficiently large to evoke slight reactions.

Following the administration of a vaccine it is believed that the quantity of opsonin in the body-fluids is temporarily decreased, and that the inoculated person is, therefore, more susceptible to infection (negative phase) (Fig. 56). It can readily be understood how a vaccine may temporarily depress the cells and defensive mechanism in general, but just how it may bring about an actual decrease in opsonin it is more difficult to understand, as the actual amount that may be used in dealing with the vaccine itself must be small. Veterinarians are careful not to expose cattle to infection immediately after they are immunized with anthrax vaccine, on account of this hypersusceptibility to infection. In general, however, I have observed that most immunizators are prone to regard the question lightly, and to neglect the importance of the negative phase, whereas some deny that it ever exists.

Contraindications to Active Immunization.--It should be emphasized that a properly prepared vaccine is a potent substance capable, when given in excessive dosage or when otherwise injudiciously administered, of doing much harm. A vaccine stimulates body-cells, and unless the cell can withstand the stimulation, the administration of vaccine may do actual harm. This is the main reason why vaccines should be used very cautiously, if at all, in the treatment of severe generalized infections. In passive immunization the conditions are different, as the body-cells are not taxed, but rather, through the neutralization of the toxic substances which they are combating, they are relieved, and an antibodyladen serum may, therefore, be freely administered in severe infections. 
In active immunization for therapeutic purposes the conditions should be carefully weighed and the treatment conducted by one who is qualified to judge of the potencies of harm and good in a vaccine, and who has had sufficient experience to guide him in dosage and frequency of inoculation, the main objects being to tide over and aid nature during an acute infection, and to arouse and stimulate her during a chronic infection.

In prophylactic immunization the physician should satisfy himself that the patient has no latent or active infection that may be rendered worse during the temporary depression that follows inoculation. It is true that this depression is fleeting and temporary, and that the possible harm incurred may be far outweighed by the ultimate good, but vaccines should be given with proper discernment and not carelessly and injudiciously. These remarks have no relation to cowpox vaccination, where the good so far overbalances the possible harm that in general all persons should be vaccinated, especially if an epidemic is impending.

(a) Tuberculosis. - There is at present some discussion relative to the harm that may be caused in tuberculosis by typhoid immunization. Probably all will agree that a patient with an active and acute tuberculous lesion should be refused inoculation, but when the lesion is quiescent or healed, or in the early latent stage, it is indeed difficult to understand how a prophylactic dose of typhoid vaccine will do more or as much harm as an attack of tonsillitis, rhinitis, or some similar acute infection.

(b) In diabetes, carcinoma, and other debilitating conditions vaccines should not be administered unless the indications or requirements are unusually urgent.

(c) Advanced nephritis, especially parenchymatous nephritis, may be regarded as contraindicating the administration of a vaccine.

\section{PROPHYLACTIC IMMUNIZATION OR VACCINATION}

\section{SMALLPOX}

Historic.-Just when and where smallpox vaccination was first practised is not known. The original method of inducing immunization against the disease by introducing the virus from a smallpox patient into a healthy person through an abrasion of the skin and thus greatly diminishing the virulence of the disease was practised by the Turks during the eighteenth century, the chief object being to preserve the beauty of the young Turkish and Circassian women. In 1878 Lady Mary Montagu, the wife of the British Ambassador at the Ottoman court 
in Constantinople, observing this practice among the Turks, had her own son and daughter inoculated and was largely instrumental in establishing the practice in Europe.

As regards the prophylactic value of this method of inoculation in England and continental Europe, statistics are incomplete, but the literature of contemporary writers shows that protection was usually complete. The induced disease was not, however, always mild, and not infrequently assumed an unexpected virulence that not only proved distressing and even fatal to inoculated individuals, but also constituted a source of infection to a community. While, therefore, the underlying principles were sound, and while these early attempts at preventive immunization mark an epoch in the history of medicine and of the world, it was not until Edward Jenner made his investigations into a theory held by farmers and by experimental evidence established it as true that a satisfactory method of immunizing the body against smallpox was introduced.

The peasantry in various parts of Europe, and especially in England, had generally observed that those who had had sores on their hands contracted from similar lesions on the teats of cows, usually escaped smallpox infection when the disease was epidemic in a community. In fact, it is said that several farmers deliberately inoculated members of their family with cowpox lesions and that these escaped smallpox.

Edward Jenner was a physician practising in Berkeley, Gloucestershire, and frequently used the method of direct inoculation from a mild case of smallpox among his patients. While a student he was impressed with the traditions of cowpox vaccination, and finding that they were largely true, determined to make experimental tests. On May 14, 1796, he vaccinated a boy, James Phipps, with virus from a cowpox lesion on the hand of a dairy maid, Sarah Nehnes, and on July 1st he inoculated the same boy with pus from a smallpox patient without resulting infection. In 1798 he furnished further proof that cowpox will afford protection against smallpox by inoculating a child direct from a vesicle on the teat of a cow, and continued the inoculation from arm to arm through a series of five children, after which all were inoculated with smallpox virus, without a single case developing. In the same year he published "An Inquiry into the Causes and Effects of the Variolæ Vaccinæ," illustrated by four plates, and within a year or two vaccination became general over Europe.

Vaccination was introduced into the United States in July, 1800, by Dr. Benjamin Waterhouse, Professor of Physic at Harvard University, 
who vaccinated his own children. At about the same time John Redman Coxe, of Philadelphia, vaccinated his oldest child and then tested the experiment by exposing him to cases of smallpox. This bold repetition of Jenner's experiment considerably strengthened public confidence in the method and the practice spread rapidly. Thomas Jefferson, writing in 1806 to Edward Jenner, said: "Future generations will know by history only that the loathsome smallpox existed and by you has been extirpated."

But Jenner and his earlier supporters met with much opposition, often bitter and unrelenting, and this is readily understood when it is realized that even at the present day, over a hundred years later, cowpox vaccination still has its opponents, in spite of the fact that the value of the method has been established, and it has been found the greatest of all boons to the human race, and notwithstanding that it has been definitely proved that a thorough and continuous practice of the operation would quickly eradicate smallpox from the face of the earth. This opposition is especially pernicious and unjust, since the practice of former years of vaccinating by direct transmission from arm to arm has been entirely abandoned, and that animal lymph, prepared and collected under strict aseptic precautions, is being used exclusively.

The Relationship of Variola and Vaccinia.-The relationship of variola to vaccinia has been discussed since Jenner's time, but no adequate explanation has been found.

According to the general belief the smallpox virus, whatever it may be, is altered in its passage through a lower animal, and loses forever its power of producing smallpox, but is still so closely related that the antibodies it produces are sufficient to protect against smallpox.

The close interrelationship existing between vaccinia and variola is shown by the presence in the virus of both, and in section of the skin of both, of microscopic cell inclusions, first described by Guarinieri in 1892 . This finding has been confirmed by Pfeiffer in Germany and Councilman and his associates in this country. These investigators have made extensive studies of these bodies, and believe them to be protozoa intimately associated with the etiology of vaccinia and variola. More recently, Fornet has described certain small, diplococcus-like bodies that were found in cowpox vaccine and in smallpox lesions. These are regarded as having an etiologic relationship to smallpox, and if these findings are confirmed, would prove the identity of variola and vaccinia.

Recent investigators, particularly Copeman, of England, and Brinkerhoff and Tyzzer, of America, have shown, by carefully conducted ex- 
periments, that vaccination will protect monkeys against subsequent inoculation with smallpox virus, and this completely confirms the early experiments of Jenner and others who proved the efficacy of vaccination by the "variolous test."

The Preparation of Cowpox Vaccine.-During the early days of vaccination it was customary to inoculate human beings with material obtained from the pustules of those previously vaccinated. The oldtime physician carefully removed choice scabs and carried them about in a special case ready for inoculation. While this method served its purpose well, there were several drawbacks to its use, the chief of which was the danger of transmitting syphilis. It has now for many years been the custom to use virus obtained from animals, the production of which can be carefully controlled and tested, any danger of transmitting syphilis being thus obviated, because the heifer or cow used in the preparation of the virus is not subject to this disease. The opponents to vaccination, however, persist in using old and obsolete statistics regarding the transmission of syphilis to support their claims, although these have absolutely no bearing upon the modern methods of preparing the virus.

Seed Virus.-This refers to the virus for vaccinating the calves or other animals used, and is a most troublesome factor to those engaged in this work. According to Park and Huddleston, a sufficient amount of vaccine virus should be on hand to vaccinate from 40 to 50 persons. Five children in good health and not previously vaccinated should then receive an inoculation, each spot being of the size of a ten-cent piece. On the fifth day after vaccination the upper layer of the resulting vesicle should be removed, and sterilized bone slips be rubbed on the base thus exposed. From 100 to 200 slips on each side of the slip may be charged from each child. The slips should be allowed to dry for a minute, and should then be placed in a sterilized box and preserved in cold storage, where they will remain active for at least two or three weeks. The aforenamed observers now use rabbits alternately to obtain seed virus.

Subsequent animals are vaccinated with any one of three vaccines(1) Slips charged from typical vesicles of a calf; (2) slips charged with the serum from a calf after removal of the vesicles; (3) the glycerinated virus may be used to vaccinate succeeding calves, but in this case it is necessary to keep the glycerinated virus for two or three months, since the use of fresh virus on a succession of calves leads to prompt degeneration of the vaccine and to the production of infected vesicles.

The New York Vaccine Laboratory produces a virus that is never 
more than four successive transfers from a human case of vaccinia, and is guaranteed to give 100 per cent. of "takes" in primary vaccination.

Animals.-Various animals have been used, but female calves from two to four months of age are preferable. Older animals may be used, and in several European institutes cows are usually employed. With properly constructed operating-tables, they may be handled with comparative ease. Rabbits have also been used, especially in propagating the seed virus and to obtain pure and highly active viruses.

The calves are kept under supervision for at least a few days. In some institutes they are tested with tuberculin, although with good veterinary inspection this test is not necessary. Soon after admission the animal is clipped and given a thorough cleansing, which includes the feet and the tail.

On the day before vaccination is to be performed the belly-wall is cleanly shaved from the cuneiform cartilage to the pubis, and well up on the inner sides of the thighs and the flanks. The skin is then thoroughly washed. Just preceding the vaccination the animal is fastened to the operating-table and the abdomen and inner surface of the thighs prepared as for an aseptic abdominal section, $i$. e., a thorough scrubbing with hot water, green soap, and soft brush, followed by alcohol and sterilized water, the parts being then dried with a sterile towel. All other parts are covered with sterile sheets, and the calf is now vaccinated under aseptic precautions.

Vaccination.-About 100 small scarifications are now made in these areas, preferably by cross-scratches or in rows of lines about one to two centimeters square and at least one to two centimeters apart. The scarification is simple, but usually brings a small amount of blood. After they have been made, they are mopped with sterile gauze and rubbed with the charged slips, using one or two slips for each small area, depending on the amount of virus each slip contains. The lesions are allowed to dry, and are then covered with sterile gauze or a simple protective paste, or are left entirely uncovered.

Precautions should be taken to keep the animals as clean as possible. Inoculated animals are to be kept in stalls or stables apart from those under observation. The stable should be so constructed that the floors can be flushed daily with a hose and hot water. Excreta should be removed promptly. No bedding is permissible, and means should be provided for fastening the legs and preventing the animal from kicking the scarifications. 
Collection.-Ordinarily, within forty-eight hours of vaccination, the scratches are pinkish, slightly raised, and papular, and within five or six days, depending upon the rate of development of the vaccine vesicles, the virus should be ready for collection (Fig. 127). The calf is killed and placed upon the operating-table. The appointments of the operating-room are usually equal to those in a well-equipped hospital

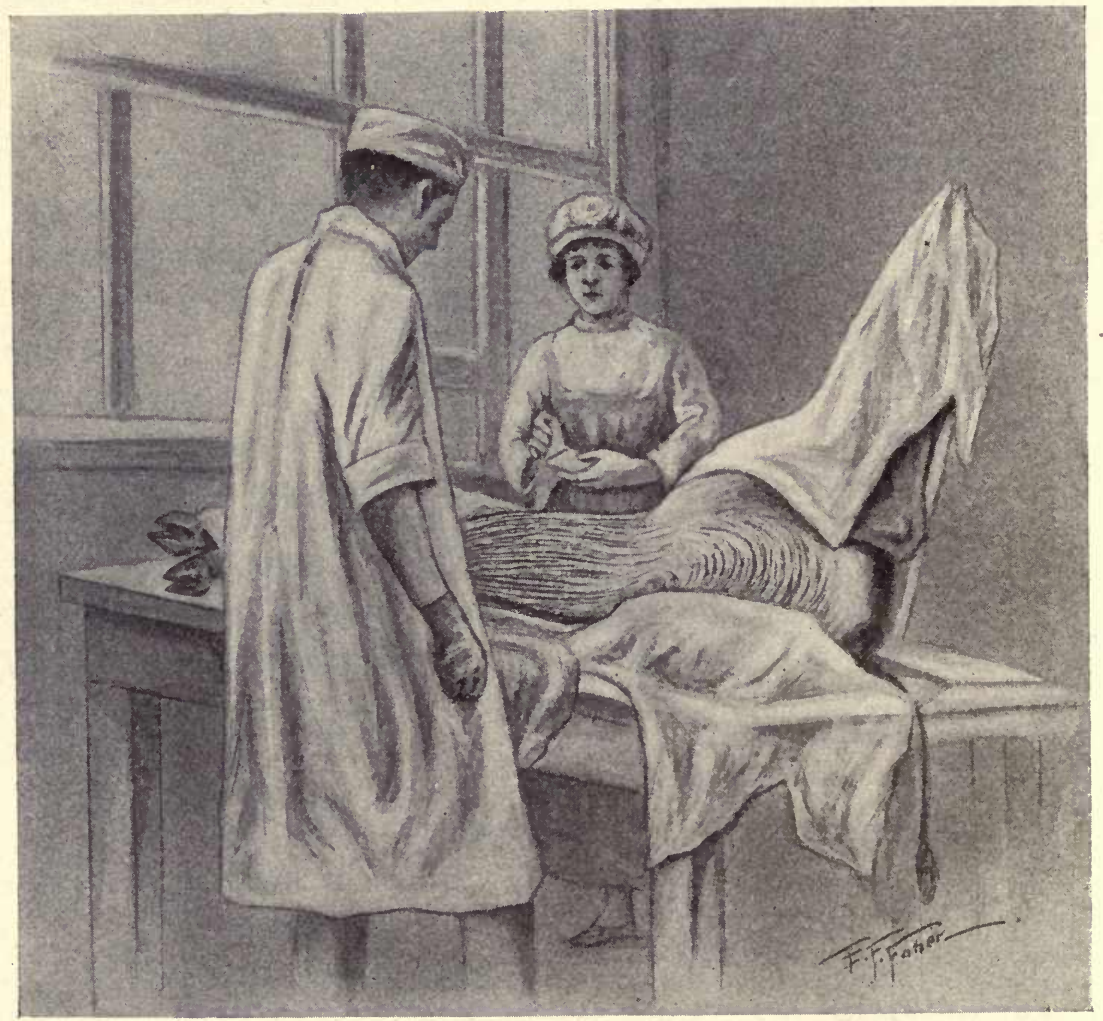

Fig. 127.-Production of Cowpox Vaccine.

Note the lines of cowpox lesions over the abdomen and flanks of the calf. The surgeon is about to cleanse this area in a thorough and careful manner, after which the cowpox material is removed with a curet and collected in a sterile vessel. All precautions are taken to insure as thorough aseptic technic as possible.

operating-room, being supplied with all conveniences and means for carrying out a careful, painstaking, and aseptic technic.

The exposed parts are covered with sterile sheets. The operator and his assistant are clad in aseptic gowns. The vaccinated field is thoroughly scrubbed with soap, sterile water, and gauze, and mopped with sterile gauze. Crusts are carefully picked off, and the soft, pulpy 
mass cureted off with a special, spoon-like curet and collected in a sterile vessel. After the curetage, serum exudes from the excoriated base of the vesicle, and ivory tips may be charged in this. The sticky and pulpy exudate is then mixed with four times its weight of glycerin and water (50 per cent. glycerin, 49 per cent. water, 1 per cent. phenol), and this is done most effectively by passing the mixture between the rollers of a Doring mill. The glycerinated pulp is allowed to stand for three or four weeks in order to allow bacteria, which are invariably present, to undergo dissolution. At the end of this time the glycerinated pulp is thoroughly titrated in specially constructed triturating machines, and put up in small capillary tubes, which are sealed, or "vaccine points" may be prepared. If properly preserved in sealed tubes in a dark, cool place the virus should remain active for at least three months.

According to Park and Huddleson, 10 grams of pulp and 200 charged slips would be an average yield from a calf, and when made up should suffice to vaccinate at least 1500 persons. Calves vary greatly in their yield of virus. Of two calves vaccinated in exactly the same manner, one may furnish material for 500 vaccinations and the other for 10,000 inoculations.

Testing the Vaccine. - The virus may be tested for its efficacy by a variety of methods. Calmette and Guerin ${ }^{1}$ inoculate rabbits upon the inner surfaces of the ears and estimate the potency of the virus from the speed of development and the size of the resulting lesions. Guerin ${ }^{2}$ estimates the potency of virus quantitatively by inoculating rabbits with serial dilutions ranging from $1: 10$ to $1: 100$. Fully potent virus should cause closely approximated vesicles in a dilution of $1: 500$, and numerous isolated vesicles in a dilution as high as $1: 1000$.

Quantitative estimation of the bacteria in the glycerinated virus is made by the plating method, and the vaccine used only when the numbers of bacteria have been greatly diminished or are entirely absent. The vaccine is also tested for tetanus by injecting relatively large quantities subcutaneously into guinea-pigs and mice.

Under the Federal Law of July 1, 1902, and the regulations framed thereunder, all firms manufacturing vaccine virus are required by the Secretary of the Treasury to obtain a license before they may sell their products in interstate commerce. The vaccine laboratories are carefully inspected by an official of the Hygienic Laboratory of the United States Public Health and Marine-Hospital Service; the inspector carries away with him as many samples of virus as he wishes, and addi-

${ }^{1}$ Ann. de l'Inst. Pasteur, 1902.

${ }^{2}$ Ann. de l'Inst. Pasteur, 1905. 
tional samples are purchased in the open market in different parts of the country. All these are subjected to a vigorous bacteriologic examination, especially for tetanus bacilli, by a laboratory worker who devotes all his time to this work. The federal regulations require each vaccine institute to perform a careful autopsy on each calf after the vaccine virus has been removed, and if any communicable disease is found or suspected in the animal, the virus must not be placed on the market, but must be destroyed. In accordance with this law, permanent records of the bacteriologic examinations of the virus and of the autopsy shall be kept in each institute.

Technic of Vaccination.-The essential part of the process of vaccination is that the virus should be introduced through the epidermis so as to be absorbed by the lymphatics and blood-vessels of the corium.

The site usually chosen is the skin of the outer side of the upper arm, over the insertion of the tendon of the deltoid muscle. Sometimes, in females, the outer side of the thigh or well above the knee on the inner aspect of the thigh is used. Vaccination on the leg, however, is never advisable, as it would appear that such vaccinations are more prone to take on an excessive inflammatory action owing to the greater congestion due to the dependent position of the lower extremities; there is also more likelihood of secondary infection and mechanical violence occurring.

The skin is washed with alcohol and finally with water and then dried, care being exercised not to rub too vigorously; if the skin is reddened, it is best to wait until the hyperemia subsides. Grasp the arm with the left hand, rendering the skin tense, and with a sterile needle or scalpel carefully remove the epidermis over a square area measuring about one-eighth of an inch (Fig. 128). Bleeding should be avoided - an abraded surface that just oozes serum is especially to be desired. The virus is then expressed or placed upon this area (never blown out), and thoroughly rubbed in with a sterilized wooden tooth-pick or the vaccine point. After allowing the lymph to dry, a light sterile gauze dressing should be applied.

Some operators abrade through the lymph; others make one or two straight lines of scratches and rub in the virus.

Another excellent method ${ }^{1}$ consists in abrading the arm with a light rotatory motion of a von Pirquet chisel (sterilized in alcohol and in a flame), measuring about $2 \mathrm{~mm}$. in width. (See Fig. 122.) The virus is then applied, and thoroughly rubbed in with a sterile tooth-pick.

${ }^{1}$ Force, J. N.: Jour. Amer. Med. Assoc., 1914, Ixii, 1466. 
Cross-scarification, which is forbidden in Germany, favors the growth of anaërobic bacteria under the crust that forms on the surface of the abrasion where the resistance is lowered by the action of the virus. The circular scarification gives more control over the dosage, and there is no tendency to the development of excessively sore areas.

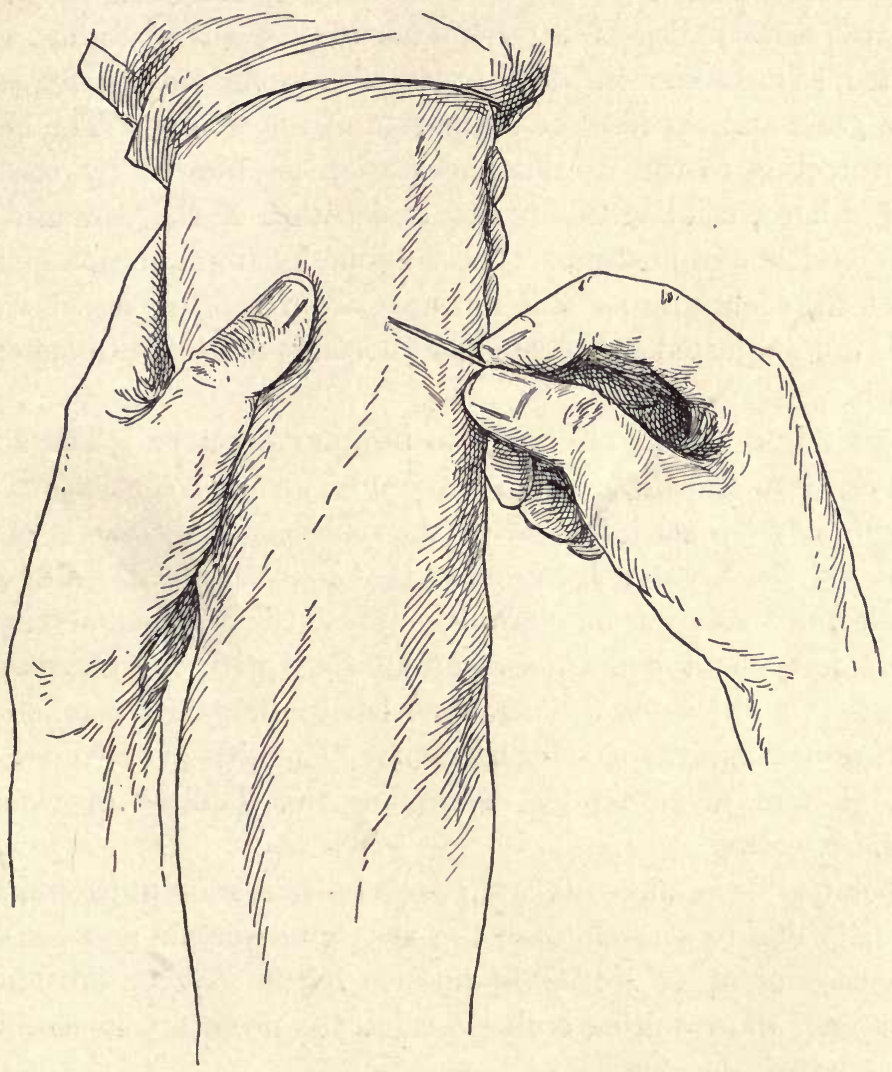

Fig. 128.-Method of Vaccination Against Smallpox.

The skin is stretched, and a series of superficial and parallel scratches made through the epidermis with a sterilized needle.

Usually one inoculation of the virus is sufficient, but in times of threatened epidemic two or more inoculations are made at the same time, not only to insure a successful result, but rapidly to immunize the patient. It would appear that the degree of immunity bears some relation to the number or size of the vaccination lesions, and this can readily be understood if the infection is local and the body-cells are stimulated by a diffusible toxin. If, however, the vaccination lesion is but the 
point of entry of what becomes a general infection, then a small lesion should suffice. This point has not been definitely settled, but statistics tend to show that persons vaccinated in two or more areas develop an immunity more quickly and that this immunity is more lasting.

The subsequent care of the wound is of considerable importance. The operation is usually regarded as a trivial one, and justly so, but the lesion requires judicious after-treatment instead of being entirely neglected, as it so often is. The severe infections are usually attributable to gross and careless contamination of the wound. The best possible protection to the vaccinial ulceration is afforded by the formation of a hard, solid crust, due to desiccation of the contents of the vaccine vesicle and pustule. Unless undue inflammation and suppuration set in, such a crust will form. Care must be taken that the crust is not subjected to mechanical violence calculated to loosen or to detach it.

Constricting shields are likely to be unsatisfactory. The adhesion of the crust to the sleeve or to a piece of protective gauze will often lead to forcible decrustation when the sleeve or the gauze is removed. Schamberg and Kolmer ${ }^{1}$ have found that daily applications of a. 4 per cent. alcoholic solution of picric acid upon the vaccinated area after the first forty-eight hours does not interfere with the success of the vaccination, and lessens the degree of local inflammatory reaction and constitutional disturbances by hardening the epithelial covering of the vaccine lesion, and thereby decreasing the liability of extraneous bacterial infection.

A point to be emphasized is that severe lesions are unnecessary, and are usually due to scratching of the vesicle or pustule and consequent introduction of dirt. No doubt tetanus bacilli may be introduced in this manner, the resulting scab affording the necessary anaërobic conditions for their development.

The Phenomena of Vaccination; Vaccinia.-Immediately following vaccination a slight redness appears, which usually subsides rapidly. After a short period of incubation-on or about the third day-a slight red elevation makes its appearance, and the lesion begins to burn and itch. On the sixth or seventh day the abrasion becomes a small, silvery gray, umbilicated vesicle with a sharply raised edge, filled with a clear serum, and surrounded by a narrow red areola (Fig. 129). By the tenth day the characteristic features are more marked, and the lesion has usually reached its height, being accompanied by a burning sensation

${ }^{1}$ Lancet, London, Nov. 18, 1911, 1397. 


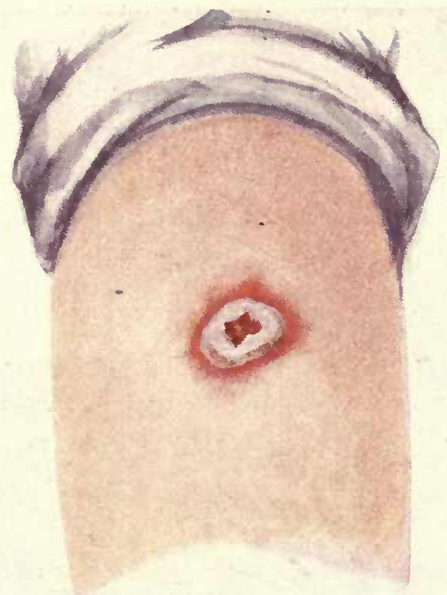

Fig. 129.-Vaccinia (Seven-day Lesion).

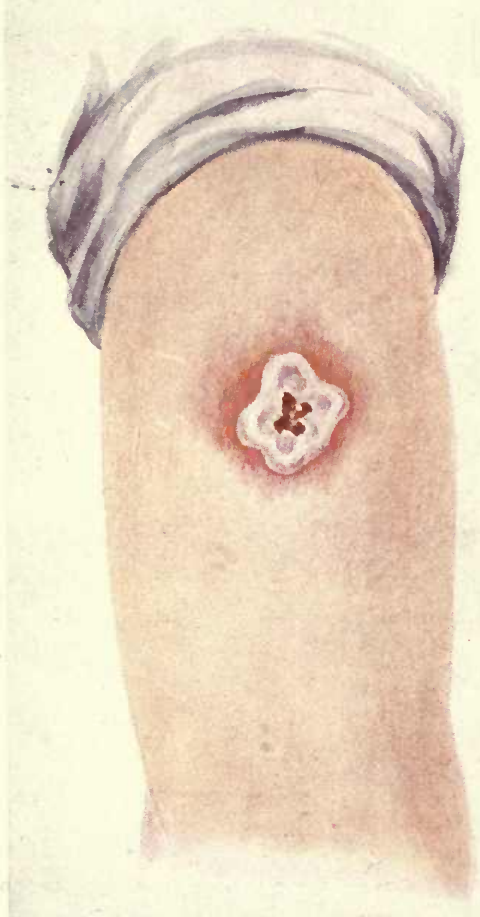

Fig. 130.-VAccinia (Nine-day Lesion).

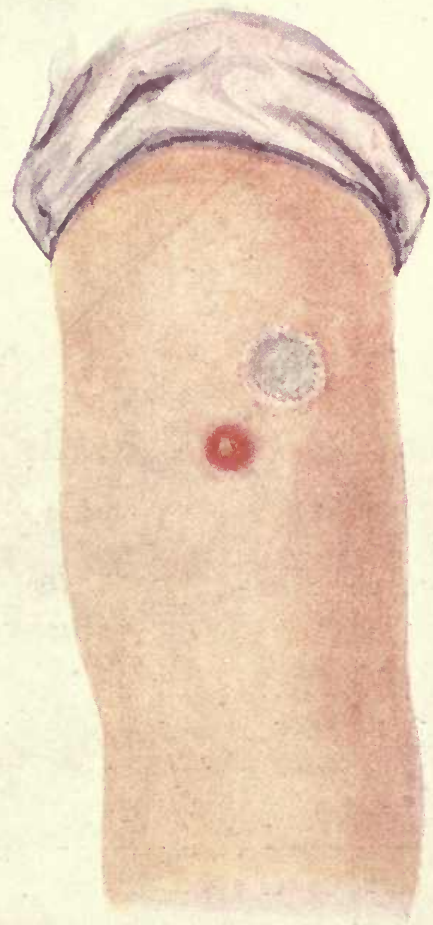

Fig. 131.-VACCINoId. A VaCCINATION SCAR.

Fig 129 shows a seven-day vaccination vesicle; Fig. 130 shows a nine-day vaccination vesicle just before pustulation occurred. Fig. 131 shows a recent vaccination scar with pitting and radiation; also a three-day "vaccinoid" or "immunity reaction" with a small vesicle. 

and an almost uncontrollable desire to scratch (Fig. 130). The areola is now quite angry in appearance, and numerous minute vesicles are seen on its surface. By the twelfth day the areola is smaller, the contents become turbid and commence to dry, so that a few days later a scab has formed that drops off in another week or two.

About the fifth day the child becomes restless and irritable and shows a slight elevation of temperature. These symptoms may become more pronounced until the end of the second week, when they subside rapidly.

Precautions should be taken to prevent scratching and infection of the vesicle. The old-time "beautiful arms," with well-marked cellulitis and adenitis of neighboring glands, were largely due to secondary infection, and are not at all necessary in the process of vaccination. Evidence would tend to indicate that the vesicle is the typical lesion of both smallpox and vaccinia, and that the pustules are simply infected vesicles. Ordinary surgical care will do much to rob vaccination of its discomfort and to render the operation a most harmless one.

The results of a vaccination, therefore, can be inspected and verified on or about the seventh to the ninth day. With persons who have been vaccinated successfully on a previous occasion the vaccinated area may show a slight areola at the end of twenty-four hours, with or without a papule, which subsides in seventy-two hours. This is called a "reaction of immunity," and is due to the presence of antibodies against the virus. Or a small, itchy, burning papule may form, which develops into a small vesicle maturing on the fifth or sixth day, and then rapidly subsiding, constituting the reaction known as vaccinoid (Fig. 131). Occasionally vaccination is followed by the appearance of various eruptions.

The appearance of the scar varies according to its age and to the degree of tissue destruction. The physician is not infrequently requested to examine a person and determine if the scar is satisfactory evidence of successful vaccination. The typical good scar is circular, and about the size of a ten-cent piece, with smooth, white, and depressed center and a raised border. The border shows numerous radiations, and the entire scar may show little pits of former hair-follicles when the lesion was sufficiently destructive to remove the upper portion of the corium. (See Fig. 131.) A burn or an ordinary pyogenic infection may leave scars quite similar to those of vaccination, and vaccination scars may show wide variation, but the circumscribed character, the raised border with radiations and depressions, and the appearance of having been stamped on the skin by a sharply cut die are quite characteristic. 
Poor scars are those that were said to have been the result of vaccination, but in very many instances they are so indistinct as to make it difficult or impossible to recognize them as vaccination-marks.

Revaccination.-One successful vaccination does not necessarily confer an absolute immunity against smallpox, and failure to recognize certain limitations in this respect has done harm by enabling antivaccinationists to create a distrust in the minds of the ignorant by pointing to individual instances of failure. That a person who has once been vaccinated may afterward suffer from smallpox is undoubted, but usually the vaccination was performed many years previously, and in any case the disease, when it does occur, is relatively mild (varioloid).

There can be no doubt but that the immunity gradually diminishes. Perhaps seven years may be taken as the average period of fairly complete protection. Children should be vaccinated within the first year after birth, revaccinated upon entering school, and again after leaving it. If smallpox is prevalent, all persons should be vaccinated, regardless of the fact that they have previously been vaccinated. Only those who have had smallpox may be excused. If, as a matter of fact, persons are still immune, the vaccination will not "take" and no harm is done, whereas if it succeeds, such persons will have the satisfaction of knowing that their immunity has been increased. Hence it cannot be too strongly emphasized that not only vaccination, but revaccination, is indicated to protect the individual and society against smallpox. Dwyer claims that a person should be revaccinated repeatedly in succession until he fails to react; even a slight "take" would indicate incomplete immunity.

Occasionally a non-immunized person refuses to "take," but vaccination should be repeated three or four times, as failure is not infrequently due to old and inactive virus, and the actual number of persons absolutely insusceptible is very small indeed.

Risks of Vaccination. - When vaccination is properly performed with a good virus, the risk of permanent injury to life or limb is almost negligible.

1. Tetanus. - The most serious of the injuries that have been attributed to vaccination is tetanus. The tetanus bacillus and its spores are so wide-spread in nature that opportunity presents itself for contamination of the vaccinal wound and the virus itself. Every precaution should, therefore, be taken in the preparation of virus, and it is especially important that physicians and laymen should realize the necessity for 
observing ordinary care, and at least ordinary cleanliness, in the treatment of the vaccinal wound.

It is exceedingly difficult to determine the source of infection in each case of vaccinal tetanus, but experimental investigations would tend to indicate that the virus itself is seldom, if ever, the vehicle of infection. John F. Anderson, Director of the Hygienic Laboratory, in testimony given before the Pennsylvania State Vaccination Commission, stated that in experiments carried out on monkeys and guinea-pigs with vaccine lymph purposely contaminated in the laboratory with countless numbers of tetanus spores, it was found impossible to communicate tetanus in this manner, although the vaccinations were more severe than the ordinary vaccinations performed on man, in that several places were inoculated and the areas abraded were large. Anderson stated that his "conclusion from these experiments is that it is almost impossible to produce tetanus, even with vaccine virus that contains tetanus germs in it, by the simple act of vaccination."

Since 1909 there were approximately 100,000 specimens of vaccine virus examined in the Hygienic Laboratory, particularly with the purpose of determining the presence of tetanus germs or their products. The vaccine was purchased in the open market, and the examinations were made as thoroughly as it was possible to make them. To use Anderson's words: "We have never succeeded in finding any evidence of the presence of the tetanus organism or its products in vaccine virus." (Report of the Commission.)

It would appear, therefore, that virus prepared according to modern methods and with all recognized precautions is safe. In view of the incidence of tetanus following other injuries, it is reasonable to conclude that most cases of vaccinal tetanus are secondary wound infections, and therefore largely preventable.

2. Syphilis.-With the use, years ago, of humanized virus, and particularly in the days of arm-to-arm vaccination, extremely rare instances of the transmission of syphilis have been known to occur. Since, however, the use of calf virus, which is the virus exclusively employed in this country, such an accident is absolutely impossible, as calves are not susceptible to luetic disease.

3. Cancer, foot and mouth disease, tuberculosis, and various chronic skin eruptions have been attributed to vaccination by its opponents; none of these claims has, however, been substantiated.

Protective Value of Vaccination.-Of the value of the protection afforded by vaccination against smallpox there can be no doubt in the 
minds of right-thinking and unbiased persons. The history of the world before the days of universal vaccination shows the wide prevalence of smallpox and its fearful mortality. It was regarded as a disease of childhood, owing to the fact that all contracted it at the earliest opportunity, and, accordingly, smallpox was the cause of a fearful infant mortality.

At the present day, owing to the general employment of vaccination, smallpox is a rare disease, but its very rarity has fostered a certain degree of false security and carelessness in carrying out the process. A young and new generation of non-vaccinated persons in any community is a source of danger, and accordingly sporadic cases are often blessings in disguise, from the fact that, when they appear, compulsory vaccination is then instituted and large numbers seek revaccination.

In Germany, where vaccination is compulsory, smallpox is now a comparatively rare disease. While the general death-rate from all diseases is lower in England and Wales than in Germany, the smallpox mortality is seven and one-half times the mortality of Germany, and, proportionate to the population, over 13 times.

Austria, one of Germany's neighbors, had, for the twenty years following 1874, almost 30 times as high a smallpox mortality as Germany. During this period 239,800 persons perished in Austria from smallpox alone.

Physicians should carefully impress upon those over whom they have any influence the necessity of being vaccinated, for only a thoroughly vaccinated population can solve the problem of exterminating smallpox as an epidemic disease.

\section{RABIES}

There are but few diseases more dreaded by the laity than rabies, or hydrophobia. Tales of the sufferings of infected persons, especially those with the furious variety of this infection, characterized by maniacal symptoms and dread of water (hydrophobia), have been thoroughly disseminated, so that the cry of "mad dog!" on the public streets is sufficient to arouse a general state of hysteric excitement in which an otherwise harmless creature may be compelled to bite or snap for selfprotection. Not all dogs under these conditions are mad or infected with rabies, and the bite of an angry dog, otherwise normal, is not necessarily dangerous from the standpoint of rabic infection. However, almost every one, upon being bitten by a dog, will promptly consult his physician, and this is proper and to be encouraged. Genuine rabies 
is an acute infectious disease in which the diagnosis is quite readily made, and whenever possible an effort should be made either to confirm or to disprove the diagnosis by making an examination of the animal's brain, and if the dog is found to have been free from rabies, this fact should be carefully impressed upon the patient, as otherwise the dread of infection may weigh heavily upon the patient and lead to distressing nervous disturbances.

While the infectiousness of rabies has been known for a great many years and was proved experimentally by Galateir ${ }^{1}$ and Pasteur, ${ }^{2}$ it was not until Negri, in 1903, described certain bodies (Negri bodies), seen by him in large nerve-cells in sections of the central nervous system, that anything was found that seemed absolutely specific for rabies. Negri regarded these bodies as specific for rabies and probably of a protozoan nature. Later investigations fully established the diagnostic value of these bodies, and their definite characteristic morphology, evidences of cyclic development, and staining qualities indicate a protozoan structure resembling members of the Rhizopoda, designated by Anna Williams in $1906^{3}$ as Neurorrhyctes hydrophobice.

Rembringer, ${ }^{4}$ Poor and Steinhardt, ${ }^{5}$ Bertarelli and Volpino ${ }^{6}$ have demonstrated the filterability of the rabic virus, and Noguchi ${ }^{7}$ has cultivated from both "street" and "fixed" virus, very minute granular and somewhat coarser pleomorphic chromatoid bodies which, on subsequent transplantation, reappeared in the new cultures through many generations and reproduced typical symptoms of rabies in dogs, rabbits, and guinea-pigs.

To Pasteur is due the credit for having discovered (1880) the fact that the disease may be prevented by conferring gradual immunization with increasing doses of the attenuated virus. This treatment, with some modification, is now used with evident success in all parts of the world.

Nature of Rabies. - The virus or parasite is contained in the saliva of the rabid animal, and infection is possible when the skin is abraded by bites and scratches. The virus travels by way of the nerve-paths

${ }^{1}$ Compt. rend. Acad. d. sc., 1879, lxxxix, 444.

${ }^{2}$ Compt. rend. Acad. d. sc., 1881, xcii, 159.

${ }^{3}$ Proc. N. Y. Path. Soc., 1906, vi, 77.

${ }^{4}$ Ann. de l'Inst. Pasteur, 1903, xvii, 834; 1904, xviii, 150.

'Jour. Infect. Dis., 1913, xii, 202.

${ }^{6}$ Centralbl. f. Bakt., Orig., 1904, xxxvii, 51. Bertarelli, ibid.

7 Jour. Exper. Med., 1913, xviii, 314. 
to the central nervous tissue, and, as in tetanus, the symptoms of the disease are due to involvement of these tissues.

The period of incubation, or the time elapsing between the time of injury and the first symptoms, is quite variable, ranging from twenty to sixty days, although it may be as short as ten days. As in tetanus, this period depends upon- $(a)$ the location of the injury; $(b)$ the quantity or dose of virus; (c) the kind of animal responsible for the injury. Bites about the face and fingers, especially if they are deep and lacerating, are especially dangerous; bites about the back and lower limbs, especially if superficial, are much less dangerous, and accompanied by a longer period of incubation. It is to be remembered that bites may be infectious as early as nine days before the dog shows well-marked symptoms of the disease. Not infrequently an animal is observed to be surly and snappy for several days before rabid symptoms develop, and a bite during this time should be regarded as dangerous.

Only about 16 per cent. of human beings bitten by rabid animals and untreated appear to contract rabies. Since the establishment of the Pasteur treatment of the disease, the percentage of developed cases after bites is much lower-about 0.46 per cent.

Diagnosis and Management of Rabies.-Even though an animal is unmistakably rabic, every effort should be made to destroy the animal, not only in order to prevent further damage, but to corroborate the diagnosis by microscopic examination of the nervous tissues for Negri bodies.

1. As a general rule, all animal bites should receive surgical attention. Wounds produced by animals clinically rabid should be cauterized at once with fuming nitric acid or pure phenol. This is done to offset the delay in securing the Pasteur treatment, and because there is evidence to show that thorough cauterization of the wound is in itself highly beneficial.

2. The animal should be promptly destroyed, not only to prevent further damage, but in order to make a microscopic diagnosis by examination of the brain for Negri bodies. This examination is highly important and should never be omitted, for if it shows the absence of bodies, this fact should be carefully impressed upon the patient, as there is no doubt that a neurotic element, amounting in many instances to actual hysteria, may cause considerable harm to the patient even though he is definitely free from rabic infection.

3. The whole dog may be packed in ice and shipped at once to a central laboratory, or the head alone may be removed and packed in 
ice or glycerin and promptly shipped. The brain should not be disturbed. When it reaches the laboratory, the diagnosis should be made at once by "smear" preparations and sections of the brain demonstrating the characteristic Negri bodies in the large ganglion-cells, and confirmed by inoculating emulsions of the brain into guinea-pigs or rabbits. The latter requires from ten to twenty days before the result may be known. A negative animal inoculation test is better evidence than a negative smear or section; obviously, these examinations are to be made only by properly trained persons.

According to Park, the value of the smear method of diagnosis may be summarized as follows:

1. Negri bodies demonstrated, diagnosis, rabies.

2. Negri bodies not demonstrated in fresh brains, very probably not rabies.

3. Negri bodies not demonstrated in decomposing brains, uncertain.

4. Suspicious bodies in fresh brains, probably rabies.

4. If the animal was clinically rabid, the Pasteur treatment should be commenced as soon as possible, without waiting for the laboratory report, if this will be delayed for several days. Wounds about the face and hands, where there is no clothing to retain the infectious saliva, should receive intensive treatment; otherwise the milder course of immunization will suffice.

5. As a general rule, it is well to send the patient to a regular Pasteur institute, where there are special facilities for the proper treatment of these cases. Otherwise the physician may treat the patient at home. Several large manufacturing firms are prepared to ship by mail the fresh daily treatments properly preserved and ready for administration.

6. The Pasteur treatment should be given to every patient bitten by a rabid animal or by one suspected of being rabid. Not all persons are necessarily infected, even by bites of rabid animals, but this should not unduly influence the physician, for he will not have fulfilled his duty unless he carefully explains the etiology of the disease and advises immediate immunization. Aside from the actual benefits of the treatment, the mental effect upon the patient is deserving of consideration. Even slight wounds by rabid animals, wherever their location, should be regarded as dangerous, and the Pasteur treatment advised in addition to routine cauterization.

7. With severe bites of angry but not necessarily clinically rabid dogs, the treatment depends upon various factors. In any case the 
wound should be thoroughly cauterized and the animal carefully guarded (not killed) for two or three weeks. If rabid symptoms appear, the animal should be destroyed, the cerebral tissues examined, and the Pasteur treatment of the patient begun.

8. All animals bitten by a rabid animal should, of course, be promptly destroyed; even those bitten by a dog suspected of being rabid should be destroyed or closely guarded until a definite diagnosis can be reached. In England, where strict laws are enforced relative to the muzzling and control of dogs, rabies is relatively infrequent, and it is especially urged that similar measures be adopted and enforced in our own communities, particularly during the summer months.

Principle of the Pasteur Treatment (Active Immunization) of Rabies. - This method is based upon the principle of stimulating the production of rabic antibodies by injecting attenuated or modified virus during the period of incubation, so that the virus introduced into the wound is destroyed, neutralized, or its effects neutralized, while the virus itself is finally destroyed. Pasteur worked out this theory and established its truth by experiments upon the lower animals before applying the treatment to man.

By passing the virus through a series of rabbits, the period of incubation is shortened to about six to seven days, and at the same time its pathogenicity for man is actually diminished (virus fixé). By drying the tissues containing the fixed virus attenuation is secured, so that it is easily possible so to modify the virus that it cannot produce rabies in man, but yet is able to produce the specific antibodies. The Pasteur treatment is, therefore, a process of active immunization with emulsions of a tissue (spinal cords of infected rabbits) in which the virus has been attenuated by a process of drying and desiccation. The early doses consist of highly attenuated cords, and succeeding doses become gradually more potent, as is usual in the technic of any method of active immunization.

Preparation of the Rabies Vaccine.-As a preliminary, it is necessary to prepare or obtain "virus fixé." This may generally be procured from a laboratory, or may be prepared by passing street virus from the medulla of a rabic cow or dog through a series of young rabbits. After from 30 to 50 passages the incubation period is gradually reduced to six or eight days ("virus fixé").

1. From an animal succumbing the day or night before, a piece of the floor of the fourth ventricle measuring about $2 \mathrm{~cm}$. in length is emulsified in 1 c.c. of sterile bouillon, and three or four drops of this 
emulsion are injected beneath the dura of a normal rabbit. In large institutes two or more rabbits are injected daily. The inoculation is quickly and easily performed by trephining a small area in the median line of the forehead and injecting the emulsion beneath the dura mater with a syringe. The whole operation must be carried out in an aseptic and practically painless manner.

2. After inoculation the animals are placed in clean cages; in from six to eight days paralytic symptoms of rabies appear, followed in three to four days by death. The hair is then sprayed with a solution of lysol and the skin removed. The cord and brain are then extracted under aseptic precautions. The cord is severed just below the medulla, a portion is snipped off into sterile bouillon for culture, and then divided into two equal pieces which are suspended by sterilized silk threads in a sterile glass jar containing flakes of caustic potash (Fig. 132). The medulla is placed in a sterile dish, and is used to continue the inoculations, as was previously described. A postmortem examination is finally performed, and any cord in which the animal is found diseased or in which the culture of the cord shows bacterial contamination is rejected.

The jars with suspended cords are kept in a special room at a temperature of about $20^{\circ}$ to $25^{\circ} \mathrm{C}$.

3. After a suitable period of drying pieces of cord are prepared for injection. This is performed in various ways at different laboratories; no attempt at exact dosage is made. In the New York Board of Health laboratories $1 \mathrm{~cm}$. of the cord is thoroughly emulsified in 3 c.c. of sterile saline solution, the process being conducted in an aseptic manner. If the material is to be shipped, an

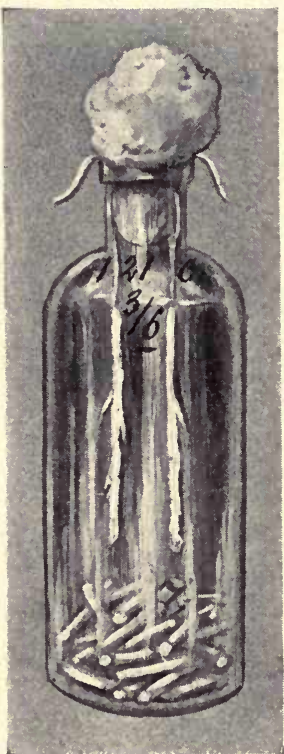

Fig. 132.-PreparaTION OF RABIES VACCINE.

Note the cords suspended within the jar by means of sterile silk threads; sticks of sodium hydroxid to absorb moisture and hasten desiccation. addition of 20 per cent. of glycerin and 0.5 per cent. of phenol is made.

Administration of the Vaccine.-Injections are given with a sterile syringe. The abdominal region of the patient is bared, a spot touched with tincture of iodin, wiped with alcohol, and the injection given subcutaneously. Keirle does not vary the dose according to the age, both the old and the young receiving the same dose. At times the injection 
is followed by redness and induration in the subcutaneous tissues, but abscesses are never formed.

Cases of severe injury, such as deep bites about the face and fingers, should be rapidly immunized (intensive treatment); in other cases the treatment may be mild (mild treatment). The uniform dose of cord emulsion, prepared as just described, is 2.5 c.c. The series of inoculations given in the Research Laboratory of New York in treating human cases after an average bite are as follows:

\section{TABLE 23.-SCHEDULE OF INOCULATIONS IN IMMUNIZATION} AGAINST RABIES

\begin{tabular}{|c|c|c|}
\hline DAX & Mild Treatment & Intensive Treatment \\
\hline First. & 14 and 13 day cord & 12 and 11 day cord. Repeated in P. M. \\
\hline Second. & 12 and 11 day cord & 10 and 9 day cord; 8 and 7 day cord P. M. \\
\hline Third......... & 10 and 9 day cord & 6 day cord \\
\hline Fourth.......... & 8 and 7 day cord & 5 day cord \\
\hline Fifth $\ldots \ldots \ldots \ldots$ & 6 day cord & 4 day cord \\
\hline Sixth. & 5 day cord & 3 day cord \\
\hline Seventh....... & 4 day cord & 2 day cord \\
\hline Eighth........ & 3 day cord & 4 day cord \\
\hline Ninth......... & 2 day cord & 4 day cord \\
\hline Tenth............ & 4 day cord & 1 day cord \\
\hline Eleventh....... & 3 day cord & 4 day cord \\
\hline Twelfth........ & 2 day cord & 3 day cord \\
\hline Thirteenth..... & 4 day cord & 2 day cord \\
\hline Fourteenth...... & 5 day cord & 4 day cord \\
\hline Fifteenth . . . . . . & 2 day cord & 1 day cord \\
\hline Sixteenth. & 4 day cord & 4 day cord \\
\hline Seventeenth..... & 3 day cord & 3 day cord \\
\hline Eighteenth. . & 2 day cord & 2 day cord \\
\hline Nineteenth..... . & 4 day cord & 4 day cord \\
\hline Twentieth & 3 day cord & 3 day cord \\
\hline Twenty-first. & 2 day cord & 2 day cord \\
\hline
\end{tabular}

Results.-According to reliable statistics, the mortality of rabies without the Pasteur treatment is about 16 per cent.; with the treatment the average mortality is about 0.46 per cent. The mortality of those bitten about the face or head is about 1.25 per cent.; of those bitten on the hand, 0.75 per cent.; of those bitten on other parts of the body, a little over 0.25 to 1 per cent. In the Pasteur Institute of Paris only such persons are treated as have been lacerated, so that the virus has gained entry into the wounds. Taking into consideration only those cases in which the diagnosis of rabies has been confirmed in the animal by a competent examiner, the mortality of the cases treated at the Pasteur Institute in Paris is only 0.6 per cent., which, compared to the average mortality of 16 per cent. without vaccine treatment, speaks most favorably for the value of Pasteur's antirabic immunization. 
The bites of wolves are more fatal than those of dogs, the mortality being about 10 per cent. in spite of the intensive treatment, and about 40 to 60 per cent. without treatment.

When symptoms of rabies have appeared, the treatment is unavailing. Antirabic serums have been prepared by immunizing animals, such as sheep and horses, and these should be tried in human patients presenting symptoms, but the results in general have not been uniformly encouraging.

\section{TYPHOID FEVER}

Our knowledge of vaccination in typhoid fever begins with the work of Pfeiffer and Kolle. ${ }^{1}$ These observers, in 1896, immunized two volunteers with heat-killed cultures, and by complete laboratory investigations demonstrated the identity of the immunity following an attack of the disease with the artificial immunity produced by inoculation. At about the same time Wright, ${ }^{2}$ of London, inoculated two men with killed cultures, and a year later published the results of the successful vaccination of 17 persons. In 1898 he continued the work in India, where 4000 soldiers were inoculated, with encouraging results. Later, during the Boer war, Wright and Leishman treated 100,000 men, and the results, while good, were not encouraging, due, as pointed out later by Leishman, ${ }^{3}$ to the fact that the vaccine was damaged during its preparation by overheating. Since 1904 an improved vaccine has been used among the British troops in India in ever-increasing quantities, with uniformly good results.

Antityphoid vaccination was begun in the United States army in 1908, the vaccine being prepared by Major Frederick F. Russel. Its value has been established so clearly that vaccination is now compulsory. The results obtained in the army have had considerable influence in establishing a wide-spread general confidence in antityphoid inoculation.

Preparation of Typhoid Vaccine.-Based upon general principles, the vaccine should be prepared of typhoid bacilli as little changed by heat or chemicals as possible. Russel has prepared the army vaccine with a single avirulent culture which proved by animal experiments and laboratory methods capable of producing large quantities of immune agglutinins and bacteriolysins. As a general rule, however, the vaccine should be polyvalent unless one obtains a culture of known value.

${ }^{1}$ Deutsch. med. Wochenschr., 1896, xxii, 735.

${ }^{2}$ Lancet, London, September 19, 1896, 907; Brit. Med. Jour., January 30, 1897, 16.

${ }^{3}$ Jour. Roy. Inst. 
The preparation of the vaccine is comparatively simple. The bacilli are grown on agar for twenty-four hours, washed off with sterile normal salt solution, standardized by counting the bacilli, and killed by heating to $56^{\circ} \mathrm{C}$. for one hour. As a matter of safety, 0.25 per cent. of tricresol is then added (Russel). The details of the technic are given in Chapter XIII.

Metchnikoff has never fully accepted the belief in the value of heatkilled vaccines, and at present is actively concerned with vaccines prepared of living bacilli sensitized with their immune serum (sensitized vaccines). Injection of these vaccines into chimpanzees is not followed by any untoward effects, and apparently the bacilli so administered are destroyed at once, as they have not been found in the blood, urine, and feces. Metchnikoff and Besredka have immunized persons according to these methods and report excellent results. Obviously, there is some reluctance in using a vaccine of living bacilli until extended animal experiments have proved that they are harmless and more efficient than the vaccines of killed bacilli.

As a general rule, the vaccine is prepared in two strengths: 500,000,000 bacteria per cubic centimeter for the first dose, and 1,000,000,000 for the second and third doses.

Method of Inoculation.-The vaccine is best administered at about 4 o'clock in the afternoon, so that the reaction appears during the night and is least likely to be disturbing. It is well to administer a cathartic the day before the inoculation is made. Inoculations should not be given during the menstrual period, as the general reaction is likely to be somewhat severer at this time.

The skin over the insertion of the deltoid muscle is touched with tincture of iodin and the injection given subcutaneously. Intramuscular injections should be avoided, as the reactions are more unpleasant and accompanied by unnecessary pain on movement. In making deep injections there is also danger of striking a nerve, a proceeding that may be followed by disagreeable neuritis.

After the injection has been given the iodin is wiped away with a pledget of cotton and alcohol; no dressing is necessary.

The syringe and needle should be sterile. Commercial firms have placed the prophylactic on the market in syringes with sterilized needles, accompanied with full instructions as to the technic of administration. The vaccine should be well shaken before the injection is given.

When a large number of injections are to be given at one time, a single syringe may be used with a large number of sterile needles, a 
separate needle being used for each person. The vaccine may be put up in individual ampules or in a stock bottle, the former being preferable.

Dosage.-Three injections are given at intervals of one week. For adults (150 pounds) the doses used in the army have been as follows:

First dose: $\quad 500,000,000$ bacilli.

Second dose: 1,000,000,000 bacilli.

Third dose: $\quad 1,000,000,000$ bacilli.

These amounts are contained in 1 c.c. (about 15 minims). Children receive doses in proportion to their weight; if the dose cannot be divided evenly, it is better to give a little more rather than a little less, for children tolerate the injections remarkably well.

Reactions.-Persons in poor physical condition are more likely than the robust to experience disagreeable after-effects.

The local reaction consists of a small red and tender area lasting about forty-eight hours. Occasionally the edema and pain may be more marked, but abscess formation is practically unknown.

The general reaction, when present, gives rise to headache, malaise, and sometimes to fever, chills, and occasionally to nausea, vomiting, or diarrhea. The severe reactions are not alarming and disappear quickly.

The inoculated person should abstain from severe exercise for the following twenty-four hours and rest; in the great majority of instances our soldiers have not been inconvenienced and were able to continue with their routine duties.

The following tables, compiled by Major Russel, ${ }^{1}$ show the proportion of reactions in adults and children:

TABLE 24.-PERCENTAGE OF GENERAL REACTIONS IN ADULTS (128,903 DOSES)

\begin{tabular}{|c|c|c|c|c|}
\hline Dose & None & MILD & Moderate & SEVERE \\
\hline $\begin{array}{l}\text { First... } \\
\text { Second. } \\
\text { Third.. }\end{array}$ & $\begin{array}{l}68.2 \\
71.3 \\
78.0\end{array}$ & $\begin{array}{l}28.9 \\
25.7 \\
20.3\end{array}$ & $\begin{array}{l}2.4 \\
2.6 \\
1.5\end{array}$ & $\begin{array}{l}0.3 \\
0.2 \\
0.1\end{array}$ \\
\hline
\end{tabular}

TABLE 25.-PERCENTAGE OF GENERAL REACTIONS IN 359 CHILDREN, TWO TO SIXTEEN YEARS OF AGE

\begin{tabular}{|c|c|c|c|c|}
\hline Dose & None & MILD & MODERATE & SEvere \\
\hline $\begin{array}{l}\text { First. . . . . . . } \\
\text { Second . . . . . . . } \\
\text { Third... }\end{array}$ & $\begin{array}{l}73.54 \\
86.26 \\
92.56\end{array}$ & $\begin{array}{r}24.51 \\
11.99 \\
0.38\end{array}$ & $\begin{array}{l}1.67 \\
1.75 \\
1.06\end{array}$ & $\begin{array}{l}0.28 \\
\ldots \\
\ldots\end{array}$ \\
\hline
\end{tabular}

1 Jour. Amer. Med. Assoc., 1913, lx, 344. 
A comparison of these tables shows that the general reaction is much more infrequent or milder in children than in adults, even after the first dose; after the second and third doses the difference is more marked.

In former years considerable stress was laid upon the possibility of a negative phase following the inoculation, during which a person was believed to be more susceptible to infection. This is now believed by Leishman, Russel, and others of extended experience to be incorrect, the more general belief being that inoculations may be made and are especially indicated during epidemics of the disease.

Duration and Degree of Typhoid Immunity.-It should be emphasized that immunity following typhoid immunization is not absolute, and an immunized person cannot afford to neglect ordinary precautions against infection. A lowered state of general body health or a large dose of infectious material may at any time result in infection.

The prophylactic treatment should be used in conjunction with well-known sanitary precautions in order to obtain the best results.

The immunity is apparently manifest soon after the first and second doses have been given. The duration is not known definitely. From the rich experience of the British army in India Colonel Firth ${ }^{1}$ concludes that immunity begins to decline in about two and one-half years after inoculation. However, even after four and five years the typhoid rate among the inoculated is, estimated roughly, one-fourth that of unprotected troops.

Results.-The value of the typhoid prophylactic therapy is best shown in the army, where conditions are better controlled than is possible in civilian life. In 1911, of a division of United States troops, about 20,000 men along the southern boundary, only two cases of typhoid fever developed and both recovered. During this same period of time 49 cases were reported in the city of San Antonio, with 19 deaths. The soldiers mixed freely in the city, ate of fruits and vegetables, drank of the same water, and in this manner were freely exposed, although the sanitary conditions in the camp were excellent.

In 1898, during the Spanish War, there were assembled at Jacksonville, Florida, 10,759 troops, among whom there were certainly 1729 cases of typhoid, and including the suspected cases, this figure reached 2693 cases, with 248 deaths. This camp continued about as long as that in 1911, the climatic conditions and water supplies being practically the same, but the sanitary conditions were bad. The remarkable difference in the typhoid rate cannot, however, be reasonably explained by

1 Jour. Roy. Army Med. Corps, 1911, xvi, 589. 
perfect camp sanitation, and the results in 1911 leave no doubt as to the value of antityphoid vaccination.

Excellent results have been reported by Spooner, Hachtel and Stoner, and others as to the prophylactic value of typhoid immunization in hospital-training schools for nurses, insane asylums, and other public institutions.

Recommendations. - In view of the satisfactory results obtained in the army, typhoid vaccination is now obligatory on all members of the army and navy corps. Protection of the individual by immunization is the only measure of protection independent of surroundings and effective under all conditions.

Typhoid inoculation in civilian practice cannot be as wide-spread or as readily performed as vaccination against smallpox, as the prophylactic must be administered subcutaneously and more than one dose is necessary.

1. Our various State and city boards of health should endeavor to educate the laity, and, if necessary, offer the prophylactic free of charge in order to build up a vaccinated community as far as this is possible.

2. Persons coming in intimate contact with typhoid patients, such as physicians, nurses, and attendants in hospitals, should be immunized. Hospital authorities are justified in making typhoid vaccination obligatory on all applicants for admission to training schools.

3. All inmates of asylums, homes, and other public institutions under forty-five years of age should be immunized and the State should be ready, if necessary, to furnish the vaccine.

4. The physician and nurse should urge vaccination upon all the members of a family when there is a typhoid patient among them.

5. The physician should especially advise immunization of those about to leave their homes for a vacation in some neighboring seashore or mountain resort.

6. In times of epidemics of typhoid fever the physician should urge vaccination of all over whom he has influence. Thorough vaccination with proper sanitary conditions offers the best hope of eradicating this dreaded disease.

\section{Plague}

In view of the frightful infectiousness and mortality of plague, prophylactic measures are highly desirable. Extermination of rats and ground squirrels, especially of the former, about the wharves of seaport cities and towns is highly essential, as the disease is transmitted by the 
fleas of these rodents. Aside from sanitary measures, plague vaccine, especially that of Haffkine, has now been used extensively, with encouraging results.

Preparation of Plague Vaccine.-The Haffkine prophylactic is prepared by growing pure cultures of Bacillus pestis in flasks of neutral bouillon to which a few drops of sterile olive oil or butter-fat have been added, to serve as floats from which the surface growth of the bacilli can take place. The flasks are cultivated at from $25^{\circ}$ to $30^{\circ} \mathrm{C}$. for five to six weeks, and are shaken every two or three days, by which the hanging, stalactite-like colonies are thrown down, so that a new crop of the bacilli can develop in contact with the air.

After growing for six weeks the purity of the culture in each flask is tested by subcultures on agar and by cirect smears. The masses of bacilli are broken up by shaking, and the material is sterilized by heating at $65^{\circ} \mathrm{C}$. for from one to three hours. Phenol is added to the point of 0.5 per cent., and the fluid is tested for sterility by culture. If it is found sterile, it is finally poured into small vials of from 10 to 30 c.c. capacity.

Kolle prepares a vaccine by cultivating the bacillus for two days in flasks of agar measuring 10 by $9.5 \mathrm{~cm}$. Each surface of agar equals about 15 ordinary agar slant cultures, and an agar slant holds about 15 loopsful of culture ( $4 \mathrm{~mm}$. loop). A loop of this size holds about 2 mg. of organisms, and, accordingly, a flask of agar contains about 225 loopsful of culture, or 225 doses of $2 \mathrm{mg}$. each. Kolle prepares the vaccine in amounts of 0.5 c.c. per dose ( $2 \mathrm{mg}$. of bacilli), and the growths in each flask are removed with 112.5 c.c. of sterile normal salt solution. The emulsion is shaken to break up clumps, heated for one hour at $70^{\circ}$ C., and tested for sterility. It is then preserved with phenol or tricresol and placed in ampules containing 0.5 c.c. each.

The German Plague Commission strongly recommended the use of twenty-four- to forty-eight-hour-old agar cultures instead of the old bouillon cultures employed in the preparation of Haffkine's vaccine.

Kolle and Strong have also employed living cultures of greatly reduced virulence for the immunization of man.

Lustig and Galeotti prepare a vaccine of the toxic precipitate produced by dissolving the bacilli in a 1 per cent. solution of caustic soda and neutralizing with 1 per cent. of acetic acid. This precipitate is dried in vacuo and redissolved in a weak solution of sodium bicarbonate, the dose for adults being $0.0133 \mathrm{gm}$. of solid substance.

Terni and Bandi inoculate rabbits or guinea-pigs intraperitoneally 
with the bacillus, and just preceding or directly after death they collect the peritoneal exudate, in which the organisms are allowed to continue growing for twelve hours more. The bacilli are then killed at a low temperature, and the fluid thus obtained, after a preservative has been added, constitutes the vaccine.

Dosage.-The ordinary dose of Haffkine's prophylactic for adult males is from 3 to 3.5 c.c.; for adult females, from 2 to 2.5 c.c. Haffkine himself has injected larger quantities without resulting harm. He recommends giving a second injection after from eight to ten days. The injections are given subcutaneously, with a sterile syringe, into the upper arm or elsewhere in areas where the skin is not tightly bound down.

The local and constitutional effects are similar to those in typhoid except that they are slightly intensified. The inoculation is followed by redness and swelling at the seat of inoculation, and general symptoms in the form of rise of temperature and a feeling of illness. The latter pass off in twenty-four hours, but the patient should rest during the first day after inoculation.

Duration of Immunity. - The immunity is apparent a few days after inoculation, but is of short duration. In India the protection is believed to last at least three months and possibly longer. In times of epidemic the inoculations should be repeated at least two or three times a year. The brief duration of the immunity is probably one reason why better results are not secured. Best results are observed during epidemics when protection is afforded for a short time, or until the danger is past. In countries or localities where the disease is endemic, persons may refuse repeated inoculation and thus become susceptible to infection.

Results. - In the pneumonic variety of plague the prophylactic does little or no good, a finding that has also been shown experimentally by Strong and Teague. ${ }^{1}$

In the bubonic variety Haffkine's vaccine has in general yielded encouraging results. The protection is not absolute; the immunity is of relatively short duration, and therefore good results are not so readily appreciated when the disease is endemic. The mortality among the inoculated is much lower, $i$. e., 11 to 41 per cent., as compared with 50 to 92 per cent. among the non-immunized. Haffkine summarized his results a few years ago as follows: Among 186,797 inoculated persons there were 3999 attacks, or 1.8 per cent.; among 639,630 uninoculated persons there were 49,433 attacks, or 7.7 per cent., with 29,733, or 4.7 per cent. of deaths.

${ }^{1}$ Philippine Jour. Sci., 1913, vii. 
The Indian Plague Commission a few years ago reported as follows:

1. Inoculation sensibly diminishes the incidence of attacks of plague. It is, however, not an absolute protection against the disease.

2. The death-rate is markedly diminished by its means, not only the incidence of the disease, but also the fatality, being reduced.

3. The protection is not conferred on those inoculated for the first few days after the injection.

4. The duration of the immunity is uncertain, but it seems to last for a number of weeks, if not for months.

After the disease has once developed, vaccination is of no avail. When there is eminent danger of infection, vaccine and antiserum should be given together.

\section{CHOLERA}

Protective inoculation against cholera was first practised by Ferran, a Spaniard, in 1884, although little definite knowledge as to the value of the procedure resulted from his work. He is said to have used impure cultures of bacilli isolated from the feces of cholera patients. Broth cultures were prepared, and the living organisms injected subcutaneously, using eight drops for the first and 0.5 c.c. for the second and third doses, the injections being given at intervals of six or eight days. While his method and results have been questioned, he was, however, the first to use a method employed later, with some modifications, by Haffkine in India with good results.

Preparation of Cholera Vaccine.-Haffkine, following Pasteur's method with anthrax, uses two vaccines, - a weaker and a stronger,living microörganisms being used in both and injected subcutaneously. Vaccine No. 1 is weaker, and is obtained by growing the bacilli on agar at a temperature of $39^{\circ} \mathrm{C}$. Vaccine No. 2 is composed of more virulent organisms, prepared by passing the vibrios through a series of guineapigs until a strain is obtained which is invariably fatal to these animals within twelve, or at least twenty-four, hours. Cultures are grown on agar, washed off with 8 c.c. of sterile bouillon or saline solution, and administered in doses of 1 c.c., which is equivalent to about two loopsful (4 mm.) or $4 \mathrm{mg}$. of living bacilli.

Kolle has shown, however, both by animal experimentation and in the human being, that heat-killed cultures are equally good, and that living cultures are unnecessary and may be undesirable.

By this method the vaccine is prepared by cultivating a virulent strain on flasks of agar for twenty-four hours, as described in the pre- 
paration of plague vaccine, and removing the growths with sufficient salt solution so that 1 c.c. shall contain one loopful $(4 \mathrm{~mm}$.) of organisms ( $2 \mathrm{mg}$.). The emulsion is then shaken to break up clumps, heated to $60^{\circ} \mathrm{C}$. for from one-half to one hour, cultured to determine sterility, and preserved with 0.5 per cent. phenol.

Strong has proposed the use of the products obtained by "autolytic digestion" of the organism, $i$. e., by incubating an emulsion of them in sterile water, in which they break up spontaneously. Twenty-four-hour agar cultures are removed with sterile water, placed in a sterile flask, and kept at a temperature of $60^{\circ} \mathrm{C}$. for twenty-four hours. The mixture is then put aside in the incubator for from two to five days. The best results are obtained apparently after five days' autolytic digestion. After such digestion the emulsion is filtered through a Reichel filter. The fluid thus obtained must, of course, be examined for sterility and carefully standardized before being used as a human vaccine.

Dosage.-Kolle's vaccine is given subcutaneously in two injections about a week apart- 1 c.c. the first time and 2 c.c. the second time.

Haffkine's vaccines are given in the same manner at an interval of five days.

The local effects are usually marked by more or less pain and edema, which subside in forty-eight hours. The constitutional effects are not infrequently severe, being marked by malaise, fever $\left(100^{\circ}-101^{\circ}\right.$ F.), nausea and vomiting, followed the next day in about 10 per cent. of persons by transient diarrhea. Usually all symptoms have disappeared within seventy-two hours.

Results.-Haffkine's prophylactic vaccine has yielded favorable results in India. Powell reports 198 cases of cholera among 6549 nonimmunized persons, with a total mortality of 124 . Of 5778 inoculated persons, there were 27 cases, with 14 deaths. Much better results were obtained with Kolle's vaccine, and it is now generally used in preference to the Haffkine vaccines.

Murata, during an epidemic in Japan in 1902, vaccinated 77,907 persons. Of these, 47 , or 0.06 per cent., developed cholera, and 20, or 0.02 per cent., died. Of 825,287 uninoculated persons, 1152 , or 0.13 per cent., died. During a recent epidemic in Russia Franschetti inoculated 11,178 persons. Of these, 8 contracted cholera and 1 died. In St. Petersburg, during $1907-08,30,000$ persons were inoculated. Of these, 12 developed cholera and 4 died. Of 10,000 uninoculated persons, 68 contracted the disease.

It appears justifiable, therefore, to conclude that inoculation confers 
some immunity. This protection may be apparent after the first dose, but is more marked after the second. The immunity conferred is far from being absolute, and it is noteworthy that while the prophylactic diminishes the liability of the inoculated person to cholera, it has less influence on the mortality when the disease occurs in those who have been vaccinated.

Used in conjunction with modern sanitary regulations, however, Kolle's vaccine certainly proves of value and should be used in combating epidemics.

\section{OTHER DISEASES}

Dysentery.-Protective vaccination against bacillary dysentery has been attempted, but has not as yet yielded satisfactory results. Shiga practised mixed active and passive immunization (vaccine plus immune serum) on 10,000 persons, and while this did not decrease the number of infections, a lower mortality resulted. The various types of the dysentery bacillus and the high toxicity of the vaccines are obstacles to the more general use of protective inoculation in this condition.

Cerebrospinal Meningitis.-Sophian and Black ${ }^{1}$ have shown experimentally that a polyvalent meningococcic vaccine, heated to $50^{\circ} \mathrm{C}$., standardized in the usual manner, and given in three injections, in doses of 100,000,000,500,000,000, and 1,000,000,000, at intervals of a week, appears to afford a high degree of protection. In the bloodserums of inoculated persons these observers were able to demonstrate opsonins, agglutinins, and complement-fixing amboceptors. All evidence points to the efficacy of prophylactic vaccination, as only a moderate degree of immunity may give complete protection against the disease. The method has not thus far received extensive trial, but in the presence of an epidemic its harmlessness and apparent value should be borne in mind.

Scarlet Fever.-Several Russian physicians, particularly Gabrickevski, ${ }^{2}$ Longovi, Nitikin, Shamarin, and others, have secured good results from a method of prophylactic vaccination against scarlet fever with a polyvalent vaccine of scarlet-fever streptococci. Heat-killed vaccines were given in three successive doses. In this country the method has been tried by Kolmer, ${ }^{3}$ who found that while inoculations with a heat-killed streptococcic vaccine cannot prevent scarlet fever

1 Jour. Amer. Med. Assoc., 1912, lix, 527. Black, ibid., 1913, lx, 1289.

${ }^{2}$ Russk. Vratch, St. Petersburg, 1906, x, 469.

${ }^{3}$ Penna. Med. Jour., February, 1912. 
itself, such inoculations may, however, prevent a severe attack of the disease by producing some immunity against secondary streptococcic infections.

\section{PROTECTIVE IMMUNIZATION AMONG THE LOWER ANIMALS}

Since discoveries in bacteriology and immunity have usually been intimately associated with animal experimentation, it is not strange that the lower animals should have been the first to benefit from the knowledge thus gained. As a consequence, vaccine therapy, both prophylactic and therapeutic, is being extensively used in veterinary practice with good results.

\section{ANTHRAX}

This was one of the first vaccines studied by Pasteur, and as a prophylactic measure, it has proved of great value. It finds its greatest field of usefulness in case of an outbreak of anthrax, when it is used to protect the uninfected members of a herd, as well as any animals pasturing on infected areas.

In preparing the vaccine Pasteur was hampered by the fact that the spores of anthrax bacilli retain the virulence of the original bacilli. As the result of extended experiments, however, he discovered a means of attenuating the virulence of cultures by growing the bacilli at a temperature of $42^{\circ} \mathrm{C}$; he also found that inoculation with these attenuated bacilli would effectively vaccinate sheep and cattle, and so protect them against an attack of the disease.

One of the most dramatic stories ${ }^{1}$ in the history of science is the account of the method by which Pasteur demonstrated his discovery to the public. Certain harsh critics, having heard of Pasteur's ability to prevent anthrax in laboratory experiments, and anxious to humiliate him, sent him a public challenge to demonstrate the experiment on a practical scale at a farm in the country. A number of farmers offered to place 60. sheep at his disposal. The challenge was immediately accepted, and Pasteur mapped out a plan of action, in which he safeguarded himself by making no half-statements, but boldly promised complete success. Of the total number, 25 sheep were to be vaccinated and 25 were to remain unvaccinated. A fortnight later all 50 were to receive a lethal dose of the fully virulent anthrax. He declared that the 25 non-vaccinated sheep would die, whereas the 25 vaccinated would remain alive and well. The remaining 10 sheep were to serve as controls. 
The challenge and its acceptance were widely advertised in the journals, and Pasteur was made the subject for many witticisms. Excitement ran high, and a large crowd, comprised of physicians, veterinary surgeons, journalists, farmers, etc., accompanied Pasteur to the farm (Pouilly le Fort) where he was to make the final test by inoculating the deadly anthrax. One vaccinated animal developed a temperature overnight, a fact that caused Pasteur much anxiety. On going to the farm the next day, however, again followed by the crowd, he found all the vaccinated animals well! Of the unvaccinated, 22 were dead, and the others died during the following night. Pasteur's triumph was complete, and the possibility of preventive vaccination was demonstrated to the world.

The vaccine is prepared of attenuated cultures of virulent anthrax bacilli.

Vaccine No. 1 is weakest or lowest in virulence, and the first to be injected. This vaccine is prepared by growing virulent anthrax bacilli at a temperature of $42^{\circ} \mathrm{C}$. for from six to ten weeks, or until $\frac{1}{10}$ loopful of the culture, when injected into rabbits, guinea-pigs, and mice, will show virulence for mice only, but not for guinea-pigs and rabbits.

Vaccine No. 2 is prepared by growing virulent anthrax bacilli at $42^{\circ} \mathrm{C}$. for about twenty days, or until $\frac{1}{10}$ loopful is virulent for mice, partly so for guinea-pigs, and not at all for rabbits.

Vaccine No. 3 is not generally used except for immunizing sheep and goats. When, however, it is required, it is made as follows: Virulent anthrax bacilli are grown at $42^{\circ} \mathrm{C}$. until $\frac{1}{10}$ loopful, when injected into mice, guinea-pigs, and rabbits, will be virulent for all the mice, all the guinea-pigs, and some of the rabbits.

The vaccines are prepared in ampules containing 1 c.c. of the emulsion, each representing one dose, to be injected subcutaneously. Vaccine No. 2 is injected twelve days after Vaccine No. 1, and No. 3 after the same interval following Vaccine No. 2. The resulting immunity usually lasts about six months.

In instances where it is desirable to immunize a herd before turning them out to pasture on infected areas it is well to inoculate the animals in the early spring, keeping them in the stable during the time required for at least two vaccinations, for the reason that, immediately after vaccination, the animals may become hypersusceptible to infection.

When conducted in a careful manner by a competent veterinarian, anthrax vaccination has proved fairly successful. 


\section{BLACKLEG}

Blackleg vaccine is used entirely as a prophylactic agent, for the disease runs too acute a course for the vaccine to exert any therapeutic influence.

The vaccine is prepared by the Bureau of Animal Industry in the following manner: The muscle tissue from a fresh blackleg tumor is ground in a mortar, extracted or macerated with a little water, and the fluid squeezed through cheese-cloth. The expressed fluid is then evaporated at a temperature of $35^{\circ} \mathrm{C}$. The dry brown scale is run through a grinding mill and heated for six or seven hours at a temperature of from $94^{\circ}$ to $96^{\circ} \mathrm{C}$. This process of heating attenuates the virulence of the bacilli present so that, when injected, they produce but a mild attack of the disease. The Department of Agriculture places the ground material in packages containing a certain number of doses. These packages are, upon request, mailed to veterinarians, who dilute the ground muscle with as many cubic centimeters of sterile water as there are doses in the package. One cubic centimeter of the suspension is injected subcutaneously in some convenient area, as, e. g., about the shoulders.

Blackleg vaccine should be applied in the spring, before young cattle are turned out to pasture on infected areas.

In case of a fresh outbreak, all the healthy animals in the herd are to be vaccinated as soon as possible.

Blackleg vaccination has been fairly successful, and usually confers an immunity lasting for a period of about six months.

\section{ACTIVE IMMUNIZATION FOR THERAPEUTIC PURPOSES-BACTERIAL VACCINE THERAPY}

Principles.-Although bacterial vaccines have been extensively employed in the treatment of various diseases, it is difficult to express an opinion as to their real value, and it is altogether impossible to make dogmatic statements as to the percentage of cases in which they will be helpful or effect a cure, or as to what result may be expected in an individual case. Following Wright's original announcements, this special therapy was enthusiastically received by the profession, and in a short space of time the method was employed experimentally in many and diverse infections. It may be stated that in many infections the vaccines may be beneficial, but they should be used only under proper conditions, as was indicated in the first portion of this chapter. 
It may be stated in general that:

1. Vaccine therapy has a special field of usefulness in the treatment of chronic infections.

2. Autogenous vaccines are to be preferred to stock vaccines, and in some infections the former must be used.

3 . It is not advisable to continue the use of the same vaccine for more than several doses if no reaction and no improvement are noted. New cultures should be made to determine if the right organism is being used, or if reinfection with another organism has occurred.

4. It is essential that the vaccine be properly prepared and not overheated.

5. It is highly important that the usual forms of treatment be employed in conjunction with the vaccine therapy. Thus abscesses should be incised; proper drainage of a discharging wound afforded; discharging ears cleansed, etc.

6. While the dose should not be too large, neither should it be too small, nor too far apart. There is a proper dose for each patient, and this may be determined by starting with a small dose and gradually increasing it until some reaction is secured. An efficient dose must necessarily produce some reaction, and increased doses are contraindicated so long as any sign of general or focal reaction is produced and so long as steady progress is maintained.

\section{DISEASES OF THE SKIN}

Furunculosis.-Furuncles are usually caused by some member of the group of staphylococci, and frequently the most rational and successful form of therapy is by means of bacterial vaccines. A stock vaccine of Staphylococcus aureus may prove satisfactory, and should be used while an autogenous vaccine is being prepared. For adults the initial dose may be $100,000,000$ cocci, succeeding doses gradually increasing until $1,000,000,000$ are given at one time. The injections may be given at intervals of from five to seven days. Following the first few doses, a slight focal and some constitutional reaction should be secured. After all the lesions have disappeared, one or two full doses at intervals of several months will continue to fortify the patient against a recurrence.

Carbuncles.-These are invariably caused by the Staphylococcus aureus, and exceptionally by a streptococcus. The urine should be examined for sugar, and even if the patient is diabetic, small doses of vaccine may be of value when used in conjunction with the customary treatment. 
Sycosis.-Sycosis vulgaris is usually caused by the Staphylococcus aureus and albus, and such patients are frequently very rebellious to the ordinary treatment. An autogenous vaccine is very helpful in some cases. Due care should be exercised in making cultures to secure pus from a well-developed lesion. Relatively large doses of vaccine are necessary, and treatment is usually prolonged, at least 12 injections being necessary before the conclusion is reached that the vaccines are of no service. As a rule, the condition will improve under vaccine therapy, but only in exceptional cases does a complete cure result.

Acne.-Acne is frequently caused by two microörganisms - a staphylococcus and the acne bacillus. In cases showing pustulation a staphylococcus is invariably present, and exceptionally the bacillus may be found alone in comedones. Cultures should be made from several lesions, being careful to secure pus that has not been contaminated by the skin. Stock vaccines may be used, although autogenous vaccines are likely to yield better results. It is well to administer a mixed vaccine of the staphylococcus and acne bacillus, especially if the lesions are pustular. It is highly essential that other forms of treatment be instituted while vaccines are being tried, as the treatment is usually prolonged. Exceptionally, however, a brilliant result may be observed after a few injections. I generally prepare a mixed vaccine of 10,000,000 acne bacilli to each $500,000,000$ cocci per 0.5 c.c. of vaccine. The first few doses consist of 0.5 c.c., and later this amount may be increased to 1 c.c. per dose, which usually contains enough bacilli and cocci for a number of injections. Doses are given every five to seven days, or just when retrogression is observed to occur. It may be necessary to use large doses, and in any case the treatment is prolonged over many weeks and months. Most cases will show improvement, but few are absolutely cured by a single series of injections.

Erysipelas.-This infection is usually caused by the streptococcus erysipelatis. In severe cases an autogenous vaccine of about $20,000,000$ cocci per cubic centimeter may be administered every three or four days, and frequently aids in reducing the severity of the inflammation and overcoming mental unrest and physical discomfort. A vaccine may be of aid in the treatment of subacute and chronic types of this disease. Stock vaccines are of little or no value.

\section{GENITO-URINARY DISEASES}

Cystitis.-Acute or subacute cystitis following catheterization after labor or surgical operations or occurring in children is usually caused by 
a member of the group of colon bacilli. It is highly essential, in order to attain success with vaccine therapy, that urine be collected aseptically and the causative microörganism secured and used in the preparation of a vaccine, as stock vaccines are of little or no value. Exceptionally the infection may be due to another microörganism, either alone or in conjunction with Bacillus coli. Treatment with an autogenous vaccine may be of distinct aid in lessening the symptoms and in reducing the amount of pus. The initial adult dose of Bacillus coli vaccine should be about from $50,000,000$ to $100,000,000$ bacilli. In subacute cystitis of the male, due to stricture of the urethra or enlarged prostate, or in the female, due to perineal injuries, not much benefit follows its use until the underlying cause is corrected or removed.

Urethritis.-There is a considerable difference of opinion as regards the efficacy of vaccines in the treatment of acute and chronic urethritis of gonorrheal origin. A polyvalent stock vaccine of gonococci of proved immunizing powers may be even more efficient than an autogenous one, especially if the latter must be prepared from a strain that has been repeatedly subcultured in order to obtain the vaccine in a pure state, or from one that has lost its virulence from long residence in the infected urethra. Owing, therefore, to the difficulty of isolating and cultivating gonococci, stock vaccines have been generally employed. In subacute urethritis the initial dose may be $25,000,000$; if complications threaten, less than this, and if no local reaction has followed more than this, is given, the object being to secure a slight increase of the secretion, which should become more purulent, and a little constitutional disturbance, followed by lessening of local pain and tenderness.

In chronic urethritis the primary infection is gonococcal, although other organisms, such as the Micrococcus catarrhalis, staphylococci, and diphtheria bacilli may have some relation to the process. A stock gonococcal vaccine may be used in sufficient dosage to evoke a reaction; not infrequently, however, a stock and an autogenous vaccine or vaccines combined yield better results. Cultures of the urethra should be made only after thorough clearising of the meatus and flushing of the urethra with sterile salt solution and massage of the prostate, cultures being made direct from the prostatic secretion or from the crypts through the urethroscope. In any case expert local treatment should be given while vaccines are being used.

Gonorrheal Arthritis.-Polyvalent stock vaccines of gonococci have generally been found to be of distinct value in the treatment of this troublesome and oftentimes chronic infection. Local treatment of the 
urethra and general therapeutic measures should be employed. It may be stated that vaccines should be used routinely in all cases, as under any condition the infection is likely to be prolonged and tedious. In the acute stages the doses should be relatively small, about $10,000,000$ cocci being given every three to five days. In the subacute stages from $50,000,000$ to $100,000,000$ may be given at intervals of from five to ten days.

Vulvovaginitis of Children.-Stock gonococcal vaccines have been used quite extensively in the treatment of this troublesome infection. On the whole, good results have been reported, although in any case final judgment must be reserved until thorough bacteriologic examination shows whether the tissues are really free from infection or whether the infection has subsided and become chronic. Smears of the secretions alone are insufficient to determine whether a cure has been effected. Injecting a solution of $1: 2000$ bichlorid of mercury in normal saline solution into the vagina, followed by immediate centrifugalization of the washings and smears of the sediment, will frequently demonstrate the presence of gonococci that will not otherwise be found. If vaccines are used, a dose of from $5,000,000$ to $10,000,000$ every five to seven days may be employed.

\section{RESPIRATORY DISEASES}

Rhinitis.-The use of mixed stock vaccines is being advocated for prophylactic immunization against recurrent attacks of acute rhinitis. With Weston, I have found autogenous vaccines of some value in lessening the severity and hastening the recovery from the acute rhinitis of scarlet fever, so potent a factor in the dissemination of that disease. In chronic rhinitis an autogenous vaccine, prepared by growing cultures with the care previously described, may be of distinct value, but only when an underlying factor, such as a malformation or adenoids, has been removed, and only when used in conjunction with efficient local treatment.

Bronchitis.-Allen speaks very highly of the value of autogenous vaccines in the treatment of acute and chronic bronchitis and bronchopneumonia of children. Various microörganisms are found in the secretions, and the vaccines used are generally mixed. The usual therapeutic measures are employed simultaneously. It is claimed that vaccines lessen the discomfort and hasten recovery.

Pertussis.-A hemophilic bacillus closely resembling the influenza bacillus has been ascribed by Bordet and Wollstein as the cause of pertus- 
sis. Several investigators who have used a stock vaccine of the pertussis bacillus claim that, used in small and appropriate doses, the severity of the paroxysms of coughing is lessened, and the whole course of the infection shortened; besides this, they assert, it decreases the danger of a complicating bronchopneumonia. When the disease is unusually severe and the prognosis is bad, a vaccine may be administered in doses of 25,000,000 if the patient is over four years of age. The pneumococcus and Bacillus influenzæ are frequently associated, and a mixed vaccine of these microörganisms may be of special aid in the later stages of the disease. The usual remedial measures should be employed while vaccines are being tried. A stock vaccine has been advocated for purposes of prophylactic immunization, especially in institutions, where pertussis among children claims a high mortality.

Otitis Media.-Autogenous vaccines prepared from carefully made cultures of the diseased tissues, secured with the aid of an ear speculum, may prove of some value in the treatment of subacute and chronic suppurative otitis media. Additional treatment may be carefully given, but not infrequently more harm than good is done by careless flushing and cleansing of the auditory canal, whereby deeper and healthy tissues become infected. Free drainage should be afforded, and in chronic otitis media necrosed ossicles and granulations may require surgical removal. Injections may be given every five to seven days. A slight increase in discharge after the first one or two doses is of good import, and indicates a slight focal reaction. With Weston, I have treated a large number of cases of suppurative otitis media following scarlet fever, and while the results were seldom brilliant, in general the duration and severity of the infections were favorably influenced.

\section{ACUTE GENERAL INFECTIONS}

Vaccines have been advocated in the treatment of typhoid fever and pneumonia. In the former an autogenous vaccine may be prepared if a blood culture is made early in the infection. Otherwise a stock vaccine prepared of a strain of known immunizing power may be used. The doses should be small, and may be given at short intervals. The object is to stimulate the body-cells to further effort in the production of antibodies. On the whole, I am not in favor of giving vaccines in typhoid fever, especially if the infection is severe or the general vitality of the patient is low, because of the danger of doing actual harm at a critical period. If vaccines are used, however, the initial dose should be less than 100,000,000 bacilli, and their effect must be very carefully observed. 
Autogenous vaccines may be of value in the treatment of delayed resolution in lobar pneumonia, cultures being secured by puncturing the lungs. Usually several microörganisms are found, and a mixed vaccine may be given.

Autogenous vaccines may also be of service in the treatment of puerperal sepsis and ulcerative endocarditis, especially after the more acute symptoms have subsided. In the former condition a streptococcus may be obtained by blood culture or by intra-uterine cultures; in the latter, the infecting microörganism is obtained only by blood culture. Stock vaccines may be administered, but are not likely to prove of value, as both infections are usually caused by streptococci, pneumococci, or some similar microörganisms showing so much difference in individual properties as to make the use of autogenous vaccines imperative. The initial doses should be small-not over 50,000,000 cocci; they may be repeated every three to five days, and are gradually increased as the conditions warrant.

\section{TUBERCULOSIS. - TUBERCULIN THERAPY}

Historic.-Within the last twenty years the subject of tuberculin therapy has elicited considerable discussion in the diagnosis and treatment of tuberculosis. The wide-spread prevalence of the disease, not only in man, but in the lower animals as well, the distressing symptoms, the gloomy prognosis, and the economic importance it possesses, are a few of the factors that have stimulated investigators the world over to zealous and persistent efforts directed toward discovering a means of preventing and curing this great scourge. Owing to the nature of the infection, which covers relatively long periods of time, and the fact that much time is required for the conduct of experimental studies, researches are of necessity prolonged, tedious, and difficult. Within a period of less than six years the cause of syphilis has been discovered and isolated, and valuable diagnostic reactions and a well-nigh specific therapy have been devised. The discovery of an early and specific diagnostic and therapeutic measure for tuberculosis will achieve still greater triumphsin fact, few could be greater or more beneficial.

Koch was the first to note the curative action of tuberculin, and it may be well to refer here to the original description of his fundamental experiments, ${ }^{1}$ which have been the basis as well as the starting-point of the entire study of tuberculins.

${ }^{1}$ Deutsch. med. Wochenschr., 1891, xvii, 101. 
"When one vaccinates a healthy guinea-pig with a pure culture of tubercle bacilli, the wound, as a rule, closes and in the first few days seems to heal. However, in from ten to fourteen days a hard nodule appears which soon breaks down, leaving an ulcer that persists to the time of death of the animal. There is quite a different sequence of events when a tuberculous guinea-pig is vaccinated. For this experiment animals are best suited that have been successfully infected four to six weeks previously. In such an animal the inoculation wound likewise promptly unites. However, no nodule forms, but on the next or second day after a peculiar change occurs. The point of inoculation and the tissues about, over an area of from 0.1 to $1 \mathrm{~cm}$. in diameter, grow hard and take on a dark discoloration. Observation on subsequent days makes it more and more apparent that the altered skin is necrotic. It is finally cast off, and a shallow ulceration remains, which usually heals quickly and permanently without the neighboring lymph-glands becoming infected. Inoculated tubercle bacilli act very differently then upon the skin of healthy and tuberculous guinea-pigs. This striking action is not restricted to living tubercle bacilli, but is equally manifested by dead bacilli, whether they be killed by exposure to low temperature for a long time or to the boiling temperature, or by the action of various chemicals.

"After having discovered these remarkable facts, I followed them up in all directions and was further able to show that killed pure cultures of tubercle bacilli ground up and suspended in water can be injected in large amounts under the skin of healthy guinea-pigs without producing any effect other than local suppuration. Tuberculous guinea-pigs, on the other hand, are killed in from six to forty-eight hours, according to the dose given, by the injection of small quantities of such a suspension. A dose which just falls short of the amount necessary to kill the animal may produce extensive necrosis of the skin about the point of injection. If the suspension be diluted until it is just visibly cloudy, the injected animals remain alive, and if the administration is continued with one to two day intervals, a rapid improvement in their condition takes place; the ulcerating inoculation wound becomes smaller and is finally replaced by a scar, a process that never occurs without such treatment; the swollen lymph-glands become smaller; the nutrition improves, and the disease process, unless it is too far advanced and the animals die of exhaustion, comes to a standstill.

"Thus was established the basis for a rational treatment of tuberculosis. However, such suspensions of killed tubercle bacilli are un- 
suitable for practical use, since they are neither absorbed nor disposed of in other ways, but remain a long time unaltered at the point of inoculation and occasion smaller or larger abscess."

Koch showed further that while the injection of tuberculous guineapigs with large doses of tubercle bacilli produced rapid death, frequently repeated small doses exerted a favorable effect upon the site of infection and the general condition of the animals. The same observer also realized that the harmful effects of injections of dead tubercle bacilli were due to the non-absorbable parts of the bacilli. He attempted to extract the immunizing substances, and in this way produced his first or Old Tuberculin. When injected into tuberculous guinea-pigs, old tuberculin produced a rapid general reaction without any local necrosis or sloughing, whereas when injected into a healthy guinea-pig, no reaction, either local or general, was produced. The fact that the general results produced by old tuberculin were analogous to those obtained by his first vaccine, except that local necrosis did not occur, induced Koch, in 1891, to promulgate it as a specific cure for tuberculosis in human beings.

It is hardly necessary to describe the hopeful anticipation with which it was received, and the keen disappointment that followed its earlier clinical use. Indiscriminate use, extravagant expectations, and excessive dosage combined to yield results so discouraging as to swing the pendulum of medical opinion so far the other way that even now the very word "tuberculin" suggests to many minds failure, and something to be avoided.

A few earlier followers of Koch continued their studies in the endeavor to discover the causes of failure in tuberculin therapy. Their researches have led to new principles in treatment and to more exact knowledge of its indications, as well as its contraindications. As now employed, its use being restricted to suitable patients and administered in safe graduated doses, and accepting as evidence only the statements of those who have used tuberculin and not of those who believe it to be dangerous and have never used it, one deduction is justified: that while tuberculin is not a specific "cure" for tuberculosis,- - any more than hygiene, diet, and climate are cures,-it helps to arrest the disease and is in general a useful factor in the treatment of certain types of the disease. Clinical studies have shown, however, that immunization of the tuberculous patient is frequently a difficult procedure, owing to the fact that such patients are prone to develop a remarkable state of hypersusceptibility, 
in consequence of which every inoculation will produce a reaction that may be injurious to the patient.

Preparation of Tuberculins.-The knowledge that tubercle bacilli and their secretions as seen in vitro contain both desirable and undesirable substances has led Koch and others to adopt different methods of preparing tuberculin in the endeavor to obtain the desirable immunizing principles in as pure a state as possible.

As a consequence, a large number of preparations have been advocated from time to time, all of which are said to possess some special properties and virtues. All tuberculins, whatever their mode of preparation and manufacture, are derived from cultures of the tubercle bacillus. So numerous have the tuberculins become, and so superior are the advantages claimed for each new product over the older ones, both for diagnostic and for therapeutic purposes, that only a few of those possessing special interest and value can here be described.

1. Old Tuberculin (O. T.).-This is Koch's original tuberculin, and is the variety regarded by many as the most useful both in diagnosis and in treatment. Its manufacture was based upon the principle that the toxins elaborated by the bacilli into the culture-medium or liberated by disintegration of the bodies were chiefly concerned in stimulating body-cells to the formation of antibodies. Since the bacillary bodies were regarded as mainly responsible for the production of abscesses at the point of inoculation, they are eliminated by a process of filtration.

Old tuberculin is prepared as follows: Large shallow flasks containing 5 per cent. of glycerin alkaline broth are inoculated with a culture of human tubercle bacilli and grown at body temperature for from six to eight weeks, at the end of which time the bacilli have grown into a flat sheet covering the surface of the fluid (Fig. 133). The entire contents are then subjected to a current of steam over a water-bath for the purposes of sterilization and for concentration into one-tenth of the original volume. The glycerin, which is not evaporated, thus constitutes 50 per cent. of the resulting mixture. The bacilli are removed by filtration through a Berkefeld or Chamberland filter. The filtrate is a clear, brown fluid, of a characteristic odor, which keeps indefinitely and is ready for use.

Koch considered the soluble toxins of the bacillus as the desirable immunizing agents, and believed that the endotoxins were responsible for the necrotic effects. Since, however, it was accepted that bacteriolytic substances would be formed only after the injection of intact or 
fragmented tubercle bacilli,-with their contained endotoxins,-Koch added T. R. and later B. E. to his list, in order to make the production of antibacterial substances still more complete. Furthermore, in order to obtain as varied a supply of antibodies as possible the use of several tuberculins, such as old tuberculin and bacillus emulsion, was recommended for use in the same patient.

2. New Tuberculin (Known as T. R. or Tuberculin Residue).-This was the next tuberculin to be promulgated by Koch, ${ }^{1}$ and is prepared as follows: Virulent cultures of human tubercle bacilli are grown in flasks of nutrient glycerin broth for from four to six weeks, the bacilli being then filtered off and dried in a vacuum. One gram of the dried tubercle

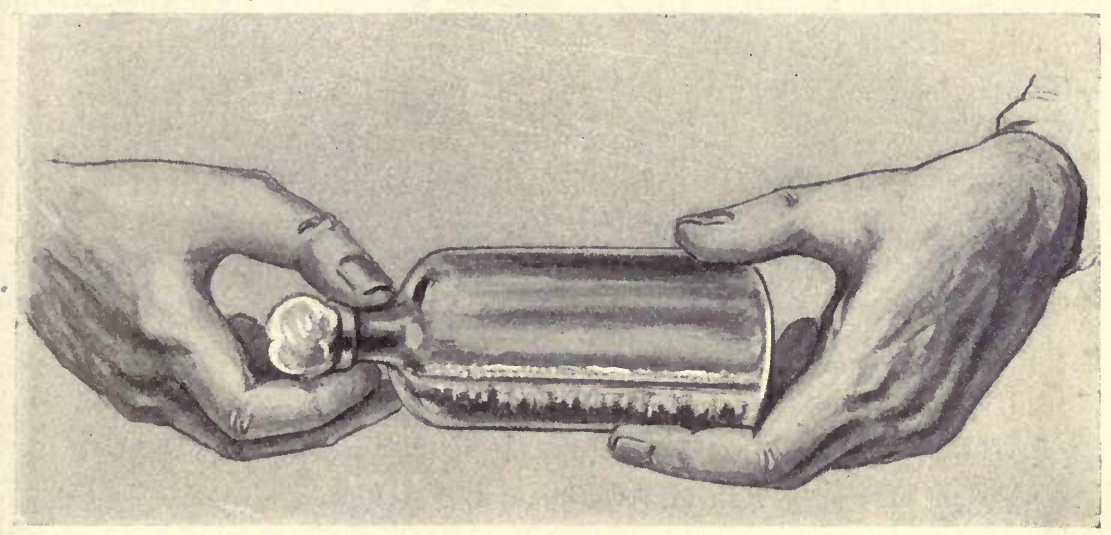

Fig. 133.-Preparation of Tuberculin.

A flask of bouillon culture of tubercle bacilli (three to four weeks). Note the surface layer of bacilli with stalactite formations.

bacilli is ground in an agate mortar until all the bacilli have been broken up. To the pulverized mass 100 c.c. of distilled water are added, and the mixture is then centrifugalized. The clear supernatant fluid is poured off, and is now known as Tuberculin Oberes (T. O., not to be confounded with $\mathrm{O}$. T.). It contains substances not precipitable by glycerin. The sediment is again dried, powdered, taken up in a small amount of water, centrifuged, the supernatant fluid poured off, and the process repeated until no sediment is precipitated except that composed of gross accidental particles. The fluids resulting from all the centrifugalizations, except the very first, are poured together and the total should not measure more than 100 c.c. This opalescent fluid is preserved with 20 per cent. of glycerin and is known as T. R. It should contain

${ }^{1}$ Deutsch. med. Wochenschr., 1897, xxiii, 209. 
in each cubic centimeter 2 milligrams of solids, representing 10 milligrams of dried tubercle bacilli.

3. Bacillen Emulsion (B. E.).-This was a still later form of tuberculin made by Koch, ${ }^{1}$ and, as its name indicates, it is an emulsion of tubercle bacilli. The culture is grown as for O. T.; the bacilli are filtered off, ground, but not washed, and one part of the pulverized material emulsified in 100 parts of distilled water; an equal part of glycerin is then added, making a 50 per cent. glycerin emulsion, each cubic centimeter of which contains the immunizing substances of $5 \mathrm{mg}$. of dried tubercle bacilli. Since B. E. was not washed, it was assumed that it would retain all extractives and the entire contents of the bodies of the tubercle bacillus.

While Koch was preparing these various tuberculins others were being made, one of which, prepared by Denys, is used extensively at present in the treatment of tuberculosis.

4. Bouillon Filtrate (B. F.).-This is practically Koch's old tuberculin unheated. It is prepared in the same manner as O. T., except that the bacillus-free filtrate-a clear fluid said to contain only the soluble secretions of the bacilli, plus the metabolized culture-mediumis not heated or concentrated and is ready for use without any further modification.

5. Beraneck's Tuberculin.-This is a preparation for which its inventor claims only minimal toxicity and a high content of specific substances. It is prepared by cultivating the bacilli on a non-peptonized 5 per cent. glycerin bouillon medium, which is not neutralized. The filtrate from this culture is known as T. B., or toxin bouillon. The residue is shaken for a long time at from $60^{\circ}$ to $70^{\circ} \mathrm{C}$. with 1 per cent. orthophosphoric acid. Equal volumes of the unheated toxin bouillon and of the acid extract of the bacillary bodies are combined to form the whole tuberculin.

6. Von Ruck's Watery Extract. ${ }^{2}$-This tuberculin has been widely used in the United States. It is prepared as follows: Concentrate a culture in vacuo at $55^{\circ} \mathrm{C}$. to one-tenth volume (this requires about one month). Filter through paper and then through porcelain. Precipitate with an acid solution of sodic bismuth iodid. Filter and neutralize the acid solution. Filter again. Precipitate with absolute alcohol to make 90 per cent. alcohol and filter. Wash the precipitate with absolute alcohol. Dry the precipitate and make a 1 per

${ }^{1}$ Deutsch. med. Wochenschr., 1901, xxvii, 839.

2 Zeitschr. f. Tuberk., 1907, xi, 493. 
cent. aqueous solution. Filter. The last filtrate is von Ruck's tuberculin.

7. Dixon's Tubercle-bacilli Extract. ${ }^{1}$-Dixon has prepared a tuberculin by treating cultures of tubercle bacilli with ether and extracting in salt solution. This has yielded good results in the treatment of tuberculosis. This product is prepared from the living organisms. The tubercle bacilli are grown on 4 per cent. glycerin veal broth for a period of from six to eight weeks at a temperature of $37.5^{\circ} \mathrm{C}$. They are removed from the incubator and collected on hard filter-paper. The filtrate of glycerin broth on which they are grown is discarded. An equal quantity, by weight, of tubercle bacilli of the bovine and human type is used. The mass of organisms is partially freed from excess of moisture by placing it between two sterile filter-papers, after which it is placed in a dish in the incubator for from twenty-four to forty-eight hours. The dried organisms are then washed in an excess of ether, which is allowed to act until it has removed all the water and glycerin. The organisms are then subjected to an excess of fresh ether and washed in this for six hours, to soften the wax of the bacilli. This fat separates so that it collects at the bottom of the vessel and is removed with a Pasteur pipet. After the second addition of ether has been removed the mass is allowed to dry until no ether odor is perceptible. After the mass of tubercle bacilli has been thoroughly dried it is ground in a mortar and suspended in physiologic salt solution in the proportion of 1 part of the ground mass to 5 parts of an 8 per cent. salt solution. This suspension is shaken in a shaking machine for from eight to ten hours, and is then allowed to stand for several days at room temperature. It is finally passed through impervious bacteria filters several times and the filtrate examined microscopically, bacteriologically, and physiologically. Culture tests are made to determine its freedom from contaminating organisms, and guinea-pigs are inoculated to ascertain that it contains no living tubercle bacilli. One cubic centimeter of this extract represents $0.5 \mathrm{gm}$. of the organisms, and is known as the stock solution, from which serial dilutions are made. This solution is sterile, but 0.5 per cent. of phenol (carbolic acid) is added as a preservative to prevent subsequent contamination.

While the tuberculins just described are those mainly used, many others have been prepared and advocated in the diagnosis and treatment of tuberculosis. The aim is always to obtain the specific substances, with as little as possible of the toxic substances-not only those con-

1 Penna. Health Bull., October, 1911. 
cerned in producing necrosis, but likewise the protein constituents responsible for specific sensitizing action and anaphylactic disturbances. For example, tuberculocidin and tuberculol are examples of attempts at isolating the pure immunizing principle; endotin, or Moeller's tuberculin, is an example of an endeavor to rid the culture fluid of protein substances. ${ }^{1}$

Action of Tuberculin.-If a healthy or a tuberculous individual is injected with old tuberculin, an immunity will be established only against the substances contained in this preparation. That this does not fulfil the requirements is proved by the fact that an animal immunized against this tuberculin will not be protected against a later infection with living tubercle bacilli. It was mainly for this reason that tubercle emulsion and new tuberculin were devised and used, in the effort to provide immunization not only against the products of the bacilli, but against the bacilli themselves, and to bring about their actual destruction.

Probably none of the various tuberculins can be considered as representing the true toxins of the tubercle bacillus, although they simulate these substances with sufficient closeness to bring about partial immunity against some of the poisonous products and to warrant their use in tuberculosis. An individual may become immunized against old tuberculin so that large doses will evoke no reaction, but this does not necessarily imply that a cure has resulted; in fact, the injection of another preparation, such as new tuberculin or bacillus emulsion, may bring about a reaction.

Partial immunization possesses, however, distinct advantages, in that it lessens some of the symptoms of tuberculosis. In addition, tuberculin immunization may give most important aid in walling off tuberculous foci with fibrous tissue, and in this manner bring about a condition of potential cure. Following the injection of tuberculin a focal reaction occurs, characterized by hyperemia and exudation about the diseased tissues. While an excessive dose of tuberculin may produce excessive hyperemia, exudation, and necrosis of tuberculous tissue and lead to actual harm, these being some of the effects that followed the early use of tuberculin and resulted in bringing it into high disfavor, smaller and carefully graduated doses tend to produce a mild inflammatory hyperemia leading to destruction of tuberculous tissue and the

\footnotetext{
${ }^{1}$ For a full account of these and other preparations I refer the reader to the book of Hamman and Wolman, "Tuberculin in Diagnosis and Treatment," 1912, Appleton \& Co.
} 
formation of connective tissue which encapsulates the focus; with this there is also associated the local production of antibodies.

The Use of Living Tubercle Bacilli.-Any vaccine that will give complete immunization against the tubercle bacillus and all its products, in addition to a healing focal reaction of the right degree, will prove of the greatest value in active prophylactic and curative immunization.

As has been stated repeatedly, this is probably best obtained by using living cultures in such form that they cannot produce the disease. Obviously, it is a difficult matter to find such a culture or to modify one to meet the requirements at hand. When this problem is solved, it may then be possible to provide universal prophylactic immunization and to aid the actually diseased in overcoming their infection. At present the tuberculins are not adapted for prophylaxis because they possess only partial immunizing powers, although these effects may be of distinct aid to the tuberculous patient, in addition to producing a focal reaction of value in walling off a lesion and producing local antibodies.

Probably the first work done with the living bacillus was that of Dixon ${ }^{1}$ with attenuated cultures. This worker found that animals inoculated with an old culture containing club-shaped and branching forms of tubercle bacilli would resist subsequent inoculation with virulent organisms. Since then numerous investigators-Trudeau, ${ }^{2}$ Pearson, ${ }^{3}$ de Schweinitz, ${ }^{4}$ McFadyean, ${ }^{5}$ Levy ${ }^{6}{ }^{6}$ Pearson and Gilliland, ${ }^{7}$ Behring, ${ }^{8}$ Thomassen, ${ }^{9}$ Neufeld, ${ }^{10}$ Theobald Smith, ${ }^{11}$ Webb and Williams, ${ }^{12}$ and others-have, either directly or indirectly, supported this original work, thus indicating that the most effectual active immunization is secured by using living cultures. The method employed by Webb and Williams is worthy of special mention, inasmuch as the ascending doses of bacilli are actually counted by an ingenious method devised by

1 Medical News, Philadelphia, October 19, 1889.

${ }^{2}$ Amer. Jour. Med. Sci., August, 1906; June, 1907; New York Med. Jour., July 23, 1893; Medical News, October 24, 1903.

${ }^{3}$ Proc. First Internat. Vet. Congress, 1893.

${ }^{4}$ Medical News, December 8, 1894.

${ }^{5}$ Jour. of Comparative Path. and Therap., June, 1901; March, 1902.

${ }^{6}$ Centralbl. f. Bakt., 1903.

${ }^{7}$ Phila. Med. Jour., November 29, 1902; Univ. of Penna. Med. Bull., 1905.

${ }^{8}$ Beiträge z. exper. Therapie, Reft. s. Marburg, 1902.

${ }^{9}$ Recuil de Med. Vet., January 15, 1903.

${ }^{10}$ Deutsch. med. Wochenschr., September 1, 1902; April 28, 1904.

"Jour. Med. Research, June, 1908.

12 Trans. of the Sixth Internat. Congress on Tuberculosis, 1908; Jour. Med. Research, 1911, xix, 1. 
Barbour. ${ }^{1}$ These observers used this method quite extensively with lower animals, and have also secured good results with persons willing and anxious to take all possible risks for the possible good that may result. In no case have harmful results followed the injections.

More recently the profession and laity have been agitated by the extravagant claims of Friedmann for a vaccine of living, acid-fast bacilli derived from the turtle. This culture is said to be avirulent for human beings, and to be capable of stimulating specific antibodies and thus bringing about a cure. The unfortunate methods by which these claims have been exploited, and the more recent investigations, tending to show that no good has followed its use, and that the vaccine is not entirely harmless, preclude any statements at this time. The principle involved, however; namely, the use of a living vaccine composed of microörganisms so altered by passage through a lower animal that they cannot produce tuberculosis in the human being, but yet resembling the human bacillus closely enough to produce specific antibodies, is sound, and should stimulate further experimental research in this direction.

Patients Suitable for Tuberculin Treatment.-In deciding which tuberculous patients are best suited to receive tuberculin treatment it must be borne in mind that tuberculin is not a form of passive immunity, depending upon antitoxins, bacteriotropins, and bactericidins, but that it serves to stimulate the body-cells to produce these protective substances. The output of antibodies provoked by tuberculin is dependent upon the condition of the body as a whole, and its administration can be of no help if the resources of the body are exhausted and the cells are incapable of beneficiary reaction. In other words, tuberculin therapy must be guided by the same considerations that influence the vaccine treatment of acute infections in general.

1. Patients afflicted with incipient tuberculosis are proper subjects for receiving tuberculin treatment, since it tends to protect them from relapse, and insures, to a greater degree, their ability to continue work.

2. Advanced and moderately advanced cases may be given tuberculin if the nutrition is fair, the febrile reaction mild, the pulse not very rapid, and if the treatment is controlled by rest. Old fibroid cases with fair nutrition are especially suitable, as such patients become capable of moderate activity and are much less likely to suffer from relapses.

3. Cases of tuberculosis of the lymphatic glands, skin, and special organs may be benefited by prolonged and careful tuberculin therapy.

4. Cases of latent tuberculosis, especially the children of infected ${ }^{1}$ The Kansas Univ. Sci. Bull., 1907, iv, No. 1. 
families who are below par physically and show tuberculin hypersensitiveness, with indefinite physical signs, are proper subjects for receiving tuberculin treatment.

5. The question arises as to whether tuberculin may be administered to ambulant patients. Tuberculin should be regarded as but one factor in the treatment of tuberculosis, and, as such, should be combined with the best therapeutic measures available. Therefore the tuberculin treatment is supplementary to rest, hygiene, and fresh air, and the benefit of the sanatorium should not be denied to patients, especially to the poorer ones. The treatment of a mildly progressive ambulant case should be undertaken only when prolonged rest in bed has had no visible effect, and when no measures can be devised for administering the tuberculin while the patient is in bed, as, e. g., - patients who have been at the sanatorium and have returned to work, or those who cannot be persuaded to enter a sanatorium or for whom no place can be found. The tuberculin treatment of more chronic or localized tuberculosis may be successfully undertaken in the clinic or office.

Contraindications to Tuberculin Therapy.-Owing to the increased focal hyperemia that follows the injection of tuberculin, hemoptysis has been considered a contraindication to its use. In such cases it is well to wait for some time at least and begin the injections with very small doses, as the ultimate effect, namely, the production of fibrous tissue, may be of great aid in prolonging life.

Various authorities have expressed different views regarding other contraindications, such as marked general weakness, fever, cardiac disease, nephritis, epilepsy, syphilis, hysteria, etc. As was stated by Hamman and Wolman, these are not contraindications, but unfortunate complications that would embarrass any form of treatment. Tuberculin may be given to any patient whose resisting powers have not been too much depressed as the result of complications. For the beginner in this form of therapy, however, it is advisable that he acquire experience by undertaking the treatment of uncomplicated cases before assuming the responsibility of treating the more difficult ones.

The fact that the ophthalmic test has been made is no contraindication to treatment by tuberculin if the reaction has subsided, since a flare-up rarely occurs except after large diagnostic doses (Hamman and Wolman).

\section{ADMINISTRATION OF TUBERCULIN}

1. Subcutaneous Injection.- Of most importance in this connection is the attitude of the therapeutist toward the question of reactions 
following the administration of tuberculin. He must know whether he does or does not wish to obtain symptoms of a tuberculin reaction during the treatment; the size of the initial and particularly of subsequent doses will depend upon his desire to obtain a reaction or upon his anxiety to avoid it.

Reactions.-At the present time tuberculin is never used for the purpose of obtaining strong reactions, such as Koch originally insisted upon getting. Koch administered a dose large enough to elicit a strong constitutional reaction, and repeated it at intervals of one or more days until that dose no longer produced a reaction, after which a still larger dose was given and the former procedure repeated. Many - too manywere unable to pass through this therapeutic furnace unsinged, and, in fact, the results obtained led to the period known as the "tuberculin delirium," ending, as Hamman and Wolman stated, "to the consequent downfall of the arrogant therapy to an humble position, whence it is but just emerging, chastened and refined, to assert its modest but now truthful claims to a therapy less spectacular but more healing, less forceful but more gently persuasive: healing a few, helping many, and hurting none."

While there is this general unanimity of opinion regarding the harmful effects of strong reactions, yet tuberculin therapeutists may be divided into those who scrupulously avoid all reaction, those who are a little bolder and do not object to a very mild reaction, and to an intermediate class. In the first group are Sahli and Trudeau; the former claims that it is essential, first of all, to do no harm, and that cases treated cautiously attain a tolerance for high doses as soon as, and even sooner than, those that are rushed. Petruschky is an exponent of the bolder method, believing that, by proceeding very cautiously, much time is wasted and not enough focal reaction is produced to promote healing. To the intermediate class, and approaching rather the timid class, are Bandelier and Roepke, Hamman and Wolman, and many others. These observers adopt no scheme of fixed dosage, but study the individual patient, remembering what is to be avoided, rather than the high dose to be reached.

The constitutional symptoms of a reaction are: temperature, loss of weight, rapid pulse, and general symptoms, such as malaise, headache, chilliness, arthritic pains, gastric or intestinal disturbances, nausea, loss of appetite, insomnia, and skin eruptions.

Of these general signs, the most important are fever, loss of weight, and symptoms of general depression. The temperature should be taken 
for several days preceding the initial dose, so that the patient's "normal" limits are known before the tuberculin is given. When the usual maximum temperature is reached, it is especially necessary to watch closely for additional signs of reaction. Without some constitutional disturbance a slight pyrexia is of less significance, and it is to be remembered that tuberculous patients may have a flare-up of fever when they are not being treated with tuberculin. Denys refuses to consider any temperature reaction as due to tuberculin that comes on more than forty-eight hours after the injection has been given. It is characteristic of tuberculin reactions that the rise is abrupt, and not in step-like progression. Hamman and Wolman would hesitate to ascribe an elevation coming on suddenly in the midst of a perfectly smooth course of tuberculin treatment, and unaccompanied by a local reaction, to the injections, when the dose has not been unduly large.

Loss of weight is a delicate sign-more valuable as a symptom of overdosage late in the treatment than as a protection against the suddenly appearing reactions.

Bandelier and Roepke regard an increase in the pulse-rate as a sign of great importance. Hamman and Wolman and Lawrason Brown have not been able to observe this sign very frequently.

The local signs are: Pain, tenderness, and swelling at the site of injection. These may consist of all gradations from simple thickening of the skin to a wide, deep, hard, and painful node, with or without involvement of the neighboring lymphatics.

The local reaction has assumed great importance in recent years, especially since Denys drew attention to it as serving as a warning of the approach of a general reaction. It is characterized by the development of pain, tenderness, and redness about the site of a former injection. It is more often solitary than any other sign, and, in the absence of a temperature record, it is safe to proceed, the sole guidance being the local reaction, both subjective and objective. A large dose of tuberculin may give a local reaction, due merely to its bulk and concentration (500 to $600 \mathrm{mg}$.), simulating a true reaction; this may be avoided by dividing the dose into two injections, which are given at the same time, but not into neighboring areas of skin.

The focal signs in pulmonary tuberculosis are: increased cough, dyspnea, expectoration, thoracic pain, hemoptysis, and extension of the physical signs.

Focal signs of a reaction are an indication that the treatment has been conducted too rapidly. 
Unless one belongs to the ultra-conservative class of tuberculin therapeutists, it is not a slight focal reaction that is to be avoided, but those reactions that are large enough to manifest themselves by changes in the physical signs or by decided symptoms. On the contrary, it is the production of such slight hyperemia about the focus of disease that constitutes the most valuable result of tuberculin therapy. It is well to start with a minute dose, and push the dosage rapidly until a slight focal reaction at the site of injection or mild pyrexia is observed. When a mild focal reaction is not produced, the patient is not receiving his due amount.

Dosage.-Since each patient is a law unto himself, the initial dose should be so small that no harm can result from its use. White and Van Norman make the initial dose equal to the quantity of tuberculin which, when applied cutaneously, will elicit a minimal reaction after seventy-two hours.

As regards the size of the initial dose, patients can be divided into three classes: (1) Children; (2) patients who exhibit a slight pyrexia or are not in good condition; (3) patients in good condition. The following table of doses is that given by Hamman and Wolman, the smaller initial dose being for classes 1 and 2, the larger for class 3 .

TABLE 26.-INITIAL AND MAXIMAL DOSES OF THE COMMONLY USED TUBERCULINS

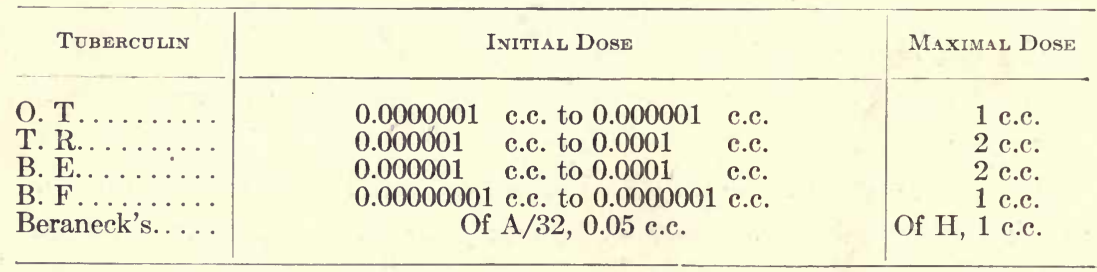

Old tuberculin (O. T.) is put up in ampules holding 1 c.c. and 5 c.c. From these, higher dilutions are prepared by adding sterile normal salt solution, using sterile glassware, starting with a $1: 10$ (A) of the original strength, then making a $1: 10$ from this (B), a $1: 10$ from that (C), a 1: 10 from that (D), and so on to the desired dilution. As 1 c.c. of the original product represents 1000 milligrams of the pure tuberculin, 1 c.c. of dilution A will contain 100 milligrams; B, 10 milligrams; C, 1 milligram; D, 0.1 milligram; E, 0.01 milligram, and F, 0.001 milligram, which is the higher of the initial doses just given.

New tuberculin (T.R.) is so prepared that 1 c.c. represents 5 milligrams of the dry powder. Dilutions may be prepared in a manner 
similar to the foregoing by diluting the original product $1: 10$ (A); 1 c.c. of this is in turn diluted to $1: 10$ (B); of this, $1: 10$ (C); and of this, $1: 10$ (D); 1 c.c. of A contains 0.5 milligram; 1 c.c. of $\mathrm{B}, 0.05$; 1 c.c. of $\mathrm{C}, 0.005$, and 1 c.c. of $\mathrm{D}, 0.0005$ milligram.

Beraneck's tuberculin is marketed, ready diluted, in a series of syringes, $\mathrm{A} / 128, \mathrm{~A} / 64, \mathrm{~A} / 32$, to $\mathrm{A} / 4, \mathrm{~A} / 2, \mathrm{~A}, \mathrm{~B}, \mathrm{C}$, to $\mathrm{H}$. $\mathrm{H}$ is the pure tuberculin. Each solution is one-half the strength of the next stronger one. The increase of dosage is usually by 0.1 c.c. until 0.5 c.c. is given. Then 0.1 c.c. of the next stronger dilution is given, and so on.

Dixon's tuberculin is supplied in syringes in the series of dilutions given in the following table, so that the doses may be increased as is found desirable:

\section{TABLE 27.-ADMINISTRATION OF DIXON'S TUBERCULIN}

\begin{tabular}{|c|c|}
\hline Dilution & $\begin{array}{l}\text { AMOUNT (IN GRAMS) OF } \\
\text { EXTRACT OF TUBERCLE }\end{array}$ \\
\hline No. & BaCILLI CoNTAINED IN \\
\hline 1. & .0 .001 \\
\hline 2. & .0 .01 \\
\hline 3. & 0.02 \\
\hline 4. & .0 .03 \\
\hline 5. & .0 .04 \\
\hline 6. & .0 .05 \\
\hline 7 & 0.06 \\
\hline & 0.07 \\
\hline 9. & 0.08 \\
\hline $10 \ldots$ & . . . . 0.09 \\
\hline
\end{tabular}

\begin{tabular}{|c|c|}
\hline & $\begin{array}{l}\text { AMOUNT (IN GRAMS) of } \\
\text { EXTRACT OF TUBERCLE }\end{array}$ \\
\hline $\begin{array}{l}\text { DILUTION } \\
\text { No. }\end{array}$ & $\begin{array}{l}\text { EXTRACT OF TUBERCLE } \\
\text { BACILLI CONTAINED IN }\end{array}$ \\
\hline 11. & $\ldots \ldots 0_{0.1}$ \\
\hline 12 . & 0.11 \\
\hline 13. & 0.12 \\
\hline 14. & .0 .13 \\
\hline 15 . & 0.14 \\
\hline 16. & 0.15 \\
\hline 17 . & ‥ 0.16 \\
\hline 18. & $\ldots \ldots \ldots 0.17$ \\
\hline 19. & $\ldots \ldots \ldots .18$ \\
\hline 20. & $\ldots \ldots \ldots 0.19$ \\
\hline
\end{tabular}

It is suggested that in ordinary cases the injection of each dilution be repeated at least five times before changing to the next number or next stronger dilution.

As a general rule, the succeeding doses of a tuberculin may be increased by 0.1 c.c., due care being exercised when the dilutions are changed. If the patient is quite sensitive, it may be well to repeat the first dose of each stronger solution once or more often as it is reached, and, instead of proceeding to 0.2 c.c., give only 0.15 c.c., thus tiding over the gap. If the patient is not so sensitive, a few leaps may be taken with the weaker dilution, so as to test the patient's tolerance, and, if it is good, the next higher dilution is given at once.

Site of Injection.-Injections are probably best given in the back, at the lower angle of the scapula. Local reactions are more reliable here than when the injections are given in the arm. All injections should be given under aseptic precautions and with a sterilized syringe; in exactly the same manner as any bacterial vaccine is given. All injections should be subcutaneous; intramuscular injections are 
to be avoided, and no great advantage is to be gained from using intravenous injections.

Time of Injection.-There is some difference of opinion as to the best time of the day for administering tuberculin therapeutically. If given in the morning, a slight febrile reaction may occur during the evening which would otherwise be overlooked. Brown is in favor of the afternoon as the most suitable time, because it affords an opportunity for omitting the dose in case there is an accidental rise of temperature on that day. On the other hand, it is contended that the rest at night would tend to prevent the occurrence of the reactions that might appear if the patient were up and about.

While it is not essential that the patient rest for a few hours after a dose has been administered, this is advisable, and where absolute rest can be enforced, the dosage may be increased with greater rapidity than in ambulant patients.

Interval Between Doses.-The interval between injections is usually from three to four days-i. e., two injections a week. This interval is merely tentative, and while it should not be shortened, it may be necessary to prolong it. While most reactions set in within from twentyfour to thirty-six hours, some may begin as late as from forty-eight to sixty hours (L. Brown). By waiting at least three days we may be assured that, if no reaction has occurred, none will take place.

The usual interval is maintained as long as the patient is doing well. After a while the patient becomes intolerant and exhibits slight reactions, depression, and loss of weight. In such instances the interval may be increased to a week and the injections continued. Hamman and Wolman find this occurrence so frequent that they advise creasing the dose interval to one week, when the dose of $100 \mathrm{mg}$. of $\mathrm{O}$. T. is reached, $200 \mathrm{mg}$. of T. R. and B. E., and $50 \mathrm{mg}$. of B. F.

2. Other Routes for the Administration of Tuberculin.-Oral Route.-It has been shown that reactions may follow the oral administration of tuberculin. But absorption is so irregular that a quantity of tuberculin may be absorbed suddenly and cause unexpected reactions. Much depends, apparently, upon the state of digestion and upon the condition of the alimentary tract. The oral route also deprives the physician of the benefits to be obtained from using the local reaction as a guide. Otherwise the method is simple and the tuberculin may be administered in the form of tablets or in capsules. It is important, however, to exercise supervision over the patient. $\mathbf{S}$. 
Solis Cohen has used a modification of Latham's method, and reports favorable results: "Tuberculin residue (T. R.) triturated with milk sugar is given with skim milk, whey, or beef-juice. The initial dose is $0.000001 \mathrm{mg}$. Both subjective and objective symptoms of reaction are watched for. The dose is repeated once or twice weekly, according to results. It is gradually increased by increments of $0.000001 \mathrm{mg}$. to the reaction point, and then dropped one point lower, and so continued for some weeks. Later, a further increase is attempted, and if reaction is not shown, is proceeded with in a similar gradual way. The arbitrary increment of $0.000001 \mathrm{mg}$. is maintained during this remittent progression until $0.0001 \mathrm{mg}$. has been reached. After that the increment may be raised to $0.00001 \mathrm{mg}$. Thus, by successive stages, a maximum dose is attained at a point determined for each individual by all the factors in the case, including the rapidity of increase, character and intensity of reaction, and maintenance of tolerance, as well as the focal and general signs of improvement. The treatment is continued with intermissions for many months, and may be resumed, if necessary, from time to time over a period of years."

Tuberculin has also been administered intrabronchially and by the rectum without good results.

Koch was the first to administer tuberculin intravenously, but this route has not come into general favor, owing to the fact that even greater control over dosage is necessary; there is, besides, no local reaction to serve as a guide, and technically the administration is more difficult than is subcutaneous injection.

The Intrafocal Route.-The use of tuberculin intrafocally, that is, a method by which the tuberculin is brought into immediate contact with the diseased area, has been advocated in the treatment of tuberculous pleurisy, tuberculous peritonitis, and tuberculosis of other serous membranes, such as those lining joints, the tunica vaginalis of the testicle etc. Senger, Crocker, and Pernet advise the intrafocal use of tuberculin in the treatment of lupus; others have applied it directly to broken-down glands and to sinuses. William Egbert Robertson ${ }^{1}$ has reported very good results in two cases of tuberculous pleurisy as a result of withdrawing a small amount of fluid and injecting $5 \mathrm{mg}$. of old tuberculin through the needle, which is left in situ for that purpose. After some hours there was a slight reaction; the remainder of the fluid was rapidly absorbed, and convalescence was promptly established,

\footnotetext{
${ }^{1}$ Personal communication.
} 
though, of course, a damaged lung remained. In a ease of hydrocele the injection of old tuberculin was followed by a sharp local and a moderate general reaction, followed by absorption, and without the slightest evidence of recurrence to date, now about a year since the injection was made.

Results of Tuberculin Therapy.-The early and disastrous results obtained with tuberculin in various types of tuberculosis cannot be used at the present day as a measure for determining the therapeutic value of tuberculin.

So much depends upon the individual case, the duration and activity of the disease, the possibility of supplementing the active immunization with sanatorium treatment, the skill and patience of the physician, etc., that, from a prognostic standpoint, every case must be judged upon its own merits. The results of the modern use of tuberculin show quite clearly that it is not a "cure" for tuberculosis, but, rather, a rational and useful therapeutic aid, the best results being secured when the treatment is carried out in special institutions or by specially trained physicians who have a practical knowledge of the difficulties, dangers, and possibilities of tuberculin therapy.

The value of this or of any therapy can be judged according to various standards: (1) Working ability; (2) duration of life; (3) the presence of tubercle bacilli in the sputum; (4) physical signs, and (5) the symptoms.

The first three are especially valuable; duration of life is, after all, the most important criterion, as anything that prolongs life is, of course, welcomed.

Kremser chose 110 patients expectorating tubercle bacilli and treated 55 unselected cases with tuberculin; of these, 22 , or 40 per cent., lost the bacilli from their sputum; of those treated without tuberculin only 16, or 29 per cent., lost their bacilli. Phillippi found that 58 per cent. of his second stage cases were rid of bacilli in the sputum under tuberculin treatment, as against 19 per cent. without. Brown reports from Saranac Lake that, in the incipient class, 67 per cent. of the tuberculin patients were rid of bacilli; of the others, 64 per cent. In the moderately advanced the figures are respectively 44 per cent. and 24 per cent. Bandelier gives the reports of 500 cases, of whom 202 had tubercle bacilli in the sputum. In the following table he compares the working capacity and sputum examinations of these patients under tuberculin treatment: 


\begin{tabular}{|c|c|c|c|c|}
\hline & Total & STAGE I & Stage II & Stage III \\
\hline $\begin{array}{l}\text { Complete earning capacity } \\
\text { on discharge............ } \\
\text { Sputum changed from pos- } \\
\text { itive to negative......... }\end{array}$ & $\begin{array}{l}500 \text { cases }(69.8 \\
\text { per cent. }) \\
202 \text { cases }(63.9 \\
\text { per cent. })\end{array}$ & $\begin{array}{r}\text { Per cent. } \\
90.4 \\
100.0\end{array}$ & Per cent. & Per cent. \\
\hline
\end{tabular}

The parallelism between the bacillary content of the sputum and the working capacity is close and shows the value, from a statistical point of view, of sputum examinations.

Healing with and without tuberculin is qualitatively the same, although, in the opinion of Ziegler, Petruschky and Rohmer, Pearson and Gilliland, Jurgens, Neumann, and others, quantitatively the results are different, all authors agreeing on the presence of more fibrosis about the lesions than is usual in the untreated cases. Autopsy findings necessarily point with less favor to tuberculin therapy than do clinical facts bearing on the rapidity and permanency with which a lesion heals.

The influence of tuberculin cannot at present be judged from the presence of antibodies in the serum, as data bearing upon the presence or absence of antitoxins, opsonins, agglutinins, and bacteriolysins are insufficient.

General symptoms and signs, such as fever, cough, loss of weight, accelerated pulse, digestive disturbances, dyspnea, and pain, if they are due to tuberculosis, as a rule improve under tuberculin treatment. Under proper conditions the incidence of hemoptysis is not usually increased, and the quantity of sputum and number of expectorated bacilli gradually decrease.

In the treatment of tuberculosis of the eye tuberculin has yielded exceptionally good results; in tuberculous adenitis considerable good may be accomplished with those glands that have not as yet softened. In bone and joint tuberculosis there is a growing opinion that surgery may be obviated or supplemented by tuberculin. In tuberculosis of the ear, tuberculin has yielded good results and should always be used. The results in tuberculosis of the skin have been generally disappointing, and when tuberculin is administered, the treatment should be prolonged and supplemented by the usual therapeutic measures. Tuberculosis of the intestine and mesenteric glands may be benefited, and in subacute tuberculous meningitis this method of treatment'may also be tried, combined with lumbar puncture to relieve intracranial pressure. In 
tuberculosis of the genito-urinary organs the prognosis is largely dependent upon the accompanying pulmonary condition and upon the extent of the local process. There is general recognition of the value of tuberculin as an adjuvant to hygienic measures, although great difference of opinion exists as to surgical intervention. If one kidney is involved, most surgeons advise early operation, followed by tuberculin treatment; if both organs are diseased, tuberculin may prove a valuable adjuvant to the treatment. 


\section{CHAPTER XXX}

\section{PASSIVE IMMUNIZATION-SERUM THERAPY}

SERUM therapy may be said to have had its origin in 1890 , when von Behring discovered diphtheria antitoxin. He found that guinea-pigs surviving a subcutaneous inoculation of living diphtheria bacilli may harbor virulent bacilli at the site of injection without showing any evidences of intoxication. Subsequent investigation showed that the blood-serum of these animals contained the protective principles, for when the serum was injected into other animals along with the diphtheria toxin, symptoms of the disease did not develop, and, indeed, as was shown later, the immune serum was found capable of neutralizing the toxin in the test-tube. Shortly afterward Kitasato made similar discoveries in studying tetanus, and these antitoxins have since proved of great importance, not only from the new light that has been thrown upon the mechanism of immunity,-and they were used as important arguments for the humoral as opposed to the phagocytic theory, and form the very basis and starting-point of Ehrlich's researches,-but also from the new and important field of therapy that was now opened, which gave promise and hope for the discovery of a specific serum treatment for each bacterial disease.

At the time it was thought possible to immunize animals with the various microörganisms known to produce disease, and that the immune serums so produced may be employed in the form of specific treatment. This theory rested on the fact that they contained the antibodies that would quickly overcome the infection. With a few of the genuinely antitoxic serums these hopes have been realized; but many other serums have not yielded the expected and wished-for results, although at the present time the reasons for failure are being recognized and gradually eliminated.

Definitions. - It will be remembered that in active immunization our own body-cells are stimulated to produce antibodies, either by reason of the presence of a disease or as the result of vaccination with the antigen of the disease in a modified and attenuated form. In passive immunization, however, our own body-cells do not produce the antibodies, 
but we receive them passively in the form of an injection of an antibodyladen serum. The antibodies are produced by active immunization of some other animal, usually a horse, and we receive the antibodies or products of this immunization in a passive manner, i. e., our body-cells receive protection against an infection and aid us in overcoming it through antibodies produced in some other animal. For this reason the process is called passive immunization; the particular kind of increased resistance afforded against infection is known as passive immunity, and since bloodserum contains the antibodies and is the usual vehicle by which they are transferred, the method is called serum therapy.

\section{PURPOSES OF PASSIVE IMMUNIZATION}

Passive immunization may be employed for two main purposes:

1. To prevent disease (prophylactic immunization).

2. To cure disease (curative immunization).

In prophylactic immunization the antibodies are introduced into our body-fluids before infection has actually occurred, or at least in the earliest stage of infection, for the purpose of placing them on guard to destroy the infecting microörganism or to neutralize its products before it has had an opportunity to produce disease. In other words, we aim to fortify our natural defenses by purchasing antibodies from another animal. From the fact that these antibodies may be introduced in a short space of time and that in this manner an immunity may be quickly gained, passive immunization for prophylactic purposes is indicated when the danger of infection is imminent, and when it is impossible, or when there is not sufficient time, for us to stimulate our own body-cells to produce our own antibodies by active immunization with a vaccine.

Since the antibodies are produced in another animal, the serum, when introduced into our body-fluids, represents a foreign protein, and, accordingly, we find that the antibodies are retained for relatively short periods of time and are quickly eliminated or destroyed. In active immunization, however, the antibodies are in native surroundings, and our body-cells continue to produce them for some time after active stimulation has ceased, in this manner insuring a higher degree of immunity and one of longer duration. For purposes of prophylaxis, therefore, active immunization is always more desirable than passive immunization; not infrequently the two forms are used simultaneously, as the antibody-laden serum will afford instant protection, while the vaccine is stimulating our body-cells to produce antibodies that will 
increase and maintain the protection over a longer period of time. This mixed form of immunization has recently received special study by von Behring in immunization experiments against diphtheria, and will be considered in detail in a later section.

In curative immunization the conditions are somewhat different. During the course of an infectious disease our body-cells are actively engaged in combating the infectious agent, so that reënforcements, in the form of specific antibodies, are indicated and welcomed for the aid they give in overcoming an infection and the relief they afford our hardpressed protective mechanism.

For these reasons it may be stated that the more acute the infection, the greater is the indication for introducing an antibody-laden serum. In chronic infections and in some acute infections we may practise active immunization by introducing a vaccine, with the purpose in mind of stimulating dormant cells to produce antibodies; but, as a rule, it is reasonable to assume that in a severe generalized infection our bodycells are doing their utmost to overcome the infection, and extra stimulation may be actually harmful. By introducing antibodies produced in some other animal, however, practically no extra strain is thrown upon the body-cells; on the contrary, they may be relieved when the new antibodies overcome the products of infection, and in this manner afford them an opportunity to recover. Unfortunately, this is actually the case in but a few infections, such as diphtheria, tetanus, and cerebrospinal meningitis, and does not apply equally well to the larger number of bacterial infections due to the pneumococcus, streptococcus, gonococcus, and similar infections. The reasons for failure of passive immunization in the cure of the last-mentioned infections are now being studied and realized, and means are being discovered for overcoming the difficulties, so that serum therapy, in a broad sense, is about to play a more important rôle in the treatment of many bacterial infections.

\section{VARIETIES OF PASSIVE IMMUNITY}

While, strictly speaking, all antibodies are probably inimical to their antigens, from the practical standpoint of passive immunization three are of primary importance, namely: (1) The antitoxins, (2) the bacteriolysins, and (3) the bacteriotropins (immune opsonins). The antitoxins neutralize their toxins; bacteriolysins cause the death of their respective bacteria if suitable complements are present, and bacteriotropins accomplish the same end by lowering the resistance of the 
bacteria, and in this manner facilitating phagocytosis. Other antibodies may be operative and prove of assistance, as, e.g., agglutinins may aid in bacteriolysis and anti-aggressins may aid in phagocytosis, but too little is known at the present time to allow fine distinctions to be made, although the indications are that not one but several antibodies are present in each immune serum, which, acting together, tend to overcome an infection.

From the practical standpoint, therefore, immune serums may be used to produce two main types of passive immunization, namely:

1. Antitoxic immunization, due to antitoxins for the true or extracellular toxins, as in diphtheria and tetanus (antitoxic immunity).

2. Antibacterial immunization, due mainly to bacteriolysins and bacteriotropins, as in meningococcus, pneumococcus, streptococcus, gonococcus, and similar infections (antibacterial immunity).

The mechanism of both of these varieties of passive immunity will be considered briefly under their respective headings.

\section{INDICATIONS FOR PASSIVE IMIMUNIZATION}

For purposes of prophylaxis only two immune serums have proved their efficiency, namely, the antitoxin of diphtheria and tetanus antitoxin.

As will be pointed out further on, diphtheria antitoxin, when administered in sufficient amounts, affords protection for at least from four to six weeks; mixed immunization, by means of the simultaneous injection of a neutral mixture of the toxin and antitoxin, as worked out by von Behring, has been found to yield equally good and more prolonged immunity, but because of certain technical difficulties has not as yet been widely adopted.

Tetanus antitoxin has its greatest value as a prophylactic. When symptoms of tetanus have once appeared, serum treatment may be of no avail, whereas it has proved its efficiency beyond doubt in neutralizing the toxin before it reaches or unites with the nervous tissue. In all wounds likely to be infected with tetanus the physician should include the administration of tetanus antitoxin as a matter of routine treatment.

Of the antibacterial serums, many have a prophylactic value in experimental animals, but none, with the exception of the antiplague serum, is in general use as a prophylactic in human practice. The reasons for this are apparent when it is remembered that pneumococcus, streptococcus, and meningococcus infections are not sufficiently epidemic 
in character to demand passive immunization. Meningococcus meningitis may, however, be an exception, but the method of active immunization advocated by Sophian is promising, easier to carry out, and should be tried during times of epidemic meningitis.

In typhoid fever, cholera, and dysentery antibacterial serums have not been generally used in prophylaxis, although it would appear that a potent anticholera serum would prove of value in preventing epidemics of this frightfully infectious disease.

With the exception, therefore, of the true intoxications, prophylaxis is more readily secured by active than by passive immunization. This is certainly true of typhoid fever, rabies, and smallpox. The antiserums of other microörganisms, such as the pneumococcus, streptococcus, and gonococcus, are being used exclusively for therapeusis, rather than for prophylaxis, of their several infections.

In veterinary practice hog-cholera serum has proved of value as a prophylactic means of combating and limiting epidemics of hog cholera. It is not definitely known whether this serum is antitoxic or antibacterial, but it is probably a combination of both.

In the treatment of disease immune serums have proved of value in diphtheria, tetanus, cerebrospinal meningitis, and, to a lesser extent, dysentery; pneumonia, streptococcus infections, and plague.

While antipneumococcus, antistreptococcus, and antigonococcus serums have proved of some value in the treatment of their particular infections, the more recent work of Neufeld and Händel, Dochez and Cole, and their coworkers in pneumonia indicates that there are wide biologic differences among various strains of these microörganisms, and that no curative properties can be expected from a given serum unless this is homologous for the type causing the infection. Further than this, it has been found impossible to secure serums as rich in antibodies as are secured with diphtheria and tetanus antitoxins, and that the serums must be given intravenously in relatively large doses. A method for the quick recognition of types of pneumococci has been worked out in the Rockefeller Hospital, and immune serums have been prepared for the main types, and the results of the serum treatment of pneumonia along these lines have been found to be most encouraging. While this method is not adapted for general use, it holds out a promise for the future of serum therapy, and opens up a wide field of investigation with the group of streptococci, gonococci, and meningococci. 


\section{CONTRAINDICATIONS TO PASSIVE IMMUNIZATION}

The chief contraindications to the therapeutic use of a serum, if any ever exist, are those dependent upon the serum itself, for, as will readily be understood, the introduction of antibodies themselves does not mean an extra strain upon our body-cells, but rather the reverse. The question before us, then, is one regarding the possible contraindications to the injection of a foreign serum, and the dangers dependent upon its use. It may be stated at once that, in the great majority of cases, the administration of a carefully prepared and properly administered serum is free from danger. Since the introduction of diphtheria antitoxin in the prophylaxis and treatment of that disease many thousands of injections have been given, in all parts of the world and under all sorts of conditions, and the number of fatalities is so small as to be regarded as almost negligible. Serum therapy should not, however, be abused to the extent of using the serum indiscriminately. I am opposed to using the serum as a prophylactic unless the indications for its employment are distinct; for example, in diphtheria it suffices to immunize only those who have been brought into immediate contact with the infection. When, however, the indications are clear and the symptoms of infection are present, I believe in using the serum early and generously.

1. Of all possible dangers consequent to the use of serum therapy, that of anaphylaxis is uppermost in the minds of practitioners. While it is true that anaphylaxis has been the cause of some fatalities, the likelihood of this accident taking place is so remote, in the great majority of cases, that it should not occupy a prominent place in the physician's mind, nor interfere with the use of the serum, as, for instance, antitoxin in the treatment of diphtheria. It is true that serum sickness is comparatively common, and while the symptoms are frequently distressing, they are not dangerous and do not constitute the dreaded and fatal anaphylaxis. With a little discrimination and care on the part of the physician the risk of anaphylaxis may be rendered still more remote if attention is given to the following questions:

(a) Is the patient sensitive to horse protein? This is probably the most important single question, as in several of the fatal cases of anaphylaxis on record it was learned afterward that the patient was usually rendered uncomfortable, and that sneezing, asthma, or even an urticarial rash would develop when the patient came into close proximity to horses, as in a stable, or when driving behind them, etc. Fortunately, these cases are very few, but several of the fatal cases of anaphylaxis on record occurred in just such persons, and at the present time a 
physician should generally be able to detect this susceptibility and avoid the dangers of anaphylaxis. In Chapter XXVIII the subject is discussed in greater detail, and a method of vaccination is described by which it may be possible to detect this condition; this consists of rubbing a little of the serum into an abrasion on the arm (Fig. 121)

(b) Has the patient been injected with a serum on any former occasion? If an injection has been given, especially a few weeks earlier, a reinjection of serum may cause well-marked serum sickness, but the possibilities of alarming anaphylaxis are so remote that serum should never be withheld if the clinical condition indicates that it should be given. Not infrequently a child receives an immunizing dose of diphtheria antitoxin, but develops the disease a month or two later, after the immunity has disappeared. Under these circumstances antitoxin should not be withheld. If time permits, the physician may inject 0.5 c.c. of the antitoxin for the purpose of producing anti-anaphylaxis, followed in two or three hours by the remainder of the serum. If it were possible to obtain it, it would be good practice to immunize the patient with an ox-serum antitoxin, and then, if it was found necessary later to use an antitoxin, the usual horse serum antitoxin could be employed. This would still further eliminate the possibility of the development of disagreeable or dangerous complications.

2. If a patient suffers from idiopathic asthma and the condition known as status lymphaticus develops, serum should be given cautiously because of the increased respiratory difficulties that may follow. It may be well to give a preliminary hypodermic injection of atropin and caffein, and then, after a few minutes, give the serum, injected slowly and subcutaneously.

3. Aside from these questions, the physician may be called upon to decide if a patient is physically able to withstand the effects of an inoculation, especially the intravenous injection of relatively large amounts of serum, such as are given in the treatment of pneumonia. In diphtheria in very young and weak children, when a large number of units or several injections are to be given, concentrated antitoxin is to be preferred, in order that injury to the subcutaneous tissues, pain, and shock may be reduced to a minimum.

\section{METHODS OF INOCULATION}

Upon the nature and severity of the infection will depend the question whether the serums are to be given subcutaneously, intramuscularly, 
intravenously, or intraspinously (subdurally). In diphtheria, the antitoxin may be given subcutaneously unless the infection is quite severe; in the latter case it should be given intramuscularly or intravenously. In tetanus the serum should be given subdurally and intravenously. In epidemic cerebrospinal meningitis the serum is always given subdurally. In pneumococcus, streptococcus, and gonococcus infections, while the serum may be given subcutaneously or intramuscularly, it is best administered intravenously. It is important for the physician to know and appreciate that the route and method of inoculation and the amount of serum administered are important factors in determining the success or failure of serum therapy.

\section{Techinic of Subcutaneous Inoculation}

Serum given subcutaneously is slowly absorbed, and a portion of the antibodies may be destroyed before they reach the blood-stream. When large quantities of serum are to be given, as in pneumonia and streptococcus infections, this method may not be permissible on account of the pain and injury to the subcutaneous tissues that may result, aside from the more important question of slow absorption and anchorage or destruction of the antibodies in various tissues before they reach the bloodstream or the focus of disease.

1. Injections should be given where the subcutaneous tissues are loose, where movement is least marked, and preferably where pressure upon the parts is least likely to occur, for some soreness, dependent upon the bulk of the injection, is bound to follow. For these reasons injections may be given in the abdominal wall; some prefer the back, in the region of the lower angle of one of the scapuloe, and the buttocks, but in a bedfast patient pressure at these points cannot readily be eliminated.

2. The skin about the site for injection may be prepared by an application of tincture of iodin; this is washed off with alcohol just before the needle is inserted. After the injection has been given the remaining iodin should be removed with alcohol, to prevent the occurrence of a dermatitis, and the puncture wound covered with cotton and collodion or with sterile gauze fastened with adhesive straps.

3. The syringe and needle should be sterile. Manufacturers of biologic supplies furnish antitoxin in syringes ready for injection, and these are usually convenient and satisfactory. The needle should be of medium size, and larger than that used for ordinary hypodermic medication. All glass or glass and metal syringes that may be boiled are to be preferred when a syringe is not furnished. Before boiling such a syringe 
the piston should be removed from the barrel, as otherwise it may expand so rapidly as to cause the latter to crack.

4. When all is in readiness, the syringe being loaded and the air expelled, the skin is pinched up between the fingers and the needle quickly inserted into the subcutaneous tissues. The injection should be given slowly, and during the operation, if the patient is a child, an assistant should be on hand to prevent struggling. In the illustration (Fig. 134) the needle is shown connected with the barrel of the syringe by means of a

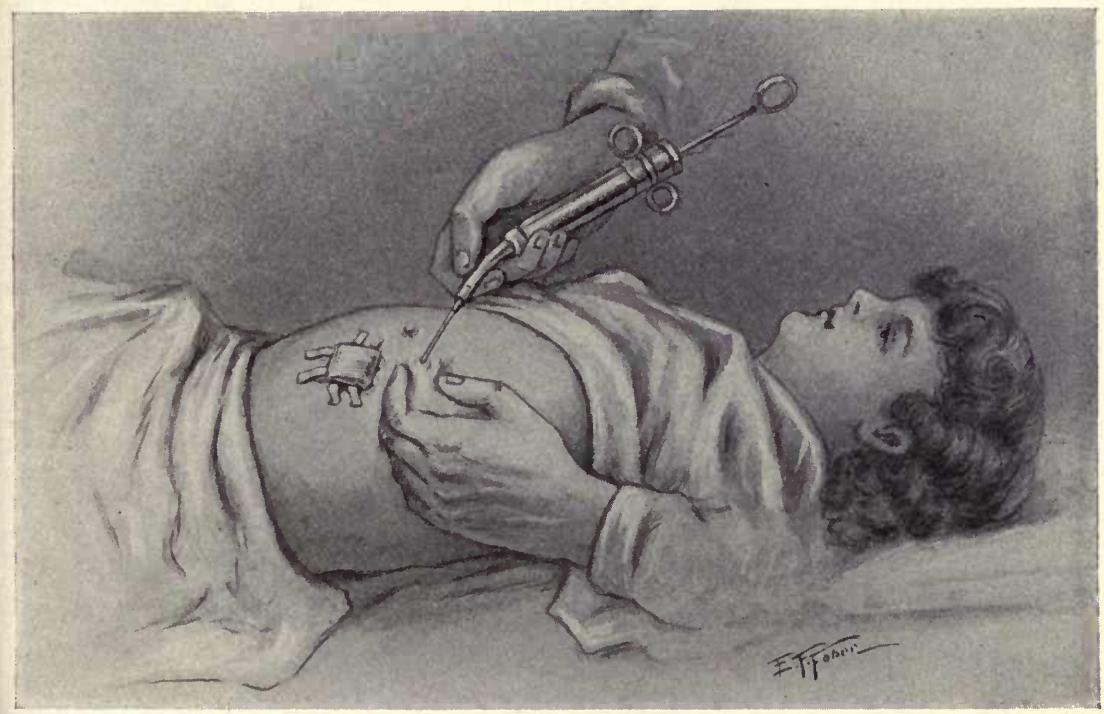

Fig. 134.-Subcutaneods Injection of Serdm.

The site of injection is painted with tincture of iodin and covered with sterile gauze fastened with straps of adhesive plaster. Just before the injection is given the iodin is wiped off with a pledget of cotton and alcohol. A fold of skin is pinched up between the thumb and forefinger of the left hand, the needle inserted, and the serum slowly injected. The needle is then quickly withdrawn, and the puncture covered with the gauze and held in place by the adhesive plaster.

short piece of rubber tubing. This permits an injection to be given without danger of the needle being broken off if the patient should struggle. Most pain is experienced when the first few drops of fluid are injected; after that the pain is not severe unless the tissues are suddenly distended, as by a quick injection.

The amount of serum that may be injected in one area depends upon the age of the patient. Due care should be exercised against injecting too much serum in one area, because of slower absorption and possible necrosis of the skin and subcutaneous tissues. 


\section{TeChNic of Intramuscular Inoculation}

As shown experimentally by Meltzer and Auer, absorption occurs much more quickly when inoculations are given into the muscles than when they are given into the subcutaneous tissues. For this reason antitoxin should be given intramuscularly in severe cases of diphtheria, as the technic is just as simple as that of a subcutaneous injection. Whenever the physician desires more speedy absorption than that which follows a subcutaneous injection, and the intravenous route cannot, for some reason, be adopted, the inoculation should be given in the muscles, preferably those of the buttocks.

The technic is the same as that employed for subcutaneous injections, except that the needle is plunged deeply into the muscles. If the most muscular portions are selected for injection, there will be little or no danger of injuring a nerve. If desired, the syringe may be detached after the needle has been inserted to ascertain if a vein has been entered, which would be shown by a flow of blood. If this occurs, the needle should be withdrawn slightly and passed in another direction.

\section{TeChNic of Intravenous Inoculation}

The necessity of administering serum intravenously in order to obtain the best results, or any result at all, is becoming more and more apparent. In severe cases of diphtheria the best results are obtained when the antitoxin is given intravenously; in the treatment of tetanus the tetanus antitoxin should be administered intravenously as well as intradurally, and both antistreptococcus and antipneumococcus serums should always be given by the intravenous route. Recent reports indicate that the proper serum treatment of these infections requires large doses given intravenously. Physicians should, therefore, be prepared to give intravenous injections. Since the use of salvarsan in the treatment of syphilis has become so popular many workers have perfected themselves in the technic of intravenous administration, but there is still great hesitancy about giving intravenous injections, although the methods are relatively simple and easily mastered.

Syringe Method.-When small amounts of fluid are to be injected, as from 5 to 20 c.c., a syringe is employed.

1. It is best to use an all-glass syringe, or at least one with a glass barrel, for the physician can then assure himself that all air has been expelled and that the fluid is free from solid particles. Further than this, a flow of blood into the syringe will indicate that the needle has entered the vein. The syringes furnished by manufacturing firms are not 
well adapted for making these injections, as the rubber plunger frequently adheres to the glass barrel, so that the injection will be jerky and difficult, and, besides, it may be difficult to determine when the vein has been entered. It is better to empty the contents of these syringes into a large, sterilized, glass-barreled syringe, such as the Record, Luer, and Burroughs-Wellcome syringes, which have a close-fitting but easily working piston, and are attached to the needle by a flange and not by a screw thread. (See Fig. 8.) The needle should be sufficiently large and have a sharp but short beveled edge. A long point may pierce the vein through and through, and permit perivascular bleeding or result in a subcutaneous injection.

2. In young children with fat arms and a weak circulation it is usually necessary to expose a vein at the elbow by making a small incision. In older children and adults a vein may stand out prominently enough to permit the needle to be inserted directly through the skin without making an incision. A firm rubber tourniquet is applied above the elbow; a very simple one is constructed by a single turn around the arm with a piece of ordinary soft-rubber tubing held in place by a hemostat. After the vein has been entered the tourniquet should be quickly removed and this is quickly and deftly accomplished by releasing the hemostat.

3. The skin about the site of injection is cleansed with soap, water, and alcohol, or merely painted with iodin, which is removed with alcohol just before the injection is to be given, in order that the vein may become visible.

4. An assistant steadies the patient's arm and should be ready to release the tourniquet.

5. The operator then steadies the skin over a vein-usually the median basilic or median cephalic-with the left thumb and forefinger, and introduces the needle into the vein. A flow of blood into the syringe indicates that the vein has been entered. The tourniquet is then released and the injection slowly given. Or the needle may be detached from the syringe and passed into the vein; when blood appears, the syringe is quickly attached and the injection made. The puncture wound is then sealed with a wisp of sterile cotton and collodion or with gauze and a bandage. The syringe shown in Fig. 135 is well adapted for the intravenous injection of serum, and was devised for the administration of concentrated solutions of salvarsan and neosalvarsan, but any reliable and large glass-barreled syringe may be used.

Gravity Method.-Larger quantities of serum or other fluid are 
better injected by the gravity method, the simple apparatus shown in Fig. 136 being quite satisfactory for the purpose. This consists merely of a graduated cylinder, which serves as a measuring funnel, rubber tubing with a pinch cock, and is furnished with a metal tip that fits the needle. The needle should be of proper size, and have a sharp but somewhat short beveled edge. It may be curved, as shown in the illustration, or may be straight. The apparatus shown in Fig. 142 is adapted

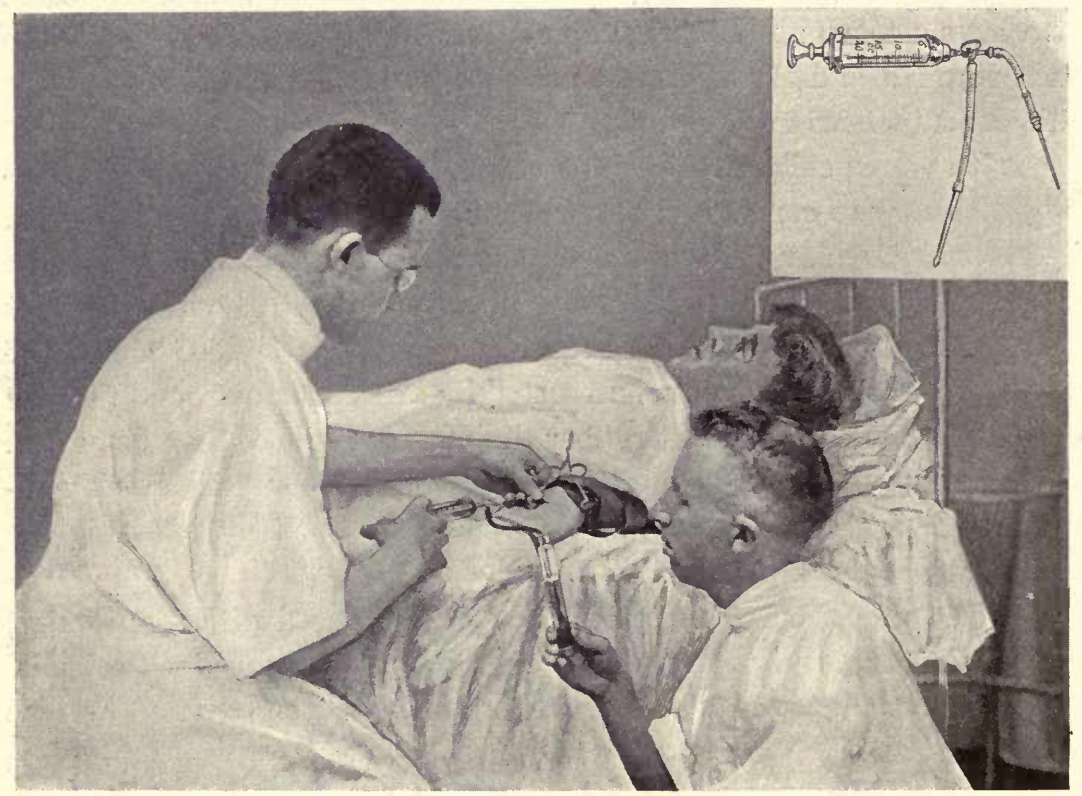

Fig. 135.-Method of Making Intravenous Injection by Means of a Syringe.

This syringe was devised for the intravenous administration of a concentrated solution of salvarsan. It is provided with a three-way cock, which permits drawing fluid into the syringe and then injecting it into a vein. This injection may also be given by any glass syringe; the particular advantage of this one is that the operator may inject more than one syringeful of fluid without removing the needle. The same syringe may be used for the intravenous injection of any serum, as diphtheria and tetanus antitoxins. (Apparatus made by B. B. Cassel, Frankfort, Germany.)

for the administration of salvarsan, and has the decided advantage of permitting the operator to give an intravenous injection to an adult person without assistance.

1. The cylinder, tubing, and needle should be sterilized by boiling prior to use.

2. The serum or other fluid may be warmed by placing the container in water of a temperature not higher than $42^{\circ} \mathrm{C}$. (just comfortably hot to hold the hand in). 
3. The injections are best given in a vein at the elbow. The arm about this region should be scrubbed with hot water and soap, followed by alcohol and 1:1000 bichlorid of mercury solution, or liberally painted with tincture of iodin. A firm tourniquet is then applied above the elbow; a single firm turn of rubber tubing held by a hemostat is quite satisfactory, as when the vein has been entered the tourniquet should be quickly released with the least movement and disturbance possible, and

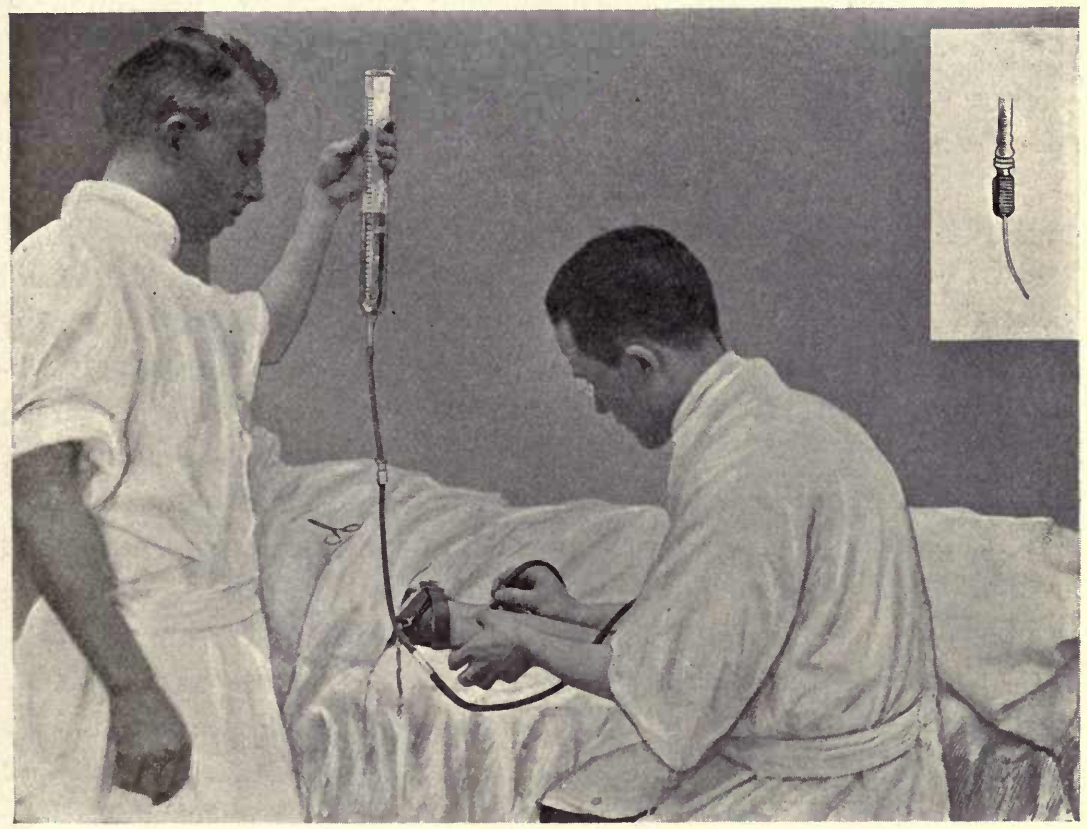

Fig. 136.-Method of Making Intravenous Injection by Gravity.

This method is suitable for the intravenous administration of salvarsan or antistreptococcus serum, etc. The needle has been entered into a prominent vein (indicated by a flow of blood); the tubing has been attached by means of a metal tip which fits the needle easily and snugly; the tourniquet has been loosened and the injection is being given.

this arrangement answers all requirements. Sterile towels should be placed about the arm and shoulder.

4. About 20 c.c. or more of sterile distilled water or normal salt solution are then poured into the cylinder, and the cock opened until all air has been expelled from the tubing. The fluid, serum, or salvarsan is then poured into the cylinder. It is a good practice to filter the fluid through several layers of sterile gauze, especially when salvarsan is being injected, in order to remove any bits of glass or other foreign bodies that may be present. 
5. An assistant holds the loaded cylinder and tubing; the operator steadies the skin over a prominent vein and quickly inserts the needle. A flow of blood indicates that the vein has been penetrated. The tubing is then quickly and carefully attached, the tourniquet released by unfastening the hemostat, and the injection given. As a rule, an elevation of the cylinder of two or three feet is sufficient. If swelling occurs about the site of puncture and the patient complains of pain, the injection is entering the subcutaneous tissue; when this occurs, the pinch cock should be closed and the needle removed. It is then necessary to make the injection into another vein or into the same vein at another site.

\section{TECHNIC OF SUBDURAL INOCULATION}

In the treatment of epidemic cerebrospinal meningitis, influenzal meningitis, and tetanus the specific serums are administered subdurally by means of a needle introduced in the lumbar region. Recently subdural injections of salvarsanized serum and weak solutions of salvarsan itself have been advocated in the treatment of cerebrospinal syphilis, tabes dorsalis, and paresis. Every practitioner should be prepared to perform lumbar puncture for the purpose of securing cerebrospinal fluid for making the Wassermann reaction and the bacteriologic, cytologic, and chemical examinations, and the administration of serum is a relatively simple matter when the puncture has been successfully made.

The technic of lumbar puncture for the purpose of securing fluid for diagnosis is described on p. 37. But when administering serum, and especially in the treatment of meningitis, the clinical condition of the patient and the danger of sudden collapse render it advisable and necessary that the inoculation be given with the patient lying on his side.

Methods.-Two methods are now being employed. The older method consists in injecting the serum by means of a syringe, and the later one is a method whereby the serum is allowed to flow in by gravity.

Not infrequently a patient will develop symptoms of collapse during a subdural injection, and these have been ascribed to undue pressure, the injurious action of trikresol or other preservative upon the respiratory centers, too rapid injection, and the introduction of too large a quantity of serum. It is now apparent that in the past too little attention has been paid to the patient while the injection was being made, and serum has usually been administered according to more or less fixed and arbitrary rules, instead of being guided by the clinical condition of the patient. 
If symptoms of collapse appear during a subdural injection, they may be relieved by allowing the fluid within the canal to flow out again, and this is best accomplished when the inoculation is given by the gravity method. The latter method has been largely worked out and is highly recommended by Sophian, these recommendations being based upon his extensive experience in the recent Texas epidemic of cerebrospinal meningitis. It is also recommended by Flexner and the Hygienic Laboratory, and is undoubtedly the method of choice.

\section{Blood-pressure as a Guide in Administering Serum Subdurally.-} According to the older and customary method of injecting serum subdurally, fluid is permitted to flow from the needle until from 15 to 20 c.c. have been removed, and an equal quantity of serum is then injected. In severe cases, with thick plastic exudates, only a few cubic centimeters of fluid may be withdrawn, and, indeed, no fluid at all may be secured. To inject arbitrarily a fixed amount of serum under such conditions may be highly dangerous to the patient, on account of increased pressure. On the other hand, when the flow is free, it may be dangerous to permit the canal to drain until intraspinal pressure is reduced to the normal, a fact indicated by the flow of a drop of fluid every three to five seconds.

With these considerations in mind, Sophian ${ }^{1}$ has studied the value of cerebrospinal fluid pressure and blood-pressure as controls on the amount of fluid that may be safely withdrawn and on the amount of serum that may be injected. During the study and treatment of 500 cases of epidemic cerebrospinal meningitis this last-named observer found that the blood-pressure was a valuable guide. Cerebrospinal fluid pressure was found to be misleading, owing probably to a local distention of the subarachnoid space at the site of injection, which resulted in readings that did not represent the true intracranial pressure.

1. Usually, upon the withdrawal of cerebrospinal fluid, a fall of blood-pressure occurs. With the ordinary blood-pressure in an adult patient-about $110 \mathrm{~mm}$. of mercury-Sophian recommends stopping the flow when there has been a drop in pressure of about $10 \mathrm{~mm}$. of mercury; in children, about $5 \mathrm{~mm}$. In a few cases there is no change in blood-pressure or even a slight rise; in these instances fluid may be removed until the flow has diminished to the rate of a drop every three to five seconds.

2. With the injection of serum the blood-pressure drops still further. Generally, the decrease in blood-pressure is proportional to the rapidity with which the serum is injected and the amount injected. By the

${ }^{1}$ Epidemic Cerebrospinal Meningitis, 1913, Mosby Co., St. Louis. 
gravity method, under ordinary conditions, at least ten minutes should be consumed in administering 15 c.c. of serum. A totäl drop of $20 \mathrm{~mm}$. of mercury indicates that sufficient serum has been injected. If it is desired to inject more, as in a severe case of meningitis, close watch should be kept for other symptoms of collapse.

3. Usually, under these conditions, less serum is administered than has been advocated heretofore. It is apparent that the more potent the serum, the less bulk is required-and the bulk alone is an important factor, for a large injection may so injure the patient as to counteract any good that the serum may do. Unfortunately, there is no accurate measure of the curative value of antimeningococcic serum. It is highly desirable that a serum be as potent as possible, and the physician must rely upon the reputation of the firm producing the serum. Efforts are being made to concentrate these serums, much as antitoxin is concentrated, and this is an end very much to be desired.

4. Blood-pressure changes are not constant in the same patient upon different occasicias. The pressure should be taken, after each puncture and inoculation, for the administration cannot be guided by observations made on a previous occasion.

Collapse during Subdural Inoculation.-Carter has shown, by experiments on dogs, that the first mechanical effects of increased intraspinal pressure were respiratory depression and marked cardiac inhibition. Sophian has found that similar effects may be produced during subdural injections of serum in the treatment of meningitis.

The symptoms of collapse, such as stupor, superficial or deep, irregular and slow respiration, and dilatation of the pupils, are foreshadowed by a marked drop in blood-pressure. The pulse may continue good or become slow and irregular. Incontinence of urine and feces may occur.

The treatment consists primarily in discontinuing the injection. By lowering the funnel, fluid is allowed to flow from the spinal canal and mix with the serum. If a syringe is being used, it should be detached from the needle or gentle suction made. After a few minutes the symptoms may disappear and the inoculation may be cautiously resumed until the desired amount of serum has been injected; otherwise the needle should be withdrawn.

In addition to this procedure atropin and caffein may be administered hypodermically in large doses, and artificial respiration resorted to if necessary. It is well to have these drugs ready for injection before the inoculation is begun, so that no time will be lost when they are needed. 


$$
8
$$



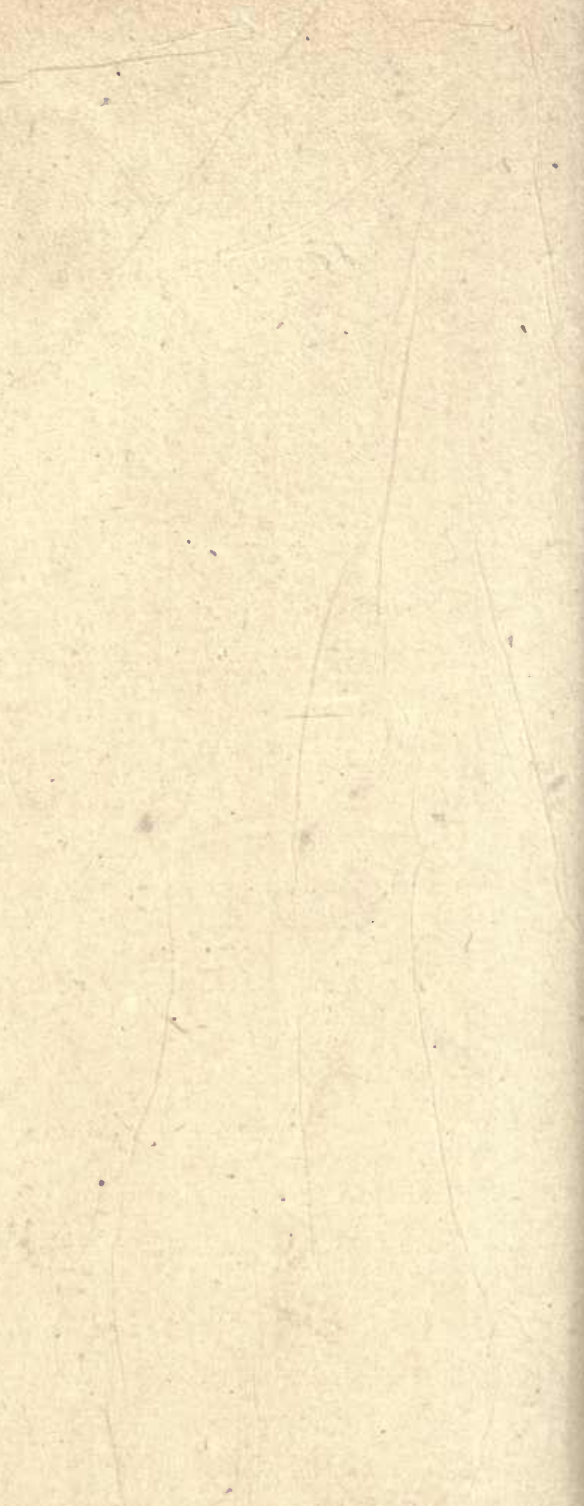
Anesthesia for Subdural Inoculation.-There is no doubt but that lumbar puncture and the subdural injection of fluid are painful, the amount of pain depending to some extent upon the degree of meningitis, the method of injection, and the skill of the operator. Severe cases of meningitis that are stuporous or moribund may not evince any evidences of added discomfort; less toxic and robust or nervous patients may, however, suffer considerably and prove difficult subjects for injection.

A local anesthetic is practically useless except for the mental effect it has upon the patient who may demand it. General anesthesia for lumbar puncture in meningitis adds a considerable element of danger, but if it is absolutely necessary, a few whiffs of ether or chloroform may be given while the needle is being inserted. In giving subdural injections in tetanus a general anesthetic is necessary.

Sophian has found that if water is given through a straw while performing lumbar puncture patients will frequently drink large quantities of it and keep very quiet.

Gravity Method.-The apparatus required is very simple, and consists essentially of a proper needle and from 12 to 16 inches of softrubber tubing attached to a container or funnel for serum and furnished with a metal tip by which it is quickly and readily attached to the needle.

Several manufacturers of biologic supplies are marketing antimeningococcic serum in a special container, fashioned after that devised by Sophian and Alexander, with the needle and tubing adapted for the administration of the serum by the gravity method. Such an apparatus is shown in Fig. 137.

The physician may, however, prepare an equally efficient apparatus, similar to that shown in Fig. 137, which consists of the glass barrel of a 20 c.c. syringe attached to 16 inches of soft-rubber tubing fitted with a metal tip that holds the needle firmly and snugly. The whole is sterilized by boiling, and any quantity of serum may be administered with it. The apparatus is adapted for the administration of antimeningococcic serum, tetanus antitoxin, influenza serum, salvarsanized serum, or any other fluid, and has given uniform satisfaction.

The needle should be from 10 to $11 \mathrm{~cm}$. in length, with a wide, rather than a narrow, lumen-about 1.5 to $2 \mathrm{~mm}$. This is important in administering serum to a case of meningitis, in which a needle with a narrow lumen may become plugged with exudate. The needle should be fitted with a trocar. The tip should have a short bevel with a sharp edge. 
Technic.-1. The serum should be warmed to body temperature by wrapping the sealed container of serum in towels wrung out of water comfortably hot for the hands (about $42^{\circ}$ C.). Cold serum possibly increases the pain caused by the injection, although the pressure upon

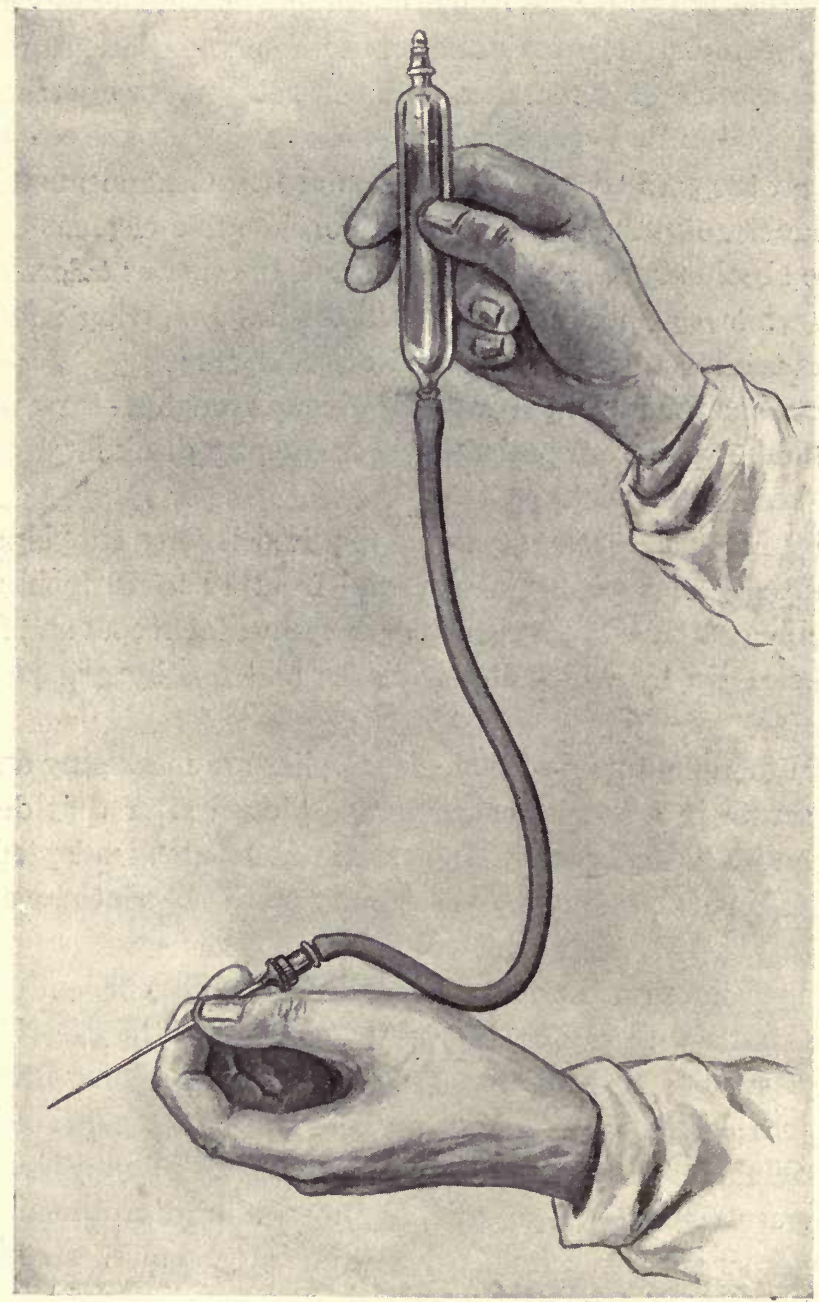

Fig. 137.-Outfit for Intraspinal Injection of Antimeningitis Serum by Gravity (Sophian).

the sensitive nerve-roots is the chief source of pain and discomfort. Due care must be exercised that the serum is not coagulated by too high temperature.

2. The funnel or syringe barrel, tubing, and needle should be steril- 
ized by boiling. The outfits furnished by the manufacturers are sterilized and ready for use. Two sterile graduated centrifuge tubes should be on hand for collecting and measuring the spinal fluid. The apparatus should be assembled and ready for injection, so that at the appointed time the tubing may be attached to the needle and the inoculation given.

3. The patient should be placed on the left side, on the edge of a bed or table. An assistant places the patient in such a manner as to arch

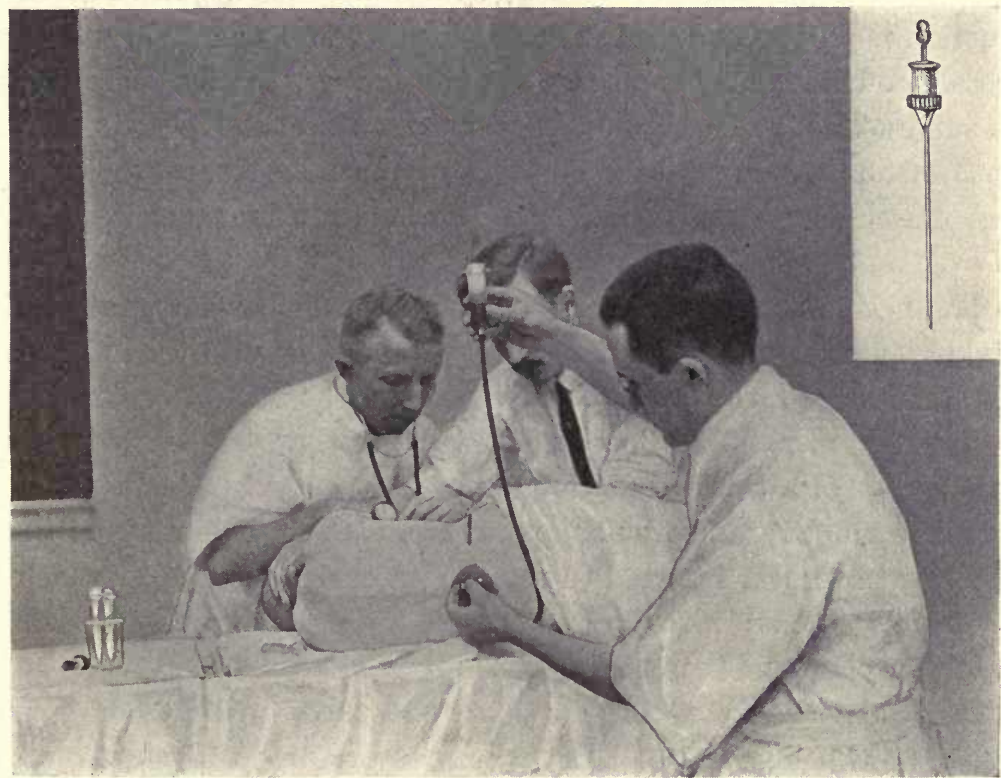

Fig. 138.-Intraspinal Injection by Gravity.

The line marks the crest of the ilium, and indicates the third lumbar interspace. The needle has been inserted and cerebrospinal fluid withdrawn. Antimeningococcus serum is being administered. The barrel of a 20 c.c. Record syringe is serving as a funnel, and is attached to the needle by means of 18 inches of soft-rubber tubing furnished with a metal tip. The needle, as shown, is reduced to about four times its actual size. This method may be used for making the subdural injection of tetanus antitoxin and salvarsanized serum.

the back as much as possible. A second assistant takes blood-pressure readings during the operation.

4. Towels wet with bichlorid are arranged about the site of inoculation. It is well for the operator to locate the site of injection by palpating the spinous processes and selecting the widest interspace, which is usually on a level with the crests of the ilia if the back is well arched. The skin is then cleansed with soap, water, and alcohol, and bichlorid solution or a coat of iodin applied. 
5. The operator must then choose between the median or lateral route of puncture. The median is the easier, and should always be adopted by the inexperienced operator.

Wash off the iodin with a pledget of cotton soaked in alcohol. Locate the chosen interspinous space, pressing well between the spines with the left thumb or index-finger, and holding the finger in place pass the needle perpendicularly in the median line between the spines, or, better still, at an angle of 45 degrees upward and inward. If an obstruction is felt, withdraw the needle slightly and pass it in a different direction until it imparts a sense of "giving way," which indicates that the subarachnoid space has been reached. Quincke has estimated the depth of lumbar puncture in adults to be usually from 4 to $6 \mathrm{~cm}$.; in large muscular men it is from 7 to $8 \mathrm{~cm}$., and in fat persons, about 10 cm.

The needle should be inserted slowly and deliberately, rather than quickly, as puncture of a bone is likely to be followed by a dull, aching pain, and, indeed, the point of the needle may be bent or broken.

The fluid may fail to flow or flow very slowly. This may be due to the presence of a thick exudate, impalement of a nerve filament, or adhesions arising from a previous puncture. The needle may be turned gently or the trocar inserted to remove an obstruction, after which the flow usually starts; if it does not do so, the needle may be withdrawn slightly or cautiously inserted a little further.

6. Fluid is collected in the centrifuge tubes while blood-pressure readings are being made. When the pressure drops $10 \mathrm{~mm}$., or if the flow is about a drop every three or five seconids, the tubing is connected and the serum injected very slowly.

As a general rule, as much fluid should be withdrawn as can be done with safety, and the maximum dose of serum given. When the flow is scanty, a larger dose of serum may be given than counterbalances the fluid removed, the injection being guided by the blood-pressure. When the total drop reaches $20 \mathrm{~mm}$. of mercury, the injection should be discontinued, or if continued, the patient should be watched closely for other symptoms of collapse.

7. After the injection has been completed the needle is quickly withdrawn and the wound covered with sterile gauze held in place by adhesive straps. All iodin should be washed off with alcohol to avoid irritation or an actual dermatitis.

Syringe Method.-1. The technic is practically the same as that just described, except that the injection is given with a syringe. 
Manufacturing concerns market their products in syringes all ready for injection. When injecting tetanus antitoxin, it is necessary to empty the syringe into another sterile syringe, as shown in the accompanying illustration (Fig. 139), which will fit an appropriate needle. Since the plunger of the purchased syringe oftentimes adheres to the barrel and renders the injection jerky and difficult, I frequently transfer the serum to a sterile, all-glass syringe which I know will work smoothly and satisfactorily.

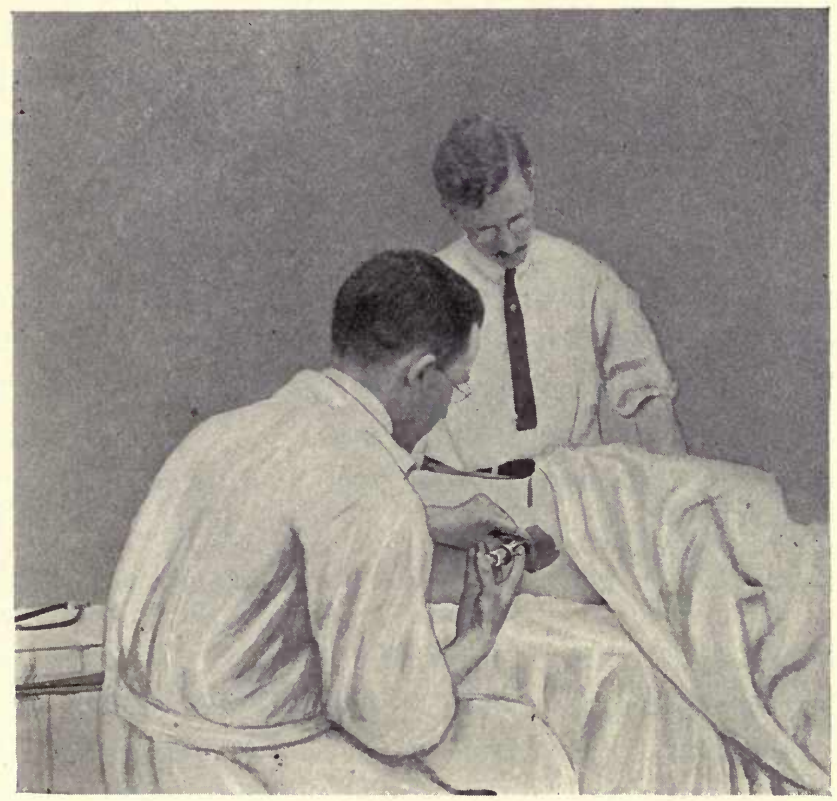

Fig. 139.-Intraspinal Injection by Means of a Syringe.

The line indicates the crest of the ilium, and usually passes between the third and fourth lumbar vertebræ, which is the proper point for inserting the needle. The site of injection has been painted with tincture of iodin after cleansing with soap, hot water, and alcohol. An assistant holds the patient to prevent sudden jerking and possible accident.

2. Lumbar puncture is performed as for the gravity method while blood-pressure observations are being made. When sufficient fluid has been removed, the loaded syringe is attached to the needle and the injection slowly given. The physician is frequently tempted to inject the serum and complete the operation quickly, but it is better to inject it in amounts of 0.5 to 1 c.c. every half to one minute, being guided by the blood-pressure readings and general condition of the patient. If the pressure falls below $20 \mathrm{~mm}$. of mercury or other symptoms of col- 
lapse appear, the fluid may be drained from the canal by gentle suction with the piston. This is usually impossible when the manufacturers' syringe is used. Otherwise the syringe is detached and the fluid collected in tubes until the patient's condition improves and the injection is resumed or the needle removed.

At times the patient is so restless that this slow method is not feasible. In such instances the physician should make the injection as slowly as possible, endeavoring to put into the canal as much serum as fluid was removed or at least a reasonable amount.

\section{ANTITOXIC IMIMUNIZATION}

\section{SERUM TREATMENT OF DIPHTHERIA}

The discovery of diphtheria antitoxin and its use in the treatment of this infection constitute one of the triumphs of modern medicine.

Twenty years ago diphtheria was one of the most dreaded of diseases, accompanied ordinarily by a mortality of at least 30 per cent., while the loss of life from the laryngeal form of the disease, particularly after tracheotomy, was simply appalling.

Shortly after Roux and Yersin (1888) had demonstrated that the symptoms of diphtheria were due largely to a soluble poison or toxin secreted by the bacilli, Ferran, and later Fraenkel and Brieger (1890), undertook experiments in active immunization against diphtheria. About the same time von Behring discovered the antitoxin, and in a series of extensive researches with Wernicke he established experimentally its prophylactic and therapeutic value in diphtheria. The first attempt to apply this discovery to the cure of this infection of the human being was made in von Bergmann's clinic (1891). The results, while encouraging, were not altogether satisfactory, owing largely to the fact that the serums were weak and the doses given too small. The discovery, however, resulted in creating an extraordinary stimulus to researches in immunity, and during the following two years more powerful serums were prepared, so that in 1896 a marked drop in the mortality of diphtheria was apparent in those places where the antitoxin was being used.

Since then diphtheria antitoxin has been the means of saving countless thousands of lives, and the treatment of diphtheria, instead of being a reproach to medicine, has become the model of what the scientific treatment of an infectious disease ought to be. Statistics and the individual experiences of those especially engaged in the treatment of diphtheria show that when the antitoxin is used on the first day of the 
disease, practically no mortality occurs. Parents and guardians should be taught this fact, and cautioned to seek medical advice promptly whenever a child complains of sore throat. While the use of diphtheria antitoxin is still decried and opposed by a few members of the medical profession,-and this is not to be wondered at when it is remembered that cowpox vaccination still has its opponents,-it is at least to be hoped that no physician will deprive a patient suffering from diphtheria of the benefits to be derived from the antitoxin treatment. In the absence of special contraindications the refusal or neglect to use antitoxin in the treatment of diphtheria would, in the opinion of most physicians, constitute an act of criminal negligence and malpractice.

Preparation of Diphtheria Antitoxin.-The methods of preparing and standardizing diphtheria antitoxin are given in Chapter XIV. Briefly, these consist in the preparation of a strong toxin by cultivating a virulent strain of the bacillus in a suitable broth for ten days or two weeks; the culture is then passed through a porcelain filter, which retains the bacilli, the filtrate being a strong solution of toxin. This is standardized by the physiologic test of determining its action upon guineapigs, the minimal lethal dose (M. L. D.) or toxin unit being the amount of toxin that will cause the death of a 250-gram guinea-pig in four days.

Strong and healthy horses that have been proved by the tuberculin and mallein tests to be free from tubercle or glanders, are then continuously immunized by a series of injections of the toxin. The first doses are guarded by the simultaneous injection of antitoxin, but after from four to six months the animals are able to tolerate enormous quantities of pure toxin. The horses are then bled aseptically from a jugular vein, and the separated blood-serum, preserved with a small percentage of an antiseptic, becomes the antitoxin of commerce.

Many manufacturing concerns market a concentrated diphtheria antitoxin prepared by precipitating the globulin fraction of the raw serum with ammonium sulphate, and redissolving it in a minimal quantity of salt solution. The globulins carry with them most of the antitoxin, and in this manner a serum may be concentrated so that a large number of units are contained in a small bulk of fluid, obviously a most desirable feature in the treatment of diphtheria. Further than this, it has been observed that these concentrated antitoxins are much less likely to produce serum sickness, a train of symptoms due to substances present alike in normal and in antitoxic horse serum, and independent of the antitoxin.

The antitoxic unit is the smallest amount of serum that will just neutralize 100 times the minimal lethal dose of toxin for a 250-gram guinea-pig. The adoption of such a standard enables us to attain some accuracy in dosage. Before its introduction antitoxin was given in so many cubic centimeters, just as antimeningococcus and antistreptococcus serums are given today, but since some horses produce more potent serums than others, the results were quite irregular. At the present time an antitoxic serum is marketed according to the number of units it contains, irrespective of the actual amount of serum, the constant endeavor being to produce as potent a product as possible.

Since antitoxin gradually deteriorates with time, physicians should carefully observe the date printed upon each package of antitoxin, which is the time limit calculated by the manufacturers beyond which they do not guarantee that the full antitoxic strength is maintained. 
Nature of Diphtheria.-In the great majority of cases diphtheria is a local infection of some portion of the upper respiratory tract. The bacilli are usually inhaled, find lodgment upon a mucous membrane, and secrete a toxin that produces necrosis of the cells of the mucosa and effectually resists phagocytosis of the bacilli. From this area of infection, which now becomes a prolific source of toxin production, the toxin or poison is absorbed by the body-fluids, and the resulting toxemia is chiefly responsible for most of the symptoms of the disease.

Other microörganisms, such as staphylococci, pneumococci, and streptococci, which may be unable to infect a healthy mucous membrane, readily multiply in the necrotic tissue and add to the severity of the local lesion, the lymphadenitis, and the toxemia.

Rarely the diphtheria bacilli gain access to the blood-stream. The severity and danger of diphtheria are dependent primarily upon the strength and amount of toxin produced by the bacilli, and secondarily upon the size and location of the primary lesion and the amount of antitoxin present in the patient's blood. A lesion in the larynx is far more dangerous than one of equal size on a tonsil, because in the former the edema and necrotic exudate obstruct the trachea and may produce death by suffocation. On the other hand, the size of the local lesion alone is not an indication of the severity of the infection, because virulent bacilli in a small patch may produce more toxin than less virulent ones in a larger area, and the degree of local tissue necrosis is not an absolute indication of the toxicity of the soluble poison. Other things being equal, the patient who has most antitoxin, either naturally or acquired as the result of a previous injection with antitoxin or of an attack of diphtheria, will present least evidences of toxemia, although the bacilli causing the infection may be most virulent. For example, the highly virulent strain of diphtheria bacillus used extensively in the past eighteen years in the production of antitoxin was isolated by Park and Williams from the throat of a patient presenting no clinical symptoms other than redness of the fauces and slight toxemia.

The primary lesion of diphtheria is usually located in the throat (tonsils, uvula, larynx), and frequently in the nose; more rarely the ears, conjunctiva, vulva, prepuce, and wounds are the seats of primary infection.

Treatment of Diphtheria. - If we were always certain of seeing our patients on the first day of their illness, and if the disease could always be diagnosed in this stage, the treatment of diphtheria would resolve itself into an immediate dose of antitoxin and rest in bed for two or three 
weeks. But patients frequently come under treatment comparatively late in the disease, or the true nature of the condition may not be fully diagnosed at first and treatment thus be delayed. Diphtheria is, therefore, still to be regarded as a dangerous infection, and while the proper use of antitoxin constitutes the most important part of the treatment, local applications, general constitutional measures, and the management of the various conditions that may complicate the disease are all to be considered. Here we will discuss only the serum treatment of the disease.

Bearing in mind the pathology of diphtheria, serum treatment aims to fulfil the following primary indications:

1. To neutralize all free toxin circulating in the body fluids, and also to neutralize, as much as possible, the toxin that has already united with the tissue-cells.

2. To cause the destruction or removal of the bacilli producing the toxin as quickly as possible.

3. To furnish the patient with sufficient excess of antitoxin to neutralize the toxin as rapidly as it is produced until the virulent bacilli disappear.

Action of Diphtheria Antitoxin.-Diphtheria antitoxin best fulfils these requirements. In fact, there are no substitutes. In former days the powerful and irritant caustics and germicides that were freely applied to the throat, instead of limiting the disease and destroying the bacilli, probably actually encouraged its extension by excoriating and depressing the resistance of the surrounding mucous membranes.

1. The chief action of antitoxin is just what its name implies, namely, a substance that neutralizes the toxin. This is regarded as a chemical reaction analogous to the neutralization of an acid by an alkali. When the antitoxin molecule has united with the toxin molecule, it is believed that the toxin is neutralized and that both are rendered inert. As the result of experimental studies, however, we know that this union is not always a firm one, and it is possible for the toxin to become dissociated and attack body-cells or other molecules of antitoxin, thus explaining in part the necessity for giving quite large doses of antitoxin-doses that are out of all proportion to what we would expect to be necessary, when considered weight for weight between guinea-pig and man, to effect complete neutralization of the toxin.

It is reasonable to presume, and may be accepted as true, that a stronger affinity exists between diphtheria toxin and antitoxin than between body-cells and toxin. Just as the union between this toxin and 
its antitoxin is somewhat unstable, so, in like manner, it is probable that the union of toxin and body-cells is not so firm but that it may, during an early stage, be dissociated to some extent by the more attractive antitoxin. This factor is to be borne in mind in the treatment of diphtheria, and is an additional argument for the administration of large doses of the serum.

2. Antitoxin pure and simple does not, however, constitute the only factor of value in antidiphtheric serum. While the pure antitoxin neutralizes the toxin, it has no injurious action on the bacilli themselves, and, indeed, it is said that the bacilli may live and multiply in the presence of large amounts of antitoxin. How, then, are we to explain the gradual disappearance of the membranous exudate and the bacilli at the local site of infection? It has been shown experimentally that virulent diphtheria bacilli resist phagocytosis. This condition is probably due to an actual leukotoxic action of the diphtheria toxin, aided by an aggressin-like action of the toxin which neutralizes opsonin, and in this manner prevents phagocytic activity. I in common with other observers, have shown that the antiserum as ordinarily produced neutralizes the antiphagocytic action of diphtheria bacilli, and enables the polynuclear leukocytes to ingest them readily. ${ }^{1}$ Whether this is brought about through neutralization of the toxin by antitoxin, or is due to the presence of an immune opsonin (bacteriotropin) that lowers the resistance of the bacilli, or to the presence of an anti-aggressin that neutralizes the intrinsic defensive mechanism of the bacilli and thus favors phagocytosis, I am unable to state, but probably all three factors are operative.

3. As ordinarily prepared, diphtheria antitoxin possesses little or no bacteriolytic activity. I have found, however, that antitoxin will fix complement with an antigen of diphtheria bacilli, ${ }^{2}$ indicating, therefore, the presence of bacteriolytic amboceptors. Serums produced by immunizing horses with unfiltered cultures of diphtheria bacilli instead of with the filtered toxin alone have been advocated for the general and the local treatment, in the hope of securing lysis of the infecting bacilli. Certainly it would appear wise to raise the bacteriotropic and bacteriolytic content of an immune serum by injecting the horses occasionally with unfiltered cultures, for it is highly probable that the action of the antiserum depends not only upon an antitoxin, but also, to some extent at least, upon a bacteriotropin and possibly a bacteriolysin.

Administration of Diphtheria Antitoxin.-Antitoxin is usually ad-

${ }^{1}$ Jour. Med. Research, 1912, xxvi, 373. $\quad 2$ Jour. Infect. Dis., 1912, xi, 44. 
ministered by subcutaneous injection into the tissues of the back, abdomen, or buttocks. Experimental studies have tended to show that complete absorption does not occur until forty-eight hours after, although it is common clinical experience to observe improvement take place during the first twenty-four hours after injection.

As will be emphasized later, it is highly desirable and necessary to get antitoxin into the circulation as soon as possible after infection has occurred. Usually. this is best accomplished by giving antitoxin to every patient even suspected of being diphtheric, and making the diagnosis afterward; the next best method is to administer the antitoxin in such manner as to favor quick absorption. For this reason intramuscular and intravenous injection should be resorted to in all severe cases. As pointed out in Chapter XXXI, the Schick reaction in diphtheria has indicated that diphtheria toxin may be dissociated from tissue-cells by large doses of antitoxin. Park and his associates have shown experimentally by this reaction that 20,000 units of antitoxin given subcutaneously were necessary to yield an effect equal to 1000 units given intravenously.

Intramuscular injections into the muscles of the buttocks are just as readily given as subcutaneous injections, and are probably no more painful to the average patient. It insures quicker absorption, and may, indeed, be adopted as a routine practice.

Intravenous injections are far more difficult, especially in children with fat arms and feeble circulations. An anesthetic or ten minutes' struggling may do the patient harm, and unless the injection can be given with little disturbance and danger, the serum should be given by intramuscular injection. Not infrequently, however, an intravenous injection yields splendid results in severe and apparently hopeless cases, and in older children and adults this route of administration should be considered.

The syringe method is well adapted for the intravenous injection of antitoxin, as the bulk method of serum is usually small, especially if a concentrated antitoxin is being used.

The technic of these injections has previously been described.

Antitoxin has also been given by the mouth and even by rectal injection. The presence of a preservative, usually phenol, renders the oral administration objectionable. While a therapeutic effect may be secured after large doses have been swallowed, there are very few occasions when this should be the method of choice.

Importance of Early Treatment.-The most important point to be observed in the treatment of diphtheria is to give antitoxin at once. It may 
be fatal to wait for the result of a culture, except perhaps in the case of the mildest of infections. In fact, the necessity for early administration of antitoxin cannot be overestimated. When once suspicion is aroused, antitoxin should be given at once and the diagnosis may follow. A few hours may make an enormous difference in the prognosis of any case, and while a dose of 2000 units of antitoxin may prove of the utmost value in checking diphtheria, it will do no harm whatever in case the disease is not present.

It is true that a physician will naturally hesitate to administer antitoxin unnecessarily; nevertheless, diphtheria is frequently a difficult disease to diagnose clinically, and is quite likely to be mistaken for tonsillitis. For this reason many physicians prefer to wait for the result of a culture, and this is proper, provided that the patient, especially in the case of a child, is given the benefit of the doubt by receiving 2000 units of antitoxin. It is to be emphasized that a single negative culture does not exclude diphtheria. As ordinarily made, about 20 per cent. of primary cultures from genuine cases of diphtheria fail to show the presence of bacilli, whereas subsequent cultures will show them to be present in large numbers. A primary negative culture is most likely to be obtained from a patient having a heavy exudate, as the physician may rub lightly over the membrane, culturing the microörganisms of secondary infection and overlooking the diphtheria bacilli in the depths of the membrane adjacent to the diseased mucous membrane. To wait another twentyfour hours for a second culture still further reduces the patient's chances for recovery. In the vast majority of instances, therefore, antitoxin should be given at once and repeated as often as is necessary until the correct diagnosis is established, and not one but at least two successive negative cultures should be obtained before diphtheria is to be excluded with any reasonable degree of safety.

The following table, compiled from the annual reports of the Philadelphia Hospital for Contagious Diseases, shows the decided influence of early treatment upon the mortality of diphtheria. This table comprises cases of diphtheria alone, and does not include cases complicated by other diseases, such as scarlet fever and measles. It is worthy of special notice that of 741 cases treated with antitoxin on the first day of the disease, not one died. Ker, ${ }^{1}$ in an exceptionally rich experience, has never seen a fatal result occur in a case that developed in a hospital and in which antitoxin was administered on the first day of the disease.

${ }^{1}$ Infectious Diseases, 1909, Oxford Med. Press. 
TABLE 28.-MORTALITY OF DIPHTHERIA ACCORDING TO THE DAY OF ADMISSION (WHICH ALSO INCLUDES THE TIME OF GIVING THE ANTITOXIN) IN THE PHILADELPHIA HOSPITAL FOR CONTAGIOUS DISEASES

\begin{tabular}{|c|c|c|c|c|c|c|c|c|c|}
\hline \multirow{2}{*}{ YeAR } & \multirow{2}{*}{$\begin{array}{c}\text { Total } \\
\text { NUMBER } \\
\text { OF CASES }\end{array}$} & \multicolumn{7}{|c|}{$\begin{array}{c}\text { Mortality According To THE Day on which ANTitoxin } \\
\text { WAS First INJECTED }\end{array}$} & \multirow{2}{*}{$\begin{array}{l}\text { AVERAGE } \\
\text { MORTALITY } \\
\text { IN DIPH- } \\
\text { THERIA } \\
\text { ALONE }\end{array}$} \\
\hline & & $\begin{array}{l}\text { First } \\
\text { Day }\end{array}$ & $\begin{array}{c}\text { Second } \\
\text { Day }\end{array}$ & $\begin{array}{l}\text { Third } \\
\text { Day }\end{array}$ & $\begin{array}{c}\text { Fourth } \\
\text { Day }\end{array}$ & $\begin{array}{l}\text { Fifth } \\
\text { Day }\end{array}$ & $\begin{array}{l}\text { Sixth } \\
\text { Day }\end{array}$ & $\begin{array}{l}\text { After } \\
\text { Sixth } \\
\text { Day }\end{array}$ & \\
\hline 1904. & 712 & 0 & 4.09 & 13.72 & 17.54 & 14.75 & 19.44 & 12.94 & 10.81 \\
\hline 1905. & 862 & 0 & 4.43 & 9.22 & 16.66 & 13.04 & 9.52 & 22.89 & 10.09 \\
\hline 1906-07. & 1239 & 0 & 3.45 & 12.90 & 10.8 & 13.08 & 29.41 & 6.09 & 8.70 \\
\hline $1908 \ldots$ & 1426 & 0 & 6.62 & 4.7 & 10.60 & 11.71 & 21.43 & 23.96 & 8.55 \\
\hline 1909. & 2153 & 0 & 4.61 & 7.13 & 10.72 & 9.35 & 7.25 & 13.33 & 6.60 \\
\hline 191 & 1870 & 0 & 5.50 & 5.13 & 8.41 & 13.74 & 6.04 & 8.33 & 6.42 \\
\hline 191 & 1895 & 0 & 6.91 & 9.41 & 7.12 & 11.04 & 7.22 & 2.66 & 6.86 \\
\hline 191 & 1676 & 0 & 2.92 & 6.33 & 8.0 & 9.53 & 14.97 & 9.63 & 6.02 \\
\hline 191 & 1273 & 3.92 & 6.51 & 6.52 & 6.87 & 4.76 & 12.5 & 18.8 & 7.63 \\
\hline Average. & 13106 & 0.4 & 5.0 & 8.3 & 10.7 & 11.2 & 14.2 & 13.1 & 7.96 \\
\hline
\end{tabular}

It is generally believed that the paralyses of diphtheria are due to a toxone, and not to the true toxin. Ehrlich believes toxone to represent a late secretory product of the diphtheria bacillus, whereas others regard it as a modified toxin. It is certainly apparent, however, that the bacilli should be gotten rid of as soon as possible, so as to eliminate the possibility of toxone production. This is best accomplished by the early use of large doses of antitoxin, aided possibly by judicious local treatment.

Dosage of Diphtheria Antitoxin.-While it is now generally agreed that the doses of from 100 to 200 units, such as were commonly given during the early years of antitoxin therapy, were far too small, there is still some difference of opinion regarding the proper doses to employ. Since the severity of the disease varies so markedly, no hard and fast rules can be given. The physician who has a clear idea of the nature of diphtheria and of the action of antitoxin, and knows what to expect of the latter in the treatment of the disease, should have no difficulty in properly treating a case of diphtheria.

It is to be emphasized, however, that while antitoxin constitutes the most important part of the treatment of diphtheria, it is not usually the whole treatment. Absolute rest in bed, a generous diet, combined with the use of tonics and local applications, are all part of the treatment. Special treatment of the laryngeal form of the disease and the treatment of complications are matters of considerable importance that influence the prognosis in a given case. I may mention, in passing, the value of 
continuous enteroclysis of normal salt solution during the early periods of severe infections; this appears to dilute the toxins and aid in their destruction and excretion.

In administering antitoxin the physician must be guided by the clinical condition of the patient, as we have as yet no practical laboratory method for estimating the degree of the toxemia. Treatment may be regarded as satisfactory when-

1. The local patch of exudate has ceased to spread and shows indications of disappearing.

2. The general condition of the patient is improved, i. e., the toxemia is decreased, the pulse grows stronger and more regular, and the patient feels more comfortable.

The temperature is not a reliable guide, for not infrequently in severe infections it may be normal or subnormal throughout.

So long as the exudate shows no signs of loosening and disappearing, but tends to spread, and so long as the general condition remains unimproved or grows worse, large amounts of antitoxin should be given. No case should be regarded as hopeless until death supervenes.

Every case of diphtheria is to be treated individually, rather than by any set rule. The amount of antitoxin given in the initial dose, and in subsequent doses as well, is dependent on the following factors:

1. The situation and extent of the lesion.

2. The general condition of the patient.

3 . The day of the disease.

4. The age of the patient.

1. The Situation and Extent of the Lesion.-In ordinary tonsillar diphtheria in which there is a small patch on one tonsil and which is first seen on the second day of the disease, the initial dose should be at least 5000 units. When the exudate involves the pillars of the fauces, the uvula, the posterior pharyngeal wall, or is well forward on the palate, this dose should be doubled, and 10,000 units be given.

Cases that present laryngeal symptoms in addition to faucial lesions should never receive less than 12,000 units. In well-marked laryngeal diphtheria with dyspnea and partial suffocation at least 20,000 units should be given, preferably by intramuscular or intravenous injection.

In nasal diphtheria a distinction must be made between those cases that exhibit merely a dirty, chronic discharge containing bacilli, in which 2000 units may suffice, and those that present an actual membrane accompanied by well-marked toxic symptoms, when a large amount of serum-at least 10,000 units-should be given. Owing to the ready 
absorption of toxin by the nasal mucosa a small patch in the nose may be accompanied by severe general toxemia and frequently requires energetic treatment.

In diphtheria of the eye, vulva, or of wounds relatively large doses should be given - at least from 10,000 to 20,000 units.

All these doses must be regarded merely as suggestions for the initial dose in cases seen on about the second day of the disease. Physicians with extensive hospital experience, for example, Woody and McCullom, generally favor large doses, and while in private practice the question of expense and economy may be a factor, the physician will be wise to err on the side of safety and give a little too much serum rather than too little, especially in the first dose, when so much depends upon how soon antitoxin is introduced into the body-fluids.

2. The general condition of the patient, or the effect which the toxemia will have on the patient, is highly important in estimating the dosage of antitoxin. A patient who is pale, drowsy, prostrated, and has a weak and irregular pulse; who has large masses of glands around the neck, or who has marked albuminuria, will require a much larger dose than one who presents none of these signs. Two persons of about the same age and suffering from the same lesions may show very different degrees of toxemia. In the severe cases we must administer the maximum dose and repeat it at suitable intervals until an effect is produced.

3. The day of illness on which the patient comes under observation is important in deciding the initial dose of antitoxin. For corresponding tonsillar lesions a dose of 2000 units on the first day may do more good than 5000 units given on the fourth day. Ker gives second-day cases of purely tonsillar diphtheria 3000 units, and adds an additional 1000 on the following day.

4. If the age of the patient exerts any influence at all on dosage, it indicates that more antitoxin should be given to children than to adults with corresponding lesions, as the disease is more fatal in children. So far as infants are concerned, Ker seldom gives more than 4000 units at a single dose, which should be an adequate amount when we consider the small size of the patient. Children over one year of age may be given from 5000 to 10,000 or more units, depending upon the location of the lesion and the degree of toxemia.

Repeating the Dose.-Whether or not subsequent doses of antitoxin will be required is dependent upon the circumstances of the individual case. In ordinary cases if on the day after treatment is commenced 
there is no diminution in the amount of membrane visible and the general symptoms have not improved, the dose should be repeated. If the membrane has spread and the toxemia is worse, the second dose should be larger than the first. In septic cases the second dose may be given in from six to ten hours after the first. If the symptoms are less urgent, the interval may be extended to twelve, but should never exceed twentyfour hours.

As to the total amount of serum to be administered, continued injections at relatively short intervals are required until improvement has taken place. So long as membrane is present and the patient is toxic it is probably worth while to push the treatment unless these show a tendency to clear away. Time must be allowed for absorption to take place, and the serum should not be pushed so far as to be wasted, and, quite possibly, excreted unchanged. The remedy is expensive, especially in private practice, and it is obviously desirable to have due regard for economy. While, as previously mentioned, physicians of such wide experience as McCullom, of Boston, and Woody, of Philadelphia, frequently give 200,000 or more units in severe cases of diphtheria, others, e. g., Ker, of Edinburgh, have never given more than 64,000 units to a single patient, and, indeed, several of my colleagues of wide experience claim that they have secured excellent results in severe infections with doses that seldom exceeded 10,000 units.

Treatment of Relapses.-Occasionally, after a patient has recovered from an attack of diphtheria the infection recurs after several weeks. It is in such cases that the physician hesitates to administer antitoxin, on account of the discomforts occasioned by serum sickness. It is true that serum sickness is more likely to follow in these than in primary cases, and the very profuse and itchy eruption, joint pains, and fever do indeed render the patient quite uncomfortable. Since a relapse is usually, although not always, comparatively mild, the serum may be dispensed with if there is no involvement of the larynx and if there is not much evidence of toxemia; otherwise full doses of antitoxin should be given without hesitation. The subcutaneous injection of 0.5 c.c. of the serum two or three hours before the main dose is given may possibly produce a condition of anti-anaphylaxis and ward off the more dangerous symptoms. If respiratory difficulties should follow, a reinjection of serum, atropin, and caffein should be administered hypodermically.

Antitoxin Sequelce.-A certain percentage of cases will present a group of symptoms that constitute the condition known as serum sick- 
ness, occurring at varying times following the administration of antitoxin. This condition has been shown to be due to certain constituents of horse serum other than the antitoxic antibodies. It is noteworthy that the serum of one horse may cause more serum sickness than that of another; in general, concentrated antitoxins produce fewer cases than do raw serum.

This condition is characterized by the development of a rash (urticarial, multiform, or scarlatiniform), mild fever, joint pains, and possibly adenitis. The scarlatiniform rash may be extremely difficult to differentiate from that of true scarlatina, especially in the wards of a diphtheria hospital, where outbreaks of scarlet fever are not uncommon.

This subject has been considered in greater detail in Chapter XXVIII on Anaphylaxis. It may be stated here that while the patient is decidedly uncomfortable, and even quite sick, for several days, serum sickness is not a dangerous condition; the treatment is largely symptomatic and palliative.

Value of Diphtheria Antitoxin.-At the present day it seems hardly necessary to introduce elaborate statistics to prove the value of antitoxin in the prophylaxis and treatment of diphtheria.

1. It is generally admitted that most of the reduction in the mortality of diphtheria cannot be attributed solely to the use of antitoxin, for unquestionably bacteriologic diagnosis has permitted the inclusion, in our statistics, of a certain number of cases that, twenty years ago, would not have been classed as diphtheria. Generally speaking, however, the mortality of diphtheria, considering all types of infection coming under observation at varying intervals after the disease has developed, is at least five times less under antitoxin treatment than when it is treated without antitoxin. This proportion is true, whether we compare the general mortality before 1896 with the present rate, or whether we take a large city and compare the mortality under both forms of treatment for a single or for several years. For example, in Philadelphia the mortality rates per 100,000 of population for the five years preceding the use of antitoxin were as follows:

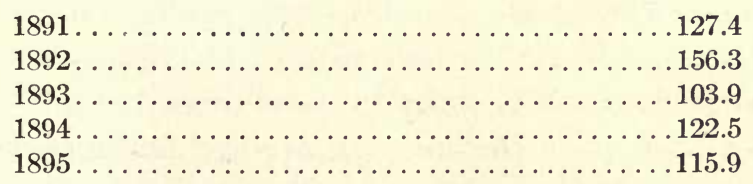

In five years following the general use of antitoxin the mortality rates per 100,000 population were as follows: 


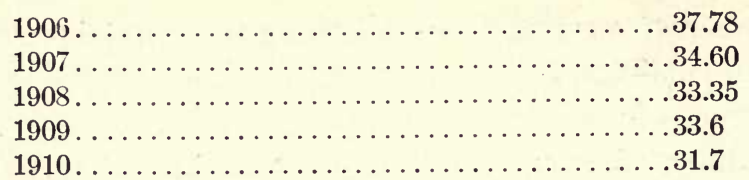

In Philadelphia, during the years 1909-10 and 1911, the average mortality of diphtheria treated with antitoxin in the Philadelphia Hospital for Contagious Diseases was 9.9 per cent., and in the private practice of physicians, 13.07 per cent. In contrast to these figures is the mortality of 40.34 per cent. in the private practice of those physicians (fortunately few) who refused to give antitoxin or in those families opposed to its use.

From Table 28 it will be seen that the average mortality in 13,106 cases of pure diphtheria treated with antitoxin in the Philadelphia Hospital for Contagious Diseases during the past ten years was only 7.96 per cent. As stated elsewhere, when this is compared with the average mortality of about 41 per cent. when no antitoxin was used, it is not difficult to appreciate the therapeutic value of the remedy.

2. As was previously stated, it is also worthy of note that there is practically no mortality in diphtheria cases receiving antitoxin on the first day of illness. During nine consecutive years (1904-1913), covering the treatment of 741 such cases in the Philadelphia Hospital for Contagious Diseases, not a single death occurred. During 1913 two of the 51 firstday cases died.

It will also be noted from Table 28 that the patient's chance for recovery grows steadily less with each day that the administration of serum is delayed, and this should be evidence enough to convince any right-minded person that we possess in antitoxin a remarkable remedy for the treatment of diphtheria.

3. The influence of antitoxin is also noted in the mortality of laryngeal diphtheria. While the mortality in this condition is still high, owing to the frequency and dangers of bronchopneumonia and the necessity for operative measures, it has been reduced at least one-half since antitoxin came into general use. Prior to 1896 the mortality was at least 70 per cent.; since then it has been reduced to 35 per cent. or less. As shown in the following table, of 1207 cases treated in the Philadelphia Hospital for Contagious Diseases, the average mortality was 35.6 per cent. 
TABLE 29.-MORTALITY OF LARYNGEAL DIPHTHERIA (INTUBATION CASES) IN THE PHILADELPHIA HOSPITAL FOR CONTAGIOUS DISEASES

(This table excludes those patients dying in the ambulance and moribund cases dying within twenty-four hours.)

\begin{tabular}{|c|c|c|c|c|c|c|c|c|c|}
\hline \multirow{2}{*}{ YEAR } & \multirow{2}{*}{$\begin{array}{c}\text { TOTAL } \\
\text { NUMBER } \\
\text { OF } \\
\text { CASES }\end{array}$} & \multicolumn{7}{|c|}{$\begin{array}{l}\text { Mortality According to THE Day UPON which INTUBa- } \\
\text { TION was PeRformed }\end{array}$} & \multirow{2}{*}{$\begin{array}{l}\text { AveraGe } \\
\text { MORTALTY }\end{array}$} \\
\hline & & $\begin{array}{l}\text { First } \\
\text { Day }\end{array}$ & $\begin{array}{l}\text { Second } \\
\text { Day }\end{array}$ & $\begin{array}{l}\text { Third } \\
\text { Day }\end{array}$ & $\begin{array}{c}\text { Fourth } \\
\text { Day }\end{array}$ & $\begin{array}{l}\text { Fifth } \\
\text { Day }\end{array}$ & $\begin{array}{l}\text { Sixth } \\
\text { Day }\end{array}$ & $\begin{array}{l}\text { After } \\
\text { Sixth } \\
\text { Day }\end{array}$ & \\
\hline 1903. & 67 & 66.6 & 25.3 & 21.8 & 30.9 & 22.5 & & 45.0 & 26.60 \\
\hline 1904. & 125 & $\ldots$. & 39.20 & 29.03 & 73.68 & 33.33 & 70.0 & 36.84 & 39.20 \\
\hline 1905. & 136 & $\ldots$ & 30.76 & 39.39 & 50.0 & 21.42 & 16.66 & 42.30 & 36.76 \\
\hline $1906-0$ & 72 & & 50.0 & 55.0 & 40.74 & 45.45 & 71.92 & 28.57 & 49.29 \\
\hline 19 & 162 & & 29.26 & 50.0 & 33.33 & 30.76 & 9.09 & 38.88 & 33.95 \\
\hline 190 & 183 & & 50.0 & 44.18 & 38.88 & 23.81 & 53.84 & 40.0 & 42.62 \\
\hline 191 & 89 & 100.0 & 55.0 & 33.33 & 28.58 & 50.0 & & 36.46 & 39.34 \\
\hline 19 & 104 & 50.0 & 76.66 & 55.55 & 52.63 & 36.36 & & 27.27 & 54.80 \\
\hline & 133 & 30.0 & 58.33 & 41.93 & 48.27 & 50.0 & 63.67 & 50.0 & 48.87 \\
\hline & 136 & 100.0 & 65.91 & 63.64 & 35.29 & 25.0 & 47.82 & 68.0 & 58.82 \\
\hline Average. & 1207 & & & & & $\ldots$ & $\ldots$ &. & 35.6 \\
\hline
\end{tabular}

Formerly when a child contracted diphtheria the parents were warned of the likelihood and danger of the infection involving the larynx and trachea; nowadays this possibility is quite remote.

4. Finally the claim of the opponents of the serum therapy of diphtheria that antitoxin increases the percentage of paralyses is without foundation. While it is true that this percentage is somewhat higher than was noted in former years, this increase is to be explained by the fact that antitoxin saves a larger number of severe cases long enough for them to manifest paralyses, and, second, by the greater attention that has recently been directed to its milder forms. Since diphtheric paralysis is regarded as caused by toxone or a later secondary toxic product of the bacilli, the indications are to rid the patient of the bacilli as quickly as possible, and this is best and most surely accomplished by the proper administration of antitoxin.

\section{Prophylactic IMmunization AgaINST DIPHTHERIA}

The subcutaneous administration of relatively small doses of antitoxin will usually confer a passive immunity against diphtheria lasting from two to four weeks.

The object is to introduce antibodies (antitoxin and opsonin) into the body-fluids in order that they may neutralize the toxin as rapidly as it is produced, aid in the destruction of the bacilli, and thus protect the 
individual in case virulent bacilli should be inspired or otherwise gain access to the tissues.

The doses advised are relatively small, and the injection does not usually produce any discomfort other than soreness about the site of inoculation. For infants under one year of age 500 units suffice; for older children and adults from 1000 to 1500 units should be given.

The duration of this passive immunity is relatively short, owing to the fact that the antitoxin is eliminated rapidly, as it is part and parcel of a foreign serum that tends to be excreted or destroyed soon after its introduction into the body. It will endure, however, for at least two weeks, and frequently longer. Since the incubation period of diphtheria is only a matter of a few days, this suffices, in the majority of instances, to protect the individual.

The indications are to immunize all persons who have come in intimate contact with a case of diphtheria. It is especially valuable in families and small communities, such as go to make up hospital wards and asylums. The physician who is attending a case of diphtheria in a private home should urge immunization upon all members of the household.

The immediate results are usually good. The main disadvantage is the short duration of the immunity, so that no matter how faithfully it is carried out, persons do not remain immune for long periods of time, and accordingly the total morbidity of the disease is not influenced to any extent. In homes from which the case of diphtheria is promptly removed to a special contagious hospital and in which the remaining members are promptly immunized the percentage of secondary cases is practically nil. Of 6772 patients who were removed to the Philadelphia Hospital for Contagious Diseases, the remaining members of the family not being immunized, secondary cases developed in 164 persons, or in 2.4 per cent. Of 4063 cases of diphtheria treated at home with antitoxin, the other members of the family not being immunized, secondary cases developed in 219 persons, or in 5.3 per cent. Of 639 diphtheric patients treated at home who did not receive antitoxin and where immunization was not practised, secondary cases developed in 151 persons, or 23.6 per cent. These figures, compiled by Dr. A. A. Cairns, chief medical inspector of Philadelphia, and taken from the annual reports of the Philadelphia Bureau of Health for the years of 1909, 1910, and 1911, show that the best results are obtained when the diphtheric patient is promptly removed to a special hospital and the remaining members of the household are immunized. Even when the patient is 
removed promptly there is some danger of other persons having been infected, and immunization should, therefore, always be promptly practised. When the patient is treated at home, other members of the household, even if immunized, are liable to develop the infection, probably owing to the fact that the patient harbors virulent bacilli for varying periods of time after the passive immunity in other persons has passed away and the quarantine is broken. Certainly in those homes where antitoxin is not used either for therapeutic or for prophylactic purposes, the percentage of secondary infections is so high as to leave no doubt as to the value of antitoxin.

In this connection I may mention the desirability of using an antitoxin prepared by immunization of cattle for the general purpose of prophylaxis, and especially for the treatment of those persons who are hypersensitive to horse serum. In these cases horse antitoxin could be used later if a person contracted diphtheria without danger of anaphylaxis.

Behring's Method of Immunization against Diphtheria.-Owing to the fact that the antibodies produced through the activities of our own body-cells (active immunization) persist for longer periods of time than those that are introduced passively (passive immunization), Behring and his assistants have been working upon a method of active immunization in diphtheria whereby our own body-cells are to be stimulated to produce our own antitoxin in sufficient amounts to protect us against the disease. It has long been known that more or less balanced mixtures of this kind produce immunity in animals, and in 1907 Theobald Smith ${ }^{1}$ suggested that it might be possible to employ this method for the purpose of producing immunity in man. Subsequently Smith ${ }^{2}$ studied the effects of injections of neutral mixtures in guinea-pigs and horses, and again pointed out the applicability of the method to human beings

Active immunization in diphtheria could probably be accomplished by the administration of minute and increasing doses of toxin, but there would be some danger of producing an acute toxemia or paralysis, and the process may require so much time as to be useless in the presence of epidemics.

Behring's method, according to his report read before the German Convention on International Medicine in 1913, is based upon the principle that the union of toxin and antitoxin is not stable, and when a neutral mixture of the two is injected into animals, sufficient toxin be-

${ }^{1}$ Jour. Med. Research, 1907, xvi, 359.

2 Jour. Exper. Med., 1909, xi, 241; Jour. Med. Research, 1910, xxiii, 433. 
comes dissociated to unite with body-cells and stimulate the production of antitoxin.

The mixture of toxin, and antitoxin is known as T.-A., and several preparations are being used; of these, T.-A. 8 possesses the lowest toxicity, and has a value equivalent to that of the standard preparation, M. M. 1, while T.-A. 7 is ten times and T.-A. 6 twenty times as strong in its toxic and immunizing power for man.

Before using it in human practice, Behring very carefully tested his mixtures on lower animals, and every new lot of T.-A. is carefully tested by the same means to make sure that it does not contain a trace of the paralyzing element of the toxin, that its toxic power is exactly determined by testing it on guinea-pigs, and finally that the mixture be known, by trial on horses, to be capable of producing antitoxin. Further, Behring requires the testing of every new lot by his standard preparation M.M.1, which has retained its toxic and immunizing properties for over a year unchanged.

The method has now been used for immunizing a large number of persons, chiefly under the supervision of von Behring and his assistants. ${ }^{1}$ The natural antitoxin content of the blood is determined, usually by the intracutaneous method on the guinea-pig, before and after immunization, and has shown uniformly a considerable increase, which persists over many months.

Local and general reactions have been observed, especially in older children and adults with doses intended for the new-born; this fact is explained on the assumption of a specific sensitization in consequence of the previous introduction of diphtheria bacilli (carriers), which latter render the individuals hypersensitive to the T.-A. Reactions that are regarded as non-specific have been observed in tuberculous and scrofulous persons, and for the present von Behring prefers that the use of the prophylactic in such persons, as well as in atrophic infants and infants less than nine months old, be regarded as contraindicated.

The fear expressed by some that the prophylactic is contraindicated in those persons who harbor diphtheria bacilli for fear of producing the disease during temporary depression of the defensive mechanism has been finally dissipated as the result of practical experience. Not one of the numerous bacillus-carriers that have been injected with T.-A. have sickened with diphtheria. Whether or not the active immuniza-

1 See Semaine Médicale, 1913, xxxiii, No. 18; Berl. klin. Wochenschr., 1914, li, 917; Therap. Monatssch., 1913, xxvii, No. 11. 
tion with T.-A. will help them to get rid of the bacilli is still an open question.

The subcutaneous injection is recommended as the best method of administration. There can be no doubt that, in many cases, a single injection produces sufficient protection. Such persons are, as a rule, those who have already been sensitized by diphtheria bacilli. For the ordinary run of cases at least two injections should be given. The first. injection then plays the part of a sensitizer. Experience shows that sensitization occurs after from ten to fourteen days, which makes it necessary that the second injection should not be given until after an interval of not less than ten days.

In this country the subject has been studied by William H. Park and his associates, who found that this form of active immunization gave rise to decided antitoxin production in 22 per cent. of susceptible persons. The interval between vaccination and the development of immunity was generally long - as a rule, not less than two weeks. Under conditions of exposure about 20 per cent. of those who failed to respond were found to develop clinical diphtheria.

The remedy has not been used sufficiently often to enable us to express an opinion as to its value. Behring believes that its proper use may thoroughly eradicate diphtheria. Obviously, its preparation must be very carefully controlled, and for the present it should be used only in institutions where thorough studies of the blood of patients may be made before and after immunization.

The Schick Test.-This consists of the intracutaneous injection of a quantity of diphtheria toxin equal to one-fiftieth of the minimal lethal dose. I dilute this in such a manner that this amount is contained in 0.05 c.c. According to Schick, onethirtieth of a unit or more antitoxin per cubic centimeter of blood is sufficient to neutralize this amount of toxin. If the patient has less than this quantity of antitoxin, the toxin is not neutralized and acts as a local irritant, producing an area of superficial inflammation. Therefore, this test is a measure of immunity in diphtheria and is advocated for testing the members of families and institutions, where it probably suffices to immunize with antitoxin those who react positively. (See p. 609.)

\section{SERUM TREATMENT OF TETANUS}

Tetanus antitoxin was discovered by Behring and Kitasato in 1892. Since then it has proved of great value in the prevention of lockjaw. While, in general, authoritative opinions regarding its curative value vary, the statistics and the individual experience of many investigators of more recent years show quite conclusively that tetanus antitoxin does. possess curative value, and is of distinct aid in the treatment of tetanus, 
especially when the nature of the disease is understood and the serum is administered accordingly.

Preparation and Standardization of Tetanus Antitoxin.-This technic has been described in Chapter XIV. Briefly, it consists in immunizing the horse with gradually increasing doses of tetanus toxin over a period of several months, until the blood of the animal contains the antitoxin in sufficient quantities for therapeutic use. The animal is then bled under aseptic precautions, and the serum, with the addition of a small amount of preservative, constitutes the antitoxin of commerce. Several manufacturers concentrate the antitoxin in the same manner as diphtheria antitoxin is concentrated. In view of the very large doses required in the treatment of tetanus this is quite desirable.

The American unit is defined as the amount of antitoxin required just to neutralize 1000 fatal doses of tetanus toxin for a 350-gram guinea-pig. The United States Government has adopted this unit, and supplies the different producers with standardized toxin for testing the antitoxin.

Action of Tetanus Toxin.-It may be well to recall briefly the main features concerning the pathogenesis of tetanus, as successful treatment depends upon a thorough understanding of these principles.

1. Tetanus is a local infection accompanied and characterized by a general toxemia. The bacilli and spores never gain access to the blood, and are never distributed through the tissues and internal organs, but reside at the local site of infection, where they produce a powerful toxin, which, when absorbed, is responsible for the main lesions and symptoms of the infection. Therefore while the blood of the tetanus patient is sterile, it usually contains the toxin. Neisser has produced tetanus in mice by giving them subcutaneous injections of the blood of a tetanus patient.

2. Tetanus toxin has a strong affinity for nerve tissue, and this constitutes the most important feature in the pathogenesis of the disease. The toxin is rapidly absorbed from the local site of infection into the blood and lymph-streams, where it is distributed to other muscles and reaches the central nervous system indirectly through absorption by the end-plates of motor nerves. As expected, absorption is most likely to occur along the motor nerves supplying the parts injured, and for this reason the muscles and nerves should be infiltrated with antitoxin as soon as possible after an injury has been received.

According to Meyer and Ransom, Marie and Morax, absorption occurs along the axis-cylinders of motor nerves, the intramuscular endings of which the toxin penetrates. The experiments of Field, Cernovodeanu, and Henni indicate, however, that the toxins are absorbed by way of the lymphatics of the nerves, and not by way of the axis-cylinders; the latter view is now most generally accepted. 
Even though the toxin gains entrance to the blood, it cannot injure the motor nerve tissue directly, as, for example, by means of the bloodvessels supplying the central nervous system. As was previously stated, the toxin in the blood and lymph channels may reach the central nervous system only in an indirect manner, through the end-plates or lymphatics of other motor nerves.

Ascending centripetally along the motor plates and lymphatics, the poison reaches the motor spinal ganglia on the side inoculated; it then affects the ganglia on the opposite side, making them hypersensitive. The visible result of this hypersensitiveness is the highly increased muscle tonus $-i$. e., rigidity. If the supply continues, the toxin next affects the nearest sensory apparatus: there is an increase in the reflexes, but only when the affected portion is irritated. In the further course of the poisoning the toxin as it ascends continues to affect more and more motor centers and also the neighboring sensory apparatus. This leads to spasm of all the striated muscles and general reflex tetanus (Park).

3. Regardless of the severity of the infection, there is always an incubation period in tetanus during which the bacilli multiply and produce toxin which is traveling toward the tissues of the central nervous system. Antitoxin in sufficient amount will neutralize the toxin as quickly as it is produced, and thus protect the infected individual until the leukocytes and other body-cells have destroyed the bacilli and spores.

In general terms, the severer the wound and the heavier the infection, the shorter will be the incubation period and the higher the mortality. In acute tetanus the incubation period is less than ten days; in chronic tetanus this period is much longer and more variable.

The toxin is produced, and may be absorbed during or at least soon after the first twenty-four hours following infection; this explains the necessity for administering antitoxin as soon as possible after the injury has been received.

Action of Tetanus Antitoxin.-1. Tetanus antitoxin will neutralize free toxin in a chemical manner similar in some respects to that by which neutralization of an acid by an alkali is effected. It is generally believed that as soon as the molecule of antitoxin has become united with a molecule of toxin, the latter is rendered inert. It may be possible, however, for the toxin molecule to become dissociated and attack nerve-cells or other molecules of antitoxin; this is one reason for the necessity of giving large doses of antitoxin in order than an excess may be at hand.

2. When tetanus toxin has once united with nerve-cells, it is difficult or impossible for the antitoxin to effect its neutralization. Hence the 
greatest value of the antitoxin lies in prophylaxis; when properly administered, however, it is possible for the antitoxin to aid in the cure of tetanus, and its use should never be omitted in the treatment of any case.

The value of antitoxin in the treatment of tetanus is probably dependent upon the following two factors: (1) Neutralization of all free toxin as quickly as it is secreted and before it is absorbed by the nervous tissue; (2) actual dissociation or neutralization of some of the toxin "loosely united" with nerve-cells or suspended in the lymph after it has left the capillaries and before it is taken up by the nerve-cells.

3. Aside from its chief antitoxic action, anti-tetanus serum probably contains anti-aggressins or bacteriotropins that aid phagocytosis by overcoming their repelling or negatively chemotactic influence. This may, however, be accomplished by simple neutralization of the toxins, which impairs their leukotoxic action sufficiently to permit living leukocytes to engulf and destroy the bacilli.

Methods of Administering Tetanus Antitoxin.-Recent investigations and case reports show quite conclusively that in the treatment of tetanus as much depends upon the method of administering antitoxin as upon the quantity administered.

1. Absorption by the subcutaneous route is so slow that it should not be depended upon in the treatment of tetanus. While it is true that the mortality of tetanus has been reduced about 20 per cent. by the administration of large amounts of serum by this route, it should be emphasized that a smaller amount, given subdurally or intravenously, will yield even better results. Knorr has shown experimentally that after subcutaneous injection the maximum quantity of antitoxin is not found in the blood until twenty-four hours have elapsed. Since every hour counts heavily in the chances for recovery when symptoms of tetanus have appeared, it may be laid down as a general rule that the first doses of antitoxin should be given subdurally or intravenously. The subcutaneous route may be chosen when serum is given for prophylactic purposes at the time of injury, but should not be relied upon in the treatment of tetanus.

2. Intramuscular injections may be given to keep up the good effect of antitoxin after the first doses have been given subdurally and intravenously, and are to be preferred to the subcutaneous route whenever the physician is unable to inject the serum subdurally and intravenously.

3. For the rapid neutralization of toxin free in the body-fluids serum should be given intravenously. In the treatment of tetanus from 10,000 to 20,000 units may be given by this route as early as possible. While 
it is conceded that when the toxin has become firmly bound to the tissues of the central nervous system dissociation by the use of antitoxin is not possible, yet one can never know, in a given case, low firm this union has become, and clinical results, supported by animal experimentation, show that life may be preserved by large doses of antitoxin injected into the blood.

4. The experimental work of Pennin, ${ }^{1}$ Park and Nicoll, ${ }^{2}$ supported by the clinical results reported by various observers, shows quite conclusively that the subdural route is a very efficacious and valuable avenue by which to administer antitoxin in the treatment of tetanus. The serum should be given by the gravity method, in exactly the same manner as in giving antimeningitic serum. To insure its thorough dissemination throughout the spinal meninges the antitoxin should be diluted, if necessary, with normal salt solution. As a rule, the amount injected should be slightly less than the amount of fluid withdrawn. In the case of a "dry tap," if the operator is reasonably sure of having entered the canal, from 3 to 5 c:c. of serum may be injected. It is generally necessary to repeat this injection within twenty-four hours.

The reason for administering antitoxin subdurally is apparent when it is remembered that neither the central nervous system nor the peripheral nerves take up antitoxin direct from the blood (Park). Only after very large intravenous doses are traces of antitoxin found in the cerebrospinal fluid, and animals passively and actively immunized may be rendered tetanic if the toxin is injected directly into the central nervous system or into the nerve. While antitoxin injected subdurally finally passes over into the blood, it will neutralize any free or dissociated toxin before the latter has developed any harmful tendency.

5. To neutralize any toxin that may have been absorbed by a nerve it may be advisable to inject antitoxin directly into the nerve, and these intraneural injections under aresthesia are advised by Ashhurst, John, and others as part of the rational treatment of tetanus.

The technic of these injections has been described elsewhere

Prophylaxis of Tetanus. - The most successful preventive treatment, and practically the only successful curative one after the disease has developed, is by means of tetanus antitoxin. As a prophylactic remedy this antitoxin exceeds in value even diphtheria antitoxin; therapeutically, however, it is far inferior to the latter, for the reason that part of

${ }^{1}$ Mitt. a. d. Grenzgebiet d. Med. und Chir., 1913. xxvii, 1.

2 Jour. Amer. Med. Assoc., 1914, lxiii, 235. 
the toxin produced by the tetanus bacilli soon unites with the nervecells of the spinal cord.

One of the greatest dangers from this terrible infection lies in the fact that while the local lesion may show no signs of disturbance, the central nervous system may suddenly manifest symptoms of poisoning. The wounds that are likely to contain the tetanus bacillus are the following: All wounds that may contain dirt contaminated by manure, such as that from the streets, stables, barns, and even fields; wounds made by firecrackers or toy pistols; gunshot wounds, especially those made by blank cartridges; crushing injuries, made by machinery or in other ways. The feet and hands are especially prone to be infected with tetanus germs. Street injuries that are not deep or perforating, but grinding and lacerating, are very likely to develop tetanus infection. It has also been stated that tetanus bacilli may be harbored in an old injury, and yet cause no symptoms until some additional injury or general disturbance of the body causes the normal protection against infection to be broken down, when toxins from the bacilli may be absorbed and tetanic symptoms develop. This theory would seem to be responsible for an otherwise apparently unaccountable development of tetanus.

From what has been said it will be seen that any injuries received on the street, or those inflicted on workers about horses or cattle and in stables, are more likely to develop tetanus than are injuries received in other ways. New-born babies may be infected through the stump of the umbilical cord. Likewise a suppurating wound, or even a fresh wound, which may be innocent at first, may become infected with the tetanus bacillus if the wound or suppurating focus is improperly cared for. Many cases of vaccinal tetanus can thus be accounted for, $i$. e., due to negligence in the care and treatment of the wound. It is now generally agreed that proper treatment of the original wound, combined with the administration of tetanus antitoxin, will surely prevent the development of lockjaw.

In former years Fourth of July wounds claimed a heavy toll of fatalities due to tetanus. Owing to the efforts of the American Medical Association municipalities have been urged to adopt legislative measures for enforcing a saner form of celebration, and efforts have been made to educate physicians in the proper care of these wounds and to impress upon them the great prophylactic value of tetanus antitoxin. These efforts have been crowned with success, as statistics collected from all parts of the country will show. In 1903 there were in the United States 406 deaths from tetanus; in 1904, 91 ; in 1905, 87; in 1906, 75; in 1907, 
73 ; in 1908, 76; in 1911, 18 cases and 10 deaths; in 1912, 7 cases with 6 deaths, and in 1913, 4 cases with 3 deaths.

While it is not within the province of this chapter to deal with surgical technic, the proper cleansing and care of a wound constitute so important a part of the prophylaxis of tetanus that I shall refer to this subject, quoting largely from the technic recently described by Ashhurst and John. ${ }^{1}$

Surgical Treatment.-1. The surrounding skin should be painted with a 3 per cent. alcoholic solution of iodin.

2. All foreign material should be removed from the wound, ana to do this properly all parts of the wound should be made accessible by wide incision, under ether, if necessary. This is especially true of a puncture wound. It should then be freed from all tags and loose shreds of tissue by means of the scissors, and the whole wound swabbed with the 3 per cent. iodin solution. The wound should next be dressed with gauze soaked in the same solution. The use of strong caustics is inadvisable, as they cause sloughing and tend to produce a good focus for the growth of tetanus bacilli.

3. The wound should be dressed daily at first, being exposed and thoroughly irrigated with hydrogen dioxid solution, and then dressed with the gauze saturated with the iodin solution. As soon as healthy granulations have formed, balsam of Peru applications should be made.

4. Antitetanic powders have been prepared, made up with antiseptics, and although experimentally their use has seemed to be successful in preventing the development or absorption of tetanus toxin, still it has not as yet shown that these results were not merely due to the strong antiseptic that was combined with the antitoxin powder. It might, however, be well to apply tetanus antitoxin and antitetanic powder to the open wound, but these remedies are not to be relied upon nor accepted as substitutes for the injection of antitoxin.

Use of Antitoxin.-The prophylactic use of tetanus antitoxin has not infrequently been unsuccessful, due probably to the fact that it was used incorrectly.

1. Antitoxin should be given as soon as possible after the wound has been inflicted, and best at the time the primary treatment is given. The antitoxin should be injected "as near the wound as possible, so as to flood the tissues in the immediate vicinity," and, if possible, it should be given intramuscularly, so that the motor nerves may absorb it rapidly.

${ }^{1}$ Amer. Jour. Med. Sci., 1913, cxlvi, No. 1. 
If any nerves are exposed, antitoxin should be injected into them. The injection of 1500 units of antitoxin is generally advised as the first prophylactic dose.

2. From the fact that tetanus antitoxin is one of the albuminous constituents of horse serum that are foreign to the human system, in the human being the antitoxin is rapidly eliminated in from eight to ten days after the injection is administered. Knorr has found, as the result of animal experiments, that by the sixth day only one-third, and by the twelfth day only one-fiftieth, of the optimum quantity remained in the blood. Hence it is important, if antitoxin is to prove useful, that it should be present in the system for two or three weeks after receipt of the injury, especially as it cannot be determined when the tetanus bacillus first gained access to the wound. There should be a second intramuscular injection of 1000 units of antitoxin about the end of the first week, and perhaps a similar dose at the end of the second week. While certain individuals may develop serum sickness, no dangerous symptoms have been observed to result from the use of tetanus antitoxin.

3. If the surgeon is first consulted several days after the injury has been inflicted, the wound should be opened and dressed as previously described, and, in addition to the intramuscular injection of 1500 units of antitoxin in the neighborhood of the wound, it will be good practice to inject an additional 5000 or 10,000 units intravenously. It requires at least twenty-four hours for the antitoxin to be absorbed from the subcutaneous tissues, and immediate neutralization of any toxin present in the blood may mean a great deal from the standpoint of prognosis if tetanus should develop.

Treatment of Tetanus.-While the great value of antitetanic serum as a preventive is unquestioned, as a specific cure it has fallen short of the earliest expectations. It has been shown experimentally, however, that tetanus antitoxin may save the lives of animals already manifesting the symptoms of an otherwise fatal intoxication. In order to accomplish this result the serum must be given in doses several hundred times the size required merely for protective purposes, and it must be injected within a short time-from twenty-four to thirty-six hours-after the onset of the tetanus. Furthermore, statistics favor the use of the serum as the mortality has been reduced from 80 to 85 per cent. to 60 or 65 per cent. in cases receiving serum treatment.

The recognition of the natural limitations of the serum treatment of tetanus will serve to emphasize the importance of its proper administration. A large number of units must be given, and must be injected 
in places best suited to secure the maximum neutralizing action of the serum.

Surgical Treatment.-1. The site of the wound should be located, and if possible incised under ether or chloroform anesthesia and thoroughly cleansed of foreign material and necrotic tissue. It should then be swabbed with the 3 per cent. alcoholic solution of iodin, washed with hydrogen dioxid solution, and packed loosely with gauze soaked in the iodin solution.

2. Cauterization with pure phenol, followed by alcohol, may be employed, but, as a rule, the weaker germicide is preferable in order not to produce necrosis of the tissues, which furnishes pabulum for bacterial growth. The wound should be dressed daily.

Serum Treatment.-A maximum amount of antitoxin should be given the patient as soon as possible, and the greater the delay in giving the antitoxin, the greater is the amount required. Since absorption after subcutaneous injection is very slow, valuable time may be lost, and since enormous amounts must be given, at great expense, this route possesses much less value than the intravenous and intraspinal methods.

1. Administer intravenously from 10,000 to 20,000 units of antitoxin at once, and repeat the dose if no effect is apparent or if the good effect wears off in about from eighteen to twenty-four hours (the technic is described on p. 690). After one or two intravenous injections the good effect may be maintained by direct intramuscular injections of from 5000 to 10,000 units for one or two doses.

2. From 3000 to 5000 units should be given intraspinally by means of lumbar puncture. This dose should be repeated every twenty-four hours unless the symptoms have markedly ameliorated. The technic of this injection is described on p. 694. A quantity of cerebrospinal fluid should be removed before the serum is injected. After the first injection the fluid may be found to have become cloudy, with a large increase of cells, especially of the polynuclear variety, although bacteriologically it may be sterile. This outpouring of leukocytes is probably a reaction to the irritant effect of the serum, and especially of the preservative it contains.

3. If a surgeon is at hand, from 500 to 1000 units of antitoxin should be injected slowly intravenously into the sheaths of the nerve-trunks leading from the infected region. These injections are directed to be made as near the trunk as possible, and to distend the nerve so as partly to neutralize and partly mechanically to interrupt the passage of toxin to the cord or brain. 
Both the intraspinal and the intraneural injections are given under light chloroform anesthesia.

General Treatment.-A large number of substances have been advocated in the treatment of tetanus; of these, the most common are injections of phenol and intraspinal injections of magnesium sulphate. While phenol may be well tolerated by tetanus patients, Ashhurst and John believe that all these treatments are of little value, and that spinal injections of magnesium sulphate are dangerous.

1. Chloral hydrate and potassium bromid should be given by mouth or by rectum, in sufficient quantities to produce sleep and quiet. Drugs, such as those of antagonistic physiologic activity, are more or less successful and frequently of aid when given in conjunction with the antitoxin.

2. While combating the disease, the general care of the patient should not be forgotten. A purgative should be administered early. Simple, nourishing, non-stimulating food should be given by the mouth, if possible, or by the nasal tube, if necessary. Absolute quiet should be maintained. Distention of the bladder from retention of urine should be guarded against. If water is not well absorbed, and especially if there is peritoneal or pelvic inflammation, saline injections into the colon should be given.

Results of the Antitoxin Treatment of Tetanus.-As was previously stated, the prophylactic value of tetanus antitoxin has been proved beyond any reasonable doubt. This does not imply, however, that the simple introduction of 1000 units of antitoxin beneath the skin will surely protect the patient, as the percentage of cases developing tetanus even after the serum has been given is altogether too high. As was pointed out under Prophylactic Treatment, the wound must receive thorough surgical attention, and the antitoxin must be injected in such places where it will have the greatest opportunity to neutralize the toxin. Even if tetanus should develop under these conditions, it is likely to be mild and the prognosis would be much more favorable.

Tetanus antitoxin has likewise been very successful in veterinary practice, especially after castration and other operations, in injuries, and among horses used for the purpose of producing diphtheria antitoxin and other immune serums.

While the curative value of tetanus antitoxin has not come up to expectations, more recent carefully prepared statistics indicate that, with serum treatment, the mortality is reduced at least 20 per cent. This includes cases treated by the subcutaneous injection of antitoxin, and it must be em- 
phasized that, in order to secure the best results, tetanus must be treated in a rational manner according to its pathology. Under these circumstances we can confidently expect a greater reduction in mortality. But at any rate no physician should withhold antitoxin in the treatment if there are any possible means of obtaining it. If only 3000 units may be had, it is far better to inject this amount intraspinally than subcutaneously.

Pennin, ${ }^{1}$ in a recent and thorough review, reaches the general conclusion that anti-tetanus serum has reduced the mortality of tetanus approximately 20 per cent. He gives figures from Denmark that are especially valuable, because they were gathered from a small area, and hence represent fairly uniform conditions: Of 199 cases not receiving serum, only 21 per cent. recovered; whereas of 189 cases treated with serum, 42.3 per cent. recovered. Of 92 acute cases with an incubation period of less than ten days 24.2 per cent. recovered when serum was used, whereas of 94 cases treated without serum only 5.3 per cent. recovered. It is significant that these Danish figures correspond closely to the American statistics and those of other countries. Irons ${ }^{2}$ has recently tabulated the results of 225 cases of tetanus treated with antitoxin collected from hospitals and private records for the years 1907 to 1913. The mortality in all cases receiving serum was 61.77 per cent.; in 21 cases without serum the mortality was 85.7 per cent. The latter figures correspond quite closely with the general mortality of about 85 per cent. of tetanus treated without serum. Irons' figures also show the influence of large doses of antitoxin; of 57 cases receiving a small dose of antitoxin (3000 units or less subcutaneously), the mortality was 73.7 per cent.; of 143 cases receiving large doses (over 3000 units subcutaneously, or 3000 or less intraspinally or intravenously), the mortality was 57.3 per cent. Magnesium sulphate was given intraspinally in 18 cases which also received serum; in 4 cases -2 acute and 2 chronicthe patients recovered, giving a mortality for the group of 77 per cent. In 2 cases death recurred shortly after injection with symptoms of respiratory failure.

In view of this evidence in favor of antitoxin in the treatment of tetanus it is apparent that the physician is compelled to give every patient with tetanus the opportunity to obtain this 20 per cent. or more benefit by administering the serum promptly and correctly.

1 Mitt. a. d. Grenzgeb. d. Med. u. Chir., 1913, xxvii.

2 Jour. Amer. Med. Assoc., 1914, lxii, 20, 25. 


\section{SERUM TREATMENT OF DYSENTERY}

Soon after the discovery of a bacillus of dysentery by Shiga in 1892 the treatment of bacillary dysentery by the use of immune serums was undertaken. Following the discovery of the etiologic importance of Shiga's bacillus in the dysenteries of Asiatic countries, similar investigations were made in various parts of the world and various bacilli were isolated. At first these microörganisms were all regarded as being identical, but further investigation has shown that marked differences are apparent, and two main types are now recognized: one type (Shiga) does not ferment mannite and produces a soluble or extracellular toxin, and a second type (Flexner-Harris, Hissy, etc.) ferments mannite and does not produce an extracellular toxin. A further discussion of these bacilli will be found in Chapter VII.

Dysentery caused by the bacilli of the Kruse-Shiga type may be regarded as a form of intoxication analogous to the intoxication of diphtheria. The intestine, where the bacilli lodge, corresponds to the throat, which is the site of infection in diphtheria; here the bacilli develop and produce their toxins, and these.toxins, when absorbed into the circulation, in turn produce the symptoms of the disease.

Antitoxin has been prepared for the bacilli of the Kruse-Shiga type, and these have yielded fairly satisfactory results in the prophylaxis and cure of this variety of bacillary dysentery. Antiserums for the mannitefermenting group of bacilli (Flexner, Harris, Hess, Duval, etc.) have not proved of much value in the treatment of these infections. Bacilli of the latter group are largely responsible for the dysenteries in this country, and also for a percentage of cases of ileocolitis of infancy. Since the antiserum of the Shiga bacillus is of practically no value in the treatment of infections caused by bacilli of other groups, the serum treatment of dysentery is employed mainly in European and Asiatic countries, where infections with this group are common. After fairly extensive trials in this country the serum treatment of infantile diarrheas and true dysenteries has proved disappointing.

The preparation and standardization of dysentery antitoxin is described in Chapter XIV.

Administration and Uses.-Dysentery antitoxin has been used both in the prophylaxis and in the cure of this infection. The doses of serum advised by various observers vary considerably, owing to the marked differences that exist in the potency of these serums. Since the various manufacturers do not employ the same standards, the physician should 
use the serum in accordance with the printed directions that accompany each package.

For prophylactic purposes, usually from 10 to 20 c.c. are recommended, and it is further advised to repeat the injection after two or three weeks, as the protection lasts only a short time.

For curative purposes, Shiga has advised 10 c.c. for mild cases and from 20 to 60 c.c. for severer cases. It may be necessary to repeat the injections several times. Vaillard and Doyle have given as much as from 80 to 100 c.c., and have repeated this dose on the following days. When the serum is being used during an epidemic, it is advisable to ascertain beforehand the nature of the infection, as the antiserum for the Shiga bacillus is highly specific and is not likely to prove of value in the treatment of infections caused by the Flexner type of bacillus.

The injections have usually been given subcutaneously. Better results would, no doubt, be obtained in the treatment of severe infections by administering large doses of serum intravenously.

Results.-Adequate statistics regarding the value of the serum in the prophylaxis and treatment of dysentery are not yet available. From the prophylactic standpoint, encouraging results have been reported by Kruse, Shiga, Vaillard and Dopter, Rosculet, and others, and it would appear that passive immunization is of value in combating localized outbreaks, such as occur in institutions and armies.

From the curative standpoint, most observers agree that the use of a potent serum will reduce tine mortality of acute cases at least from 30 to 50 per cent. Shiga reports a drop in the mortality in Japan of from 22 to 26 per cent. to 9 to 12 per cent.; Kruse obtained a reduction in mortality of about 10 per cent. Vaillard and Dopter ${ }^{1}$ treated 96 cases, with but 1 death; Rosenthal ${ }^{2}$ treated 157 cases with 7 deaths,- - a mortality of 4.5 per cent. as compared with that of 10 to 11 per cent. occurring in other German hospitals. Coyne and Auche ${ }^{3}$ treated 11 cases due to the Flexner type of bacillus and report good results. In a thorough investigation made in the United States in 1903 by the Rockefeller Institute, under the direction of Flexner, it was found that the results of the serum treatment of ileocolitis, among children at least, were quite disappointing. This is largely due to the fact that several different strains of bacilli may be the cause of an infection, and unless a corresponding antiserum is employed for the particular type causing the

1 Ann. Inst. Past., 1906, xx, 321; 1907, xxi, 241.

${ }^{2}$ Deut. med. Wochenschr., 1904, xxx, No. 19.

${ }^{3}$ Compt. rend. Soc. Biol., 1906, lx, No. 26. 
infection in a given case, good results cannot be expected. Probably if some means were discovered for making a prompt bacteriologic diagnosis, and if several immune serums were on hand for the treatment of infections caused by the main types, after the methods worked out by Neufeld, Dochez, and Cole in the treatment of pneumonia, good results may be obtained.

The curative effect of dysentery antitoxin is shown by a reduction in the number of stools, by the fact that blood and pus disappear from the discharges, pain and tenesmus are relieved, the temperature becomes normal, and the patient gains in weight. Individual observers are frequently enthusiastic over the results obtained in individual cases, and no doubt these are striking in those instances where the antiserum appears to be specific for the particular form of infection.

\section{THE SERUM TREATMENT OF HOG CHOLERA}

While the cause of hog cholera has not as yet been discovered, it is a well-known fact that the virus is present in the blood of infected animals, and it is possible, by immunizing healthy hogs with gradually increasing doses of infected blood, to prepare a potent immune serum that will prove of value in the prophylaxis and cure of hog cholera. The nature of this serum is unknown. It possesses many of the features of an antitoxin, and for the present may be classed with these.

Production and Standardization of Hog Cholera Serum.-Healthy hogs weighing about 100 pounds are selected, and injected subcutaneously with 40 c.c. of hog cholera serum per hundred pounds of weight. Two or three days following this protecting dose they are injected intravenously with 3 or 4 c.c. of sterile, defibrinated blood, obtained from an animal suffering from the disease; or the animals may be exposed in pens known to be infected. If the animals live for one month without showing evidences of toxemia, they receive another injection of 5 c.c. of infected blood (virus). Two or three weeks later they receive another injection of from 15 to 20 c.c. of infected blood; in from fifteen to twenty-one days after this inoculation they receive a final injection of from 4 to 5 c.c. of virus per pound of body weight. Animals tolerating the last injection are said to be hyperimmune, and are bled in ten days. In hyperimmunizing the animal some prefer to inject the virus intraperitoneally instead of intravenously. If this method is adopted, about double the dose of infected blood (virus) is required. About 5 per cent. of animals succumb during the period of immunization.

The immunized hogs are bled aseptically from the tail by snipping off the tip, and 5 c.c. of blood per pound of weight is collected in sterile vessels. Each animal is bled once a week until three or four bleedings have been made.

In about one week after the last bleeding they are hyperimmunized again by giving them 4 or 5 c.c. of infected blood per pound of body weight, and additional bleedings are made so long as the tail lasts. 
The serums secured in the several bleedings are mixed together. The third or fourth bleeding is said to be most potent or to contain the greatest number of antibodies.

In testing and standardizing the immune serum, six hogs, weighing about 100 pounds each, are placed in an infected pen. No. 1 receives no serum and is a control; No. 2 receives 15 c.c. of serum; No. 3 receives 20 c.c.; No. 4 receives 30 c.c.; No. 5 receives 35 c.c.; and No. 6 receives 40 c.c. of immune serum. The animals are allowed to remain in the pens until the control succumbs and the protecting dose of serum has been determined. In this manner we can determine just about the amount of serum required to protect 100 pounds of hog. The Pennsylvania Live Stock Sanitary Board has found that it does not require quite 40 c.c. of serum, but this amount is recommended for safety, and because the weight may not be judged accurately.

Hog-cholera serum is used in both the prophylaxis and the therapeutic management of this disease. For prophylactic purposes for each 100 pounds of hog 40 c.c. of serum are injected. These injections are usually given subcutaneously and occasionally intramuscularly. In some instances active and passive immunization is practised by the simultaneous injection of 2 c.c. of virus, together with 40 c.c. of immune serum per 100 pounds of weight. Owing to the danger of spreading the disease, this method is not generally employed.

The results of prophylactic immunization are excellent, and the method is valuable in checking epidemics. Immunity is said to last for six months after vaccination.

For curative purposes the serum has yielded good results, providing it is administered not later than the fourth day after the animal shows evidences of the disease. Several injections may be required, and the intramuscular route should be chosen, because quicker absorption is thus insured.

Calf cholera serum has been prepared by immunizing horses with strains of colon, paracolon, and other bacilli belonging to these groups, isolated from calves dying of calf cholera. The immune serum should agglutinate the microörganisms used in its production in dilutions of 1:2000 to $1: 500$.

This serum has proved of value in the prevention of calf cholera, and may be of benefit in the treatment, providing that it is prepared with the same strain or strains of microörganisms responsible for the infection.

\section{SERUM TREATMENT OF SNAKE-BITES}

The nature of snake venom is discussed in Chapter VII, and the method of preparing antivenomous serum is described in Chapter XIV. 
Calmette's antivenene for cobra venom is useful in the treatment of cobra envenomation, but is not serviceable for the treatment of other snake-bites, as shown by Martin for Australian serpents and by McFarland for American snakes. In the venoms of our snakes, as, for example, the rattlesnake, copper-head, and moccasin, the poison is essentially locally destructive, the respiratory poison being of secondary importance. McFarland failed to immunize horses against this locally destructive poison. Later Noguchi and Madsden succeeded in producing an antiserum, prepared by immunizing horses with venom after the toxophorous groups of the molecules had been destroyed, capable of neutralizing the hemorrhagin of the Crotalus venom.

The serums of Calmette, Noguchi, and others are useful in the treatment of their respective envenomations, but aside from India and a few other reptile-infested countries, as well as in zoölogical gardens and laboratories where snakes are kept, the serums have a very limited sphere of usefulness.

\section{SERUM TREATMENT OF HAY-FEVER}

The nature of pollen toxin has been discussed in Chapter VII, and the preparation of antitoxin in Chapter XIV.

Dunbar has prepared an antitoxin for certain pollen; this may be obtained commercially. The method of administration consists in dropping the serum into the eyes and sniffing it into the nose at the onset of an attack. It is necessary for the patient to carry the serum and dropper about, as the effects produced are of short duration, and the patient is subject to repeated reinfections. Subcutaneous injections are not advisable, as considerable local edema is produced, and the amount of protection afforded is uncertain.

Many observers, as, for example, Semon, ${ }^{1}$ McBride, ${ }^{2}$ Knight, ${ }^{3}$ Throst, ${ }^{4}$ Weichardt, ${ }^{5}$ and others, report that the serum affords a temporary relief which is grateful to the patient, but which cannot be said to be curative of the disease. In some instances it fails altogether, and in these it is reasonable to assume that the antitoxin has not been prepared from the particular pollen that infected the patient. An intolerance to the serum may be excited.

More recently the possibilities of effecting active immunization

1 Brit. Med. Jour., 1903, ii, 123, 220.

${ }^{2}$ Edin. Med. Jour., 1903, ii, 7.

${ }^{3}$ Med. Record, March 10, 1906.

${ }^{4}$ Münch. med. Wochenschr., June 9, 1903.

${ }^{5}$ Centralbl. f. Bakt., Abst., 1906, xxxviii, 493. 
against hay-fever have been shown by Claves ${ }^{1}$ with the pollen of ragweed. The method is still in the experimental stage, but it is reasonable to assume that vaccines may be prepared for the pollen of various plants usually responsible for the hay-fever and autumnal catarrhs of this country.

\section{ANTIBACTERIAL IMMUNIZATION}

General Considerations. - It may be stated that antibacterial serums have not been found of equal value to the antitoxins, either in the prophylaxis or in the treatment of their respective infections. It is true, however, that antimeningococcus serum has reduced the mortality of epidemic cerebrospinal meningitis from 75 to 90 per cent. to 30 per cent. and less, and has thereby firmly established its value in the treatment of this dreaded infection. Recent work in pneumonia has developed a method of serum therapy that has proved of value in the treatment of this disease, and it is likewise true that antistreptococcus and antigonococcus serums yield at times and in individual cases most prompt and happy results. But the expectations for serum therapy that were aroused in 1894 with the discovery of diphtheria antitoxin have not been fully realized, although at the present time the reasons for failure are being studied, understood, and gradually overcome.

Granting that serum therapy could be reduced to the simple proposition of bringing specific antibodies into relation with the microorganisms producing a given infection, the process remains quite intricate, largely owing to the fact that although different strains of the same microörganism may possess identical morphologic and biologic characteristics, yet they vary not only in pathogenicity, but also in the specificity of the antibodies that they stimulate the body-cells to produce. In other words, serurn therapy is more specific than it is generally considered to be. For instance, the antibodies of one strain of pneumococcus may have little or no action upon another strain, and the same is probably true of the various pathogenic bacilli and groups of streptococci, gonococci, and to a lesser extent also of meningococci. This fact has long been known, and an effort has been made to overcome the difficulty by immunizing horses with a large number of different strains of the same microörganism in the hope that the polyvalent serum so produced would contain sufficient antibodies for all or most infections of the various strains of the particular microörganisms in question. With diphtheria and tetanus bacilli, the soluble toxin is apparently quite

1 Proc. Soc. Exper. Biol. and Med., 1913, x, 69. 
similar for all strains, so that immunization with the toxin of one yields an antitoxin capable of neutralizing the toxins of all. With dysentery bacilli, snake venoms, and pollens, however, the toxins are more specific, and produce more specific antitoxins.

Recent work in pneumonia by Cole and Dochez and their coworkers in the Rockefeller Institute, and Neufeld in Germany, indicates a more promising future for antibacterial immunization. These investigators have been able to divide pneumococci into four main groups, have worked out a relatively quick method of determining the group to which a particular pneumococcus belongs, and have prepared immune serums for these main groups. By injecting the serum corresponding to the strain producing a given infection, encouraging results have been obtained in the treatment of pneumonia, whereas the polyvalent serums have been found, after quite extensive use, to yield indifferent results, due in part to a relatively low content in the particular antibodies for that certain strain causing a given infection.

These investigations in pneumonia are of great importance because they reveal an immense field of interesting and similar researches in streptococcus, gonococcus, meningococcus, typhoid, and other infections. While it is obviously impossible to prepare an immune serum for each and every strain of microörganism, it may be possible to subdivide strains into a few main groups and then discover a method for quickly determining to which group a particular culture belongs, so that the corresponding immune serum may be administered. In this manner we may be able to reduce the 30 per cent. mortality still remaining in epidemic meningitis and otherwise place the treatment of specific infections upon a more strictly scientific basis, as in the serum treatment of diphtheria.

As previously stated, the method and route of injecting an immune serum are of considerable importance in serum therapy. Large doses of serum should be given until the desired effect is secured, or until it becomes evident that more can be produced. In the mean time manufacturers should make every effort to produce potent serums and to concentrate them, if possible, just as diphtheria antitoxin is concentrated.

\section{THE SERUM TREATMENT OF MENINGOCOCCUS MENINGITIS}

During the pandemic of meningococcal cerebrospinal meningitis in 1904-05 several laboratories sought to produce an immune serum for the purpose of treating human cases of this infection. 
After pursuing experimental studies on the subject on the lower animals, Jochmann, ${ }^{1}$ in 1905 , immunized a horse and used the serum in the treatment of 38 cases of epidemic meningitis. At first he employed the subcutaneous method of injection, and later he used the intraspinal method. The results were quite encouraging, and during the following year 30 more cases were treated, with a resulting mortality of 27 per cent., as against a mortality of 53 per cent. in untreated cases.

At about the same time Kolle and Wassermann ${ }^{2}$ reported that they had prepared an antimeningococcus serum, which had not, however, up to that time been used in the treatment of human infections. A year later serum was administered subcutaneously and then intraspinally, with encouraging results.

In this country Park had, in 1905, prepared an antimeningococcus serum, which was used in the treatment of 20 cases in Hartford, Conn., by subcutaneous injection, but without beneficial results. Jochmann had, in the mean time, shown the superiority of intraspinal injections, and this method soon supplanted the subcutaneous method.

In 1905 Flexner began a series of studies regarding experimental meningococcus infections in the lower animals, and the therapeutic value of antimeningococcus serum. These valuable experiments attracted the attention of the world, and placed this method of treatment upon a firm basis. In 1906 Flexner $^{3}$ proved that a specific immune antimeningococcus serum could be produced that, if injected intraspinally, would save the lives of monkeys. Later horses were immunized and the serum used in the treatment of human infections during an epidemic in Akron, Ohio, in May, 1907. In a short time the serum was used extensively in other epidemics, and a report of these early cases was made by Flexner ${ }^{4}$ in 1907. A later report by Flexner and Jobling, ${ }^{5}$ covering the treatment of 400 cases, showed that the mortality had been reduced from 75 per cent. to below 30 per cent. In 1909 they reported ${ }^{6}$ upon 712 cases treated with the serum, with a mortality of 31.4 per cent. In a more recent report Flexner ${ }^{7}$ reviewed all the cases-1300 in number - gathered from all parts of the world, treated with serum prepared in the Rockefeller Institute. The general mortality rate is given as 30.9 per cent., as against 75 to 80 per cent. among cases not receiving serum

${ }^{1}$ Deut. med. Wochenschr., 1906, xxii, No. 20, 788.

${ }^{2}$ Deut. med. Wochenschr., 1906, xxxii, No. 16.

${ }^{3}$ Jour. Amer. Med. Assoc., 1906, xlvii, 560.

${ }^{4}$ Jour. Exper. Med., 1907, ix, 168.

5 Jour. Exper. Med., 1908, x, 141.

${ }^{6}$ Jour. Amer. Med. Assoc., 1909, liii, 1443.

${ }^{7}$ Jour. Exper. Med., 1913, xvii, 553. 
treatment. Of 1394 cases treated with serum during the Texas epidemic $^{1}$ (1912), the mortality was 37 per cent., as compared with a mortality of 77 per cent. among 562 cases receiving no serum.

Good results have been reported by many observers with Jochmann's, Kolle and Wassermann's, Ruppel's, Paltauf's, and Dopter's serums, and the serums have been prepared by several commercial biologic laboratories, so that the curative value of antimeningococcus serum is definitely established.

Nature of Meningococcus Meningitis.-Bacteriologic and pathologic evidence indicates that the first stage of meningococcus meningitis consists of a meningococcus bacteremia, the virulent meningococci gaining access to the blood-stream through the upper air-passages. Later the infection becomes localized in the spinal and cerebral meninges. It is probable that the microörganism causes a primary nasopharyngitis, and in some instances the meninges may be infected by direct extension through the sphenoid and ethmoid sinuses. The main symptoms and lesions of the disease, and several of the complications, for example, the paralyses, eye complications, deafness, hydrocephalus, and mental disturbances, are probably directly due to suppuration of the meninges, with involvement of accessory and motor nerve-roots, meningeal irritation, and pressure from the accumulation of exudate in the ventricles and subarachnoid space. Complications, such as arthritis, pyelitis, endocarditis, adenitis, etc., are due to the bacteremia, which may become chronic and be accompanied by deposits of meningococci in the various tissues and organs. In addition to these complications there is probably a varying degree of general toxemia, due to a soluble toxin or endotoxin liberated through lysis of the cocci.

Treatment of Epidemic Meningitis.-Although cerebrospinal meningitis may be considered primarily as a general infection, in the majority of instances local suppuration of the meninges constitutes the main lesion. For anatomic and physiologic reasons, however, it is impossible to treat the disease according to the ordinary principles governing the treatment of localized suppuration, as, for example, by continuous drainage and by cleansing the affected parts with germicidal solutions. Unaided, the leukocytes and body-fluids are generally unable to destroy the cocci and terminate the infection before serious harm to important nerves and nerve-centers has resulted, so that epidemic meningitis, with a mortality of from 75 to 90 per cent., and followed by more or less

${ }^{1}$ Sophian: Epidemic Cerebrospinal Meningitis, St. Louis, 1913. Report of Dr. Steiner, President of the Texas State Board of Health. 
serious sequelæ, which few survive, is one of the most dreaded of infections.

In administering antimeningitic serum we aim to assist the patient's leukocytes and body-fluids to overcome the infection. Repeated spinal punctures remove portions of the infective material mechanically, but the greatest dependence in bringing about quick destruction of the cocci and effecting recovery of the patient is to be placed upon the serum.

\section{Preparation of the Antimeningococcus Serum.-Method of Flexner and Jobling.-} 1. Many strains of meningococcus are used in order that a polyvalent serum may be prepared. Fresh strains from new epidemics and sporadic cases are constantly added. "Fast" strains, or those isolated from cases in which the serum has produced no beneficial effect, are especially desirable.

2. Immunization is performed first with an autolysate of the meningococci, and later with living cultures.

3. Stock cultures are kept alive by transplanting them every four days in slants of ascitic glucose agar, neutral to phenolphthalein.

4. In preparing the autolysate, the cultures are subcultured first on glucose-agar slants without serum. After twenty-four hours' growth about 3 c.c. of salt solution are added to each slant, and the culture emulsified. One cubic centimeter is then poured over the surface of glucose-agar slants in large 500 c.c. Blake bottles. After twenty-four hours' incubation heavy, uniform, and diffuse growths are secured.

Add 10 c.c. of normal salt solution to each bottle and wash off the culture. If necessary, a long heavy platinum loop may be used. Each bottle is tested for contamination by staining a smear according to the method of Gram. Each bottle is emptied into a common vessel; 2 per cent. toluol is added, mixed well, and incubated for from eighteen to twenty-four hours. The toluol is then allowed to evaporate, or it may be immediately filtered off through sterile gauze saturated with salt solution. The preparation is kept in a refrigerator and should be prepared fresh every month.

5. The injections are given subcutaneously about the neck and abdomen. Young and healthy horses are selected for the purpose. The first dose consists of 2 c.c. of the autolysate, and this is gradually increased, depending on the manner in which the animal reacts, until 10 c.c. are given in a single dose. Then inject 2 c.c. of living culture diluted with two parts of salt solution, and increase the doses, the same as with the autolysate, until 10 c.c. of culture are given at one injection. Next inject living cultures and autolysate alternately, until a maximum of from 30 to 35 c.c. are given in one dose; this last is then used as the constant dose until the immunization has been completed.

Injections are given every five to seven days until the large doses are reached, when they are given every ten days or two weeks.

Horses usually show quite marked reactions, such as fever, depression, and induration about the site of injection, but if due care is exercised, few animals are lost during the immunization.

6. Bleedings are usually begun about the fourth month after immunization has been instituted. The horses are bled aseptically from a jugular vein about every two weeks, from six to eight liters of blood being removed at each sitting. The serum is separated and preserved with trikresol. Recent investigations indicate that trikresol may be partly responsible for paralysis of the respiratory centers, and at present every effort should be made to collect and market the serum in a strictly aseptic manner, so that 
none or but very little preservative is required. ${ }^{1}$ Efforts are being made at present to discover an efficient volatile antiseptic that may be driven off by warming the serum at body temperature.

Method of Kolle.-Kolle prepares antimeningococcus serum by immunizing horses with heated and then with living cultures or with bacterial extracts. All injections are given intravenously.

(a) With Cultures.-The first dose equals half a culture removed with normal salt solution from a test-tube of slanted agar after twenty-four hours' incubation, and heated at $60^{\circ} \mathrm{C}$. for half an hour. A week later the dose equals that of a whole slant. The dose is increased each week by one slant until 10 are given. The eleventh dose equals one agar slant of living culture, and each week the dose is increased until the growths from 10 slants are given at one time. Fourteen days after the last injection the horses are bled and the serum is tested.

(b) With Bacterial Extracts.-Horses are first injected with two doses of heated cultures, as just described. The third dose consists of 0.1 c.c. of bacterial extract. Every two weeks the dose is increased by 1 c.c. of extract until 5 c.c. are given at one time.

Standardization of Antimeningococcus Serum.-An accurate method of standardizing antimeningococcus serum has not as yet been devised. In the selection of a serum physicians must, therefore, be guided by the reputation of the manufacturers.

An antimeningococcic serum of high antibody content has antitoxic, bacteriotropic, and bactericidal properties. Kraus and Dorr consider that the chief function of the serum is antitoxic; Flexner and Jobling, Neufeld, Jochmann, and Wassermann believe that its bacteriotropic properties are its most important qualities.

The following methods for testing an immune serum are in use or have been advocated; none of them is, however, sufficiently reliable to serve as a definite measure of antibody content or of curative value; two of them, the bacteriotropic and the complement-fixation test, are most widely used in laboratories for the purpose of estimating the antibody content of a serum.

1. Bacteriotropic Titration.-While the antimeningitic serum was being prepared at the Rockefeller Institute, Jobling ${ }^{2}$ used the opsonic test in standardization as the method of choice, on account of the part taken by specific opsonins in promoting recovery from meningococcus infections. As a definite and suitable standard of strength Jobling has suggested that a serum be accepted as satisfactory when it shows unmistakable phagocytic activity in dilutions up to 1:5000. The method of Neufeld is used, as described on page 200.

2. Complement-fixation Tests.- The advantages of these tests are that the same polyvalent antigen may be used as is employed for purposes of immunization; the technic is simple, and the reactions are usually sharp and definite. According to Sophian, in a series of comparisons with opsonic and complement-fixation tests the results corresponded in every instance, a high opsonic content being accompanied by a high complement-fixation reading. The latter indicates, at least, that the horse has responded to immunization and that curative antibodies probably are present. Laboratories usually adopt their own standards in preparing antimeningococcic serum. In the complement-fixation test Kolle requires complete inhibition of hemolysis with 0.1 c.c. of serum.

The technic of these tests is given on page 491 .

3. Agglutination Tests.-These tests are readily conducted with the polyvalent antigen used in immunization, a macroscopic technic, as that described on page 284,

${ }^{1}$ Hall, W.: Bull. No. 91, Hyg. Lab., U. S. P. H. S., 1914.

2 Jour. Exper. Med., 1909, xi, 6, 4. 
being employed. Kolle regards a serum as satisfactory when it causes agglutination of meningococci in dilutions up to $1: 5000$.

4. Animal Inoculation Tests.-These tests have been found quite irregular and impracticable for general use. As stated by Jobling, not only does the pathogenicity of the meningococcus vary considerably from day to day, but the resistance of animals to this microörganism is also quite variable. By preparing a large quantity of bacterial emulsions and using sufficient controls to determine the fatal dose, and by employing a standard lethal dose of emulsions mixed with varying quantities of serum injected intraperitoneally into 250-gram guinea-pigs, some conception of the protective value of a serum may be obtained.

Action of Antimeningococcus Serum.-As previously stated, experiments in vitro show that a potent antimeningitic serum possesses three chief antibodies upon which its curative powers probably depend, namely: (1) Bacteriotropins (immune opsonins), which lower the resistance of the meningococci and facilitate their phagocytosis; bactericidins, which kill the cocci extracellularly, either with or without final lysis; and (3) antitoxins, which neutralize the true extracellular toxin, which some strains of-meningococci apparently produce in varying degree. Other than these are the agglutinins, which probably aid in bacteriolysis, and anti-aggressins, which may assist in the process of phagocytosis.

Microscopic examination of a direct stained smear of the sediment of cerebrospinal fluid obtained from fresh cases will show large numbers of polynuclear leukocytes and cocci, the majority of the latter being extracellular. As the case improves, whether under serum treatment or spontaneously, the microörganisms diminish in number and become intracellular, frequently appearing clumped and failing to grow in culture. It would appear, therefore, that a cure is brought about partly by means of phagocytosis aided by bacteriotropins; by bacteriolysis through the agency of specific bacteriolytic amboceptors in the immune serum and complements in the spinal fluid and blood-serum, and to some extent by neutralization of a toxin with antitoxin.

A potent antimeningococcus serum furnishes these main antibodies, and since the first two must act locally upon the cocci infecting the meninges, the serum must be applied locally and directly by intraspinous and subdural injection, since only traces of immune serum could eventually find their way into the cerebrospinal fluid if the serum were injected subcutaneously or intravenously. On the other hand, in the treatment of meningococcus bacteremia and toxemia the serum should be injected intravenously and subcutaneously.

Unfortunately, an immune serum may not contain the antibodies 
for the cocei producing a given infection, and hence the serum, even though it is skilfully administered in large doses, will have no influence upon the disease. Apparently the cocci of these resistant or "fast" strains are uninjured by the antibodies in the serum. To overcome this difficulty, a large number of different strains of meningococci are used in immunizing horses. If, however, the serum of one laboratory is found to exert no beneficial effect, the physician should use the serum of another, for different laboratories probably immunize their horses with cultures not in common use. It is highly desirable to secure cultures of these "fast" strains. These should be sent at once to laboratories engaged in the production of antimeningitic serum, for the larger the number of these strains used in immunization, the more potent and valuable will the serum be.

Administration and Dosage of Antimeningitic Serum.-In Acute Meningitis.-As a rule, serum should be injected into the spinal canal as early in the disease as possible, and in such maximum amount as is compatible with safety. Intraspinal injection is absolutely necessary, for the serum must be brought into contact with the infected membranes, and only a trace would reach the spinal fluid if the serum were injected subcutaneously or intravenously. The advantages of early administration are obvious, and if the symptoms are indefinite, the physician should not hesitate to perform lumbar puncture and to secure fluid for microscopic examination, just as he would take a throat or nose culture to aid in the diagnosis of diphtheria. The maximum, or at least an adequate, amount of serum should be injected, care being observed to avoid undue pressure as a result of injecting too quickly or too large an amount. This administration of antimeningitic serum is, therefore, an important and delicate, though relatively simple, procedure.

1. The technic of intraspinal injection has been described on p. 694 . Whenever possible, the serum should be injected by the gravity method, and the amounts of fluid withdrawn and serum injected controlled by blood-pressure readings.

2. Lumbar puncture is performed, and the fluid collected in graduated tubes. In the ordinary case, fluid may be allowed to drain until the blood-pressure drops about $10 \mathrm{~mm}$. of mercury, or if the pressure is unchanged or rises, until the fluid flows about one drop every three seconds, provided there are no other evidences of collapse, such as faintness, headache, and great restlessness.

3. As a rule, the amount of serum injected should be slightly less than the amount of fluid withdrawn. When the injection is controlled 
by the blood-pressure readings, - the amount varies considerably,-usually the injection should stop when the pressure falls another 10 or 15 $\mathrm{mm}$. For adults, the dose of serum should be about 30 c.c.; for an infant, about 15 c.c.

The serum should be allowed to flow in slowly, an ordinary injection consuming at least ten or fifteen minutes. If symptoms of collapse should appear before an adequate amount of serum has been injected, the funnel may be lowered and the spinal fluids allowed to flow out. When the symptoms have disappeared, the injections may be continued and satisfactorily completed.

3. When the physician cannot administer the serum by the gravity method or under blood-pressure control, the injection may be given by means of a syringe (see p. 700). It should be given slowly, and the patient observed closely in order to detect the general symptoms of collapse. The amount of serum injected should not be larger than the amount of cerebrospinal fluid withdrawn. According to Sophian, the average doses are as follows:

\begin{tabular}{|c|c|c|}
\hline & Dose of Antimeningitic & $\begin{array}{l}\text { AMOUNT OF FLUID } \\
\text { WITHDRAWN }\end{array}$ \\
\hline 1 to 5 years. & . . 3 to 12 c.c & 12 c.c. \\
\hline 5 to 10 years. & . . 5 to 15 c.c. & 15 c.c \\
\hline 10 to 15 years. & $\ldots .10$ to 20 c.c. & 20 c.c. \\
\hline 15 to 20 years. & . . 15 to 25 c.c. & 30 c.c. \\
\hline 20 years and ov & $\ldots .20$ to 30 c.c. & 40 c.c. \\
\hline
\end{tabular}

The injection of too large a dose of serum may be followed by headache, pain in the back and legs, and restlessness. When the amount of serum injected exceeds the amount of spinal fluid withdrawn the symptoms just named must be regarded as the signal to stop; otherwise they may be disregarded.

Intravenous Injection.-During epidemics of meningitis it may be possible to detect cases in the bacteremic stage when meningococci are present in the blood and clear fluid is collecting in the ventricles. In these and in all severe fulminant infections it is good practice to inject from 30 to 100 c.c. of serum intramuscularly or intravenously. It is advisable to secure a culture of blood in ascites dextrose broth in all cases in adults and older children. If sufficient serum may be obtained and the expense is a secondary consideration, an intravenous or intramuscular injection, given at the outset and once or twice during the acute stage, may benefit the patient by neutralizing toxins and possibly prevent complications due to the entrance of meningococci into the bloodstream. 
Repeating Doses of Serum.-It is the general rule to give an intraspinal injection of serum every day for four days, and then on alternate days until the acute symptoms have subsided, and to resume the treatment if an exacerbation or a relapse occurs. In severe fulminant infections, and especially if the exudate is thick and only small amounts of serum can be introduced under pressure, it is well to repeat the injection every twelve hours until several doses have been given. Some cases require daily consecutive injections for six or more days; the average case will require from four to six injections if the treatment is begun during the acute stage; in the subacute and chronic cases many more treatments are required. There are two main indications and guides:

1. The condition of the cerebrospinal fluid.

2. The clinical condition of the patient.

1. In most instances the cerebrospinal fluid tends to clear up macroscopically as the disease improves. This is, however, occasionally misleading, as the fluid may become more turbid as the result of an aseptic meningitis or excitation of a polynuclear leukocytosis due to the serum, while in reality the numbers of meningococci are diminishing and the patient is improving.

More accurate information is obtained by the microscopic examination of a stained smear of the sediment of the cerebrospinal fluid withdrawn. In fresh acute cases the cocci are numerous and mostly extracellular; improvement is indicated by a diminution in their number, and by the fact that they are mostly intracellular. I generally determine the phagocytic index or the relative proportion of leukocytes that have engulfed cocci and the opsonic index or the relative number of cocci per leukocyte as determined by counting a large number. When many cocci are present, or if they are few in number but mostly extracellular, the indications are to puncture next day, even if the clinical condition of the patient is good and the temperature is lower. The number and position of cocci are, therefore, of more importance as a guide to subsequent injections than is the total number of pus-cells.

2. As an indication for repeating the doses of serum the clinical condition is of most value when combined with the examination of the cerebrospinal fluid. Occasionally the patient's condition may seem to improve, although the fluid may show numerous cocci, which will subsequently aggravate the clinical condition unless the serum is administered. In favorable cases there is usually a lower temperature the day following an injection, and frequently delirium becomes less marked and there is some return to consciousness. The complexion, which is often 
cyanosed at first, regains a healthy color; the pain in the head, neck, and limbs becomes less severe, although the neck and spine may remain stiff for several days. Finally the mind becomes clear and the patient is cheerful, and no longer irritable, apathetic, and hypersensitive. $\mathrm{He}$ feels better and his appetite returns. When this favorable outcome supervenes, the serum injections may be discontinued, to be resumed, however, upon the first evidence of a relapse. The physician should be on his guard for the appearance of acute hydrocephalus, which condition is relieved by repeated lumbar puncture.

The Serum Treatment of Cases with Thick Elastic Exudate.-In very severe cases the exudate may be so thick that it will not flow from the needle. In these cases the serum should be injected in small doses under pressure, and the injections repeated every eight to twelve hours. As they are likely in any case to terminate fatally, the physician is justified in taking the risk of increasing intracranial pressure. It may be well carefully to inject a small amount of warm sterile salt solution, which will dilute the exudate and possibly start a flow; or a second needle may be inserted higher up, when a thinner exudate is found, or washing may be possible by injecting salt solution in the upper needle and draining through the lower.

The Serum Treatment of Cases with a Dry Canal and Cases of Posterior Basal Meningitis.-Occasionally a patient improves clinically and the amount of cerebrospinal fluid becomes very scanty, the spinal tap being dry, although it is certain that the needle has entered the subarachnoid space. In such instances a small amount of serum may be injected, or the injection may be dispensed with if the clinical condition continues to improve. If, however, cases with dry canals present evidences of toxemia and general aggravation of symptoms, small doses of serum should be injected under pressure and the injections repeated as often as necessary. The physician must be very cautious, however, for if there are clinical evidences of severe intracranial pressure, it is probable that there is an encapsulation of fluid within the ventricles, and shutting off of the communication between the ventricles and the subarachnoid space. In this posterior basic meningitis intraspinal injections are dangerous and aggravate the process. In infants it is necessary to puncture the ventricles through the anterior fontanel and in older children and adults by trephining at Kocher's point, removing the fluid, and if it is found to be cloudy or purulent, injecting serum. It may be necessary to tap both ventricles alternately at intervals of several days, depending upon the reaccumulation of fluid and pressure symptoms. 
Permanent drainage may be instituted in severe cases by means of small catheters. While the operation is not usually dangerous, the ultimate prognosis is very unfavorable.

The Serum Treatment of Subacute and Chronic Meningitis.-If there is no evidence of sepsis; if the mind is clear and the neck limber; if the general conditions are good and the cerebrospinal fluid is practically cleared up, the affection is most likely hydrocephalitic, and may be relieved by repeated spinal punctures, with removal of as much fluid as is safe, using blood-pressure as an index. If meningococci are present in cultures of the fluid, small amounts of serum may be injected. The prognosis in these cases, however, is generally bad, as the process is prolonged and the patient finally succumbs.

In the second form of chronic meningitis, when the meningeal symptoms are active, intensified, and persistent, serum should be administered every few days in the same manner and in the same dosage as in the acute cases. Improvement is, however, usually temporary, and the ultimate prognosis is very grave.

Serum Sickness.-Intraspinal injections of serum result in the sensitization of the patient in just the same manner as if serum were injected by other routes. The percentage of cases developing serum sickness is likely to be high, since antimeningitic serum is not refined (Sophian reports 60 per cent. of his cases as developing the condition), and while the symptoms are distressing, they are seldom alarming, and fatal anaphylaxis is extremely rare. Occasionally the onset of serum sickness may be confusing, but if the meningeal condition has been responding as well as could be expected, it is wise to let the patient alone, rather than to make additional punctures and cause further depression. Local sedatives, laxatives, atropin, sedatives, and, at times, morphin, are indicated.

Results of the Serum Treatment on Meningococcus Meningitis.(a) Upon the Course of the Disease. - In the majority of cases the subdural injection of a potent antimeningitic serum is followed by some immediate improvement in the local suppurative meningitis and general sepsis, for the temperature usually drops, the mental condition improves, and delirium is diminished, although the Kernig sign may persist, partly as the result of meningeal irritation and partly on account of fear. Hydrocephalus is generally relieved, as indicated by lessening of the pressure symptoms, as, for example, severe headache, vertigo, and vomiting; breathing becomes more regular, and the pulse also becomes slower and more regular. The duration of the illness is usually shortened. 
According to Holt, in the New York epidemic of 1904-05, antedating the use of serum, among 350 cases that recovered the duration in 3 per cent. was one week or less, and in 50 per cent. five weeks or longer. Of 288 cases reported by Flexner and Jobling, the average duration of active symptoms in those receiving serum was eleven days. Sophian, in an experience of several hundred cases, reports that many acute cases were relieved in five or six days and discharged as cured in two weeks.

(b) Upon Complications.-Next to its influence upon mortality, the good effects of antimeningitic serum are apparent in that it lessens the incidence and severity of the terrible complications of this disease. The most severe and permanent sequels are those resulting from affections of the internal ear and the essential structures of vision. A conservative estimate of the incidence of the former among cases not receiving serum treatment is 12 per cent. (Göffert), whereas Flexner's ${ }^{1}$ analysis of 1300 cases treated with serum shows that deafness occurred in but 3.5 per cent. of cases. At least from 12 to 24 per cent. of cases not receiving serum treatment will develop more or less serious eye complications, whereas Flexner's report shows that impairment of vision among serumtreated cases occurred in about 0.9 per cent. of cases. The latter report shows the occurrence of arthritis in but 0.9 per cent. of cases, whereas among cases untreated with serum this is a frequent complication. Whereas chronic meningitis is relatively common among cases treated without serum, it is uncommon among those treated with serum.

(c) Upon Mortality.-The gross mortality among cases treated without serum varies from 70 to 90 per cent.; among serum-treated cases the mortality is about 30 per cent. For example, of 1294 cases treated with serum prepared in the Rockefeller Institute, the general mortality was 30.9 per cent.

The importance of early diagnosis and prompt institution of serum treatment is shown in the following table:

TABLE 30.-MORTALITY ACCORDING TO THE PERIOD OF INJECTION OF SERUM

\begin{tabular}{|c|c|c|c|c|}
\hline \multirow{2}{*}{ Trme of Injection } & \multicolumn{4}{|c|}{ Mortality, Per Cent. } \\
\hline & $\begin{array}{l}\text { Flexner, } \\
1294 \text { Cases }\end{array}$ & $\begin{array}{l}\text { Dopter, } \\
402 \text { Cases }\end{array}$ & $\begin{array}{c}\text { Netter and } \\
\text { Debre, } 99 \text { Cases }\end{array}$ & $\begin{array}{l}\text { Sophian, } \\
161 \text { Cases }\end{array}$ \\
\hline $\begin{array}{l}\text { First to third day ........ } \\
\text { Fourth to seventh day . . } \\
\text { Later than seventh day ... } \\
\text { Average mortality . . . . . }\end{array}$ & $\begin{array}{l}18.1 \\
27.2 \\
36.5 \\
30.8\end{array}$ & $\begin{array}{l}8.2 \\
14.4 \\
24.1 \\
16.44\end{array}$ & $\begin{array}{l}20.9 \\
33.3 \\
26.0 \\
28.0\end{array}$ & $\begin{array}{r}9.0 \\
14.9 \\
22.6 \\
15.5\end{array}$ \\
\hline
\end{tabular}

${ }^{1}$ Jour. Exper. Med., 1913, xvii, 553. 
The influence of age upon mortality was early pointed out by Flexner. The very high mortality in infants and in old persons is due to their lowered vitality and enfeebled resistance. An additional factor in young children is their greater tendency to develop extreme hydrocephalus and convulsions.

TABLE 31.-MORTALITY ACCORDING TO AGE

\begin{tabular}{|c|c|c|c|c|}
\hline \multirow{2}{*}{$\mathrm{AGE}$} & \multicolumn{4}{|c|}{ REPORTED BY } \\
\hline & Flexner & Dopter & Netter & Sophian \\
\hline 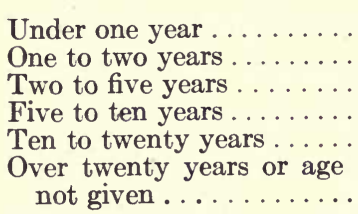 & $\begin{array}{l}49.6 \\
31.0 \\
28.4 \\
15.1 \\
29.4 \\
\\
38.2\end{array}$ & $\begin{array}{r}48.6 \\
20.1 \\
9.3 \\
8.5 \\
10.2 \\
14.1\end{array}$ & $\begin{array}{r}50.0 \\
0.0 \\
16.6 \\
12.5 \\
0.0 \\
\\
0.0\end{array}$ & $\begin{array}{r}50.0 \\
21.2 \\
17.5 \\
9.0 \\
18.0 \\
\\
32.0\end{array}$ \\
\hline
\end{tabular}

The statistics show indubitably that the mortality of epidemic meningitis can be greatly reduced by the administration of serum treatment. While the ordinary type of epidemic meningitis responds best to the specific treatment, the fulminant cases may also receive some of the beneficial influence of the serum. To quote from what Flexner wrote in 1909, and repeated in 1913: "In view of the various considerations presented, the conclusions may be drawn that the antimeningitis serum, when used by the subdural method of injection, in suitable doses and at proper intervals, is capable of reducing the period of illness; of preventing in large measure the chronic lesions and types of the infection, of bringing about complete restoration of health, thus lessening the serious, deforming, and permanent consequences of meningitis; and of greatly diminishing the fatalities due to the disease."

Prophylactic Immunization in Meningococcus Meningitis.-In Chapter XXIX mention has been made of the probable value of active immunization against epidemic meningitis by the subcutaneous injections of three doses of a polyvalent meningococcus vaccine at intervals of a week. Sophian and others have shown experimentally that opsonins, bacteriolysins, agglutinins, and other antibodies are produced, and while meningococcus meningitis is ordinarily but mildly infectious (about 5 per cent. of secondary cases in homes), the method is practically devoid of danger and worthy of trial, especially for physicians, nurses, and members of a household who are exposed to the infection over a period of many weeks. 
Passive immunization by means of the subcutaneous injection of antimeningitic serum was advised by Jochmann in 1906, but has not come into general use. The immunity resulting from the injection of from 10 to 20 c.c. of serum is only temporary, and probably lasts about a month. Another drawback is the resulting serum sensitization, which renders subsequent injections of serum more likely to be followed by serum sickness. In the presence of an active epidemic, such as that which occurred in Texas during 1912, immediate passive immunization of physicians, nurses, and attendants by the subcutaneous injection of 15 c.c. of serum, followed by active immunization with three doses of vaccine $(500,1000$, and 1000 millions) at intervals of a week, may be advisable. While there are no available statistics to prove the value of this procedure, it is, at least, a rational one, and since there is danger of contracting the disease, especially after prolonged contact, physicians should practise immunization during epidemics of this dreaded disease. In mixed passive and active immunization the serum probably affords immediate protection, and tides over any temporary negative phase or period of lowered resistance following the injections of vaccine.

\section{THE SERUM TREATMENT OF INFLUENZAL MENINGITIS}

Since lumbar puncture as an aid to the diagnosis of meningitis is coming into more general use, the important fact has been revealed that the influenza bacillus is not an infrequent cause of severe, and usually fatal, seropurulent cerebrospinal meningitis. In 1911 Wollstein $^{1}$ collected 58 cases of this infection, all but 6 ending fatally, and as the bacterial diagnosis of meningitis is becoming more widely known and more commonly employed, the number of reported cases is increasing rapidly. The mortality of 90 per cent., which is exceeded only by the tuberculous and pneumococcus infections of the meninges, and the encouraging results following the use of a specific anti-influenzal serum in experimental infections in monkeys, render this subject one of great importance from the standpoint of serum therapy.

Influenzal Meningitis.-Like the acute meningeal infections in general, influenzal meningitis is more prevalent among children than among adults.

Infection of the meninges is probably always secondary to infection of the respiratory tract with virulent influenza bacilli, the route of infection being chiefly through the blood-current. Direct infection from

${ }^{1}$ Jour. Exper. Med., 1911, xiv, 73; Amer. Jour. Dis. Child., 1911, i, 42. 
the nose cannot be excluded, and should be considered a possibility. However, all or nearly all cases of spontaneous influenzal meningitis in human beings are the result of influenzal bacteremia, since the bacilli have been cultivated in large numbers from the heart's blood before and after death. The same is true of experimental influenzal meningitis in the monkey.

According to Flexner, ${ }^{1}$ the cerebrospinal fuid removed by lumbar puncture from human patients is always turbid, and deposits a yellowish or whitish sediment on standing. "As the disease advances, the fluid becomes more heavily charged with pus-cells, until toward the end, and as late as the seventh day of illness, the puncture may yield merely a viscid mass of purulent matter. The number of influenza bacilli present in the fluid is usually large, and the bacilli lie chiefly extracellular, among the pus-cells, although a variable but small number is usually found ingested by the leukocytes. In morphology the bacilli vary somewhat, and in this respect the observer may readily be deceived as to the nature of the bacteria present. While some of the fluids contain the typical, minute rods, others show quite irregular and knobbed or even filamentous bacteria that have little resemblance to the influenza bacillus as seen in recent cultivations. These bizarre or involution forms, however, are met in old and exhausted cultures; and when they are recultivated on a suitable hemoglobin medium, they yield the typical minute rods."

The cerebrospinal fluid removed from monkeys inoculated by subdural injection with virulent cultures of the influenzal bacillus resembles in all essential particulars the fluid removed from patients with spontaneous infections.

The bacteriologic diagnosis can usually be made by microscopic examination of stained smears of the fluid, but whenever possible, the diagnosis should be confirmed by cultural methods.

Anti-influenza Serum.-After having satisfactorily demonstrated experimental influenza meningitis in the monkey, Flexner and Wollstein prepared an immune serum and showed that the experimental infection could be controlled and cured by injecting the serum directly into the seat of disease by intraspinal inoculation. The immune serum was prepared by the ordinary methods, first a goat and then a horse being injected with non-virulent and finally with virulent bacilli, covering a period of many months, until their serums showed the presence of agglutinins and bacteriotropins. The serum lacked bacteriolytic prop-

1 Jour. Amer: Med. Assoc., 1913, lxi, 1872. 
erties, and did not give rise to complement fixation in dilutions greater than $1: 100$.

Following the administration of serum, the cerebrospinal fluid tends to clear up; the bacilli become fewer in number and are mostly ingested by the phagocytes; the pus-cells become less numerous, and while the bacilli may persist in the fluid for a longer period, they ultimately disappear.

Administration of Anti-influenza Serum.-The Rockefeller Institute has distributed serum throughout different parts of the country, and is prepared to furnish it to physicians upon request. Physicians, and especially pediatrists, should resort to lumbar puncture early in all suspected cases of meningitis, for only in this manner may influenzal meningitis be detected early enough to derive any possible benefit from serum treatment. The serum should be injected directly into the spinal canal by the gravity or syringe method in exactly the same manner and with the same precautions as are observed in administering serum in the treatment of epidemic meningitis. Since the disease is usually accompanied by a bacteremia, it is well to inject serum intravenously, although serum injected intraspinally soon finds its way into the blood-stream. All data, including the clinical history and the records of bacteriologic examinations of cerebrospinal fluid and blood, the amounts of serum injected; and the results obtained, should be sent to the Director of the Rockefeller Institute.

\section{THE SERUM TREATMENT OF PNEUMOCOCCUS MENINGITIS}

Meningitis is caused more frequently by the pneumococcus than by the influenza bacillus. Its mortality is certainly no less than in influenzal meningitis.

The few instances in which antipneumococcic serum has been employed have not yielded results that inspire confidence in its employment alone. As will be emphasized later, in considering the serum treatment of pneumonia, an antipneumococcus serum is at best active only against the homologous organism or organisms, the types of which have been employed in its preparation. Even when the homologous serum is used in treating experimental pneumococcus meningitis in monkeys, the fatal termination may be delayed, but is not prevented. For this reason the outlook for its successful employment alone in human infections is not encouraging. Recent investigations of Lamar $^{1}$ have ${ }^{1}$ Jour. Exper. Med., 1911, i; ibid., 380; xiv, 256; 1912, xvi, 581. 
shown, however, that mixtures of homologous antipneumococcus serum, sodium oleate, and boric acid exert a marked and decided curative influence upon a virulent experimental meningitis, and while this method has not thus far been generally applied in the treatment of the disease in humans, it is deserving of trial and offers considerable encouragement for an otherwise highly fatal infection.

Pneumococcus Meningitis.-This infection is usually secondary, and follows on pneumonia or on inflammations of serous membranes by indirect transmission by the blood or by direct infection from the nasopharynx, mastoid cells, frontal, sphenoid and ethmoid sinuses, and internal ear.

The diagnosis is usually made as the result of microscopic examination of stained smears of cerebrospinal fluid removed by lumbar puncture. Large numbers of polynuclear leukocytes with intracellular and extracellular Gram-positive diplococci, occurring in pairs or in short chains, usually indicate a pneumococcus infection. Whenever possible, the diagnosis should be confirmed by making cultures of the fluid on dextrose blood agar, and by injecting portions intraperitoneally and subcutaneously in mice.

Pneumococcus infections of the cerebral meninges have been found experimentally to be more refractory to treatment than infections of the spinal meninges, hence human infections following injuries to the head, or occurring as the result of direct extension from neighboring sinuses, are likely to be more refractory than indirect infections by way of the blood.

Serum Treatment.-Numerous investigations by Conradi, ${ }^{1}$ Korschun and Morgenroth, ${ }^{2}$ Levaditi, ${ }^{3}$ and Noguchi ${ }^{4}$ have shown that substances may be obtained directly from tissue-cells and leukocytes or after autolysis which are bactericidal and hemolytic, and, as shown by Noguchi, are largely in the nature of higher saturated fatty acids or their alkaline soaps. (For an account of their similarity to complements see Chapter XVIII.) As shown by Klotz, ${ }^{5}$ soaps occur in inflammatory foci, and the origin of the fatty acids and soaps is readily accounted for since Achaline ${ }^{6}$ has shown the presence of lipase in such foci. With the death of leukocytes in an inflammatory focus, brought about by a bacterial poison, leukocidins, or lack of nutriment, disintegration occurs, and by auto-

${ }^{1}$ Beit. z. chem. Phys. u. Path., 1902, i, 193.

${ }^{2}$ Berl. klin. Wochenschr., 1902, xxxix, 870.

${ }^{3}$ Ann. de l'Inst. Pasteur, 1903, xvii, 187.

- Biochem. Zeitschr., 1907, vi, 327.

${ }^{5}$ Jour. Exper. Med., 1905, vii, 633.

${ }^{6}$ Compt. rend. Soc. de biol., 1899, li, 568. 
lysis and lipolysis fatty acids and soaps are produced that in themselves seem to exert a destructive action upon the infecting bacteria.

With these considerations in mind, Lamar investigated the influence of soaps upon pneumococci. Solutions of 0.5 to 1 per cent. of sodium oleate were found rapidly to kill pneumococci; much weaker solutions, as, e. g., 0.1 per cent., or even 1 part of soap in 10,000 parts of water, were found to lessen their virulence, and, what is more important and significant, to render the organisms peculiarly susceptible to lysis by a homologous antipneumococcus serum.

A serious drawback to the application of these discoveries was that protein constituents of serum and exudates were found to inhibit the bacteriolytic and hemolytic action of unsaturated fatty acid soaps, as was shown by Noguchi ${ }^{1}$ and then by von Liebermann. ${ }^{2}$ The latter and von Fenyvessy ${ }^{3}$ later found that this inhibition can be prevented in the test-tube and also in the animal body by adding a minute quantity of boric acid, which prevents the union of soap and protein matter when the latter is not too greatly in excess.

The experiments of Lamar with mixtures of homologous antipneumococcus serum, sodium oleate, and boric acid in the treatment of pneumococcus meningitis in the monkey have yielded excellent results, especially when used early in the infection. These experiments have proved that sodium oleate lowers the virulence of pneumococci and renders them peculiarly and highly susceptible to solution by bacteriolysins present in the serum, and that boric acid largely prevents the inhibitory action of protein constituents upon this sensitizing action of sodium oleate.

Practical Applications. - One drawback to the use of this method in the treatment of human infections is the necessity of using an antipneumococcus serum corresponding to the organism causing the infection. In the Rockefeller Hospital a method has been worked out whereby the type of infection is quickly determined by agglutination reactions. Fortunately, the number of types of pneumococci are relatively few, and it is to be hoped that an efficient polyvalent serum will soon be made available by the Rockefeller Institute. Until this desideratum is attained, the commercial serums at present on the market may be used, with the addition of sodium oleate and boric acid.

It is highly desirable that the treatment be administered as early as possible, when the exudate is largely serous or at most seropurulent.

Loc. cit.

${ }^{2}$ Biochem. Zeitschr., 1907, iv, 25

${ }^{3}$ Zeitschr. f. Immunitätsforsch., orig., 1909, ii, 436. 
The mixture should be injected into the spinal canal after the withdrawal of the fluid by the gravity or syringe method and under bloodpressure control, as previously described. The amount injected and the number of injections depend upon the clinical condition of the patient, and in general may be administered in the same way as is antimeningitic serum.

The initial dose may be 20 c.c., and is prepared as follows:

Antipneumococcus serum (sterile) ............. 4 c.c.

5 per cent. aqueous solution of boric acid (sterile)...... . 15 c.c.

- 2 per cent. aqueous solution of sodium oleate (Kahlbaum's or Merck's) (sterile) . . . . . . . . . . . .

\section{THE SERUM TREATMENT OF OTHER LOCALIZED PNEUMOCOCCUS INFECTIONS}

Lamar has also suggested that mixtures of antipneumococcus serum, sodium oleate, and boric acid may be used in the treatment of pneumococcus pleuritis, peritonitis, or other localized infections, such as arthritis and sinusitis, where the exudate may be removed and the serum be brought into contact with the infected tissues.

\section{The Serum Treatment of PNeumonia}

Acute lobar pneumonia, with its clear-cut clinical course, unsatisfactory and difficult treatment, uncertain prognosis, and high mortality, was one of the diseases in which the earliest efforts were directed toward discovering a specific serum therapy. Since the pioneer work of the Klemperers in 1891, numerous investigators have prepared serums that have yielded either indifferent results or proved beneficial in but a limited number of cases, so that there has been no well-established form of specific therapy.

Recent investigations by Neufeld and Händel ${ }^{1}$ in Germany, and by Dochez, ${ }^{2}$ Cole $^{3}$ and Gillespie ${ }^{4}$ in the Rockefeller Institute, have disclosed several reasons for the failure of serum therapy in pneumonia, and have emphasized the importance of the following factors:

1. The serum should correspond to the type of pneumococcus causing the infection.

${ }^{1}$ Zeitschr. f. Immunitätsforsch. 1909, iii, 159; Arb. a. d. k. Gesundheitsamte, 1910, xxxiv, 169; ibid., 1910, xxxiv, 293; Berl. klin. Wochenschr., 1912, xlix, 680.

${ }^{2}$ Jour. Exper. Med., 1912, xvi, 665, 680, and 693; Jour. Amer. Med. Assoc., 1913, lxi, 727.

${ }^{3}$ Jour. Exper. Med., 1912, xvi, 644; Arch. Int. Med., 1914, xiv, 56.

${ }^{4}$ Jour. Amer. Med. Assoc., 1913, Ixi, 727; Jour. Exper. Med., 1914, xix, 28. 
2. The serum must be administered in large doses, and preferably intravenously.

3. To be most effective the treatment should be given as early as possible.

The investigators in the Rockefeller Institute have divided the pneumococci causing lobar pneumonia into four main groups, and have worked out a method for the rapid identification and classification of the particular pneumococcus that is the etiologic factor in a given infection, so that with the proper administration of the corresponding immune serum very encouraging results have been obtained in the serum treatment of pneumonia. These researches are of importance not only in this connection, but also from the fact that they may have disclosed the reasons for failure in the treatment of streptococcus and other infections, and that similar studies in these conditions may insure for serum therapy a definite and valuable rôle in the treatment of disease.

The Nature of Lobar Pneumonia.-The frequency with which the Diplococcus pneumonice is found in the local lesion and in severe cases in the blood-stream of pneumonia patients, and the more recent experimental studies of Wadsworth, ${ }^{1}$ Meltzer, ${ }^{2}$ Wollstein and Meltzer, ${ }^{2}$ Winternitz, Kline and Hirschfelder, ${ }^{3}$ leave little doubt regarding the etiologic relationship of this microörganism to lobar pneumonia. Much still remains to be learned, however, regarding the method of infection and the nature of the resulting disease. While pneumococci are to be found living in the upper air-passages as harmless parasites, it is probable that those causing infection differ inherently as regards adaptation or virulence for man. In addition, it is likely that general resistance is lowered in some more or less peculiar manner, and experimental studies in animals, as well as the course of the disease in man, suggest most strongly that local changes in the respiratory tract may precede the infection, so that a combination of factors, such as the virulence of the organisms and the diminished general and local resistance, plays a part in the production of lobar pneumonia.

In whatever manner produced, the disease is finally to be regarded as a general infection, with localization of the process in the lung. While pneumococci may be found in the blood of the most severe cases, the general symptoms are apparently due to intoxication with a poison or toxin derived primarily from the pneumococci, and secondarily from the

${ }^{1}$ Amer. Jour. Med. Sci., 1904, exxvii, 851. 2 Jour. Exper. Med., 1912, xv, 133.

${ }^{3}$ Jour. Exper. Med., 1913, xvii, 353, RWR; ibid., 1913, xviii, 548.

${ }^{4}$ Jour. Exper. Med., 1912, xvii, 657; ibid., 1913, xviii, 50. 
exudate in the local lesions. The studies of Rowntree, ${ }^{1}$ Medigreceanu, ${ }^{2}$ and Peabody, ${ }^{3}$ showing chlorin retention; of Peabody, ${ }^{4}$ showing progressive loss in the oxygen-combining power of the hemoglobin, due to the formation of methemoglobin; of Medigreceanu, ${ }^{5}$ showing a deficiency of oxydase or lessened power of the tissues to carry on proper oxidation, and of Neufeld and Dold, ${ }^{6}$ Rosenow, ${ }^{7}$ Cole, ${ }^{8}$ Jobling and Strouse, ${ }^{9}$ indicating the presence of endotoxins within the pneumococci-all these support the view that in pneumonia there is well-marked intoxication, and this, in addition to the effects of the local pulmonary consolidation on the heart, respiration, and nervous system, constitute the main features of the infection.

Regarding the mechanism of recovery from pneumonia, there is little definite information. The recent studies of Neufeld, Dochez, and Clough indicate that antibodies are produced at or about the time of the crisis, and that these are probably responsible for the destruction of the bacteria in the circulating blood, and, to a greater extent, in the local lesion. In the resolution of the local lesion it is probable that ferments play an important part. That resolution does not occur earlier may be due to the overbalancing of the leukocytic ferments by the antiferments of the serum, and the lytic ferments become active only when they reach a point of excess over the antiferments, causing a solution of the fibrin, relieving tension, and affording an outlet for the exudate. According to Vaughan, the pneumococci may be considered as furnishing a ferment that brings about the production of a specific antiferment, capable of reacting upon its substratum, the ferment and the new bacterial tissue, and causing its destruction by a process of solution. Pneumococci in the resolving lesion are probably destroyed by leukocidins released through disintegration of leukocytes, by fatty acids, and probably by antibacterial substances in the blood.

While it is true that immunity does not usually follow an attack of pneumonia, and, indeed, the patient is apparently hypersusceptible, it has been found experimentally that the antibodies are highly specific for the particular organism causing an infection. Reinfection is, therefore, possible with an organism belonging to another group, and lia-

${ }^{1}$ Bull. Johns Hopkins Hosp., 1908, xix, 367.

${ }^{3}$ Jour. Exper. Med., 1913, xvii, 71.

5 Jour. Exper. Med., 1914, xix, 309.

- Berl. klin. Wochenschr., 1911, xlviii, 1069.

${ }^{7}$ Jour. Infec. Dis., 1911, ix, 190.

๑ Jour. Exper. Med., 1913, xviii, 597.
2 Jour. Exper. Med., 1911, xiv, 289.

${ }^{4}$ Jour. Exper. Med., 1913, xviii, 7.

${ }^{8}$ Jour. Exper. Medl., 1912, xvi, 644. 
bility to reinfection may be increased because of lowered local and general resistance due to the previous attack.

Antipneumococcus Serum.-The indications of specific serum therapy, are, therefore, mainly twofold: first, to destroy any pneumococci present in the blood and in the local lesion; or if the latter is impossible because of mechanical obstacles that interfere with the circulation and prevent access of the antibodies to the cocci, to at least prevent extension of the lesion by preventing the multiplication of organisms at its margin; second, to neutralize the toxins produced during the course of the disease.

It would, of course, be highly desirable to have at our command a serum that would cause solution of the local exudate and bring about a crisis and a cure. It is hardly reasonable to expect, however, that a serum can be produced that will contain digestants for fibrin and leukocytes. The local lesion is most likely to be harmful because of the toxic substances that emanate from it, and not because so large an area of lung is temporarily incapacitated and the heart embarrassed. A serum that will prevent general bacteremia, limit the extension of the local lesion, and neutralize the toxins while nature is preparing to react upon the exudate with a ferment, is probably fulfilling all that may be expected of a specific serum therapy.

Groups of Pneumococci.-Neufeld and Händel have shown that an immune serum produced by the injection of a given variety of pneumococci into an animal was not effective against all forms of pneumococci. In the Rockefeller Hospital a serum, known as Serum 1, prepared by immunizing a horse with a culture obtained from Neufeld, was found to protect against only about one-half the types of pneumococci (Group 1) studied. By immunizing rabbits to each of the types that were not acted upon by Serum 1, and testing the immune serum against all strains by cross-agglutination and by cross-protection experiments, it was found that a number of the serums possessed the same properties, thus indicating that their respective cultures belonged to the same general group (Group 2). By immunizing a horse with one of these, Serum 2 was produced. In Group 3 are placed all the organisms of the so-called Pneumococcus mucosus type. In Group 4 are included all the varieties of pneumococci against which Serums 1 and 2 are not effective, and which, from their other properties, do not belong in Group 3. Animals may readily be immunized to any member of this Group 4, and the serum of the immunized animal is protective against the strain used for immunization, but in no instance has this serum been found effective against any other member of this group or against the organisms of the other groups. While no cultural or morphologic differences between the members of Group 1, 2, and 4 exist, it has been found possible to group them by the agglutination reaction in exactly the same manner as by protection experiments. Of 24 strains studied in the Rockefeller Hospital, 47 per cent. belonged to Group 1, 18 per cent. to Group 2, 13 per cent. to Group 3, and 22 per cent. to Group 4.

Determining the Type of Pneumococcus.-For this purpose, Cole has given the following method: "When a patient with pneumonia is admitted to the hospital, a 
culture is immediately made from the blood and also one from a portion of sputum coughed up from the lung, or, when this is not obtainable, a culture is made directly from the lung by the insertion of a needle. This procedure seems to be without danger. When there are large numbers of organisms in the sputum, a culture may be obtained most rapidly by injecting the washed sputum into the abdominal cavity of a mouse. After four or five hours the peritoneal cavity may be washed out with salt solution and the cells thrown down in the centrifuge; a suspension of the organisms is thus obtained. In whatever way the culture is obtained, the agglutination test is at once applied. If the organism fails to agglutinate with either Serum 1 or Serum 2 , it is, of course, useless to undertake serum treatment. If, however, one of the serums agglutinates the organism, treatment may be commenced at once with the appropriate one."

Preparation of Antipneumococcus Serum.-Horses are immunized with dead and then with virulent living cultures. In view of the fact that the serum should possess some antitoxic value, it is desirable that the animals be immunized also with autolysates. The whole process may be conducted after the method described for the production of antimeningococcus serum.

In the Rockefeller Institute different horses are immunized with strains belonging to Groups 1 and 2. It would appear possible to produce a potent polyvalent serum, and this is very much to be desired, especially if further studies continue to show that 65 per cent. of infections are caused by organisms belonging to these two groups.

Kolle, by immunizing horses with cultures secured from pneumonia patients, produces an antipneumococcic serum. These cultures are grown in broth for fortyeight hours, heated to $60^{\circ} \mathrm{C}$., and 5 c.c. injected intravenously into a horse. The dose is increased each week until 120 c.c. are given at one time. Then 5 c.c. of living culture is injected, and the doses increased in a similar manner until 120 c.c. are given at one time. About six months are consumed in the process of immunization, and two weeks after the last injection has been given the serum is tested.

Standardization of Antipneumococcus Serum.-As previously mentioned, there is at present no accurate method for standardizing an antibacterial serum. It is possible, however, to obtain some measure of its protective and curative power by employing various tests

1. Protective Value.-The lethal dose of a living pneumococcus culture for mice is determined, and from 10 to 100 times this amount of culture is mixed with decreasing doses of immune serum and the mixtures injected subcutaneously or intraperitoneally into a series of mice in order to determine the dose of serum that will protect. Dochez has found that when these mixtures are injected at once and in the same place, the serum will obey the law of multiple proportions up to a certain limit.

Merck's antipneumococcus serum is so standardized that 0.01 c.c. injected subcutaneously protects a mouse inoculated intraperitoneally twenty-four hours later with from 10 to 100 times the lethal dose of a living virulent culture. This is known as a normal serum, 1 c.c. containing an immunity unit (I. U.). The serum is marketed in vials containing from 20 to 40 c.c. Kolle regards as satisfactory a serum that, in doses of 0.001 c.c. and less, will protect mice.

2. Bacteriotropic Value.-Neufeld lays considerable stress upon this point. The technic of this titration has been described elsewhere.

3. Complement-fixation and Agglutination Tests.-While these tests are frequently sharply cut, and while they serve as a measure for record in the laboratory, they do not necessarily indicate the therapeutic value of the serum. 
Action of Antipneumococcus Serum.-The curative and protective value of this serum depend mainly upon bacteriolysins, bacteriotropins, and antitoxins. The first are readily demonstrated in protection experiments and also in pneumonic patients when pneumococci in the blood-stream are destroyed. Bacteriotropins may likewise be demonstrated experimentally and in the blood of patients if the corresponding organism is used in the tests (Neufeld, Strouse). The antitoxic properties are shown clinically and also in vitro by neutralization of the hemotoxic poison obtained by dissolving pneumococci in bile.

Administration of Antipneumococcus Serum.-To obtain the best results, the serum should be given as early as possible and intravenously. In young children, when the giving of an intravenous injection is quite difficult or impossible, the muscles of the buttocks should be substituted. Certainly small doses given subcutaneously are almost devoid of effect. The procedure in use in the Rockefeller Institute consists in injecting 0.5 c.c. of serum subcutaneously to discover if hypersensitiveness exists and to produce anti-anaphylaxis. As soon as the type of organism has been determined, from 50 to 100 c.c. of the serum, diluted one-half with salt solution, are injected intravenously. The condition of the patient serves as a guide in the later treatment. Usually the serum is not administered oftener than once every twelve hours.

It has been shown experimentally that, in the presence of a maximum degree of infection, no amount of serum, however large, is effective. This suggests that the body must furnish a second substance to act with the antibodies in the serum, and indicates the early administration of serum before the infection has reached too extreme a grade. I would also suggest that the body may be deficient in bacteriolytic complements, and that the effect of a serum may be enhanced by adding fresh sterile guinea-pig serum-say 5 c.c. to each 100 c.c. of immune serum-just prior to administration.

Results in the Serum Treatment of Pneumonia.-It is hardly necessary to review the numerous reports that have been made in past years, because in most instances the serum was administered subcutaneously and in too small doses to be of value, even granting that it contained antibodies for the particular infection. Of 23 patients, all seriously ill, treated in the Rockefeller Institute during the past year, the results were as follows: Of 15 cases due to pneumococcus 1 , all recovered but one-a mortality of 6.6 . per cent., as compared with the mortality of 24 per cent. among 34 patients not receiving serum treatment; of 8 cases due to Type 2 , all recovered but two, one of these refusing to con- 
tinue the treatment-a mortality of 25 per cent. as compared to 61 per cent. among 13 patients not receiving serum.

Aside from this decided influence upon mortality, the general effects of the serum were good. In 10 cases pneumococci were isolated from the blood before the treatment was begun. In all these patients the blood had become sterile after the first treatment. Following the injection of serum all the patients seemed to feel better, and in a number of them there was an apparent lessening in the degree of intoxication. While in no case was one injection sufficient to bring about a crisis, in all except the fatal cases the serum had apparently an ultimate favorable effect, lowering the temperature and shortening the course of the disease.

\section{THE SERUM TREATMENT OF STREPTOCOCCUS INFECTIONS}

The acute character of streptococcus infections and their relative frequency and severity have made them the subject of numerous efforts on the part of various investigators toward developing an efficient serum therapy. To Marmorek belongs the credit of first attempting, in 1895, to prepare a curative serum on a large scale. Since then Aronson, Tavel, Krumbein, Moser, Meyer-Ruppel, Menzer, and others have prepared immune serums with various cultures and according to various methods. While many antistreptococcus serums will show undoubted protective value, especially against their homologous cultures as tested in experimental animals, the general opinion regarding their curative value in streptococcus infections of man have been conflicting and as a rule unfavorable. Occasionally the rapid improvement of a patient following an injection of the serum would indicate that it has proved beneficial, and the same is occasionally true of a particular group of infections treated with a specially prepared serum. The tendency of acute streptococcus infections to end spontaneously by crisis must, however, be borne in mind, and the good result observed in individual cases may be coincident with, rather than the result of, the administration of the serum.

Several causes for the failure of antistreptococcus serum therapy are now understood, and if these can be eliminated, the value of this form of therapy will be greatly augmented.

1. The serum should be given in large doses, and by intramuscular and intravenous injection. In a true streptococcic infection the cocci are likely at some time to be found in the blood-stream, and an attempt to destroy these organisms or to limit a local infection by injecting 10 c.c. 
of serum in the subcutaneous tissues is almost sure to result in failure. As in the serum treatment of pneumonia, at least 100 c.c. of serum should be given intravenously and the dose repeated if necessary.

2. The serum should be used as early in the disease as possible, instead of waiting until the patient has become moribund. In puerperal sepsis and scarlet fever, for instance, the question of serum therapy should be considered early, for when properly administered, the serum will at least do no harm and may prove efficacious. In order to determine the value of the serum a bacteriologic diagnosis should always be attempted, especially by means of blood cultures obtained by placing from 2 to 5 c.c. of blood in a flask containing at least 100 c.c. of dextrose broth just prior to injecting the serum.

3. The serum should be polyvalent. Marmorek maintains that all streptococci are alike, and he has, accordingly, prepared his serum from a single highly virulent strain. Other investigators question this assertion, and at present the consensus of opinion is agreed that streptococci from different infections, such as puerperal sepsis, scarlet fever, ulcerative endocarditis, and erysipelas, exhibit certain immunologic differences, even though their biologic and morphologic characters are quite similar. Thus far no adequate methods for differentiating between these organisms have been discovered, but it is likely that future researches will show that streptococci from different infections, and even from cases of scarlet fever, possess different immunologic characters similar to the variations observed among the pneumococci causing lobar pneumonia. If this is found to be true, under these conditions, a similar serum treatment, while complicated, is likely to prove valuable in the treatment of streptococcus infection. For the treatment of streptococcus infection in scarlet fever the serum should be prepared of numerous strains isolated from patients having this disease. What has just been said is also true of the other three infections so frequently streptococcal, namely, puerperal sepsis, phlegmonous cellulitis, and ulcerative endocarditis. If not these four, at least two antistreptococcus serums should be available: one for scarlet fever and the other for other infections; both, and especially the latter, should be prepared by immunizing horses with a large number of various strains.

Mode of Action of Antistreptococcus Serum.-Virulent streptococci exert a powerful negative chemotactic influence upon leukocytes, repelling them and effectively resisting phagocytosis for varying periods of time. The early researches of Bordet showed that antistreptococcus serum neutralizes this influence and promotes phagocytosis. Since 
then numerous investigators have supported Bordet's findings, so that it may be accepted as true that one of the chief antibodies in antistreptococcic serum is of the nature of a bacteriotropin or immune opsonin. A potent serum also contains an antitoxin, as may be shown experimentally by neutralization of the hemotoxic poison of streptococci, and also clinically, when the rapid subsidence of fever and general improvement of the patient are probably due, in part, to neutralization of streptococcal toxins. Thus far the presence of bacteriolysins has not been definitely proved, although they may be present and operative in vivo. I have found that antistreptococcus serum contains an antibody capable of fixing complement with streptococcus antigens. ${ }^{1}$ It may be stated, therefore, that the action of antistreptococcus serum is dependent primarily upon bacteriotropins, and secondarily upon antitoxins and, possibly, bacteriolysins.

Preparation of Antistreptococcus Serum.--Some differences of opinion have been expressed regarding the advisability of passing cultures that are being used for purposes of immunization through a lower animal in order to increase their virulence. For example, the unsatisfactory results that have followed the use of Marmorek's serum have been ascribed not only to the fact that it is monovalent, but also to possible alteration of the strain in its biologic characteristics by animal passage, so that its virulence for the human being was diminished or lost, and, accordingly, while the antiserum is protective for the animals through which the passage has been conducted, it is inactive for the human being. Tavel, Krumbein, and Paltauf have prepared polyvalent serums with different strains from human infections without animal passage. Menzer has prepared a serum with strains of cocci derived from acute rheumatic fever, and Moser with strains obtained from scarlet fever, which have also not been passed through animals. Aronson has attempted a combined procedure, making use of passed and unpassed cultures conjointly, and this appears to be the method of choice. In other words, those who prepare antistreptococcus serums should use as many fresh strains as possible, and in several of the older cultures the virulence should be increased from time to time by passage through animals.

In preparing the serum young and healthy horses should be used. The injections should first commence of dead cultures given subcutaneously, then of autolysates, and finally of living cultures administered intravenously. Occasionally severe local and general reactions are observed, and the whole procedure should be conducted under careful supervision.

First Method.-Cultures are grown on a solid medium, and an emulsion and autolysate prepared as described for immunizing with meningococci. Begin by injecting subcutaneously 5 c.c. of emulsion heated to $60^{\circ} \mathrm{C}$. for an hour, and increasing the dose each week by 5 c.c. until 100 c.c. are given at one time. If the reactions are mild (general and local), the doses may be increased more rapidly. Then begin with 2 c.c. of living culture and increase the dose each week. When a dose of 10 c.c. is reached, inject with autolysate and living cultures alternately, gradually increasing the dose until a dose of 50 c.c. is reached. Living cultures are then given intravenously and the dose rapidly increased until 100 c.c. and more are given at

${ }^{1}$ Arch. of Int. Med., 1912, ix, 220. 
one time. Intravenous injections are not infrequently tolerated better than subcutaneous injections. The horses may be bled several times during the course of immunization and their serums tested. When the serum is to be used therapeutically, the animals should not be bled in less than from ten to fourteen days after the last injection was given. In view of the large doses required, a concentrated serum is advisable (Heinemann and Gatewood ${ }^{1}$ ). Since trikresol has an inhibiting influence on phagocytosis (Weaver and Tunnicliff ${ }^{2}$ ), the minimal quantity ( 0.2 per cent. or less) should be used, or preferably no preservative at all.

Second Method.-Kolle prepares a polyvalent serum with cultures derived from cases of erysipelas, puerperal sepsis, scarlet fever, etc. Their virulence is increased from time to time by passage through rabbits.

Horses are used for immunization purposes and all injections are given intravenously at intervals of a week. Cultures are grown on test-tubes containing a solid medium, and immunization is started with half a culture, heated. The dose is increased each week with an additional culture until it equals 16 cultures. Then living and killed cultures are mixed, giving in one week 2 living and 14 killed cultures and so on until 10 living and 6 killed cultures are given at a single dose. The horses are bled two weeks after the last dose is administered.

Standardization of Antistreptococcus Serum.-There is at present no single satisfactory method for standardizing these serums, although a satisfactory method is a desideratum for testing serums placed on the market. In order to obtain an approximate idea as to the value of a serum, the following tests may be employed:

1. Protective Value.-At the Serum Institute in Vienna a passed culture (one used in the process of immunization) is selected, and that dose which will kill a mouse at the expiration of or just preceding the end of four days is regarded as a single lethal dose. In testing an antiserum 10 times this quantity of culture is used with decreasing doses of immune serum injected twenty-four hours previously or simultaneously. A normal serum is one of which 0.01 c.c. will afford protection, and 1 c.c. of such a serum is said to be one immunity unit, $i$. e., it affords protection against 1000 lethal doses of culture.

2. Bacteriotropic Value.-The technic of Wright or Neufeld may be employed with a virulent culture and human leukocytes. Weaver and Tunnicliff have observed better results when using one part of immune serum reactivated with nine parts of fresh guinea-pig serum.

3. Complement-fixation Tests.- These tests may be employed with the bacterial emulsion or autolysate used in immunization as the antigen.

The serum should be kept in a cool, dark place. After a few months it loses some of its protective value, and much of it on the market is worthless.

\section{Administration of Antistreptococcus Serum.-It must be emphasized} here that, in order to obtain the best results, antistreptococcus serum must be given intravenously. In an adult patient with a severe general infection from 30 to 100 c.c. of serum, diluted with an equal amount of sterile normal salt solution, may be given in one dose. If improvement follows, subsequent doses should be given subcutaneously or intramuscularly in order to prolong the action of the serum. If no improvement follows in from twelve to twenty-four hours, or if an acute exacerbation sets in, a second dose should be given intravenously. Since the various manu-

${ }^{1}$ Jour. Infect. Diseases, 1912, x, No. 3. $\quad{ }^{2}$ Jour. Infect. Diseases, 1911, ix, 130. 
facturers use different cultures in the preparation of these serums, it would be well to use a different brand of serum if the first does not exert a beneficial effect. In patients with severe infections the activity of the serum may be enhanced by adding, just before injection, 5 c.c. of fresh sterile guinea-pig serum to each 50 c.c. of the immune serum.

In the treatment of localized streptococcus infections, such as meningitis and sinusitis, it may be well to mix antistreptococcus serum with sodium oleate and boric acid, as previously described.

Value of Antistreptococcus Serum.-Although the serum has been in use for almost twenty years, the true exact value of the remedy has not as yet been estimated. It may be stated that a carefully prepared and properly administered serum will do no harm and may do good, and that its use should form a part of the treatment of severe streptococcal infections.

In some cases of wound infections with severe cellulitis and septicemia the serum may at times exert a most pronounced and happy effect. In other cases, and especially in those in whom the cocci are found in the blood, repeated injections may be of no value.

In severe anginose or malignant scarlet fever large doses of serum from horses especially immunized with strains of streptococci from scarlet-fever patients have, on the whole, yielded favorable results. Not all cases of severe scarlet fever, however, are due to secondary streptococcal infections: those patients who are overwhelmed and prostrated at the very outset are probably intoxicated with the true scarlatinal virus, whatever that may be, and such cases are not likely to be benefited by serum treatment. The patients most likely to improve under serum therapy are those who become severely ill after the onset of the disease and the appearance of the eruption.

In puerperal sepsis and endocarditis of streptococcal origin the results of serum treatment have not been uniform, but are generally unfavorable. If serum is administered at all, it should be given early, in large doses, and intravenously. Not all cases of puerperal sepsis are streptococcic, and while the physician may not be justified in withholding serum until a bacteriologic diagnosis has been made, this factor must be considered when estimating the value of a serum.

In erysipelas the results have been very indifferent, and the same may be said of bronchopneumonia, laryngeal diphtheria, smallpox, and tuberculosis. 


\section{THE SERUM TREATMENT OF GONOCOCCAL INFECTIONS}

In 1906 Torrey and Rogers ${ }^{1}$ described the preparation of an antigonococcus serum and advocated its use in the treatment of gonococcal infections, and especially of its various complications and metastases. In the following year these observers reported ${ }^{2}$ favorably upon the results of serum treatment in gonorrheal arthritis, and to a lesser extent in infections of the genito-urinary organs. Uhle and Mackinney ${ }^{3}$ used the serum in the treatment of 23 cases of gonococcal infection, and found it beneficial in three cases of gonorrheal arthritis and in one of myositis, whereas in epididymitis and urethritis no appreciable effects were observed. Herbst and Belfield, ${ }^{4}$ Schmidt, ${ }^{5}$ and Swinburne ${ }^{6}$ agree as to the value of the serum in gonorrheal arthritis, whereas in other complications they secured somewhat conflicting results. In all instances the serum was used in relatively small doses, namely, 2 c.c., injected subcutaneously each day or every other day, the number of injections depending on the clinical condition of the patient.

Recently Corbus ${ }^{7}$ has reported more favorable results in the treatment of 24 cases of gonococcus infection by using larger doses of serumfrom 36 to 45 c.c.-injected intramuscularly. This observer advocates the use of the complement-fixation test as a reliable guide to the administration of the serum: the more intense the reaction, the more efficient will the serum prove; if the reaction is negative, the serum should not be used.

Antigonococcus serum is advocated in the treatment of the following conditions:

1. In gonococcus bacteremia.

2. In those infections arising by extension through the lymphatics or the circulatory system, as, for example, arthritis, iritis, endocarditis, and pleuritis.

3. In those acute infections arising by direct extension from the urethra, such as acute prostatitis and epididymitis; acute orchitis and probably cystitis in the male; and acute salpingitis in the female.

The serum has not proved of value in the treatment of gonorrheal conjunctivitis, although it would appear advisable to employ it in large doses administered intravenously or intramuscularly.

${ }^{1}$ Jour. Amer. Med. Assoc., 1906, xlvi, 261, 273.

2 Jour. Amer. Med. Assoc., 1907, xlix, 918.

${ }^{3}$ Jour. Amer. Med. Assoc., 1908, li, 105. ${ }^{4}$ Illinois Med. Jour., 1908, xiii, 689.

5 Therap. Gaz., 1909, xxvi, 609.

${ }^{6}$ Trans. Amer. Urol. Assoc., 1909, iii, 170.

${ }^{7}$ Jour. Amer. Med. Assoc., 1914, lxii, 1462. 
Preparation of Antigonococcus Serum.-Torrey's serum is prepared by immunizing rams with gradually increasing intraperitoneal doses of dead, and later of living, cultures of gonococci. Larger amounts of serum may be secured by immunizing horses according to the methods described for the preparation of meningococcus and streptococcus immune serums, and in view of the larger doses now advocated, this is advisable. This serum has been successfully concentrated in the same manner as is diphtheria antitoxin.

Mode of Action of Antigonococcus Serum.-According to Torrey, this serum is largely bacteriolytic in nature. The presence of antitoxins has not been demonstrated; small amounts of bacteriotropins are present, so that a potent serum probably destroys the cocci by extracellular lysis and phagocytosis.

Administration of Antigonococcus Serum.-The amount of serum used and the method of inoculation are very important factors in the success or failure of the treatment. If the serum is used at all, it should be given in large doses. The original method of giving 2 c.c. subcutaneously has been found inadequate in most instances.

In acute gonococcal metastases in the joints or in the endocardium or other serous membrane, from 30 to 50 c.c of serum should be injected intravenously, or at least intramuscularly. When gonococci are found in the blood, or when the patient is profoundly septic, from 50 to 100 c.c. of serum should be given intravenously. In epididymitis, orchitis, and other local complications from 30 to 50 c.c. of serum should be given intramuscularly or intravenously. Other forms of treatment should be instituted simultaneously. If necessary, the serum injections should be repeated in twenty-four hours, and if a good primary effect follows an intravenous injection, it may be prolonged by one or more, subcutaneous or intramuscular injections on subsequent days

\section{THE SERUM TREATMENT OF STAPHYLOCOCCUS INFECTIONS}

While several attempts have been made to treat staphylococcus infections with an immune serum, the investigations have been too few and too brief to warrant a statement in regard to the value of serum therapy in these infections. Thomas ${ }^{1}$ prepared a serum by immunizing a ram with 18 different strains of Staphylococcus pyogenes aureus, and reported good results in the treatment of 28 cases of furunculosis and carbuncles.

It is probable that, with more extensive use of antistaphylococcus serum, its value will be proved, especially in the treatment of severe

\footnotetext{
${ }^{1}$ Jour. Amer. Med. Assoc., 1913, lx, 1070.
} 
furunculosis of infants as well as of adults, when the low general vitality of the patient contraindicates the use of a bacterial vaccine. With extended use it may also be found to be of benefit in staphylococcus bacteremia, osteomyelitis, arthritis, carbuncle, and other severe infections. Following recovery or relief from an acute infection it would seem to be wise actively to immunize the patient with a vaccine, or serum and vaccine may be used conjointly.

The activity of the serum is probably largely dependent upon the presence of bacteriotropins and antitoxins, the former promoting phagocytosis and the latter neutralizing the staphylolysins or hemotoxic poisons produced by staphylococci.

\section{THE SERUM TREATMENT OF ANTHRAX}

Sclavo, ${ }^{1}$ Mendez, ${ }^{2}$ and Deutsch ${ }^{3}$ have prepared anti-anthrax serums by immunizing sheep, goats, asses, and horses with virulent cultures of anthrax bacilli. In the treatment of human infections, only Sclavo's serum has been used, the others being used in the treatment of anthrax among the lower animals. An anthrax serum prepared by American manufacturers is also on the market.

Sclavo's serum has been shown to possess protective and therapeutic properties in experimental infections, and favorable results have been recorded in cases of human anthrax. According to Sclavo's statistics, the serum has reduced the average mortality of anthrax from 24 per cent. to 5.3 per cent. Cigognani, Legge, Lockwood and Andrewes, Stretton and Mitchell, and others have reported favorably as to the value of the serum.

It is somewhat difficult to prepare a potent serum, and horses should be immunized with a large number of strains from human infections over a long period of time. The serum is probably largely bacteriotropic and bacteriolytic in nature.

Administration of Anti-anthrax Serum.--In the Philadelphia Hospital for Contagious Diseases a number of anthrax cases are treated every year. Whenever blood-cultures have revealed the presence of the bacilli in the circulation, the patient has usually succumbed to the disease in spite of serum treatment; the doses employed-10 to 20 c.c.-may, however, have been too small. I would recommend the following course of treatment in these cases:

${ }^{1}$ Berl. klin. Wochenschr., 1901, 18 and 19, 481, 520.

${ }^{2}$ Centralbl. f. Bakt., 1899, xxvi, Nos. 21 and 22.

${ }^{3}$ Impfstoffe u. Sera, Leipzig, 1903. 
1. If the lesion is relatively small and accompanied by but slight glandular involvement, with little or no evidences of toxemia, a blood culture should first be made by placing from 2 to 5 c.c. of blood in a flask of neutral bouillon, followed by an intravenous or intramuscular injection of from 20 to 50 c.c. of serum. The object sought is to introduce serum before the lesion is handled, and the blood culture is the best indication for subsequent injections of serum and serves as a guide to prognosis

2. The lesion should then be excised, leaving a wide margin so as to include infected lymphatic channels. It should be handled as little as possible. The wound is then dusted lightly with powdered calomel and next heavily powdered with ipecac. Edema soon subsides, and the wound usually heals rapidly, with surprisingly little scar-tissue formation. If the edema does not subside and the infection is spreading at the margins of the wound, more tissue should be excised or multiple local injections with phenol and anti-anthrax serum should be made.

3. The blood culture may be examined within twenty-four hours. If anthrax bacilli are present, from 100 to 200 c.c. of serum should be given intravenously, the injection being repeated in twenty-four hours. Daily blood cultures should be made, and the serum injections continued until the blood becomes sterile. Salvarsan may also be injected intravenously. In our experience, cases with sterile blood cultures have invariably recovered.

4. In internal anthrax all the physician can do is to administer large doses of the serum intravenously. Salvarsan may also be tried. (See Chapter on Chemotherapy.)

\section{THE SERUM TREATMENT OF TYPHOID FEVER}

The serum of Chantemesse is the only serum that has been used on a large scale in the treatment of typhoid fever in man.

The serum is derived from horses that have been immunized for several years with bouillon filtrates containing typhoid toxin, chiefly endotoxin, and with typhoid bacilli. Kraus and von Stenitzer, Meyer, Bergell, and Aronson use bouillon filtrates and aqueous bacterial extracts; Besredka injects dead and then living cultures; MacFadyen uses an endotoxin secured by breaking up cultures frozen at very low temperature. It would appear that a serum should be bacteriolytic and endotoxic, and this probably is best secured by prolonged intravenous immunization of horses with a large number of dead cultures and then with autolysates and living cultures conjointly. 
According to Chantemesse, the subcutaneous injection of a few drops of his serum produces leukocytosis and raises the opsonic index of the patient's serum. He emphasizes the fact that the serum should be given early,- - before the seventh day,-and reports that, by its use, the mortality has been reduced from 17 per cent. to 4.3 per cent. These results have not been generally confirmed, and the subject is still sub judice.

\section{THE SERUM TREATMENT OF PLAGUE}

Of the various antipest serums that have been prepared, that of Yersin is probably best known. This serum, it would appear, possesses some prophylactic and therapeutic value. For purposes of prophylaxis from 10 to 20 c.c. of serum may be injected subcutaneously or intravenously; the period of protection is short, averaging from ten to fourteen days. Combined active and passive immunization, effected by means of injections of a pest vaccine and an antipest serum, will probably exert a protective action of several months' duration, and should be used by physicians, nurses, and others during epidemics of plague. When used for therapeutic purposes, the results have been quite variable. If serum is used in the treatment of plague, it should be given as early as possible, in the form of intravenous or intramuscular injections of from 50 to 150 c.c., if this amount is available. Injections should be continued at twelve- to twenty-four-hour intervals for two or more days until suppuration has been controlled and the disease shows signs of abating. The Plague Commission of India has not issued very favorable reports upon the use of either this serum or that of Lustig.

(a) In addition to Yersin's serum, which is prepared at the Pasteur Institute of Paris by immunizing horses with dead and then with living cultures of pest bacilli, other serums have been prepared. For example:

(b) Kolle immunizes horses with intravenous injections of heat-killed cultures, beginning with $1 / 4$ agar slant culture and doubling the dose each week until 15 cultures are given at one time. The horses are bled fourteen days after the last dose is given.

(c) Lustig immunizes horses with pest-nucleoproteins, obtained by breaking up the bacilli with 1 per cent. of potassium hydroxid and precipitating the proteins with acetic acid. These are then suspended in sterile normal salt solution, as in the preparation of Lustig's vaccine.

(d) Terni-Bandi immunizes donkeys and sheep with aggressins obtained by intraperitoneal injection of guinea-pigs with pest bacilli.

(e) Markl immunizes horses with filtrates of old pest bouillon cultures. He believes that the value of pest serum is largely dependent upon antitoxins.

The serums are usually tested by injecting mice with lethal doses of pest culture and decreasing doses of antiserum. The agglutinin content may also be measured: 
Whenever cultures are used in immunization, the serum should always be cultured carefully and tested by animal inoculation to guard against the possibility of living bacilli being present.

\section{THE SERUM TREATMENT OF CHOLERA}

In some respects cholera would seem to be due mainly to a toxin elaborated by the bacilli in the intestinal tract of infected persons, similar to the action of the toxin of the Kruse-Shiga type of dysentery bacillus. Various attempts have been made to prepare an efficient anticholera serum, but the only one that has yielded encouraging results in experimental infections as well as in cholera of human beings is that prepared by Kraus. This serum is prepared by immunizing horses with a true toxin derived from a cholera-like vibrio isolated by Gottschlich from the intestinal contents of pilgrims dying at El Tor from a dysentery or cholera-like infection. According to Kraus, this antiserum is largely antitoxic, and serves to neutralize the toxin of true cholera more effectively than does the antiserum resulting from immunization with cholera cultures. A serum that is antitoxic and is obtained by prolonged immunization of horses by intravenous injections with dead cultures of cholera, and later with living cultures, bacterial extracts, and filtrates of old bouillon cultures conjointly, would seem to be a desideratum.

Reports from Russia, where Kraus' and Kolle's serums have been employed, indicate that a reduction of about 10 to 20 per cent. in the mortality has been accomplished. Jegunoff's method of administering the serum seems quite rational, and consists in making intravenous injections of 140 c.c. of serum with 500 to 700 c.c. of sterile salt solution, followed by a similar or slightly lower dosage in from six to twenty-four hours after the first dose. The intravenous administration of salt solution alone has proved of value in the treatment of cholera, and it is reasonable to assume that a potent serum may be of service by neutralizing toxins, destroying the bacilli, and at least furnishing additional fluids for the depleted tissues and circulation.

There are no available statistics as regards the prophylactic value of anticholera serum, but combined active and passive immunization by means of a subcutaneous injection of from 10 to 20 c.c. of serum, followed by three doses of vaccine, would appear to be a rational procedure in the presence of an epidemic or of a threatened epidemic of cholera. 


\section{THE SERUM TREATMENT OF TUBERCULOSIS}

While numerous efforts have been made to prepare an efficient antituberculosis serum, only two-those of Maragliano and Marmorekhave been studied and are familiar.

Maragliano's serum is prepared by immunizing horses for from four to six months with a mixture of a toxin prepared by the filtration of cultures only a few days old and concentrated in vacuo at a temperature of $30^{\circ} \mathrm{C}$., mixed with that obtained by aqueous extraction of killed virulent cultures and concentrated by heating on a water-bath at $100^{\circ} \mathrm{C}$. for three or four days. Maragliano assumes that the antiserum possesses antitoxic, bactericidal, and agglutinating properties. One cubic centimeter of this serum is injected every other day for one and a half months. The favorable action of the serum is reported on, especially by Mircoli and other Italian physicians, but in Germany and France proof of its value could not be established.

Marmorek's serum is now prepared by immunization of horses with young tubercle bacilli, whose acid-fast character is still very slight or entirely absent. When the horses have attained a high degree of immunity, they receive injections of various strains of pure cultures of streptococci obtained from the sputum of tuberculous patients. The serum of these animals is, therefore, antituberculous and also antistreptococcic, and is serviceable against a mixed infection.

The serum is administered daily, either by subcutaneous injection, in doses of from 5 to 10 c.c., or by the rectum in doses of from 10 to 20 c.c. The latter form of administration is quite objectionable to most patients, but is the one least likely to produce serum sickness.

While this serum has been used quite extensively, the evidence at present is too conflicting to permit definite conclusions to be drawn as to its value in treatment. It would, however, seem to be worthy of further trial in cases of localized bone and joint tuberculosis and in the incipient stage of pulmonary tuberculosis. Citron recommends its use in patients who evince persistent rise of temperature, and in the very severe but not hopeless cases where tuberculin therapy cannot be undertaken. In some of these cases he has obtained very encouraging results. Citron occasionally begins with the serum treatment, and later combines tuberculin administration with it, finally omitting the serum altogether. 


\section{CHAPTER XXXI \\ SERUM THERAPY (Continued)}

NORMAL SERUM THERAPY

THE field of serum therapy has been extended in recent years by the successful use of normal serum in the treatment of various pathologic conditions, particularly the hemorrhagic diseases, some toxicoses of pregnancy, and certain skin affections.

While human blood has also been administered, usually by direct transfusion, in the treatment of pernicious anemia, the leukemias, and pseudoleukemia, permanent good results have not been secured, although the immediate effects, owing probably to the introduction of large numbers of normal erythrocytes, may be satisfactory.

\section{Normal Serum IN the Treatment of HemorRhage}

Numerous reports by Welch, ${ }^{1}$ John, ${ }^{2}$ Franz,${ }^{3}$ Nohlia,${ }^{4}$ Reichard, ${ }^{5}$ Perkins, ${ }^{6}$ Claybrook, ${ }^{7}$ and others have shown that injections of normal human, horse, or rabbit serum are of considerable value in the treatment of melena neonatorum, hemophilia, purpura hœmorrhagica, hemorrhagic retinitis, intestinal bleeding in typhoid fever and in connection with cirrhosis of the liver, pulmonary tuberculosis, in some cases of uterine hemorrhage, and in surgical operations upon icteric persons. Barringer ${ }^{8}$ has reported the successful treatment, by injections of fresh normal human serum, of unilateral kidney hemorrhage in a hemophiliac, and advises that this simple treatment should be tried in similar cases of varicose veins of the renal papilla. Levison ${ }^{9}$ has reported the successful checking of hemorrhage from the urinary bladder following a simple op-

${ }^{1}$ Amer. Jour. Obst. and Dis. of Women, etc., 1912, lxv, No. 412.

${ }^{2}$ Münch. med. Wochenschr., 1912, lix, No. 4.

${ }^{3}$ Münch. med. Wochenschr., 1913, lix, 2905.

${ }^{4}$ La Presse Médicale, 1913, xxi, No. 20.

5 Jour. Amer. Med. Assoc., 1912, lix, 1539.

${ }^{6}$ Jour. Amer. Med. Assoc., 1912, lix, 1539.

${ }^{7}$ Jour. Amer. Med. Assoc., 1912, lix, 1540.

${ }^{8}$ Jour. Amer. Med. Assoc., 1912, lix, 1538.

9 Jour. Amer. Med. Assoc., 1913, lx, 721. 
eration by performing cystostomy, removing clots, and filling the bladder with sterile horse serum.

This form of treatment is so simple and has been so successful in checking hemorrhage in melena neonatorum and in other hemophilias following injuries or operations that it should never be omitted.

Defibrinated human blood, human serum, or the serum of the horse and rabbit may be employed. The doses advised have been from 10 to 20 c.c. for infants and children and from 20 to 50 c.c. for adults.

The technic is very simple. Sterile normal horse serum ready for injection may be purchased in the open market. Human serum may be secured by withdrawing blood into large centrifuge tubes (see p. 33) and allowing the serum to separate, or the clot may be broken up after an hour and the serum secured by rapid centrifugalization. For intravenous medication the serum should be free from particles of fibrin. Indeed, the whole operation may be conducted at the bedside by withdrawing blood from the donor into a flask containing sterile glass beads, and after a few minutes of vigorous shaking the defibrinated blood is injected subcutaneously or intramuscularly. Whenever human serum or blood is used and time permits, a Wassermann reaction should be performed beforehand, and it should be determined, by hemolytic and agglutination tests, that the donor's serum does not hemolyze or agglutinate the recipient's erythrocytes. (See p. 290.) Of course, all procedures should be conducted in an aseptic manner.

\section{Normal Serum in the Treatment of the Toxicoses of Pregnancy}

Feiux, Freund, Rongy, and others have found injections of fresh normal human serum from pregnant women, serum from placental blood, and even horse serum useful in the treatment of the vomiting of pregnancy. Freund has likewise observed that injections of Ringer's and Locke's solutions are sometimes efficacious; he has found injections of serum of some value in eclampsia, and tentatively advises its use in this condition. The same observer has employed injections of normal serum for the relief of the itching of pregnancy, and reports success. Similar observations have been made by Veiel ${ }^{1}$ and Wolf, ${ }^{2}$ especially after injections of serum secured from other healthy pregnant or recently delivered women.

To obtain placental serum, the following technic may be employed: After the delivery of the child the cord is sponged with bichlorid solu-

1 Münch. med. Wochenschr., 1912, lix, No. 35.

${ }^{2}$ Berl. klin. Wochenschr., 1913, 1, No. 36. 
tion, cut, and the maternal end bled into a sterile, wide-mouthed flask. This is placed in the refrigerator until there is complete separation of serum. In pipeting off the serum due care must be exercised not to include corpuscles. If the serum is not perfectly clear, it should be centrifuged with aseptic precautions. It is well to have each serum tested by the Wassermann reaction and 1 c.c. cultured in 100 c.c. of glucose bouillon to test its sterility. The serum is then preserved in bottles or vials, with the addition of two drops of 5 per cent. phenol to each 10 c.c. of serum.

\section{Normal Serum IN THE TREaTMENT OF Skin Diseases}

In addition to the good results obtained in the treatment of general pruritus of pregnancy with normal serum, Linser, ${ }^{1} \mathrm{Hench},{ }^{2}$ and Prætorius ${ }^{3}$ have had favorable results from injections of normal human serum or horse serum in the treatment of urticarial and chronic obstinate itching affections, especially senile pruritus, and also in malignant pemphigus. Even better results have been observed in the treatment of pemphigus, psoriasis, and other skin diseases by injections of the patient's own serum. This subject will be referred to further on.

Mention may also be made of the results observed in the treatment of acute and chronic nephritis by injections of blood-serum from the renal vein of the goat, dog, or sheep. Teissier ${ }^{4}$ was probably the first to apply this form of therapy, and reports favorable results in the treatment of seven cases. Spillman, ${ }^{5}$ Bisso, ${ }^{6}$ and Dominquez ${ }^{7}$ have also published favorable reports. The treatment is based upon the assumption that the blood in the renal vein contains some of the internal secretion of the kidney, which acts favorably upon the liver and emunctories in general. This method of treatment has been advocated by the previously mentioned observers in the acute exacerbations of chronic nephritis, in acute or chronic nephritis with threatening uremia, and in arterial hypertension presumably of renal origin. Amounts of serum ranging from 10 to 50 c.c. have been injected subcutaneously, and repeated on several days or every other day until several doses have been given.

${ }^{1}$ Dermat. Wochenschr., March 30, 1912.

${ }^{2}$ Münch. med. Wochenschr., 1913, lix, No. 48.

${ }^{3}$ Münch. med. Wochenschr., 1913, lx, No. 16.

${ }^{4}$ Bull. de l'Acad. de Méd., 1908, lxxii, No. 31.

5 La Presse Médicale, 1909, xvii, No. 86.

${ }^{6}$ Semana Med., Buenos Aires, 1912, xix, No. 7.

' Rev. de Med. y Cir., Havana, 1913, xvii, No. 24. 


\section{AUTOSERUM THERAPY}

A number of observers have reported favorable results following the administration of the patient's own serum in the treatment of various diseases. In most instances serum is secured by withdrawing blood into a sterile container, and defibrinating it with glass beads; this is followed by thorough centrifugalization, or the serum is secured after the blood has been allowed to coagulate spontaneously. In other instances the serum has been obtained from blisters purposely produced by the application of cantharides; in still others, and especially in tuberculosis of serous membranes, good results have been observed to follow subcutaneous injections of small amounts of the patient's pleural or peritoneal fluid.

\section{AUTOSERUM IN THE TREATMENT OF SkIN Diseases}

Sprethoff, ${ }^{1}$ Strumpke, ${ }^{2}$ Gottheil and Latenstein, ${ }^{3}$ and other observers have secured favorable results from this form of serum therapy in the treatment of obstinate and chronic dermatoses, due to general, rather than to local, causes, in which the usual therapeutic measures are ineffectual or only partially successful, as, for example, psoriasis, dermatitis herpetiformis, pemphigus, lichen ruber, lichen planus, urticaria, squamous eczema, etc. Blood is withdrawn from the patient in amounts of from 50 to 100 c.c. While still fresh, the serum is separated and injected intravenously in doses of from 30 to 40 c.c. These treatments are repeated from two to six times at intervals of from three to five days.

\section{Autoserum IN THE TREATMENT OF ACUTE INFECTIOUS Diseases}

Favorable results have also been reported in the treatment of acute infections, as, for example, scarlet fever with the serum of convalescent scarlet-fever patients. Thus Reiss and Jungmann, ${ }^{4}$ and later Reiss, ${ }^{5}$ have observed a marked amelioration in the general symptoms of severe cases of scarlet fever following the intravenous injection of from 50 to 100 c.c. of serum removed from two or more third to fourth week convalescents. These observers use this form of therapy in severe cases on or before the fourth day of the disease. After blood is withdrawn from convalescents the serums are separated, tested by the Wassermann reaction, mixed, cultured to determine their sterility, put up in amounts of

${ }^{1}$ Med. Klin., 1913, ix, No. $24 . \quad 2$ Deut. med. Wochenschr., 1913, xxxix, No. 30.

${ }^{3}$ Jour. Amer. Med. Assoc., 1914, lxiii, 1190.

${ }^{4}$ Deut. Archiv f. klin. Med., 1912, cvi, Nos. 1, 2.

5 Therap. Monatsschr., 1913, xxvii, No. 6. 
50 c.c. in ampules with the addition of 5 drops of 5 per cent. phenol, and kept on ice.

Teissier ${ }^{1}$ has had favorable results from the subcutaneous and intravenous administration of serum from smallpox convalescents in the treatment of severe and hemorrhagic smallpox. The injections must be given early in the disease. Improvement in the general symptoms, diminished suppuration, and consequent lessened scar formation are some of the good effects ascribed to this treatment.

Favorable results have also been observed in the treatment of leprosy with injections of serum secured by raising a blister with cantharides. Similar reports have been made by $\mathrm{Jez}^{2}$ in the treatment of erysipelas, and by Mordinos ${ }^{3}$ in that of typhoid fever, influenza, and Malta fever. Other observers have also reported good results following the injection of from 5 to 10 c.c. of the patient's serum in the treatment of gonorrheal arthritis, typhoid fever, pneumonia, and other infections. Robertson ${ }^{4}$ states that he has never observed the slightest influence of autoserum injections in the treatment of typhoid fever and pneumonia.

\section{Autoserum (SALVARSANIZED) IN THE TREATMENT OF SYPHILIS OF THE BRAIN AND SPINAL CORD}

The treatment of syphilis of the central nervous system has always been unsatisfactory. With the discovery of salvarsan and neosalvarsan, the hope was fostered that these remedies would prove of therapeutic value in the treatment of tabes dorsalis, paresis, and cerebrospinal syphilis. Experience has shown, however, that while the progress of tabes dorsalis may be arrested in the early stages by vigorous treatment, in paresis the prognosis is much less hopeful. As these diseases are now known to be truly syphilitic, the presence of Treponema pallidum having been actually demonstrated in the cerebral cortex, spinal cord, and cerebrospinal fluid by Noguchi and Moore, Nichols, Graves, Marie, Levaditi, and others, the cause for failure in the treatment of these infections must be ascribed largely to the fact that the choroid plexus filters out salvarsan and mercury, as well as antibodies, and prevents these remedies from reaching the cerebrospinal fluid, just as it prevents the entrance of serum, albumin, sugar, urea, ammonia, etc. Although there can be no doubt as to the spirocheticidal properties of salvarsan, and as to its ability to kill the treponema in the tissues, this much-desired action does not seem to occur mainly because the drug cannot gain ac-

${ }^{1}$ Quoted in Jour. Amer. Med. Assoc., 1913, lx, 380.

${ }^{2}$ Wien. klin. Wochenschr., August 31, 1901.

${ }^{3}$ La Presse Médicale, 1911, xix, No. 96, 1009.

${ }^{4}$ Personal communication. 
cess to the parasites. For similar reasons antimeningococcic serum must be injected intraspinally, because when given intravenously, the plexus appears to withhold the antibodies. The same is true of tetanus antitoxin.

Shortly after the discovery of salvarsan and neosalvarsan hope was entertained that these remedies, when injected intraspinally, would bring the drug into direct contact with the infected tissues. Investigations by Wechselmann, ${ }^{1}$ Marinesco, ${ }^{2}$ and Ellis and Swift ${ }^{3}$ have shown, however, that even minute quantities of either drug may be irritating and may prove dangerous.

Plaut 4 showed early that the serum of patients who have received salvarsan exerts a definite antisyphilitic effect, whereas normal serum displays no such activity. Meirowsky and Hartman ${ }^{5}$ and Gibbs and Calthrop ${ }^{6}$ observed good results in the treatment of syphilis from subcutaneous injections of serum from other patients who had received salvarsan. Swift and Ellis ${ }^{7}$ then showed that serum taken from a patient within six hours after the salvarsan was injected inhibited the growth of Treponema pallidum, but that if taken before the salvarsan was administered, or within from six to twenty-four hours after treatment, there was no inhibition. These last-named observers also reported beneficial effects following the intraspinal injection of salvarsanized serum, with but slight irritative phenomena. Further experimental studies on the spirocheticidal activity of salvarsanized serum were made by Gonder, ${ }^{8}$ Castelli, ${ }^{9}$ and especially by Swift and Ellis, ${ }^{10}$ the last-named investigators also noting that heating the serum at $56^{\circ} \mathrm{C}$. for half an hour markedly increased its activity, which was due in part to the destruction of some inhibiting substance.

Shortly after this Swift and Ellis ${ }^{11}$ published a report of the treatment of a number of cases of tabes dorsalis and other syphilitic infections of the central nervous system with intraspinal injections of salvarsanized serum. In practically all these cases clinical improvement was observed, with total or partial disappearance of the positive sero1446.

1 "Ehrlich-Hata 606," Berlin, 1911, 11. Deut. med. Wochenschr., 1912, xxxviii,

${ }^{2}$ Diätet. u. physik. Therap., 1913, xvii, 194.

${ }^{3}$ Jour. Exper. Med., 1913, xviii, 428.

${ }^{4}$ Deut. med. Wochenschr., 1910, xxxvi, 2237.

${ }^{5}$ Med. Klin., 1910, vi, 1572.

${ }^{6}$ Brit. Med. Jour., 1911, 1, 360.

${ }^{7}$ New York Med. Jour. 1912, xcvi, 53.

${ }^{8}$ Zeitschr. f. Immunitätsf., Orig., 1912, xv, 57.

9 Zeitschr. f. Chemotherap., Orig., 1912, 1, 122 and 167.

${ }^{10}$ Jour. Exp. Med., 1913, xviii, 435.

${ }^{11}$ Arch. Int. Med., 1913, xii, 331. 
biologic findings in the cerebrospinal fluids. Subsequent reports by Hough,, ${ }^{1}$ McCaskey, ${ }^{2}$ Riggs, ${ }^{3}$ Cutting and Mack, ${ }^{4}$ Eskucken, ${ }^{5}$ Boggs and Snowden, ${ }^{6}$ and Mapothes and Beaton ${ }^{7}$ of a number of cases of tabes dorsalis, paresis, and other syphilitic infections of the central nervous system showed that this method of treatment possesses distinct value. It was advocated by Swift and. Ellis in the treatment of these diseases as an adjuvant to intravenous injections of salvarsan, as a part of an intensive medication aiming to bring salvarsan into intimate contact with the parasites in the most direct and safest manner. While this form of treatment has, in a large percentage of cases, effected a marked improvement in the subjective symptoms and has modified the underlying pathologic tissue changes, as evidenced by the disappearance or improvement of objective signs and serobiologic findings in the cerebrospinal fluid, it must still be considered in the experimental stage, for sufficient time has not yet elapsed to permit an estimate of its ultimate effect to be made. It is especially indicated in early and incipient cases of syphilitic infections of the nervous system. It is selfevident that it cannot be expected to cure cases in which marked tissue destruction has occurred, but if it serves to cure early and incipient cases of tabes and paresis, or at least tends to arrest their progress and possibly the further progress of more chronic cases, and gives symptomatic relief, then this mode of therapy is a valuable one. At present it is apparent that, in the hands of careful and competent persons, and with the strict observance of an aseptic technic, the method is relatively devoid of danger and constitutes a new, rational, and valuable addition to the treatment of diseases that may otherwise prove intractable to the ordinary antisyphilitic measures.

Technic.-From 0.6 to $0.9 \mathrm{gm}$. of salvarsan or neosalvarsan is injected intravenously. One hour later 40 c.c. of blood are withdrawn directly into centrifuge tubes and allowed to coagulate, after which it may be centrifugalized. The following day 12 c.c. of serum are pipeted off and diluted with 18 c.c. of sterile normal salt solution. This 40 per cent. serum is then heated at $56^{\circ} \mathrm{C}$. for one-half hour. After lumbar puncture the cerebrospinal fluid is withdrawn until the pressure is reduced to $30 \mathrm{~mm}$. cerebrospinal fluid pressure. The barrel of a 20 c.c. Luer syr-

${ }^{1}$ Jour. Amer. Med. Assoc., 1914, lxii, 183.

${ }^{2}$ Jour. Amer. Med. Assoc., 1914, lxii, 187.

${ }^{3}$ Jour. Amer. Med. Assoc., 1914, lxii, 1888.

${ }^{4}$ Jour. Amer. Med. Assoc., 1914, lxvii, 903.

${ }^{5}$ Münch. med. Wochenschr., 1914, li, No. 14.

${ }^{6}$ Arch. Int. Med., 1914, xiii, 970.

${ }^{7}$ Lancet, London, April, 1914, 18, 1. 
inge (which has a capacity of about 30 c.c.) is attached to the needle by means of a rubber tube about $40 \mathrm{~cm}$. long. The tubing is allowed to fill with cerebrospinal fluid, so that no air will be injected. The serum is then poured into the syringe, and permitted to flow slowly by means of gravity into the subarachnoid space. At times it is necessary to insert the plunger of the syringe to inject the last 5 c.c. of fluid. It is important that the larger part of the serum should be injected by gravity, and if the rubber tubing is not more than $40 \mathrm{~cm}$. long, the pressure cannot be higher than $400 \mathrm{~mm}$. Usually the serum flows in easily even under a lower pressure. By the gravity method the danger of suddenly increasing the intraspinous pressure to the danger-point, such as might occur with rapid injection with a syringe, is avoided (Swift and Ellis).

The method of injection by gravity is described on p. 694. In the absence of a suitable manometer for estimating cerebrospinal fluid pressure the blood-pressure may be taken as a guide; in any event the serum should be injected slowly.

McCaskey consumes about six or seven minutes in administering the salvarsan intravenously, and advises withdrawing the blood twenty minutes thereafter, instead of waiting an hour in order to secure a larger quantity of the drug in the serum. He injects 15 c.c. of serum in 50 per cent. dilution intraspinally, and has not observed any increased irritative effects.

Swift and Ellis inject the serum in strengths of 50 to 60 per cent. or even higher in patients who do not exhibit reactions following the injection of 40 per cent. serum. Boggs and Snowden withdraw from 75 to 100 c.c. of blood one hour after the salvarsan injection, secure the serum, heat it at $56^{\circ} \mathrm{C}$. for one-half hour, and inject the undiluted serum in a dose equal to the amount of fluid withdrawn, whether this is only a few or as much as 30 c.c.

The method employed by Marinesco and Minea, ${ }^{1}$ whereby the patient's serum is salvarsanized in vitro, is not to be regarded as the same as the method of Swift and Ellis. The first-named investigators sought to administer larger doses of salvarsan by this method, but in a preliminary report, covering the treatment of 20 cases receiving injections every seven to eight days, the results are said to have been disappointing. The recent unfortunate outcome of this form of therapy in the County Hospital of Los Angeles is ascribed to the oxidation and consequent toxicity of the neosalvarsan as a result of allowing the salvarsanized serum to stand for twenty hours before injecting it.

${ }^{1}$ Bull. de l'Acad. de Méd., 1914, lxxvii, No. 7. 
After-treatment.- The patient should be kept in bed for twentyfour hours and the foot of the bed should be elevated for part of this time.

Usually the temperature reaction is mild. There is frequently some pain in the legs, which appears a few hours after the injection is given. The pain is more often noticed in tabetics than in patients with cerebrospinal syphilis. It can usually be controlled by means of phenacetin and codein, but occasionally morphin is required.

In a few instances violent maniacal symptoms have developed, due possibly to the direct irritant action of the drug on the tissues, aided by the sudden liberation of endotoxins from myriads of killed spirochetes.

Serobiologic Findings in the Cerebrospinal Fluid.-In all cases before the treatment is undertaken a Wassermann reaction should be performed with the blood and cerebrospinal fluid of the patient. A total cell count should also be made with a specimen of fluid, the percentage of lymphocytes ascertained, and a Noguchi butyric-acid-globulin test performed. Normally, the cells number about 8 per cubic millimeter of fluid (counted with a Fuchs-Rosenthal counting chamber); the presence of more than 15 cells in this quantity of fluid may be regarded as bordering on the pathologic. In tabes and paresis the cells may vary in number from 50 to more than 100 , and are mostly small lymphocytes. A normal cerebrospinal fluid remains clear or shows a faint opalescence when tested by the Noguchi butyric-acid test. In tabes, paresis, etc., varying degrees of cloudiness and the presence of precipitates are observed.

Repeating the Dose.-Usually a number of treatments are required, and these may be given at from one to three weeks' intervals, depending upon the condition of the patient. The results are estimated from the subjective and objective symptoms and from an examination of the cerebrospinal fluid. A decrease in the degree of positiveness of the Wassermann reaction and diminution in cells and in globulins are favorable signs. Theoretically, treatment should be continued until the Wassermann reaction becomes negative, the cells reach normal proportions, and the globulins show no increase. Practically, it may be impossible to secure these results; in many instances the Wassermann reaction is the first to disappear. A total cell count of the fluid is not to be depended upon without a differential count with stained smears, for the injections may produce a form of aseptic meningitis, accompanied by an outpouring of polynuclear leukocytes. This subject has previously been referred to in a consideration of the serum treatment of epidemic meningitis. 


\section{Autoserum IN THE TREATMENT OF Tuberculosis of Serous Membranes}

Numerous investigators, as, for example, Gilbert, ${ }^{1}$ Marcon, ${ }^{2}$ Schnutgen, ${ }^{3}$ Fishberg, ${ }^{4}$ Pfender, ${ }^{5}$ Robertson, ${ }^{6}$ and others, have reported favorable results in the treatment of tuberculous pleurisy with effusion, cases that arise either insidiously or abruptly with pain in one already tuberculous, or in one in whom tuberculosis is suspected, following withdrawal of a portion of the fluid and immediate injection of from 2 to 5 c.c. into the subcutaneous tissues. In these cases there is usually a sharp reaction, consisting of a rise in temperature, occasionally accompanied by chill, lassitude, and, in the majority of cases, diuresis or, more rarely, diarrhea, followed by gradual absorption of the fluid within the following few days up to two or three weeks. Fishberg mentions the disappearance of pain, dyspnea, and prostration within two or three days in favorable cases.

It is difficult to state whether the improvement is due to autotherapy or simply to the puncture and removal of so much fluid. Eisner ${ }^{7}$ has seen a leukocytosis follow injection of serum in experimental tuberculous infections of rabbits and guinea-pigs, and believes that this explains the good results in this particular form of therapy. Zimmermann ${ }^{8}$ has expressed a similar opinion. Other investigators assert their belief in the presence of aggressins, bacteriolytic amboceptors, complements, and endolysins from disintegrated leukocytes as explaining the results. It is more likely that these fluids contain the bacilli or their products, and constitute a form of vaccine or auto-tuberculin, stimulating body-cells to produce antibodies largely in the nature of bacteriotropins and bacteriolysins. Levy, Valenzi and Ponzin, ${ }^{9}$ Szurek, ${ }^{10}$ and Arnsperger ${ }^{11}$ are inclined to believe that the beneficial results are obtained independently of. the injections, and while the procedure is quite generally regarded as perfectly safe, Jousset ${ }^{12}$ has recorded a case of cold abscess following an injection. This mode of treatment seems to have failed in about 10 to 15 per cent. of cases.

The technic is very simple, and the injections may be given by any

1 Gaz. des Hôpit., 1894, 560.

${ }^{2}$ La Presse Médicale, 1909, No. 71, 627.

${ }^{3}$ Berl. klin. Wochenschr., 1909, No. 3, 97.

${ }^{4}$ Jour. Amer. Med. Assoc., 1913, lx, 962.

${ }^{5}$ Wash. Med. Ann., 1914, xiii, 83.

${ }^{6}$ Personal communication.

${ }^{7}$ Zeitschr. f. klin. Med., 1912, lxxvi, 34.

${ }^{8}$ St. Peters. med. Wochenschr., 1909, No. 34, 461.

${ }^{9}$ Bull. et. Mem. de la Soc. d. Hôp. d. Paris, 1910, xxvii, 265.

${ }^{10}$ Med. Klinik, 1909, No. 44, 1665.

11 Therap. d. Gegenwart, 1911, lii, 495.

12 Arch. Gén. de Méd., 1912, xci, 141. 
physician who can make an ordinary exploratory puncture. In all cases where puncture shows the presence of a serous fluid, the needle should not be withdrawn completely, but when its point has reached the subcutaneous tissues, from 2 to 5 c.c. should be injected then and there. In some patients it will be necessary to repeat the treatment several times every two or three days before any effect becomes evident. Caforio ${ }^{1}$ has reported good results following the tapping of a bilateral hydrocele and injection of a portion of the fluid into the subcutaneous tissues. It would appear that this procedure may be successful in hydrocele of tuberculous origin, but good results are not to be expected in cases due to contusion, puncture wounds, gonorrheal epididymitis, or orchitis.

In view of the fact that the fluid may contain a sufficient number of living tubercle bacilli to produce secondary infection, it would appear advisable to withdraw fluid into an equal volume of 2 per cent. sodium citrate in normal salt solution, heat at $60^{\circ} \mathrm{C}$. for one-half to one hour, and preserve in a sterile container with the addition of a few drops of 5 per cent. phenol until ready for subcutaneous injection in doses of from 2 to 5 c.c.

It would appear, therefore, that reintroduction into the body of fluids obtained from the serous cavities (pleural, peritoneal) of tuberculous patients may be of value in treatment, and, as a general rule, the earlier in the course of the disease that the autoserum therapy is practised, the better will the results be. A similar treatment may be carried out in the subacute or chronic forms of tuberculous meningitis. Fluid is to be collected in sodium citrate solution, heated, and preserved with phenol, as just described. The dose may vary from 0.5 to 2 c.c., according to age and clinical conditions.

\section{AUTOSERUM IN THE TREATMENT OF NON-TUBERCULOUS EFFUSIONS}

In pleural and peritoneal effusions of renal, cardiac, or hepatic origin, this method of autotherapy has generally failed.

Following the apparent success of Hodenpyl ${ }^{2}$ in the treatment of a case of cancer with injections of the patient's ascitic fluid, the autopsy subsequently showing the presence of metastatic cancer not demonstrable during life, Risely ${ }^{3}$ treated 65 cases of cancer with ascitic fluids obtained from cancer patients in all stages of the disease, and also with various normal and abnormal body fluids from other than cancerous conditions. None of these various transudates was found to exert any

${ }^{1}$ Riform. Méd., 1912, xxviii, 1009.

${ }^{8}$ Jour. Amer. Med. Assoc., 1911, lvi, 1383.

${ }^{2}$ Med. Rec., 1910, lxxvii, 359. 
effect in retarding the growth of cancer in mice, and while in a small percentage of cases large doses of ascitic fluid of cancerous origin may relieve pain and retard the growth of the cancer for from one to five months, no permanent effects, either preventing or checking the disease, were apparent.

Leavy and Hastings ${ }^{1}$ and Carter ${ }^{2}$ have drawn attention to the apparent improvement in marasmic infants following the daily injection, for a number of doses, of an ounce of sterile ascitic fluid (the result of cardiac or renal disease) into the subcutaneous tissues of the gluteal region or abdominal wall. As these fluids are non-toxic, reinjection is a justifiable procedure after the fluids have been subjected to the Wassermann reaction and to careful cultural and animal injection tests to prove their sterility.

${ }^{1}$ Bost. Med. and Surg. Jour., 1910, clxiii, 293.

${ }^{2}$ Amer. Jour. Med. Sci., 1911, cxlii, 241. 


\section{CHAPTER XXXII}

\section{CHEMOTHERAPY}

From the earliest times, healers of the sick have sought specific remedies in the form of drugs or methods that would always and unfailingly effect the cure of a certain disease, and indeed this search has been extended for a single remedy that will cure all diseases, regardless of their origin and nature. To this end, throughout the ages experimentation, conscious or otherwise, has gone hand in hand with medicine. Countless substances gathered from the vegetable, animal, and mineral kingdoms have been experimented with, but for the most part have been discarded. Despite this persistent search for specifics, until comparatively recent times but two remedies have been found worthy of being regarded as specifics, namely, cinchona bark for malaria, a remedy discovered by the South American Indians, and mercury for syphilis.

With discoveries in bacteriology and the establishment of the etiologic relationship of various microörganisms to certain diseases, testtube experiments soon demonstrated that certain substances could quickly and easily destroy these microparasites. It was apparent, however, that in the animal body conditions were different; here the germicide, even when administered in doses sufficiently large to prove dangerous to life, usually failed to kill the microörganisms. The exceptions were quinin and mercury, which we now know are specifically germicidal for the plasmodium and spirochete respectively, a fact that was suspected long before the parasites themselves were discovered.

In the latter part of the eighties it was discovered that the blood possessed germicidal powers, and rapid advances were soon made in our knowledge of the defensive mechanism of the animal body, and the means afforded for preventing infection, and even of successfully overcoming it if, by any chance, microörganisms passed the normal barriers and gained a foothold on the tissues proper. Here indeed was a more or less specific therapy that was not suspected until 1894, when diphtheria antitoxin was discovered. It was then speedily realized that body-cells could be made to produce a specific remedy for a certain disease, and it was naturally assumed that this was possible for all those 
diseases in which the specific microörganism was known and could be cultivated.

In the foregoing chapters we have reviewed our knowledge of the nature of these specific remedies produced by the body-cells and called antibodies. In Chapter XXX we have also reviewed the applieation of these remedies in the treatment of various infections, and have pointed out their limitations and the difficulties encountered in their production. Naturally, in the early days it was deemed necessary and advisable to utilize a lower animal for the manufacture of these antibodies. With the discovery of a means of attenuating or modifying a disease-producing parasite it was found possible to inject these vaccines into our own bodies, and thus stimulate our own cells to produce the specific antibody - a method that had been introduced empirically years before by Jenner in vaccination against smallpox.

As was previously stated, scientists have long believed that it was possible to find or produce chemical substances that would not unite with the blood albumin or body-cells, but would have a highly selective and germicidal action on microparasites and prove capable of killing these in the living body. Curiously enough Ehrlich, to whom we are already indebted for much of our knowledge of the intricate problems of cell life, and especially the mechanism of their defense and offense against parasitic invaders, has again taken the lead and set about testing hundreds of compounds in a painstaking, logical, and scientific manner, in the hope of finding one that would prove most germicidal for various trypanosomes and spirochetes, and at the same time be least toxic for the body-cells. These researches finally culminated in the discovery of the arsenical compound now popularly known as salvarsan. This discovery constitutes a great triumph for medical science, not only because of the intrinsic value of the drug in the treatment and cure of syphilis and frambesia, but because it demonstrates the truth of a principle, and has opened up vast possibilities for future investigation.

\section{PRINCIPLES OF CHEMOTHERAPY}

Organotropism and Parasitotropism.-The guiding principle in chemotherapy is to imitate nature's method of overcoming an infection by the aid of substances that destroy the microörganisms, while the body-cells of the host are left unharmed. To this end the chemical agent employed must possess a much stronger affinity for the microorganisms than for the body-cells-to quote Ehrlich, it must be more 
parasitotropic than organotropic. If a given chemical agent shows a greater affinity for the albumins of the body-juices and for the cells than it does for the protoplasm of the microparasites, that is, if it is more organotropic than parasitotropic, it is evident that it is not suitable for therapeutic purposes, especially if, at the same time, the toxic dose for the host should be smaller than that for the microörganism.

The object of chemotherapeutic research is, therefore, to discover chemicals that have a greater parasitotropic than organotropic activity, and the greater the difference between these, the more valuable do the substances become.

There is only one way of determining these values, and that is by animal experimentation. Rats, mice, guinea-pigs, rabbits, and fowls have been most generally employed for this purpose, and most work so far has been done with protozoan parasites, such as the organism of chicken spirilloses, the spirillum of recurrent fever, various trypanosomes, and the spirochete of syphilis. These were selected because they are readily found in the blood or in lesions, and are rapidly pathogenic.

As a result of the study of several hundreds of different products by Ehrlich and his collaborators and by many other noted investigators, it was found that there are, after all, comparatively few that exert a parasitotropic effect in animals; these are, however, well characterized chemically, and have been classified into three main groups:

1. The group of arsenical compounds-arsenious acid (arsenic trioxid), atoxyl, arsacetin, arsenophenylglycin, dioxydiamido-arsenobenzol dichlorhydrate (popularly known as "606," or salvarsan), and various antimony compounds.

2. Certain azo-dyes of the benzidin group, for example, trypan red, trypan blue, and trypan violet.

3. The group of basic triphenylmethane dyes, such as parafuchsin, methyl-violet, pyronin, and others.

Newly discovered drugs are administered in increasing amounts to experimental animals until the toxic dose (dosis lethalis), the tolerated dose (dosis tolerata), and the curative or sterilizing dose (dosis curativa or sterilisana) have been determined.

While chance may succeed in giving us a drug fulfilling the requirements, so far it has had little influence, and it is difficult to realize the tremendous amount of work done by Ehrlich and the members of his institute before salvarsan was discovered. The following table illustrates the relation of the tolerated to the curative doses of a few chem- 
ical substances, including salvarsan, given by intramuscular injection, in spirillosis of fowls, and illustrates the particular value of "606," as dependent upon the fact that it is highly parasitotropic in an amount that is far below the organotropic or toxic dose:

\begin{tabular}{|c|c|c|c|}
\hline Chemical & $\begin{array}{c}\text { Maximum } \\
\text { Tolmerated } \\
\text { Dose }\end{array}$ & $\begin{array}{c}\text { STERILIZING } \\
\text { OR CURATIVE } \\
\text { Dose }\end{array}$ & Proportion \\
\hline 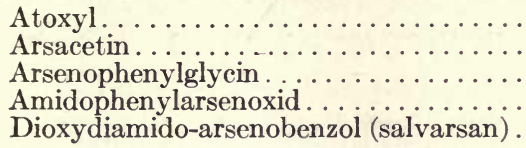 & $\begin{array}{l}0.06 \\
0.1 \\
0.4 \\
0.03 \\
0.2\end{array}$ & $\begin{array}{l}0.03 \\
0.03 \\
0.12 \\
0.0015 \\
0.0035\end{array}$ & $\begin{array}{l}1: 2 \\
1: 3.3 \\
1: 3.3 \\
1: 20 \\
1: 58\end{array}$ \\
\hline
\end{tabular}

Chemoreceptors.- Of considerable interest in this connection is the question of how substances can be modified in order to render them progressively more parasitotropic and less organotropic in action. When ordinary germicides, such as phenol, mercury bichlorid, formalin, etc., are added to suspensions of bacteria, we believe that the latter are killed mainly as the result of poisoning of their protoplasm, and that the chemical substance enters into chemical union with the bacterial albumins by direct toxic action, accompanied by such physical changes as that of coagulation, and alters bacterial metabolism and brings about death of the cells. Such germicides do not appear to exert any selective action on any particular albumin. When mercury bichlorid is added to a mixture of bacteria in a serum, many of the microörganisms may escape destruction through the formation of protective envelops of an albuminate of mercury formed with the serum albumins; a similar action may be noted with phenol.

In chemotherapeutic research, therefore, it is the aim to start with a substance that primarily shows a more marked affinity for the protoplasm of the parasite than it does for the body-cells, and then, by subtracting or adding to the molecule or by inducing an intramolecular rearrangement, an effort is made to develop its parasitotropic action. The question then arises, does the increased parasitotropism of the chemical substance depend upon its higher direct and simple action upon the protoplasm of the microparasite, or is this effect to be explained upon its increased combining power or affinity for the molecules of the parasites or other cells because the latter are provided with special groups for effecting the union? Ehrlich has endeavored to answer this question by maintaining that both body-cells and microparasites possess special receptors or side-arms by which chemical substances may be bound; 
these he terms chemoreceptors. While Ehrlich originally assumed that the so-called side-arms of the protoplasmic molecule served primarily for the process of nutrition, he now believes that these special chemoreceptors do exist. He suggests that they may probably possess a less complex structure, similar to the receptors of the first order for simple toxins, that they are more firmly attached to the cell, and that, accordingly, they are less readily cast off, thus explaining why crystalline chemical substances are, as a general rule, incapable of eliciting the production of corresponding antibodies. This theory is based upon the discovery that certain strains of a microparasite develop a state of "resistance" or "fastness" to a particular substance, and that this acquired characteristic may be transmitted from generation to generation. This subject will be further discussed elsewhere.

According to Ehrlich's postulate, therefore, toxic agents cannot act on microörganisms unless they are fixed by suitable receptors (corpora non agunt nisi fixata). This conception is similar in every way to his conception of the processes of infection and immunity and the development of antibodies; that is, the toxic agent must first be "fixed" or "anchored" to the molecule of a cell by suitable receptors by a process of chemical interaction before damage can be inflicted. Chemoreceptors differ from other receptors in being more firmly attached to cells, so that while the cell may become immune to the toxic effects of the agent, the blood-serum may not contain the immune bodies.

When arsenic is introduced into the body, it is "fixed" by the receptors of certain cells; mercury in turn is fixed by other receptors, and so on through the list. The basic principle of chemotherapy is that it is possible to produce chemical substances that carry side-arms capable of being fixed by microparasites, and to a much less extent by the body-cells.

It is not necessary that the whole molecule of a toxic substance possess a combining affinity for certain receptors: if one or more atom groups becomes attached, it is presumed that it carries with it the remainder of the molecule. Moreover, that atom group that is anchored or is responsible for the anchorage of the entire molecule need not possess any of the properties of the entire molecule or of any part thereof. For example, when the molecule of salvarsan is anchored to the spirochete of syphilis by its $\mathrm{OH}$ or its $\mathrm{NH}_{2}$ side-chain, or by both, the spirochete must later contend with two molecules of arsenic, which, being in a trivalent condition, can exercise its toxic effects to a marked degree upon the parasite. The side-arms as they exist in salvarsan are much more readily taken up by the spirochete than by the body-cells, which is shown by 
the marked difference between the lethal and tolerated doses and the curative dose.

Drug "Fastness." - In the foregoing chapters we have sought to emphasize the importance of the microörganism in the processes of infection and immunity from the standpoint of the possibilities of these cells immunizing themselves against the deleterious agencies of the host, and particularly the antibodies, as explained in the hypothesis of Welch. (See p. 103.) It soon appeared that the problem of chemotherapy was greatly complicated by these activities on the part of the parasite, so that the hypothesis appears to be further supported as a result of chemotherapeutic studies. If the dose of a chemical is just small enough to allow a few microörganisms to escape, these immediately fortify ("immunize") themselves against the drug and become invulnerable to its effects. It was found that these microparasites were then able to multiply, even in the presence of the drug, and, further, that this property of "drug resistance" was transmitted from one generation to another. If, for example, the trypanosomes in a mouse have become resistant to trypan red, a quantity of these trypanosomes may be inoculated into another mouse, and from this one to another, and so on, for many generations, and it would finally be found that the trypanosomes still retained their immunity to the action of trypan red.

This acquired resistance or "fastness" is in a large measure specific. A strain of trypanosomes resistant to the benzidin dyes is non-resistant to arsenic and the triphenylmethane dyes, whereas one resistant to arsenic is not resistant to the dyes, etc. As Levaditi and Fraser have shown, the antibody-resistant trypanosomes do not anchor the antibody, hence it is probable that the analogy holds for the drug-resistant microparasites.

As was previously stated, Ehrlich has explained this phenomenon on the basis of chemoreceptors. He has ascertained that "fastness" for a certain chemical agent does not depend on atrophy of the corresponding receptors, but upon a modification in their structure, as is evidenced by the fact that, by changing the structure of the chemical, it may still find suitable receptors and lead to the destruction of the parasite. For example, mice that have been infected with arsenic-fast trypanosomes may be cured by an injection of arsenophenylglycin, even at a time when death is imminent. It would appear, therefore, that the arsenic-fast receptors of the trypanosomes were but slightly altered, and were still capable of uniting with an allied product.

This factor greatly complicates chemotherapy. If, for instance, the 
destruction of trypanosomes by an arsenical preparation has not been complete, and if the antibodies produced by the body-cells do not succeed in destroying the remainder, there is a strong probability that a new strain will now develop that will be resistant not only to the particular arsenical preparation used, but will also be proof against the serum antibodies. This new strain may now cause a relapse of the disease. Another chemical is now injected, but if this likewise fails to kill all the trypanosomes, the remainder will generate still another strain "resistant" to the preparation and the antibodies. This may continue as long as the parasite is able to produce new receptors; when this limit is reached, its nutrition will be impaired and the serum antibodies, alone or aided by another chemical substance, may finally destroy all trypanosomes, the infection "dying out," so to speak, or proving completely vulnerable to a chemical agent.

These considerations have an actual experimental basis, and natural examples of acquired serum-resistance or "fastness" are to be found in relapsing fever, syphilis, sleeping sickness, and possibly malaria. In relapsing fever the clinical course of the disease would indicate that only three or four serum-fast strains can be produced, and we accordingly find that, after a patient has withstood a number of relapses corresponding to the number of antibody-fast strains that the spirillum may produce, spontaneous recovery occurs, there being then antibodies that the strain cannot resist, and the infection "dies out," due in part to destruction of the antibodies and in part to starvation of the parasite because the number of receptors is insufficient to carry on nutrition. In the mean time, however, the patient may succumb to the disease before the spirillum has "played its last card."

In syphilis conditions are different, and the phenomenon of "resistant races" further explains many conditions not previously understood. The spirochete is apparently capable of repairing its receptors to a remarkable degree, especially after injury received from the antibodies produced by body-cells, and, further, is able to maintain its nutrition with a number of different food-stuffs, so that in the untreated person relapse follows relapse, the offensive forces of the spirochete being held in abeyance by the defenses of the host over long periods of time (latent periods), but the vital parts of the host being gradually damaged and the defenses weakened so that death or serious symptoms may supervene long before the disease has "worn itself out," if, indeed, this ever occurs in syphilis.

This phenomenon also explains why the syphilitic person cannot be 
reinoculated with syphilis while his disease is active. That he is vulnerable to syphilis is evidenced by the activities of the spirochetes within his tissues and body-fluids. But this is so because a race is at work that is resistant to the antibodies first produced, whereas these antibodies are still potent and capable of killing a fresh, non-resistant race of spirochetes.

The theory of chemoreceptors affords an explanation of this phenomenon, just as the side-chain theory affords an explanation of the formation of cellular antibodies and the processes of immunity. In adopting it we must be prepared to believe that the number of receptors and the possibilities of their repair or modification are practically unlimited, as evidenced by the large number of substances capable of exerting toxic action. Syphilis, for example, is probably a disease of great antiquity, handed down from person to person, from generation to generation, during which transmission the receptors of the spirochete must have undergone innumerable transformations and alterations, yet finding suitable receptors in the cells of practically all nonsyphilitic persons, although it is now apparent that strains have gradually been evolved

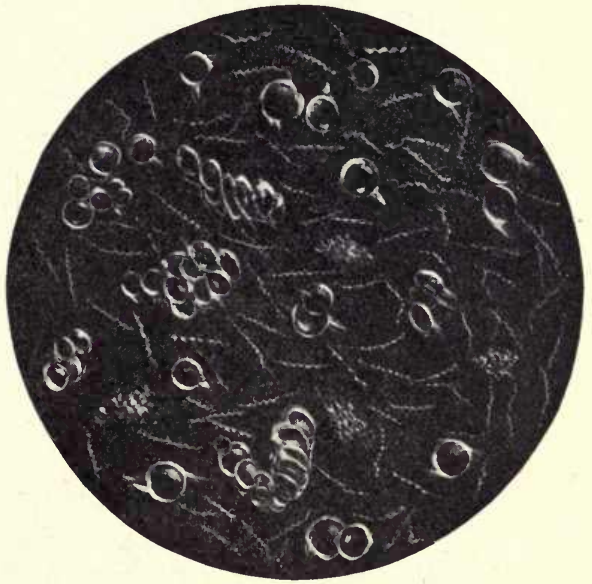

Fig. 140.-Blood of a Rat INFECted with SPIROCHETA RECURRENTIS.

(Drawing made from dark-field illumination just before administration of salvarsan.) that possess receptors with marked affinity for certain tissues, as, for example, those for the central nervous system, cardiovascular system, etc.

Therapia Magna Sterilisans.-As the development of resistant strains is thus one of the possibilities and impediments to successful specific therapy, whether with chemicals (chemotherapy) or with antibodies (serum therapy), our efforts should be directed toward discovering chemical substances and a method of administration that will completely sterilize the individual at one time (Ehrlich's therapia magna sterilisans). This possibility has been amply demonstrated experimentally by Ehrlich, and it has occasionally been accomplished in the 1 "Die Behandlung der Syphilis" (Königsberg Versammlung), Leipzig, 1910, 930. 
early stages of human syphilis by means of salvarsan administered early in the proper manner and in correct dosage, but, unfortunately, it is not true of the majority of cases, the difficulties increasing with the duration of the infection. Fortunately, the investigations of Margulies have shown that while resistant races of trypanosomes are easily evolved, the evidence is entirely against the probability of the development of arsenicresistant syphilitic spirochetes as the result of prolonged treatment with nonsterilizing doses of salvarsan.

With this brief discussion of the primary principles of chemotherapy, which is really a very ancient method of treatment and dates from the

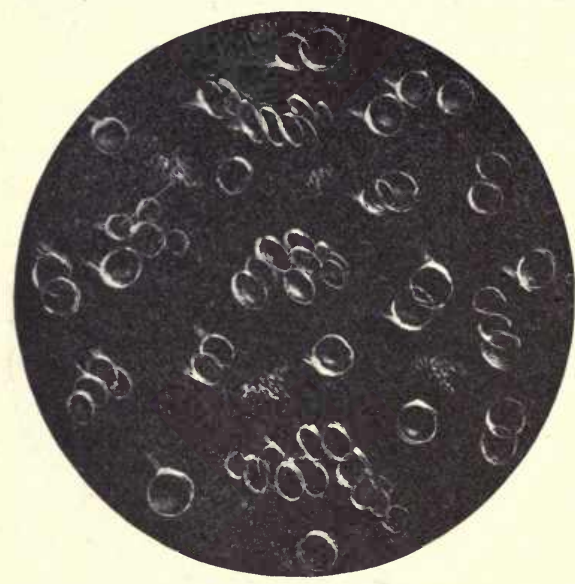

Fig. 141.-Blood of Same Rat EighTEEN Hodrs aFTer InTRavenods InJECTION OF 0.0008 MG. Salva RSAN. time that chemicals were first used for treating the sick, but which has now emerged from the darkness of pure empiricism into the light of a science, we shall pass on to a consideration of salvarsan and neosalvarsan. These two substances were not discovered as the result of accident, but were the outcome of exact knowledge, logical thinking, and carefully planned experimentation. It is impossible to tell what these discoveries presage; certainly they open up vast possibilities in the development of a specific therapy for all infections. One fact is certain: that while chance must ever play some rôle, future discoveries will probably result only from prolonged, patient, and laborious study.

\section{SALVARSAN AND NEOSALVARSAN IN THE TREATMENT OF SYPHIIIS}

Historic.-The administration of arsenic in protozoan infections has long been a recognized method of treatment. The organic compound of arsenic known as atoxyl (the sodium salt of para-aminophenylarsenic acid) was first used in the treatment of trypanosomiasis, and although this drug did not produce the results that were anticipated, it formed the starting-point for important researches in the preparation of organic compounds of arsenic and their use in protozoan infections. On the 
strength of Schaudinn's statement regarding the close biologic relationship of the spirochetes of syphilis to trypanosomes, Uhlenhuth was led to employ atoxyl in the treatment of experimental spirillosis in fowls. These experiments, in addition to those of Weisser and Metchnikoff, demonstrated beyond doubt the curative and prophylactic properties of atoxyl in spirillar infections. The drug was, therefore, administered in cases of syphilis in the human subject. It was found to exert a very beneficial effect, especially in malignant forms of the disease. Further experience, however, demonstrated that atoxyl was too toxic for use in the human subject, for digestive disturbances, nephritis, and especially optic atrophy could be traced to its use, even in small doses. As a therapeutic agent, therefore, it has gradually come into disfavor.

Ehrlich and Bertheim then made the valuable discovery that the chemical constitution of atoxyl was not, as had been supposed, that of a metarsenate, but that it was in reality that of a para-amidophenylarsenate. Working on this basis, these observers prepared the substance known as arsacetin (the sodium salt of acetylpara-amidophenylarsenic acid), which, although less toxic than atoxyl, did not fulfil the requirements of a remedy, and its use was discontinued because of its toxic effects on human tissues. To lessen the relatively great toxicity of these compounds for human tissues was a problem that Ehrlich set out to solve. A most important advance in this direction was made when it was discovered that the unsaturated trivalent arsenic in arsenobenzol and arsenophenylglycin has a greater parasiticidal power relative to its toxic action on the tissues of the host than the pentavalent arsenic compounds, such as atoxyl and arsacetin.

Finally, after testing hundreds of these arsenical compounds, it was found that in dioxydiamidoarsenobenzol we had a substance that approached the ideal in chemotherapy, since it is a drug that possesses a maximum degree of parasitotropism and a minimum degree of organotropism. Ehrlich and Hata designated this light yellow, readily oxidizable powder as No. 592. Its hydrochloric acid salt was designated No. 606 , and constitutes the well-known salvarsan.

In the published account of their researches Ehrlich and Hata ${ }^{1}$ describe an extensive series of investigations in experimental relapsing fever, spirillosis of fowls, and syphilis of rabbits. These go to prove the high curative value of salvarsan, its relatively feeble toxicity for the

1 "Die experimentelle Therapie der Spirillosen," Berlin, 1910; Zeitschr. f. Immunitätsf., Ref. 1910, 1123. Numerous publications from the Institute of Experimental Therapy, Frankfort. 
tissues of the infected animals, and its great superiority over all other substances that possess spirillicidal properties.

Clinical evidence in the treatment of human syphilis supported the experimental findings. On account of the disadvantages of salvarsan, due to its insolubility and the necessity for using the hydrochlorid, and also in an effort still further to lessen its toxicity, Ehrlich conducted further researches to discover a neutral salt that would be more soluble and less toxic. As a result of these efforts neosalvarsan, or "914," has been produced. This number is significant of the number of experiments performed since the discovery of " 592 " and "606."

Properties of Salvarsan.-Salvarsan is the dihydrochlorid of dioxydiamidoarsenobenzol, and occurs as a yellow, crystalline, hygroscopic powder, very unstable in air, and easily oxidized to poisonous compounds. It is marketed commercially put up in small sealed vacuum tubes. It contains 31.57 per cent. of arsenic, is readily soluble in water, particularly in hot water, and yields a solution having an acid reaction. If the acidity is neutralized by the addition of caustic soda solution, the unsoluble base (dioxydiamidoarsenobenzol) is precipitated. If only half of this amount of alkali is added, then the monohydrochlorid of dioxydiamidoarsenobenzol is formed. If, in addition to the amount of caustic soda necessary to precipitate the base, a further quantity of alkali is added, the hydrogen atoms of the phenol hydroxyls become replaced by $\mathrm{Na}$ and the compound goes into solution as the disodium salt of dioxydiamidoarsenobenzol:

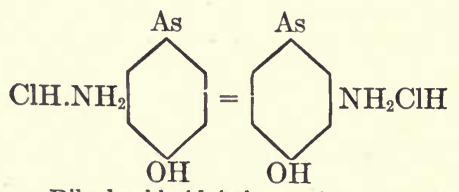

Dihydrochlorid (salvarsan)

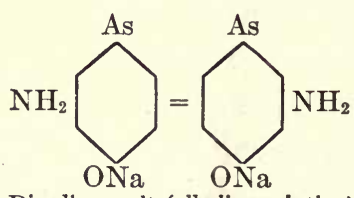

Disodium salt (alkaline solution)

Test-tube experiments showed less spirillicidal power of the drug than is demonstrated in the living animal. The following table shows the toxicity of the preparation (Ehrlich and Hata):

\begin{tabular}{|c|c|c|}
\hline Animal & Method of Administration & Maximum Tolerated Dose \\
\hline 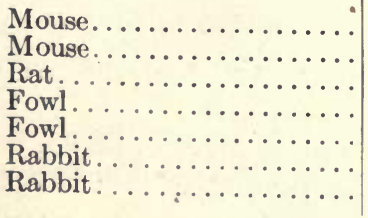 & $\begin{array}{l}\text { Subcutaneous } \\
\text { Intravenous } \\
\text { Subcutaneous } \\
\text { Intramuscular } \\
\text { Intravenous } \\
\text { Intravenous } \\
\text { Subcutaneous }\end{array}$ & $\begin{array}{l}\text { 1:300 per } 20 \text { grams } \\
1: 350 \text { per } 20 \text { grams } \\
0.2 \text { gram per kilogram } \\
0.25 \text { gram per kilogram } \\
0.08 \text { gram per kilogram } \\
0.1 \text { gram per kilogram } \\
0.15 \text { gram per kilogram }\end{array}$ \\
\hline
\end{tabular}


In experimental syphilis of rabbits the minimal dose necessary to produce a complete cure was found to be between 0.01 and 0.015 gram per kilogram. The tolerated dose by intravenous injection is 0.1 gram; the curative dose of salvarsan in syphilis of rabbits is, therefore, only from one-seventh to one-tenth of the tolerated dose.

Properties of Neosalvarsan.-This is an orange-yellow powder possessing a peculiar odor. It is very unstable in the air and is readily soluble in water, yielding a yellow solution that is neutral to litmus. Its structure is somewhat more complex than that of salvarsan, being a condensation-product of the latter and hydraldit (formaldehyd sulphoxylate of sodium), the reaction taking place according to the following equation:

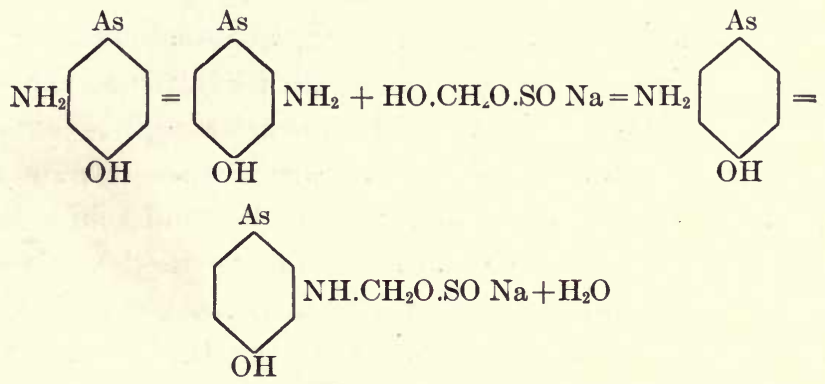

While neosalvarsan is less toxic than salvarsan, and although it is much more easily administered and largely free from irritative effects, recent clinical reports would tend to show that its spirocheticidal properties are somewhat less than those of salvarsan.

Methods of Preparing Salvarsan for Administration.-Soon after the introduction of the drug several methods of preparation and administration were suggested. Many of the disadvantages attached to salvarsan treatment, and many of the bad results and complications reported, are to be attributed to defective methods of preparation and administration of the drug.

Because of the marked stability of salvarsan and neosalvarsan, the following points must be borne in mind:

1. The ampule containing the drug must be intact.

2. The powder must be of a yellow and not of a gray or brownish color.

Each ampule must be carefully examined before the contents are administered.

3. The drug should be prepared for injection just prior to administration. 
It is not necessary to describe the earlier methods, because these are now largely only of historic interest, and it is quite generally accepted, from the point of view both of efficient treatment and of the comfort of the patient, that the intravenous injection of a dilute solution of the disodium salt is the best form of administration. For this reason I shall briefly mention the other methods, and describe the method of intravenous injection of the alkaline solution in greater detail further on.

The Acid Solution.-When salvarsan is dissolved in warm water or warm normal saline solution, a strongly acid solution is obtained. In this form the drug is most irritating and also most toxic, and when injected subcutaneously and intramuscularly, produces severe pain and necrosis. This method is seldom if ever used at the present time.

The Mono-acid Solution.-If to the watery acid solution half the amount of alkali necessary to produce complete neutralization is added, a solution of the mono-acid compound will be formed. This solution has been given intramuscularly, but is also extremely irritating.

The Neutral Suspension.-This is the drug in the form of a precipitate of the base, prepared by adding to the original acid solution just sufficient caustic soda to neutralize it. The method was devised by Michaëlis and Wechselmann, and for some time was the form of administration most generally employed for subcutaneous and intramuscular injection. It is probably less irritating than the acid and clear alkaline solution, but occasionally there resulted encapsulation of masses of necrotic tissue containing considerable quantities of arsenic.

Other Suspensions.-Suspensions of salvarsan in liquid paraffin, sterile olive oil, oil of sesame, or almond oil are said to keep for some time if placed in dark containers. This cannot, however, be always depended upon, and the slightest decomposition of the original drug is capable of producing marked toxic symptoms. These suspensions are said to be comparatively non-irritating, but they may cause local necrosis of tissues, and on account of their slow absorption, only small amounts gain access to the general circulation at one time.

This method may, however, be of value, especially in infants and in cases where slow absorption is desired in order to prolong the effect of the drug. Analgesics, such as eucain, creosote, or an essential oil, may be incorporated in the suspension in order to lessen the pain.

The suspension is so prepared that each cubic centimeter contains 0.1 gram of the drug.

Alkaline Solution of the Disodium Salt.-This is the form in which the drug should be administered by intravenous injection. It is this solu- 
tion which Hata used in his original experiments, and it was the form recommended by Ehrlich. I shall, therefore, describe this method in detail a little further on.

\section{ADMINISTRATION OF SALVARSAN AND NEOSALVARSAN}

\section{INTRAVENOUS INJECTION}

Dosage of Salvarsan.-Males receive in general about 0.3 to $0.6 \mathrm{gm}$.; female patients are usually given from 0.25 to $0.5 \mathrm{gm}$. In the case of weak and poorly nourished adult patients it in inadvisable to give more than 0.3 or $0.4 \mathrm{gm}$. Since it has been generally impossible to cure a patient with a single larger dose, the practice among syphilologists at present is to give smaller doses frequently repeated.

For infants suffering from congenital syphilis the dose is from 0.006 to $0.01 \mathrm{gm}$. of salvarsan for every two pounds of body weight, so that a child of eight pounds would receive from 0.024 to $0.04 \mathrm{gm}$. of salvarsan. To older children, weighing from 40 to 60 pounds, 0.2 to $0.3 \mathrm{gm}$. may be given.

Dosage of Neosalvarsan.-This preparation is less toxic than salvarsan, and may be administered in larger doses, as from 0.6 to $1 \mathrm{gm}$. The same general rule as to the physical condition of the patient should apply here in deciding the dosage. At the present time the tendency is to give adult patients about $0.6 \mathrm{gm}$. for three, four, and more injections at intervals of a week or so.

Frequency of Injections; Intensive Treatment.-As was just stated, there is a distinct tendency among those of large experience to regard salvarsan as a more potent spirocheticid than neosalvarsan. As previously mentioned, the original idea of sterilizing the patient with one large dose of the drug has been largely abandoned, especially in the treatment of syphilis in any but the earliest stages. A large number of smaller doses are being given, and the results are controlled by the Wassermann reaction with blood and cerebrospinal fluid, in addition to the cytologic changes in the latter. Thus from 0.3 to $0.5 \mathrm{gm}$. of salvarsan or neosalvarsan is given every week or twice a week for three, four, or ten doses and more, depending upon the clinical results and the serologic findings. In this connection it must be remembered that a negative Wassermann reaction is of little value if blood has been withdrawn within two weeks of the last treatment. (See Chapter XXIII.) While it is the common practice to administer a number of doses of salvarsan or neosalvarsan, and to follow this with mercury and then with 
salvarsan again, this method must be regarded as containing an element of danger, especially since Wechselmann has drawn attention to the fact that mercury acts as an irritant to the kidneys. It may be stated that, in general, most cases of syphilis require a number of injections of salvarsan; this number depends upon the age and nature of the infection, and should be controlled by the Wassermann reaction with both blood and spinal fluid.

Preparation of the Patient.-In view of the fact that many of the fatalities due to salvarsan have been ascribed to defective kidneys, especially to kidneys damaged by the previous administration of mercury, it should be a routine measure to have the urine thoroughly examined for sugar, albumin, and casts previous to the administration of salvarsan or neosalvarsan.

While thousands upon thousands of injections have been made without ensuing untoward results, still the administration of this drug is not without danger, and the physician should familiarize himself with the contraindications, and subject his patient to a careful physical examination before undertaking this form of therapy. This subject will be discussed further under the head of Contraindications to Salvarsan Therapy.

In the majority of cases, however, no contraindications exist and no elaborate preparations are necessary. The rectum should be emptied before the injection is given, and it is best to administer the drug on an empty stomach. After receiving salvarsan the patient should rest overnight under direct supervision, as in a hospital, the injection being given during the afternoon or early evening hours. The same practice should be followed with neosalvarsan, although in thousands of instances patients have received an intravenous injection, rested for an hour or so, and then returned to their homes.

Preparation of Salvarsan Solution.-As previously mentioned, the physician should examine the ampule containing the drug to convince himself that it is intact. The solution should be prepared just before it is injected. On account of the oxidation that occurs it is unsafe to use the drug if the ampule has been open for a number of hours, and for the same reason it is not good practice to prepare a bulk solution for a number of patients unless the physician is certain that he will be able to make the injections quickly and without interruption.

The clear alkaline solution is most generally used. It is prepared as follows:

1. The diluent should consist of sterile and freshly distilled water. 
Many of the toxic and other ill effects attending salvarsan therapy have been attributed to the use of raw and stale water. Experiments conducted by Yakinoff show that the presence of the endotoxins of such microörganisms as Bacillus coli, Bacillus pyocyaneus, and staphylococci in the water, increases the toxicity of salvarsan from two to eight times, the water alone and the salvarsan alone being without effect. In office practice physicians may distil water by means of some simple apparatus, such as that of Muencke. The distilled water is then sterilized in an Arnold sterilizer for one-half hour, or by boiling for ten or fifteen minutes.

2. All glassware used should be carefully sterilized, usually by boiling, as in the physician's ordinary office sterilizer.

3. The ampule containing the drug is wiped off with alcohol, the neck filed across and broken off, and the contents emptied into a sterile 50 c.c. glass-stoppered mixing eylinder containing preferably a number of small glass beads. From 15 to 20 c.c. of hot (about $50^{\circ}$ C.) sterile distilled water are added, and with shaking the drug passes into solution. A 15 per cent. solution of caustic soda is now added drop by drop to the solution in the cylinder. A precipitate of the base is first deposited, and on further addition of caustic soda, aided by shaking, this is again brought into solution, the fluid being strongly alkaline. The amount of alkali necessary is about four drops of a 15 per cent. solution for each $0.1 \mathrm{gm}$. of salvarsan; thus, for $0.6 \mathrm{gm}$., 1.14 c.c., or about from 23 to 45 drops of 15 per cent. solution of caustic soda, would be required. Citron gives the following table:

$0.2 \mathrm{gm}$. salvarsan requires 0.38 c.c. of 15 per cent. sodium hydroxid $=8$ drops.

$0.3 \mathrm{gm}$. salvarsan requires 0.54 c.c. of 15 per cent. sodium hydroxid $=12$ drops.

$0.4 \mathrm{gm}$. salvarsan requires 0.76 c.c. of 15 per cent. sodium hydroxid $=15$ or 16 drops.

$0.5 \mathrm{gm}$. salvarsan requires 0.95 c.c. of 15 per cent. sodium hydroxid $=19$ or 20 drops.

$0.6 \mathrm{gm}$. salvarsan requires 1.14 c.c. of 15 per cent. sodium hydroxid $=23$ to 24 drops.

A drop more of the alkali than is just necessary to produce the clear solution should be added. If this is not done, on cooling the solution may show a precipitate; this can be redissolved by the addition of a drop of alkali. The drug is now in a solution of about 20 c.c., and if an intramuscular injection is to be given, this is the form to be preferred. Intramuscular injections are, however, likely to be painful, and should be given only in those cases where it is practically impossible to give the drug intravenously, as in the case of infants. For intravenous injection the solution of 20 c.c. is diluted with warm sterile distilled water to make 300 c.c. in a second sterile cylinder or beaker, which is graduated 
or bears a 300 c.c. mark; with $0.6 \mathrm{gm}$. in solution, each 50 c.c. contain $0.1 \mathrm{gm}$., so that the dose given may be controlled by the amount of fluid injected. This flask is thoroughly shaken to insure an even diffusion of the drug, and the contents poured into the cylinder from which it is administered, being filtered into this cylinder through a piece of sterile gauze in order to remove any bits of broken glass from the ampule that may have gained access or any other insoluble particles. (See Fig. 142.)

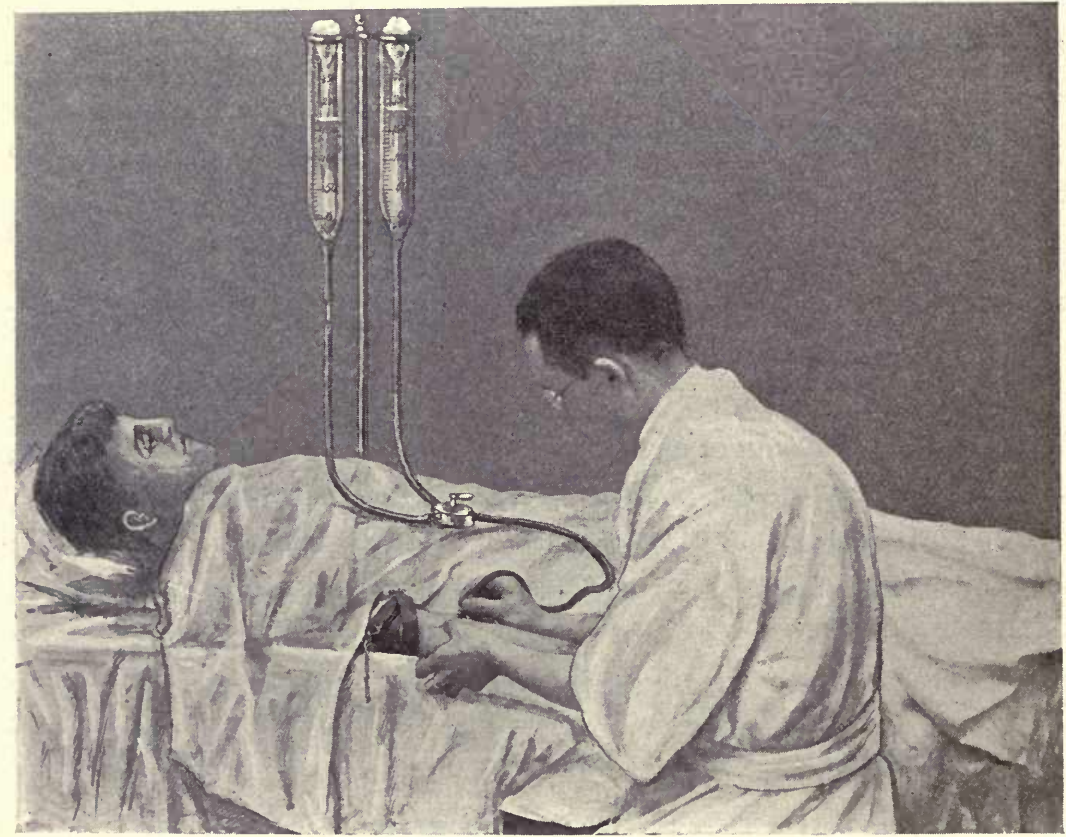

Fig. 142.-Intravenods Injection of Salvarsan.

With this apparatus the operator may give an injection without assistance. Notice the three-way cock, which permits the flow of salt solution or salvarsan solution at will. Usually a tourniquet composed of a simple rubber tubing and held in position by a hemostat is better than the one shown, as the operator can quickly release it with least disturbance and loss of time. Note the funnels in both containers for straining the salvarsan solution and distilled water or salt solution. (After the apparatus of Boehm.)

Preparation of Neosalvarsan Solution.-This drug is readily soluble in water, forming a clear solution which is ready for use. File the neck of the ampule, cleanse it with alcohol, and break open. The contents are emptied directly into a flask containing 200 c.c. of warm, sterile, freshly distilled water. On gentle agitation the drug rapidly dissolves. Hot water should not be used, nor should a solution be heated once it has been made. From the mixing flasks the solution is poured into the cylinder through a piece of sterile gauze, which filters out any bits of 
glass or other insoluble particles. For administration any simple apparatus, such as that shown in Fig. 143, answers all purposes.

Neosalvarsan can also be given intravenously in a concentrated form by dissolving the drug in 20 c.c. of sterile distilled water. It is injected by means of an ordinary glass syringe or by the special syringe devised for this purpose shown in Fig. 135.

Injecting Apparatus.-A number of different apparatus for the ad-

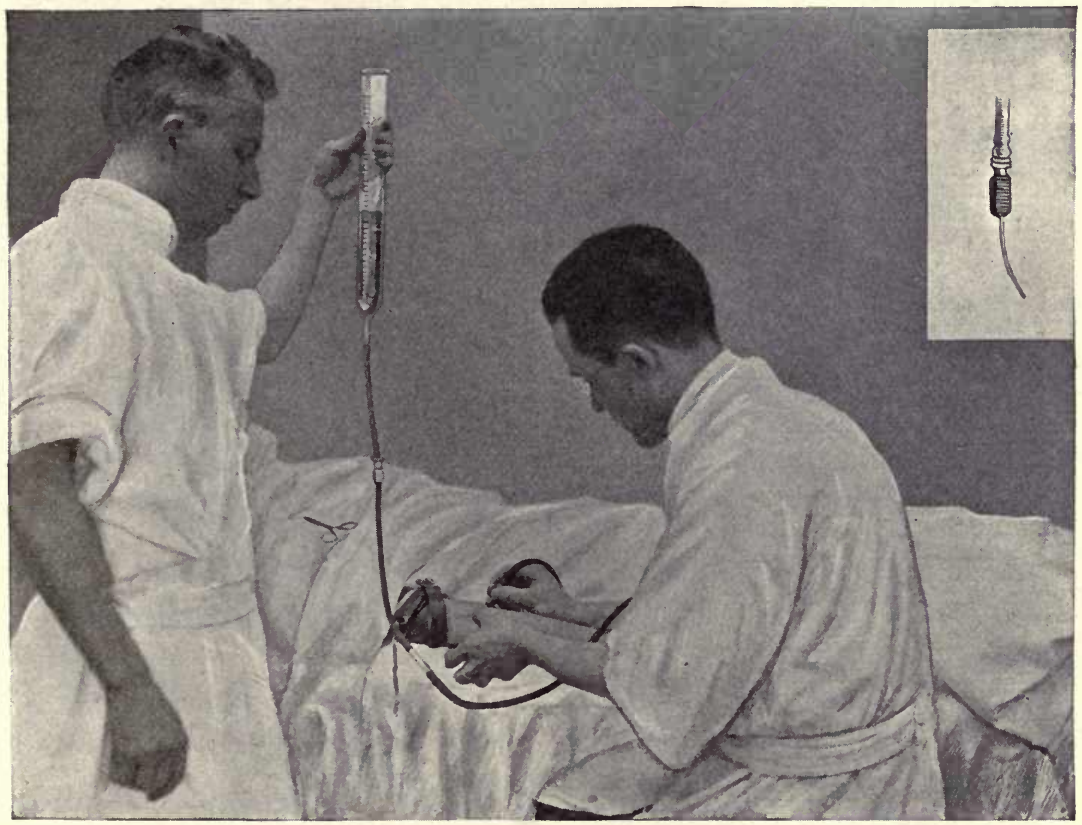

Fig. 143.-Method of Making Intravenods Injection by Gravity.

This method is suitable for the intravenous administration of salvarsan or antistreptococcus serum, etc. The needle has been entered into a prominent vein (indicated by a flow of blood); the tubing has been attached by means of a metal tip which fits the needle easily and snugly; the tourniquet has been loosened and the injection is being given.

ministration of salvarsan and neosalvarsan have been invented and marketed. That shown in Fig. 142 is well adapted for the purpose; one cylinder carries sterile normal salt solution and the second holds the salvarsan or neosalvarsan solution. By fastening the cylinders to an upright rod attached to the side of the table their height may be adjusted as desired, and by means of the special cock the operator may insert the needle and allow the salt solution to flow in until he is sure of having satisfactorily penetrated the vein, when he may turn on the salvarsan 
solution. In this manner the physician is enabled to give an injection without assistance.

Or, if desired, the simpler apparatus shown in Fig. 143 may be used. This consists of a single cylinder to the narrow lower end of which about five feet of rubber tubing are attached. A piece of glass tubing is inserted at the lower end to serve as a window, and at the end there is an arrangement whereby it can easily be attached to the needle used for making venipuncture. A clamp is placed on the tubing at some convenient place, where it may be operated by an assistant.

Whatever apparatus is employed, it should be sterilized before use. The cylinders and needle are boiled in an office sterilizer, and the tubing is cleansed by running sterile salt solution or water through it. The needle should have a sharp point with a short beveled edge, as a longpointed needle may pierce the vein through and through. Sufficient sterile distilled water or normal salt solution is added to fill the rubber tubing, while the cylinder should contain an additional 10 or 15 c.c. If the double cylinders are used (Fig. 142), one should contain from 20 to 30 c.c. of salt solution or water and the second the solution of the drug. The solution of drug is then filtered into the second cylinder, and the pinch cock opened for a minute to be sure that all air has been expelled.

2. The patient should lie on a bed or on an operating table. An arm - usually the patient's left in the case of right-handed operators-is prepared by placing a few towels around it and a firm tourniquet is applied above the elbow. Whatever material is used, whether rubber or a broad muslin bandage, it should be fastened with a hemostat, for when it becomes necessary to unfasten it, this may be done quickly and with least disturbance by the operator, who simply unfastens the hemostat. The skin over a prominent vein may be cleansed with green soap and water, followed by alcohol and 1:100 bichlorid solution, or simply by adding one or two coats of 10 per cent. tincture of iodin, which is washed off with cotton and alcohol just before the needle is inserted. In fat subjects a vein can frequently be felt when it cannot be seen. Occasionally it may be necessary to infiltrate the skin with sterile eucain solution and expose the vein by incision.

3. The operator now passes the needle into a vein. With experience, considerable skill is gained in performing this little operation. A free flow of blood indicates that the needle has been properly inserted, and one can easily tell by the sense of touch whether the needle is free in the lumen. The tourniquet is then quickly and deftly released by the operator, the clamp on the tube is opened, and while the blood is flowing from 
the vein and the saline solution or water from the tubing the nozle of the latter is fitted into the needle quickly and securely while the latter is held firmly in the vein. The appearance of bulging at the side of the vein and the occurrence of pain indicate that the needle has not been properly inserted. In this event the clamp should be fastened, the needle withdrawn, the tourniquet adjusted, and another vein punctured. It is useless to attempt to enter the same vein at the same point.

4. The introduction of a full dose of salvarsan or neosalvarsan will usually take from ten to twenty minutes or thereabouts. If the flow is retarded, the needle may be turned gently and slightly so as to change the relation of the bevel to the wall of the vein.

5. When salt solution forms the first portion of the injection, no harm has been done if perivascular infiltration occurs. This method gives added assurance to the operator; indeed, it should never be omitted when salvarsan is being injected, and it is a good general rule to have the first portion of the injection consist of normal salt solution.

6. After the requisite dose has been injected, a few cubic centimeters of salt solution are again permitted to flow into the vein, so that the tubing and needle are washed free from salvarsan, and at no time does the drug come in contact with the tissues.

7. The needle is then quickly withdrawn, and the site of the puncture sealed with collodion and cotton after the iodin has been removed by washing with alcohol.

After-care of the Patient.-In the majority of instances the administration of salvarsan is not followed by unpleasant symptoms. This depends, however, to a considerable extent upon the nervous constitution of the patient. Many persons will complain of a feeling of fullness and may perspire freely for a short time. There may be slight pain at the site of injection and in the axilla of the injected side. As was previously mentioned, salvarsan should be administered at the patient's home or in a hospital, followed by rest in bed until the next morning. When neosalvarsan is injected, robust persons may, after resting for an hour or so, travel homeward. Occasionally severer reactions follow salvarsan administration, and these may be considered under the head of after-effects.

After-effects of Salvarsan.-Within an hour or two after its administration arsenic is excreted by the kidneys and bowels and nausea may be complained of. If catarrh of the stomach is present, severe vomiting may ensue. The nausea is relieved by sipping a little hot water; hot applications over the stomach and small amounts of carbonated water or cham- 
pagne will usually control the vomiting. It may occasionally be necessary to administer $1 / 8$ grain of morphin hypodermically, especially if the patient is of a neurotic temperament. Headache may occasionally develop, and is due to a neurotic condition, constipation, anxiety, hunger, or possibly to an increase in the exudate of the syphilitic process. Diarrhea may be observed in a few cases; this is readily controlled by the administration of bismuth. Chills and fever are more infrequent at the present time, the result probably of using freshly distilled water and somewhat smaller doses of the drug. In some cases an arsenic rash, in the form of an erythema, may follow injection. This is not the Jarisch-Herxheimer reaction, which is found only in syphilis, the arsenic rash having been observed in non-syphilitic persons injected with the drug. There is no evidence to show that salvarsan or neosalvarsan will injure healthy kidneys, although a mild transient albuminuria may follow the injections in some instances. When, however, the kidneys have been damaged by mercury, salvarsan may give rise to an acute irritation, and, indeed, Wechselmann ascribes many of the salvarsan casualties to this condition, and issues the warning that, while mercury may follow salvarsan, it should never precede it. The good general effects following the administration of salvarsan are manifested, as a rule, in a sense of well-being, and not infrequently patients who are anemic, poorly nourished, and despondent in a short time become healthy, active, and cheerful. This may be due in part to a psychic effect, but there is frequently evidence of a far-reaching change in the nutrition of the patient.

The Jarisch-Herxheimer Reaction.-This reaction manifests itself in the development of a rash, the extension or aggravation of an existing eruption, or an inflammatory reaction in any syphilitic tissue the result of treatment. It has been observed in the course of mercurial treatment, and before the discovery of the Spirocheta pallida and the Wassermann reaction it was regarded as of considerable diagnostic importance. Any aggravation of syphilitic symptoms following the administration of salvarsan or mercury has been interpreted as a Herxheimer reaction. The cutaneous reaction is manifested by edema, redness, pain, and the mucous patches show a similar reaction. Gummas become swollen, may ulcerate, and show increased exudation. The lancinating pains of locomotor ataxia may be augmented, and various paralyses, due to pressure, may follow in those nerves that traverse bony canals. These effects are also known under the name of neurorelapses. They usually appear two or three months or even four or five months after treatment, and 
they were at first believed to be due to the contained arsenic and were regarded as constituting a special danger attending the use of salvarsan.

Various explanations for this phenomenon have been offered: Ehrlich believes that it indicates failure of the injected dose to produce complete destruction of the spirochetes, with temporary stimulation of the microorganisms to increased multiplication and activity. He very pertinently compares the neurorelapse or Herxheimer reaction to the extensive development of individual bacterial colonies on agar plates, when but few microörganisms are present, as contrasted with their small size when the number is large. The so-called provocative positive Wassermann reaction may be considered as a part of this reaction.

\section{INTRAMUSCULAR INJECTION}

As was previously stated, this route of administration is not generally employed at the present time because of the local irritative effects of salvarsan especially. When slow absorption and elimination are desired, or when intravenous injections are impossible, as in the case of very young children, this method may be adopted. When salvarsan is to be given, the clear, concentrated alkaline solution is generally used. Neosalvarsan is to be preferred, because it is less irritating than salvarsan. It may be given in a concentrated aqueous solution or suspended in sterile oil with the addition of a local anesthetic, such as eucain or creosote. The injections are made, under strict antiseptic precautions, in the gluteal region, into the upper and outer quadrant of the muscles. The syringe and the needle should be sterilized and the injection prepared. After cleansing the skin with alcohol the needle is quickly and boldly plunged into the deep tissues. The syringe may then be detached to ascertain if blood flows, which would show that a vein has been punctured and require a reinsertion; if blood does not appear, the barrel should be reattached and the injection slowly given.

Intraspinous Injection of Neosalvarsan.- Owing to the fact that a drug injected intravenously may not reach the tissues of the central nervous system, salvarsan and neosalvarsan have not fulfilled the early expectations, apparently chiefly for the reason that they cannot reach the spirochetes. It would seem, therefore, that adequate treatment of syphilis of the central nervous system consists in the direct application of the remedy to the infected tissues themselves. Wechselmann ${ }^{1}$ and Marinesco ${ }^{2}$ have injected salvarsan, and Marie and Levaditi ${ }^{3}$ and others

${ }^{1}$ Deutsch. med. Wochenschr., 1912, 38, 1446.

2 Zeitschr. f. phys. u. Therap., 1913, 17, 194.

${ }^{3}$ Bull. et Soc. med. d. hôp., Paris, November 18, 1913. 
have given neosalvarsan, directly into the spinal canal. The results, however, were either dubious or frankly dangerous, and the injections were followed by severe and alarming symptoms, due mainly to the production by the drug of direct irritation upon the sensitive nervous system. Ravaut ${ }^{1}$ has found that the use of hypertonic solutions of neosalvarsan are better borne and of value in the treatment of special cases. Wile $^{2}$ has also employed this method, and has found it of some value in cerebrospinal syphilis. Tabetics presenting no bladder or rectal symptoms were found to do especially well. As is to be expected, the earlier the treatment is instituted, the better are the results. This form of treatment is, however, to be regarded as dangerous, and is to be used only in selected cases, and with a full understanding of the risksincurred. Wile has given the following technic:

"The solution used for injection consists of a 6 per cent. solution of neosalvarsan in distilled water. This solution is hypertonic, and made of such concentration that each minim must contain $3 \mathrm{mg}$. of the drug. The dosage injected is from 3 to 12 mg. - that is, from one to four drops of the solution, which is made up as follows:

"An ampule containing $0.3 \mathrm{mg}$. of neosalvarsan is dissolved in 5 c.c. of freshly distilled water. If the ampule contains $0.6 \mathrm{gm}$., 10 c.c. of water are used. In both solutions each drop will contain $3 \mathrm{mg}$. of the drug. The syringe employed for the injection is accurately graduated in drops. The patient is placed in a position for lumbar puncture, either sitting or lying, according to the choice of the operator. The puncture is then made with the needle, the end of which fits the graduated syringe. After a few drops of the spinal fluid have flowed out of the cannula, or a greater quantity if a diagnostic puncture is desired at this time, the syringe is fitted into the needle, and the fluid is allowed to run back into the syringe barrel, thus mixing with the amount of the drug in the barrel. The mixed spinal fluid and drug are then gently forced into the canal, and slight suction is made on the syringe to withdraw a second amount of fluid, which washes out the needle. This is then reintroduced, the needle is quickly withdrawn, and the patient placed in the Trendelenburg position, in which position he is allowed to remain for at least one hour."

\section{INTRASPINOUS INJECTION OF SALVARSANIZED SERUM}

In this method the salvarsan or neosalvarsan is injected intravenously in the usual manner, and shortly afterward blood is withdrawn, the serum separated, heated to $55^{\circ} \mathrm{C}$. for half an hour, and a portion injected intraspinously. In this technic, which has been worked out largely by Swift and Ellis, the drug is highly diluted, and therefore is not likely to produce much irritation. In addition, some of the good effects may be due to the presence of antibodies in the serum itself. This method constitutes the most useful form of intraspinous medica-

${ }^{1}$ Ann. de Med., 1914, 1, 49.

2 Jour. Amer. Med. Assoc., 1914, lxii, 1165; ibid., 1914, lxiii, 137. 
tion thus far proposed. The details of this technic are given in Chapter XXXI.

Mention has also been made, in the preceding chapter, of a method of intraspinous medication consisting in the injection of a combination of the patient's serum and salvarsan or neosalvarsan mixed in vitro. This method is to be regarded as more dangerous and probably less efficient than that of Swift and Ellis, and as yet in the experimental stage. Recently Fordyce ${ }^{1}$ has given the following technic (Ogilvie):

"Fifty c.c. of blood are drawn into a centrifuge bottle and centrifuged twice. It is important to have the serum clear and free from fibrin and blood-cells. To obtain the requisite amount of the drug old salvarsan is mixed in the usual way, in the proportion of $0.1 \mathrm{gm}$. to 40 c.c. of fluid, care being taken not to overalkalinize; 0.4 c.c. of this solution is the equivalent of $1 \mathrm{mg}$., and is taken as the standard for measuring the dosage. For this purpose a 1 c.c. pipet graduated in hundredths should be employed. The desired amount of salvarsan is added to from 12 to 15 c.c. of the serum, shaken to and fro to mix thoroughly, and then placed in the incubator at $37^{\circ} \mathrm{C}$. $\left(98.6^{\circ} \mathrm{F}\right.$.) for one hour, after which it is inactivated for half an hour at $56^{\circ} \mathrm{C}$. $\left(132.8^{\circ} \mathrm{F}\right.$.). The latter is the most important step in the technic; the spirocheticidal properties of the serum are markedly increased by heating.

"Salvarsanized serum, prepared according to this method, must be used fresh, that is, within three hours of the time that it is made up. Patients should be prepared for its administration as for intravenous injection, with a laxative the night before and only a light meal two hours prior to the treatment.

"A lumbar puncture is made, and an amount of fluid equivalent to the amount introduced is withdrawn. While the needle is in situ, the barrel of a Luer syringe is connected by means of a piece of rubber tubing. Spinal fluid is allowed to fill this to expel the air, and the serum is then permitted to flow in by gravity. After its administration the patient should lie flat without any pillows, the foot of the bed being kept elevated for several hours. He must be kept in bed for at least twentyfour hours, in some cases forty-eight or seventy-two hours. Failure to do this may result in unpleasant symptoms, as pain in the extremities, headache, anesthesia, and bladder and rectal paresis."

The proper dose is still a matter of doubt, and a note of warning must be sounded against employing too large amounts. Fordyce believes that the limit of safety lies within $0.5 \mathrm{mg}$. It is better to begin with a dose of $0.25 \mathrm{mg}$., repeating or gradually increasing this according to the tolerance of the patient. The intervals between doses should be two weeks or longer.

In the treatment of syphilis of the central nervous system the intravenous method may first be tried, giving a series of six or eight injections of salvarsan, beginning with a dose of from 0.25 to $0.3 \mathrm{gm}$., and injecting it at intervals of from one to two weeks. At the end of this time the blood and spinal fluid should be tested, and if it is found that

${ }^{1}$ Jour. Amer. Med. Assoc., 1914, lxiii, 552. 
no marked effect has been obtained either clinically or serologically, a course of intraspinous injections may be considered.

\section{CONTRAINDICATIONS AND PRECAUTIONS IN SALVARSAN THERAPY}

When it is remembered that millions of doses of salvarsan and neosalvarsan have been given by thousands of different physicians, by many and diverse methods, the relative safety of the drug is apparent. The fact remains, nevertheless, that patients have succumbed soon after receiving an injection who would not otherwise have died at that time. These fatalities cannot be explained on a purely toxicologic basis. Recently Wechselmann ${ }^{1}$ has reviewed the deaths following salvarsan therapy and has arrived at the conclusion that "insufficiency of the kidney, and not hypersensitiveness of the brain, is the point of the entire question of salvarsan fatalities." He is very emphatic in warning against mixed mercurial and salvarsan treatment, especially if salvarsan is given after a course of mercury, for the latter drug is a renal irritant and may lead to prolonged retention of the salvarsan, which may undergo a process of reduction in the tissues and form one of the poisonous products that Ehrlich has called "arsenoxid."

Contraindications to salvarsan therapy may be divided into two main groups:

1. Those to whom the injection may be dangerous on account of the reaction that may follow, as, for example, cases of early cerebral lues with cranial nerve manifestations of an exudative character; also cases of tabes with beginning optic atrophy. In these patients salvarsan or neosalvarsan should be given with extreme caution and only in small doses, and frequent examinations of the eyes should be made.

2. Those with extensive disease of the circulatory system, such as severe uncompensated heart disease, coronary sclerosis, and extensive aneurysm; also cases of diabetes mellitus, severe nephritis, ulceration of the stomach, and advanced tuberculosis or carcinoma.

In this connection I may cite here the precautions laid down by Wechselmann, director of the dermatological department of the Rudolf Virchow Hospital, in whose clinic over 25,000 doses of salvarsan have been given. These precautions are:

1. To use the most exact technic.

2. To employ a dose of the drug carefully adapted to the individual case.

1 "The Pathogenesis of Salvarsan Fatalities," translated by Martin, 1913, Fleming Smith Co., St. Louis. 
3. To make careful observation by the most exact chemical and microscopic examination of the urinary secretion when employing salvarsan. This holds good particularly when the combined treatment is employed.

4. The conjoint use of salvarsan with heavy mercurial treatment is dangerous! If one will use the combined treatment, then let him give mercury very carefully many days after giving the last salvarsan injection, but he must never reverse this rule.

5. Consider carefully every general reaction or rise of temperature following the use of salvarsan, and make a thorough investigation as to its causes.

\section{VALUE OF SALVARSAN AND NEOSALVARSAN IN THE TREATMENT OF SYPHIIIS}

It is too soon to judge of the ultimate value of salvarsan in the treatment of syphilis. It may be stated, however, that the immediate effects are so beneficial that the drug is to be regarded as the best we possess for the treatment of lues. As is to be expected, the best results are secured when the remedy is administered early, and, of course, when tissue changes have occurred no drug can be expected to restore a lost function, although it may bring about considerable improvement by causing the disappearance of syphilitic tissue. In long-standing cases salvarsan may, at least, hold the symptoms in abeyance, and effectively prevent progress of the disease. It is especially valuable in patients who cannot tolerate mercury. The remarkable efficacy of salvarsan is attested by the rapidity with which spirochetes disappear from primary sores and from secondary lesions, the manner in which they disappear being frequently little short of marvelous. There can be no doubt but that salvarsan is a powerful spirocheticide, having but remarkably little toxic effect upon the body-cells. It would appear that in order to rid the tissues of the spirochetes it is only necessary to bring the drug into contact with them. For this reason newer and better methods of administration are bound to increase the value of the drug. Barring the wellrecognized contraindications, salvarsan may be used in practically all stages of the disease. In the later stages of the infection, in which the central nervous tissue is involved, the physician must bear in mind the possible danger of a neurorelapse or a Herxheimer reaction occurring. While salvarsan may do no good in late cases, since it cannot restore lost nerve tissue, it may alleviate symptoms and prevent progress of the in- 
fection. In the treatment of any case of syphilis the best results are not secured from following any course of treatment according to a rule of thumb; they are obtained only by a suitable combination of expert clinical knowledge and training in serologic technic. All possible aid should be enlisted in the treatment of the disease.

It is beyond the scope of this book to present either case reports or a lengthy discussion of salvarsan in the treatment of the various stages of syphilis; suffice it to say that, in the absence of contraindications, the drug may be administered whenever living spirochetes are present in a patient's tissues, as evidenced either by clinical symptoms or a positive Wassermann reaction. The method of administration selected should be that which will best bring the drug into contact with the diseased tissues.

\section{SALVARSAN IN THE TREATMENT OF NON-SYPHIIITIC DISEASES}

While salvarsan therapy has achieved its greatest success in the treatment of syphilis, its influence on certain non-syphilitic diseases has been so pronounced that it is being used in an ever-increasing number of diseases, the aim being to thus enlarge its sphere of usefulness. A recent systematic study of the subject has been made by Best. ${ }^{1}$ Good results have been reported by various observers in the following diseases:

Frambesia (Yaws).- The results achieved in this disease from the use of salvarsan have equaled those obtained in syphilis. Two thousand four hundred and thirty cases have been reported, a cure having been effected in all but a few instances. Since the use of salvarsan has come into favor hospitals for the treatment of frambesia have been closed.

Relapsing Fever.-In the treatment of this disease the drug has likewise had a remarkable effect. Of 195 cases reported, in most of them the microörganisms could not be found in the blood after an injection, and in none of the cases were relapses observed to occur.

Filariasis.-Salvarsan has been found effective in killing the Filaria sanguinis hominis and in ridding the blood of these parasites. Of course, in elephantiasis it is impcssible to restore the affected parts to their normal appearance.

Vincent's Angina.-An immediate improvement and rapid healing have been reported as following either an intravenous injection or the local application of the drug in the form of a dusting-powder or in suspension in glycerin.

${ }^{1}$ Jour. Amer. Med. Assoc., 1914, lxiii, 375. 
Duhring's Disease (Dermatitis Herpetiformis), Scurvy, Chorea, Malaria, Acanthosis Nigricans, Ulcus Tropicum, Variola, and Verruca Plana. -In all these affections salvarsan has been found to exert a beneficial and curative effect, either by direct action upon the microörganisms present or as the result of the alterative and stimulating effect of the arsenic.

In Aleppo boil, leprosy, lupus vulgaris, tuberculosis, anemia, keratosis follicularis, lichen planus, mycosis fungoides, pellagra, and pityriasis rubra, good or indifferent results have been reported. In many conditions it would appear that salvarsan exerts a direct germicidal effect, and in others the beneficial results appear to be dependent upon a certain tonic, stimulating, and alterative effect of the arsenic. In chancroid, scarlet fever, Hodgkin's disease, psoriasis, trichinosis, and trypanosomiasis the drug does not appear to exercise any influence, which is due probably, in the last-mentioned disease, to the fact that trypanosomes are much more likely to become arsenic-fast than are the spirochetes.

\section{CHEMOTHERAPY IN BACTERIAL DISEASES}

While studies in chemotherapy have been largely confined to the protozoan infections, similar investigations are being made in bacterial infections, especially in tuberculosis and the pyogenic disorders.

As has previously been stated in Chapter XXIX, progress in this direction has been made by Lamar in the treatment of pneumococcus infections with mixtures of pneumococcus immune serum, sodium oleate, and boric acid. Morgenroth has conducted similar researches in animal infections with the pneumococcus, finding that whereas quinin, hydroquinin, and hydrochlorisoquinin have no effect on the pneumococcus, ethyl hydrocuprein was capable of arresting the infection in 50 per cent. of the animals when given six hours after inoculation, that is, at a time when the animals were severely infected.

It is to be hoped, therefore, that further researches will result in the discovery of substances that have a marked bactericidal action and that are yet but slightly, if at all, toxic for the body-cells, that is, substances in which bacteriotropism greatly exceeds organotropism. It would appear that this discovery is possible and probable, but it can be accomplished only as the result of persistent and prolonged research. Probably the most wonderful discovery possible in medicine would be a specific remedy for tuberculosis, and this may be within the realms of chemotherapy. 


\section{CHEMOTHERAPY IN MALIGNANT DISEASE}

Brief mention may be made of certain recent advances that have been made in the experimental chemotherapy of cancer in the rat and mouse. While the pathogenesis of malignant disease is still unknown, specific therapeutic measures of this kind have been undertaken on the assumption that the cancer-cell is different from the normal cell from which it originated, and that certain substances will show a selective affinity for them; in other words, that specificity may be present among organotropic substances. Here, of course, the difficulties are great, because the structural, biologic, and functional differences between the normal cell and the cancer cell may be slight, and substances must be found that possess a high affinity for the cancer-cell only.

That this condition may indeed exist has been indicated by the experiments of Wassermann and his collaborators. These investigations were based upon the discovery, by Gosio, that sodium selenate and sodium tellurate are more rapidly reduced by cancer-cells than by normal cells, and that this reduction takes place within the bodies of the cells. Experiments on mouse tumors showed that the injection of these substances into the growths may actually lead to their destruction, the explanation, according to Neuberg and Caspani, being that certain compounds of the heavy metals in colloidal form favor self-digestion (autolysis) of the tumor cells.

The problem now resolved itself into finding some substance that would carry the metals to the tumors, or, as Wassermann said, "the building of rails which would reach the tumor and by which the selenium could travel," as local injection was obviously out of the question from the practical standpoint. Eosin, being a substance endowed with great powers of diffusion, was selected for the purpose, and a number of eosinselenium compounds were tried out. The results were encouraging, as in a number of instances the tumors became soft and sloughed away or their further growth was checked. "If three consecutive daily intravenous injections of the eosin-selenium compound are given in $2.5 \mathrm{gm}$. doses for 15-gram mice, a distinct softening and elasticity of the tumor are noticed on the fourth day; on the fifth day a fourth injection of the same dose is given, after which there is no longer the feeling of a solid tumor, but rather that of a fluctuating cyst in which small, movable tumor particles can be discovered. After the fifth injection on the seventh day this soft mass becomes smaller, the capsule becomes lax, and the configuration of a circumscribed tumor can no longer be dis- 
tinguished, but only a long edematous cord can be felt. Usually, as a result of the sixth injection, in favorable cases, the absorption and diminution proceed so that one gets the feeling of an empty sac. In case no intercurrent disease occurs, the animal is cured in about ten days, with a disappearance of all remnants of the tumor."

As these results were observed after intravenous injection of the mixtures, and as no injury to the body-cells was apparent, it seems that a step forward has really been taken; at least, these investigators have shown that it is possible for chemical substances to pass from the blood and attack tumor cells. Copper and tin have been found to possess a more marked affinity for tumor cells, and the whole subject is probably just at the threshold of further discoveries that may be applied with great benefit to the treatment of human malignant disease. 


\section{PART V \\ EXPERIMENTAL INFECTION AND IMMUNITY}

INTRODUCTORY

Methods.-The exercises and experiments herein outlined are for the purpose of teaching the principles of infection and immunity by actual laboratory work, whereby the student performs the experiments and is taught to observe the results. At the same time a knowledge of the technic of immunologic methods is obtained. The instructor in charge of such an experimental course may choose certain experiments in outlining a course according to the allotment of time and purpose of the instruction.

In all instances I have outlined the experiments according to average conditions. It is readily understood that differences in the virulence of a certain culture or the weight and physical condition of an experimental animal will require that the doses advised be changed to meet the conditions.

As a general rule, a course should be concentrated, with exercises at least three times a week in order that the student may follow his work closely and have an opportunity for making adequate and accurate observations.

An attempt is made to bring out the important points of an experiment by a few pertinent questions. It should be impressed upon the student that the mind should be held open for observation and that unexpected and untoward results may be obtained which, however, are always of interest and always instructive when the experiment is conducted in a careful, methodical, and conscientious manner. Frequent general discussions should be held for a general review of the subject and correlation of facts and observations. In my experience students are eager for the work and seldom fail to suggest additional work in the nature of original research.

The Student.-1. The student should work protected by an apron or gown with short sleeves; he should be careful of the hands and avoid abrasions and cuts and carefully wash and disinfect the hands after the work of each day has been completed. 
2. The working table should be set in order after each day's work; pipets and soiled glassware should be properly disposed of, instruments thoroughly cleansed, and the table wiped off with 1 per cent. formalin solution. It should be impressed upon the student that good and accurate work is seldom done amid disorderly and dirty surroundings.

3. The student must never sacrifice accuracy for speed; painstaking and accurate work is always to be desired; speed is gained only with practice.

Records.-Each worker should record in writing in a suitable notebook his observations of the various tests and experiments. Not infrequently unexpected results are obtained, and it is important to understand and explain these as correctly as possible. Note-books should be subject to frequent inspection; it is not necessary to write out a description of the technic, but the results of the experiment and answers to the questions should be set forth clearly and with sufficient detail.

Animal Experiments and Autopsies.-In all experiments calling for operative procedure an anesthetic is to be used in order that unnecessary pain be avoided. Ordinarily, ether is to be employed, or in rabbits the rectal injection of 0.5 to 1 gram of chloral hydrate dissolved in 5 to 10 c.c. of water. Autopsies are to be carefully conducted, and the lesions described in writing. After autopsy the table is to be scrubbed and cleansed with a solution of formalin, and the carcass disposed of by incineration or placing in a solution of formalin until removed and otherwise disposed of.

\section{EXERCISE 1.-ACTIVE IMMUNIZATION OF ANIMALS}

Experiment 1.-Production of Agglutinins, Bacteriolysins, and Opsonins

1. Secure a culture of Bacillus typhosus, an old laboratory culture of cholera bacilli, and a culture of Staphylococcus aureus.

2. Proceed to immunize two rabbits with each culture by intravenous injections according to the technic described on page 168 .

3. One week after the last injection the animals are to be bled and the serums secured after the first method described on page 42 .

(a) Define the meaning of antigen.

(b) What are antibodies?

(c) What is meant by active immunization?

(d) What precautions should be observed in handling these antigens?

(e) What precautions are to be observed in giving intravenous injections? 
(f) What antibodies do you suspect are present in these immune serums?

\section{Experiment 2.-Production of Precipitins}

1. Immunize a rabbit with sterile horse serum by intravenous injections after the first method as described on page 70; immunize a second rabbit after the second method.

2. Immunize two rabbits with sterile human serum after the first method.

3. Immunize two rabbits with cow milk by intravenous injections after the third method.

4. Bleed the animals from the carotid artery one week after the last injection and preserve the serums in a sterile condition.

\section{Experiment 3.-Production of Hemolysins}

1. Immunize a rabbit with washed sheep corpuscles by intravenous injections after the first method as described on page 72 .

2. Immunize a second rabbit with sheep cells after the second method.

3. Immunize a third rabbit with washed human cells after the third method.

4. Immunize a fourth rabbit with washed human cells by intraperitoneal injections as described on page 73 .

5. Bleed the animals in four days to a week after the last injection and separate the serums. 70.

6. Prepare a portion of human amboceptor dried on paper as described on page

7. Preserve the balance of the serum and the other serums with equal parts of neutral glycerin.

\section{Experiment 4.-Production of Cytotoxin}

1. Immunize two rabbits with dog kidney after the method described on page 73.

2. After the immunization has been completed, preserve the serum with 0.2 per cent. phenol in sterile ampules.

While these various immune serums are being prepared the student is engaged with the work on infection, or if the subject of immunity is taken up at once, immune serums should be furnished by the instructor.

\section{EXERCISE 2.-INFECTION}

\section{Experiment 5.-Experimental Pneumonia}

1. Grow a virulent culture of pneumococcus in glucose serum broth for fortyeight to seventy-two hours at $37^{\circ} \mathrm{C}$. until a good rich growth is secured. Prepare and stain smears by Gram's method.

2. Pass a large catheter which has its tip cut off into the trachea of a dog until it has passed into one of the primary bronchi. By means of a syringe inject quickly 15 c.c. of culture; remove the catheter and mouth-gag. Take the rectal temperature and leukocyte count previous to inoculating.

3. Inject a second dog in the same manner with 2 c.c. of culture.

4. Inject a third dog with 5 c.c. of culture intravenously. 
5. Observe all animals for forty-eight hours, taking the rectal temperature night and morning. Make leukocyte counts every four hours during the day. Make physical examination of the chest.

6. Autopsy the animals under aseptic precautions and with complete anesthcsia.

7. Culture the heart's blood of each in serum bouillon.

8. Culture the pulmonary lesions in serum bouillon.

9. Prepare smears of the heart's blood and lesions and stain with methylene-blue and Gram's stain.

10. Remove consolidated portions of lung and place in 5 per cent. formalin. After twenty-four hours, cut sections which are passed through by the paraffin method and stained with hematoxylin and eosin, methylene-blue, and Gram's stain.

(a) Did any of the animals show evidences of infection?

(b) Are there evidences of pneumonia? How do these lesions compare with those of human pneumonia?

(c) How do you explain their production?

(d) Does the animal receiving the smaller dose of pneumococci intrabronchially show evidences of pneumonia? If not, why not? Does this show a numerical relationship of bacteria to infection?

(e) Were the temperature changes similar to those observed in human lobar pneumonia?

(f) Did leukocytosis occur and if so, why?

(g) Are there any evidences of pleuritis and if so, how do you explain its production?

(h) Did the dog receiving the pneumococci intravenously show evidences of pneumonia? Does this bear any relation to the question of the route of introduction of bacteria to infection?

(i) Are the pneumococci seen in the smears of the lesions and blood encapsulated? If so, what is the significance of these capsules? Compare these cocci with those shown in the smear of the culture before injection? Are the capsules lost in the artificial culture-media?

(j) Discuss the question of the possible modes of infection in human lobar pneumonia, especially inspiratory pneumonia.

\section{EXERCISE 3.-TOXINS}

\section{Experiment 6.-Diphtheria Toxin}

1. Inoculate tubes of neutral or slightly alkaline bouillon with a virulent culture of diphtheria bacilli (Park-Williams Bacillus No. 8 being especially desirable).

2. Grow at $35^{\circ} \mathrm{C}$. for five days and filter the culture through a Berkefeld filter.

3. Inject a 250 - to 300 -gram guinea-pig in the median abdominal line with $\mathbf{0 . 5}$ c.c. of the filtrate (toxin).

4. Heat 1 c.c. of toxin at $60^{\circ} \mathrm{C}$. for an hour and inject 0.5 c.c. into a second guineapig subcutaneously. 
5. Observe the animals for at least four days, especially for the development of a characteristic edema about the site of injection.

6. After death perform a careful autopsy, paying particular attention to the bloody edema at the site of injection and marked hyperernia of the suprarenal glands. Make cultures on Loeffler's blood-serum medium of edematous area, peritoneum, and heart blood.

(a) To what has death been due?

(b) Has diphtheria toxin a selective affinity for any particular tissue?

(c) Has heat any effect upon diphtheria toxin?

(d) Is there a period of incubation before symptoms develop and why?

\section{Experiment 7.-Method of Testing the Virulence and Toxicity OF Diphtheria Bacilli}

1. Make a culture of a patient harboring the bacilli on a tube of Joeffler's serum medium. Inoculate at $35^{\circ} \mathrm{C}$. for from eighteen to twenty-four hours; prepare a smear and stain with Loeffler's methylene-blue. If diphtheria bacilli are present, they must be isolated in pure culture. Never attempt a guinea-pig test with an impure culture.

2. Isolate by the "streak" method, on plates of blood-serum.

3. Inoculate a tube of 1 per cent. glucose bouillon, which is neutral or slightly alkaline, with several different colonies.

4. Incubate at $35^{\circ} \mathrm{C}$. for three days, keeping the tube in a slanted position in order to give the culture as much oxygen as possible. If a good growth does not appear in twenty-four hours, transplant to another tube of bouillon until the bacilli have been "educated" to grow on the medium.

5. Examine for purity. Select a 250- to 300-gram guinea-pig and inject 2 c.c. of the unfiltered culture in the median abdominal line. Animals over the weight specified are more resistant and less reliable for test. The unfiltered culture is used, since toxin is but one element of the disease-producing power of diphtheria bacilli, and toxin production in bouillon may not be a true index of the toxin production in mucous membranes.

6. Carefully observe the animal for at least four days. Even slight toxemia, especially if accompanied by edema at the site of injection, should be regarded as a positive result.

7. After death perform a careful autopsy. Make cultures of the edematous area, peritoneum, and heart blood. Observe whether acute hyperemia of the suprarenal glands is present.

8. Not infrequently animals showing mild or even an absence of the symptoms of toxemia developparalysis of the hindquarters two or three weeks later. According to Ehrlich, this paralysis is due to the action of "toxon," a toxic substance secreted by the bacillus or, as believed by others, a modified form of toxin.

9. To prove that diphtheria was the cause of the toxemia or death, mix 2 c.c. of the culture in a test-tube with 1 c.c. of diphtheria antitoxin (500 units). After standing aside for an hour at room temperature, inject the mixture subcutaneously in the median abdominal line of a 250- to 300-gram guinea-pig.

(a) Is the diphtheria bacillus aggressive?

(b) What evidence have you that the lesions and death are due to a toxin? 
(c) Why does the use of diphtheria antitoxin make the test conclusive? Would tetanus antitoxin be capable of neutralizing diphtheria toxin?

\section{Experiment 8.-Tetanus Toxin}

Tetanus toxin is composed of two distinct poisons of different properties. One, tetanospasmin, has a great affinity for the central nervous system, and is largely responsible for the symptoms of tetanus infection (neurotoxic); the second, tetanolysin, is thermolabile and is hematoxic. Tetanus toxin is very labile, and when in solution soon becomes attenuated. For these experiments it is necessary to use either fresh toxin or that which has been recently precipitated and dried.

1. Secure some dried tetanus toxin and dissolve in sterile salt solution. The toxin may be secured from an antitoxin laboratory and preserved indefinitely in the refrigerator. Since the strength varies with different products, the lethal dose for a 300-gram guinea-pig should be ascertained from the laboratory furnishing the toxin.

2. Secure 2 grams of fresh normal guinea-pig brain and liver. Crush in separate sterile mortars.

3. Add a lethal dose of fresh tetanus toxin and 5 c.c. sterile salt solution to each. Mix thoroughly and place in the incubator for an hour. Remove and carefully transfer to sterile centrifuge tubes. Centrifuge thoroughly.

4. Inject two 300-gram guinea-pigs subcutaneously in the median abdominal line with the supernatant fluids.

5. Inject a third guinea-pig with a similar dose of toxin and 5 c.c. salt solution (control).

6. Mark pigs carefully and observe for several days.

(a) What are the symptoms of tetanus?

(b) To what constituents of tetanus toxin are the chief symptoms due?

(c) Does the pig which received the toxin-brain mixture show symptoms? How do you explain the result?

(d) Does the pig which received the toxin-liver mixture show symptoms? How do you account for the result?

(e) Is the tetanus bacillus characterized by its toxicity or aggressiveness?

(f) Is there a period of incubation before symptoms develop and why?

(g) Autopsy the animal. Are there any definite lesions of the tissues and internal organs?

\section{Experiment 9.-Tetanus Toxin}

1. Secure some fresh tetanus toxin.

2. Prepare a 1 per cent. emulsion of washed sheep corpuscles (washed three times in an excess of normal salt solution). 
3. Into a series of six test-tubes place 1 c.c. of the corpuscle emulsion and increasing doses of toxin: 0.1 c.c., 0.2 c.c., 0.4 c.c., 0.8 c.c., 1 c.c., 2 c.c. Add sufficient normal salt solution to bring the total volume to 3 c.c.

4. Place 1 c.c. of the corpuscle suspension in 2 c.c. salt solution as a control to make sure that the salt solution is isotonic.

5. Heat a portion of toxin at $60^{\circ} \mathrm{C}$. for an hour in a water-bath and set up a similar set of tubes.

6. Incubate all tubes for two hours at $37^{\circ} \mathrm{C}$.

7. Read results at the end of this time and again after the tubes have settled in the refrigerator overnight.

(a) Has hemolysis occurred in any of the tubes?

(b) What is meant by hemolysis?

(c) What constituent of tetanus toxin is responsible for hemolyzing these cells?

(d) Does this experiment show the selective affinity of a toxin for certain cells?

(e) How is tetanus toxin produced?

(f) Does heat destroy the hemotoxic agent?

\section{EXERCISE 4.-TOXINS (Continued)}

\section{Experiment 10.-Botulism Toxin}

1. Prepare toxin by cultivating the Bacillus botulinus in an alkaline bouillon made in the form of an infusion from ham with the addition of 1 per cent. of glucose, 1 per cent. of peptone, and 1 per cent. of sodium chlorid. Strict anaërobic cultures should be grown for four weeks and filtered through a Berkefeld filter. The toxin may be preserved in brown, sealed vials, and kept on ice, or in a dried form in vacuum.

2. Dilute 0.2 c.c. of the toxin with 3 or 4 c.c. salt solution and administer, per os, to a cat by means of a small catheter passed into the stomach.

3. Inject 0.1 c.c. of the toxin subcutaneously in the abdominal wall of a rabbit.

4. Observe animals closely for several hours following administration of the toxin, for some products are so highly toxic that symptoms may appear within a few hours.

(a) What are the symptoms of botulism infection?

(b) Do these symptoms show any selective action of botulism toxin for certain tissues?

(c) Autopsy the animals. Are there any gross tissue changes?

\section{Experiment 11.-Dysentery Toxin}

1. Inoculate a flask of moderately alkaline bouillon with a culture of dysentery bacilli, preferably of the Shiga-Kruse type. Inoculate at $37^{\circ} \mathrm{C}$. for two weeks and pass it through a bacterial filter.

2. Inoculate three rabbits intravenously with 1,2 , and 5 c.c. of the filtered toxin.

3. Give a fourth rabbit 2 c.c. of the toxin by mouth through a small catheter passed into the stomach.

4. Observe the animals very closely. 
(a) What are the symptoms of dysentery intoxication?

(b) Observe the temperature at frequent intervals. Are there any changes?

(c) Does the animal which received the toxin by mouth show symptoms? How do you explain the result?

(d) Autopsy the animals with particular attention to the gastrointestinal tract. Are there any evidences of a selective action of the toxin?

\section{EXPERIMENT 12.-STAPHYLOTOXIN}

Inject 2 c.c. of a twenty-four-hour bouillon culture of Staphylococcus aureus in the ear vein of a rabbit. Take the temperature before and every twelve hours. after injection. Autopsy the animal seventy-two hours later under aseptic precautions.

(a) What lesions are found?

(b) Where are these chiefly situated and why?

(c) Culture the heart's blood on tubes of agar, also several of the lesions.

(d) After twenty-four hours' incubation examine your cultures.

(e) What was death due to?

(f) Define bacteremia.

(g) Prepare and stain sections of the kidney, including a lesion. What are the histologic changes? How do you explain the production of these tissue changes?

\section{EXPERIMENT 13.-STAPHyLOTOXIN}

1. Inoculate a flask of neutral bouillon with a virulent culture of Staphylococcus aureus and grow for two weeks at $37^{\circ} \mathrm{C}$. Filter through a Berkefeld filter.

2. Prepare a 1 per cent. suspension of washed rabbit erythrocytes in normal salt solution.

3. Into a series of six test-tubes place 1 c.c. of the corpuscle suspension and increasing amounts of staphylococcus filtrate: 0.1 c.c., 0.2 c.c., 0.4 c.c. 0.8 c.c., 1 c.c., and 2 c.c. Add normal salt solution to bring the total volume to 3 c.c.

4. Prepare a control by placing 1 c.c. of corpuscle suspension in 2 c.c. salt solution.

5. Incubate tubes for two hours at $37^{\circ} \mathrm{C}$. and read results.

6. This test will be referred to again in the technic for determining the antilysin in blood-serum.

(a) Has hemolysis occurred in any of the tubes?

(b) To what constituent of the filtrate are these results due?

(c) Does this experiment show the selective action of a toxin?

(d) Do the results have any bearing upon the anemia and jaundice of severe staphylococcus infections? 


\section{EXERCISE 5.-TOXINS (Continued)-PLANT AND ANIMAL TOXINS}

\section{EXPERIMENT 14.-STREPTOTOXIN}

1. Cultivate a strain of virulent streptococci in tubes of slightly alkaline serum or ascites bouillon for forty-eight hours at $37^{\circ} \mathrm{C}$.

2. Inoculate a guinea-pig intraperitoneally with 1 or 2 c.c. of the unfiltered culture.

3. Autopsy aseptically eighteen or twenty-four hours later.

(a) What are the gross features of the exudate?

(b) Prepare smears of the exudate and stain with methylene-blue. Are there many cells present? How do you explain the cellular content?

(c) Are streptococci present? Are these inclosed by any of the cells? How do you explain the condition?

(d) Is the exudate bloody? If so, why?

(e) Examine your blood-agar plates. Are there any peculiar changes around the colonies? To what are these changes due? Does this have any connection with the bloody character of the exudate?

(f) Why are streptococcus infections so virulent and spreading in character?

(g) Is the streptococcus an aggressive microörganism?

(h) Do streptococci contain an endotoxin?

\section{Experiment 15.-Phytotoxins}

1. Secure $0.01 \mathrm{gm}$. ricin or abrin and dissolve in 10 c.c. normal salt solution or distilled water.

2. Inject 1 c.c. intravenously into a rabbit. Place the animal in a metabolic cage and collect urine, or catheterize every twelve hours or at death.

3. Examine the urine for hemoglobin and erythrocytes.

4. Prepare a 1 per cent. suspension of washed rabbit and guinea-pig corpuscles.

5. Into a series of six small test-tubes place increasing doses of the ricin or abrin solution as follows: $0.1,0.2,0.3,0.4,0.5$, and 0.8 c.c. Add 1 c.c. of rabbit-cell emulsion to each and sufficient normal salt solution to make the total volume in each tube equal to 2 c.c. A seventh tube is the corpuscle control and contains 1 c.c. of the erythrocyte suspension and 1 c.c. of salt solution.

6. Prepare a similar series of tubes with the guinea-pig erythrocyte suspension.

7. Shake the tubes gently and incubate for two hours.

(a) Do any of the tubes show hemolysis or hemagglutination?

(b) Is the action the same with both bloods?

(c) Does the plant toxin show a selective affinity?

(d) Does the rabbit show any evidences of hemolytic jaundice? Are there blood elements in the urine? 
(e) Autopsy the animal, paying particular attention to the kidneys and gastro-intestinal tract? What changes have occurred?

\section{Experiment 16.-Zoötoxin (CoBra Venom)}

1. Collect about 2 c.c. of normal human blood and place in 5 c.c. of 2 per cent. sodium citrate in 0.85 per cent. sodium chlorid solution. This mixture must not be shaken and the cells should be washed at least four times with normal salt solution, after which they are made up to a 4 per cent. suspension.

2. Prepare a 4 per cent. suspension of sheep corpuscles in the same manner.

3. Prepare a stock dilution of cobra venom by dissolving $0.005 \mathrm{gm}$. dried venom in 10 c.c. of normal salt solution. This solution will keep about one week in the refrigerator.

4. Prepare a solution of venom $1: 10,000$ by placing 2 c.c. of the stock solution in 8 c.c. salt solution. Prepare a 1:20,000 dilution by placing 2 c.c. of dilution $1: 10,000$ in 2 c.c. salt solution.

5. Place 1 c.c. of each corpuscle suspension into two small test-tubes and add 1 c.c. and 0.5 c.c. each venom dilution. Shake the tubes gently and place in the incubator for an hour and then in the refrigerator overnight.

6. Inject 2 c.c. of the $1: 10,000$ dilution intravenously into a rabbit and 1 c.c. intravenously into a guinea-pig.

7. Observe the animals closely, particularly the urine.

(a) Does the venom show a hemotoxic action? Does it act the same on rabbit and guinea-pig corpuscles? If not, why not?

(b) How do you explain venom hemolysis?

(c) What are the evidences of venom hemolysis in vivo?

(d) Is the hemotoxic poison the most dangerous constituent of venom?

\section{EXERCISE 6.-ENDOTOXINS AND AGGRESSINS}

\section{Experiment 17.-Endotoxins}

1. Incubate eight agar slants with Bacillus typhosus and cultivate at $37^{\circ} \mathrm{C}$. for seventy-two hours.

2. Wash off the growths with 5 c.c. distilled water for each tube.

3. Place the emulsion in a sterile bottle with glass beads and shake for twentyfour hours at room temperature. At the same time inoculate six more slants of agar and wash off the growths at the end of twenty-four hours with 3 c.c. of normal salt solution for each tube.

4. Filter the emulsion, which has been shaken, through a Berkefeld filter.

5. Inject two rabbits intravenously with 2 c.c. each of the filtrate (endotoxins) and unfiltered culture.

6. Observe influence on body-weight and temperature and for symptoms of toxemia.

7. Inject three pigs intraperitoneally as follows:

1st pig: 2 c.c. of emulsion of typhoid bacilli.

2d pig: 2 c.c. of filtrate.

$3 \mathrm{~d}$ pig: 1 c.c. each of emulsion and filtrate. 
(a) Are there symptoms of toxemia in the rabbits? Are these symptoms alike in both animals? Which is more toxic, the filtrate or the whole culture?

(b) Does the filtrate enhance the effect of the whole culture?

(c) If any of the animals succumb, autopsy under aseptic precautions. Prepare cultures of the peritoneum and heart's blood on agar slants or in neutral bouillon. Is the typhoid bacillus markedly aggressive?

(d) If there is a peritoneal exudate, prepare smears and stain with carbolfuchsin $(1: 10)$. What type of cell predominates? Are any of the bacilli engulfed in the cells?

(e) Do any of the bacilli show signs of disintegration? If so, do you know what these changes are due to?

\section{Experiment 18.-Natural Aggressins}

1. Inject a guinea-pig intraperitoneally with 3 c.c. of twenty-four-hour bouillon culture of Bacillus typhosus.

2. After twenty-four hours, anesthetize the animal and remove the exudate aseptically into 5 c.c. of a 2 per cent. solution of sodium citrate to prevent coagulation.

3. Filter.

4. Inject three guinea-pigs intraperitonealy as follows:

1st pig: 3 c.c. of filtrate.

$2 \mathrm{~d}$ pig: 2 c.c. of twenty-four-hour bouillon culture of typhoid bacilli.

3d pig: 1 c.c. each of culture and filtrate.

5. Observe animals for several days, particularly the temperature reaction and weights.

(a) Is the filtrate alone toxic?

(b) Does the filtrate appear to enhance the effects of the unfiltered culture?

(c) If the animals succumb, autopsy aseptically. Make cultures of the peritoneum and heart's blood on agar slants or in bouillon. Prepare smears of the exudate and stain with 1:10 carbolfuchsin. Do any of the animals show a typhoid bacteremia? Does the filtrate appear to prevent phagocytosis?

EXERCISE 7.-BACTERIAL PROTEIN; PTOMAINS; MECHANICAL ACTION OF BACTERIA

Experiment 19.-Bacterial Protein (Vaughan)

1. Inoculate 10 slants of 2 per cent. neutral agar in large bottles with a culture of Bacillus coli and grow at $37^{\circ} \mathrm{C}$. for four days.

2. Add about 10 c.c. of distilled water to each bottle and gently remove the 
growth with a sterilized platinum wire, being particularly careful not to remove fragments of agar.

3. Pipet the heavy emulsion to a large centrifuge tube. Another cubic centimeter or two of water is added to each bottle to remove the balance of the growth.

4. Centrifuge at high speed until the bacilli are thoroughly settled. Decant off the supernatant fluid and add 50 per cent. alcohol; mix and centrifuge. Decant and add 95 per cent. alcohol; mix and centrifuge.

5. Remove the sediment of bacteria to a small flask with 50 c.c. of absolute alcohol and set aside at room temperature for a day. Decant off the alcohol and add 50 c.c. of ether. Mix and set aside for another twenty-four hours. Decant off the ether that remains and remove sediment to a porcelain or agate mortar. Place in the incubator for a few hours until thoroughly dry.

6. Grind the dry mass very thoroughly, the operator wearing a mask, until a fine powder is produced. Place the powder of bacterial substance in a wide-mouthed dark-glass bottle and preserve in a dark closet. A portion will be needed later for experiments in anaphylaxis.

7. Mix $0.02 \mathrm{gm}$. of the dry powder with 10 c.c. salt solution.

8. Inject 2 c.c. of this emulsion intraperitoneally in a 300 -gram guinea-pig.

9. Heat 2 c.c. of the emulsion at $60^{\circ} \mathrm{C}$. for two hours and inject intraperitoneally in a guinea-pig.

10. Inject a third pig intraperitoneally with 2 c.c. of a four-day bouillon culture of Bacillus coli.

(a) Could endotoxins withstand these various manipulations?

(b) Does the heated extract kill the animal more quickly than the unheated, and if so, why?

(c) What are the symptoms produced?

(d) Autopsy the animals. Are there evidences of peritonitis? Are there differences in the lesions of the three animals? If so, how do you explain them?

(e) The bacterial split portion will be studied later under Anaphylaxis.

\section{Experiment 20.-PTomains}

1. Procure 4 ounces of beef and mince.

2. Place in a flask with 250 c.c. tap water and inoculate with a culture of Bacillus coli. Fit the flask with a rubber stopper with a glass tube to carry off gases.

3. Inoculate a flask of neutral bouillon at the same time with the same culture.

4. Cultivate both at $37^{\circ}$. C. for two weeks.

5. Filter both through a coarse Berkefeld filter.

6. Concentrate both filtrates to one-half their volume at a low temperature on a water-bath.

7. Inject two guinea-pigs with each preparation, giving 1 c.c. subcutaneously and 0.5 c.c. intraperitoneally.

8. Observe the four animals closely.

(a) What symptoms develop in both sets of animals?

(b) What are ptomains? What rôle do they play in the production of disease?

(c) Can antibodies be produced for true ptomains? 


\section{Experiment 21.-Mechanical Action of Bacteria}

1. Inject a 300-gram pig intraperitoneally with 2 c.c. of a forty-eight-hour-old bouillon culture of Bacillus anthracis. Great care must be exercised in handling and injecting this culture.

2. Autopsy the animal at the end of forty-eight hours and make cultures on agar slants of the heart's blood, liver, and spleen. Remove the heart, lungs, liver, spleen, and kidneys, and place in 2 per cent. formalin. Prepare smears of the blood and stain with Gram's method.

3. After fixing for twenty-four hours, sections of these organs are to be prepared and stained with methylene-blue and with Gram's stain.

4. Examine the cultures at the end of twenty-four hours.

5. Examine the sections.

(a) Is the anthrax bacillus aggressive?

(b) Does the anthrax bacillus produce much toxin?

(c) Did the animal show any symptoms of its infection?

(d) Are there evidences of peritonitis?

(e) How do you explain the probable causes of death in human anthrax?

\section{EXERCISE 8.-KINDS OF IMMUNITY}

\section{Experiment 22.-Phagocytosis in Natural Immunity}

1. Inject a healthy guinea-pig intraperitoneally with 1 c.c. of a twenty-fourhour or forty-eight-hour glucose bouillon culture of streptococci of low or moderate virulence. A culture of staphylococci may be used instead, although the former is preferable.

2. At the end of eighteen hours study the peritoneal fluid. Prepare smears and stain with methylene-blue or other suitable stain. Prepare cultures in glucose bouillon of the heart's blood.

(a) Did the animal show any evidences of infection?

(b) Has phagocytosis occurred in the peritoneal cavity?

(c) What constituent of normal serum aids phagocytosis?

(d) Did a bacteremia develop?

\section{Experiment 23.-Natural Antibacterial Immunity}

1. Inject a 250-gram guinea-pig subcutaneously with 1 c.c. of a twenty-four-hour bouillon culture of Bacillus anthracis.

2. Inject a large albino rat with the same dose and in the same manner.

3. Observe the animals for twenty-four to forty-eight hours. At autopsy prepare smears and cultures of the heart blood of each. Stain the smears with methyleneblue and according to the method of Gram.

Do the animals present symptoms of infection? Are there any differences between the two? 


\section{Experiment 24.-Relative Factors in Natural Immunity}

1. Place a frog in a shallow vessel of warm water in the incubator at $37^{\circ} \mathrm{C}$.

2. After twenty-four hours give a subcutaneous injection of tetanus toxin equal to one-tenth the fatal dose for a 300-gram guinea-pig.

3. Inject a second frog with an equal amount of toxin and keep it at ordinary room temperature in cold water.

4. Observe both animals.

(a) Do symptoms of tetanus develop in any of the animals?

(b) Why does heat favor tetanus infection?

(c) Give further examples of relative natural immunity.

(d) Are anthrax bacilli found in the smears and cultures of both animals? Which animal is immune? Could phagocytosis play a rôle in this immunity?

\section{Experiment 25.-Influence of Temperature Upon Natural Im- MUNITY}

1. Procure dried tetanus toxin and dissolve sufficient in 6 c.c. normal salt solution equivalent to 6 lethal doses for a 350-gram guinea-pig.

2. Inject 2 c.c. into the pectoral muscles of a young hen. Take the rectal temperature.

3. Inject 2 c.c. into a second hen. Take the rectal temperature and place her in cold water until the temperature has dropped several degrees.

4. Place hen No. 1 in a cage and keep her at ordinary laboratory temperature.

5. Place hen No. 2 in a cage and keep her in a cold place or renew the bath several times in order to keep her temperature subnormal.

(a) What is the normal temperature of the hen?

(b) Do both animals present symptoms of tetanus?

(c) How do you explain the difference?

6. If hen No. 1 does not show symptoms of tetanus by the second day, reinject her with an amount of toxin equal to 10 lethal doses for a guinea-pig.

(a) Does this hen present evidences of tetanus?

(b) If so, how do you explain the result?

\section{EXERCISE 9.-ACQUIRED IMMUNITY}

\section{Experiment 26.-Acquired Active (Antibacterial) Immunity}

1. Immunize a rabbit with typhoid bacilli as described in the chapter on Active Immunization.

2. Inject this immune rabbit intravenously with 6 loopfuls of a twenty-fourhour culture of Bacillus typhosus thoroughly emulsified in 2 c.c. sterile salt solution.

3. Inject a normal rabbit of equal weight with an equal dose and in the same manner.

4. Both animals are weighed and their temperatures recorded before the injec- 
tions are given. If possible, take temperature every four hours. Observe both animals for symptoms of infection. If one or both succumb, autopsy aseptically and culture the heart blood in neutral bouillon or bile.

(a) Are there differences in the clinical condition of the two animals?

(b) To what do you ascribe these differences?

(c) What chief antibody is responsible for the destruction of the bacilli in the immune animal?

(d) Has typhoid bacteremia developed?

\section{PASSIVE ACQUIRED IMMUNITY}

Experiment 27.-Acquired Passive (Antitoxic) Immunity

1. Inject a 250 - to 300 -gram guinea-pig subcutaneously with 1 c.c. (500 units) of diphtheria antitoxin (pig No. 1).

2. Secure diphtheria toxin in which the lethal dose (L. D.) for a 250- to 300-gram guinea-pig is known.

3. Place this dose of toxin in a small test-tube or, better, in the barrel of a precision syringe (Hitchens), and add 1 c.c. antitoxin (500 units). Mix thoroughly and keep at room temperature for an hour.

4. Inject pig No. 1 with an amount of toxin equal to the L. D. dose.

5. Inject the toxin and antitoxin mixture into a second pig of 250 to 300 grams.

6. Inject the same amount of toxin into a third pig of equal weight as a control.

7. Observe animals closely for at least four or five days.

(a) What are the chief symptoms of diphtheria intoxication of the guinea-pig?

(b) Do all animals show these symptoms?

(c) How do you explain the absence of symptoms in pig No. 1? How in pig No. 2? Is the mechanism of protection the same in both?

(d) What bearing has this experiment upon the treatment of diphtheria?

\section{Experiment 28.-Acquired Passive (Antitoxic) Immunity}

The above experiment may be conducted in exactly the same manner, using tetanus toxin and antitoxin.

\section{Experiment 29.-Acquired Passive (Antibacterial) Immunity}

1. Secure a culture of pneumococcus, and if its lethal dose for white mice is unknown, determine this by injecting a series of mice with decreasing doses of a fortyeight-hour serum bouillon culture.

2. Secure a few cubic centimeters of antipneumococcus serum, especially a serum prepared with the culture being used, or at least one that is polyvalent.

3 . Inject three mice subcutaneously with $0.1,0.5$, and 1 c.c. of the serum. Then inject them subcutaneously with double the lethal dose of pneumococci. Inject a fourth mouse with culture alone (control).

4. Observe the animals for several days, and at autopsy culture the heart blood in tubes of serum bouillon and prepare smears which are to be stained according to Gram. 
(a) Has the serum served to protect any of the mice?

(b) Is this protection due to bacteriolysins, bacteriotropins, or both? Do antitoxins play any part?

(c) Why is it preferable to use homologous culture and immune serum? What bearing has this upon the treatment of human pneumococcus infections?

(d) Do you know a method for quickly isolating and identifying pneumococci from sputum?

Note.-This experiment may be conducted with rabbits; also a culture of streptococcus and its immune serum may be used.

\section{EXERCISE 10.-PHAGOCYTOSIS}

\section{Experiment 30.-Phagocytosis (Macrophages)}

1. Secure pigeons' blood and defibrinate. Wash the corpuscles several times and prepare a 5 per cent. suspension.

2. Inject a guinea-pig intraperitoneally with 3 c.c. of this corpuscle suspension.

3. After three hours withdraw a small amount of peritoneal exudate by means of a capillary pipet. Examine with hanging-drop preparations and prepare smears and stain with Wright's stain.

4. Make similar preparations twelve, eighteen, twenty-four, and forty-eight hours after injection.

(a) Has phagocytosis occurred?

(b) Which cells have become phagocytes?

(c) Do the pigeon cells appear as if undergoing digestion?

(d) Which portion of the pigeon cell resists digestion?

(e) Explain mechanism of intracellular digestion.

\section{Experiment 31.-Phagocytosis (Microphages)}

1. Place a drop of blood in the hollow cell of a ground-out slide such as is used for hanging-drop preparations. Cover with a clean slide, seal with a ring of vaselin, and place in a large Petri dish containing pieces of filter-paper moistened with water (moist chamber). Place in the incubator for fifteen minutes.

2. Remove the cover-glass and carefully wash cover-glass and cell with normal salt solution. The erythrocytes are removed and the leukocytes left adherent to the cell and cover-glass.

3. Fill the cell with fresh serum and add a quantity of culture, preferably a loopful of a twenty-four-hour bouillon culture of non-virulent anthrax bacilli. Apply the cover-glass and vaselin the margins to prevent evaporation.

4. If at all possible, employ a warm stage and watch the process under the microscope.

(a) Does phagocytosis occur?

(b) Which cells are acting as phagocytes? 
(c) Can you make out the phase of fixation and phase of ingestion?

(d) By what agencies do leukocytes kill engulfed bacteria?

\section{Experiment 32.-Phagocytosis}

1. Inject a guinea-pig intraperitoneally with 5 c.c. of finely divided cinnabar suspension and 1 c.c. subcutaneously on each side in the region of the inguinal lymphglands.

2. Autopsy the animal at the end of twenty-four hours. Prepare hanging-drop preparations and smears of the peritoneal exudate (stained lightly with methyleneblue). Prepare sections of the inguinal and abdominal lymphatic glands.

(a) Has phagocytosis occurred in the peritoneal cavity? Which cells have become phagocytic?

(b) Do you find phagocytes in the lymph-glands? Where are the leukocytes situated? Which cells have become phagocytes?

(c) How did the material reach the glands?

(d) How do you explain the presence of cinnabar in the endothelial cells of the gland?

\section{EXERCISE 11.-CHEMOTAXIS}

\section{Experiment 33.-Positive Chemotaxis}

1. Inject a guinea-pig intraperitoneally with 1 or 2 c.c. of a twenty-four-hour culture of Staphylococcus aureus.

2. Autopsy the animal eighteen hours later and prepare smears of the exudate. Fix with methyl alcohol for five minutes, dry, and stain with carbol-thionin, Wright's or Loeffler's methylene-blue, counterstained with eosin.

3. Prepare cultures of the peritoneal exudate.

(a) Describe the exudate.

(b) Has phagocytosis occurred?

(c) Which cells are actively phagocytic?

(d) Are all the cocci engulfed?

(e) Are there any evidences of the cocci undergoing intracellular digestion?

(f) Could a sterile substance call forth an exudation of leukocytes when injected into a serous cavity?

\section{Experiment 34.-Negative Chemotaxis}

1. Inject a guinea-pig intraperitoneally with 0.5 to 1 c.c. of a forty-eight-hour serum bouillon culture of virulent streptocacci.

2. Autopsy at the end of eighteen to twenty-four hours if death has not already occurred.

3. Prepare cultures in serum bouillon and a number of smears. Stain the latter with methylene-blue or according to Gram. 
(a) Describe the exudate. How does it differ from that found in the preceding experiment?

(b) How do you explain the serous character of the exudate?

(c) Has phagocytosis occurred?

(d) Do you think the streptococci have multiplied in the peritoneal cavity?

(e) What means could you suggest for overcoming this action of virulent streptococci?

\section{EXERCISE 12.-OPSONINS}

\section{EXPERIMENT 35.-Normal Opsonins}

1. Prick the finger and secure 1 c.c. of blood in a small test-tube. Also 1 c.c. in a centrifuge tube containing 2 c.c. of sodium citrate solution. After coagulation remove the serum from the first tube.

2. Divide the serum into two portions and heat one portion at $56^{\circ} \mathrm{C}$. for thirty minutes.

3. Prepare an emulsion of leukocytes by centrifuging the blood collected in the citrate solution, removing the supernatant fluid, adding normal salt solution, and centrifuging again. Repeat this step once more in order to wash the cells thoroughly and after the last centrifuging remove the supernatant fluid and add sufficient salt solution to make the total volume 1 c.c. and mix thoroughly.

4. Prepare an emulsion of staphylococci which is homogeneous and free of clumps.

5. Mark two capillary tubes with a wax pencil about an inch from the tip; fit rubber teats to the other end.

6. With pipet No. 1 take up a volume of blood-cells; allow a bubble of air to enter and then an equal volume of bacterial emulsion; bubble of air and an equal volume of the fresh unheated serum. Mix well by alternate expulsion and aspiration on a clean slide. Then draw the whole into the stem of the pipet and seal the tip in a flame.

7. Repeat with pipet No. 2, using the heated serum.

8. Incubate both pipets at $37^{\circ} \mathrm{C}$. for half an hour.

9. Remove the pipets from the incubator, break off the tips, mix the contents, and prepare smears.

10. Fix the smears with a saturated solution of bichlorid of mercury for one minute; wash in water and stain with carbol-thionin for two minutes; wash in water and dry.

11. Examine with oil-immersion lens.

(a) What are the requisites of a satisfactory opsonic preparation?

(b) Is there any difference in the amount of phagocytosis between the heated and unheated serums? If so, how do you explain the result?

(c) Do normal opsonins play any rôle in natural immunity?

(d) Give the properties of normal opsonins. 


\section{EXPERIMENT 36.-IMMUne Opsonins (BACTERIOTROPIN)}

1. Secure 1 c.c. of serum from a rabbit immunized with staphylococci and heat 0.5 c.c. at $56^{\circ} \mathrm{C}$. for thirty minutes.

2. Using the same blood and bacterial emulsion as prepared in the preceding experiment, prepare two opsonic preparations with the heated and unheated immune serum.

(a) Is phagocytosis more marked than in the preceding experiment? If so, why?

(b) Is there any difference in the degree and extent of phagocytosis with the heated and unheated serum?

(c) Give the properties of immune opsonin or bacteriotropin.

\section{EXPERIMENT 37.-HEMOPSONIN}

1. Secure 0.5 c.c. serum from a rabbit immunized with sheep cells and heat at $56^{\circ} \mathrm{C}$. for half an hour to destroy hemolytic complement.

2. Prepare a 5 per cent. suspension of sheep erythrocytes in normal salt solution after washing them three times.

3. Prepare an emulsion of rabbit leukocytes, or the emulsion of human cells in the preceding experiments may be used.

4. Take a capillary pipet; make a mark about an inch from the tip and fit the other end with a rubber teat.

5. Draw up equal volumes of leukocytic emulsion, sheep cell suspension, and serum. Mix well, seal the pipet, and inoculate for an hour at $37^{\circ} \mathrm{C}$.

6. Prepare smears and stain with Wright's blood-stain.

(a) Has phagocytosis occurred?

(b) Which cells have become phagocytes?

(c) Has hemolysis occurred in the mixtures and if not, why not?

\section{EXERCISE 13.-OPSONINS (Continued)}

\section{Experiment 38.-Mechanism of Action of Opsonins}

1. Secure serum from a rabbit immunized with Staphylococcus pyogenes aureus.

2. Prepare a leukocytic suspension from normal rabbit blood.

3. Prepare an emulsion of eighteen-hour culture of Staphylococcus aureus.

4. Make the following mixtures in capillary pipets:

No. 1: Equal parts of leukocytes, serum, and bacterial emulsion.

No. 2: Equal parts of leukocytes and bacterial suspension.

5. In two small test-tubes mix:

No. 3: 0.5 c.c. each of leukocytic suspension and serum.

No. 4: 0.5 c.c. each of serum and bacterial emulsion.

6. Incubate pipets 1 and 2 and tubes 3 and 4 for fifteen minutes at $37^{\circ} \mathrm{C}$.

7. Prepare smears of capillary tubes 1 and 2 and stain with carbol-thionin.

8. To tubes 3 and 4 add 15 c.c. normal salt solution, mix, and centrifuge thoroughly. Decant off the supernatant fluid and restore volume in each tube to 1 c.c.

9. Prepare capillary pipets as follows: 
No. 3: Equal parts of contents of tube 3 and bacterial emulsion.

No. 4: Equal parts of contents of tube 4 and leukocytic mixture.

10. Incubate both pipets for fifteen minutes, prepare smears, and stain with carbol-thionin.

(a) Carefully compare the degree and extent of phagocytosis in the four mixtures.

(b) Has leukocytosis occurred in mixture No. 2? If so, what special term is applied to phagocytosis in the absence of serum?

(c) What rôle does serum play in phagocytosis? Explain the mechanism involved.

(d) Discuss the question of "stimulin" and opsonin as demonstrated by the results in preparations Nos. 3 and 4 .

\section{EXPERIMENT 39.-SPECIFICITY OF OPSONINS}

1. Secure 1 c.c. of the serum of a rabbit immunized with staphylococci and heat at $56^{\circ} \mathrm{C}$. for thirty minutes. Divide into two portions in separate small test-tubes.

2. To No. 1 add 1 c.c. of normal salt solution and 4 or 5 loopfuls of a twentyfour-hour agar slant culture of staphylococci. Incubate for thirty minutes; centrifuge thoroughly and remove the supernatant fluid (diluted serum) to a separate tube. Call this "treated" serum.

3. To the untreated serum add 1 c.c. salt solution, so that both are diluted equally.

4. Prepare an emulsion of rabbit or human leukocytes.

5. Prepare an emulsion of staphylococci, homogeneous and free of clumps.

6. Prepare two opsonic mixtures: No. 1 containing equal parts of blood suspension, bacterial emulsion, and untreated serum; No. 2 containing equal parts of blood, bacterial emulsion, and treated serum.

7. Incubate for thirty minutes. During this interval proceed as follows:

8. Prepare an emulsion of typhoid bacilli, homogeneous and free of clumps.

9. Secure 0.5 c.c. of serum from a rabbit immunized with typhoid bacilli and heat at $36^{\circ} \mathrm{C}$. for thirty minutes.

10. Prepare the following mixtures in capillary pipets:

No. 3 blood-cells + typhoid serum + typhoid bacterial emulsion.

No. 4 blood-cells + typhoid serum + staphylococcus bacterial emulsion.

No. 5 blood-cells + staphylococcus serum + typhoid emulsion.

11. Incubate for fifteen minutes.

12. Prepare smears of all and stain with carbol-thionin.

(a) Is there any difference in the degree of phagocytosis in mixtures No. 1 and 2 ? If so, why?

(b) Are opsonins specific?

(c) Has phagocytosis occurred in the cross-mixtures of staphylococci with typhoid serum, and typhoid bacilli with staphylococcus serum? If so, how do you explain this apparent lack of specificity?

(d) What may happen in a mixture of fresh unheated typhoid serum, typhoid bacilli, and leukocytic emulsion? 


\section{EXERCISE 14.-OPSONIC INDEX}

\section{Experiment 40.-Determining the Opsonic Index}

1. Secure a small quantity of blood from a guinea-pig or rabbit which has been immunized with Staphylococcus pyogenes aureus. Also two specimens from normal animals to serve as control serums. Remove the serums, being careful to keep them marked and separate. The two normal serums may be mixed in a watch-glass or small test-tube (pooled).

2. Prepare a leukocyte mixture, using normal guinea-pig or rabbit blood according to the immune animal used.

3. Prepare a bacterial emulsion of an eighteen-hour culture of Staphylococcus pyogenes aureus.

4. Proceed to determine the phagocytic and opsonic index as per technic given in the text (page 195).

(a) What constitutes a satisfactory bacterial emulsion?

(b) What constitutes a satisfactory leukocytic suspension? Name several methods of obtaining leukocytes for this technic.

(c) What constitutes a satisfactory phagocytic film?

(d) Should the serum be fresh?

(e) How do you determine the phagocytic index?

(f) How do you determine the opsonic index?

(g) What is the relation between the opsonic and phagocytic indices?

(h) Give the practical value of the opsonic index in disease.

(i) Give the practical value of the opsonic index as a measure of immunity.

Note.-If any of the students have received typhoid vaccine, the opsonic index with his serum may be determined.

\section{Experiment 41.-Quantitative Estimation of Bacteriotropins}

1. Secure a culture of pneumococcus.

2. Secure a few cubic centimeters of antipneumococcus serum, especially a serum corresponding to the culture.

3. Prepare a leukocytic emulsion.

4. Conduct the test after the technic given in the text (page 200).

(a) Give the value of a bacteriotropic estimation of a serum.

(b) What relation do bacteriotropins bear to immunity?

(c) In what class of diseases are bacteriotropins of most value?

\section{EXERCISE 15.-BACTERIAL VACCINES}

\section{Experiment 42.-Preparation of Typhoid Vaccine}

1. Prepare two to six agar slant cultures of Bacillus typhosus and grow for fortyeight hours at $37^{\circ} \mathrm{C}$.

2. Remove cultures, prepare a suspension count by the method of Wright, sterilize, dilute, and prepare vaccine for administration as given in the text. 
3. Prepare two emulsions: one to contain about 500 million bacilli in each cubic centimeter (first dose), and the second 1000 million per cubic centimeter (second and third doses).

\section{Experiment 43.-Preparation of Staphylococcus Vaccine}

1. Prepare two to six agar slant cultures of Staphylococcus aureus and grow for twenty-four hours at $37^{\circ} \mathrm{C}$. If a patient with furunculosis is available, make cultures of pus and secure staphylococcus, of which a vaccine is prepared.

2. Proceed in the preparation of the vaccine as given in the text, placing each dose in separate ampules, and so diluting that each dose is of one cubic centimeter and contains 1000 million of cocci. Count by the method of Wright and by the counting chamber method.

\section{EXERCISE 16.-ANTITOXINS}

\section{Experiment 44.-Standardizing Diphtheria Antitoxin}

1. Prepare a strong diphtheria toxin with the Park-Williams Bacillus No. 8, after the technic given in the text.

2. Secure some of the dried Standard Antitoxin and dilute so that 1 c.c. is equal to one immunity unit.

3. With this antitoxin determine the $\mathrm{L}+$ dose of the toxin prepared accordin " to the technic given, using six guinea-pigs and the following doses of toxin: 0.1 c.c., 0.12 c.c., 0.15 c.c., 0.18 c.c., 0.2 c.c., 0.25 c.c.

4. Secure a sample of diphtheria antitoxin in the open market containing about 4 c.c. serum and 2000 units of antitoxin. If the titration given on the label is still correct, one would expect about 500 units of antitoxin per cubic centimeter of serum. Carefully remove 1 c.c. of serum and dilute with 19 c.c. salt solution (1:20). From this stock dilution prepare the following dilutions (taken from Bulletin No. 21, Hygienic Laboratory, M. J. Rosenau):

1 c.c. +14 c.c. $\mathrm{NaCl}$ solution 1 c.c. $=.00333$ or $\frac{1}{30}=300$ units per c.c.
1 c.c. +16 c.c. $\mathrm{NaCl}$ solution 1 c.c. $=.00294$ or $\frac{1}{3} \frac{1}{4}=340$ units per c.c.
1 c.c. +18 c.c. $\mathrm{NaCl}$ solution 1 c.c. $=.00263$ or $\frac{1}{38}=380$ units per c.c.
1 c.c. +20 c.c. $\mathrm{NaCl}$ solution 1 c.c. $=.00238$ or $\frac{1}{420}=420$ units per c.c.
1 c.c. +22 c.c. $\mathrm{NaCl}$ solution 1 c.c. $=.00217$ or $\frac{1}{460}=460$ units per c.c.
1 c.c. +24 c.c. $\mathrm{NaCl}$ solution 1 c.c. $=.002$ or $\frac{1}{500}=500$ units per c.c.

5. Mix 1 c.c. of these various dilutions with the $\mathrm{L}+$ dose of toxin; stand aside for an hour and inject subcutaneously in median abdominal line of 250- to 300-gram guinea-pig as per the technic already given.

6. Carefully observe all animals for a period of four days at least. Autopsy those that succumb, paying particular attention to the condition of the abdominal wall and suprarenal glands. If the serum should contain less than 300 units of antitoxin per cubic centimeter of serum, the test should be repeated with lower dilutions.

7. Inject 1 c.c. of the serum subcutaneously into a white mouse to test for excess of preservative. It requires 1 c.c. of a 0.5 per cent. solution of tricresol or 0.5 c.c. of a 0.5 per cent. phenol solution to kill a medium-sized mouse. If the mouse shows trembling, it would indicate that the serum contains nearly this percentage of tricresol (Bulletin No. 21, Hygienic Laboratory).

8. Inoculate 1 c.c. of the serum in a flask containing 100 c.c. sterile neutral bouillon. Incubate at $37^{\circ} \mathrm{C}$. for-at least four days. This will test the sterility of the product. 
(a) Define the unit of diphtheria antitoxin.

(b) Of what practical value is the measurement of diphtheria antitoxin?

(c) Is there any practical method of determining the quantity of toxin in the blood of a diphtheric patient?

(d) How would you determine the amount of natural antitoxin in the blood of a person?

\section{Experiment 45.-Standardizing Tetanus Antitoxin}

The technic of standardizing tetanus antitoxin may be carried out in a similar manner with dried Standard Toxin and an antitoxin purchased in the open market.

\section{EXERCISE 17.-ANTITOXINS (Continued)}

\section{Experiment 46.-SPecificity of Antitoxins}

1. Secure small quantities of fresh diphtheria and tetanus toxins; the $\mathrm{L}+$ dose of each should be known.

2. Secure small quantities of diphtheria and tetanus antitoxins; the number of units per cubic centimeter of serum should be known.

3. Into four precision syringes place the following mixtures. After standing an hour at room temperature, inject subcutaneously into 300 -gram guinea-pigs.

No. 1: L+dose of diphtheria toxin +100 units of diphtheria antitoxin. Inject into pig No. 1.

No. 2: $\mathrm{L}+$ dose of diphtheria toxin +100 units of tetanus antitoxin. Inject into pig No. 2.

No. 3: $\mathrm{L}+$ dose of tetanus toxin +100 units of tetanus antitoxin. Inject into pig No. 3.

No. 4: $\mathrm{L}+$ dose of tetanus toxin +100 units of diphtheria antitoxin. Inject into pig No. 4.

(a) What do you observe regarding the specificity of antitoxins?

(b) What are the main symptoms of diphtheria and tetanus in the guinea-pig?

(c) Even though a pig injected with diphtheria toxin shows no general symptoms of intoxication, what local sign may be present?

(d) What is the nature of the toxin-antitoxin reaction?

\section{Experiment 47.-Nature of the Toxin-antitoxin Reaction. ACTION OF ANTI-TETANOLYSIN}

1. Secure fresh tetanus toxin and determine the dose producing complete hemolysis of 1 c.c. of a 1 per cent. suspension of rabbit corpuscles in two hours at $37^{\circ} \mathrm{C}$.

2. Place double this dose of toxin in a series of six small test-tubes and add increasing doses of fresh tetanus antitoxin: 0.001 c.c., 0.005 c.c., 0.01 c.c., 0.05 c.c., 0.1 c.c., 0.2 c.c. Add salt solution to bring the total volume to 1 c.c.; incubate at $37^{\circ} \mathrm{C}$. for an hour. Add 1 c.c. of 1 per cent. suspension of rabbit corpuscles to each tube. Prepare two controls, one with the dose of toxin and corpuscles but to which no serum 
is added, the second with 0.2 c.c. serum and dose of corpuscles. Shake tubes and incubate for two hours. Make a preliminary reading and again after tubes have settled twenty-four hours in the refrigerator.

3. The first control should be completely hemolyzed, indicating that a sufficient lytic dose of toxin was employed.

(a) What constituent of tetanus toxin has a marked affinity for erythrocytes?

(b) Is this agent thermostabile? How can you determine this?

(c) What rôle does it play in tetanus intoxication?

(d) How is this hemotoxic agent neutralized by tetanus antitoxin?

(e) Would anti-tetanospasmin neutralize the hemotoxic activity of tetanus toxin?

(f) Would diphtheria antitoxin neutralize this hemotoxic agent? If not, why not?

(g) Explain the mechanism of neutralization of a toxin by antitoxin in vitro? Is it the same as that occurring in vivo?

\section{EXPERIMENT 48.-ANTISTAPHYLOLYSIN}

1. Prepare a staphylolysin by growing a culture of Staphylococcus aureus in bouillon for two or three weeks. Pass through a Berkefeld filter and preserve the filtrate with 0.5 phenol. Determine the lytic dose for 1 c.c. of a 1 per cent. suspension of rabbit corpuscles.

2. Secure serum from a rabbit immunized with staphylococci. Heat at $56^{\circ} \mathrm{C}$. for thirty minutes.

3. Secure normal horse serum. Heat it at $56^{\circ} \mathrm{C}$. for thirty minutes.

4. Secure normal rabbit serum. Heat at $56^{\circ} \mathrm{C}$. for thirty minutes.

5. Into a series of six small test-tubes place the lytic dose of staphylolysin and increasing amounts of rabbit immune serum as follows: $0.001,0.005,0.01,0.05,0.1$, 0.2 c.c. Arrange a similar series, using normal horse serum and normal rabbit serum. Prepare a control containing the lytic dose of toxin. Add 1 c.c. of a 1 per cent. suspension of rabbit cells to each tube and sufficient normal salt solution to make the total volume equal 2 c.c. Shake each tube gently and incubate at $37^{\circ} \mathrm{C}$. for two hours.

6. Inspect the tubes. The smallest amount of normal horse serum inhibiting hemolysis is taken as 1 , or the unit. Compare the values of the immune and normal rabbit serums with this unit.

(a) Why is normal horse serum adopted as the standard?

(b) What is antistaphylolysin?

(c) What are the various agents produced by staphylococci and responsible for the lesions and symptoms of staphylococcus infections?

(d) Would the antilysin neutralize the leukocidin?

(e) Explain the mechanism of lysin-antilysin action.

(f) Which rôle does the lysin play in staphylococcus infections?

(g) Of what value would be the titration of antistaphylolysin in the serum of the patient? 


\section{EXERCISE 18.-FERMENTS AND ANTIFERMENTS}

\section{EXPERIMENT 49.-TRYPtic Ferment of Leukocytes}

1. Collect 0.5 c.c. of blood in each of two small test-tubes by puncture of a finger; set aside until coagulation has occurred. Add several changes of warm distilled water until the red corpuscles are hemolyzed and colorless clots of leukocytes, fibrin platelets, and detritus are secured.

2. Place one clot in the bottom of a tube of sterile and slanted Loeffler bloodserum medium which is fairly dry and firm.

3. Place the second clot in a second tube of this medium and add 0.2 to 0.4 c.c. of fresh serum.

4. Plug these tubes firmly, paraffin the stoppers to prevent evaporation, and incubate along with a non-inoculated control at $50^{\circ} \mathrm{C}$. for two days.

(a) In which tube do you note evidences of digestion?

(b) Describe the appearance of the digested medium.

(c) Does the tube containing serum show digestion? How do you explain the result?

(d) What rôle may this ferment play in infection?

(e) Why do not the leukocytes digest themselves?

(f) What is the nature of the ferment?

(g) May this ferment play a rôle in the disposal of old and dead leukocytes?

\section{Experiment 50.-Testing the Antitryptic Power of Blood- Serum (After the Marcus Modification of the Method of MÜLler AND JochmanN)}

1. Prepare a solution of trypsin by thoroughly shaking $0.1 \mathrm{gm}$. of Kahlbaum's trypsin with 5 c.c. glycerin and 5 c.c. distilled water. Incubate at $55^{\circ} \mathrm{C}$. for half an hour, shake thoroughly, filter, and preserve in the refrigerator.

2. Secure six Petri dishes of Loeffler blood-serum culture-media which have been sufficiently dried to drive off the water of condensation and with a firm elastic surface. serum.

3. Collect 1 c.c. of blood from a patient three hours after last meal; separate the

4. In a watch-glass or hanging-drop slide mix one drop of serum with an equal sized drop of trypsin solution. Mix well and transfer five loopfuls to an area on a Petri dish of medium. With a blue-wax pencil draw a circle on the cover of the plate to include the site of planting and mark No. 1.

5. Prepare serial dilutions with one drop of serum with two, three, four, five, six, seven, eight, nine, and ten drops of trypsin solution. Mix well and plant five loopfuls of each dilution on the medium in five dishes. Two plantings may be made in each plate and with care they will not become confluent. Mark each plate.

6. Inoculate the sixth plate with five drops of trypsin solution without serum (control).

7. Incubate all tubes at $37^{\circ} \mathrm{C}$. for six to twelve hours until the control shows well-marked digestion.

8. Wherever the trypsin has not been neutralized by the serum, shallow liquefied 
dimples appear on the surface of the Loeffler medium. The greater the amount of trypsin required to cause digestion, the higher the titer of antitrypsin in the blood. By conducting a control series with the two normal pooled serums one may determine whether in a given case the antitryptic power of the blood is normal, increased, or decreased.

(a) Does this test possess any practical value?

(b) Discuss the presence of antitrypsin in the blood-serum.

\section{EXERCISE 19.-FERMENTS (Continued)}

\section{Experiment 51.-Abderhalden Sero-enzyme Reaction in Preg- NANCY}

The technic of this test is very exact. The glassware should be sterile and all manipulations carried out carefully and exactly as laid down by Abderhalden. Consult the text for the technic (Chapter $\mathrm{XV})$.

1. Test five Schleichter and Schull shells No. 579a for permeability to albumin, using 5 per cent. egg-albumen water and the biuret or ninhydrin reaction.

2. Those shells which prove impermeable to albumin are now tested with a 1 per cent. solution of silk peptone (Höchst), using ninhydrin as the indicator. The shells impermeable to albumin and permeable to peptone are suitable for the main test.

3. Secure a fresh human placenta; wash thoroughly to remove blood; cut into pieces about the size of a dime; wash and rewash until perfectly white; search carefully for tiny blood-clots; boil repeatedly until the water reacts negatively to ninhydrin. Consult the text-book for the exact technic. Abderhalden lays a great deal of stress upon the proper preparation of the substratum.

4. Secure blood from a patient in advanced pregnancy; also a specimen from a male. After a few hours, separate the serums and centrifuge thoroughly until free of cells. The serums should not be over twelve hours old.

5. Conduct a reaction after the technic given in the text-book, including the controls.

(a) Describe the biuret and ninhydrin reactions.

(b) Why must the shells be so carefully tested?

(c) Why must the placenta be free of blood and boiled previous to the test?

(d) Why should an aseptic technic be employed?

(e) What are the prevailing views regarding the specificity of the ferments?

(f) Has the pregnancy test a practical value?

(g) Give the principles of the optical method.

(h) Why is the serum control so important?

(i) Enumerate the principal sources of error in the technic of the dialysation method. 


\section{EXERCISE 20.-AGGLUTININS}

\section{Experiment 52.-The Gruber-Widal Reaction in Typhoid Fever ("Wet" Method). Microscopic Reaction}

1. Collect blood in a Wright capsule or small test-tube from a typhoid convalescent patient. Rabbit immune serum may be used instead. Separate theserum from the clot.

2. Prepare two dilutions with hanging-drop slides, $1: 50$ and 1:100, and a culture control. Use a twenty-four-hour bouillon culture of Bacillus typhosus-one free of clumps and in which the bacilli are long and motile.

3. It is well to prepare a similar test with a known positive typhoid serum and a normal negative serum.

4. Dilution and slides are prepared according to the technic given in the textbook. A second set of tests in dilutions of 1:40 and 1:80 may be prepared with the aid of the white corpuscle pipet.

5. Place slides away from direct sunlight and examine at the end of an hour. Examinations are readily made with the $1 / 6$ objective, the light being well cut off. Examine the culture control first, then the higher and lower dilutions.

(a) Describe the phenomenon of agglutination.

(b) Is it necessary for all bacilli to be agglutinated to constitute a positive reaction?

(c) What are the features of a doubtful reaction? Of a negative reaction?

(d) Does the normal serum contain agglutinin for the typhoid bacillus?

(e) Why is it necessary to use high dilutions? What dilution is the lower limit of practical safety?

(f) Does the control show agglutination? If so, by what term is this agglutination known?

(g) What are the characteristics of a satisfactory culture for the microscopic agglutination test?

(h) Would a dead culture be serviceable in this reaction?

(i) What practical value has the Widal reaction in the diagnosis of typhoid fever?

(j) What value has the agglutination reaction in determining the degree of immunity following typhoid immunization?

Note.-If the students are immunized with typhoid vaccine, they may use one another's serum in this and following experiments.

\section{Experiment 53.-Grubler-Widal Reaction in Typhoid Fever ("Dry" Method)}

1. Prepare smears of blood of a typhoid convalescent patient on clean glass slides or collect a few drops on partially glazed paper, as prescription blanks. Allow blood to dry and do not apply heat. 
2. Using a good twenty-four-hour-old culture of Bacillus typhosus, prepare an agglutination test after the technic given in the text-book. Be particularly careful not to transfer paper fiber to the slide-apply the salt solution and dissolve the blood by gently rubbing with a small platinum loop $(2 \mathrm{~mm}$.). Mix the blood in the loopful of culture with the slide held or placed over a white surface so that the proper delicate orange tint is secured.

3. Prepare culture control as usual; also similar tests with a known positive and negative serum.

4. Examine at the end of an hour.

(a) What special precautions are to be observed in this method?

(b) What is the particular practical value of this reaction?

(c) Are accurate dilutions possible and if so, by what technic?

(d) Are agglutinins resistant to deleterious influences?

(e) Compare the value of this method with the one used in the preceding experiment.

\section{EXERCISE 21.-AGGLUTININS (Continued)}

\section{Experiment 54.-Macroscopic Agglutination Reaction}

1. Secure serum from a rabbit which has been immunized with Bacillus typhosus. Serum of a typhoid convalescent may be used instead.

2. Prepare a series of serum dilutions in small narrow test tubes in amounts of 1 c.c., ranging from $1: 10$ up to $1: 640$.

3. Add to each $1 \mathrm{c}$ c. of the bacillary emulsion of a good twenty-four- to fortyeight-hour bouillon culture of Bacillus typhosus or an emulsion prepared by washing twenty-four-hour growths from agar slant cultures with normal salt solution, according to the technic given in the text. This doubles the dilutions, which now range from 1:20 up to 1:1280. Prepare the culture control.

4. Shake gently and incubate for two hours at $37^{\circ} \mathrm{C}$. and then record results after tubes have been standing at room temperature for six hours. Reëxamine tubes with the agglutinoscope and note the higher delicacy of such readings.

5. If a typhoid immune serum of unknown titer is used and agglutination is complete in the highest dilution, the test must be repeated with still higher dilutions in order to determine the agglutinin titer of the serum.

(a) Has agglutination occurred in the control tube? Why is this control so important in this and all agglutination reactions?

(b) Is agglutination as complete in the lower as in the higher reaction? If not, how do you explain this result? Of what practical import is this phenomenon?

(c) Describe the appearance of macroscopic agglutination.

(d) Are the agglutinated bacilli dead? To determine this, pipet off the supernatant fluids of several tubes into a germicidal solution; then add an excess of sterile normal salt solution to the sediment of agglutinated bacilli, stir up the sediment, and transfer with a sterile pipet to a sterile centrifuge tube; centrifuge thoroughly; remove the supernatant 
fluid with a sterile pipet and plant several loopfuls of bacteria on slants of agar and in neutral bouillon. Why is it advisable to wash the sediment?

(e) What advantages has the macroscopic over the microscopic method?

\section{Experiment 55.-Macroscopic Agglutination Reaction (Kolle)}

1. Prepare dilutions in amounts of 1 c.c. of a typhoid immune serum in proper test-tubes, ranging from 1:20 up to $1: 1280$.

2. Add one loopful of a twenty-four-hour culture of Bacillus typhosus to each tube, being careful to emulsify thoroughly on the side of the test-tube according to the technic given. This does not materially alter the degree of dilution. Prepare the culture control as usual.

3. Incubate and examine tubes as in the previous experiment.

What are the advantages and disadvantages of this method?

\section{Experiment 56.-Macroscopic Agglutination Reaction (Killed Culture)}

1. Inoculate a flask containing 200 c.c. of neutral broth with Bacillus typhosus and incubate for forty-eight hours. At the end of this time a good rich growth is usually secured. Shake gently to stir and break up any clumps of bacilli and heat at $60^{\circ} \mathrm{C}$. for one hour on a water-bath, gently shaking the flask once or twice during this time. Add 5 c.c. formalin; shake, and place in the refrigerator for three days; stopper the flask with a rubber stopper, and before using shake well in order thoroughly to mix the dead bacilli which drop to the bottom of the flask.

2. Prepare a series of dilutions of typhoid immune serum and conduct this experiment according to the macroscopic technic, using the dead instead of a living bacillary emulsion.

(a) What are the advantages of using a killed culture?

(b) Is this method as delicate as when living cultures are used?

(c) Is spontaneous agglutination likely to occur?

(d) What constitutes a satisfactory killed culture for this reaction?

\section{EXERCISE 22.-AGGLUTININS (Continued)}

\section{Experiment 57.-Group Agglutination}

1. Prepare five series of test-tubes containing 1 c.c. of dilutions ranging from $1: 20$ to $1: 640$ of a typhoid immune serum.

2. To the first series add 1 c.c. of a thirty-six-hour bouillon culture of Bacillus typhosus; to the second series, Bacillus paratyphosus "B"; to the third, Bacillus paratyphosus "A"; to the fourth, Bacillus enteritidis (Gärtner); to the fifth, Bacillus coli. The dilutions are thus doubled. Prepare culture controls of all five cultures, and be careful that tubes are properly labeled.

3. Incubate for two hours and record reactions at the end of further six hours at room temperature. patient.

4. This experiment may be repeated with the serum of a typhoid convalescent 
(a) In which series of tubes is agglutination most complete?

(b) Discuss the question of specificity of the agglutinins.

(c) How do you explain group agglutination?

(d) Are group agglutinins present to the same degree as the main agglutinin? How may the group agglutinins be eliminated?

(e) Of what value is the agglutination reaction in showing the biologic relationship of bacteria?

(f) How would the agglutination reaction be used in the diagnosis of an unknown microörganism?

\section{Experiment 58.-Pro-Agglutination-Agglutinoids}

1. This very important phase of agglutination may have been noted in the previous experiments, especially if old immune serums were used, when there is a possibility that agglutinin has degenerated in agglutinoids. Agglutinoids having a stronger affinity than agglutinin for the agglutinogen, and having lost the agglutinophore group, produce little or no agglutination and prevent the action of agglutinin until diluted out of action in the higher serum dilutions. In this way agglutination may be poor or absent in low and present in higher dilutions, an important practical fact to be remembered.

2. The action of agglutinoids may be seen by conducting a macroscopic test with an old immune serum.

3. Otherwise some agglutinoid may be produced by heating an immune serum to $60^{\circ} \mathrm{C}$. for an hour on the water-bath and conducting the test with dilutions of the heated serum.

4. Repeat the tests with old and heated immune serums in dilutions of $1: 40$, $1: 80,1: 160,1: 320$, using the macroscopic and microscopic technic, as this phenomenon of pro-agglutination is not infrequently found in the routine Widal reaction in typhoid fever.

\section{Experiment 59.-The Absorption or Saturation Agglutination Reaction (Castellani)}

1. Immunize a rabbit with three intravenous injections of a heated emulsion of Bacillus typhosus and three of Bacillus paratyphosus " $\mathrm{B}$ " or Bacillus coli, according to the technic given under Active Immunization and Methods of Animal Inoculation.

2. With this immune serum conduct a test according to the technic given in the text.

(a) Of what practical value is this method?

(b) What are "major" and "minor" agglutinins?

\section{EXERCISE 23.-AGGLUTININS (Continued)}

\section{Experiment 60.-Hemagglutinins}

1. Secure 0.1 c.c. of antihuman hemolysin prepared by giving a rabbit a series of injections of washed human erythrocytes. Inactivate the serum by heating to $55^{\circ} \mathrm{C}$. for half an hour. Dilute $1: 100$ by adding 9.9 c.c. salt solution.

2. Prepare 10 c.c. of a 1 per cent: suspension of washed human erythrocytes. 
3. Into a series of six small test-tubes place 0.1 c.c., 0.2 c.c., 0.4 c.c., 0.6 c.c., 0.8 c.c., 1 c.c. of the diluted immune serum; add 1 c.c. of suspension of blood-cells and 1 c.c. of salt solution to each tube. As a control, place 1 c.c. of corpuscle suspension and 1 c.c. of normal salt solution into a seventh tube.

4. Shake gently and place in the incubator for an hour.

(a) Describe agglutination of red corpuscles.

(b) Are the clumps easily broken up?

(c) What would have occurred if complement were present?

(d) Why was it necessary to heat the fresh immune serum? Is it necessary to heat an old immune serum?

(e) Examine a loopful of the sedimented corpuscles microscopically.

\section{Experiment 61.-Blood Transfusion Tests}

Several students may contribute a few cubic centimeters of their blood and agglutination and hemolysin tests made as described in the text (Chapter XVI).

Of what value are agglutination and hemolytic tests preliminary to blood transfusion?

\section{EXERCISE 24.-PRECIPITINS}

\section{Experiment 62.-Titration of a Precipitin Serum}

1. Secure 1 c.c. of antihorse immune serum prepared by immunizing a rabbit with normal horse serum.

2. Secure 1 c.c. of normal horse serum and place 2 c.c. of the following dilutions made with normal salt solution into a series of six narrow test-tubes: 1:100, 1:500, $1: 1000,1: 2000,1: 5000$, and $1: 10,000$. shaken.

3. To each tube add 0.1 c.c. of the immune serum. The tubes must not be

4. Place tubes in an incubator at $37^{\circ} \mathrm{C}$. and observe every thirty minutes for two hours and again after standing in a refrigerator overnight.

(a) Describe the phenomenon of precipitation.

(b) What are the requisites of a satisfactory precipitin serum?

(c) What are the requisites of a satisfactory preparation of the antigen for this reaction?

(d) Discuss the delicacy of this reaction.

\section{Experiment 63.-Titration of a Precipitin Serum} serum.

Repeat this titration, using antihuman immune serum and normal human

\section{Experiment 64.-Specificity of Precipitins}

1. Secure 1 c.c. each of clear antihorse and antihuman serums.

2. Secure 1 c.c. each of normal horse, normal human, and normal guinea-pig 
serum. Prepare 1:100 dilutions with normal salt solution; set up the following in long narrow test-tubes:

Tube 1: 2 c.c. of normal horse serum $(1: 100)+0.1$ c.c. antihorse serum.

Tube $2: 2$ c.c. of normal horse serum $(1: 100)+0.1$ c.c. antihuman serum.

Tube $3: 2$ c.c. of normal human serum $(1: 100)+0.1$ c.c. antihuman serum.

Tube 4: 2 c.c. of normal human serum $(1: 100)+0.1$ c.c. antihorse serum.

Tube 5: 2 c.c. of normal guinea-pig serum $(1: 100)+0.1$ c.c. antihorse serum.

Tube $6: 2$ c.c. of normal guinea-pig serum $(1: 100)+0.1$ c.c. antihuman serum.

Tube 7: 2 c.c. of normal salt solution +0.1 c.c. antihorse serum (control).

Tube 8: 2 c.c. of normal salt solution +0.1 c.c. antihuman serum (control).

3. Do not shake the tubes; place them in the incubator at $37^{\circ} \mathrm{C}$.; inspect every thirty minutes for two hours.

(a) Discuss the question of specificity of precipitins.

(b) Of what practical value are precipitin reactions?

(c) Enumerate the chief points in the technic of a precipitin reaction in the differentiation of proteins.

\section{EXERCISE 25.-PRECIPITINS (Continued)}

\section{Experiment 65.-Forensic Blood Test}

1. Secure from the instructor two pieces of muslin or gauze containing respectively a stain of human and horse blood. These are to be numbered and the source of each stain known only to the instructor. Secure 1 c.c. each of normal human and horse serum and dilute 1:1000.

2. Prepare extracts of each stain as described in the text (Chapter XVII).

3. Secure 1 c.c. of clear antihuman and antihorse immune serum.

4. Set up the following in long narrow test-tubes:

Tube 1: 2 c.c. of extract No. 1 in dilution of $1: 1000+0.1$ c.c. of antihuman serum. Tube 2: 2 c.c. of extract No. 1 in dilution of $1: 1000+0.1$ c.c. of antihorse serum.

Tube 3: 2 c.c. of extract No. 2 in dilution of $1: 1000+0.1$ c.c. of antihorse serum.

Tube 4: 2 c.c. of extract No. 2 in dilution of $1: 1000+0.1$ c.c. of antihuman serum.

Tube 5: 2 c.c. of normal human serum in dilution $1: 1000+0.1$ c.c. antihuman serum (control).

Tube 6: 2 c.c. of normal horse serum in dilution of $1: 1000+0.1$ c.c. antihorse serum (control).

Tube $7: 2$ c.c. of normal salt solution +0.1 c.c. of antihuman serum.

Tube 8: 1 c.c. of normal salt solution +0.1 c.c. of antihorse serum.

5. Do not shake tubes; place in the incubator at $37^{\circ} \mathrm{C}$. and inspect every thirty minutes for two hours and after standing in the refrigerator overnight.

(a) Inspect the controls. Are the antiserums potent and satisfactory? Why is it advisable to have these controls?

(b) Are you able to diagnose the source of each stain?

(c) In a medicolegal test, if these reactions were negative, how would you proceed further in your efforts to establish the identity of a particular stain? 


\section{EXERCISE 26.-PRECIPITINS (Continued)}

\section{EXPERIMENT 66.-MILK-PRECIPITIN. (LACTOSERUM)}

1. Secure a cubic centimeter of anticow serum previously prepared by immunizing rabbits with injections of fresh cow's milk.

2. Prepare a 1:50 dilution of cow's milk (precipitinogen) by mixing 0.2 c.c. milk with 9.8 c.c. normal salt solution. Prepare a similar dilution of human or goat milk for control tests.

3. Into a series of six small test-tubes place 2 c.c. of the following dilutions of cow's milk: $1: 50,1: 100,1: 200,1: 400,1: 800$, and $1: 1600$.

4. Into a second series of three tubes place 2 c.c. of the following dilutions of control milk: 1:50, 1:100, 1:200.

5. Into the nine tubes of these series add 0.1 c.c. cow lactoserum.

6. A third series of six tubes containing 2 c.c. of the above dilutions of cow's milk plus 0.1 c.c. of normal rabbit serum may be set up as further controls.

7. Keep the tubes at room temperature and inspect at the end of fifteen minutes; half an hour; one hour, and two hours.

(a) Describe a positive reaction.

(b) Discuss the specificity of lactoserums.

(c) Of what practical value are lactoserum reactions?

\section{Experiment 67.-Bacterial Precipitins}

1. Inoculate two flasks each containing 50 c.c. of sterile neutral bouillon with cultures of Bacillus typhosus and Bacillus coli and cultivate at $37^{\circ} \mathrm{C}$. for three weeks. Filter cultures through a sterile Berkefeld filter until clear.

2. Into a series of four small test-tubes place 2 c.c. of the following dilutions of the typhoid filtrate (precipitinogen) undiluted: $1: 2,1: 4$, and 1:10. Prepare a second series of tubes with similar amounts of the same dilutions of Bacillus coli filtrate.

3. Add 0.1 c.c. of potent typhoid immune serum to all tubes of both series. A serum with high agglutinin titer will be satisfactory.

4. Place 2 c.c. of the undiluted typhoid and coli filtrates in separate tubes as controls. Prepare an additional control by placing 0.1 c.c. of the typhoid immune serum in 2 c.c. normal salt solution. hours.

5. Observe the tubes at the end of half an hour, and after one, two, and six

(a) Describe a positive bacterial precipitin reaction.

(b) Has a precipitate formed with the Bacillus coli filtrate? With what microörganism would typhoid immune serum be likely to produce a precipitate?

(c) Discuss the relative delicacy of precipitation and agglutination reactions in the differentiation of bacteria.

\section{Experiment 68.-Noguchi Globulin Reaction}

1. Secure 1 c.c. each of several cerebrospinal fluids from cases of paresis, tuberculous meningitis, serous meningitis, and normal persons.

2. Conduct this floccule-forming reaction after the technic given in the text.

3. Make total cell counts on each fluid (see text). 

(a) Explain the appearance of a positive reaction.
(b) What constitutes a negative reaction?
(c) What is the diagnostic value of this reaction in syphilis?
(d) What other value has this reaction in diagnosis?
(e) What relation does this reaction bear to total cell counts?
(f) Explain the mechanism of the reaction.

\section{EXERCISE 27.-AMBOCEPTORS AND COMPLEMENTS.-HEMOLYSINS}

\section{Experiment 69.-Resistance of Red Blood-corpuscles to Salt Solution of Various Tonicities. Non-specific Hemolysis}

1. Arrange a series of twenty-three small sterile test-tubes in a rack; to each of the first twenty-one tubes add 3 c.c. of various dilutions of salt solutions ranging from 0.6 per cent. to 0.2 per cent. in steps of 0.02 per cent. Large amounts of these solutions should be at hand, preserved in bottles fitted with tight cork stoppers to prevent evaporation, or prepared at the time according to the technic given in the text (page 380). To tube No. 22 add 3 c.c. distilled water and to No. 23 the same amount of normal salt solution ( 0.85 per cent.).

2. Secure blood-corpuscles after the method given in the text. Dog blood may be substituted for human blood. After the final washing, add 0.05 c.c. to each tube. Shake gently.

3. Make a preliminary reading at once; the final reading is made after the tubes have been standing in the refrigerator overnight.

4. Note the appearance of hemolyzed blood. Prepare a color scale by placing 1 c.c. blood-corpuscles in 30 c.c. distilled water, which represents a standard of 100 per cent. hemolysis. From this solution prepare dilutions with distilled water to represent 80,60, 40 and 20 per cent. hemolysis. For example, 4 c.c. of the stock solution plus 1 c.c. distilled water equals 5 c.c. of an 80 per cent. solution; 3 c.c. of the stock plus 2 c.c. water equals a 60 per cent. solution; 2 c.c. of stock plus 3 c.c. of water equals a 40 per cent. solution, and so on. Carefully compare the volume of non-hemolyzed cells in the bottom of some of the test-tubes with the amount of color in the supernatant fluid. A Duboseq colorimeter may be used for making comparisons with the controls.

5. Determine the minimal and maximal resistance of the corpuscle employed.

(a) What is the appearance of hemolyzed blood?

(b) What is the meaning of the term hemolysis?

(c) Why is this called non-specific hemolysis?

(d) What does a normal salt solution mean?

(e) What is the importance of using a normal salt solution in hemolytic experiments?

(f) How is hemolysis produced by hypotonic solutions?

(g) What would be the objections of using a hypertonic salt solution?

(h) How is an isotonic salt solution prepared?

(i) What is the meaning of the terms minimal and maximal resistance of red blood-corpuscles? .

(j) Of what practical value is this test? 


\section{Experiment 70.-Serum Hemolysis in Vitro}

1. Secure 2 c.c. of blood from the ear of a rabbit which has received at least two intravenous injections of sheep cells. Separate the serum and divide into two portions. Inactivate one portion (A) by heating in a water-bath for half an hour at $56^{\circ} \mathrm{C}$.

2. Place 0.1 c.c. and 0.2 c.c. of the fresh unheated serum of portion (B) in two test-tubes. Likewise the same amounts of the heated serum (A) in two more tubes. Add 1 c.c. of a 1 per cent. suspension of washed sheep cells to each tube and sufficient salt solution to bring the total volume to 4 c.c. As a control, place 1 c.c. of corpuscle suspension and 3 c.c. salt solution in a tube. Shake gently, and incubate for an hour.

(a) Which tubes show hemolysis?

(b) What substances are concerned in serum hemolysis?

(c) Why did hemolysis occur with the unheated and not with the heated serum? What is the meaning of inactivation of a serum?

(d) Why is this called serum hemolysis?

(e) What is the appearance of the control tube? Why is this control included? What would have happened if a hypotonic salt solution had been used?

\section{Experiment 71.-Serum Hemolysis in Vivo}

1. Immunize a rabbit with three intravenous injections of 5 c.c. each of a 10 per cent. suspension of washed cat erythrocytes in sterile salt solution. Give the injections each day; four days after the last injection the rabbit blood is titrated and usually contains a fair amount of anticat hemolysin.

2. Heat 2 c.c. of the immune serum at $56^{\circ} \mathrm{C}$. for thirty minutes and inject into the external jugular vein of a cat.

3. Place the animal in a metabolic cage and collect the urine.

4. After two or three days, autopsy, removing the spleen, liver, and kidneys. Place in 5 per cent. formalin and prepare and stain sections with hematoxylin and eosin and Giemsa solution.

(a) Has blood destruction occurred? What are the evidences?

(b) Would hemolysis occur in the test-tube with a heated immune serum? If not, why not?

(c) How then do you explain hemolysis in vivo with a heated serum?

(d) Examine sections of the spleen, liver, and kidneys. Are there any evidences of phagocytosis of blood-cells, focal necrosis, and nephritis? Explain the probable mechanism of the production of these changes.

\section{EXERCISE 28.-AMBOCEPTORS AND COMPLEMENTS. HEMOLYSINS}

\section{Experiment 72.-Titration of a Hemolytic Amboceptor}

1. Secure 1 or 2 c.c. of blood from the ear of a rabbit which has been immunized with injections of sheep cells. Separate the serum and inactivate by heating to $56^{\circ}$ 
C. for half an hour. Dilute $1: 100$ by adding 0.1 c.c. serum to 9.9 c.c. normal salt solution.

2. Prepare 40 c.c. of a 5 per cent. dilution of fresh guinea-pig serum to be used for complement. Prepare 40 c.c. of a $2 \frac{1}{2}$ per cent. suspension of washed sheep cells by adding 1 c.c. of corpuscles to 39 c.c. of normal salt solution.

3 . Proceed with the titration as given in the text on page 375 . If the smallest dose, viz., 0.1 c.c. of the $1: 100$ dilution, completely hemolyzes the corpuscles, it will be necessary to retitrate with a dilution of $1: 1000$.

(a) Is it necessary to use exactly the same amounts of complement and corpuscles in all tubes and if so, why?

(b) If hemolysis did not occur at all in this experiment, what factors may be at fault?

(c) If the corpuscle control were completely hemolyzed, what deduction would you draw?

(d) What is the amboceptor unit of this serum?

\section{- Experiment 73.-Quantitative Factors in Serum Hemolysis}

1. Having determined the amboceptor unit of the above antisheep immune serum, proceed as follows:

2. To a series of four test-tubes add an amount of immune serum equaling one, two, three, and five amboceptor units respectively. To each tube add 0.5 c.c. of the $1: 20$ dilution of complement serum. This amount is just half the dose of complement used in titrating the amboceptor. Add 1 c.c. of a $21 / 2$ per cent. suspension of sheep cells to each tube and sufficient salt solution.

3. In a second series of four test-tubes place one half an amboceptor unit and the following amounts of diluted complement serum: 1 c.c., 2 c.c., 3 c.c., and 4 c.c. Add 1 c.c. of the suspension of sheep corpuscles and sufficient salt solution to make the total volume in each tube about equal. Incubate for one hour and read the results.

4. In a third series of four test-tubes place one amboceptor unit, 1 c.c. of complement serum (1:20), and the following amounts of corpuscle suspension: 1 c.c., 2 c.c., 3 c.c., and 4 c.c. Add sufficient salt solution to make the total volume in each tube about equal.

5. Prepare a corpuscle control with 1 c.c. of suspension and 4 c.c. normal salt solution.

6. Shake all tubes gently and incubate for two hours at $37^{\circ} \mathrm{C}$.

(a) Can an excess of amboceptor make up for a deficiency in complement?

(b) Can an excess of complement make up for a deficiency of amboceptor?

(c) What happens when an excess of corpuscle suspension is used?

(d) Discuss the importance of quantitative factors in serum hemolysis. 


\section{EXERCISE 29.-AMBOCEPTORS AND COMPLEMENTS (Continued)}

\section{Experiment 74.-Rôle of Amboceptor and Complement in Hem- OLYSIS}

1. Prepare a $2 \frac{1}{2}$ per cent. suspension of washed sheep corpuscles. Bleed a healthy guinea-pig under ether anesthesia, separate the serum, and dilute 1:20 with normal salt solution (complement). Secure antisheep hemolytic serum (inactivated) whose hemolytic titer is known (consult instructor).

2. Proceed to set up a series of four test-tubes as follows:

Tube 1: 1 c.c. corpuscle suspension+sufficient normal salt solution to make the total volume about 4 c.c.

Tube 2: 1 c.c. corpuscle suspension +1 c.c. complement serum $(1: 20)+$ sufficient salt solution.

Tube 3: 1 c.c. corpuscle suspension + two hemolytic doses of antisheep hemolysin + salt solution.

Tube 4: 1 c.c. corpuscle suspension +1 c.c. complement serum + two doses of hemolysin + salt solution.

3. Shake each tube gently and incubate at $37^{\circ} \mathrm{C}$. for one or two hours.

(a) In which tube has hemolysis occurred? Explain the results.

(b) What does inactivation of a serum mean?

(c) Could some hemolysis occur, using the complement serum and corpuscles without immune hemolysin?

\section{EXPERIMent 75. - Specificity of Amboceptors}

1. Prepare a $2 \frac{1}{2}$ per cent. suspension of washed sheep corpuscles and a 1 per cent. suspension of washed human corpuscles. Bleed a guinea-pig under ether, separate serum, and dilute 1:20 with normal salt solution. Secure antisheep and antihuman hemolytic serums (inactivated) whose hemolytic titers are known.

2. Proceed as follows:

Tube 1: 1 c.c. sheep corpuscles +1 c.c. complement $(1: 20)+$ two doses of antisheep hemolysin + salt solution up to 4 c.c.

Tube 2: 1 c.c. sheep corpuscles +1 c.c. complement $(1: 20)+$ five doses of antihuman hemolysin.

Tube 3: 1 c.c. human corpuscles +1 c.c. complement $(1: 20)+$ two doses of antihuman hemolysin.

Tube $4: 1$ c.c. human corpuscles +1 c.c. complement $(1: 20)+$ five doses of antisheep hemolysin.

Tube 5: 1 c.c. sheep corpuscles +3 c.c. salt solution (control).

Tube 6: 1 c.c. human corpuscles +1 c.c. salt solution (control).

3. Shake tubes gently and incubate for an hour or two.

(a) In which tubes has hemolysis occurred?

(b) What does this experiment teach as to the specificity of these amboceptors?

(c) Discuss the specificity of hemolytic and bacteriolytic amboceptors. 
Experiment 76.-General Properties of Amboceptors

1. Secure antisheep amboceptor so diluted that 1 c.c. contains 1 hemolytic unit.

2. Prepare a $2 \frac{1}{2}$ per cent. suspension of sheep corpuscles.

3. Secure fresh guinea-pig complement serum and dilute 1:20.

4. Place two units of amboceptor into each of six test-tubes. Place these in a water-bath and heat at $65^{\circ} \mathrm{C}$.

5. Remove a tube from the water-bath after fifteen minutes, thirty minutes, forty-five minutes, one hour, one and one-half hours, and two hours. After allowing them to cool, add 1 c.c. corpuscle suspension and 1 c.c. complement serum (1:20).

6. Set up a tube containing two units of amboceptor (not heated) +1 c.c. corpuscle suspension +1 c.c. complement serum (control). Shake each tube gently and incubate for an hour at $37^{\circ} \mathrm{C}$.

(a) Has the amboceptor deteriorated by exposure to this degree of heat?

(b) Discuss the stability of amboceptors to temperature, age, germicides, and drying.

(c) Discuss the relative stability of amboceptors and complements.

\section{EXERCISE 30.-AMBOCEPTORS AND COMPLEMENTS.-HEMOLYSINS (Continued)}

\section{Experiment 77.-Mechanism of Amboceptor ACtion}

1. Secure antisheep amboceptor so diluted that 1 c.c. contains one hemolytic unit.

2. Prepare a $21 / 2$ per cent. suspension of sheep corpuscles.

3. Secure fresh guinea-pig serum and dilute 1:20.

4. Into two centrifuge tubes place two units of amboceptor and 1 c.c. of corpuscle suspension. Mix, and place one tube in a glass of cracked ice, leaving the other at room temperature. After one hour centrifuge both. Pipet the supernatant fluids into two separate test-tubes.

5. To the sedimented corpuscles in both centrifuge tubes add 1 c.c. corpuscle suspension and 1 c.c. diluted complement serum. Mix well. Incubate tubes for one hour at $37^{\circ} \mathrm{C}$.

6. To the supernatant fluid of each tube add 1 c.c. corpuscle suspension and 1 c.c. of diluted complement serum. Mix. Incubate tubes for an hour.

(a) In which tubes has hemolysis occurred?

(b) How do you explain the results?

(c) Discuss the prevailing views of amboceptor action.

\section{Experiment 78.-A Further Study of the Mechanism of Ambo-} CEPTORS

1. In a centrifuge tube place six hemolytic doses of antisheep hemolysin and 2 c.c. of fresh guinea-pig complement diluted 1:20. Place in a glass of cracked ice until the mixture is thoroughly chilled. Then add 2 c.c. of a $2 \frac{1}{2}$ per cent. suspension of sheep cells (also chilled). Mix and keep at a low temperature for an hour, centrifuge thoroughly, and pipet the supernatant fluid to a separate test-tube. 
2. In a second centrifuge tube place 2 c.c. of diluted complement and 2 c.c. corpuscle suspension. Keep at room temperature for one hour, centrifuge, and pipet the supernatant fluid into a test-tube.

3. Proceed as follows: To 2 c.c. of the supernatant fluid from the first centrifuge tube add 1 c.c. corpuscle suspension; to the remaining 2 c.c. add 1 c.c. corpuscle suspension and two units of amboceptor; to the sedimented corpuscles add 2 c.c. of diluted complement serum.

4. To 2 c.c. of the supernatant fluid from the second centrifuge tube add 1 c.c. corpuscle suspension; to the remaining 2 c.c. add 1 c.c. corpuscle suspension and two units of hemolytic amboceptor.

5. To the sedimented corpuscles in both centrifuge tubes add 1 c.c. complement serum and 1 c.c. salt solution. Mix well.

6. Shake all tubes gently and incubate for one hour at $37^{\circ} \mathrm{C}$.

(a) In which tubes has hemolysis occurred?

(b) Does complement unite directly with corpuscles?

(c) What evidence have you that amboceptors unite directly with corpuscles?

(d) What is meant by the term "sensitizing corpuscles"?

(e) Is complement-amboceptor activity apparent at low temperature?

(f) What temperature best favors complement-amboceptor activity?

\section{EXERCISE 31.-AMBOCEPTORS AND COMPLEMENTS.-HEMOLYSINS (Continued)}

\section{Experiment 79.-Natural Hemolysins. Removal of Natural Hemolysins}

1. Secure 1 c.c. of serum from the blood of five different persons and inactivate in the water-bath.

2. Remove 0.5 c.c. of serum from each to five separate centrifuge tubes and add 4.5 c.c. of $2 \frac{1}{2}$ per cent. suspension of sheep cells to each. Shake gently and after half an hour at room temperature centrifuge thoroughly and pipet the supernatant dilute serum to separate tubes. Do not discard the corpuscles in the centrifuge tubes.

3. To each of the remaining 0.5 c.c. amounts of serum add 4.5 c.c. salt solution and place 1 c.c. and 2 c.c. into two test-tubes respectively ( 0.1 c.c. and 0.2 c.c. undiluted serum).

4. Into two more test-tubes place 1 and 2 c.c. respectively of the diluted serum which has been treated with corpuscles.

5. Add to each tube 1 c.c. of guinea-pig complement (1:20), 1 c.c. of $2 \frac{1}{2}$ per cent. suspension of sheep cells, and sufficient salt solution. Shake gently and incubate for one hour.

6. To the sedimented corpuscles in the centrifuge tubes add 2 c.c. of diluted complement serum and sufficient salt solution. Shake gently and incubate for one hour.

(a) Has hemolysis occurred with the untreated serums?

(b) How do you explain the results?

(c) Has hemolysis occurred with the treated serums and if not, why not? 
(d) Has hemolysis occurred with the corpuscles used in treating the serums and why?

(e) Of what practical importance are natural hemolysins?

(f) Is natural antihuman hemolysin ever found in human serums? What are they called?

Note.-The quantity of natural amboceptor in any of these serums may be determined by the method of titration given in the text. In choosing five serums at random it may be possible that some will not show an appreciable amount of natural antisheep amboceptor.

\section{EXERCISE 32.-AMBOCEPTORS AND COMPLEMENTS (Continued)}

\section{EXPERIMANTT 80.-Hemolytic CoMplement}

1. After giving a rabbit three intravenous injections of 5 c.c. of a 10 per cent. suspension of washed sheep cells at intervals of three days, remove three cubic centimeters of blood from an ear and separate the serum. Dilute the serum 1:10 with salt solution.

2. Intothour test-tubes place 1 e.e. of a $21 / 2$ per cent. suspension of sheep corpuscles

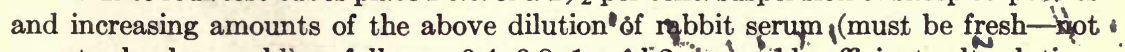
over twelve hours old) as follows: $0.4,0.8,1$, and 2 c.c.) '́dd sufficient salt solution. Shake and incubate for one hour.

(a) Has hemolysis occurred?

(b) How do you explain the reaction?

(c) From where was the complement derived?

(d) Are complements found in the bloods of all animals?

(e) Discuss the question of the multiplicity of complements.

\section{Experiment 81.--Inactivation and Reactivation of Complement}

1. Heat 1 c.c. of the $1: 10$ dilution of fresh rabbit immune serum used in the preceding experiment to $56^{\circ} \mathrm{C}$. for half an hour (in a water-bath).

2. In a test-tube place a cubic centimeter of this heated serum and 1 c.c. of a $2 \frac{1}{2}$ per cent. suspension of sheep cells and sufficient salt solution. Shake gently and incubate for one hour.

(a) Does hemolysis occur?

(b) How do you explain the result?

(c) What is meant by inactivation of complement?

(d) Is complement thermostabile?

3. Add to the tube 1 c.c. of fresh guinea-pig serum diluted 1:10 and reincubate for an hour.

(e) Has hemolysis occurred?

(f) How do you explain the result?

(g) What is meant by reinactivation? 


\section{Experiment 82.-General Properties of Complement}

1. Secure antisheep amboceptor the hemolytic unit of which is known by previous titration.

2. Prepare a $2 \frac{1}{2}$ per cent. suspension of washed sheep corpuscles.

3. Into a series of four test-tubes place the following:

Tube 1: 1 c.c. of fresh guinea-pig serum diluted 1:20.

Tube 2: 1 c.c. of guinea-pig serum (1:20) which has been kept at room temperature for two days.

Tube 3: 1 c.c. of guinea-pig serum (1:20) three days old.

Tube 4: 1 c.c. of guinea-pig serum (1:20) five days old.

Add 1 c.c. of a $2 \frac{1}{2}$ per cent. suspension of sheep corpuscles, two hemolytic units of antisheep amboceptor, and sufficient normal salt solution to each tube. Shake gently and incubate at $37^{\circ} \mathrm{C}$. for one hour.

4. Into a series of six test-tubes place 1 c.c. of fresh guinea-pig complement serum diluted 1:20. Place these in a water-bath at $56^{\circ} \mathrm{C}$. At intervals of ten, twenty, thirty, forty, fifty, and sixty minutes remove a tube, add 1 c.c. corpuscle suspension, two units of amboceptor, and sufficient salt solution. Shake gently each tube and incubate for one hour at $37^{\circ} \mathrm{C}$.

(a) Record the results. Does hemolytic complement deteriorate readily at room temperature?

(b) What are complementoids?

(c) What practical significance has this experiment? Should a complement serum be fresh when used in hemolytic work?

(d) Is complement thermolabile? How long does it take to destroy a diluted complement at $56^{\circ} \mathrm{C}$.?

(e) In inactivating a serum why do we not use a higher temperature?

\section{EXERCISE 33.-AMBOCEPTORS AND COMPLEMENTS (Continued)} Experiment 83.-Titration of Hemolytic Complement

1. Prepare 20 c.c. of complement $1: 20$; prepare a $2 \frac{1}{2}$ per cent. suspension of sheep cells.

2. To a series of 10 test-tubes add increasing doses of diluted complement serum: $0.1,0.2,0.4,0.5,0.6,0.7,0.8,0.9,1$, and 1.5 c.c.; add $11 / 2$ units of hemolytic amboceptor (determined by previous titration); 1 c.c. of corpuscle suspension and sufficient salt solution to bring the total volume in each tube to 3 c.c.

3. Shake gently and incubate for one hour at $37^{\circ} \mathrm{C}$.

(a) Record the results. What is the complement unit of this serum?

(b) What animal serum is best adapted for complement in hemolytic work?

(c) Is the complement content of the serums of different animals constant? Does it vary in animals of the same species? In the same animal at different times? 


\section{Experiment 84.-Phenomenon of Complement Fixation}

This experiment is introduced here to show the Bordet-Gengou phenomenon of complement fixation. The exact technic of complementfixation reactions as conducted for the diagnosis of syphilis and other infections will be given in subsequent exercises.

1. Use the same complement serum, amboceptor and corpuscle suspension as used in the preceding experiment. The unit of complement is now known.

2. Secure 1 c.c. of an antigonococcus and a normal serum and heat at $56^{\circ} \mathrm{C}$. for thirty minutes.

3. Secure an emulsion of gonococci which is now called the antigen: for the proper dose to use consult the instructor.

4. Proceed as follows:

Tube 1: 0.2 c.c. antigonococcus serum + dose of antigen +2 units of complement + normal salt solution.

Tube 2: 0.2 c.c. antigonococcus serum +2 units of complement + normal salt solution (serum control).

Tube 3: 0.2 c.c. normal serum + doses of antigen +2 units of complement + normal salt solution.

Tube 4: 0.2 c.c. normal serum +2 units of complement + normal salt solution (serum control).

Tube 5: dose of antigen +2 units of complement + normal salt solution (antigen control).

Tube 6: 2 units of complement + normal salt solution (hemolytic control).

Tube 7: 1 c.c. corpuscle suspension + normal salt solution (corpuscle control).

Plug tube with cotton as it is finished.

5. Shake all tubes gently and incubate for one hour at $37^{\circ} \mathrm{C}$.

6. Add $11 / 2$ units of amboceptor and 1 c.c. corpuscle suspension to all tubes except corpuscle control. Shake gently and reincubate for one hour.

(a) Examine tubes and record your results.

(b) Why was the serum control on each serum necessary?

(c) Why was the hemolytic system control necessary?

(d) Why was the antigen control necessary?

(e) What is meant by non-specific complement fixation?

(f) What is meant by specific complement fixation? Explain the phenomenon. Which tube of this series shows specific complement fixation and why? Is there any evidence of non-specific fixation in any of the tubes? If so, what bearing would this have on the results of this test?

(g) What is meant by complement deviation?

(h) Upon what does the specificity of complement fixation depend? 


\section{EXERCISE 34.-ANTIGENS FOR THE WASSERMANN REACTIONS}

Experiment 85.-Preparation of Antigens for the WassermanN REACTION

1. Prepare 50 c.c. of an alcoholic extract of syphilitic liver (see page 420 ).

2. Prepare an extract of acetone-insoluble lipoids from 20 grams of beef heart (see page 422 ).

3. Prepare 50 c.c. of a cholesterinized alcoholic extract of human heart (see page 421).

(a) Are antigens for the Wassermann syphilis reaction specific?

(b) Upon what does the high specificity of the Wassermann reaction depend?

(c) What constitutes a specific antigen for the Wassermann reaction?

(d) Discuss the important relation of antigen to the Wassermann reaction.

\section{EXERCISE 35.-ANTIGENS (Continued)}

\section{Experiment 86.-Methods of Titration of Antigens}

While the above extracts are in the course of preparation, stock extracts may be used for titration. After the student has finished the above three extracts, they are titrated in a similar manner.

1. Dilute 1 c.c. of an alcoholic extract of syphilitic liver in a test-tube $1: 10$ by slowly adding 9 c.c. of normal saline solution. Dilute a cholesterinized extract $(1: 20)$ by slowly adding 9.5 c.c. salt solution to 0.5 c.c. of extract.

2. Conduct the anticomplementary titration of each (page 428).

3. Conduct the antigenic titration of each (page 431).

(a) What is meant by the anticomplementary dose of an antigen?

(b) What is meant by the antigenic dose of antigen?

(c) Why are these titrations so important?

(d) In a complement-fixation test what would be the result if the antigen were used in the anticomplementary dose? What would be the result if the antigen were used in less than the antigenic dose?

(e) What constitutes a satisfactory antigen for any complement-fixation test?

(f) In conducting a diagnostic reaction, should the antigen be used in exactly one antigenic dose or double or treble this amount? Why and under what conditions would this be a safe procedure?

(g) Why should the antigen be diluted slowly with salt solution?

(h) Why is a serum control so important in these titrations? What other controls are used and why? 
(i) Which is more important, the anticomplementary or antigenic titration and why?

\section{EXERCISE 36.-WASSERMANN REACTION}

\section{Experiment 87.-ANTicomplementary ACtion of Serums}

1. Secure 1 c.c. of five different specimens of human serums which have been standing in the laboratory for one, two, four, six, and ten days respectively. The last specimen should be intentionally infected with a culture of staphylococcus.

2. Divide each serum into two portions and heat portion " $\mathrm{B}$ " to $56^{\circ} \mathrm{C}$. for half an hour.

3. Place 0.2 c.c. of each specimen (unheated) in a series of five test-tubes.

4. Place 0.2 c.c. of each specimen (heated) in a second series of five test-tubes.

5. To each tube add 1 c.c. of complement 1:20 and sufficient salt solution to bring the total volume to 3 c.c. Shake gently and incubate for half an hour. Add $11 / 2$ units of amboceptor and 1 c.c. corpuscle suspension to each tube and incubate for one hour.

6. A hemolytic system control (complement, corpuscles, and amboceptor) should be included; also a corpuscle control.

(a) Record your results. Are any of the serums anticomplementary? How do you determine this?

(b) What is meant by the terms anticomplementary action of a serum?

(c) What significance has this phenomenon in complement-fixation work?

(d) Are anticomplementary bodies thermolabile or thermostabile? May both exist? Under what conditions are each most likely to be present?

(e) How are thermolabile anticomplementary bodies removed or their influence overcome? When a serum is three or more days old, what is the chief object of heating it before it is used in a complementfixation test?

(f) Does complement keep for three days?

(g) Is it possible to remove the thermostabile anticomplementary action of a serum? Could such a serum be used in a complement-fixation reaction? How is this condition of the serum detected?

(h) Under what conditions is a serum likely to become anticomplementary in action? Is a sterile serum likely to become anticomplementary?

\section{EXERCISE 37.-WASSERMANN REACTION (Continued)}

Experiment 88.-Wassermann Reaction (First Method)

1. Secure four specimens of blood: one from a known syphilitic person; the second from a known normal person, the third and fourth specimens for diagnosis. Also a specimen of cerebrospinal fluid. 
2. Conduct a Wassermann reaction with each serum after the first method, using an antigen of alcoholic extract of syphilitic liver.

(a) Record your results. In case the hemolytic system control was not completely hemolyzed, what may be the reasons and what influence would this result have on interpreting the reactions?

(b) In case the antigen control was not completely hemolyzed, what influence would this result have on the reactions?

(c) If a serum control were incompletely hemolyzed, what influence would this have on the results of the test?

(d) Is this method a quantitative reaction?

(e) What is the nature of the antibody in a syphilitic serum?

(f) How are these reactions recorded? How should they be reported to a clinician?

(g) Discuss the value of the Wassermann reaction in the various stages of syphilis as a guide to treatment.

\section{EXERCISE 38.-WASSERMANN REACTION (Continued)}

\section{Experiment 89.-Wassermann Reaction (Second Method)}

1. Conduct a Wassermann reaction with each of five specimens of serum after the second method, using an alcoholic extract of syphilitic liver, an extract of acetoneinsoluble lipoids, and an alcoholic extract of heart reënforced with cholesterin. One of these serums should be from a syphilitic person (positive control) and one from a normal person (negative control).

(a) What are the advantages of using more than one antigen?

(b) Discuss the relative value of these three antigens.

(c) Under what conditions may a cholesterinized extract be used?

\section{EXERCISE 39.-WASSERMANN REACTION (Continued)}

\section{Experiment 90.-Wassermann Reaction (Third Method)}

Conduct a Wassermann reaction with each of four specimens of serum after the third method, using an extract of acetone-insoluble lipoids. One of these serums should be a positive control and one a normal or negative control.

What is the advantage of using a quantitative test?

\section{EXERCISE 40.-WASSERMANN REACTION (Continued)}

\section{Experiment 91.-Wassermann Reaction (Fourth Method)}

Conduct a Wassermann reaction with a known positive, a known negative, and two unknown serums after the fourth method, using an alcoholic extract of syphilitic liver.

What are the main advantages of this technic? 


\section{EXERCISE 41.-NOGUCHI MODIFICATION OF THE WASSERMANN RE- ACTION}

\section{Experiment 92.-Titration of Antihuman Hemolysin}

1. Secure 0.1 c.c. of inactivated antihuman rabbit amboceptor serum and dilute 1:100 by adding 9.9 c.c. normal saline solution.

2. To a series of six small test-tubes add increasing amounts of diluted amboceptor as follows: $0.1,0.2,0.4,0.6,0.8$, and 1 c.c., add 0.1 c.c. of 40 per cent. complement, 1 c.c. of 1 per cent. human corpuscle suspension, and sufficient saline solution to bring the total volume to 2 c.c.

3. Incubate for one to two hours, shaking the tubes once or twice during this time.

(a) Are there any evidences of hemagglutination?

(b) What constitutes the unit of hemolytic amboceptor?

(c) Is an antihuman hemolytic system more delicate than an antisheep system in complement-fixation work?

(d) What are the advantages of using an antihuman hemolytic system?

\section{Experiment 93.-Technic of the Noguchi Modification}

1. Secure five specimens of blood not over twenty-four hours old, including at least one positive and one negative serum.

2. Conduct the Noguchi reaction with each serum in the unheated or active state, using an antigen of acetone-insoluble lipoids. (See page 450.)

3 . Conduct the reactions with the same antigen, using the serums after heating to $56^{\circ} \mathrm{C}$. for half an hour.

(a) Record your results. Are they equal in both series?

(b) What is meant by proteotropic reaction?

(c) What effect has heat upon syphilis reagin?

(d) Does an active serum react more delicately than an inactivated one?

(e) In performing this test with unheated serum what precautions are to be observed?

\section{EXERCISE 42.-WASSERMANN AND NOGUCHI REACTIONS}

EXPERIMENT 94.-CoMparison of Methods

1. Secure five serums and a cerebrospinal fluid.

2. Conduct a Wassermann reaction with each after the second method.

3. Conduct a Noguchi test with each serum inactivated and using an extract of acetone-insoluble lipoids.

(a) How do the results compare?

(b) Which reactions are more easily read?

(c) Discuss the relative delicacy and value of the Wassermann and Noguchi reactions with both active and inactivated serum in the latter. 


\section{EXERCISE 43.-GONOCOCCUS COMPLEMENT-FIXATION REACTION}

EXPERIMENT 95.-Titration of Gonococcus Antigen

1. Secure 1 c.c. of gonococcus antigen and dilute 1:10 by adding 9 c.c. normal saline solution.

2. Secure 1 c.c. of an antigonococcus serum or the serum of a person who reacts positively and inactivate. 479.

3. Conduct an anticomplementary and antigenic titration as described on page

4. A similar titration may be conducted with glanders antigen and antiserum.

(a) Give three methods of preparing a bacterial antigen.

(b) Is it advisable to make polyvalent antigens and why?

(c) Which is of more practical value, the anticomplementary or antigenic titration and why?

(d) What part of the anticomplementary dose of an antigen may be safely used in a diagnostic test?

(e) Is it advisable to titrate a bacterial antigen at frequent intervals?

\section{EXERCISE 44.-GONOCOCCUS COMPLEMENT-FIXATION REACTION} Experiment 96.-Technic of the Gonococcus Reaction

1. Secure six specimens of serum from a genito-urinary clinic, particularly of men suffering with chronic gonococcus infections. Also a known positive and a known normal serum for controls.

2. Conduct the reactions after the method described on page 478 .

(a) Is the gonococcus reaction specific?

(b) Is complement fixation in bacterial infections likely to be as well marked as in syphilis?

(c) In what cases of gonococcus infection is the reaction likely to be negative? Likely to be positive? tive?

(d) How soon after infection is the reaction likely to become posi-

(e) Discuss the practical value of the gonococcus complement-fixation test.

\section{EXERCISE 45.-GONOCOCCUS COMPLEMENT-FIXATION TEST}

\section{Experiment 97.-TeChNic of the Gonococcus Reaction}

1. Using a similar set of serums as in the preceding experiment, conduct the reactions with the one-tenth technic as described on page 481 .

(a) What is the main advantage of this technic?

(b) By which method are the reactions more easily read and recorded? 


\section{EXERCISE 46.-COMPLEMENT FIXATION IN THE DIFFERENTIATION OF PROTEINS}

Experiment 98.-Titration of Immune Serums

1. Secure 1 c.c. each of antihuman and antihorse serum. Inactivate both.

2. Secure 0.1 c.c. each of fresh human and horse serum and dilute 1:1000.

3. Conduct an antigenic titration of each immune serum as described on page 495.

(a) Explain the mechanism of this reaction.

(b) Are protein amboceptors specific?

(c) Discuss the question of inhibition of hemolysis due to a precipitin reaction.

\section{EXERCISE 47.-COMPLEMENT FIXATION IN THE DIFFERENTIATION OF PROTEINS}

\section{Experiment 99.-Technic of the Forensic Blood Test}

1. Secure from the instructor two pieces of gauze stained respectively with human and horse blood. These are to be numbered and their identity known only to instructor.

2. Secure 1 c.c. each of antihuman and antihorse serum.

3. Secure 0.1 c.c. of known human and horse serum for controls.

4. Proceed with the test for identifying these bloods as described on page 494 .

(a) Discuss the specificity of this test.

(b) Is this test more delicate than the precipitin reaction?

(c) Which is more liable to error in technic?

(d) Discuss the applicability of the complement-fixation technic to the differentiation of proteins in general, as animal, vegetable, and bacterial proteins.

\section{EXERCISE 48.-VENOM HEMOLYSIS}

\section{Experiment 100.-Venom Hemolysis in Syphilis}

1. Secure 1 c.c. of venom solution $1: 1000$.

2. Secure four specimens of blood (for technic see page 386) from cases of late secondary or tertiary syphilis and one specimen of normal blood. Also a known normal blood for a control.

3. Prepare the subdilutions of venom and conduct the tests after the technic described on page 387 .

(a) Discuss the prevailing views regarding the mechanism of venom hemolysis.

(b) Discuss the value of the venom test in the diagnosis of syphilis.

(c) Discuss the main points in the technic. 


\section{EXERCISE 49.-BACTERIOLYSIS}

\section{Experiment 101.-Pfeiffer Bacteriolytic Test}

1. Secure 1 c.c. of serum from a rabbit which has been immunized with typhoid bacilli. By working with cholera and a highly potent serum better results are secured, but as there is probably more danger connected with the handling of cholera cultures, typhoid may be substituted with fairly good results. Inactivate by heating to $56^{\circ}$ C. for half an hour.

2. Prepare a twenty-four-hour-old agar culture of a suitable strain of Bacillus typhosus.

3. Prepare a 1:100 dilution of the immune serum and place 3 c.c. in a small testtube. Add three loopfuls of culture and emulsify thoroughly. Inject a guinea-pig intraperitoneally with 2 c.c. of the emulsion.

4. At intervals of ten, twenty, forty, and sixty minutes withdraw small amounts of exudate and study bacteriolysis; prepare hanging-drop preparations which may be compared with a similar control on the culture; prepare smears of the culture and peritoneal exudate and stain with carbol-thionin or carbolfuchsin.

5. If desirable, the bacteriolytic titer of the serum may be determined after the method given in the text.

(a) Describe the phenomenon of bacteriolysis.

(b) Are bacteriolysis and hemolysis similar processes? Give the source of complement in this reaction.

(c) Discuss the specificity of bacteriolytic reactions.

(d) Discuss the practical value of the bacteriolytic test in the diagnosis of a microörganism.

\section{EXERCISE 50.-BACTERIOLYSIS}

\section{Experiment 102.-Microscopic Method of Measuring the BaC- teriolytic Power of the Blood}

This method may be employed for a rapid estimation of the bacteriolysin produced in the blood in response to an inoculation of typhoid vaccine.

1. Secure a small quantity of the patient's serum by collecting blood aseptically in a Wright capsule. About 1 c.c. of serum will be sufficient. Secure a control serum, preferably a "pooled serum," in the same manner. Prepare dilutions of the patient's serum in the following manner: Place a series of six small test-tubes (sterile) in a rack; add 1 c.c. sterile broth to each tube; into the first tube place 1 c.c. of the fresh serum from the patient (1:2 dilution); mix well and transfer 1 c.c. to the second tube; mix and transfer 1 c.c. to the third, and so on to the last tube, when 1 c.c. is discarded.

2. Secure a twenty-four-hour culture of typhoid bacilli in neutral bouillon.

3. Take a simple capillary pipet with a long stem, plugged with cotton and sterilized, and make a mark about $3 \mathrm{~cm}$. from the end. - Draw up the highest dilution of serum to the pencil mark, then a bubble of air, and finally an equal volume of culture. Thoroughly mix by carefully aspirating and driving out the contents on a hollow 
ground slide or in a small tubule. Aspirate the mixture into the middle portion of the stem and seal the pipet in a flame. Label with the final dilution (1:128).

4. Proceed in the same manner with the remaining five dilutions of the patient's serum, which then, mixed with an equal quantity of culture, equals $1: 64,1: 32,1: 16$, $1: 8$, and 1:4. Place these pipets in a large test-tube and label with the patient's name and time when placed in the incubator.

5. Prepare a similar set of pipets using the control serum.

6. Prepare a culture control by mixing equal volumes of culture and sterile broth.

7. Place all pipets in the incubator at $37^{\circ} \mathrm{C}$. for two hours.

8. Prepare hanging-drop preparations of each pipet by breaking off the tip and placing a drop of the contents (after mixing) on a cover slide and suspending in the usual manner. First examine the culture control and then each of the various dilutions, noting in each case the dilution in which there is the first bacteriolytic effect, and the dilution in which there is a complete effect; arrive at the bacteriolytic index by comparing the patient's and the control blood just as the opsonic index is stated.

(a) What are the first evidences of bacteriolysis?

(b) Are endotoxins neutralized when bacteriolysis occurs?

\section{EXERCISE 51.-BACTERIOLYSIS}

Experiment 103.-Method of Measuring the Bactericidal ACtivity of the Blood in Vitro (Method of Stern and Körte)

With a culture of typhoid bacilli, rabbit typhoid immune serum, and rabbit complement serum, a test may be carried out after the method given in the text.

(a) What main precautions are to be followed in this method?

(b) Discuss complement deviation.

(c) Discuss the practical value of this method.

\section{EXERCISE 52.-CYTOTOXINS}

\section{EXPERIMENT 104.-ACtion OF NePhrotoxin}

1. Prepare an emulsion of dog kidney as described on page 73 and immunize a rabbit.

2. Inject a dog intravenously with 2 c.c. of rabbit immune serum per kilo of body weight.

3. Place the animal in a metabolism cage, collect all urine, and carefully examine for albumin, casts, and hemoglobin; make quantitative albumin determinations.

4. After three days autopsy and examine the kidneys and liver histologically.

5. Heat the immune serum to $56^{\circ} \mathrm{C}$. for thirty minutes and place increasing amounts in a series of test-tubes as follows: $0.01,0.05,0.08,0.1$, and 0.2 c.c.; add 1 c.c. of fresh guinea-pig complement serum $1: 20$, and 1 c.c. of $21 / 2$ per cent. suspension of washed dog erythrocytes. Incubate for two hours.

(a) Describe the changes occurring in the liver and kidneys. Give the prevailing views regarding the mechanism of their production.

(b) Are there any evidences of a hemolytic action of the serum in vivo? In vitro? 
(c) How do you explain the presence of hemolysin in this immune serum?

(d) Discuss the specificity of cytotoxins in general.

(e) How may a hemolysin be removed from a nephrocytotoxic serum?

(f) Have the cytotoxins any practical value in the treatment of disease?

\section{EXERCISE 53.-MIOSTAGMIN REACTION}

\section{Experiment 105.-Technic of the Miostagmin Reaction}

1. Secure a portion of dry pulverized cancer tissue and prepare an antigen after the technic described in the text.

2. Titrate this antigen.

3. Secure four specimens of serum: one from a known case of cancer; one from a normal person, and two for diagnosis.

4. Proceed with the reaction as described in the text.

(a) Discuss the principles of this reaction.

(b) Discuss the practical value of this reaction in the diagnosis of cancer.

\section{EXERCISE 54.-ANAPHYLAXIS}

For experiments in anaphylaxis sensitize a series of animals as follows:

(a) Give seven young guinea-pigs an intraperitoneal injection of 0.01 c.c. horse serum (1 c.c. of a $1: 100$ dilution).

(b) Give three young guinea-pigs an intraperitoneal injection of 0.01 c.c. of human serum.

(c) Give two young guinea-pigs an intraperitoneal injection of 0.1 c.c. egg-albumen (1 c.c. of $1: 10$ dilution).

(d) Give two rabbits 1 c.c. of horse serum intravenously every three days for three doses.

(e) Give a dog 10 c.c. of horse serum súbcutaneously.

EXPeriment 106.-Anaphylaxis in the Guinea-Pig. Specificity OF ANAPHYLAXIS

1. Two weeks after the sensitizing injections proceed as follows:

(a) Inject a guinea-pig sensitized to horse serum with 1 c.c. horse serum intraperitoneally.

(b) Inject a guinea-pig sensitized to human serum with 0.1 c.c. of human serum intravenously.

(c) Inject a guinea-pig sensitized to egg-albumen with 1 c.c. of diluted (1:4) albumin intraperitoneally. 
(d) Inject a guinea-pig sensitized to horse serum with 1 c.c. of diluted (1:4) eggalbumen.

(e) Inject a guinea-pig sensitized to human serum with 0.1 c.c. horse serum intravenously.

(f) Inject a guinea-pig sensitized to horse serum with 1 c.c. human serum intraperitoneally.

2. Carefully study the symptoms of anaphylaxis. After death autopsy the animals.

(a) Describe acute anaphylactic shock in the guinea-pig. Does death always occur?

(b) How soon after the intraperitoneal injection of the intoxicating dose do symptoms develop? After the intravenous? Why the difference?

(c) Is anaphylaxis a specific reaction?

(d) What are anaphylactogens?

(e) Discuss the prevailing views regarding the mechanism of the anaphylactic reaction.

(f) Describe the anatomic changes occurring in acute and fatal anaphylaxis in the guinea-pig. Discuss the cause and nature of these changes.

\section{EXERCISE 55.-ANAPHYLAXIS (Continued)}

\section{Experiment 107.-NATURE of THE ANAPHylatoxin}

1. Place 15 grams of coli bacterial substance prepared as described on page 126 in a distilling flask and add 250 c.c. of a 2 per cent. caustic soda in absolute ethyl alcohol. Place on a water-bath attached to a reflux condenser and collect the distillate.

2. Evaporate the distillate to dryness and for each 10 c.c. of the distillate add 5 c.c. of water. Neutralize with $\frac{\mathrm{n}}{20}$ hydrochloric acid.

3. Inject 5 c.c. intraperitoneally into young guinea-pigs. Inject 1 or 2 c.c. intravenously.

(a) Do anaphylactic symptoms develop?

(b) Are the anatomic changes in the lungs similar to those observed in serum anaphylaxis?

(c) Discuss the prevailing views of the nature of anaphylatoxins.

(d) Discuss the prevailing views of the anaphylaxis antibody, toxogen, or anaphylactin.

\section{EXERCISE 56.-ANAPHYLAXIS (Continued)}

\section{EXPERIMENT 108.-ANAPHyLAXIS IN THE Dog}

1. Five weeks after sensitization anesthetize a dog with ether and connect the carotid or femoral artery with a mercurial manometer and arrange for a kymographic tracing. 
2. After recording the normal blood-pressure tracing, inject 5 c.c. of homologous serum (horse) into a jugular vein.

3 . Record the blood-pressure.

4. Study the coagulation time of the blood.

5. Autopsy.

(a) Describe the blood-pressure changes. To what may they be ascribed?

(b) Is blood coagulation delayed? Give a reason for this change.

(c) Do anatomic changes occur in anaphylaxis of the dog?

\section{EXERCISE 57.-ANAPHYLAXIS (Continued)}

\section{Experiment 109.-PASsive ANAPHylaxis}

1. Secure 1 c.c. of serum from a rabbit sensitized to horse serum five weeks previously and inject 0.5 c.c. intraperitoneally into each of two guinea-pigs.

2. Twenty-four and forty-eight hours later inject both animals intravenously with 0.2 c.c. of horse serum.

(a) Are anaphylactic symptoms in evidence?

(b) Discuss the nature of passive anaphylaxis.

(c) If allowed to live, would these animals become sensitized to rabbit serum?

\section{EXERCISE 58.-ANAPHYLAXIS (Continued)}

\section{EXPERIMENT 110.-ANTI-ANAPHYLAXIS}

1. Eight days after sensitizing a guinea-pig with horse serum, inject 1 c.c. of horse serum subcutaneously.

2. Fifteen days after sensitizing a guinea-pig with horse serum, inject 2 c.c. of serum into the rectum.

3. Fifteen days after sensitizing a guinea-pig with horse serum, inject 0.0001 c.c. serum subcutaneously every fifteen minutes for six doses.

4. On the sixteenth day after sensitizing, inject a guinea-pig with 1 c.c. of horse serum. If this animal develops anaphylactic shock, inject the other three guineapigs in a similar manner.

(a) Do anaphylactic symptoms develop? If not, why not?

(b) Discuss the importance of quantitative factors in producing anti-anaphylaxis.

(c) Discuss the importance of anaphylaxis and anti-anaphylaxis in serum therapy.

(d) What method is generally employed in the effort to induce an anti-anaphylactic state in serum therapy?

(e) What drug has been found experimentally of value in ameliorating or preventing the pulmonary changes of anaphylaxis? 
(f) Do anesthetics prevent anaphylaxis?

(g) Under what conditions would you expect anaphylaxis in persons?

\section{EXERCISE 59.-ANAPHYLAXIS (Continued)}

\section{Experiment 111.-Local Anaphylactic Reactions}

1. Shave the abdomen of the rabbit sensitized to horse serum five weeks after the time of sensitization.

2. Make two superficial abrasions over the upper portion of the abdomen; into one rub horse serum, and in the other, normal salt solution.

(a) Does a local reaction occur? If so, describe the lesion.

3. Then inject 0.01 c.c. of serum subcutaneously. If acute anaphylactic death does not follow, observe the animal during the next twenty-four hours.

(a) Does a local Arthus reaction follow this injection of serum? If so, describe the reaction.

(b) Explain the mechanism of a local anaphylactic reaction.

(c) Are the tuberculin, luetin, and mallein reactions anaphylactic? Explain their mechanism and discuss their practical value in diagnosis.

4. Inject 1 c.e. horse serum intravenously. If anaphylaxis occurs, record the symptoms. Autopsy with particular attention to the heart.

\section{EXERCISE 60.-CHEMOTHERAPY}

\section{EXPERIMENT 112.-SALVARSAN}

1. Secure a white rat infected with Sp. recurrens (Sp. Obermayer) and one with $\mathrm{T}$. equiperdum. Also two normal rats.

2. Examine a drop of blood from the tail of each infected rat and form a rough estimate of the number of parasites per microscopic field.

3. Inject both rats intravenously with $0.001 \mathrm{gm}$. salvarsan freshly prepared.

4. Examine the blood at the end of two hours; after forty-eight, seventy-two, and ninety-six hours.

5. Inject a control rat intravenously with $0.01 \mathrm{gm}$. salvarsan and the second with $0.05 \mathrm{gm}$.

(a) Do the control rats survive?

(b) Are the infected rats sterilized?

(c) What is meant by the dosis lethalis? By the dosis curativa By the dosis tolerata?

(d) Discuss organotropism and parasitotropism.

(e) Discuss "drug fastness."

(f) Discuss the question of chemoreceptors. 



\section{INDEX}

ABDERHALDEN's serodiagnosis of pregnancy, 252

dialyzation method, 252, 253

blood-serum, 258

glassware, 253

ninhydrin, 254

precautions, 254

preparation of placental tissue, 257

reading reaction, 259

reagents, 254

sources of error, 260

testing dialyzing shell, 253

shell for non-permeability to albumin, 255

for permeability to peptone, 256

experimental work, 839

optical method, 252, 261

placental peptone, 261

polariscope, 262

reading reaction, 262

testing peptone, 262

practical value, 263

principles, 252

Abortion, contagious, complement-fixation test in, 486

Abrin, 222

toxin, 118

Absorption agglutination reaction, experimental work, 843

in mixed infection, 288

by colloids, 515

methods for differentiating between

mixed and single infection, 272

Abwehrfermente, 249

Acanthosis nigricans, salvarsan in, 811

Acetone-insoluble lipoids for Wassermann reaction, 422

Acid-fast bacilli, fixing and staining, 197

Acne, vaccine therapy, 657

Actionocongestin, 533

Adenitis, tuberculous, tuberculin treatment, 679

Adulteration, meat, detection of, biologic blood test for, 310 technic, 312

Agglutinating power of serum, variation, 274

serums, 68

Agglutination, 266

group, experimental work, 842

mechanism of, 270
Agglutination, mechanism of, Bordet's theory, 271

Gruber theory, 270

Paltauf's theory, 270

test, 278

absorption, experimental work, 843 in mixed infection, 288

in cerebrospinal meningitis, 277

in cholera, 277

in diagnosis of disease, 275

in dysentery, 276

in glanders, 277

in identification of microörganism, 277

in Malta fever, 277

in measuring immunizing response, 278

in plague, 277

in pneumonia, 277

in single or mixed infection, 278

in tuberculosis, 277

in typhoid fever, 275, 279, 282

macroscopic, 279

experimental work, 841,842

Kolle and Pfeiffer's method, 288 technic, 284

microscopic, 279

dry method, technic, 283

experimental work, 840

wet method, technic, 282

practical applications, 275

precautions, 281

requisites for, 279

Agglutinins, 152, 266

absorption methods for differentiating between a mixed and single infection, 272

action of, based on colloidal reactions, 517

definition, 266

experimental work, 815,840

formation, 268

group, 271

history, 266

immune, 268

in immunity, rôle of, 274

nature, 270

normal, 268

origin, 269

partial, 271

preservation of, in dried paper form, 79

production of, 68

intraperitoneal method, 69 
Agglutinins, production of, intravenous method, 68

properties, 270

specificity, 271

Agglutinogen, 269

experimental work, 843

Agglutinoids, 268

Agglutinoscope, 288

Aggressins, 108, 122, 182

anti-, 126

artificial, 103, 125

in relation to infection, 103

natural, 125

experimental work, 824

nature of, 124

Albuminolysin, 553

Alcoholic extracts of normal organs for-Wassermann reaction, 420

reënforced with cholesterin for

Wassermann reaction, 421

of syphilitic livers for Wassermann reaction, 420

Aleppo boil, salvarsan in, 811

Aleuronat, preparation of, 339

Alexin. See Complement.

Allergen, 536, 543

Allergic reactions, 582. See also Anaphylactic reaction.

Allergin, 552

Allergy, 534, 536

Amboceptoids, 321

Amboceptor, 154, 157, 185, 316, 319, 326, 337, 338

and complement, quantitative relationship, 374

anti-, 325

bacteriolytic, 155,319

titration of, 325

formation, 322

hemolytic, 319,362

and complement, quantitative relationship, 374

for Noguchi's modification of Wassermann reaction, 451

for Wassermann reaction, 415

preservation of, 79

titration of, 325

experimental work, 848

history, 319

mechanism of action, 321 experimental work, 851

native, 324

natural, 324

properties, 321

experimental work, 851

quantitative estimation, 325

rôle of, in hemolysis, experimental work, 850

specificity, 323

experimental work, 850

structure, 320

unit of serum, 374

Amebadiastase, 183

Amorphous colloids, 512
Ampules, vaccine, conversion of testtubes into, 24

making of, 24

Anaphylactic reactions, 582

as measure of immunity, 610

experimental work, 867

in diphtheria, 609

in gonococcus infections, 609

in leprosy, 610

in pregnancy, 610

in sporotrichosis, 610

in typhoid fever, 607

prognostic value, 610

Anaphylactin, 552, 557, 563

nature, 553

terminology, 552

Anaphylactogens, 536, 542, 543

bacterial, 547

endotoxins as, 548

non-protein, 543

physical state, 546

protein, chemistry of, 544

Anaphylactotoxins, 542

Anaphylatoxin, 536, 548

Anaphylaxis, 531

accelerated, in infectious diseases, 568 antibody, 552, 563

Arthus phenomenon, 533

Besredka's theory, 557

cellular theory, 555

chronic, 540

definition, 535

experimental work, 864

fall in blood-pressure in, 541, 542

Friedberger's theory, 558

Gay and Southard's theory, 557

Hamburger and Moro's theory, 556

heterologous, 559

history, 532

homologous, 559

immediate, in infectious diseases, 568

in cats, 539

in cold-blooded animals, 541

in cows, 541

in dogs, 540

experimental work, 865

in guinea-pig, 537 experimental work, 864

in hens, 541

in horse, 541

in $\operatorname{man}, 537$

in pigeons, 541

in rabbits, 539

in relation to immunity, 565

to infection, 565

in serum treatment, 686

in sheep, 541

in white mice, 540

rats, 540

indirect, 543

lipoid, 544

mechanism, 541, 561

nature of, experimental work, 865

Nolf's theory, 558

passive, 548,559 
Anaphylaxis, passive, production of, 560, 866

physical theory, 558

relation of, to infectious diseases, 566 to non-infectious diseases, 570

Richet's studies on, 533 theory, 556

Smith's phenomenon, 535

specificity, 563

terminology, 536

theories, 556

torpid early, in infectious diseases, 568

Vaughan and Wheeler's theory, 557

von Pirquet's studies on, 533

Anemia, salvarsan in, 811

Anesthesia for subdural inoculation of serum, 697

in lumbar puncture, 39

Wassermann reaction after, 466

Angina, Vincent's, salvarsan in, 810

Animal blood. See Blood, Animal.

cold-blooded, anaphylaxis in, 541

conjunctival tuberculin test in, 600

cutaneous tuberculin test in, 601

experiments, 815

immunization, active, general technic, 66

methods for effecting, 65

inoculation, 53

intracardial, 62

of guinea-pigs, 62

intramuscular, 56

intraperitoneal, 64

of guinea-pig, 64

of rabbit, 64

intravenous, 56

of dog, 62

of goats, 62

of guinea-pig, 57

of horse, 61

of mice, 60

of rabbit, 56

of rats, 60

of sheep, 62

subcutaneous, 54

with fluid inoculum, 54

with solid inoculum, 55

technic, 53

general rules, 53

intracutaneous tuberculin test in, 601

parasites, aggressiveness, 133

infection with, 132

mode, 132

production of disease by, 133

preparation of, for vaccination, 627

protective immunization, 653

subcutaneous tuberculin test in, 600

tuberculin reaction in, 600

vaccination of, 627

preparation for, 627

Anopheles maculipennis, 166

punctipennis, 166

Antenatal infection, 90

Anthrax, internal, salvarsan in, 768 serum treatment, 767,768
Anthrax, vaccination, 653

Antiaggressins, 126

Antiamboceptors, 325

Antianaphylaxis, 561, 687

experimental production, 562, 866

mechanism, 563

Anti-anthrax serum, 767 administration, 767

Antibacterial immunity, 684 acquired, 171

experimental work, 826

immunization, 684, 735

Antibody, 65, 66, 148, 149, 161, 317

anaphylaxis, 552,563

and antiferments, similarity between, 247

of first order, 149

of second order, 152

of third order, 154

specificity of, 162

Anticholera serum, 770

$$
\text { Kraus', } 770
$$

Anticomplementary action of serums, experimental work, 857

titration of antigens in Wassermann reaction, 428

Anticomplements, 327 auto-, 327

Anticytotoxic serums, 506

Antidiphtheric serum, production of, 227

Antidysenteric serum, collecting and testing, 238

production of, 236

culture, 236

immunizing animals, 237

Antiferments, 246

and antibodies, similarity between, 247

experimental work, 838

in disease, 247

simple, 149

Antigen, 65, 66, 67, 148, 159

bacterial, identification of, complement-fixation test for, 498

preparation of, in complement fixation, 473

principles of complement fixation with, 476

standardizing, in complement fixation, 475

determination of, by complement fixation, 494

for gonococcus complement-fixation test, 478

for Noguchi's modification of Wassermann reaction, 453

titration of, 453

for Wassermann reaction, 417 anticomplementary titration, 428 antigenic titration, 431

experimental work, 856

hemolytic titration, 430

method of diluting, 427

of titrating, 428

preparation, 419 
Antigen, gonococcus, titration of, experimental work, 860 non-protein, 159

Antigenic dose of syphilitic serum, 418 titration in Wassermann reaction, 431 values of various extracts in serum diagnosis of syphilis, 424

Antigonococcus serum, 765 action, 766

administration, 766 preparation, 766

Antihemolysins, 372

Anti-influenza serum, 750 administration, 751

Antilactase, 246

Antilysin test for antistaphylococcus serum, 239

Antimeningococcus serum, 736 action, 741

administration, 742

dosage, 74

repeating, 744

Flexner and Jobling's method of preparing, 739

intravenous injection, 743

Kolle's method of preparing, 740

preparation, 739

repeating doses, 744

serum sickness from, 746

standardization, 740

subdural inoculation, 742

Antipepsin, 246

Antiplague serum, 769

Antipneumococcus serum, 757

action, 759

administration, 759

intravenous injection, 759

Kolle's method of preparing, 758

preparation, 758

results from, 759

standardization, 758

Antiprecipitin, 296

Antirennin, 246

Antisensibilisin, 557

Antiseptics, preservation of serum by, 76

Antistaphylococcus serum, 238, 766 antilysin test for, 239 preparation, 239

Antistaphylolysin, experimental work, 837

method of titrating, in serum, 240

Antisteapsin, 246

Antistreptococcus serum, 760 action, 761

administration, 763

in bronchopneumonia, 764

in diphtheria, 764

in endocarditis, 764

in erysipelas, 764

in puerperal sepsis, 764

in scarlet fever, 764

in smallpox, 764

in tuberculosis, 764

in wound infections, 764

intravenous injection, 763
Antistreptococcus serum, Kolle's method of preparing, 763

preparation, 762

standardization, 763

value, 764

Antitetanic serum, production of, 234

Antitetanolysin, action of, experimental work, 836

Antitoxic immunity, 684

acquired, 171 passive, 173

immunization, 684, 702

Antitoxin, 149, 220

action of, based on colloidal reactions, 516

on toxin, 224

antidysenteric, 236

antistaphylococcus, 238

botulinus, 236

definition, 220

diphtheria, 702. See also Diphtheria antitoxin.

dysentery, 730

administration and uses, 730

experimental work, 835

formation, 221

hay-fever, 242, 734

history, 220

immunity, natural, 169

measure of, 242

natural, 224

pollen, 242, 734

practical application, 243

production of, 68

for therapeutic purposes, 226

properties, 223

relation of, to proteids, 223

specificity, 224

experimental work, 836

structure, 223

tetanus, 719 . See also Tetanus antitoxin.

unit, 42

Behring-Ehrlich, 231

Antitrypsin, 246

test, 250

Bergmann and Meyer's, 250

Antituberculosis serum, 771

Antityphoid serum, 768

Antityrosinase, 246

Antiurease, 246

Antivenene, Calmette's, 734

Antivenin, preparation of, 241

production of, 241

Apotoxin, 556

Aqueous extract of pallidum culture for Wassermann reaction, 424

of syphilitic livers for Wassermann reaction, 419

Arsenoxid, 808

Arthritis, gonorrheal, autoserum treatment, 776

vaccine therapy, 658

Arthus phenomenon of anaphylaxis, 533

Artificial aggressins, 103, 125 
Ascoli and Izar's miostagmin test, 526 . See also Miostagmin reaction.

Asthma, horse, 578

in serum treatment, 687

Athrepsia, 170

Athreptic immunity, 170

Atmosphere, bacteria in, 85

Atrophy, receptoric, 80, 171

Atropin sulphate as preventive of serum disease, 576

Auto-anticomplements, 327

Autocytotoxins, 505

Autogenous bacterial vaccines, 620

Autolysins, 363

Autonephrotoxins, 505

Autopsies, 815

Autoserum treatment, 775

of acute infectious diseases, 775

of cancer, 782

of erysipelas, 776

of gonorrheal arthritis, 776

of hydrocele, 782

of influenza, 776

of leprosy, 776

of Malta fever, 776

of marasmus, 783

of non-tuberculous effusions, 782

of pneumonia, 776

of scarlet fever, 775

of skin diseases, 775

of smallpox, 776

of tuberculosis of serous membranes, 781

of tuberculous meningitis, 782

pleurisy, 781

of typhoid fever, 776

salvarsanized, in syphilis of brain, 776

after-treatment, 780

repeating dose, 780

serobiologic findings in cerebrospinal fluid, 780

technic, 778

Autumnal catarrh, 242

Ayer and Gay's method of titration of bacteriolytic complement, 334

BaCILlen emulsion, tuberculin, preparation, 666

Bacillus, acid-fast, fixing and staining, 197

botulinus, 116

diphtheria, 112

virulence and toxicity, method of testing, 818

dysentery, 116

tetanus, 115

tubercle, living, in treatment of tuberculosis, 669

Bacteremia, 93

Bacteria, 83

agglutination reaction in identification of, 277

aggressiveness of, 95
Bacteria, Bail's classification, 124

defensive mechanism of, in relation

to infection, 102

effect of opsonins on, 189

fixing and staining, 197

in atmosphere, 85

in foods, 85

in milk, 85

in placenta, 86,90

in water, 85

mechanical action, 108, 131 experimental action, 826

morphologic changes, in relation to infection, 102

non-agglutinable species, 274

non-pathogenic, 84

numeric relationship, to infection, 99

on skin, 85,87

pathogenic, 83,84

physiologic changes, in relation to infection, 102

recovered from feces, water-supplies, etc., bacteriolytic test for identification, 343

toxicity of, 94,95

transmission of, by suctorial insects, 86 virulence of, 94

decrease, 95

increase, 96

by addition of animal fluids to culture-medium, 97

by passage through animals, 96

by use of collodion sacs, 97

Bacteria-free filtration, preservation of serum by, 76

Bacterial anaphylactogens, 547

antigens, identification of, complement-fixation test for, 498

preparation, in complement fixation, 473

principles of complement fixation with, 476

standardizing, for complement fixation, 475

diseases, chemotherapy in, 811 complement fixation in, 473

emulsion in opsonic index, 194

ferments, 244

invasion, factors preventing, 90

mechanism, 91

normal defenses against, 90

precipitinogens, preparation, 313

precipitins, experimental work, 846

precipitin test with, 298 technic, 313

production of, 71

proteins, 108, 126

action, 128

experimental work, 824

nature, 127

Vaughan's theory, 128

split proteins, 126

toxins, 108. See also Toxins.

vaccines, 206, 613. See also Vaccines, bacterial. 
Bactericidal power of blood, capillary pipet method of measuring, 355

Neisser and Wechsberg's method of measuring, 349

plate culture method for measuring, 349

technic, 350

Stern and Körte's method of measuring, 349 experimental work, 863

Töpfer and Jaffe's method of measuring, 352

Wright's capillary pipet method of measuring, 355

Bactericidins, 337

Bacterin, 613

therapy, 611, 614, 617

history, 611

Bacteriolysins, 154, 318, 336

and hemolysins, analogy between, 367 definition, 337

experimental work, 815

history, 336

influence of, on endotoxins, 340

normal, 341

origin, 338

practical applications, 341

production of, 69

properties, 341

specificity, 341

Bacteriolysis, 146, 336

and hemolysis, analogy between, 367

experimental work, 862

mechanism, 340

Bacteriolytic amboceptor, 155, 319

titration of, 325

complement, titration of, 334

Gay and Ayer's method, 334

power of blood, microscopic method of measuring, 862

serum in treatment of disease, 359

method of titrating, 344

production of, 69

test for identification of bacteria recovered from feces, water-supplies, etc., 343

method of testing virulence of culture, 343

of titrating bacteriolytic serum, 344

Pfeiffer's, 342

experimental work, 862

in diagnosis of disease, 348

technic of, 347

preparing immune serum, 343

technic, 342

Bacteriotropins, 145, 185, 188

experimental work, 832

quantitative estimation, experimental work, 834

Neufeld's technic, 220. See also Neufeld's quantitative estimation.

Simon's method, 203

Bail's classification of bacteria, 124
Bail's hypothesis, 123

Bandi and Terni's plague vaccine, 648

Bauer's modification of Wassermann reaction, 456

B. E. tuberculin, preparation of, 666

Behring-Ehrlich antitoxin unit, 231

Behring's method of immunization against diphtheria, 717

Beraneck's tuberculin, dose of, 675 preparation of, 666

Bergmann and Meyer's antitrypsin test, 250

Berkefeld filter, 77

Besredka's theory of anaphylaxis, 557

B. F. tuberculin, preparation of, 666

Biologic blood test for detection of bloodstains, 303

technic, 308

of meat adulteration, 310 technic, 312

Blackfan's apparatus for collecting blood, 36

Blackleg vaccination, 655

Blood, animal and human, differentiation, precipitin test for, 303

obtaining large amounts, from dog, 51

from guinea-pig, 46

from hog, 50

from horse, 52

from monkey, 50

from rabbit, $42-46$

Nuttall's method, 42

from rats, 46

from sheep, 48

small amounts, 41

from dog, 51

from guinea-pig, 41

from horse, 52

from monkey, 50

from rabbit, 41

from rats, 46

from sheep, 42,49

bactericidal power, capillary pipet method of measuring, 355

Neisser and Wechsberg's method of measuring, 349

plate culture method for measuring, 349

technic, 350

Stern and Körte's method of measuring, 349

experimental work, 863

Töpfer and Jaffe's method of measuring, 352

Wright's capillary pipet method of measuring, 355

bacteriolytic power, microscopic method of measuring, experimental work, 862

Blackfan's apparatus for collecting, 36

capsules, Wright's, making of, 23

method of sealing, 34

removing serum from, 34 
Blood, collecting of, for Wassermann reaction, 408

New York Board of Health outfit, 410

films for phagocytic counts, 199

method of preparing, 198

human and animal, differentiation, precipitin test for, 303

obtaining large amounts, 33

phlebotomy, 33

wet cupping, 36

small amounts, 32

from infants and children, 33

Keidel tube for collecting, 35, 36

methods of obtaining, 28

placental, method of obtaining, 37

plasma, obtaining, 30

precipitin test for, 303

technic, 308

Blood-corpuscles and blood-serum, obtaining, 30

obtaining, 28

red. See Erythrocytes.

test, biologic, for detection of bloodstains, 303

technic, 308

of meat adulteration, 310 technic, 312

Teichmann's, 303

transfusion, 273

experimental work, 844

tests before, for isohemagglutinins and isohemolysins, 290

Blood-pressure as guide in administering serum subdurally, 695

fall in, in anaphylaxis, 541, 542

Blood-serum. See Serum.

Blood-stains, biologic test for, 303 technic, 308

experimental work, 845,861

identification of, complement-fixation

test for, 494

Body-fluids, relation of, to phagocytosis, 184

Boil, Aleppo, salvarsan in, 811

Bones, tuberculosis of, tuberculin test in diagnosis of, 590

treatment, 679

Bordet-Gengou phenomenon of complement fixation, 332, 391

as colloidal reaction, 519

determination of antigen by, 494

experimental work, 855

for identification of bacterial antigens, 498

of blood-stains, 494

of meats, 498

in bacterial diseases, 473

in cancer, 499

in contagious abortion, 486

in dourine, 487

in echinococcus disease, 492

in glanders, 484
Bordet-Gengou phenomenon of complement fixation in gonococcus infections, 477. See also Gonococcus complement-fixation test. in horse syphilis, 487

in standardization of immune serums, 491

in tuberculosis, 490

in typhoid fever, 489

mechanism, 395

non-specific, 396

original, 394

practical application, 399

preparation of bacterial antigens, 473

principles, 391

with bacterial antigens, 476

protein differentiation by, 494

quantitative factors in, 397

standardizing bacterial antigens, 475

technic, 401, 473

Bordet's theory of mechanism of agglutination, 271

Botulinus antitoxin, 236

Botulism toxin, 116

experimental work, 820

Bouillon filtrate, preparation of, 666

Brain, syphilis of, salvarsanized autoserum in, 776

after-treatment, 780

repeating dose, 780

serobiologic findings in cerebrospinal fluid, 780

technic, 778

Brezovsky and Detre's modification of Wassermann reaction, 459

Bronchitis, vaccine therapy, 659

Bronchopneumonia, antistreptococcus serum in, 764

Browning and Mackenzie's modification of Wassermann reaction, 459

test for estimating amount of complement absorbed in Wassermann reaction, 434

Bubonic plague, serum treatment, 769 vaccination, 647

Buckwheat idiosyncrasy, 578

Butyric-acid test, Noguchi's, for protein, 300

Cadaver serums, testing, in Wassermann reaction, 411

Calf cholera serum, 733

Calmette's antivenene, 734

conjunctival tuberculin test, 587,598 dangers, 592

in animals, 600

Cancer, autoserum treatment, 782 chemotherapy in, 812

complement-fixation test in, 499

contraindications to vaccines in, 623

eosin-selenium compound in, 812

epiphanin reaction in, 526 
Cancer, Freund and Kaminer's cytolytic test for, 509

miostagmin reaction in, 528,530

precipitin test in, 314

Freund and Kaminer's, 314

sero-enzymes in, 264

venom hemolysis in, 390

von Dungern's test in, 500

Capillary pipet for counting bacterial vaccine, 210

for opsonic index determination, 196 making of, 18

method of sealing, 198

rubber teats for, 20

Capsules; blood, Wright's making of, 23 method of sealing, 34 removing serum from, 34

Carbuncle, antistaphylococcus serum in, 766

vaccine therapy, 656

Castellani's agglutination reaction, experimental work, 843

Cataphoresis, 513

Catarrh, autumnal, 242

Cats, anaphylaxis in, 539

Cells, functions of, 147

heart failure, 178

Cellular theory of anaphylaxis, 555 of immunity, 143

Centrifuge, 17

care of, 17

electric, 18

Cerebral syphilis, Wassermann reaction in, 462

Cerebrospinal fluid for Wassermann reaction, 411

method of securing, 37

meningitis, agglutination test in, 277

precipitin test in diagnosis, 298

serum treatment, 736

vaccination, 652

Chancroid, salvarsan in, 811

Chantemesse's serum, 768

Chemoreceptors, 787

Chemotaxis, 179

experimental work, 830

negative, $145,179,182$

experimental work, 830

positive, 145,179

experimental work, 830

Chemotherapy, 784

experimental work, 867

in bacterial diseases, 811

in cancer, 812

in malignant disease, 812 principles, 785

Chills and fever after intravenous injection of salvarsan, 804

Cholera, agglutination test in, 277

hog, serum treatment, 732

serum, calf, 733

hog, production of, 732

standardization of, 732

treatment, 770

vaccination, 650
Cholera, vaccination, dosage of vaccine, 651

Haffkine's vaccine, 650

Kolle's vaccine, 650

preparation of vaccine, 650

results, 651

Strong's vaccine, 651

Cholesterin and lecithin for Wassermann reaction, 423

Chorea, salvarsan in, 811

Cobra hemotoxin, 120

lecithid, 330

venom, experimental work, 823

preparation, 385

tests, 383 . See also Venom hemolysis.

Cobralecithinase, 384

Cold-blood animals, anaphylaxis in, 541

Cole's method of determining type of pneumococcus, 757

Collapse in subdural inoculation of serum, 694, 696

symptoms, 696

treatment, 696

Colles' law, Wassermann reaction and, 464

Collodion sacs for increasing virulence of bacteria, 97

Colloidal reactions, action of agglutinins based on, 517

of antitoxins based on, 516

of hemolysins based on, 518

of precipitins based on, 517

complement-fixation test as, 519

immunity reactions and, analogy between, 515

Wassermann test as, 520

solutions, 512

suspensions, 512

Colloids, absorption by, 515

amorphous, 512

nature, 511

osmotic pressure, 512

physical structure, 513

precipitation, 513

properties, 511

relation of, to immunity, 511

surface tension, 512

varieties, 511

Comer's automatic pipet, 215

Complement, 146, 154, 157, 185, 316, $318,326,337,362$

action, 329

and amboceptor, quantitative relationship, 374

bacteriolytic, titration of, 334

definition, 326

deflection of, 333

deviation of, 333

dominant, 321,366

endocellular, 330

fixation, Bordet-Gengou phenomenon, 332, 391. See also Bordet-Gengou phenomenon. 
Complement fixation reactions, experimental work, 855

in differentiation of proteins, experimental work, 861

non-specific, 396

practical applications, 399

principles of, 391

quantitative factors in, 397

technic, 401

for Noguchi's modification of Wasser-

mann reaction, 450

for Wassermann reaction, 411

titration, 413

hemolytic, experimental work, 853

titration of, 334

experimental work, 854

history, 326

inactivation of, experimental work, 853

multiplicity, 328

nature, 329

non-dominant, 321

origin, 328

properties, 326

experimental work, 854

reactivation of, experimental work, 853

rôle of, in hemolysis, experimental work, 850

splitting, 331

structure, 326

titration of, quantitative, 334

Complementoid, 327

Congenital mental deficiency, Wassermann reaction in, 465

syphilis, Wassermann reaction in, 463 , 464

Congestion, 556

Conjunctival tuberculin test of Calmette, 587, 598

dangers, 592

in animals, 600

of Wolff-Eisner, 587, 598

dangers, 592

in animals, 600

Contagious abortion, complement-fixation test in, 486

diseases, 84

Contamination, 81

Cow-disease, 613

Cowpox, 613

vaccine, preparation, 626

animals, 627

collection of virus, 628

seed virus, 626

testing virus, 629

Cows, anaphylaxis in, 541

Crotin toxin, 118

Cryptogenic infections, 92

Crystalloids, 511

Culex, 166

Cupping, wet, 36

Cutaneous reaction in typhoid fever, 608 tuberculin reaction of von Pirquet, 587,596

in animals, 601
Cyst, echinococcus, complement-fixation test in, 492

Cystitis, vaccine therapy, 657

Cytase, 144, 157, 318, 337, 338

Cytolysins, 316, 502

definition, 317

nomenclature, 318 -

varieties, 318

Cytotoxic reactions, 509

in diagnosis, 509

of Freund and Kaminer for cancer, 509

Cytotoxins, 154, 318, 502

experimental production, 816

in diagnosis, 509

in immunity, rôle of, 508

in treatment, 508

methods of studying, 503

nature, 502

nomenclature, 502

practical applications, 508

preparation, 503

production of, 73

Pearce's method, 73

properties, 502

specificity, 504

varieties, 506

Dementia, paralytic, Wassermann reaction in, 462

Dermatitis herpetiformis, salvarsan in, 811

Detre and Brezovsky's modification of Wassermann reaction, 459

Deuterotoxin, 115

Deviation of complement, 333

Diabetes, contraindications to vaccines in, 623

Diarrhea after intravenous injection of salvarsan, 804

Diet as predisposing to infection, 101

Digestive tract as portal of entrance for bacteria, 88

Diphtheria, allergic reaction in, 609

antistreptococcus serum in, 764

antitoxin, 702

action, 705

administration, 706

dosage, 709

in diphtheria of eye, 711

of vulva, 711

of wounds, 711

in nasal diphtheria, 710

in tonsillar diphtheria, 710

influence of age, 711

repeating, 711

total, 712

early use, importance, 707

intramuscular injection, 707

intravenous injection, 707

mor ality after use, 714

before use, 713

oral method of administering, 707

preparation, 703 
Diphtheria antitoxin, production of, 227 collecting serum, 230

immunizing animals, 228

rectal method of administering, 707 sequels, 712

serum sickness from, 712

standardizing, 231

experimental work, 835

subcutaneous injection, 707

total amount to be administered, 712 treatment of relapses with, 712

unit of, 242

value, 713

bacillus, 112

virulence and toxicity, method of testing, 818

Behring's method of immunization against, 717

nasal, dosage of antitoxin in, 710

nature of, 704

of eye, dosage of antitoxin in, 711

of vulva, dosage of antitoxin in, 711

of wounds, dosage of antitoxin in, 711

prophylactic immunization against, 715

serum treatment, 702. See also Diphtheria antitoxin.

tonsillar, dosage of antitoxin in, 710

toxin, 112

experimental work, 817

limes death dose, 232

zero dose, 232

method of testing virulence, 113

production of, 227

standardizing, 114

testing, 228

toxoid, 115

treatment of, 704. See also Diphtheria antitoxin.

Diplococcus pneumoniæ, 755

Disease, antiferments in, 247

bacteriolytic serums in treatment of, 359

diagnosis of, Pfeiffer bacteriolytic test, 348

ferments in, 248

Pfeiffer's bacteriolytic test in diagnosis of, 348

production of, 107

sero-enzymes in, 264

Dixon's tuberculin, dose of, 675 preparation, 667

Dog, anaphylaxis in, 540

intravenous inoculation, 62

obtaining large amounts of blood from, 51

small amount of blood from, 51

Donath and Landsteiner's method of serum diagnosis of paroxysmal hemoglobinuria, 379

Dose, intoxicating, 536

Dourine, complement-fixation test in, 487

Drug fastness, 789

idiosyncrasy, 578
Duhring's disease, salvarsan in, 811

Dungern's complement-fixation test in cancer, 500

modification of Wassermann reaction, 459

Dysentery, agglutination test in, 276

antitoxin, 730

administration and uses, 730

serum treatment, 730 results, 731

toxin, 116 experimental work, 820

vaccination, 652

EAR, tuberculosis of, tuberculin test in diagnosis of, 590

treatment, 679

Echinococcus disease, complement-fixation test in, 492

miostagmin reaction in, 530

Effusions, non-tuberculous, autoserum treatment, 782

Egg-albumen idiosyncrasy, 578

Ehrlich's method of serum diagnosis of paroxysmal hemoglobinuria, 379

theory of immunity, 146 and Metchnikoff's theory, compatibility, 155

therapia magna sterilisans, 791

Electric centrifuge, 18

Emulsion, bacterial, in opsonic index, 194

Emulsions, 511

Endemic infection, 82

Endocarditis, antistreptococcus serum in, 764

ulcerative, vaccine therapy, 661

Endocellular complement, 330

Endocomplement, 330

Endogenous infection, 85, 86

Endolysins, 183, 339

Endotoxic substances, method of obtaining, 121

Endotoxins, 108, 120, 182

as anaphylactogens, 548

experimental work, 823

influence of bacteriolysins on, 340

method of obtaining, 121

nature of, 121

Enteritis anaphylactica, 540

Enzoötic infection, 82

Enzymes, 244

sero-, in disease, 264. See also Seroenzymes.

Eosin-selenium compound in cancer, 812

Epidemic cerebrospinal meningitis, treatment, 738

infection, 82

Epiphanin reaction, 522

in cancer, 526

in malignant disease, 526

in pregnancy, 526

in sarcoma, 526

in syphilis, 526

principle, 522 
Epiphanin reaction, reading results, 525 specificity, 523

Epitheliotoxin, 506

Epizoötic infection, 82

Erysipelas, antistreptococcus serum in, 764

autoserum treatment, 776

vaccine therapy, 657

Erythrocytes for Wassermann reaction, 416

method of determining resistance, 380

obtaining of, 28

resistance of, to salt solution, experimental work, 847

washing of, 28

Exhaustion theory of immunity, 144

Exogenous infection, 85

Experimental immunity, 814

infection, 814

Experiments, animal, 815

Exposure as predisposing to infection, 102

Eye, diphtheria of, dosage of antitoxin in, 711

tuberculosis of, tuberculin test in diagnosis of, 590

treatment, 679

Familial susceptibility, 100

Fastigium period of infection, 135

Fastness, drug, 789

Feces, bacteria recovered from, bacteriolytic test for identification of, 343

Ferment reactions, 230

Ferments, 244

and toxins, similarity between, 244

bacterial, 244

experimental work, 838

in disease, 248

in pregnancy, 248

Fever and chills after iptravenous injection of salvarsan, 804

of infection, 137

protein, 137

Filariasis, salvarsan in, 810

Film, blood, for phagocytic counts, 199 method of preparing, 198

Filter, 78

Berkefeld, 77

Uhlenhuth, 305

Filtration, bacteria-free, preservation of serum by, 76

Finger pricking, method of, 31

Fixateur. See Amboceptor.

Fixator, 322

Flexner and Jobling's method of preparing antimeningococcus serum, 739

Floccule-forming precipitin tests, 299

Fomites, 84

Foods, bacteria in, 85 idiosyncrasy, 578

Fordyce's technic of intraspinous injection of salvarsanized serum, 807

Forensic blood test, 498
Fornet's ring test for syphilis, 299

Frambesia, luetin reaction in, 605 salvarsan in, 810

Wassermann reaction in, 465,466

Freezing serum, preservation by, 78

Freund and Kaminer's cytolytic cancer diagnosis, 509

precipitin test in cancer, 314

Friedberger on anaphylatoxin, 550

theory of anaphylaxis, 558

Friedmann's treatment of tuberculosis, 670

Frigo, 79

Fruits, idiosyncrasy, 578

Fulminating infection, 136

Furunculosis, antistaphylococcus serum in, 766

vaccine therapy, 656

Galeotri and Lustig's plague vaccine, 648

Gastrotoxin, 507

Gay and Ayer's method of titration of bacteriolytic complement, 334

Gay and Southard's theory of anaphylaxis, 557

Genital organs as portal of entrance for bacteria, 89

Genito-urinary diseases, vaccine therapy, 657

tuberculosis, tuberculin test in diagnosis of, 590

treatment, 680

Glanders, agglutination reaction in, 277 complement-fixation test in, 484

mallein reaction for, 606 . See also Mallein reaction.

Glandular tuberculosis, tuberculin test in diagnosis of, 590

Globulins, Noguchi's butyric-acid test for, 300

Goats, intravenous inoculation, 62

Gonococcal infections, serum treatment, 765

Gonococcus antigen, titration of, experimental work, 860

complement-fixation test, 477

antigen for, 478

experimental work, 860

hemolytic systems, 478

practical value, 482

specificity, 482

technic, 478

using one-tenth usual amounts, 481

infections, allergic reaction in, 609

complement fixation in, 477

Gonorrheal arthritis, autoserum treatment, 776

vaccine therapy, 658

Graduated pipets, 21

Gravity method of intravenous injection of salvarsan, 801

of serum, 691

of subdural injection of serum, 697 
Group immune bodies, 323 precipitins, 293, 295

Gruber-Widal reaction in typhoid fever, $268,275,279,282$

experimental work, 840

Gruber's theory of mechanism of agglutination, 270

Guinea-pig, anaphylaxis in, 537 complement serum for Wassermann reaction, 412

intracardial inoculation, 62

intraperitoneal inoculation, 64

intravenous inoculation, 57

obtaining large amount of blood from, 46

small amount of blood from, 41

HAFFKInE's plague vaccine, dosage, 649 effects, 649

preparation, 648,650

results, 649

Hamburger and Moro's theory of anaphylaxis, 556

Hamman and Wolman's method of preparing tuberculin for subcutaneous tuberculin test, 592

plan of dosage for tuberculin in subcutaneous test, 594

Haptines, 147

Haptophore group of toxin, 110

Hay-fever, 242, 578

antitoxin, 734

serum treatment, 734

toxin of, 119

Headache after injection of salvarsan, 804

Heart failure cells, 178

Hecht-Weinberg modification of Wassermann reaction, 457

Hemagglutinins, 272 experimental work, 843

Hemocytometer chamber, counting bacterial vaccines with, 211

Hemoglobinuria, paroxysmal, serum diagnosis, 379

Hemolysins, 154, 318, 361

action of, based on colloidal reaction, 518

and bacteriolysins, analogy between, 367

definition, 362

experimental production, 816

history, 361

immune, 363

method of titration, 375

production of, 371

methods for removing, from serum, 378

natural, 368

experimental work, 852

method of determining, in serum, 378

removal of, experimental work, 852 nature, 363
Hemolysins, nomenclature, 363

normal, 368

practical applications, 373

preservation of, in dried paper form, 79

production of, 71

intraperitoneal method, 72

intravenous method, 72

properties, 371

sources, 372

specific, 363

specificity, 368

Hemolysis, 361

and bacteriolysis, analogy between, 367

non-specific, 380

relation of lipoids to, 521

rôle of amboceptor and complement in, experimental work, 850

serum, experimental work, 848

quantitative factors, experimental work, 849

venom, 383, 521. See also Venom hemolysis.

Hemolytic amboceptor, 319, 362

and complement, quantitative relationship, 374

for Noguchi's modification of Wassermann reaction, 451

for Wassermann reaction, 415

preservation of, 79

titration of, 325 experimental work, 848

complement, experimental work, 853

titration of, 334 experimental work, 854

jaundice, 372

titration of antigens in Wassermann reaction, 430

Hemophilia, normal serum treatment, 772

Hemopsonins, 188

experimental work, 832

Hemorrhage, normal serum treatment, 772

Hemorrhagin, 734

Hemotoxin, 117

cobra, 120

Hens, anaphylaxis in, 541

Hepatotoxin, 507

Herman-Perutz test for syphilis, 299

Herxheimer-Jarisch reaction, 804

Heterolysins, 363

Histamin, 552

Hitchens syringe, 233

Hodgkin's disease, salvarsan in, 811

Hog cholera serum, production, 732 standardization, 732 treatment, 732

obtaining large amount of blood from, 50

Hopkins' method of counting bacterial vaccines, 212

tube for standardizing bacterial vaccine, 213

Horse, anaphylaxis in, 541 
Horse asthma, 578

intravenous inoculation, 61

obtaining large amount of blood from, 52

small amount of blood from, 52

syphilis, complement-fixation test in, 487

Humoral theory of immunity, 143, 146

Hydatid disease, complement-fixation test in, 492

Hydrocele, autoserum treatment, 782

Hydrocephalus in meningococcus meningitis, effect of serum treatment on, 746 Hydrophobia, 636 . See also Rabies.

Hypersensitiveness, 531, 577. See also Idiosyncrasy.

Hypothesis of Bail, 123

of Welch, 103

IDIOSYNCRASY, 531, 577

buckwheat, 578

drugs, 578

egg-albumin, 578

foods, 578

fruits, 578

hay-fever, 578

horse asthma, 578

iodoform, 578

pork, 578

strawberries, 578

vegetables, 578

Immune agglutinins, 268

bodies, 319,362

group, 323

partial, 233

hemolysins, 363

method of titration, 375

production of, 371

opsonins, experimental work, 832 production of, 69

precipitins, 294

serums and normal serums, difference between, 324

methods for making, 66

precipitin, 70

preservation of, 76

in dried paper form, 79

in fluid form, by bacteria-free filtration, 76

by freezing, 78

with antiseptics, 76

in living animal, 80

in powder form, 79

standardization of, complement-fixation test in, 491

Immunity, 138

acquired, 170

active, 170

by vaccination, 171

causes of, 170

experimental work, 827

antibacterial, 171

antitoxic, 171

experimental work, 827
Immunity, acquired, passive, 172 antitoxic, 173 experimental work, 828

Vaughan's theory, 174 active, 65

agglutinins in, rôle of, 274

anaphylaxis in relation to, 565

antibacterial, 684

experimental work, 826

antitoxic, 684

antitoxin, natural, 169

athreptic, 170

cellular theory, 143

cytotoxins in, rôle of, 508

definition of, 140

Ehrlich's theory, 146

and Metchnikoff's theory, compatibility, 155

exhaustion theory, 144

experimental, 814

history of, 140

humoral theory, 143, 146

individual, 166

Metchnikoff's theory, 144

and Ehrlich's theory, compatibility, 155

natural, 165

antitoxin, 169

causes of, 167

influence of temperature on, experimental work, 827

phagocytosis in, 168 experimental work, 826

relative factors in, experimental work, 827

Vaughan's theory, 174

opsonins in, 190

passive, 682

varieties of, 683

phagocytosis theory, 144

and side-chain theory, compatibility, 155

precipitins in, rôle of, 297

racial, 166

reaction, 569

colloidal reaction and, analogy between, 515

relation of colloids to, 511

of infection to, 83

of lipoids to, 520

of phytotoxins to, 118

retention theory, 144

side-chain theory, 146

and phagocytic theory, compatibility, 155

species, 166

theories of, 138, 144

types of, 165

Vaughan's theory, 173

Immunization, active, 611

contraindications to, 622

for prophylaxis, 616

for therapeutic purposes, 655

for treatment of disease, 617

history of, 611 
Immunization, active, mechanism of, 616 negative phase, 621

of animals, 653

general technic, 66

methods for effecting, 65

antibacterial, 684,735

antitoxic, 684,702

Behring's method, against diphtheria, 717

curative, 682,683

passive, 681

contraindications to, 686

indications for, 684

purposes of, 682

prophylactic, $580,623,682$. See also vaccination.

therapeutic, 580

Incubation period of infection, 134

Index, opsonic, 191. See also Opsonic index.

phagocytic, 200

Individual immunity, 166

predisposition, 100

Infection, 81

acute, 136

sero-enzymes in, 265

vaccine therapy, 660

aggressins in relation to, 103

anaphylaxis in relation to, 565

antenatal, 90

avenue of, tissue susceptibility and, 97

avenues of, 86

chronic, 136

complications, 136

course, 134

cryptogenic, 92

defensive mechanism of microörganism in relation to, 102

definition of, 81

diet as predisposing to, 101

endemic, 82

endogenous, 85, 86

enzoötic, 82

epidemic, 82

epizoötic, 82

exogenous, 85

experimental, 814,816

exposure as predisposing to, 102

fever of, 137

fulminating, 136

general susceptibility in relation to, 99

gonococcus, allergic reaction in, 609 serum treatment, 765

grades of, 136

intoxications as predisposing to, 101

malignant, 136

malnutrition as predisposing to, 101

mechanism of, 94

mixed, 105

absorption agglutination test in, 288

morbid conditions as predisposing to, 102

morphologic changes of microörganisms in relation to, 102

numeric relationship of bacteria to, 99
Infection, pandemic, 82

period of convalescence, 136

of decline, 135

of fastigium, 135

of high fever, 135

of incubation, 134

of prodromal symptoms, 135

physiologic changes of microörgan.sms in relation to, 102

pneumococcus, serum treatment, 754

previous, as predisposing to infection, 101

relapse of, 136

relation of, to immunity, 83

remittent, 136

sequels of, 136

sources of, 83

sporadic, 82

stages of, 134

staphylococcus, serum treatment, 766

streptococcus, serum treatment, 760

systemic reaction to, 137

trauma as predisposing to, 102

with animal parasites, 132

modes, 132

wound, antistreptococcus serum in, 764

Infectious diseases, 84

accelerated anaphylaxis reaction in, 568

acute, autoserum treatment, 775

anaphylaxis, torpid, early reaction in, 568

immediate anaphylaxis reaction in, 568

production of, 107

relation of anaphyiaxis to, 566

Infestation, definition of, 81

Infestment, definition of, 81

Inflammation, behavior of leukocytes in, 180

Influenza, autoserum treatment, 776

Influenzal meningitis, serum treatment, 749

Inoculation, animal, 53. See also Animal inoculation.

Insects, suctorial, transmission of bacteria by, 86

Interbody, 154, 319

Intestine, tuberculosis of, tuberculin treatment, 679

Intoxicating dose, 536

Intoxications as predisposing to infection, 101

Intrabronchial route for tuberculin treatment, 677

Intracardial inoculation of animals, 62 of guinea-pigs, 62

Intracutaneous tuberculin test of Mantoux, 587, 595

of Mendel, 587, 595

in animals, 601

Intrafocal route for tuberculin treatment, 677

Intramuscular injection of animals, 56 
Intramuscular injection of diphtheria antitoxin, 707

of neosalvarsan in syphilis, 805

of salvarsan in syphilis, 805

of serum, treatment, 690

of tetanus antitoxin, 722

Intraneural injection of tetanus antitoxin, 723

Intraperitoneal inoculation of animals, 64 of guinea-pig, 64

of rabbit, $64^{-}$

method of production of agglutinins, 69

of hemolysins, 72

Intravenous injection of animals, 56 of antimeningococcus serum, 743 of antipneumococcus serum, 759 of antistreptococcus serum, 763 of diphtheria antitoxin, 707 of $\mathrm{dog}, 62$

of goats, 62

of guinea-pig, 57

of horse, 61

of mice, 60

of neosalvarsan in syphilis, 797

- after-care, 803

apparatus, 801

dosage, 797

frequency, 797

gravity method, 801

intensive treatment, 797

preparation of patient, 798

of rabbit, 56 of solution, 800

of rat, 60

of salvarsan in syphilis, 797

after-care, 803

after-effects, 803

apparatus, 801

chills and fever after, 804

diarrhea after, 804

dosage, 797

frequency, 797

gravity method, 801

headache after, 804

Herxheimer reaction after, 805 intensive treatment, 797

Jarisch-Herxheimer reaction after, 804

preparation of patient, 798 of solution, 798

of serum, gravity method, 691

syringe method, 690

treatment, 690

of sheep, 62

of tetanus antitoxin, 722

method of producing hemolysins, 72

serum precipitins, 70

route for tuberculin treatment, 677

Invasion, 82

Iodoform idiosyncrasy, 578

Isocytotoxins, 506

Isohemagglutinins, 273

tests for, before transfusion of blood, 290
Isohemolysins, 370

tests for, before transfusion of blood, 290

Isolysins, 363

Isoprecipitin, 295

Izar and Ascoli's miostagmin test, 526.

See also Miostagmin reaction.

JAFFE and Töpfer's method of measuring bactericidal power of blood, 352

Jarisch-Herxheimer reaction, 804

Jaundice, hemolytic, 372

Jenner on vaccination, 141, 624

Jennerian vaccination, 171

Jobling and Flexner's method of prepar-

ing antimeningococcus serum, 739

Joints, tuberculosis of, tuberculin test in diagnosis of, 590

treatment, 679

Kaminer and Freund's cytolytic cancer diagnosis, 509

precipitin test in cancer, 314

Keidel tube for collecting blood, 35, 36

Keratosis follicularis, salvarsan in, 811

Koch's subcutaneous tuberculin test, 587,592

in animals, 600

Kolle and Pfeiffer's macroscopic agglutination test, 288

and Strong's plague vaccine, 648

Kolle's cholera vaccine, preparation, 650

method of counting bacterial vaccines, 212

of preparing antimeningococcus serum, 740

antipneumococcus serum, 758

antistreptococcus serum, 763

plague serum, 769

plague vaccine, preparation, 648

Kolmer's method of testing virulence and toxicity of diphtheria bacilli, 113

Körte and Stern's method of measuring bactericidal power of blood, 349

experimental work, 863

Kraus' anticholera serum, 770

Kretz, paradox of, 548

LACTOSERUM, 293

experimental work, 846

production of, 71

Landsteiner and Donath's method of serum diagnosis of paroxysmal hemoglobinuria, 379

Larynx, tuberculosis of, tuberculin test in diagnosis of, 590

Law, Colles', Wassermann reaction and, 464

Profeta's, Wassermann reaction and, 464

Lecithid, 521 
Lecithid, cobra, 330

Lecithin, 384, 521

and cholesterin for Wassermann reaction, 423

mono-fatty-acid-, 384

Leistenkern, 147

Leitenketter, 147

Leprosy, allergic reactions in, 610

autoserum treatment, 776

luetin reaction in, 605

salvarsan in, 811

Wassermann reaction in, 465, 466

Leukins, 338

Leukocidin, 117॰

Leukocytes, behavior of, in inflammation, 180

in quantitative estimation of bacteriotropins, 201

obtaining of, 28

washed, in opsonic index, 195

Leukocytic extracts, 338 , preparation, 339

Leukotoxins, 506

Lichen planus, salvarsan in, 811

Limes death dose of diphtheria toxin, 232 zero dose of diphtheria toxin, 232

Lipoid anaphylaxis, 544

Lipoids, 160, 404

acetone-insoluble, for Wassermann reaction, 422

relation of, to hemolysis, 521

to immunity, 520

to Wassermann reaction, 522

Livers, syphilitic, alcoholic extracts, for

Wassermann reaction, 420 aqueous extracts, for Wassermann reaction, 419

Lobar pneumonia, nature, 755 serum treatment, 754

Locomotor ataxia, Wassermann reaction in, 462

Looped pipets, 20 making of, 20

Luetin, preparation of, 602 reaction, 601

in frambesia, 605

in leprosy, 605

in yaws, 605

method of application, 502

negative, 603

normal, 603

papular form, 603

positive, 603

practical value, 605

preparation of luetin, 602

pustular form, 604

results, 605

torpid form, 604

Lumbar puncture, 37

after-treatment of patient, 41

anesthesia in, 39

contraindications, 37

disposal of fluid, 41

injection of antimeningococcus serum by, 742
Lumbar puncture, injection of neosalvarsan by, 805

of serum by, 694

of tetanus antitoxin by, 723

preparation of patient, 38

technic, 39

value, 37

Lupus vulgaris, salvarsan in, 811

Lustig and Galeotti's plague vaccine, 648

Lustig's method of preparing plague serum, 769

Lymphocytosis, 178

Lysins, 73, 154

MACKENZIE and Browning's modification of Wassermann reaction, 459

test for estimating amount of complement absorbed in Wassermann reaction, 434

Macrocytase, 157, 183

Macrophages, 145, 177

experimental work, 829

Malaria, salvarsan in, 811

Wassermann reaction in, 465

Malignant disease, chemotherapy in, 812 epiphanin reaction in, 526

infection, 136

Mallein reaction, 606 ophthalmic, 607

preparation of mallein, 606

subcutaneous, 607

Malnutrition as predisposing to infection, 101

Malta fever, agglutination test in, 277 autoserum treatment, 776

Mantoux's intracutaneous tuberculin test, 587,595

Maragliano's tuberculosis serum, 771

Marasmus, autoserum treatment, 783

Marcus modification of Müller and Jochmann's method of testing blood-serum, experimental work, 838

Markl's method of preparing plague serum, 769

Marmorek's tuberculosis serum, 771

Measles, effect of, on tuberculin reaction, 586

Meat adulteration, detection, biologic blood test for, 310 technic, 312

Meats, identification of, complementfixation test for, 498

Mendel's intracutaneous tuberculin test, 587,595

in animals, 601

Meningitis, cerebrospinal, agglutination test in, 277

precipitin test in diagnosis, 298

serum treatment, 736

vaccination against, 652

influenzal, serum treatment, 749

meningococcus, epidemic, treatment of, 738

nature of, 738 
Meningitis, meningococcus, prophylactic immunization in, 748, 749

serum treatment, 736

cases of posterior basal meningitis, 745

with dry canal, 745

with thick elastic exudate, 745

chronic cases, 746

results, 746

serum sickness in, 746

subacute cases, 746

pneumococcus, 752

serum treatment, 751,752

tuberculous, autoserum treatment, 782

tuberculin test in diagnosis of, 591 treatment, 679

Meningococcus meningitis, epidemic, treatment of, 738

nature, 738

prophylactic immunization in, 748, 749

serum treatment, 736

cases of posterior basal meningitis, 745

with dry canal, 745

with thick elastic exudate, 745

chronic cases, 746

results, 746

serum sickness in, 746

subacute cases, 745

Mental deficiency, congenital, Wassermann reaction in, 465

diseases, sero-enzymes in, 265

Mercury treatment of syphilis, effect of, on Wassermann reaction, 466

Mesenteric glands, tuberculosis of, tuberculin treatment, 679

Metchnikoff's theory of immunity, 144 and Ehrlich's theory, compatibility, 155

of phagocytosis, 176

Meyer and Bergmann's antitrypsin test, 250

Mice, intravenous inoculation, 60 white, anaphylaxis in, 540

Microcytase, 157, 183

Microörganisms, 83. See also Bacteria.

Microphages, 144, 177

experimental work, 829

Milk, bacteria in, 85

precipitins, experimental work, 846

production of, 71

Miostagmin reaction, 526

experimental work, 864

in cancer, 528,530

in echinococcus disease, 530

in paratyphoid fever, 530

in syphilis, 530

in tuberculosis, 530

in typhoid fever, 530

practical value, 529

principles, 526

technic, 527

Mixed infection, 105
Monkey, obtaining large amount of blood from, 50

small amount of blood from, 50

Mono-fatty-acid-lecithin, 384

Morbid conditions as predisposing to infection, 102

Moro and Hamburger's theory of anaphylaxis, 556

Moro's percutaneous tuberculin test, 587,599

Much's psycho-reaction, 388 technic, 388

Müller and Jochmann's method of testing blood-serum, Marcus modification, experimental work, 838

Multiform rashes in serum disease, 574

Mycosis fungoides, salvarsan in, 811

NASAL diphtheria, dosage of antitoxin in, 710

Nastin, 160

Negri bodies in rabies, 637

Neisser and Wechsberg's method of measuring bactericidal power of blood, 349

Neosalvarsan in syphilis, 792

administration, 797

effect of, on Wassermann reaction, 469

history, 792

intramuscular injection, 805

intravenous injection, 797. See also Intravenous injection of neosalvarsan.

subdural injection, 805

Wile's technic, 806

value, 809

properties of, 795

Nephritis, contraindications to vaccines in, 623

normal serum treatment, 774

Nephrotoxic serum, production of, 73

Nephrotoxin, 506

action of, experimental work, 863

Neufeld's quantitative estimation of bacteriotropins, 200

controls, 202

culture, 202

leukocytes, 201

precautions, 203

readings, 202

serum, 201

Neurorrhyctes hydrophobiæ, 637

Neurotoxin, 508

New York Board of Health outfit for collecting blood for Wassermann reaction, 410

Ninhydrin, 254

Noguchi's butyric-acid test for protein, 300

globulin reaction, experimental work, 846

luetin reaction, 601. See also Luetin reaction. 
Noguchi's modification of Wassermann reaction, 449

antigen for, 453

titration of, 453

complement for, 450

experimental work, 859

fluid to be tested, 455

hemolytic amboceptor for, 451

human corpuscles for, 451

technic, 450

test, 455

Nolf's theory of anaphylaxis, 558

Nucleoproteins, production of, 74

Nuttall's method of obtaining large amount of blood from rabbit, 42

Oрнтнацмic mallein, preparation, 607 reaction, 607

reaction in typhoid fever, 608

Opsonic index, 191

as diagnostic procedure, 204

as guide to size and frequency of doses of bacterial vaccines, 205

bacterial emulsion in, 194

collection of patient's and control serum, 193

definition, 191

determining, experimental work, 834

in prognosis, 204

limitation, 192

practical value, 203

precautions in technic, 193

principle, 191

purpose, 192

technic, 193

precautions in, 193

washed leukocytes in, 195

Opsonification, susceptibility to, 189

Opsonins, 145, 185, 187

action of, mechanism, experimental work, 832

definition of, 188

effect of, on bacteria, 189

experimental work, 815,831

history of, 187

immune, experimental work, 832 production of, 69

in immunity, 190

nature of, 188

normal, experimental work, 831

properties of, 188

source of, 189

specificity of, experimental work, 833

Oral method of administering diphtheria antitoxin, 707

route for tuberculin treatment, 676

Organotropism, 785

Osmotic pressure of colloids, 512

O. T. tuberculin, dose of, 674 preparation of, 664

Otitis media, vaccine therapy, 660

Overproduction theory of Weigert, 148

Overwork as predisposing to disease, 100
Paltauf's theory of mechanism of agglutination, 270

Pandemic infection, 82

Paradox of Kretz, 548

Paralysis, general, Wassermann reaction in, 462

Paralytic dementia, Wassermann reaction in, 462

Parasites, 83

animal, aggressiveness of, 133

infection with, 132

modes, 132

production of disease by, 133

half, 124

partial, 124

true, 124

Parasitotropism, 785

Parasyphilitic diseases, Wassermann reaction in, 462

Paratyphoid fever, miostagmin reaction in, 530

Paroxysmal hemoglobinuria, serum diagnosis, 379

Pasteur on immunity, 141, 142

treatment of rabies, intensive, 642

mild, 642

principle, 640

results, 642

Pasteurian vaccination, 171

Pearce's method of producing nephrotoxic serum, 73

Pellagra, salvarsan in, 811

Wassermann reaction in, 466

Pelvic tuberculosis, tuberculin test in diagnosis of, 590

Percutaneous tuberculin test of Moro, 587,599

Peritonitis, tuberculous, tuberculin test in diagnosis of, 591

Pertussis, vaccine therapy, 659

Pfaundler's reaction, 267

Pfeiffer and Kolle's macroscopic agglutination test, 288

Pfeiffer's bacteriolytic test, 342 experimental work, 862 in diagnosis of disease, 348 technic, 347

Phagocytes, 144, 176 varieties, 177

Phagocytic index, 200

Phagocytosis, 145, 175

experimental work, 829, 830

history, 175

in natural immunity, 168

experimental work, 826

Metchnikoff's theory, 176

relation of body-fluids to, 184

of cell types to infection, 178

results of, 182

revised theory of, 186

spontaneous, 187

theory of immunity, 144

and side-chain theory, compatibility, 155

Phenomenon, Arthus, of anaphylaxis, 533 
Phenomenon, Bordet-Gengou, 332, 391. See also Bordet-Gengou phenomenon. Smith's, of anaphylaxis, 535

Phlebotomy, 33

in children, 33

Phogosin, 180

Physical theory of anaphylaxis, 558

Phytoprecipitin, 292

Phytotoxins, 109, 118

experimental work, 822

general properties, 118

relation to immunity, 118

Pigeons, anaphylaxis in, 541

Pipets, 18

capillary, for counting bacterial vaccine, 210

for opsonic index determination, 196 making of, 18

method of sealing, 198

rubber teats for, 20

Comer's automatic, 215

for Wassermann reaction, 407

graduated, 21

looped, 20

making of, 20

sterilization of, 22

Piroplasma bigeminum, 171

Pirquet's cutaneous tuberculin test, 587, 596

in animals, 601

studies on anaphylaxis, 533

Pityriasis rubra, salvarsan in, 811

Placenta as portal of entrance for bacteria, 90

bacteria in, 86,90

Placental blood, method of obtaining, 37 serum, method of obtaining, 773

L Plague, agglutination test in, 277

serum treatment, 769

vaccination, 647

dosage of vaccine, 649

duration, 649

effects, 649

Haffkine's vaccine, 648

dosage, 649

effects, 649

results, 649

Kolle and Strong's vaccine, 648

Kolle's vaccine, 648

Lustig and Galeotti's vaccine, 648

preparation of vaccine, 648

results, 649

Terni and Bandi's vaccine, 648

Plants, higher, toxins of, 118

Plasma, blood, obtaining of, 30

Pleurisy, tuberculous, autoserum treatment, 781

tuberculin test in diagnosis of, 591

Pneumococcus, Cole's method of determining type, 757

infections, serum treatment, 754

meningitis, 752

serum treatment, 751,752

Pneumonia, agglutination test in, 277 autoserum treatment, 776
Pneumonia, bacterial vaccines in, 660, 661

experimental, 816

nature of, 755

serum treatment, 754

results, 759

Poison, protein, 548

Polariscope, 262

Pollen antitoxin, 224, 734

toxin, 119

Polyceptor, 320

Porges-Meier test for syphilis, 299

Pork idiosyncrasy, 578

Precipitate, 294

Precipitation of colloids, 513. See Precipitin test.

Precipitin, 152, 292

action of, based on colloidal reactions, 517

bacterial, experimental work, 846 precipitin test with, 298 technic, 313

production of, 71

definition, 292

experimental production, 816

work, 844

formation, 295

group, 293, 295

history, 292

immune, 294

in immunity, rôle of, 297

milk, experimental work, 846

production of, 71

nomenclature, 294

normal, 294

production of, 70

intravenous method, 70

properties, 294

protein, precipitin test with, 301

serum, production of, 70

titration, experimental work, 844

specificity, 296

experimental work, 844

structure, 294

test, floccule-forming, 299

for blood-stain, 303

technic, 308

for detection of meat adulteration, 310 technic, 312

for differentiation of human and animal blood, 303

Fornet's, for syphilis, 299

Herman-Perutz, for syphilis, 299

in cancer, 314

Freund and Kaminer's, 314

mechanism, 294

Porges-Meier, for syphilis, 299

practical applications, 298

technic, 303

with bacterial precipitins, in diagnosis of cerebrospinal meningitis, 298

practical application, 298

technic, 313 
Precipitin test with protein precipitins, practical application, 301

Precipitinogen, 294

bacterial, preparation of, 313

Precipitoid, 294, 295

Predisposition, 100. See also Susceptibility.

Pregnancy, Abderhalden's serodiagnosis of, 252. See also Abderhalden's serodiagnosis of pregnancy.

allergic reaction in, 610

epiphanin reaction in, 526

ferments in, 248

toxicoses of, normal, serum treatment, 773

vomiting of, normal, serum treatment, 773

Preparator, 319

Pricking finger, method, 31

Pro-agglutination, 288

experimental work, 843

Pro-agglutinoids, 268

Prodromal symptoms of infection, 135

Profeta's law, Wassermann reaction and, 464

Prophylactic immunization, 580, 623, 682. See also Vaccination.

Protective substances, 65

Proteids, relation of antitoxins to, 223

Protein anaphylactogens, chemistry, 544 bacterial, 108, 126

action of, 128

experimental work, 824

nature of, 127

split, 126

Vaughan's theory, 128

complement fixation in differentiation of, experimental work, 861

differentiation by complement fixation, 494

fever, 137

increased amount, Noguchi's butyricacid test for detection, 300

poison, 548

precipitins, precipitin test with, 301

Protoxin, 115

Provocatory stimulation of Wassermann reaction, 469

Psoriasis, salvarsan in, 811

Psycho-reaction of Much, 388 technic, 388

Ptornains, 108, 129 experimental work, 825

Puerperal sepsis, antistreptococcus serum in, 764 vaccine therapy, 661

Pulmonary tuberculosis, tuberculin test in diagnosis of, 589

Pump, suction, 29

Puncture, lumbar, 37. See also Lumbar puncture.

spinal, 37. See also Lumbar puncture.

Pyemia, 93

Pyocyanase, 244
RABBIT, anaphylaxis in, 539

intraperitoneal inoculation, 64

method of production of agglutinins in, 69

intravenous inoculation, 56

obtaining large amounts of blood from, $42-46$

Nuttall's method, 42

small amounts of blood from, 41

Rabies, 636

diagnosis, 638

incubation period, 638

management, 638

nature, 637

Negri bodies, 637

Pasteur treatment, intensive, 642 mild, 642

principle, 640

results, 642

vaccine, preparation, 640, 641

virus fixé of, 640

Rachicentesis, 37. See also Lumbar puncture.

Racial immunity, 166

susceptibility, 100

Rashes in serum disease, 573-575

multiform, 574

scarlatiniform, 575

urticarial, 574

Rats, intravenous inoculation, 60

obtaining large amount of blood from, 46

small amount of blood from, 46

white, anaphylaxis in, 540

Reaction, Abderhalden's pregnancy, 252. See also Abderhalden's serodiagnosis of pregnancy.

agglutination, 278. See also Agglutination test.

allergic, 582. See Anaphylactic reaction.

anaphylactic, 582. See Anaphylactic reaction.

antilysin, for antistaphylococcus serum, 239

antitrypsin, 250

Bergmann and Meyer's, 250

Ascoli and Izar's miostagmin, 526. See also Miostagmin reaction.

bacteriolytic, 342 . See also Bacteriolytic test.

Bauer's modification of Wassermann, 456

Bergmann and Meyer's antitrypsin, 250

biologic blood, for detection of bloodstains, 303

technic, 308

of meat adulteration, 310 technic, 312

blood, biologic, for detection of blood.stains, 303

technic, 308

of meat adulteration, 310 technic, 312 
Reaction, blood, Teichmann's, 303

body, 553

Bordet-Gengou, 332, 391. See also Bordet-Gengou phenomenon of complement fixation.

Browning and Mackenzie's modification of Wassermann, 459

Calmette tuberculin, 587, 598 dangers, 592

in animals, 600

Castellani's saturation, 288

cobra venom, 383 . See also Venom hemolysis.

complement-fixation, 332, 391 . See also Bordet-Gengou phenomenon of complement fixation.

cutaneous, in typhoid fever, 608

cytotoxic, 509

in diagnosis, 509

of Freund and Kaminer, for cancer, 509

Detre and Brezovsky's modification of Wassermann, 459

epiphanin, 522. See also Epiphanin reaction.

for seminal stain, 304

Fornet's ring, for syphilis, 299

Freund and Kaminer's cytolytic cancer, 509

precipitin, for cancer, 314

gonococcus complement-fixation, 477.

See also Gonococcus complement-fixation test.

Gruber-Widal, in typhoid fever, 268, $275,279,282$

Hecht-Weinberg modification of Wassermann, 457

Herman-Perutz, for syphilis, 299

immunity, 569

Jarisch-Herxheimer, 804

Koch's tuberculin, 587, 592 in animals, 600

Kolle and Pfeiffer's macroscopic agglutination, 288

luetin, 601. See also Luetin reaction. mallein, 606. See also Mallein reaction.

Mantoux tuberculin, 587, 595

Mendel tuberculin, 587, 595 in animals, 601

miostagmin, 526. See also Miostagmin reaction.

Moro tuberculin, 587, 599

Much's psycho-, 388

Noguchi's butyric-acid, for protein, 300

luetin, 601. See also Luetin reaction.

modification of Wassermann, 449 . See also Noguchi's modification.

ophthalmic, in typhoid fever, 608

Pfeiffer's bacteriolytic, 342 experimental work, 862 in diagnosis of disease, 348 technic, 347
Reaction, Porges-Meier, for syphilis, 299 precipitin. See Precipitin test.

psycho-, of Much, 388

saturation, of Castellani, 288

Seifert's epiphanin, 526

Stern's modification of Wassermann, 458

Tchernogubou's modification of Wassermann, 458

Teichmann's blood, 303

toxin-antitoxin, nature of experimental reaction, 836

tuberculin, 582. See also Tuberculin reaction.

typhoidin, 608, 609

vaccinoid, 569

von Dungern's, in cancer, 500

modification of Wassermann, 459

von Pirquet's tuberculin, 587, 596 in animals, 601

Wassermann, in syphilis, 401. See also Wassermann reaction.

Weichardt's epiphanin, 522. See also Epiphanin reaction.

Widal, in typhoid fever, experimental work, 840

Wolff-Eisner tuberculin, 587, 598 dangers, 592 in animals, 600

Reagin, syphilis, 405

Receptoric atrophy, 80, 171

Receptors, 147

of first order, 149, 223

of third order, 317

three orders, 149

Rectal injections of serum as preventive of serum disease, 576

method of administering diphtheria antitoxin, 707

Relapsing fever, salvarsan in, 810

Wassermann reaction in, 465

Remittent infection, 136

Resistance, drug, 789

Resistant races, 790

Respiratory diseases, vaccine therapy, 659

organs as portal of entrance for bacteria, 88

Retention theory of immunity, 144

Revaccination, smallpox, 634

Rhinitis, vaccine therapy, 659

Richet's studies on anaphylaxis, 533

theory of anaphylaxis, 556

Ricin toxin, 118

Ring test, Fornet's, for syphilis, 299

Rubber teats for capillary pipets, 20

Ruck's tuberculin, preparation of, 666

SALT solution, 27

resistance of erythrocytes to, experimental work, 847

Salvarsan, 785

experimental work, 867

in acanthosis nigricans, 811 
Salvarsan in Aleppo boil, 811

in anemia, 811

in chancroid, 811

in chorea, 811

in dermatitis herpetiformis, 811

in Duhring's disease, 811

in filariasis, 810

in frambesia, 810

in Hodgkin's disease, 811

in internal anthrax, 768

in keratosis follicularis, 811

in leprosy, 811

in lichen planus, 811

in lupus vulgaris, 811

in malaria, 811

in mycosis fungoides, 811

in non-syphilitic diseases, 810

in pellagra, 811

in pityriasis rubra, 811

in psoriasis, 811

in relapsing fever, 810

in scarlet fever, 811

in scurvy, 811

in smallpox, 811

in syphilis, 792

acid solution, 796

administration, 797

alkaline solution of disodium salt, 796

contraindications, 808

effect of, on Wassermann reaction, 469

history, 792

intramuscular injection, 805

intravenous injection, 797. See also Intravenous injection of salvarsan.

methods of preparing, for administration, 795

mono-acid solution, 796

neutral suspension, 796

precautions, 803

value, 809

in trichinosis, 811

in tropical ulcer, 811

in trypanosomiasis, 811

in tuberculosis, 811

in verruca plana, 811

in Vincent's angina, 810

in yaws, 810

properties of, 794

Salvarsanized autoserum in syphilis of brain and spinal cord, 776

after-treatment, 780

repeating dose, 780

serobiologic findings in cerebrospinal fluid, 780 technic, 778

serum, intraspinous injection, 806

Saponin, 521 Fordyce's technic, 807

Sapremia, 93

Saprophytes, 83, 124

Sarcoma, epiphanin reaction in, 526
Saturation agglutination reaction, experimental work, 843

test of Castellani, 288

Scar in smallpox vaccination, 633

Scarlatiniform rashes in serum disease, 575

Scarlet fever, antistreptococcus serum in, 764

autoserum treatment, 775

salvarsan in, 811

vaccination, 652

Wassermann reaction in, 465

Sclavo's serum, 767

Scurvy, salvarsan in, 811

Seifert's epiphanin reaction, 526

Selenium-eosin compound in cancer, 812

Seminal stains, test for, 304

Sensibilisin, 553, 557

Sensibilisinogen, 557

Sensitization, 536

Sensitized vaccines, 620

bacterial, preparation of, 216

Sensitizers, 322

Sensitizing substance, 146

Sepsis, puerperal, antistreptococcus serum in, 764

vaccine therapy, 661

Septicemia, 94

Serobacterin, 620

Serodiagnosis of pregnancy, Abderhalden's, 252. See also Abderhalden's serodiagnosis of pregnancy.

Sero-enzymes in acute infections, 265

in cancer, 264

in disease, 264

in mental diseases, 265

in syphilis, 265

in tuberculosis, 265

Serous membranes, tuberculosis of, autoserum treatment, 781

tuberculin test in diagnosis of, 591

Serum, active, 328

agglutinating, 68

power, variation in, 274

amboceptor unit, 374

and corpuscles, obtaining of, 30

anti-anthrax, 767

administration of, 767

anticholera, 770

anticomplementary action, experimental work, 857

anticytotoxic, 506

antidiphtheric, production of, 227

antidysenteric, collecting and testing, 238

production of, 236

culture, 236

immunizing animals, 237

antigonococcus, 765

action of, 766

administration of, 766

preparation of, 766

anti-influenza, 750

administration of, 751 
Serum, antimeningococcus, 736. See also Antimeningococcus serum.

antiplague, 769

antipneumococcus, 757. See also Antipneumococcus serum.

antistaphylococcus, 238, 766

antilysin test for, 239

preparation of, 239

antistreptococcus, 760. See also Antistreptococcus serum.

antitetanic, production of, 234

antitryptic power, testing, experimental work, 838

antituberculosis, 771

antityphoid, 768

auto-, treatment with, 775 . See also Autoserum treatment.

bacteriolytic, in treatment of disease, 359

method of titrating, 344

production of, 69

cadaver, testing of, in Wassermann reaction, 411

calf cholera, 733

Chantemesse's, 768

cytotoxic, production of, 73

diagnosis of paroxysmal hemoglobinuria, 379

disease, 534, 537, 571

accelerated reaction in, 573

atropin sulphate as preventive, 576

from antimeningococcus serum, 746

from diphtheria antitoxin, 712

immediate reaction in, 573

multiform rashes in, 574

nature, 572

prevention, 576

rashes in, 573-575

rectal injections of serum as preventive, 576

scarlatiniform rashes in, 575

severe, 575

symptoms, 573

treatment, 577

urticarial rashes in, 574

doses of, in Wassermann reaction, 411

hemolysis, experimental work, 848

quantitative factors, experimental work, 849

hemolytic, production of, 71

hog cholera, production of, 732 standardization of, 732

immune, and normal serum, difference between, 324

methods for making, 66

preservation of, 76

in dried paper form, 79

in fluid form, by bacteria-free filtration, 76

by freezing, 78

with antiseptics, 76

in living animal, 80

in powder form, 79

standardization of, complement-
Serum, inactivated, 327, 328

intramuscular inoculation, technic, 690

intravenous inoculation, gravity method, 691

syringe method, 690

technic, 690

Kraus' anticholera, 770

Maragliano's tuberculosis, 771

Marmorek's tuberculosis, 771

methods of inoculation with, 687

nephrotoxic, production of, 73

normal, 772. See also Serum treatment, normal.

and immune serum, difference between, 324

preservation of, 75

obtaining of, 30

patient's own, 775. See also Autoserum treatment.

placental, method of obtaining, 773

precipitins, 70

experimental work, 844

production of, 70

preservation of, 75

reactivated, 328

rectal injections, as preventive of serum disease, 576

salvarsanized, intraspinous injection, 806

Fordyce's technic, 807

Sclavo's, 767

sickness, 534, 537, 571. See also $\mathrm{Se}$ rum disease.

subcutaneous inoculation, technic, 688

subdural inoculation, technic, 694 .

See also Subdural inoculation.

treatment, 614, 681

anaphylaxis in, 686

asthma in, 687

contraindications to, 686

indications, 684

intramuscular inoculation, technic, 690

intravenous inoculation, gravity method, 691

syringe method, 690

technic, 690

methods of inoculation, 687

normal, 772

in hemorrhage, 772

in nephritis, 774

in skin diseases, 774

in toxicoses of pregnancy, 773

in vomiting of pregnancy, 773

of anthrax, 767

of bubonic plague, 769

of cerebrospinal meningitis, 736

of cholera, 770

of diphtheria, 702. See also Diphtheria antitoxin.

of dysentery, 730

results, 731

of gonococcal infections, 765

of hay-fever, 734

of hog cholera, 732 
Serum treatment of influenzal meningitis, 749

of internal anthrax, 768

of meningococcus meningitis, 736

cases of posterior basal meningitis, 745

with dry canal, 745

with thick elastic exudate, 745

chronic cases, 746

results, 746

serum sickness in, 646

subacute cases, 746

of plague, 769

of pneumococcus infections, 754

meningitis, 751, 752

of pneumonia, 754

results, 759

of snake-bites, 733

of staphylococcus infections, 766

of streptococcus infections, 760

of tetanus, 719. See also Tetanus antitoxin.

of tuberculosis, 771

of typhoid fever, 768

purposes, 682

status lymphaticus in, 687

subcutaneous inoculation, technic, 688

subdural inoculation, technic, 694. See also Subdural inoculation.

Sheep, anaphylaxis in, 541

intravenous inoculation, 62

obtaining large amount of blood from, 48

small amount of blood from, 42,49

Side-chain theory of immunity, 146

and phagocytic theory, compatibility, 155

Simon and Lamar's modification of Wright's method for opsonic index, 200

Skin as portal of entrance for bacteria, 87

bacteria on, 85,87

diseases, autoserum treatment, 775

normal serum treatment, $\mathbf{7 7 4}$

salvarsan in, 811

vaccine therapy, 656

tuberculosis of, tuberculin test in diagnosis of, 590

treatment, 679 Smallpox, antistreptococcus serum in,

autoserum treatment, 776

revaccination, 634

salvarsan in, 811

vaccination, 623 . See also Vaccination, smallpox.

vaccinia and, relationship, 625

Smith's phenomenon of anaphylaxis, 535

Snake venoms, 119, 241 nature of, 120 properties of, 119

Snake-bites, serum treatment, 733

Sodium citrate, 27

Solutions, 27

colloidal, 512
Southard and Gay's theory of anaphylaxis, 557

Species immunity, 166

susceptibility, 100

Spermatotoxin, 506

Spinal cord, syphilis of, salvarsanized autoserum in, 776

after-treatment, 780

repeating dose, 780

serobiologic findings in cerebrospinal fluid, 780 technic, 778

puncture, 37. See also Lumbar puncture.

Splitting of complement, 331

Spontaneous phagocytosis, 187

Sporadic infection, 82

Sporotrichosis, allergic reactions in, 610

Stain, blood, biologic test for, 303 technic, 308

experimental work, 845

identification of, complement-fixation test for, 494

test for, experimental work, 861

seminal, test for, 304

Staining acid-fast bacilli, 197

bacteria, 197

Stalagmometer, Traube's, 527

Standardization of antimeningococcus serum, 740

of antipneumococcus serum, 758

of antistreptococcus serum, 763

of bacterial antigens in complement fixation, 475

vaccines, 210

of diphtheria antitoxin, 231 experimental work, 835

toxin, 114

of hog cholera serum, 732

of immune serums, complement-fixation test in, 491

of tetanus antitoxin, 234, 720 experimental work, 836

Staphylococcus, 117

infections, serum treatment, 766

yaccine, preparation, experimental work, 835

Staphylolysin, 117

method of titrating, 240

preparation of, 239

Staphylotoxin, experimental work, 821

Status lymphaticus in serum treatment, 687

Sterilization of bacterial vaccines, 213 testing, 213

of pipets, 22

of syringes, 27

of test-tubes, 25

Stern and Körte's method of measuring bactericidal power of blood, 349

experimental work, 863

Stern's modification of Wassermann reaction, 458

Stichreaktion, 586 
Stimulation, provocatory, of Wassermann reaction, 469

Stimulins, 145, 187

Stock bacterial vaccines, 620

Strawberries, idiosyncrasy, 578

Streptococcus, 117

infections, serum treatment, 760

Streptolysin, 117

Streptotoxin, experimental work, 822

Strong and Kolle's plague vaccine, 648

Strong's cholera vaccine, preparation, 651

Subcutaneous injection of animals, 54 with fluid inoculum, 54 with solid inoculum, 55

of diphtheria antitoxin, 707

of serum, technic, 688

of tetanus antitoxin, 722

of tuberculin, 671,675

mallein reaction, 607

tuberculin reaction, 587,592 in animals, 600 .

Subdural injection of antimeningococcus serum, 742

of neosalvarsan in syphilis, 805

Wile's technic, 806

of salvarsanized serum, 806

of serum, anesthesia for, 697

blood-pressure as guide, 695

collapse in, 694, 696

symptoms, 696

treatment, 696

gravity method, 697

syringe method, 700

technic, 694

of tetanus antitoxin, 723

Subinfection, 81, 89

Substance sensibilisatrice, 185, 319, 322, 337,362

Suction pump, 29

Suctorial insects, transmission of bacteria by, 86

Susceptibility, acquired, 100

familial, 100

general, in relation to infection, 99

individual, 100

inherited, 100

racial, 100

species, 100

Suspensions, 511

colloidal, 512

Sycosis, vaccine therapy, 657

Synocytotoxin, 507

Syphilis after smallpox vaccination, 635

Bauer's modification of Wassermann reaction, 456

Browning and Mackenzie's modification of Wassermann reaction, 459

complement fixation in, 401. See also Wassermann reaction.

Detre and Brezovsky's modification of Wassermann reaction, 459

epiphanin reaction in, 526

Fornet's ring test for, 299

Hecht-Weinberg modification of Wassermann reaction, 457
Syphilis, Herman-Perutz test for, 299

horse, complement-fixation test in, 487

luetin reaction for, 601. See also Luetin reaction.

mercury treatment, effect of, on Wassermann reaction, 466

miostagmin reaction in, 530

neosalvarsan in, 792. See also Neosalvarsan in syphilis.

Noguchi's modification of Wassermann reaction, 449. See also $\mathrm{No}$ guchi's modification.

of brain, salvarsanized autoserum in, 776

after-treatment, 780

repeating dose, 780

serobiologic findings in cere-

brospinal fluid, 780

technic, 778

of spinal cord, salvarsanized autoserum, 776

after-treatment, 780

repeating dose, 780

serobiologic findings in cerebrospinal fluid, 780

technic, 778

Porges-Meier test for, 299

reagin, 405

salvarsan in, 792. See also Salvarsan in syphilis.

sero-enzymes in, 265

Stern's modification of Wassermann reaction, 458

Tchernogubou's modification of Wassermann reaction, 458

venom hemolysis in, 385

experimental work, 861

practical value, 388

technic, 385

von Dungern's modification of Wassermann reaction, 459

Wassermann reaction in, 401. See also Wassermann reaction.

Syphilitic livers, alcoholic extracts, for Wassermann reaction, 420

aqueous extracts, for Wassermann reaction, 419

Syringe, 26

for administration of bacterial vaccines, 217

Hitchens', 233

method of intravenous inoculation of serum, 690

of subdural inoculation of serum, 700

sterilization of, 27

Systemic reaction to infection, 137

TABES dorsalis, Wassermann reaction in, 462

T. B. tuberculin, 666

Tchernogubou's modification of Wassermann reaction, 458

Teats, rubber, for capillary pipets, 20 
Technic, general, 17

Teichmann's blood test, 303

Temperature, influence of, on natural immunity, experimental work, 827

Terni-Bandi's method of preparing plague serum, 769 plague vaccine, 648

Tertiary syphilis, Wassermann reaction in, 641

Test. See Reaction.

Test-tubes, conversion of, into ampules for holding vaccines, etc., 24

for immunologic work, 25

for Wassermann reaction, 407 sterilization of, 25

Tetanolysin, 111, 115, 819

Tetanospasmin, 111, 115, 819

Tetanus after smallpox vaccination, 634 antitoxin, 719,726

action, 721

intramuscular injection, 722

intraneural injection, 723

intravenous injection, 722

method of titrating, 235

methods of administering, 722

preparation, 720

production of, 234

collecting serum, 234

immunizing animals, 234

prophylactic administration, 723, 725

results of treatment, 728

standardization, 234, 720 experimental work, 836

subcutaneous injection, 722

subdural injection, 723

unit of, 242

bacillus, 115

prophylaxis, 723

serum treatment, 719. See also

Tetanus antitoxin.

surgical treatment, 725, 726

toxin, 115

action, 720

experimental work, 819

production of, 234

treatment of, 719,726 . See also

Tetanus antitoxin.

Therapeutic immunization, 580

Therapia magna sterilisans, 791

Thyrotoxins, 508

Tissue susceptibility, avenue of infection and, 97

T. O. tuberculin, 665

Tonsillar diphtheria, dosage of antitoxin in, 710

Töpfer and Jaffe's method of measuring bactericidal power of blood, 352

Toxemia, 94

Toxicity of bacteria, 94, 95

Toxicoses of pregnancy, normal serum treatment, 773

Toxin, 73,108

abrin, 118

and ferment, similarity between, 244
Toxin, botulism, 116

experimental work, 820

crotin, 118

diphtheria, 112

experimental work, 817

limes death dose, 232

zero dose, 232

production of, 227

standardizing, 114

testing, 228

virulence, 113

dysentery, 116

experimental work, 820

exogenous, 108

extracellular, 108, 109

chemical properties, 110

general properties, 109

haptophore group, 110

nature of, 110

nomenclature, 108

of hay-fever, 119

of higher plants and animals, 118

pollen, 119

principal, special properties, 112

ricin, 118

selective action, 111

soluble, 108

chemical properties, 110

general properties, 109

structure of, 110

tetanus, 115

action of, 720

experimental work, 819

production of, 234

toxophore group, 110

Toxin-antitoxin reaction, nature of, 224 experimental work, 836

Toxogen, 553

Toxogenin, 556

Toxoid, 110

diphtheria, 115

Toxon, 111

Toxophore group of toxin, 110

T. R. tuberculin, dose of, 674

preparation of, 665

Transfusion, blood, 273

experimental work, 844

tests before, for isohemagglutinins and isohemolysins, 290

Traube's stalagmometer, 527

Trauma as predisposing to infection, 102

Treatment, cytotoxins in, 508

effect of, on Wassermann reaction, 466

Treponema pallidum, aqueous extract of culture, for Wassermann reaction, 424

Trichina spiralis, 167

Trichinosis, salvarsan in, 811

Tritotoxin, 115

Tropical ulcer, salvarsan in, 811

Trypanosomiasis, salvarsan in, 811

Tubercle bacilli, living, in treatment of tuberculosis, 669

Tuberculin, action of, 668 administration of, methods, 671,676 
Tuberculin, bacillen emulsion, preparation of, 666

B. E., preparation of, 666

Beraneck's, dose of, 675 preparation of, 666

B. F., preparation of, 666

bouillon filtrate, preparation of, 666

delirium, 672

Dixon's, dose of, 675

preparation of, 667

new, dose of, 674

preparation of, 665

old, dose of, 674 preparation of, 664

O. T., dose of, 674 preparation of, 664

preparation of, 664

reaction, 582

Calmette's, 587, 598

dangers, 592

in animals, 600

comparative delicacy and relation, 587

conjunctival, of Calmette, 587, 598 dangers, 592 in animals, 600

of Wolff-Eisner, 587, 598

dangers, 592

in animals, 600

cutaneous, of von Pirquet, 587, 596 in animals, 601

dangers, 591

delicacy, 587

effect of measles on, 586

error in, sources, 585

false negative, 585

positive, 585

in animals, 600

in diagnosis of pelvic tuberculosis, 590

of pulmonary tuberculosis, 589

of tuberculosis of bones, joints, and glands, 590

of eye, ear, and larynx, 590

of genito-urinary system, 590

of serum membrane, 591

of skin, 590

of tuberculous meningitis, 591

peritonitis, 591

pleurisy, 591

value, 589

in prognosis, value, 591

intracutaneous, of Mantoux, 587, 595

of Mendel, 587, 595

in animals, 601

Mantoux's, 587, 595

Mendel's, 587, 595

in animals, 601

methods of conducting, 587

Moro's, 587, 599

nature, 583

percutaneous, of Moro, 587, 599

relation, 587

specificity, 583
Tuberculin reaction, subcutaneous, 587, 592

in animals, 600

von Pirquet's, 587, 596

in animals, 601

Wolff-Eisner's, 587, 598

dangers, 592

in animals, 600

residue, preparation, 665

subcutaneous injection, 671

T. O., 665

T. R., dose of, 674

preparation of, 665

treatment, 661

action of tuberculin, 668

contraindications, 671

dosage, 674

history, 661

in tuberculosis of bones and joints, 679

of ear, 679

of eye, 679

of genito-urinary organs, 680

of intestine, 679

of mesenteric glands, 679

of skin, 679

in tuberculous adenitis, 679

meningitis, 679

intrabronchially, 677

intrafocal route, 677

intravenous, 677

living tubercle bacilli, 669

methods of administration, 671,676

oral route, 676

patients suitable, 670

preparation of tuberculin, 664

reaction in, 672

constitutional symptoms, 672

focal signs, 673

local signs, 673

results, 678

subcutaneous injection, 671,675

von Ruck's, preparation of, 666

Tuberculonastin, 160

Tuberculosis, agglutination test in, 277

antistreptococcus serum in, 764

complement-fixation test in, 490

contraindications to vaccines in, 623

Friedmann's treatment, 670

genito-urinary, tuberculin test in diagnosis of, 590

living tubercle bacilli in treatment of, 669

miostagmin reaction in, 530

of bones and joints, tuberculin treatment, 679

tuberculin test in diagnosis of, 590

of ear, tuberculin test in diagnosis of, 590

treatment, 679

of eye, tuberculin test in diagnosis of, 590

treatment, 679

of genito-urinary organs, tuberculin treatment, 680 
Tuberculosis of glands, tuberculin test in diagnosis of, 590

of intestine, tuberculin treatment, 679 of joints, tuberculin test in diagnosis of, 590

of larynx, tuberculin test in diagnosis of, 590

of mesenteric glands, tuberculin treatment, 679

of serous membranes, autoserum treatment, 781

tuberculin test in diagnosis of, 591

of skin, tuberculin test in diagnosis of, 590

treatment, 679

pelvic, tuberculin test in diagnosis of, 590

pulmonary, tuberculin reaction in diagnosis of, 589

salvarsan in, 811

sero-enzymes in, 265

serum treatment, 771

tuberculin treatment, 661. See also Tuberculin treatment.

venom hemolysis in, 390

Tuberculous adenitis, tuberculin treatment, 679

meningitis, autoserum treatment, 782 tuberculin test in diagnosis of, 591 treatment, 679

peritonitis, tuberculin test in diagnosis of, 591

pleurisy, autoserum treatment, 781 tuberculin test in diagnosis of, 591

Typhoid fever, agglutination test in, 275, 279,282

allergic reactions in, 607 autoserum treatment, 776 complement-fixation test in, 489 cutaneous reaction in, 608

Gruber-Widal reaction in, 268, 275, 279,282

miostagmin reaction in, 530

ophthalmic reaction in, 608

serum treatment, 768

vaccination, 643

dosage of vaccine, 645

duration and degree, 646

method of inoculation, 644

preparation of vaccine, 643

reactions, 645

recommendations, 647

results, 646

vaccine therapy, 660

Widal reaction in, experimental work, 840

vaccine, preparation, experimental work, 834

Typhoidin reaction, 608, 609

Typho-protein, 608

UFFENHEIMER's method of determining presence of diphtheria toxin, 114

Uhlenhuth filter, 305
Ulcer, tropical, salvarsan in, 811

Ulcerative endocarditis, vaccine therapy, 661

Umstimmung, 601

Unit, amboceptor, of serum, 374 antitoxin, 242

of diphtheria antitoxin, 242

of tetanus antitoxin, 242

Urethritis, vaccine therapy, 658

Urticarial rashes in serum disease, 574

Vaccination, 65, 611, 613, 614, 623, 682

active acquired immunity by, 171

anthrax, 653

blackleg, 655

bubonic plague, 647

cerebrospinal meningitis, 652

cholera, 650

dosage of vaccine, 651

Haffkine's vaccine, 650

Kolle's vaccine, 650

preparation of vaccine, 650

results of, 651

Strong's vaccine, 651

diphtheria, 715

dysentery, 652

history of, 611

Jennerian, 171

meningococcus meningitis, 748, 749

of animals, 627

preparation for, 627

Pasteurian, 171

plague, 647

dosage of vaccine, 649

duration of, 649

effects of, 649

Haffkine's vaccine, 648

dosage, 649

effects, 649

results, 649

Kolle and Strong's vaccine, 648

Kolle's vaccine, 648

Lustig and Galeotti's vaccine, 648

preparation of vaccine, 648

results of, 649

Terni and Bandi's vaccine, 648

preparation of animals for, 627

rabies, 636. See also Rabies.

scarlet fever, 652

smallpox, 623

history of, 623

phenomena of, 632

preparation of vaccine, 626

protective value, 635

revaccination, 634

risks of, 634

scar in, 633

subsequent care of wound, 632

syphilis after, 635

technic of, 630

tetanus after, 634

typhoid fever, 643 . See also Typhoid fever vaccination. 
Vaccine, 613

ampules, conversion of test-tubes into, 24

making of, 24

anthrax, 653

autogenous, 620

bacterial, 206, 613

administration of, 217

frequency, 218

syringe for, 217

autogenous, 620

counting of, 210

Hopkins' method, 212

Kolle's method, 212

with hemocytometer chamber, 211

Wright's method, 210

definition of, 206

dosage of, 218

opsonic index as guide to, 206

experimental work, 834

in acne, 657

in acute infections, 660

in bronchitis, 659

in carbuncles, 656

in cystitis, 657

in erysipelas, 657

in furunculosis, 656

in genito-urinary diseases, 657

in gonorrheal arthritis, 658

in otitis media, 660

in pertussis, 659

in pneumonia, 660,661

in puerperal sepsis, 661

in respiratory diseases, 659

in rhinitis, 659

in skin diseases, 656

in sycosis, 657

in typhoid fever, 660

in ulcerative endocarditis, 661

in urethritis, 658

in vulvovaginitis of children, 659

in whooping-cough, 659

inoculation with, dosage, 218

effects of, 218

method of making, 217

sensitized, preparation of, 216

standardization of, 210

stock, 620

technic for preparing, 206

diluting and adding preservative, 214

emulsion, 208

procuring infected material, 206

pure cultures, 207

sterilization, 213

testing, 213

treatment by, 655

blackleg, 655

cerebrospinal, 652

cholera, preparation of, 650

contraindications to, 622

in cancer, 623

in diabetes, 625

in nephritis, 623
Vaccine, contraindications to, in tuberculosis, 623

cowpox, preparation of, 626 animals, 627 collection of virus, 628 seed virus, 626

testing virus, 629

dead, 620

dysentery, 652

Haffkine's cholera, preparation, 650

plague, 648

dosage, 649

effects, 649

results, 649

in prophylaxis of disease, 611

Kolle and Strong's plague, 648

Kolle's cholera, preparation, 650 plague, 648

living, 620

Lustig and Galeotti's plague, 648

nomenclature, 613

plague, preparation of, 648

preparation of, method, 614

rabies, administration of, 641

preparation of, 640

scarlet fever, 652

sensitized, 620

staphylococcus, experimental work, 835

stock, 620

Strong's cholera, preparation, 651

Terni and Bandi's plague, 648

treatment, $611,614,617$

history, 611

typhoid, experimental work, 834

preparation of, 643

Vaccinia, 612, 613, 632

smallpox and, relationship, 625

Vaccinoid, 633

reaction, 569

Variola. See Smallpox.

Varioloid, 634

Vaughan and Wheeler on anaphylatoxin, 548,549

Vaughan and Wheeler's theory of anaphylaxis, 557

Vaughan's theory of bacterial proteins, 128

of immunity, 173

Vegetables, idiosyncrasy, 578

Venesection, 33

in children, 33

Venom, cobra, experimental work, 823 preparation of, 385

hemolysis, 383,521

in cancer, 390

in syphilis, 385

experimental work, 861

practical value, 388

technic, 385

in tuberculosis, 390

nature, 383

snake, 119,241

properties of, 119

nature of, 120 
Verruca plana, salvarsan in, 811

Vincent's angina, salvarsan in, 810

Virulence of bacteria, 94

decrease, 95

increase, 96

by addition of animal fluids to culture-medium, 97

by passage through animals, 96

by use of collodion sacs, 97

Virus fixé of rabies, 143, 640

Vomiting of pregnancy, normal serum treatment, 773

von Dungern's complement-fixation test in cancer, 500

modification of Wasserman reaction, 459

von Pirquet's cutaneous tuberculin test, 587,596

in animals, 601

studies on anaphylaxis, 533

von Ruck's tuberculin, preparation, 666

Vulva, diphtheria of, dosage of antitoxin in, 711

Vulvovaginitis of children, vaccine

therapy, 659

Washed leukocytes in opsonic index, 195 Washing erythrocytes, 28

Wassermann reaction after anesthesia, 466

Colles' law and, 464

in cerebral syphilis, 462

in congenital mental deficiency, 465 syphilis, 463,464

in frambesia, 465,466

in general paralysis, 462

in latent syphilis, 462

in leprosy, 465,466

in malaria, 465

in paralytic dementia, 462

in parasyphilitic diseases, 462

in pellagra, 466

in primary syphilis, 459

in relapsing fever, 465

in scarlet fever, 465

in secondary syphilis, 460

in syphilis, 401

acetone-insoluble lipoids for, 422

alcoholic extracts of normal organs for, 420

reënforced with cholesterin for, 421

of syphilitic livers for, 420

antigen for, 417

anticomplementary titration, 428

antigenic titration, 431

experimental work, 856

hemolytic titration, 430

method of diluting, 427

titrating, 428

preparation, 419

antigenic dose of serum, 418

antisheep amboceptor for, 415
Wassermann reaction in syphilis, aqueous extract of pallidum culture for, 424

of syphilitic livers for, 419

as colloidal reaction, 520

Bauer's modification, 456

Browning and Mackenzie's modification, 459

cerebrospinal fluid for, 411

collecting blood for, 408

New York Board of Health outfit, 410

Colles' law and, 464

comparative antigenic values of

various extracts, 424

complement for, 411

titration, 413

congenital, 463, 464

Detre and Brezovsky's modification, 459

doses of serum in, 411

effect of mercury treatment on, 466

of neosalvarsan on, 469

of salvarsan on, 469

of treatment on, 466

erythrocytes for, 416

experimental work, 857,858

first method, 432 technic, 435

fluid to be tested, 408

fourth method, 434 reading results, 449 technic, 446

glassware for, 407

guinea-pig complement serum for, 412

Hecht-Weinberg modification, 457 hemolytic amboceptor for, 415

history, 401

latent, 462

lecithin and cholesterin for, 423

methods for conducting, 432

modifications, 449

Noguchi's modification, 449. See also Noguchi's modification.

original, 432,435

technic of, 435

pipets for, 407

practical value, 469

primary, 459

principles and theories, 404

Profeta's law and, 464

provocatory stimulation, 469

reading and recording, 439

red blood-corpuscles for, 416

relation of lipoids to, 522

second method, 433

reading results, 442

technic, 441

secondary, 460

serum for, 408

specificity, 465

Stern's modification, 458

Tchernogubou's modification, 458 
Wassermann reaction in syphilis, technic, 407

tertiary, 461

testing cadaver serums, 411

test-tubes for, 407

third method, 434

reading results, 444

technic, 443

various stages, 459

von Dungern's modification, 459

in tabes dorsalis, 462

in tertiary syphilis, 461

in various stages of syphilis, 459

in yaws, 465

Profeta's law and, 464

Water, bacteria in, 85

Water-supplies, bacteria recovered from, bacteriolytic test for identification of, 343

Wechsberg and Neisser's method of measuring bactericidal power of blood, 349

Wechselmann's method of converting negative syphilitic serums to positive syphilitic serums, 410

Weichardt's epiphanin reaction, 522 . See also Epiphanin reaction.

Weigert's overproduction theory, 148

Welch, hypothesis of, 103 .

Wet cupping, 36

Wheeler and Vaughan's theory of anaphylaxis, 557

White mice, anaphylaxis in, 540 rats, anaphylaxis in, 540

Whooping-cough, vaccine therapy, 659

Widal reaction in typhoid fever, 268, 275, 279,282
Widal reaction in typhoid fever, experimental work, 840

Wile's technic of intraspinous injection of neosalvarsan, 806

Wolff-Eisner conjunctival tuberculin test, 587,598

dangers, 592

in animals, 600

Wolman and Hamman's method of preparing tuberculin for subcutaneous tuberculin test, 592

plan of dosage for tuberculin in subcutaneous test, 594

Wounds, diphtheria of, dosage of antitoxin in, 711

infection, antistreptococcus serum in, 764

Wright's blood capsules, making of, 23 method of sealing, 34 removing serum from, 34

capillary pipet method of measuring bactericidal power of blood, 355

method of counting bacterial vaccines, 210

YAws, luetin reaction in, 605

salvarsan in, 810

Wassermann reaction in, 465

Yersin's plague serum, 769

ZoÖPRECIPITIN, 292

Zoötoxins, 109, 119

experimental work, 823 

9 


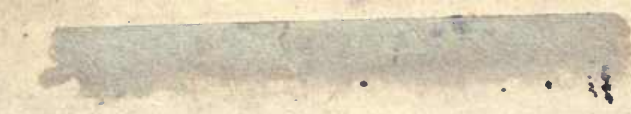

I!

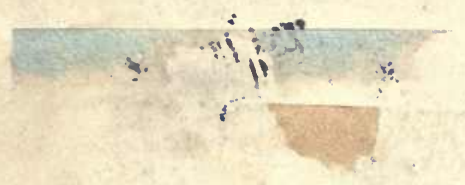

$i$

$$
\vdots+j
$$
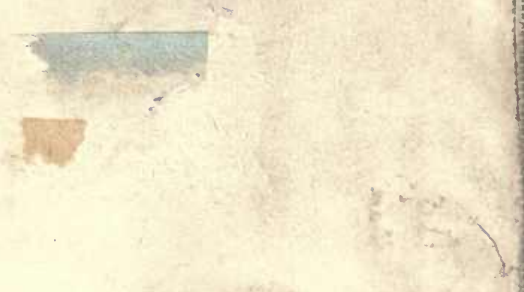
$n+\quad \because$.
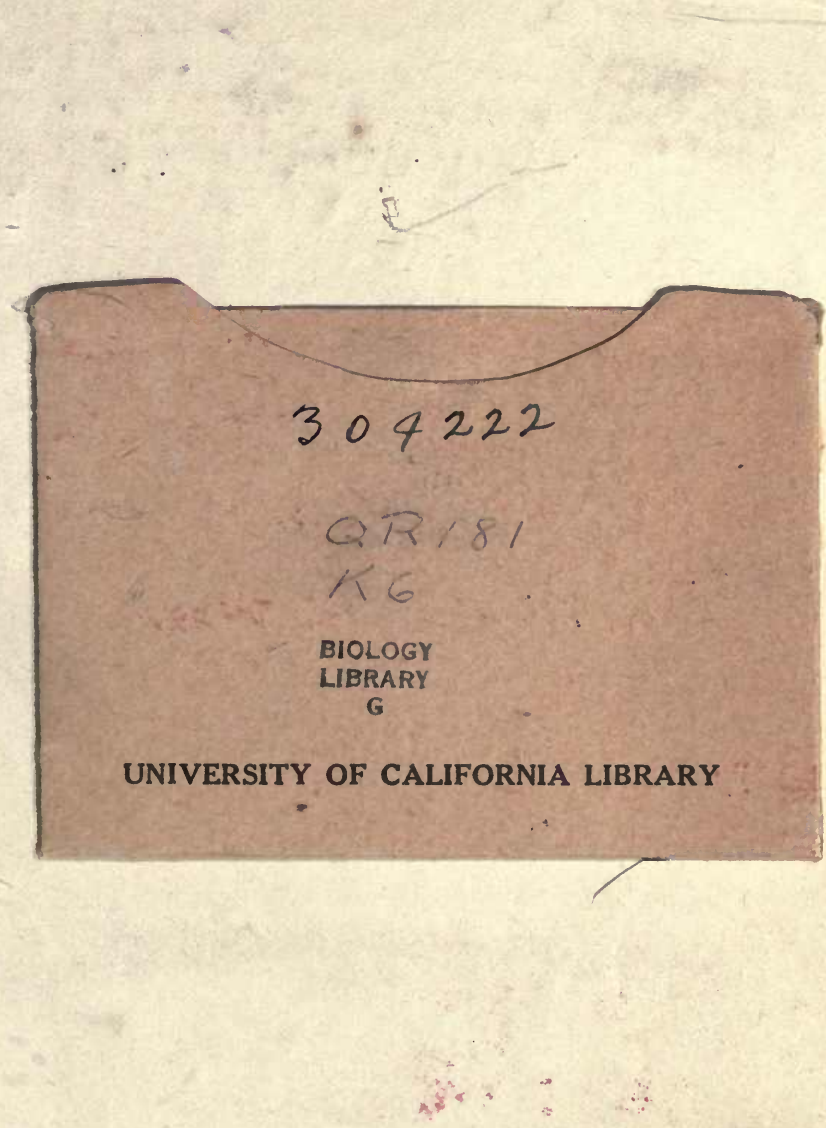


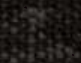

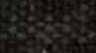

6 